\title{
Standards Referenced in Model, State, and City Building Codes
}

John N. Brewer, Jr.

Building and Fire Research Laboratory

Gaithersburg, Maryland 20899

QC
100
U56
4952
1992

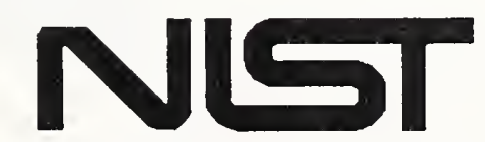

United States Department of Commerce Technology Administration

National Institute of Standards and Technology 


\section{Standards Referenced in Model, State, and City Building Codes}

John N. Brewer, Jr.

September 1992

Building and Fire Research Laboratory

National Institute of Standards and Technology

Gaithersburg, MD 20899

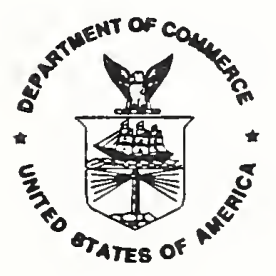

\section{U.S. Department of Commerce}

Barbara Hackman Franklin, Secretary

Technology Administration

Robert M. White, Under Secretary for Technology

National Institute of Standards and Technology

John W. Lyons, Director 



\begin{abstract}
This publication provides a listing of the standards that are referenced in the building codes promulgated by: (1) the three model building code organizations; i.e., Building Officials and Code Administrators International, Inc. (BOCA), International Conference of Building Officials (ICBO), and the Southern Building Code Congress International, Inc. (SBCCI); (2) the 29 states that have mandatory state building codes; and ( 3 ) 35 selected U.S. cities. In addition to identifying each standard referenced in the above-named codes, this publication lists the current date of the standard, its title, the codes referencing it, the date of the code, the locations within the code where the standard is referenced, and the date of the standard referenced in the code. This publication is intended to provide assistance to the building community in updating, using and maintaining the standards referenced in building codes.
\end{abstract}

Key Words: Building codes; building regulations; building regulatory systems; building technology; standards; standards organizations 

The author is indebted to Emil Braun, Physicist, and to Thomas A. Somers, Computer Specialist, Fire Measurement and Research Division, Building and Fire Research Laboratory (BFRL), for the computer programming and formatting of the list of standards referenced in this report, and to Carolyn L. Flood, Secretary, BFRL, for data entry and processing the report for publication. James G. Gross, Assistant Director, BFRL, is gratefully acknowledged for his constructive suggestions and overall direction. 
Page

Abstract . . . . . . . . . . . . . . . . . . . . $i i$

Acknowledgments . . . . . . . . . . . . . . . . . . . . iii

I. Background . . . . . . . . . . . . . . . . . . . . 1

II. Adoption of Building Codes . . . . . . . . . . . . . . . . 2

III. Standards Referenced . . . . . . . . . . . . . . . 2

IV. Content and Use of Tables . . . . . . . . . . . . . . . 4

V. Building Codes Included in Study . . . . . . . . . . . . . 6

VI. Format of Tabular Presentation . . . . . . . . . . . . 7

Figure 1 - Explanation of Tables . . . . . . . . . . . . . 9

Appendix A - Standards Generating Organizations Included in Study . . 10

Index of Standards Generating Organizations . . . . . . . . . . . . 16

List of Standards Referenced in Model, State and Major City

Building Codes . . . . . . . . . . . . . . . . . . . . . Al-A467 


\section{BACKGROUND}

Building codes are comprehensive legal documents, or ordinances, establishing minimum requirements for the design, construction, alteration or repair of buildings to assure a high level of safety, health and well-being for the occupants. They are established through legislative action of the state, city, or other jurisdictional entity to which they apply.

Three model building codes are developed, published and available for states and cities to adopt and may be adopted as written or with amendments and modifications to suit local conditions. The National Building Code (NBC) is published by the Building Officials and Code Administrators International, Inc. (BOCA), which was founded in 1915 and is dedicated to professional code administration and enforcement for the protection of public health, safety and welfare. ${ }^{1}$ The Uniform Building Code (UBC) was first enacted by the International Conference of Building Officials (ICBO) in 1927. It has as its purpose the development of better building construction and greater safety of the public by uniformity in building laws. ${ }^{2}$ The Southern Building Code Congress International, Inc. (SBCCI), founded in 1940, has been publishing the Standard Building Code (SBC) since 1945, with its purpose to protect the public's life, health and welfare in the built environment. ${ }^{3}$ All three model codes are updated annually and published on a three-year cycle. Although these three model codes are available for adoption by states and cities, some states and cities prefer to develop and use their own, individual building codes.

Building codes reflect the results of research in building and construction and include the opinions of the Nation's experts in all aspects of building technology related to safety, health, comfort and performance. These findings are developed into standards and made available by such nationally recognized organizations as the American National Standards Institute (ANSI), the American Society for Testing and Materials (ASTM), the National Fire Protection Association (NFPA), and many others. Building codes refer to or include portions of those nationally recognized standards.

It is the aim of national standards generating organizations to provide up-todate information in the standards they produce and offer to the building community. Consequently, standards referenced in building codes are frequently updated, revised, replaced or withdrawn. Promulgators of building codes, then, are faced with a continuing problem of updating their codes to incorporate current, valid, and applicable standards. Even as a building code, or publication such as this one, is being printed and distributed, some of the standards referenced will be replaced, withdrawn, or otherwise changed. It is important that the year of issue of the standard be given in the building code.

Along with the importance for building codes to reference current, up-do-date standards, there are a number of technical judgments, assessments of variations

1 The BOCA National Building Code/1990.

2 The Uniform Building Code/1991.

3 The Standard Building Code/1991. 
due to geographical or urban differences, and other factors, that present major obstacles to the individuals responsible for upgrading building codes. While this publication does not address those complexities, it is intended that the compilation and comparative listing of all referenced standards will be of assistance to those working toward greater building code uniformity, correctness and effectiveness.

In making the decision to develop this publication, it was determined that a service could be provided by offering pertinent current information regarding standards referenced in various building codes as follows: (1) the three model building codes (i.e., the National Building Code, the Standard Building Code, and the Uniform Building Code), the building codes in those 29 states which have mandatory statewide building codes, and the building codes of 35 cities selected by population and geographic distribution (identification of each building code is identified in Part V); (2) identification of the current date and status of the standards referenced; (3) identification of the appropriate section, or sections, of each code in which a standard is referenced; and (4) the date of the standard referenced in each code.

\section{ADOPTION OF BUILDING CODES}

In 1976, 18 states had mandatory statewide building codes. By 1992, the number had increased 61 percent, to 29 . This increase is positive recognition by the officials and legislatures of the additional states of the importance of using consistent statewide building codes to regulate building construction for the public's safety and welfare.

Of these 29 states, 27 have adopted one of the three model codes, either entirely or as a basis for their own codes. Most of these state codes are required as a minimum throughout the state, with local governments allowed to amend if more stringent. Some cities, New York and Milwaukee, for example, are exempt from their state code, using their own locally-developed code instead. Milwaukee's code, however, is based on the Wisconsin state code. In those states where building codes are not mandatory statewide, or where no codes exist at all, all major cities have their own codes for building design and construction. of the 35 cities selected by population and geographic distribution to be included in this report, 15 are located in the remaining 21 states with no mandatory statewide building code. Of these 15 cities, 14 have chosen to use one of the three model codes, either entirely or with amendments, rather than develop their own codes.

The 18 states with building codes in 1976 were using codes which were 2.2 years old, on average. In 1992, the average age of the building codes of the 29 states with codes was 1.9 years, reflecting an increased interest by the states to maintain up-to-date codes.

\section{STANDARDS REFERENCED}

A typical building code may reference on the order of 350 standards developed and offered by as many as 50 standards generating organizations. These referenced standards, along with other provisions contained in a building code, become the 
legal minimum criteria which building designers and constructors must meet for design, construction, modification, and repair of buildings.

The application of some standards is so widespread that they may be referenced in several chapters, and sections of chapters, covering different but related components of a building. For instance, the American Concrete Institute standard, "Building Code Requirements for Reinforced Concrete," is referenced 844 times in the 67 building codes included in this report. The second most referenced standard is the "Test Method for Surface Burning Characteristics of Building Materials," offered jointly by the American Society for Testing and Materials, the National Fire Protection Association and the Underwriters Laboratories. Other more frequently referenced standards are the National Fire Protection Association's "National Electrical Code," the American National Standards Institute/American Society of Mechanical Engineers" standard, "Safety Code for Elevators and Escalators," and the National Fire Prevention Association's "Installation of Sprinkler Systems." It is noted that these standards with the highest frequencies of reference are all related to the structural integrity, fire, electrical and mechanical safety aspects of the building codes. A similar pattern exists for other standards included in this report. This may be as expected and is consistent with the purpose of building codes to assure a high level of safety, health and well-being for the occupants.

At the same time, it must be recognized that the frequency of use of a standard may depend on other factors. For instance, the way a particular building code is organized, developed and written also has some bearing on the number of times a standard is referenced. Some codes may reference a standard only once or twice, specifically, in a given chapter or section, relying upon detailed information from that standard to describe the code's requirements for design and construction. Another code may choose to refer to the standard repeatedly instead of describing the standard's contents in the code itself, all as a matter of choice and style.

As stated earlier, the three model building codes are revised every year and reprinted every three years to address new and changing conditions and to update the references to standards. Many state and city codes are likewise revised every few years. However, it is evident from the information reported in this publication that, in spite of all the efforts to include the latest in safety, health, and comfort in the design and construction of buildings, many building codes continue to reference outdated, obsolete standards.

For instance, in the 3 model codes, 29 state and 35 city codes included in this study, a combined total of 1723 standards are referenced. Of these, 235, or 13 percent, have been withdrawn, canceled, revised, renumbered, or, for some other reason, are not current standards. As such, those standards are no longer generally available to the designers and constructors charged with the responsibility of complying with the particular building code in question; nor are they available to the local officials responsible for enforcing the building code.

The referencing of outdated standards is found in the three model codes as well as in those building codes that are individually developed and administered by states and cities. In the 1991 edition of the Standard Building Code, for instance, 332 standards are referenced. Of these, 38, or 11 percent, were 
revised before that edition was published. The Uniform Building Code uses its own standards which are revised or updated with each new edition. As such, the current date of these standards is the date of the building code itself. However, in preparing its standards, the Uniform Building Code makes use of, to some extent, standards developed by such national standards generating organizations as ANSI, ASTM, NFPA, etc., and includes references to any such standards in its building code. In its 1991 edition, reference is made to 154 of these national standards. Thirty-one of these, or 20 percent, were outdated when that edition was printed.

In this same connection, the National Building Code references 358 standards in the 1991 supplement to its 1990 edition. Thirteen of these, or less than 4 percent, had been withdrawn or replaced before that edition was prepared for printing.

The states and cities that adopt any of the three model codes, either in whole or in part, can be expected to follow similar patterns as regards referencing outdated standards. Of the states and cities that develop and promulgate their own building codes, two of the largest cities in the Nation continue to reference outdated standards, even though the building codes themselves were recently revised. New York City, in its 1990 edition, references 403 standards of which 89 , or 22 percent, are obsolete or otherwise not current. Some of these standards were withdrawn or canceled over 20 years ago.

The City of Chicago, which also produces its own building code, references a comparatively small number of standards, 128, in its 1990 edition. However, 39, or 30 percent, were not current and several of them have not been current for more than 20 years.

This compilation of standards referenced in building codes is built upon earlier work, accomplished some years ago and published as NBSIR 76-1140, ${ }^{4}$ which offers a similar listing of standards referenced in various building codes. Since that time, the building codes used throughout the Nation have expanded greatly in coverage with a resultant increase in the number of standards referenced in most building codes. In addition, and as suggested earlier, it has been found that a number of building codes are vastly outdated as regards referencing obsolete or modified standards.

\section{CONTENT AND USE OF TABLES}

Consistent with the earlier publication, the following data relative to standards utilization in building codes and their potential use to the building regulatory community are included: (1) location of each standard referenced in each building code to facilitate amendment when the date of the standard changes; (2) data base for determining those standards that are in widespread use; (3) identification of seldom used standards so studies can be initiated to determine the reason(s) that a standard is used in one code and not in another; (4) a compilation of the utilization of standards by various building regulatory

4 NBSIR 76-1140, "Standards Referenced in Selected Building Codes," Bertram M. Vogel, P.E., October 1976. 
authorities which provides an important data base for the promulgation of future Federal regulations; (5) identification of those standards that have the highest priority for conversion to metric (SI); and (6) a data base for organizations such as the Council of American Building Officials (CABO), the National Conference of States on Building Codes and Standards (NCSBCS), the individual model code organizations, as well as individual regulatory agencies, in working towards improvement of building codes through use of up-to-date standards.

This publication provides information relative to standards use for the various segments of the building community listed below.

For the Building Regulatory Community

- Current date of referenced standards

- Correct title of standard

- Organization generating the standard and the correct address of the organization

- Location in building code where the standard is referenced

- Date of the standard referenced in the building code, thus identifying a need for updating the reference

- Standards which have been discontinued or superseded

- Standards which should be considered for future reference in the building code

- Basis for establishing a standards reference library

For Standards Generating Organizations

- User, or nonuser, of a particular standard

- Adequacy of the referenced standard relative to date

- Adequacy of standard reference in the building code

- A basis for comparing codes for uniformity

- Areas where no standards exist

For Designing Professionals

- Basis for identifying design standards in other jurisdictions

- Basis for establishing a standards reference library

- Current assessment of the state-of-the-art on design standards

- Areas of concern in the design of projects in other jurisdictions

- Guide to the standards activities of professional societies

For Federal Government Agencies

- Possible conflicts between Federal and local regulations

- Codes which will probably comply with Federal programs

- Codes which may require updating

- Identification of areas where no applicable standards exist

For Industrial Organizations (including trade associations)

- Utilization of standards developed by these organizations

- Determination of standards which should be developed

- Adequacy of standards referenced in building codes 


\section{BUILDING CODES INCLUDED IN STUDY}

The following building codes are included in this compilation:

1. Model Building Codes (abbreviations and current date)

a. National Building Code (NBC - 1990 w/1991 Supplement), as promulgated by the Building Officials and Code Administrators International, Inc. (BOCA)

b. Standard Building Code (SBC - 1991), as promulgated by the Southern Building Code Congress International, Inc. (SBCCI)

c. Uniform Building Code (UBC - 1991), as promulgated by the International Conference of Conference of Building Officials (ICBO)

2. State Building Codes (abbreviations) - dates of codes
a. Alaska (AK) - 1991
b. Arkansas (AR) - 1991
c. California (CA) - 1989
d. Connecticut (CT) - 1989
e. Florida* (FL) - 1991
f. Georgia (GA) - 1991
g. Indiana (IN) - 1989
h. Kentucky (KY) - 1991
i. Massachusetts (MA) - 1990
j. Michigan (MI) - 1989
k. Minnesota (MN) - 1990
1. Montana (MT) - 1989
m. New Hampshire (NH) - 1992
n. New Jersey (NJ) - 1991
o. New Mexico (NM) - 1988
p. New York (NY) - 1990
q. North Carolina (NC) - 1991
r. North Dakota (ND) - 1991
s. Ohio $(\mathrm{OH})-1989$
t. Oregon (OR) - 1990
u. Rhode Island (RI) - 1990
v. Tennessee (TN) - 1988
w. Utah (UT) - 1990
x. Vermont (VT) - 1989
y. Virginia (VA) - 1990
z. Washington (WA) - 1991
aa. West Virginia (WV) - 1989
bb. Wisconsin (WI) - 1992
cc. Wyoming (WY) -1992

*Does not include Dade County, Broward County, and EPCOT. 
3. Major City Building Codes* - dates of codes

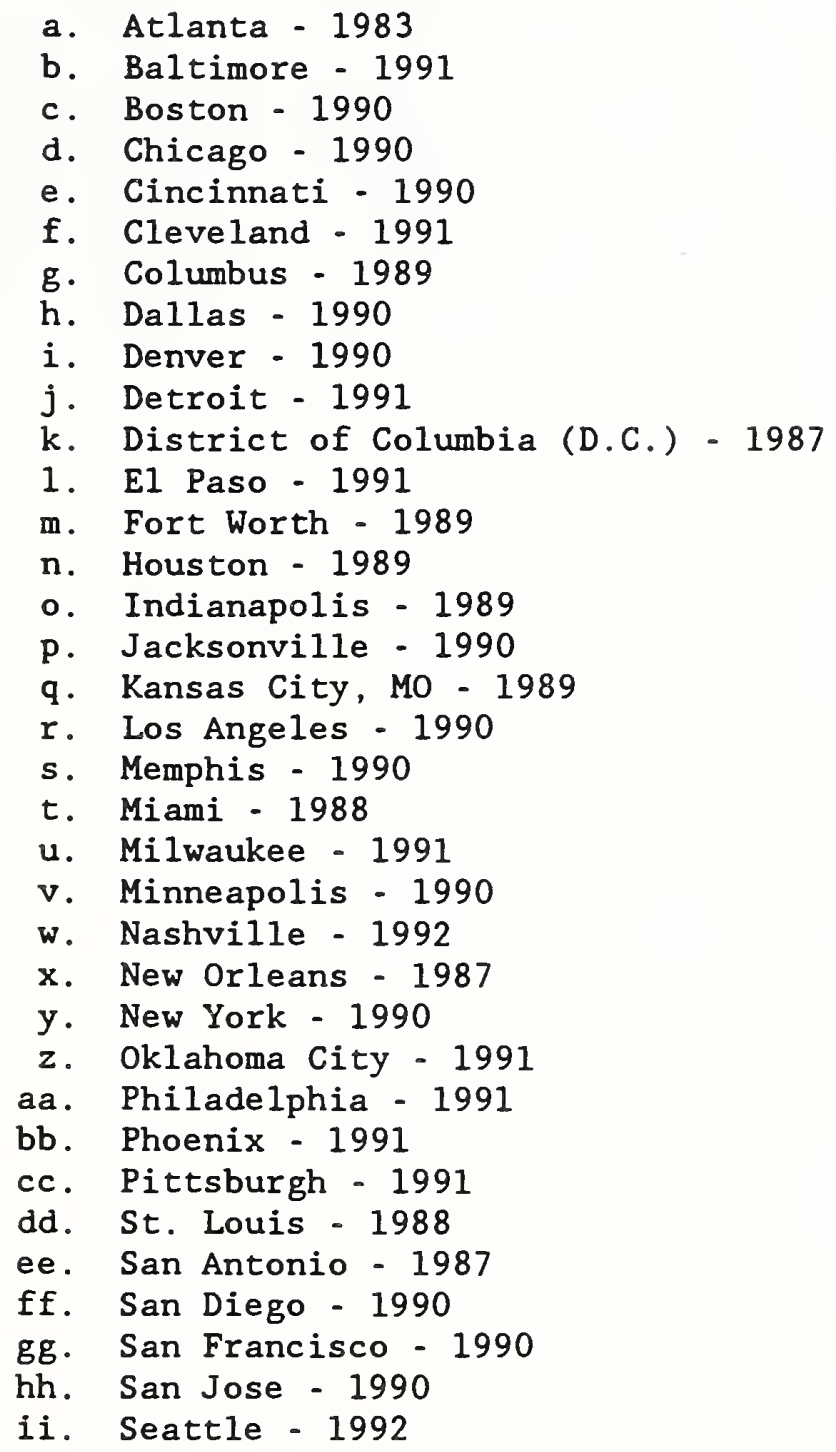

* Some large cities are not included since they are in states requiring the cities within those states to use the state code with no amendments. Typical, but not all inclusive, of the cities falling within this category are little Rock, Arkansas; Louisville, Kentucky; Trenton, New Jersey; Portland, Oregon; and Richmond, Virginia.

\section{FORMAT OF TABULAR PRESENTATION}

The tabular presentation of the standards referenced in building codes is based on the alphabetical listing, by acronym, of the standards generating organizations. Appendix A contains the addresses and acronyms for the 120 organizations which have developed standards included in the study. 
As illustrated in Figure 1, each standards generating organization is listed along with the standards which are included in at least one building code. In general, these standards are listed by the standard numbers, or letters, and title assigned by the generating organization. This is not always the case since several standards generating organizations do not assign numbers or letters to their standards. When this occurs, identifying letters and numbers have been assigned arbitrarily for identification by the computer. Additional information provided for each referenced standard includes: (1) current date of the standard (if the standard is still active), as well as the date referenced in the building codes; (2) names and current dates of the building codes which reference the standard; and ( 3 ) section, or sections, in each included code where the standard is referenced. 


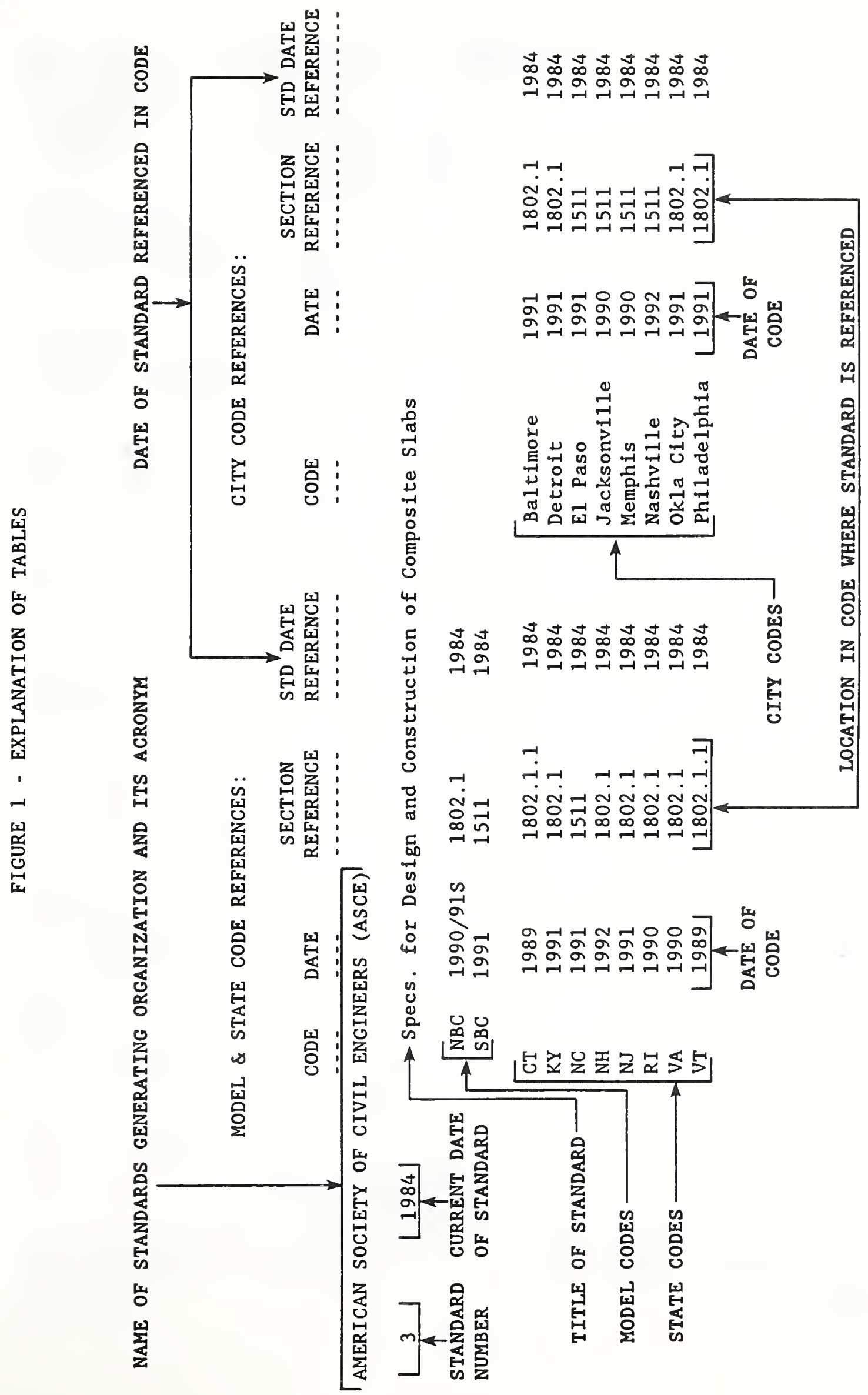




\section{APPENDIX A}

\section{STANDARDS GENERATING ORGANIZATIONS INCLUDED IN STUDY}

Aluminum Association (AA)

900 19th Street, NW.

Washington, DC 20006

(202) $862-5100$

American Architectural Manufacturers Association (AAMA)

1540 East Dundee Road

Palatine, IL 60067

(708) 202-1350

American Association of State Highway and

Transportation Officials (AASHTO)

444 North Capitol Street, NW.

Washington, DC 20001

(202) 624-5800

Air Conditioning Contractors of America (ACCA)

1513 16th Street, NW.

Washington, DC 20036

(202) $483-9370$

American Concrete Institute ( $\mathrm{ACl})$

P.O. Box 19150

Detroit, MI 48219

(313) $532-2600$

Architectural Fabric Structures Institute (AFSI)

c/o Industrial Fabric Association International

345 Cedar Street, Suite 800

St. Paul MN 55101

(612) 222-2508

American Hardboard Association (AHA)

520 N. Hicks Road

Palatine, IL 60067

(708) $934-8800$

American Institute of Architects (AIA)

Order Department

9 Jay Gould Court

P.O. Box 753

Waldorf, MD 20604

(301) 645-6936

American Institute of Steel Construction, Inc. (AISC)

The Wrigley Building, Eighth Floor

400 North Michigan Avenue

Chicago, IL 60611

(312) $670-2400$

Association of Iron and Steel Engineers (AISE)

Three Gateway Center, Suite 2350

Pittsburgh, PA 15222

(412) $281-6323$
American Insurance Services Group, Inc. (AISG)

Engineering \& Safety Service

85 John Street

New York, NY 10038

(212) $669-0400$

American Iron and Steel Institute (AISI)

1133 15th Street, NW.

Washington, DC 20005

(202) $452-7100$

American Institute of Timber Construction (AITC) 11818 S.E. Mill Plain Boulevard, Suite 415

Vancouver, WA 98684

(206) 254-9132

American National Standards Institute (ANSI)

11 West 42nd Street

New York, NY 10036

(212) $642-4900$

American Plywood Association (APA)

7011 S. 19 th Street

P.O. Box 117

Tacoma, WA 98411-0700

(206) $565-6600$

American Public Health Association (APHA)

1015 15th Street, NW.

Washington, DC 20005

(202) $789-5600$

American Petroleum Institute (API)

1220 L Street, NW.

Washington, DC 20005

(202) $682-8000$

American Railway Engineering Association (AREA)

50 F Street, NW.

Washington, DC 20001

(202) 639-2190

Air Conditioning and Refrigeration Institute (ARI)

1501 Wilson Boulevard

Arlington, VA 22209

(703) $524-8800$

American Society of Civil Engineers (ASCE)

United Engineering Center

345 East 47th Street

New York, NY 10017

(212) $705-7496$ 
American Society of Heating, Refrigerating and Air-Conditioning Engineers (ASHRAE)

1791 Tullie Circle, NE.

Atlanta, GA 30329

(404) $636-8400$

American Society of Mechanical Engineers (ASME) United Engineering Center

345 East 47th Street

New York, NY 10017

(212) $705-7722$

American Society of Sanitary Engineering (ASSE)

P.O. Box 40362

Bay Village, $\mathrm{OH} 44140$

(216) $835-3040$

American Society for Testing and Materials (ASTM)

1916 Race Street

Philadelphia, PA 19103-1187

(215) 299-5585

American Wood Preservers Association (AWPA) P.O. Box 849

Stevensville, MD 21666

(301) $465-3169$

American Wood Preservers Bureau (AWPB)

P.O. Box 5283

Springfield, VA 22150

(703) $339-6660$

American Wood Preservers Institute (AWPI)

1945 Old Gallows Road, Suite 550

Vienna, VA 22182

(703) $893-4005$

American Welding Society (AWS)

P.O. Box 351

Miami, FL 33135

(305) $443-9353$

American Water Works Association (AWWA)

6666 West Quincy Avenue

Denver, CO 80235

(303) 794-7711

Builders Hardware Manufacturers Association, Inc. (BHMA)

355 Lexington Avenue

New York, NY 10017

(212) $661-4261$

Brick Institute of America (B|A)

11490 Commerce Park Drive, Suite 300

Reston, VA 22091

(703) 620-0010
Building Officials and Code Administrators

International, Inc. (BOCA)

4051 West Flossmoor Road

Country Club Hills, IL 60478-5795

(708) $799-2300$

Council of American Building Officials (CABO)

5205 Leesburg Pike, Suite 201

Falls Church, VA 22041

(703) $931-4533$

Compressed Gas Association (CGA)

Crystal Gateway One, Suite 501

1235 Jefferson Davis Highway

Arlington, VA 22202

(703) $979-0900$

Canadian General Standards Board (CGSB)

Technical Information Unit

Ottawa, Ontario, Canada K1A $1 \mathrm{G} 6$

(613) $956-0400$

Cast Iron Soil Pipe Institute (CISPI)

5959 Shallowford Road, Suite 419

Chattanooga, TN 37421

(615) $892-0137$

California Lathing and Plastering Contractors Association (CLPCA)

c/o Western Lath/Plaster/Drywall Industries

Association

8635 Navajo Road

San Diego, CA 92119

(619) $466-9070$

Crane Manufacturers Association of America (CMAA) 8720 Red Oak Boulevard, Suite 2010

Charlotte, NC 28217

(704) $522-8644$

Concrete and Masonry Industry Firesafety Committee (CMIFC)

c/o Portland Cement Association

5420 Old Orchard Road

Skokie, IL 60077-1083

(708) 966-9559

U.S. Consumer Product Safety Commission (CPSC)

5401 Westbard Avenue, Room 408

Bethesda, MD 20816

(301) $492-6800$

Concrete Reinforcing Steel Institute (CRSI)

933 N. Plum Grove Road

Schaumburg, IL 60173-4758

(708) $517-1200$ 
Canadian Standards Association (CSA)

Certification Division

Rexdale, Ontario, Canada M9W 1R3

(416) $747-4300$

Cedar Shingle and Shake Bureau (CSSB)

(Formerly Red Cedar Shingle and Handsplit

Shake Bureau)

515 116th Avenue, NE., Suite 275

Bellevue, WA 98004

(206) 453-1323

U.S. Department of Agriculture (DA)

1776 F Street, NW.

Washington, DC 20437

(202) 447-8732

Department of Health - Rules and Regulations (DHRR)

Now York State

c/o Lenz \& Riecker, Inc.

One Columbia Place

Albany, NY 12207

(518) $436-8647$

U.S. Department of Commerce (DoC)

National Institute of Standards and Technology

Gaithersburg, MD 20899

(301) $975-4029$

U.S. Department of Energy (DoE)

1000 Independence Avenue, SW.

Washington, DC 20402

(202) $586-8800$

U.S. Department of Transportation (DoT)

Research and Special Programs Administration 4007 th Street, SW.

Washington, DC 20402

(202) $366-4934$

Electronics Industries Association (EIA)

2001 Eye (I) Street, NW.

Washington, DC 20006

(202) $457-4900$

Energy Testing Laboratory of Maine (ETLM)

South Maine Vocational Technical Institute

South Portland, ME 04106

(207) $799-7303$

Federal Communications Commission (FCC)

1919 M Street, NW.

Washington, DC 20554

(202) $632-7000$
Federal Emergency Management Agency (FEMA)

Printing and Publications

P. O. Box 818

Washington, DC 20024

(202) $646-3484$

U.S. Department of Transportation

Federal Highway Administration (FHWA)

Construction and Maintenance Division

400 7th Street, SW., Room 3203

Washington, DC 20590

(202) 426-0677

Factory Mutual Engineering Corporation (FM)

Standards Laboratories Department

1151 Boston Providence Turnpike

Norwood, MA 02062

(617) $762-4300$

General Services Administration

Federal Specifications (FS)

Specifications Section

7th \& D Streets, SW., Room 6654

Washington, DC 20407

(202) 472-1701

Florida Solar Energy Center (FSEC)

300 State Road 401

Cape Canaveral, FL 32920

(904) $783-0300$

Gypsum Association (GA)

810 First Street, NE., Suite 510

Washington, DC 20002

(202) $289-5440$

Hardwood Plywood Manufacturers Association (HPMA)

1825 Michael Faraday Drive

P.O. Box 278

Reston, VA 22090

(703) $435-2900$

Health and Rehabilitation Services (HRS)

Division of Health

State of Florida

P.O. Box 21

Jacksonville, FL 32201

(904) 359-6046

U.S. Department of Housing and Urban Development (HUD)

820 1st Street, NE.

Washington, DC 20002-4205

(202) $275-4251$

International Conference of Building Officials (ICBO)

5360 South Workman Mill Road

Whittier, CA 90601

(213) 699-0541 
International Masonry Institute (IMI)

823 15th Street, NW., Suite 1001

Washington, DC 20005

(202) $783-3908$

Industrial Staple, Nail and Tool Association (ISANTA) 435 North Michigan Avenue, Suite 1717

Chicago, IL 60611

(312) 644-0828

International Organization for Standardization (ISO) c/o American National Standards Institute

11 West 42nd Street

New York, NY 10036

(212) $642-4995$

Kentucky Department of Housing, Buildings and

Construction (Ky. Bldg. Code) (KBC)

The 127 Building

1047 U.S. 127 South

Frankfort, KY 40601

(502) $564-8090$

Metal Building Manufacturers Association (MBMA) 1230 Keith Building

Cleveland, $\mathrm{OH} \quad 44115-2180$

(216) 241-7333

Maple Flooring Manufacturers Association (MFMA)

60 Revere Drive, Suite 500

Northbrook, IL 60062

(708) $480-9138$

Masonry Institute of America (MIA)

2550 Beverly Boulevard

Los Angeles, CA 90057

(213) 388-0472

Naval Supply Depot

Military Specifications-Standardization Document

Order Desk (MIL)

700 Robbins, Building 4D

Philadelphia, PA 19111

(215) 697-2179

Manufacturers Standardization Society of the Valve and Fittings Industry, Inc. (MSS)

127 Park Street, NE.

Vienna, VA 22046

(703) $281-6613$

National Association of Plumbing, Heating, Cooling Contractors (NAPHCC)

$180 \mathrm{~S}$. Washington Street

Falls Church, VA 22046

(703) $237-8100$
Naval Facilities Engineering Command (NAVFAC) Naval Publications and Forms

5801 Tabor Avenue

Philadelphia, PA 19120-5099

(215) $697-4374$

The National Board of Boiler and Pressure Vessel Inspectors (NBBPVI)

1055 Crupper Avenue

Columbus, $\mathrm{OH} 43229$

(614) 644-2236

National Concrete Masonry Association (NCMA) 2302 Horse Pen Road

P.O. Box 781

Herndon, VA 22070-0781

(703) $713-1900$

National Council on Radiation Protection and Measurements (NCRP)

NCRP Publications

7910 Woodmont Avenue, Suite 800

Bethesda, MD 20814

(301) 657-2652

Nickel Development Institute (NDI)

15 Toronto Street, Suite 402

Toronto, Ontario, Canada M5C 2E3

(416) $362-8850$

National Environmental Balancing Bureau (NEBB) 1385 Piccard Drive

Gaithersburg, MD 20850

(301) $977-3698$

Northeastern Lumber Manufacturers Association

(NELMA)

272 Tuttle Road

P.O. Box 87A

Cumberland Center, ME 04021

(207) 829-6901

National Forest Products Association (NFoPA)

1250 Connecticut Avenue, NW., Suite 200

Washington, DC 20036

(202) $463-2700$

National Fire Protection Association (NFPA)

Batterymarch Park

Quincy, MA 02269

(617) $770-3000$

Northern Hardwood and Pine Manufacturers

Association (NHPMA)

c/o Northeastern Lumber Manufacturers Association

272 Tuttle Road

P.O. Box 87A

Cumberland Center, ME 04021

(207) 829-6901 
National Lumber Grades Authority (NLGA)

260-1055 West Hastings Street

Vancouver, B.C., Canada V6E 2E9

(604) 689-1563

National Oak Flooring Manufacturers Association, Inc. (NOFMA)

22 N. Front Street, Suite 600

Memphis, TN 38103

(901) 526.5016

National Particleboard Association (NPA)

18929 Premiere Court

Gaithersburg, MD 20879

(301) 670-0604

National Pest Control Association, Inc. (NPCA)

8100 Oak Street

Dunn Loring, VA 22027

(703) $573-8330$

National Sanitation Foundation (NSF)

P.O. Box 130140

Ann Arbor, MI 48113-0140

(313) $769-8010$

National Spa and Pool Institute (NSPI)

2111 Eisenhower Avenue

Alexandria, VA 22314

(703) 838-0083

National Wood Window and Door Manufacturers

Association (NWWDA)

1400 East Touhy Avenue

Des Plaines, IL 60018

(708) 299-5200

Office of Civil Defense Management (OCDM)

c/o Federal Emergency Management Agency

Washington, DC 20024

(202) 646-3484

Office of the Chief of Engineers (OCE)

Department of the Army

Attention: ENGMC-ES

1000 Independence Avenue, SW.

Washington, DC 20314

(202) 272-0660

\section{U.S. Department of Labor}

Occupational Safety and Health Administration (OSHA)

Francis Perkins Building

200 Constitution Avenue, NW.

Washington, DC 20210

(202) 523-7251
Portland Cement Association (PCA)

5420 Old Orchard Road

Skokie, IL 60077-1083

(708) $966-9559$

Prestressed Concrete Institute (PCI)

175 West Jackson Boulevard

Chicago, IL 60604

(312) 786-0300

Plumbing Drainage Institute (PDI)

$1106 \mathrm{~W}$. 77th Street, South Drive

Indianapolis, IN $46260-3318$

(317) 251-6970

Post-Tensioning Institute (PTI)

1717 W. Northern Avenue, Suite 218

Phoenix, AZ 85021

(602) $870-7540$

Research Council on Structural Connections (RCSC) c/o Stanley D. Lindsey \& Associates, Lid.

1906 West End Avenue

Nashville, TN 37203-2371

(615) 244-2211

Redwood Inspection Service (RIS)

405 Enfrente Drive, Suite 200

Novato, CA 94949

(415) 382-0662

Rubber Manufacturers Association (RMA)

$1400 \mathrm{~K}$ Street, NW.

Washington, DC 20005

(202) $682-4800$

Southern Building Code Congress International, Inc. (SBCCl)

900 Montclair Road

Birmingham, AL 35213-1206

(205) 591-1853

Structural Clay Products Institute (SCPI)

c/o Brick Institute of America

11490 Commerce Park Drive

Reston, VA 22091

(703) 620-0010

U.S. Department of Agriculture

Southeastern Forest Experiment Station (SFES)

Forest Service

200 Weaver Boulevard

P.O. Box 2680

Asheville, NC 28804

(704) $257-4390$ 
Steel Joist Institute (SJI)

1205 48th Avenue North, Suite A

Myrtle Beach, SC 29577

(803) 449-0487

Sheet Metal and Air-Conditioning Contractors

National Association, Inc. (SMACNA)

4201 Lafayette Center Drive

Chantilly, VA 22021

(703) $803-2980$

Society of Motion Picture and Television

Engineers (SMPTE)

595 West Hartsdale Avenue

White Plains, NY 10607-1824

(914) $761-1100$

Society of the Plastics Industry, Inc. (SPI)

1275 K Street, NW., Suite 400

Washington, DC 20005

(202) $371-5200$

Southern Pine Inspection Bureau (SPIB)

4709 Scenic Highway

Pensacola, FL 32504

(904) 434-2611

Single Ply Roofing Institute (SPRI)

20 Walnut Street, Suite 208

Wellesley, MA 02181

(617) 237-7979

Tile Council of America (TCA)

P.O. Box 326

Princeton, NJ 08542

(609) 921.7050

Texas Forest Service (TFS)

Forest Products Laboratory

P.O. Box 310

Lufkin, TX 75902-0310

(409) $639-8180$

Truss Plate Institute (TPI)

583 D'Onofrio Drive, Suite 200

Madison, WI 53719

(608) 833-5900

Uniform Building Code (UBC)

International Conference of Building Officials

5360 South Workman Mill Road

Whittier, CA 90601

(213) 699-0541

Underwriters Laboratories, Inc. (UL)

333 Pfingsten Road

Northbrook, IL 60062-2096

(708) $272-8800$
Underwriters Laboratories of Canada (ULC)

7 Crouse Road

Scarborough, Ontario, Canada M1R 3A9

(416) $757-3611$

United States Diving, Inc. (USD)

$901 \mathrm{~W}$. New York Street

Indianapolis, IN 46202

(317) 237-5252

Waferboard Association (WA)

c/o Structural Board Association

45 Sheppard Avenue East

Willowdale, Ontario, Canada M2N 5W9

(416) $730-9090$

Washington State Building Code Council (WAC) Department of Community Development Ninth and Columbia Building

Olympia, WA 98504-4151

(206) 586-3423

West Coast Lumber Inspection Bureau (WCLIB) 6980 S.W. Varnes Road

P.O. Box 231

Portland, OR 97223

(503) 639-0651

\author{
Western Lath/Plaster/Drywall Industries \\ Association (WLPDIA) \\ 8635 Navajo Road \\ San Diego, CA 92119 \\ (619) $466-9070$
}

Wire Reinforcement Institute (WRI)

1101 Connecticut Avenue, NW., Suite 700

Washington, DC 20036

(202) 429-5125

\author{
Western Wood Products Association (MWPA) \\ 1500 Yeon Building \\ 522 SW Fifth Avenue \\ Portland, OR 97204-2122 \\ (503) $224-3930$
}




\section{INDEX OF STANDARDS GENERATING ORGANIZATIONS}

Aluminum Association (AA) . . . . . . . . . . . . . . . . . . . . . . A 1

American Architectural Manufacturers Association (AAMA) . . . . . . . . . A 2

American Association of State Highway and Transportation Officials (AASHTO) . . . . . . . . . . . . . . . . . . . . A 4

Air Conditioning Contractors of America (ACCA) . . . . . . . . . . . . . A 5

American Concrete Institute (ACI) . . . . . . . . . . . . . . . . . . A 6

Architectural Fabric Structures Institute (AFSI) . . . . . . . . . . . . A 18

American Hardboard Association (AHA) . . . . . . . . . . . . . . . . . A 19

American Institute of Architects (AIA) . . . . . . . . . . . . . . . . . A 20

American Institute of Steel Construction, Inc. (AISC) . . . . . . . . . A 21

Association of Iron and Steel Engineers (AISE) . . . . . . . . . . . . . A 23

American Insurance Services Group, Inc. (AISG) . . . . . . . . . . . . . A 24

American Iron and Steel Institute (AISI) . . . . . . . . . . . . . . . A 25

American Institute of Timber Construction (AITC) . . . . . . . . . . . . A 28

American National Standards Institute (ANSI) . . . . . . . . . . . . . A 32

American Plywood Association (APA) . . . . . . . . . . . . . . . . . A 68

American Public Health Association (APHA) . . . . . . . . . . . . . A 73

American Petroleum Institute (API) . . . . . . . . . . . . . . . . . . . A 74

American Railway Engineering Association (AREA) . . . . . . . . . . . . . A 75

Air Conditioning and Refrigeration Institute (ARI) . . . . . . . . . . . A 76

American Society of Civil Engineers (ASCE) . . . . . . . . . . . . . . . A 77

American Society of Heating, Refrigerating and Air

Conditioning Engineers (ASHRAE) . . . . . . . . . . . . . . . . . . A 80

American Society of Mechanical Engineers (ASME) . . . . . . . . . . . A 83

American Society of Sanitary Engineers (ASSE) . . . . . . . . . . . . . . A 88

American Society for Testing and Materials (ASTM) . . . . . . . . . . . . A 90

American Wood Preservers Association (AWPA) . . . . . . . . . . . . . . . A 186

American Wood Preservers Bureau (AWPB) . . . . . . . . . . . . . . . A 195

American Wood Preservers Institute (AWPI) . . . . . . . . . . . . . . . A 200

American Welding Society (AWS) . . . . . . . . . . . . . . . . . A 201

American Water Works Association (AWWA) . . . . . . . . . . . . . . . . A 203

Builders Hardware Manufacturers Association, Inc. (BHMA) . . . . . . A 205

Brick Institute of America (BIA) . . . . . . . . . . . . . . . . . . . A 206

Building Officials and Code Administrators International,
Inc. (BOCA). . . . . . . . . . . . . . . . . . . . . 208

Council of American Building Officials (CABO) . . . . . . . . . . . . . A 210

Compressed Gas Association (CGA) . . . . . . . . . . . . . . . . . . . . A 211

Canadian General Standards Board (CGSB) . . . . . . . . . . . . . . . . A 212

Cast Iron Soil Pipe Institute (CISPI) . . . . . . . . . . . . . . . . A 213

California Lathing and Plastering Contractors Association (CLPCA) . . . . A 214

Crane Manufacturers Association of America (CMAA) . . . . . . . . . . . . A 215

Concrete and Masonry Industry Firesafety Committee (CMIFC) . . . . . . A 216

Consumer Product Safety Commission, United States (CPSC) . . . . . . . . A 217

Concrete Reinforcing Steel Institute (CRSI) . . . . . . . . . . . . . . A 219

Canadian Standards Association (CSA) . . . . . . . . . . . . . . . . . A 220

Cedar Shingle and Shake Bureau (CSSB). . . . . . . . . . . . . . . A 221

Department of Agriculture, United States (DA) . . . . . . . . . . . . . . A 222

Department of Environmental Conservation-Rules and Regulations,

New York State (DECRR) 
Department of Health, New York State (DH)

Department of Health - Rules and Regulations, New York

State (DHRR)

Department of Labor - Rules and Regulations, New York

State (DLRR)

A 226

Department of Commerce, United States (DoC) . . . . . . . . . . . A 227

Department of Energy, United States (DoE) . . . . . . . . . . . . . . A 231

Department of Transportation, United States (DoT) . . . . . . . . . . A 232

Electronics Industries Association (EIA) . . . . . . . . . . . . A 233

Energy Testing Laboratory of Maine (ETLM) . . . . . . . . . . . . . . . A 234

Federal Communications Commission (FCC). . . . . . . . . . . . . . . . . A 235

Federal Emergency Management Agency (FEMA) . . . . . . . . . . . . A 236

Federal Highway Administration, U.S. Department of

Transportation (FHWA) . . . . . . . . . . . . . . . A 237

Factory Mutual Engineering Corporation (FM) . . . . . . . . . . . A 238

Federal Specifications, General Services Administration (FS) . . . . . A 240

Florida Solar Energy Center (FSEC) . . . . . . . . . . . . . . . A 245

Gypsum Association (GA) . . . . . . . . . . . . . . . . . . . . . A 246

Hardwood Plywood Manufacturers Association (HPMA) . . . . . . . . . . A 248

Health and Rehabilitation Services, State of Florida (HRS) . . . . . . A 249

Housing and Urban Development, U.S. Department of (HUD) . . . . . . . . A 250

International Conference of Building officials (ICBO) . . . . . . . . . . A 251

International Masonry Institute (IMI) . . . . . . . . . . . . . . A 252

Industrial Staple, Nail and Tool Association (ISANTA) . . . . . . . . A 253

International Organization for Standardization (ISO) . . . . . . . . . . A 254

Kentucky Department of Housing, Buildings and Construction (KBC) . . . . A 255

Metal Building Manufacturers Association (MBMA) . . . . . . . . . . A 256

Maple Flooring Manufacturers Association (MFMA) . . . . . . . . . . . A 257

Masonry Institute of America (MIA) . . . . . . . . . . . . . A 258

Military Specifications, Naval Supply Center (MIL) . . . . . . . . . A 259

Manufacturers Standardization Society of the Valve and Fittings

Industry, Inc. (MSS) . . . . . . . . . . . . . . . . . A 260

National Association of Plumbing, Heating, Cooling Contractors

(NAPHCC)

A 261

Naval Facilities Engineering Command (NAVFAC) . . . . . . . . . . . . A 262

National Board of Boiler and Pressure Vessel Inspectors (NBBPVI) . . . A 263

National Concrete Masonry Association (NCMA) . . . . . . . . . . . . . . A 264

National Council on Radiation Protection and Measurements (NCRP) . . . A 266

Nickel Development Institute (NDI) . . . . . . . . . . . . . . A 268

National Environmental Balancing Bureau (NEBB) . . . . . . . . . . . A 269

Northeastern Lumber Manufacturers Association (NELMA) . . . . . . . . . A 270

National Forest Products Association (NFoPA) . . . . . . . . . . . A 271

National Fire Protection Association (NFPA) . . . . . . . . . . . . . . . A 276

Northern Hardwood and Pine Manufacturers Association (NHPMA) . . . . . A 321

National Lumber Grades Authority (NLGA) . . . . . . . . . . . . . . . . A 322

National Oak Flooring Manufacturers Association, Inc. (NOFMA) . . . . A 323

National Particleboard Association (NPA) . . . . . . . . . . . . . A 324

National Pest Control Association, Inc. (NPCA) . . . . . . . . . . . . A 325

National Sanitation Foundation (NSF) . . . . . . . . . . . . A 326

National Spa and Pool Institute (NSPI) . . . . . . . . . . . . . . . . A 327

National Wood Window and Door Manufacturers Association (NWWDMA) . . . A 328 
Office of Civil Defense Management (OCDM) . . . . . . . . . . . . . A 329

Office of the Chief of Engineers, U.S. Department of the Army (OCE) . . . . . . . . . . . . . . . . . . . A 330

Occupational Safety and Health Administration, U.S. Department of Labor (OSHA) . . . . . . . . . . . . . . . . . . . . A 331

Portland Cement Association (PCA) . . . . . . . . . . . . . . A 332

Prestressed Concrete Association (PCI) . . . . . . . . . . . . . A 333

Plumbing Drainage Institute (PDI) . . . . . . . . . . . . . . . . . A 334

Public Service Commission-Rules and Regulations, New York

State (PSCRR) . . . . . . . . . . . . . . . . . . . A 335

Post-Tensioning Institute (PTI) . . . . . . . . . . . . . . . . . A 336

Research Council on Structural Connections (RCSC) . . . . . . . . . . . . A 337

Redwood Inspection Service (RIS) . . . . . . . . . . . . . . . . . . . A 338

Rubber Manufacturers Association (RMA) . . . . . . . . . . . . . . A 339

Southern Building Code Congress International, Inc. (SBCCI) . . . . . . . A 340

Structural Clay Products Institute (SCPI) . . . . . . . . . . . . . . . . A 343

State Energy Office-Rules and Regulations, New York State (SEORR) . . . . A 344

Southeastern Forest Experiment Station, U.S. Department of

Agriculture (SFES) . . . . . . . . . . . . . . . . . . A 345

Steel Joist Institute (SJI) . . . . . . . . . . . . . . . . . . A 346

Sheet Metal and Air-Conditioning Contractors National

Association, Inc. (SMACNA) . . . . . . . . . . . . . . . . . A 347

Society of Motion Picture and Television Engineers (SMPTE) . . . . . . . A 348

Society of the Plastics Industry, Inc. (SPI) . . . . . . . . . . . . . . A 349

Southern Pine Inspection Bureau (SPIB) . . . . . . . . . . . . . . . . . A 350

Single Ply Roofing Institute (SPRI) . . . . . . . . . . . . . . . . . A 351

Tile Council of America (TCA) . . . . . . . . . . . . . . . . . . . . A 352

Texas Forest Service (TFS) . . . . . . . . . . . . . . . . . A 353

Truss Plate Institute (TPI) . . . . . . . . . . . . . . . . . . . A 354

Uniform Building Code $(U B C)$. . . . . . . . . . . . . . . . . A 356

Underwriters Laboratories, Inc. (UL) . . . . . . . . . . . . . . . A 447

Underwriters Laboratories of Canada (ULC) . . . . . . . . . . . . . . . A 460

United States Diving, Inc. (USD) . . . . . . . . . . . . . . . A 461

Waferboard Association (WA) . . . . . . . . . . . . . . . . . . . A 462

Washington State Building Code Council (WAC) . . . . . . . . . . . . . A 463

West Coast Lumber Inspection Bureau (WCLIB) . . . . . . . . . . . . . . . A 464

Western Lath/Plaster/Drywall Industries Association (WLPDIA) . . . . . A 465

Wire Reinforcement Institute (WRI) . . . . . . . . . . . . . . . . . A 466

Western Wood Products Association (WWPA) . . . . . . . . . . . . . . A 467 


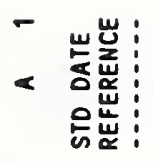

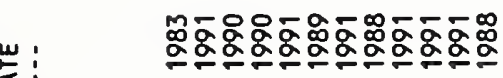

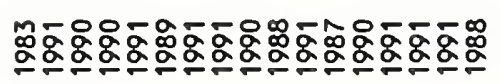

岁:

政: 


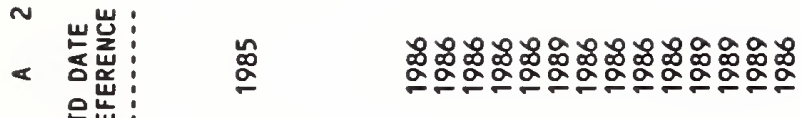

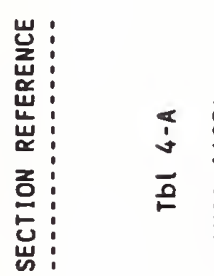

芑

w:

ָิ

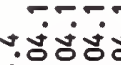

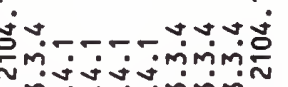

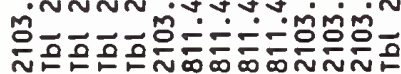

\&

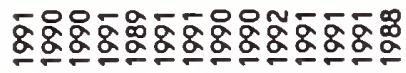

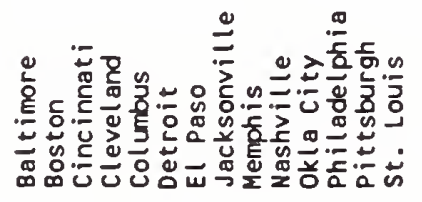

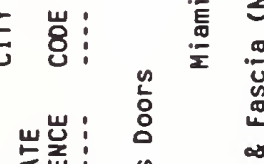

㟧

影

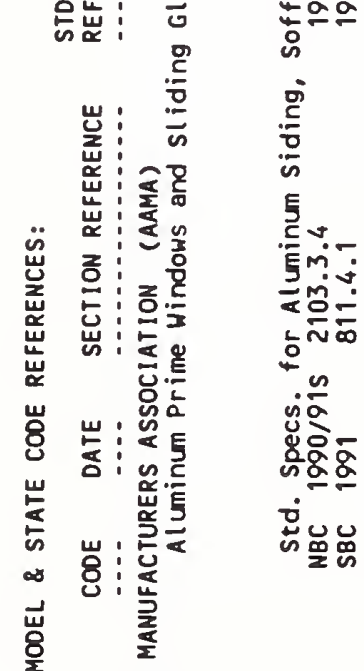

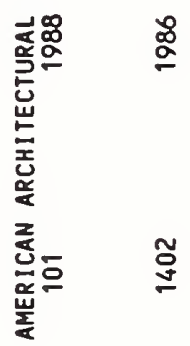

$\frac{x}{2}$ โกั

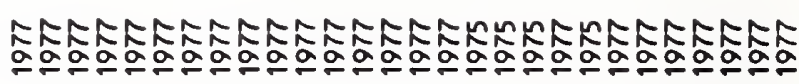

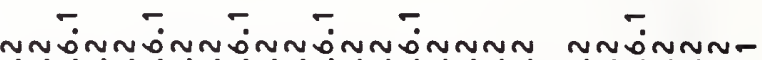

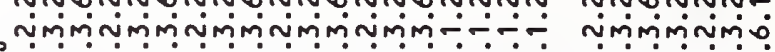

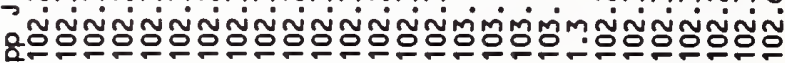

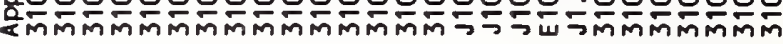

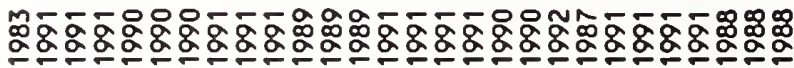

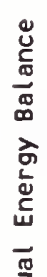

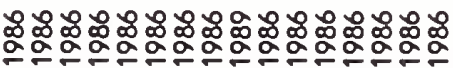

ํ.

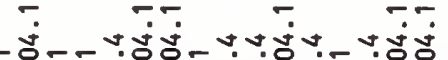

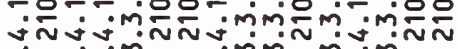

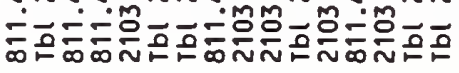

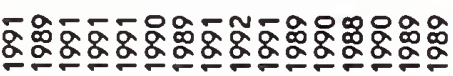

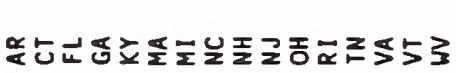

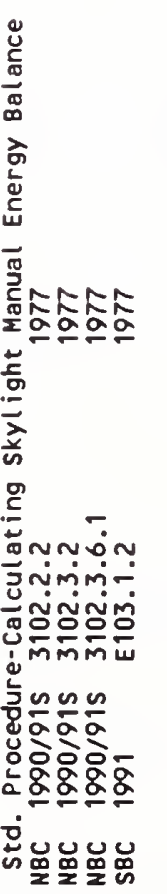

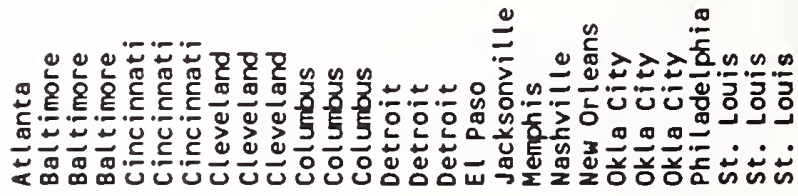

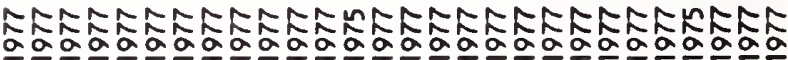

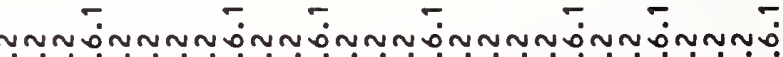
-nmm- nmm nmm-nmminmminmm-nmm

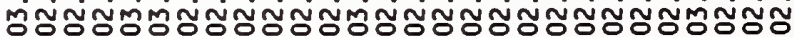

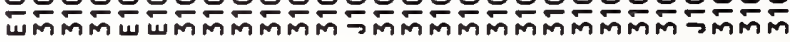

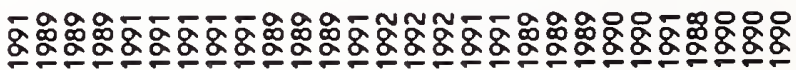

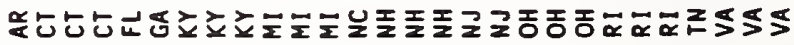




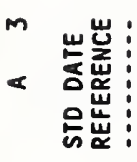

$$
\begin{aligned}
& \text { : } \\
& \text { 怘 } \\
& \text { 总 } \\
& \text { ర్ } \\
& \text { 릉 岂: } \\
& \text { 岂: }
\end{aligned}
$$

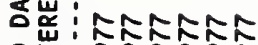

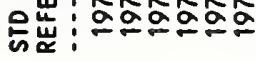

$$
\begin{aligned}
& \text { 岕 } \\
& \text { iิ } \\
& \text { zo nimiminim } \\
& \text { 岃 } \\
& \text { 岁 w a a ara }
\end{aligned}
$$

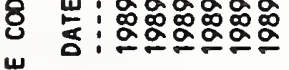

$$
\begin{aligned}
& \text { 岁:5553引3 } \\
& \text { 항 } \\
& \text { 옹 } \\
& \text { 苈 }
\end{aligned}
$$




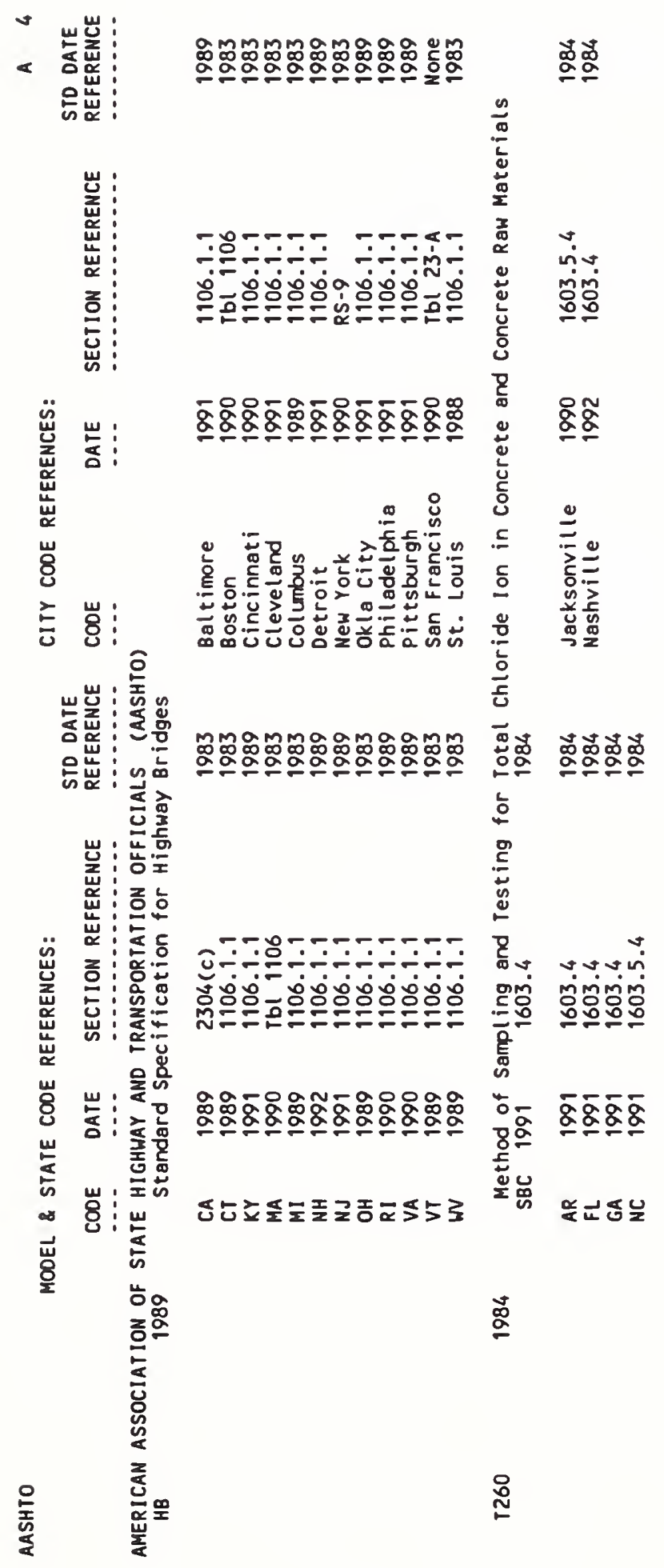




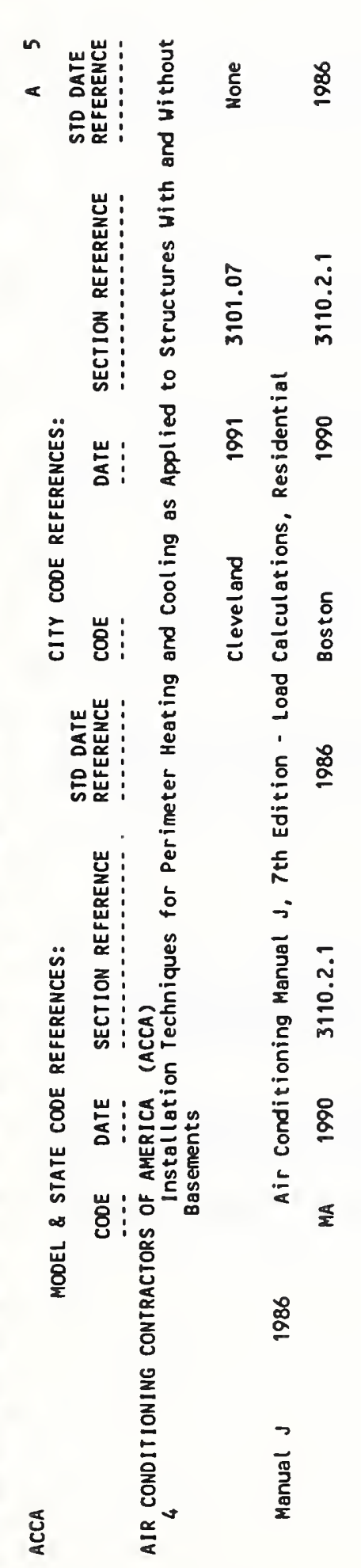




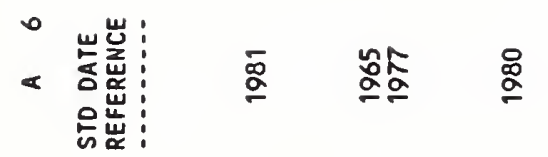

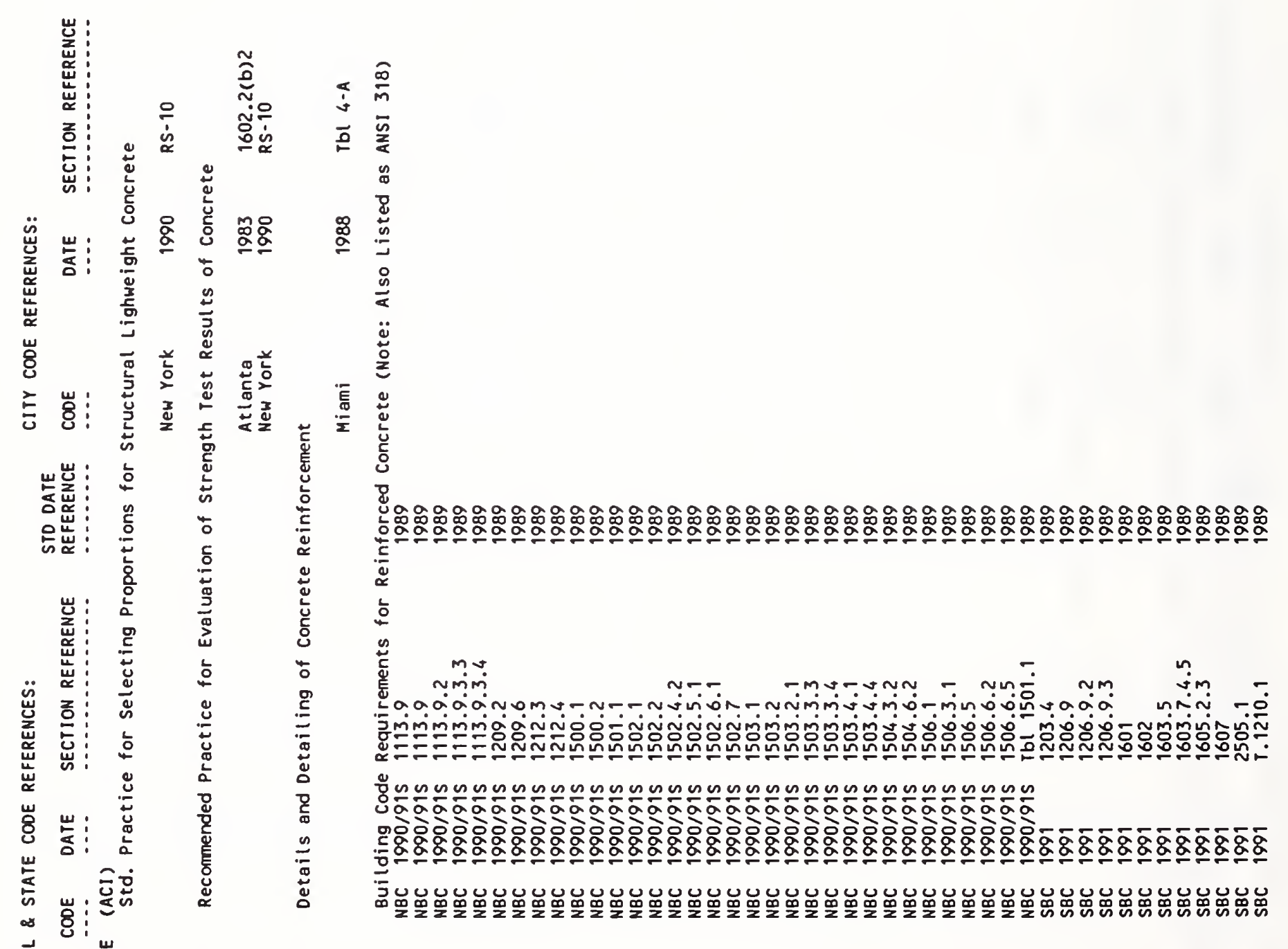

岕

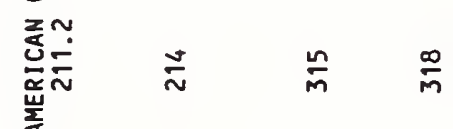




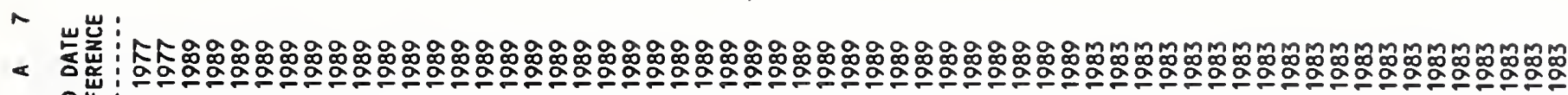
㩆:

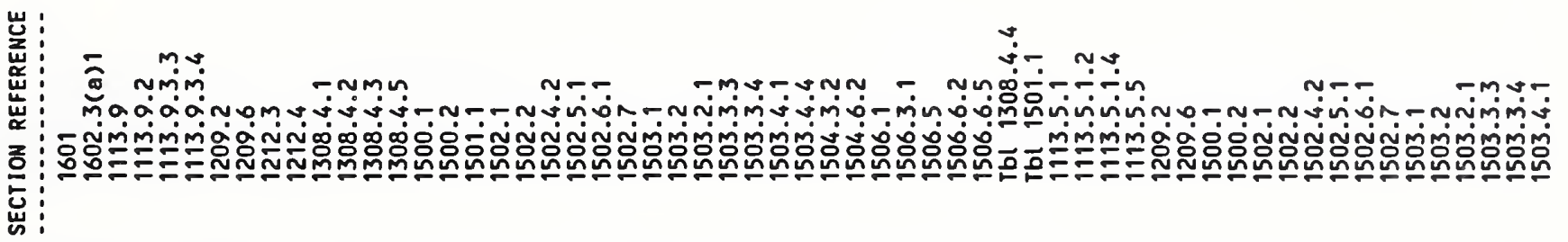

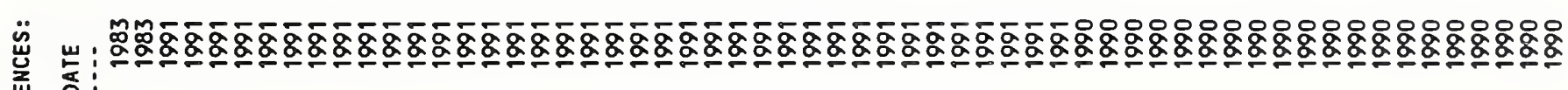
ธ。

- 山. U

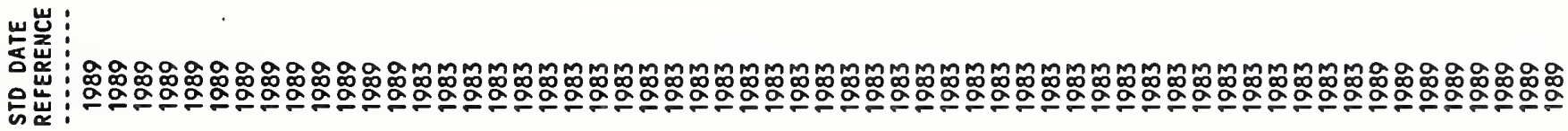

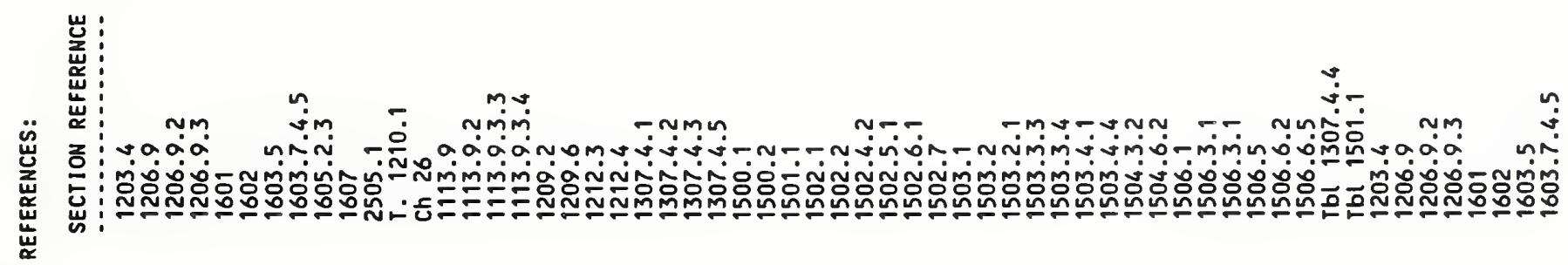

莞

岁

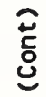


๔ 领:

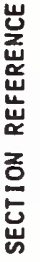

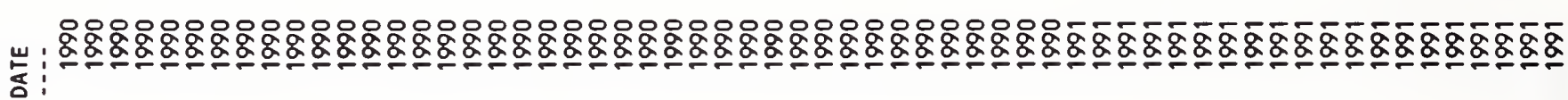

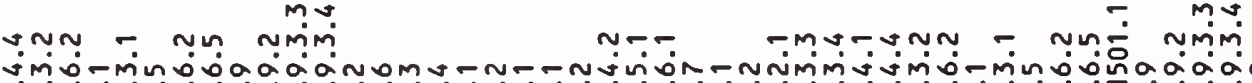

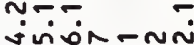

mó

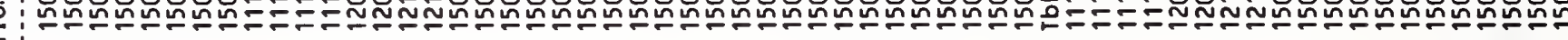

崖:

莙 过

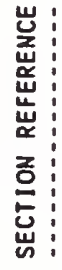

m

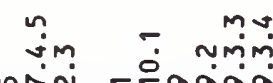

ñ-

-moj

inno

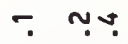

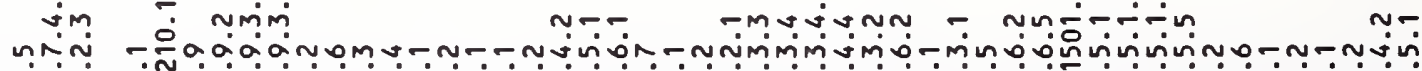
nлиำ

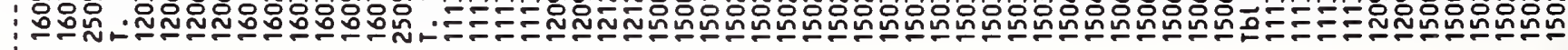

岩 岩

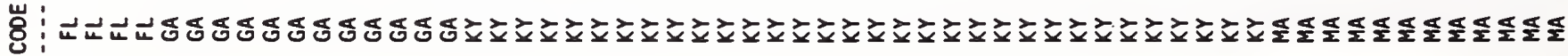

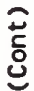

$\ddot{\Xi} \quad \stackrel{\infty}{m}$ 

家崖:

岕

芦 $\underset{\varpi}{\varpi}$

岁

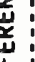
å

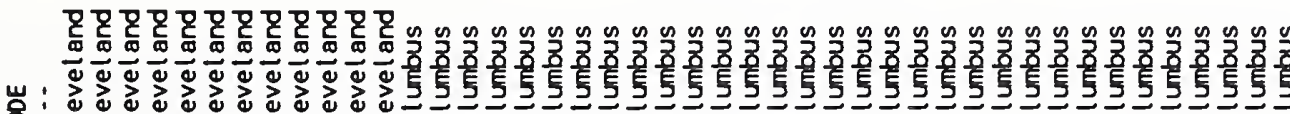

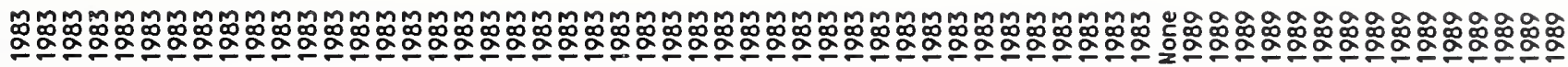

mu-vñ ก--

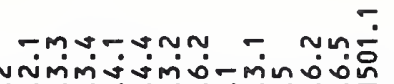

กับัต Trmm z

岁

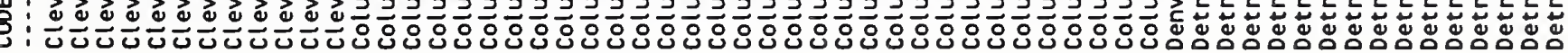

岁:

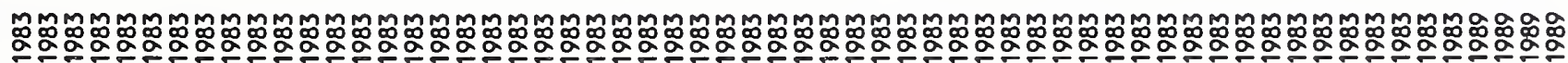

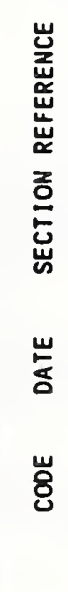

芒 


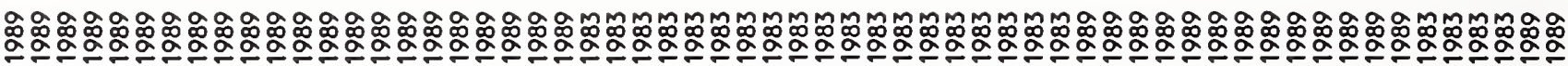
焉

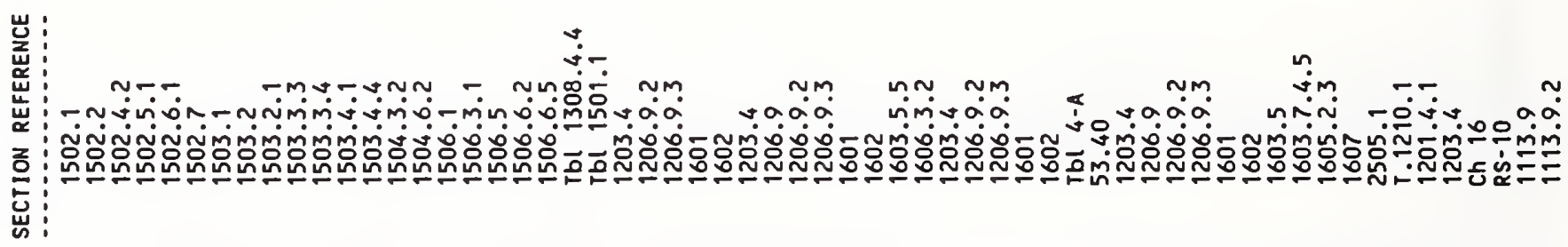

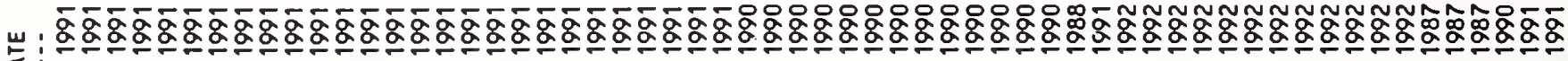
玄

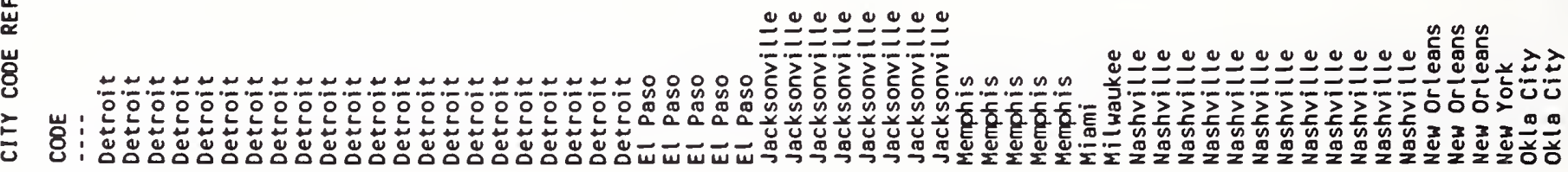
는

范 它岕: บ.-

mus. -nMm บัrimivis óñ்

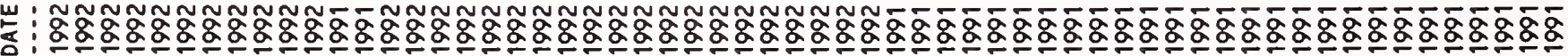

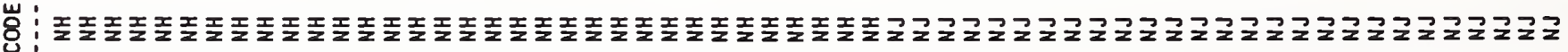<smiles>[CH]=C</smiles> 


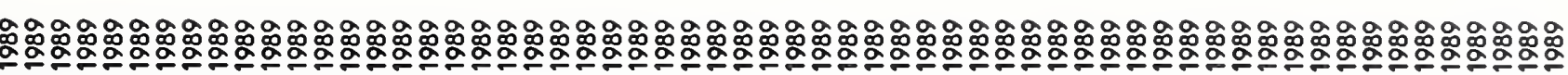
这崖

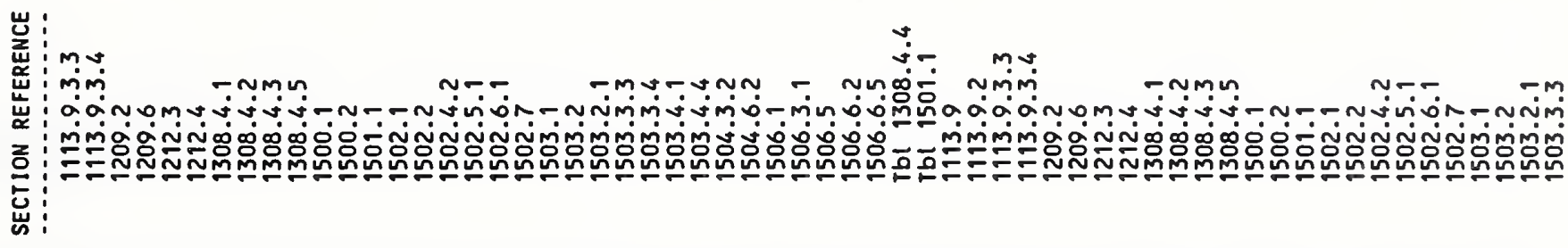

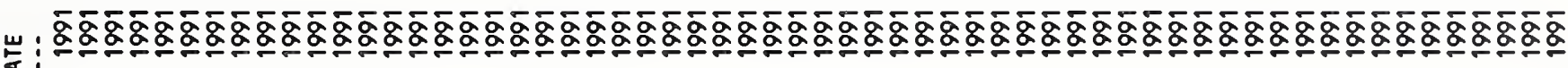
:

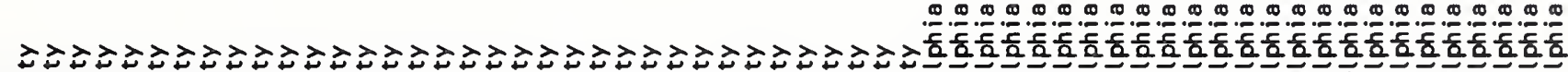

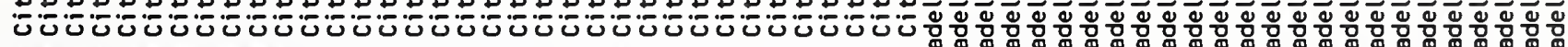

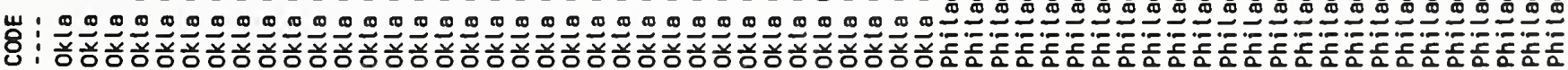

山㟧:

客密: 运蓆

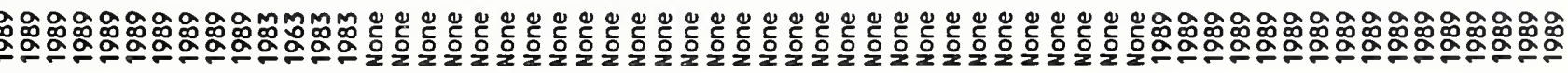

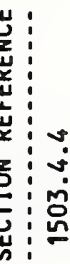

i.

nmim

n-t

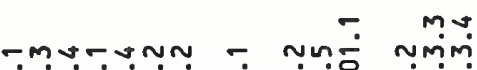
-

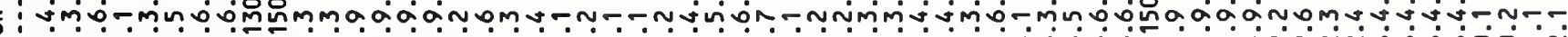

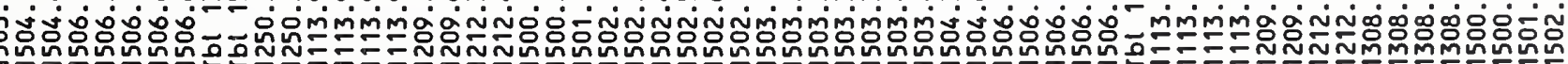

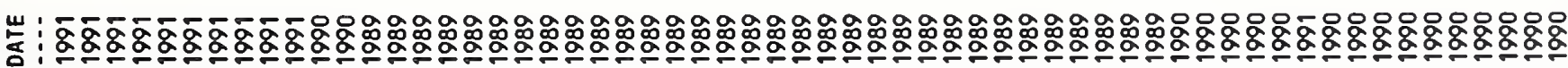

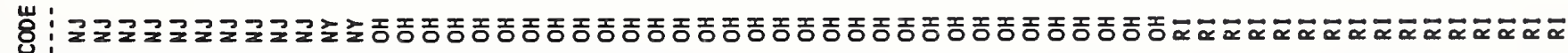

额 
$\simeq$ шّّ :

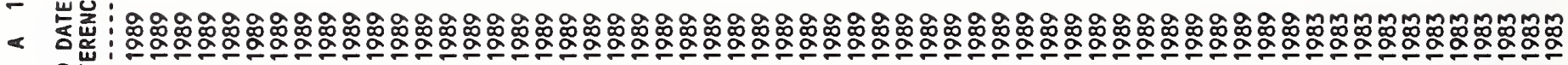
运崫 :

岁

य mujmomin

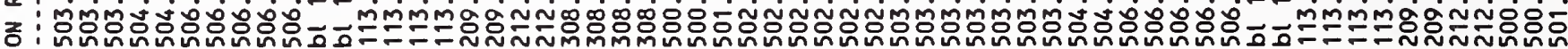

总

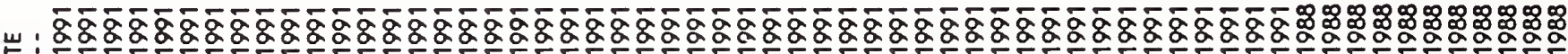
安:

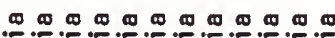

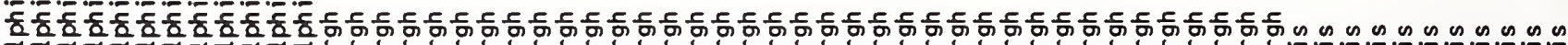

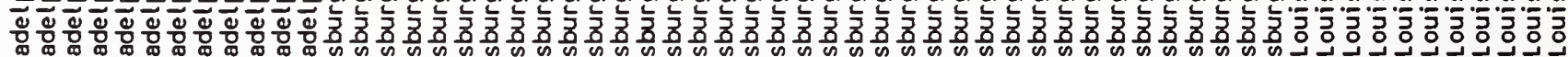

定

шّ山ّ :

:

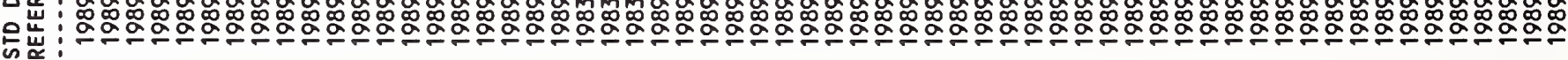

㟧

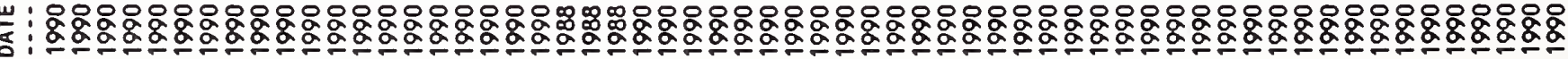

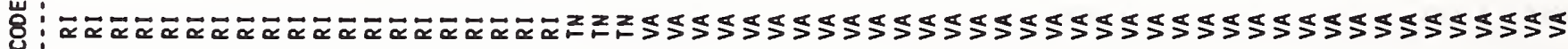

¿ัٌ

$\stackrel{\infty}{m}$ 


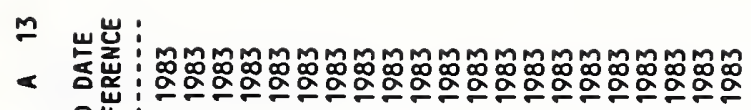
舟崖?

:

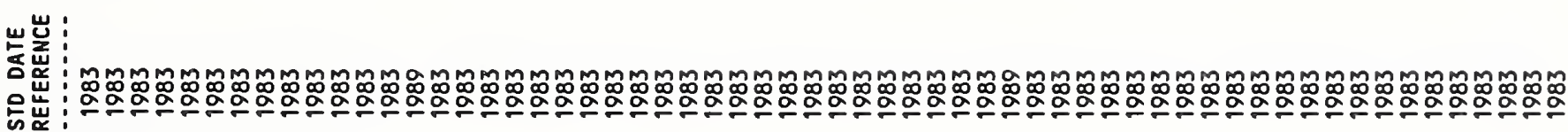

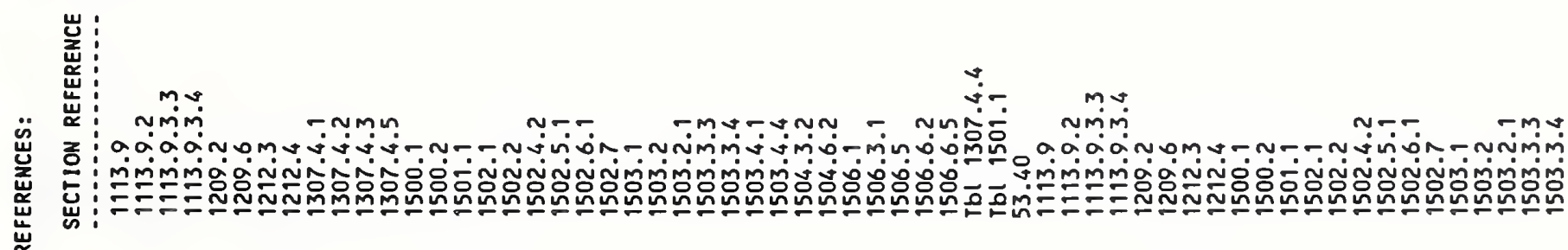

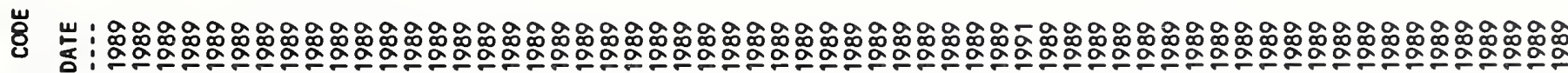

崖

$\infty$

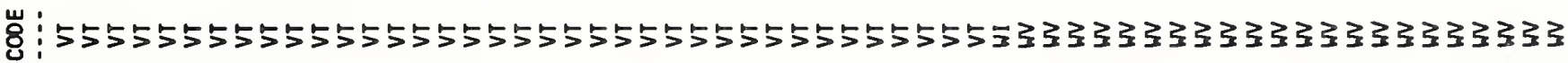

总

$\bar{\Xi} \quad \stackrel{\infty}{m}$ 


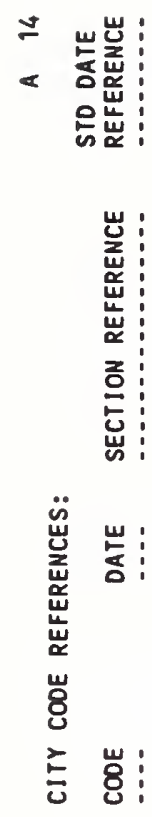

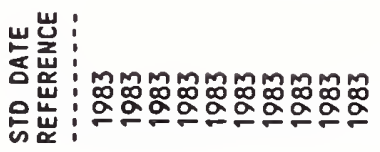

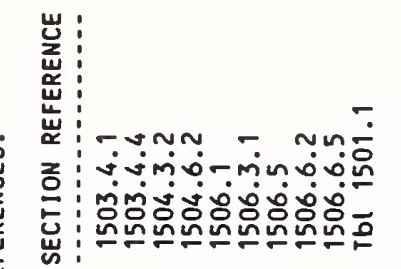

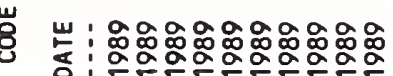

崖

岁:

嵌

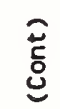

$\frac{\infty}{m}$

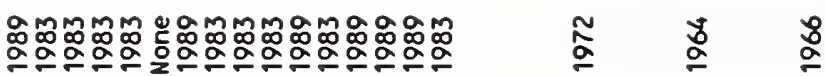

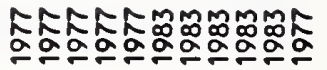

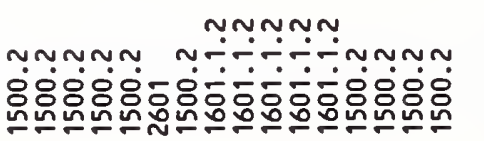

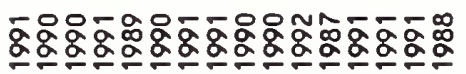

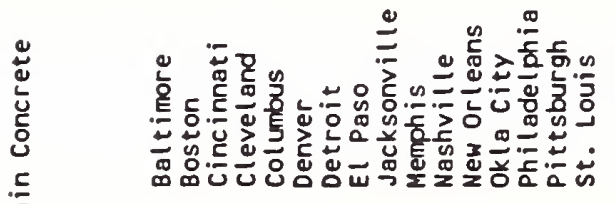

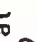

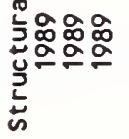

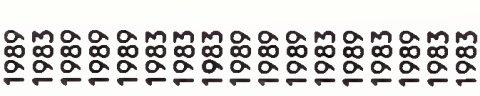

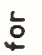

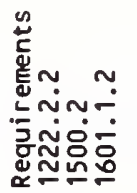

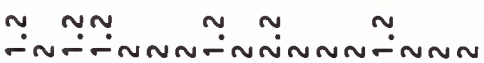

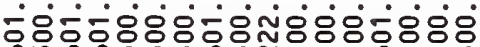

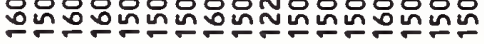

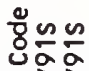

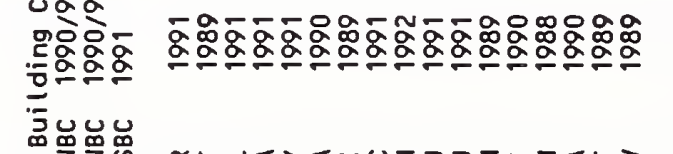

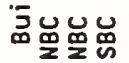

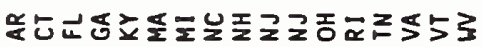

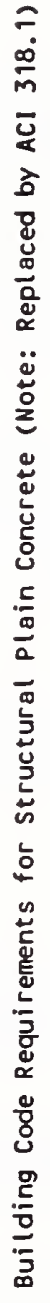

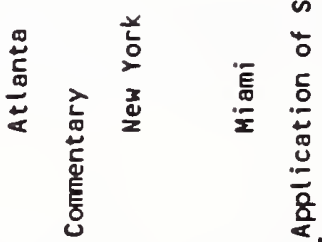

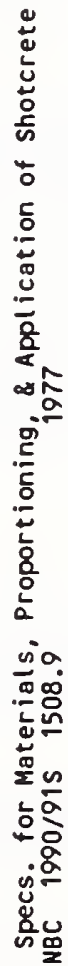
ด.9090.9090.

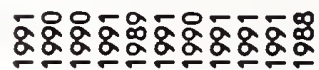

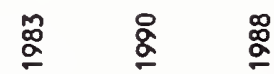

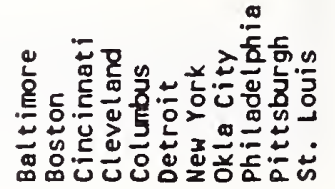
NลNAMNANNAN

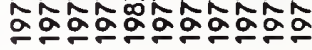

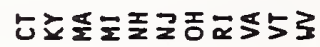

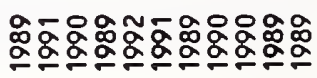

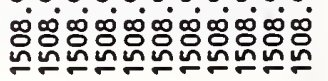




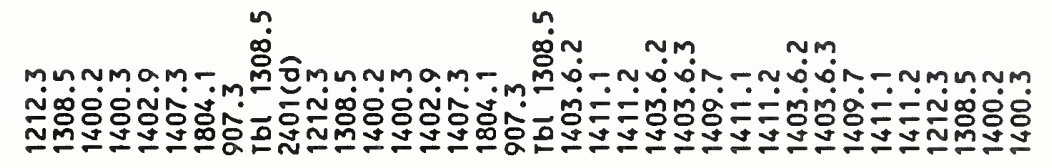

岂:

崖: 要

중

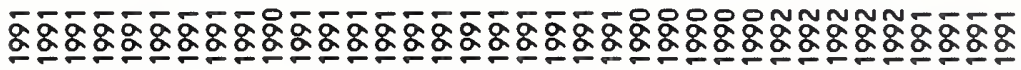

崖:

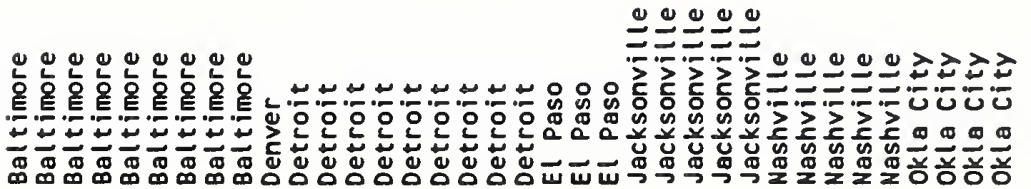

崖岂:

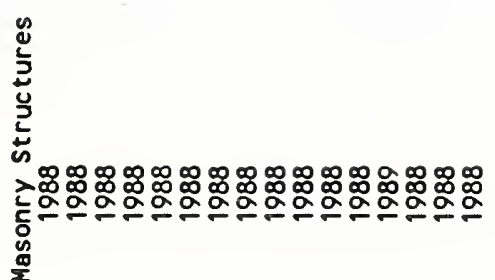

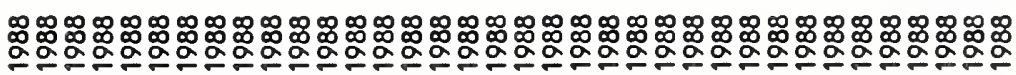

岁:

$\stackrel{2}{\circ}$

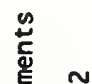

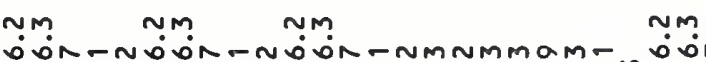

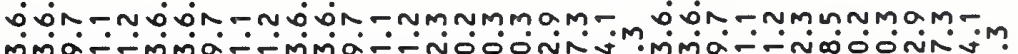

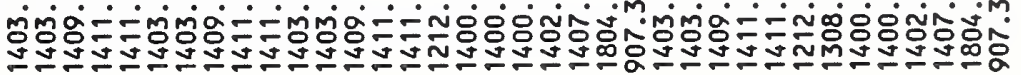

峞

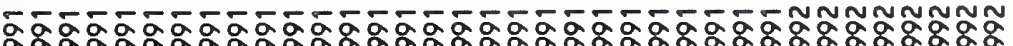

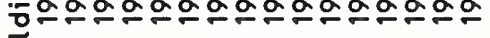

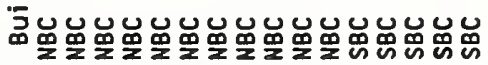




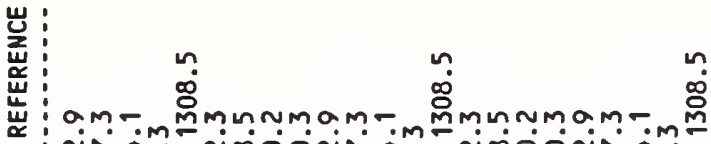
z:

总:

岕

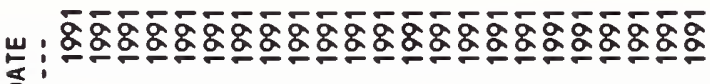
ธ์

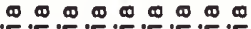

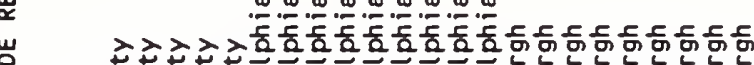

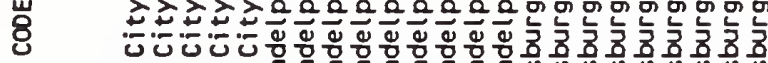
乞

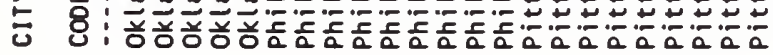

岂:

은

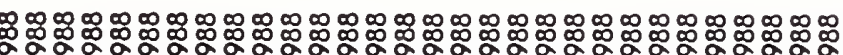
is $\propto$

嵌

岂

㟧:

in

ஸ்

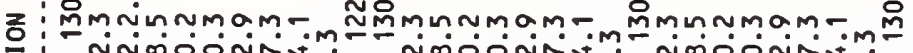

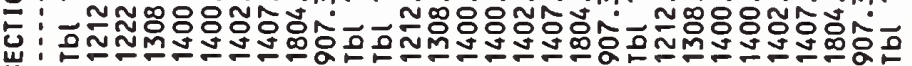

u

1

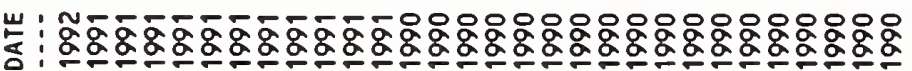

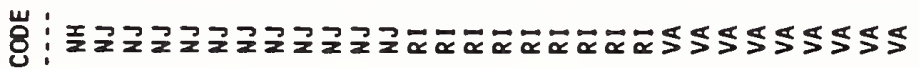

苍

$\underset{\mathbb{Z}}{\mathscr{g}}$

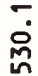

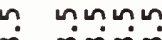

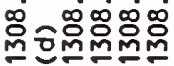

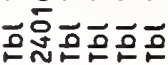

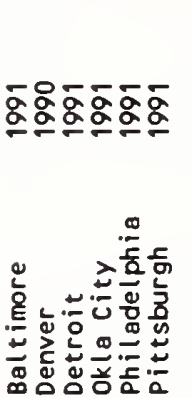

造

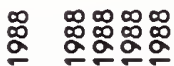

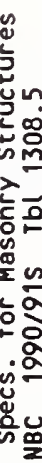

nฺn

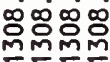

드응

ペロロロ

종양ㅇㅇ

폻

g
ปิน

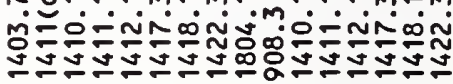

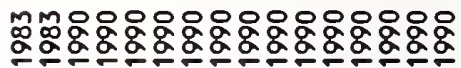

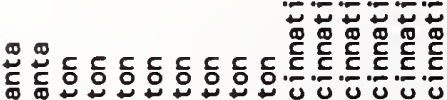
Ф

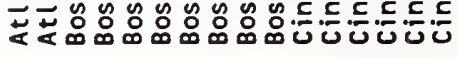

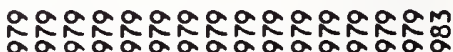

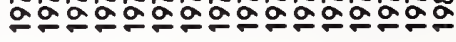

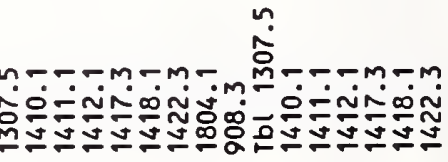

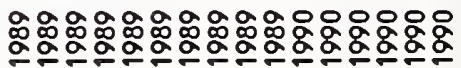

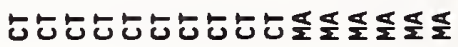




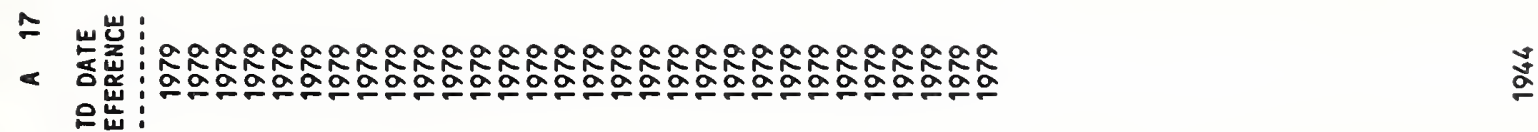
的崖:

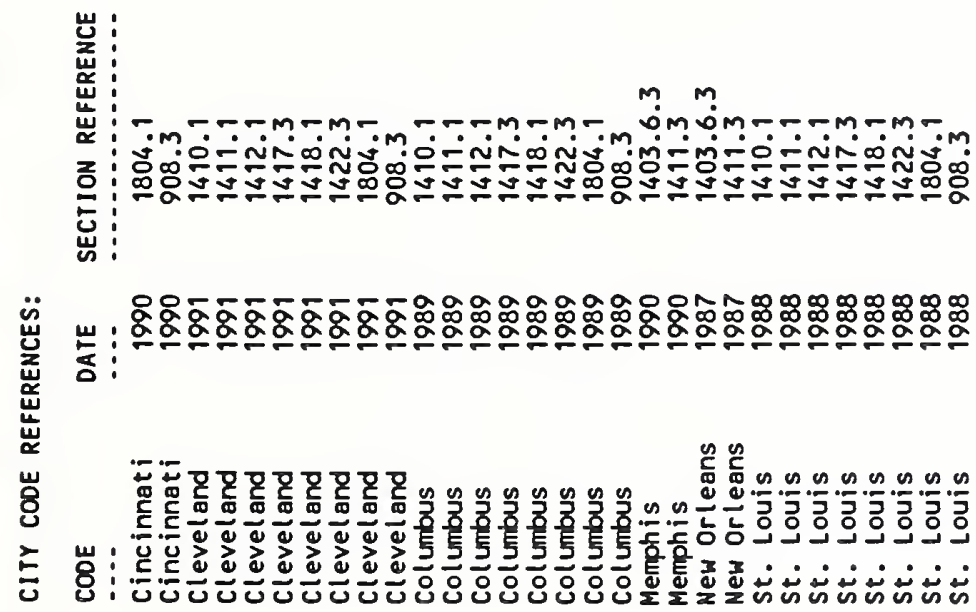

产

$\tilde{\alpha}$

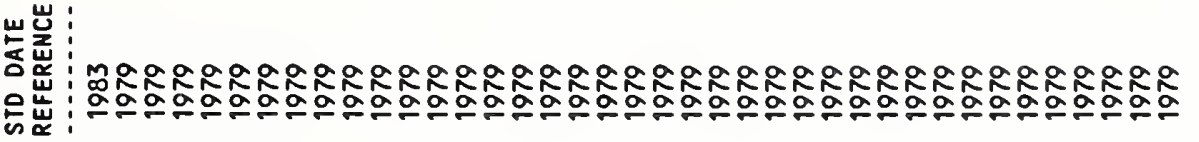

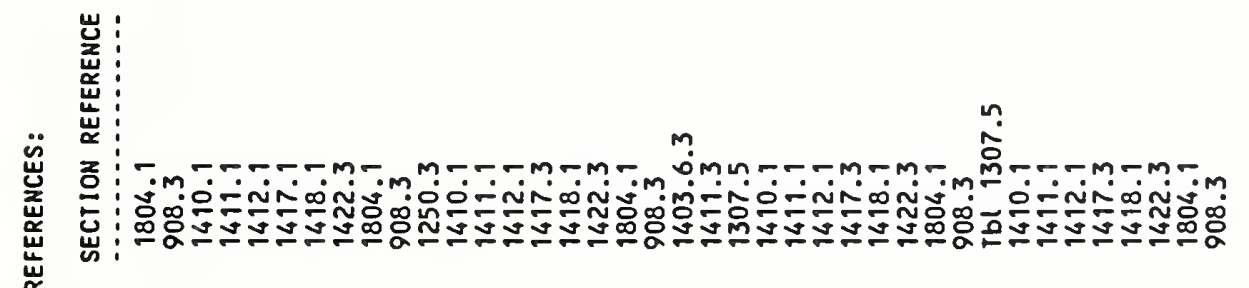

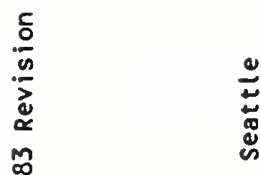

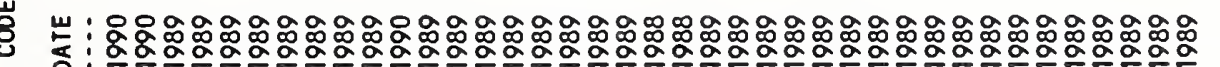

崖

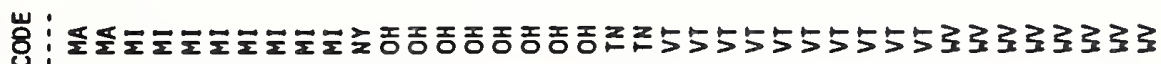

ㅇำ

ํํำ

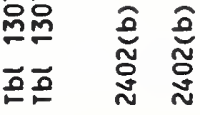

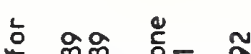

$\dot{\square}$

형

ヒ5 离品

할

ڤ્ڤ

产

छ

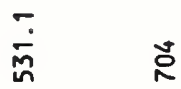




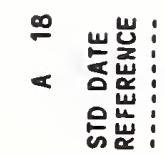

:

芯岩:

岩

힝

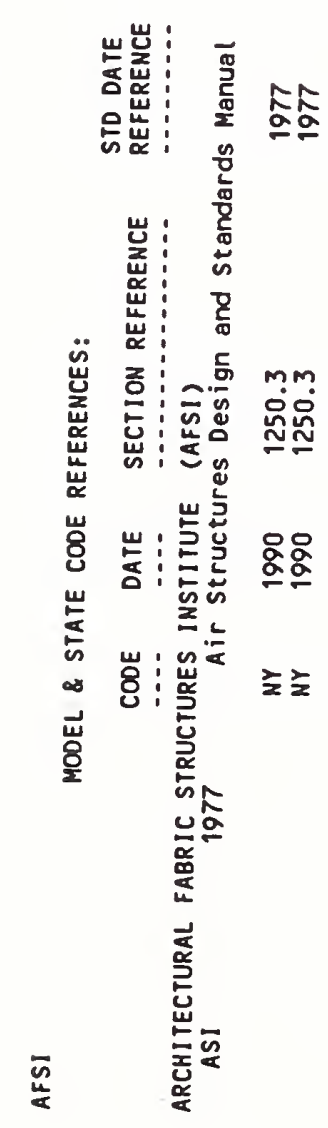




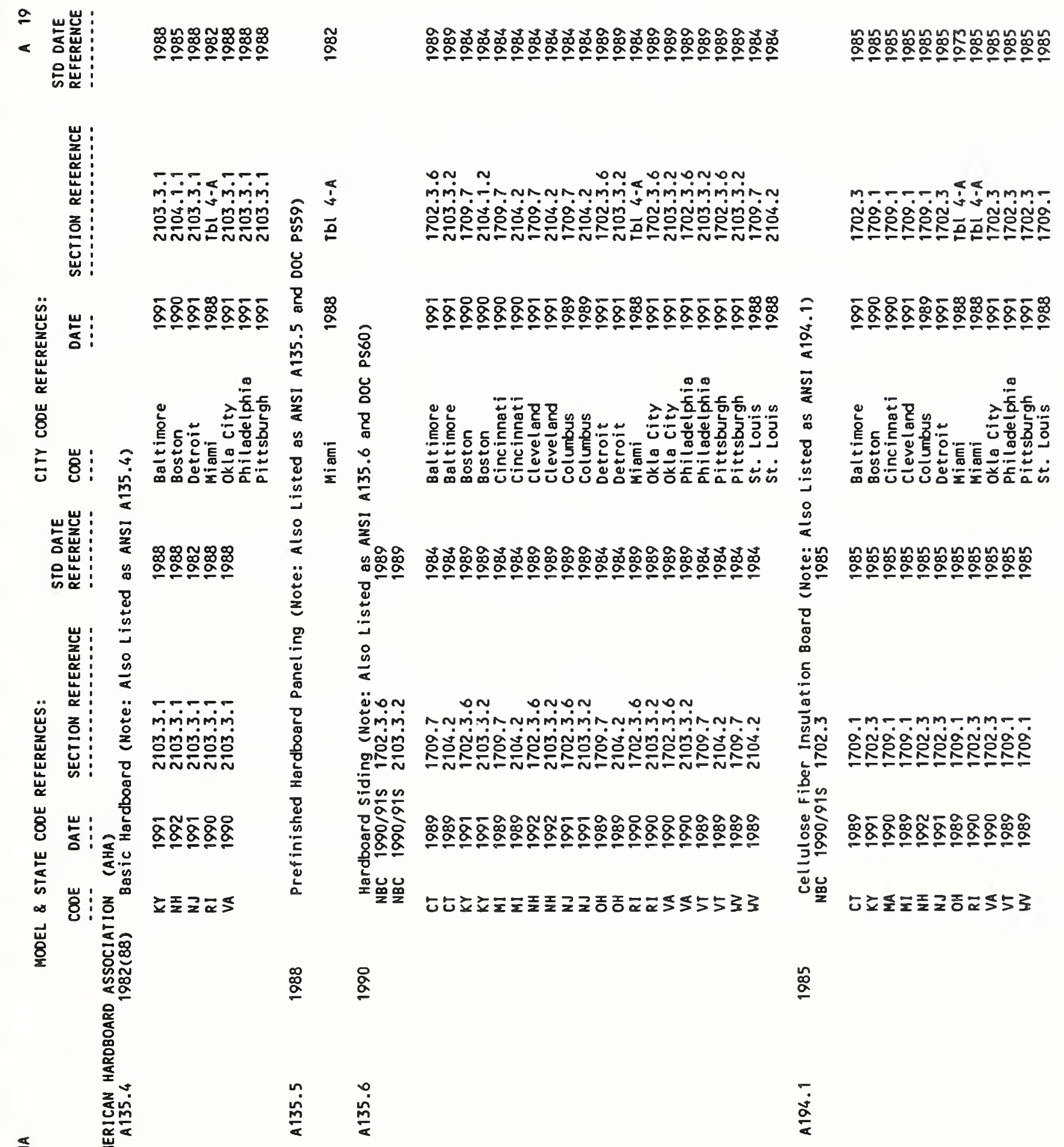




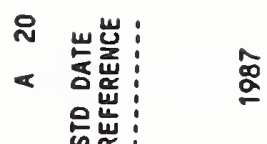

$$
\begin{aligned}
& \text { 岁: 䓵 } \\
& \text { 离 } \\
& \text { z } \\
& \text { 总: 芯 }
\end{aligned}
$$

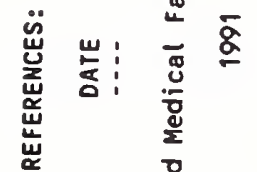

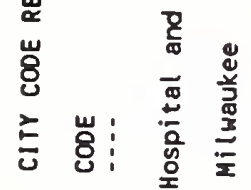

$$
\begin{aligned}
& \text { 岕岕: 它 }
\end{aligned}
$$

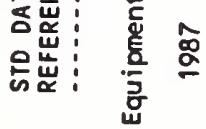

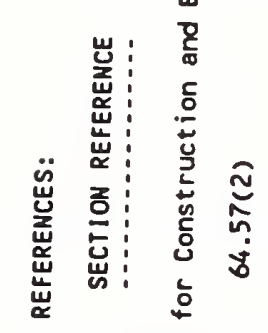

$$
\begin{aligned}
& \text { 岧岕: }
\end{aligned}
$$

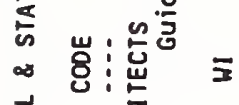

$$
\begin{aligned}
& \text { 펼 폴 } \\
& \text { 䇏鱼 } \\
& \text { 崖 } \\
& \text { 喜品 }
\end{aligned}
$$




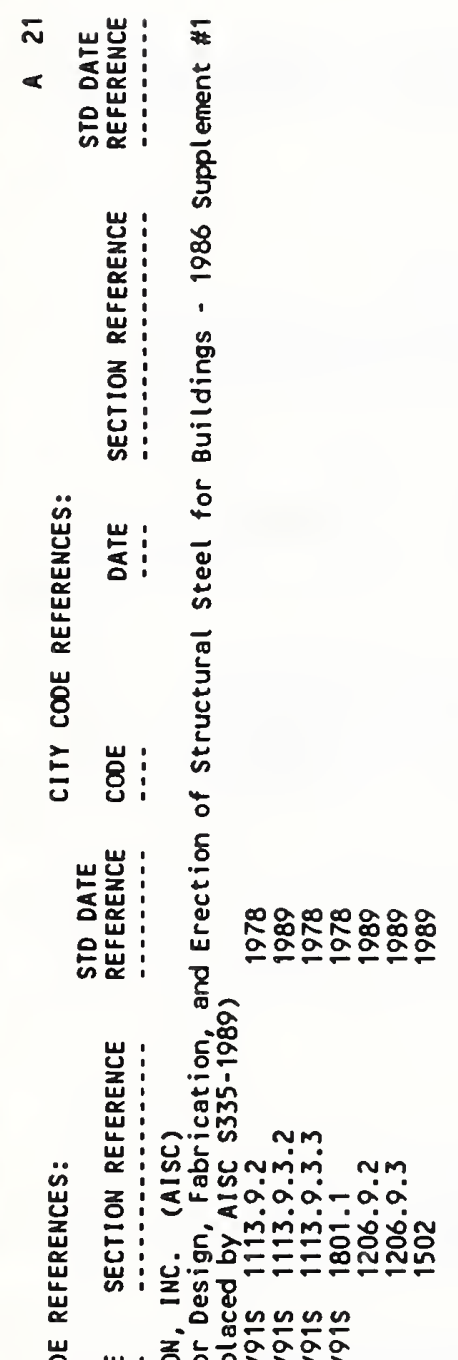

8्

岕

닝 : 远

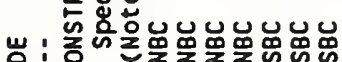

8 :

壳

岂

w

疍

些

$\stackrel{\leftrightarrow}{\alpha}$

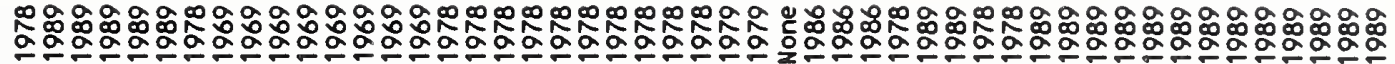

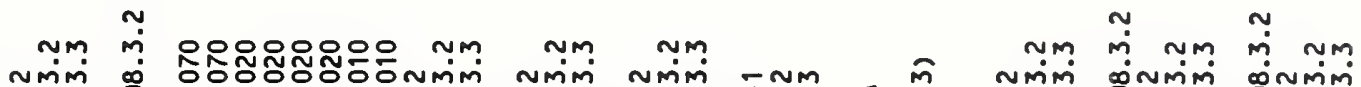
Nmm Dै-

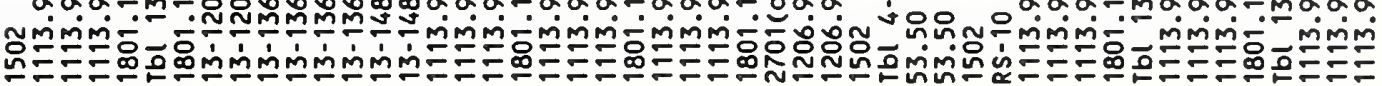

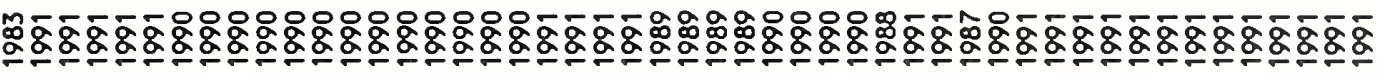

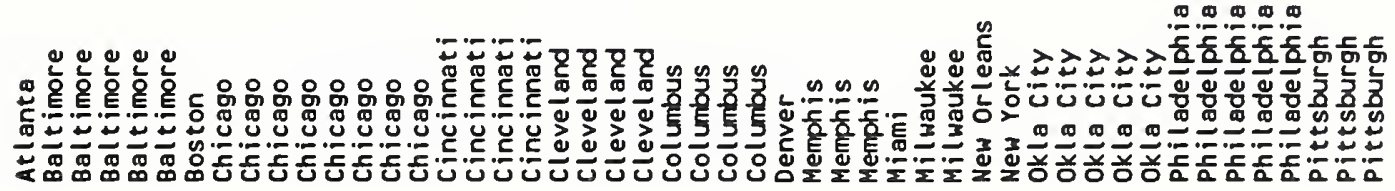

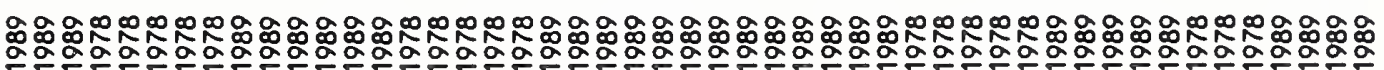

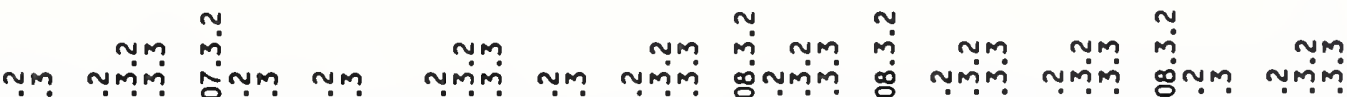

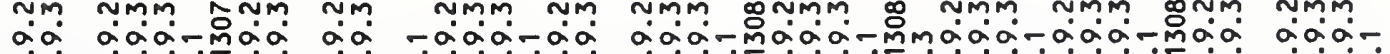

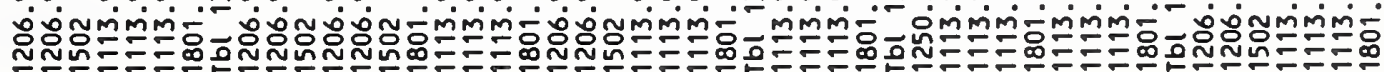

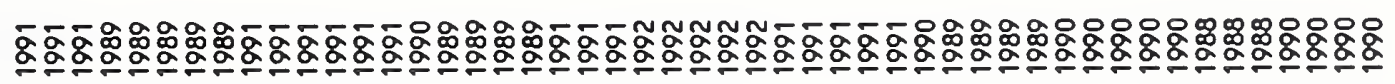

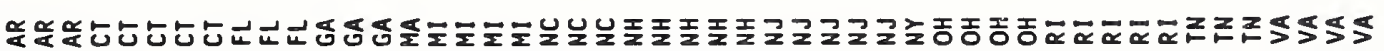


$\approx$ 떤 :

<

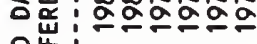
응

: :

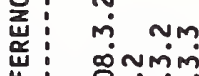

岃 rmaaa-

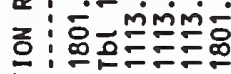

出:

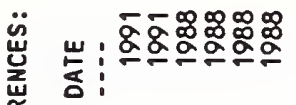

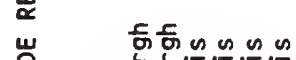

8्ठ

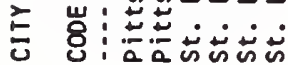

崖:

完

岀:

تิ

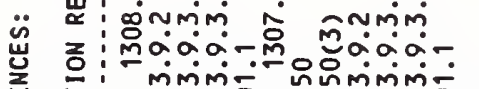

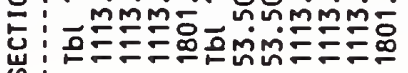

के

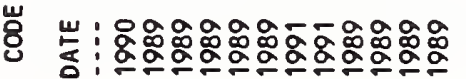

䋇

岂: \55555ミ33333

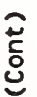

$\stackrel{ఖ}{2}$

్ָల్ల

产

造

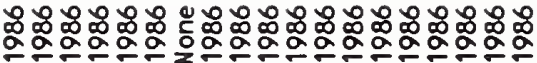

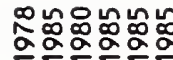

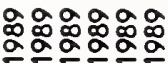

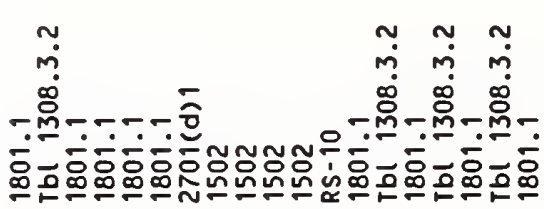

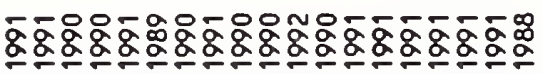

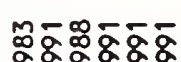

mंडिmंm

象

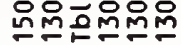

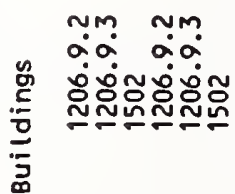

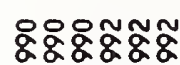

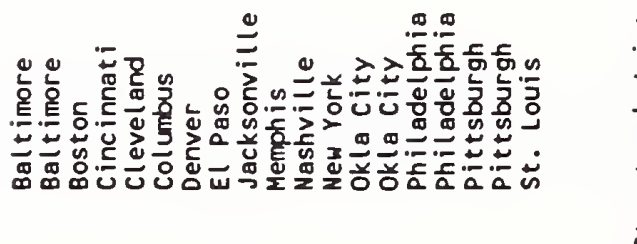

苂

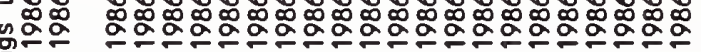

吾

in

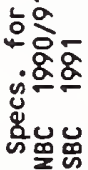

กี กั.

mั่

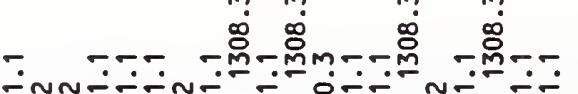

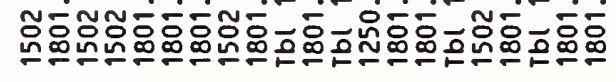

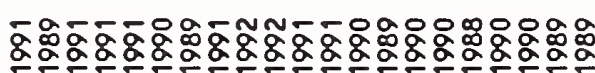

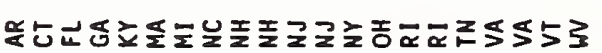

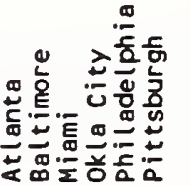

里品

o o o

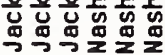

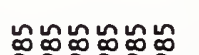

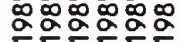

क여염

mimimimim

minimimim

品品哈

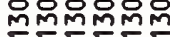

acai-

mimin:

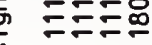

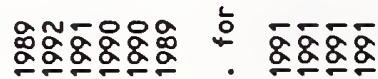

는줋

立立立立

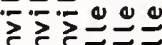




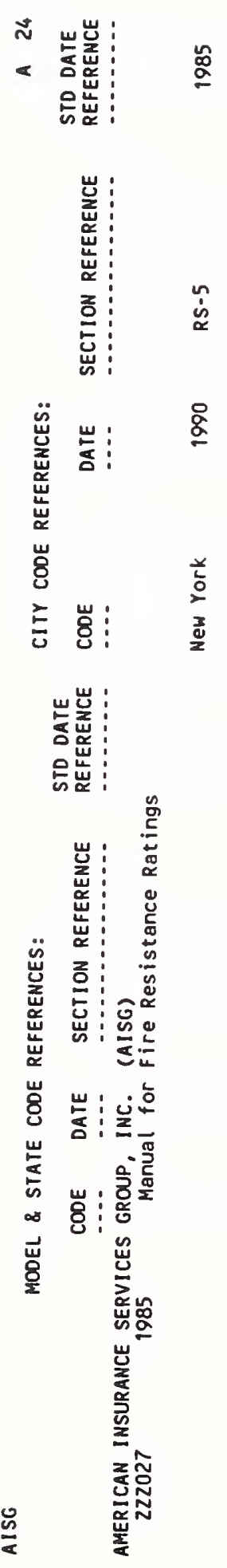


๙

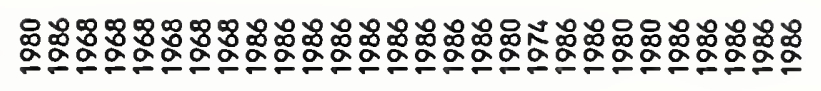

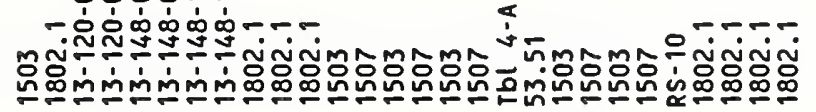

융슝융융응

넌 :
$\frac{2}{\frac{8}{2}}$

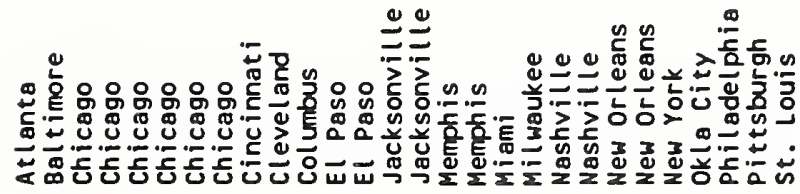

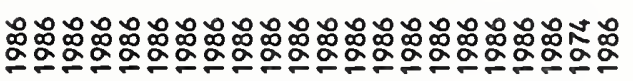

㩆:

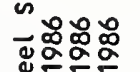
岕

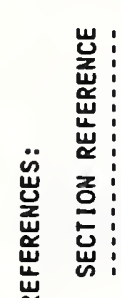

$$
\text { 若 }
$$$$
\text { 웅 }
$$

\section{o-ju}

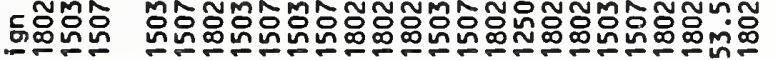
品

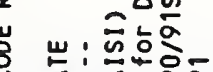$$
\text { 㟥 }
$$

岩:

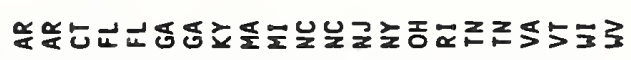

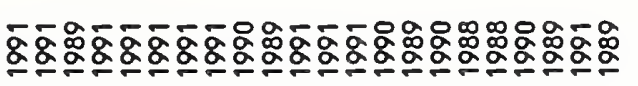

岕察

울

중

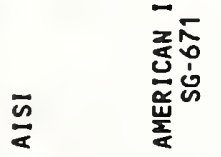

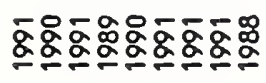

$\stackrel{\circ}{\circ}$

$\dot{N}$

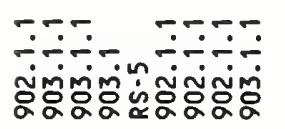

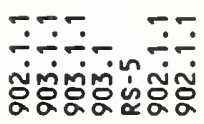

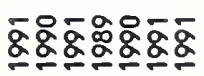

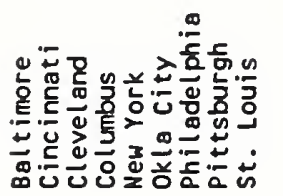

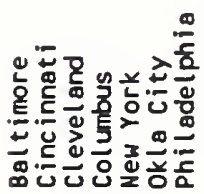

象。

요욤욤요

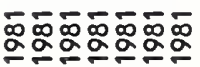

岕

$\stackrel{5}{\circ}$

음

:

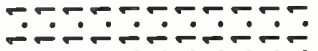

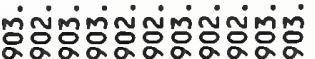

产乐

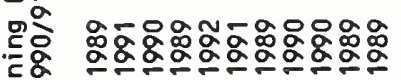
造

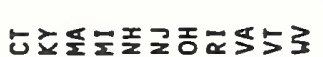

$$
\begin{aligned}
& \text { 岕 } \\
& \text { 웅 } \\
& \text { 웜. }
\end{aligned}
$$

$\div \div \div \div$

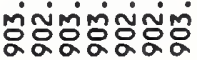

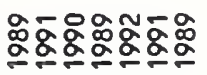

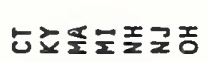

⿷匚

$\check{\sim}$ 


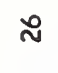

$<$ <

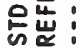

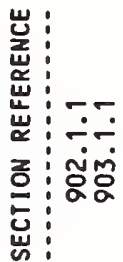

峞

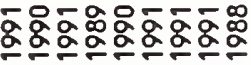

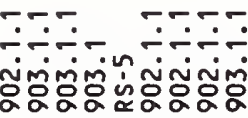

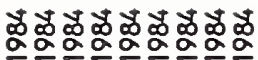

$\underset{\sim}{\alpha}$

岁

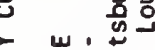

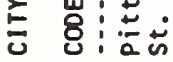

宸:

的:

㟧:

苨

z :

กิกंm

出 응ㅇㅇ

$\therefore$

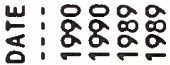

岁: ‘枋

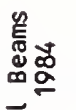

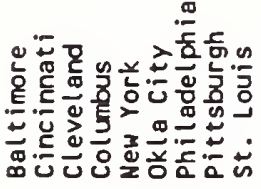

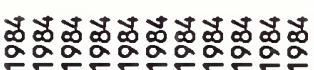

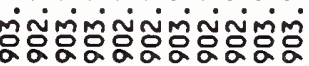

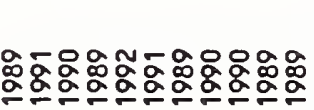

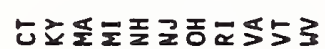

范

융 울

$\stackrel{\bar{\Phi}}{\underline{a}}$

$\frac{5}{4}$

ลิ

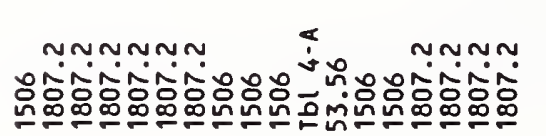

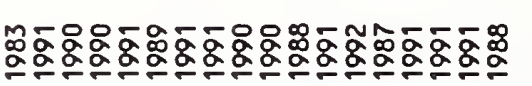

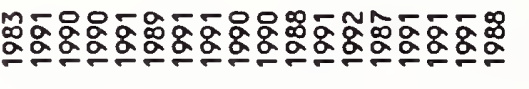

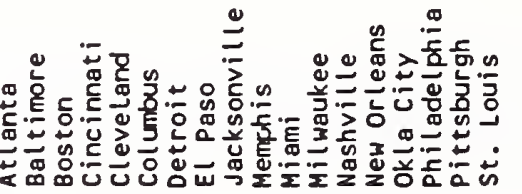

造

$\overline{\mathrm{d}}$

峁

inم

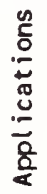

ฐก

ป气ํํำ

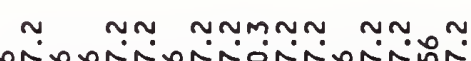

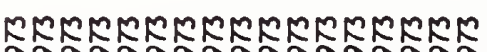

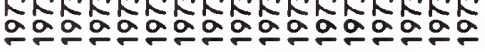

药

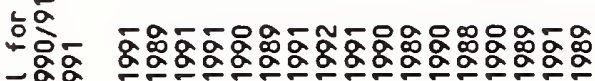

๓⿴囗十

要鮕

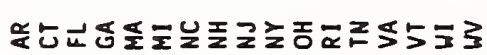

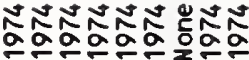

ई

ஸั

产

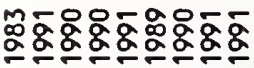

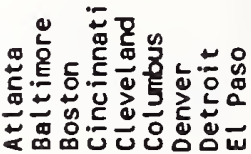

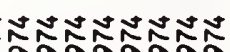

으으으흐으으웜

兽

t- $\quad-\overline{-}$

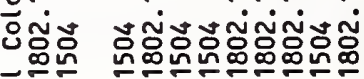
बे 站

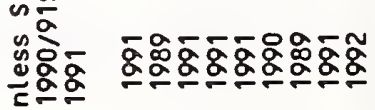
密品

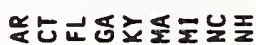




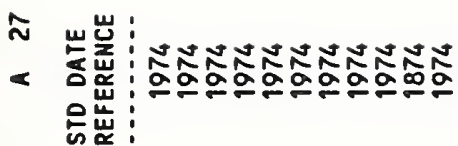

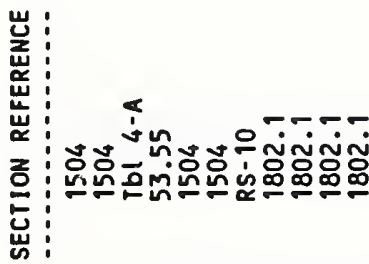

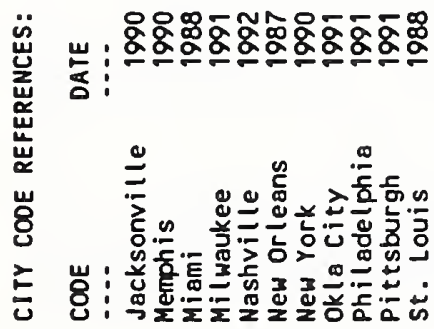

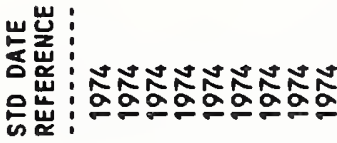

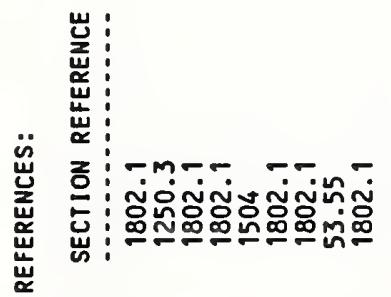

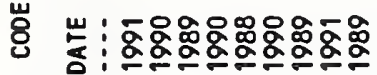

放

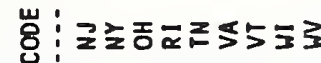

㝕

$\stackrel{\stackrel{0}{0}}{\mathbb{N}}$ 


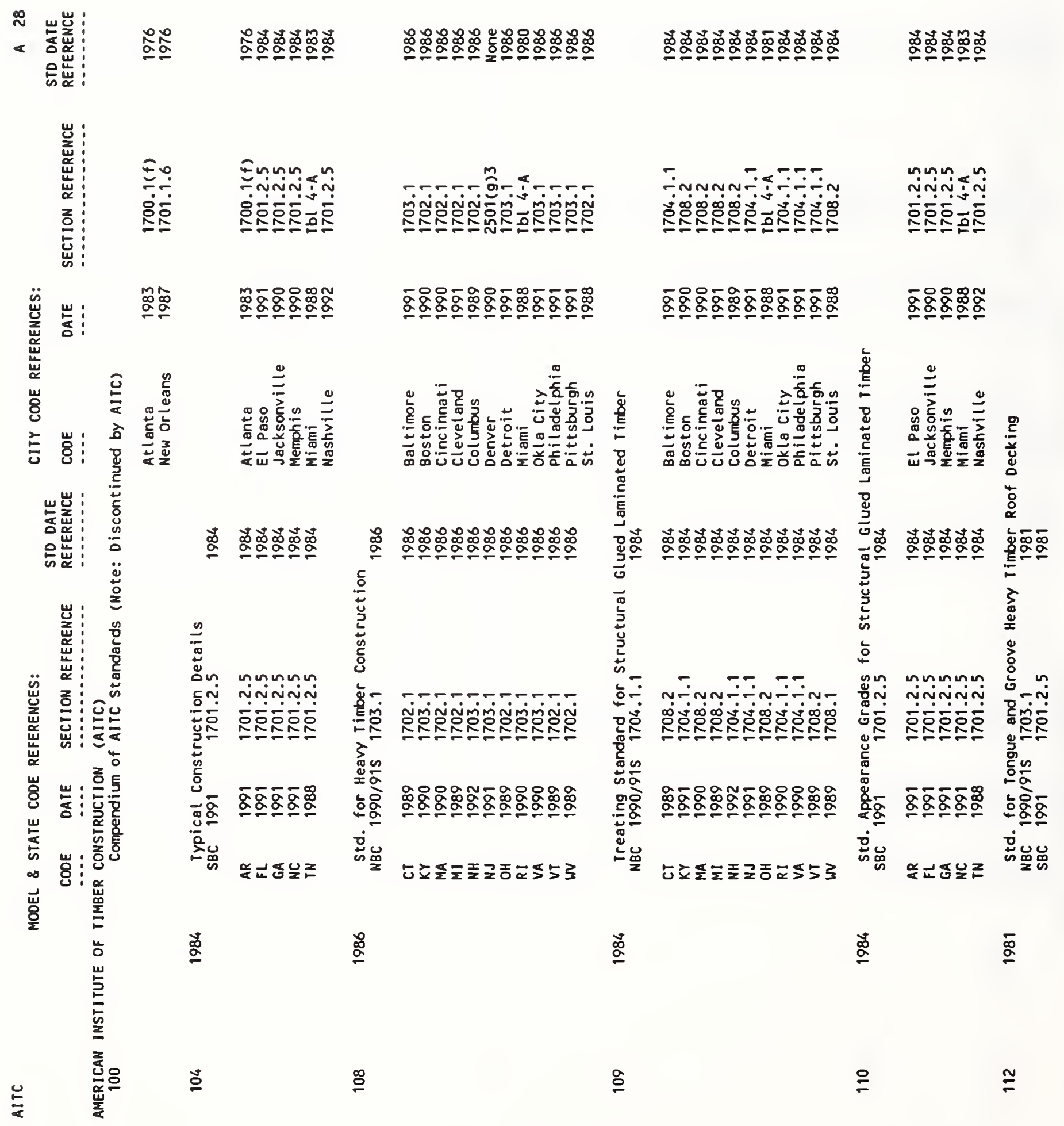



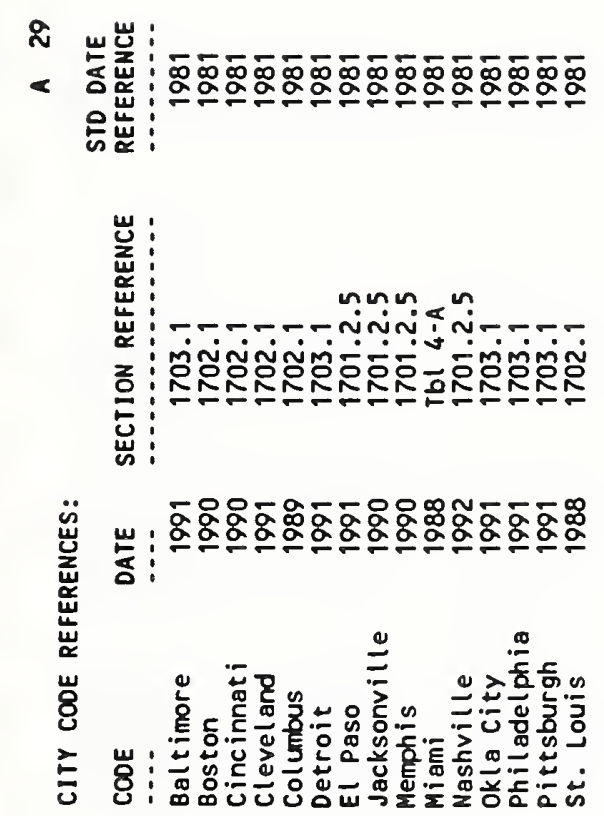

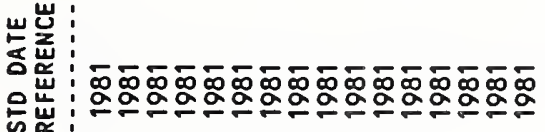

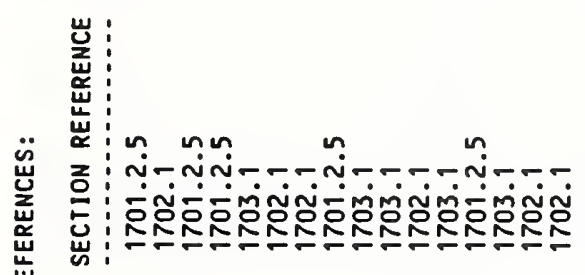

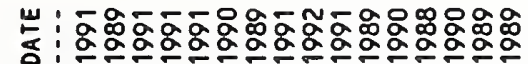

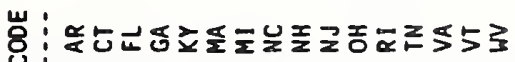

㟧

ํㅗㅇ

$\cong$ mammañ

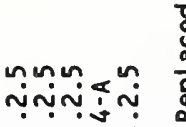

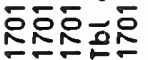

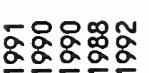

-

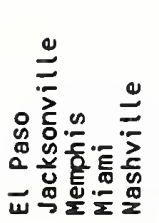

兽

-

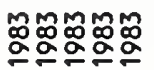

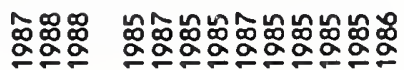

nunumin

nininin

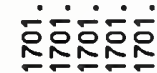

ธे

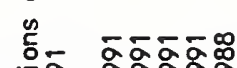

包金

惫 떤정존

Е

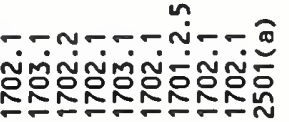

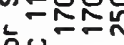

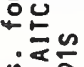

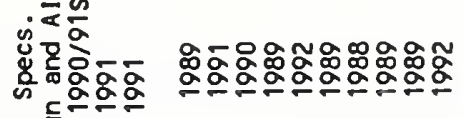

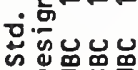

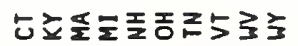

shing

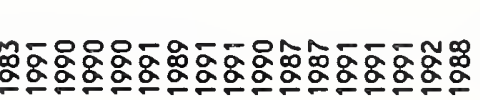

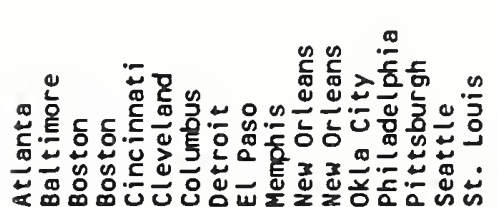

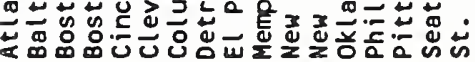

$\stackrel{m}{g}$

$\stackrel{m}{=}$

$\cong$

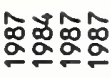

nุำกิ

옹ำ

ㅇㅑㅀㅀㅜ웜

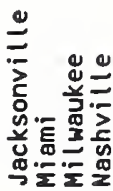

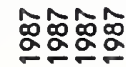

焉

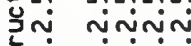

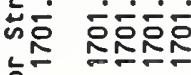

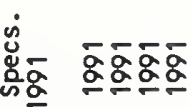

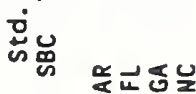

ฒิ

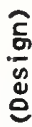

$\cong$ 


\section{店}

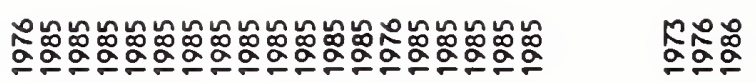

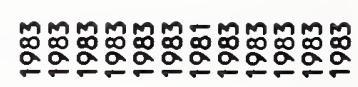

触:

.

山्山

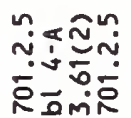

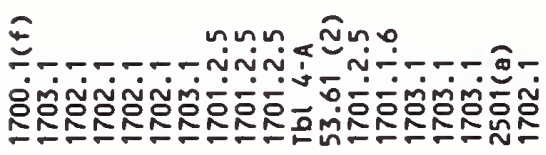

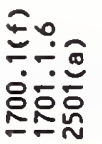

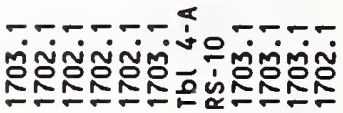

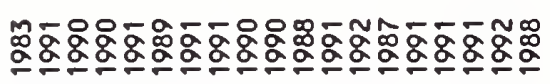

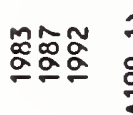

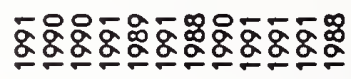

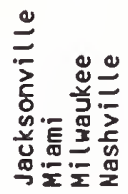

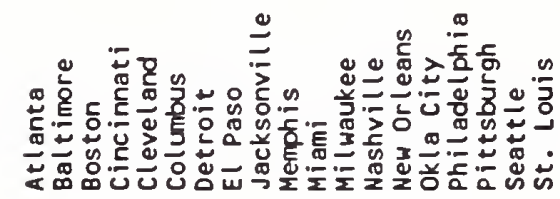

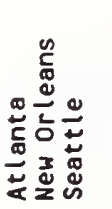

$\overline{\frac{n}{\alpha}}$

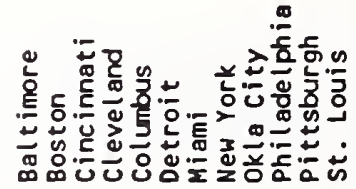

产

额

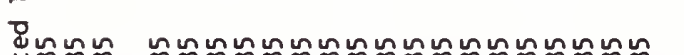

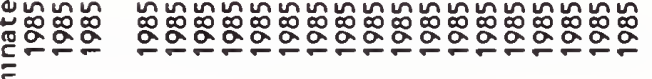

总

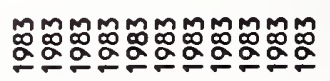

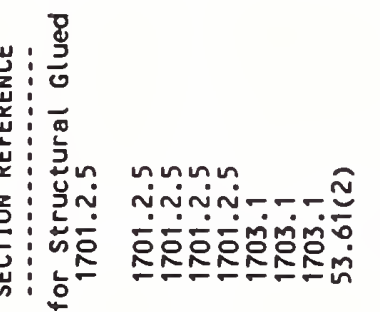

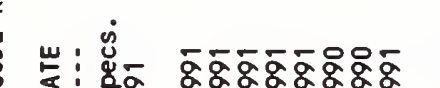

弯

㟧

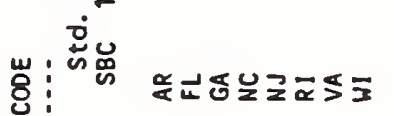

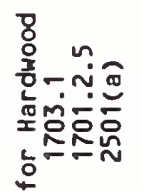

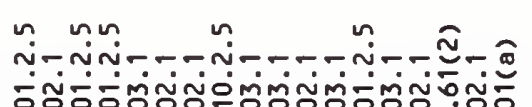

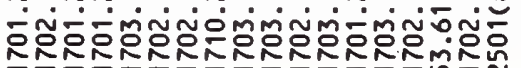

@

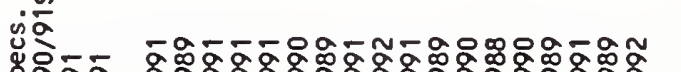

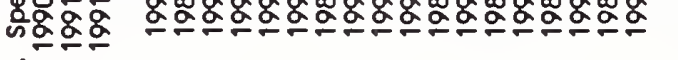
离品朂

훙

$\stackrel{\infty}{\circ}$

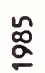

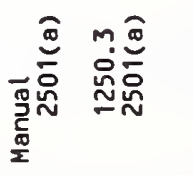

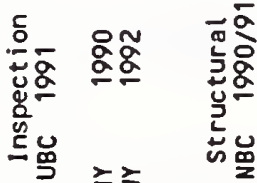

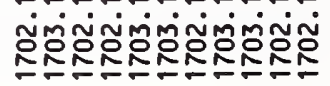

离

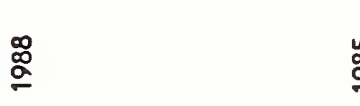

2

$\stackrel{\mathrm{a}}{\mathrm{g}}$

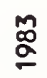

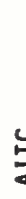

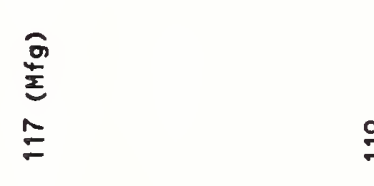

$\stackrel{9}{=}$

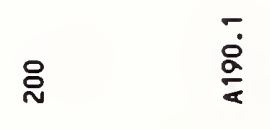




\section{- 造岕}

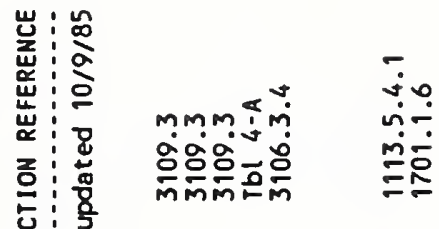

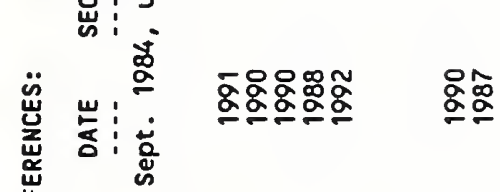

.

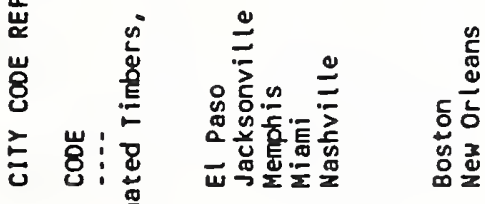

岂: :

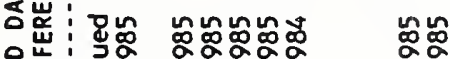

응

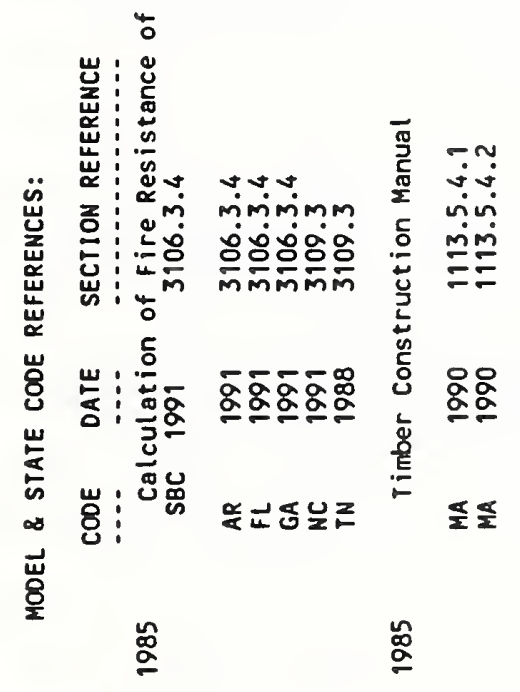

$0 \quad \frac{\bar{N}}{2}$ 


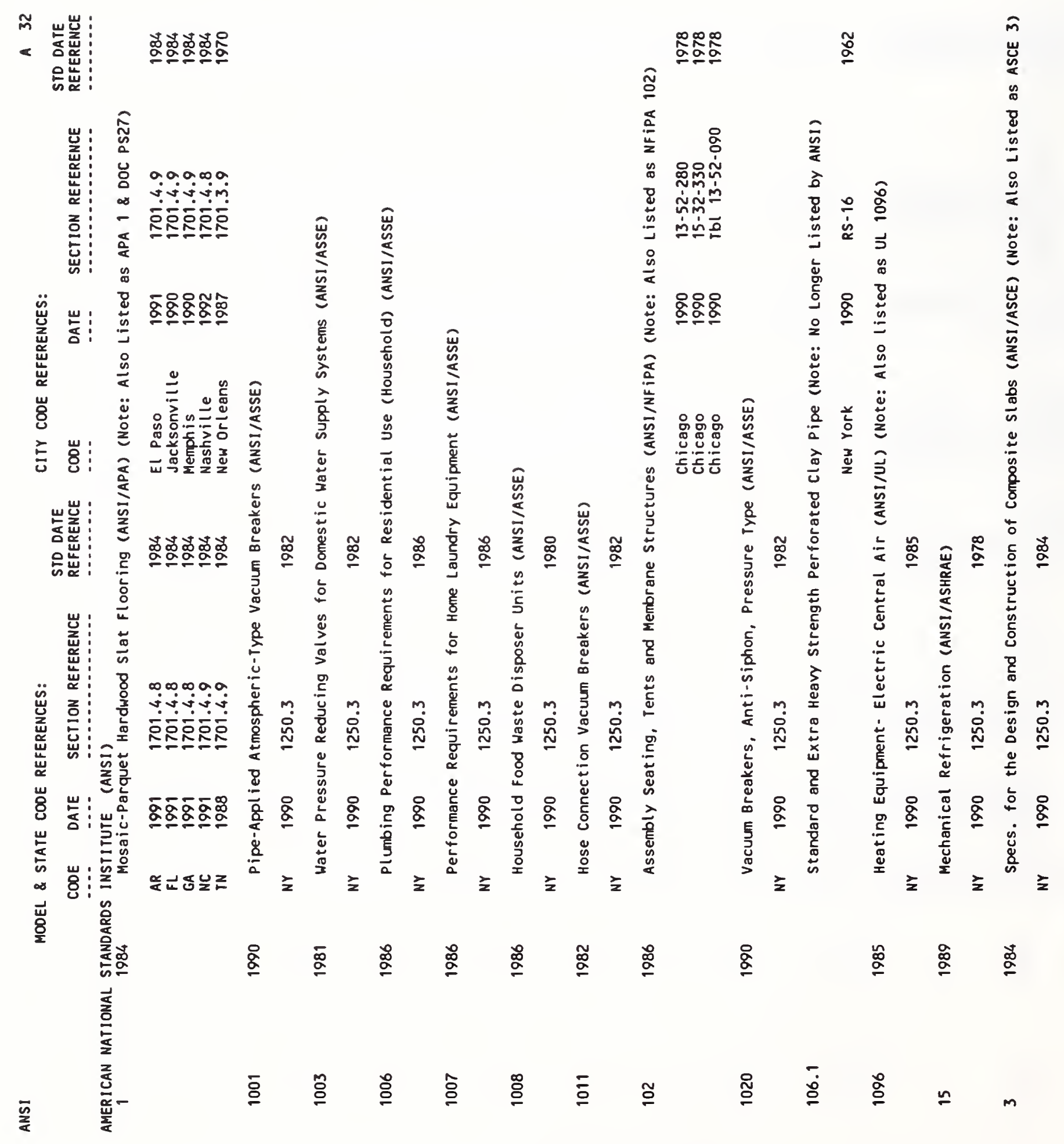


in m

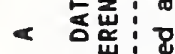

㩆: 兽

状:

差:

峞:

흘

跑:

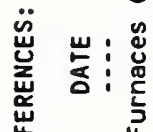

峞

¿

岁:

แ山ّ :

实

㩆:

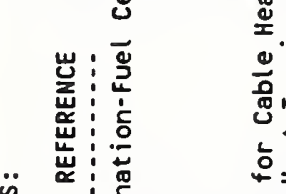

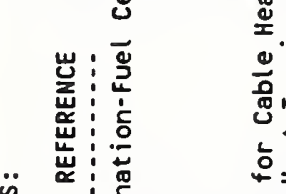

졸

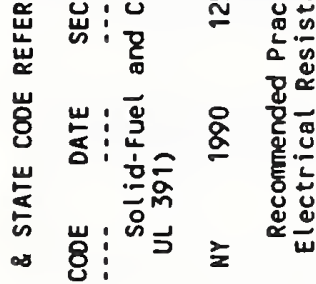

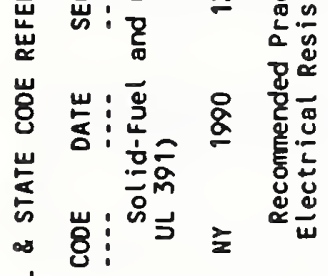

풀 $\stackrel{2}{2}$

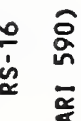

둴ㅆ

要

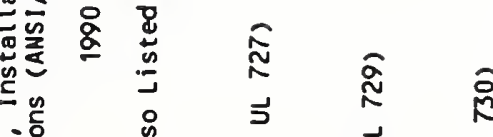

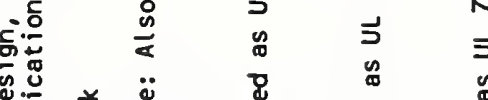

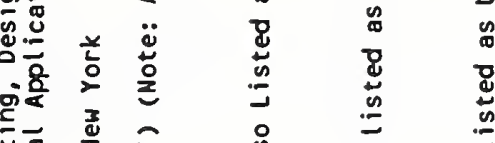

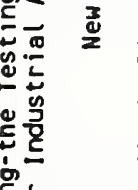

高

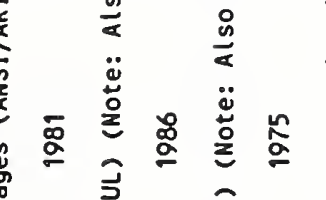

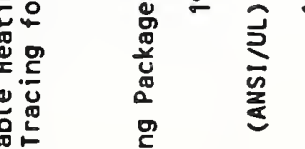

变

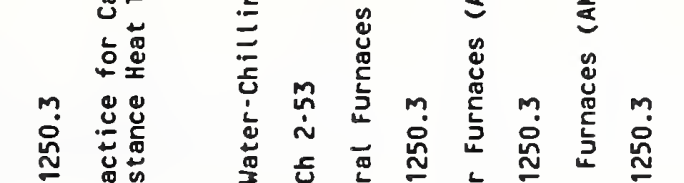

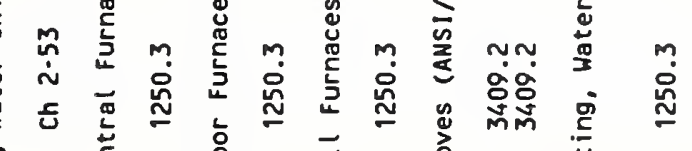

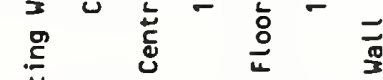

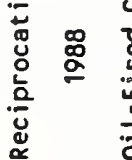

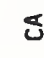

s

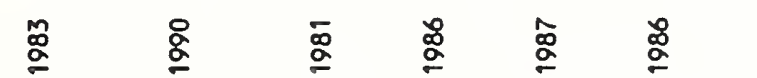

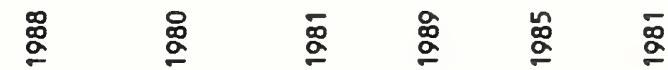

$\overline{\frac{\pi}{2}}$

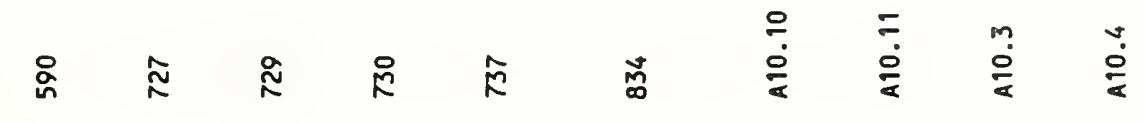

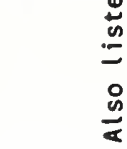

范

$\stackrel{\square}{\circ}$

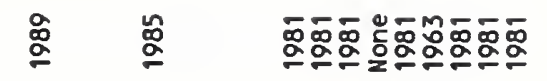

$\frac{0}{8}$

至

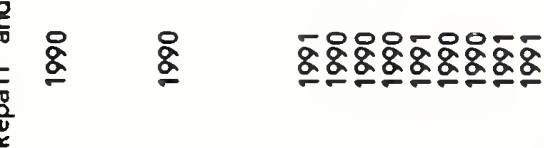

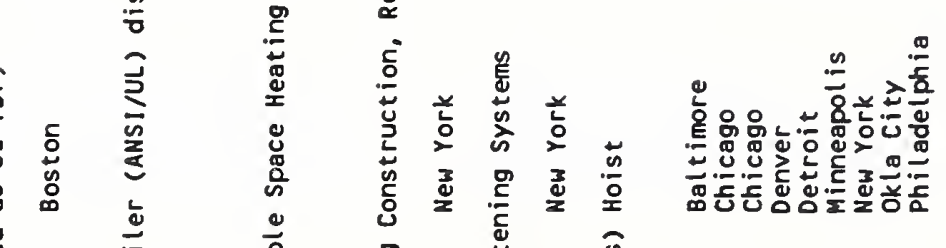

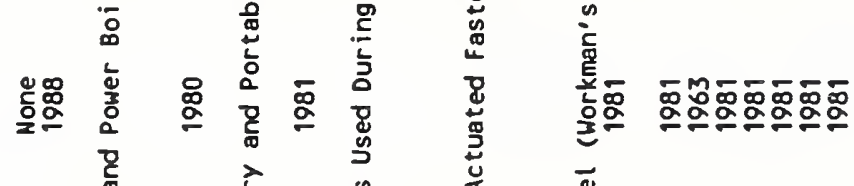

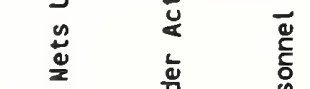

弯

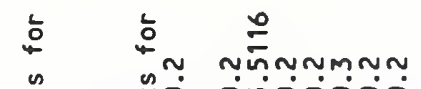

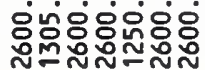

产 趂

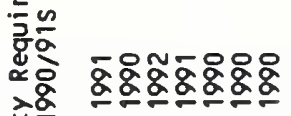

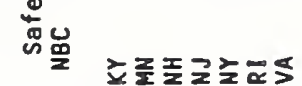




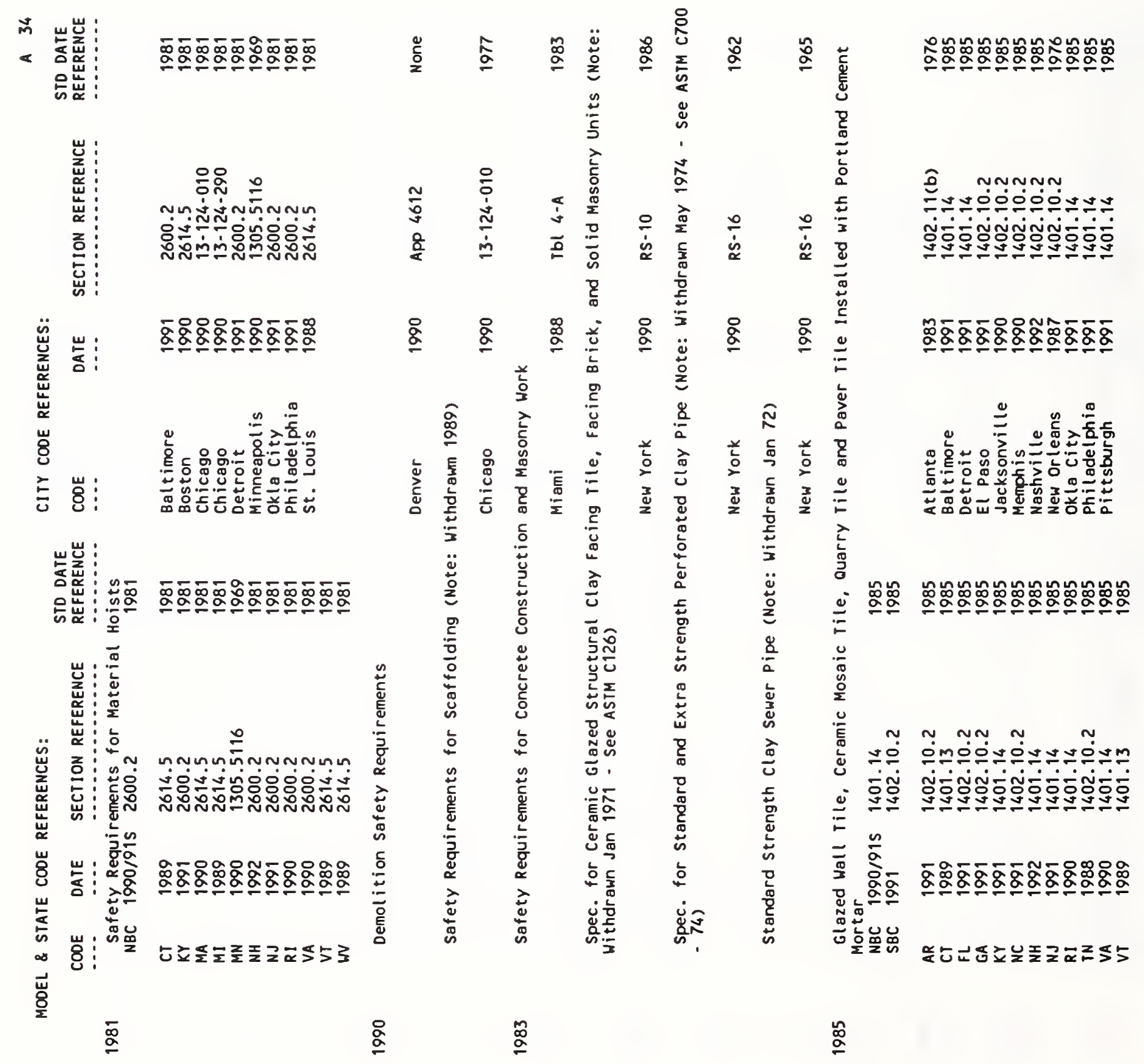




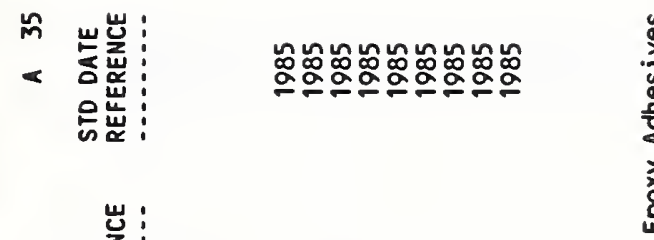

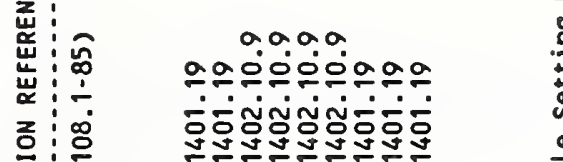

5

出:

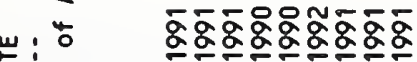

落:

品

岁

‡

崖

运: .

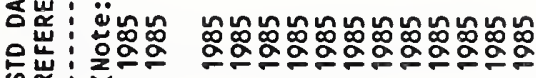

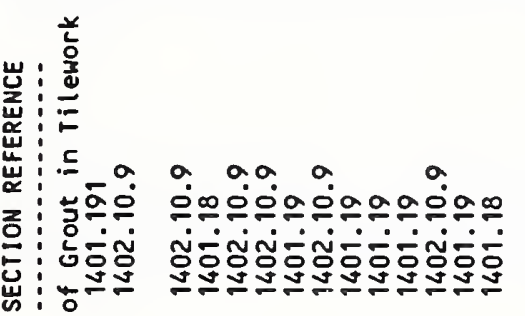

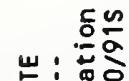

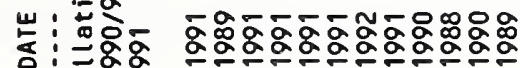

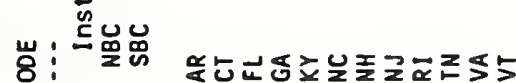

齐

తิ

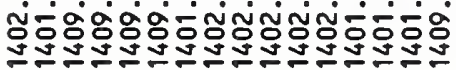

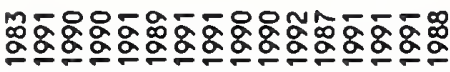

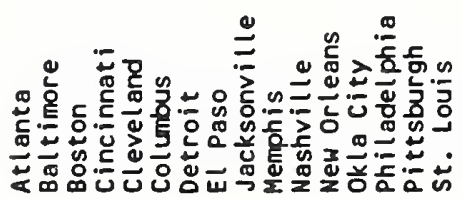

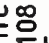

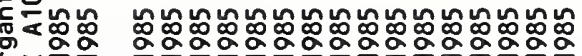
क⿺⿻一⿰冫⿰亅⿱丿丶丶⿱⿰㇒一乂 高告

产岕

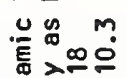

ड़

$\div$

동ำ

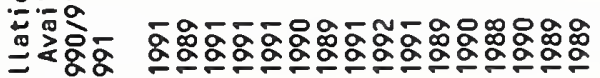

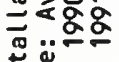

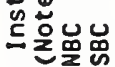

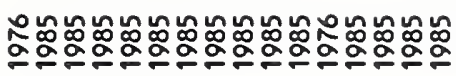

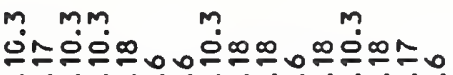

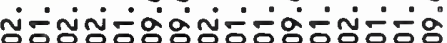

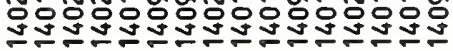

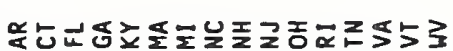

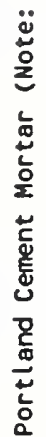

蔍

要

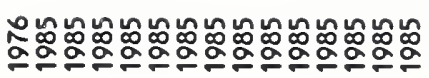

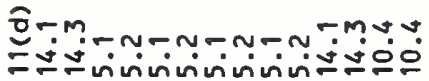

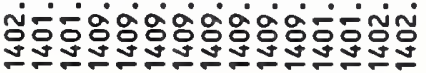

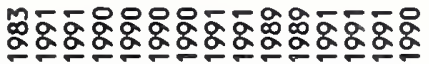

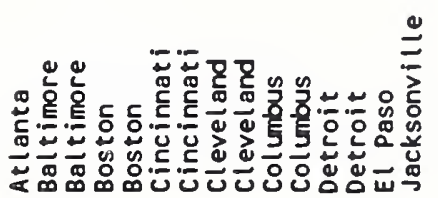

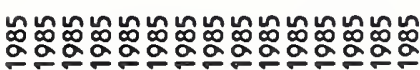

5. जั: 妾高 उั0-mo

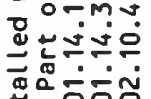

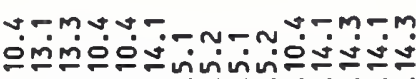
กั-ส่ร்

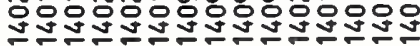

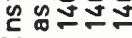

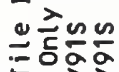

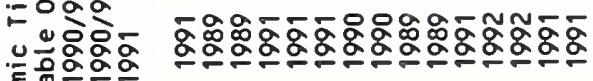

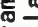

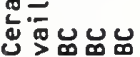

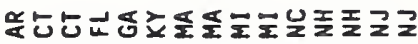

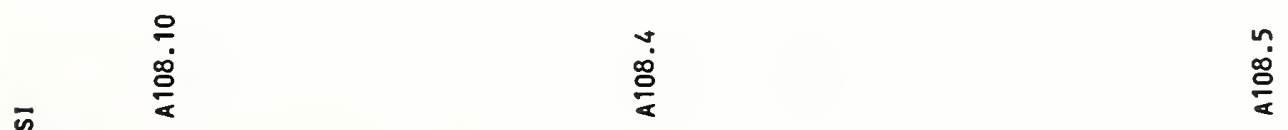




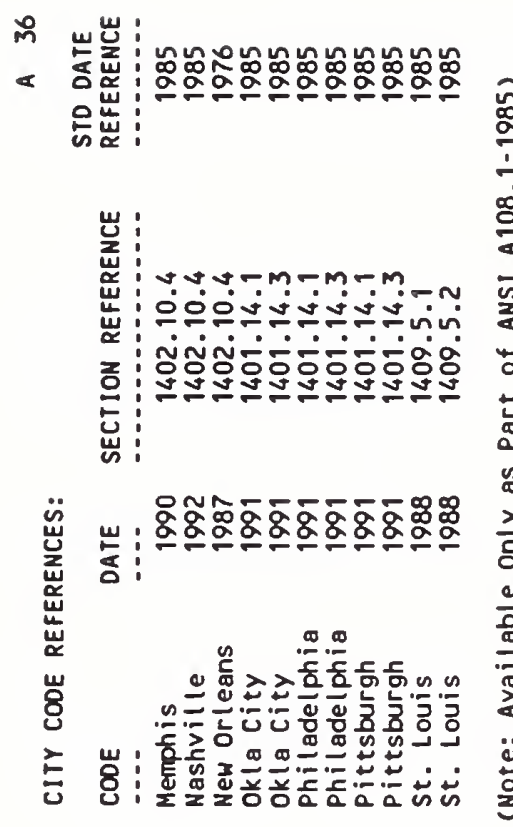

崖: : 究

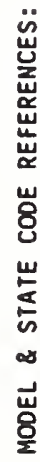

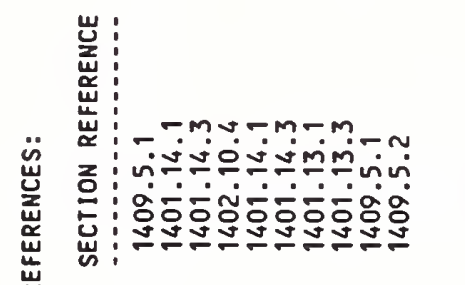

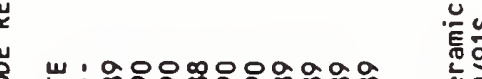

$\frac{\omega}{5}$

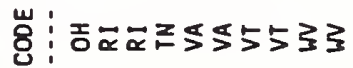

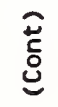

$\bar{n}$ כ $\frac{1}{2}$

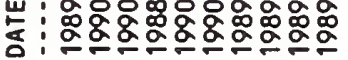

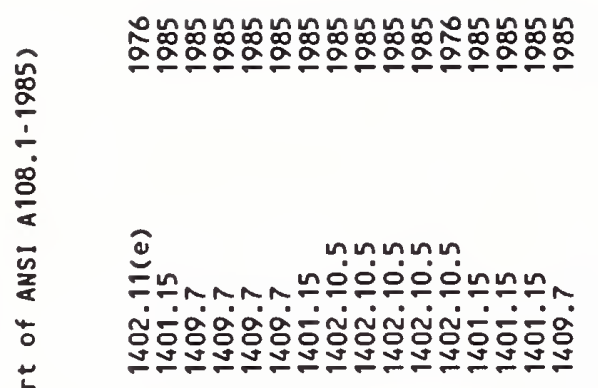

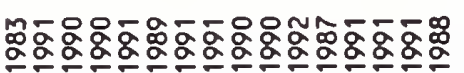

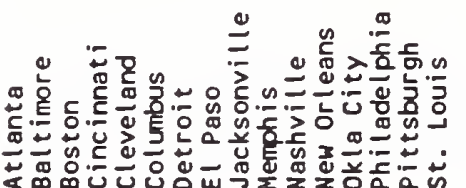

定

ํㅗㄷㅇㅝ

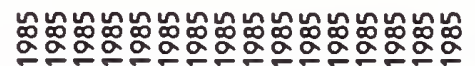

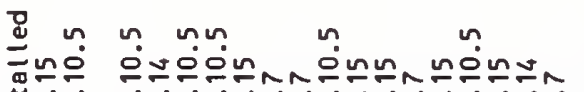
कें

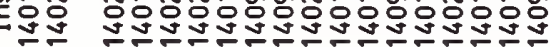
产高 迹客 告

可商品

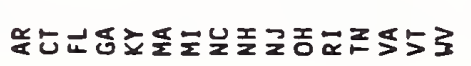

올

음

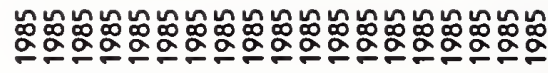

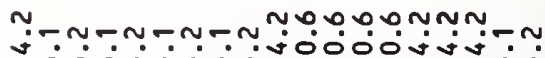

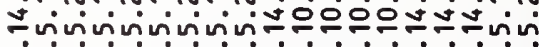

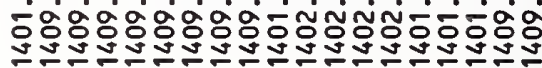

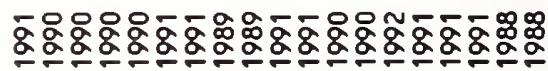

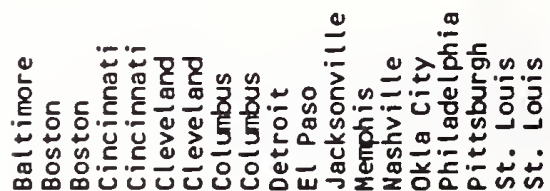

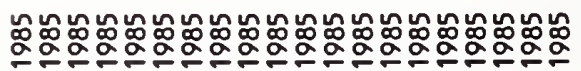

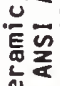

aㅁ S世 $\sum_{\frac{1}{4}}^{\infty}$

䓀

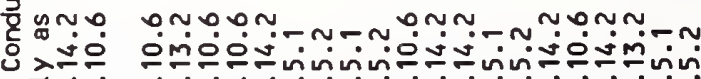

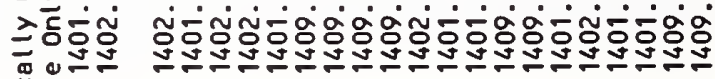
요의 눙음

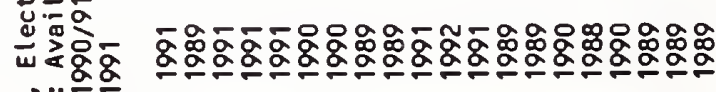
$a^{-0}$

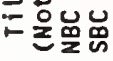

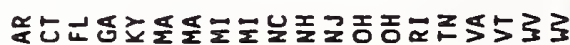




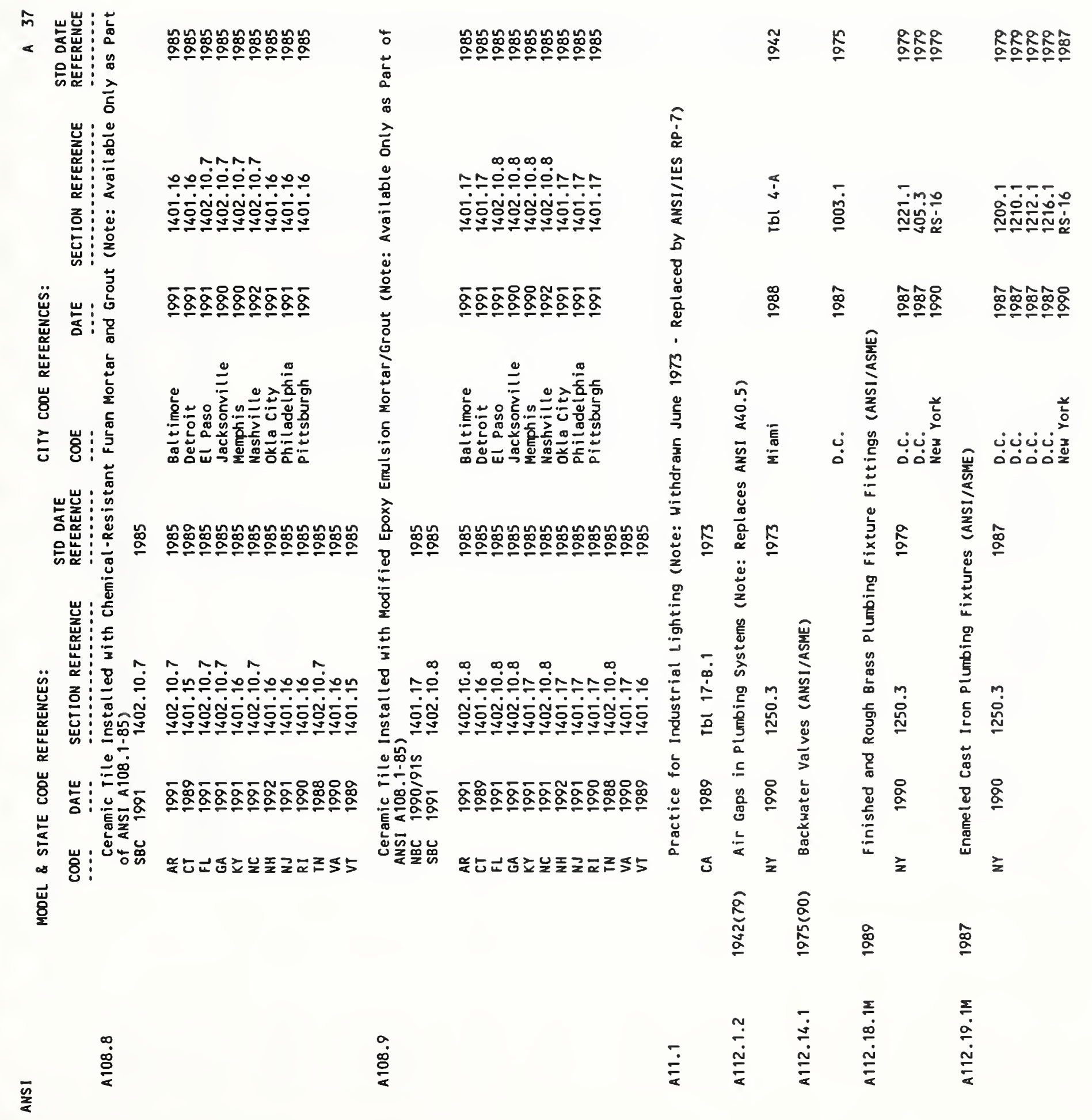




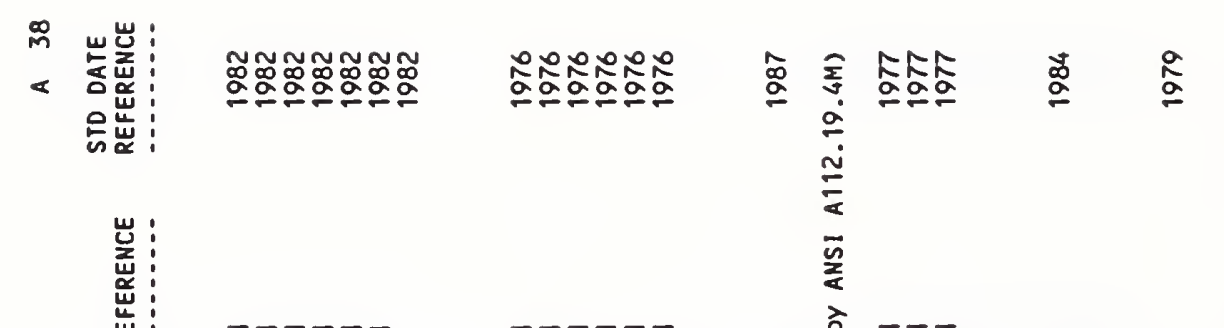




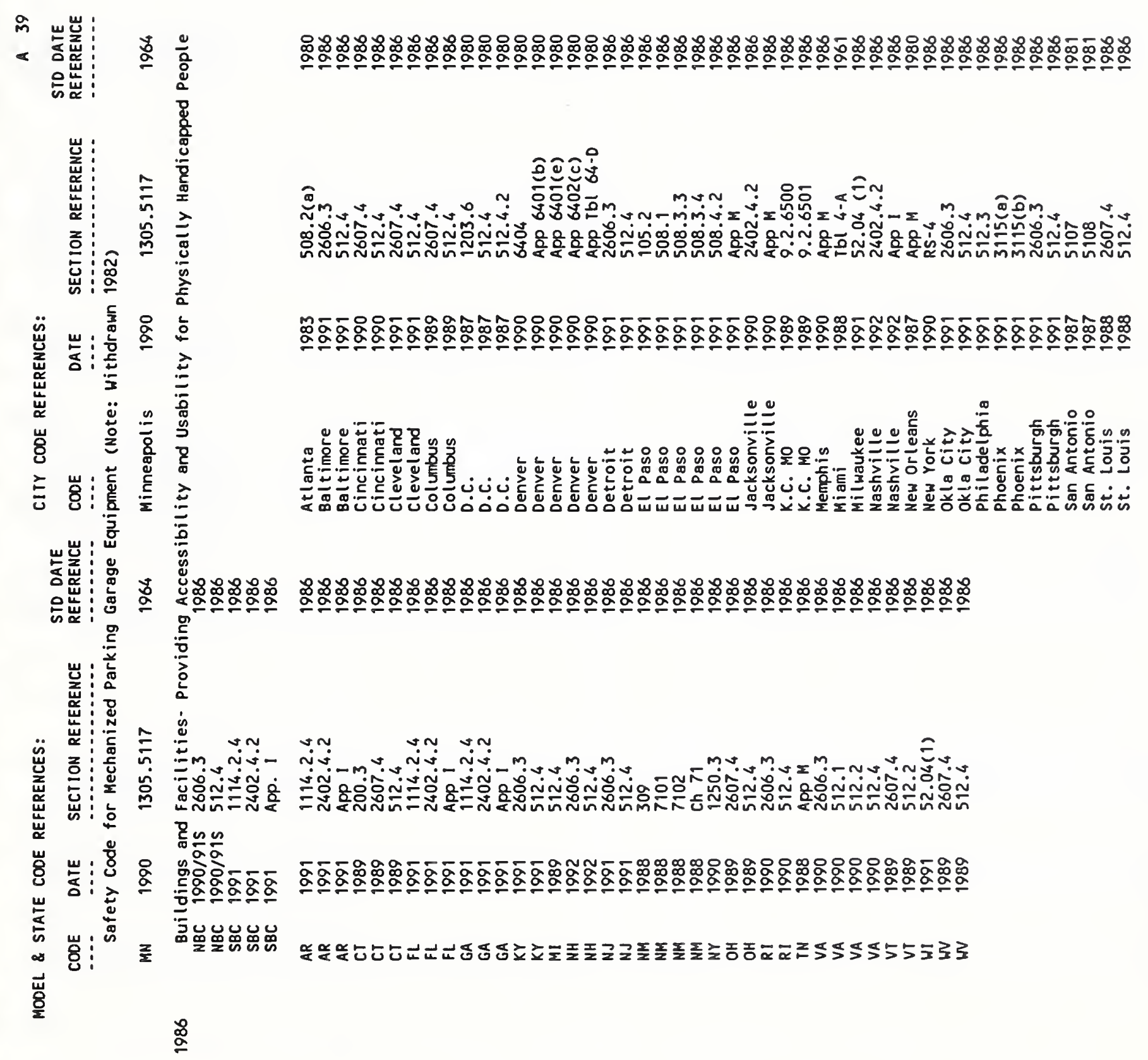




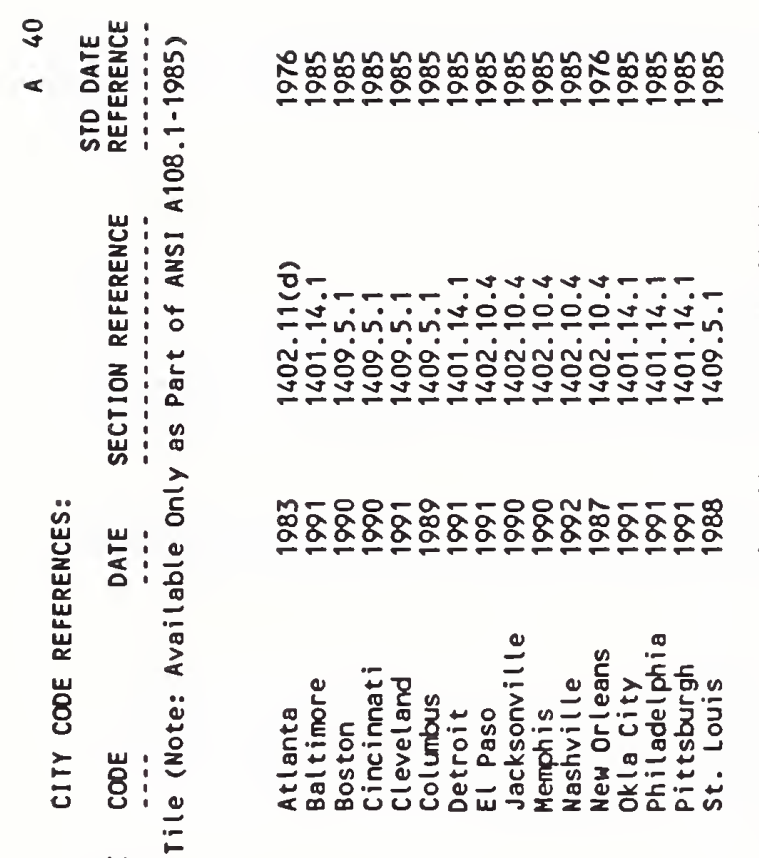

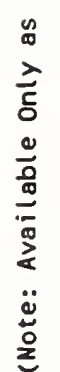

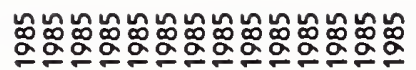

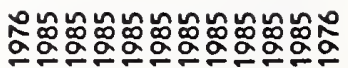

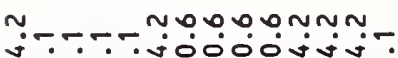

$\stackrel{0}{=}$

ทุกบบทุ

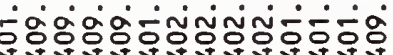

= - an

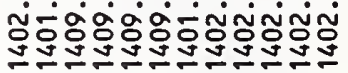

$\stackrel{0}{=}$

ànazanazazana

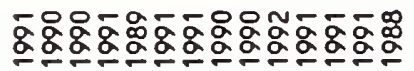

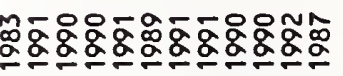

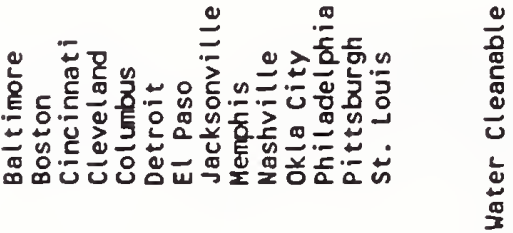

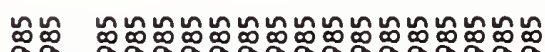

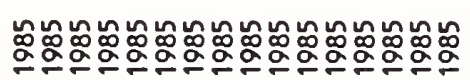

㩆:

㟧 :

密:

热

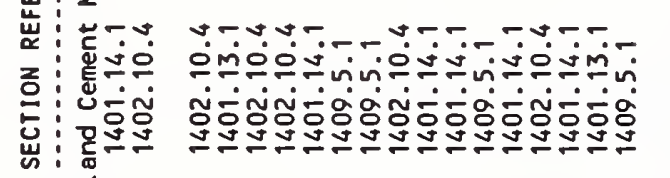

w : 눙

这

岁: 苔毠邑

8 :

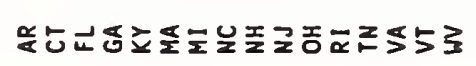

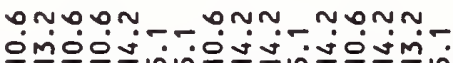

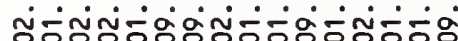

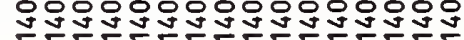

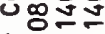
5⿺辶一

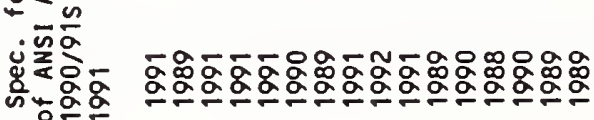

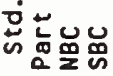

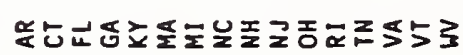

$\stackrel{\infty}{2}$

$\frac{\infty}{\frac{\infty}{\alpha}}$

$\stackrel{\sim}{\stackrel{\infty}{\sigma}}$

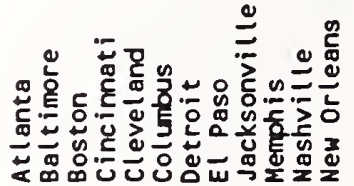

\section{蒙莕}

总高恕

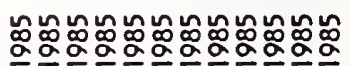

\&ें

密新

Ut $n n$ un $n$

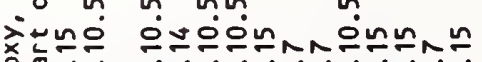

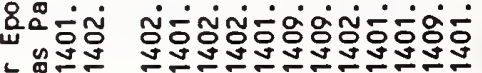

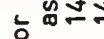
में

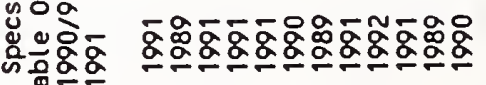
范莨眊品

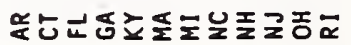




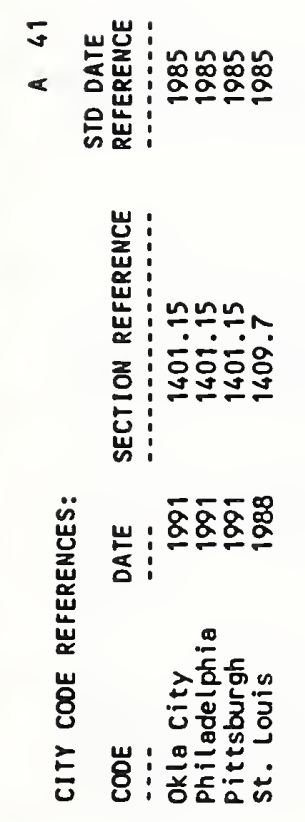

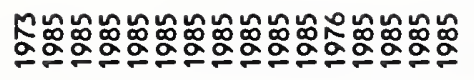

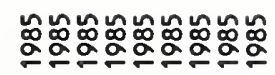

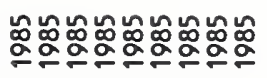

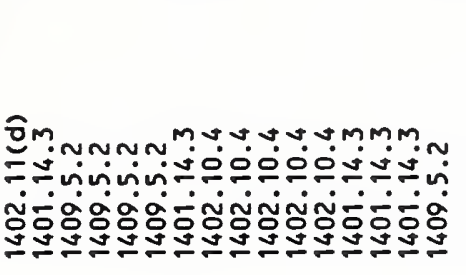

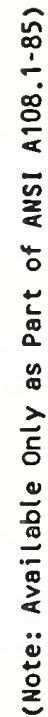

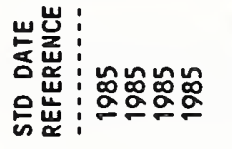

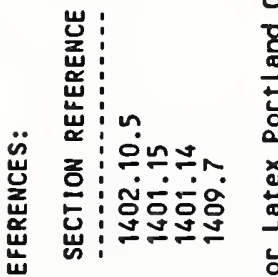

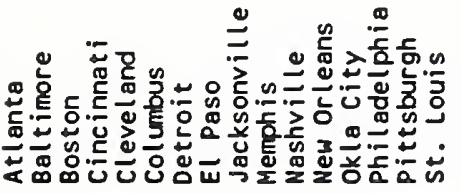

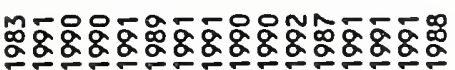

느응ㅇㅇ으는

0000

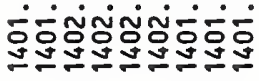

으우우우우으으

is

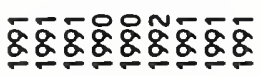

年

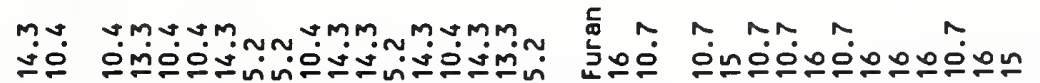

-

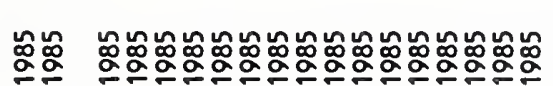

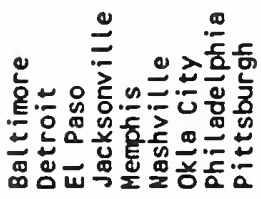

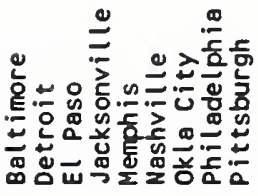

范

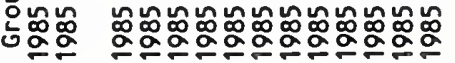

뭏

要

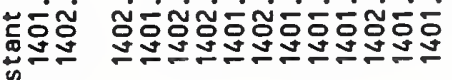

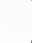

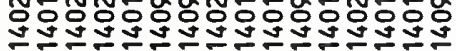

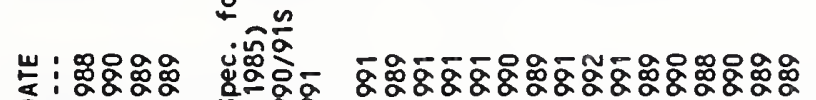

농:오으

岁: $: ₫ 53$

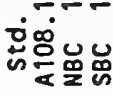

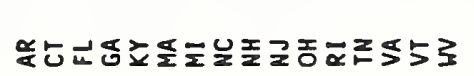

$\frac{m}{8} \underline{x}$

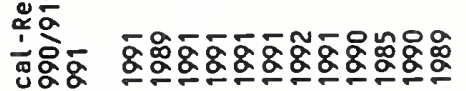
额品

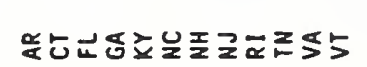

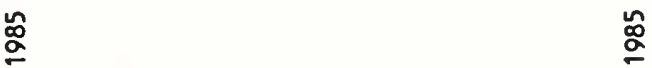

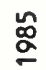

言

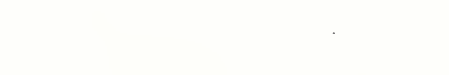

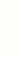

$\bar{m} \quad \frac{m}{\frac{\infty}{c}} \quad \stackrel{\overrightarrow{0}}{\frac{\infty}{\alpha}}$

$\stackrel{n}{\frac{\infty}{x}}$

$\frac{\infty}{2}$ 


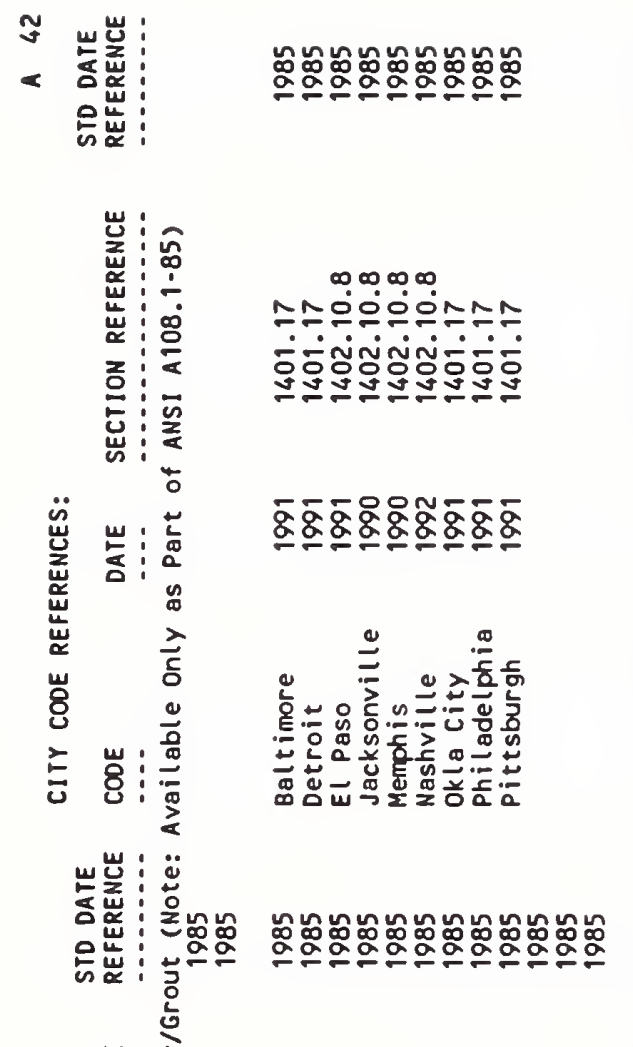

岂:

岃:

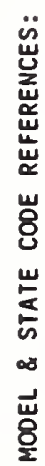

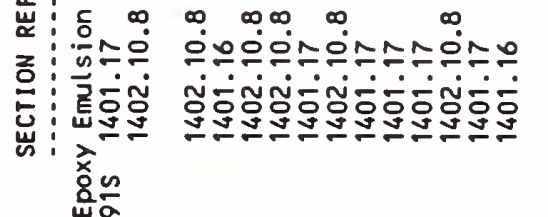

蒫

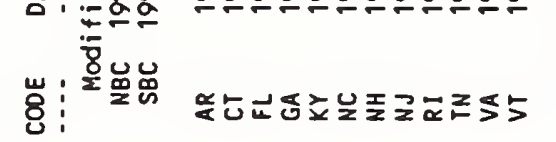

吕

$\bar{n} \frac{\infty}{\infty}$

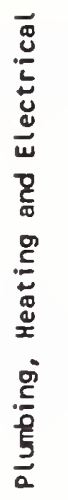

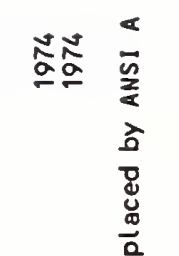

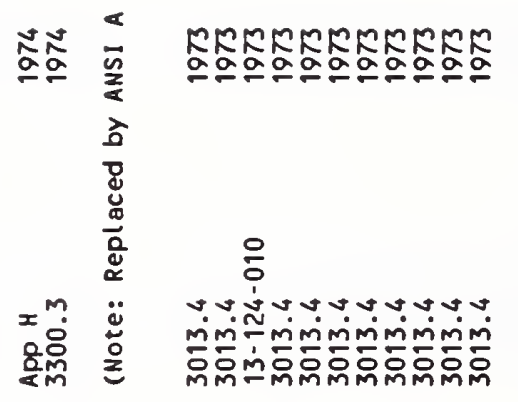

这

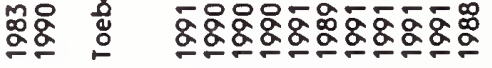

क्ष

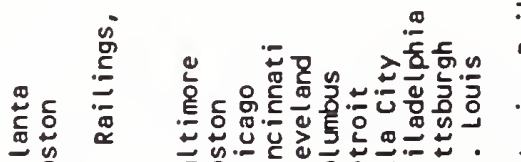

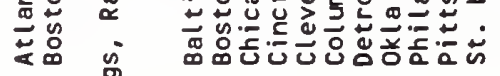

参

$\stackrel{\underline{a}}{=}$

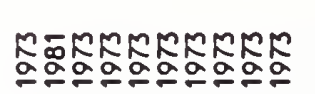

도ำ

高

起亭

m.

务

웡

要密

min̄minimimimm

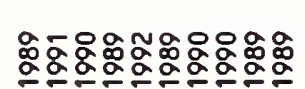

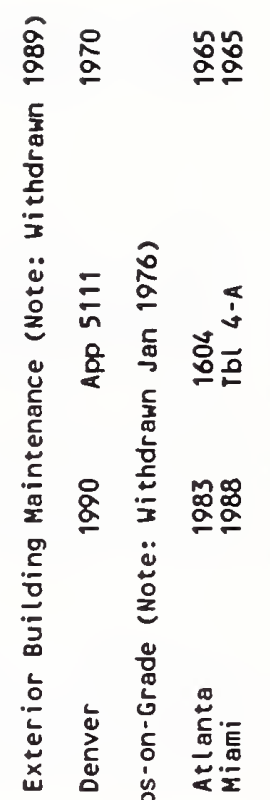

$\stackrel{0}{0}$

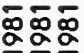

ทंvivin

мิํํ용

양ㅎㅀ음

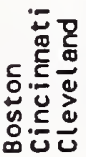

宸

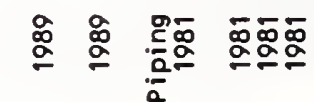

$\leftarrow$

$\stackrel{5}{2}$

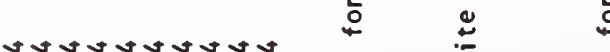

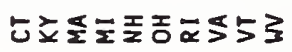




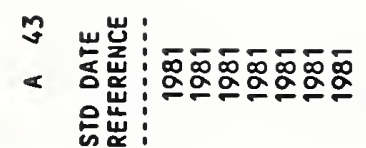

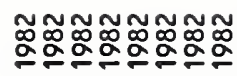

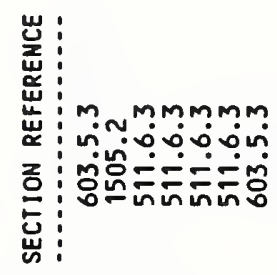

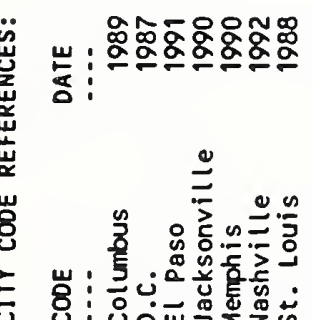

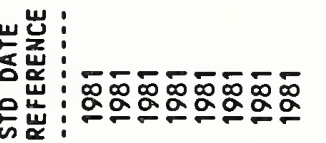

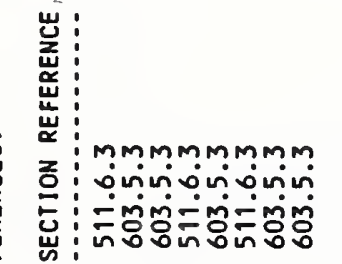

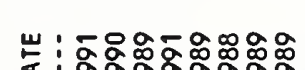

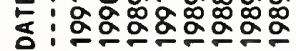

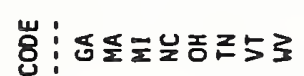

ญ્ટ

产 $\quad \frac{m}{<}$

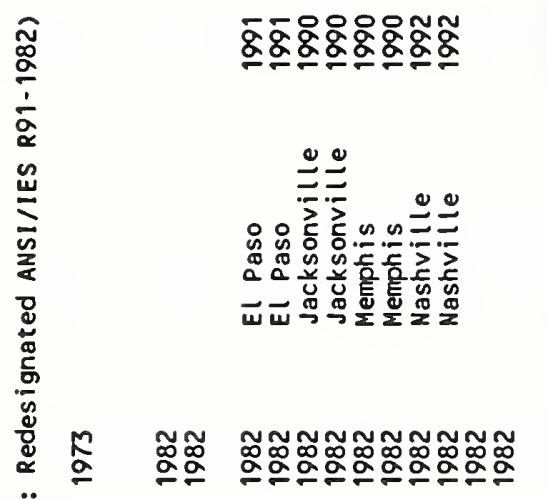

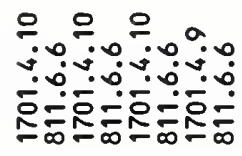

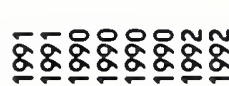

aิ

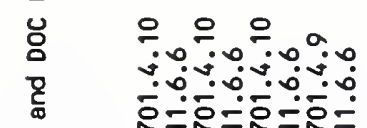

in $5=\infty$

竞

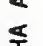

告

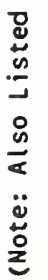

논

导

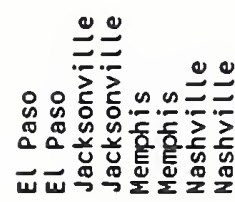

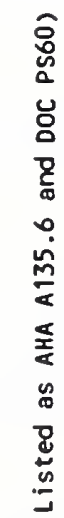

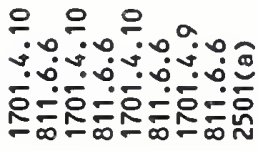

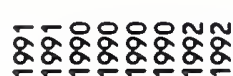

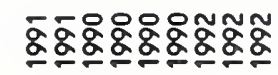

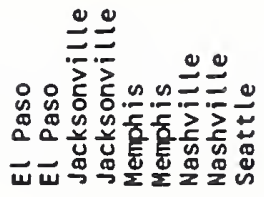

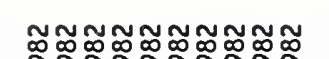

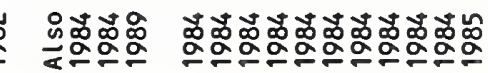

苛

旁

$\stackrel{\infty}{\check{1}}$

용

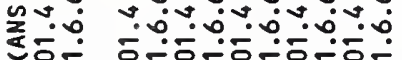

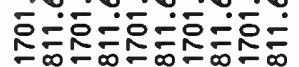

कर्क

a

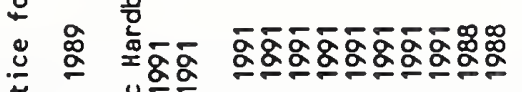

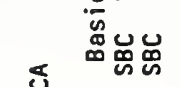

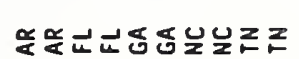

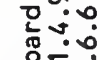

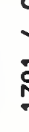

官.

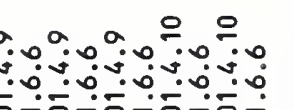

$\infty=\infty \div \infty=\infty$

10

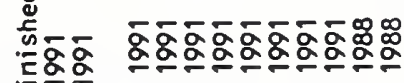
究品品

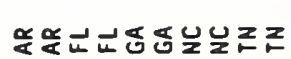

훙ㅎㅎㅎㅁ

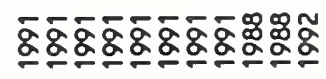

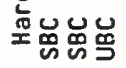

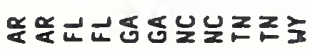

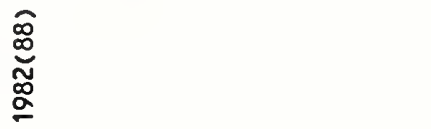

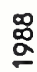

$\stackrel{\circ}{\circ}$

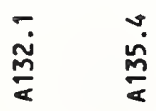

$\stackrel{n}{\frac{n}{2}}$

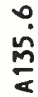




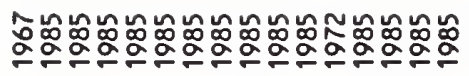

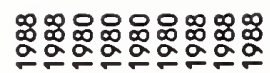

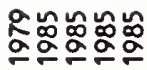

5

的岕:

岁:

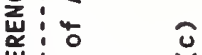

崖:

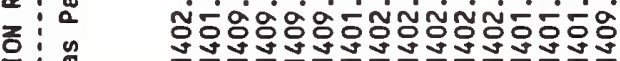

惫

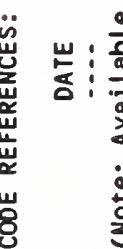

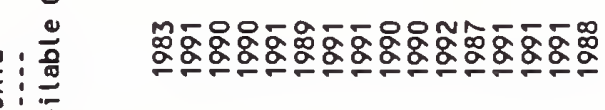

mm웜ำm

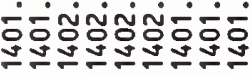

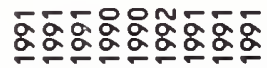

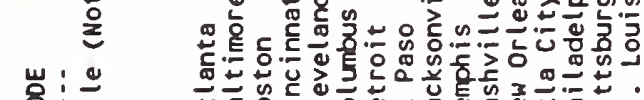

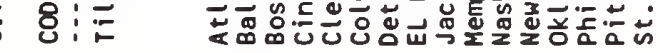

岃: 蒙

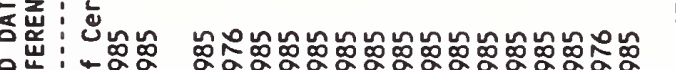

姜

要: $m \mathrm{mmm} m \mathrm{~mm}$

z

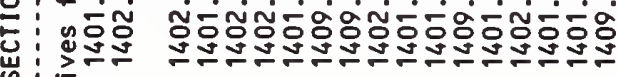
造

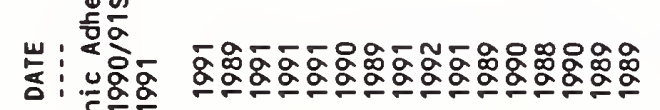

: ‘㸺品

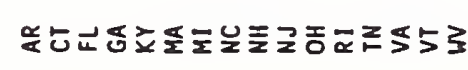

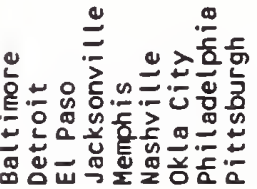

$\overline{\hat{m}}$

๓ัญ

舟

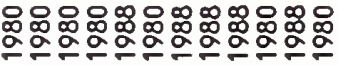

总

- - - - -

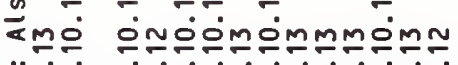

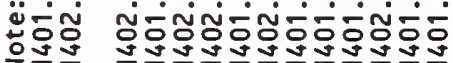

$\frac{20}{2}$

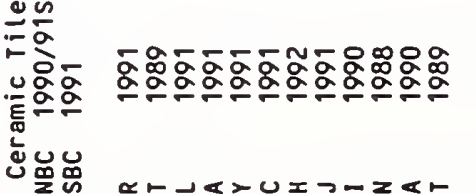

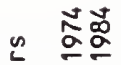

总

tommmn

ô ơmimimen

兽

ถั่

옹

웋ㅇㅎㅇㅎㅁ

잉

总

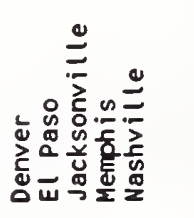

:

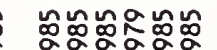

으으음

¿

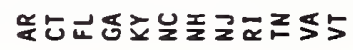

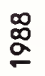

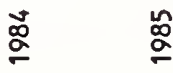

$\overline{\frac{n g}{z}}$

$\overline{\frac{m}{2}}$

$\frac{\bar{m}}{\frac{m}{\alpha}}$

m.

$\frac{\square}{6}$ 
$\because$

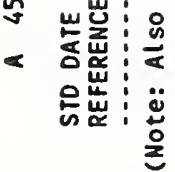

名 :

岃:

娄: ज

중

运

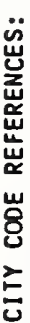

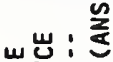

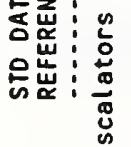

닌

密:

昰

흥

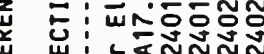

岕

商

$\overrightarrow{\underline{s}}$ का , 要

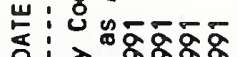

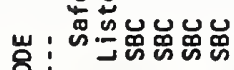

$\overrightarrow{5}$

$\stackrel{-}{\leftarrow}$

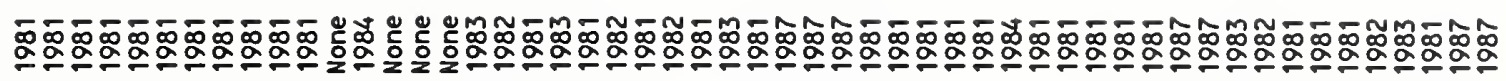

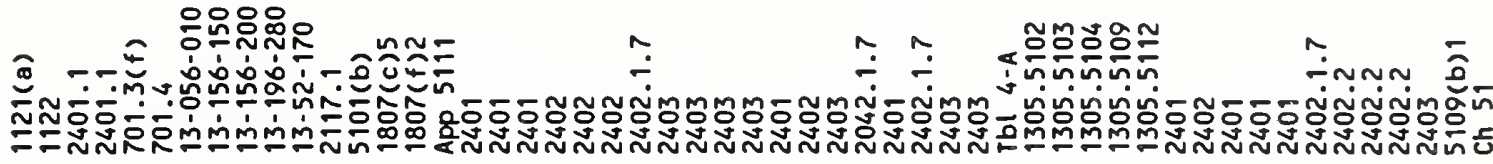

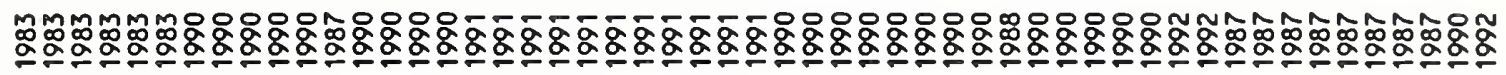

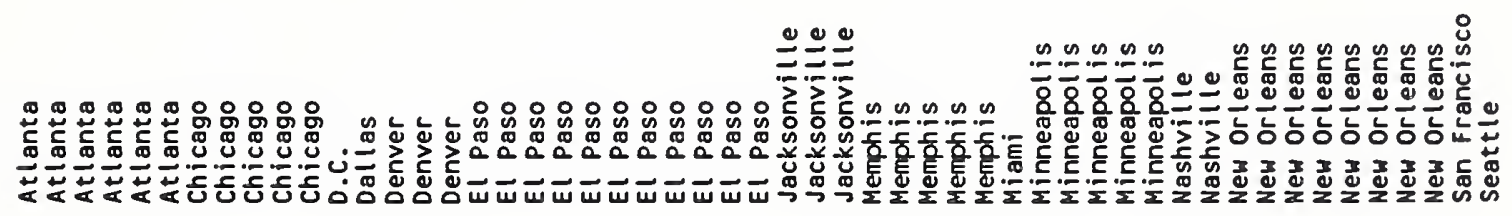

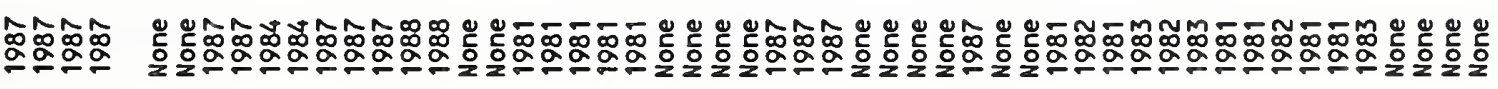

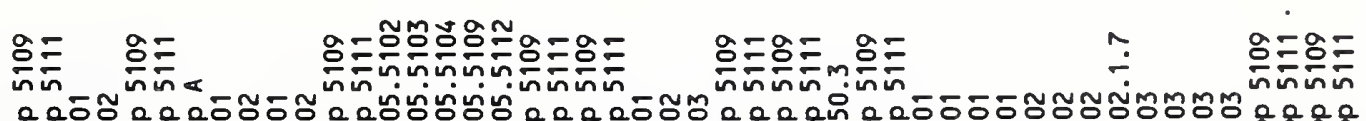

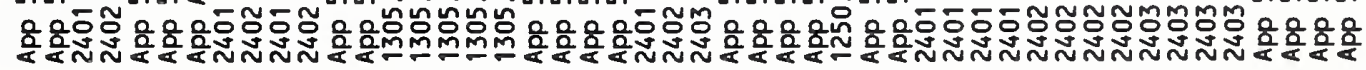

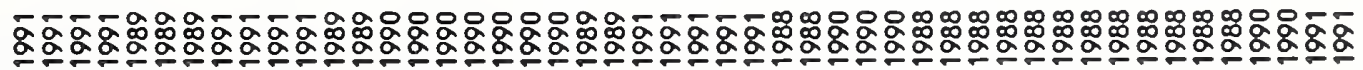

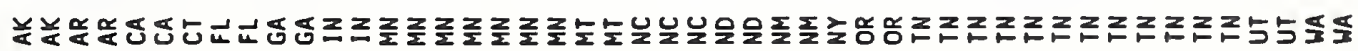

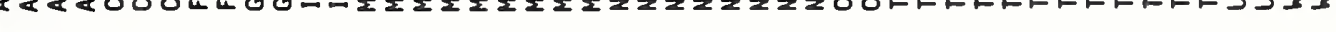




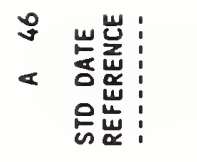

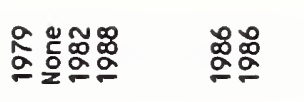

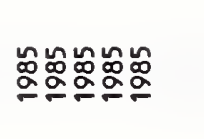

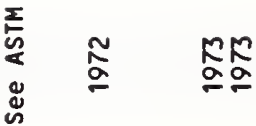

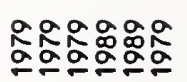

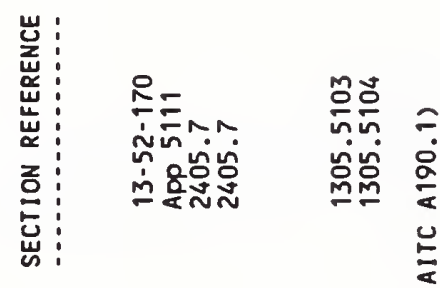

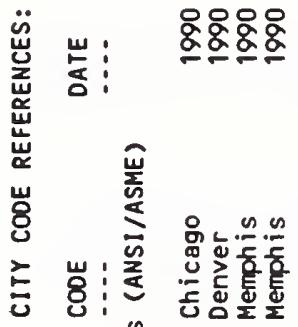

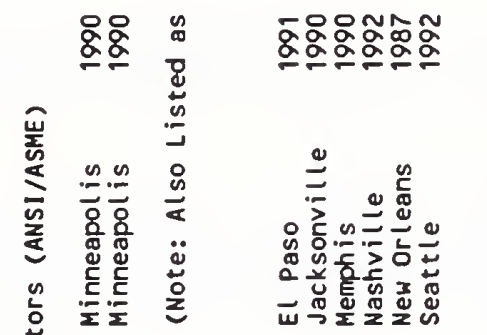
س : :
要
道崖:
可

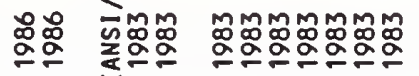

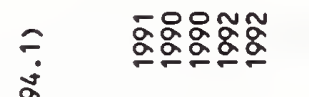

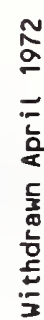

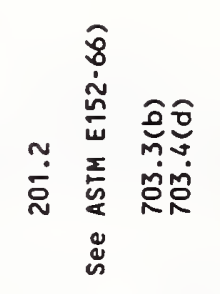

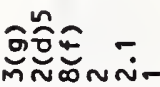

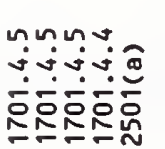
m

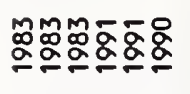
负ำกำ

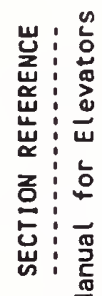
(1)
栾

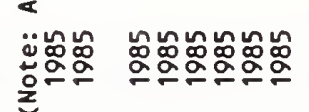
离

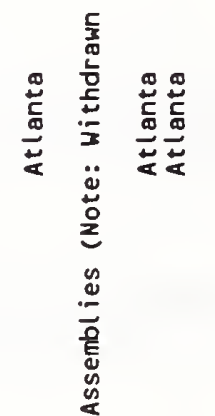

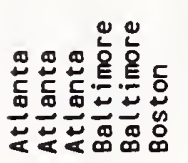

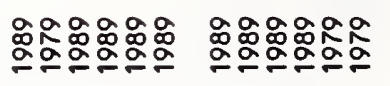

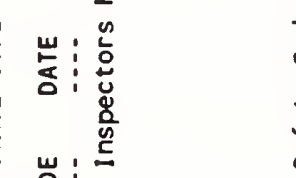
岩 :

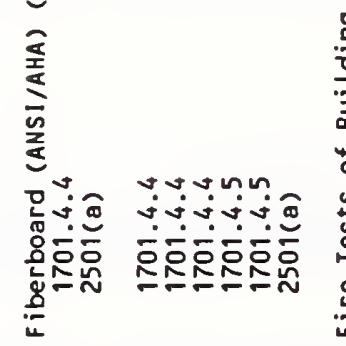

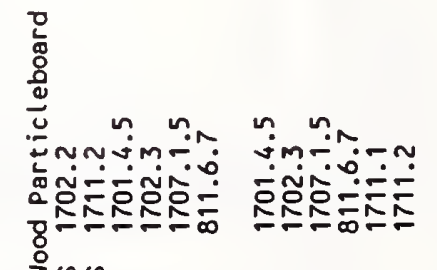

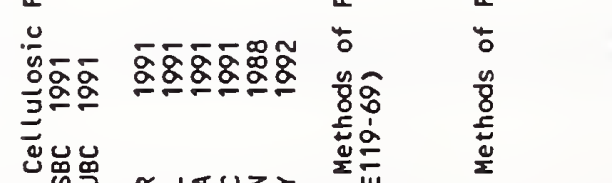

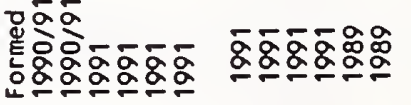

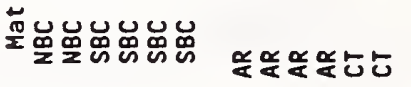
$\stackrel{\frac{m}{2}}{\frac{m}{\alpha}} \frac{\dot{\delta}}{\frac{0}{\alpha}}$
$\frac{2}{\frac{a}{2}}$

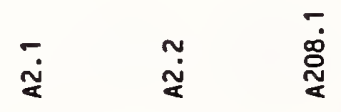


< 菏 战?

岂:

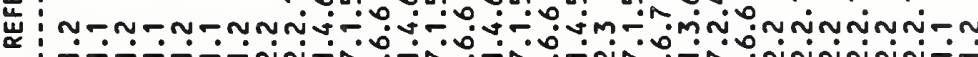

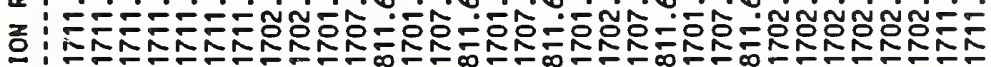

:

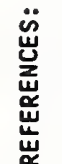

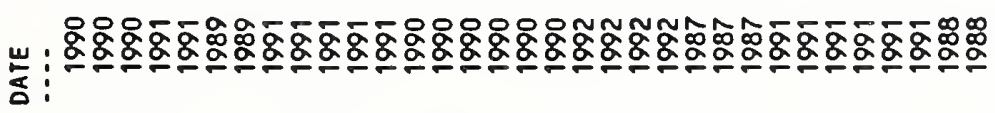

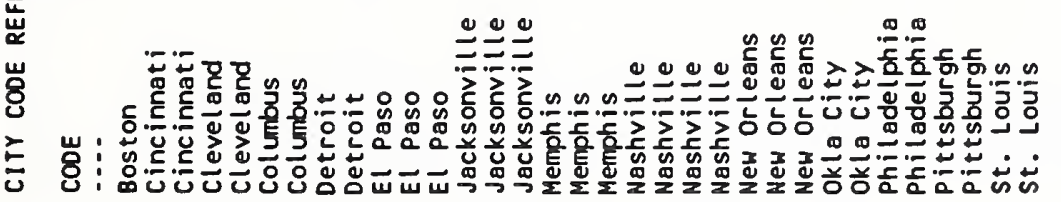

岂:

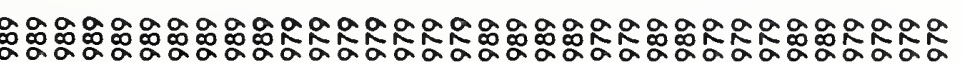

岁

崫

$\ddot{s}$

a nn un n

zo

出:

岁

崖

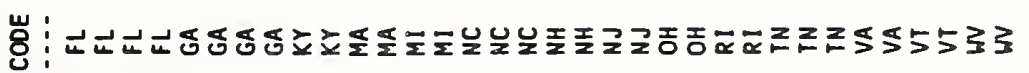

ڤัa

$\stackrel{\circ}{\circ}$

$\stackrel{\stackrel{8}{\circ}}{\frac{8}{5}}$

弯

호응

突它

m

क्षेत्र

石

动

든.옹

䆛

跑。

疎 品

은

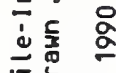

$\circ$

发三

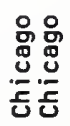

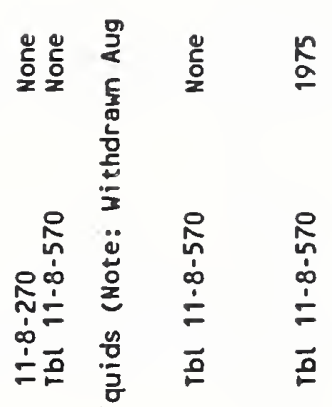

$\stackrel{8}{\circ}$

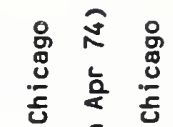

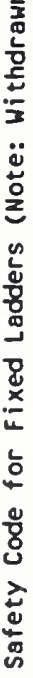

突

एक

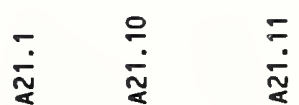

$\frac{\cong}{\dddot{\pi}}$

$\stackrel{m}{\frac{m}{2}}$ 
幽岕: 捁

$<$ 客崖:

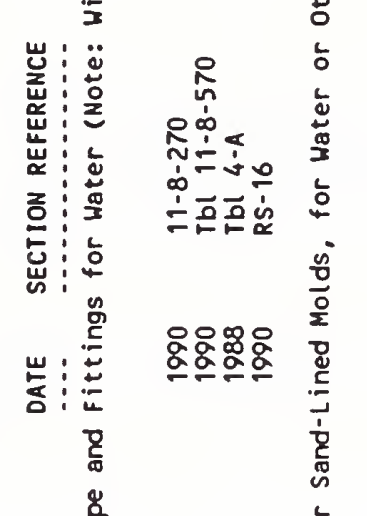

岁 :

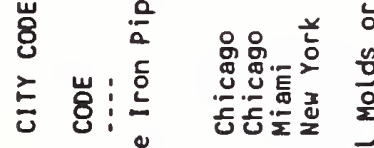

嵌:

和崖

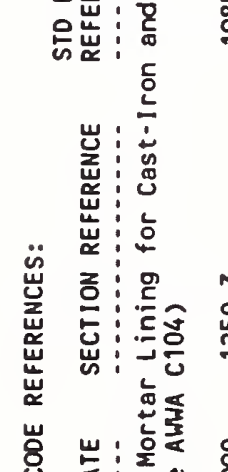

.

แ : U

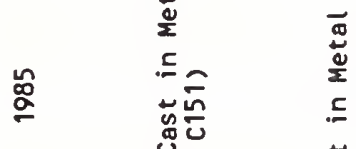

窟

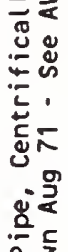

들

凉竞

产

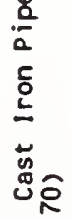

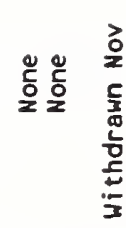

官

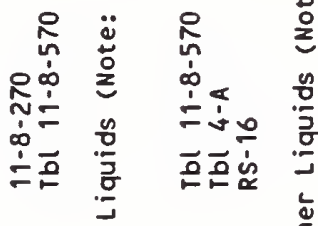

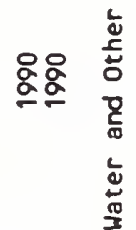

용융

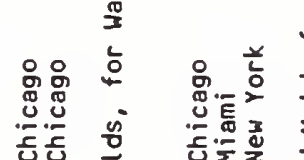

양ㅉㅇㅇㅛ

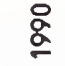

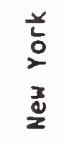<smiles>[TeH]</smiles>

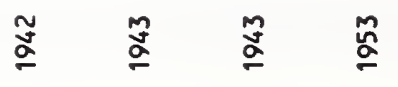

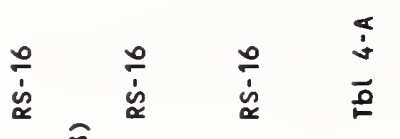

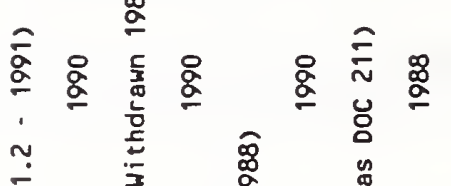

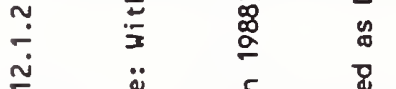

至

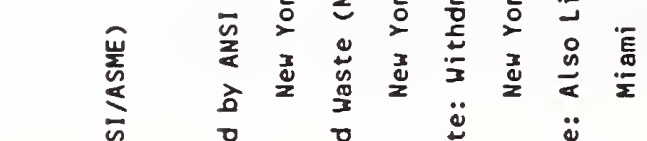

$\stackrel{0}{\circ}$

.

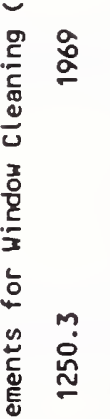

药

$\stackrel{\text { ฒ }}{\stackrel{\alpha}{\sigma}}$

$\stackrel{8}{\circ}$

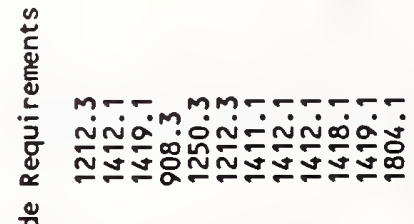

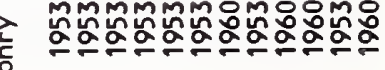

o

8

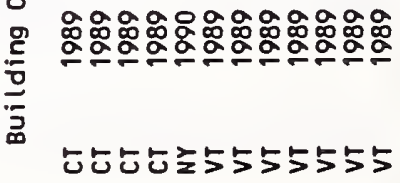

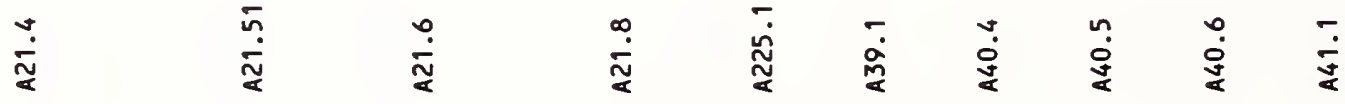




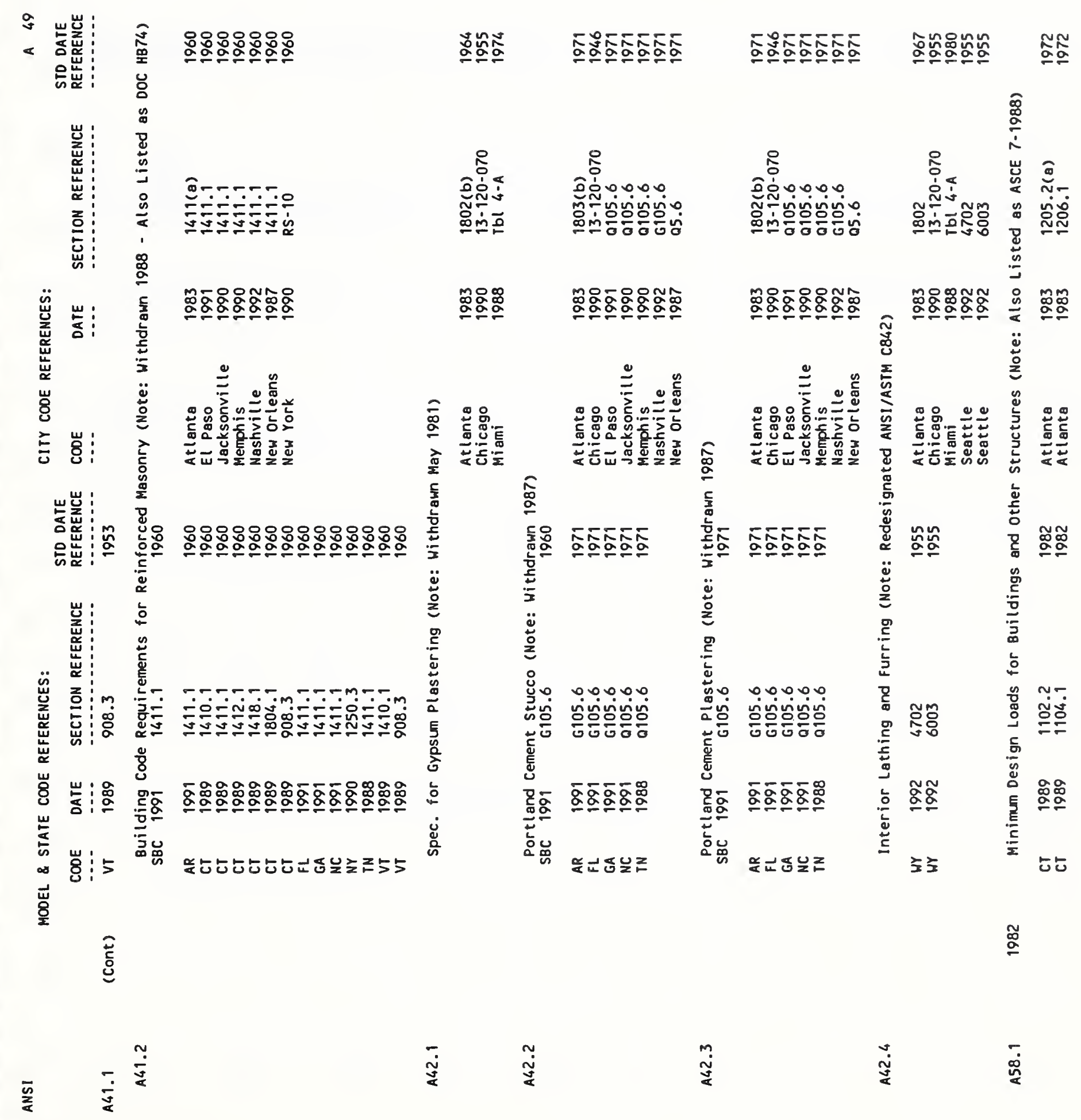



员岕:

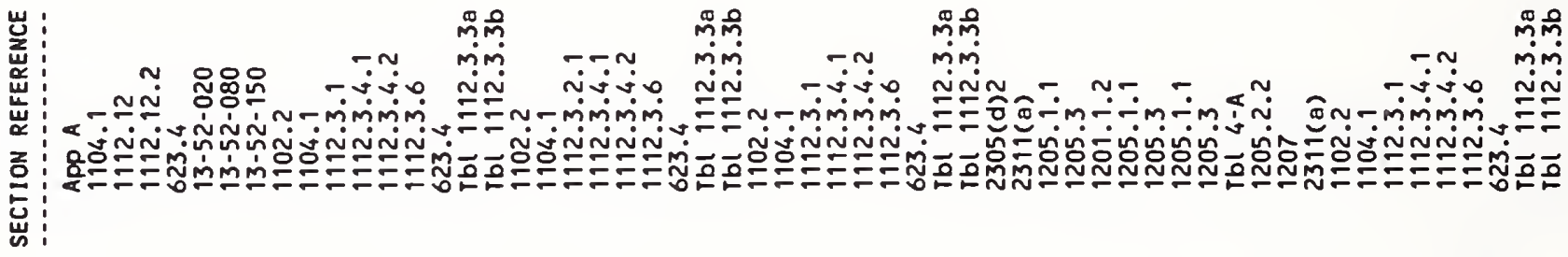$$
\text { 芯 }
$$

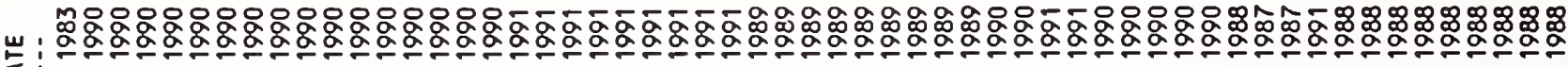
a :

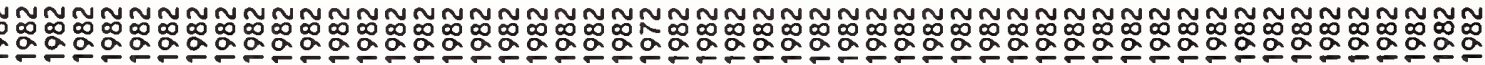

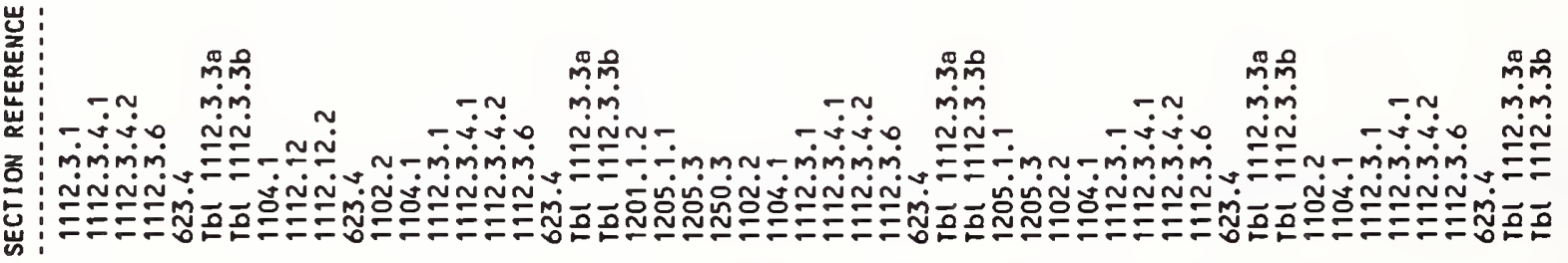

سب

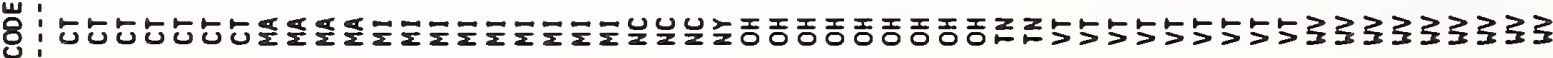

옹 


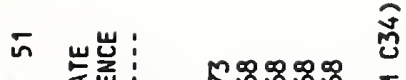

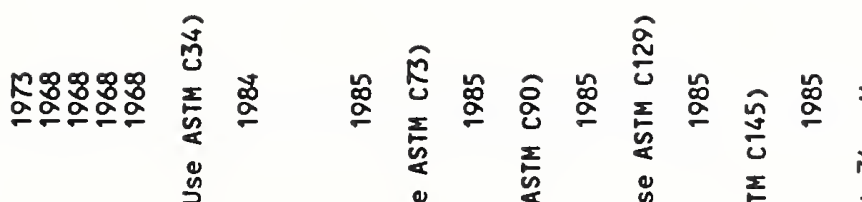

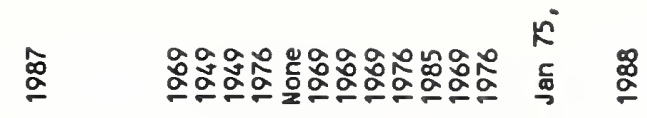

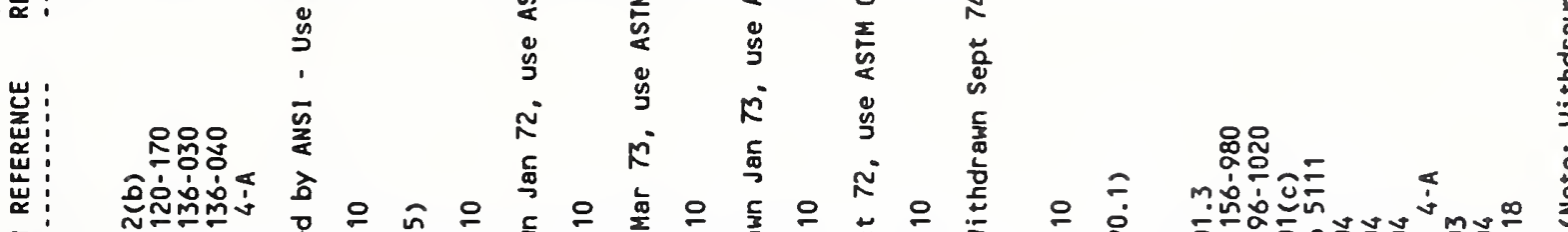

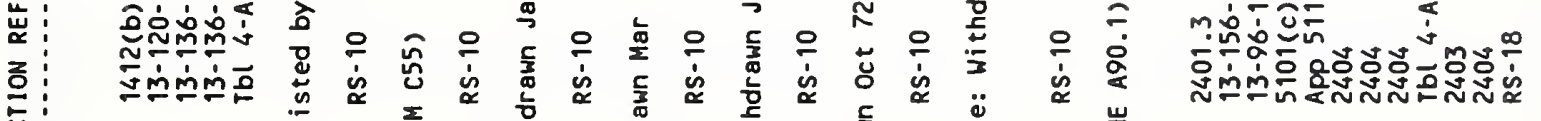

点:

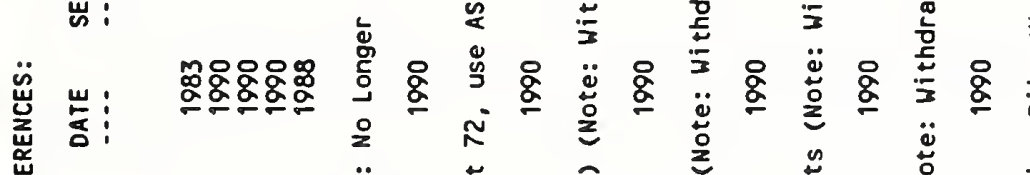

崫

\&

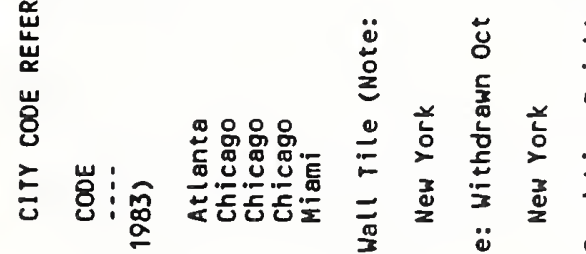

岁:

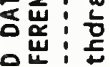

的岲?

岁

造

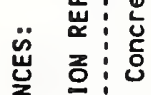

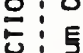

出:

岁 岕:

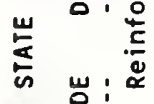

岁 !

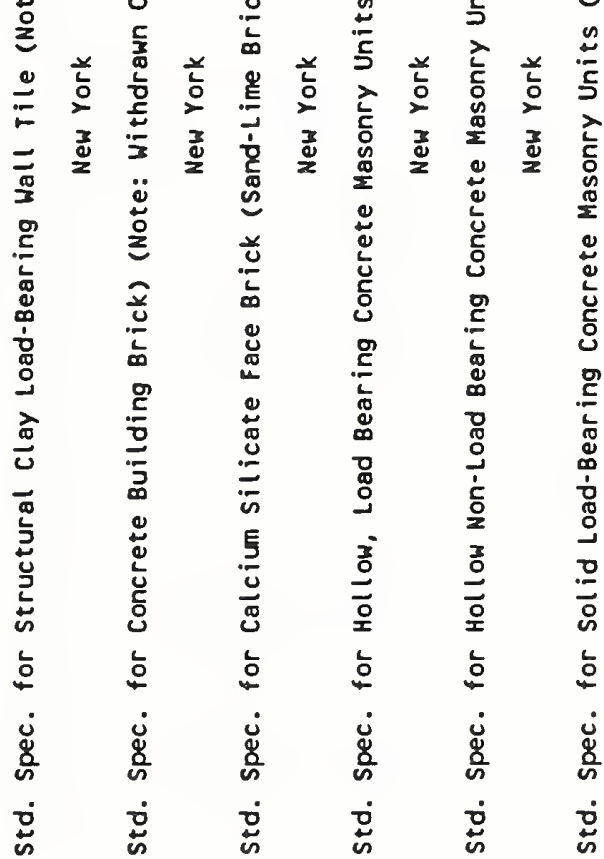

일

용

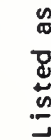

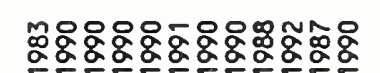

ฮे 임

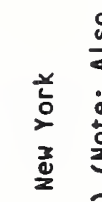

\section{。}

我

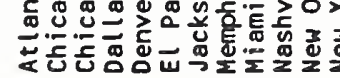

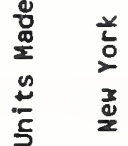

弟

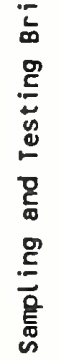

ธ

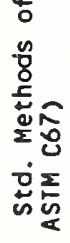

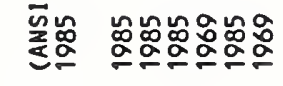

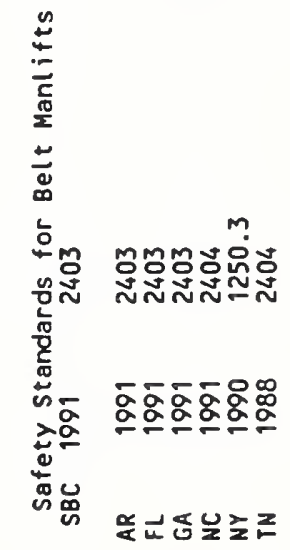

$\frac{0}{\dot{s}}$

$\stackrel{\leftrightarrow}{\underline{\alpha}}$

产

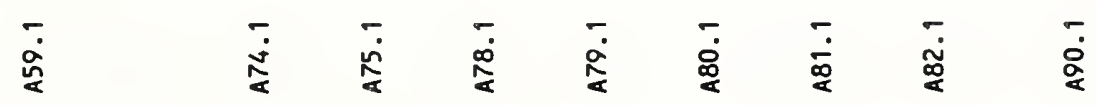

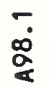




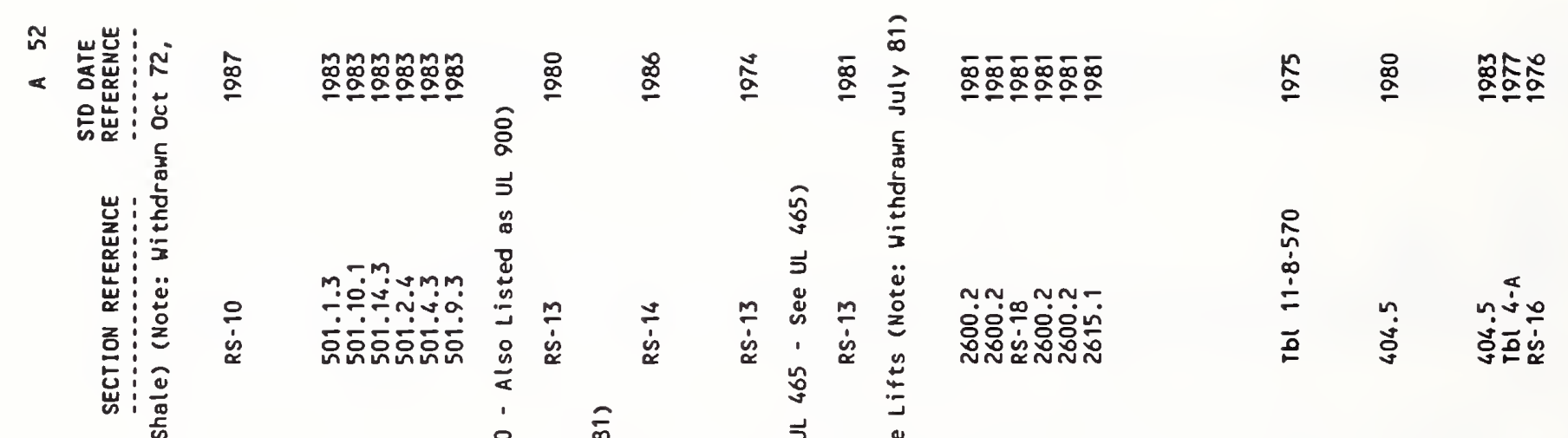

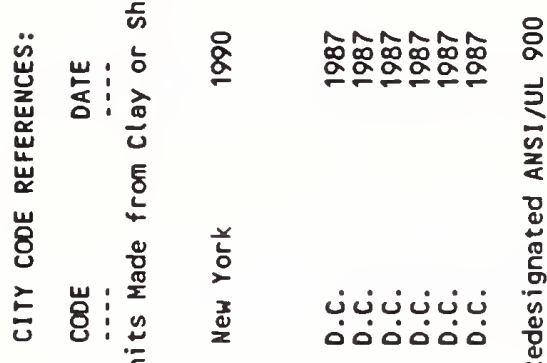

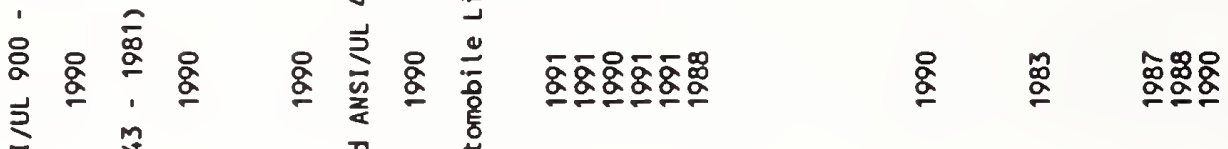

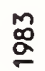
$\stackrel{\stackrel{g}{\mathrm{~g}}}{\stackrel{0}{\mathrm{O}}}$ 


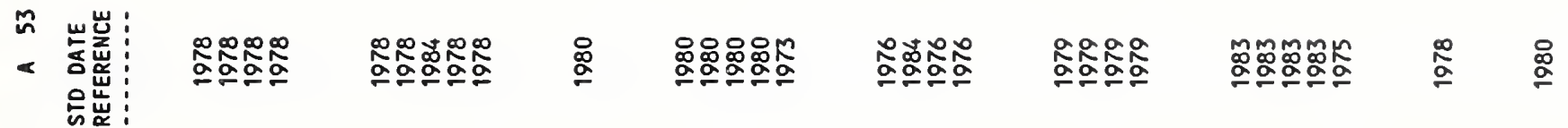

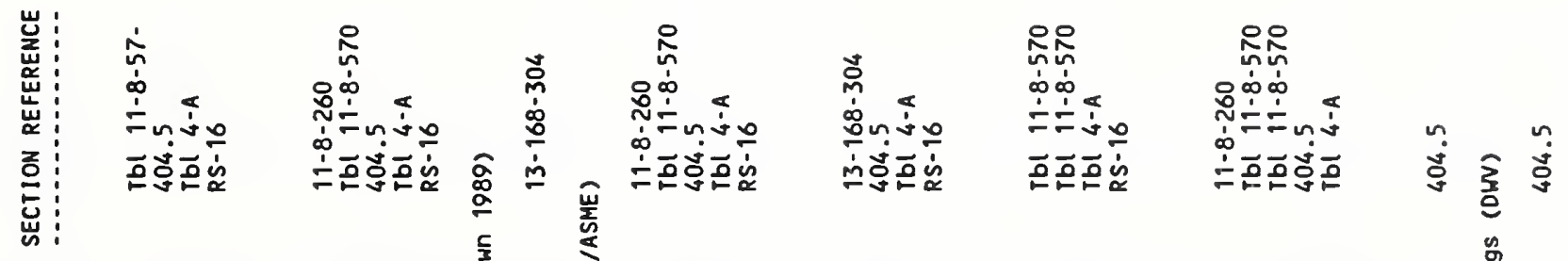
薯:

崫<smiles>C1CCCCC1</smiles>

น

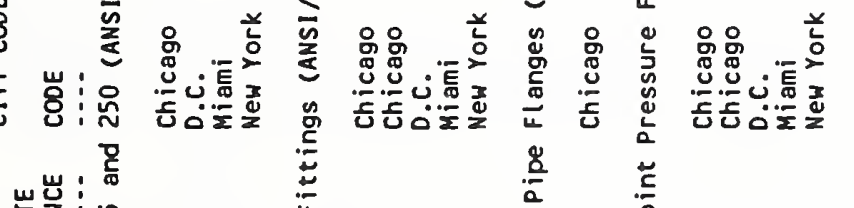
峞总: 은형

: 岀:

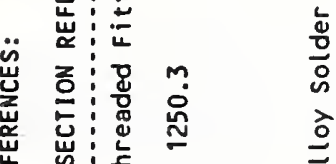

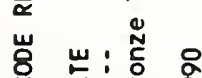

岁:

:

。्ठ 竞

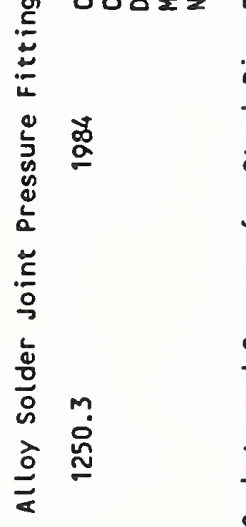

(1)
$\stackrel{\check{g}}{\varrho}$<smiles>[CH]C</smiles>
$\stackrel{\varpi}{\sigma}$

产 용

음

帝

잉

ò

产

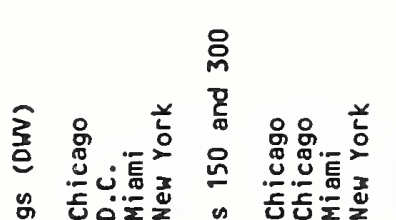

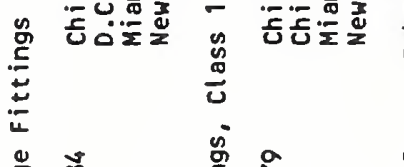

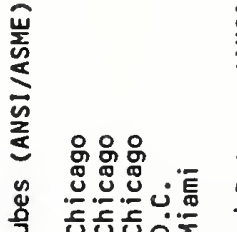

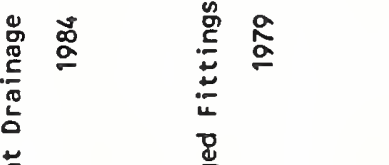

응

它

\%ั.

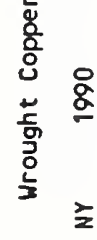

$\stackrel{\stackrel{\circ}{\circ}}{\circ}$

id

$\stackrel{a}{\circ}$

ळ

$\underset{2}{2}$

$\stackrel{\varpi}{\stackrel{0}{0}}$

ळ

牙

$\frac{n}{\infty} \quad \frac{\infty}{\infty}$

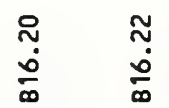

$\stackrel{m}{\stackrel{m}{0}}$

$\underset{\substack{0 \\ \hdashline}}{\stackrel{0}{0}}$

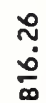

$\begin{array}{ll}\stackrel{\infty}{1} & \stackrel{0}{0} \\ \stackrel{0}{\infty} & \frac{0}{\infty}\end{array}$ 


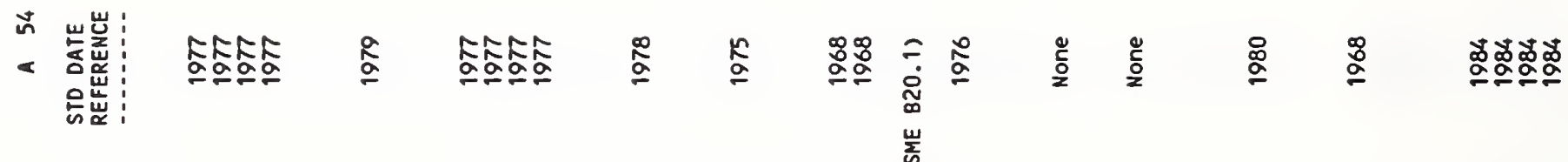

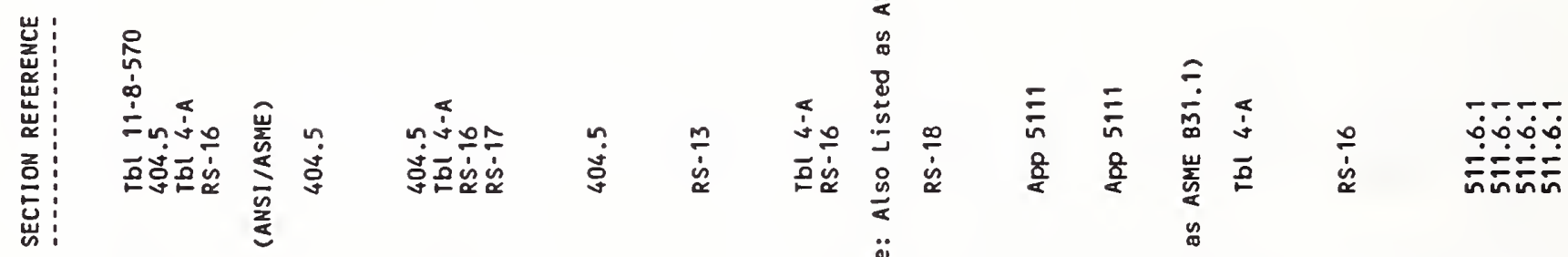

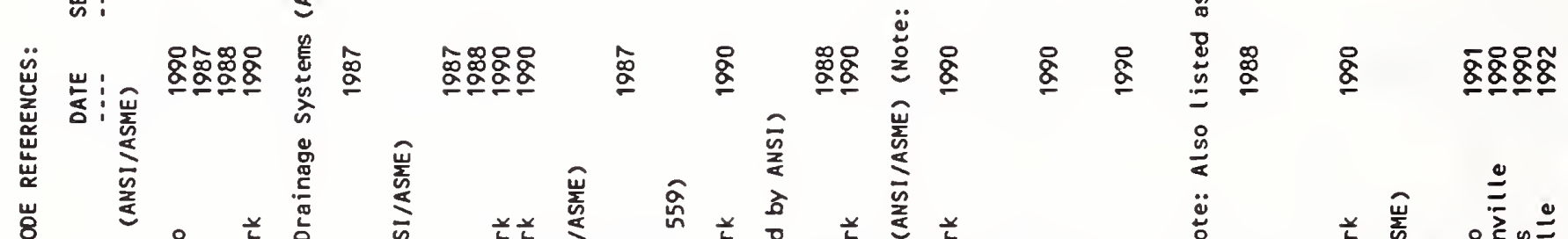

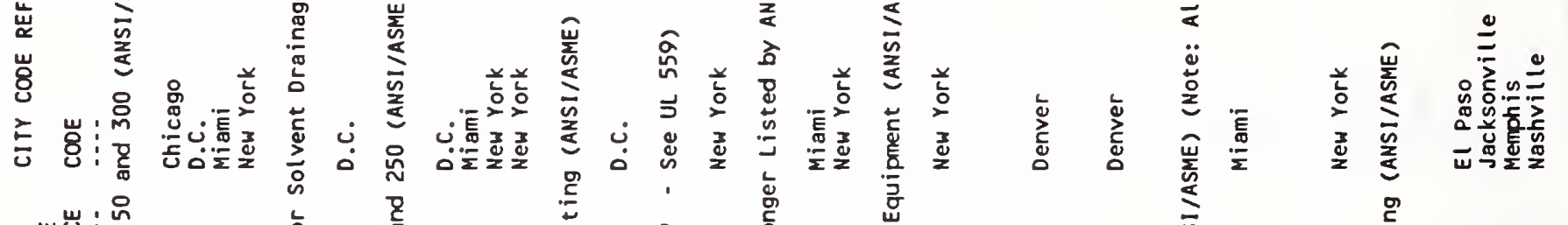

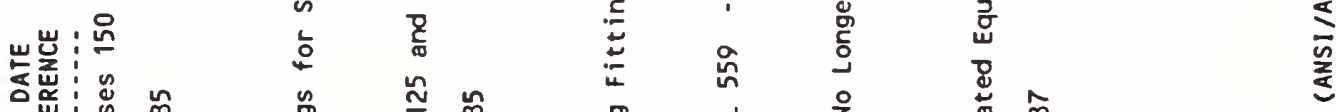

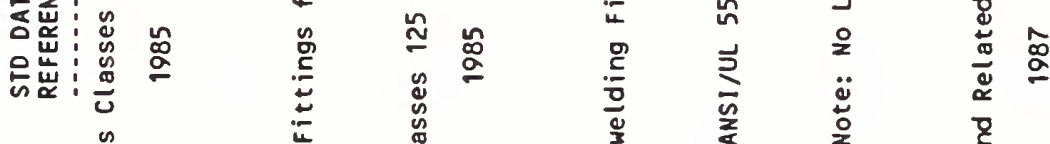

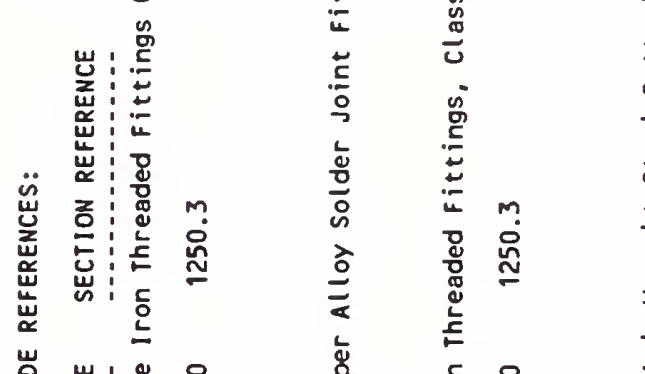

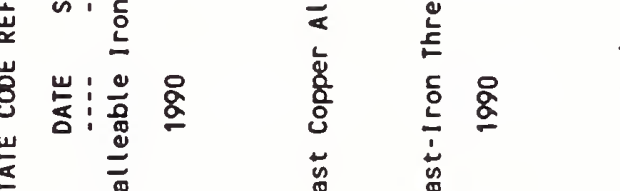

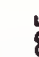

岁!

药
离
$\dddot{2}$

菅

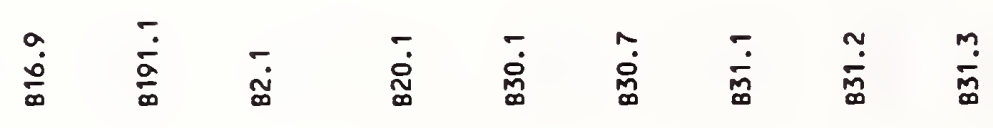




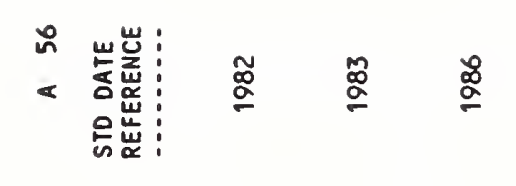

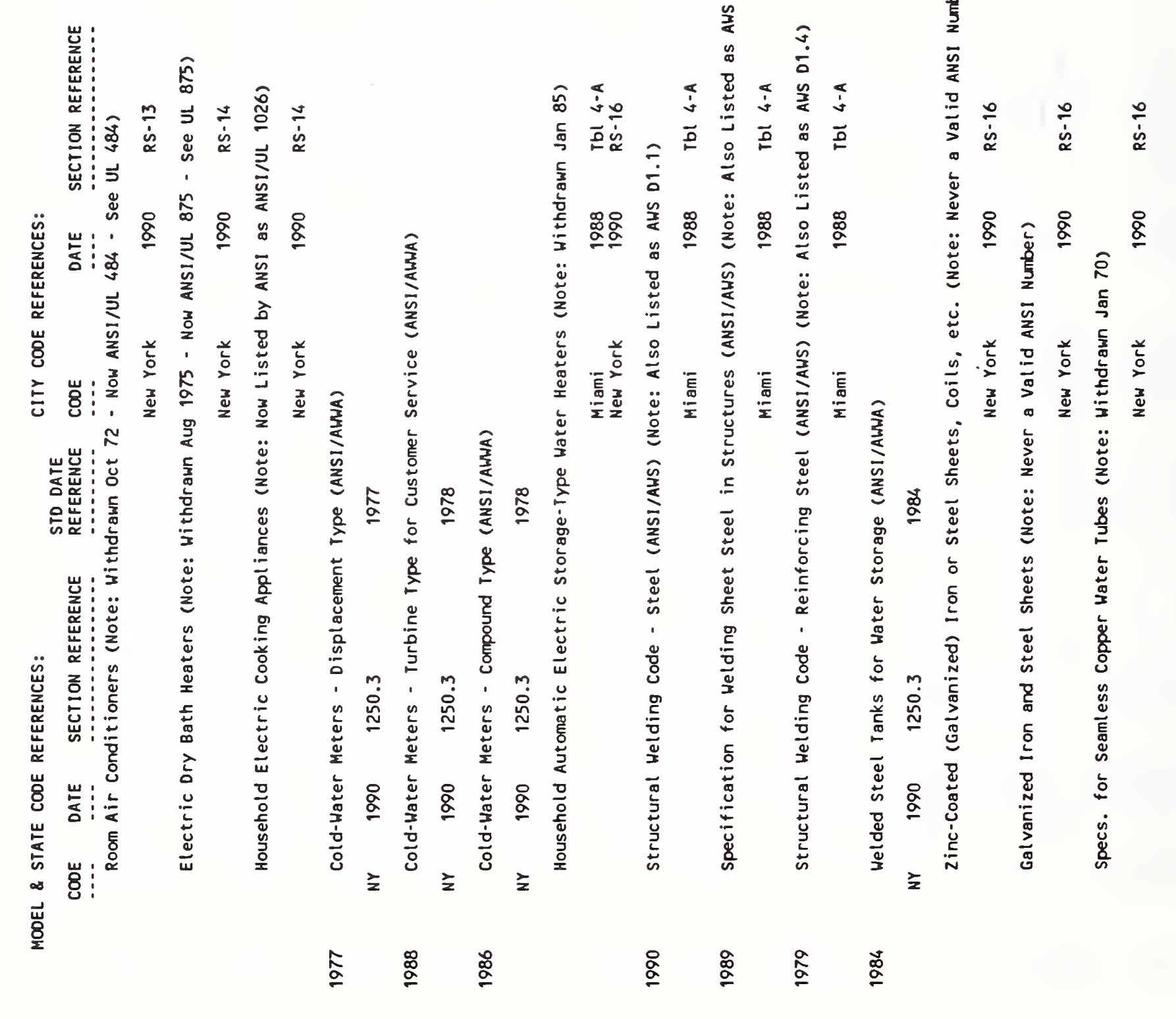

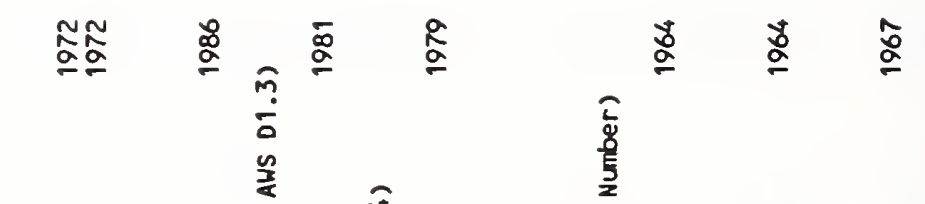

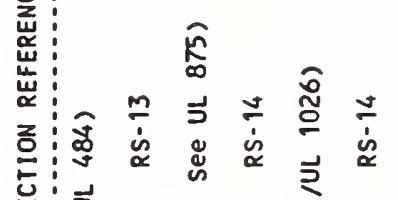

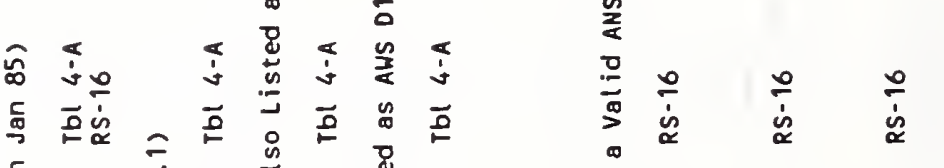

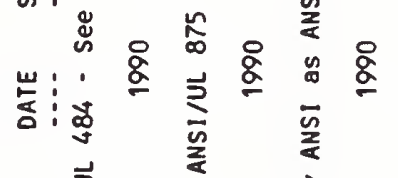

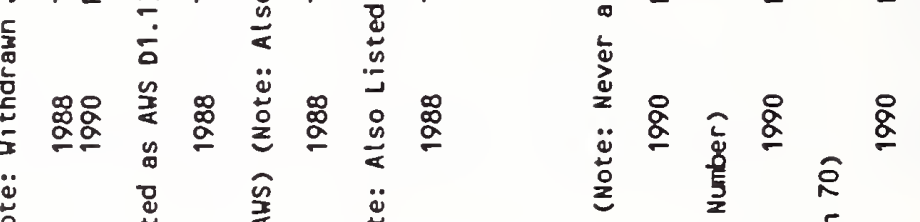

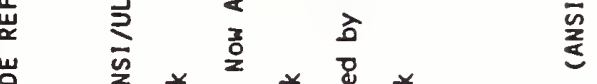

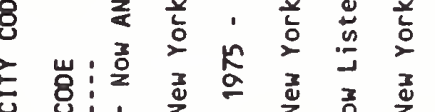

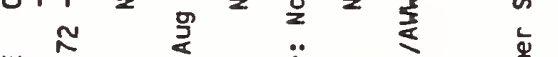

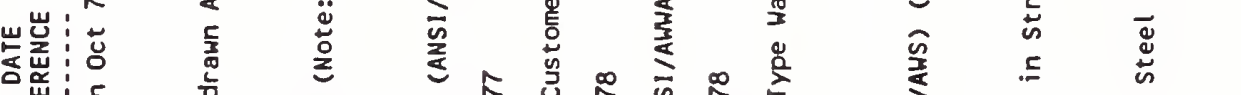

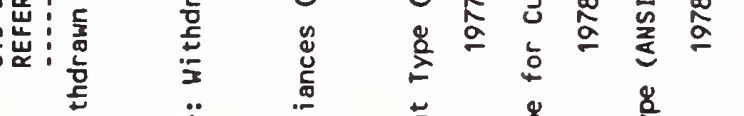

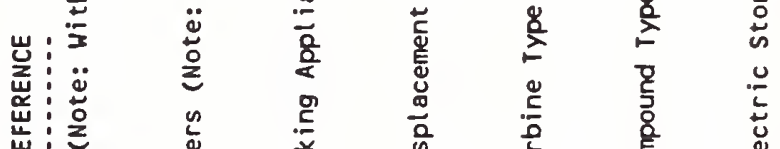

증 


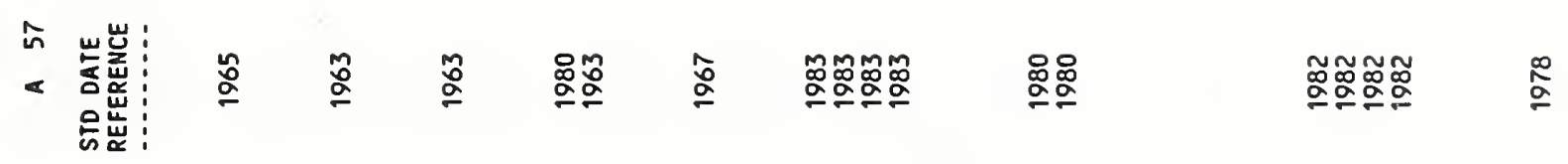

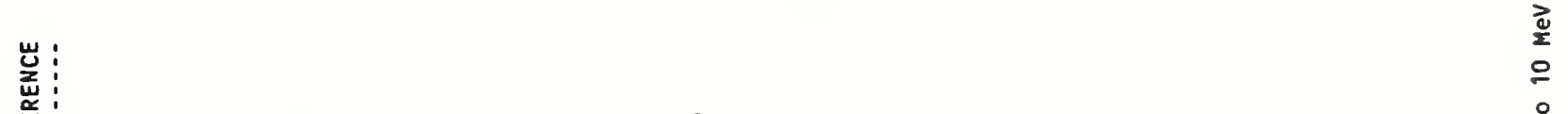

逆:

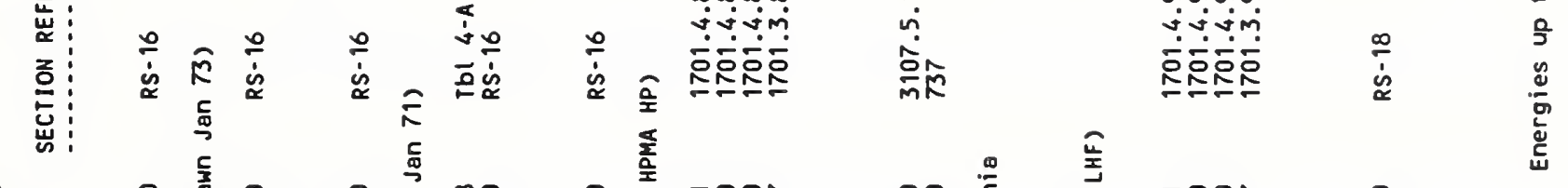

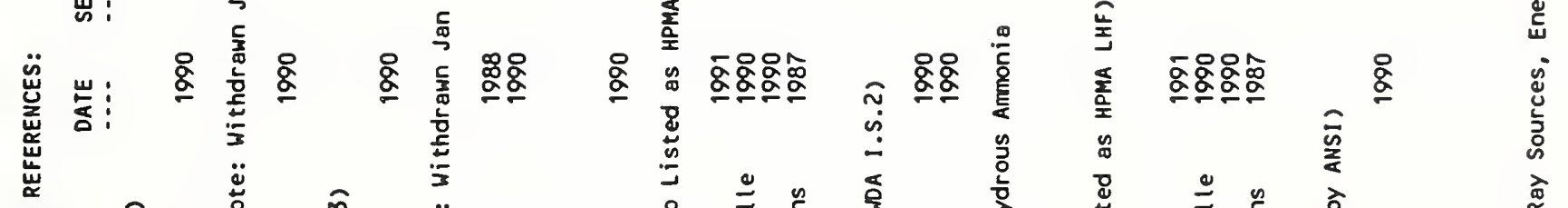

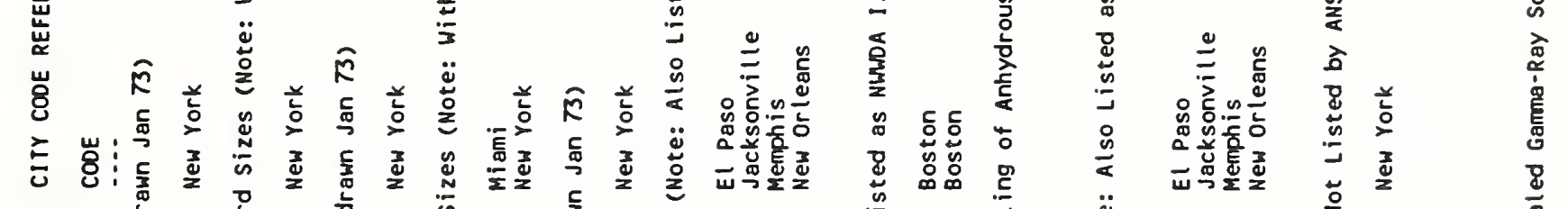

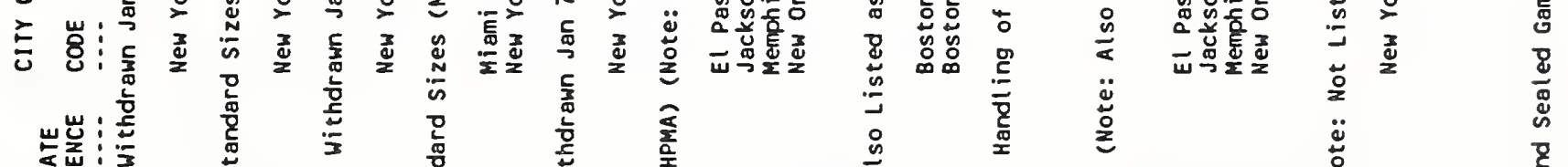

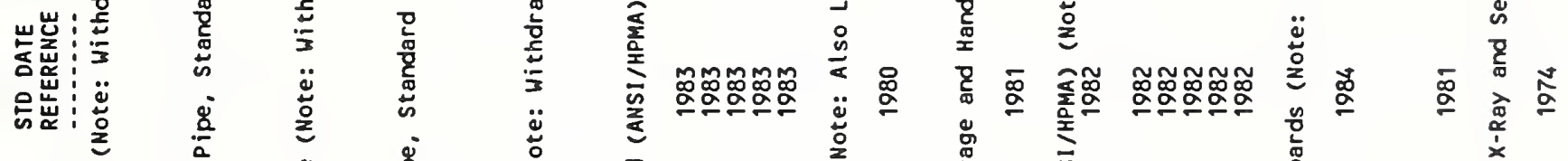

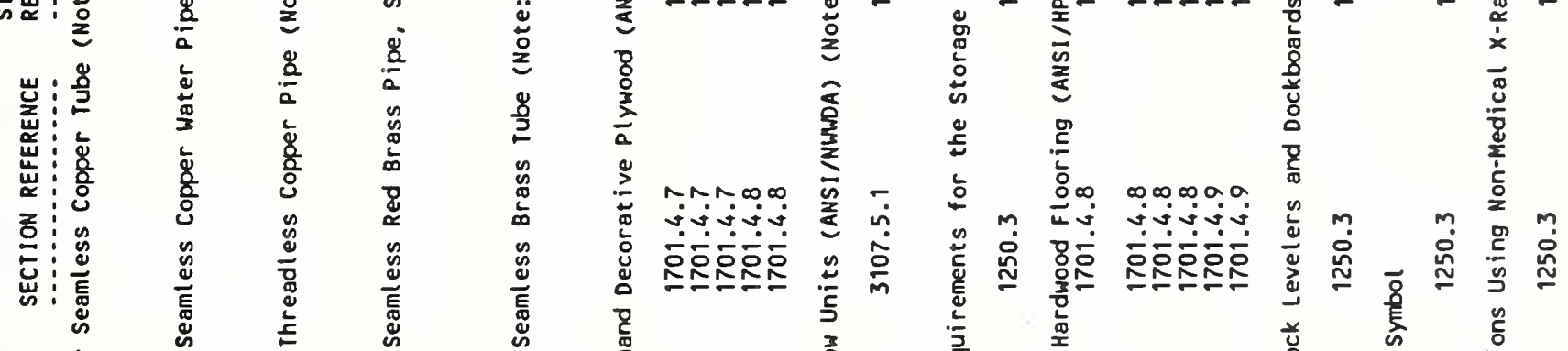

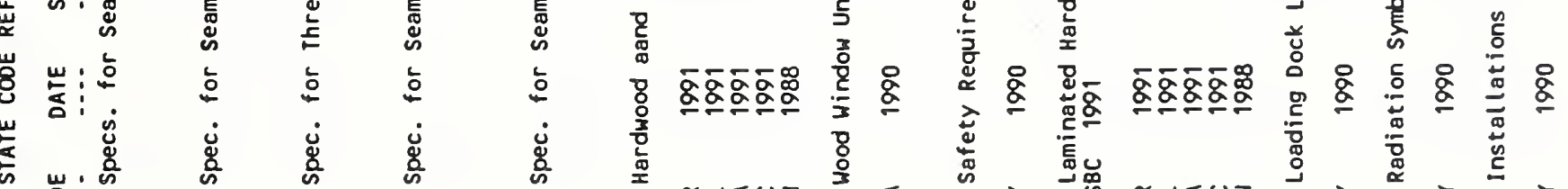

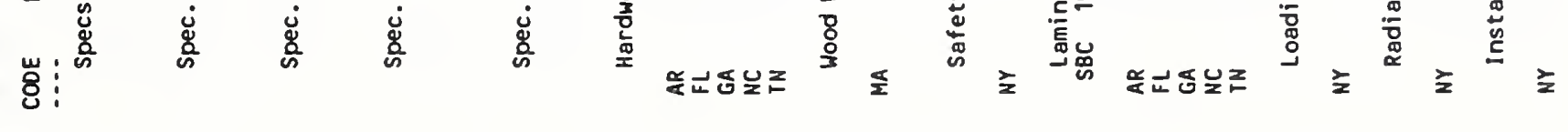

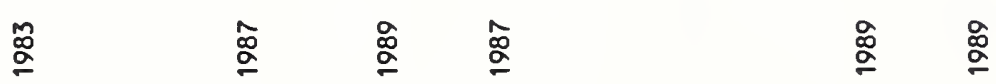

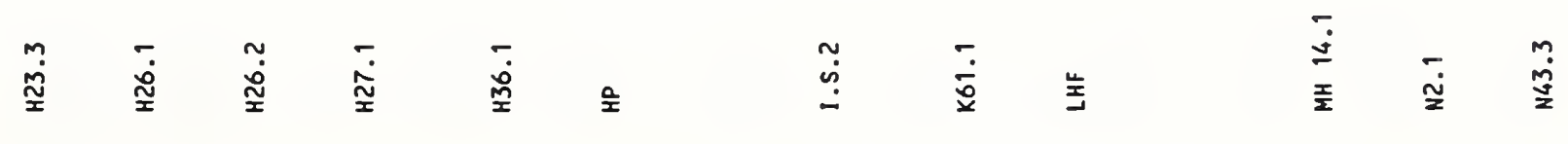




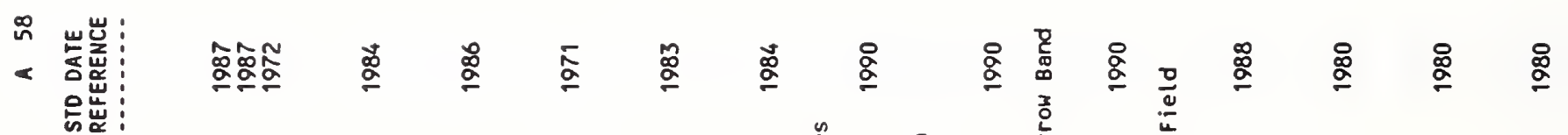

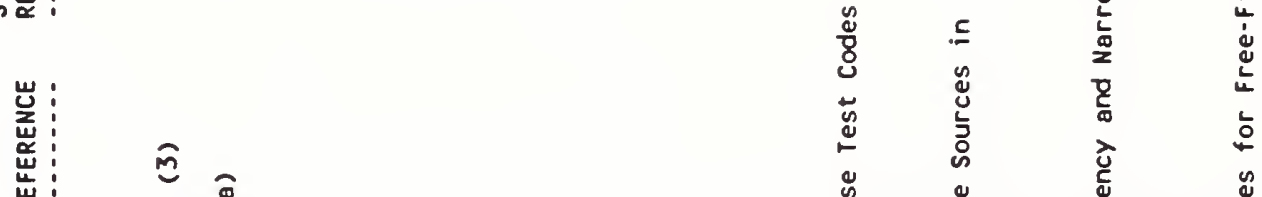

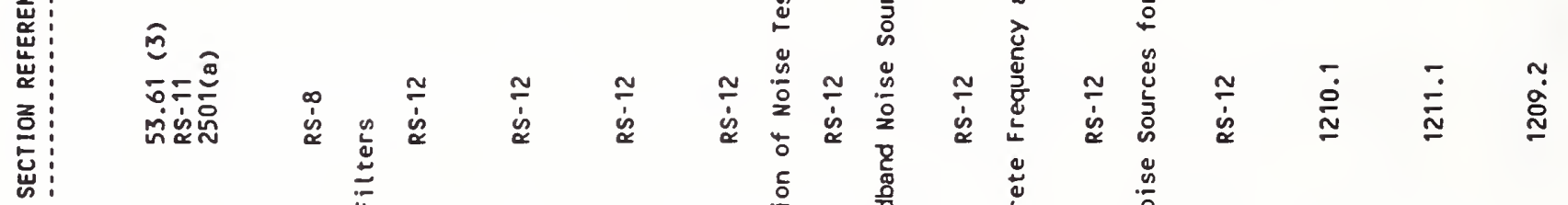

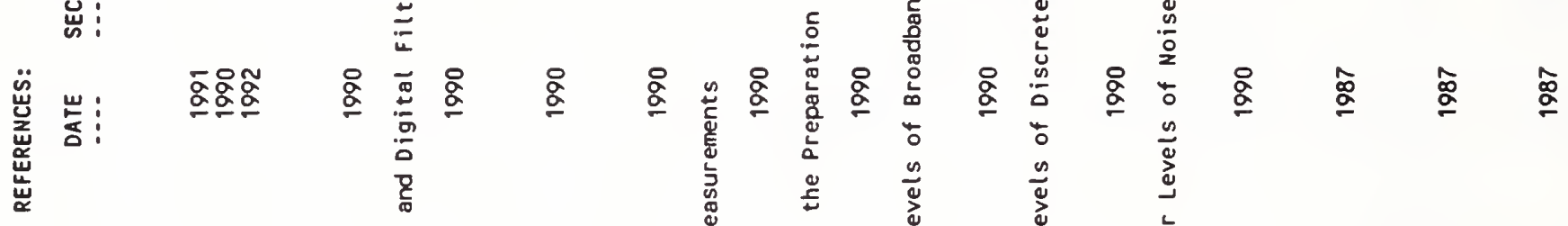

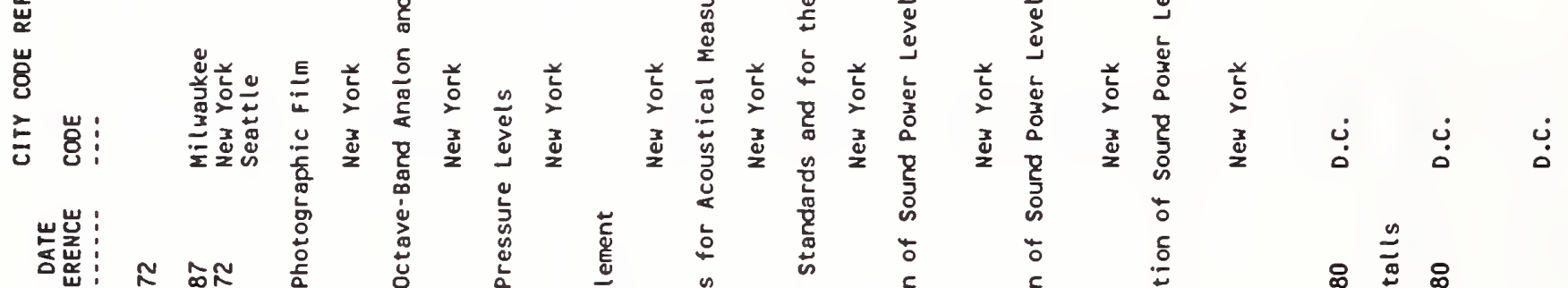

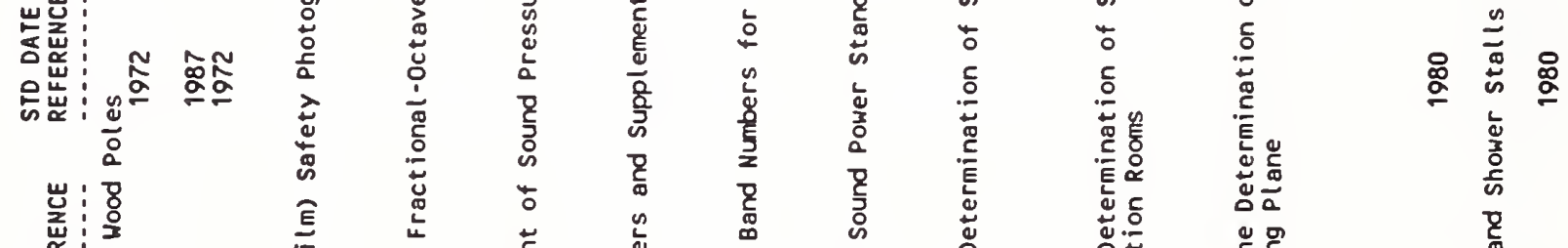

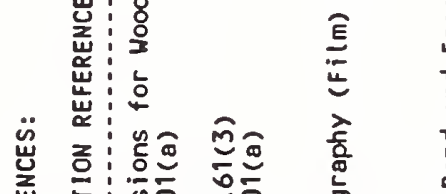

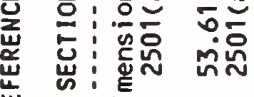

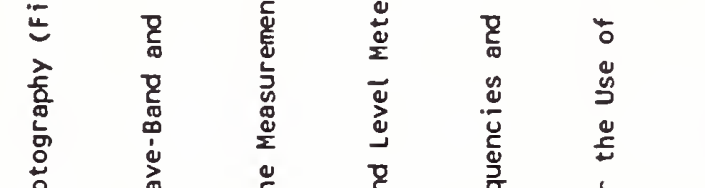

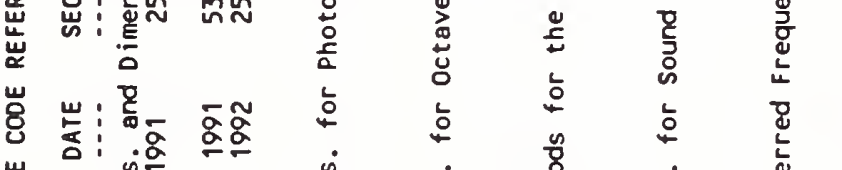

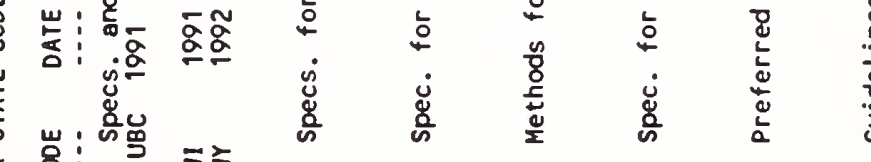

8ㄴํ의

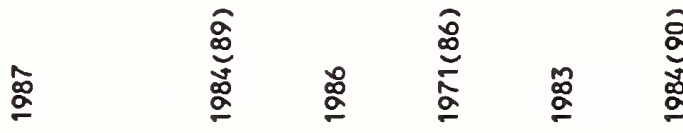

高 $\stackrel{\circ}{\circ}$

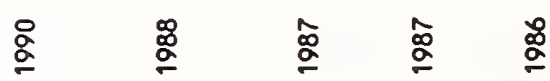

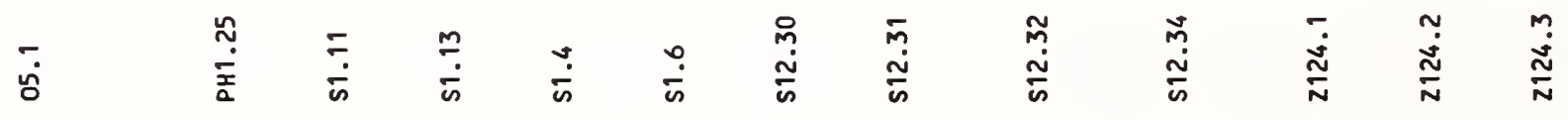




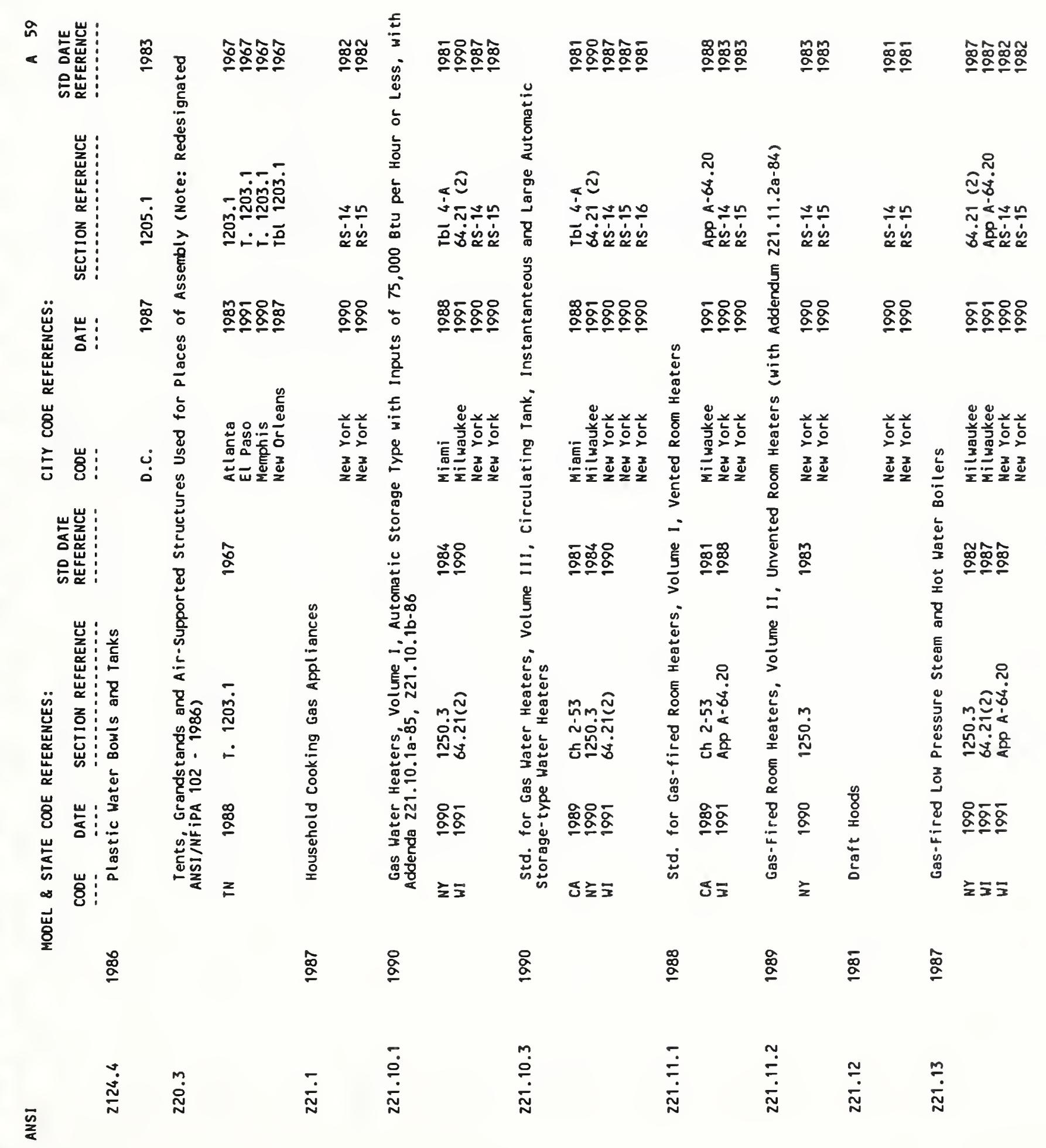




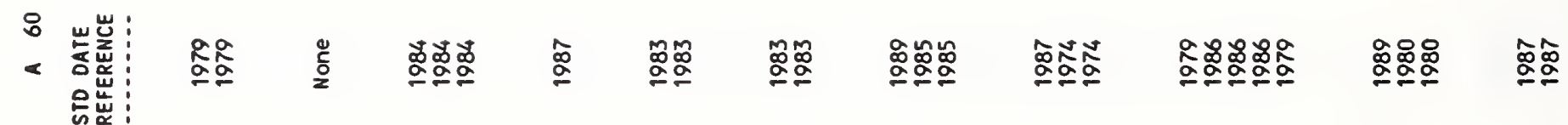

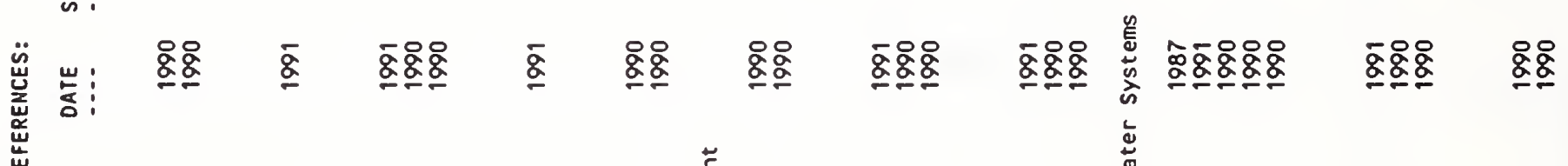

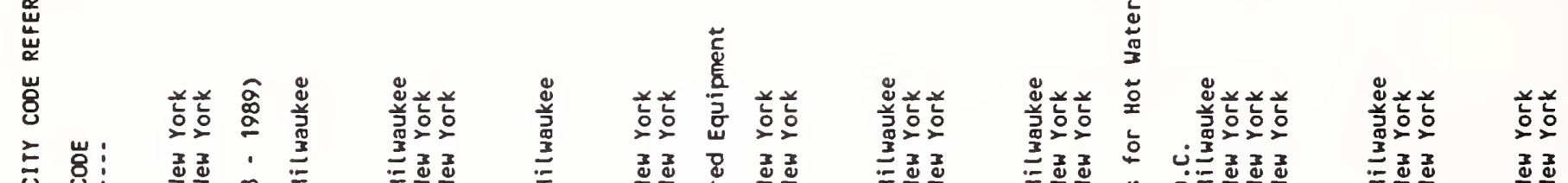

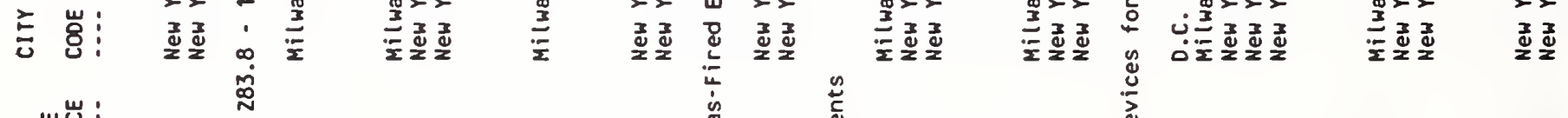

政

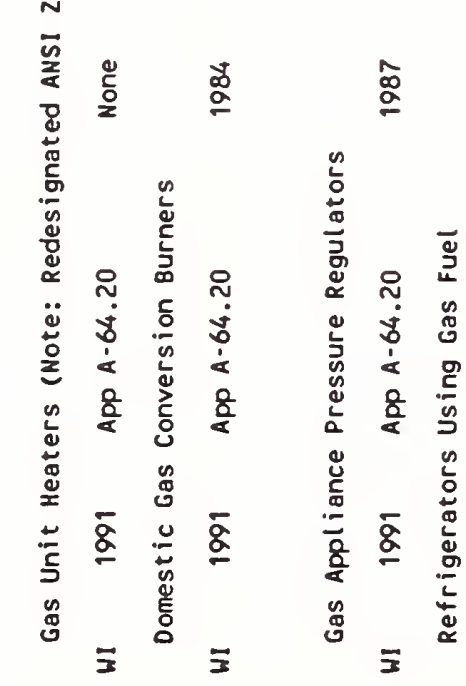

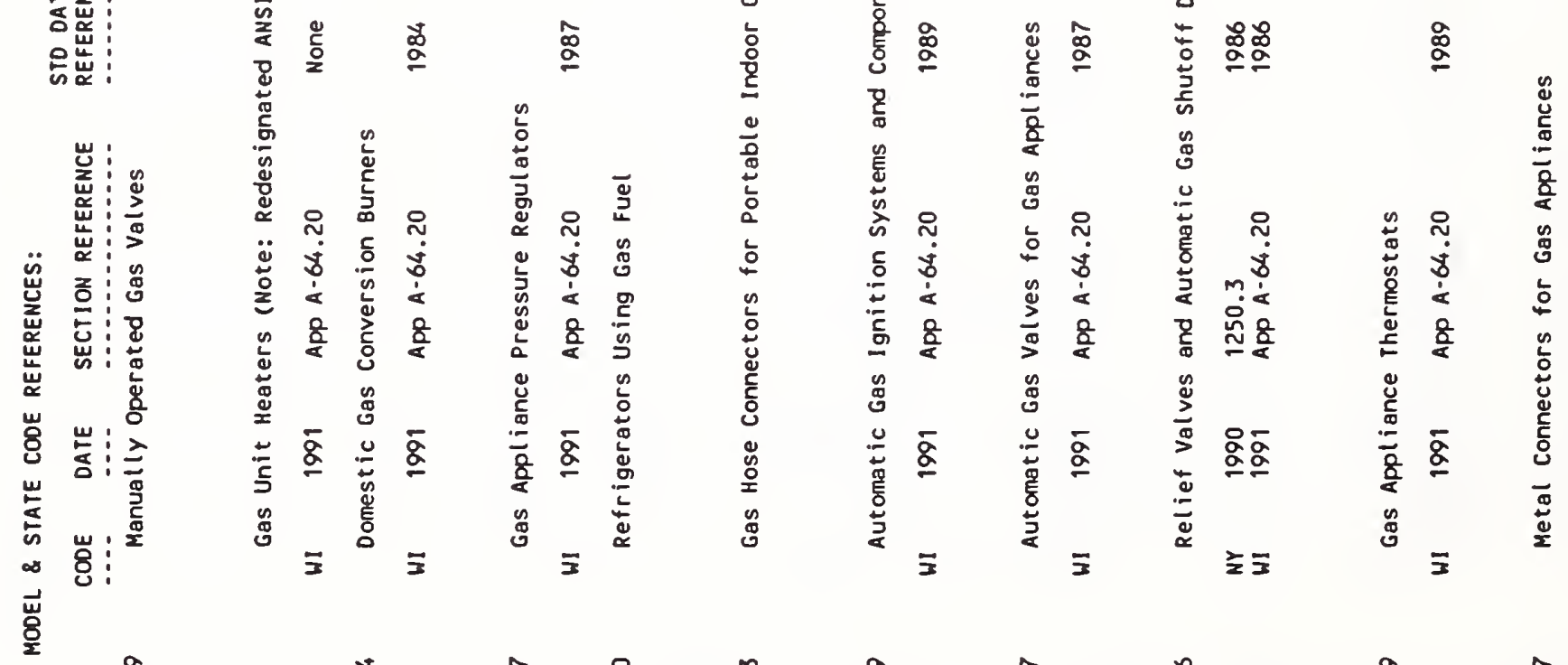

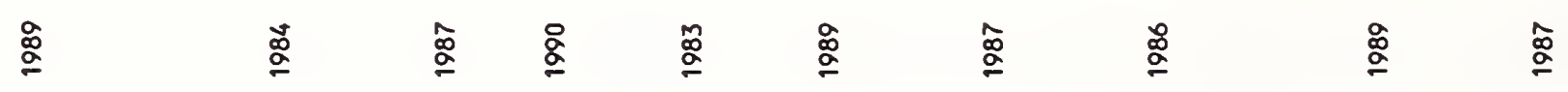

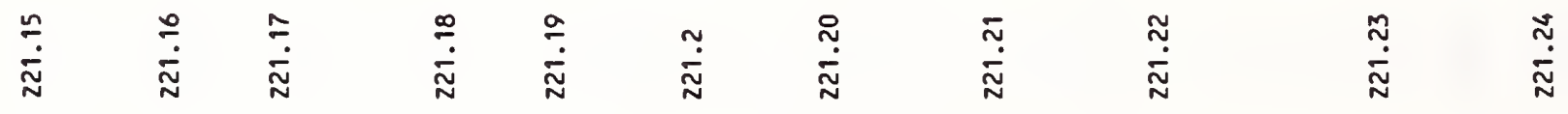




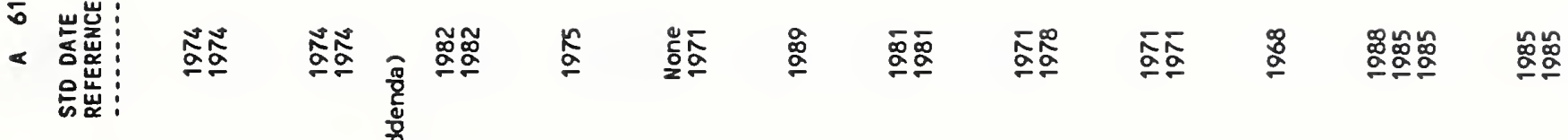<smiles>C1CCCC1</smiles>

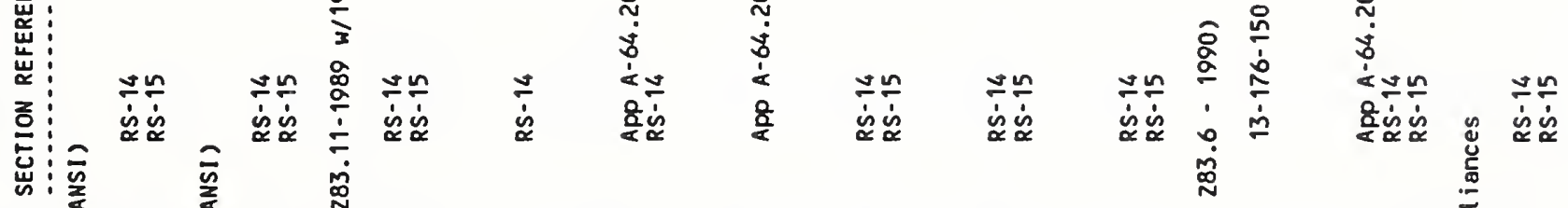

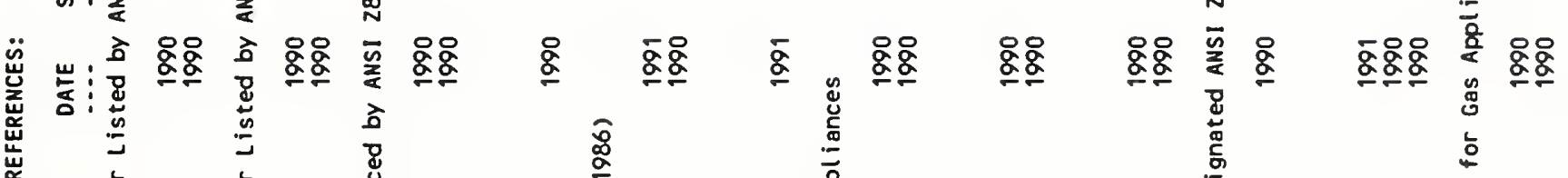

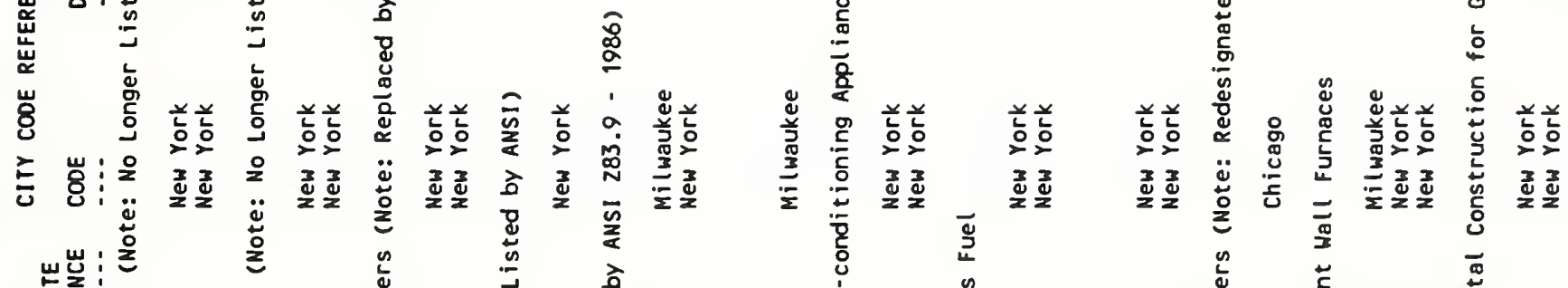

政:

施:

崖:

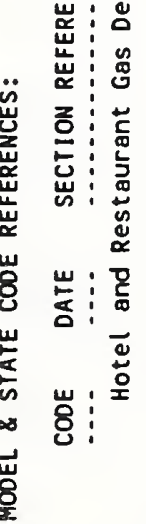




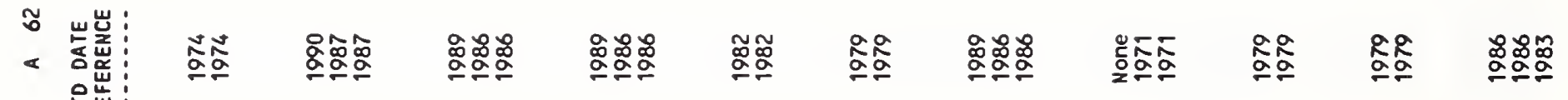

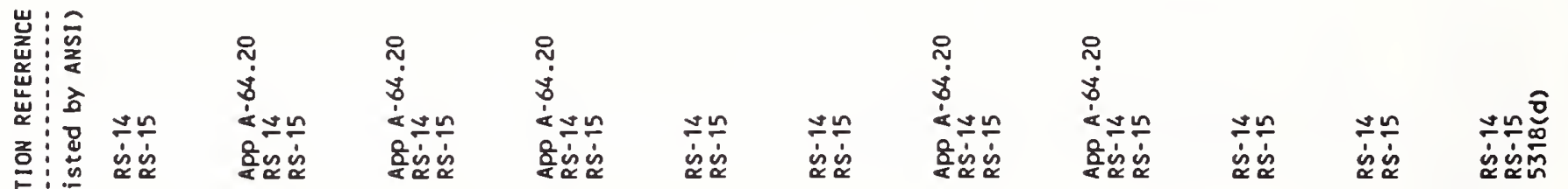

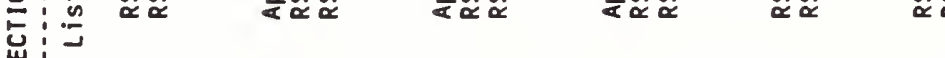

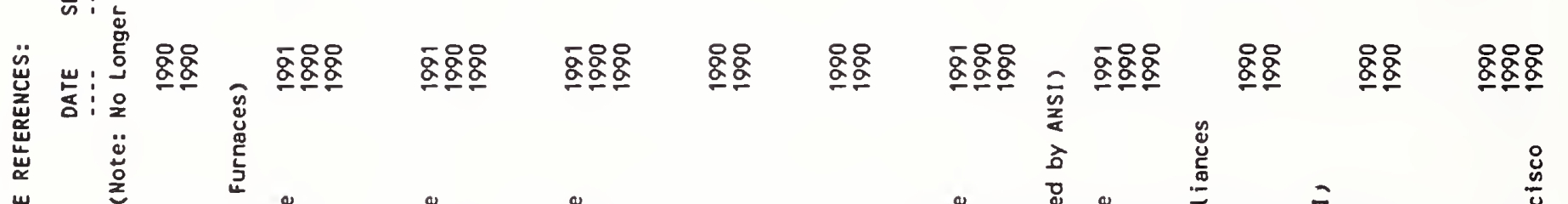

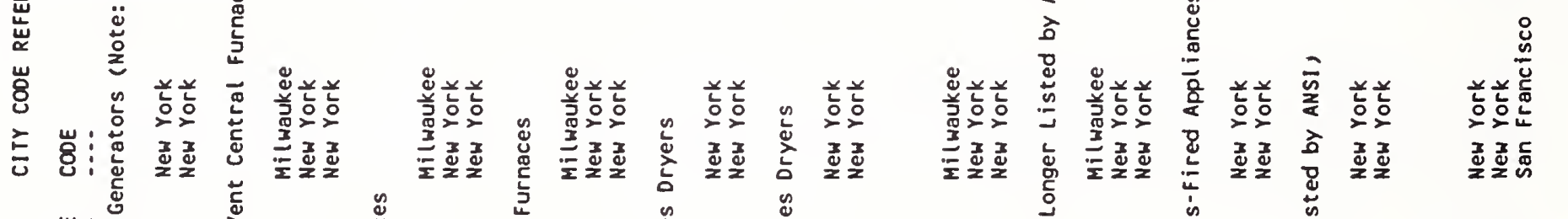

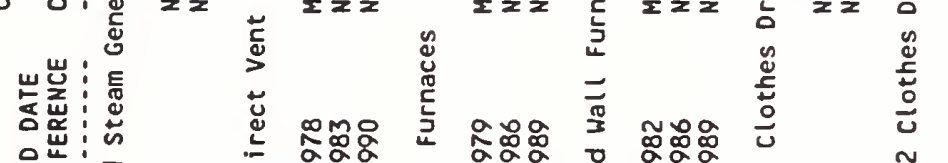

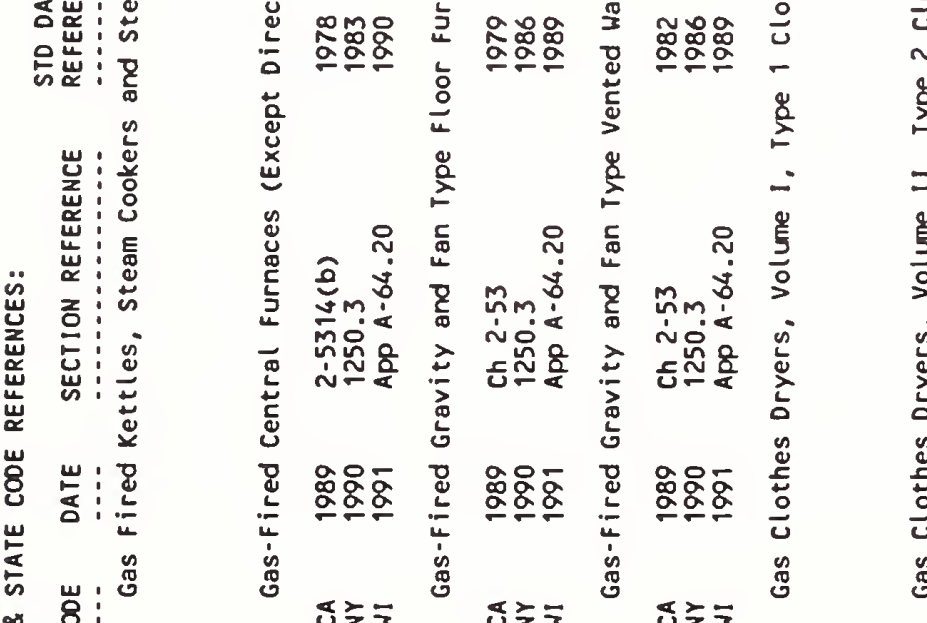
愛 8

$$
\text { 品 }
$$<smiles>[TlH]</smiles>

ڤ

๕

ฐ $\cong$

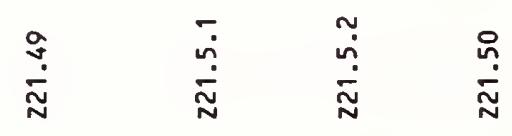

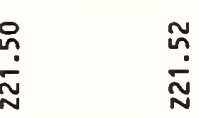

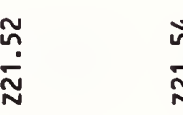

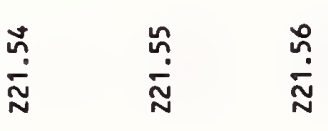

$\stackrel{\leftrightarrow}{3}$

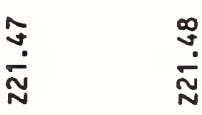

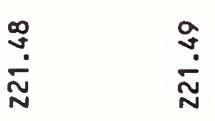

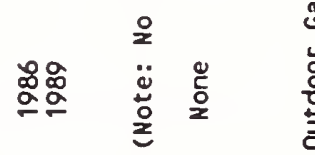

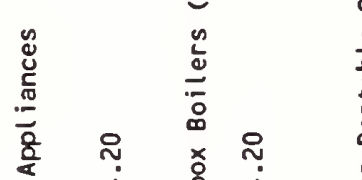

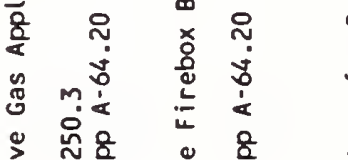

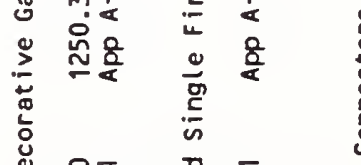

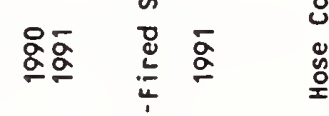

立嘀

畜产

ฒำ

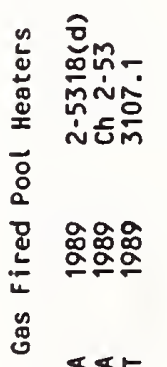

ธระ 


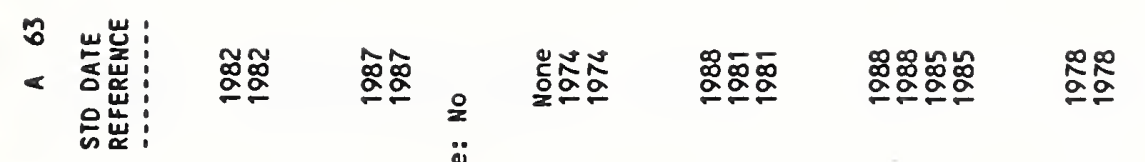

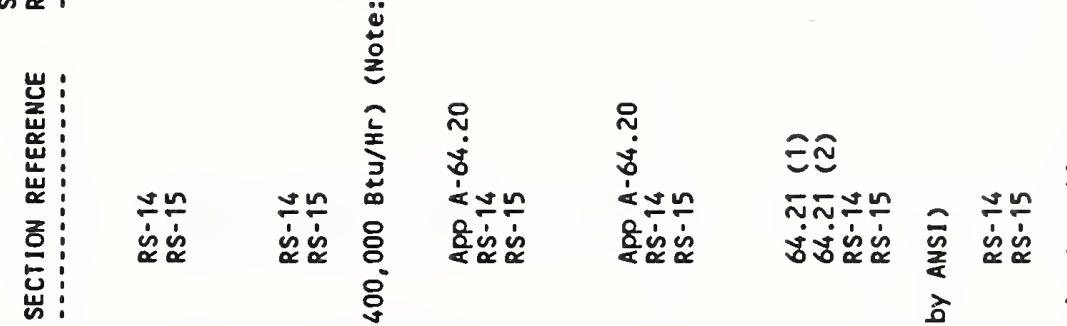

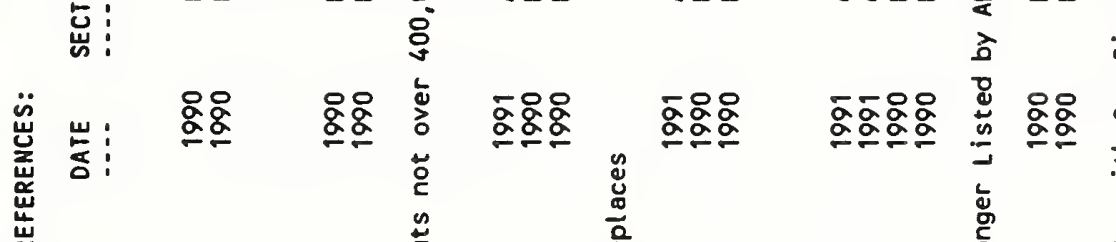

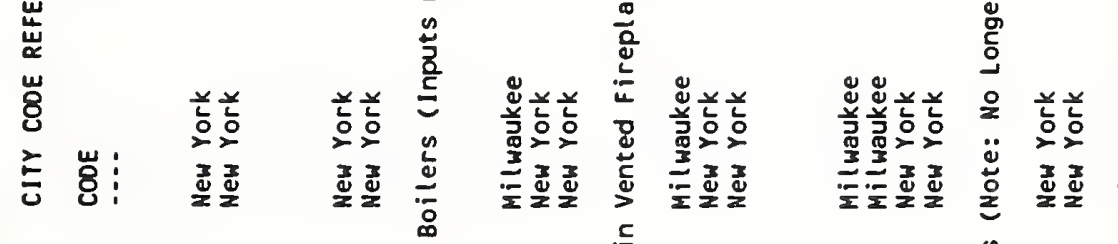

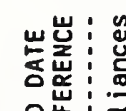

㩆

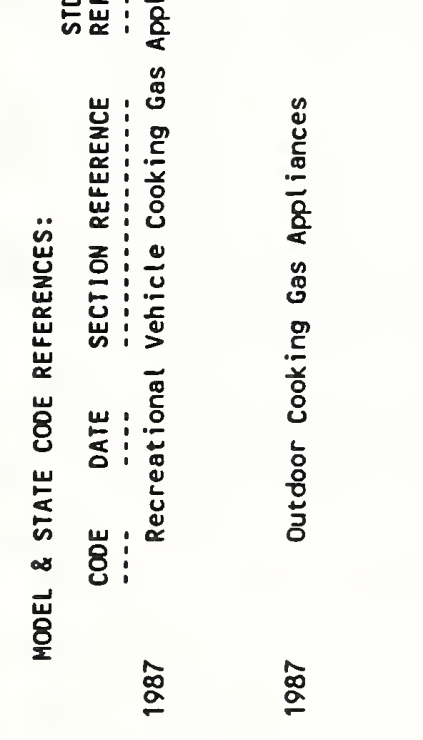

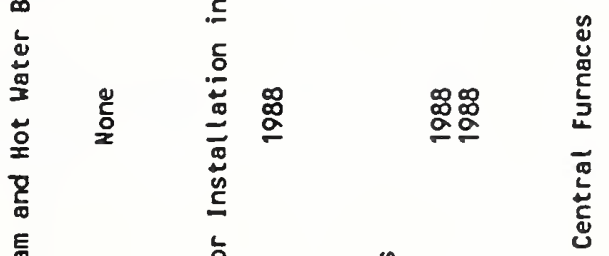

at

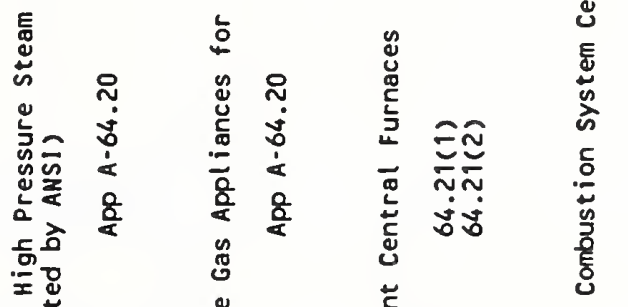

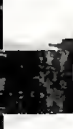

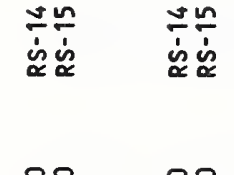

I

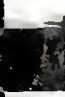

8

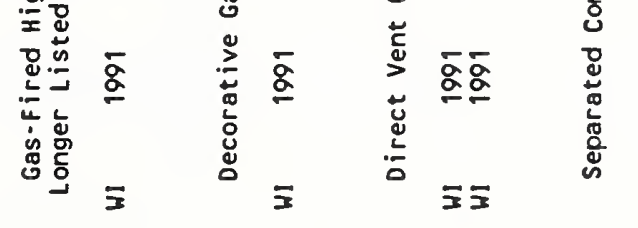

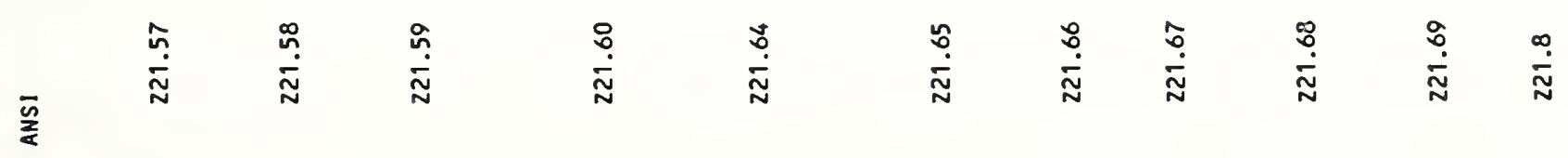

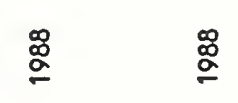

$\underset{\Xi}{2}$

$\stackrel{\mathrm{a}}{\mathrm{\sigma}}$ 


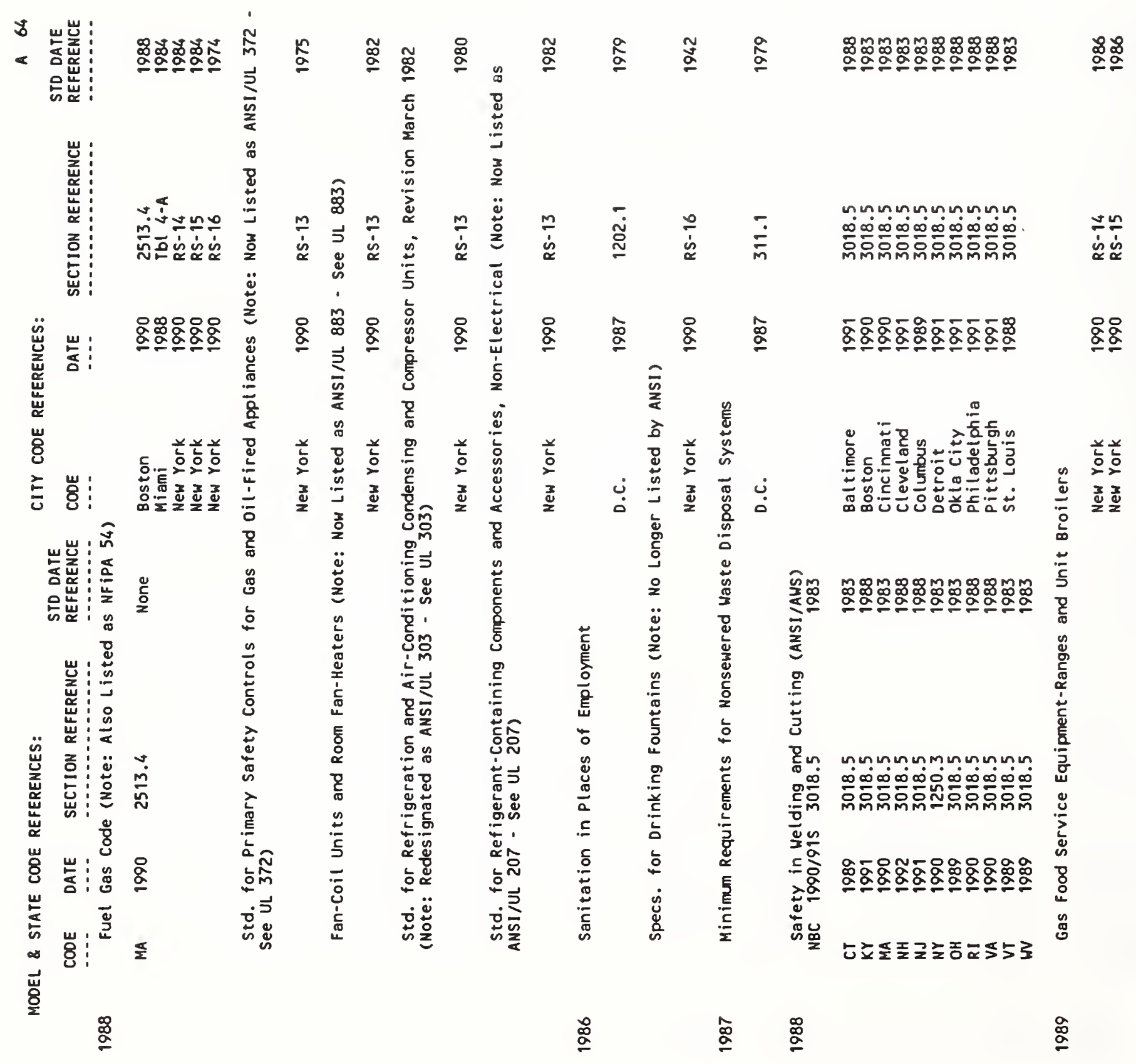
产

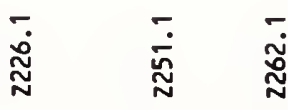

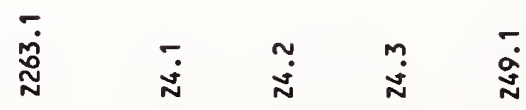

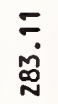




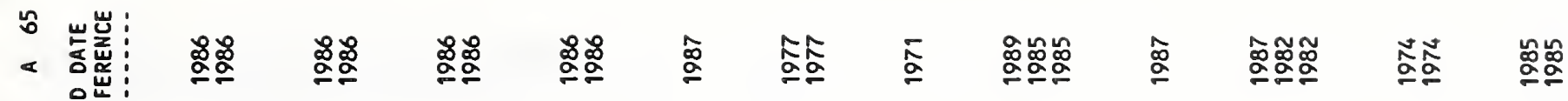

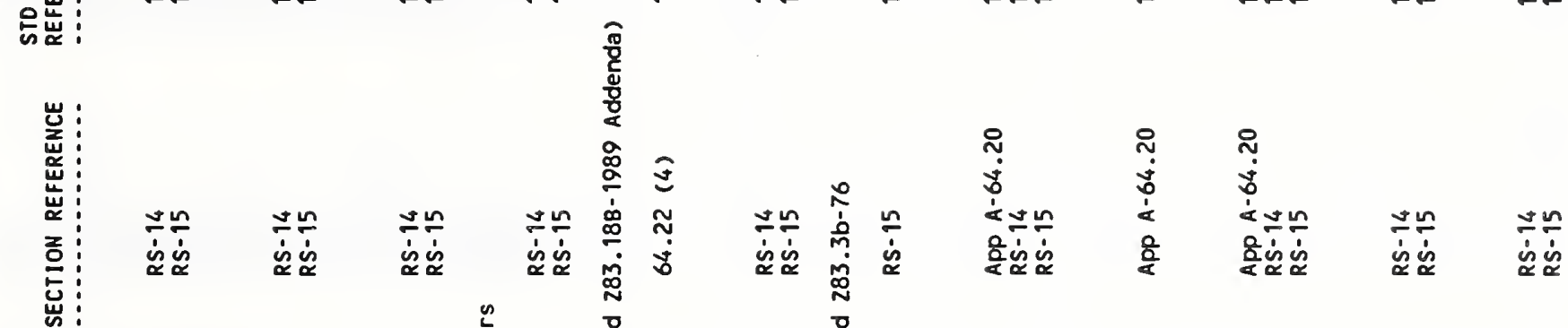

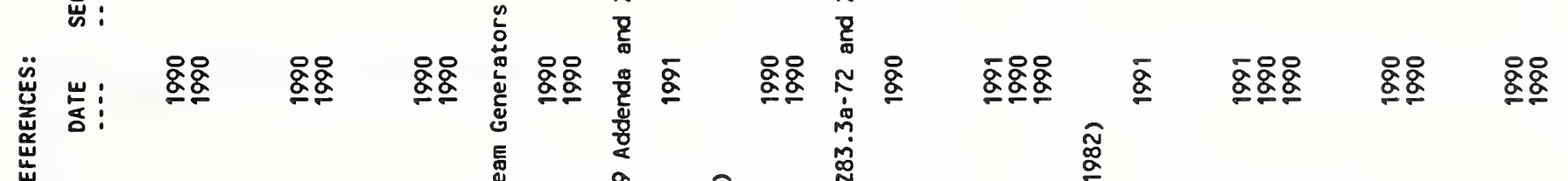

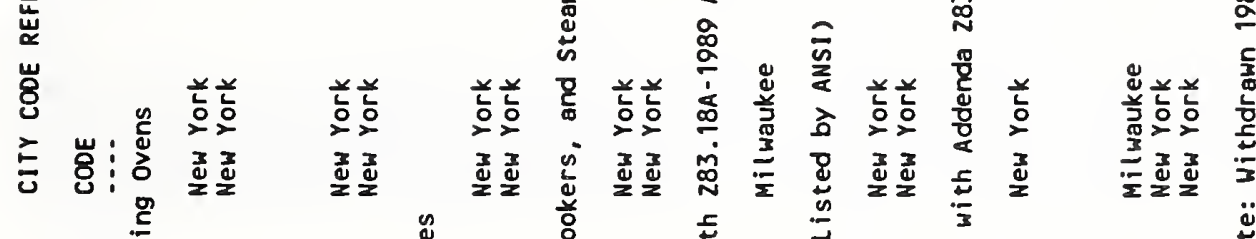

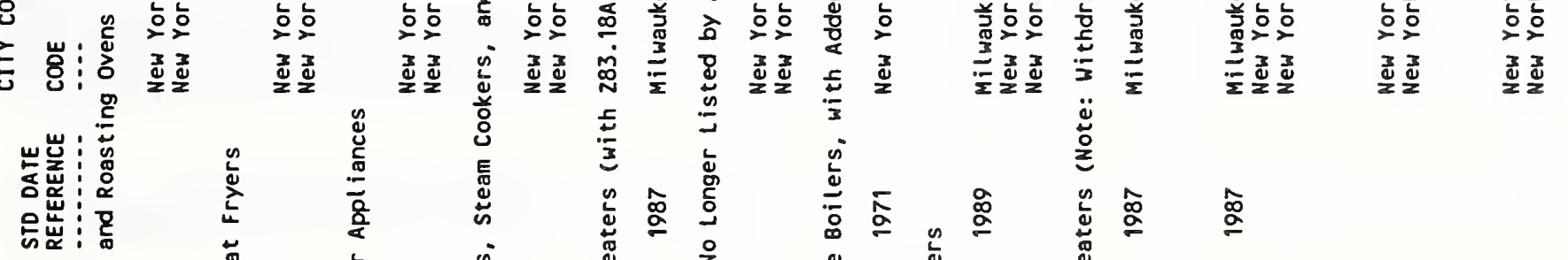

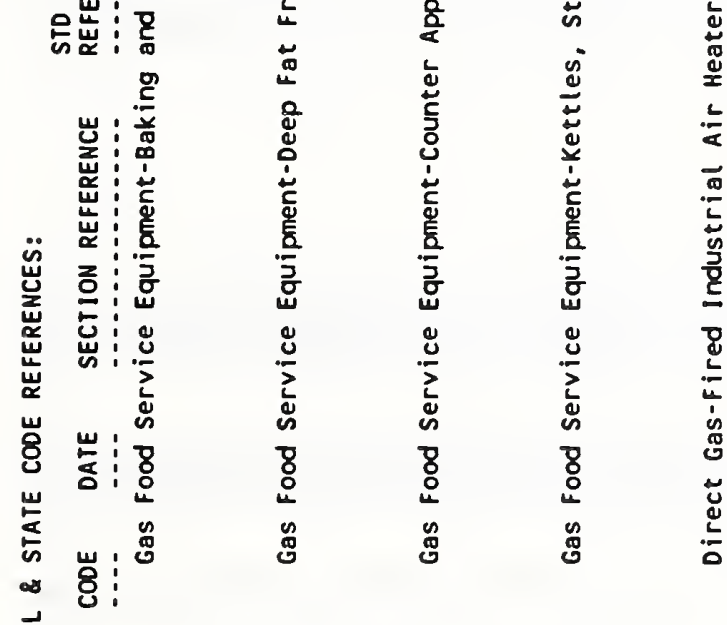

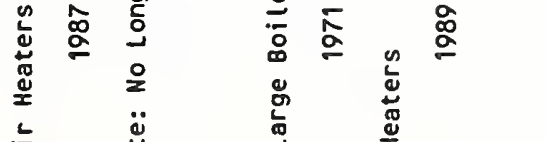

高

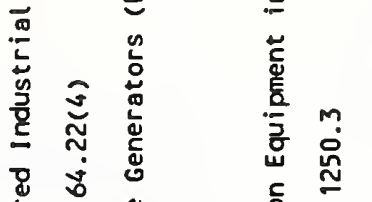

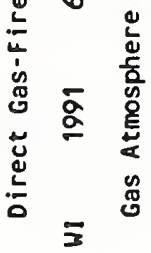

@

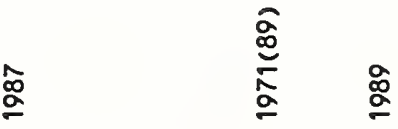

@

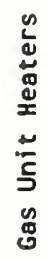

$\overline{\underline{z}}$

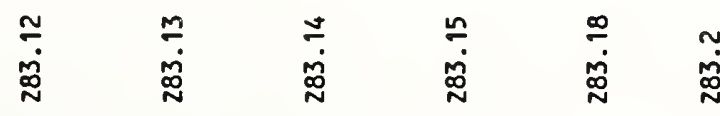

艿

莡

鱼总 


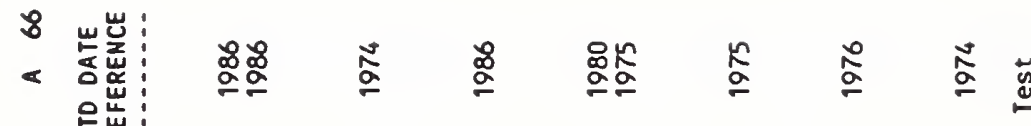

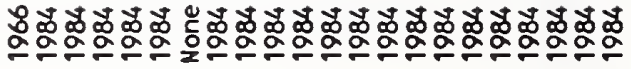

范

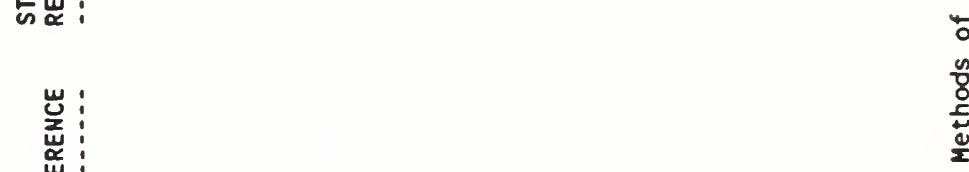

至

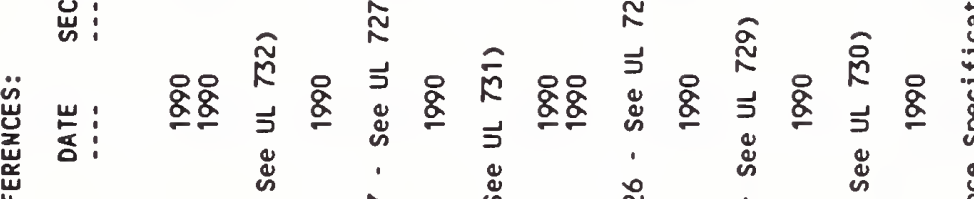

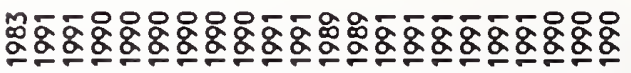

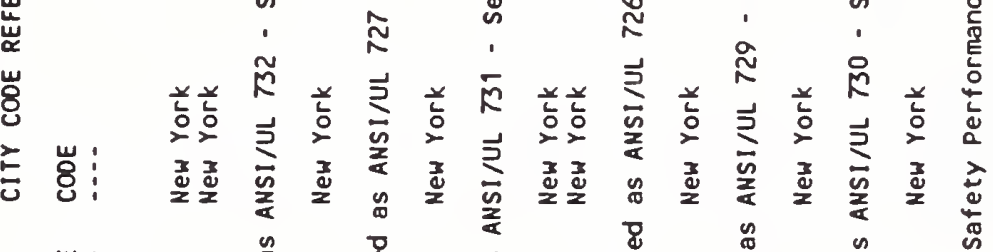

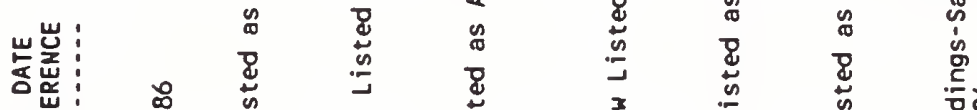

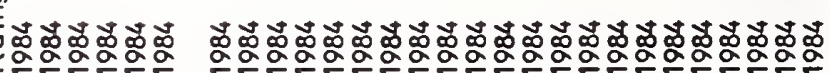

岂

일

క్न

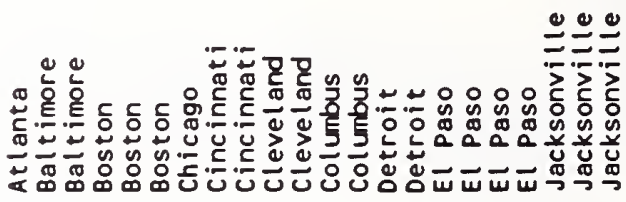

.

总

峞

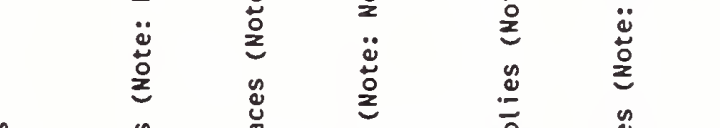

造

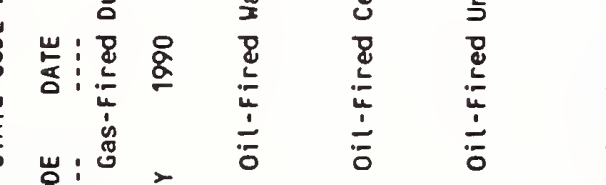

岁: z

$\stackrel{2}{2}$

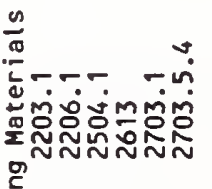

वंmminmo

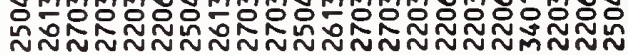
$\frac{n}{2} \frac{\omega}{a}$

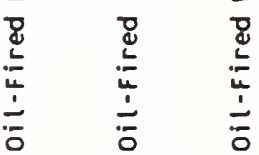

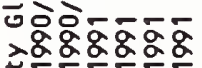

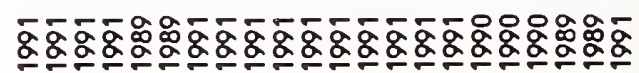

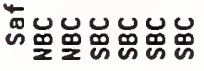

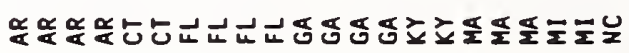

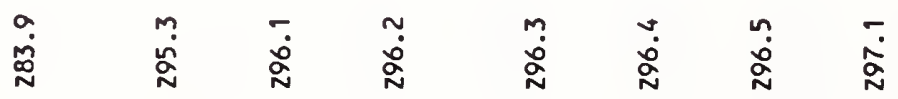




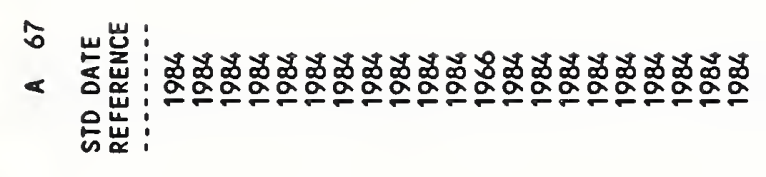

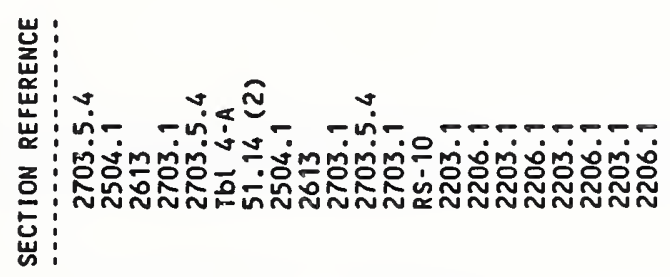

䓛

崖 兰

岁 齐

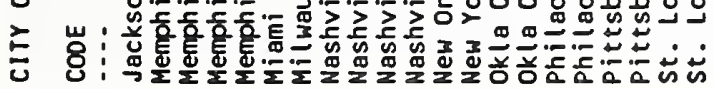

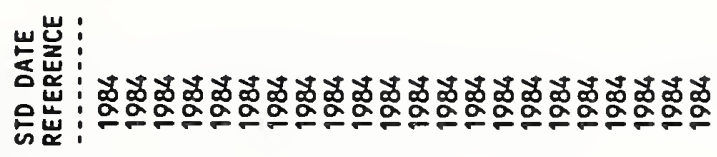

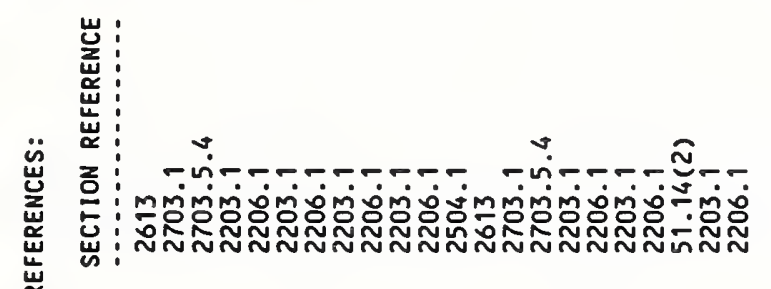

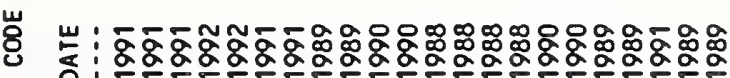

岕

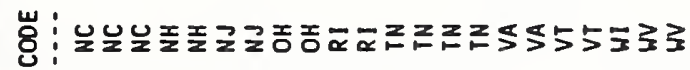

훙

ક્ટ

$\sum_{\frac{\pi}{2}}^{\grave{a}}$ 


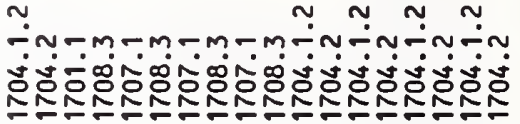

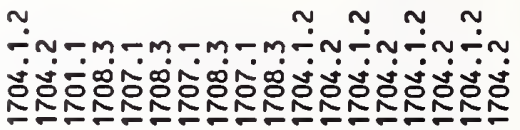

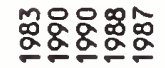

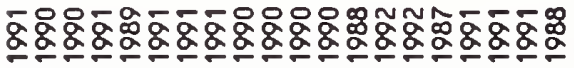

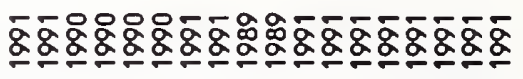

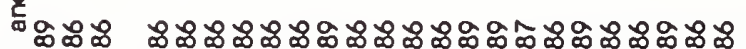

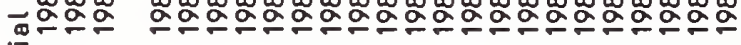

ஐa

,

苞

nกNก n

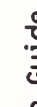
mmmmmm ะำํํำำำ

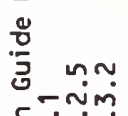

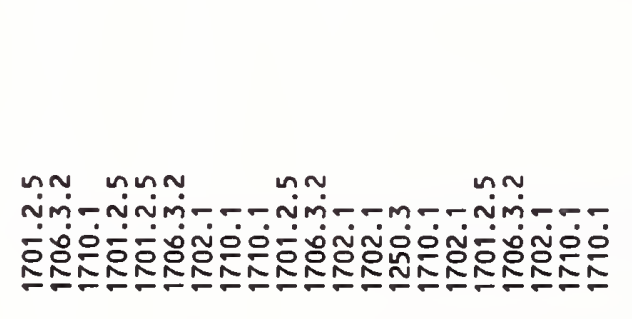

$\stackrel{\infty}{m}$

$\dot{0}$

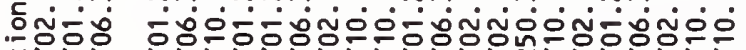
고도

\section{象向}

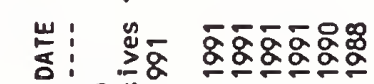

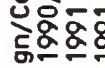

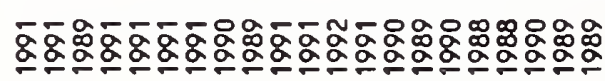
造总总惫

山 : 政邑

8

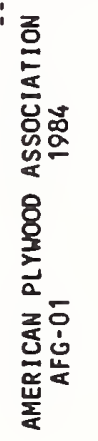

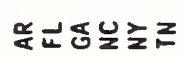

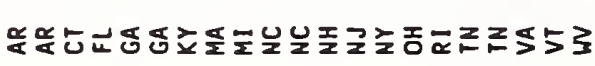

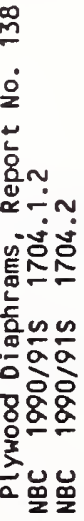

$\stackrel{\Xi}{\sigma}$

$\stackrel{8}{2}$

䓢

$\frac{n}{m}$ 


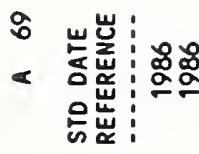

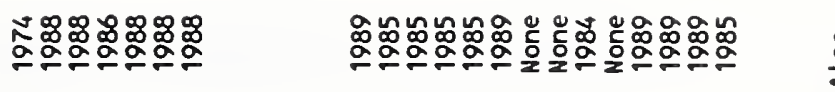

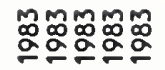

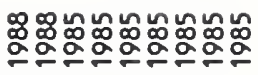

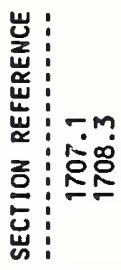

$$
\begin{aligned}
& \text { 岂 }
\end{aligned}
$$

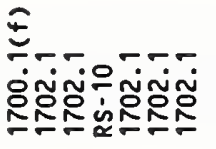

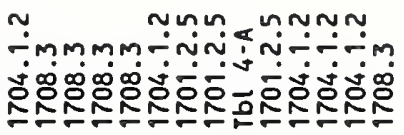

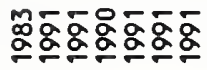

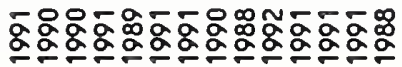

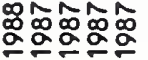

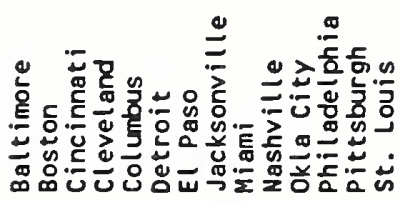

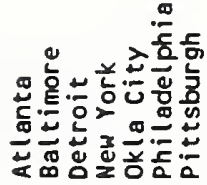

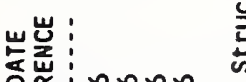

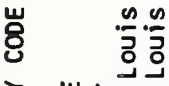

$$
\begin{aligned}
& \text { 岩额 } \\
& \text { 就 } \\
& \text { 㧱 }
\end{aligned}
$$

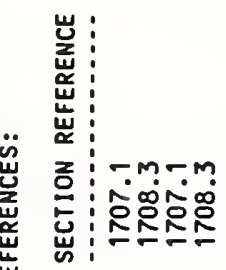

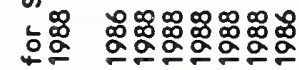

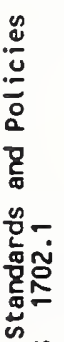
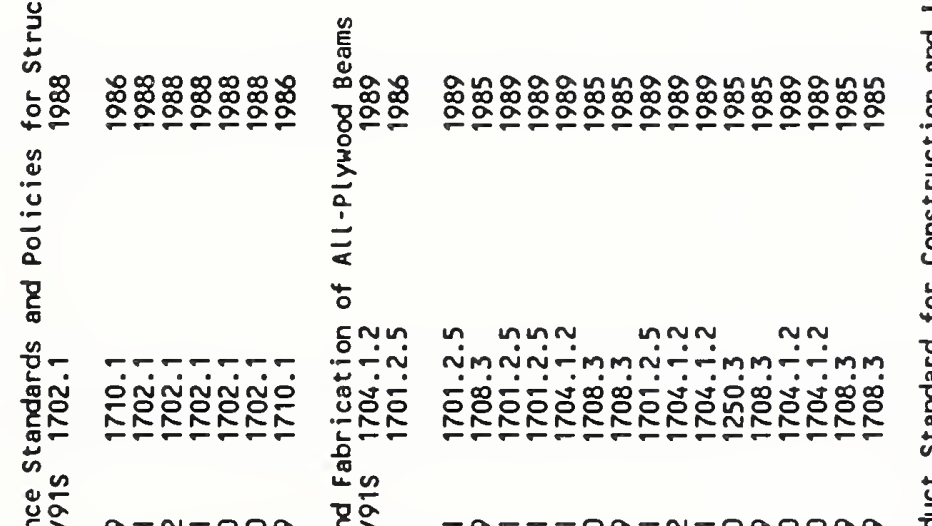

$$
\begin{aligned}
& \text { t。 }
\end{aligned}
$$

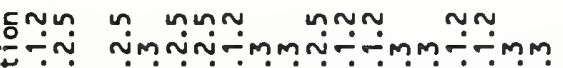

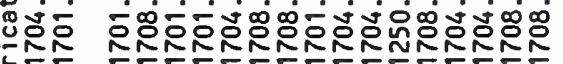

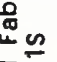

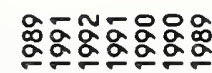

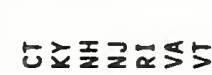

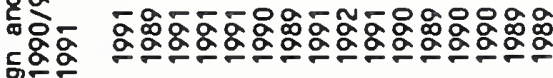

$$
\begin{aligned}
& \text { 品品 }
\end{aligned}
$$

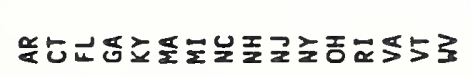

$$
\begin{aligned}
& \text { 产 } \\
& \bar{\sigma} \quad \bar{\alpha} \\
& \text { 密 } \\
& \stackrel{\dddot{a}}{\stackrel{\Xi}{\alpha}}
\end{aligned}
$$

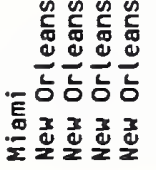

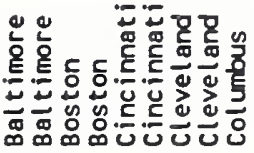

$$
\begin{aligned}
& \text { 을 } \\
& \dddot{\circ}
\end{aligned}
$$

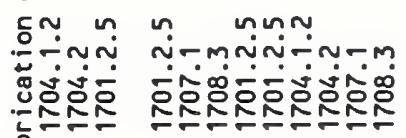

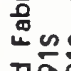

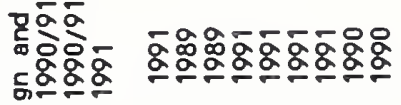

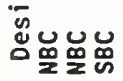

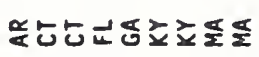

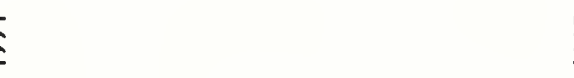

$$
\begin{aligned}
& \stackrel{\circ}{\circ}
\end{aligned}
$$




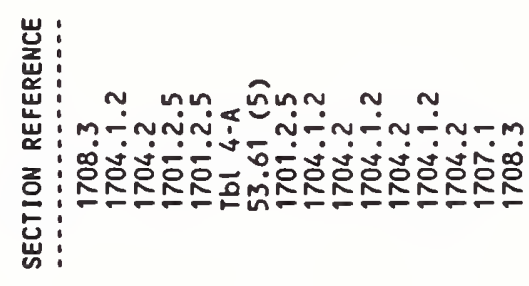

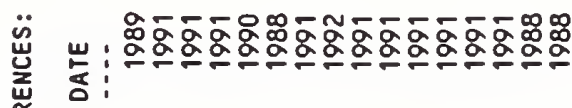

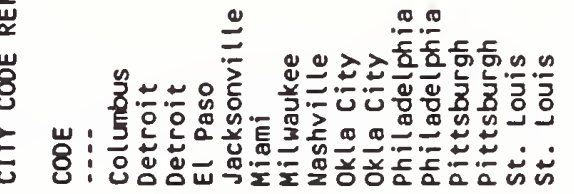

世ّ山心 :

ธิ 눈
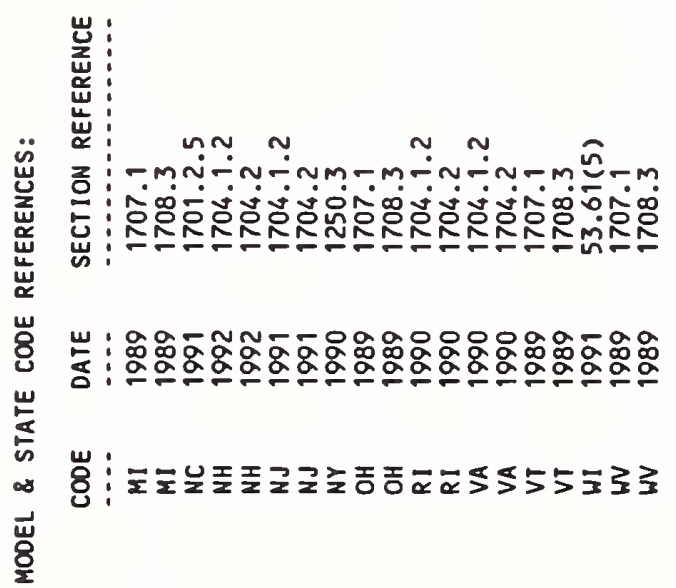

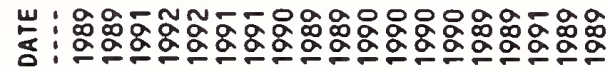

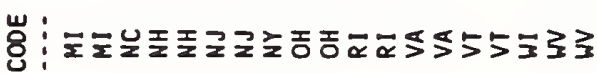

总

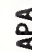

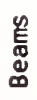

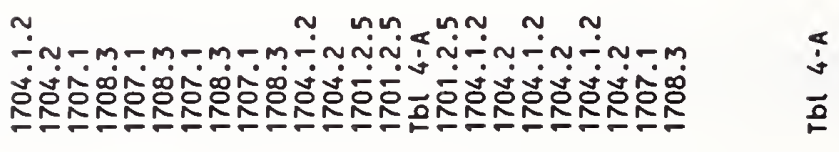

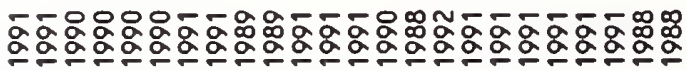

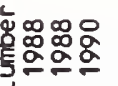

$$
\text { 음 }
$$

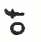

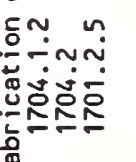
递电

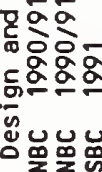

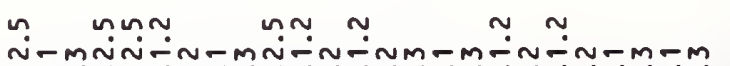

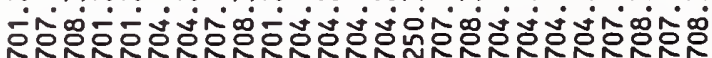

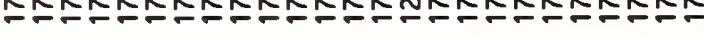

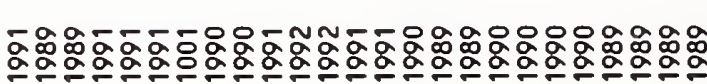

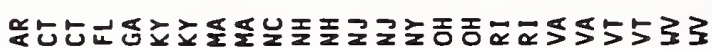

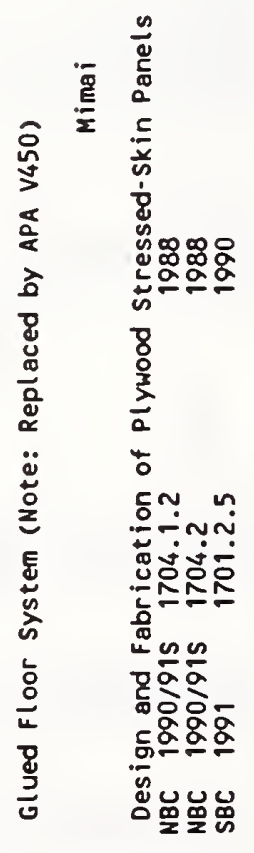

$\stackrel{2}{\circ}$

$\stackrel{m}{s} \stackrel{m}{g}$ 


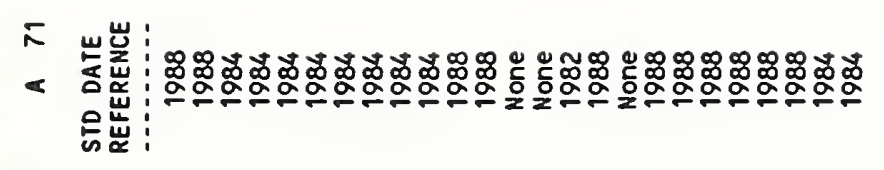

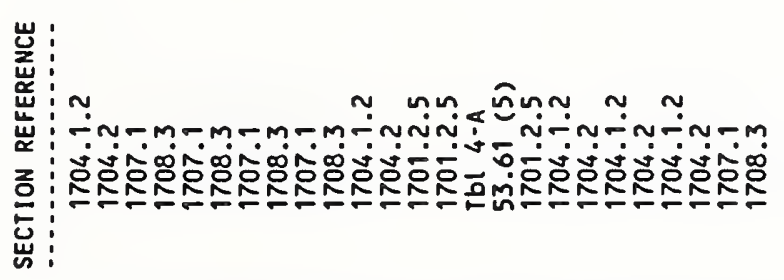
落 \&:

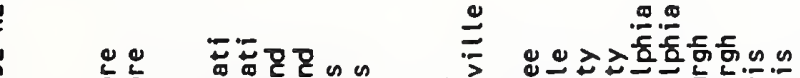

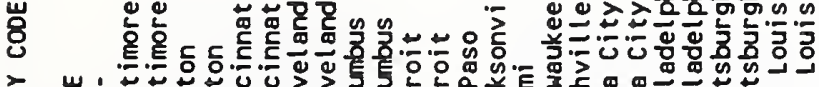

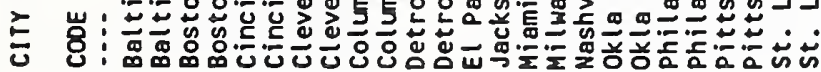

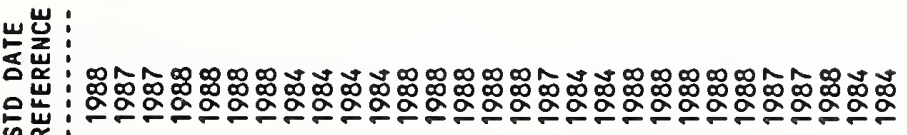

雚

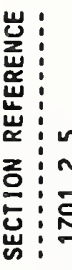

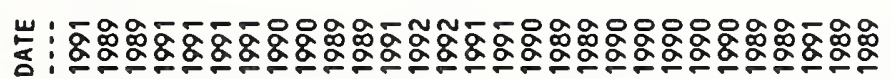

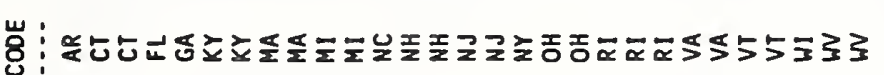

芒

$\stackrel{m}{\frac{m}{g}}$

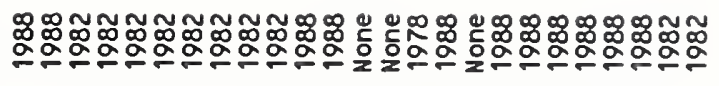

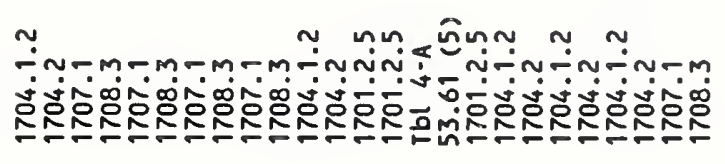

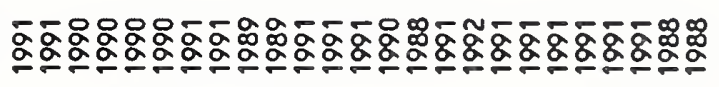

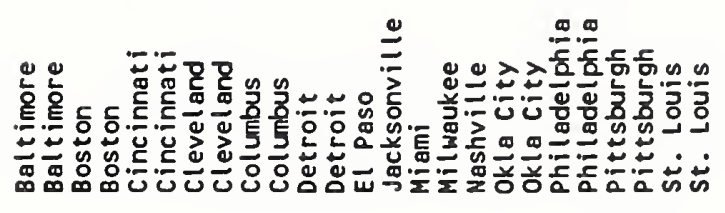

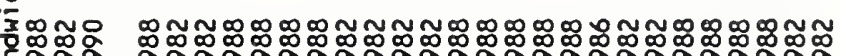
క్థ

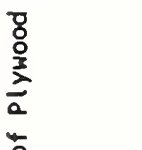

ธุ ?

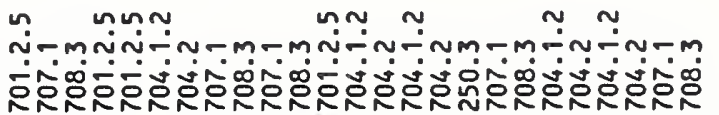

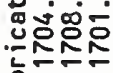
แேะே

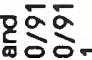

등으음

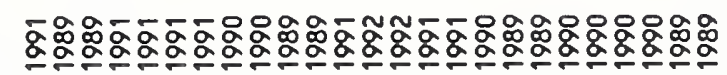

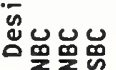

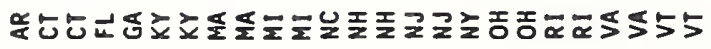




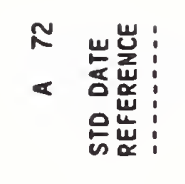

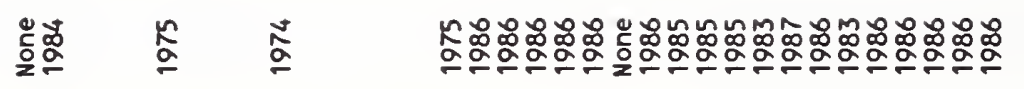

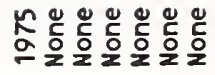

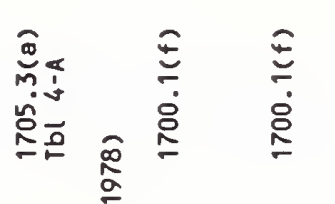

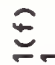

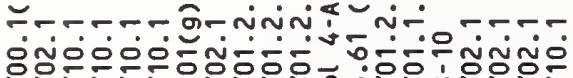

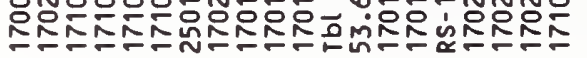

苞岕:

岁

莺 岁:

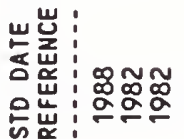

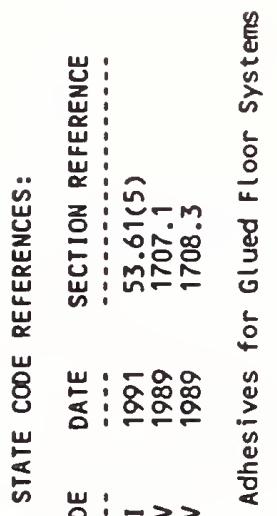

岁: $: 33$

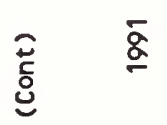

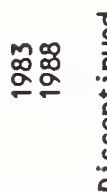

离 $\stackrel{\infty}{\infty}$ $\frac{a}{a} \stackrel{2}{a}$

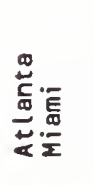

造

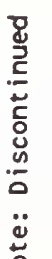

단

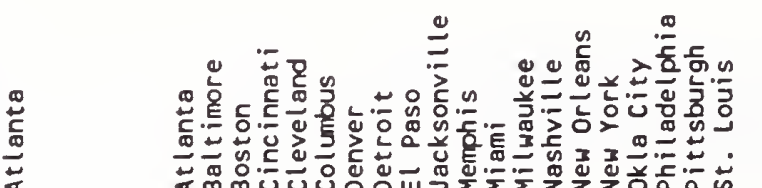

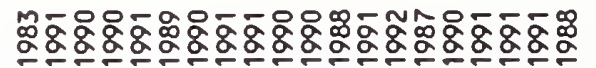

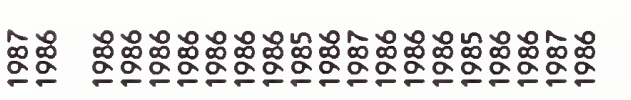

-

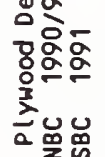

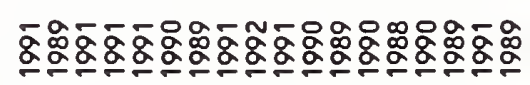

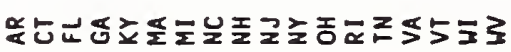

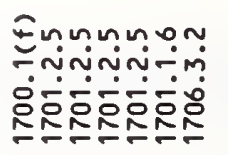

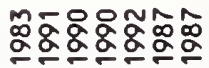

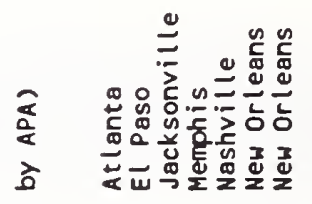

芠

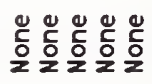

$\stackrel{\ddot{0}}{\stackrel{0}{z}}$

跑

जิn iñinin

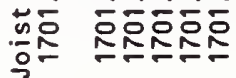

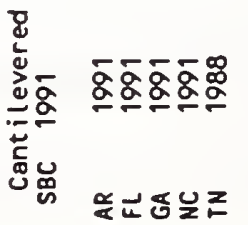



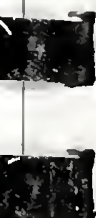

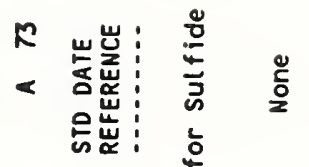

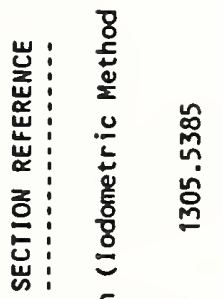

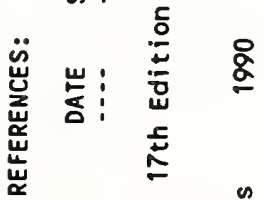

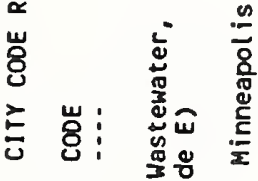

岂:

和崫:

文

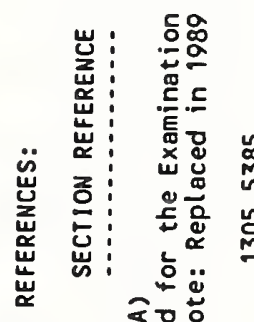

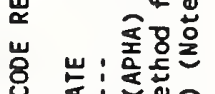

庹

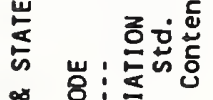

\& 否:

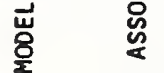

吾

74

产

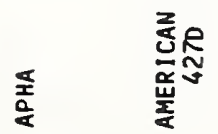


\& 訔:

占岩?

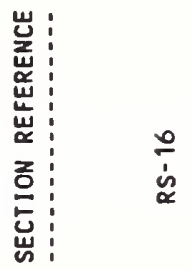

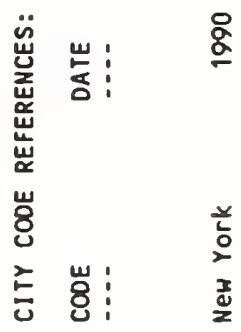

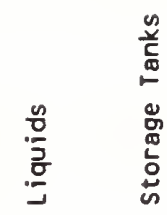

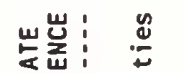

稳:

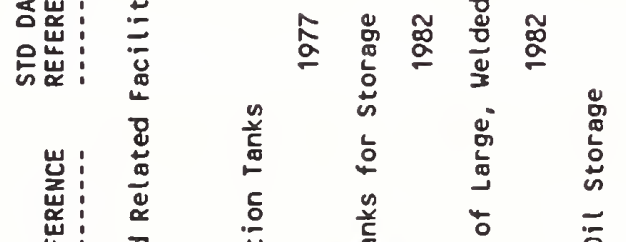

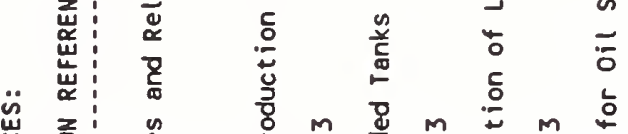

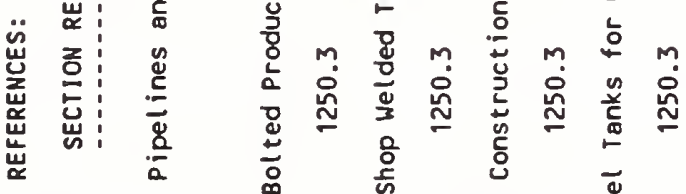

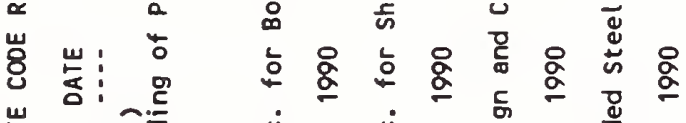

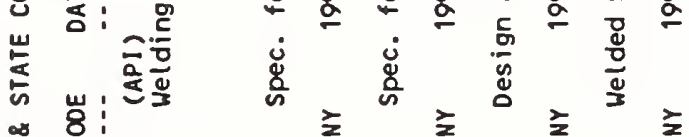

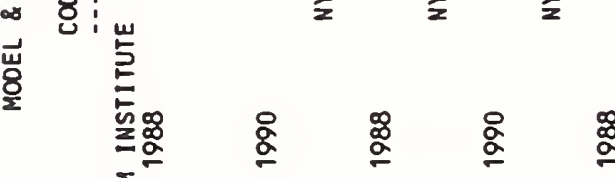

喜

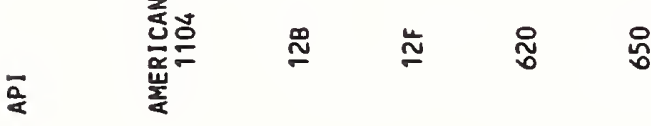




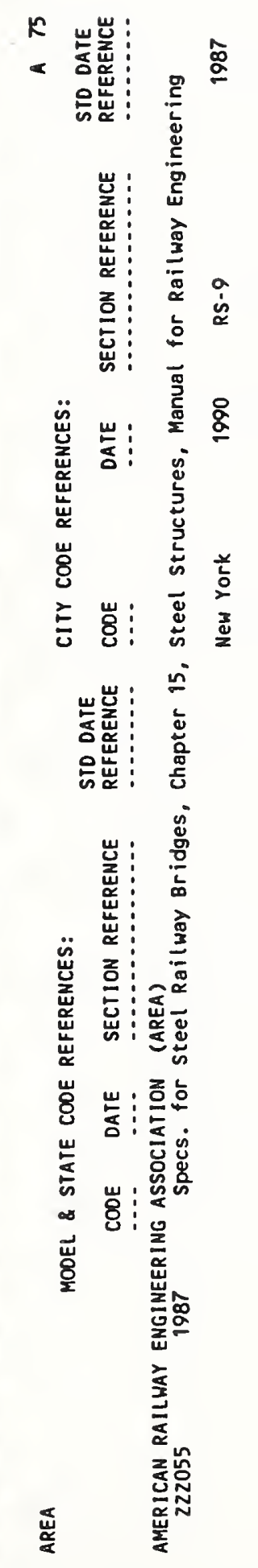




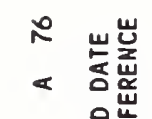
要 $\quad \frac{\mathfrak{g}}{3}$
密
\$

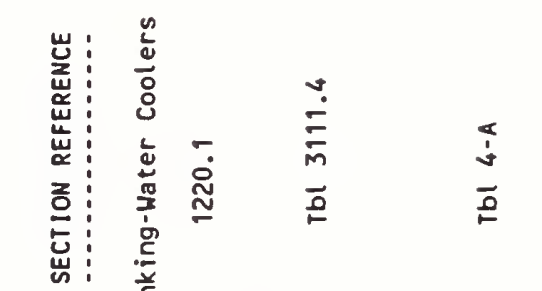

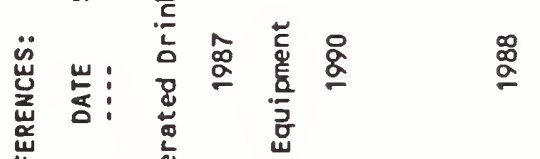

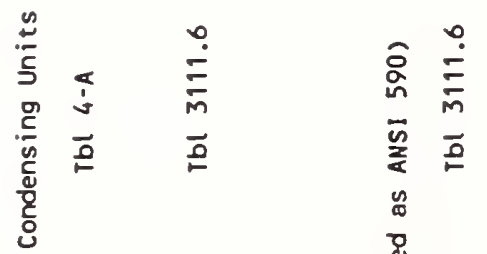

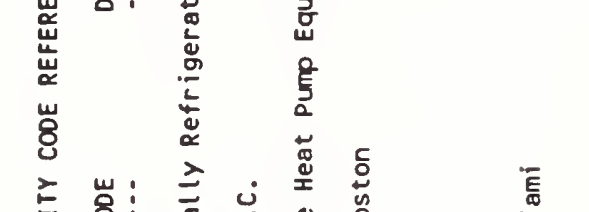

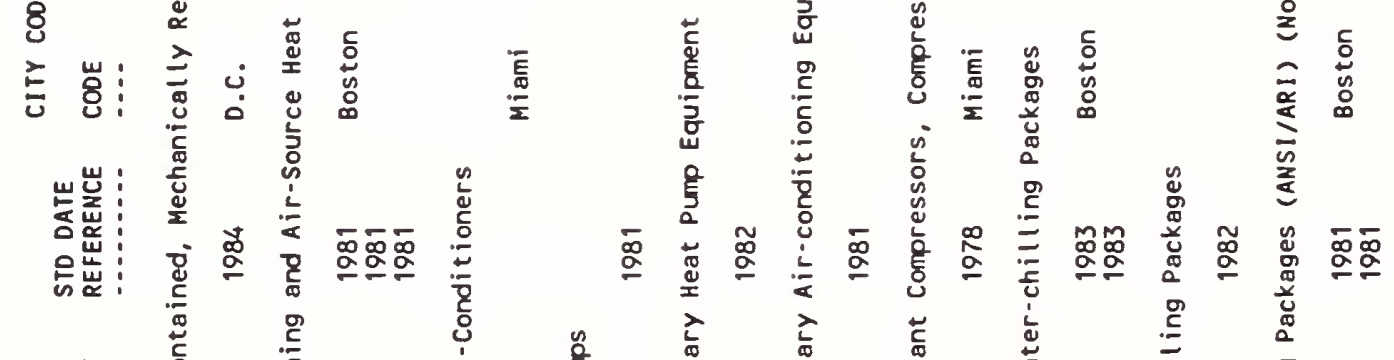

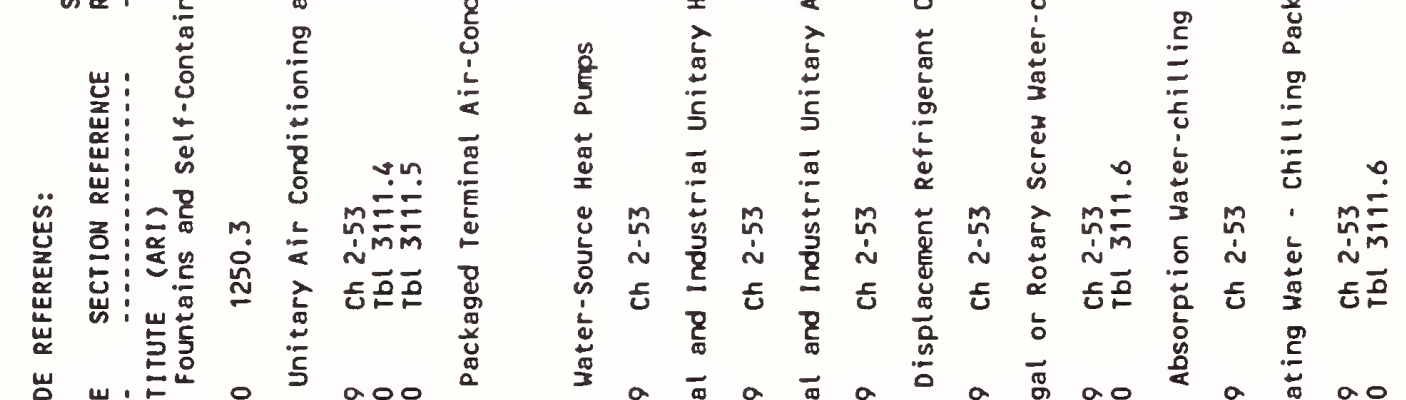

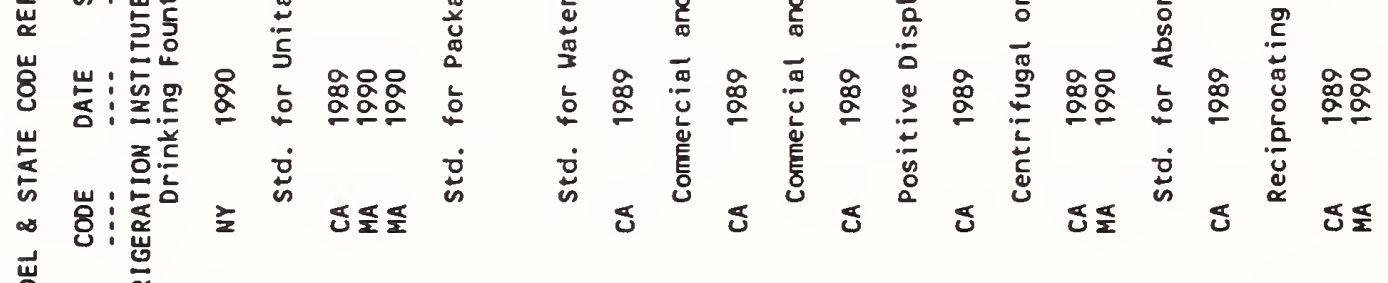
蓉

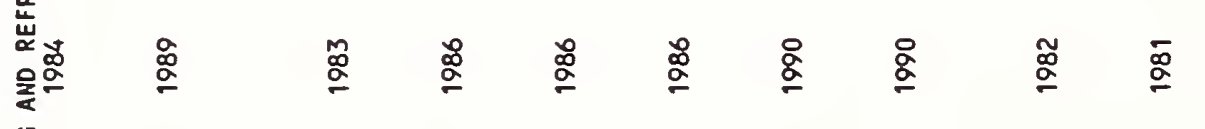

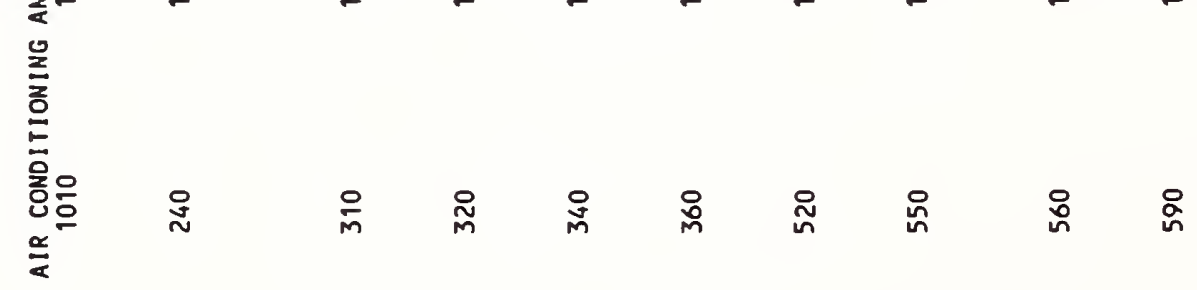




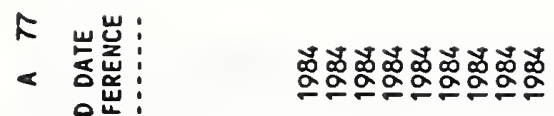

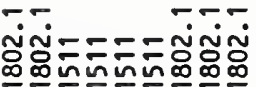

总

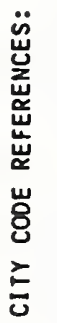

峞 :

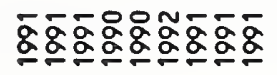

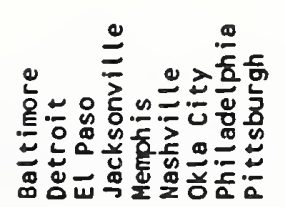

岁: 旁:

运崫

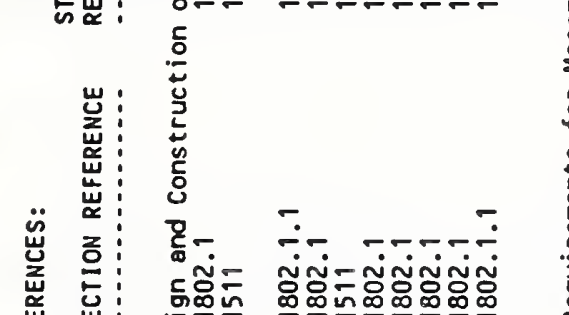

:

జ

岁

崖

家:

悹品

崖㟧曼邑

过 :

울

(a)

苛产

임

点

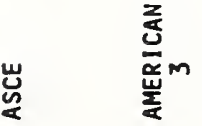

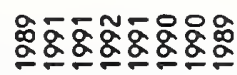

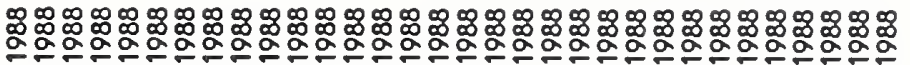

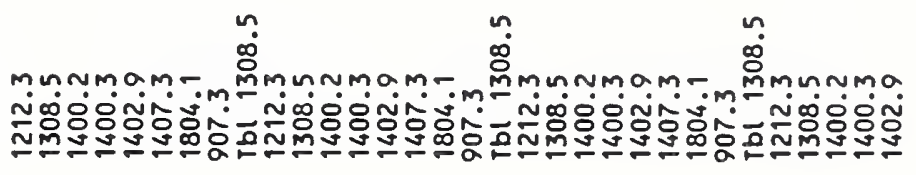

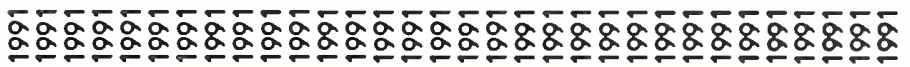

$\omega \omega \omega \omega$

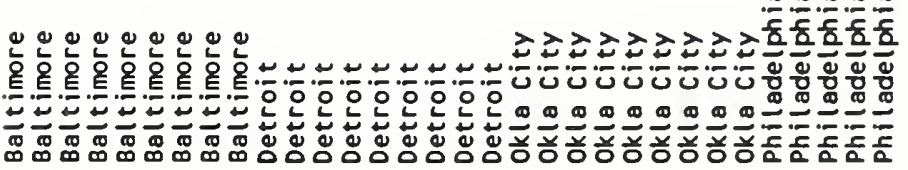

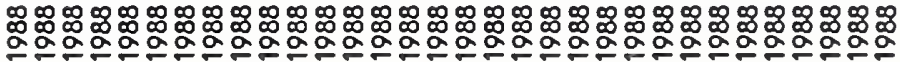

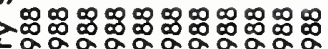

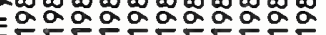
禹

高

$\stackrel{4}{n}$

崖 n Emninnmam-

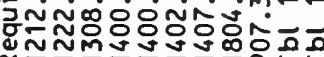
(1)

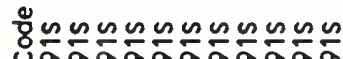

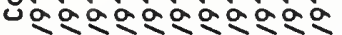

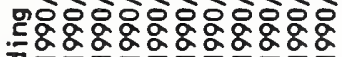

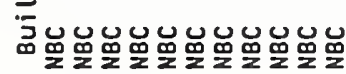

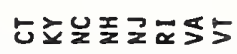

๓ก)

กับ

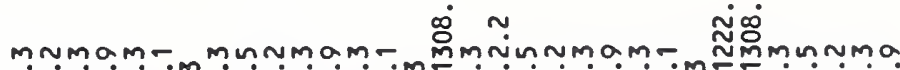

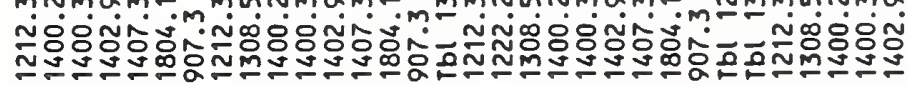

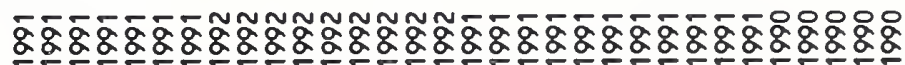

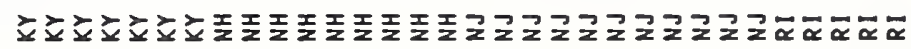




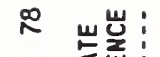

< 预 온

崖 总:

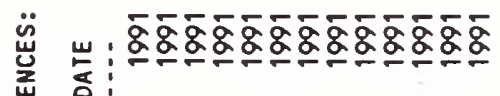
:

\section{Ф.๑.๑}

岁

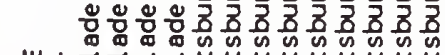

o

岀:

䟘 운

.

讪

岁

晨

$\infty$

㟧

岁: $\bar{\alpha} \bar{\alpha} \bar{\alpha} \bar{\alpha} \leq \$ \leq \$ \leq \$ \leq \ \leq$

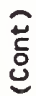

岕

in

z:

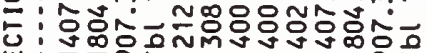

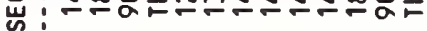

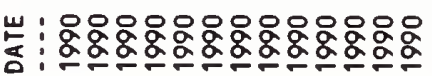

$\underset{8}{\mathscr{8}}$

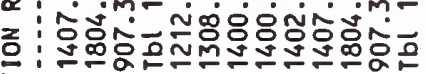

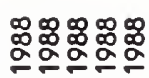

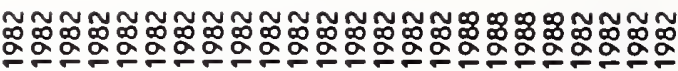

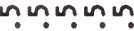

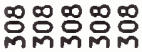

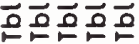

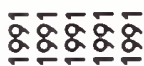

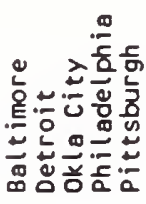

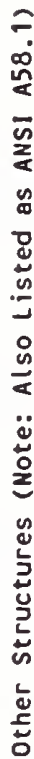

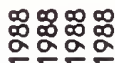

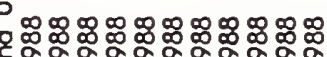

政

$\frac{2}{9}$

ஸ்றோயா

ํㅡㄹㅡำ

공몽혼용

ธ。

穿焉

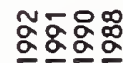

$\frac{1}{\bar{z}} \bar{x} \bar{x}$

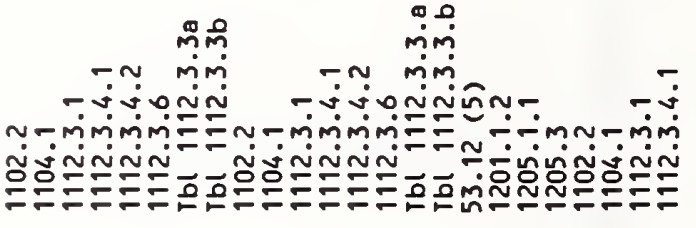

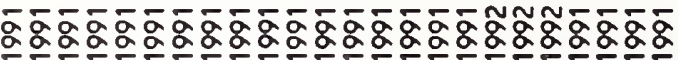

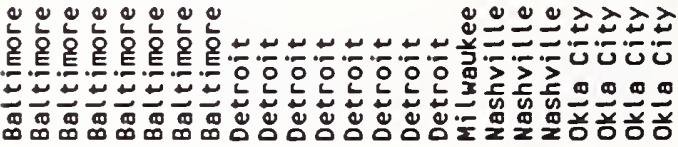

Nom

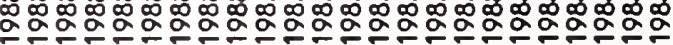

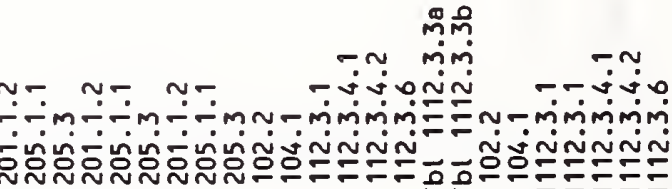

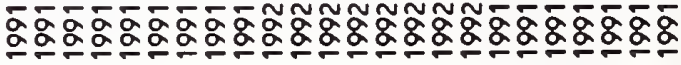

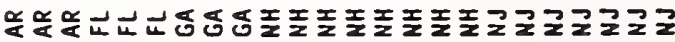


2 L 运岕?

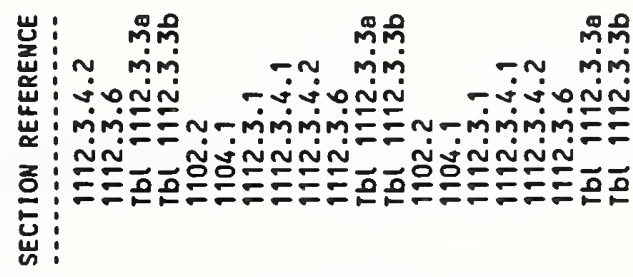

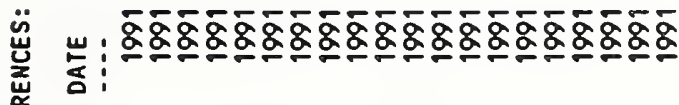
ㅎ..............

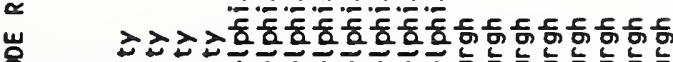

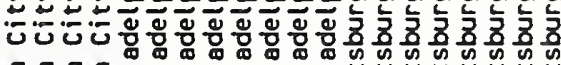

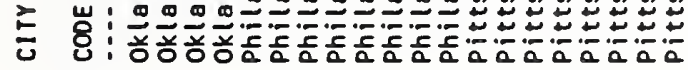

넌

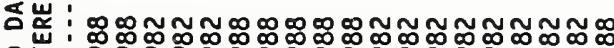

웅 :

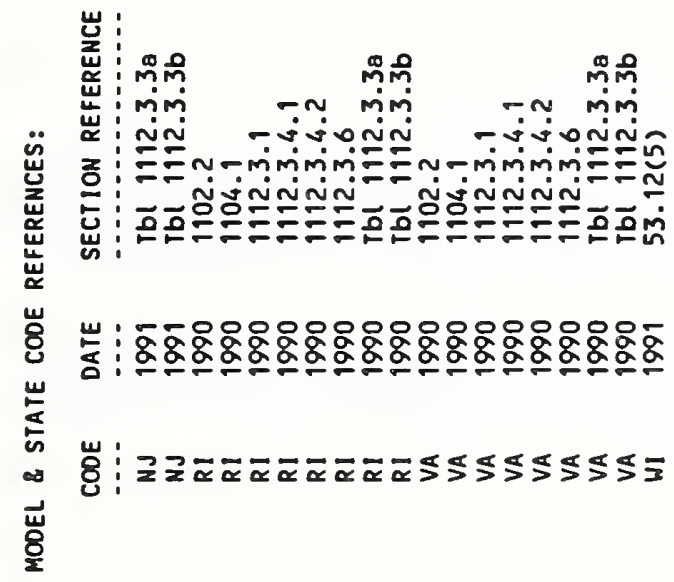

㝕

岕 


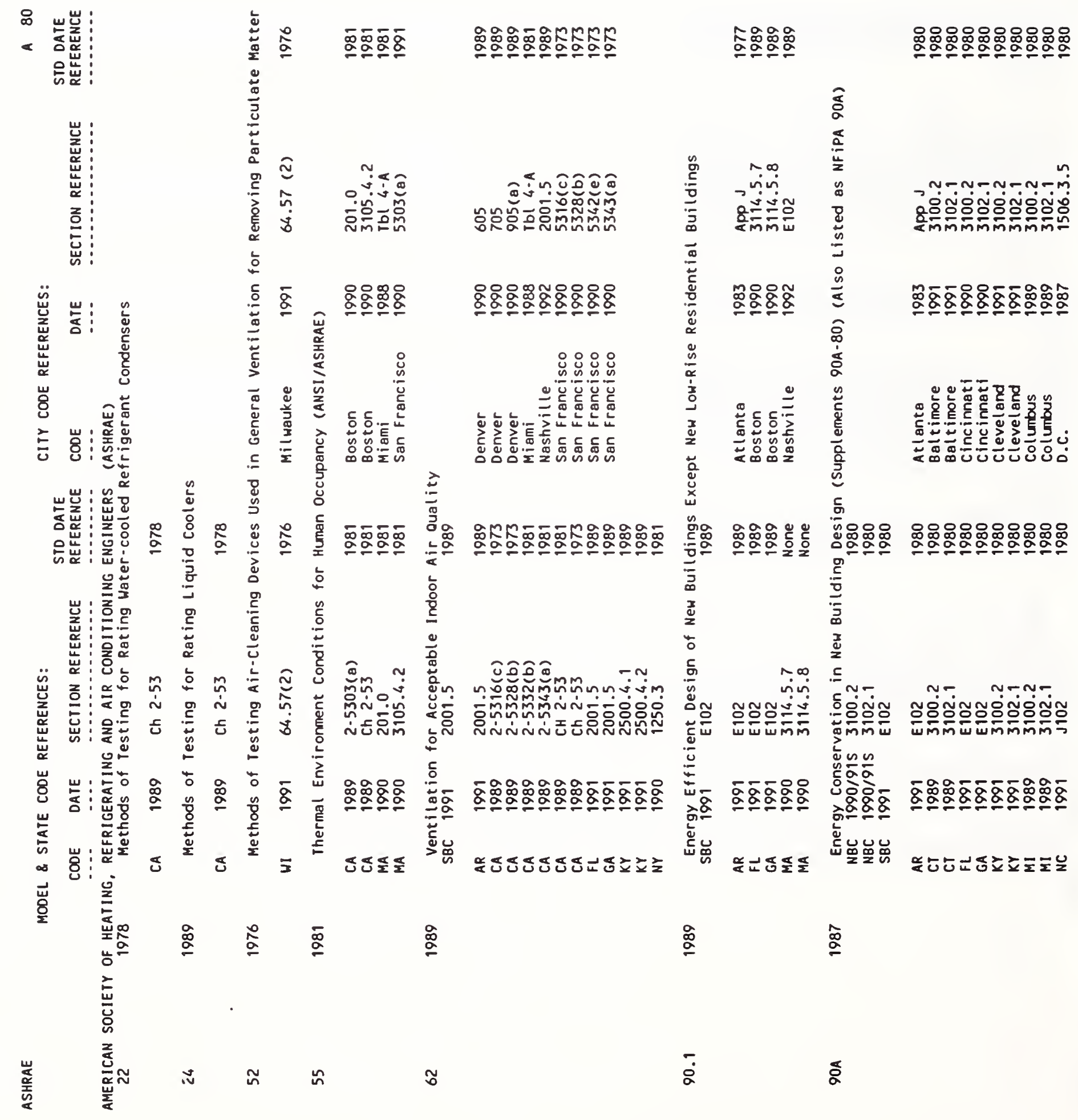




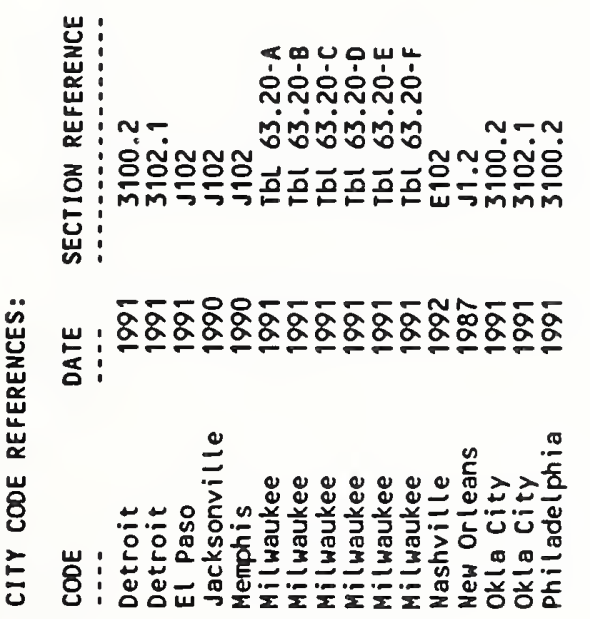

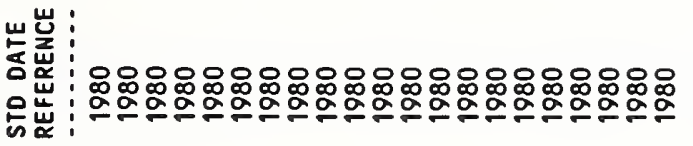

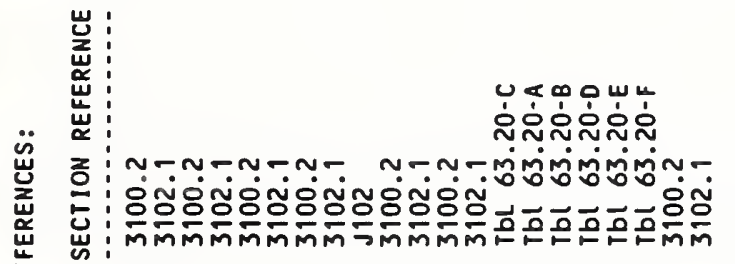

岁 : :

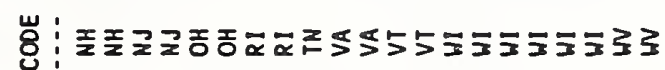

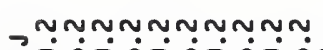

nก⿻

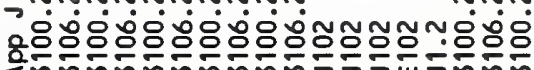

क̊요 $\frac{a}{2}$

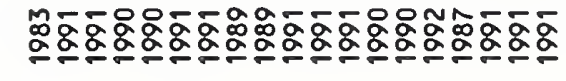

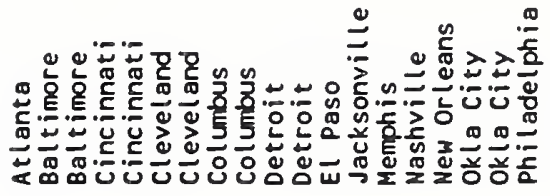

동 동 동 용영

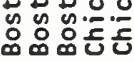

동

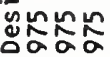
를 $\Xi$ N ํํํํํำ n

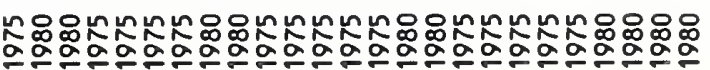

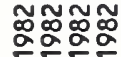

양영영여

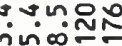

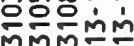
这里 cํㅇㅇㅎㅇ 공ㅎㅁ음 空品总邑

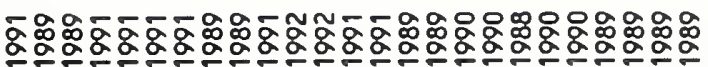

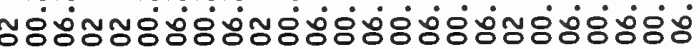

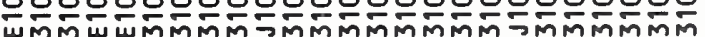

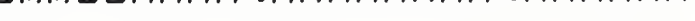

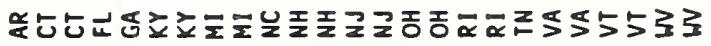

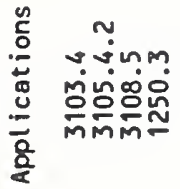
: 응양요용

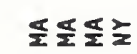




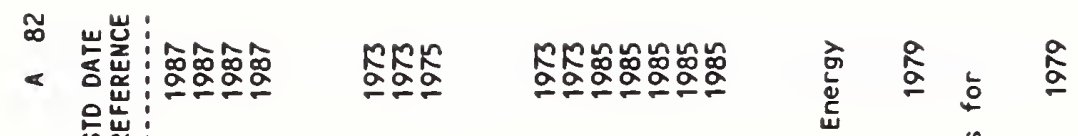
的岩?
嵓 :
:
zำ
出:

密:

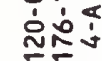
ต่m

苞

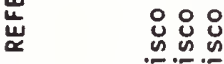

岩 产

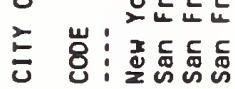

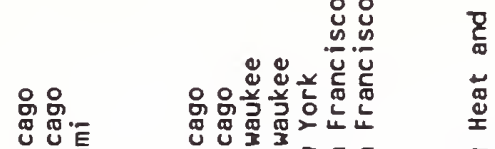

옹으 ิㅡㄴ

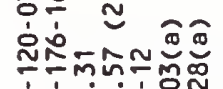

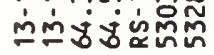

空

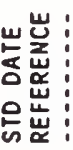

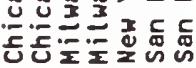

${ }^{6} \bar{x}^{\overline{1}}$

œ

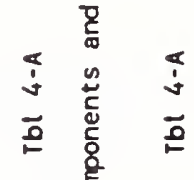

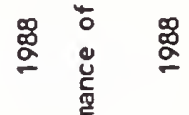

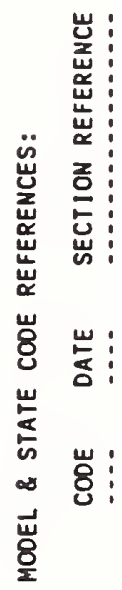

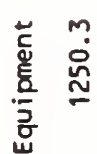

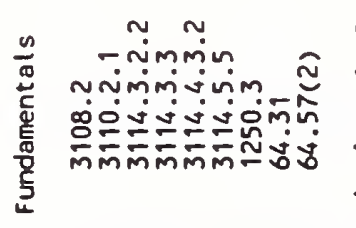

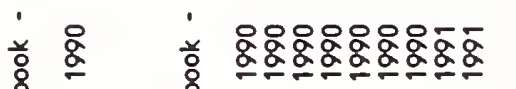

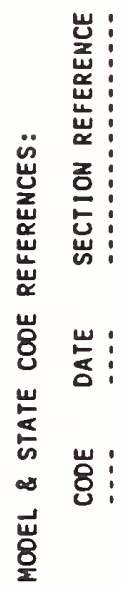

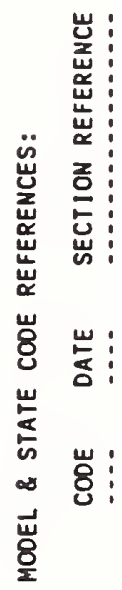

递

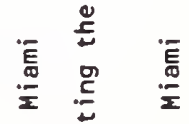

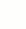

$\frac{\infty}{\bar{z}}$

co

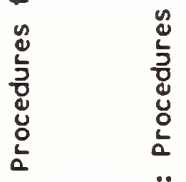

$\ddot{\Xi}$

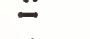

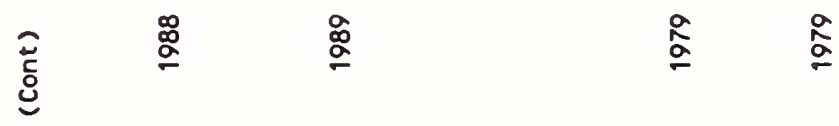

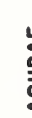

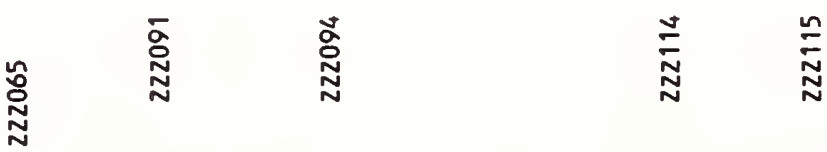




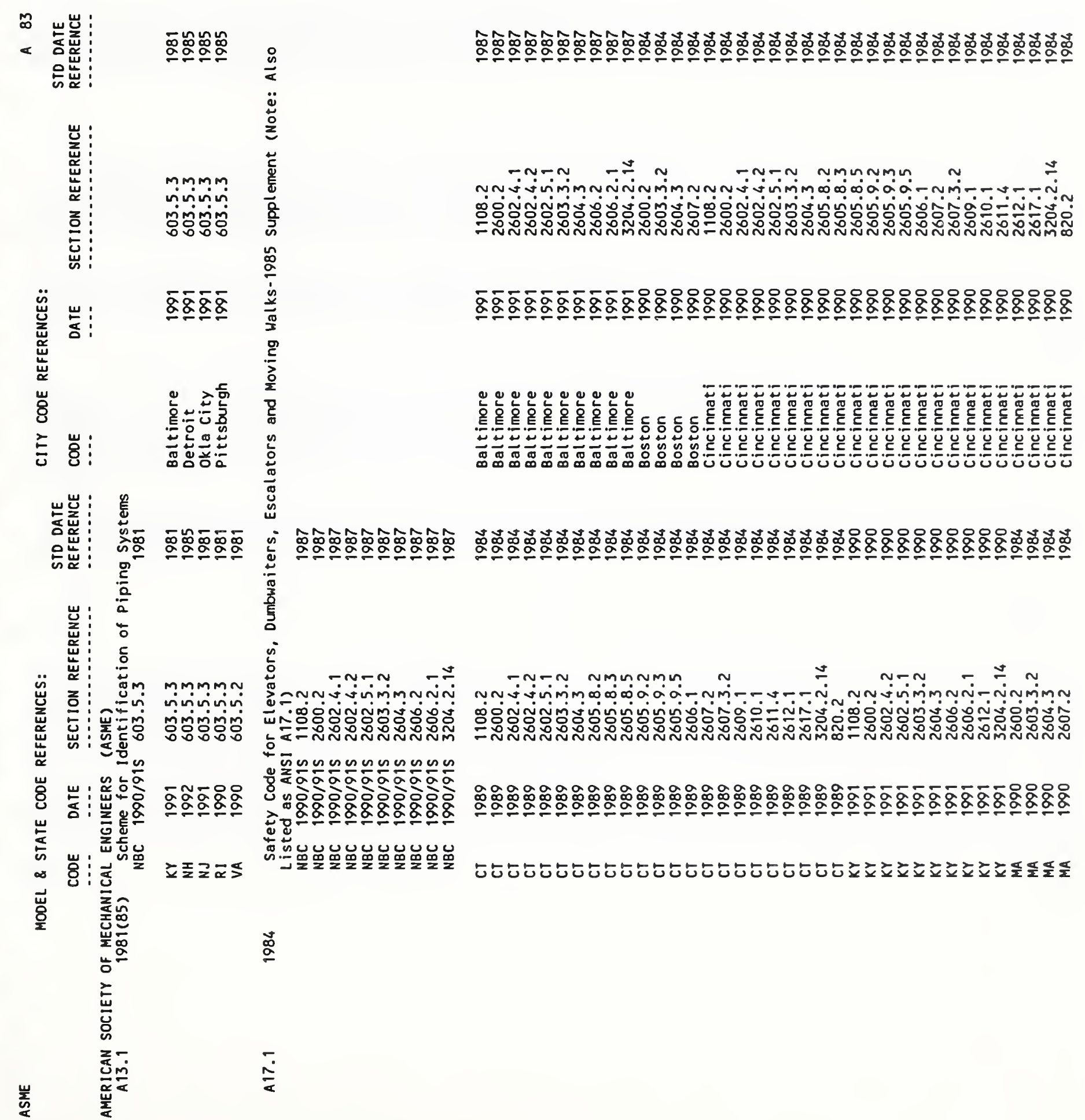


Ф

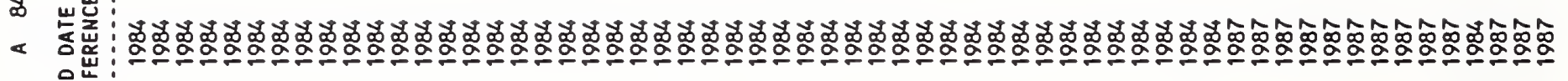
道压 :

岂:

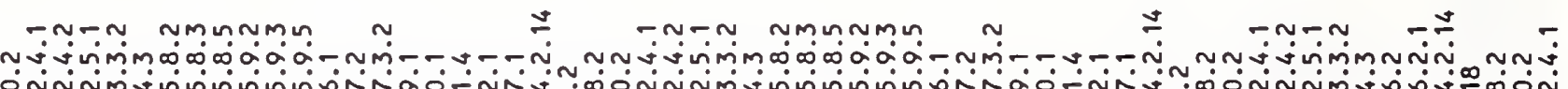
z : : 出:

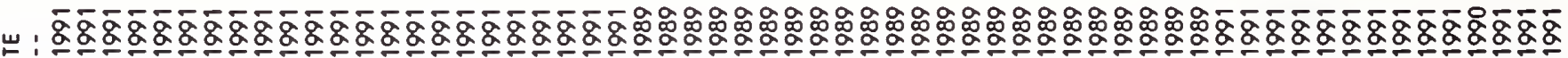
:

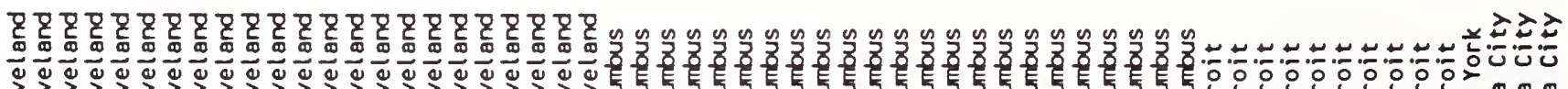
岁:

崖:

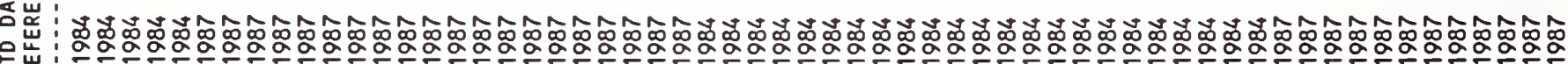
은

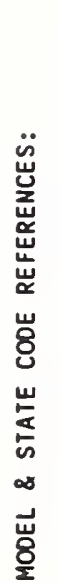

思

$\sim 2$

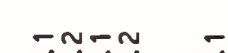

:

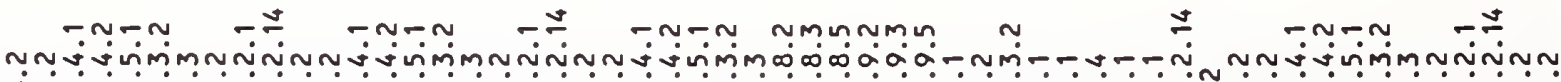

๒

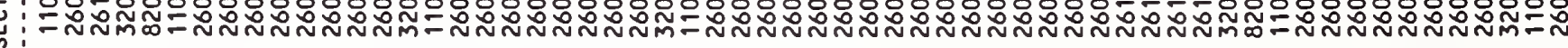

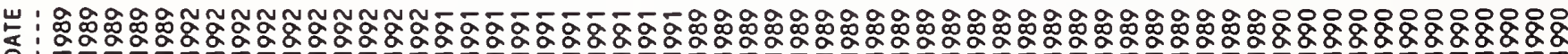

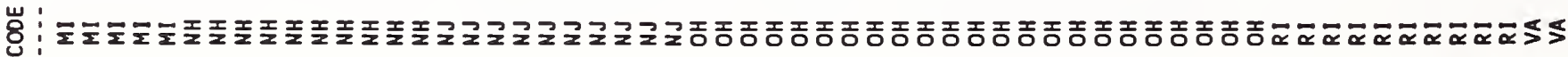

苍

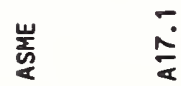


ڤ ฌిజిజ 造崖 :

岕

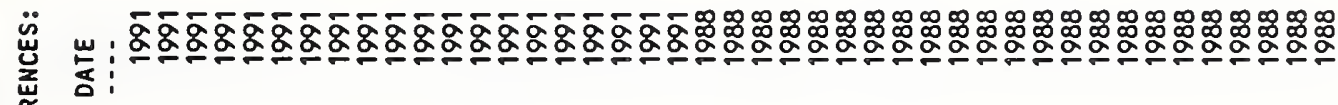

กุบฺฺ

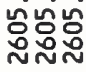

iñ

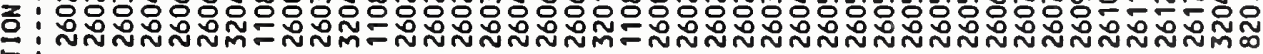

$\underset{\sim}{\stackrel{w}{\alpha}}$

岁

$\therefore . \oplus . \oplus$

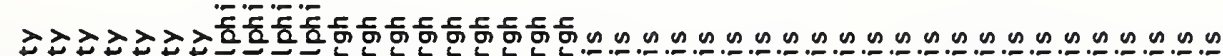

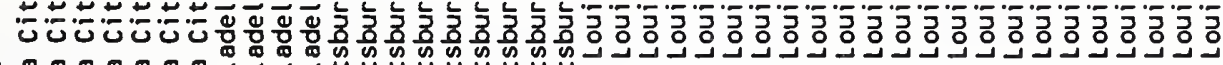

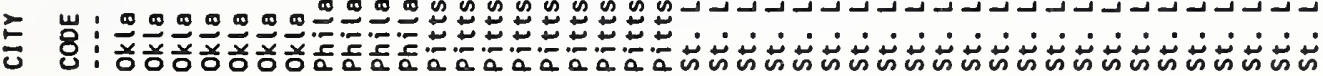

岕岕:

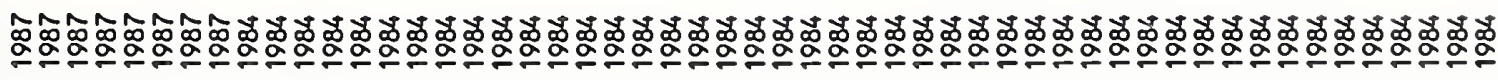

的岕:

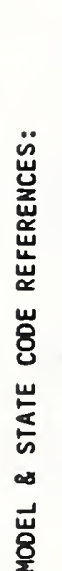

岕

:

- n-

$-z$

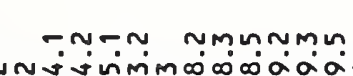

$\underset{1}{2}$

-nT? nmunmen n

$\underset{12}{2}$

กิ่ง

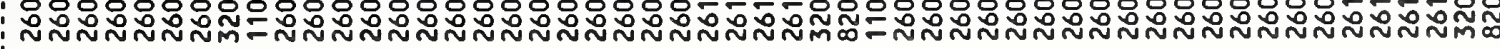

닌

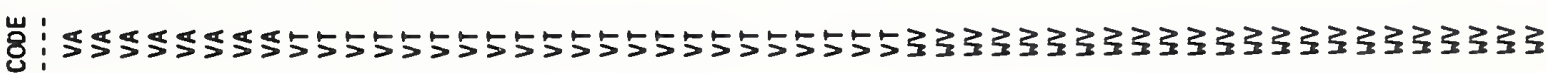

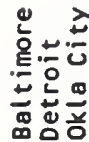

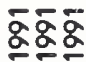

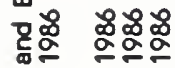

㽞

.

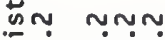

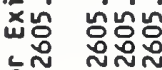

象

ชัํํ응

京喿京

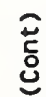

๕̊

崖毞

$\stackrel{m}{\frac{m}{2}}$ 


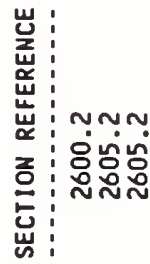

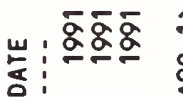

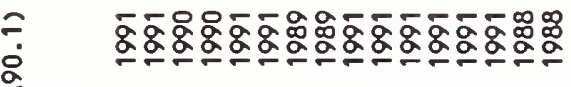

$\therefore$.

흥흥하

过总

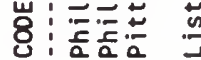

년 $\quad \frac{\text { के }}{x}$

客

은 녔: 응

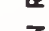

z:

更为为

w:

烗

ठै:

㟧

䓵

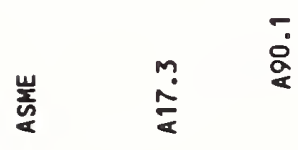

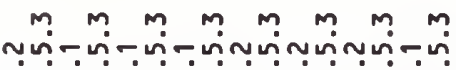

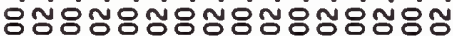

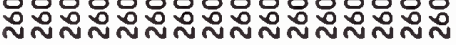

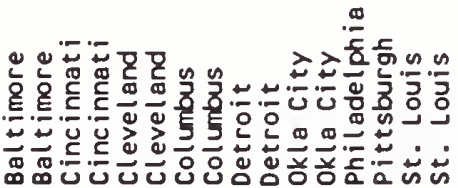

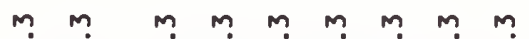

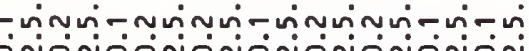

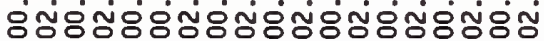

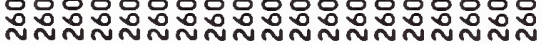

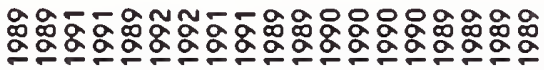

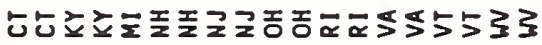

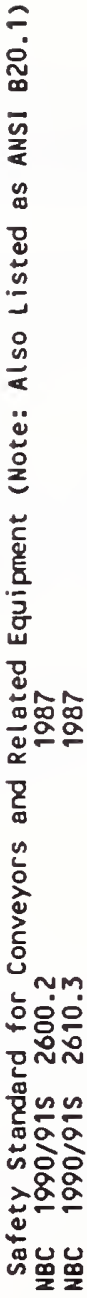

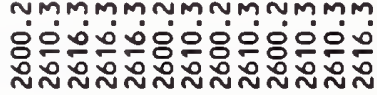

nunnununum

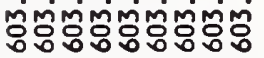

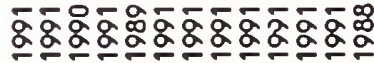

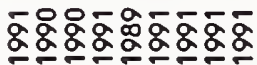

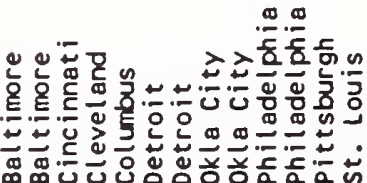

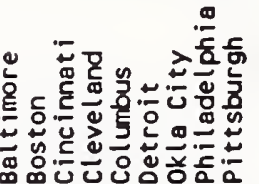

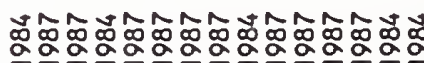

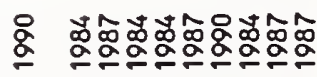

MNMmกMNMMNMNMmM

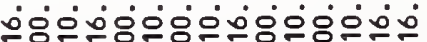

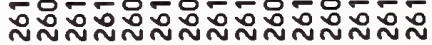

n nunununumun

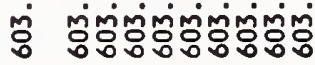

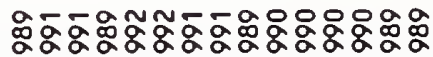

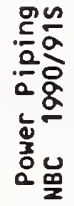

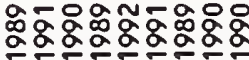

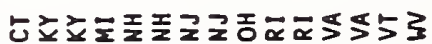




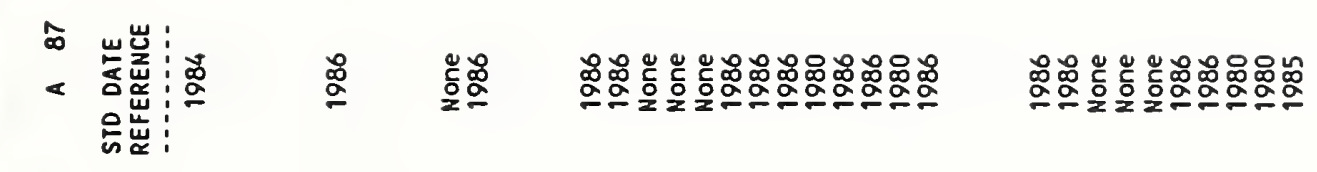

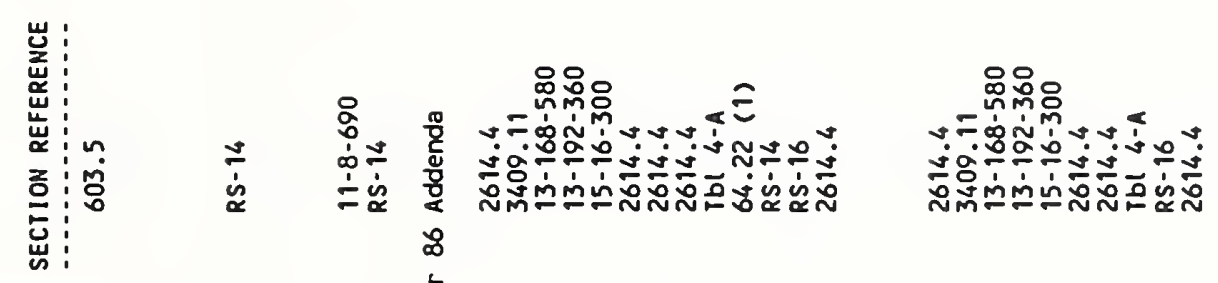

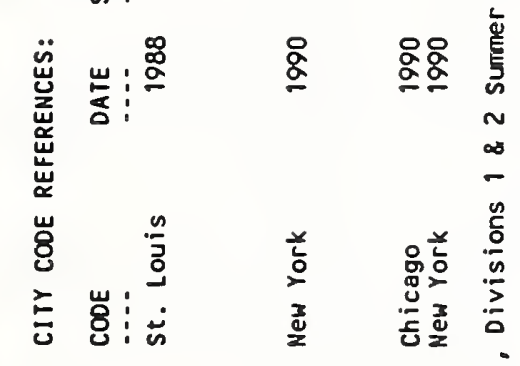

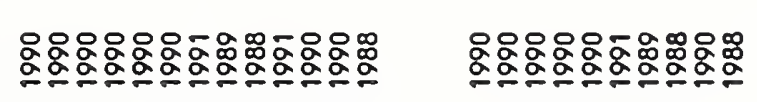
世岕 引

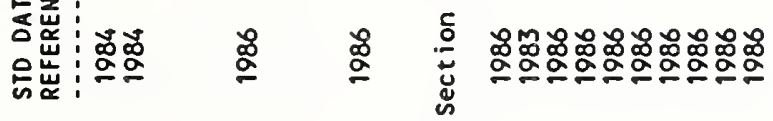

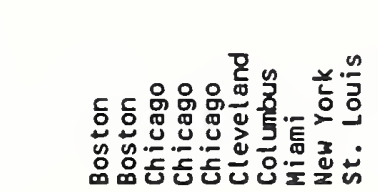

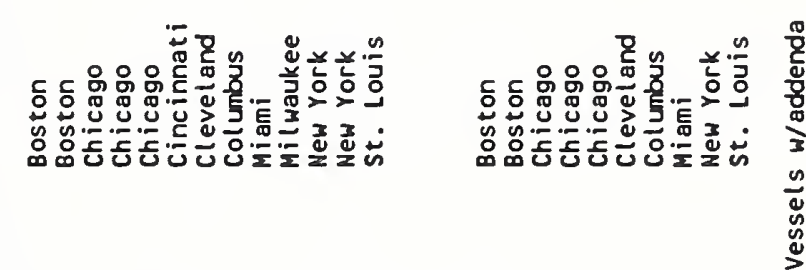

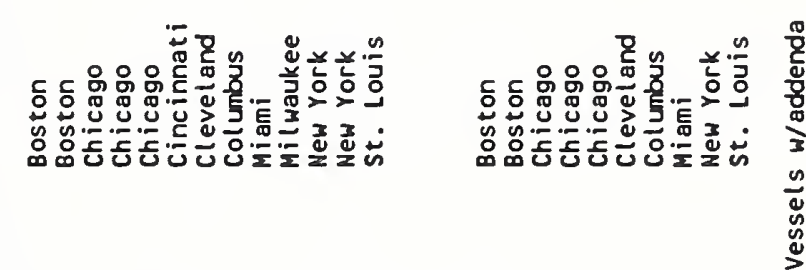

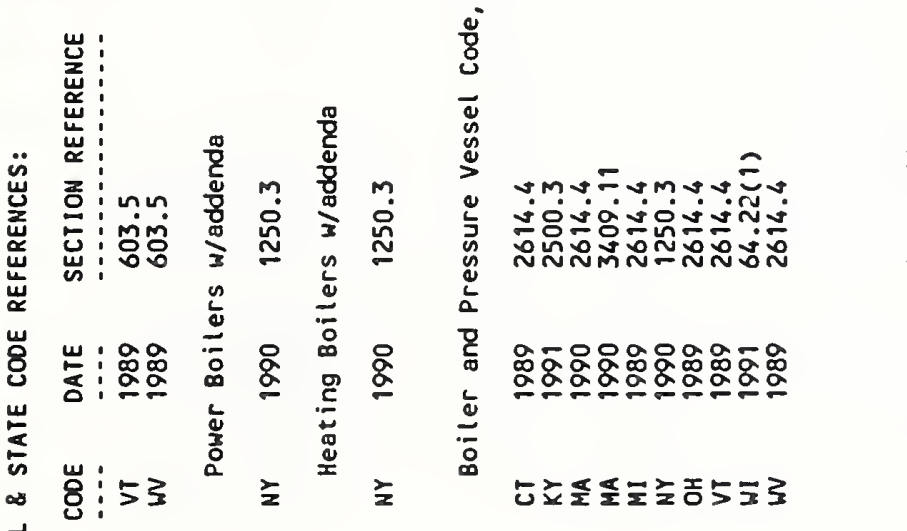

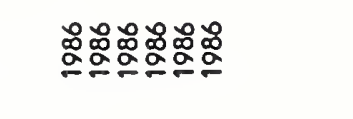

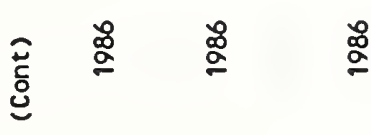

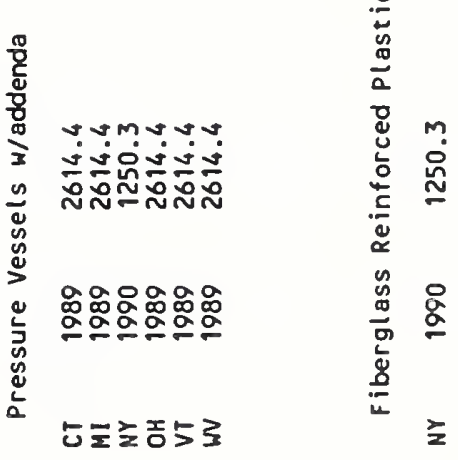

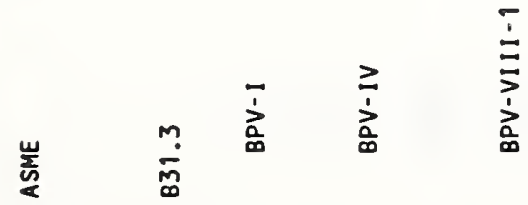

ఖ

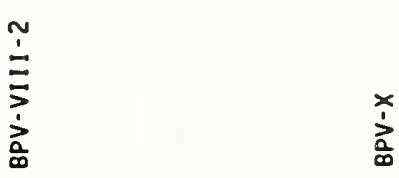




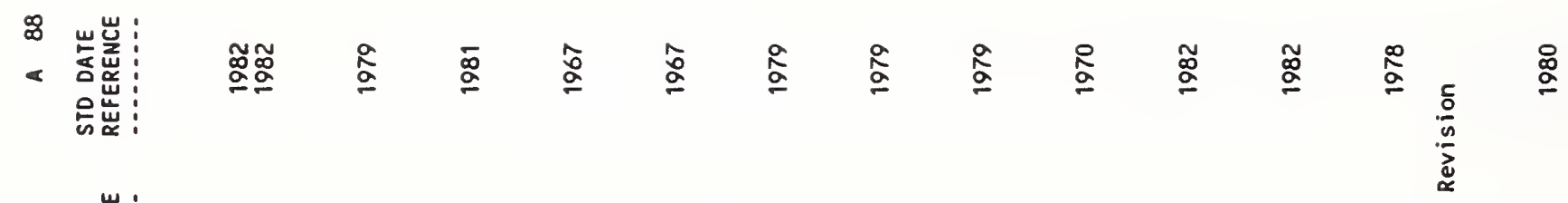

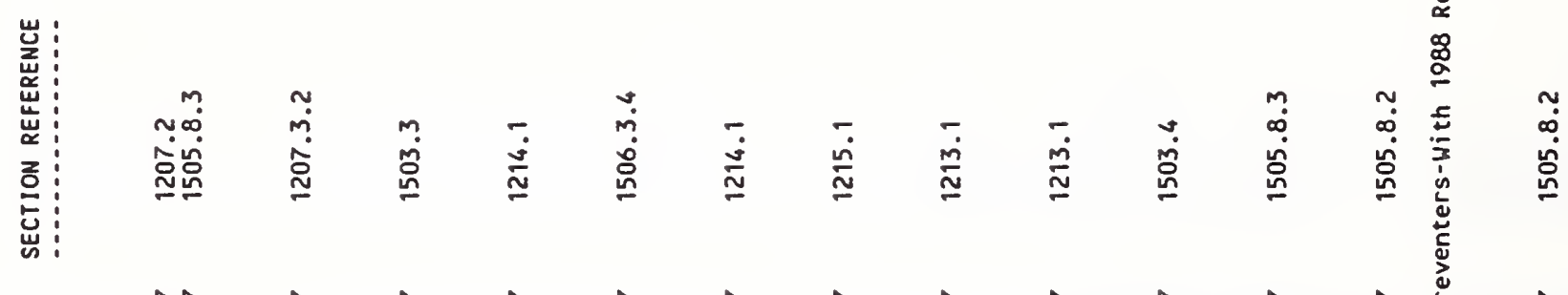
蒿萦:

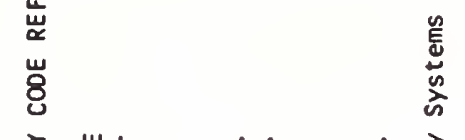

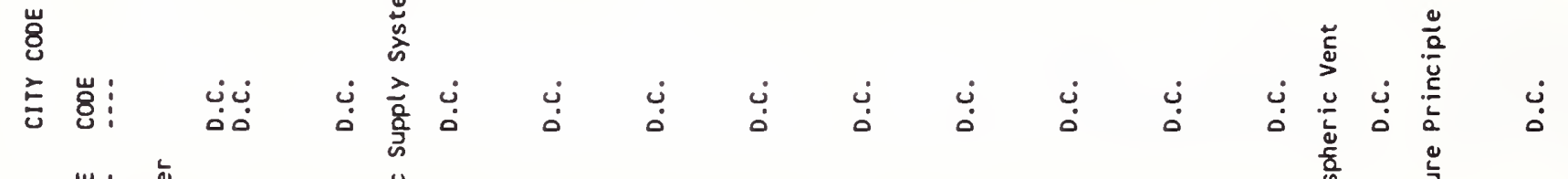

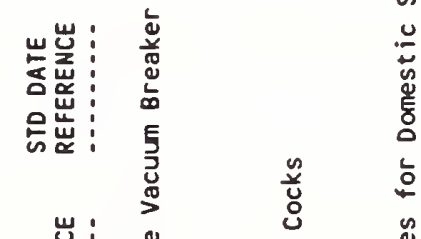

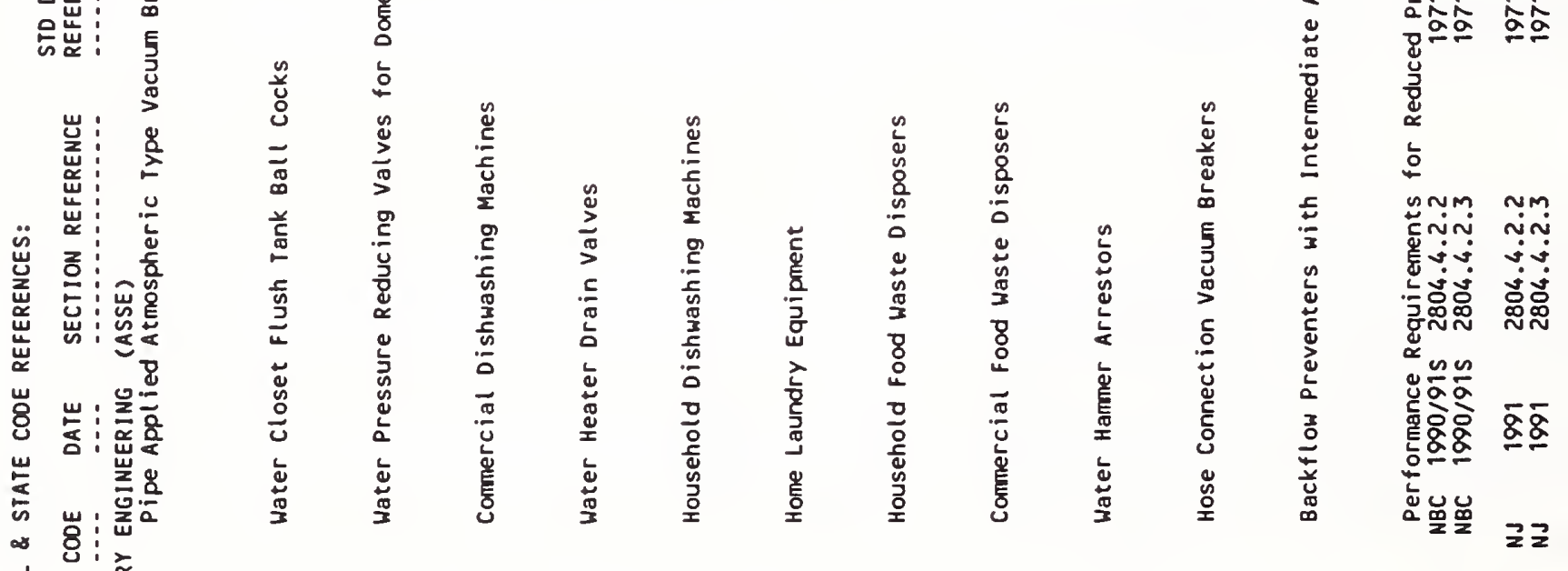

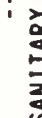

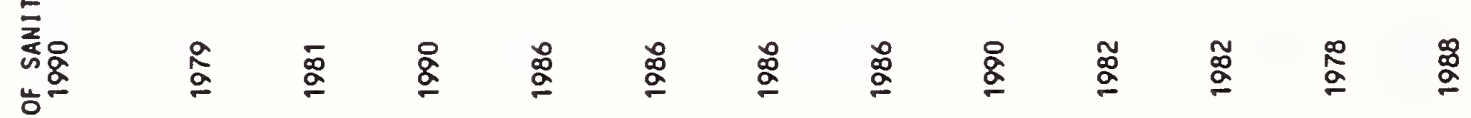
言

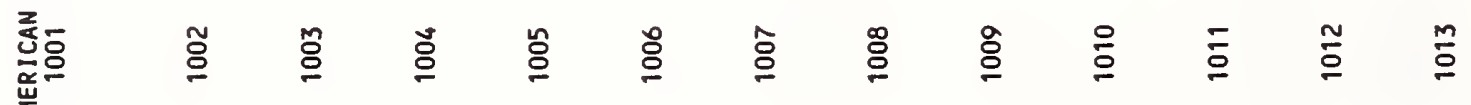




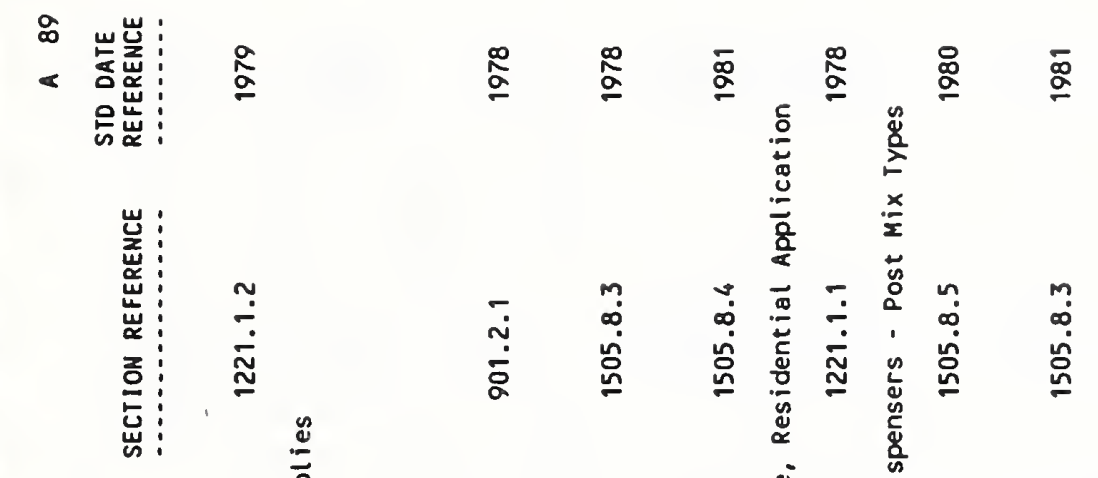

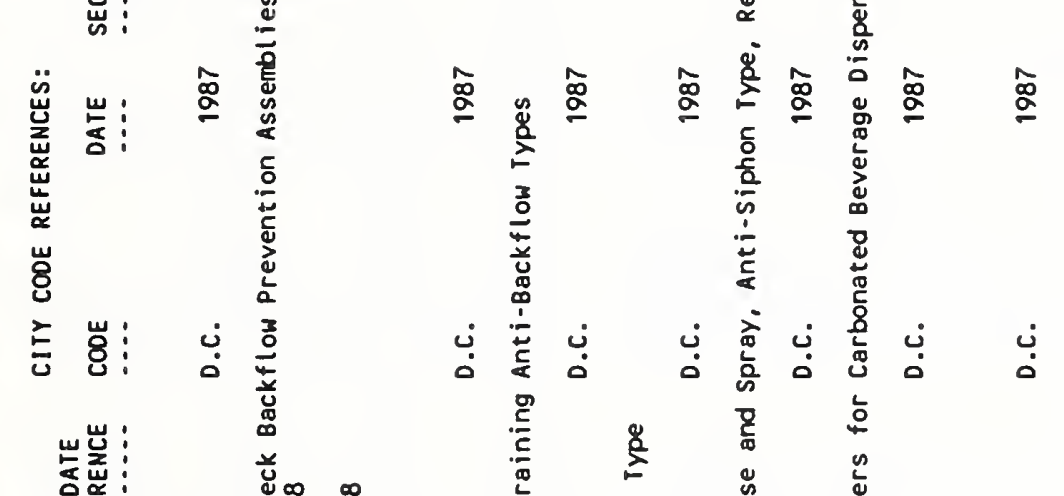

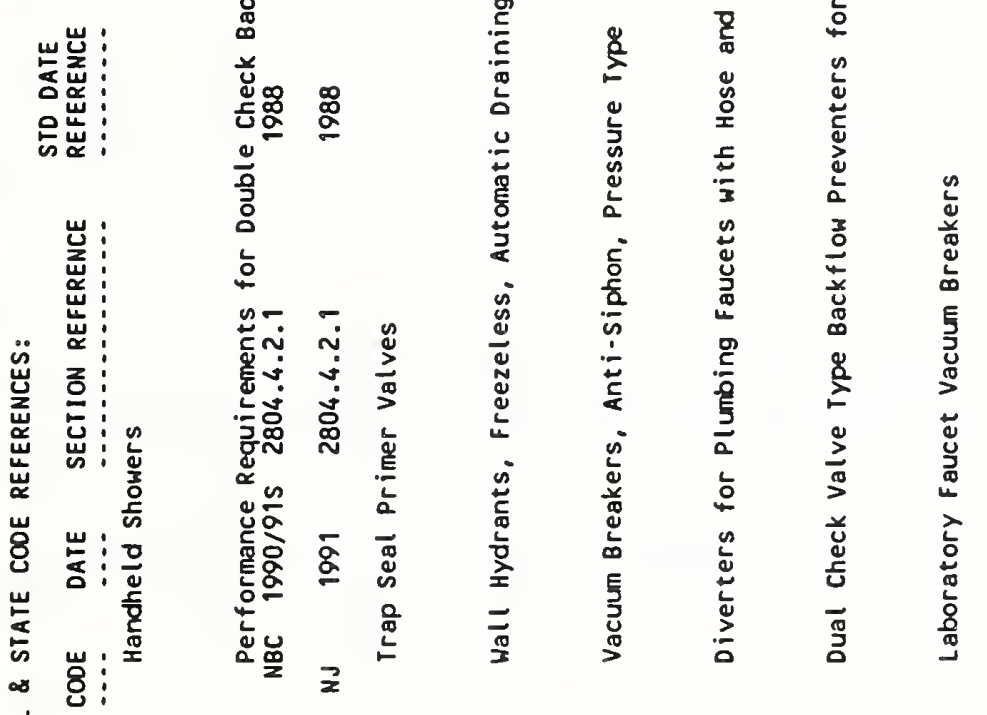
㟧

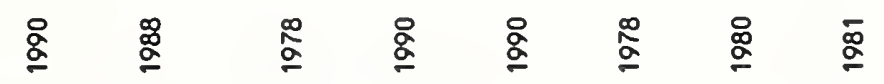
ఏ 


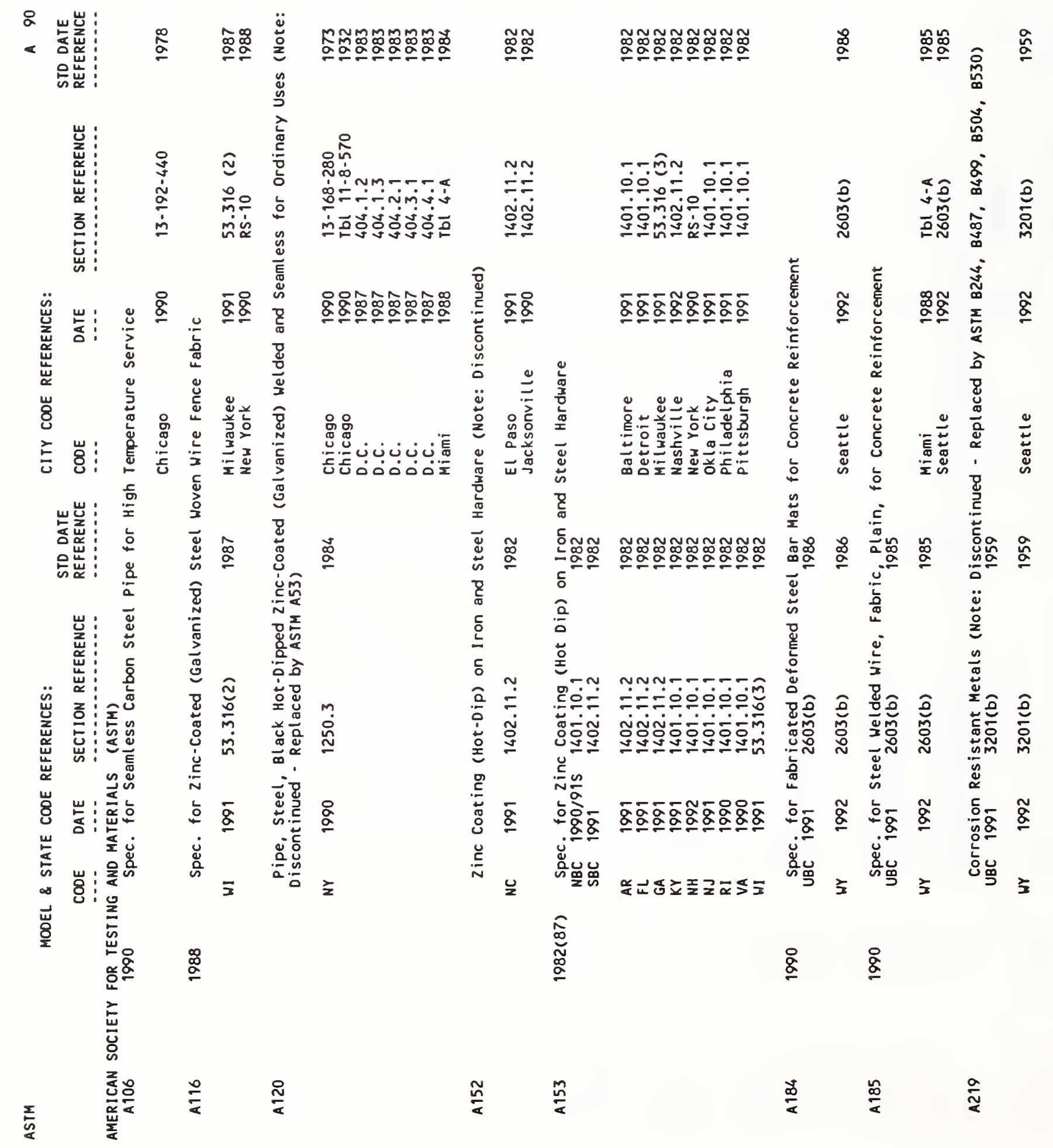




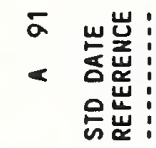

ฆ

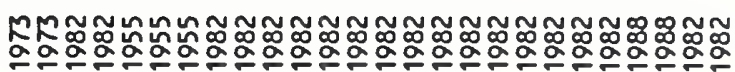

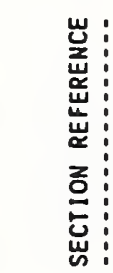

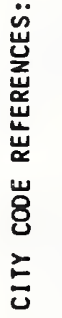

崖:

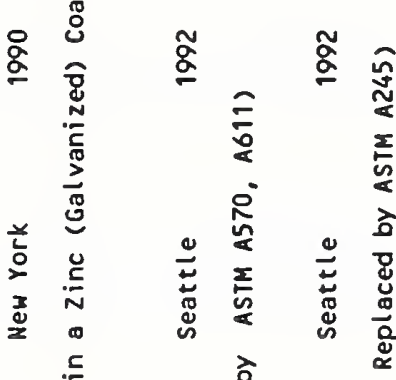

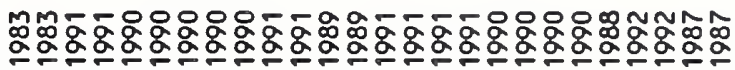

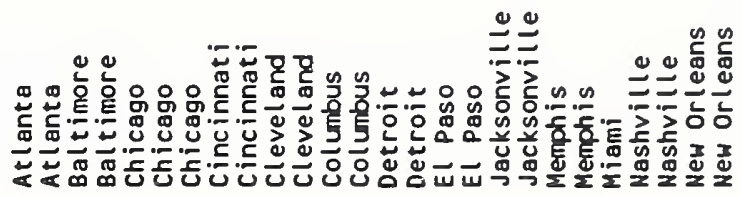

$\frac{9}{2}$

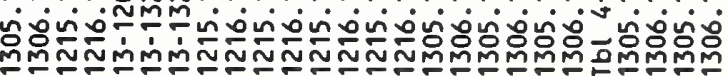

is c i

옹연원

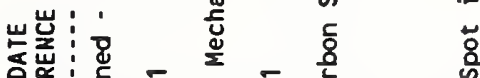

范

ล

它

点要

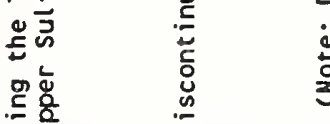

崖:

敋

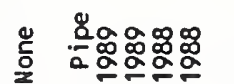

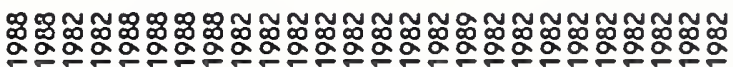

迎望

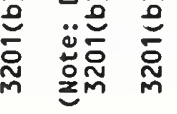

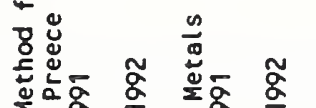

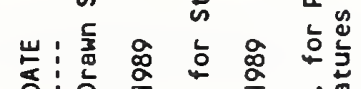

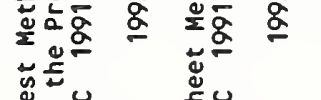

.

㟧

ธ

它萝

w邑

영 용

$\stackrel{9}{\circ}$

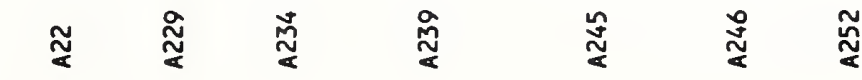

紊

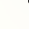


논

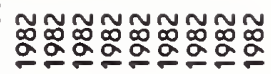
员岕:

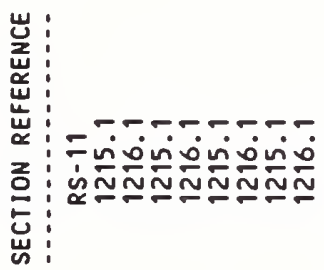

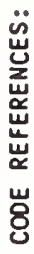

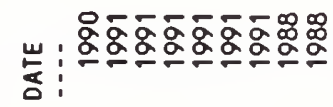

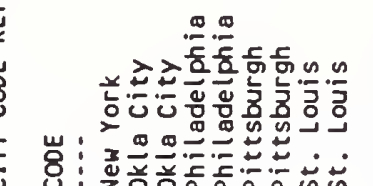

는 :

ธิ

它岕:

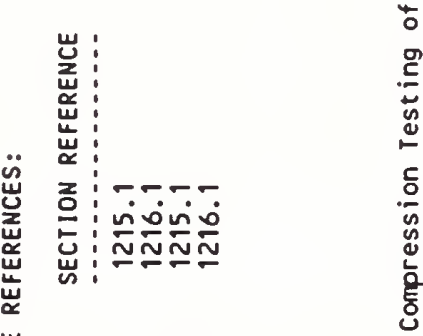

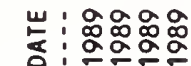

岁:

ְ̃

ָั

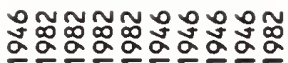

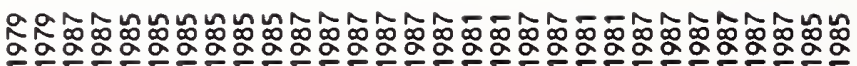

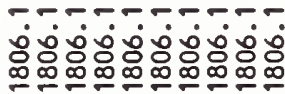

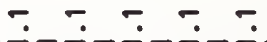

Vion

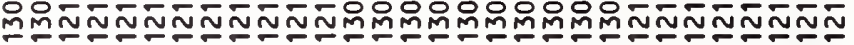

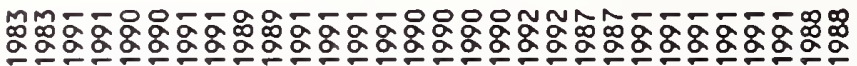

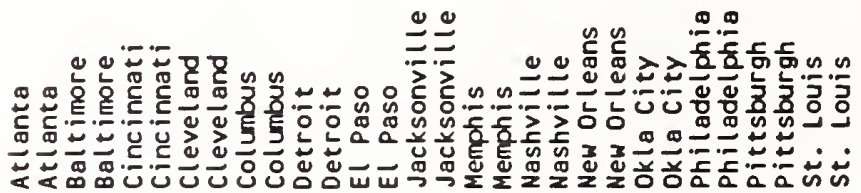

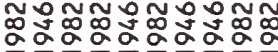

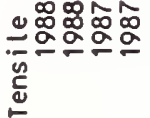

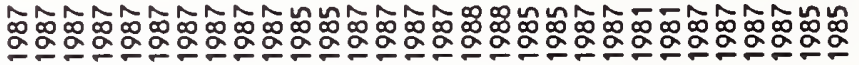

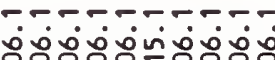

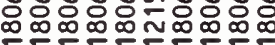

畩

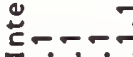

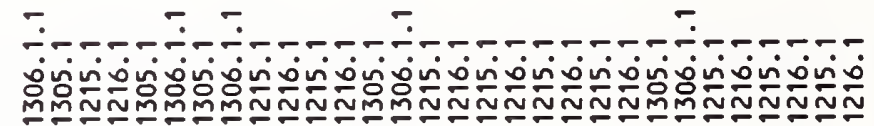

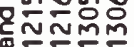
주의 $\frac{2}{2}$

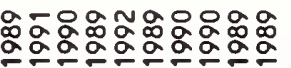

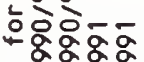

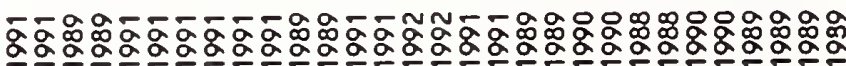
过u保

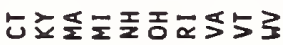

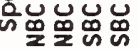

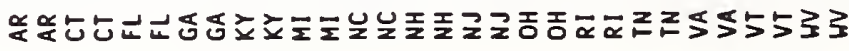




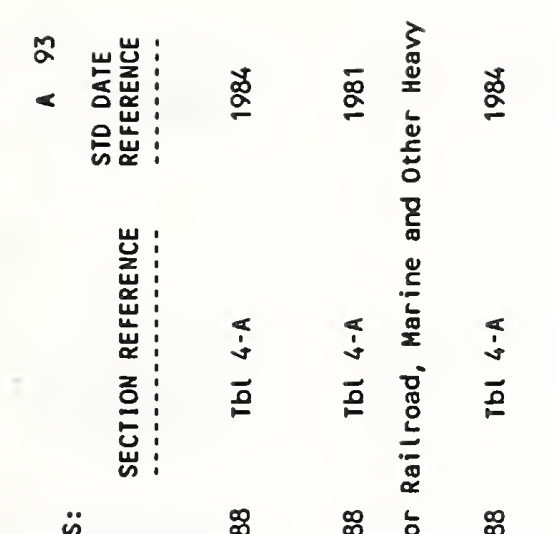

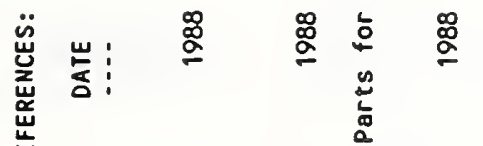

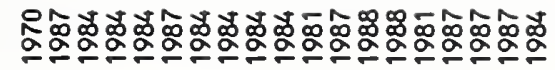

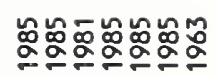

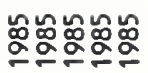

$\frac{5}{5}$

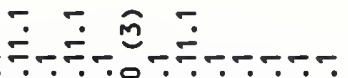

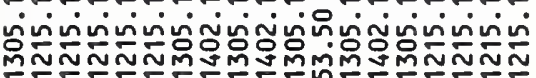

:

mim jommine

요룰

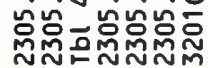

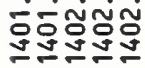

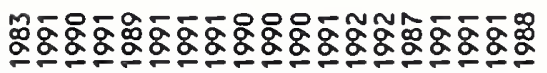

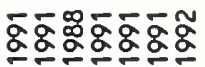

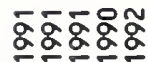

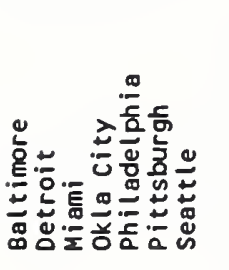

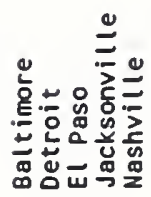

ш 山्व

愘:

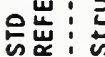

岁客

崖:

空

종 :

出

宛

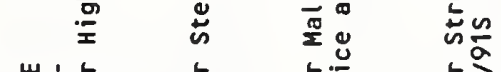

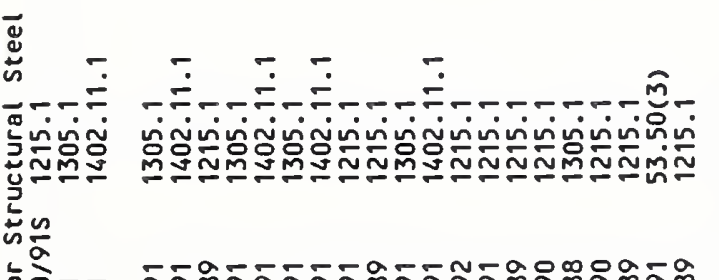

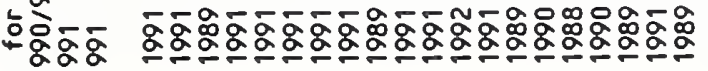

岁品品品

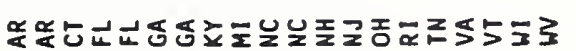

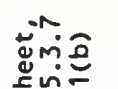

mimiminima

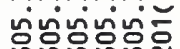

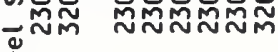

$\stackrel{\Delta}{\sim} \frac{\pi}{a}$

๕:

岁:

岁密

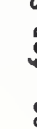

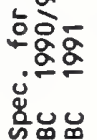

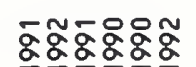

우요

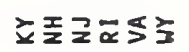

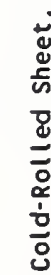

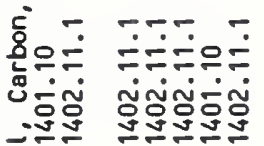
ఖ is $\frac{1}{2}$

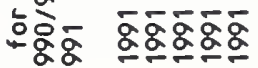
这造品

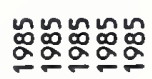
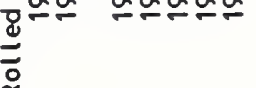

高

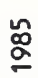

运近氐立呈

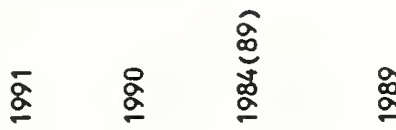

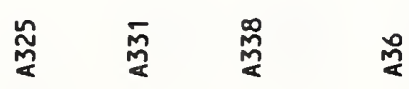

串

胥

$\frac{2}{2}$ 


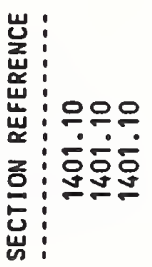

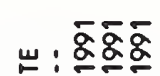

宅:

$\therefore \quad$ ๘

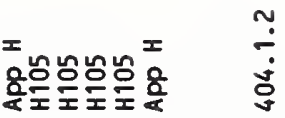

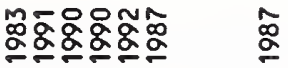

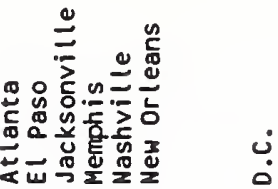

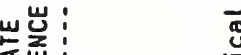

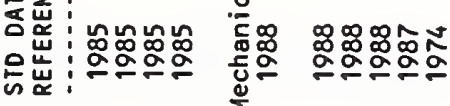

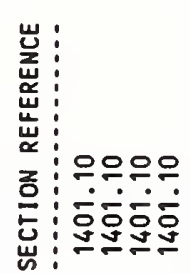

ᄒ

$\stackrel{\text { c }}{\circ}$

$\bar{z}$

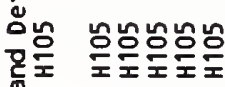

(n)

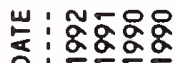

कृ

西

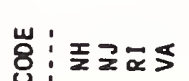

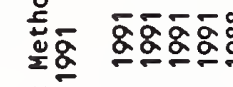

芯邑

뚠떤웛즌

용

$\stackrel{\circ}{\circ} \stackrel{\circ}{\circ}$

足

會 $\frac{0}{8}$

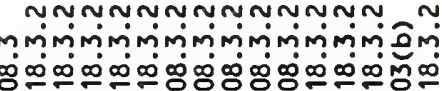

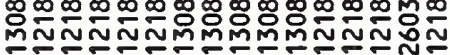

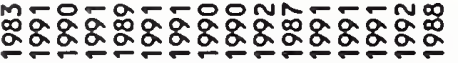

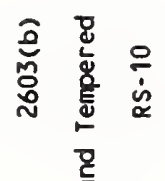

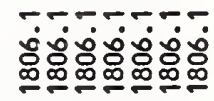

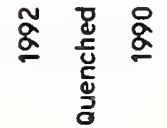

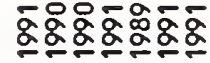

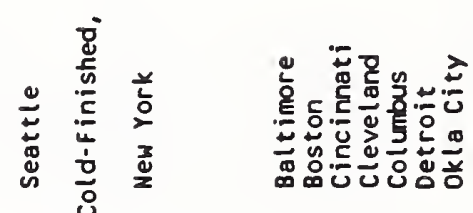

ปัँ

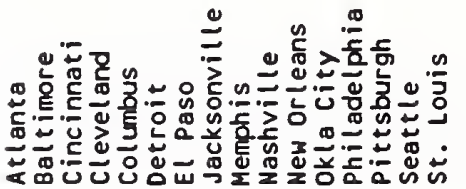

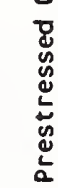

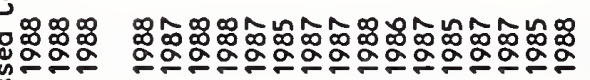

흔

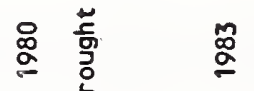

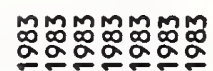

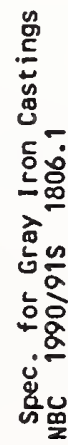

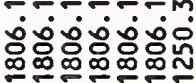

$\infty \infty \infty \infty \infty \omega \infty \infty \infty \infty \infty$

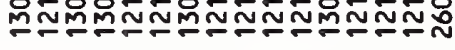

战

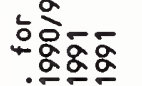

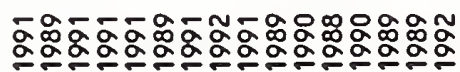

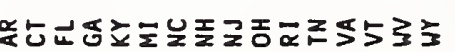

亏

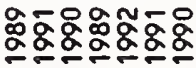

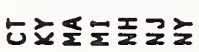

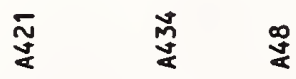




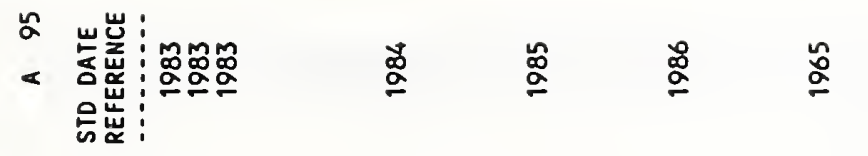

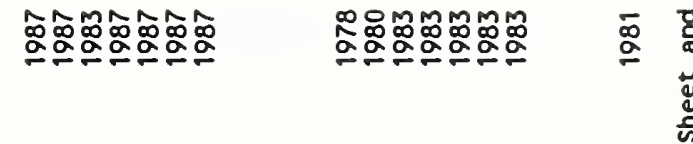

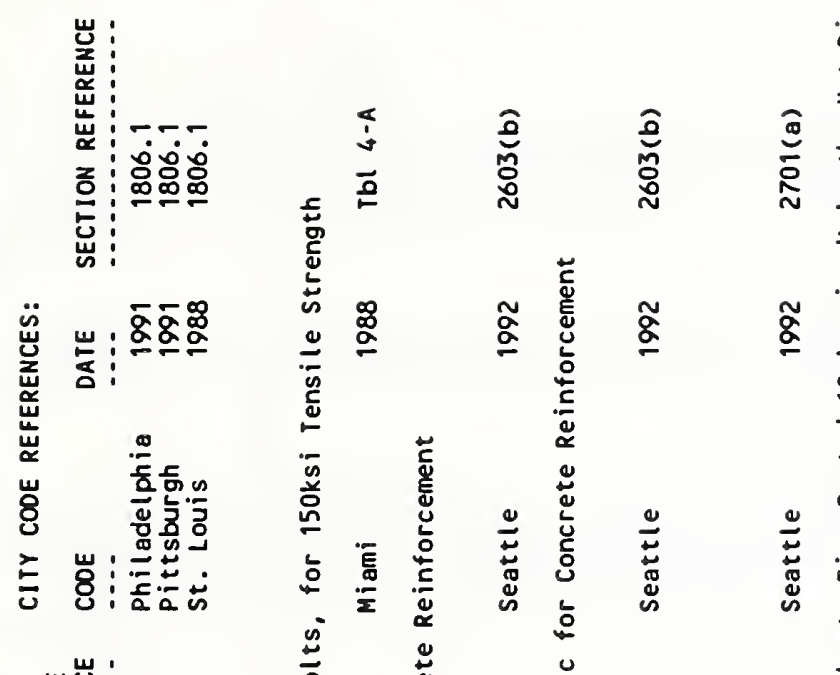

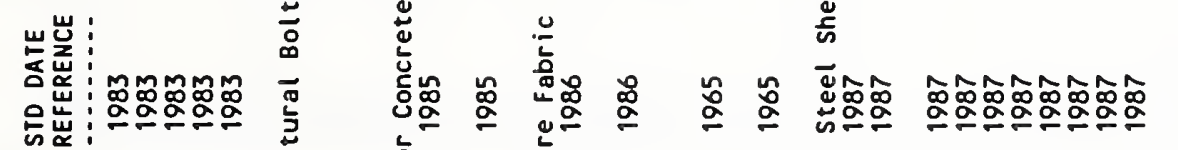

임

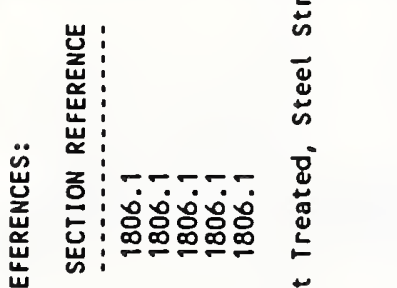

要

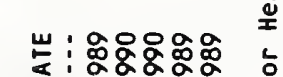

崖

岁: ‘车引

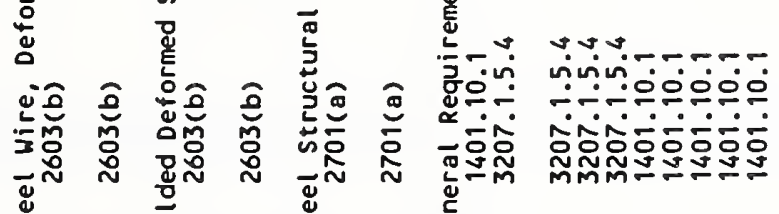

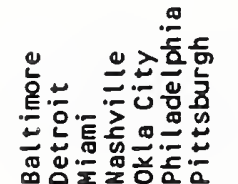

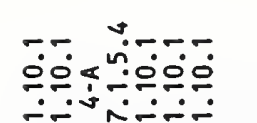

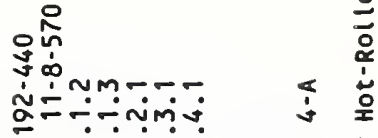

๓ீ:ต்

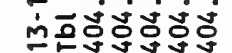

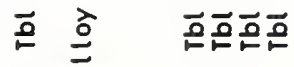

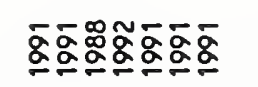

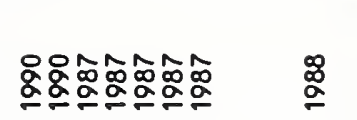

$\bar{\alpha} \bar{\alpha} \bar{\alpha} \bar{\alpha}$

$\frac{8}{9}$

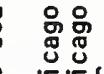

نبن نبن

总

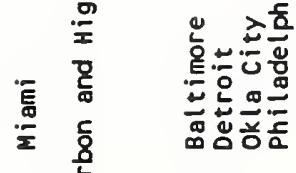

-

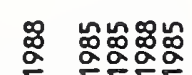

인

蒿

悹

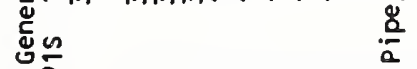

\&

‘-

sơn

亏 ญัँ

苂号

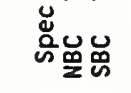

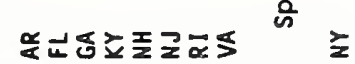

$\stackrel{8}{2}$

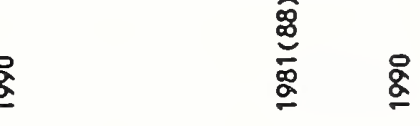

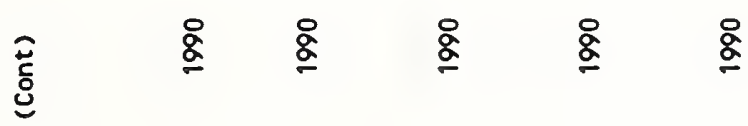

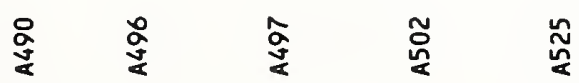

管

嵒总

要粱 


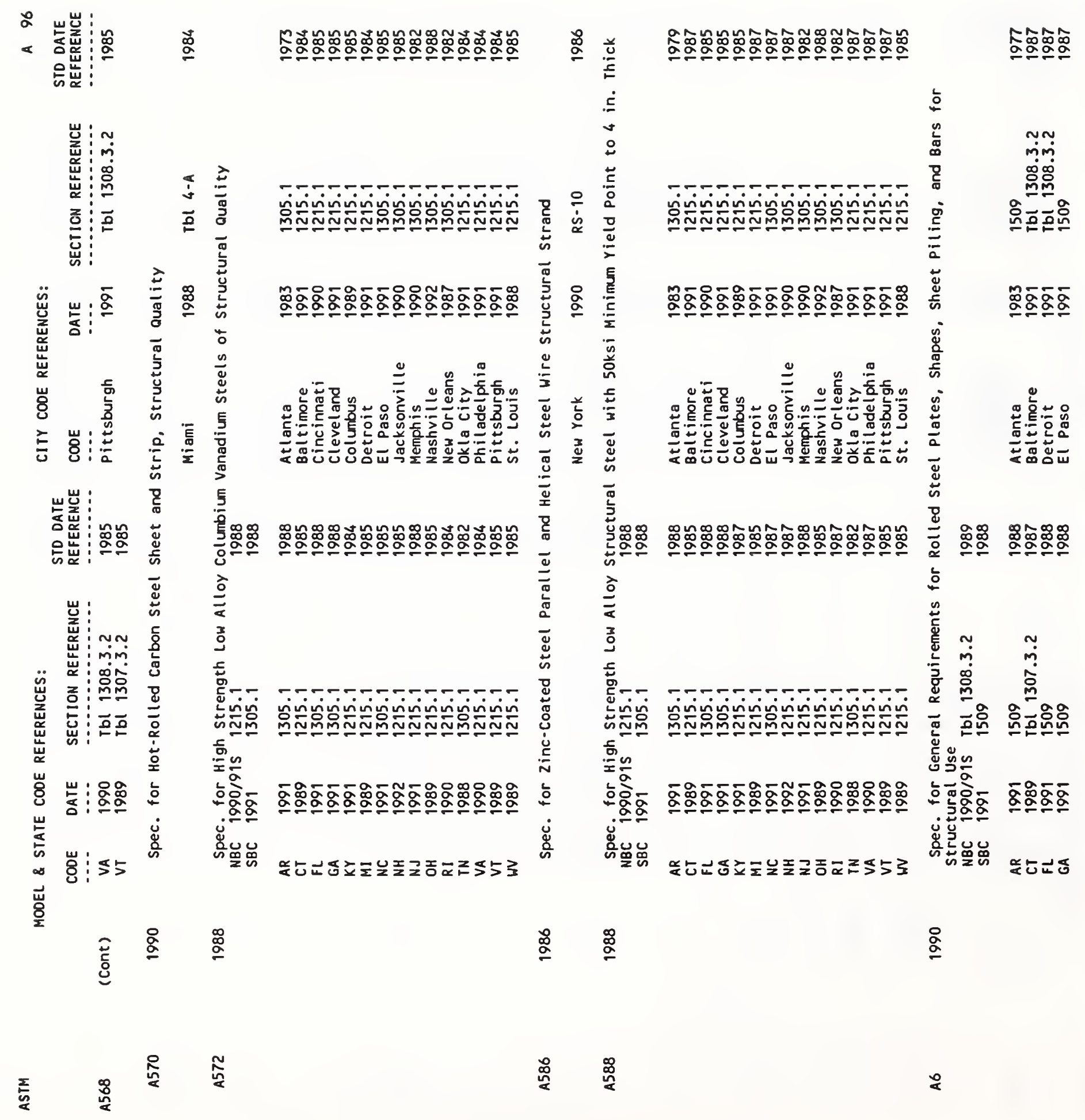


a w 뼈

แับ

क्षै mimin

岃

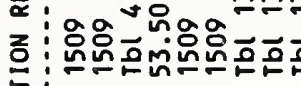

出:

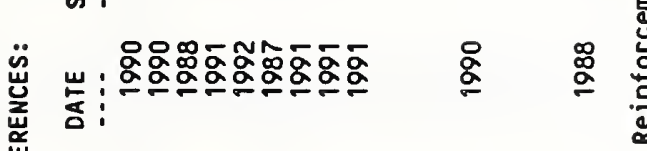

$ઈ \quad \approx$

$\frac{2}{i} \quad \frac{5}{\dot{2}}$

뚜으

=ำió

ตำำ

ตำำ

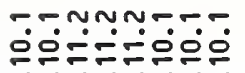

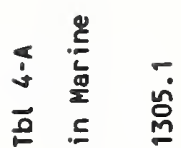

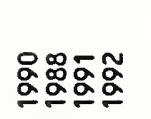

$\ddot{F}$

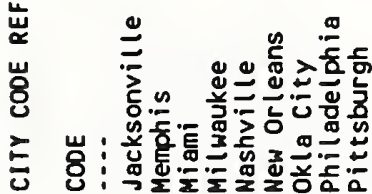

岕岂:

웅

니 :

NnN NN

范 范

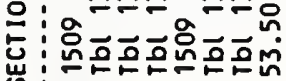

密

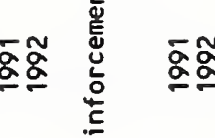

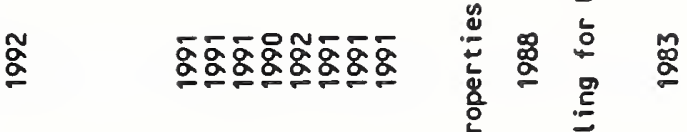

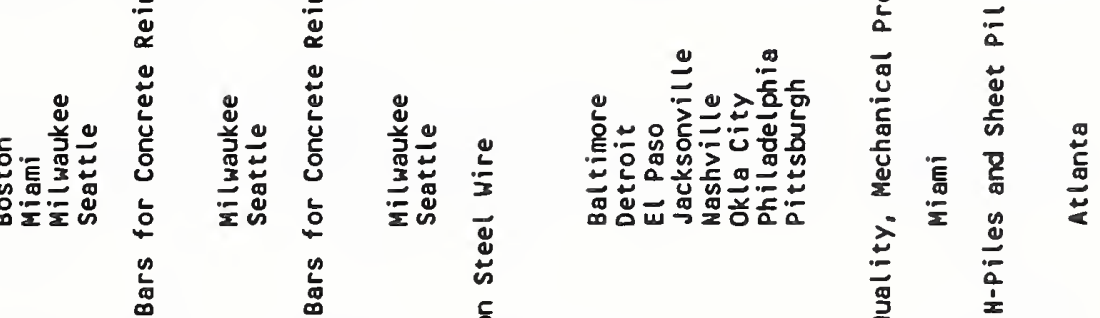

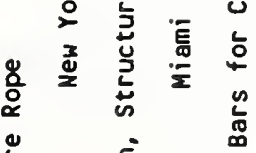

¿ 8

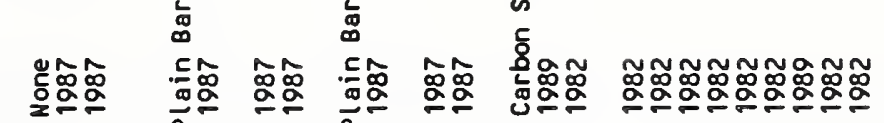

总

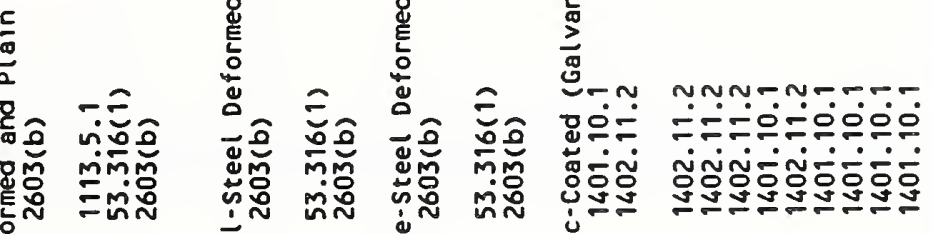

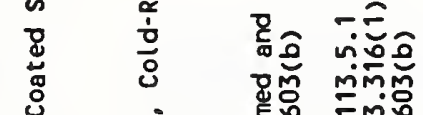

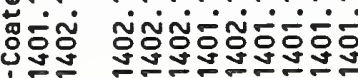

产乐

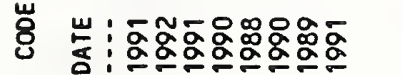

䋇

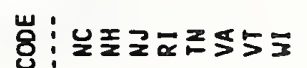

\&

$\frac{\overrightarrow{\mathrm{U}}}{2}$



용 용

용

$\stackrel{9}{g}$

$\stackrel{g}{g}$

美

菖

$\frac{0}{8} \quad \frac{1}{8}$

范

m. 


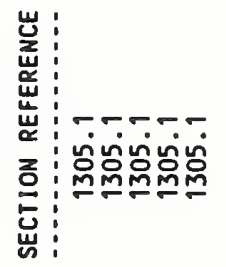

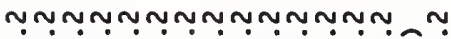

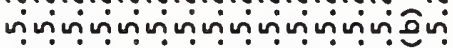

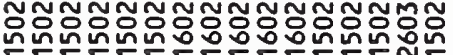

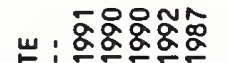

号:

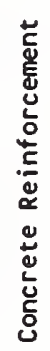

崖总 京

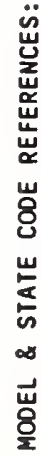

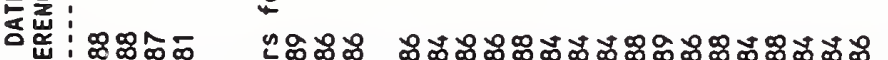

은

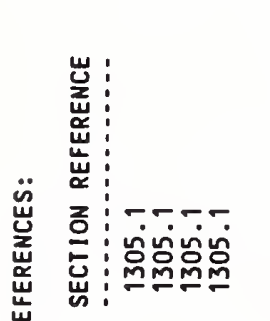

山1--

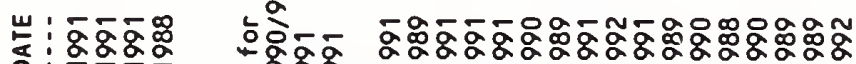

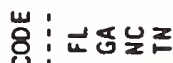
密岩品

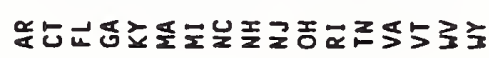

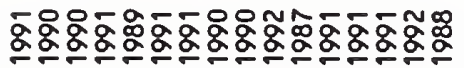

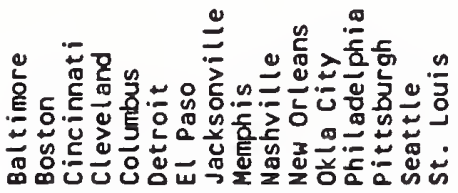

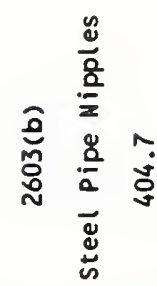

$\tilde{g}$

๙<smiles>C1CCC(C2CCCCC2)CC1</smiles>

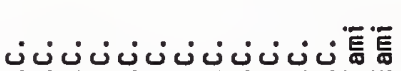
بان

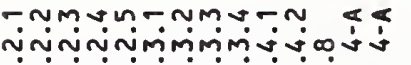

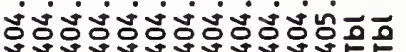

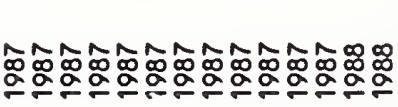

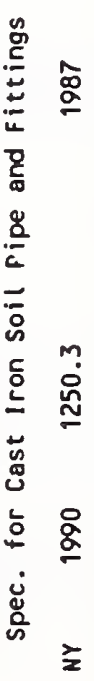

总 茓 วิे

㞗高 过 胥

ब号

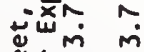
穴.气ำ

选 N 艾幺 눙ㅎㅇ 品 解毠 z

$\stackrel{\circ}{\circ} \stackrel{\hat{\alpha}}{\circ}$

$\stackrel{\circ}{\check{0}}$

홍 용

$\underset{8}{\stackrel{8}{\alpha}}$ 


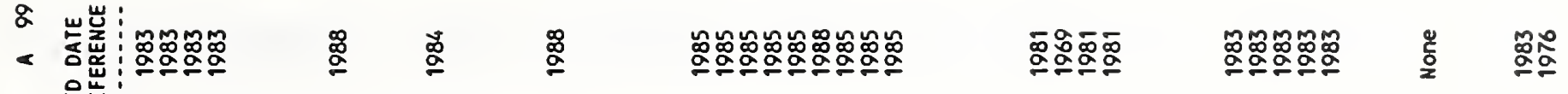

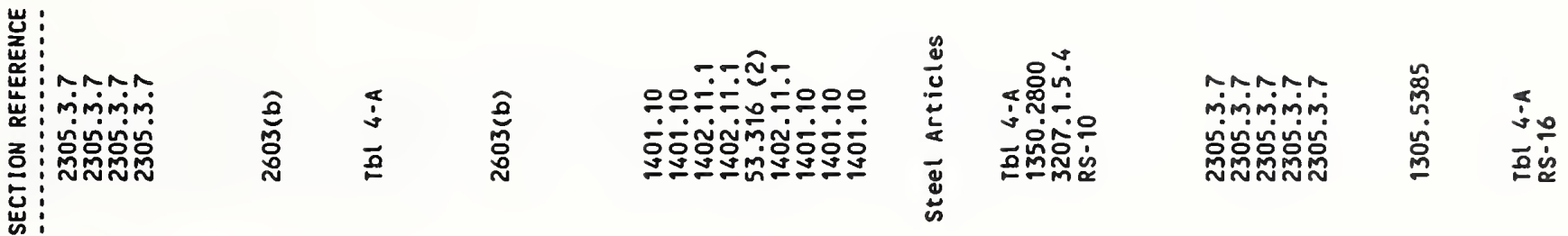

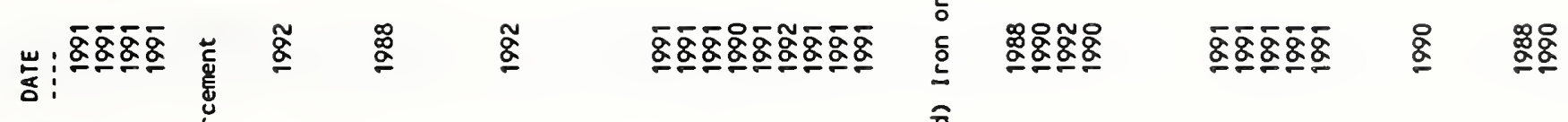

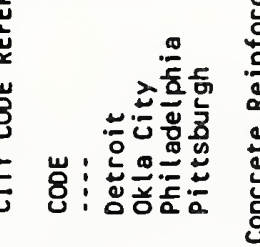

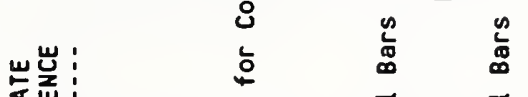

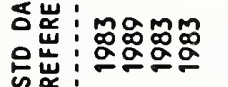

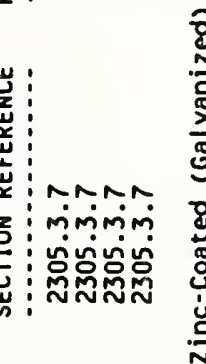

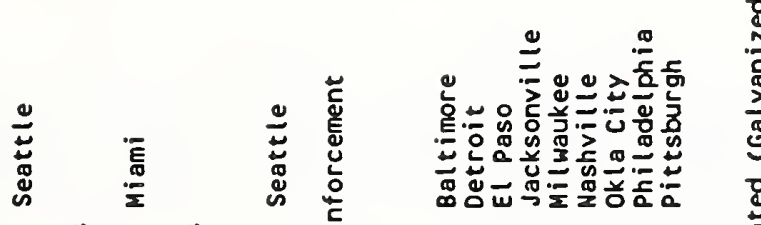

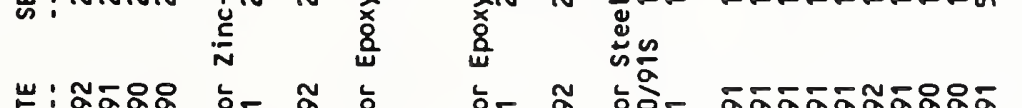

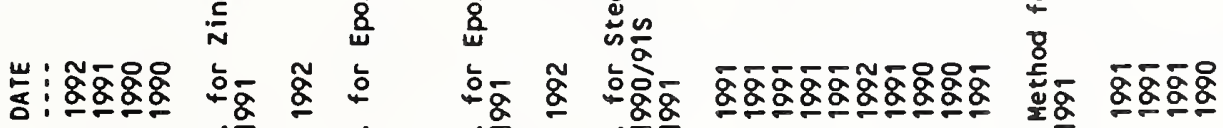

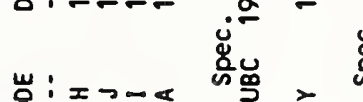

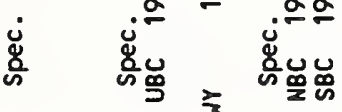

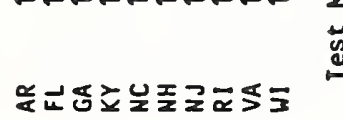

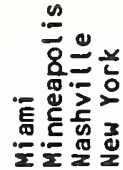

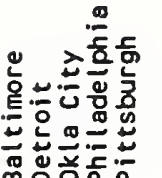

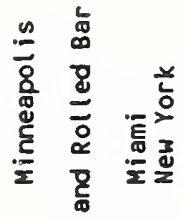

ฐँّ

突

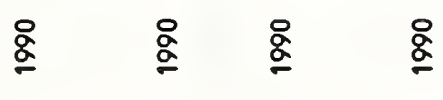

$\stackrel{5}{2}$

然

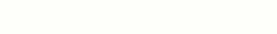

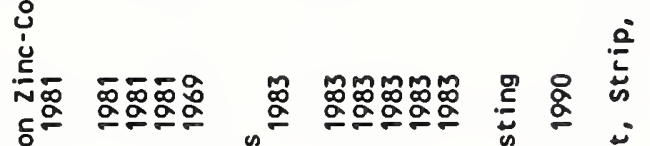
ب⿱宀⿻三丨口

-

峁

$\frac{5}{\frac{c}{a}}$

㿟

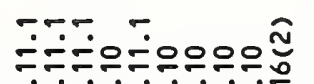

蒙

nทn一용

mimimimin

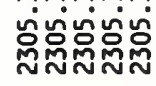

N

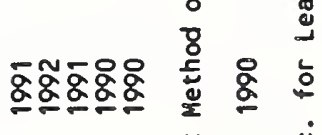

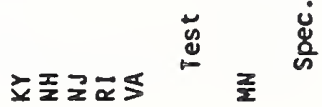

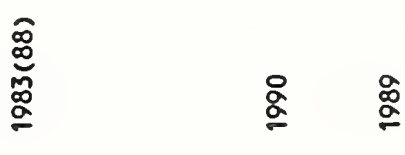

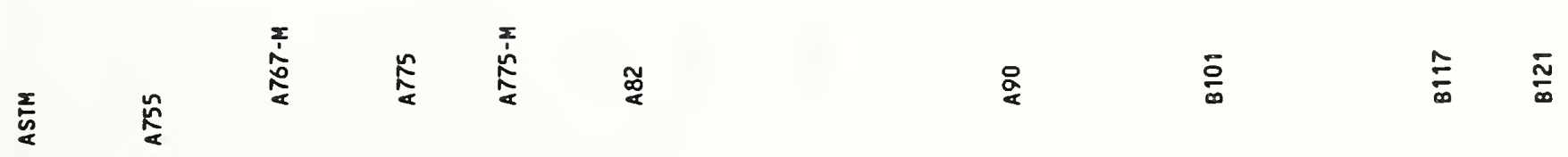




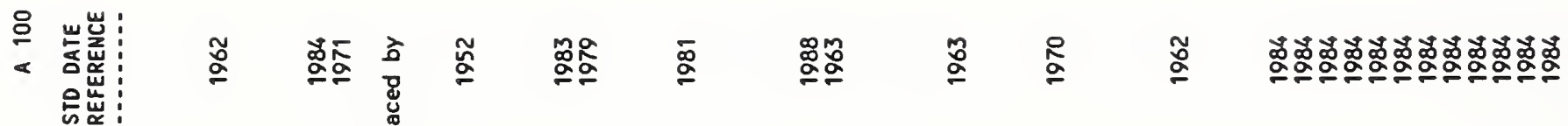
艾:

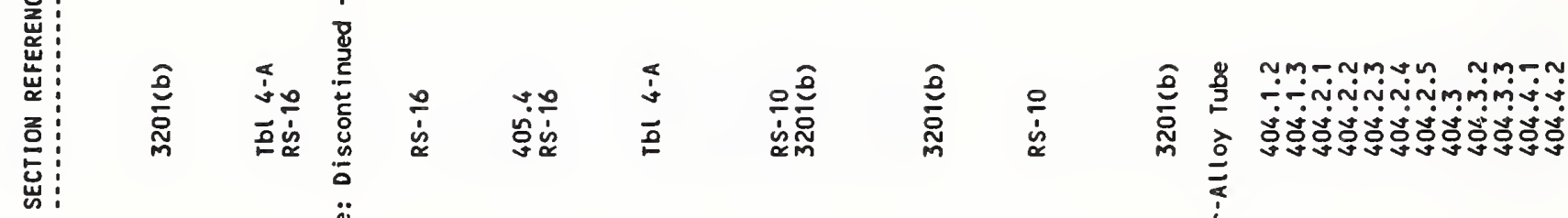

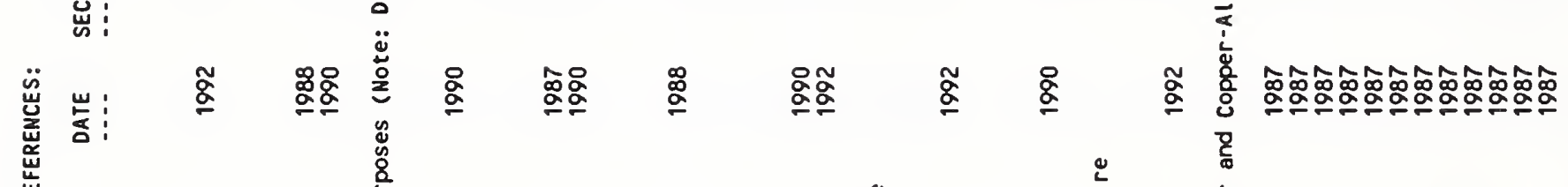

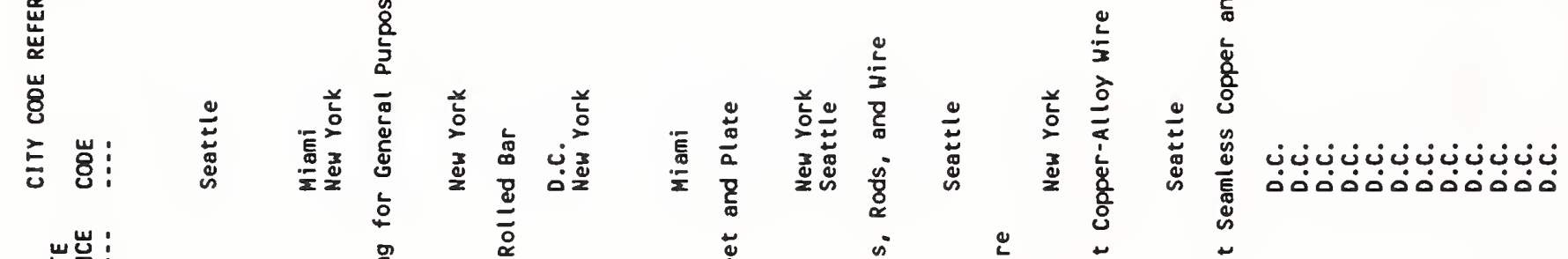

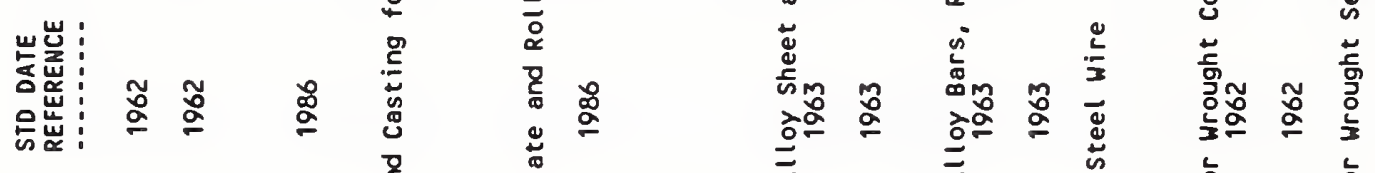

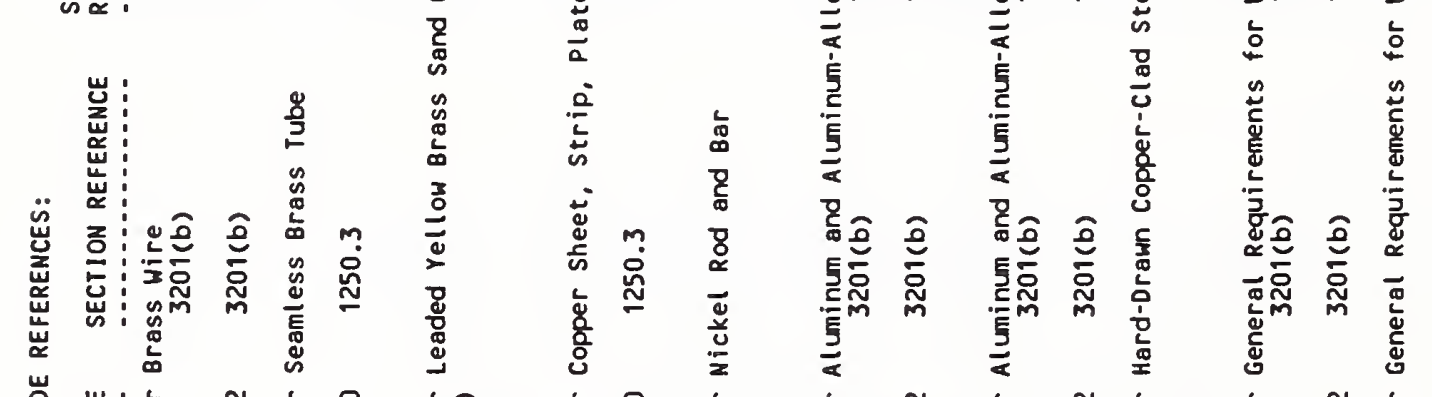

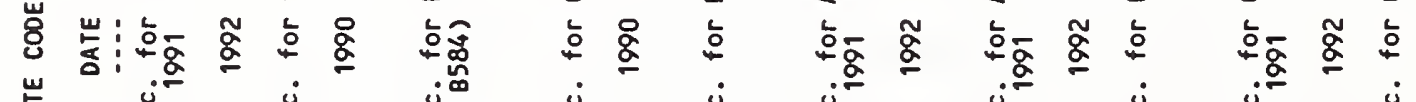

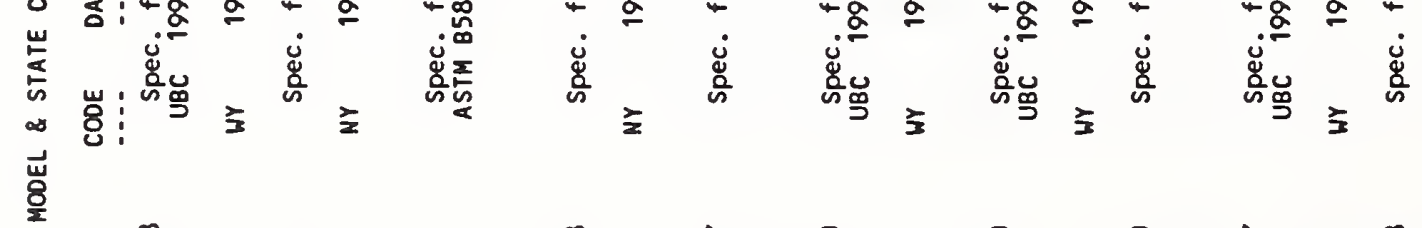
\$

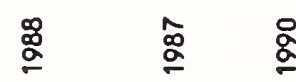
$\stackrel{g}{g}$
$\stackrel{g}{g}$

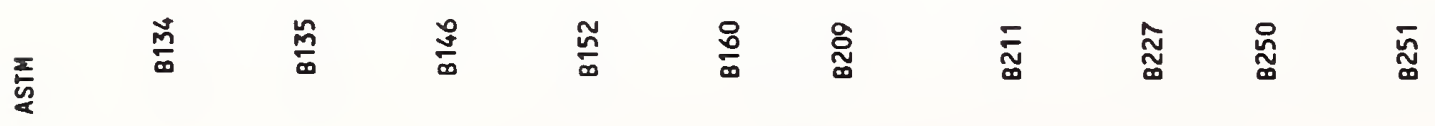



豆容:

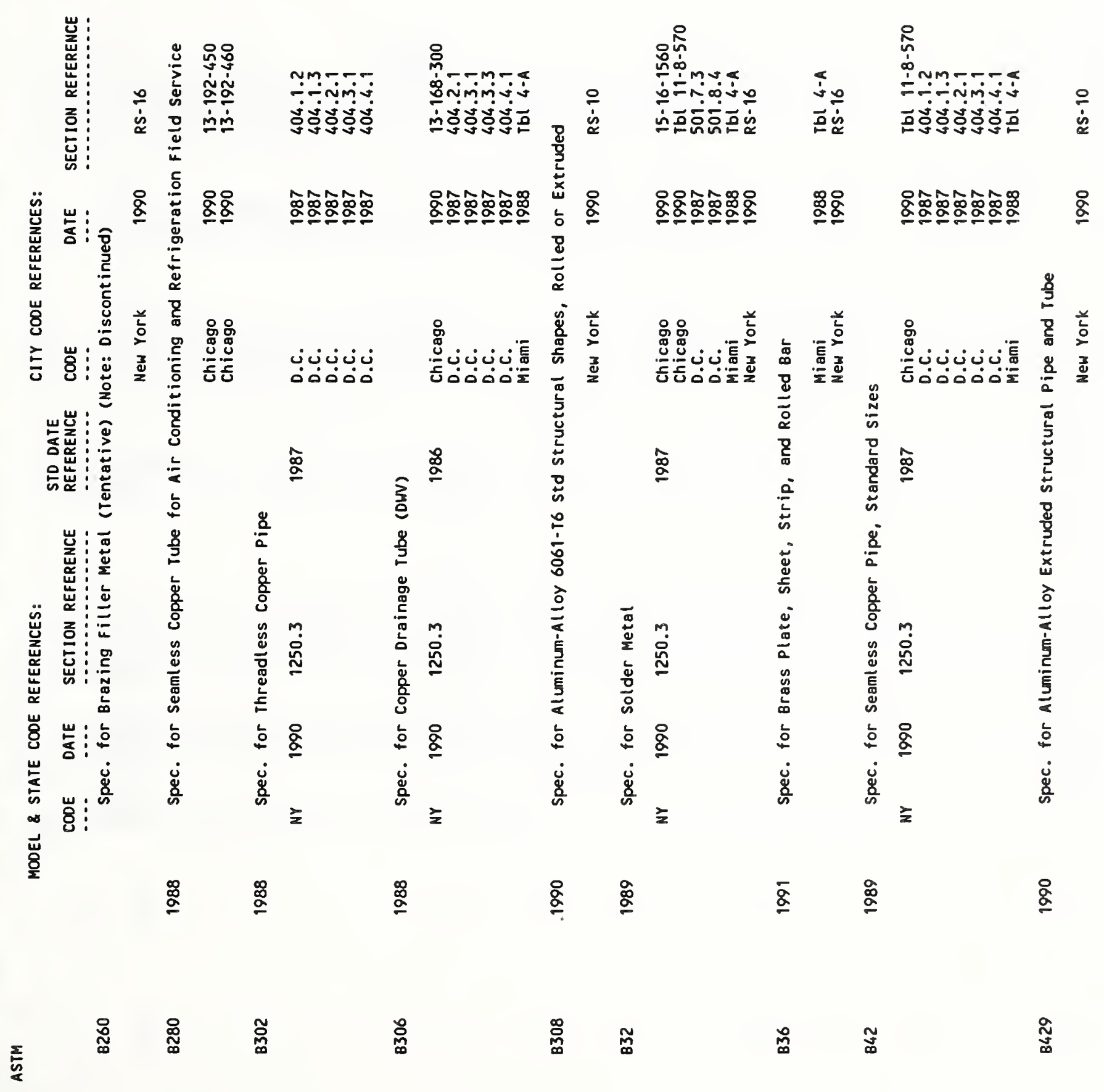




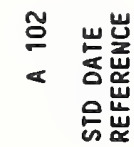

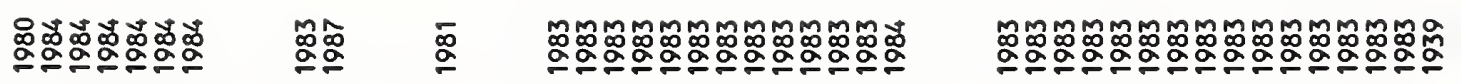

$\stackrel{ฒ}{\stackrel{\Xi}{\Xi}}$

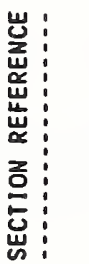

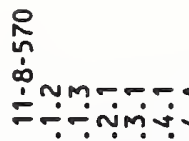

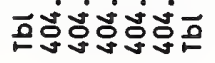

jo

ก.

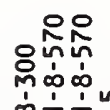

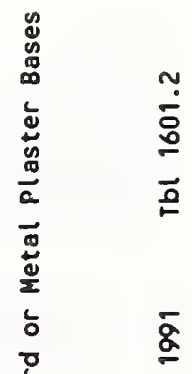

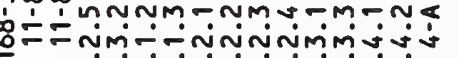

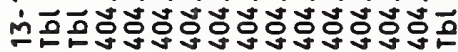

芯总

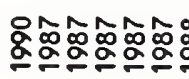

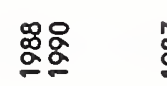

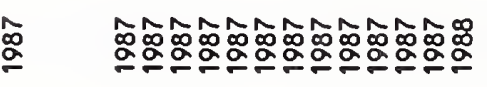

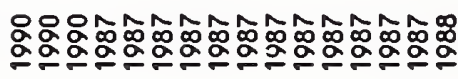

$\bar{\S}$

岁

ठ

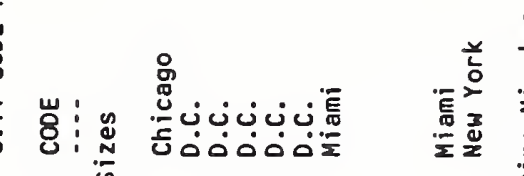

岁:

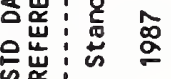

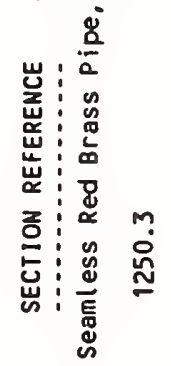

这京市

و ن

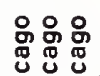

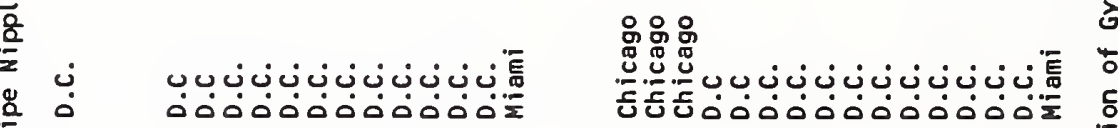

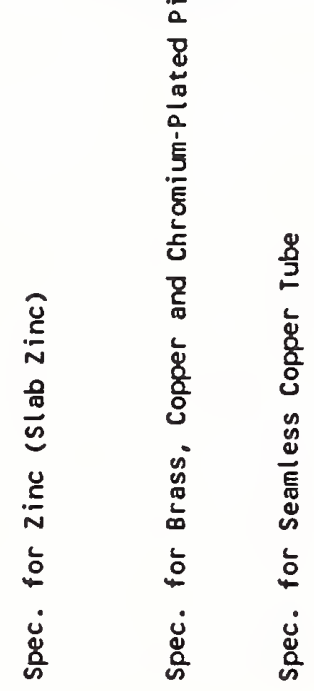

$\stackrel{\$}{\circ} \stackrel{8}{2}$

㟧

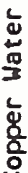

(2)

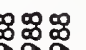
요요

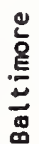
눙<smiles>C1CCCC1</smiles>

要

岕ก

m.

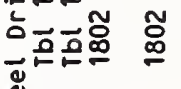

岁舟告

용

就㝘察

岁岛诘

岁:

㟧

总总怘

\&

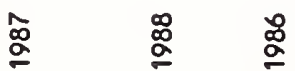

$\stackrel{0}{\circ}$

$\underset{8}{\mathscr{0}}$

章

m

ஃ

密 命

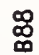

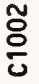


$\underline{9}$

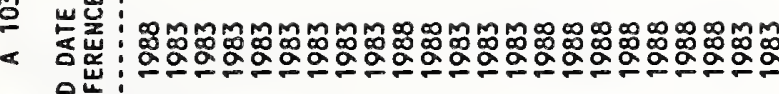
的峞

㟧 :

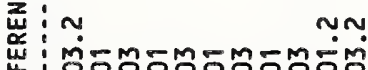

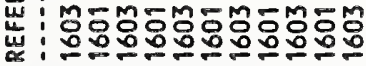

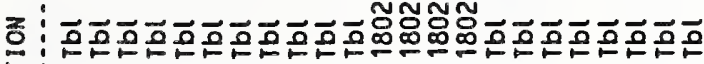

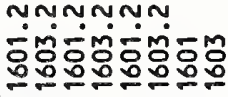

崫:

莡

品

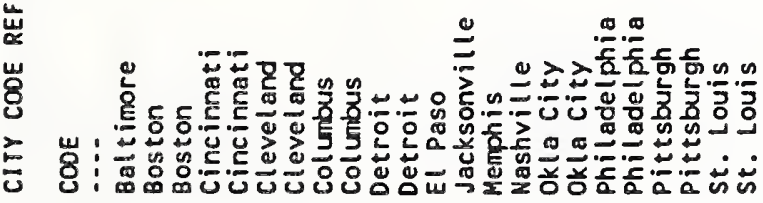

넝:

व

낸

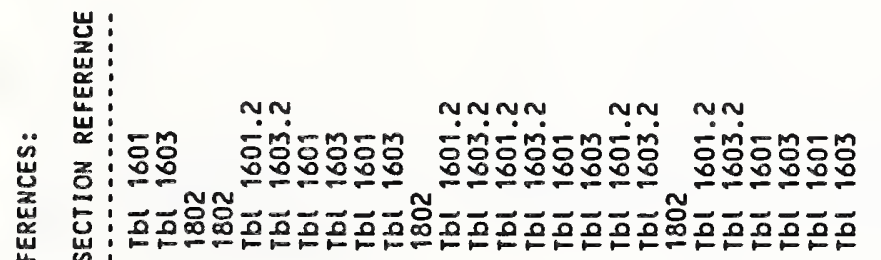

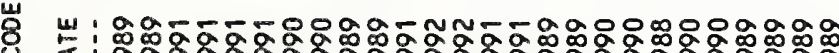

농

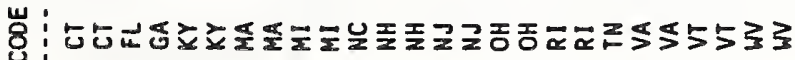

苑

萝

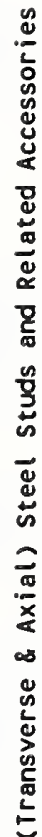

?

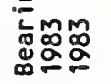

总

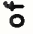

$\frac{5}{2}-$

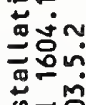

气ू은

는

这玄

sisu

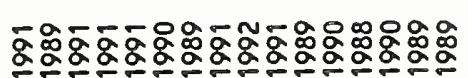

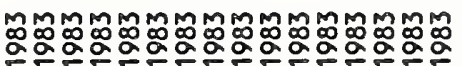

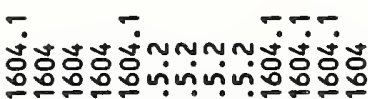

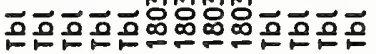

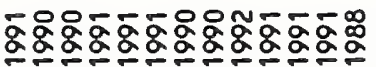

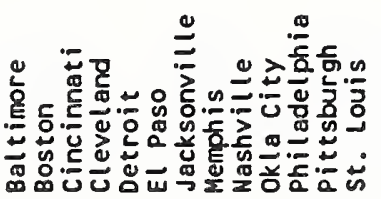

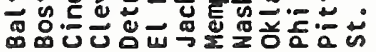

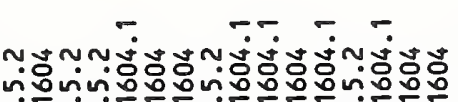

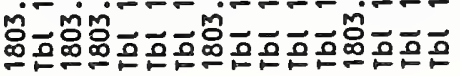

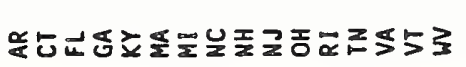

高

ธิธํํ
ఏ్

$\stackrel{\Xi}{\Xi}$

$\frac{c}{2}$

ธั่

它

$\stackrel{2}{\circ}$

玄荢

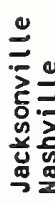

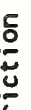

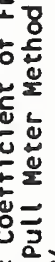

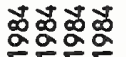

눙

总

웅후은웅

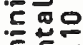

튼응

농ํํㅇ

ㅇํㄴ

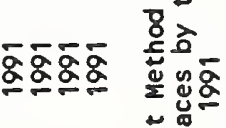

范

떤정을

政兽

$\stackrel{g}{g}$

$\stackrel{\Xi}{0}$ 
岂 岂

< 온

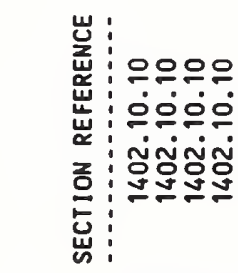

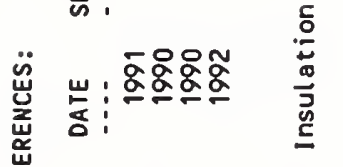

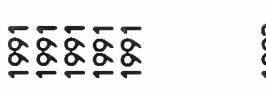

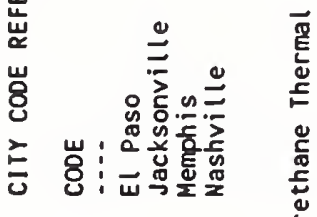

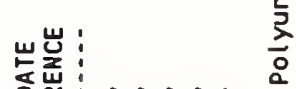

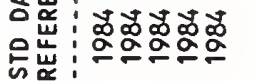

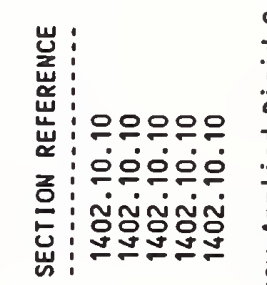

高乐

岁

峁

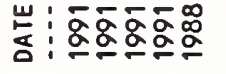

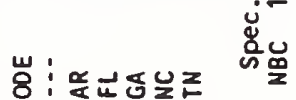

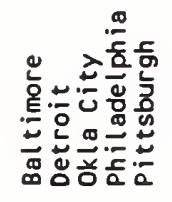

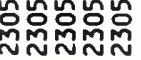

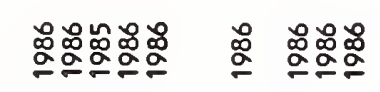

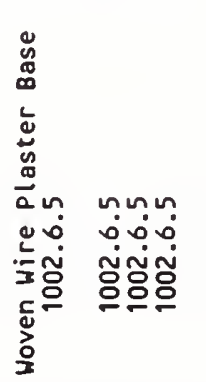

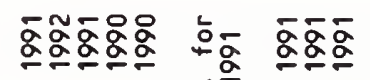

西

$\approx$

状

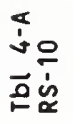

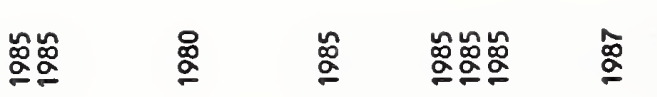

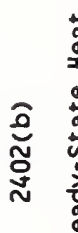

ฉू용

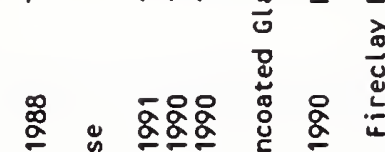

ळ.

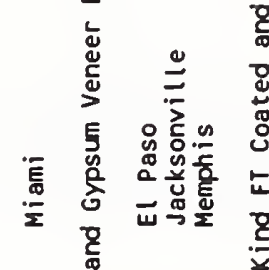

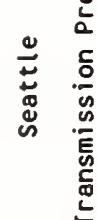

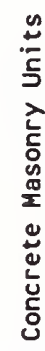

mimmm

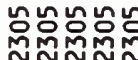<smiles>C1CCC1</smiles>

范

떤워

高

$\stackrel{\circ}{\circ}$

笓

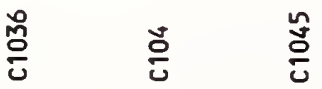

ชิํำ

产 产

$\frac{8}{3}$

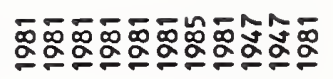

กี่

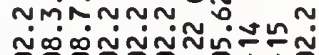

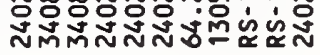

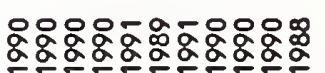

(a)

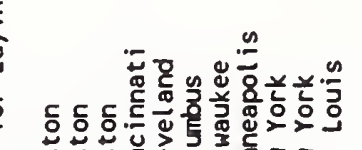

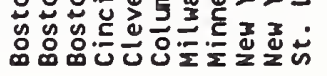

울

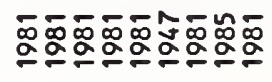

里

ब

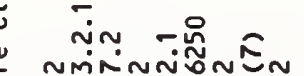

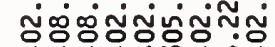

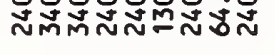

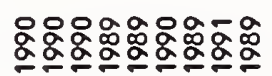

䢰

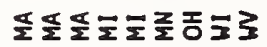

\begin{tabular}{ll}
$\Sigma$ & $\stackrel{0}{0}$ \\
\hdashline & $\frac{0}{0}$
\end{tabular} 


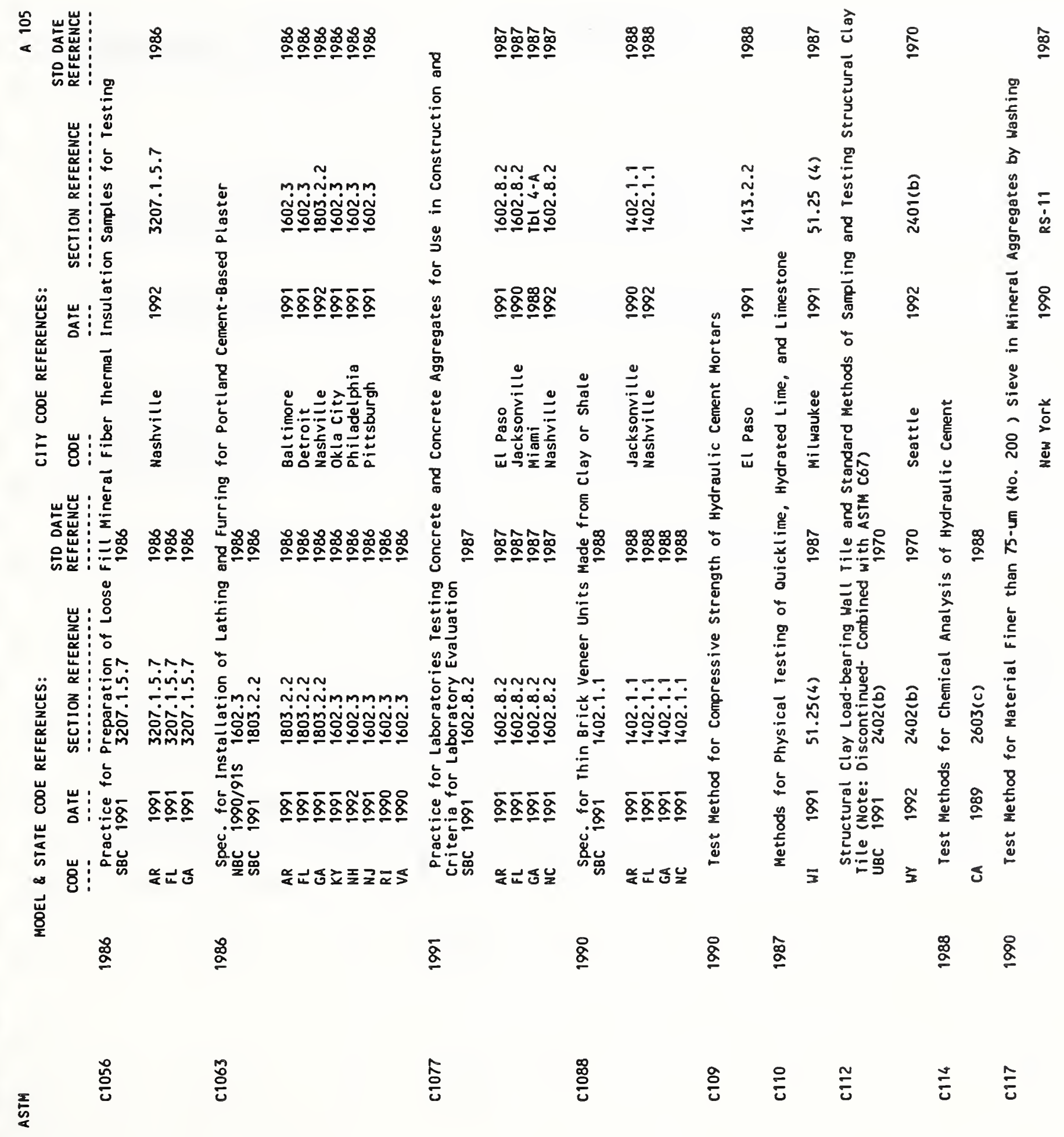




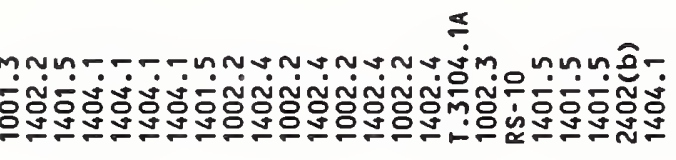

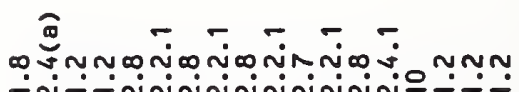

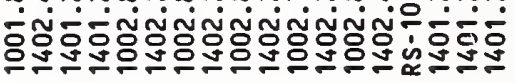

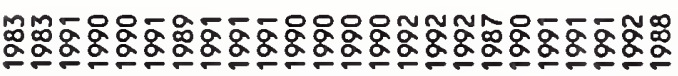

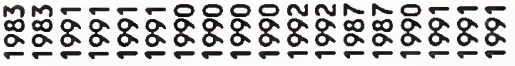

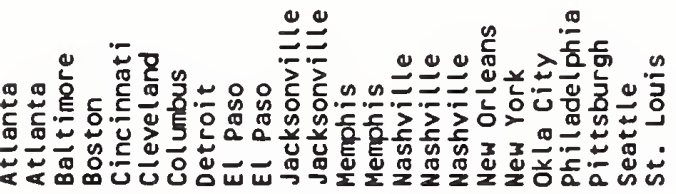

$\stackrel{\circ}{\&}$

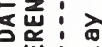

运崫:

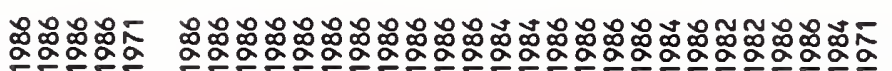

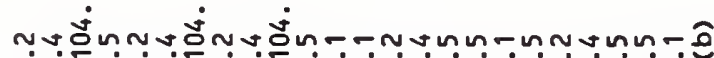

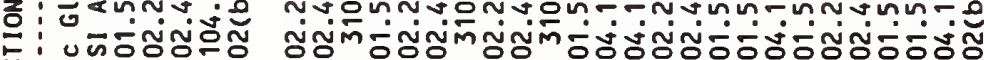

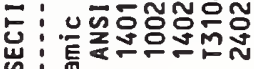

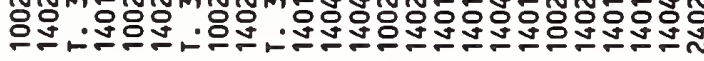

บำ

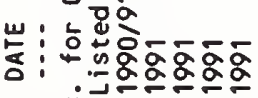

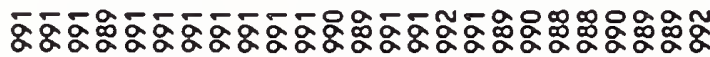

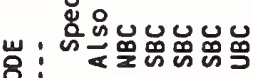

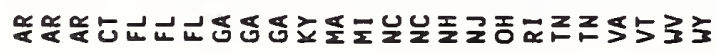

离

:웡ํํㅇ

M

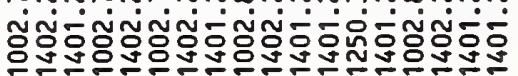

家

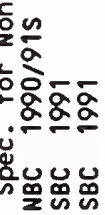

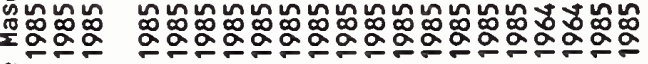

芩
$\stackrel{\Xi}{2}$

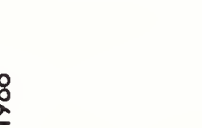
ठㅇㅇ: 
¿

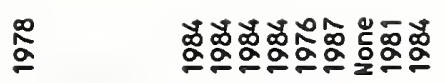

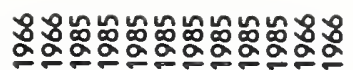

步㟧: 婴

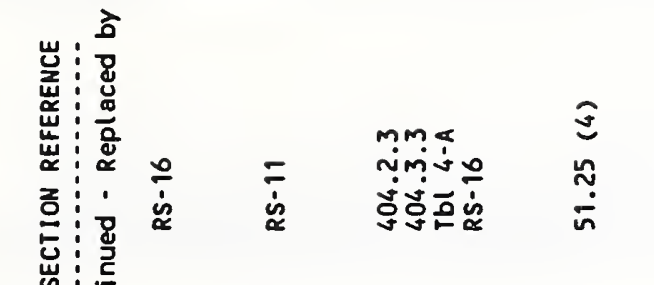

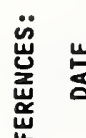

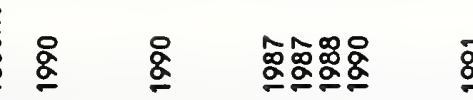

ธ。์

g-

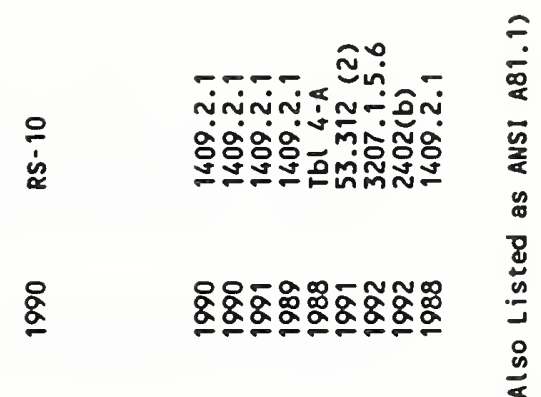

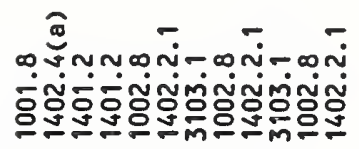

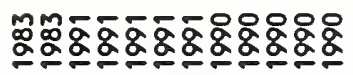

品

岁

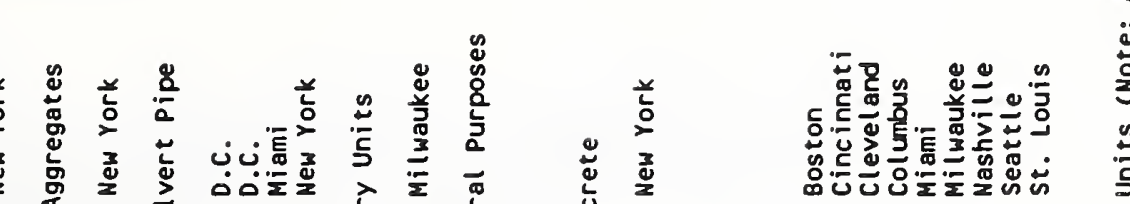

山 : :

뜬원

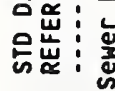

嵌 :

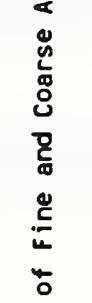

要,

焉

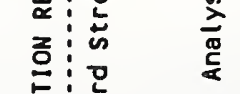

点:

㟧出

岁 山:

委

है

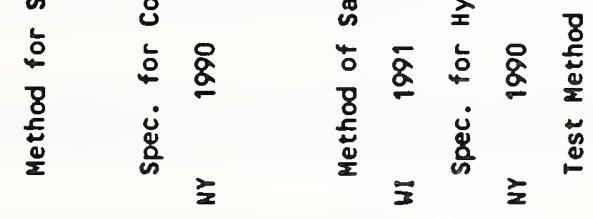

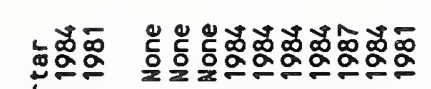

(2)

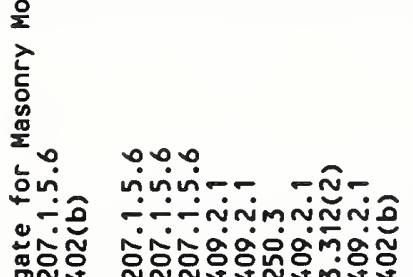

बกำ

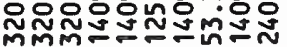

음

훌

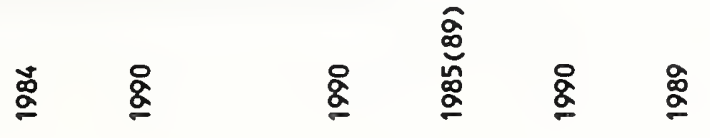

$\stackrel{ณ}{\alpha}$

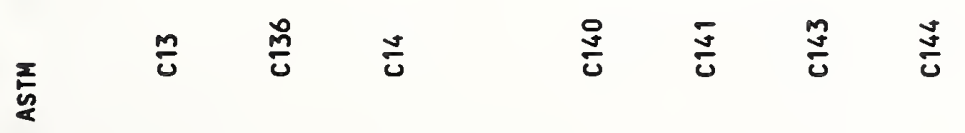

$\frac{n}{0}$ 


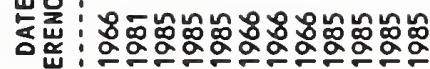
은
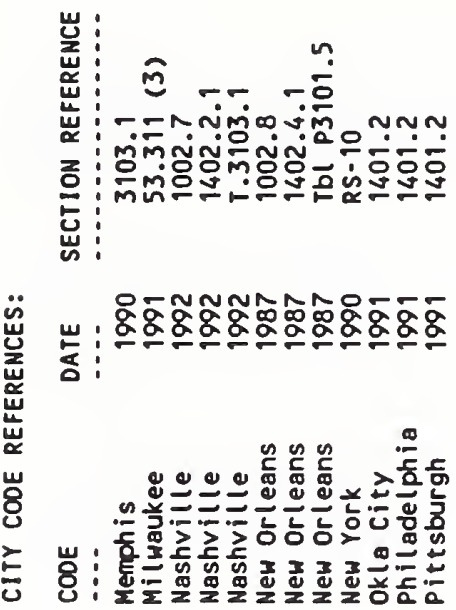

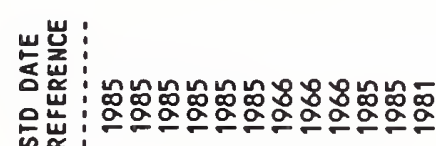

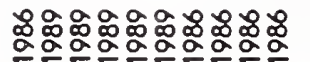

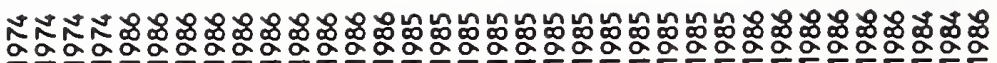

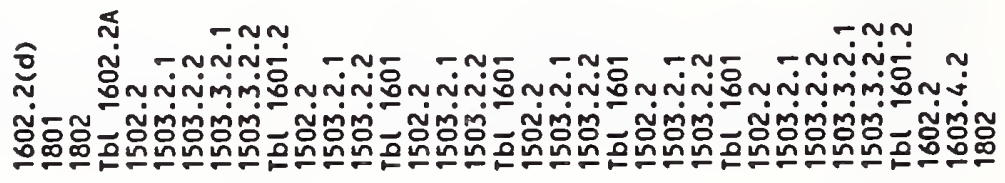

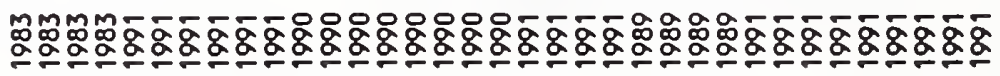

等

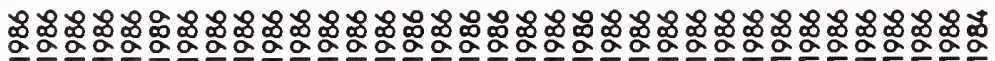

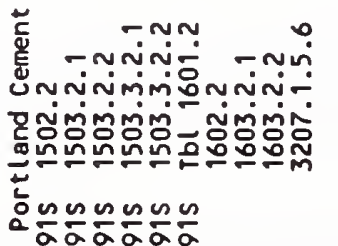

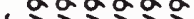

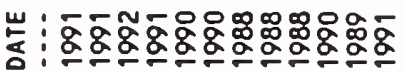

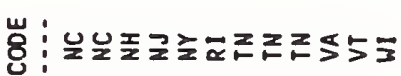

કัญ

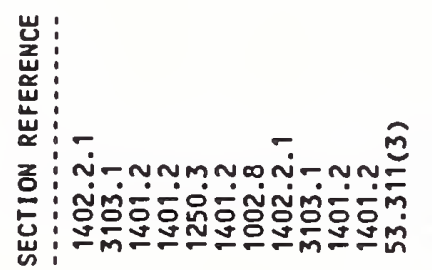

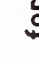

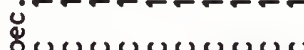

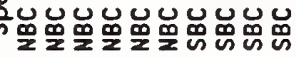

$\stackrel{\Xi}{\Xi}$

ํํㄴ

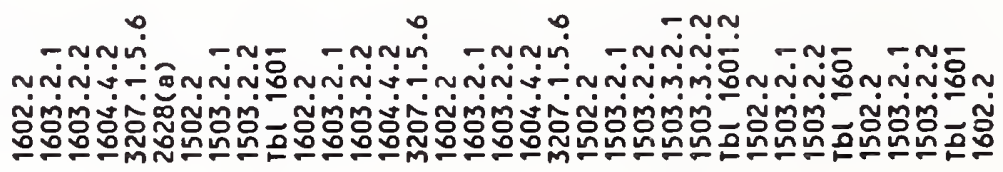

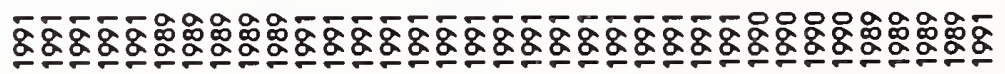

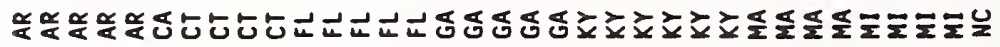


일
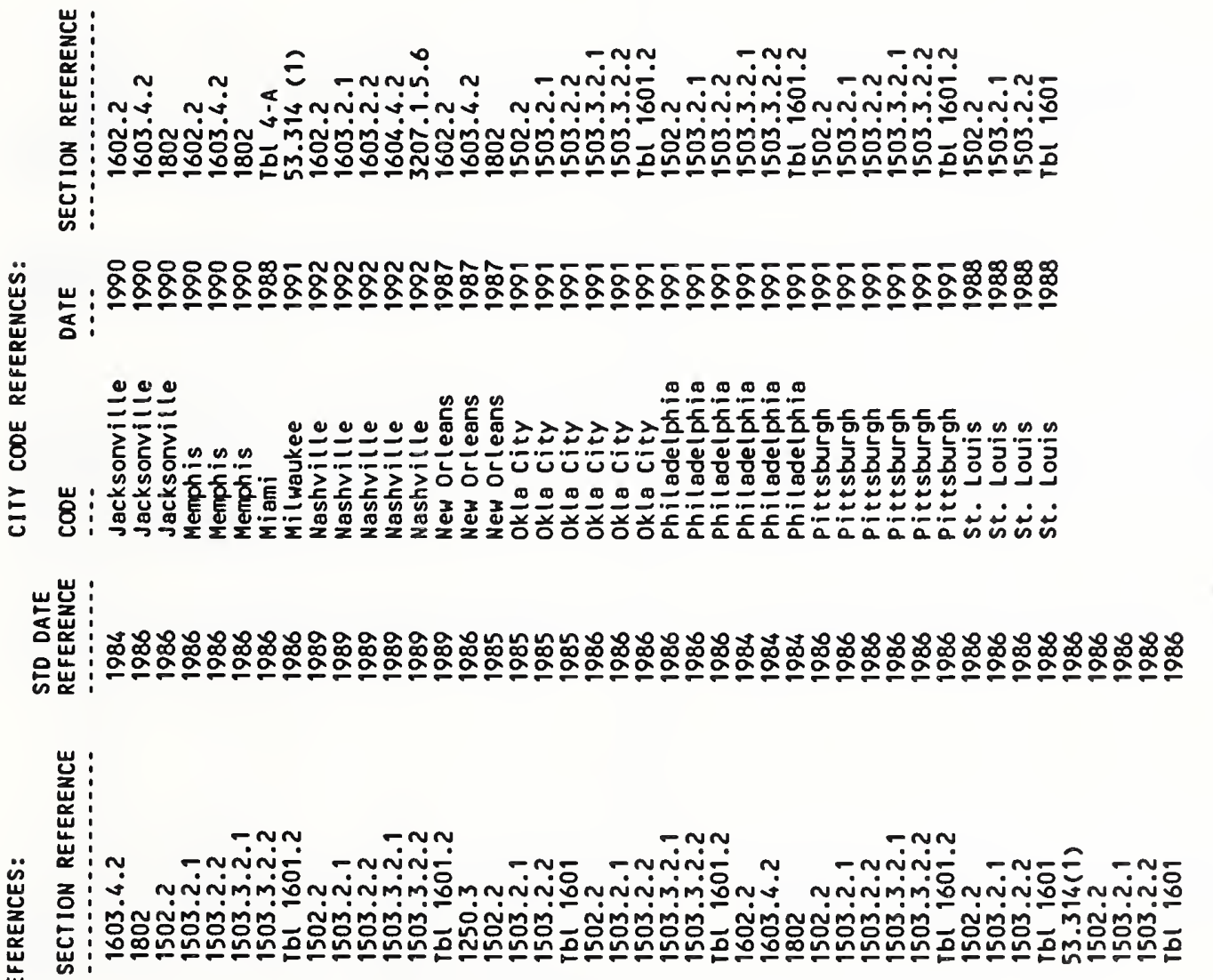

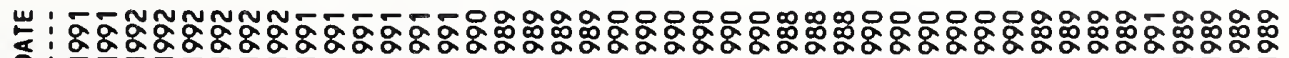

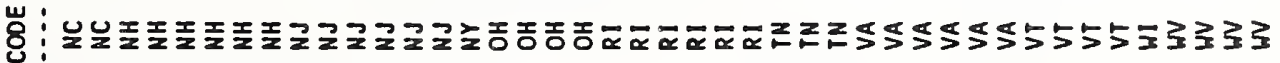

ํํํ

$\stackrel{\circ}{\circ}$

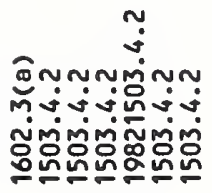

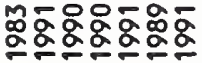

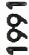

旁

ڤ్

过

ปे

言皮

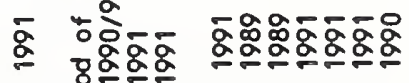

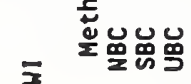

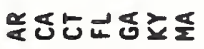




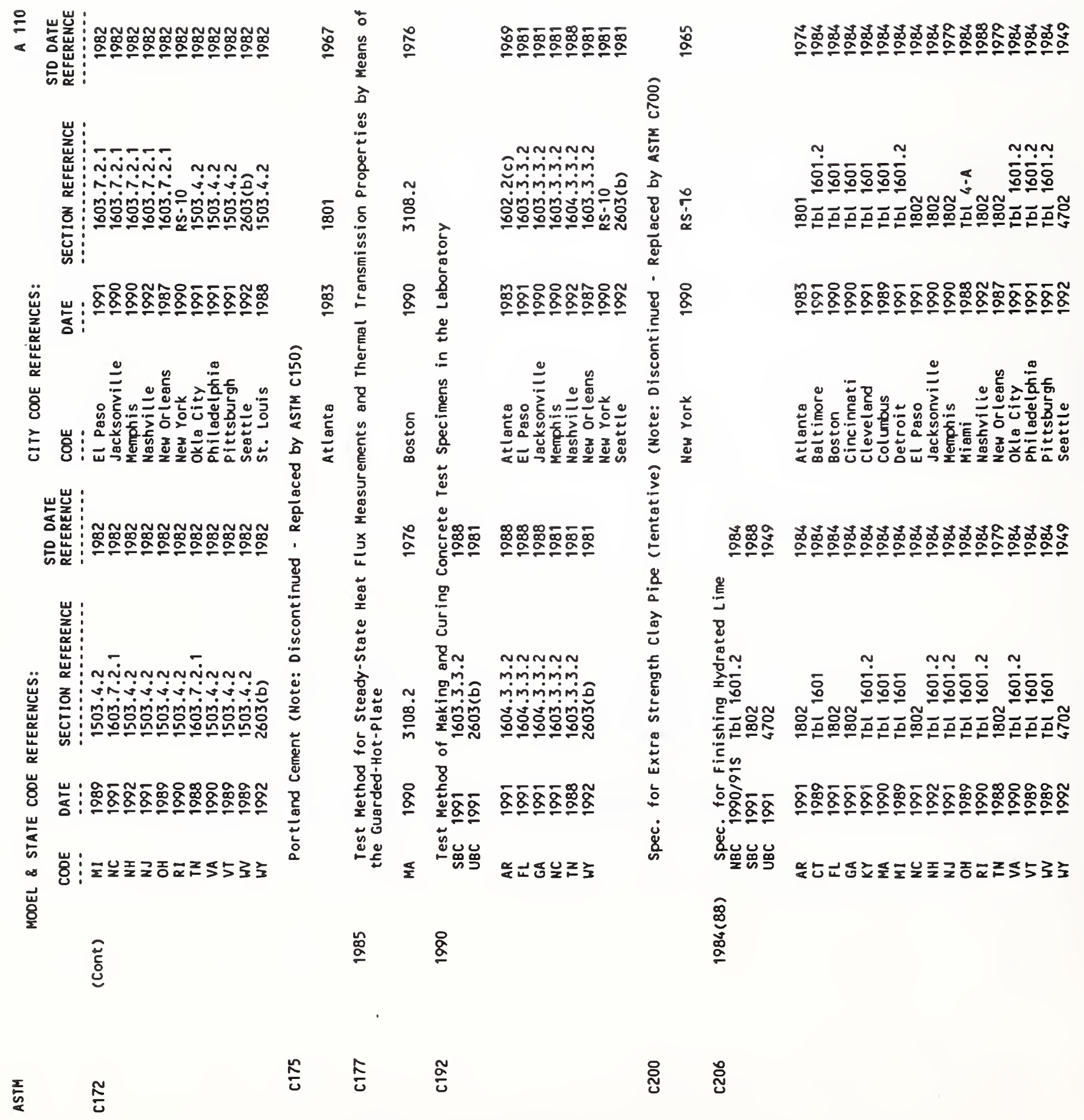




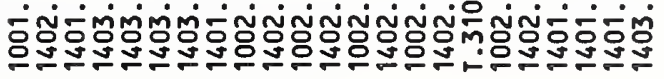

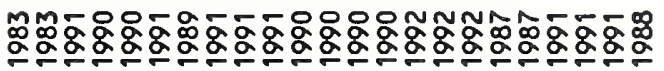
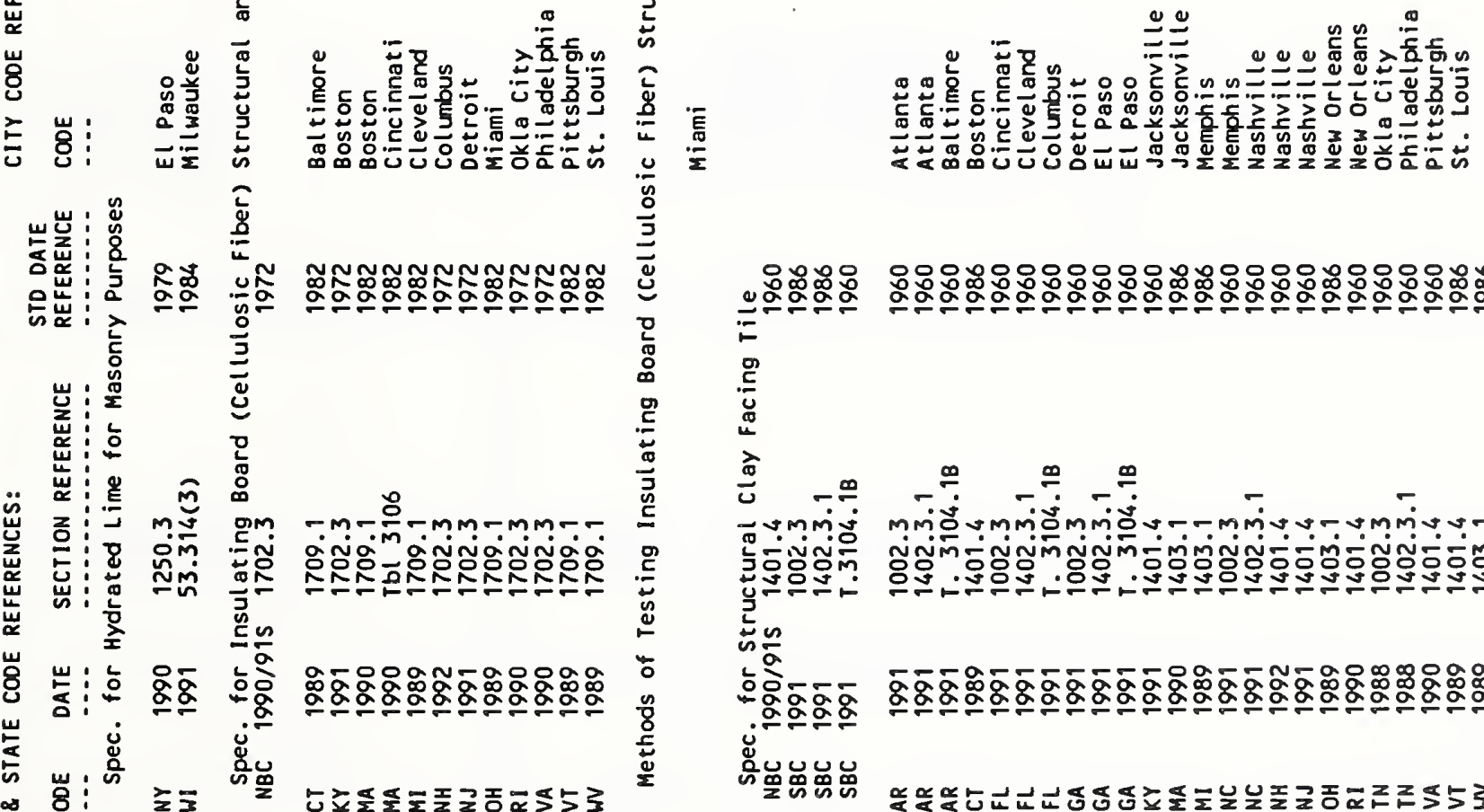

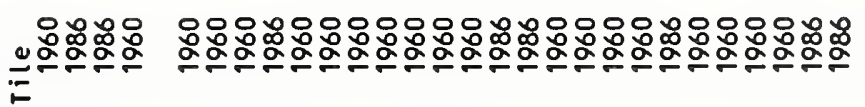
$\frac{\mathfrak{g}}{\mathscr{G}}$

ฮิ

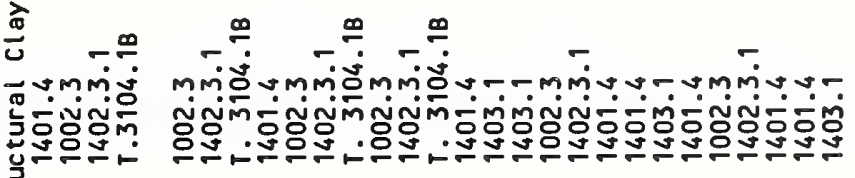
究 
ฮ

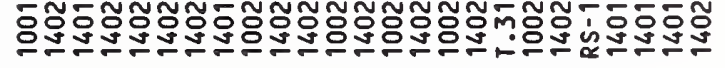

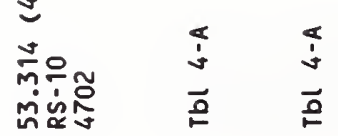

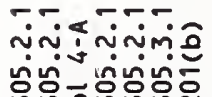

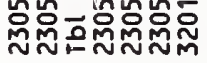

훙요

$\stackrel{\Xi}{g} \stackrel{\Xi}{\square}$

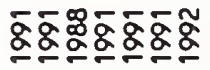

崖:

它崖:

品器产

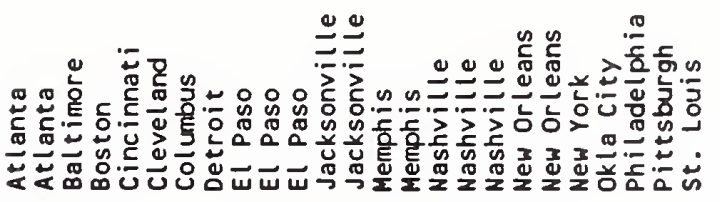

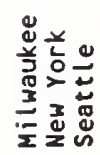

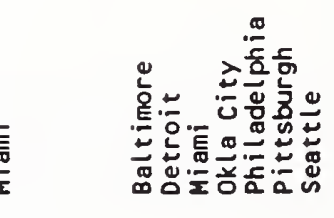

岁:

焉

$\ddot{u}$

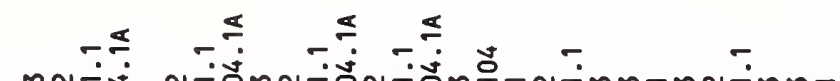

它

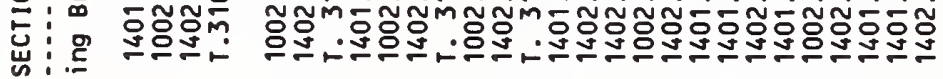
这 $\frac{n}{2}$

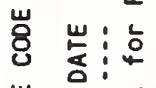

$\frac{1}{\alpha}$

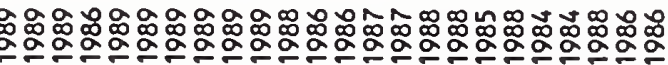

员 吆器

空

$\frac{\bar{E}}{\frac{5}{2}}$

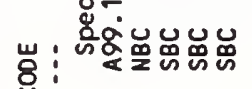

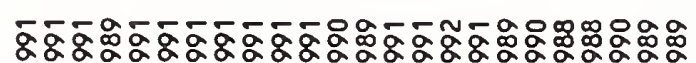

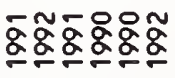

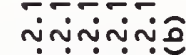

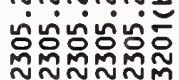

 


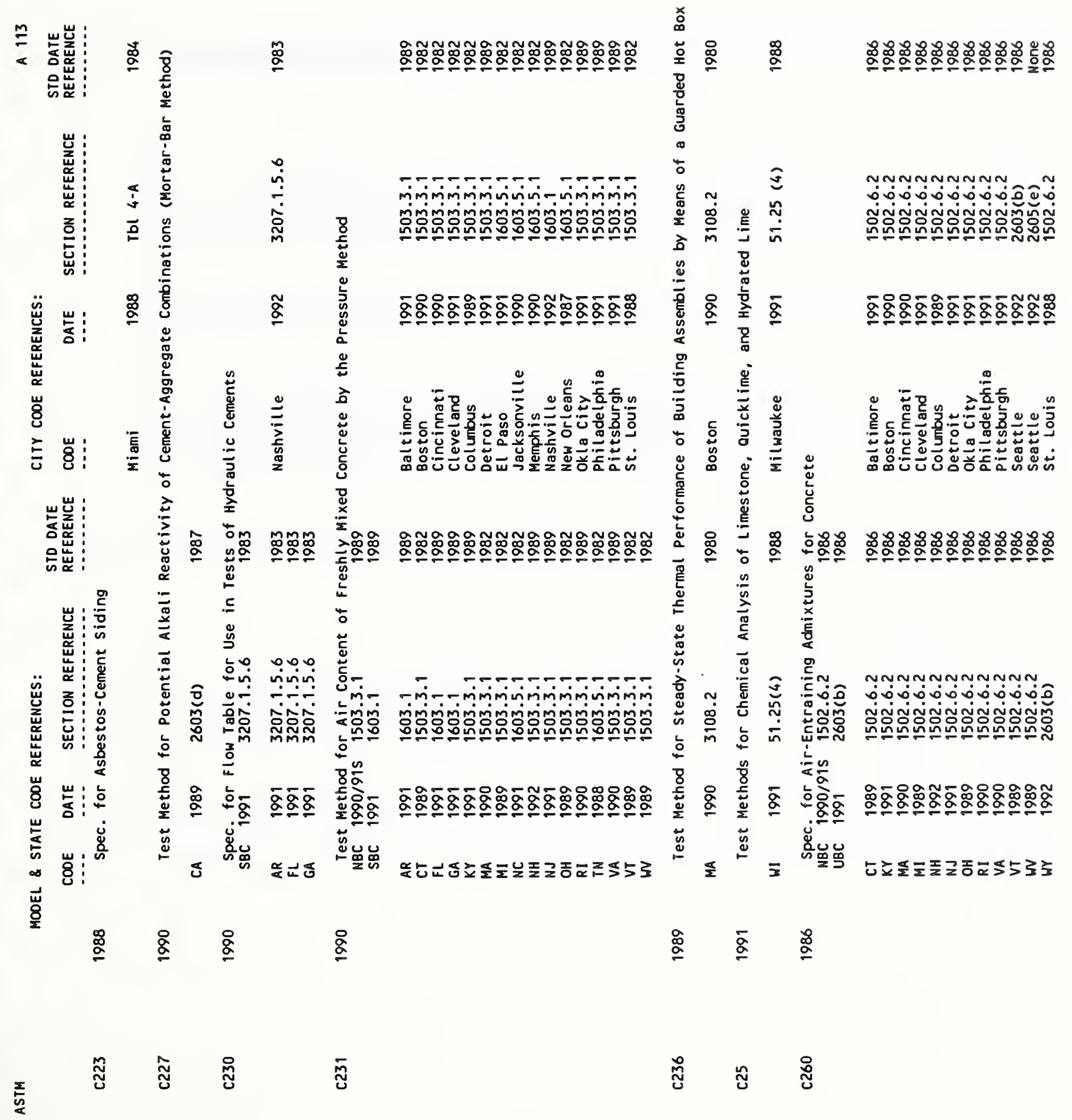




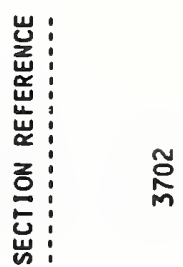

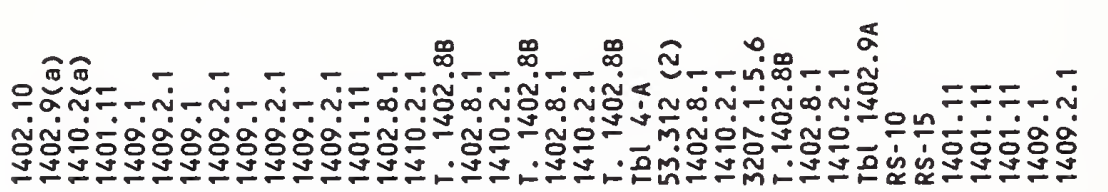

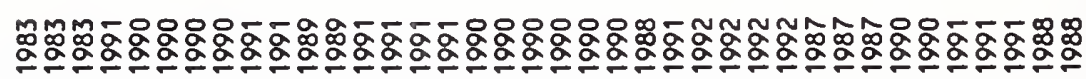

岕:

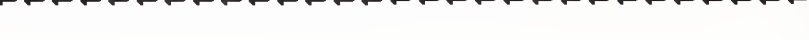

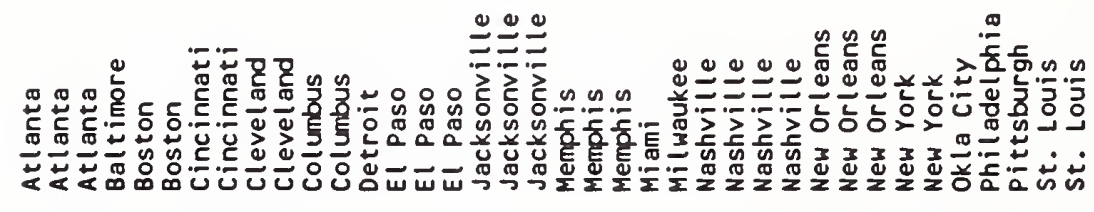

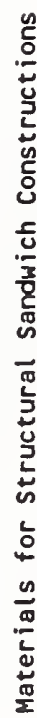

шّ :

造密:

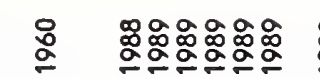

ఏåå

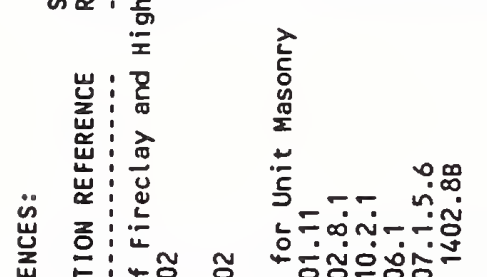

首:

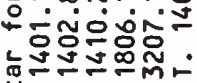

我

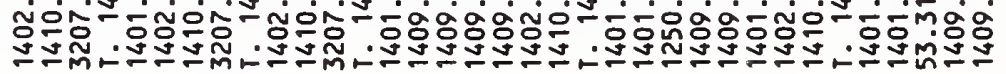
$\frac{0}{2} \frac{\infty}{2}$

岩

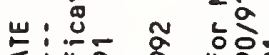

至

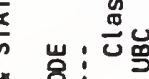

\&

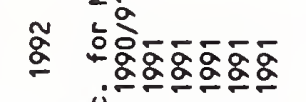

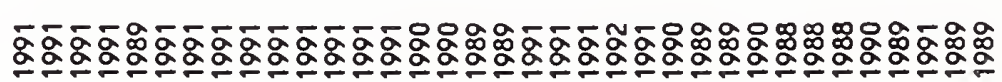

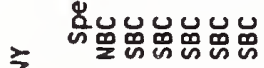

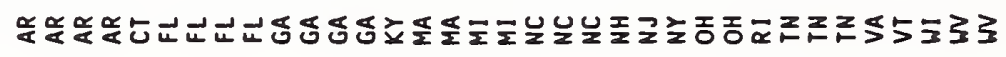

혈

ฆั

总

I 


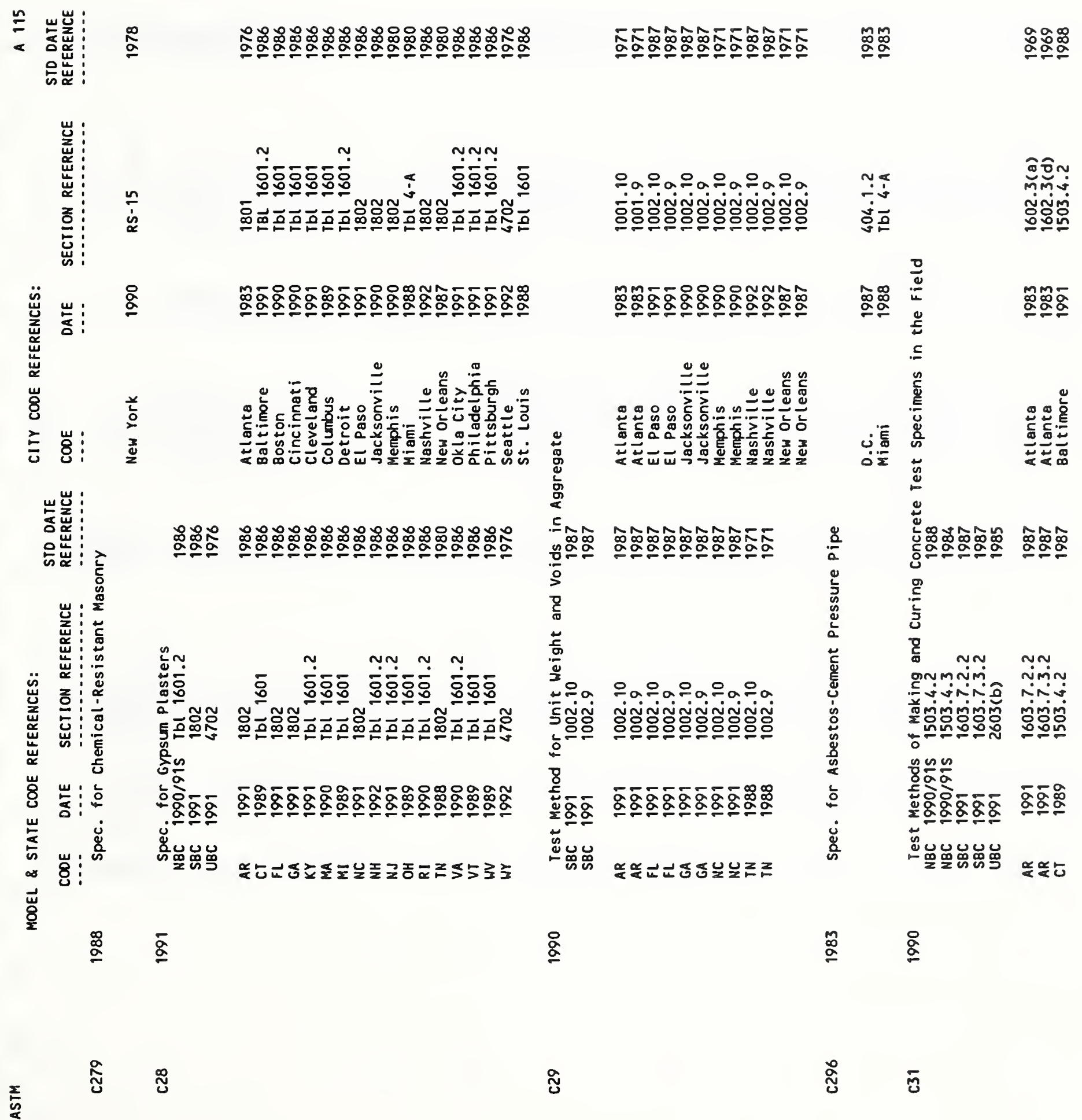




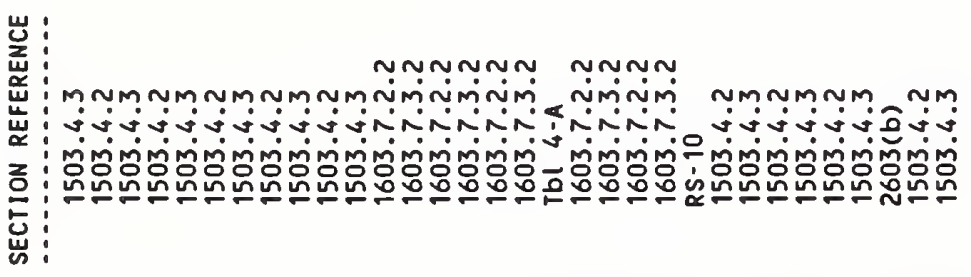

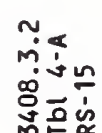

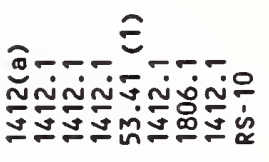

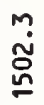

苍

용요

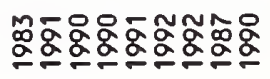

$\overline{\underline{\alpha}}$

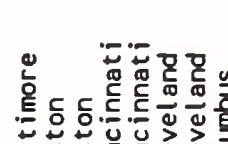

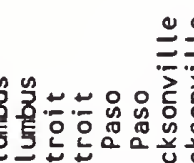

$\stackrel{0}{=}=$

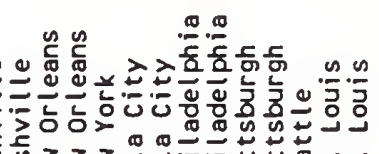

先

崖:

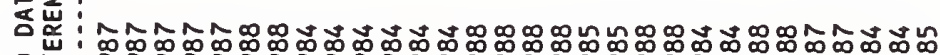
位岕:

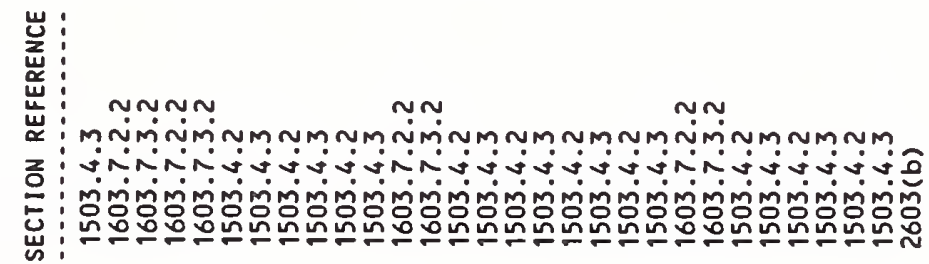

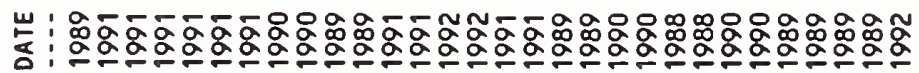

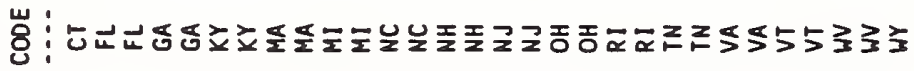

窇

$\stackrel{5}{\circ}$

岁 줄도를<smiles>C1=CCCC1</smiles>

范

영요

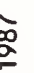

$\frac{n}{3}$

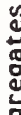

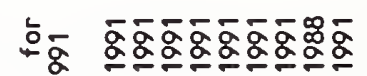
离.

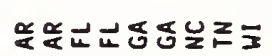

임 


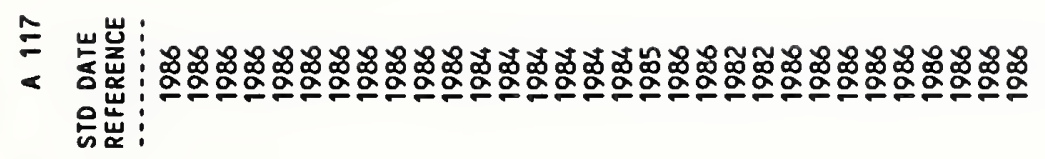

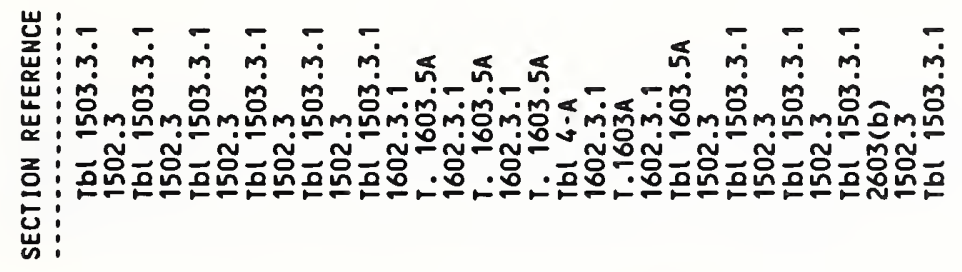

蒿

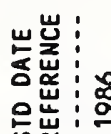

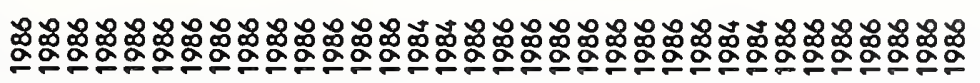

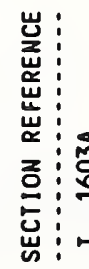

in.<smiles>[C]1C=[Te]C1</smiles><smiles>C=CC=CCCCC</smiles><smiles>C1CCCCC1</smiles>
ณ में<smiles></smiles>

\section{(i.}

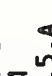
เุ $\dot{m}$ $\dot{m} \dot{m}$

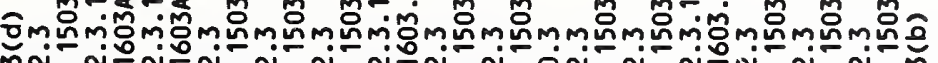

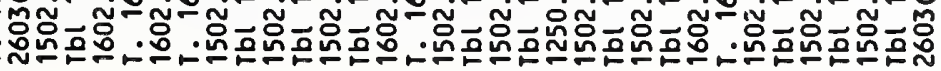

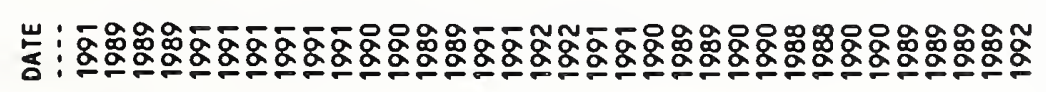

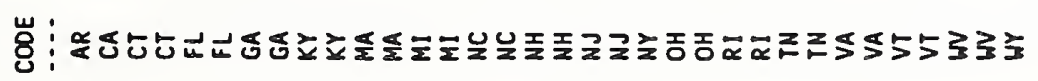

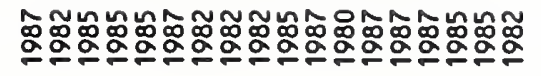
mmmmmmmim

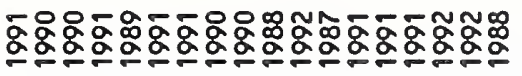

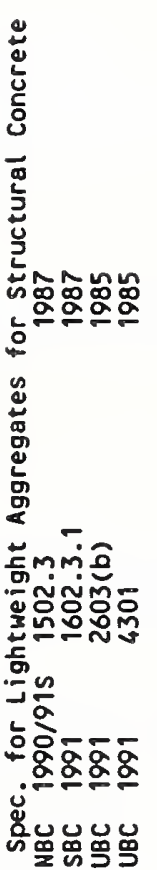

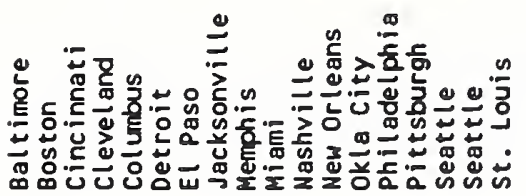

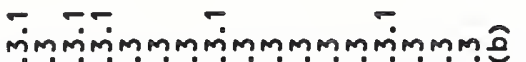

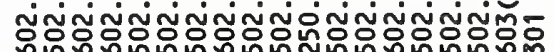

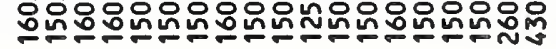

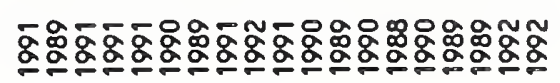

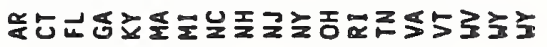




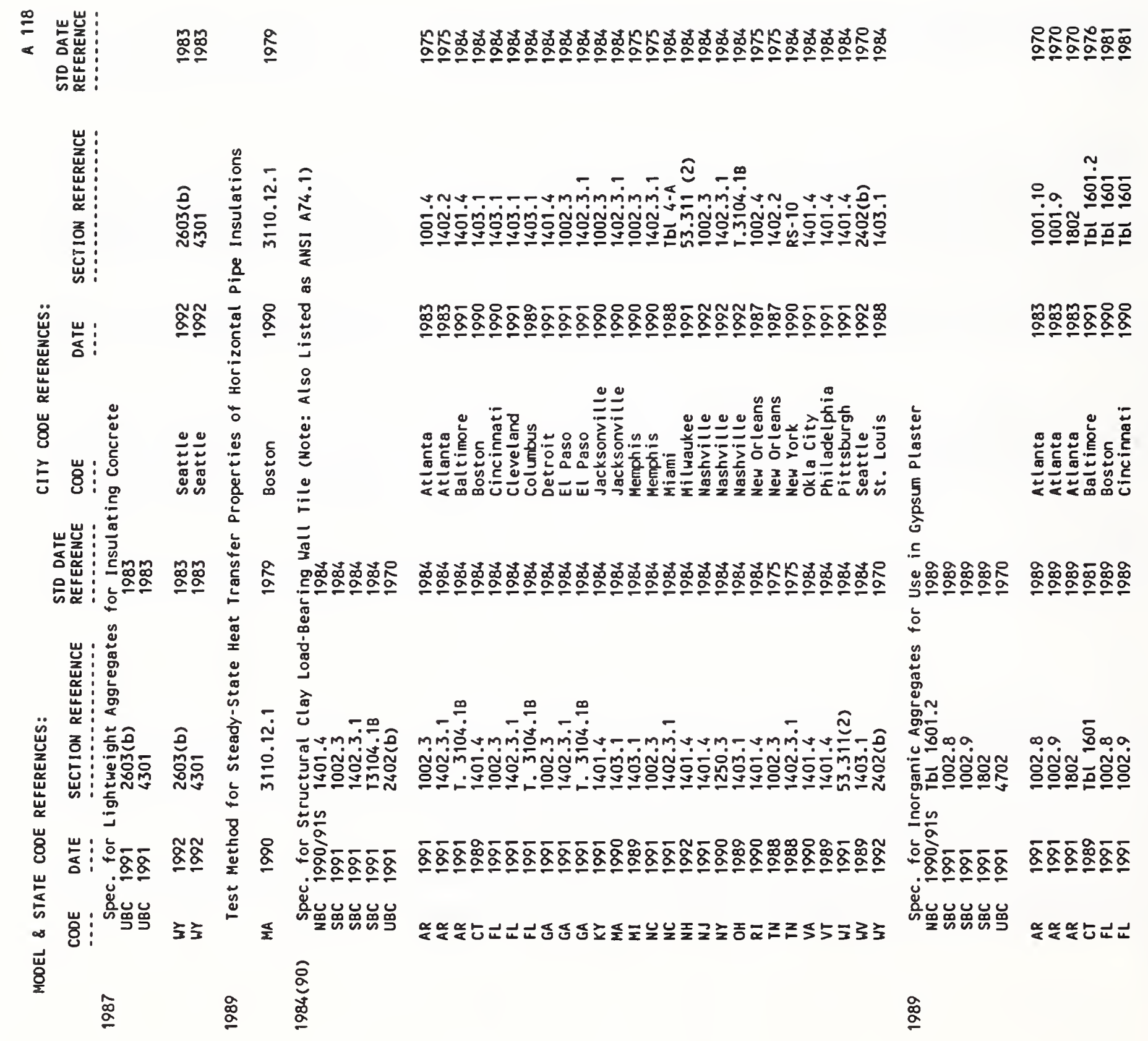


$\cong$ w

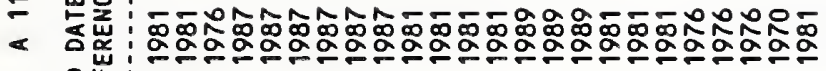
的岕

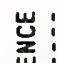

:

嵌

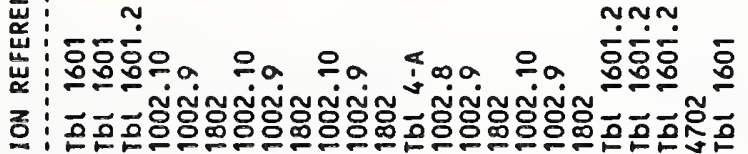

总

䓛

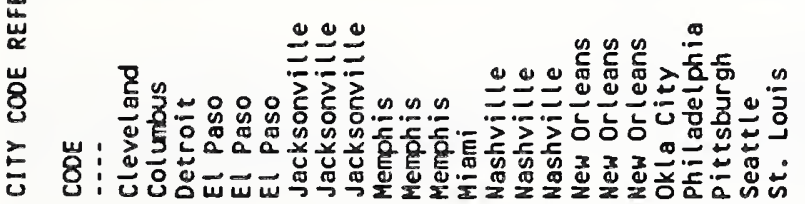

ㄴّㄴ :

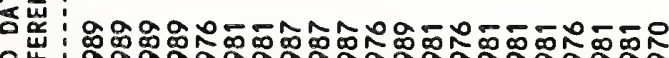

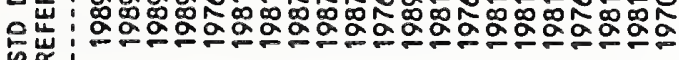

岁

б

:

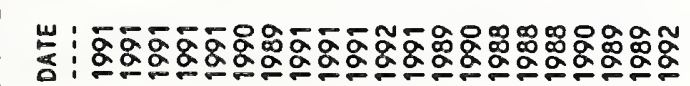

岕

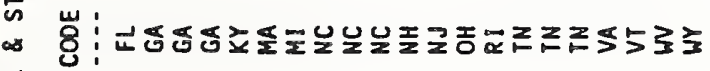

崩

萨

点 留

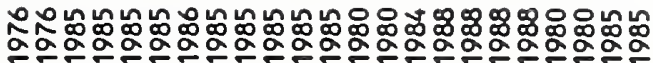

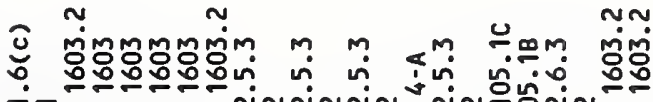

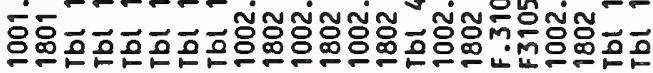

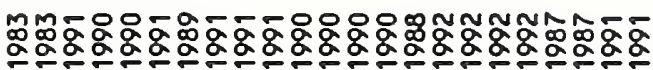

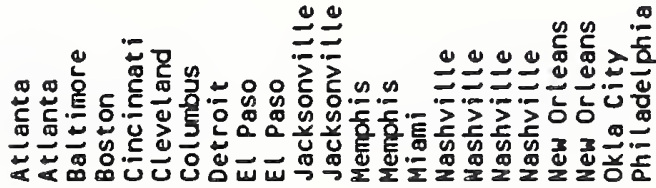

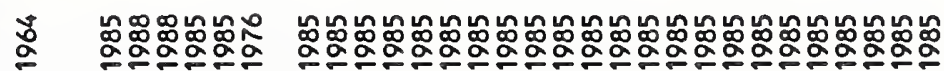

$\stackrel{5}{\frac{5}{5}}$

竞

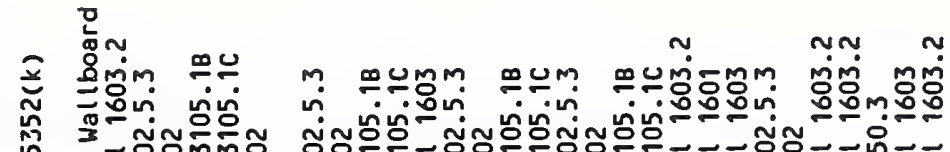

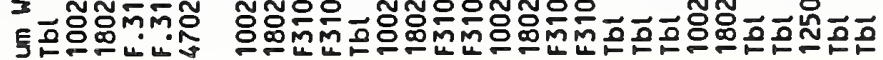

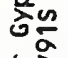

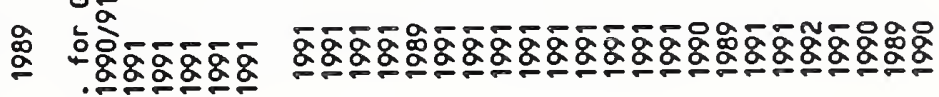
宑-

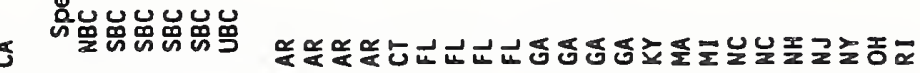

$\bar{\alpha}$

荅 กั 
\&

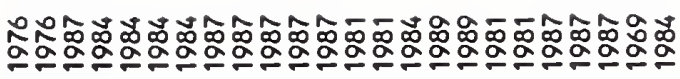

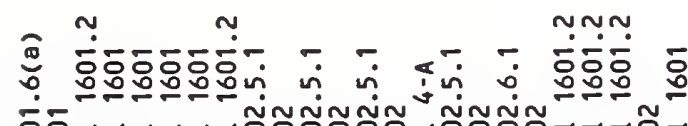

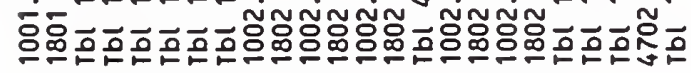

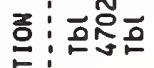

出:

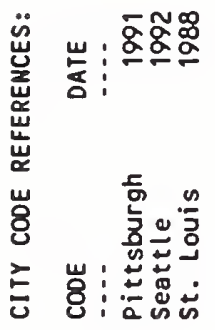

띨

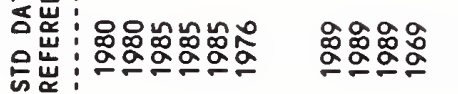

널 :

产

岩: 즌조농

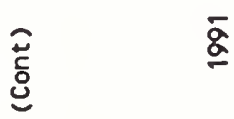

密

लิ
崖 $\sim$

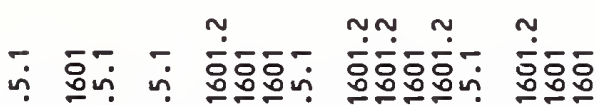

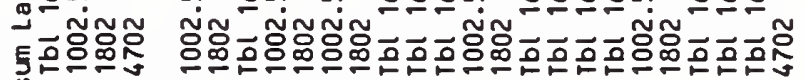

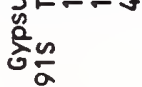

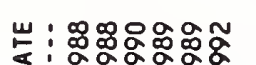

현 으의으으읭

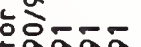

앙의영

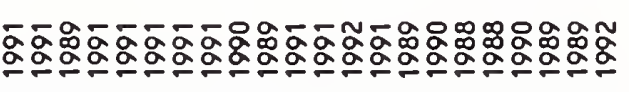

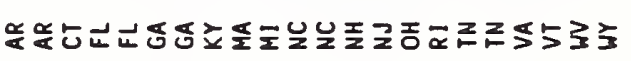

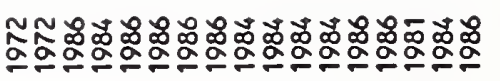

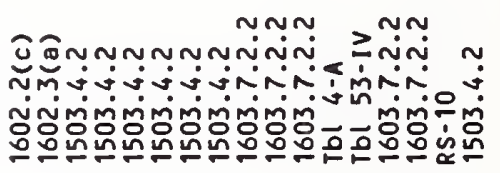

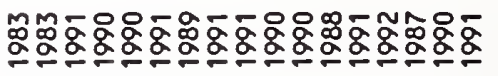

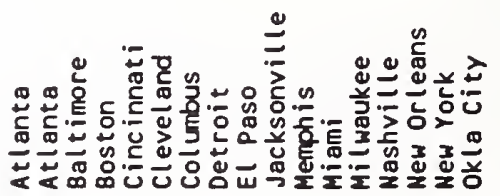

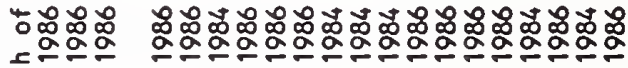

离

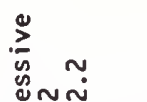

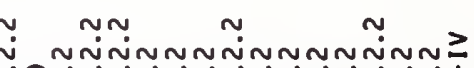

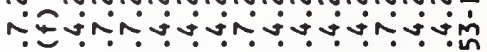

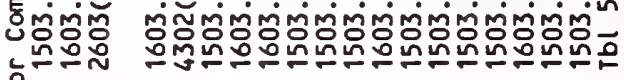
$8 \frac{\pi}{2}$

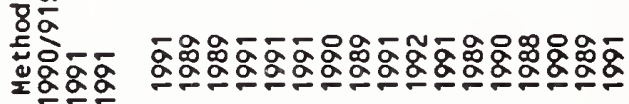
苟品品品

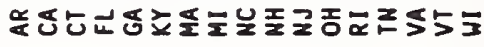




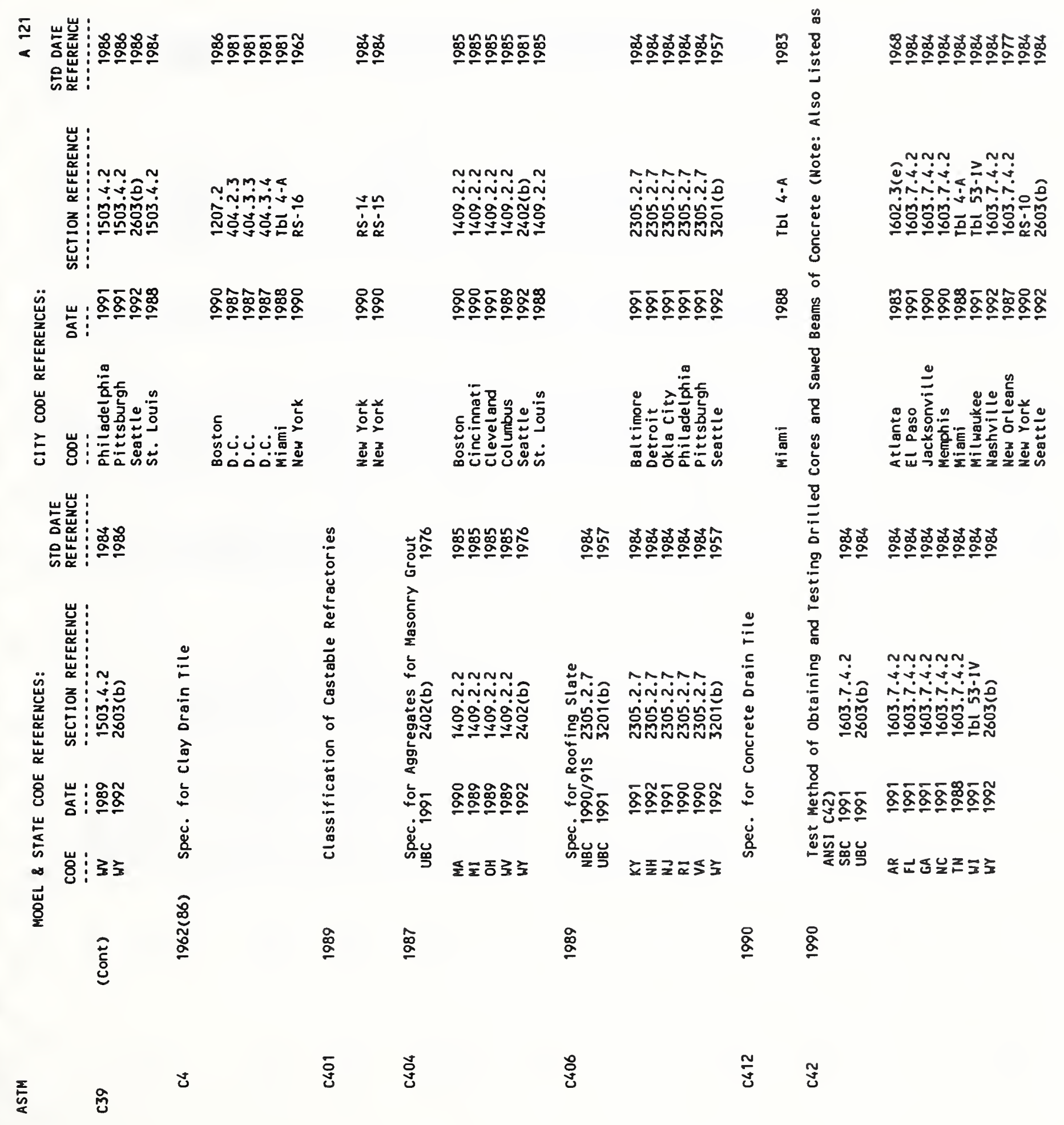




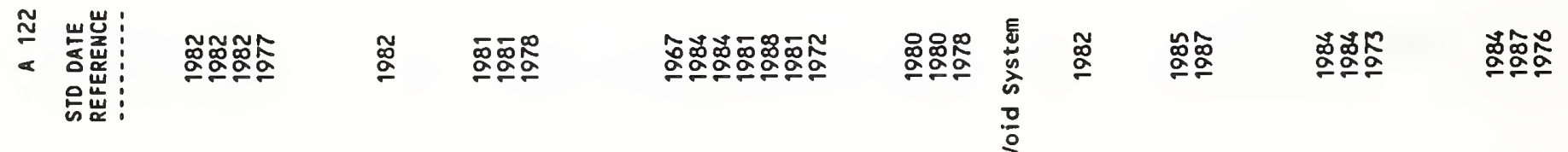

岂:

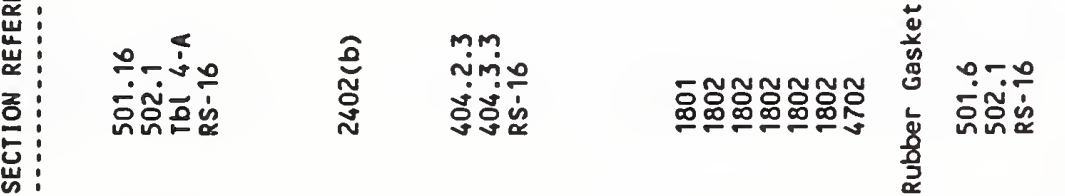

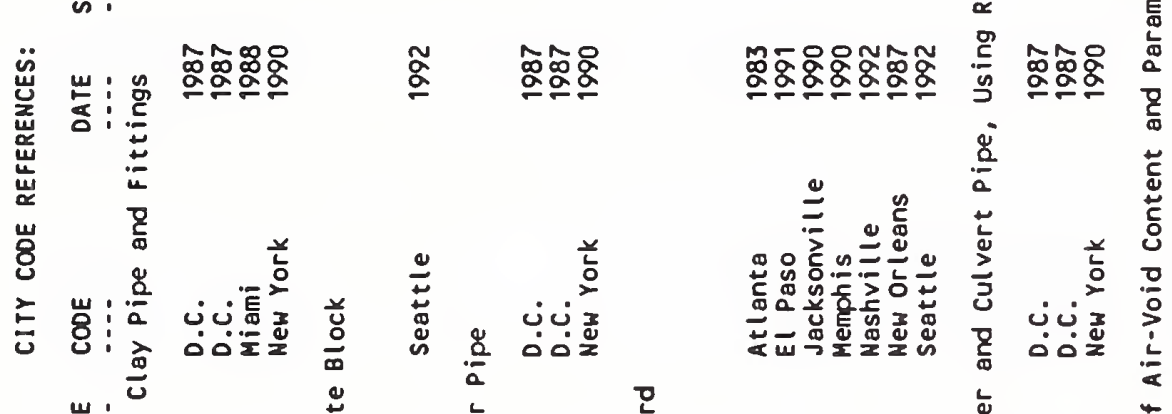

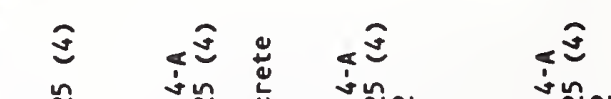

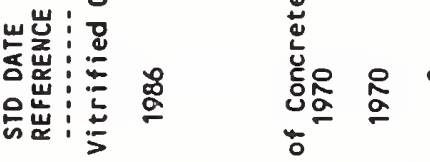

눙

\&

w :

空:

岕: ‘

종: $: \frac{1}{0}$ ?

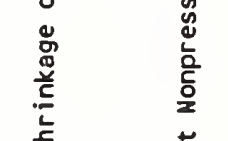

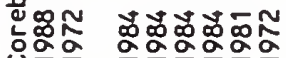

동

基

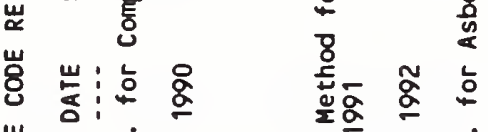

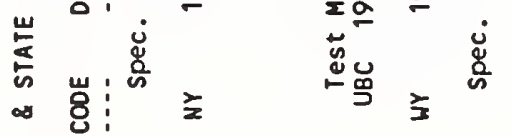

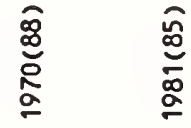

$\stackrel{\widetilde{్}}{0}$

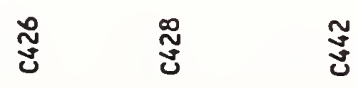

鱼

$\sqrt{\frac{3}{N}}$

$\underset{v}{5}$

I 


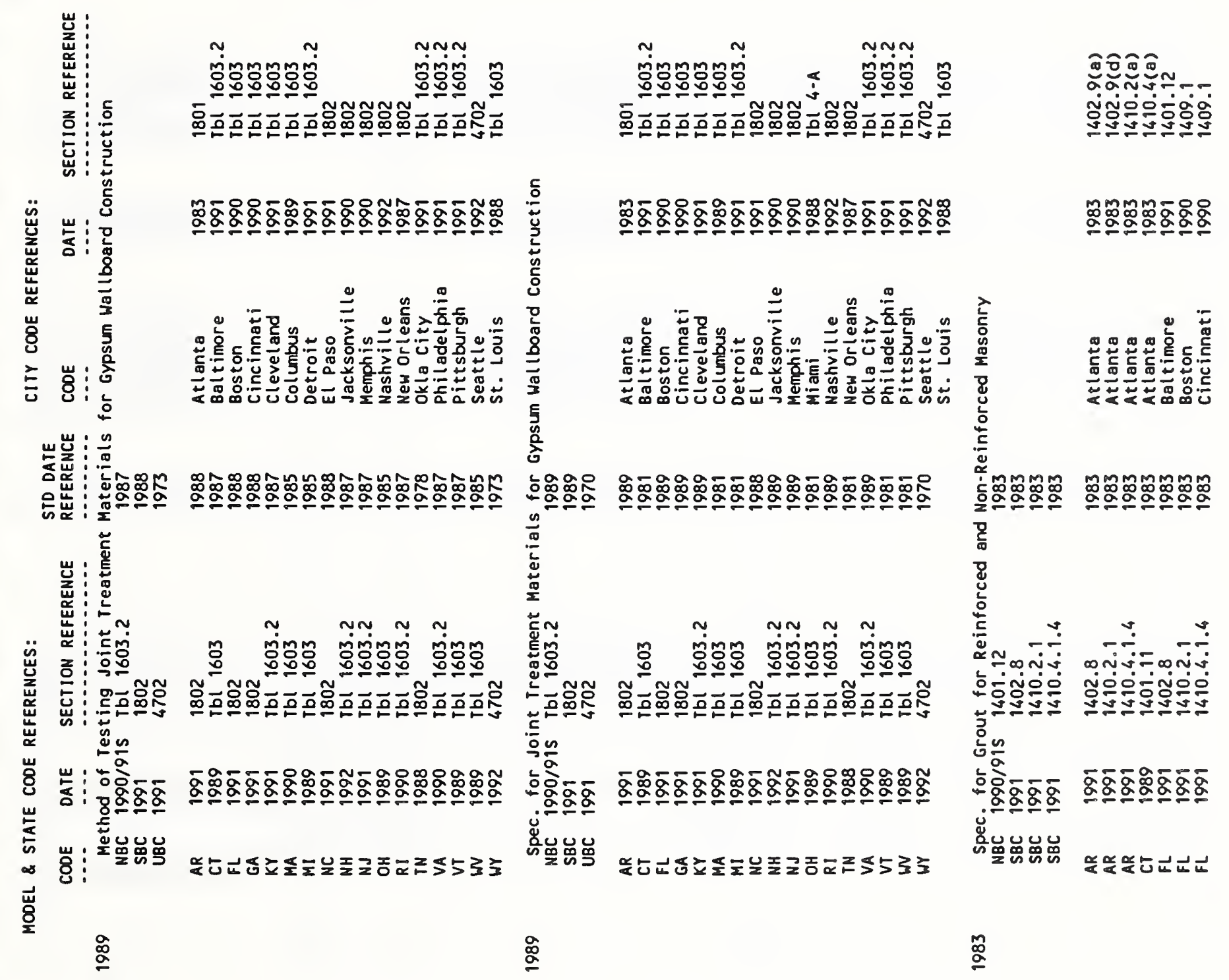


눈 战崖!
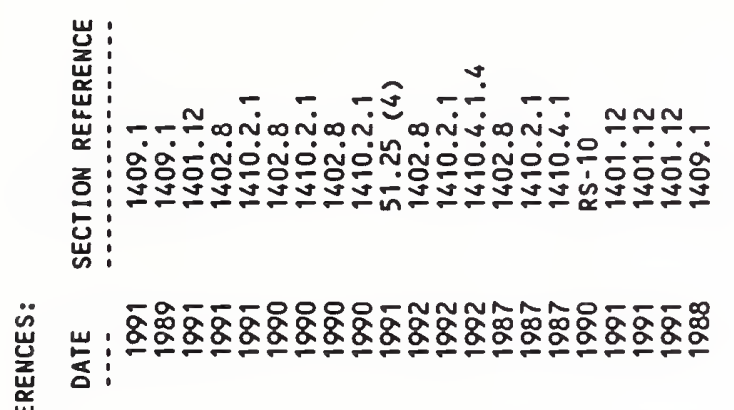

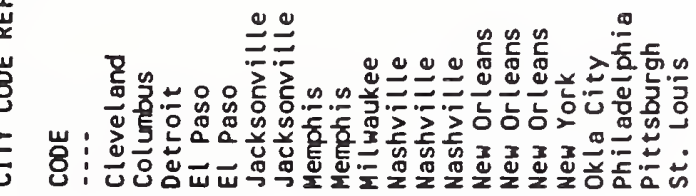

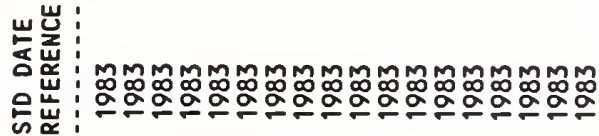

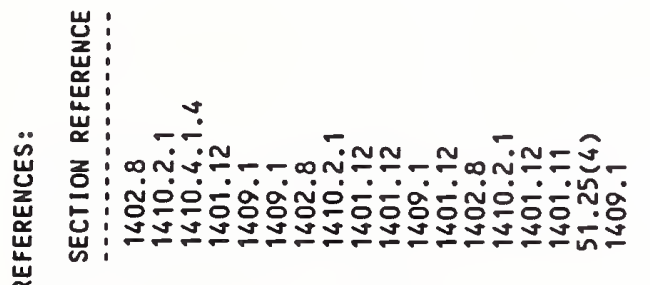

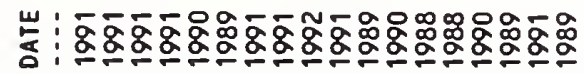

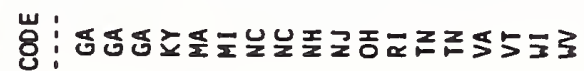

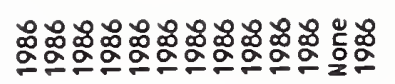

$\stackrel{n}{a}$

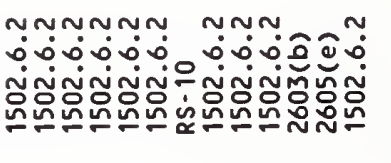

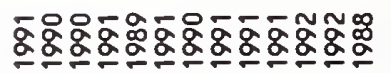

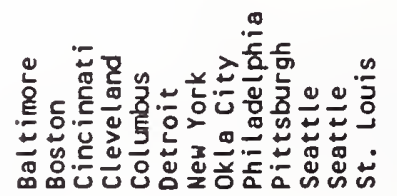

$\stackrel{\square}{\square}$

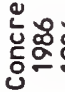

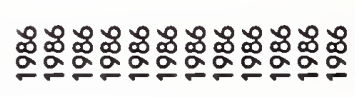

ᄒํ

峁

莫

ฮำำ

妾里

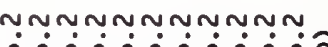

0000000000

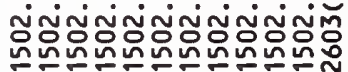

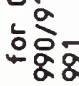
通品品

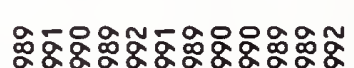

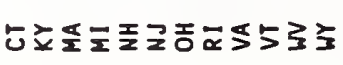

임

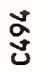

恣

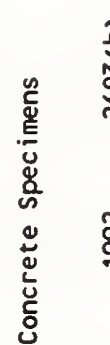

总

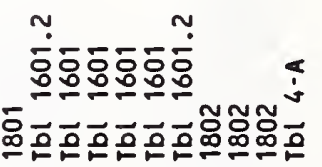

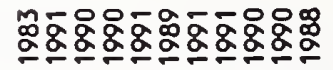

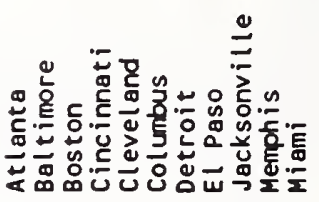

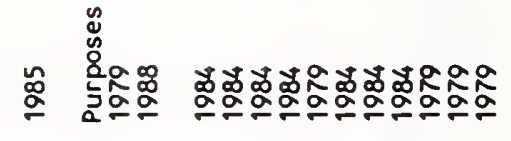
๘

$\stackrel{0}{2}$

里

劳总

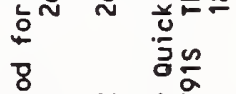

总宫

总

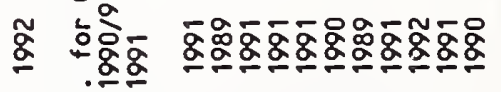

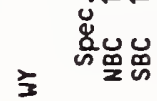

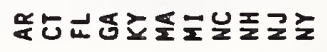

苍

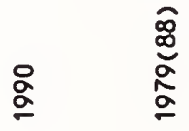

芠

ฯ 


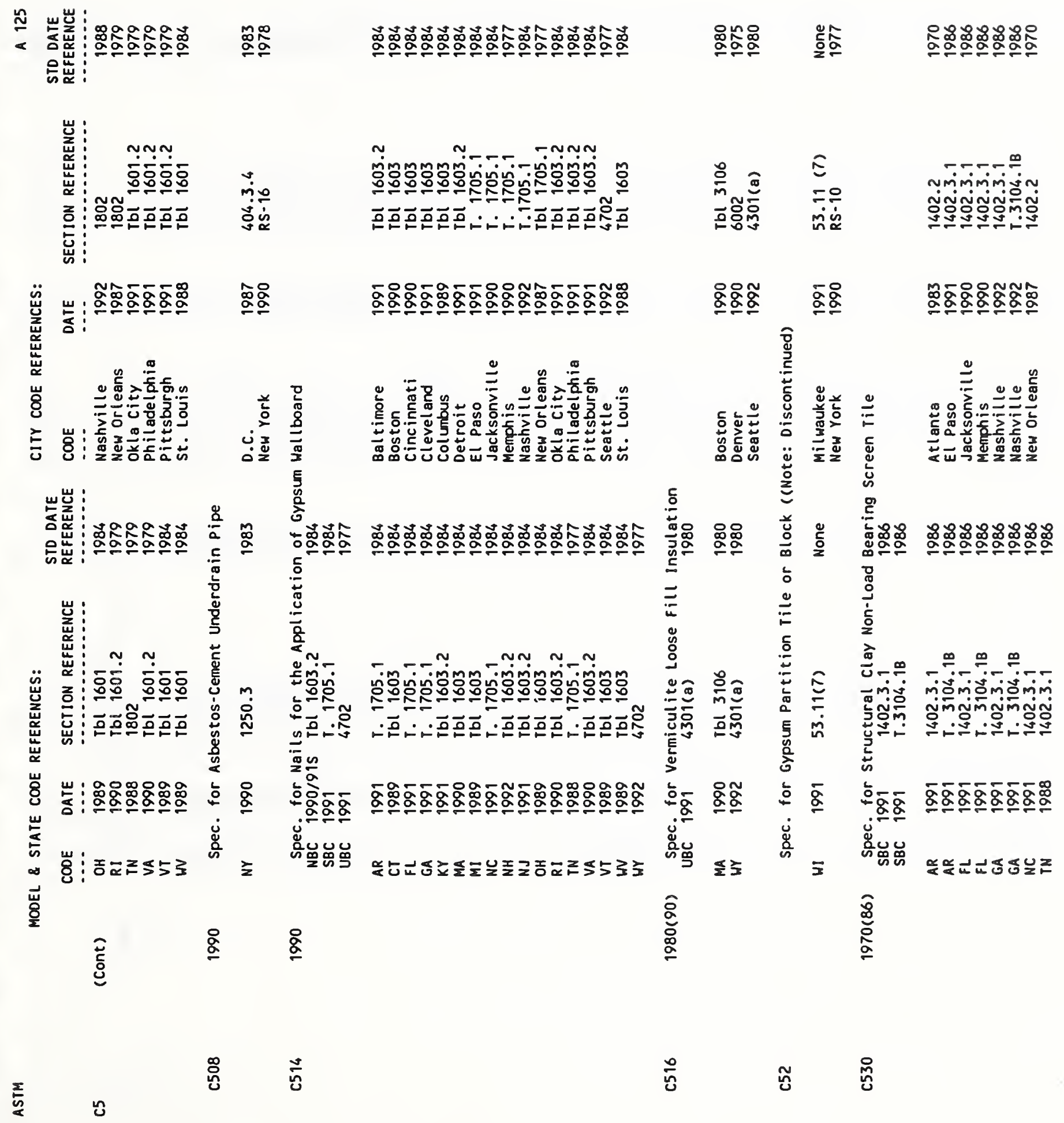




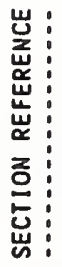

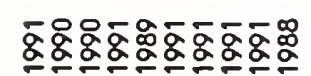

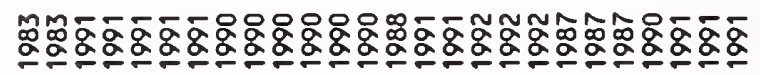

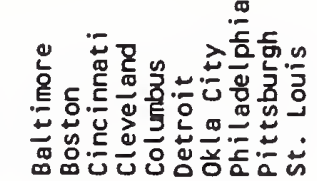

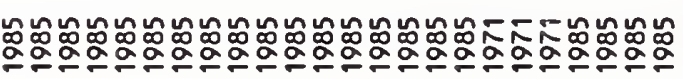

品

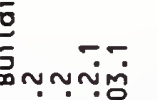

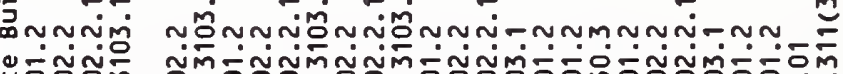

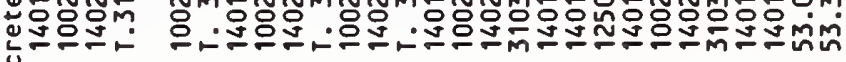
은<smiles>[CH]1CCCC2C=C12</smiles>

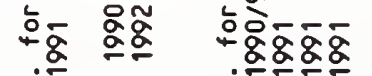

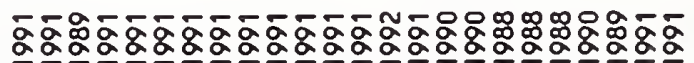
范㗞品品品

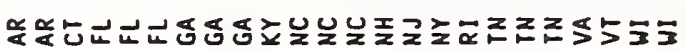
$\stackrel{\check{0}}{\underline{\underline{g}}}$

जิ $\tilde{ธ}$ 
^

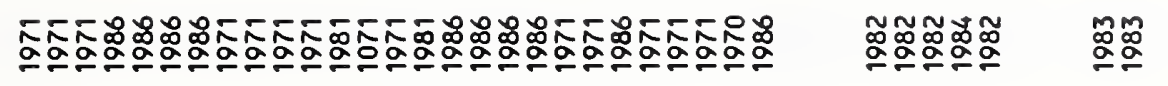

토옹

is $x$ :

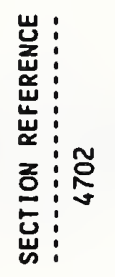

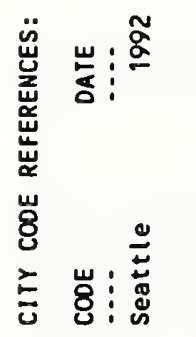

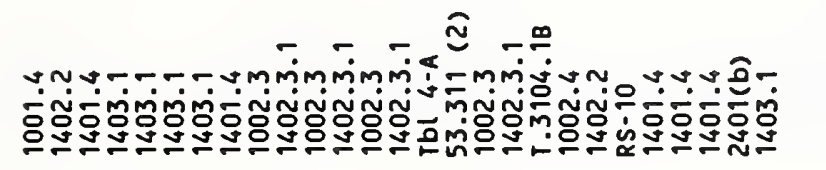

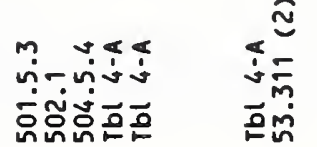

莕

\section{m-}

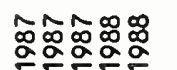

\&ूळ

ma:

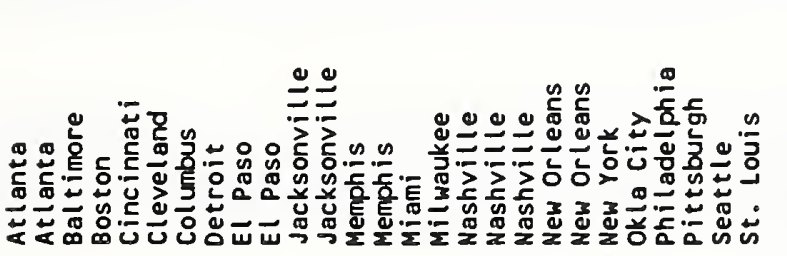

$\stackrel{2}{*}$

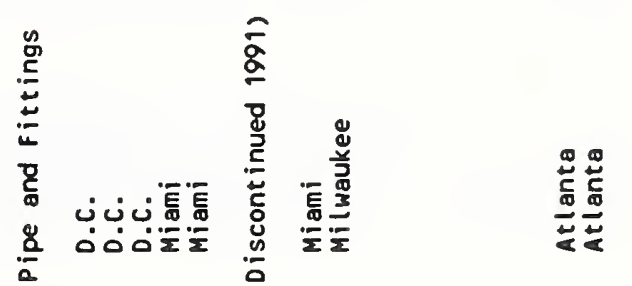

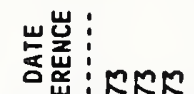

:

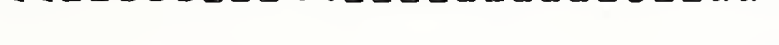

:

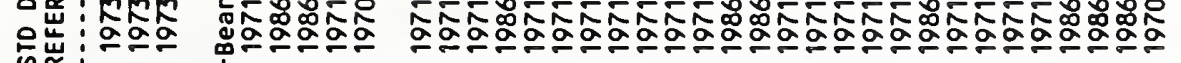

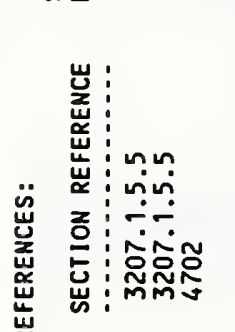

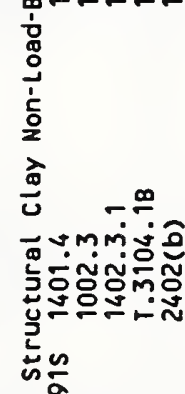

西

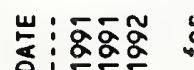

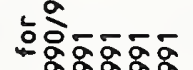

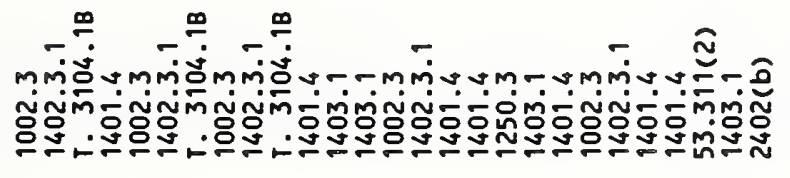

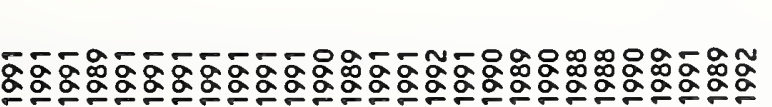

岁

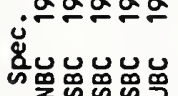

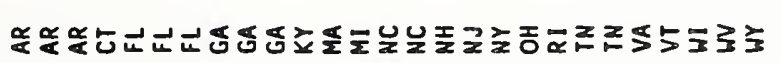

竞

怘

$\stackrel{2}{\circ}$

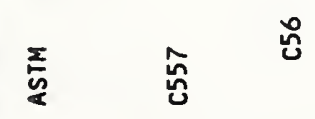

นั้

nิ 
๔ 온

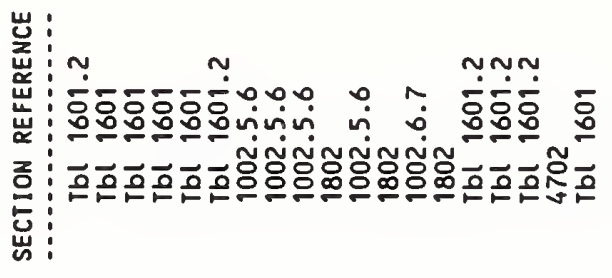

怘

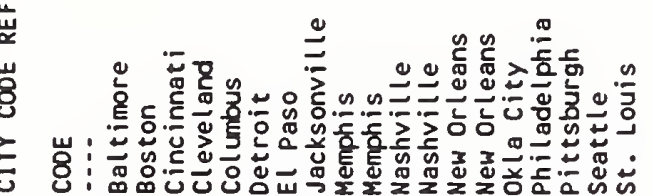

崇:

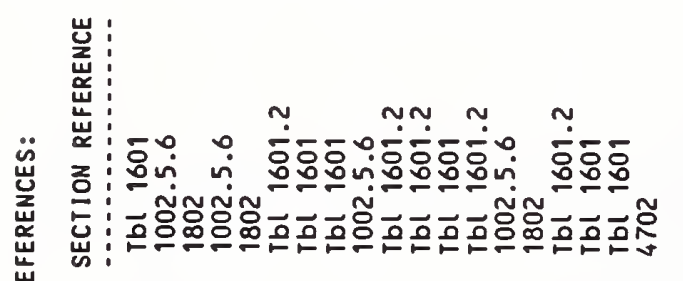

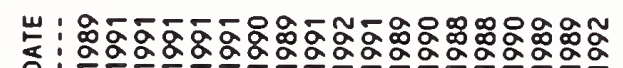

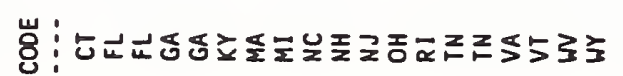

ํㅗㅇ

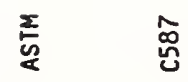

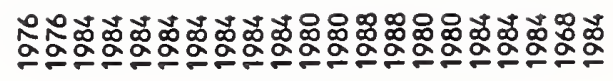

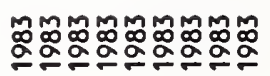

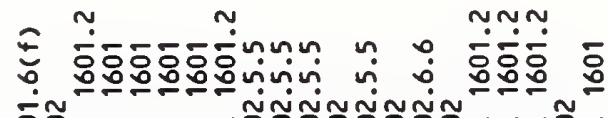

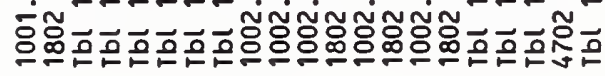

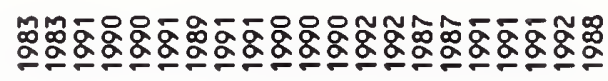

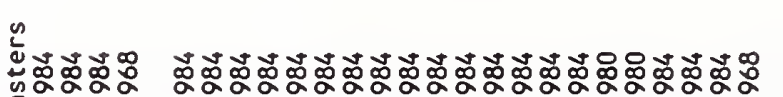
a

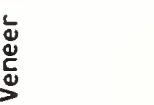

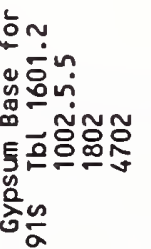

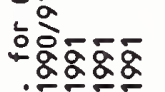

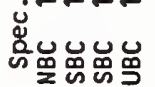

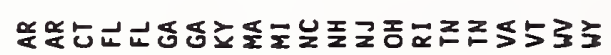

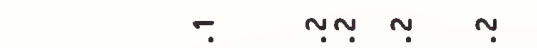

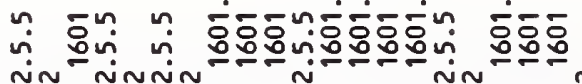

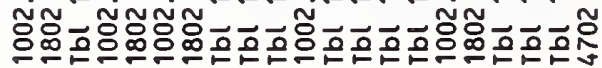

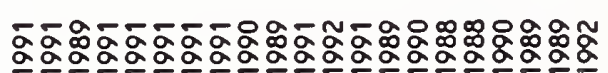
으.

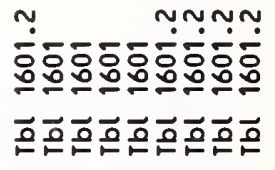

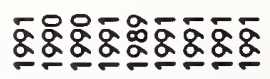

离

a

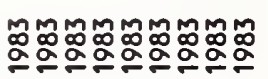

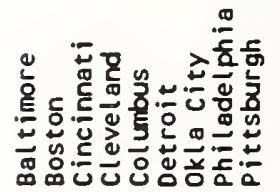
离 $\bar{\alpha}$

怘

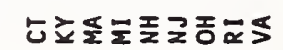

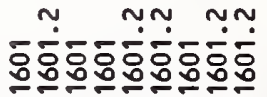

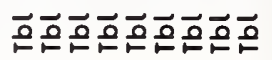

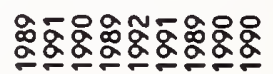

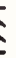




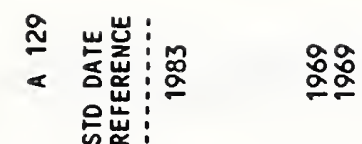

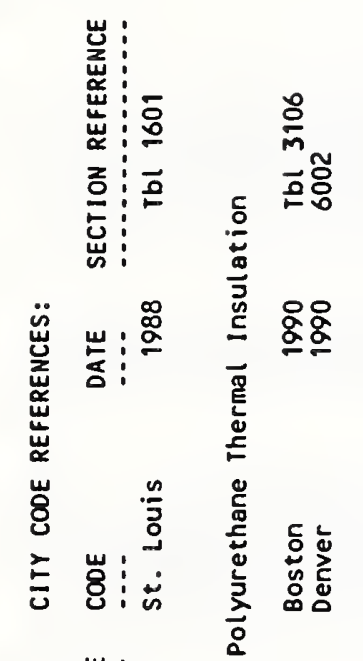

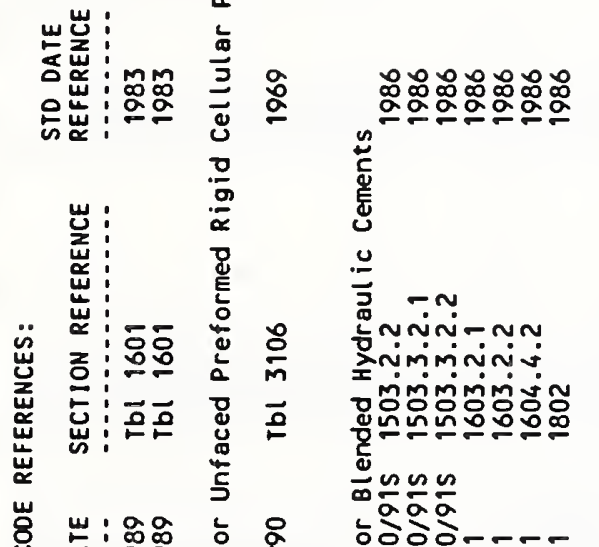

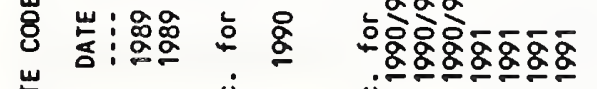

岕就

$\infty$

풀

잉:53 के ț:

\begin{abstract}
ron

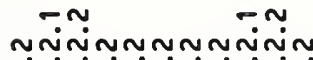

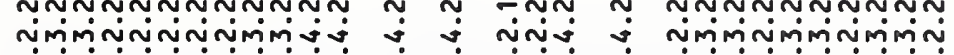

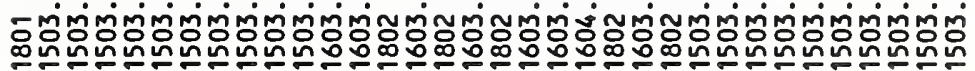

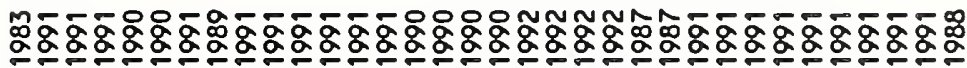

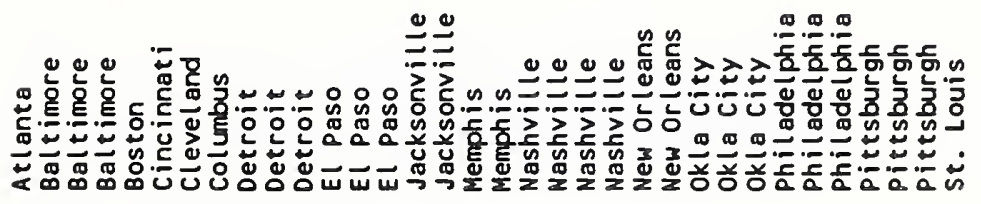

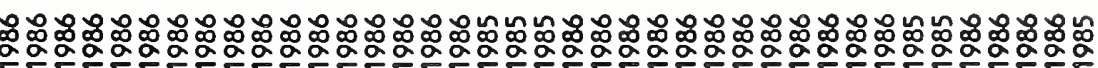

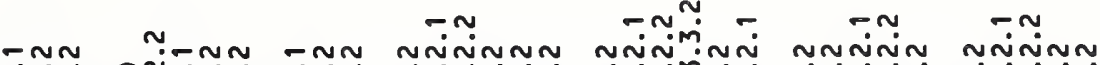

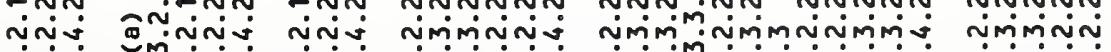

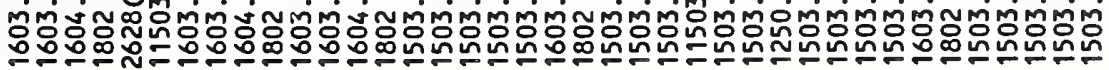

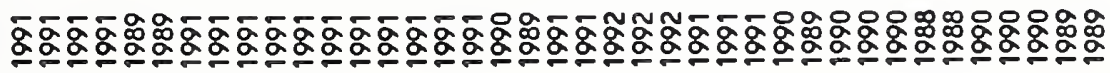

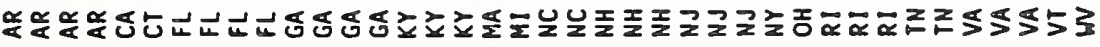

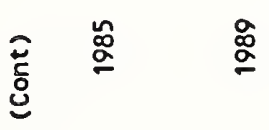

紊突

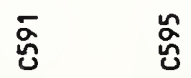




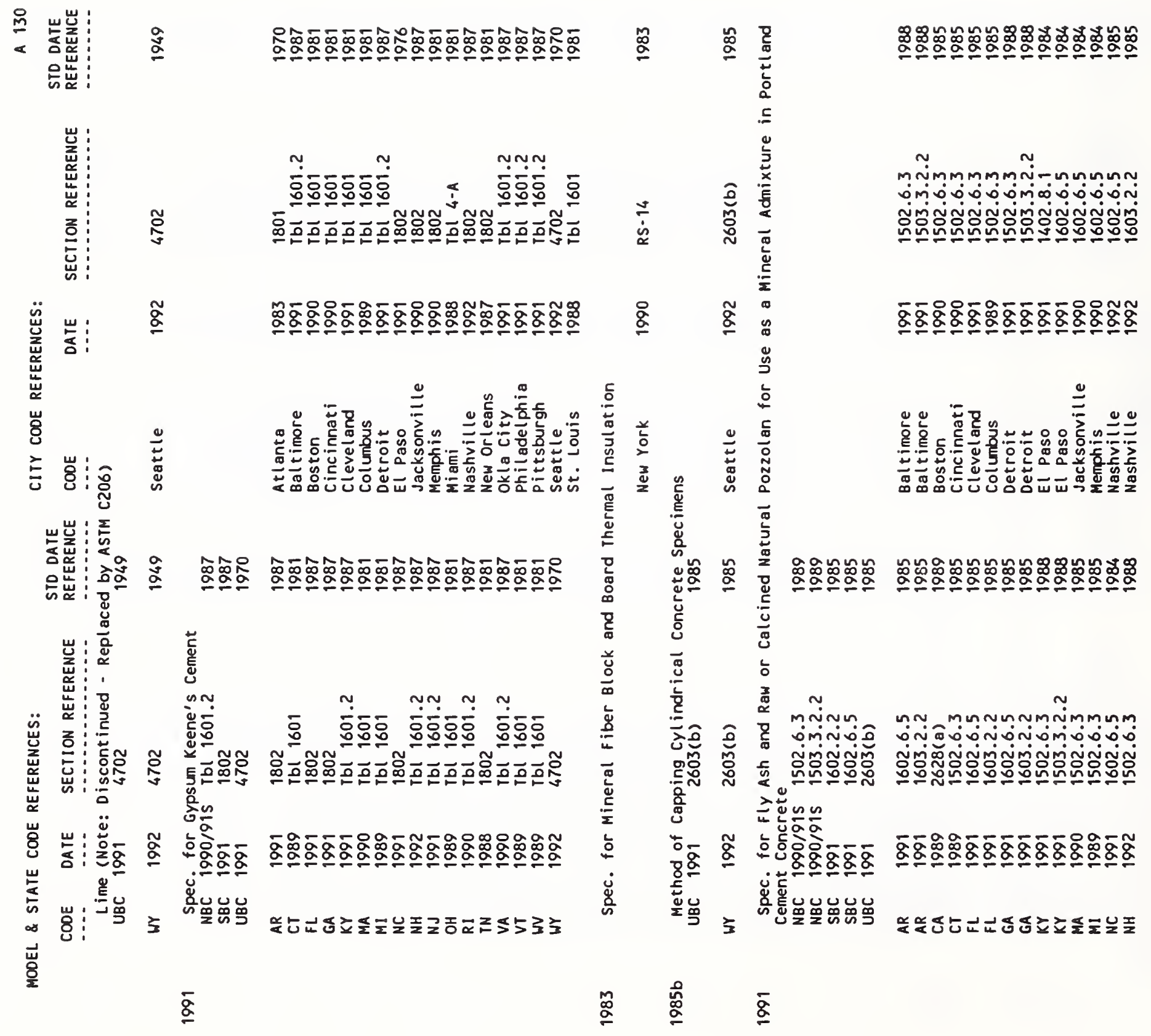


든 的㟧:

:

葆 $\Xi$ :

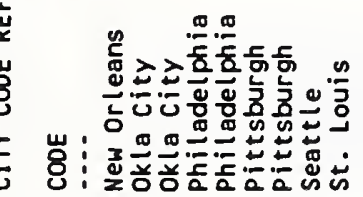

닐 :

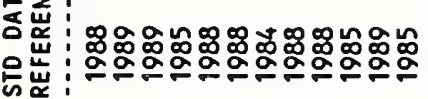

峁

热:

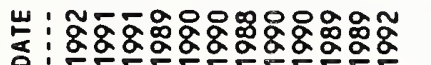

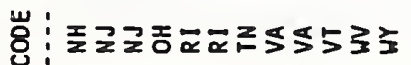

¿્ટ

$\sum \frac{\infty}{2}$

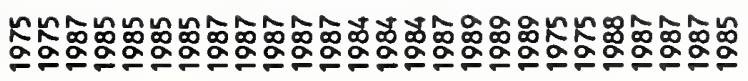

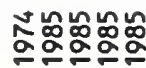

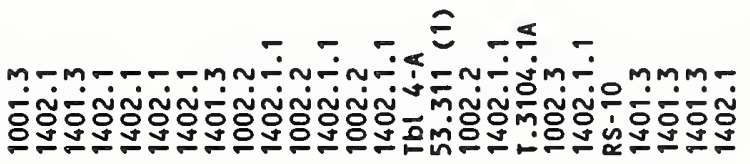

ํํㅇํำ

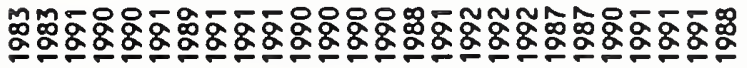

함ㅎㅇ용

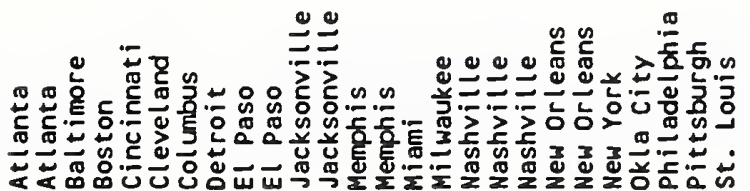

운

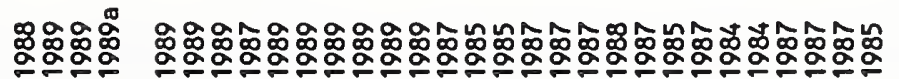

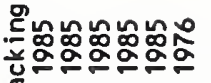

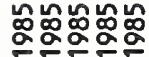

高

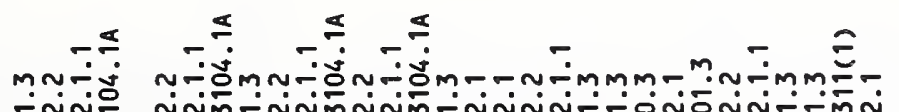

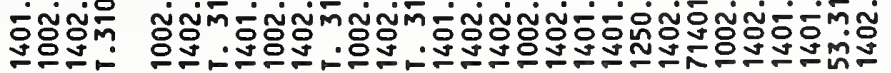

\section{$\frac{\infty}{2}$}

염염

象品品品品罟

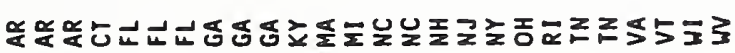

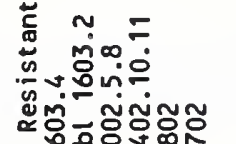

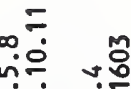

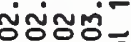

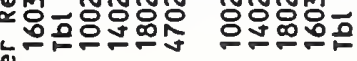
这 $\frac{\pi}{2} \frac{\infty}{a}$

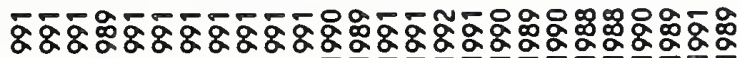

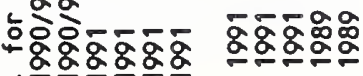
$\frac{\infty}{8}$ ชั 
㚇 容 䟻

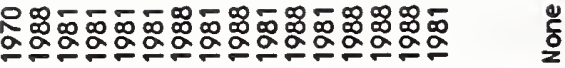

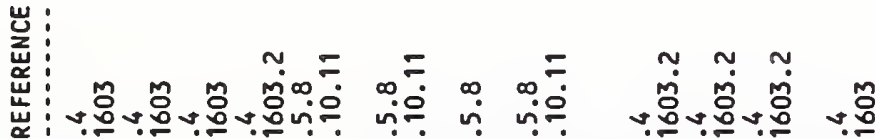

zo

岕!

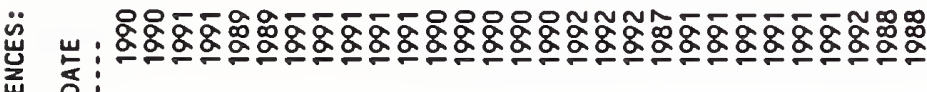

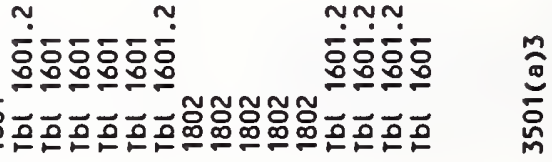

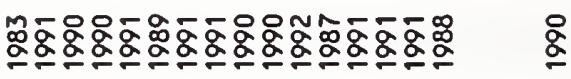

宸

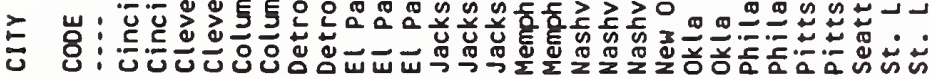

шU山 :

它芯

운

ü :

岂

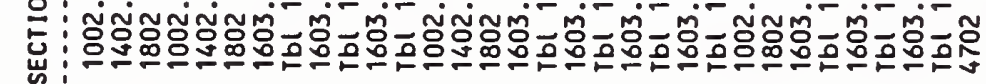

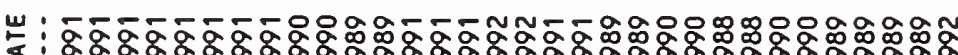

宸

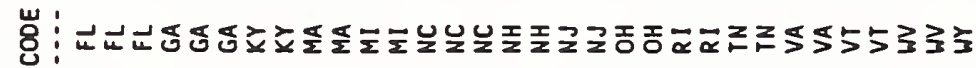

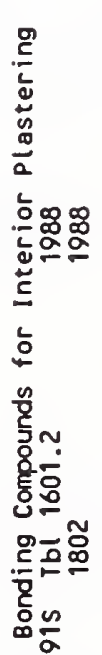

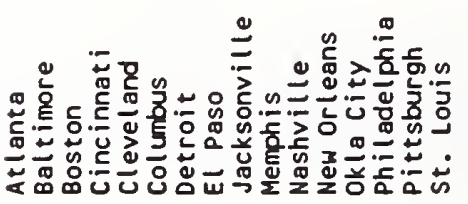

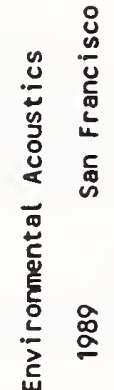

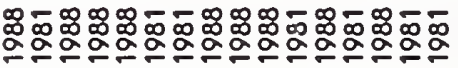

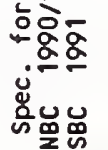

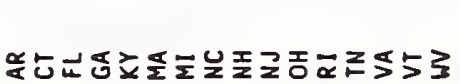

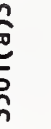

\section{(1)}

(n)

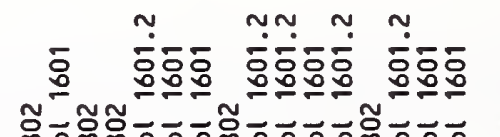

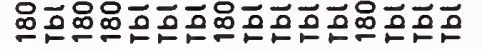

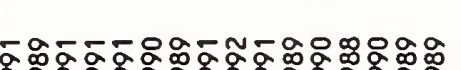

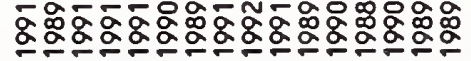

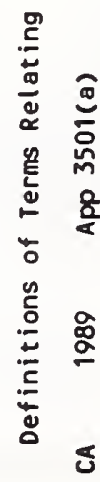

:

$\stackrel{\mathscr{8}}{\stackrel{8}{\square}}$

$\stackrel{\mathscr{g}}{\mathrm{g}}$ 


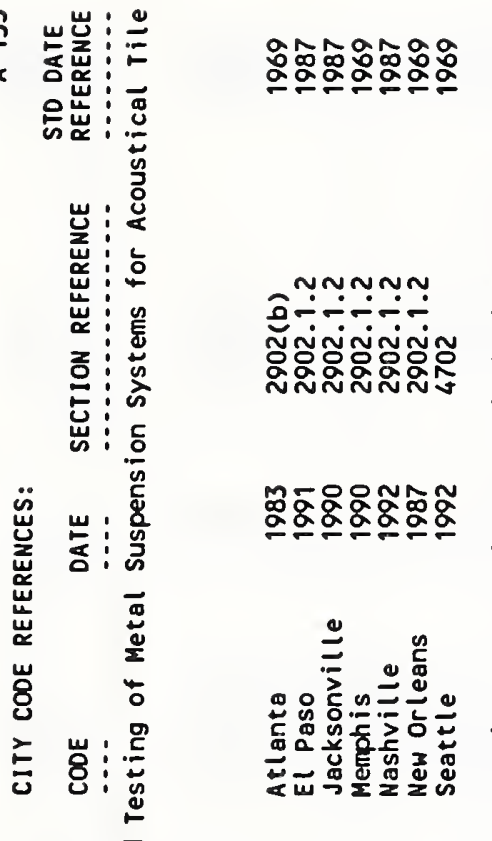

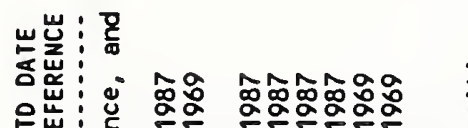

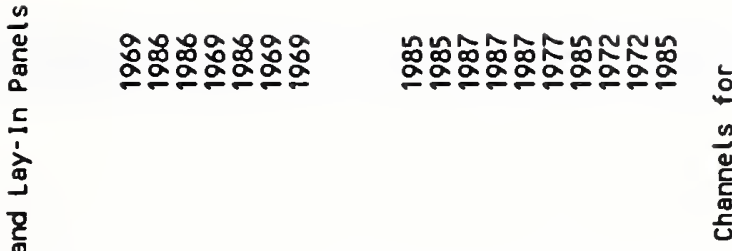

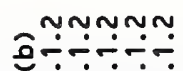

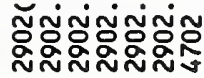

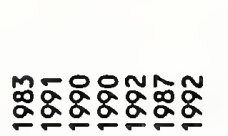

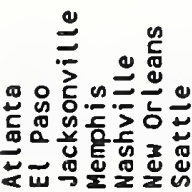

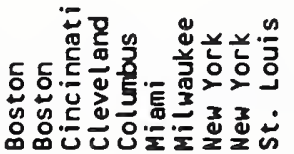

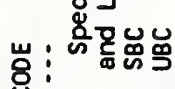

ஷேす比るう

$\bar{\alpha}$

药

ณั.

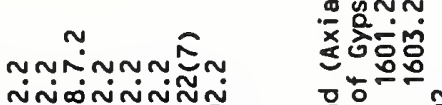

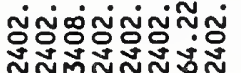

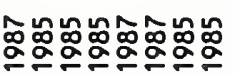

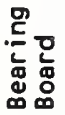

密

ง

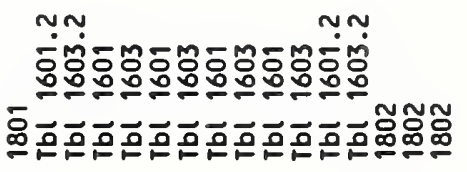

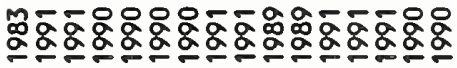

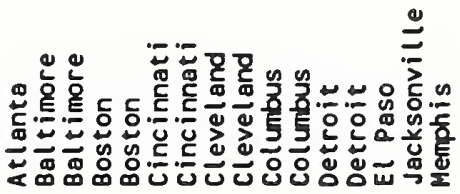

:

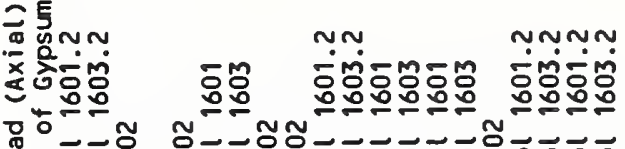

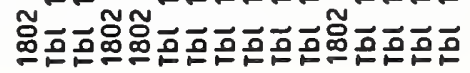

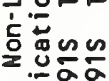

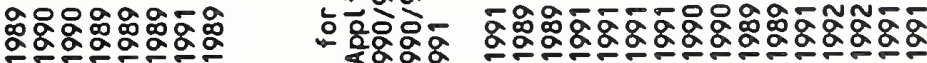
ن

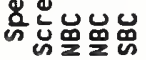

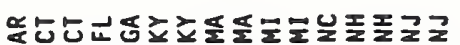




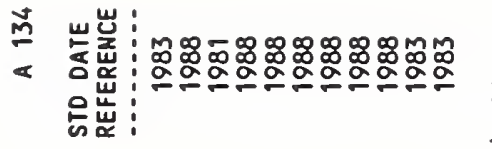

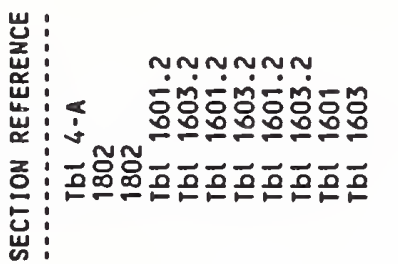

芦

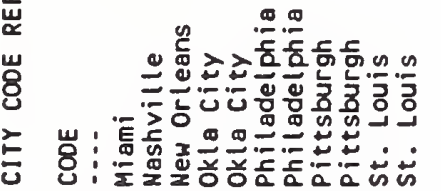

ш 는 :



눈

岂

บก ก บ

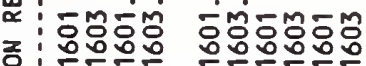

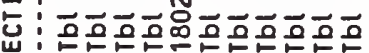

世

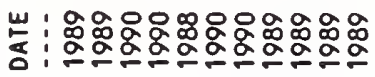

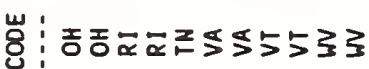

룰

કัธ

ए

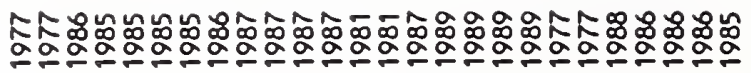

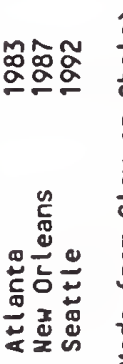

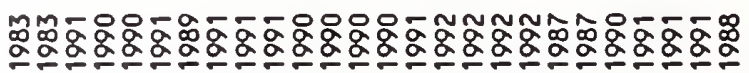

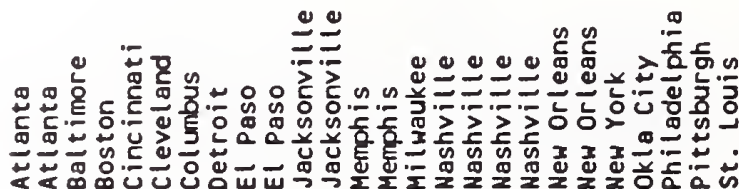

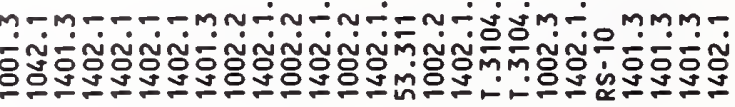

$\frac{8}{m}$

$\stackrel{0}{\sim}$

\&

ᄃ્

$\stackrel{\infty}{\stackrel{\infty}{\alpha}}$

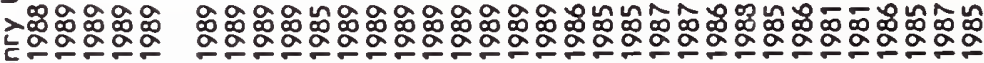
愛

$\stackrel{\text { I }}{\underline{0}}$

-

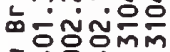

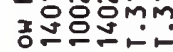

옹

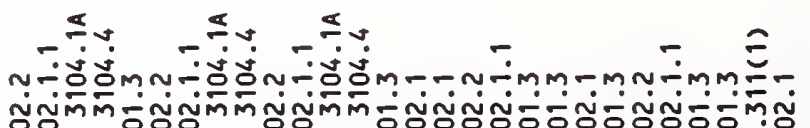

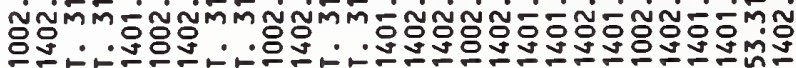

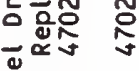
is g 뉸.

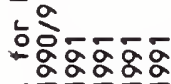

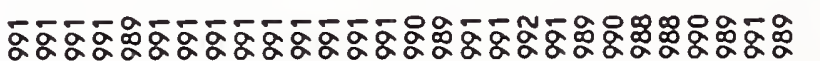

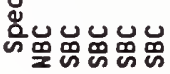

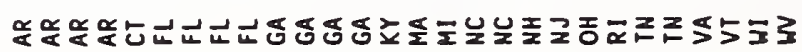
$\frac{\alpha}{2}$ 总品品 立 


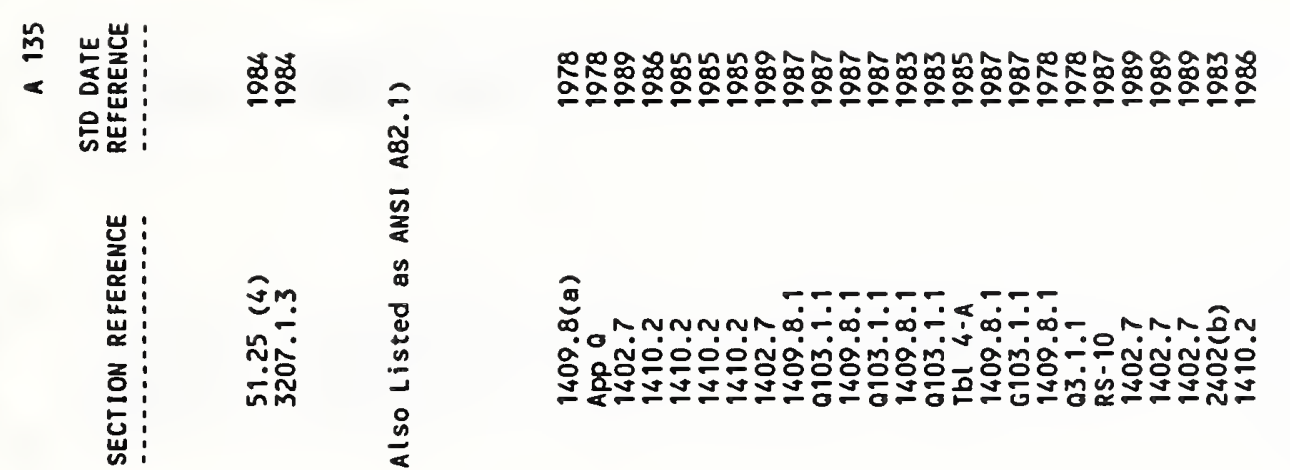

䓛

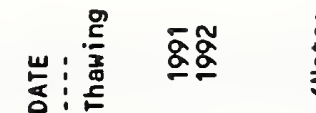

0

岁

它



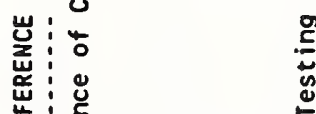

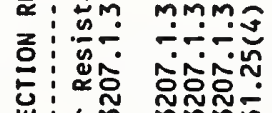

岃: ‘

แ :

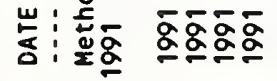

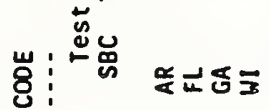

요용

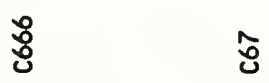

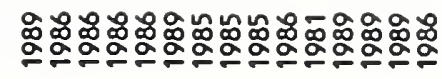

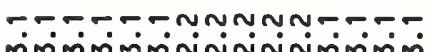
نें

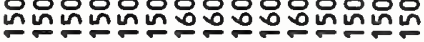

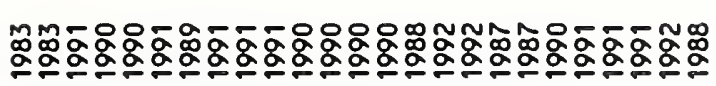

$\frac{\text { 它 }}{\frac{x}{2}}$

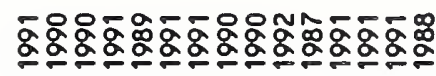

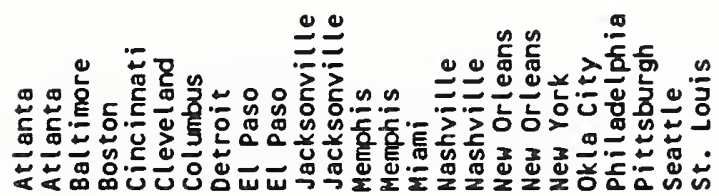

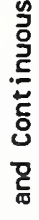

量

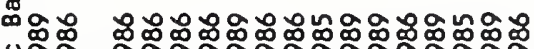

ㄴ.

$\frac{2}{5}$

ลे

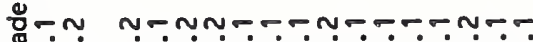
玉min nininimiminimiminimim ะป่

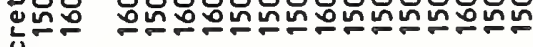
는

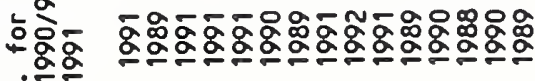
岁诘品

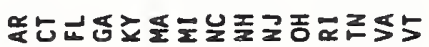




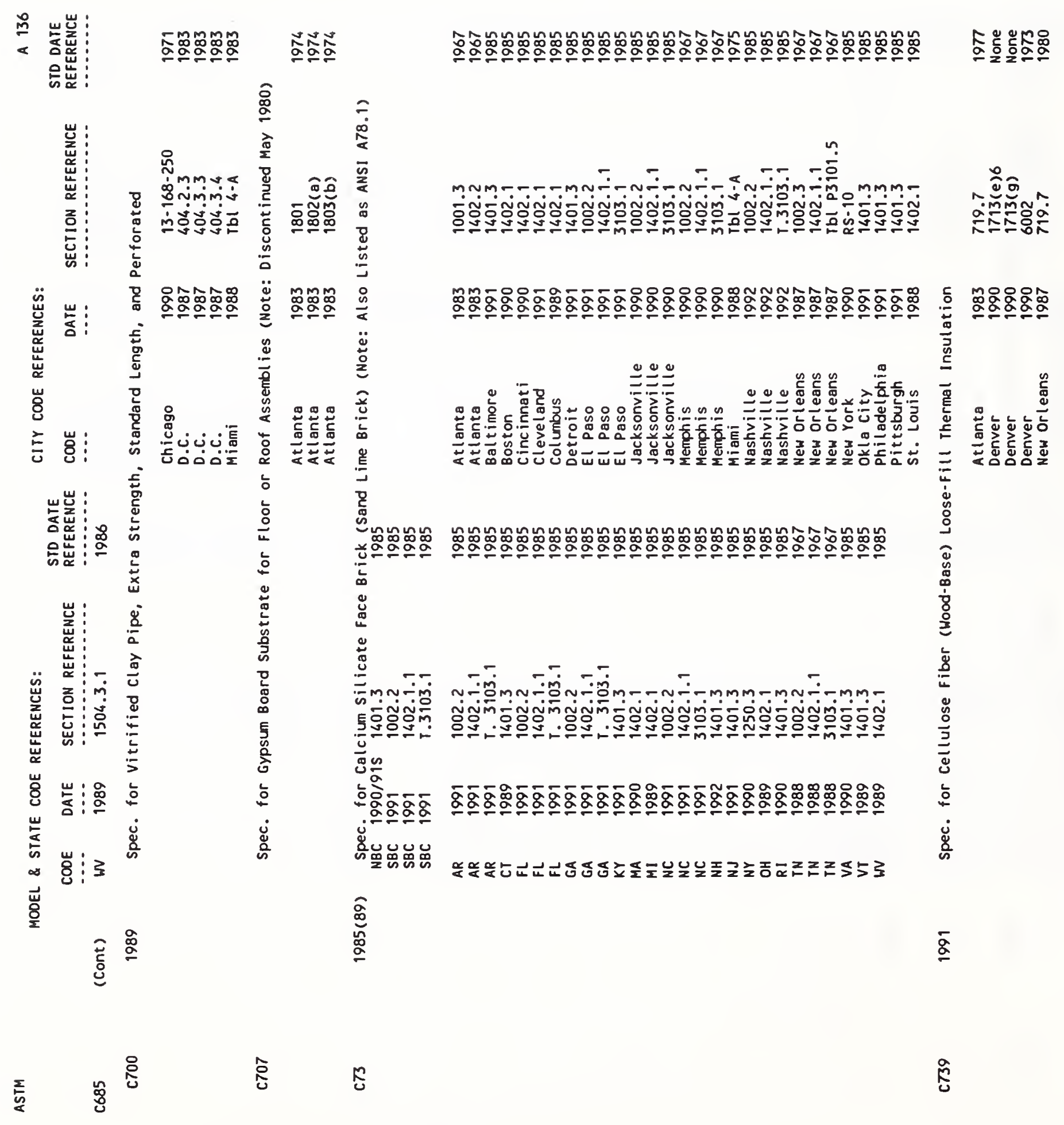




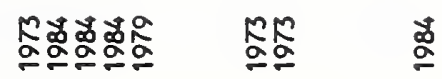

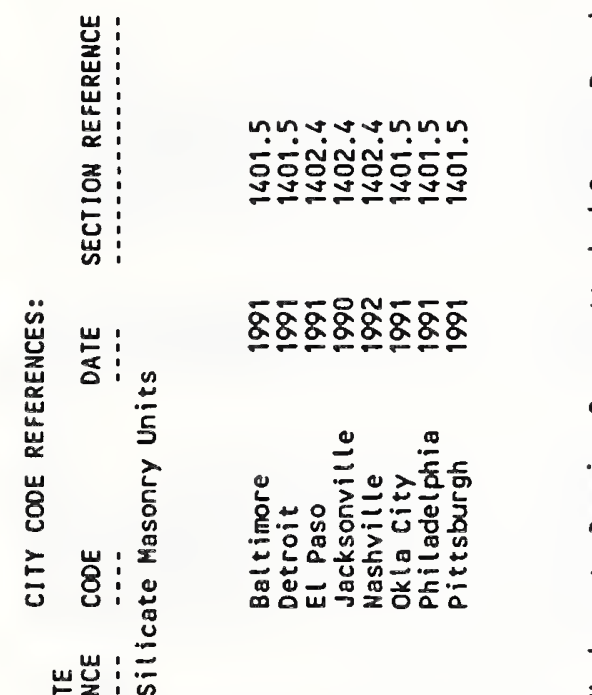

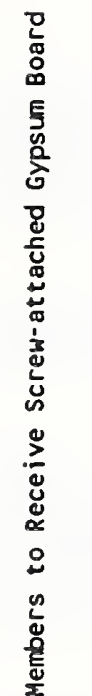

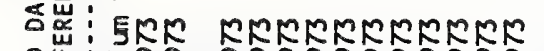

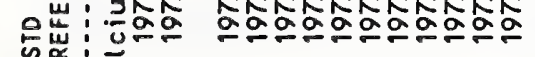

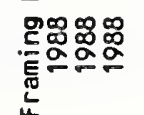

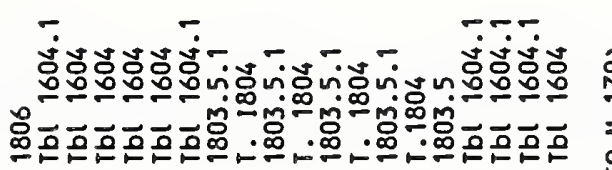

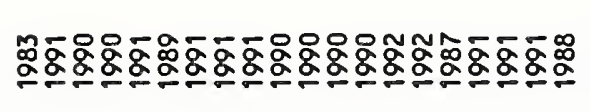

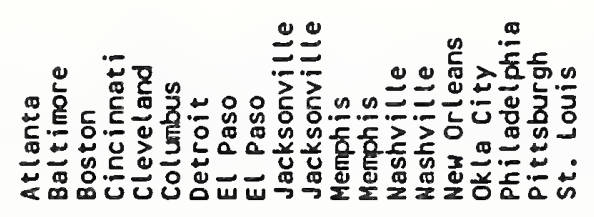

జజ్

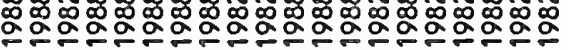

离

ড-

ㄷำ

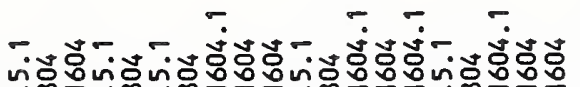

in

㟧 : 总

岕

o

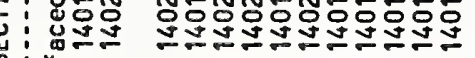

à

ठัّ

崖

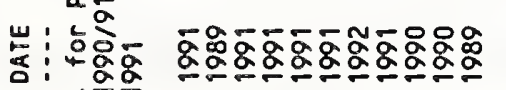
당

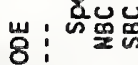

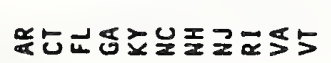

命

$\stackrel{\mathscr{0}}{2}$

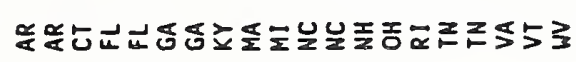

$\frac{0}{3}$

किmiñ

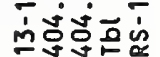

号

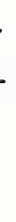

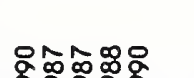

箸

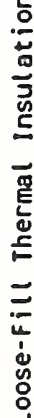

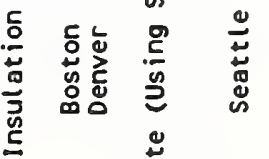

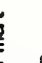

א

E

范

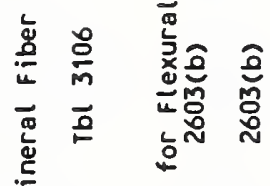

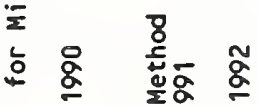

这

峛

$\leqq$

密

亏

이효

ธุ

ปั่ 


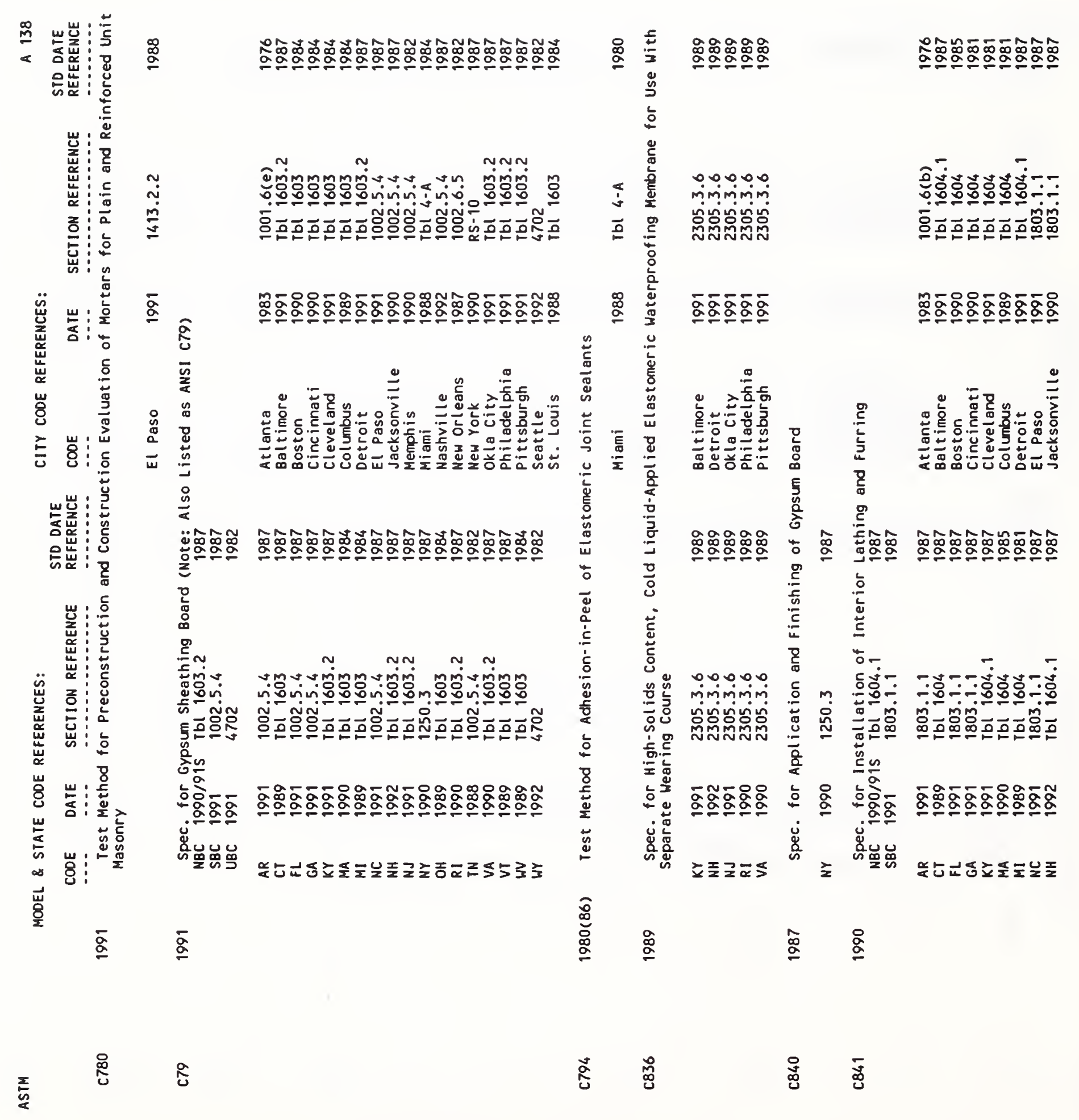




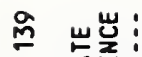

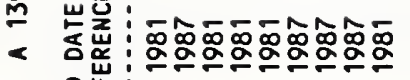
题岕:

㟧 :

勇 :

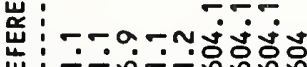

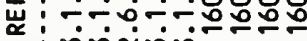

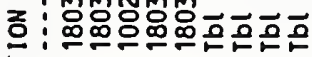

出:

莩

ర్ర

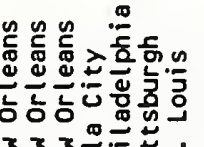

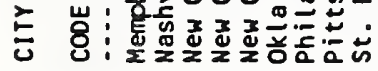

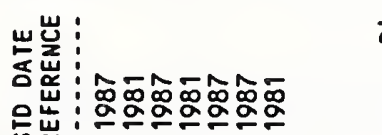

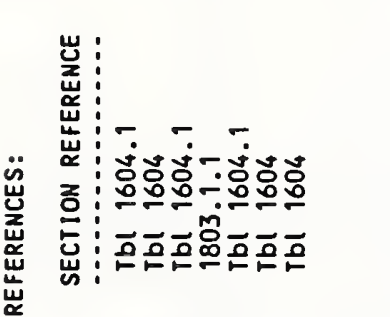

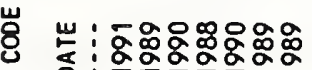

崖

↔ 岂: :

일

空

జัฒ

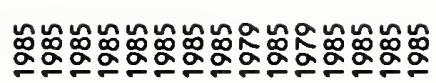

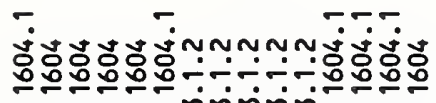

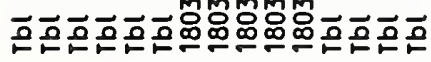

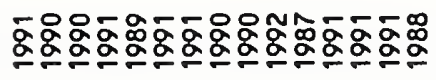

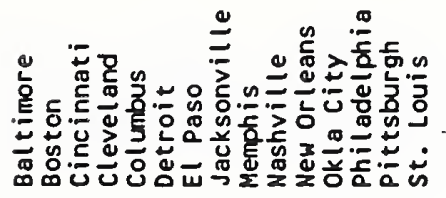

离

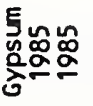

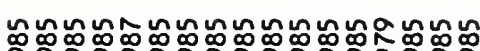

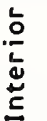

ํำำ

뭉

这皆

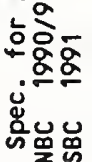

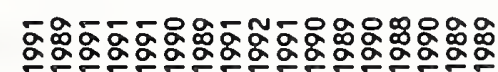

\%

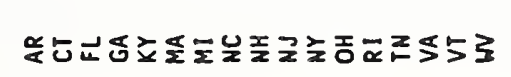

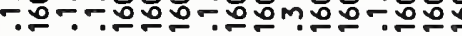

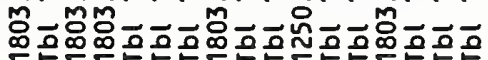

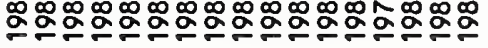

离

焉

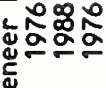

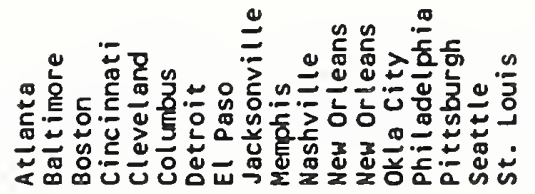

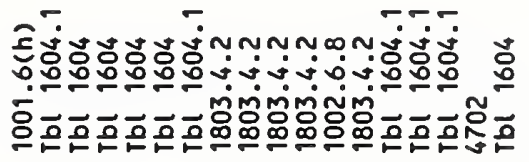

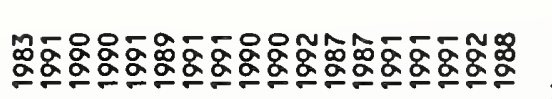

离

芯

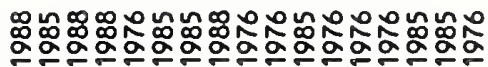

点

กับ

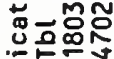

후음

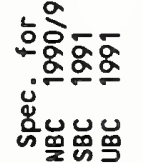

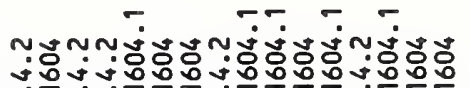

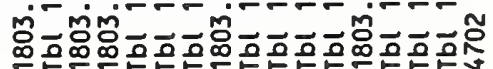

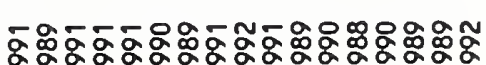

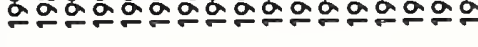

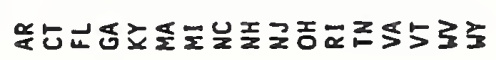

命

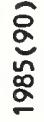

总

点 总 


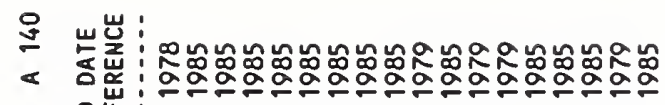
演:

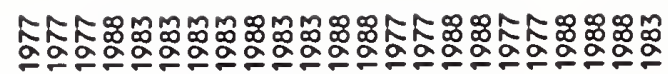

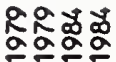

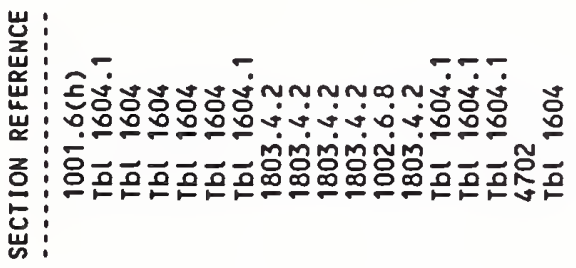

莖ㄴ

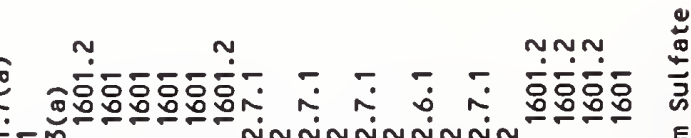

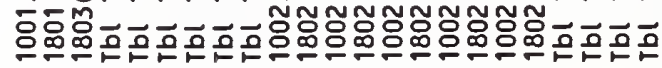

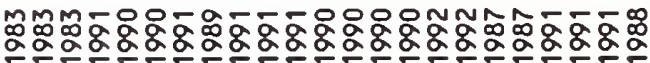

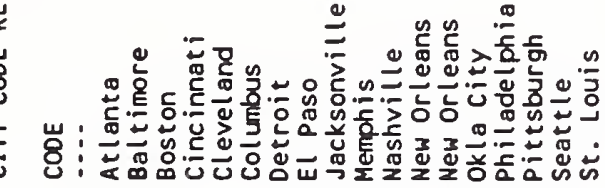

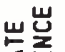

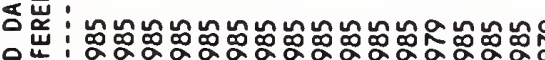

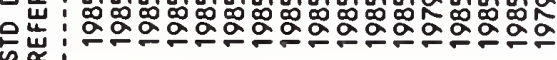

\$ू్

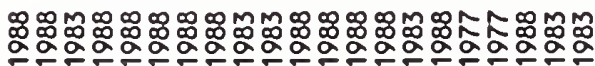

岀

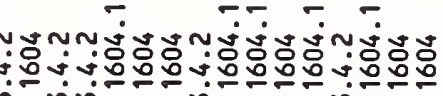

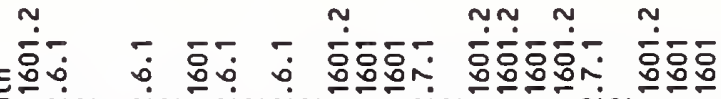

总:

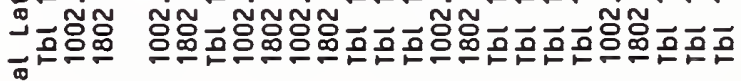

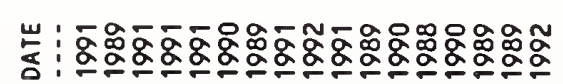

产我

畜

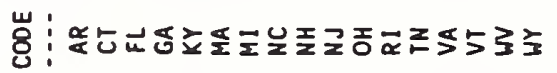
亭京京

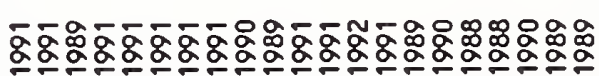

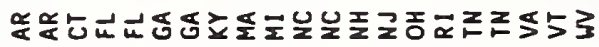

동

\&

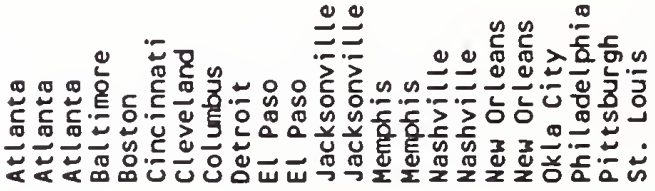

i

minn

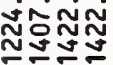

홓ㅇㅇㅇㅇㅣ

욜

$\stackrel{\Phi}{\vec{B}}$

$\frac{5}{8}$

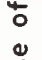

芩

合

m

$\stackrel{0}{2}$

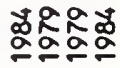

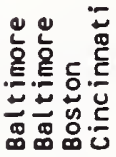

政

$\stackrel{\sim}{\dddot{n}}$ กminn ูํํำำ

焉

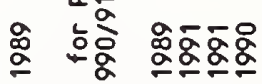
宛

ヒ立立乐

훙

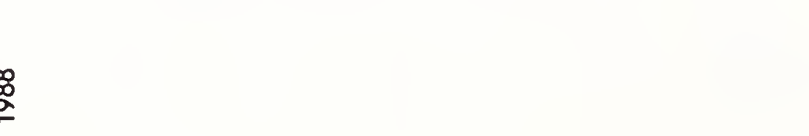

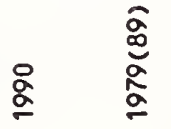

ఫ్ర

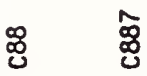

紊哥 
$\bar{\Xi}$

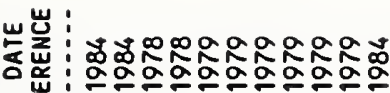
옳

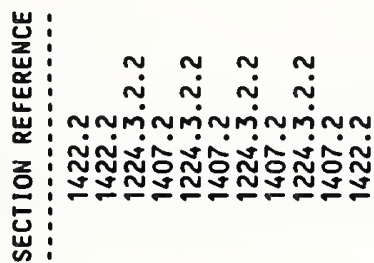

芦

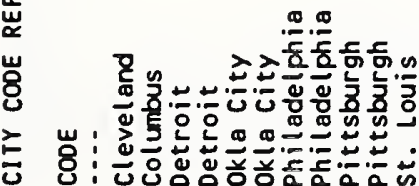

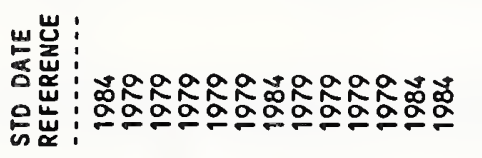

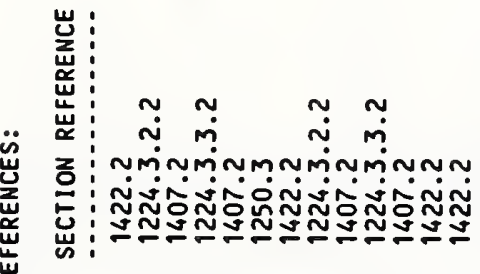

mi

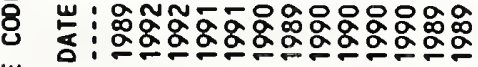

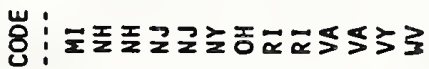

芒

$\underset{\frac{5}{0}}{\stackrel{0}{8}}$

๙ิ̊

音

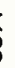

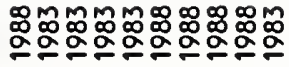

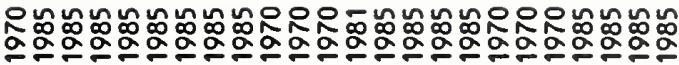

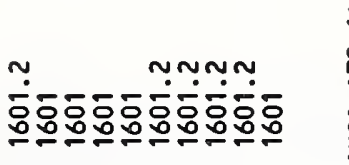

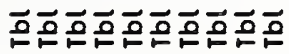

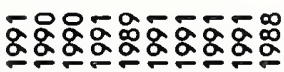

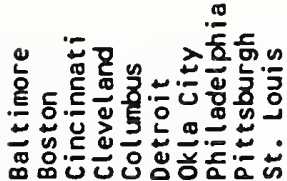

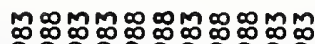

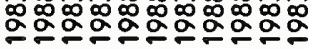

มñ

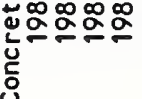

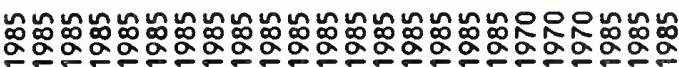

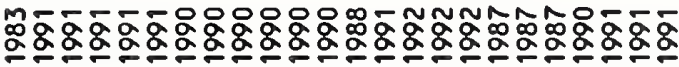

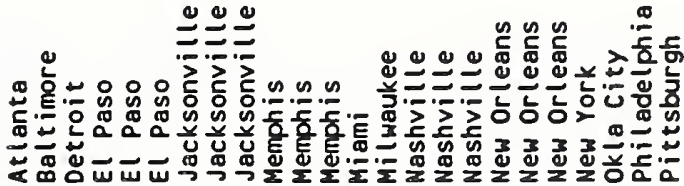

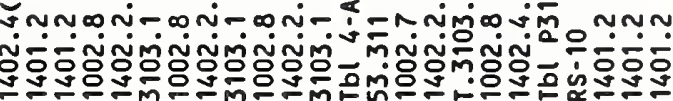

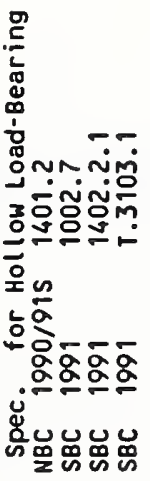

Бัตั

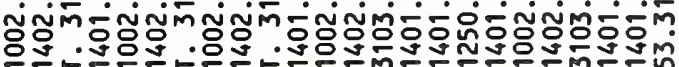

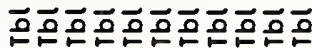

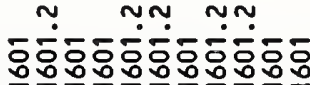

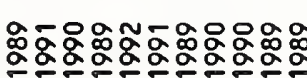

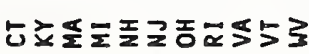

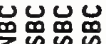

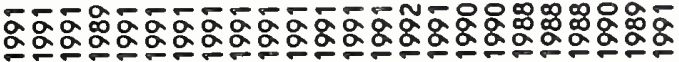

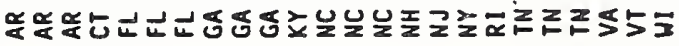

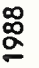

$\stackrel{8}{\circ}$

웅 


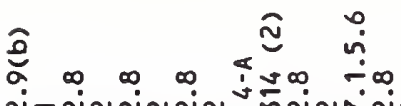
กัธ్์

m-m-m-m-m

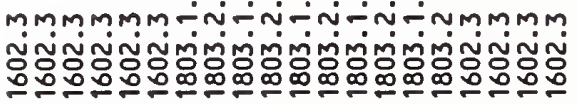

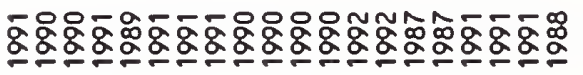

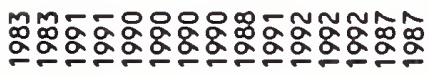

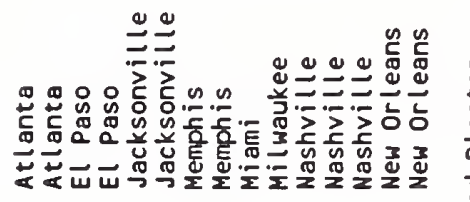

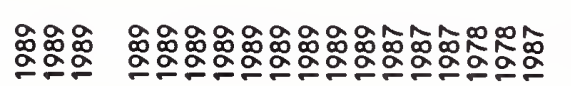

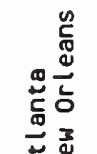

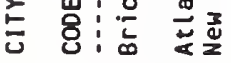

崖:

崖: 䓂

:

㱏:

즈: 도

는

出: :

崖京

西

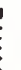

山्य

ơ

항

$\stackrel{\bar{\alpha}}{\circ}$

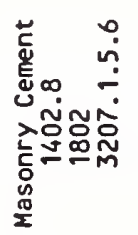

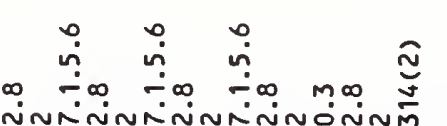

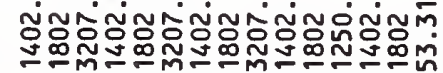

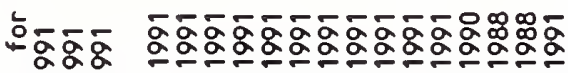

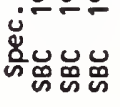

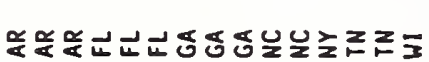

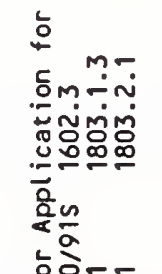

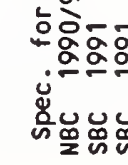

m-im-m-m-

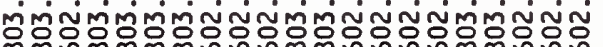

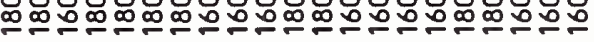

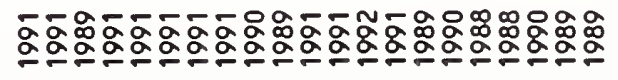

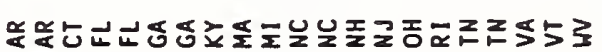

\&

紊

ธั

$\overline{8}$

๕̊
ळ

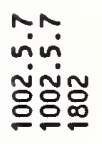

홍요

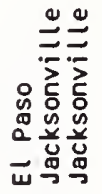

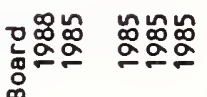

范

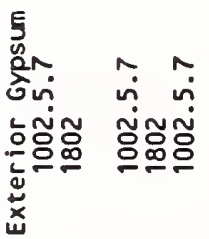

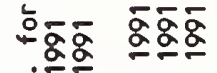

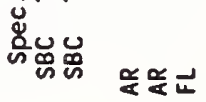

离 
尔

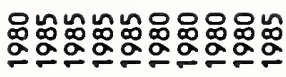

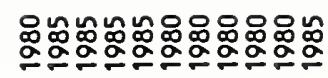

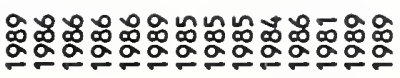

केष :

岂:

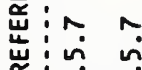

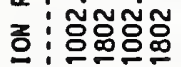

氙:

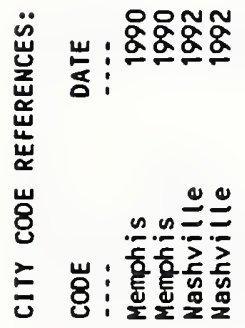

山्山: :

它密

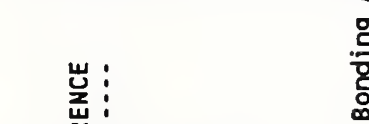

岂

岁

㠇

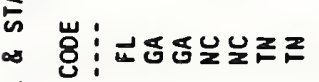

봉

宅

2

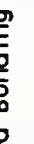

วิ

กับกับกับ

:

虫:

(1..

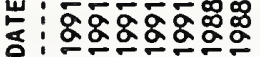

훙응

通.

ఏ̊

ผั

g

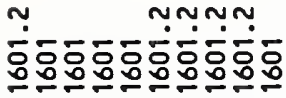

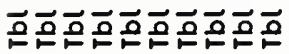

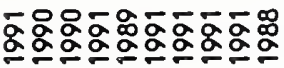

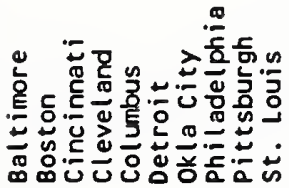

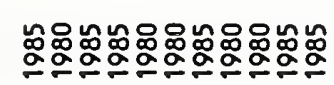

임요 mimmmm

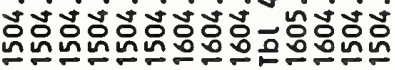

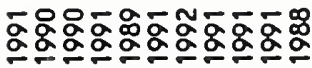

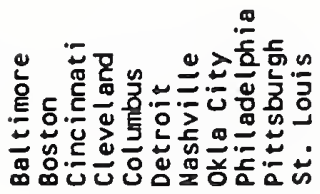

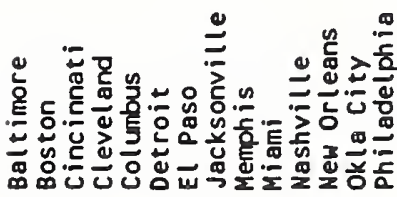

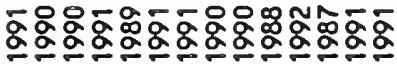

ㅇํㅇ

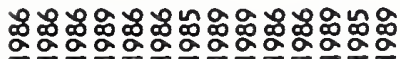

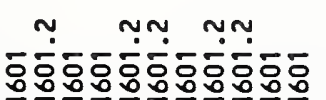

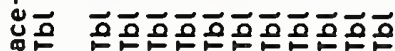

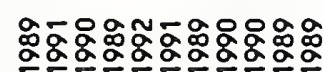

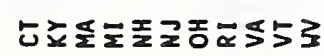

m

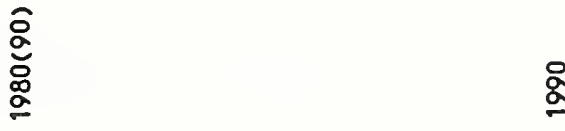

㽞?

ง

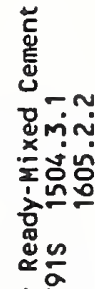

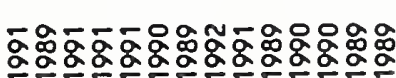

就市

遇品品

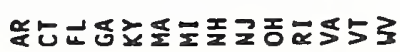

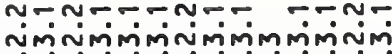

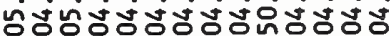

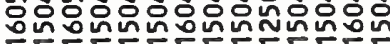

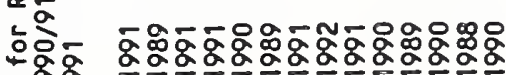

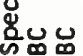

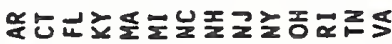

용

d 


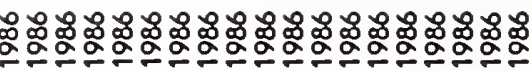

응ㅎㅆ

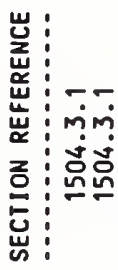

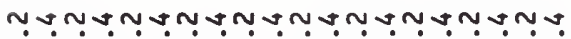

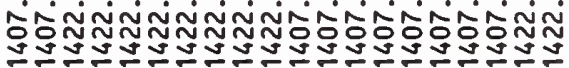

กับ

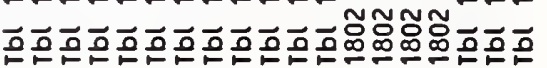

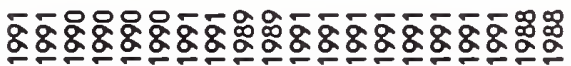

\&

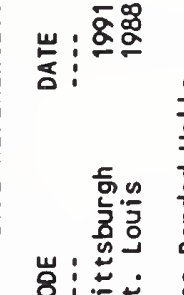

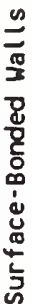

岩岂:

舟岕: 웅

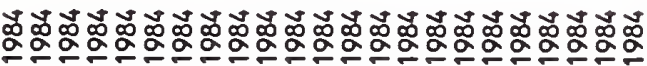

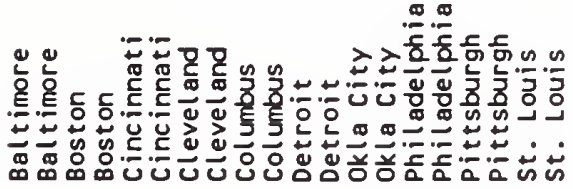

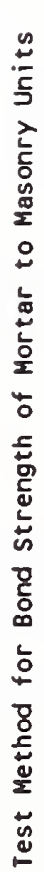

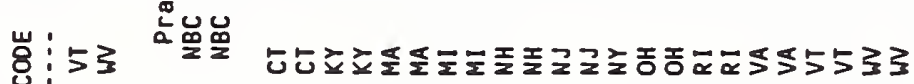

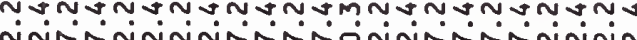

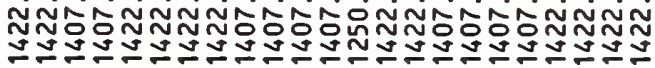

:

ш :a

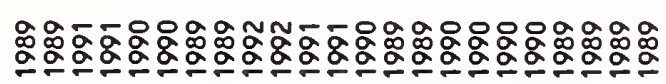

ธำ

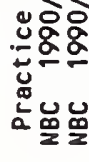

离

ฐ

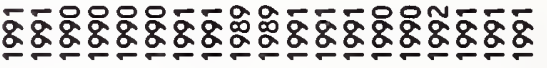

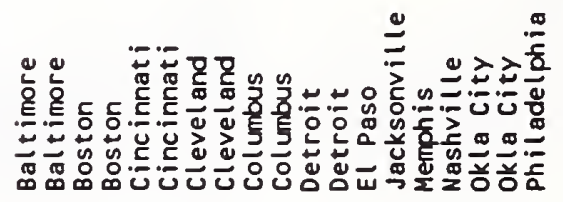

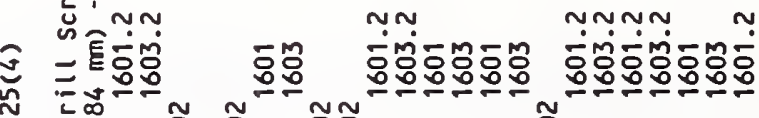

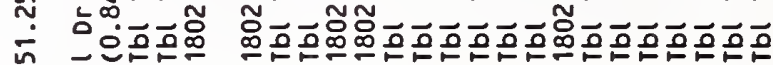
is.

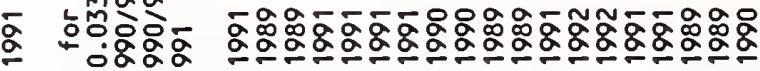

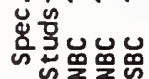

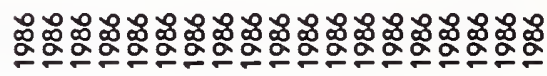
这

는

$\leftarrow \simeq$

वิํำ

$\Sigma$

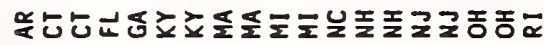

舀

สู 


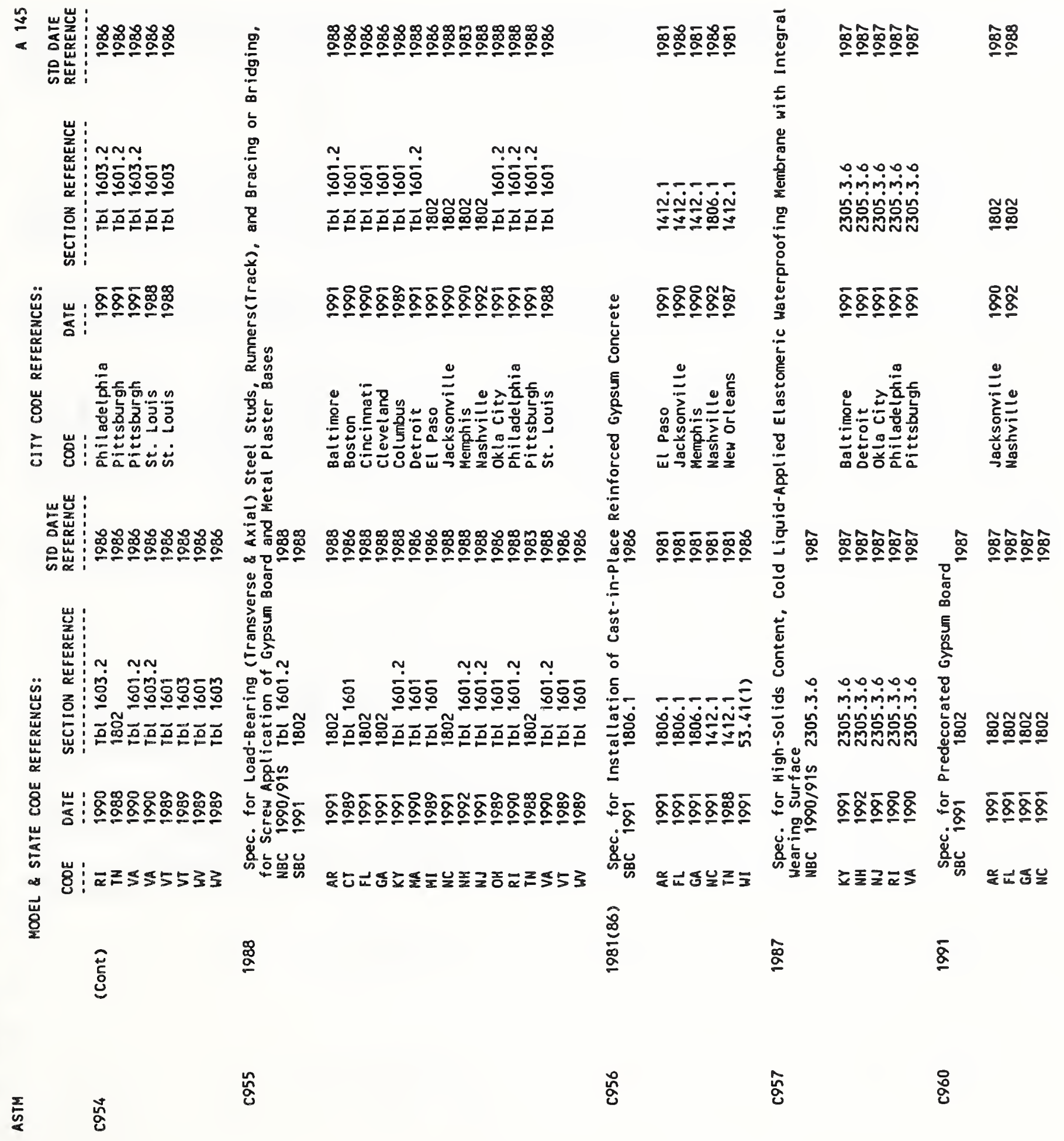




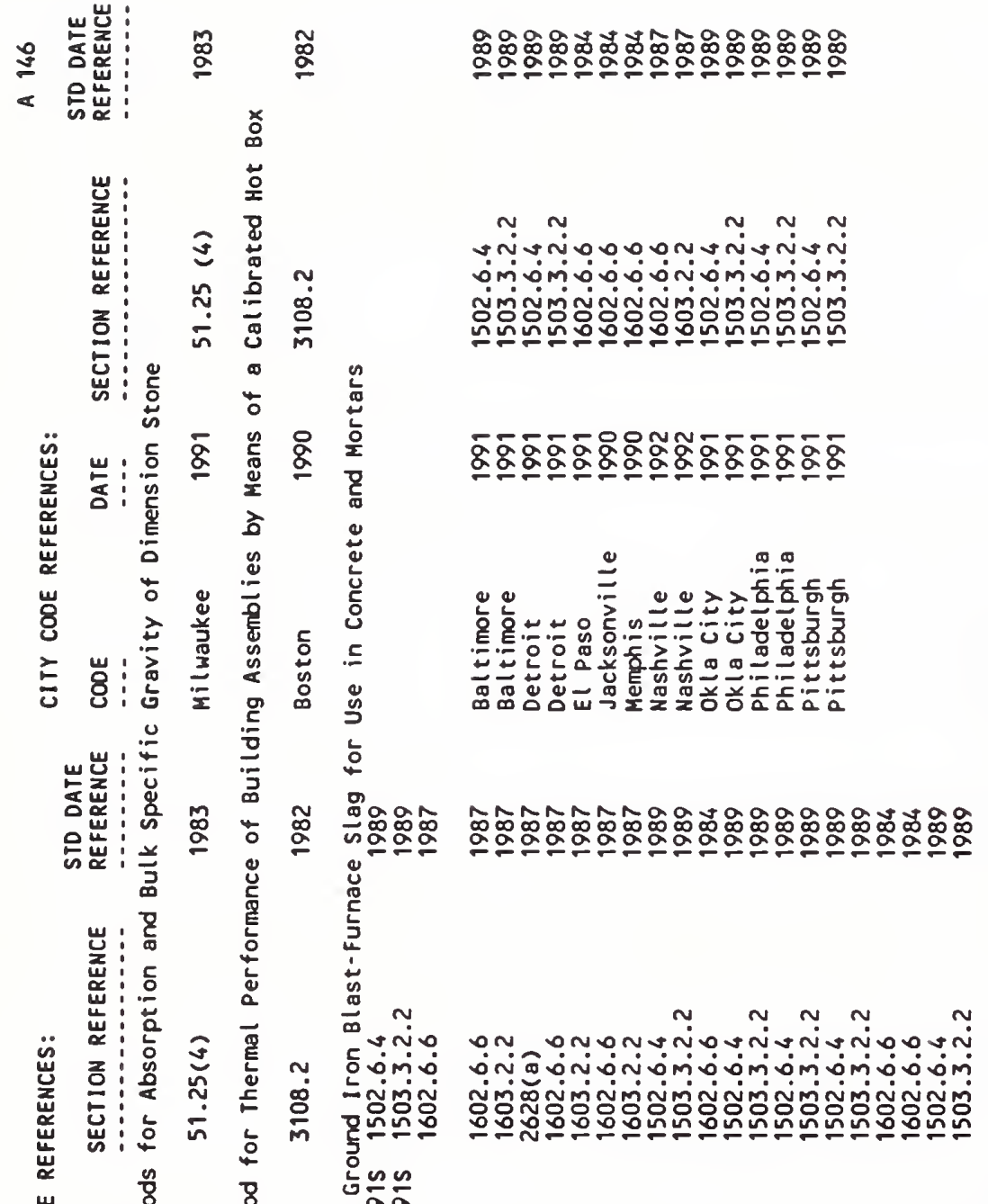

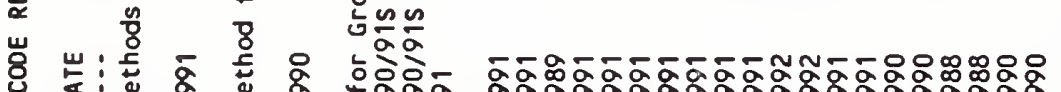

安

压

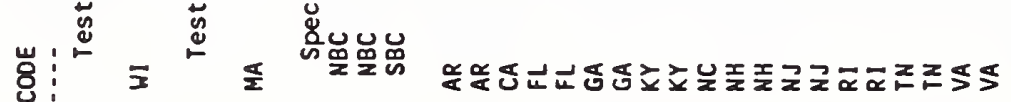

崖

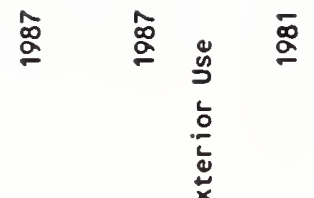

ฐ

$\tilde{g}$

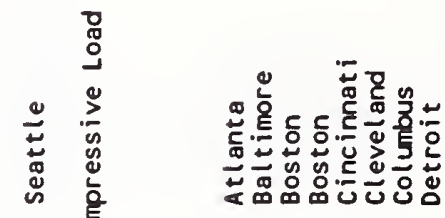

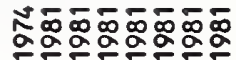

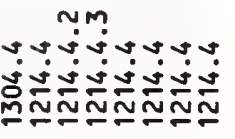

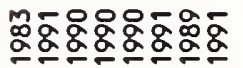

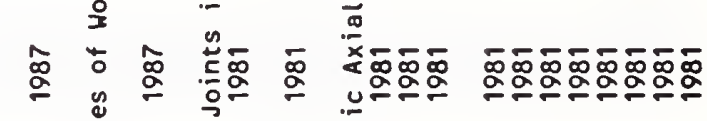

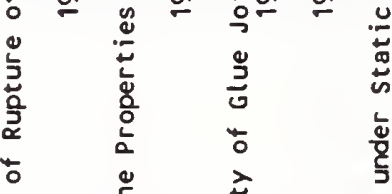

¿

$\frac{2}{3} \stackrel{5}{\frac{5}{2}}$

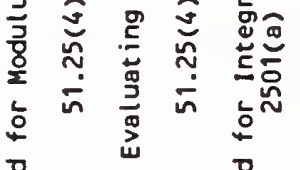

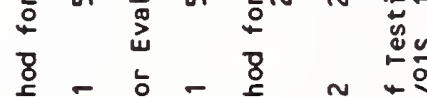

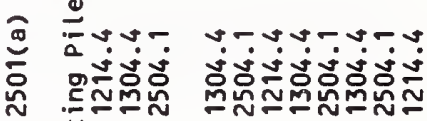
约

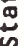

产

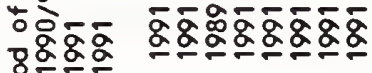

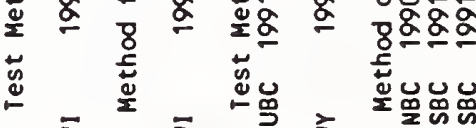

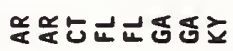

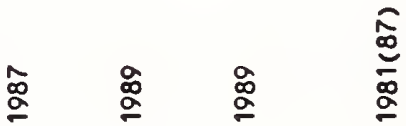

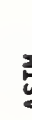

a

g. 


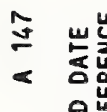

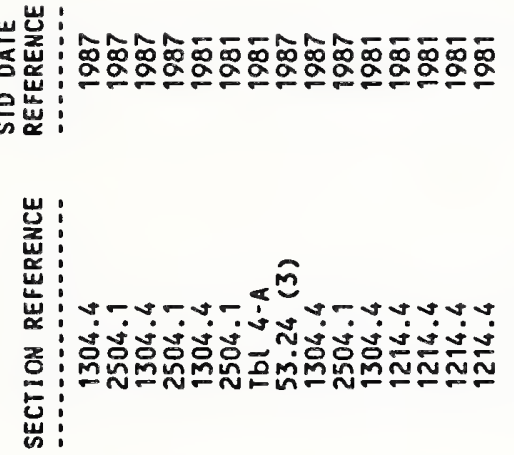

క

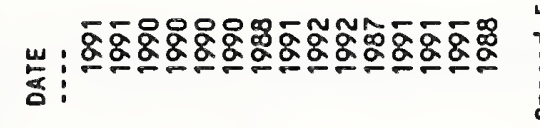

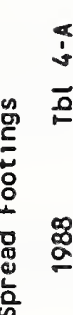

j.

mீimpon

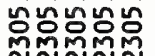

용용

号

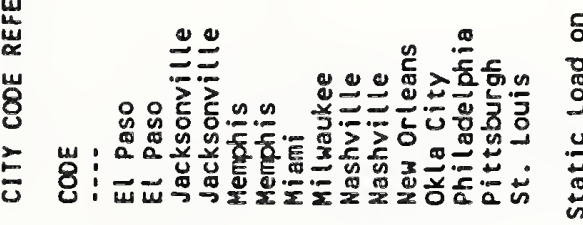

岩蔗:

온 :

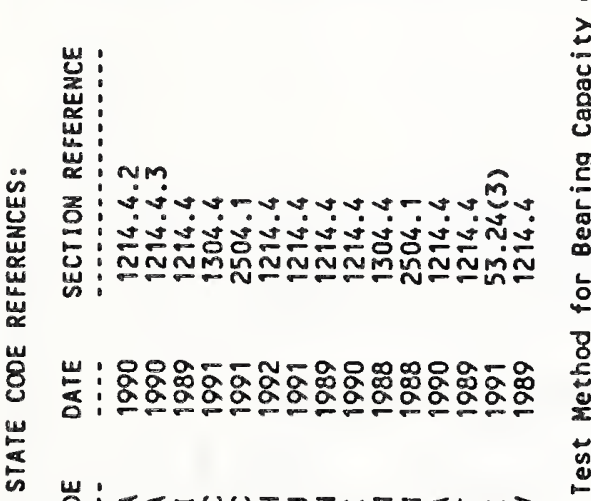

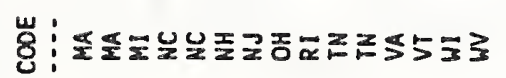

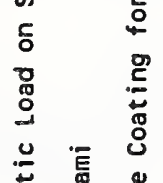

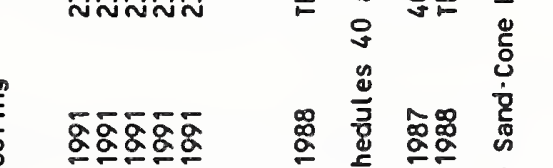

o 空

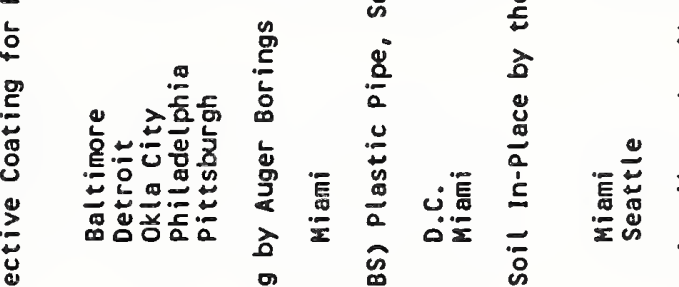

ळ

œू

흐요

$\overline{\overline{8}}$

mị

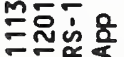

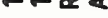

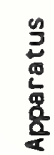

뭉

紊

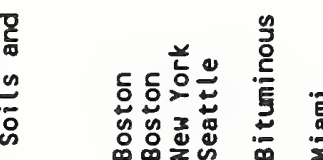

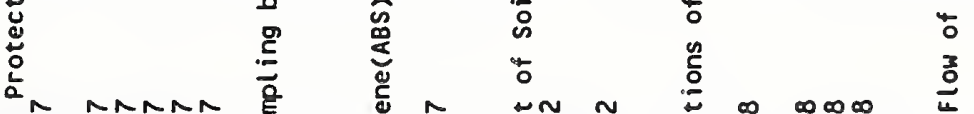

$\overline{8}$

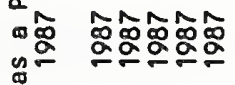

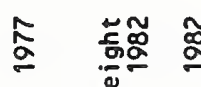

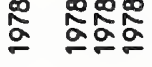

马्g

$\stackrel{\infty}{3}$

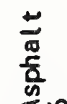

\&o 00000

Imं mimimim

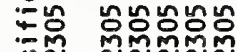

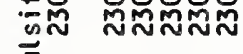

丞乐

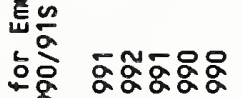

㟧哭

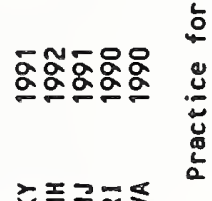

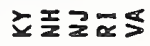

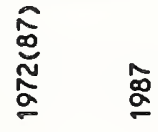

ळัँ

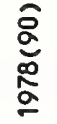

졸조프

miñ

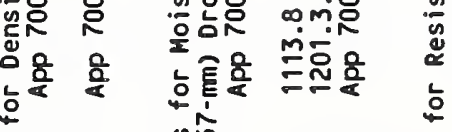

○

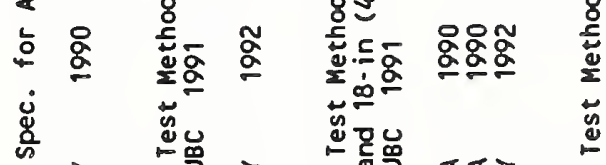

范

$\frac{\Xi}{\frac{\pi}{0}}$

$\frac{\tilde{N}}{0} \frac{\tilde{n}}{0} \frac{\tilde{n}}{0}$

$\frac{n}{n}$

층

$\frac{x}{2} \frac{m}{\frac{2}{5}}$ 


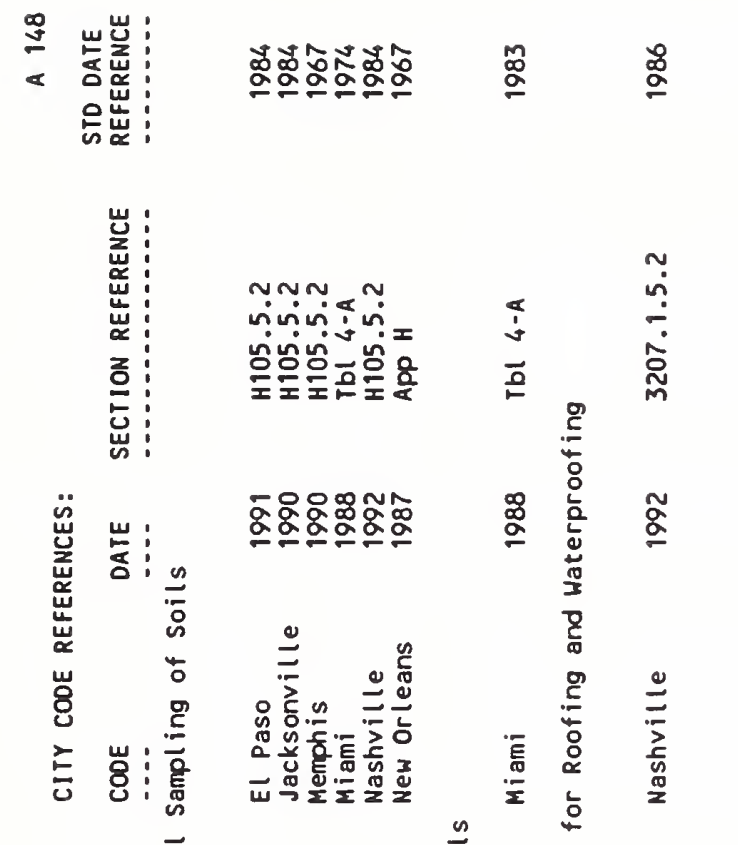

岀: 岸: 离

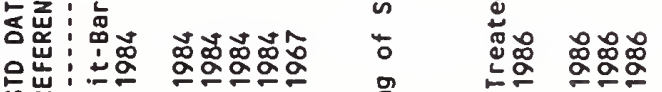

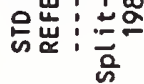

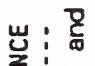

崖:

焉

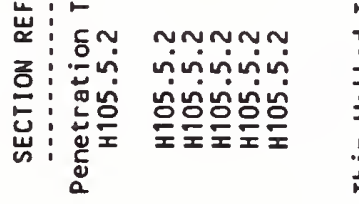

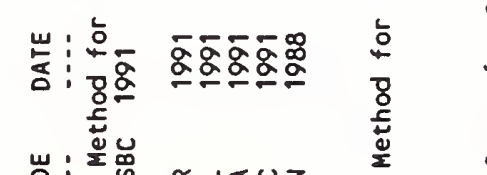

亮

产

岩:

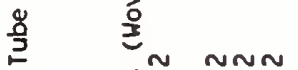

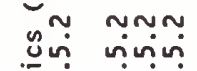

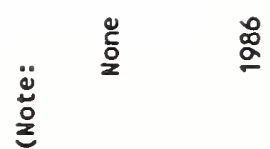

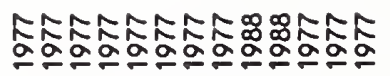

$\stackrel{\dddot{\Xi}}{\stackrel{\Xi}{g}}$

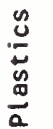

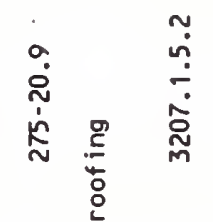

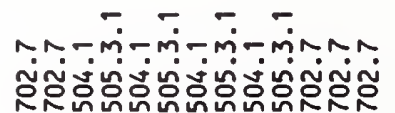

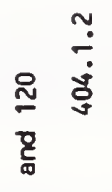

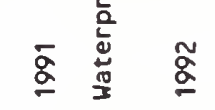

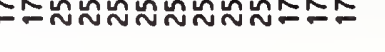

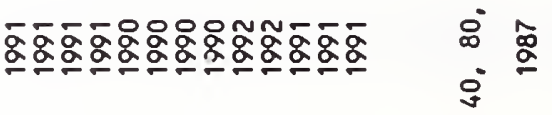

$\stackrel{0}{\circ}$

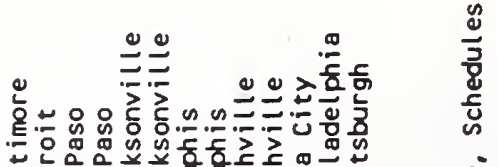

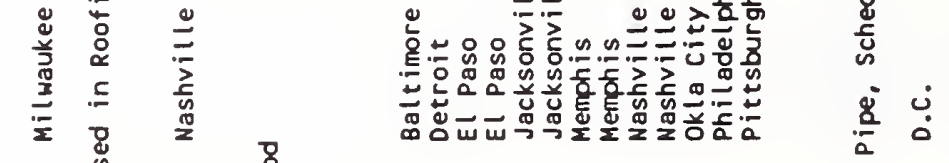

잉

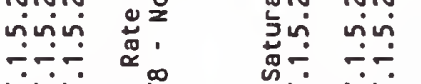

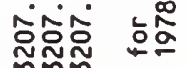

萂:

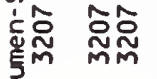

空

s-̄ $\bar{\alpha} \bar{\alpha} \bar{\alpha} \bar{\alpha}$

荢品

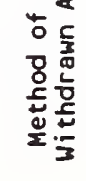

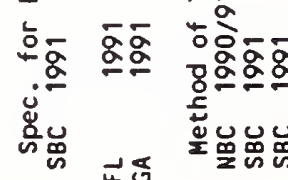

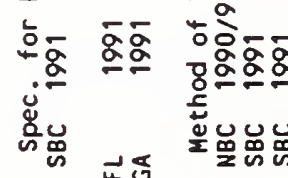

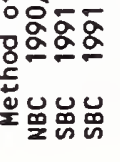

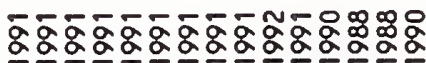

㟧

要

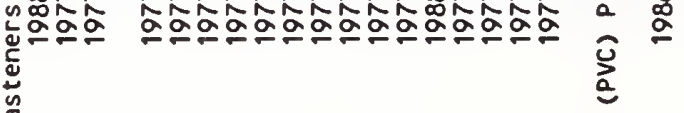

离

䓵

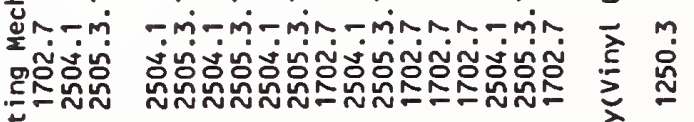

政

영

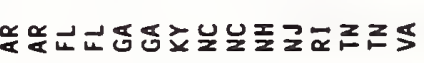<smiles>[13CH3]</smiles>

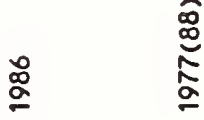

$\stackrel{9}{\stackrel{\alpha}{\alpha}}$

$\frac{1}{2}$

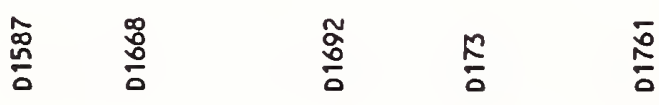

$\frac{n}{\frac{n}{0}}$ 


\section{舀管:}

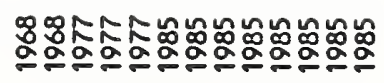

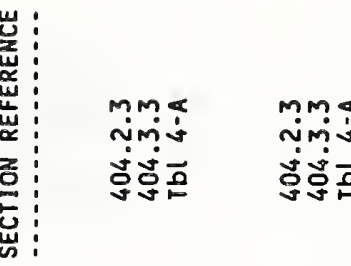

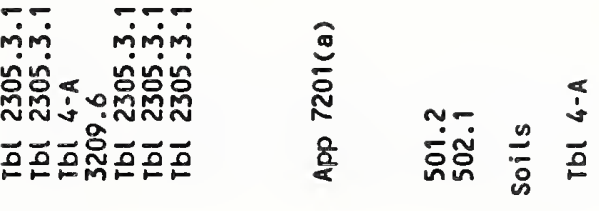

ฮำ

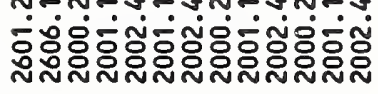

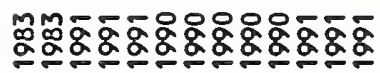

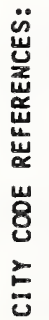

눙

ิㅗㅀำ

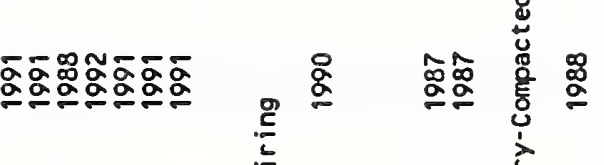

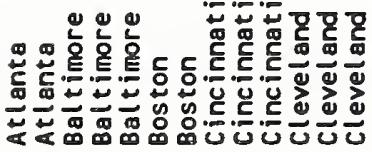

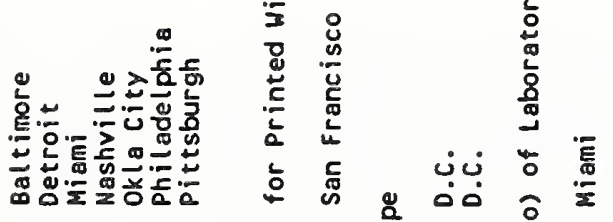

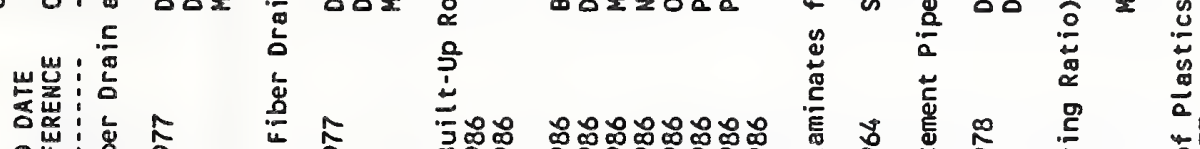

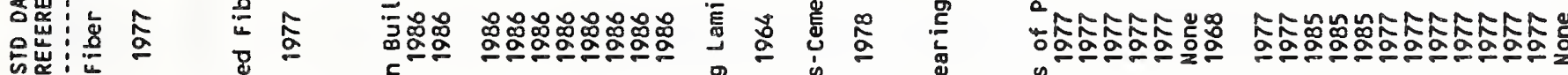

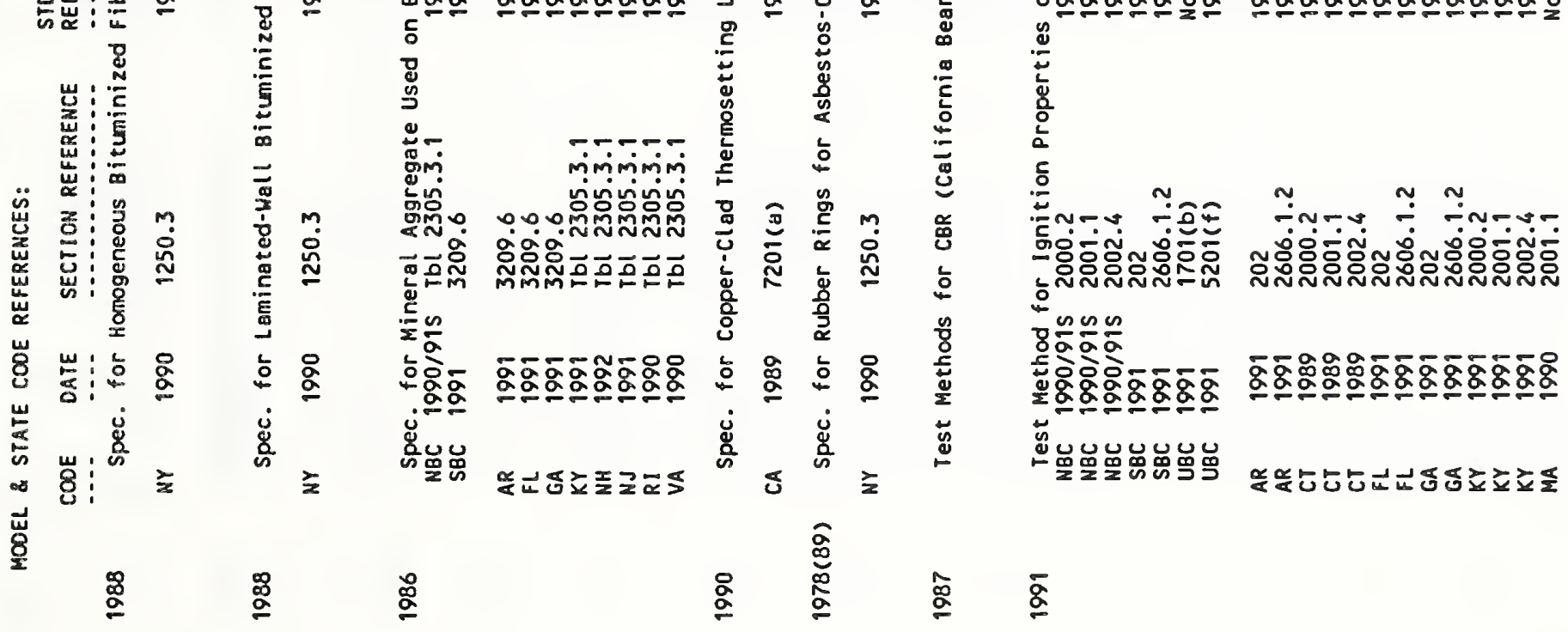

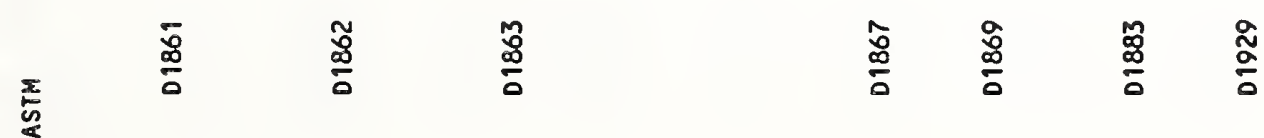




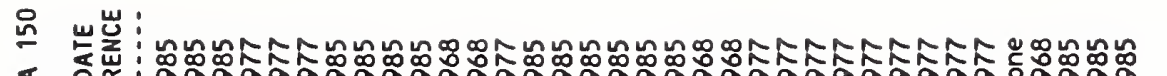
은 क⿻ ㇒⿺

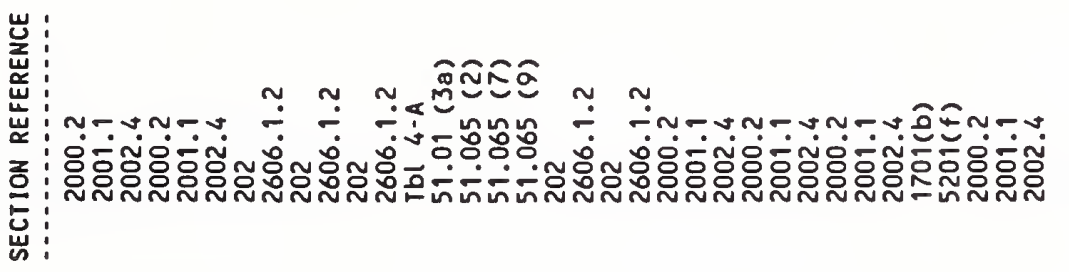

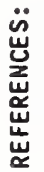

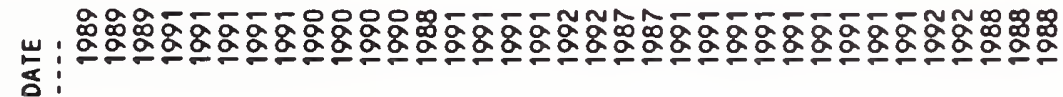

ర్

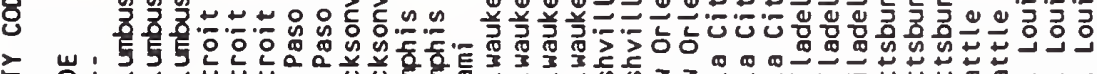

む

w :

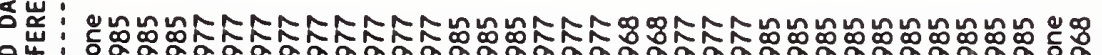
ㅇ⿺ㄴ

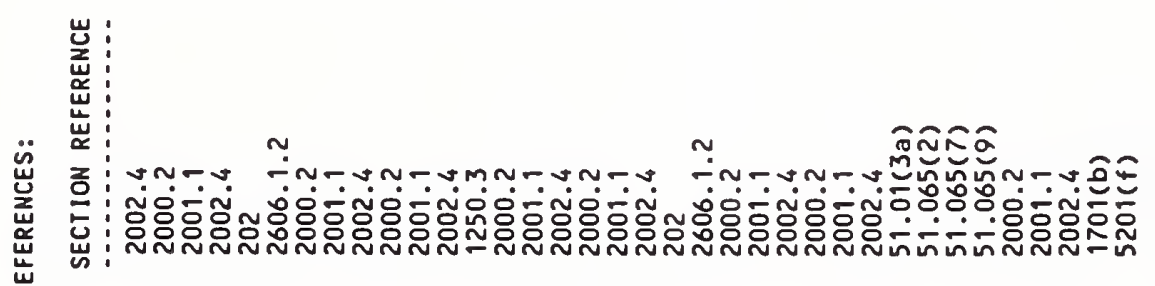

要

㟧

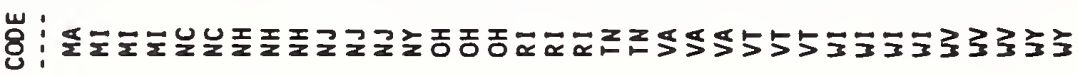

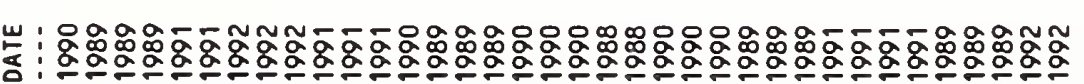

$\stackrel{\sim}{\underline{a}}$

ฐัญ

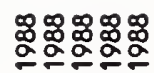

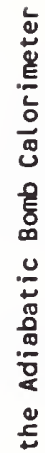

a

范

产

品

4

$\frac{5}{2}$

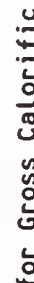

范

$\bar{g} \stackrel{\circ}{\circ}$

ํㅗㅇ
产

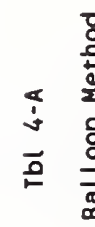

용

minminल

营总跑点

돈웅

호몬흔흐는

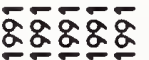

唋

.

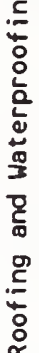

$\stackrel{8}{\circ} \stackrel{\square}{\alpha}$

్ㅕㅇ명명명

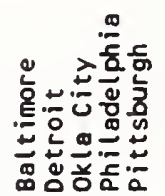

s

Ð

-

تिimin:

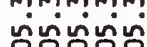

Nํำ

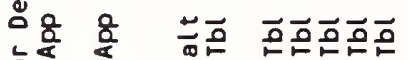

造乐

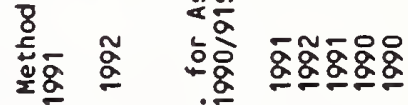

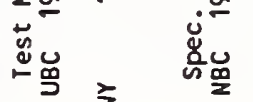

흐돌조

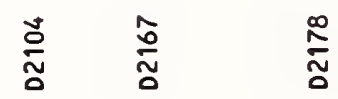

跑管 


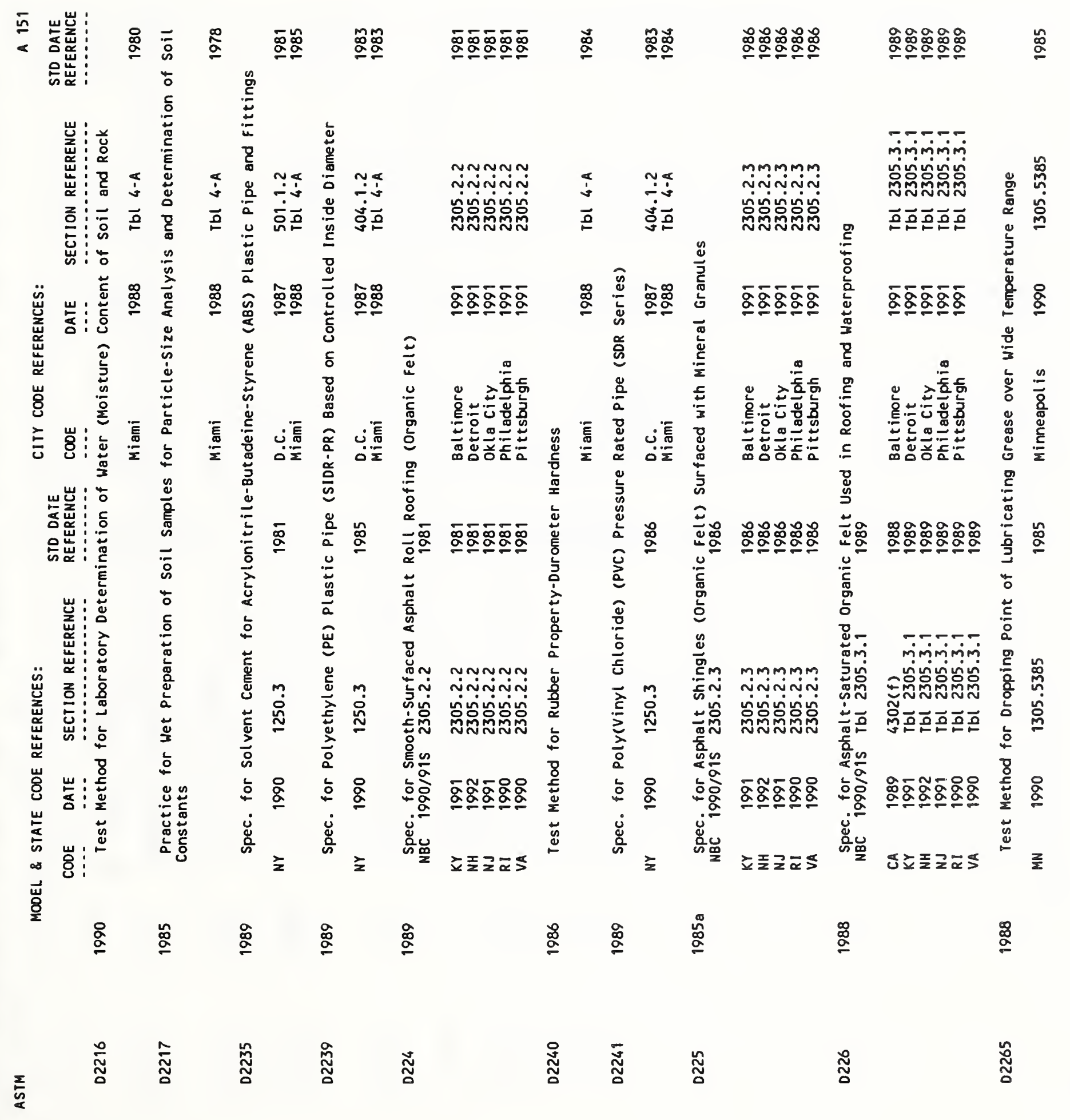




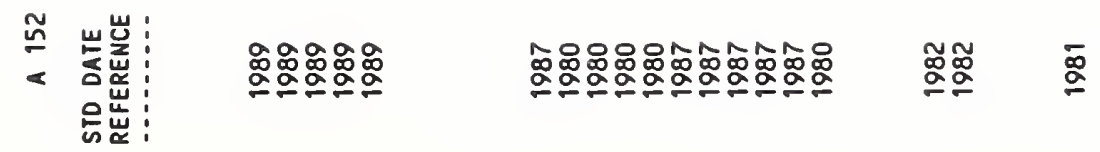

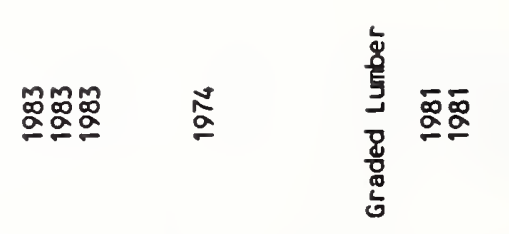

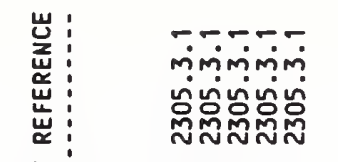

:

:

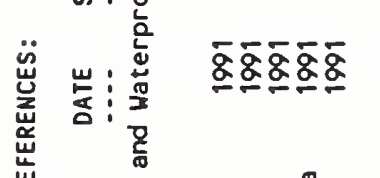

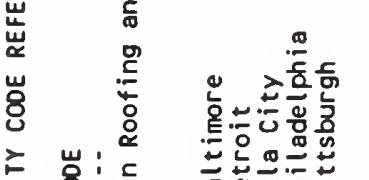

츤

w

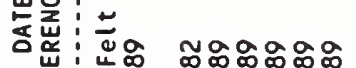

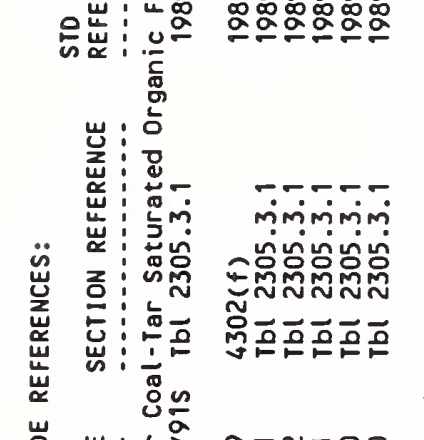

혼

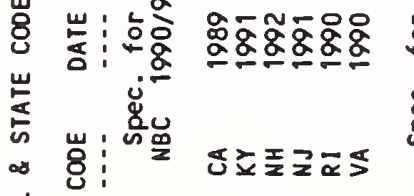

(2)

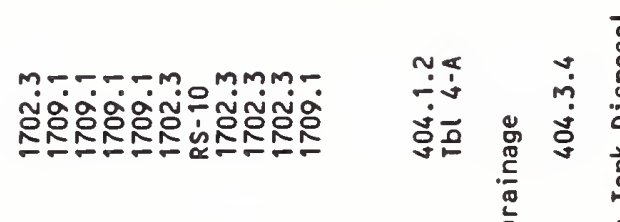

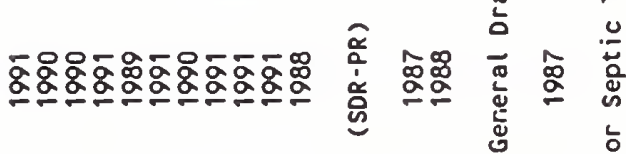

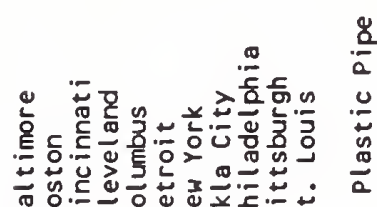

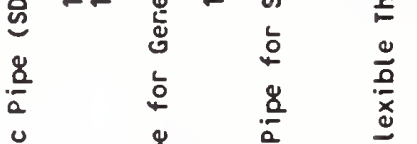

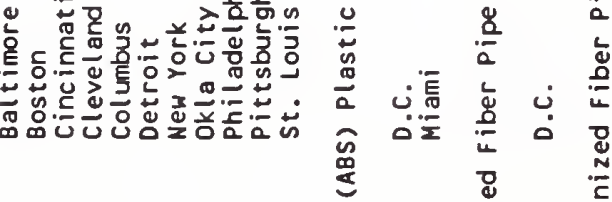

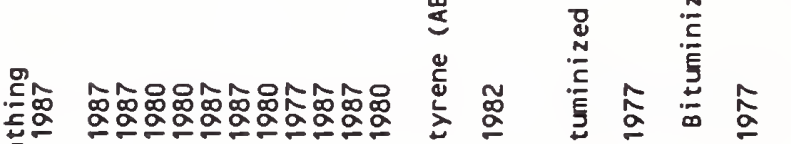

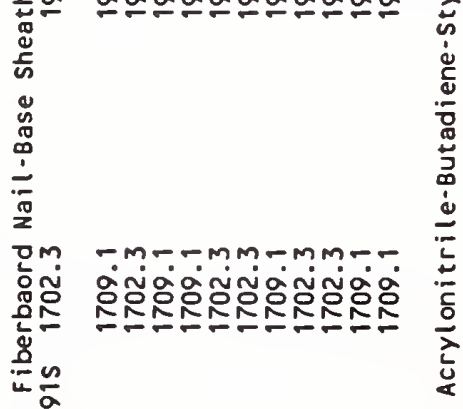

崩

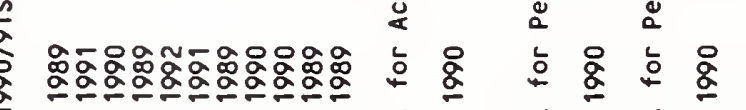

密苨

岁客客安

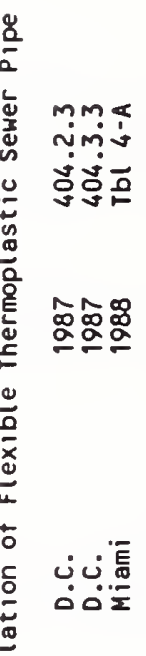

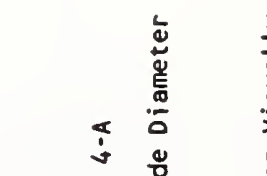

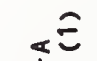

욤ํํ

产

怘

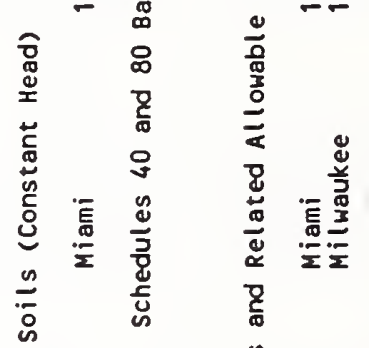

$\stackrel{\widehat{\alpha}}{\stackrel{0}{\infty}}$

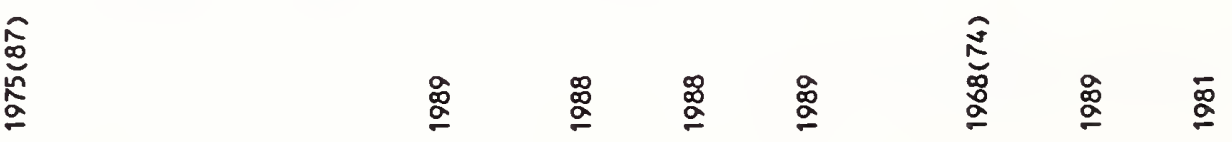

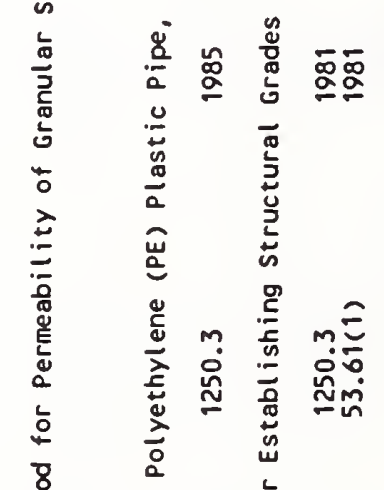

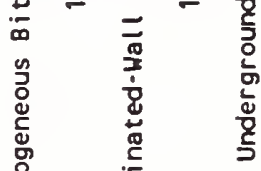

올 宊

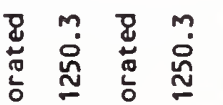

范

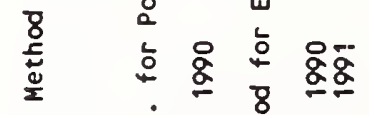

兽

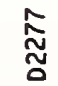

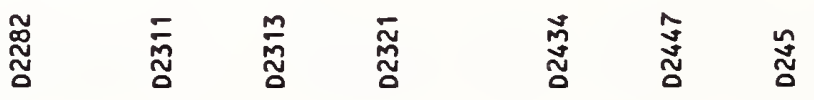




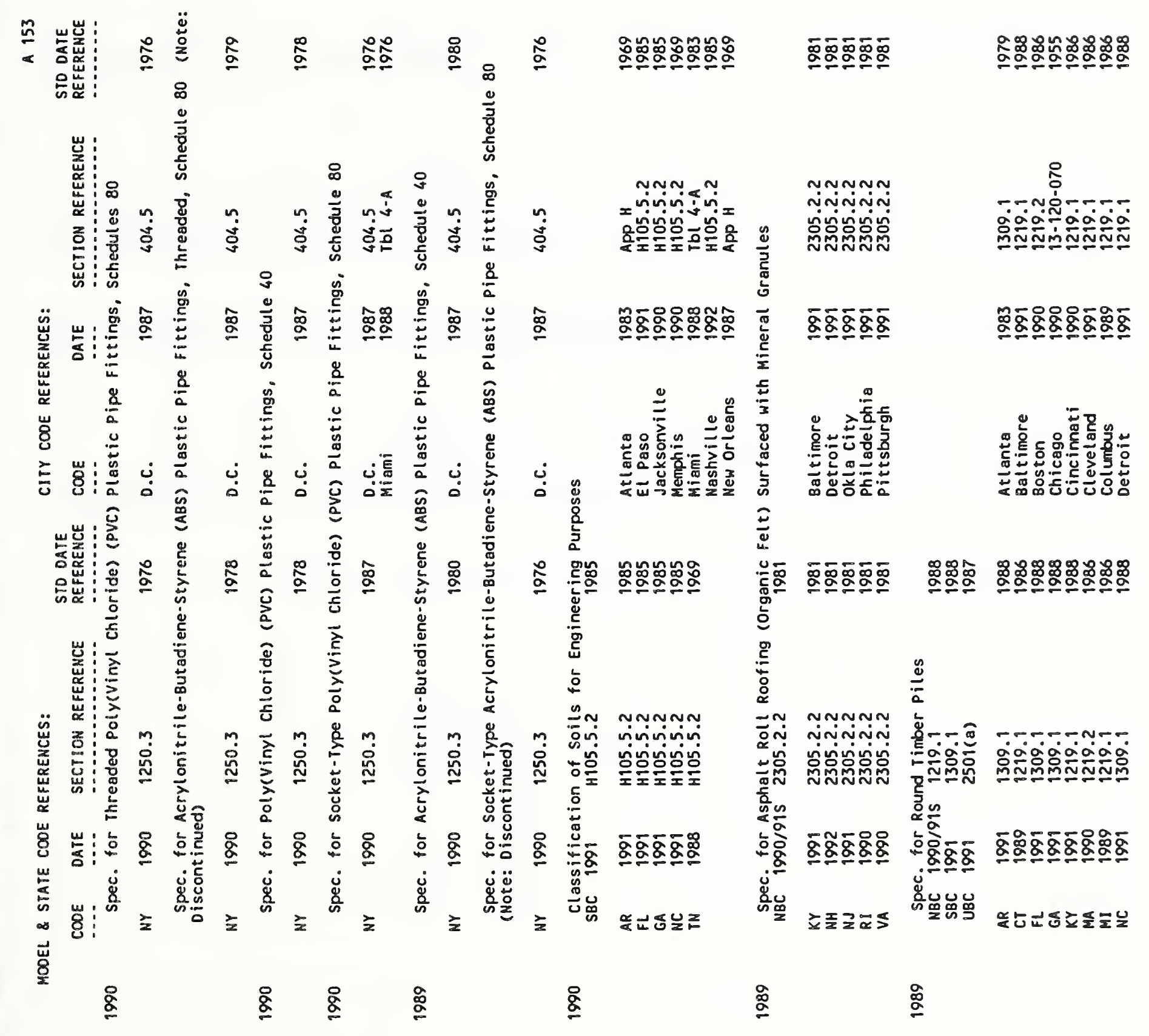


ڤั

< 的岕:

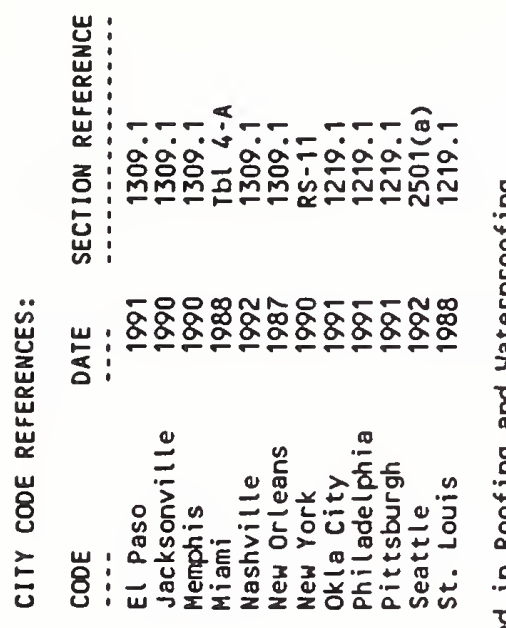

岕:

嵪:

Und

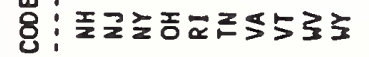

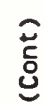

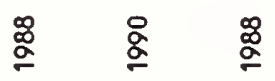

怘 吾 莡

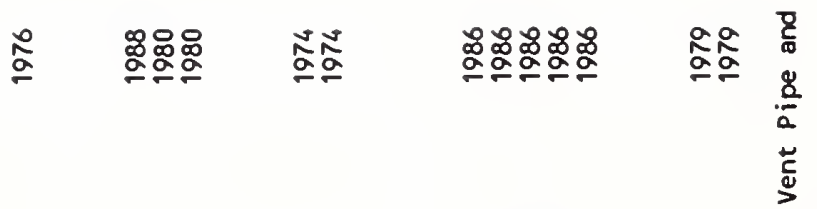

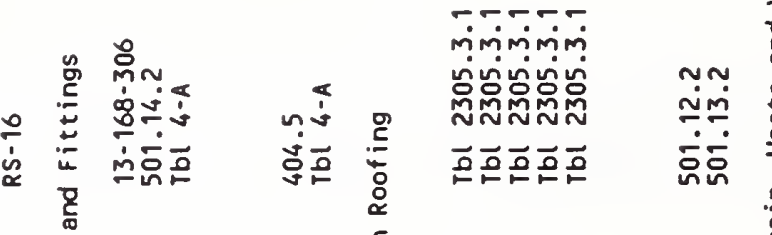

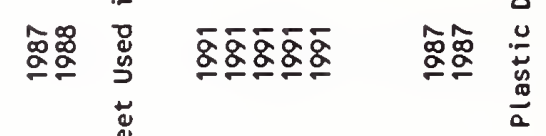

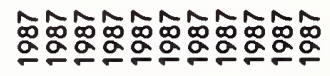

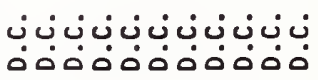

ذن.

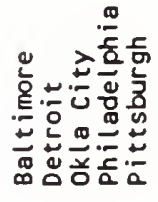

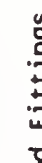

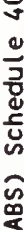

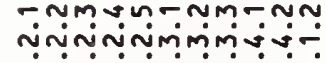

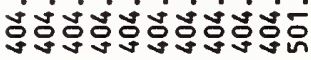

$\nsubseteq$

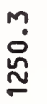

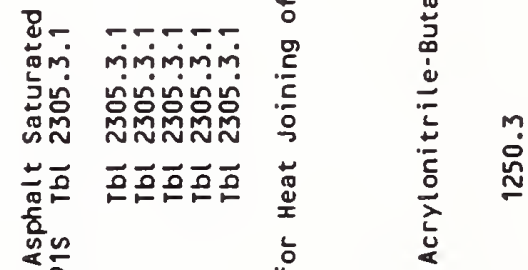

要量

立更文 $\bar{\alpha} \$

$\stackrel{\circ}{\stackrel{\mathscr{g}}{9}}$

$\stackrel{\circ}{\circ}$

莡 


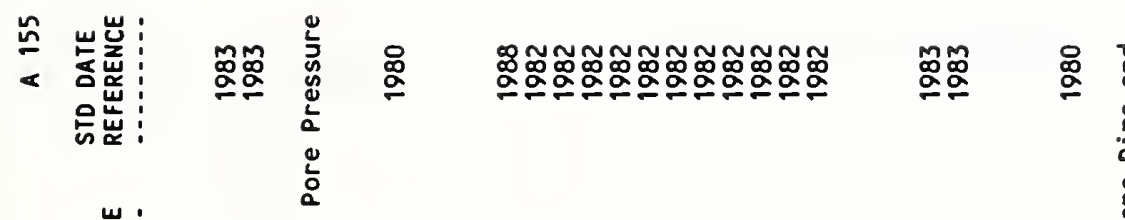

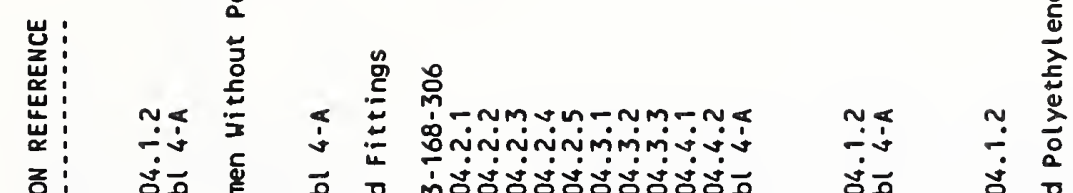

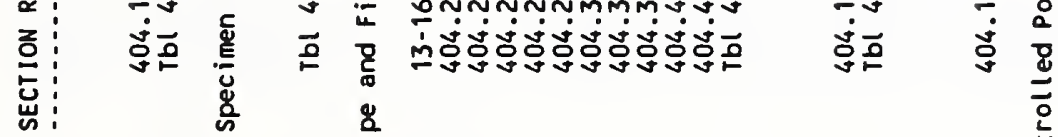

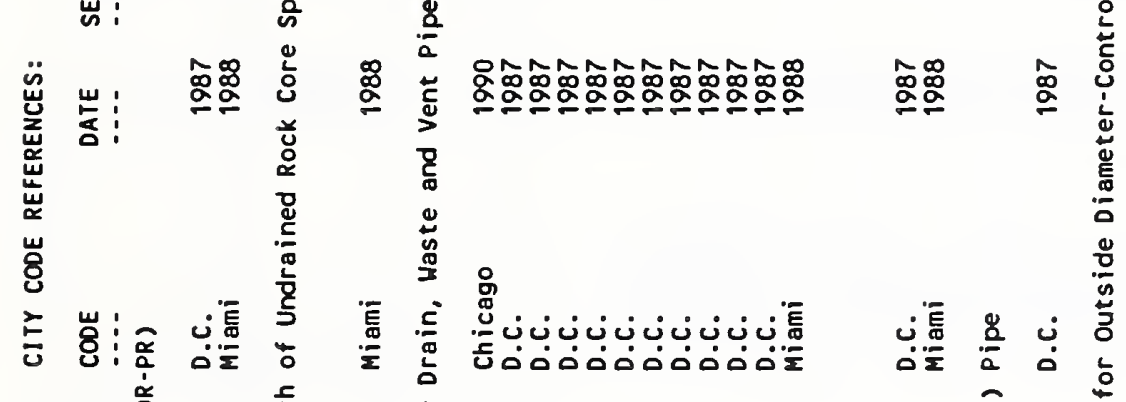

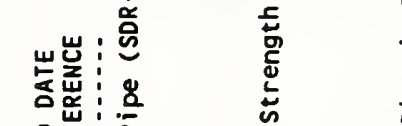

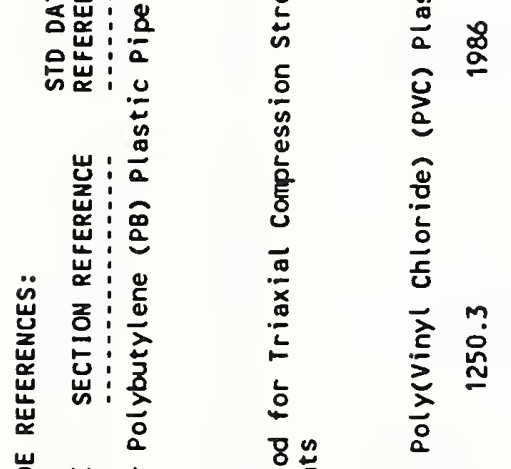

岁岩:

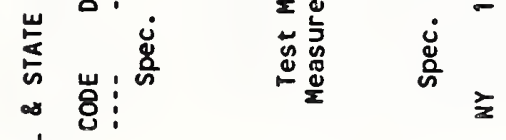

물

$\stackrel{\infty}{\stackrel{\infty}{g}} \quad \stackrel{\bar{g}}{\sigma}$

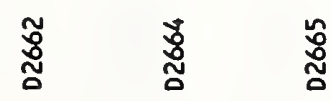

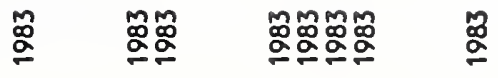

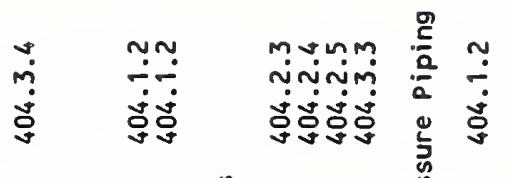

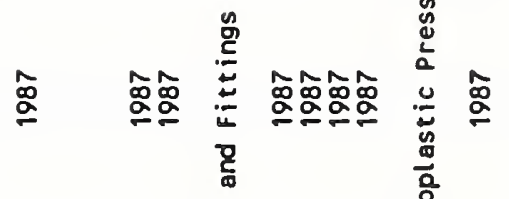

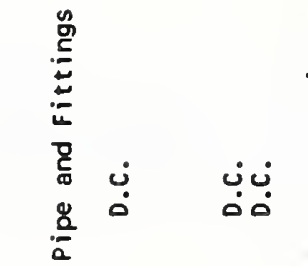

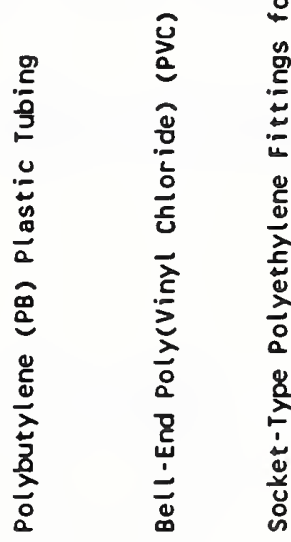

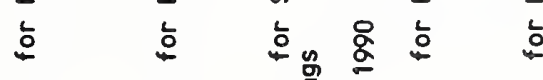

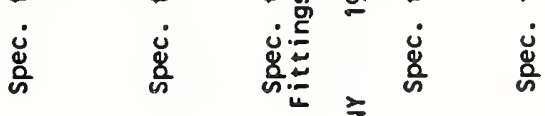

兽 $\frac{\widehat{0}}{\frac{0}{2}} \quad \frac{a}{a}$

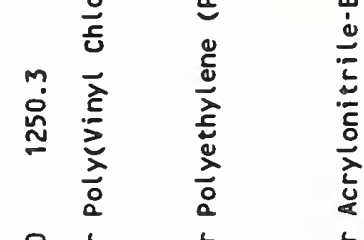
ن

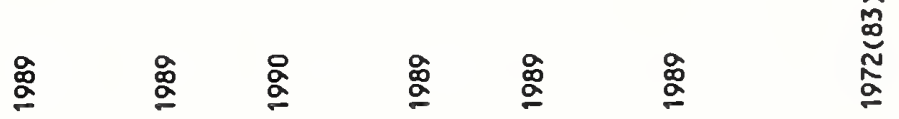

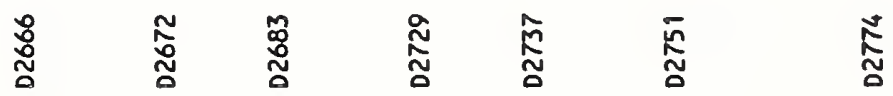




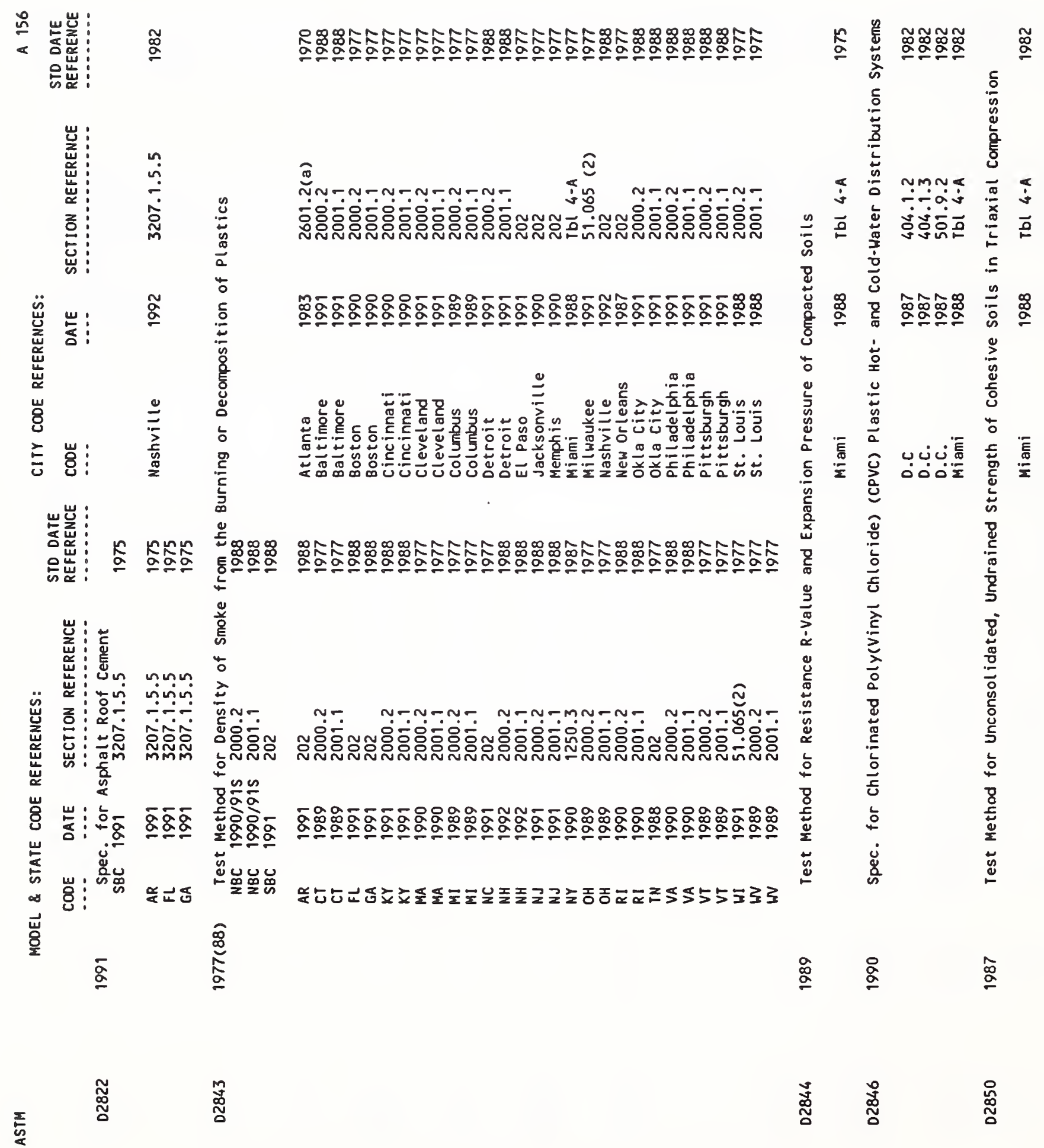




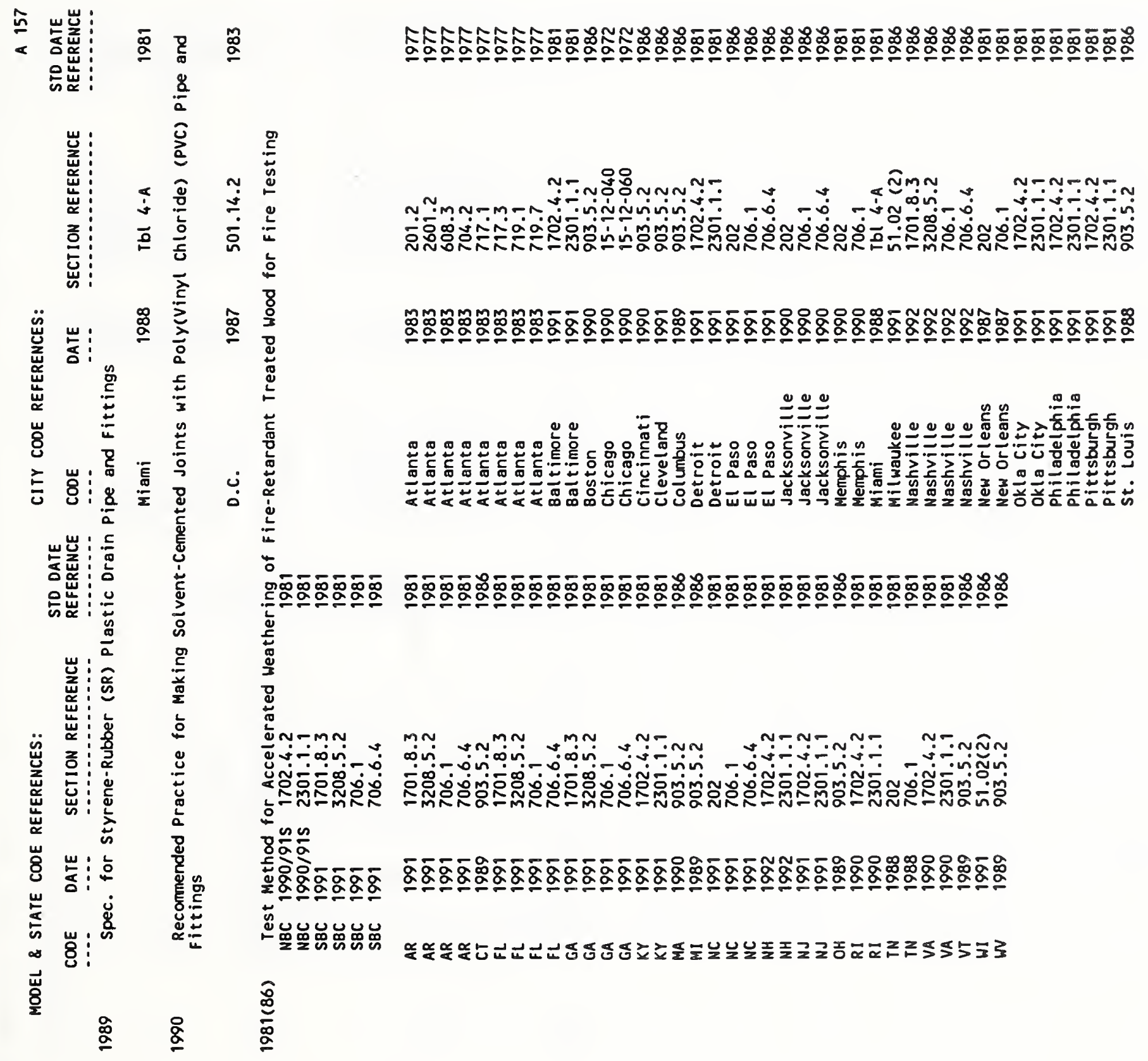




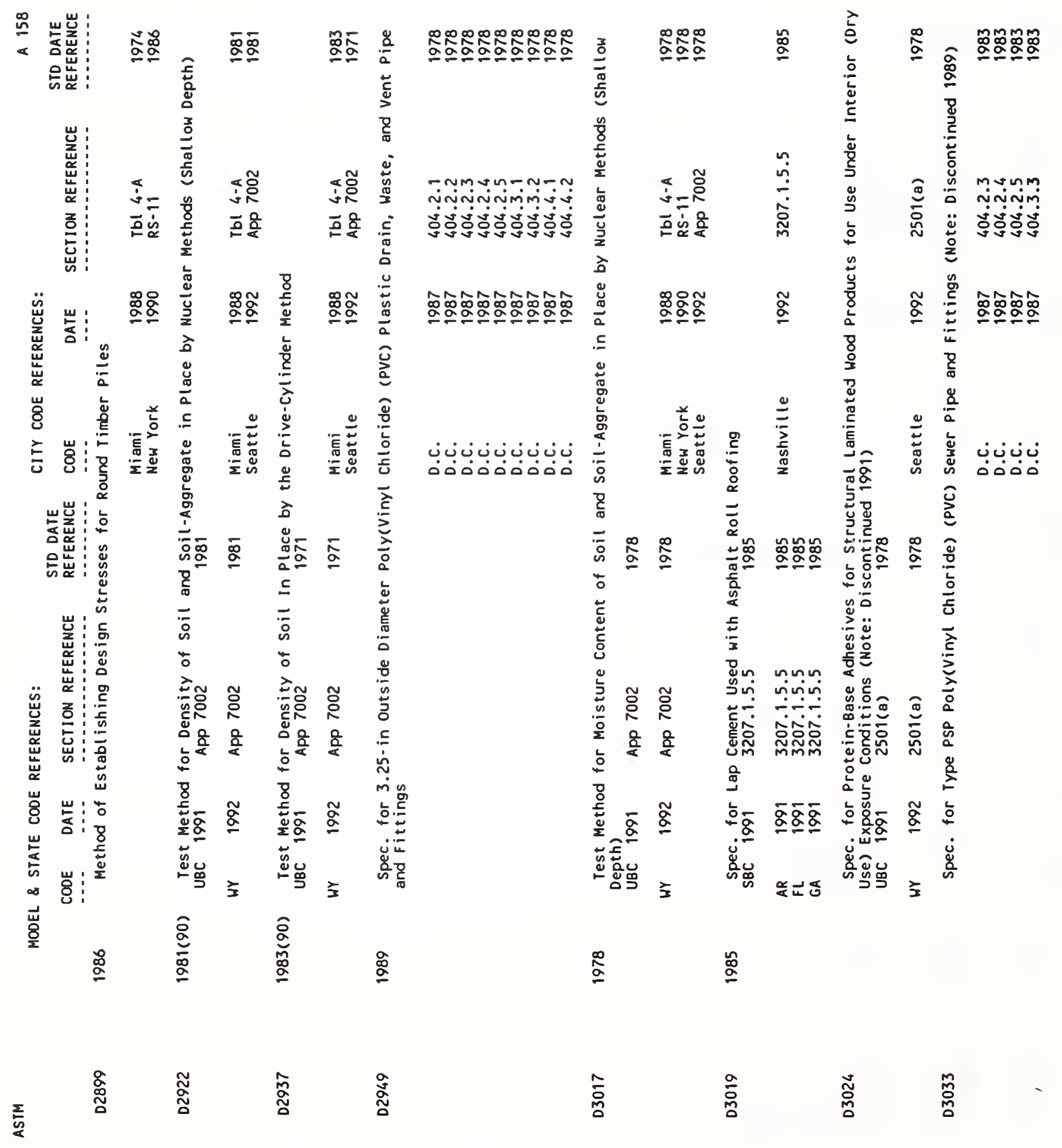




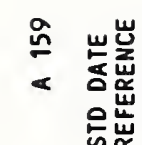

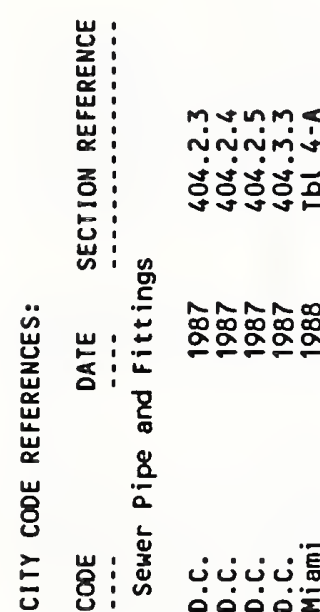

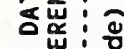

吢岕: :

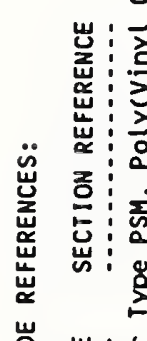

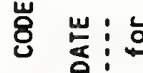

崖

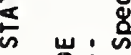

\&

훌

$\stackrel{a}{\varrho}$

誉

$\frac{\Sigma}{2}$

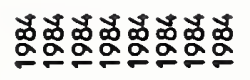

mim mimm

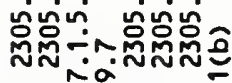

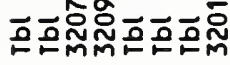

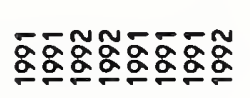

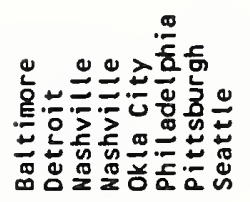

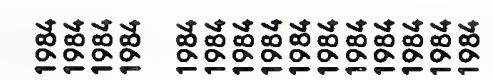

$\stackrel{\text { : }}{\stackrel{9}{\circ}}$

șin

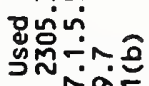

ธ듀ำ

这告

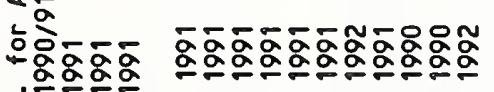

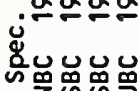

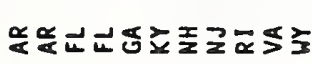

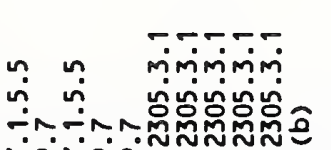

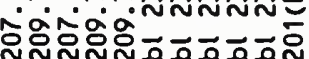

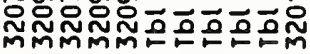

a

$\stackrel{\sim}{\frac{m}{0}}$

mmmmm

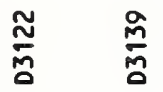

高 $\frac{\overline{0}}{\mathrm{~m}}$

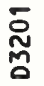

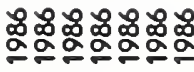

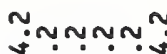

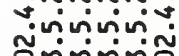

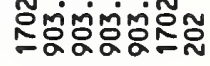

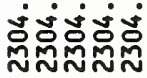

$\frac{\Phi}{0}$
$\frac{1}{8}$
$\frac{1}{3}$

$\bar{\alpha} \bar{g} \bar{\alpha} \bar{g} \bar{\alpha}$

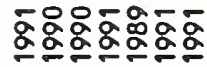

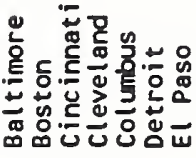

ن

岁

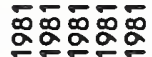

i

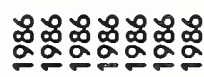

产

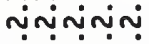

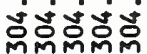

ผึผผ

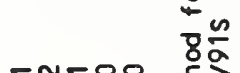

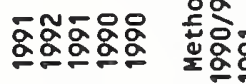

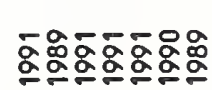

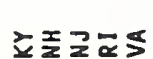

苍员

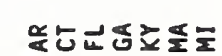

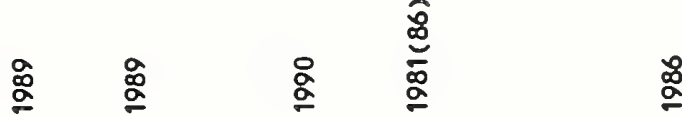

$\stackrel{8}{2}$

MNT. ThN

ن.

응오옹ํㅇㅇ 


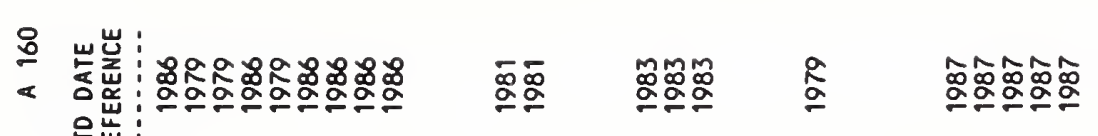
的岕:

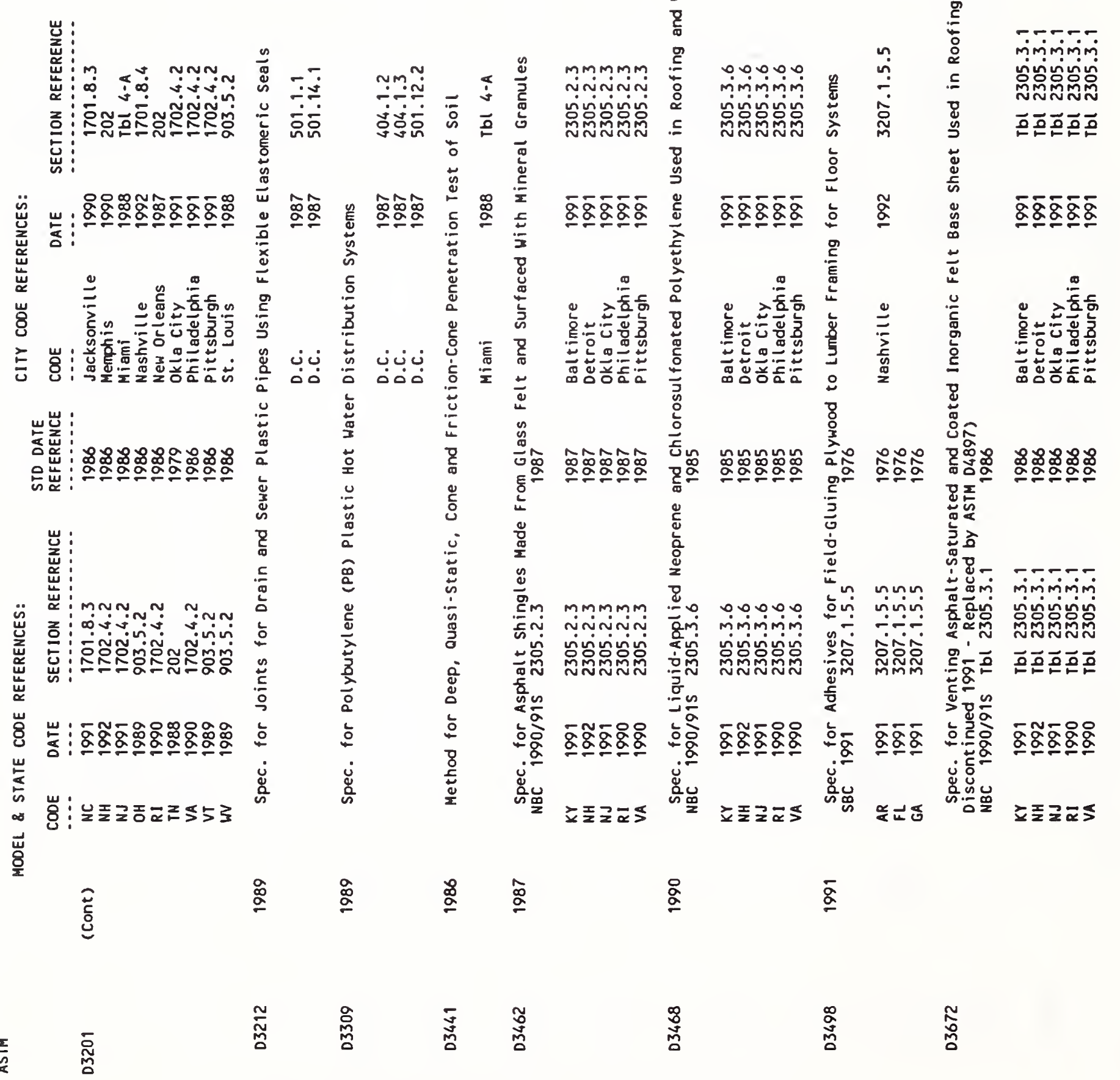




\section{巨峞
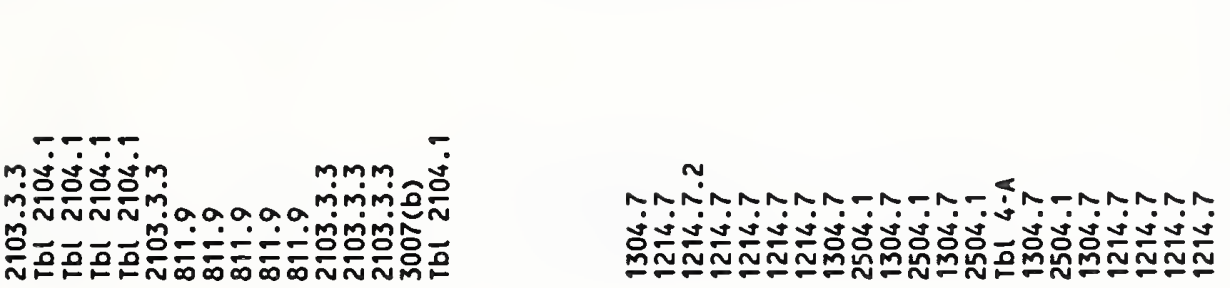

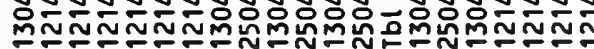

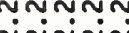

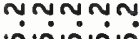

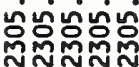

종무

แ

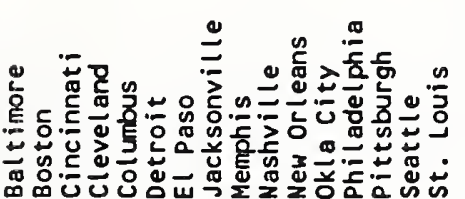

w본

为竞:

은현

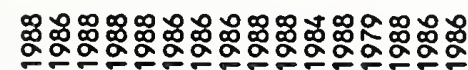

, 은

널 :

芯:

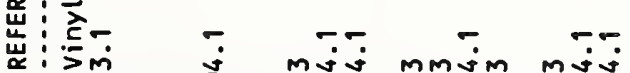

is $\approx: \geq m$

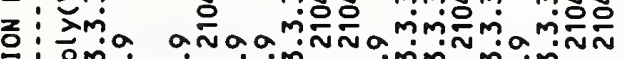

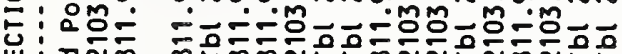

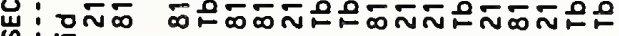
$\frac{\pi}{\alpha} \frac{\pi}{2}$

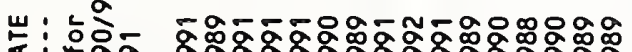

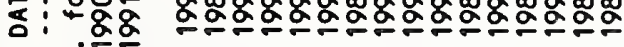

: 究毠品

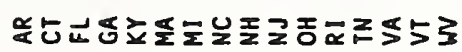

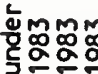

$\stackrel{\mathscr{E}}{=}$

몸

है?

कํํㅇํㅇ

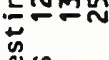

$\stackrel{\infty}{\infty} \frac{\pi}{2}$

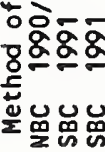

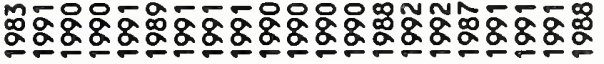

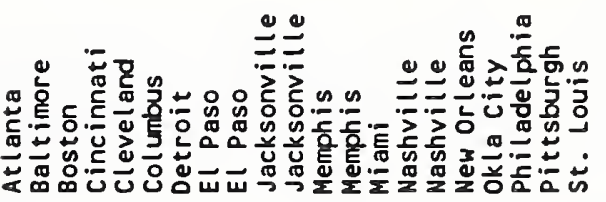

mmmmmm

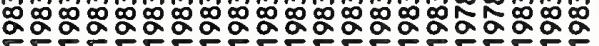
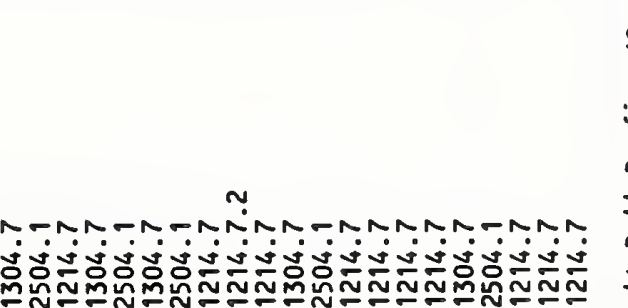

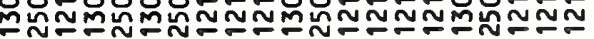

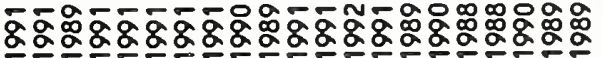

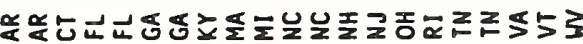

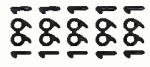

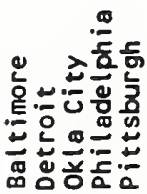

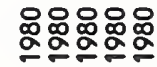

(o)

nก⿻上丨 กับบที ํํㅇํํㅇํㅇ응

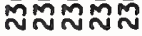
客的

푸요용<smiles></smiles>

ㅊㅗㄹㅀㅀㅗ 


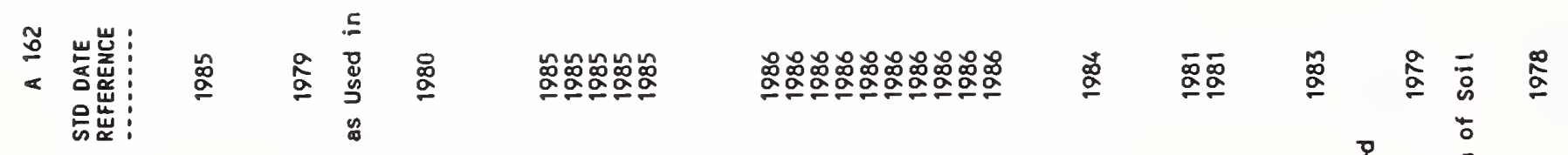

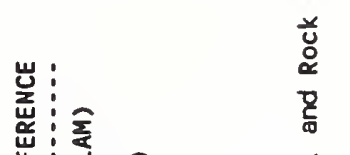

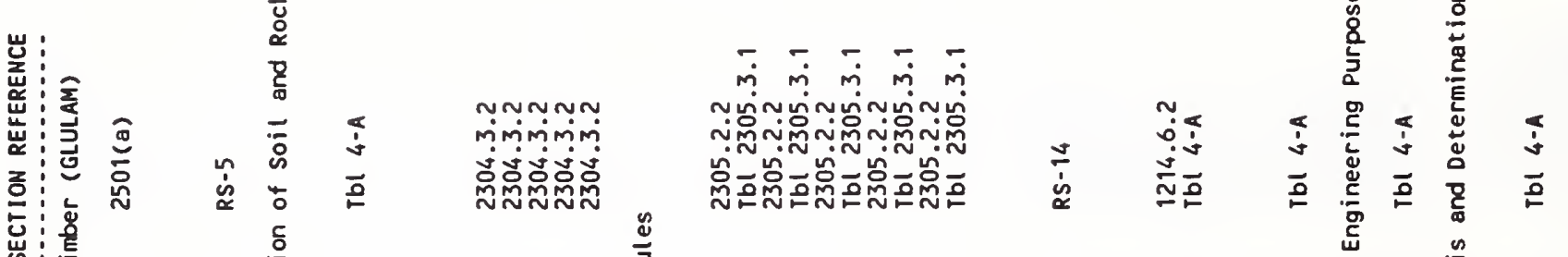

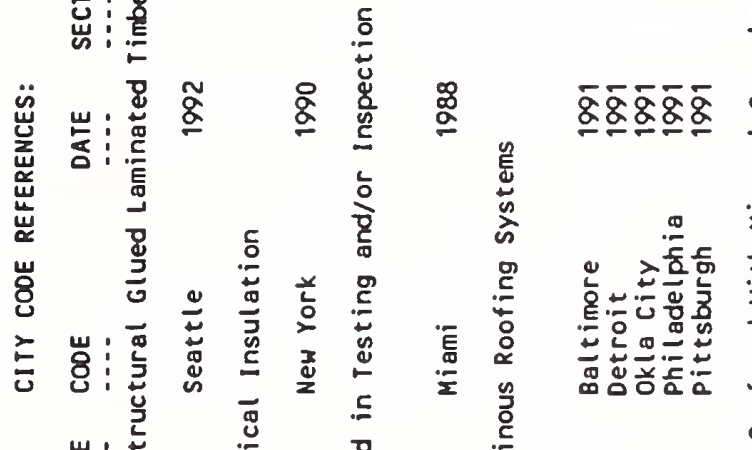

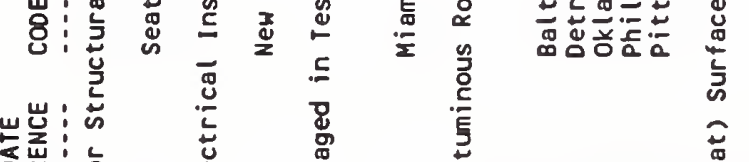

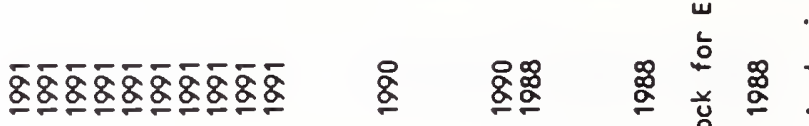

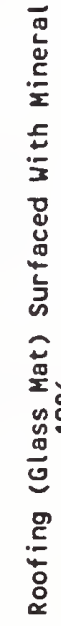

政

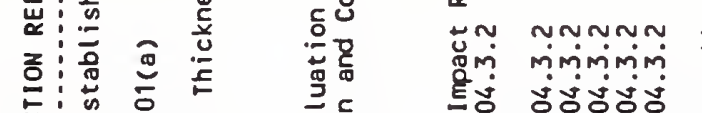

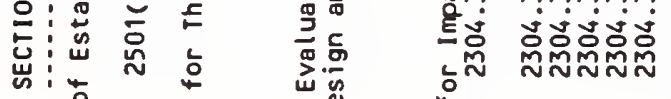

然

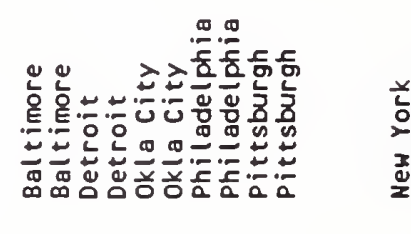

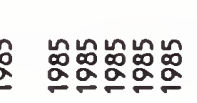

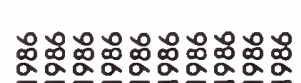

竞

雚

离

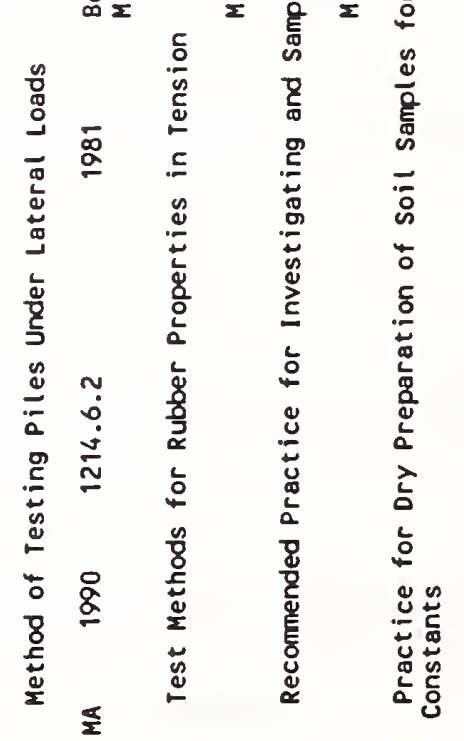

푱

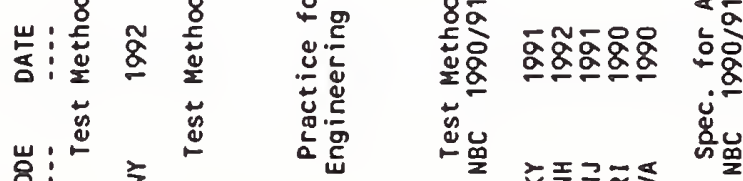

$\overline{\dot{m}} \overline{\dot{m}} \overline{\dot{m}} \overline{\dot{m}} \bar{m}$

年

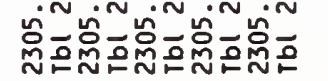

产

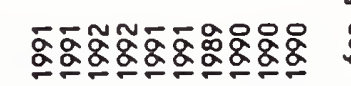

8

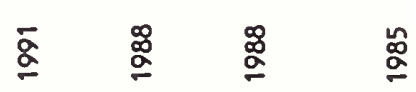

$\stackrel{\mathbb{\Xi}}{2}$

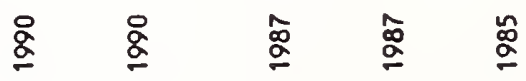

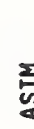

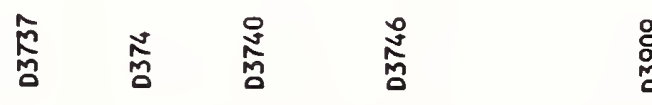

高

登 


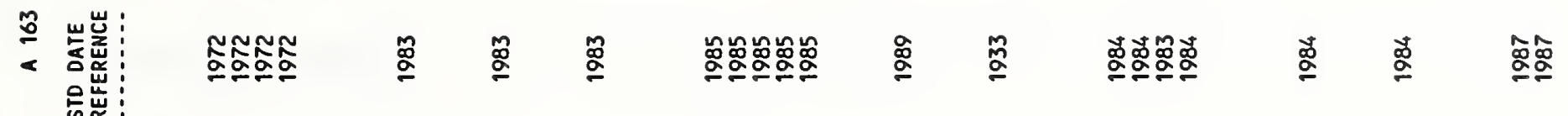

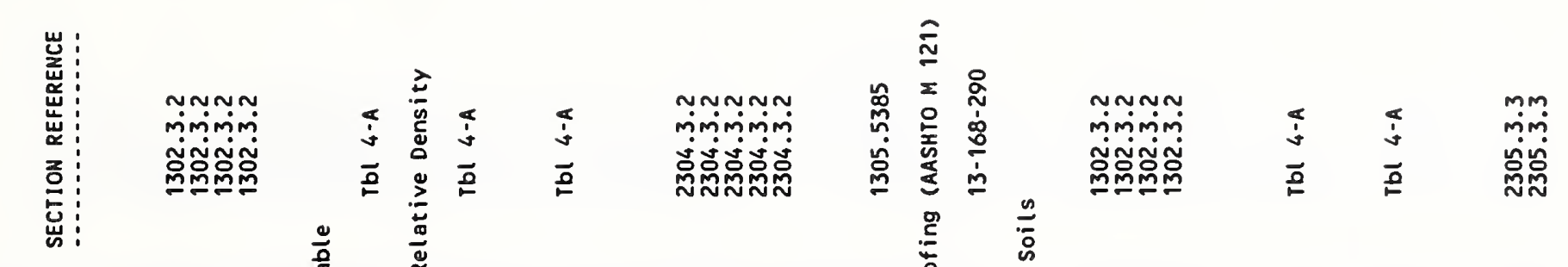

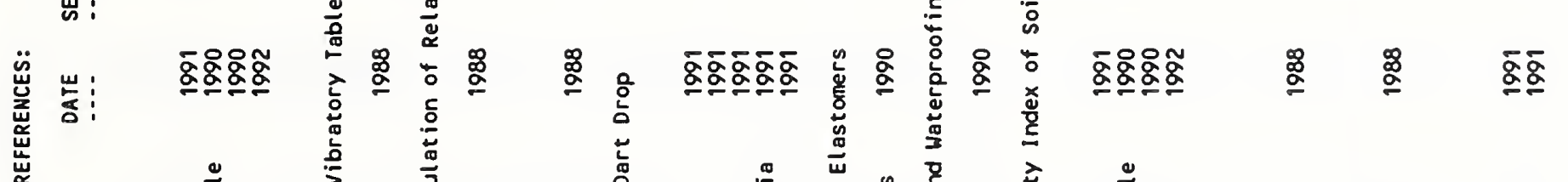

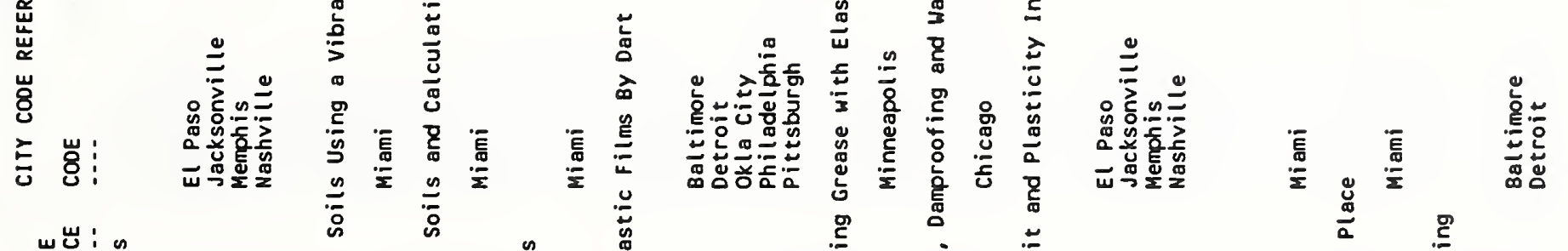

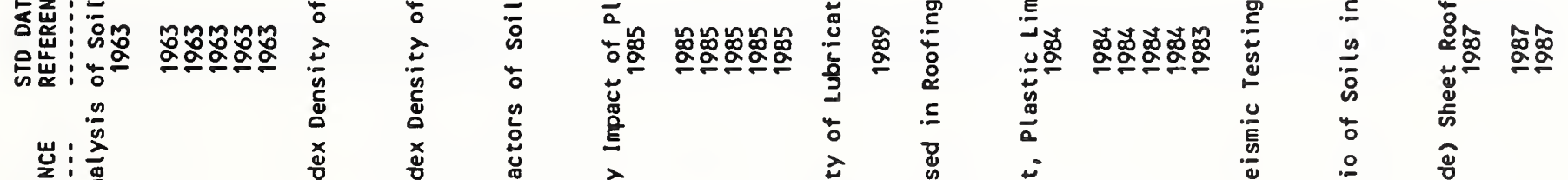

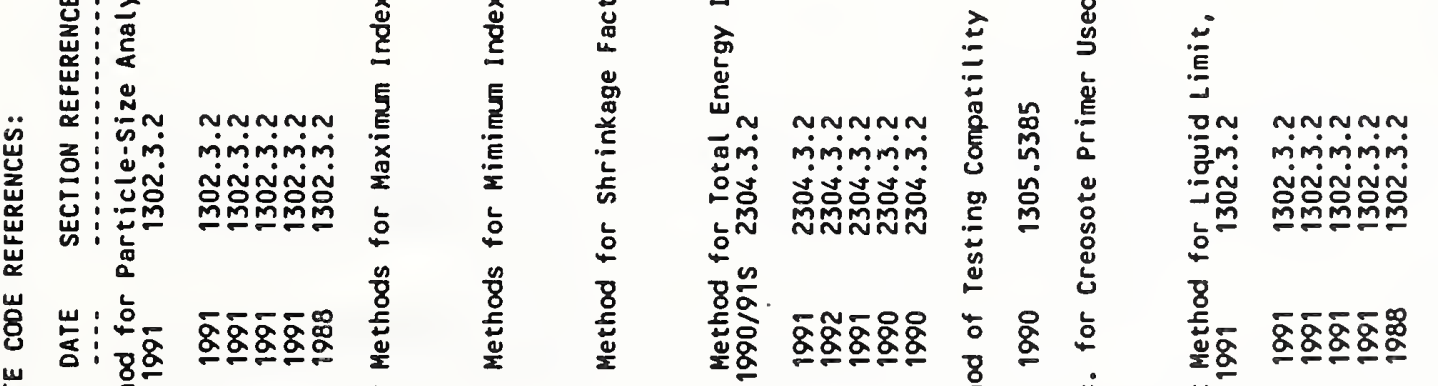

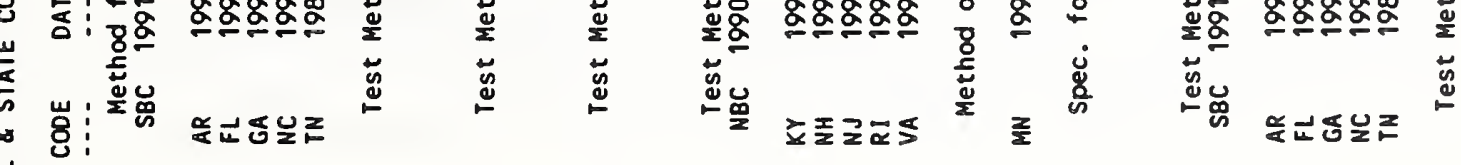

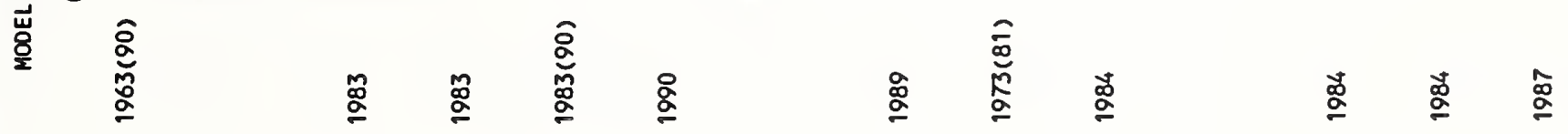

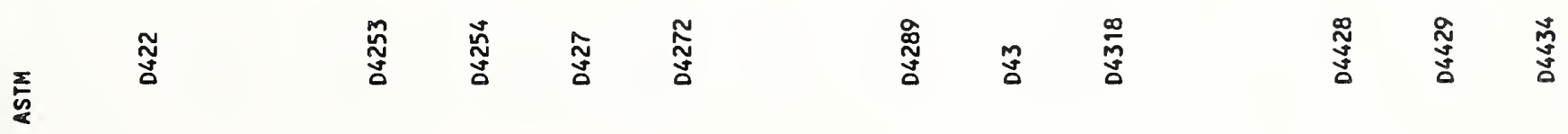




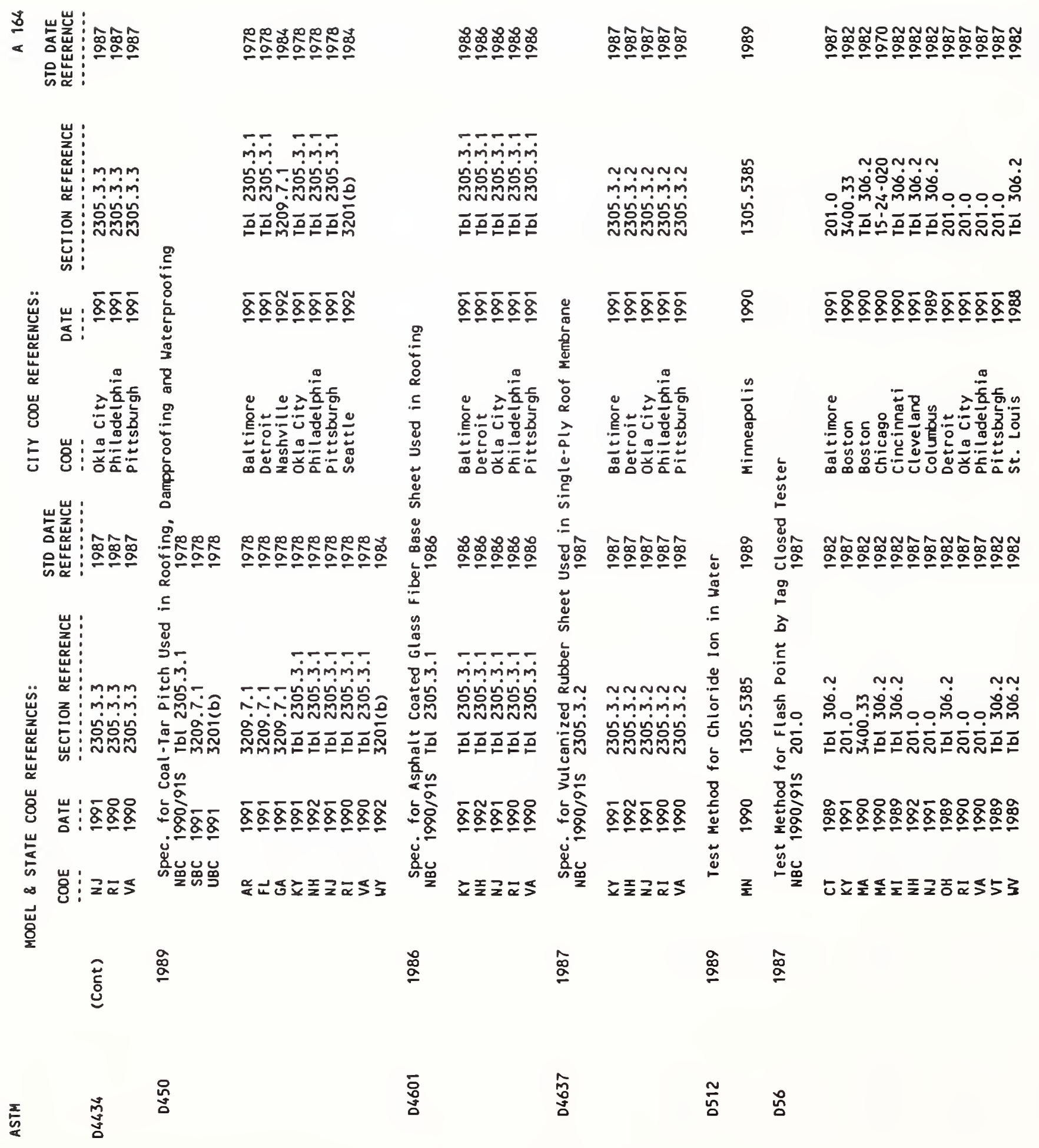




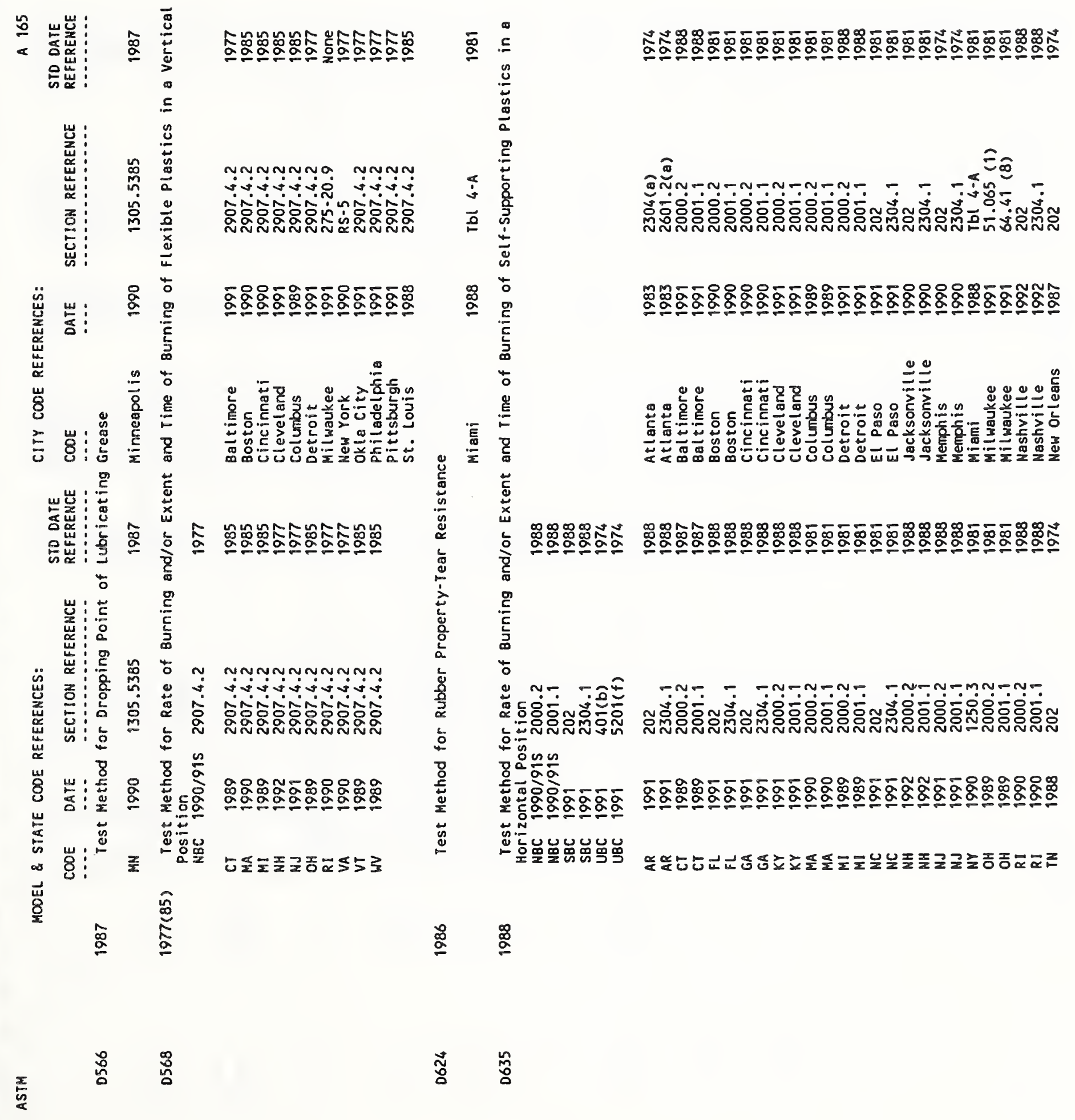




\section{\&}

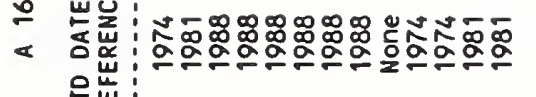

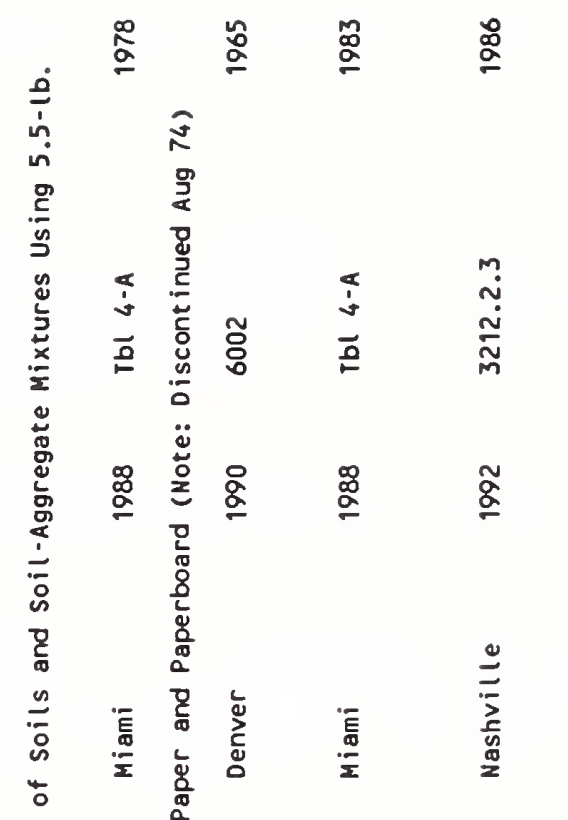

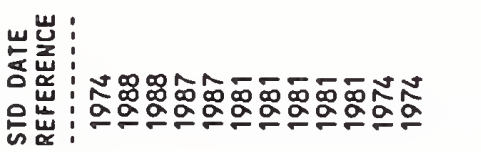

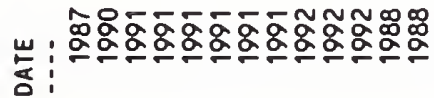

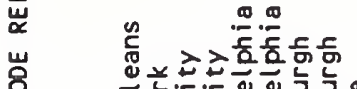

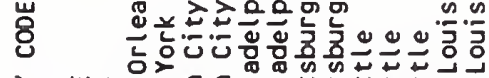

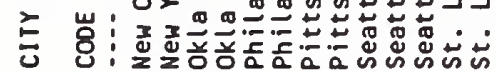

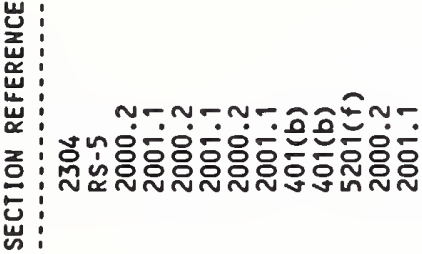

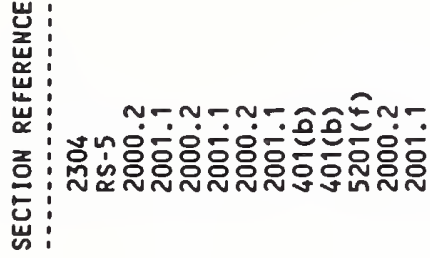

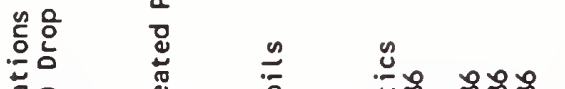

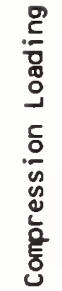

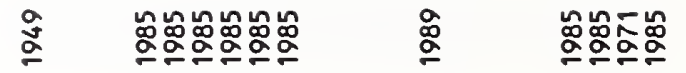

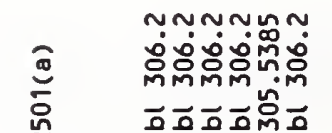

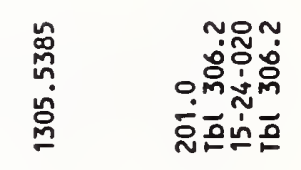

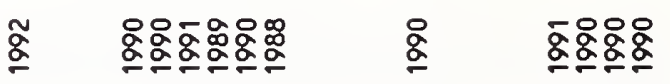

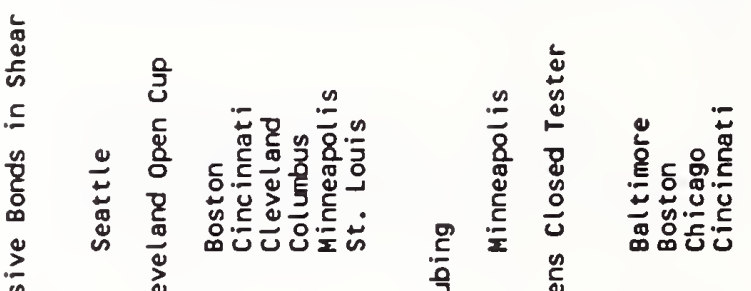

:

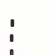

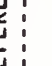

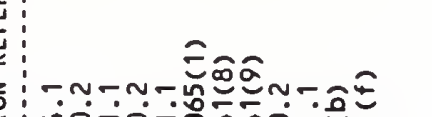

ํํㅇㅇㅇㅇㅇ

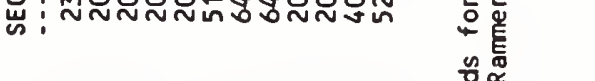

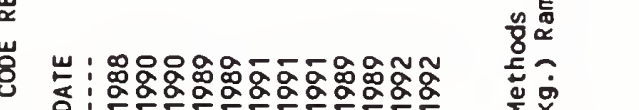

感

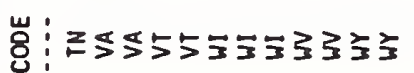

焉

岁
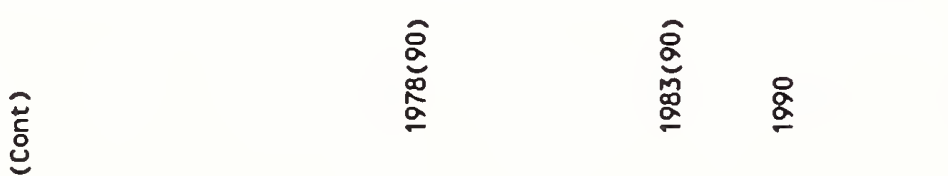

$\stackrel{\circ}{\circ} \stackrel{\circ}{\circ}$

$\stackrel{\circ}{\circ} \stackrel{\circ}{\circ}$

焉管

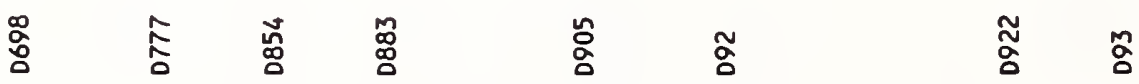




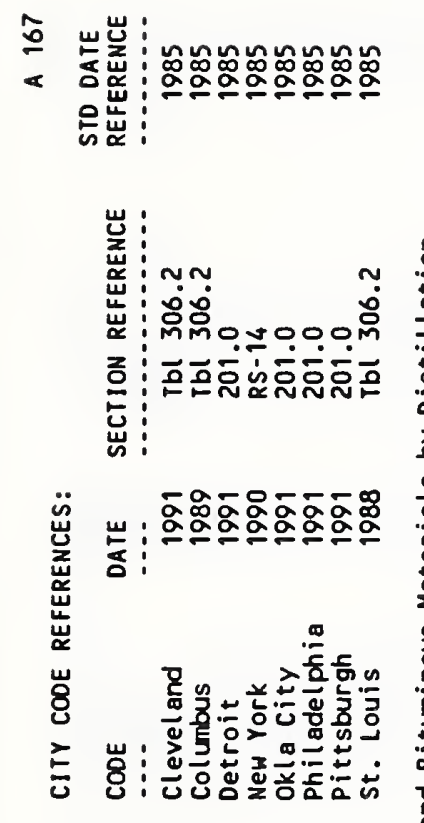

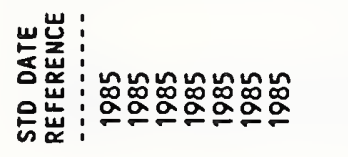



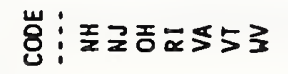

芒

高哀

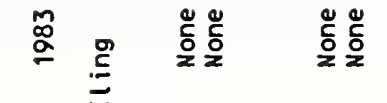

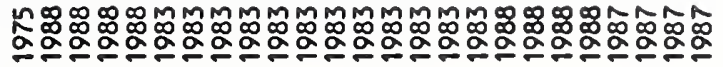

誉商商商商

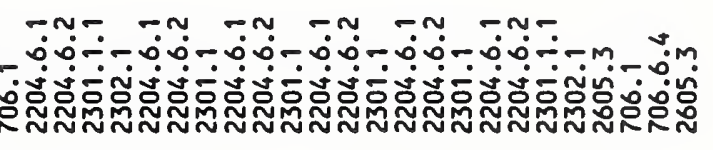

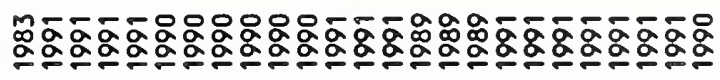

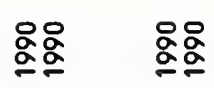

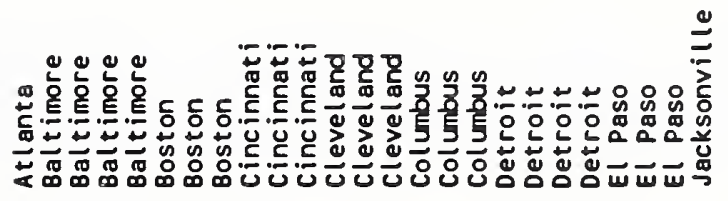

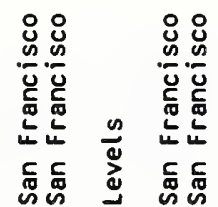

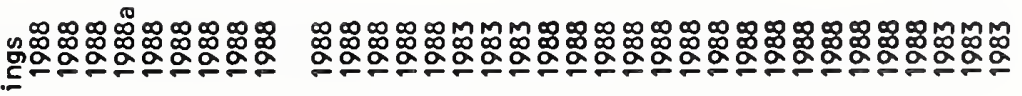

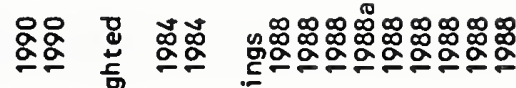

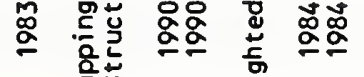
㝘 范葶

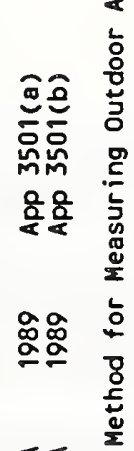
ธธ์

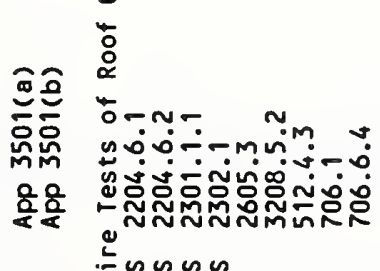

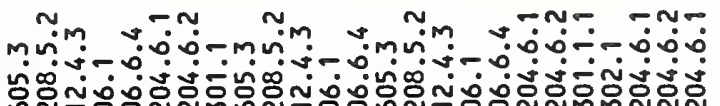

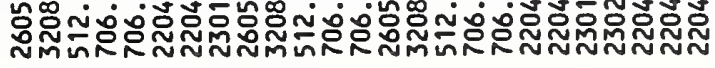
$5 \frac{5}{2} \frac{0}{2} \frac{n}{2} \frac{n}{2}$

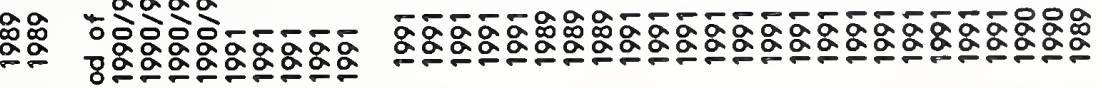

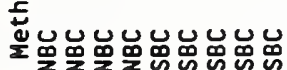

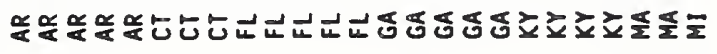

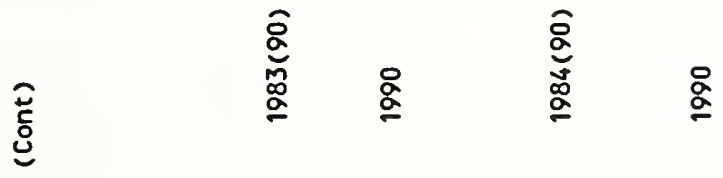

ڤ 


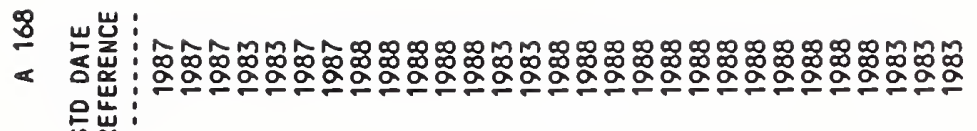

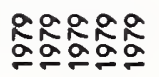

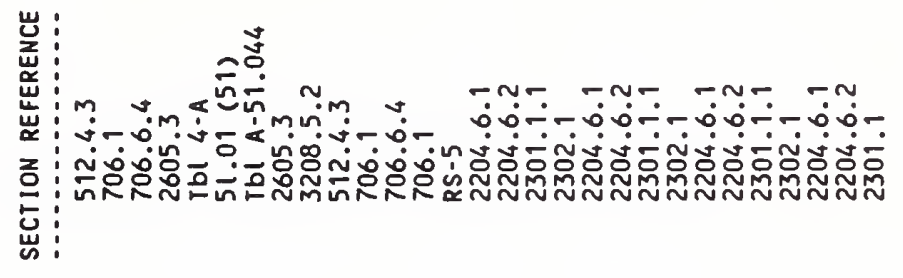

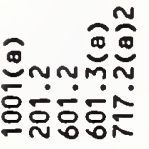

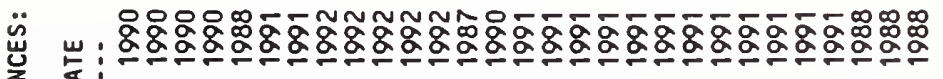

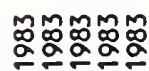

ธ。:

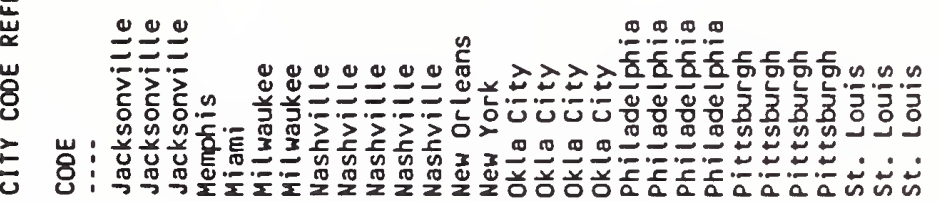

шّ느 :

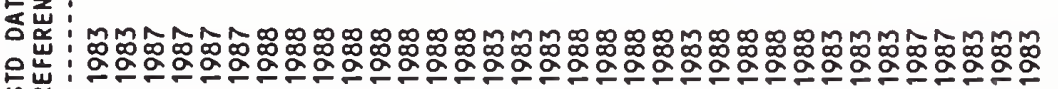

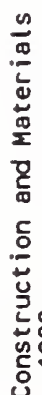

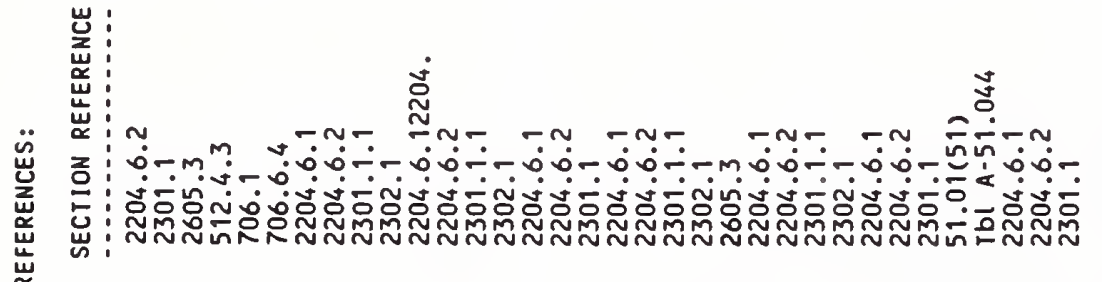

:

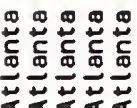

我

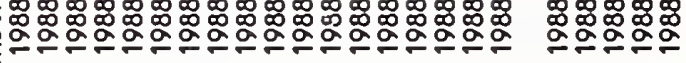

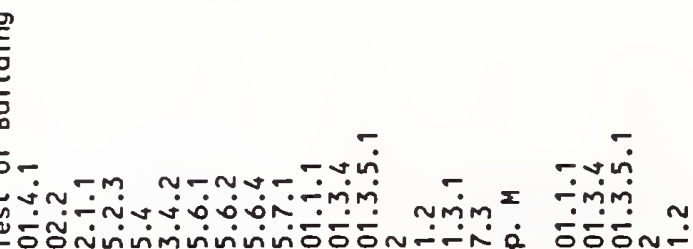

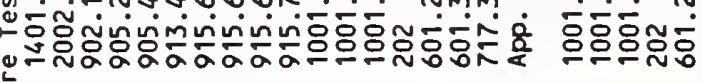
Lnesusensuses

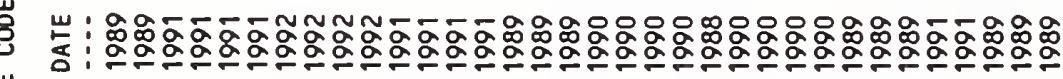

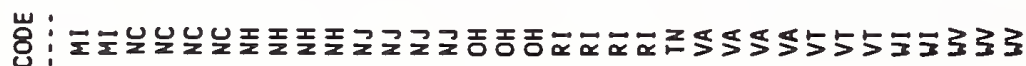

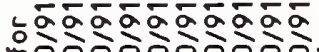

خ

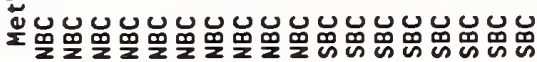

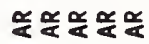

$\stackrel{\Xi}{0}$

$\stackrel{\infty}{\frac{9}{4}}$

$\frac{a}{w}$ 


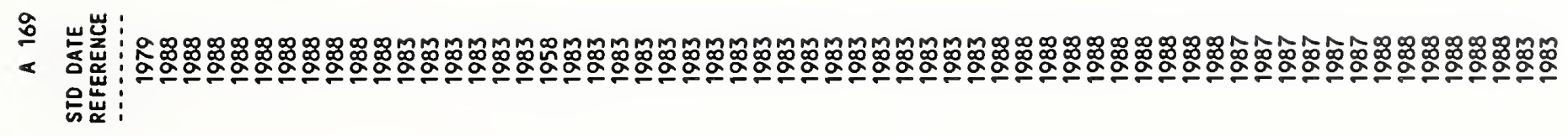

빤

要

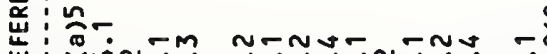

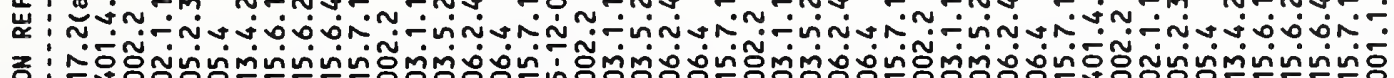

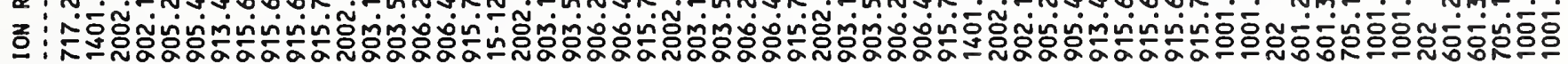
:

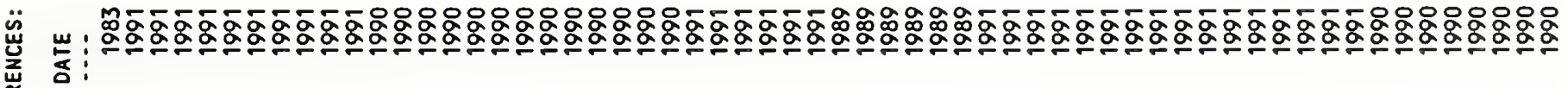

$\stackrel{4}{\propto}$

岁

$\stackrel{2}{=}$

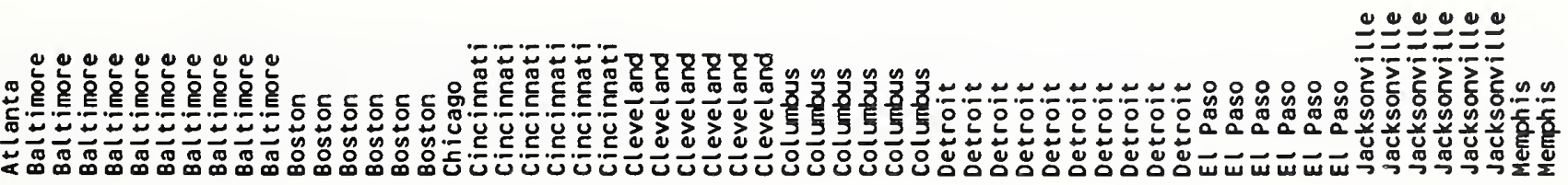

닌

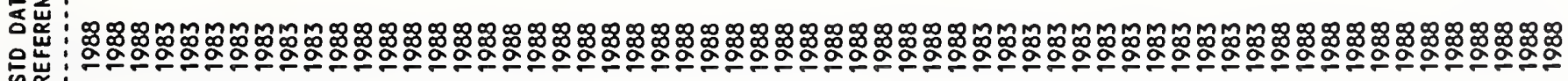
.

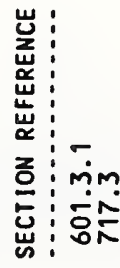
岩

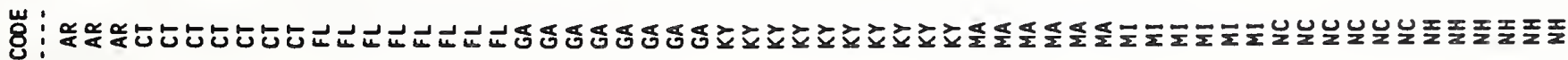

它

紊

$\frac{a}{i}$ 

䢘崖

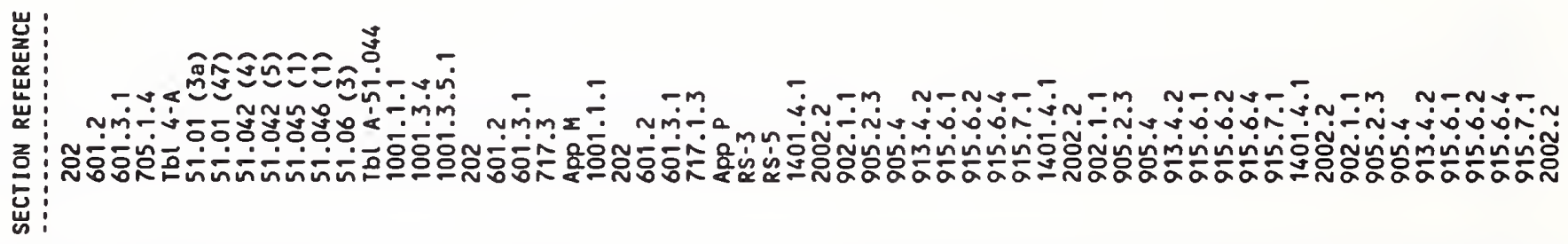

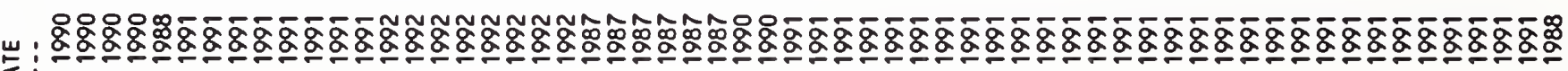
:

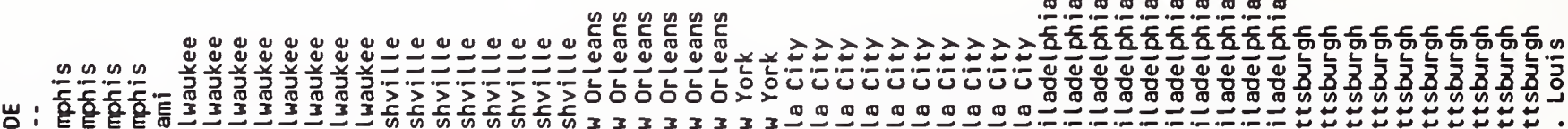
岁

w는

代

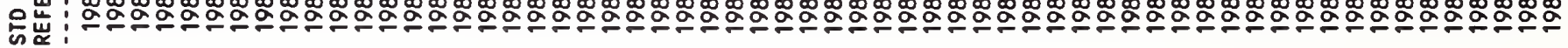

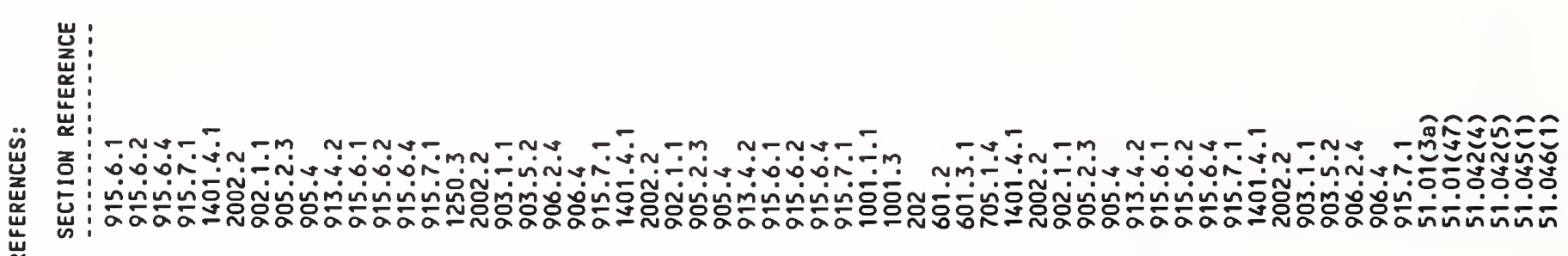

岩

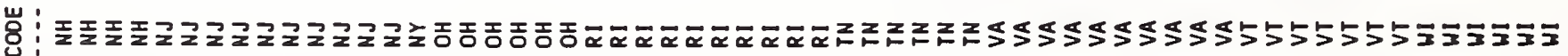

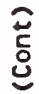




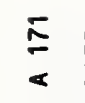
mamma 造宸:

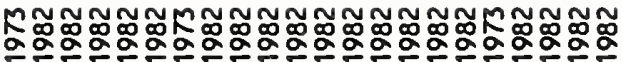

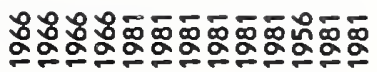

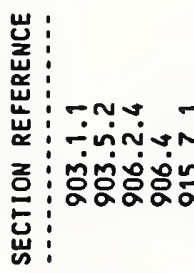
苞

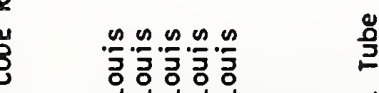

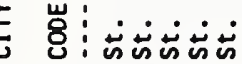

닌 :

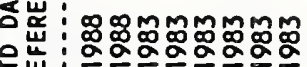

은

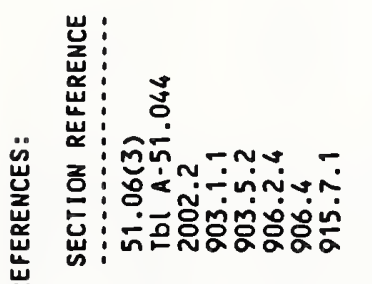

峞

8

崖

$\infty$

㟧

岁:

产

ळ

$\stackrel{m}{m}$

妾

$\frac{a}{w}$

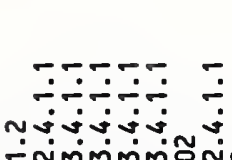

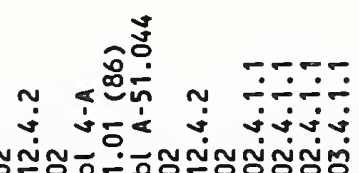

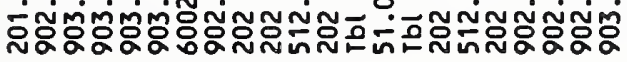

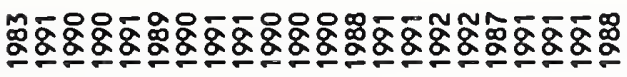

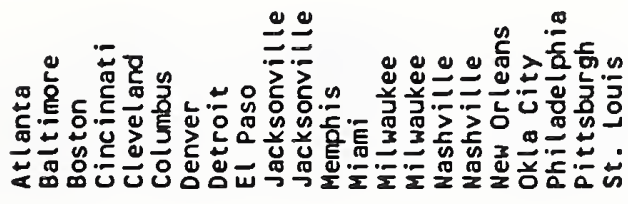

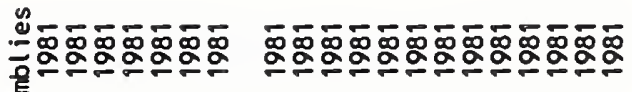

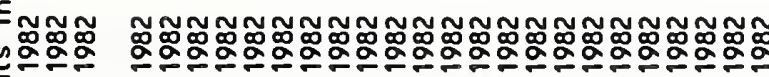
$\stackrel{\square}{\frac{1}{2}}$

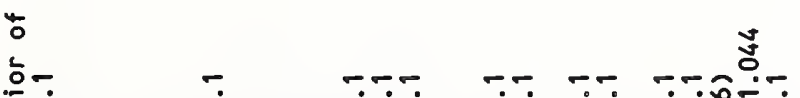

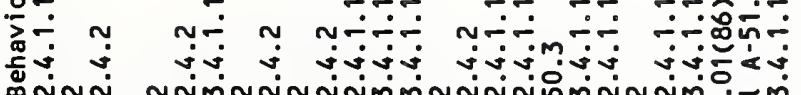

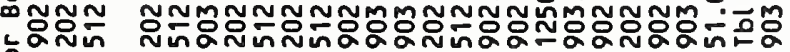

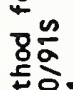

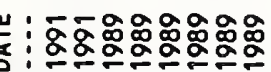

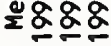

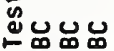

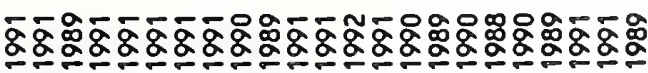

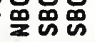

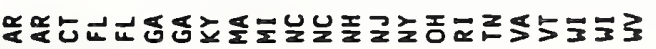

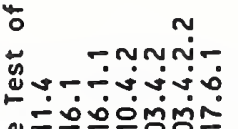

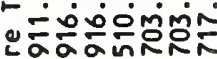
는

능응

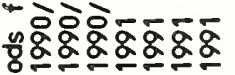

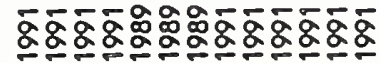

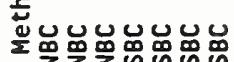

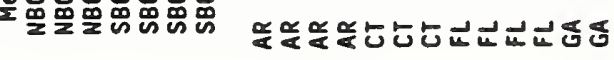

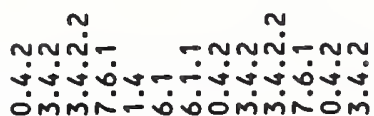

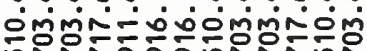

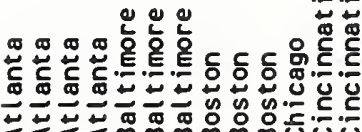

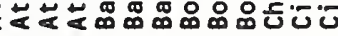

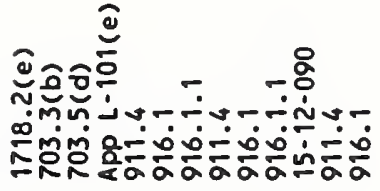

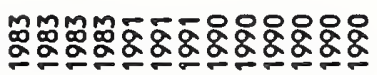

$\stackrel{\infty}{\infty}$

$\underset{\text { กิ }}{\tilde{m}}$ 
$\cong$

崖岕:

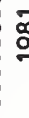

:

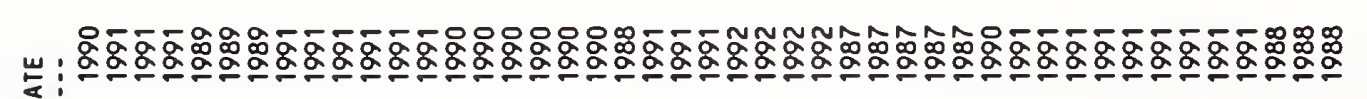
和:

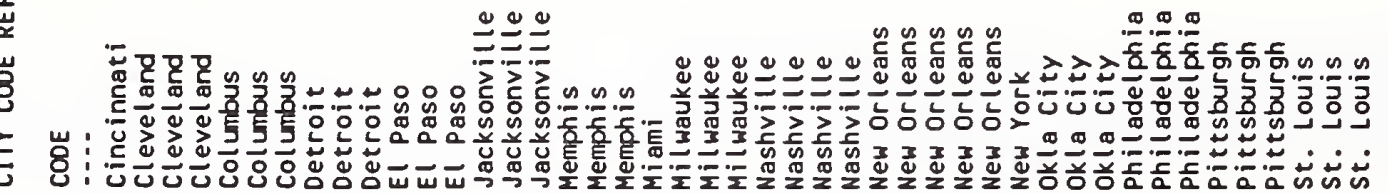

넌

顿

空岩:

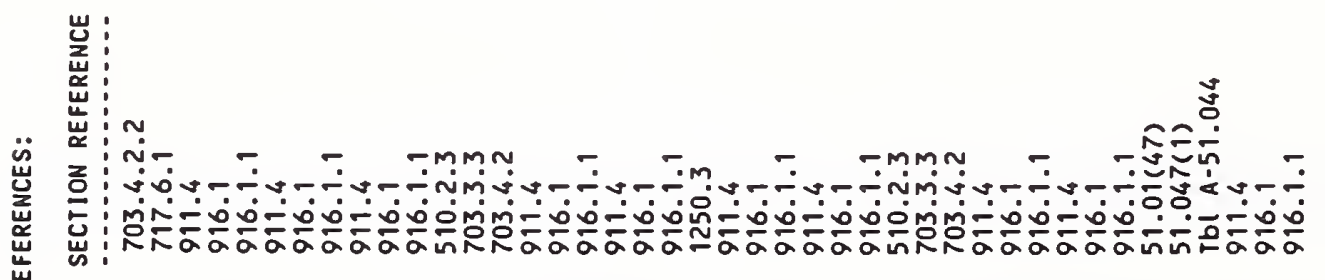

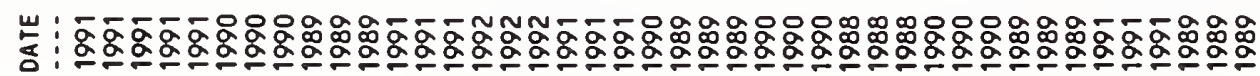

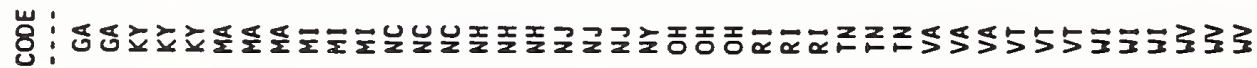

$\stackrel{8}{2} \stackrel{8}{2}$

$\frac{2}{2}$

$\tilde{\dot{s}}$

蒿

ì

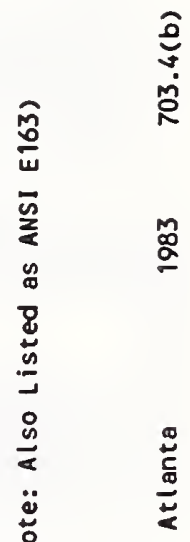

茫

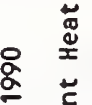

은

(2)

는

旁

.

¿ \& $\frac{7}{8}$ $\div$ \& 우ำ ํํㅇ 는

8
$\frac{5}{2}$
$\frac{0}{2}$ 
뜬

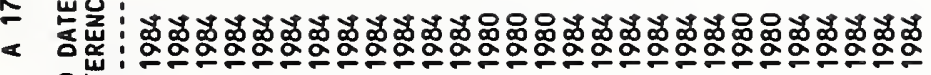

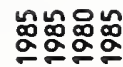

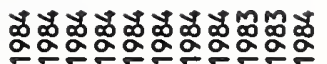

:

$\varepsilon=\frac{100}{20}$

z

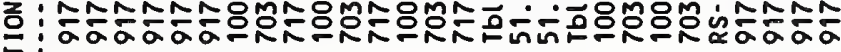

总:

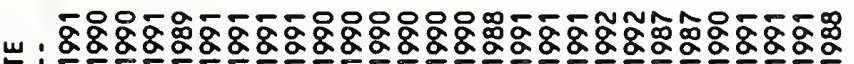
:

菂

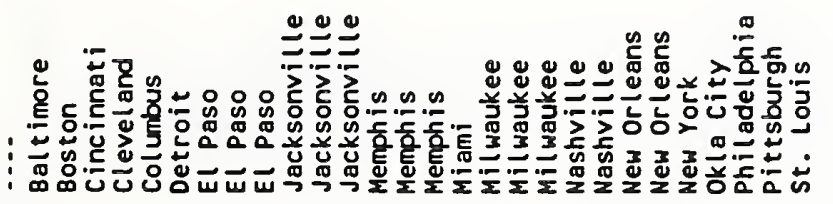

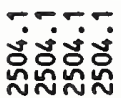

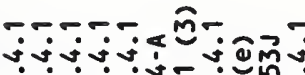

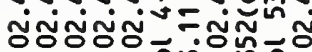

monmonis

(a)

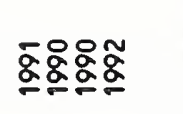

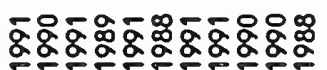

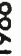

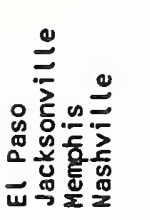

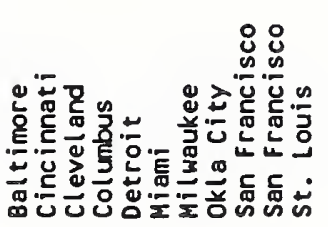

岧

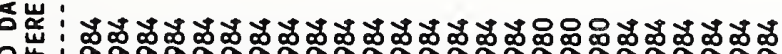

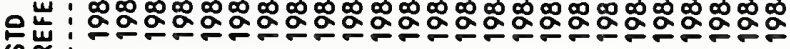

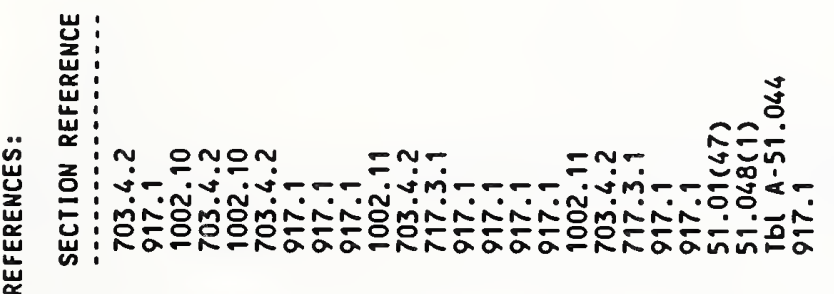

岁

岕

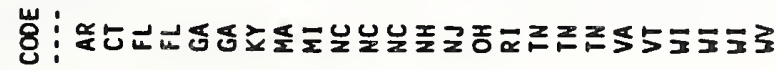

옹

$\underset{\frac{5}{n}}{\text { mo }}$

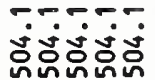

กำ

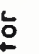

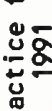

递品

茴

딴전존

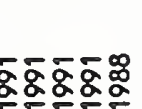

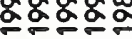

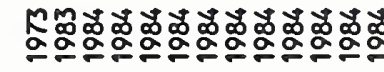

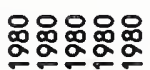

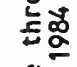

马ूर

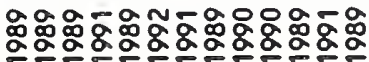

nُ

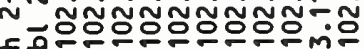

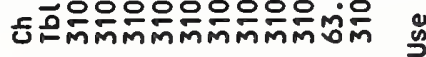




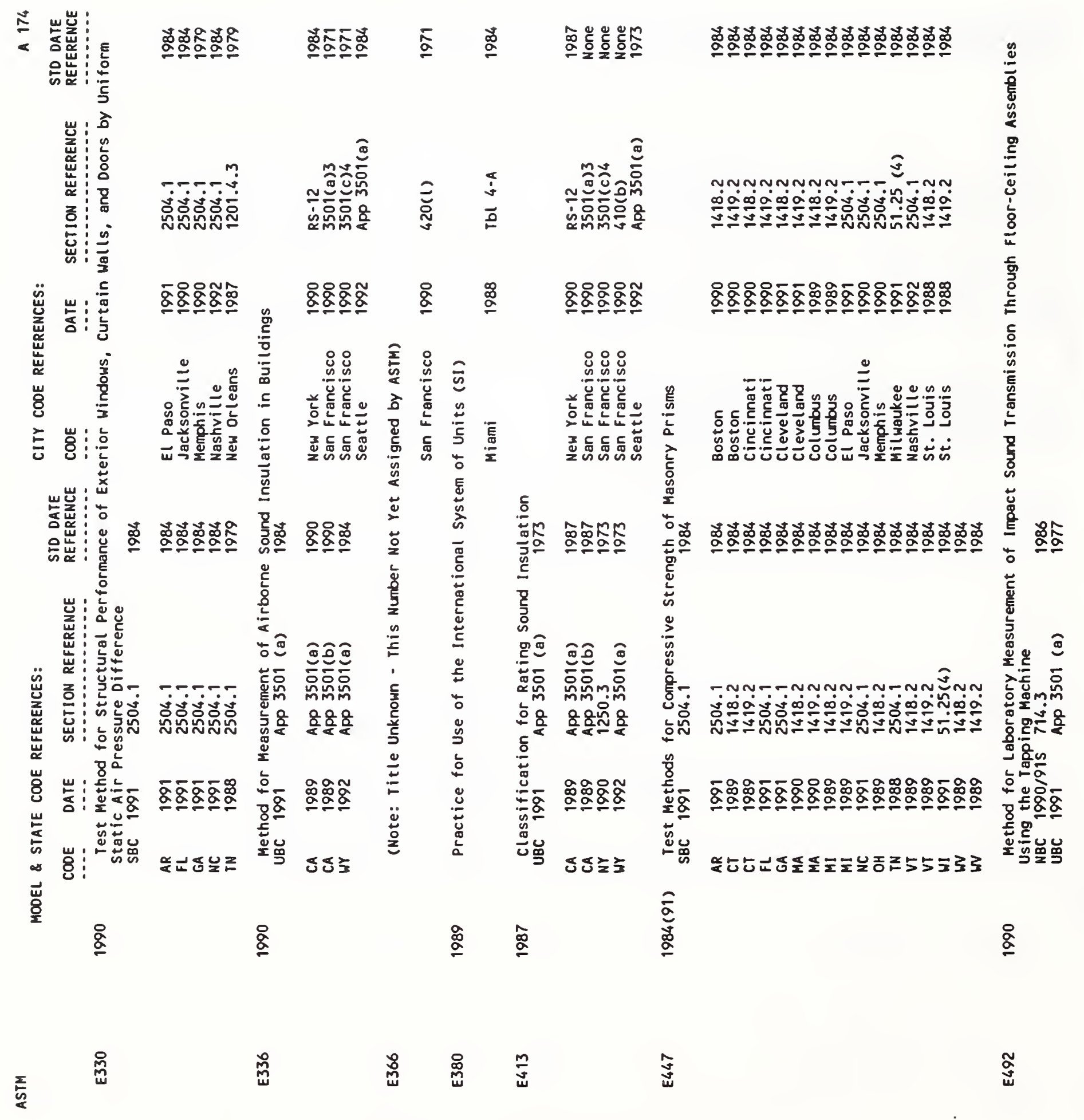



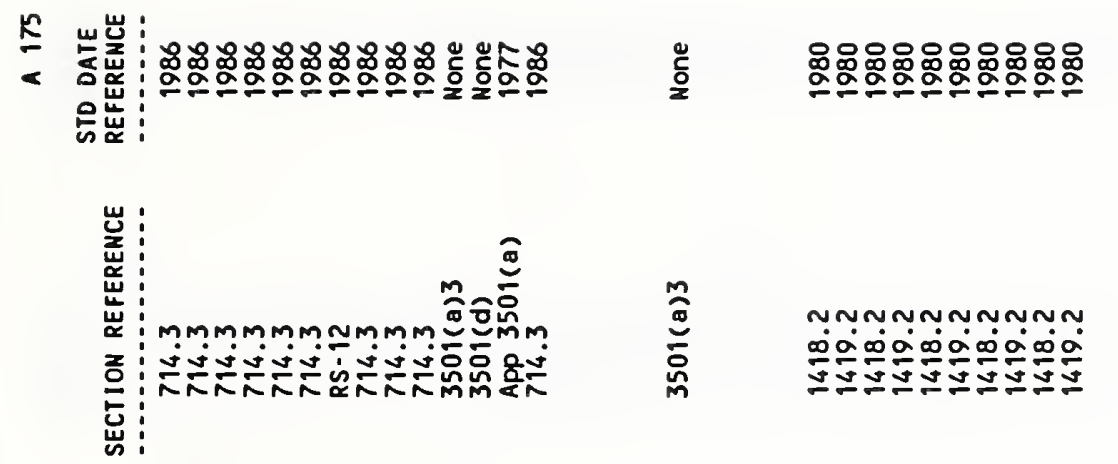

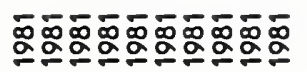

器器

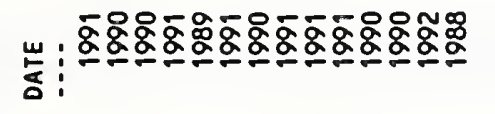

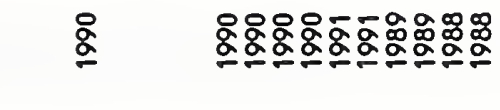

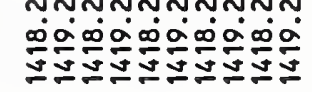

กกบกNกNบNก

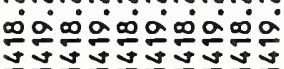

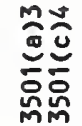

๙ิ

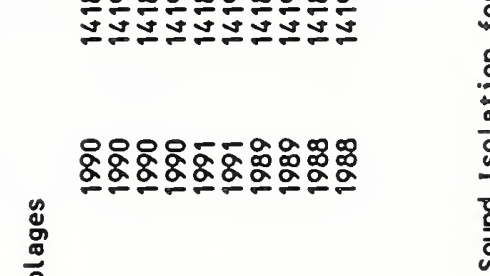

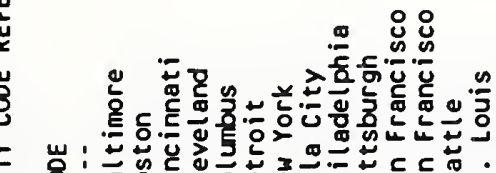

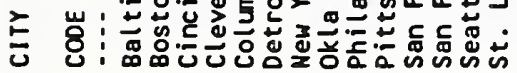

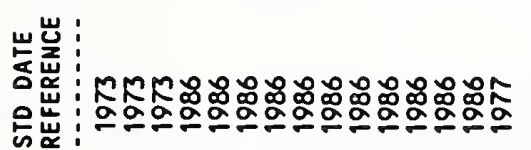

岁:

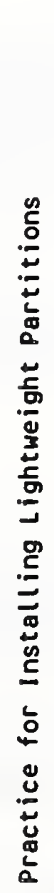

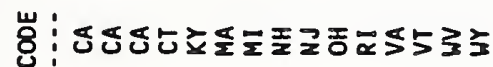

总

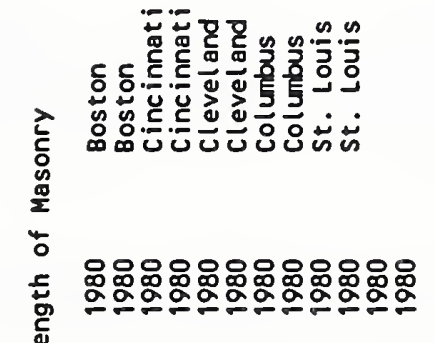

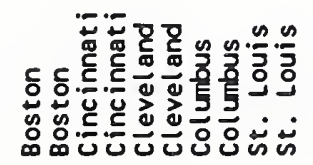

通

产

立立

覒点

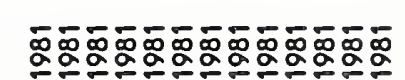

פ흐

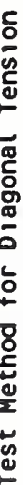

ธธ

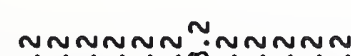

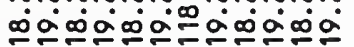

nunnannunnanan

$\infty \dot{\alpha} \omega \dot{\alpha} \omega \dot{0} \omega \dot{\alpha} \omega \dot{\alpha} \omega \dot{\alpha}$

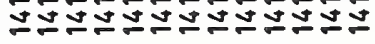

目婁

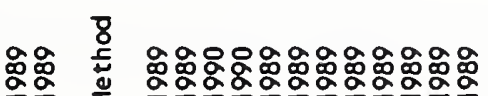

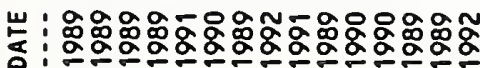

핼

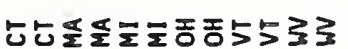

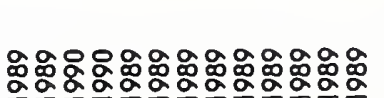

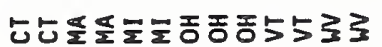

区ভ

范

$\stackrel{\text { ă }}{\circ}$

$\frac{a}{\stackrel{0}{0}}$

오을ํํำ

울울

웡

영

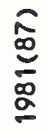

命

$\frac{a}{n}$

忩

$\underset{⿱ 亠 乂}{\tilde{w}}$ 


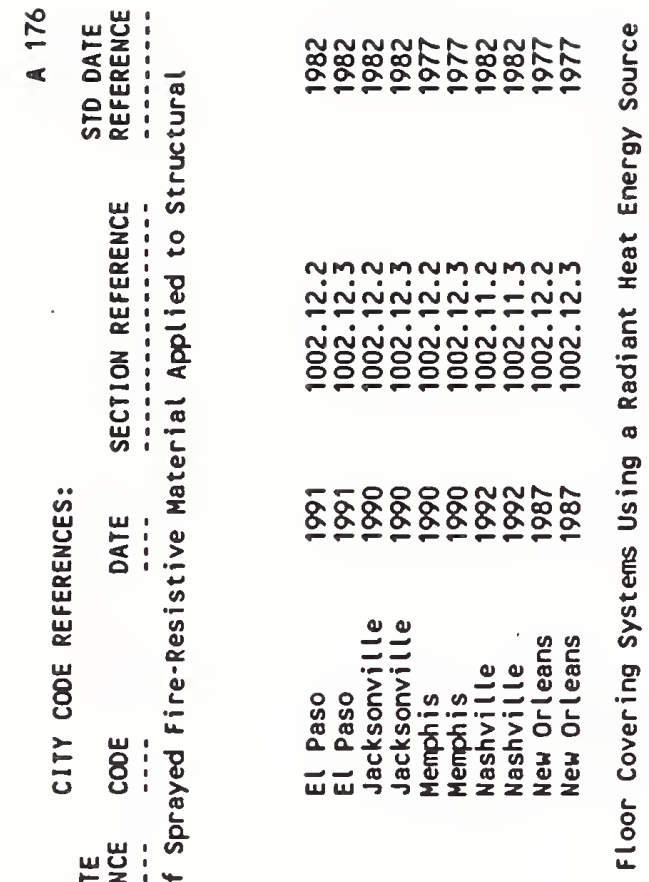

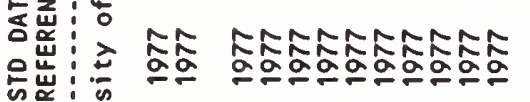

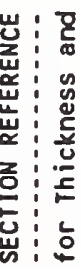

岁: $: \frac{0}{\delta}$

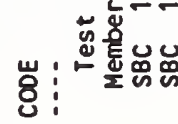

$\stackrel{\hat{a}}{\underline{a}}$

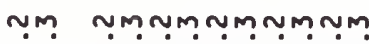

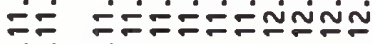

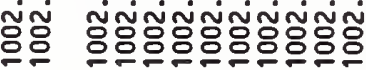

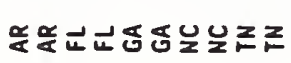

$\bar{\alpha}$

荢

莡

ำ

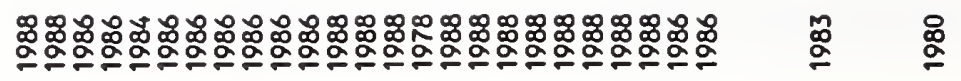

송응

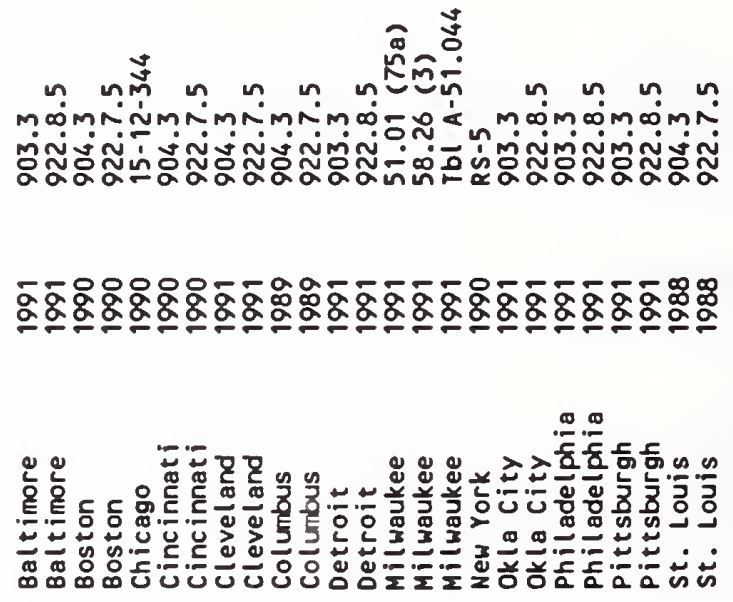

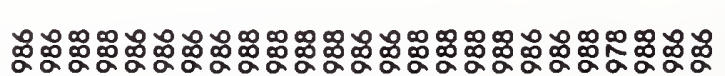

等

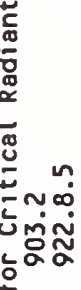

$8 \frac{\pi}{a} \frac{\pi}{a}$

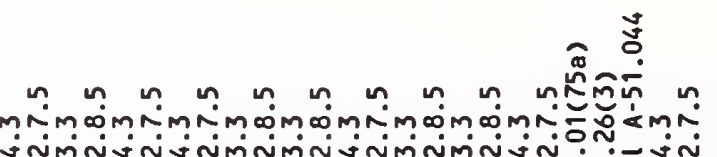

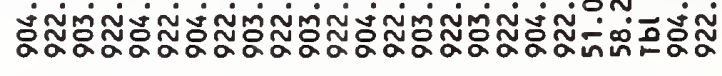

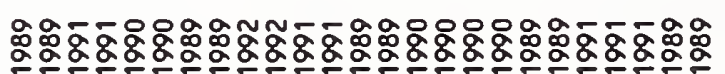

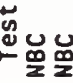

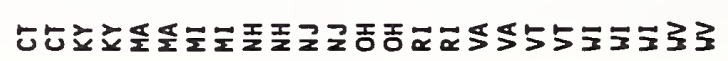

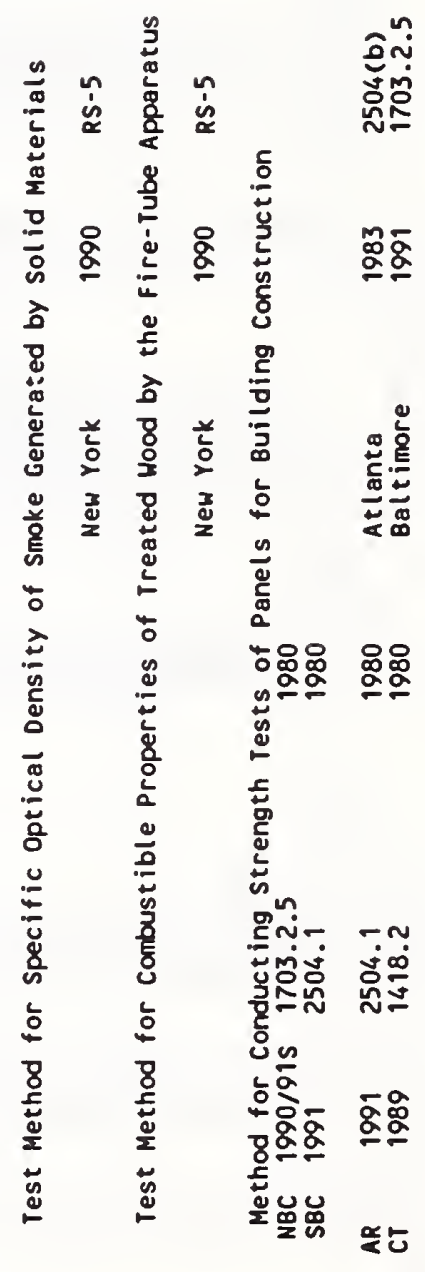

$\stackrel{m}{\stackrel{0}{g}} \stackrel{\circ}{\circ}$

ช్ 
₹

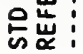

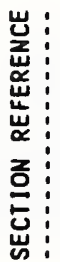
nกm

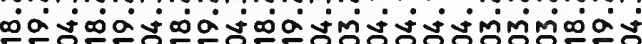
:

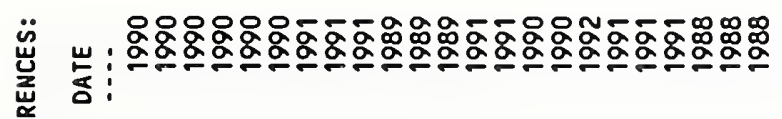

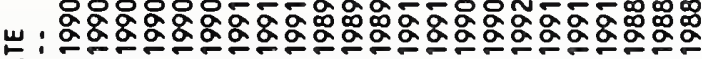
ธั
}

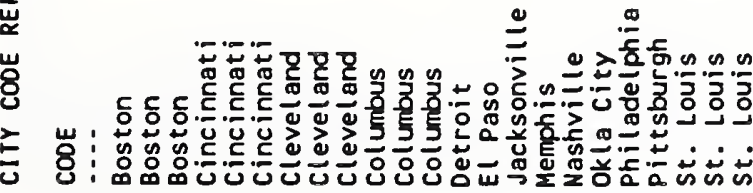

뜬 :

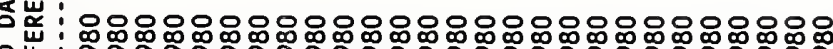

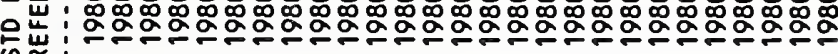

芯

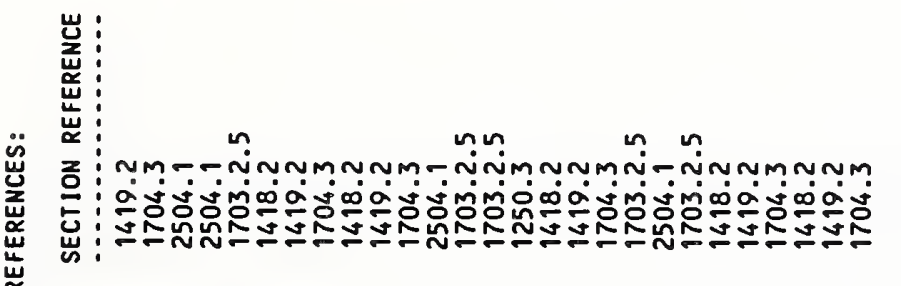

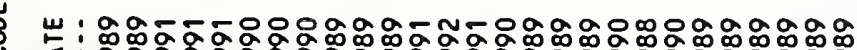

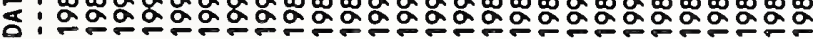

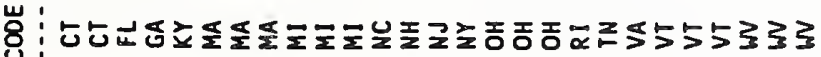

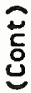

$\underset{\frac{1}{4}}{\frac{\pi}{N}}$

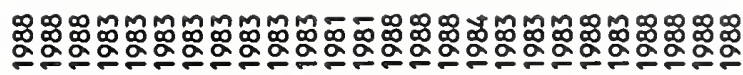

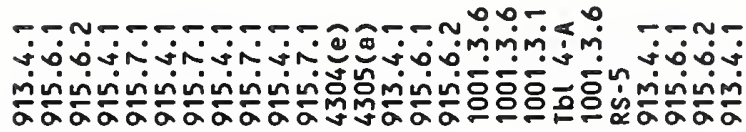

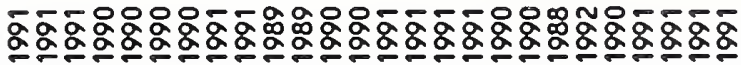

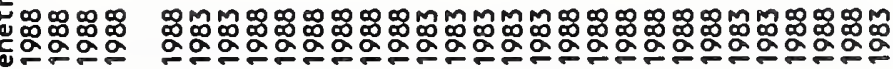
家

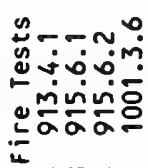

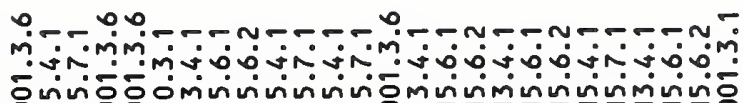

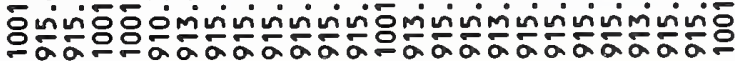
느는

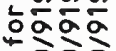
항영음음

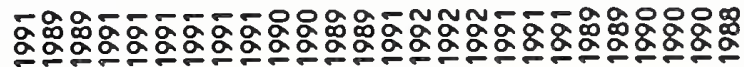

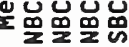

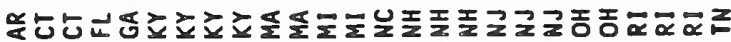




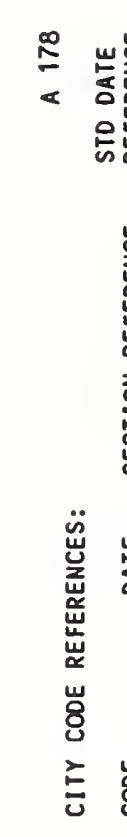
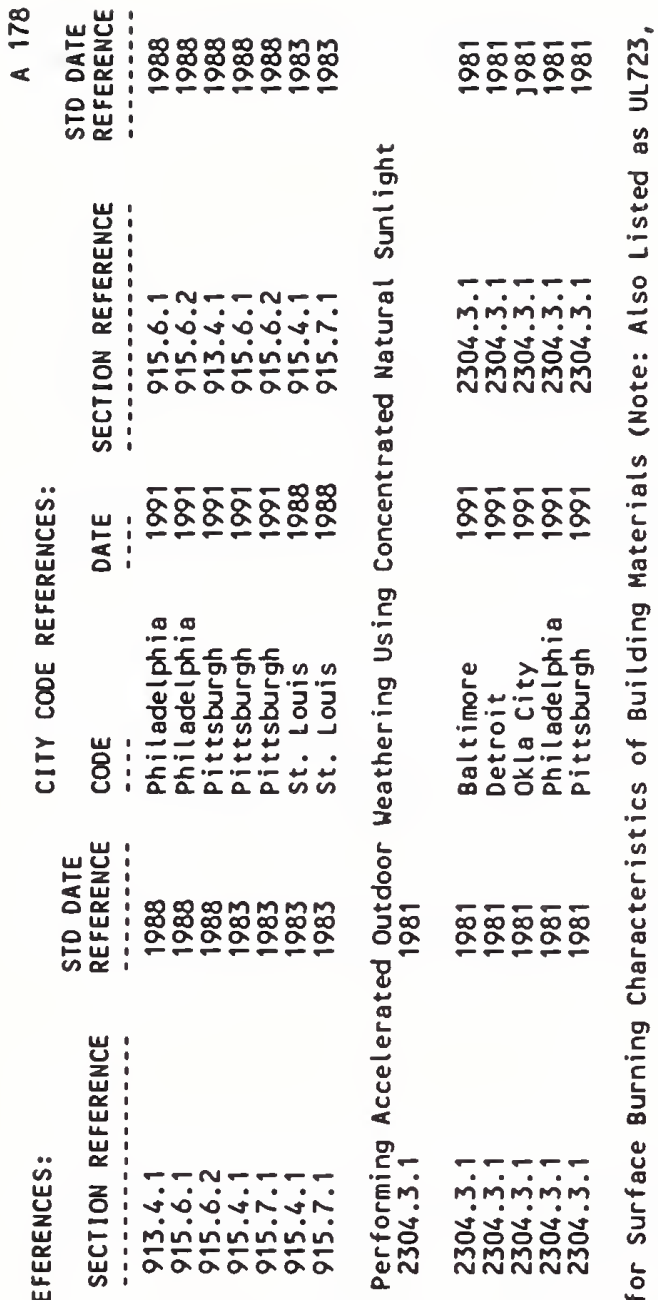

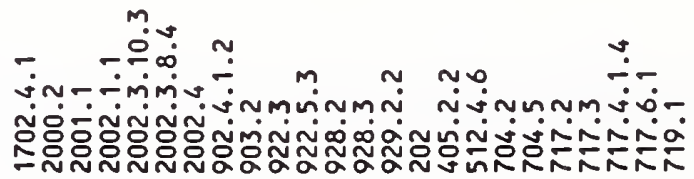

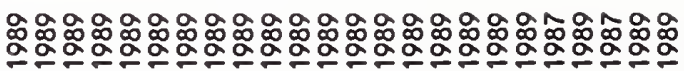

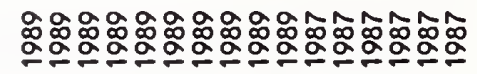

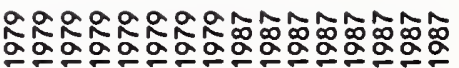

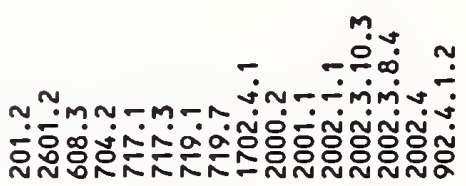

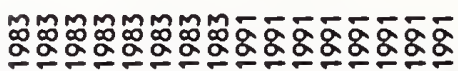

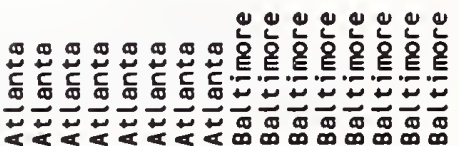

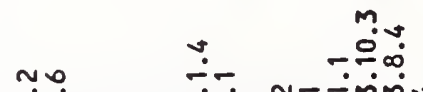

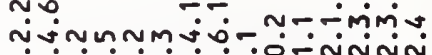

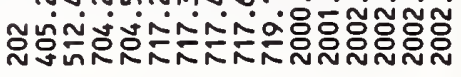

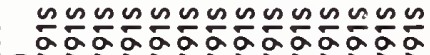

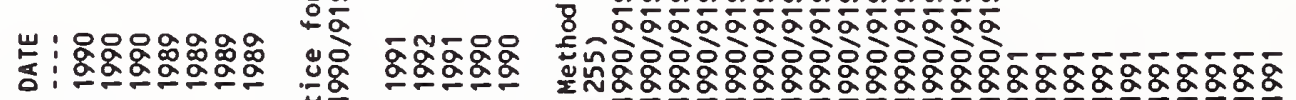
w⿺尢丶

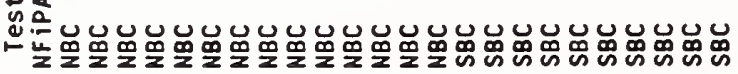

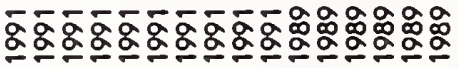

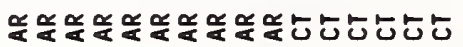




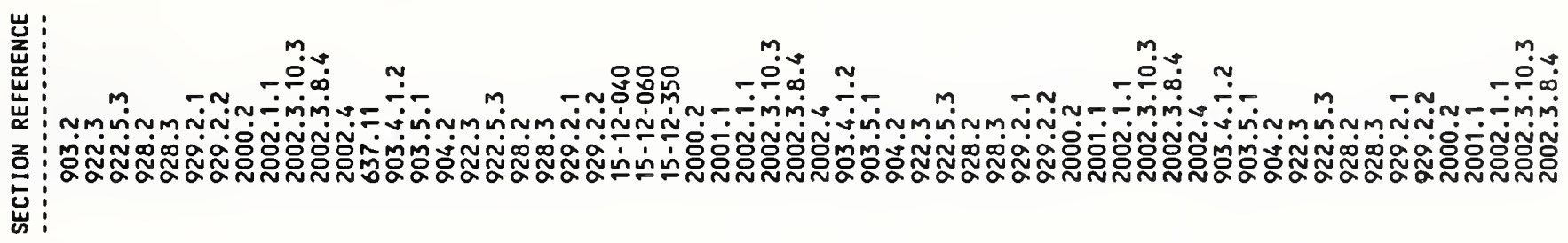

\footnotetext{
䓛
}

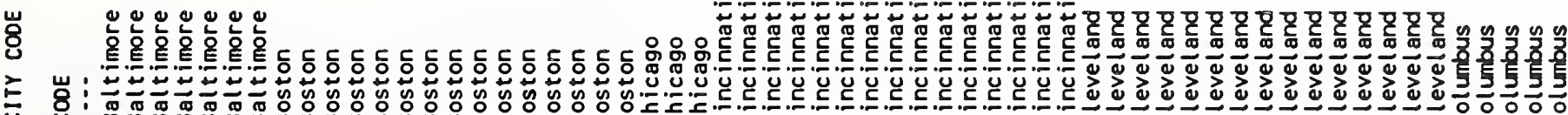

与

논

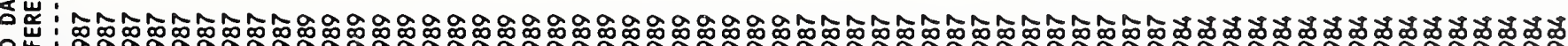
苮

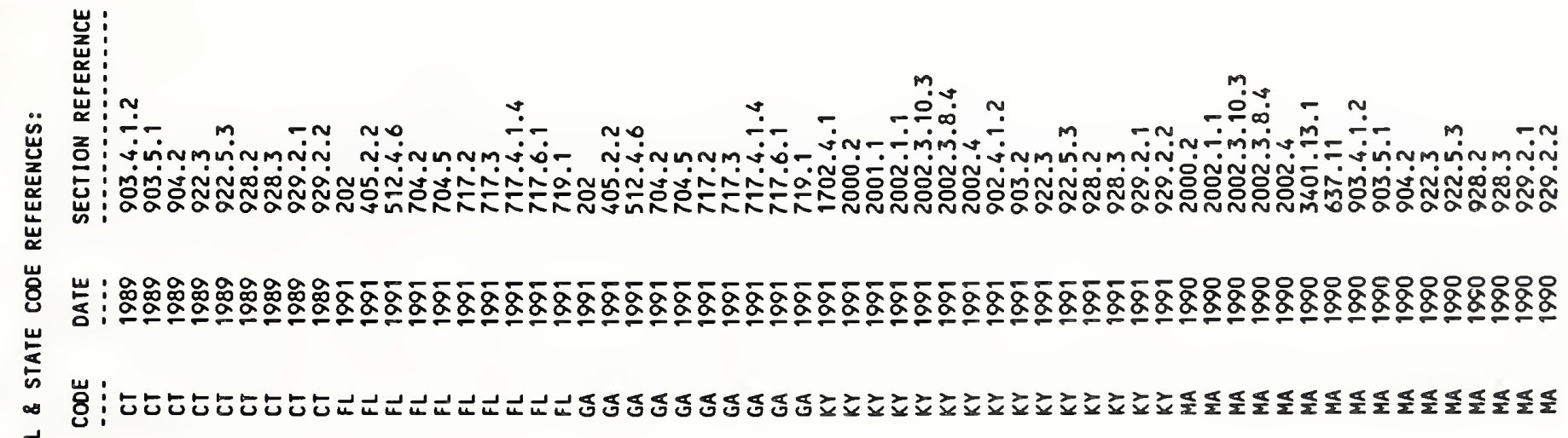

总 


\section{Ф

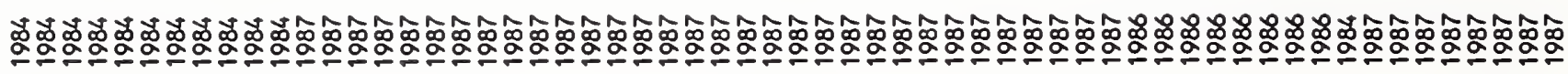 查崖?}

㑾 ш

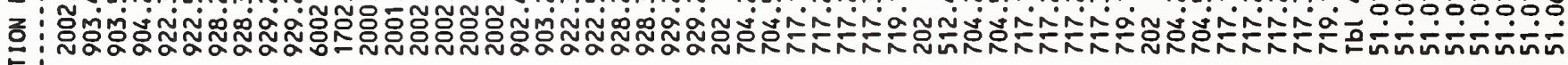
岕:

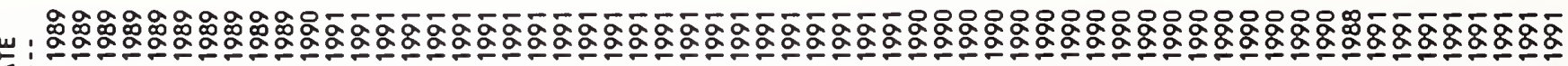
ธ:

떤

崖

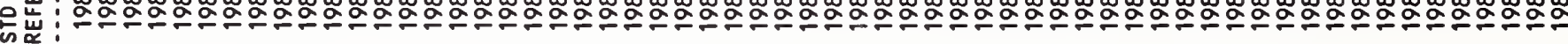

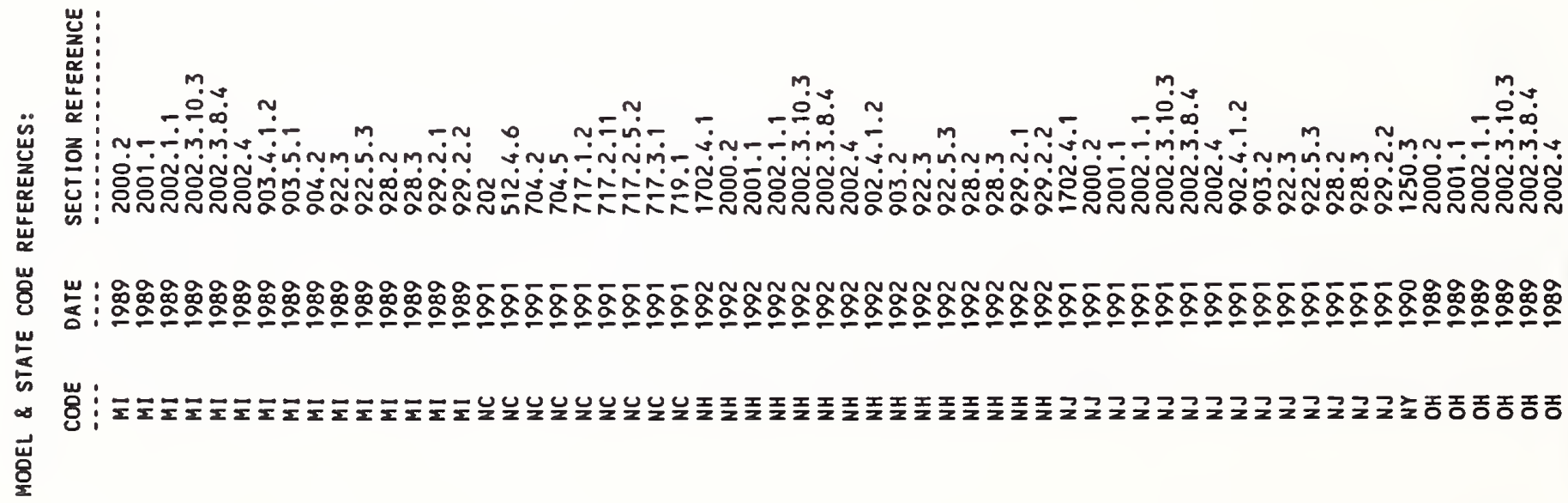

巳ัٌ

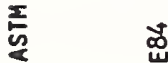



题压?

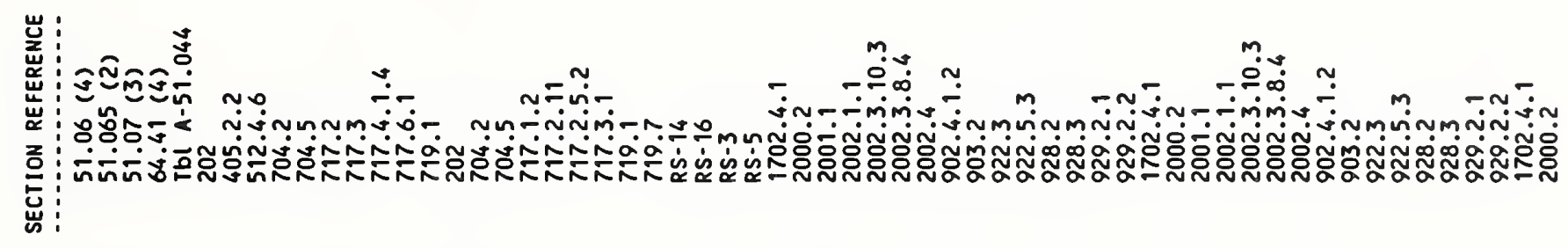

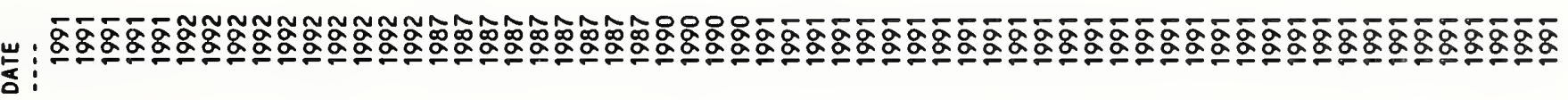

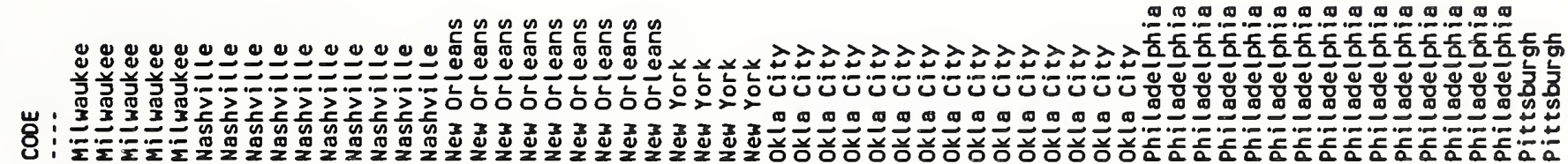
崖空:

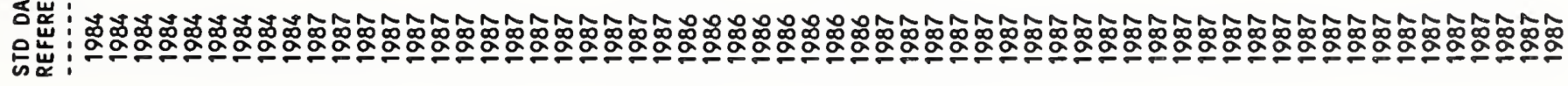

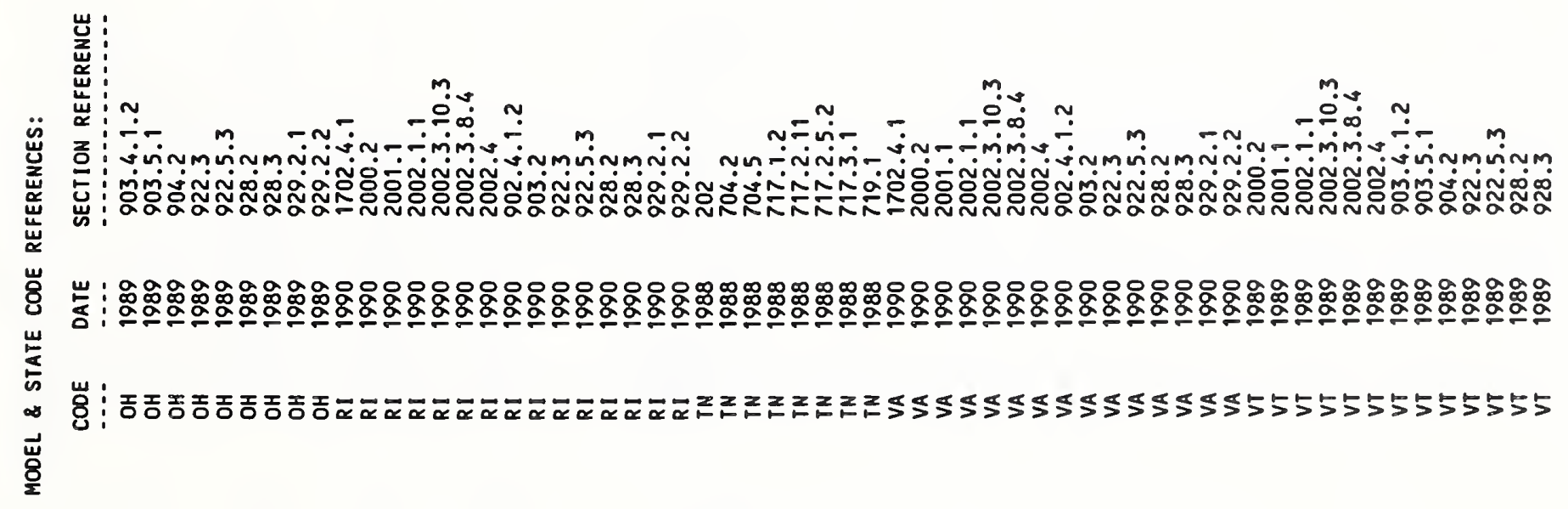

点 


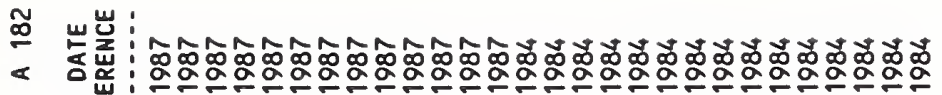

은

岂:

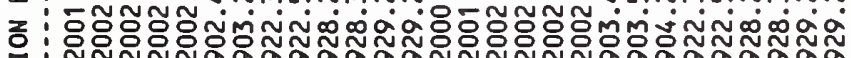

㟧

$$
\text { 芦 }
$$

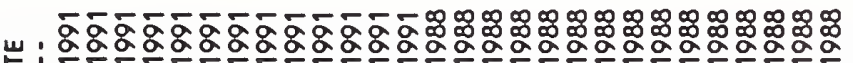
点!

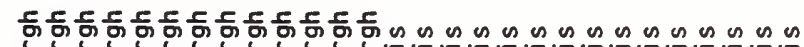

ठั

¿

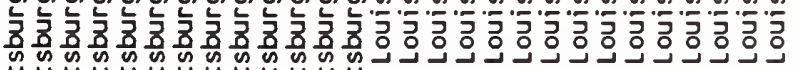

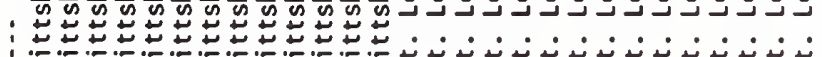

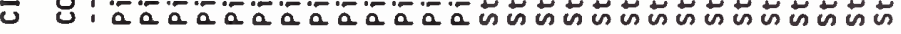

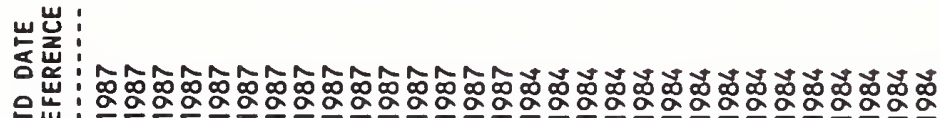
的崖。

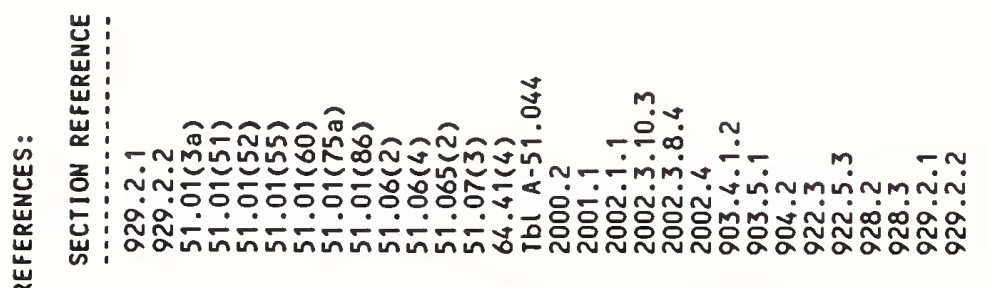

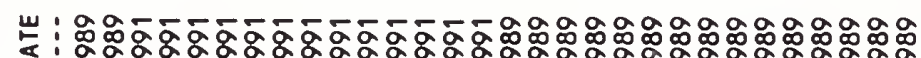

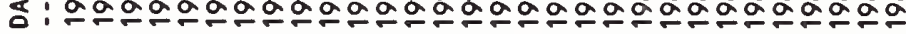

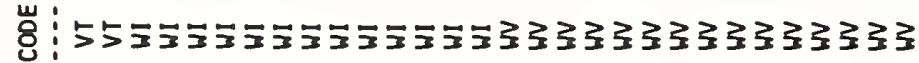

્ْટ

$\underset{5}{\frac{2}{n}}$

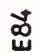

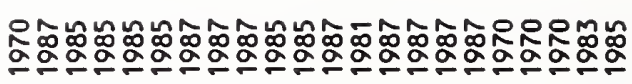

$\frac{2}{2}$

$\frac{9}{\frac{9}{3}}$

ֻั)

$\frac{8}{6}$

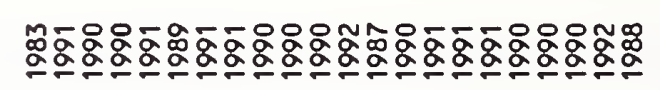

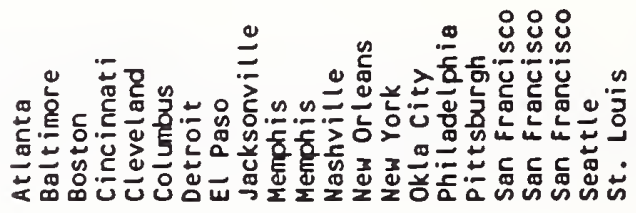

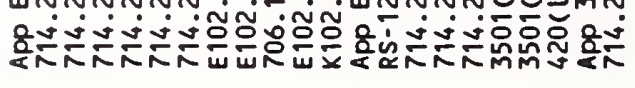

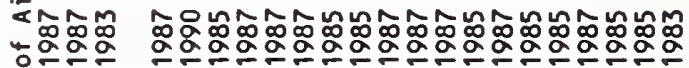

芒

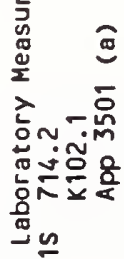

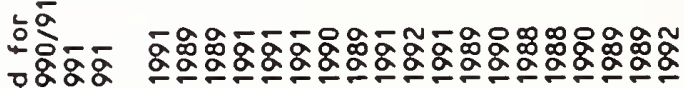

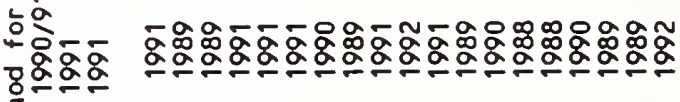

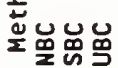

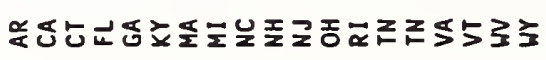

$\stackrel{8}{\circ}$

$\stackrel{8}{8}$

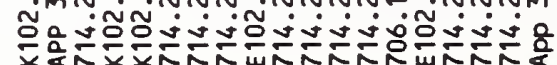




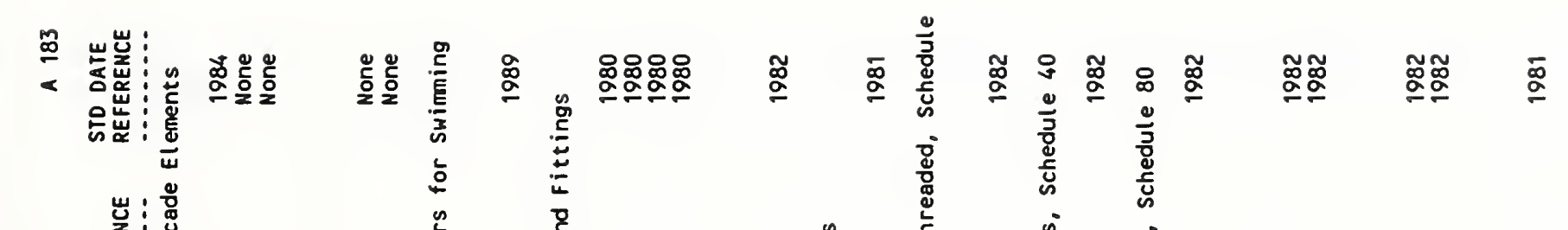

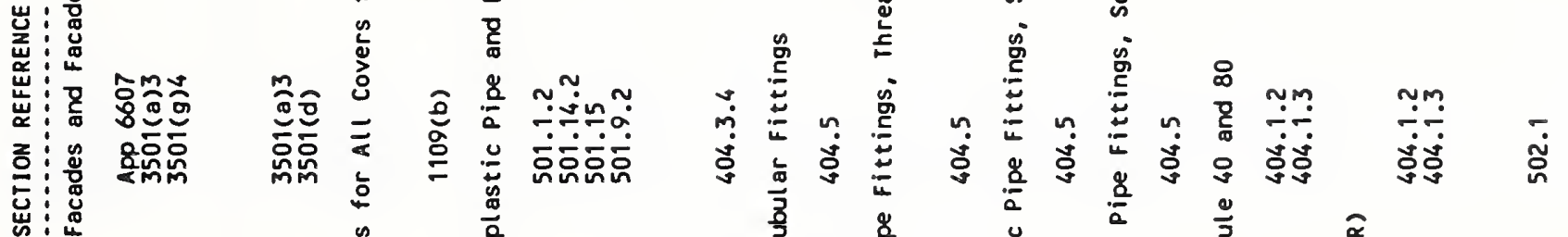

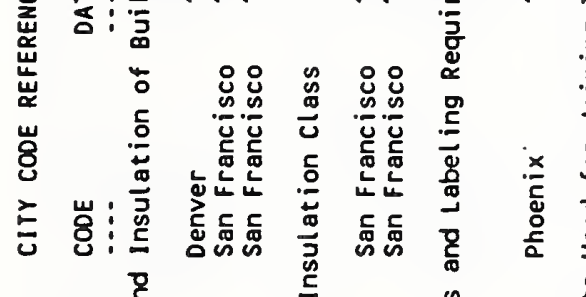

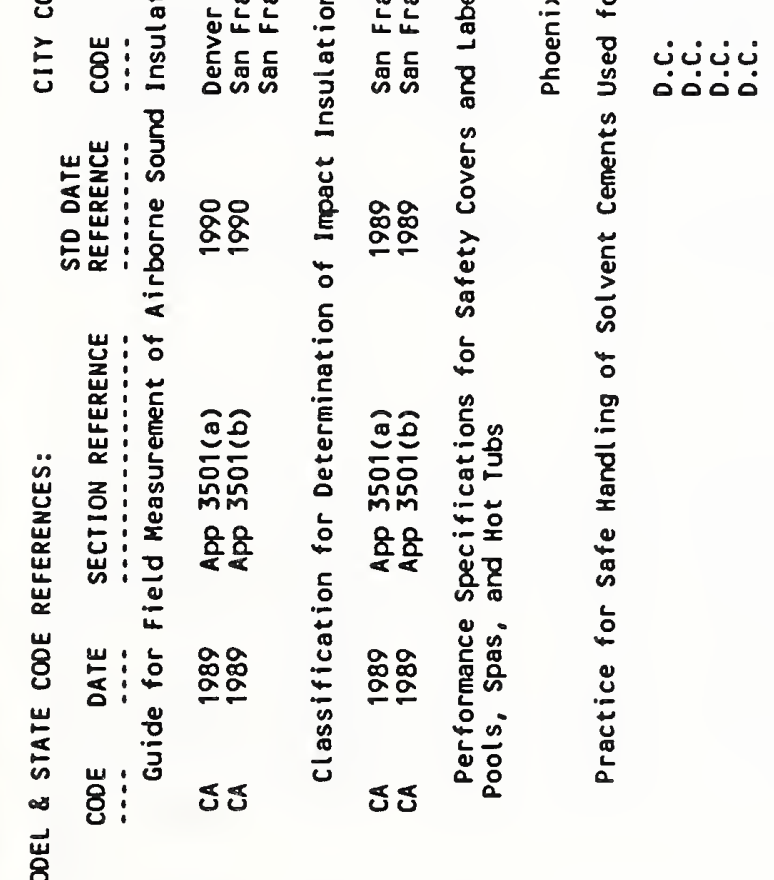

害

\&

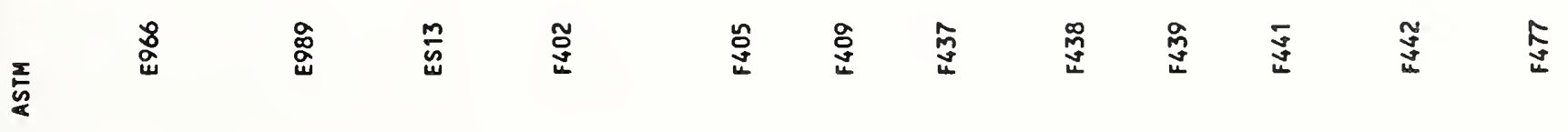









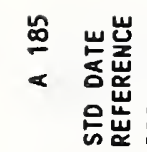

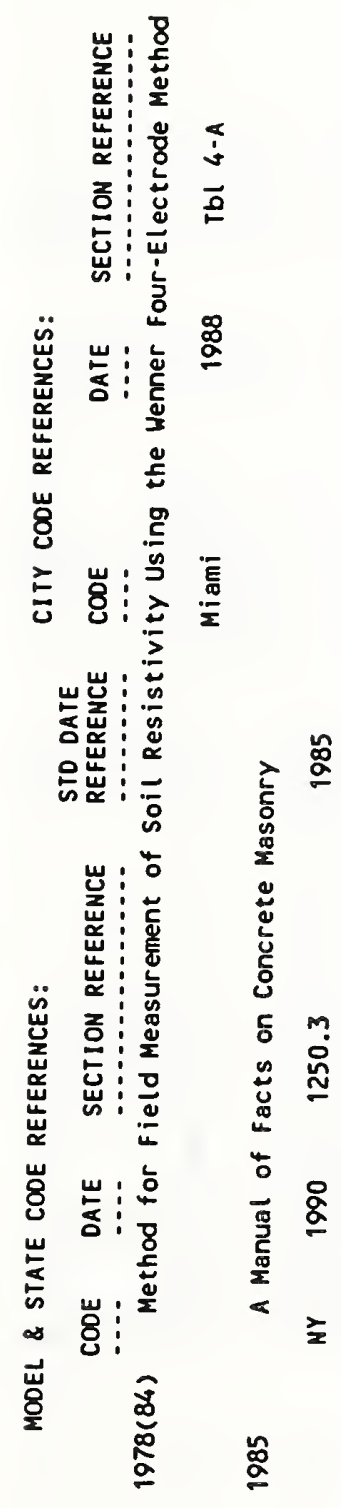

焉芺慈 


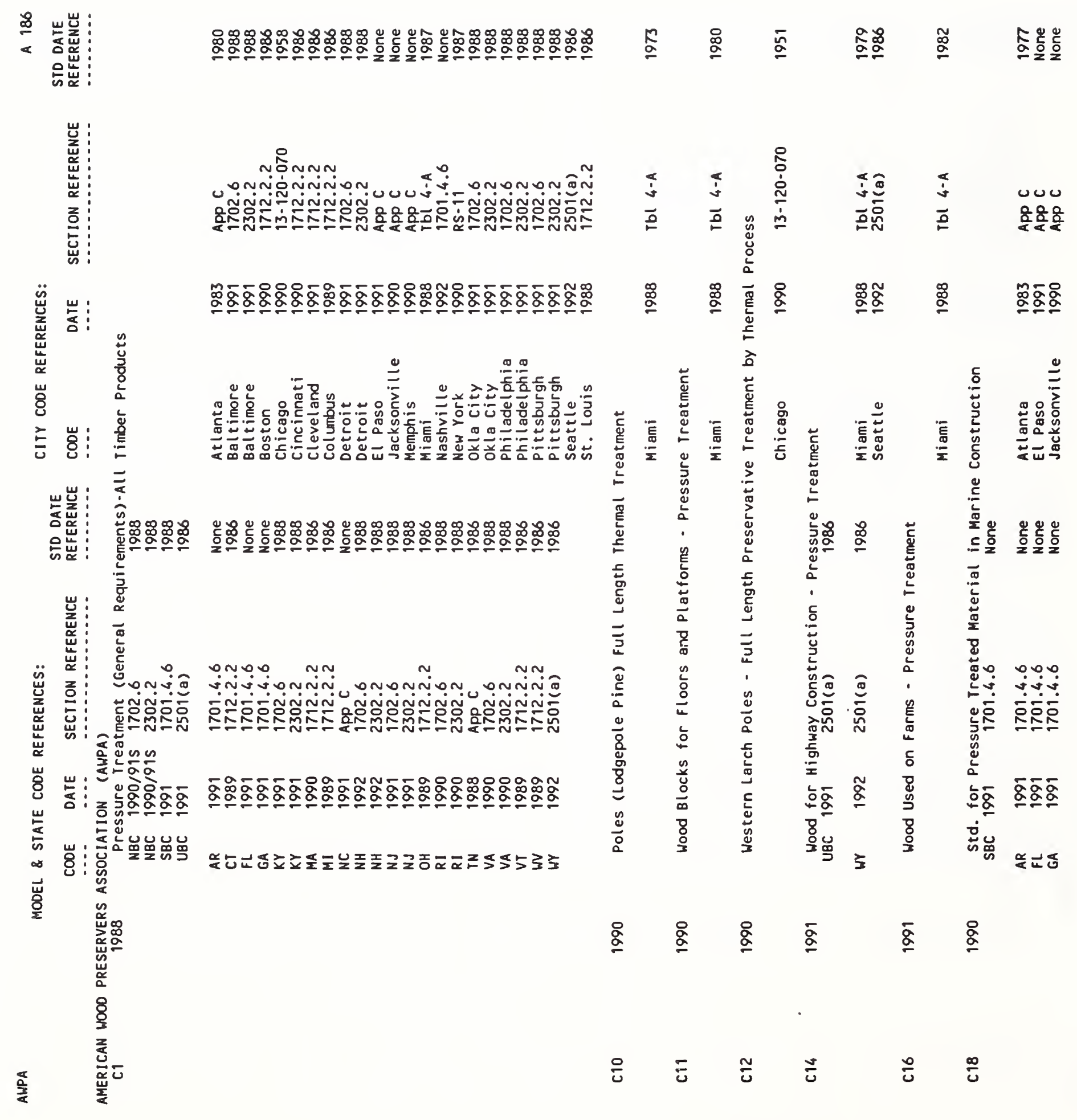




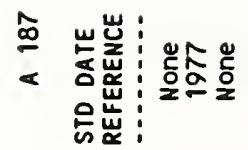

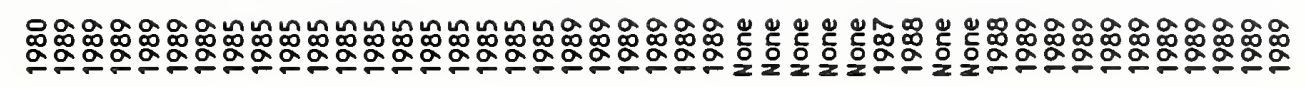

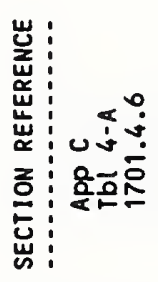

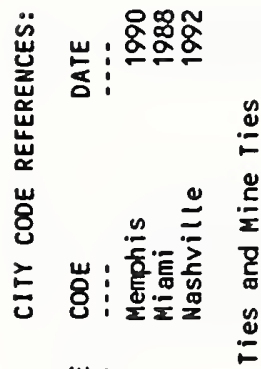

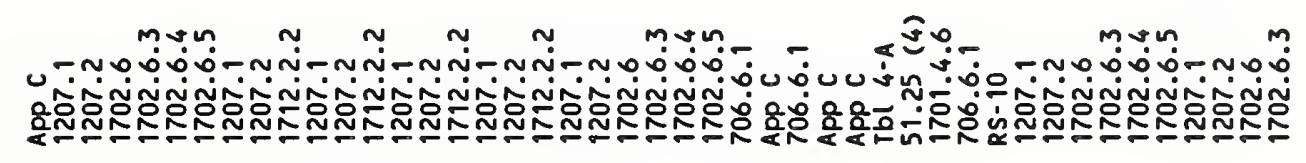

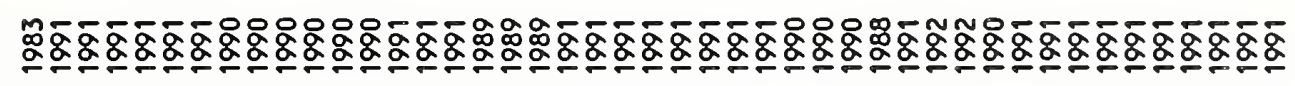

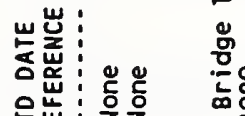

क्ष:

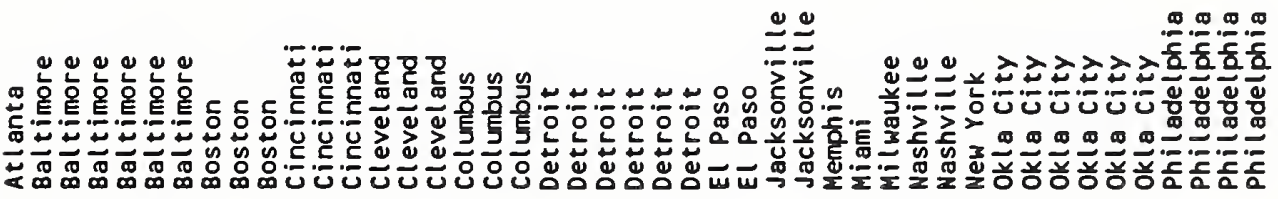

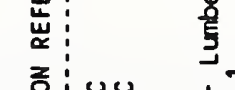

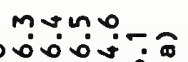
-

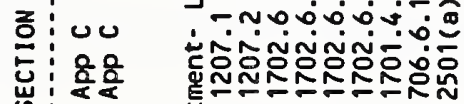
는

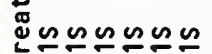

\section{는 \\ 농 :}

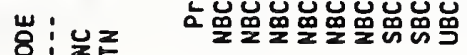

$\infty$ 8 : 노요

苍

$\stackrel{a}{\frac{a}{2}}$

U

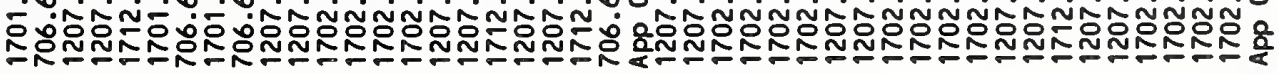

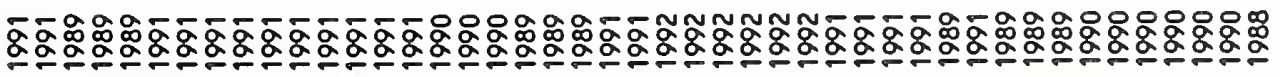

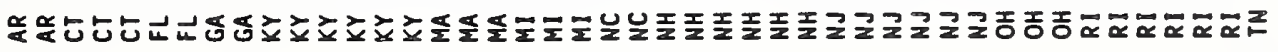




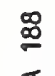

$\propto$

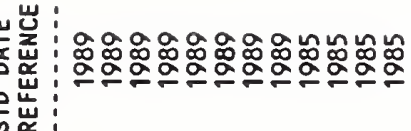

畩:

-nnnm

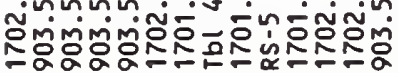

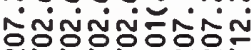

岕

萑

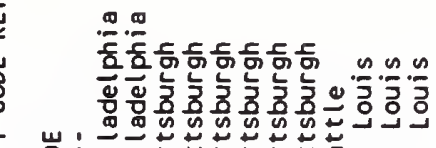

志

닐 :

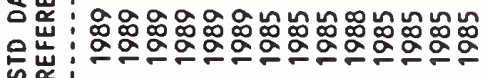

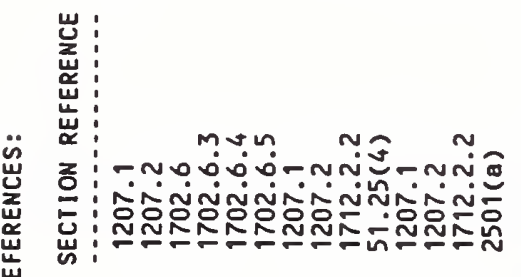

ठั

崖

.

鄫

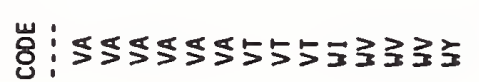

容

บิ

重

$\widetilde{บ}$

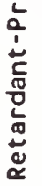

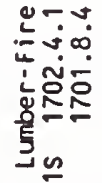

部岩最

$\bar{\Xi}$

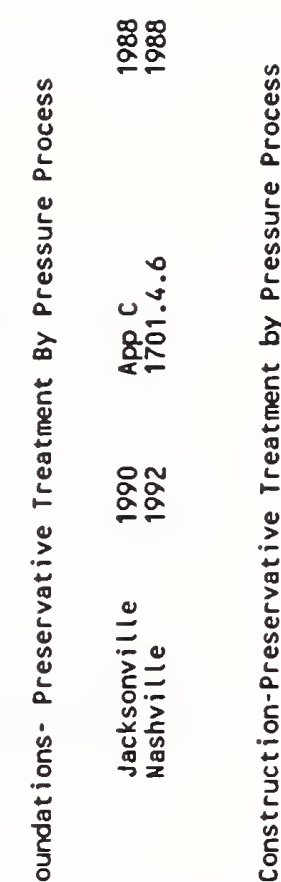

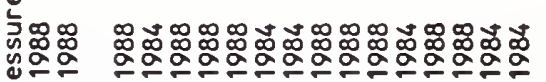

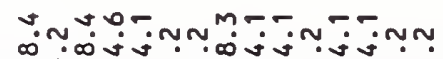

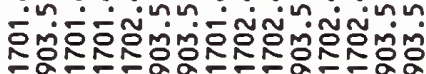

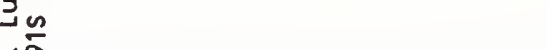

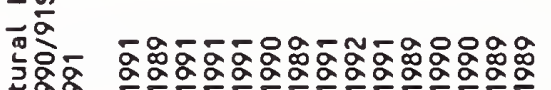

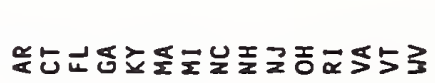

$\stackrel{ฌ}{2}$

ธี
ま

๕ి

ֻ

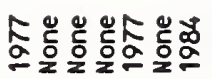

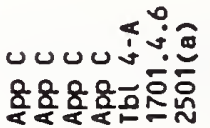

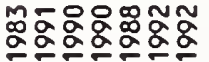

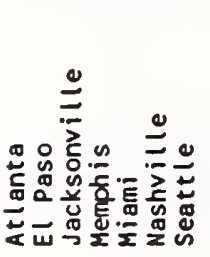

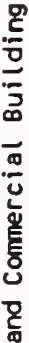

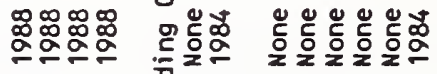

范

둥

$\ldots$

家家

为。

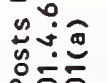

후ㅇㅠㅠ

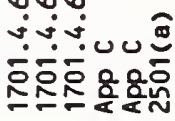

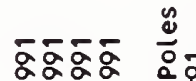

혀웡

딴정느

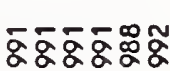

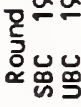

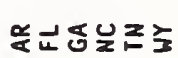

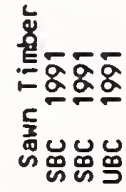

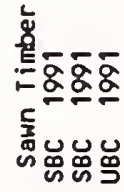

ב

ป 


\section{⿳⺈⿴囗十⺝⿱ 我岕 :}

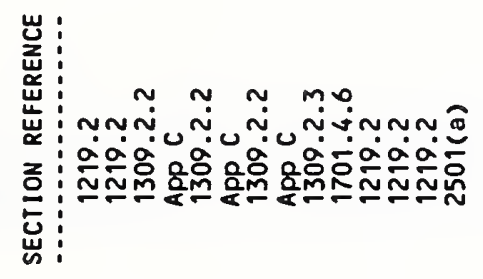

䓛

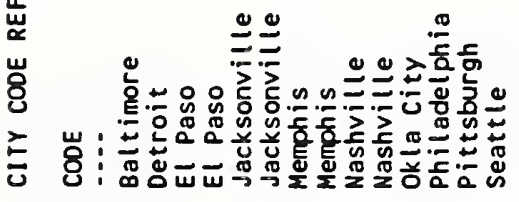

犗:

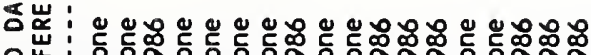

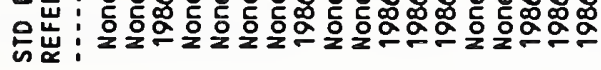

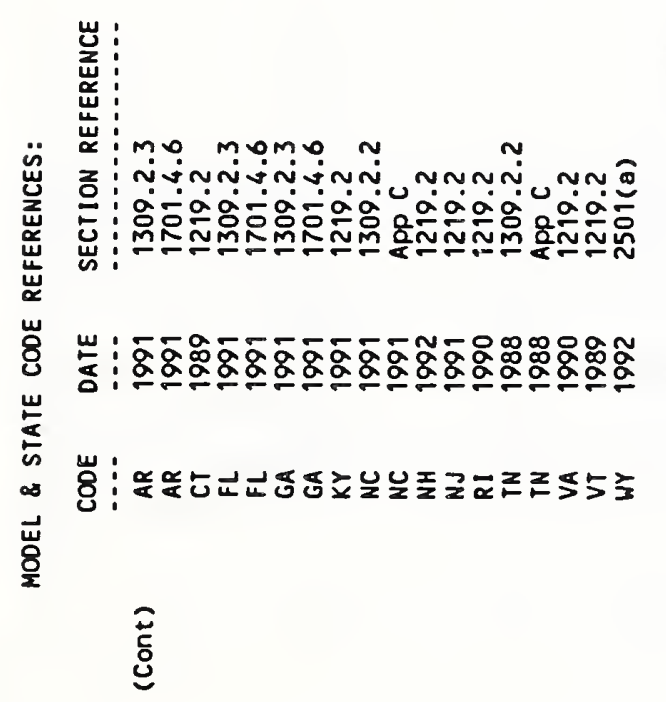

妾

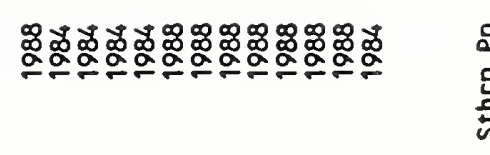

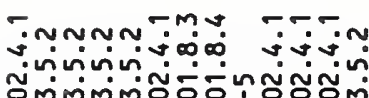

$\begin{array}{lll}5 & 5 \\ j & j\end{array}$

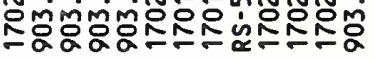

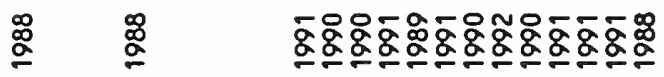

离 $\frac{\bar{E}}{\bar{\Sigma}}$

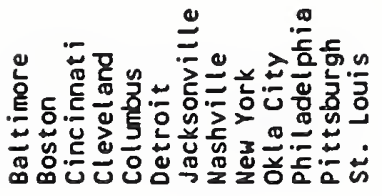

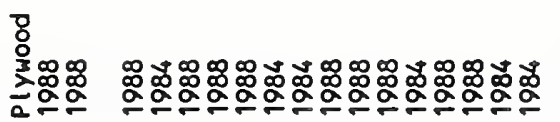

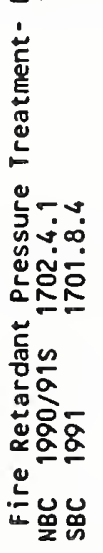

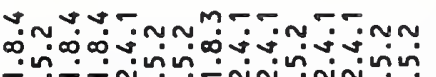

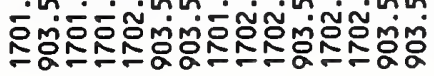

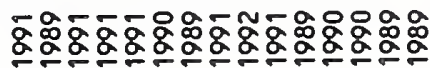

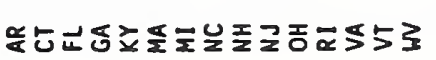

$\stackrel{\check{a}}{\stackrel{\Xi}{\Xi}}$

$\bar{\alpha}$

บับ

ஸับ
5

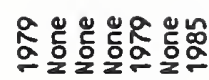

טuvữ

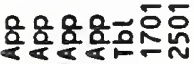

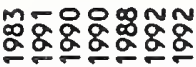

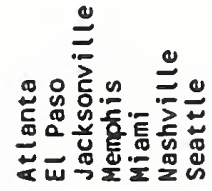

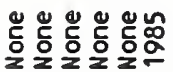

든

出定

苏尊

cos 000

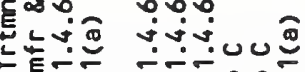

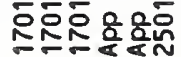

政

这

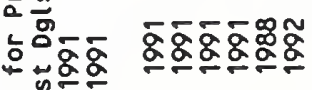
iं

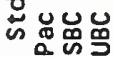

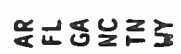




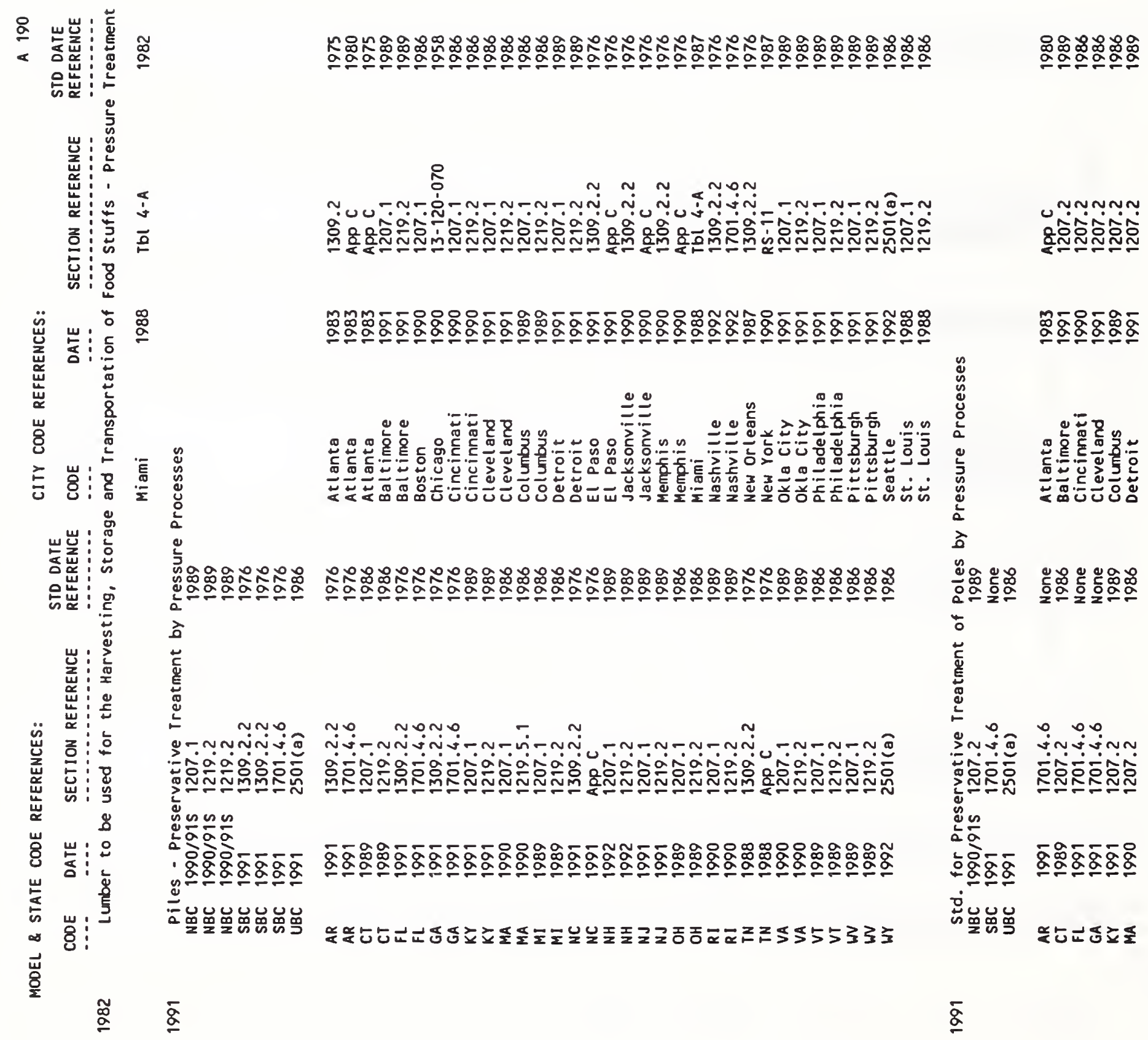

突 


\section{ฐุ.}

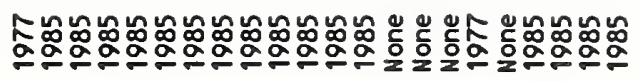

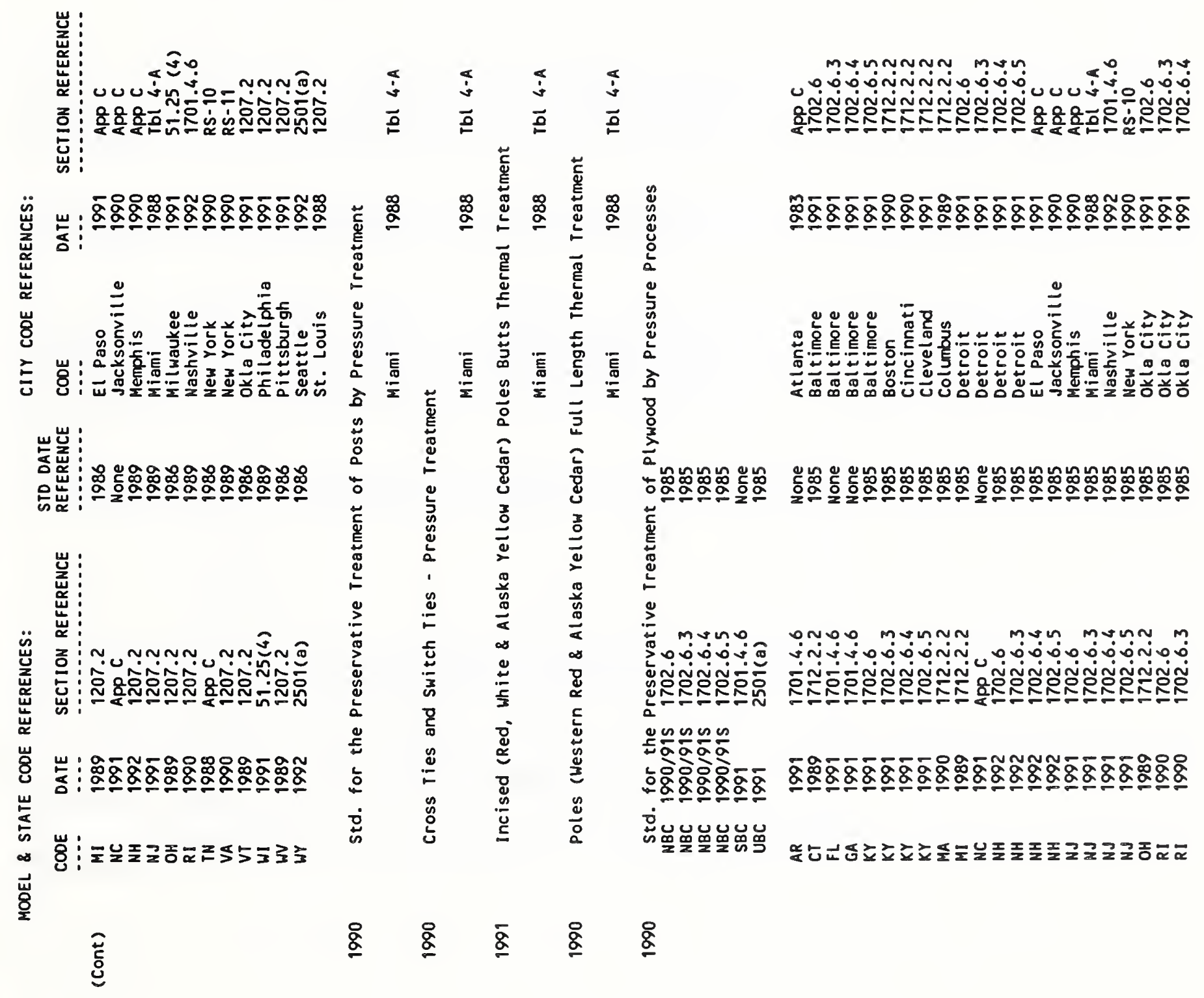

$\frac{\sqrt[3]{2}}{d}$

ก ษ ษ ษ 
$\approx$

岕:

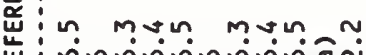
\%

ว

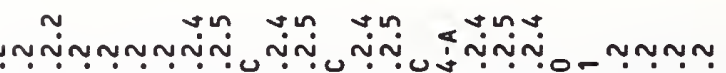

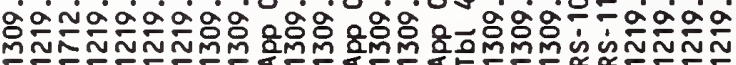

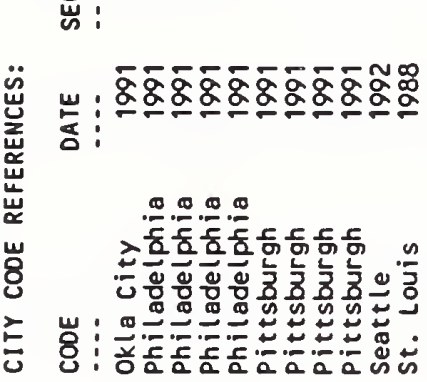

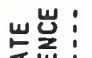

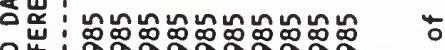

운

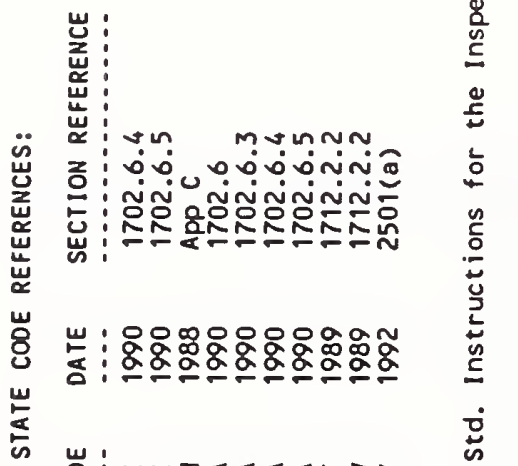

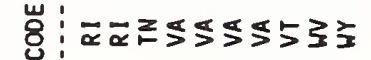

$\stackrel{8}{8}$

$\stackrel{\circ}{2}$

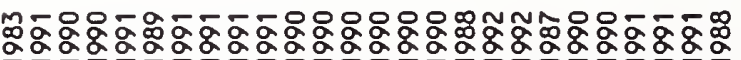

(2)

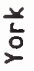

$\stackrel{3}{2}$

莡哭恕

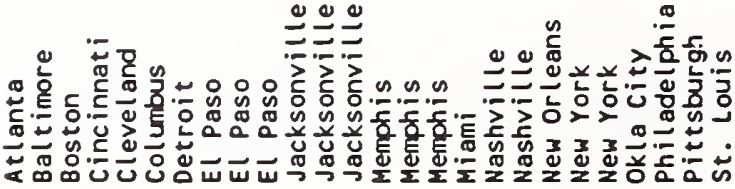

芩

:

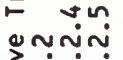

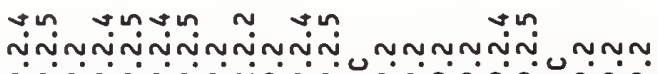

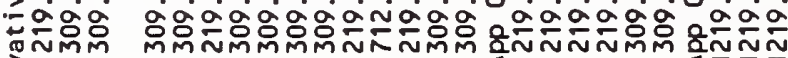

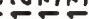

㟧的

훙

형혀음

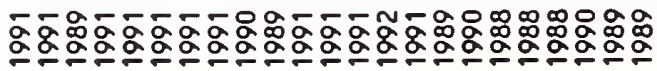

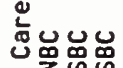

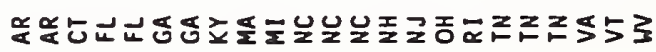

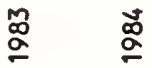

宅

a

$\frac{N}{\frac{1}{2}}$

$\bar{a}$

$\frac{\bar{g}}{\frac{1}{a}}$

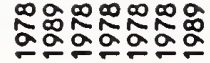

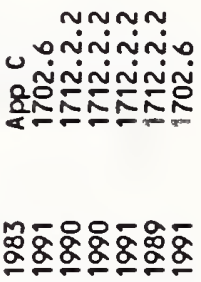

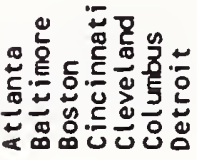

ذ్

L D. OnDo nN

3.5. univinin

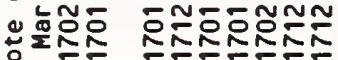

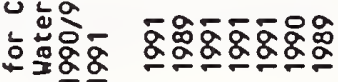

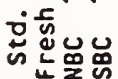

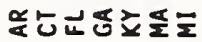




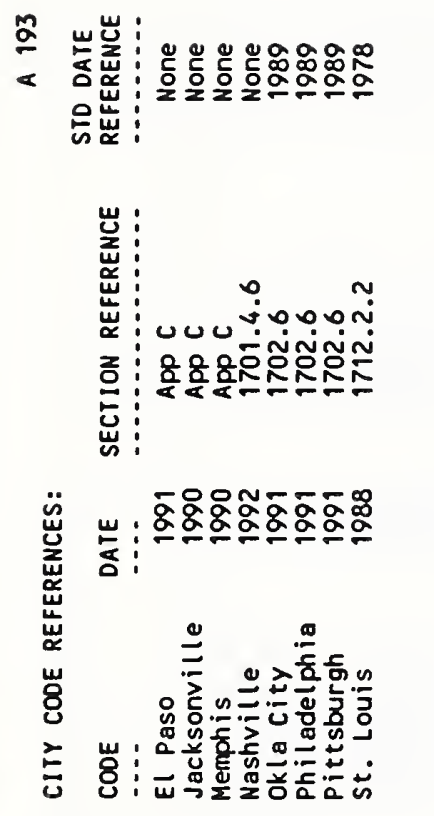

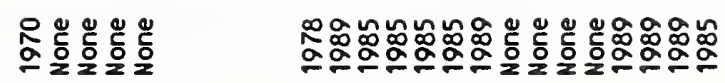

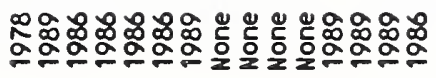

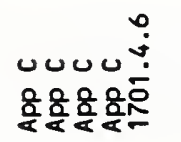

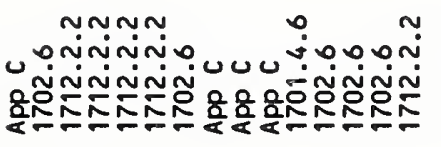

\section{쫑몽요요}

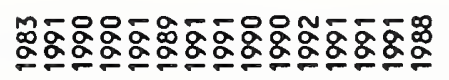

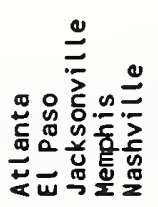

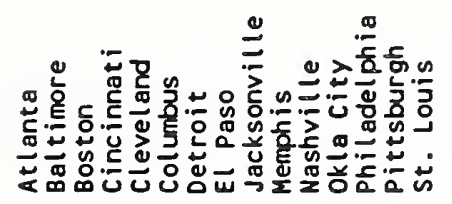

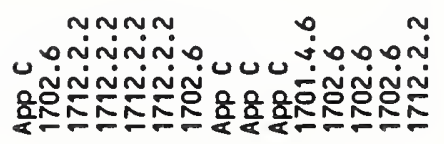

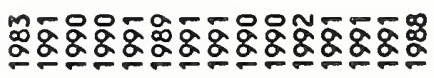

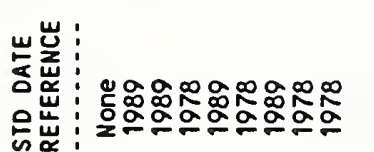

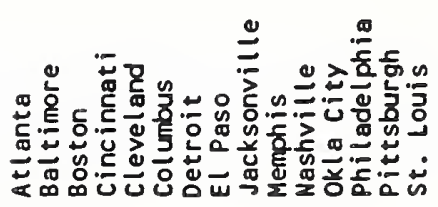

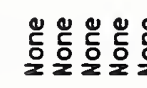

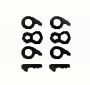

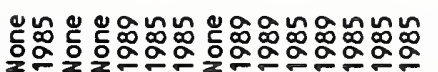

まま



ક્ّ

$\bar{\Sigma}$

范

نư

도원연

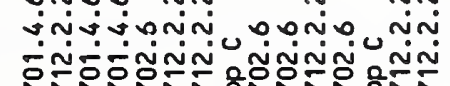

崩

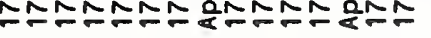

$\overline{\mathrm{g}}_{\mathrm{a}}^{\mathrm{a}}$

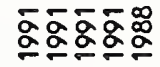

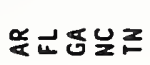

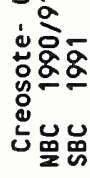

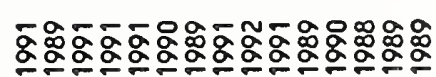

-

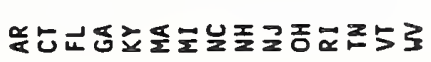

a

cก่ -

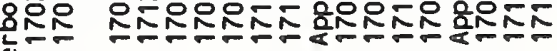

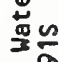

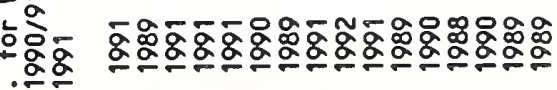

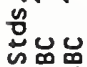

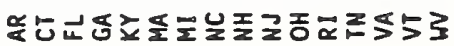

$\stackrel{0}{\varrho}$

$\bar{g}$

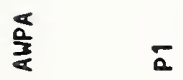

$\bar{a}$

ก

ก 


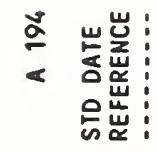

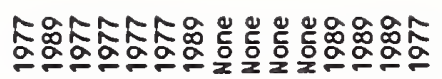

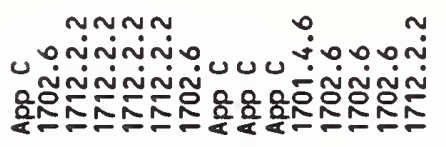

苨崖

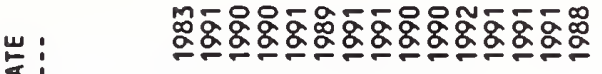

崖

岁

崖

崖岕

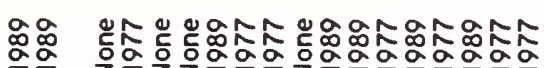

岁:

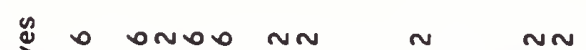
0.

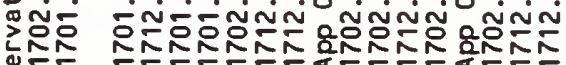
造。

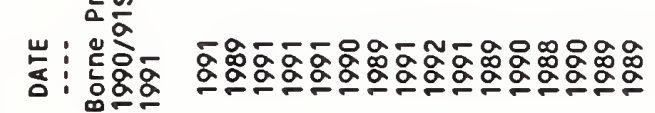

岩: “睘出

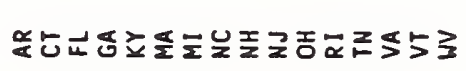

$\stackrel{\Xi}{\circ}$

$\infty$

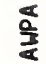

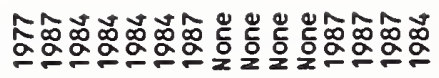

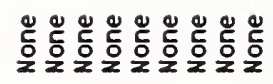

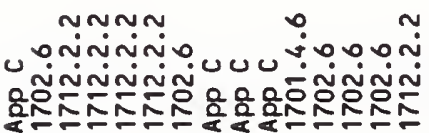

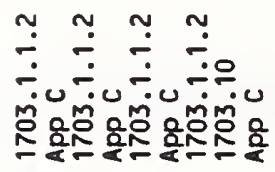

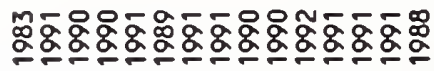

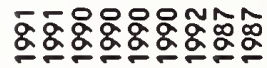

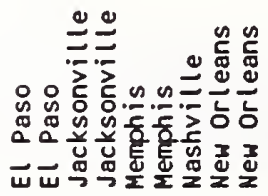

ัૅ

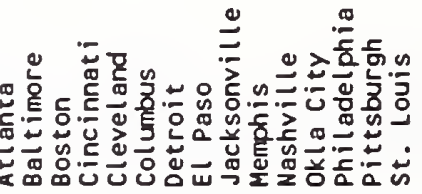

$\frac{5}{4}$

(2)

نَ

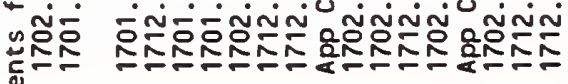

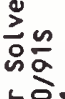

홓ㅁㅇ

焉员品

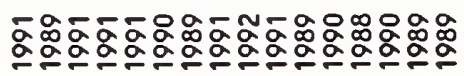

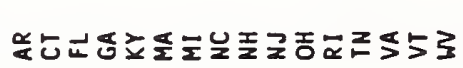

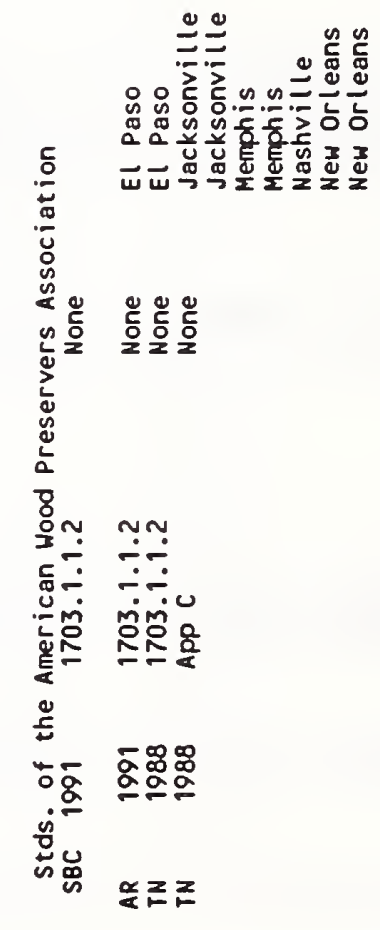

ฒ

$\bar{g}$

$a$

$\stackrel{\text { N }}{\text { N }}$ 


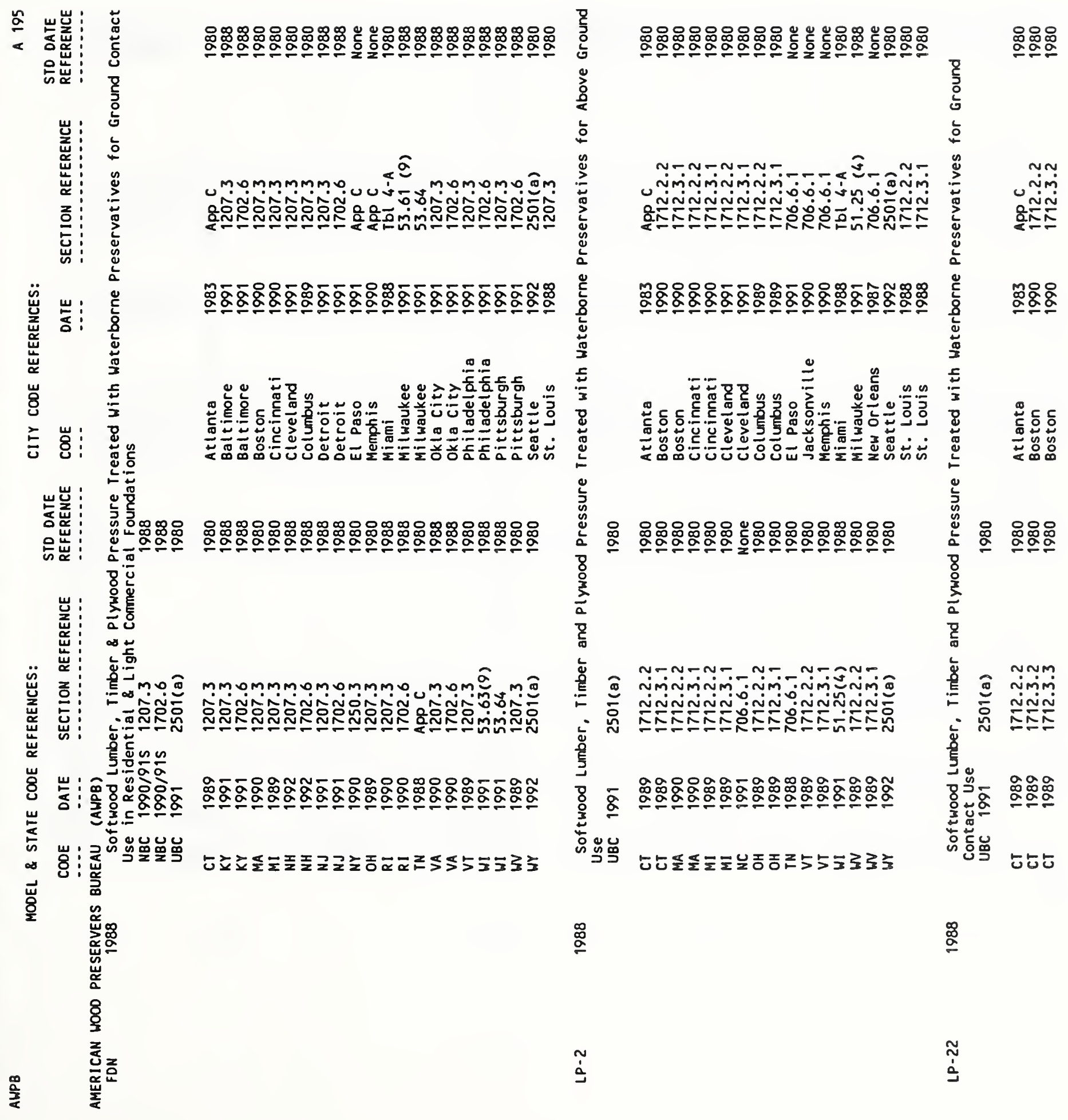


\& 岕 趾:

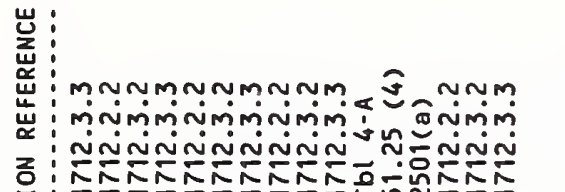

出:

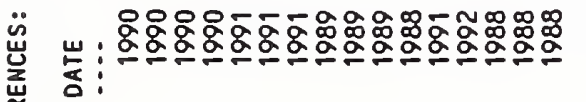

崩

岁

¿

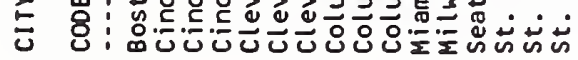

岕 :

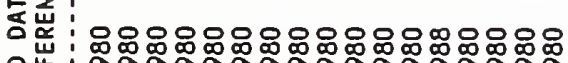

운

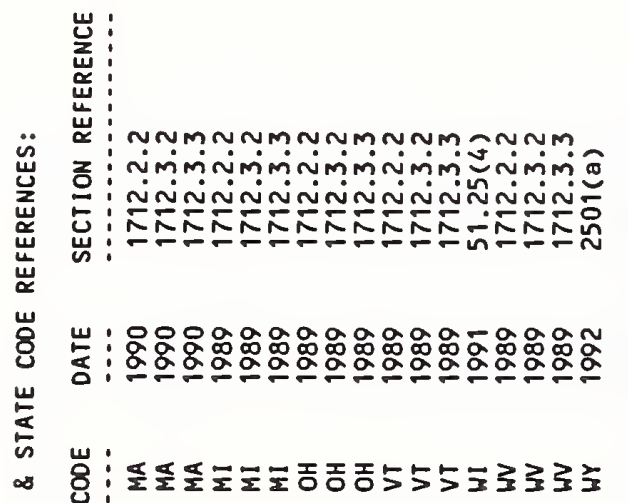

岁

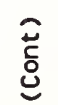

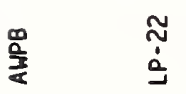

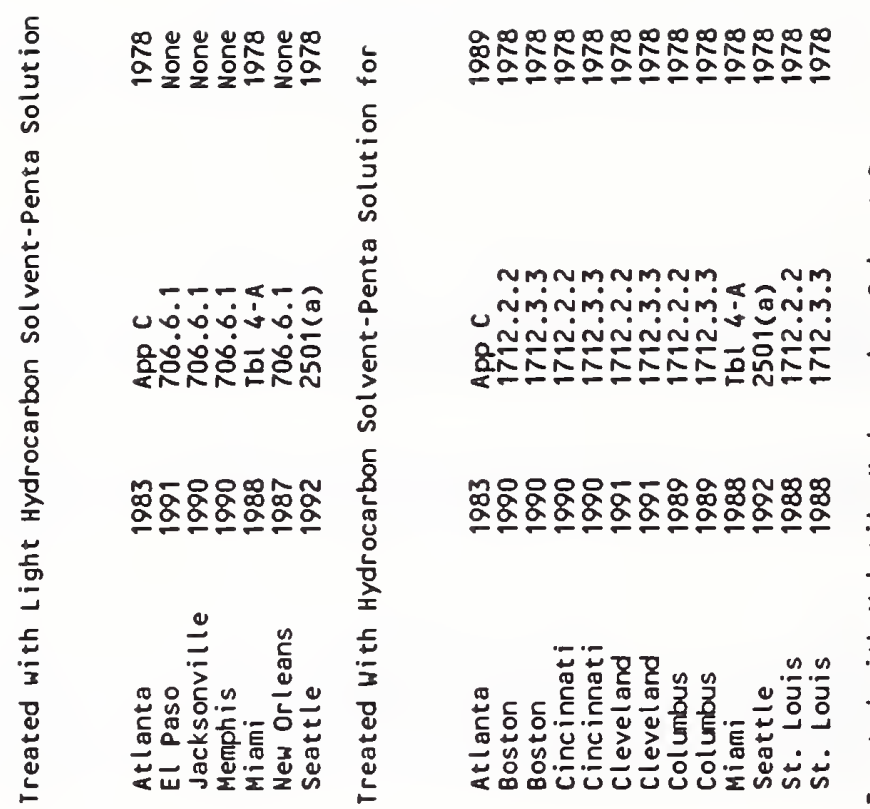

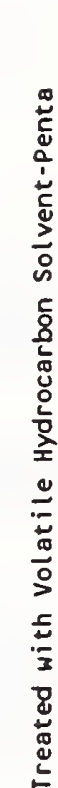

象象串

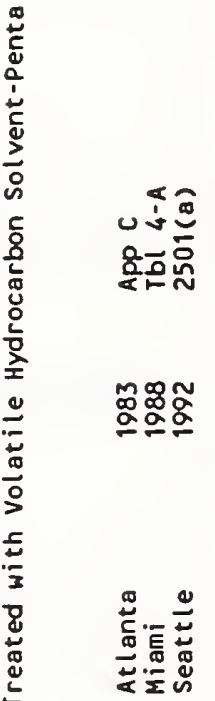

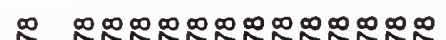

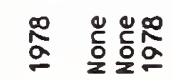

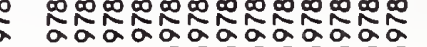

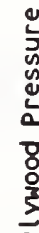

$\stackrel{\infty}{a} \stackrel{\infty}{a}$

ไั้ง

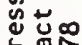

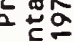

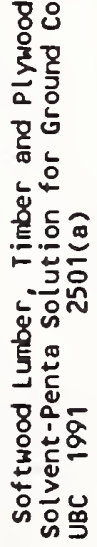

늘즌조

$\div 0 \frac{0}{0}$

ํํํํำ

尊家

ờnmmmmmmm

垔苟

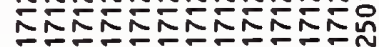

藏

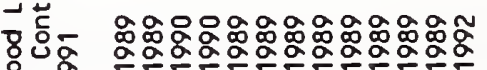

문은

宫说怘

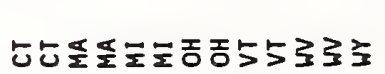

ร

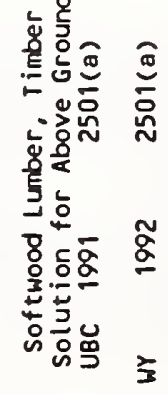

$\underset{\mathscr{a}}{\stackrel{\mathscr{g}}{\sigma}}$

$\stackrel{9}{\mathbb{2}}$

$\stackrel{a}{a}$ 
ڤ

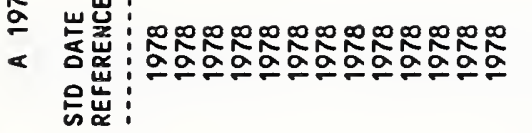

㟧 :

:

偻

3 NㅗNNNNำ

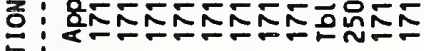

出: 芦

过

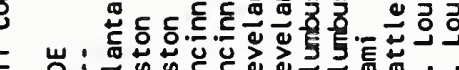

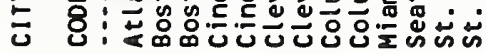

닐 :

ธك $\infty \infty \infty \infty \infty \infty \infty \infty \infty \infty \infty \infty$

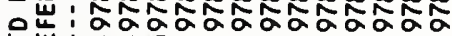

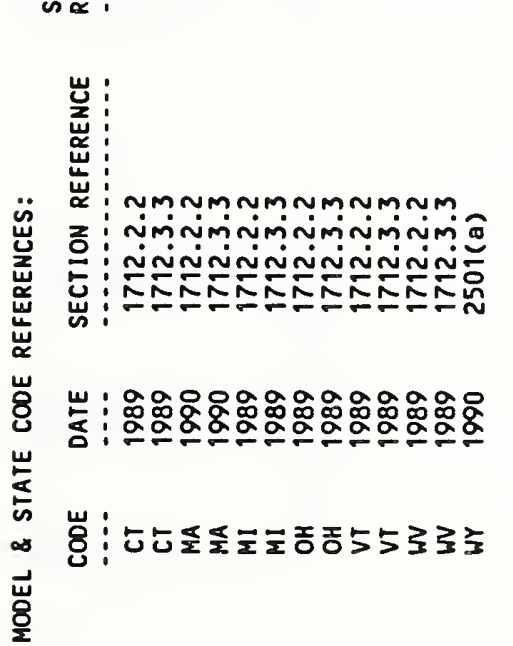

它

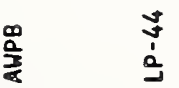

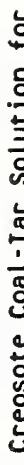

ᄂ

ฆ뽕

ن⿺

웅혼유

NMNMNMNM NM

Nimininiminis बิ

iniñniniñ

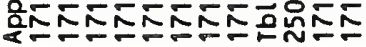

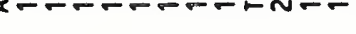

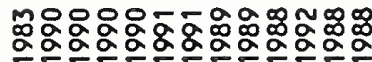

西

葛

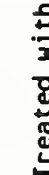

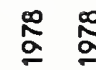

일

每

$\infty \infty \infty \infty \infty \infty \infty \infty \infty \infty \infty$

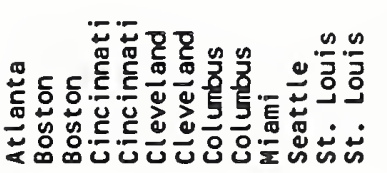

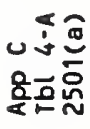

$\stackrel{\infty}{\infty}$

으므으

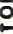

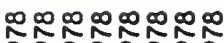

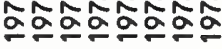

iminiminim

uniñniñ

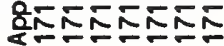

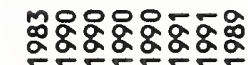

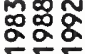

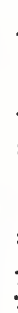

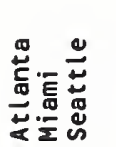

క

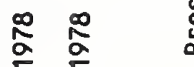

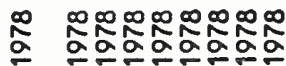

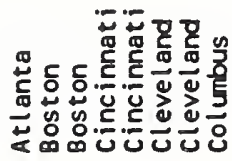
tons 


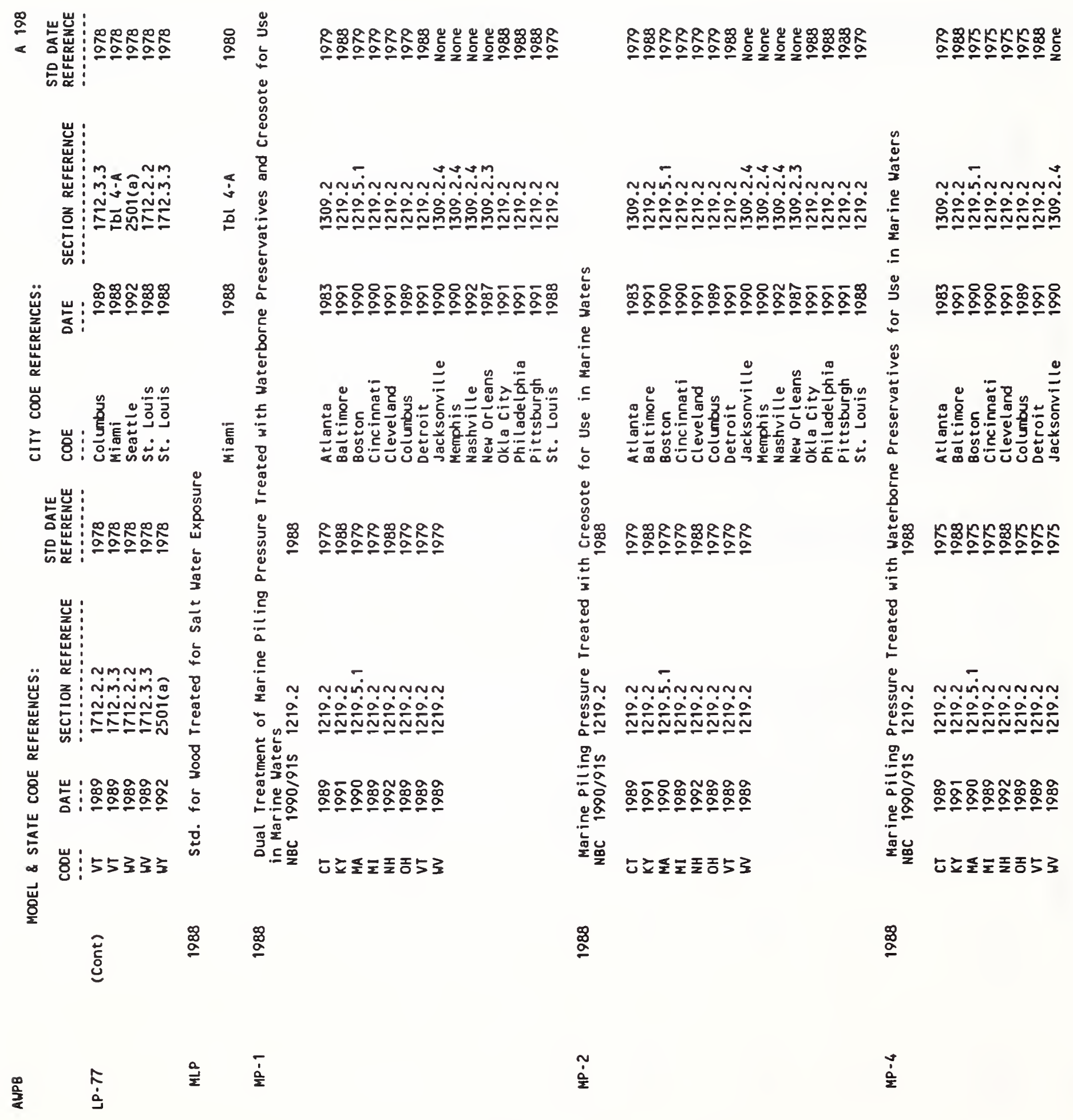


\& w 뿬

< 咥 은

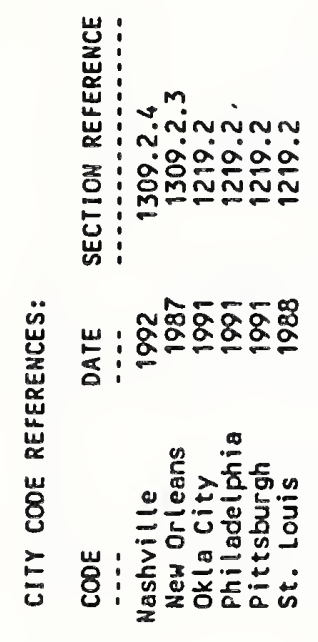

岕岕:

岕:

岁岕:

宸

œ 岁!

㞵

蒙

$\frac{\infty}{3} \frac{a}{2}$ 


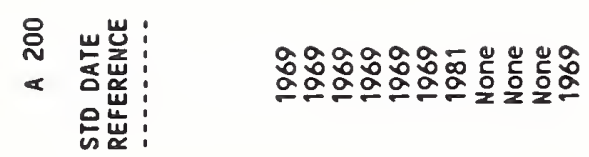
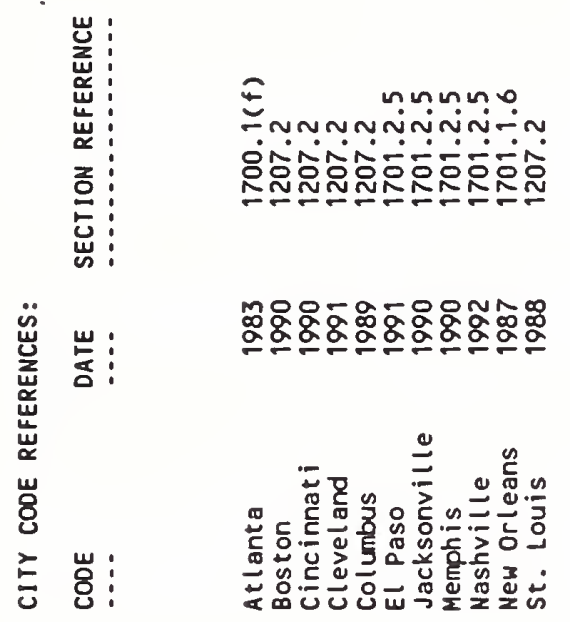

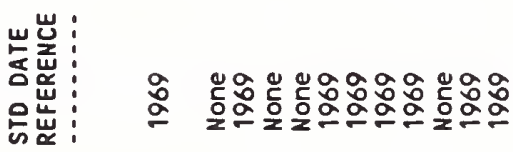

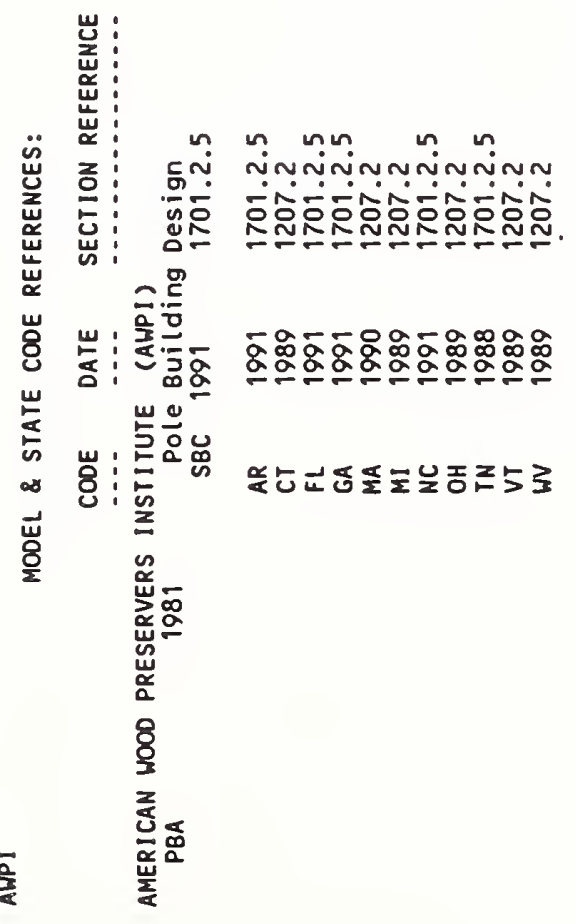




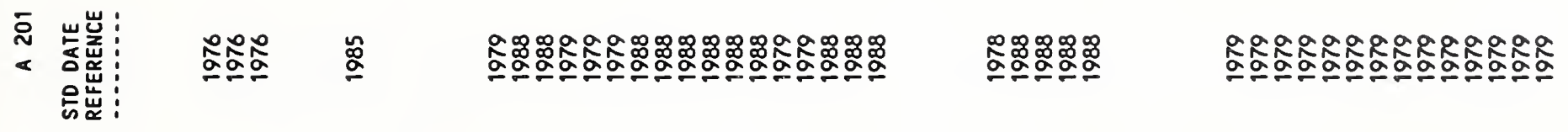

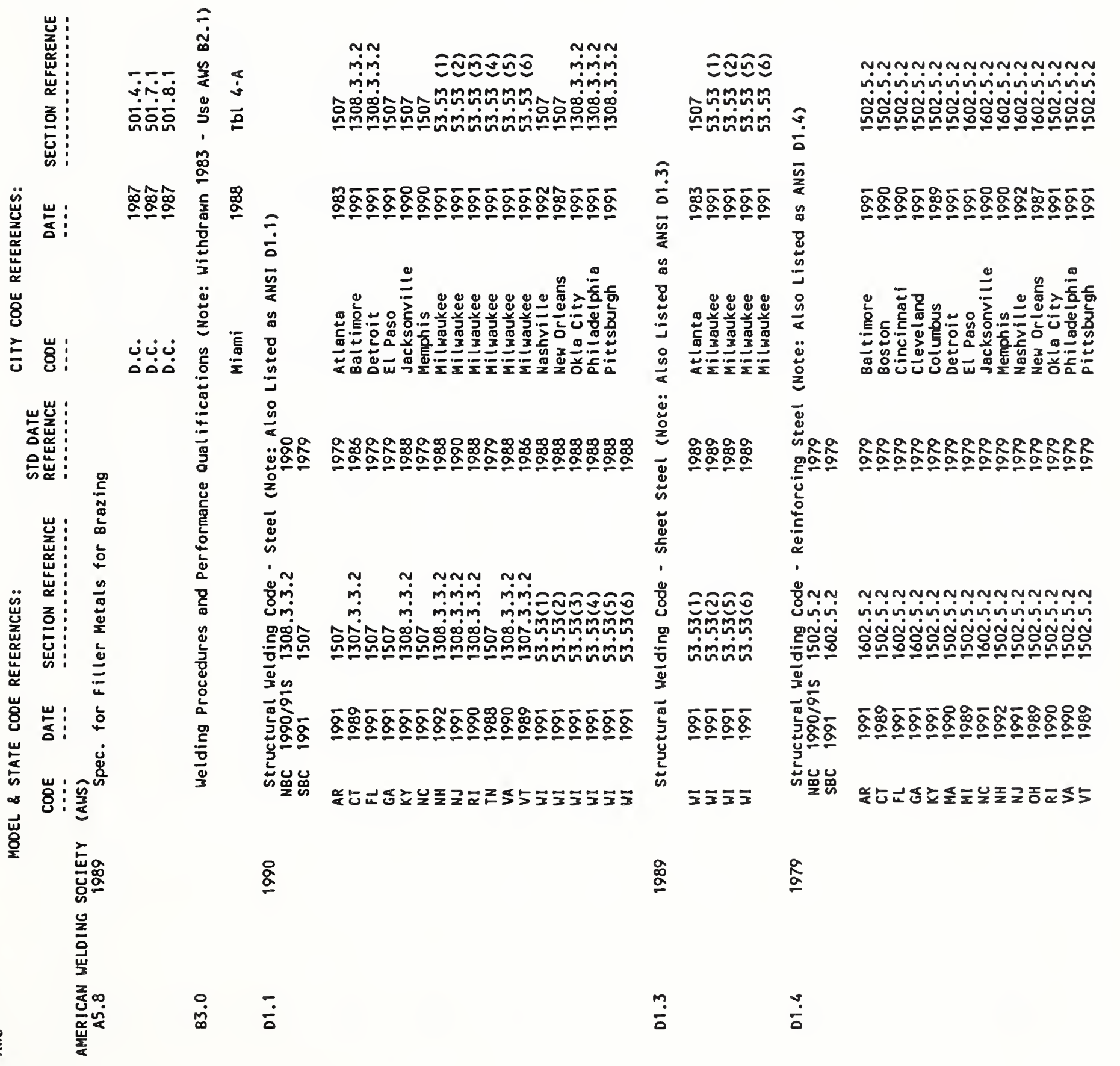


芯岕: 옹

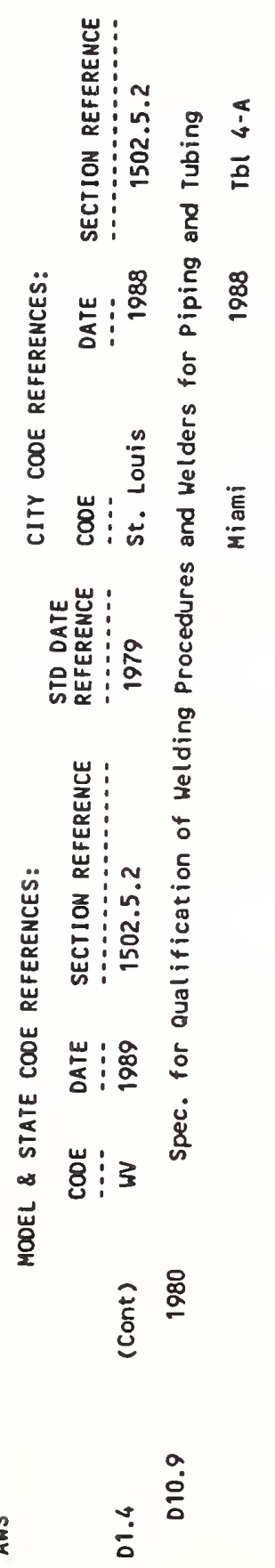




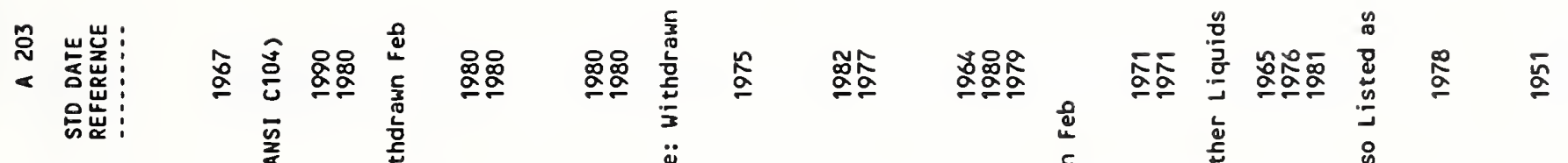

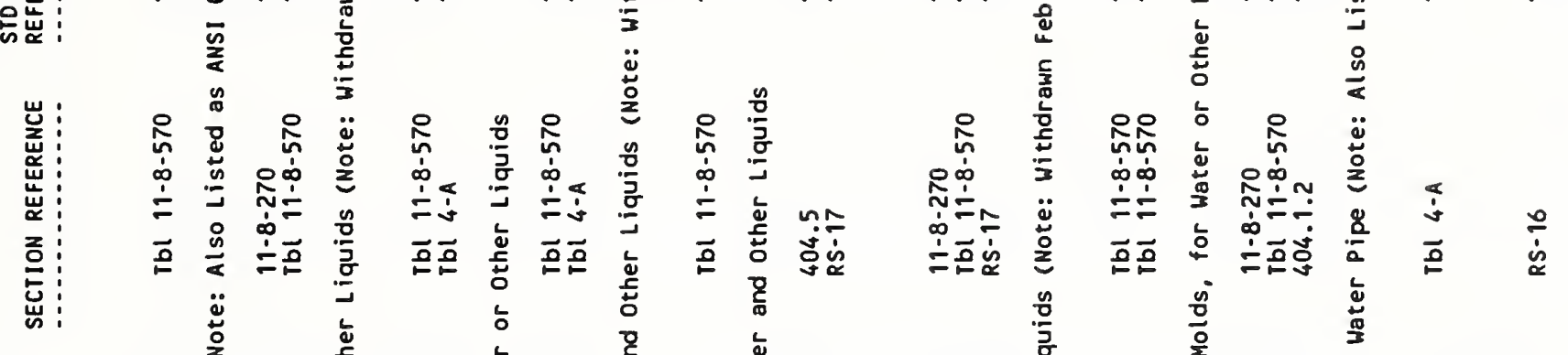

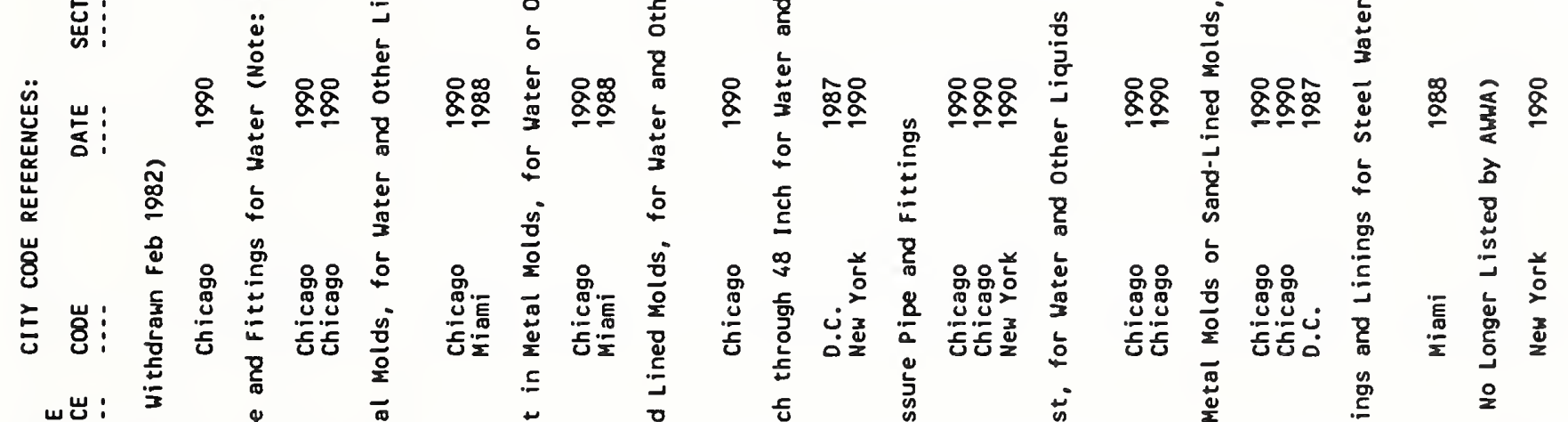

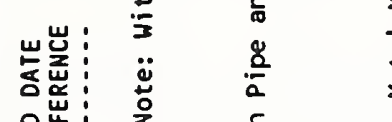

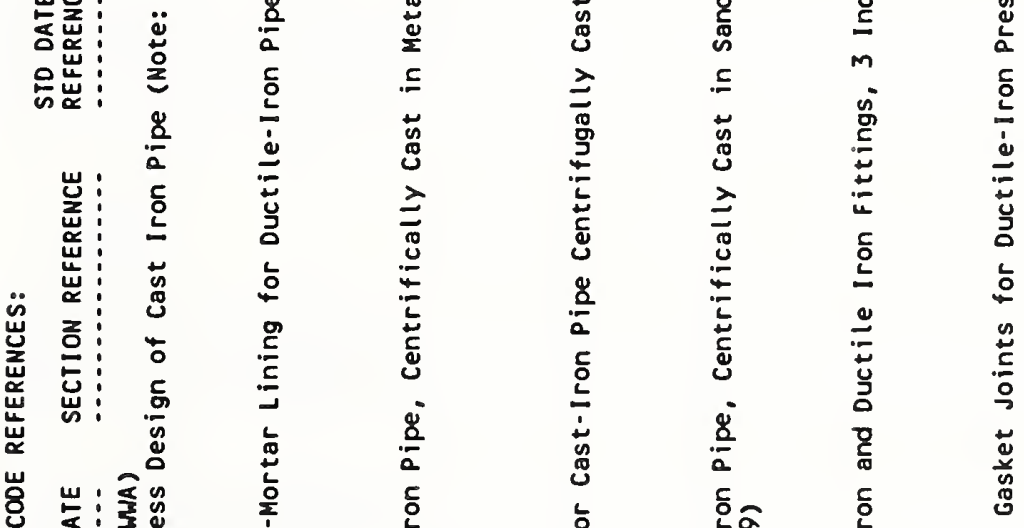

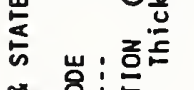

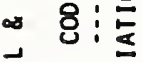

离<smiles>C1CC2CCC12</smiles>

ฐ

ฐ

$\stackrel{g}{2}$

$\stackrel{\Xi}{2}$

巠

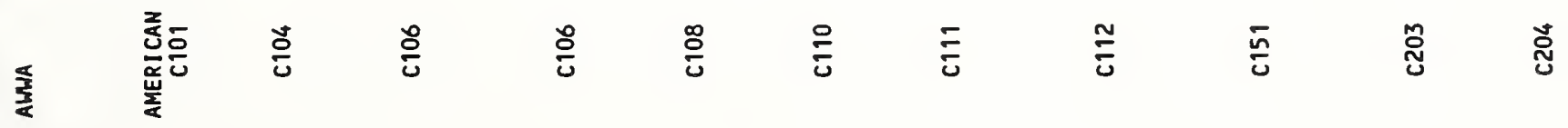




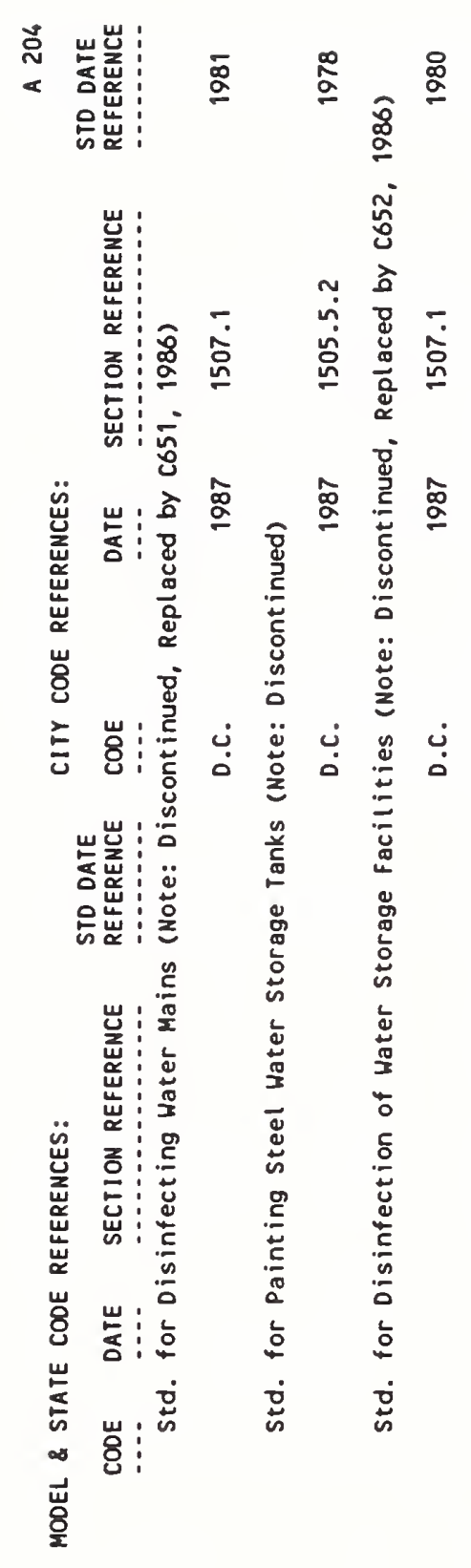

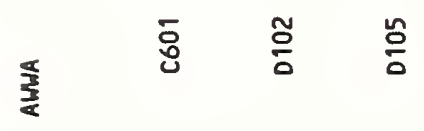


总容疋

的岕:

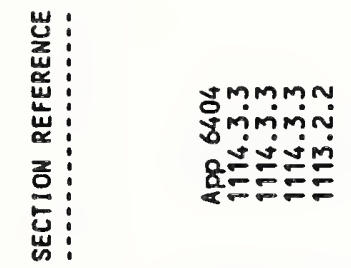

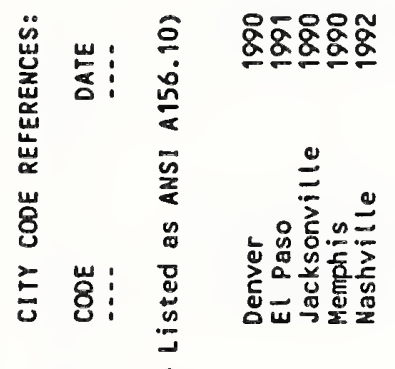

岂闿:

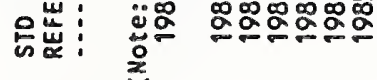

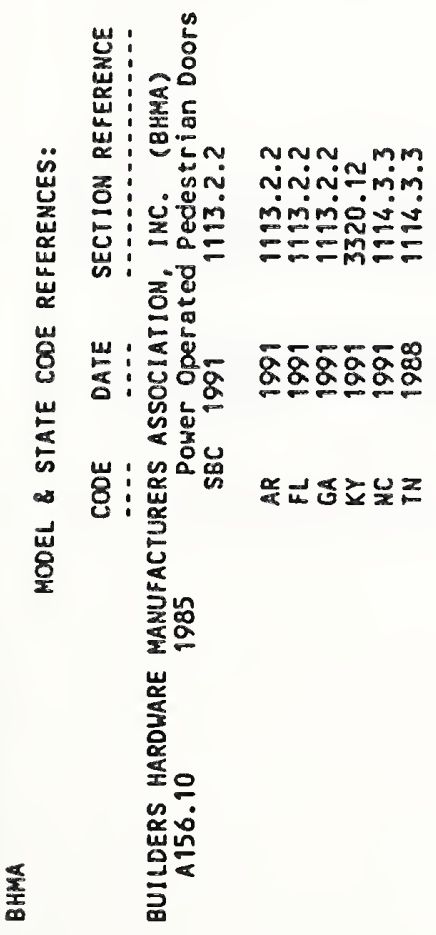




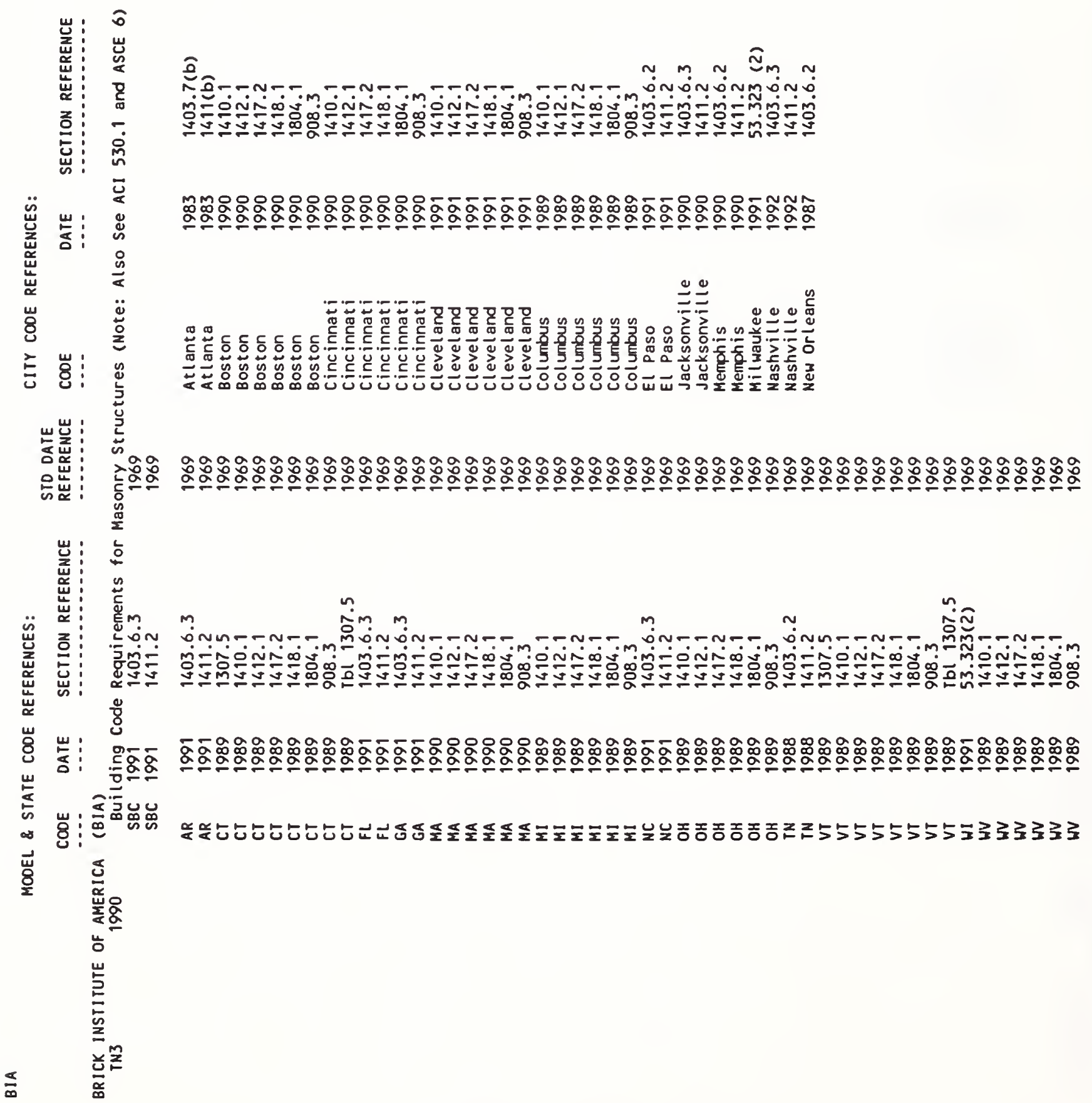




\section{全 员岕:}

:

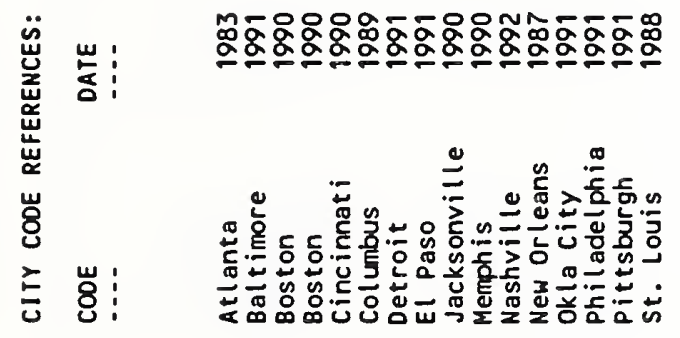

岕:

준

은

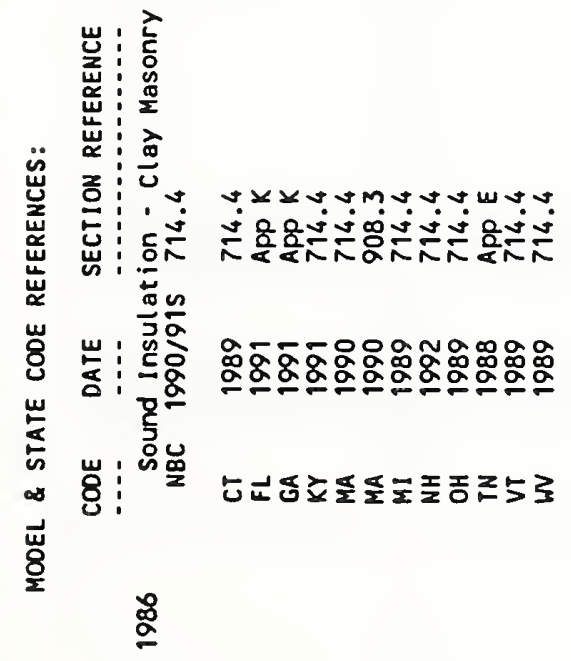

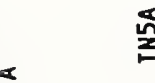

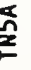




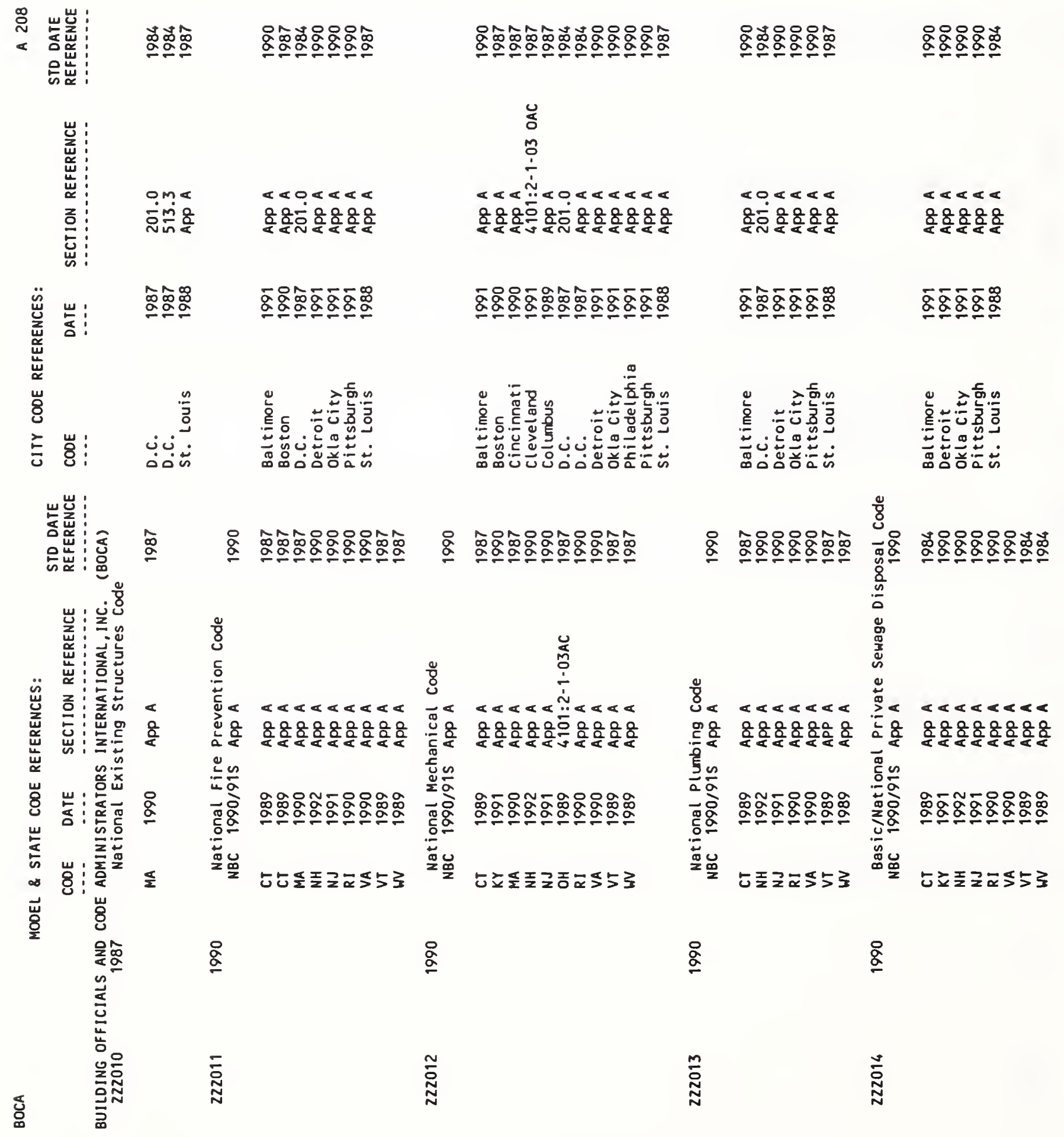




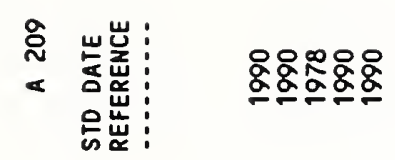

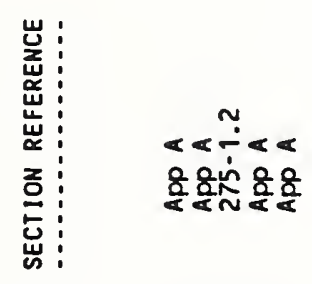

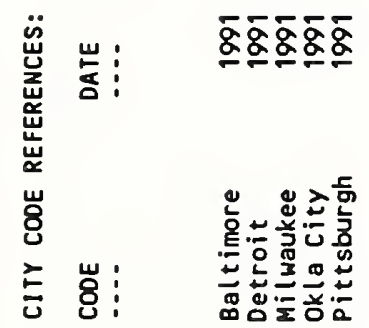

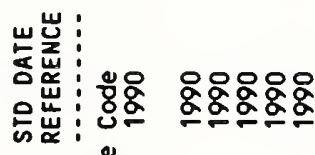

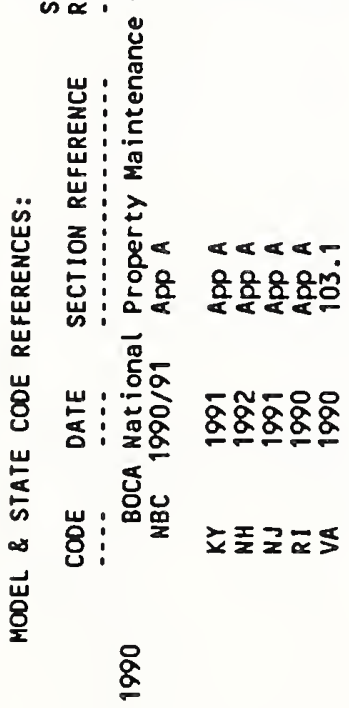

ญั 

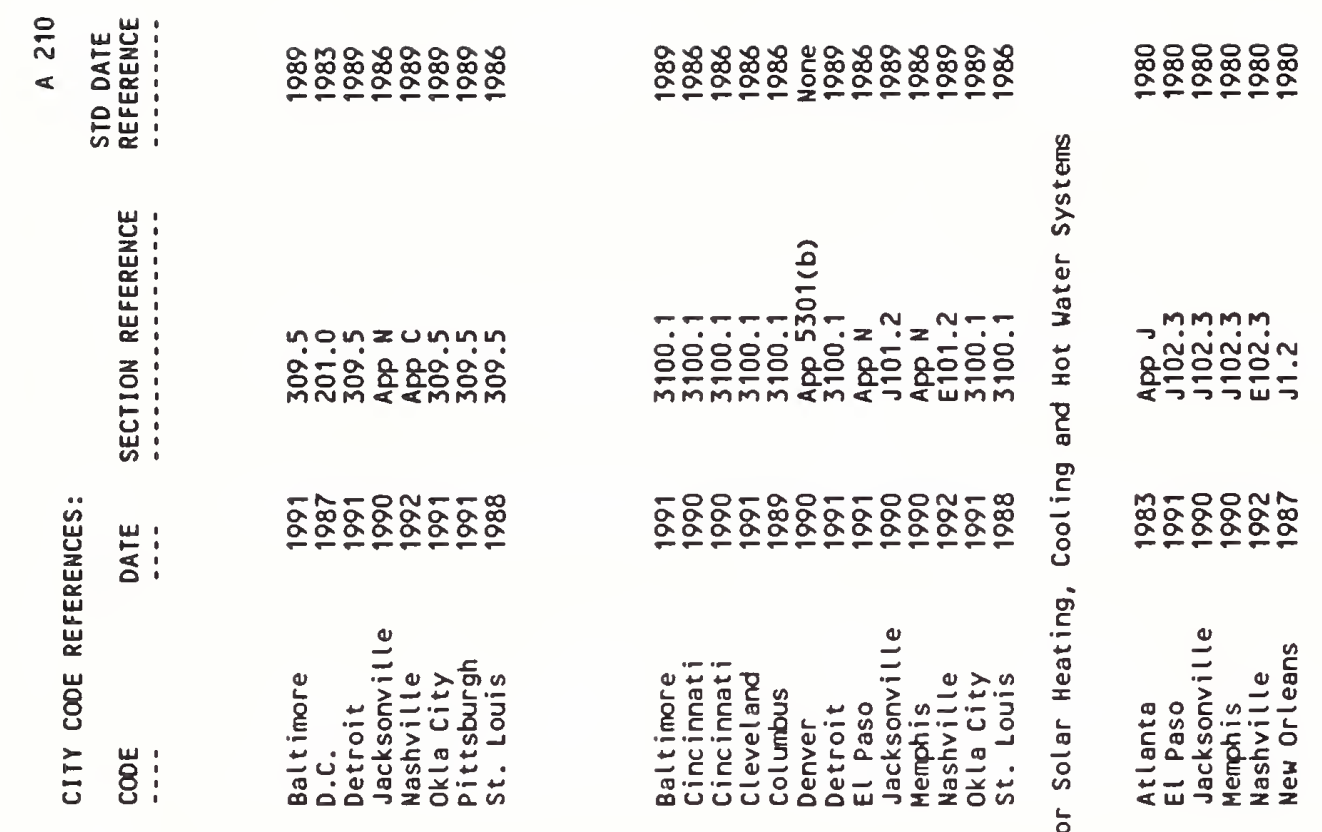

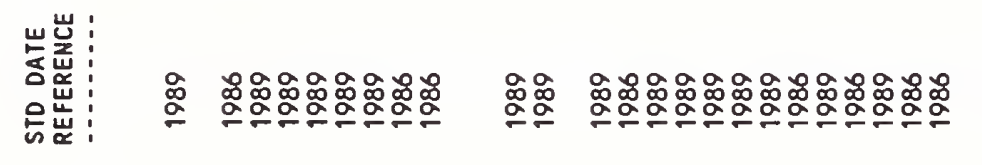

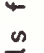

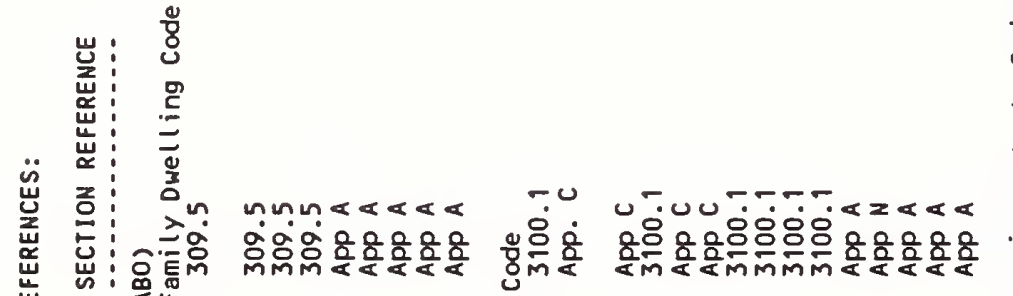

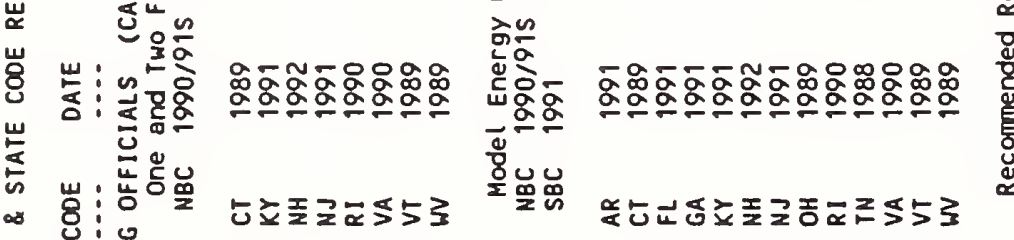

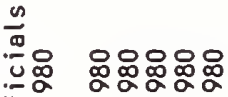

8

풍

紊

$\stackrel{g}{\stackrel{\Xi}{2}}$

$\stackrel{\circ}{2}$

mmmmm

ํํำํํำำำำ

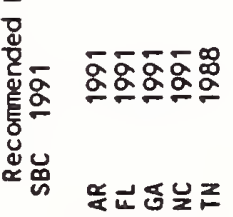

适

แั

$\frac{0}{\stackrel{0}{N}}$

令 
₹ 㩆 :

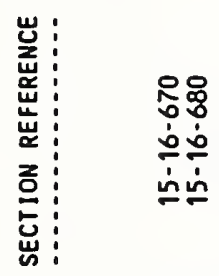

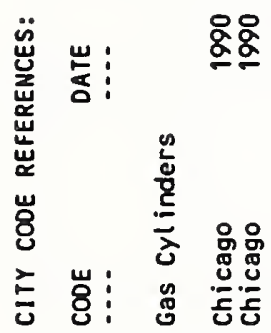

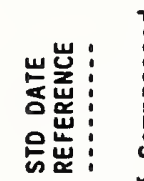

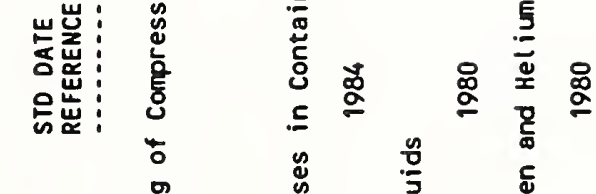

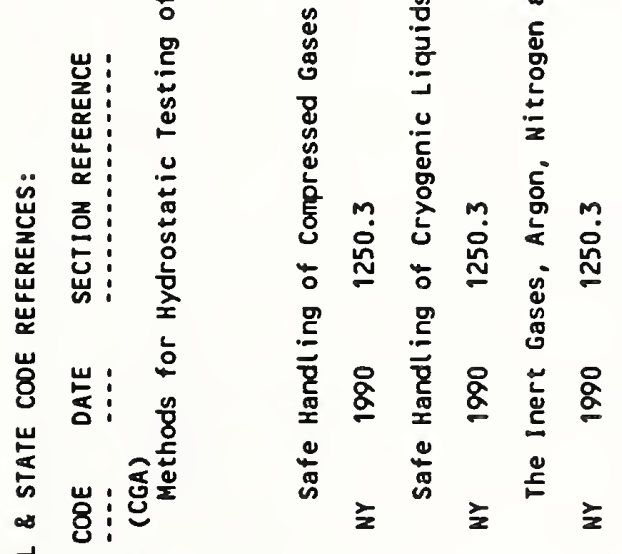

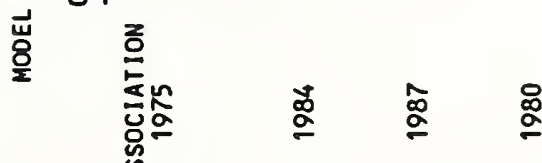

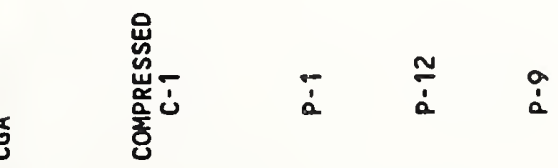

ฮิ 


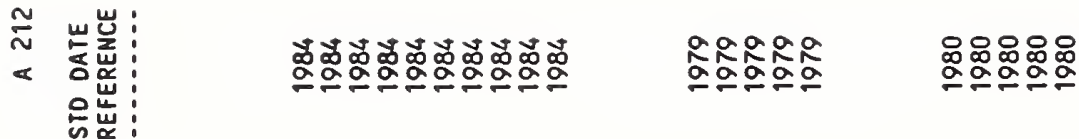

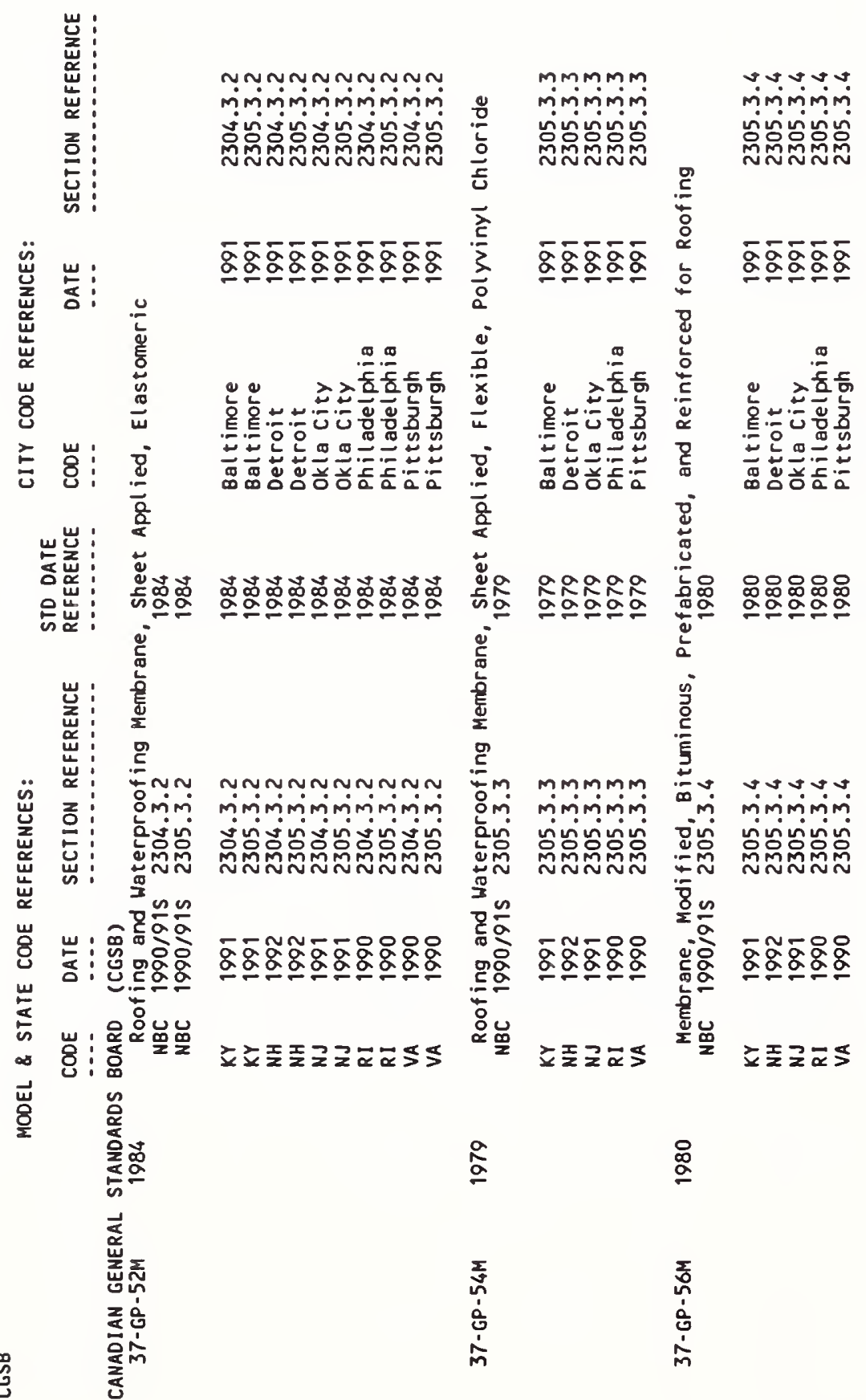




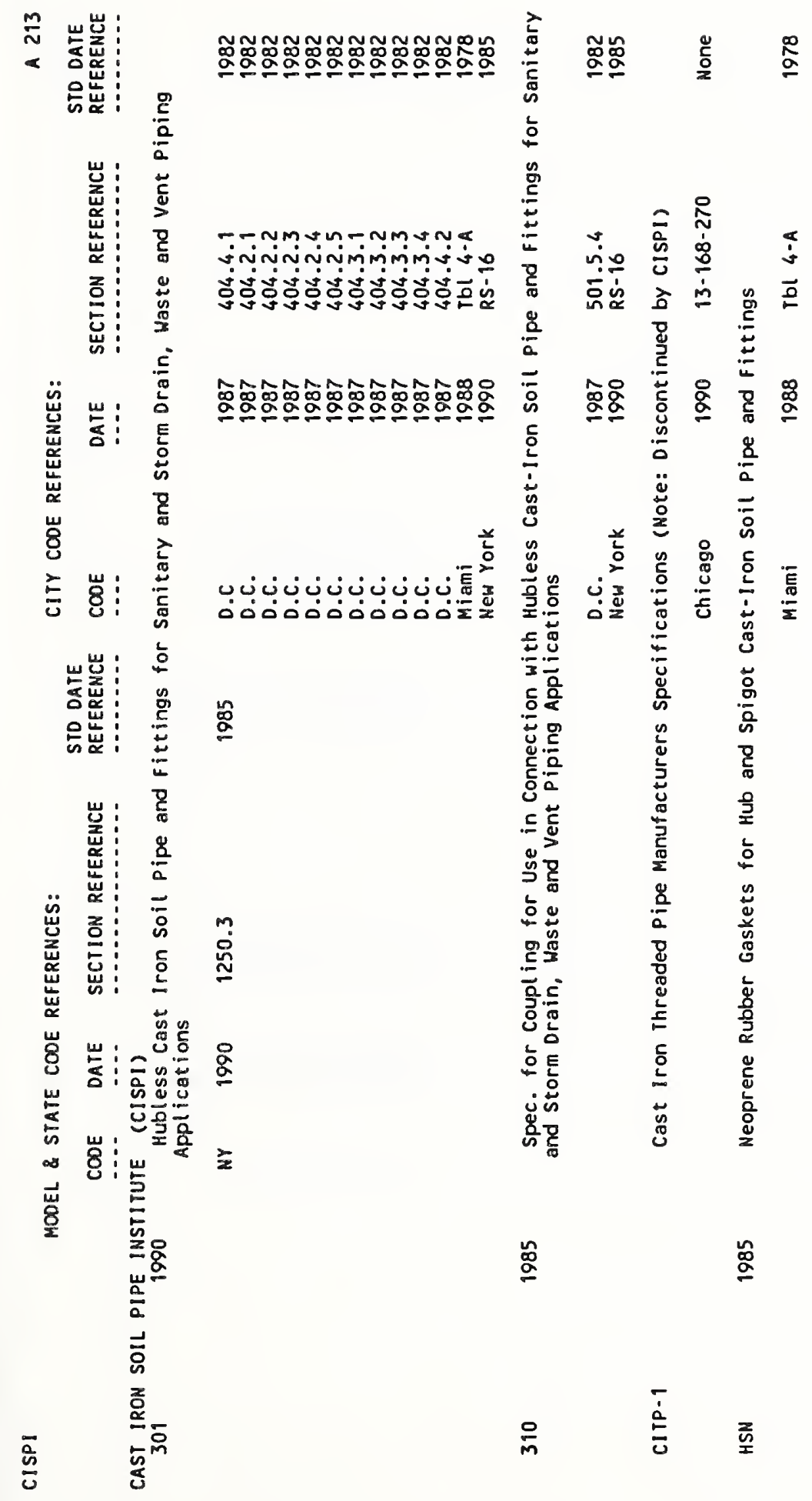




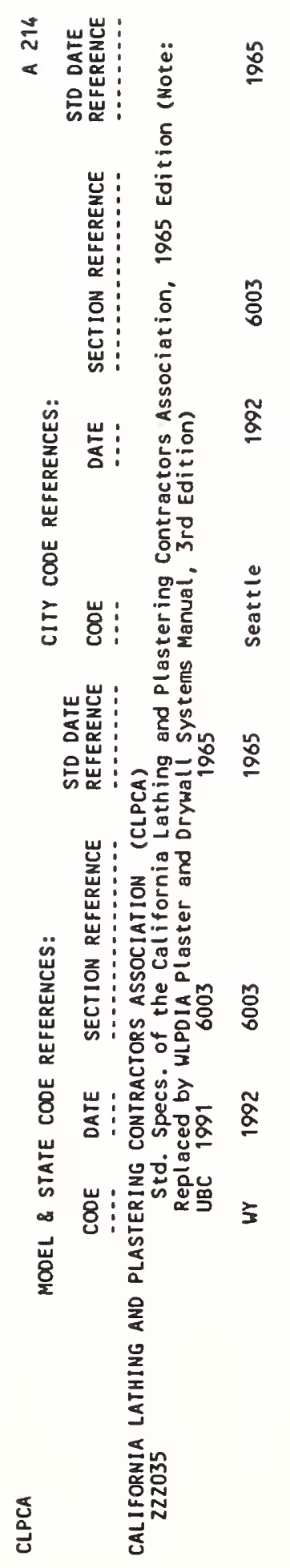



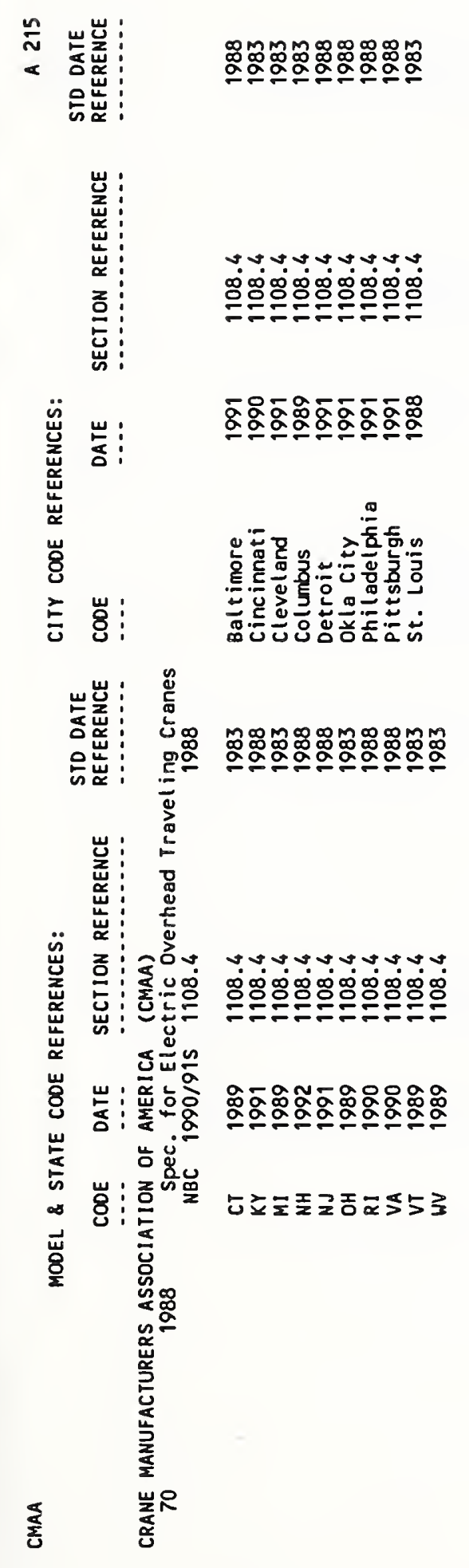


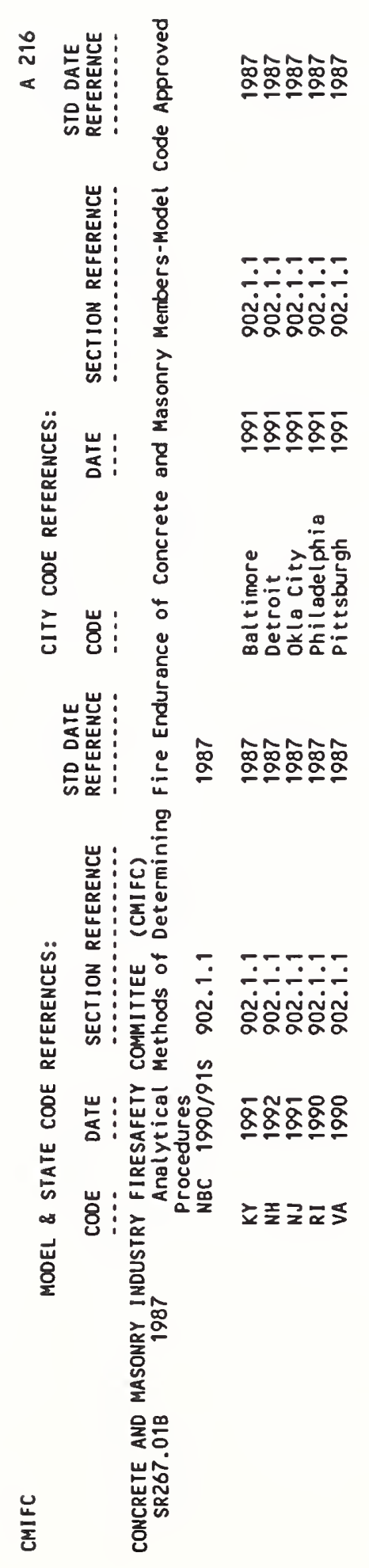




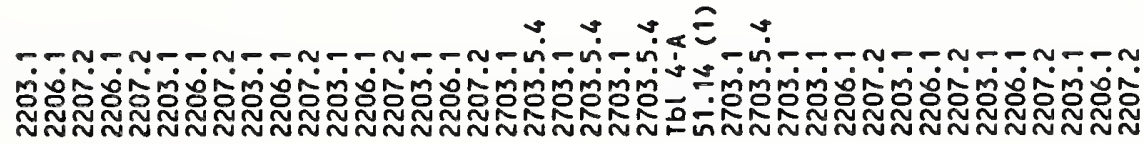

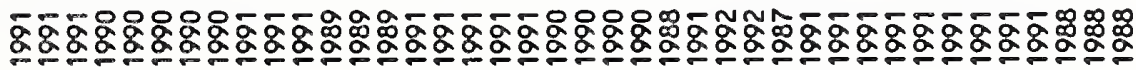

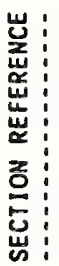

范岕:
㟧

岁

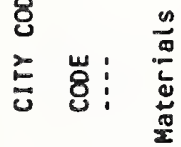

널:

各焉:

点:

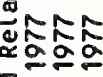
幽

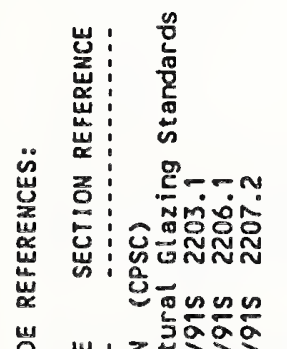

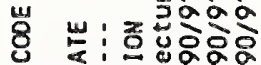

w

is

$\infty$

할

ธ : 돈

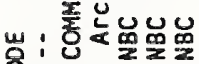

8

㟧

ড়్ㅀ

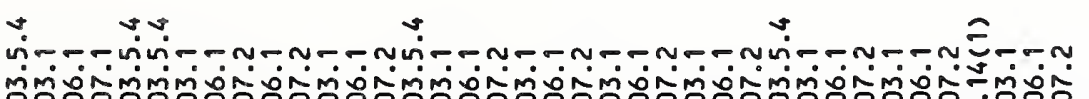

ผ

-

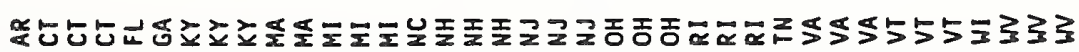

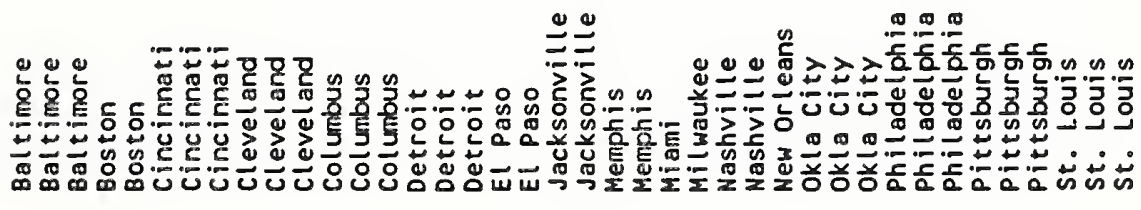

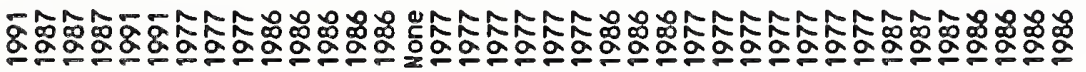

జّó

蛋근

部总

嵓

iे
웅

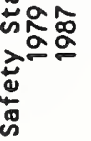

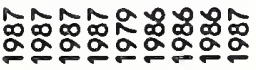

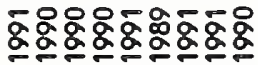

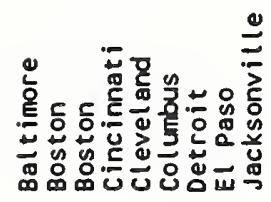

苙

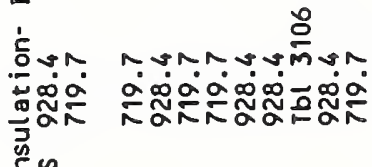

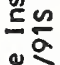

:

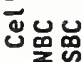

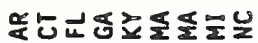




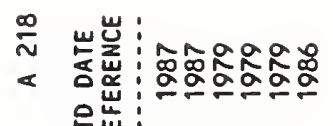

क它:

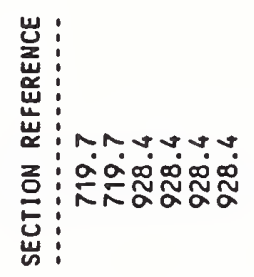

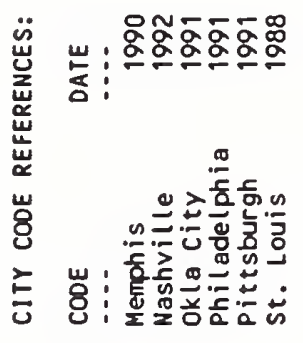

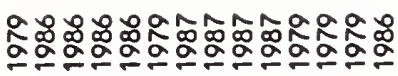

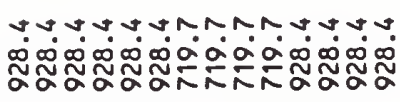

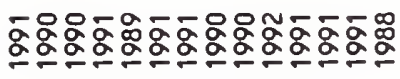

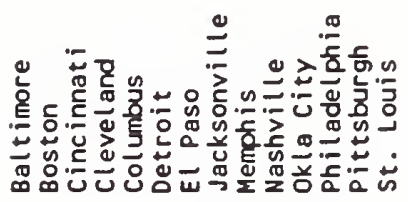

岕:

政

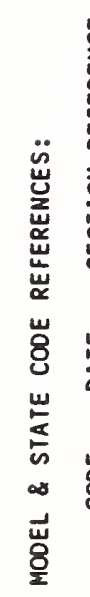

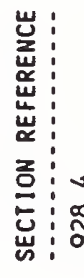

山 N-a 0 - 00

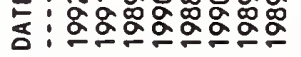

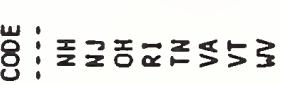

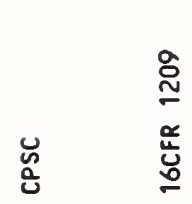

ํํㅇ

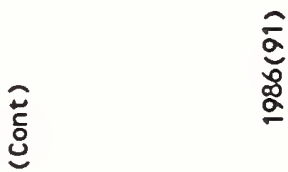

2
0

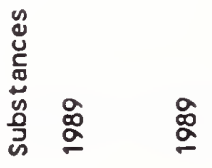

范

茞

เ

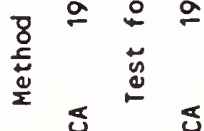

$\bar{\alpha} \quad \bar{g}$

j)

范

苑 范 

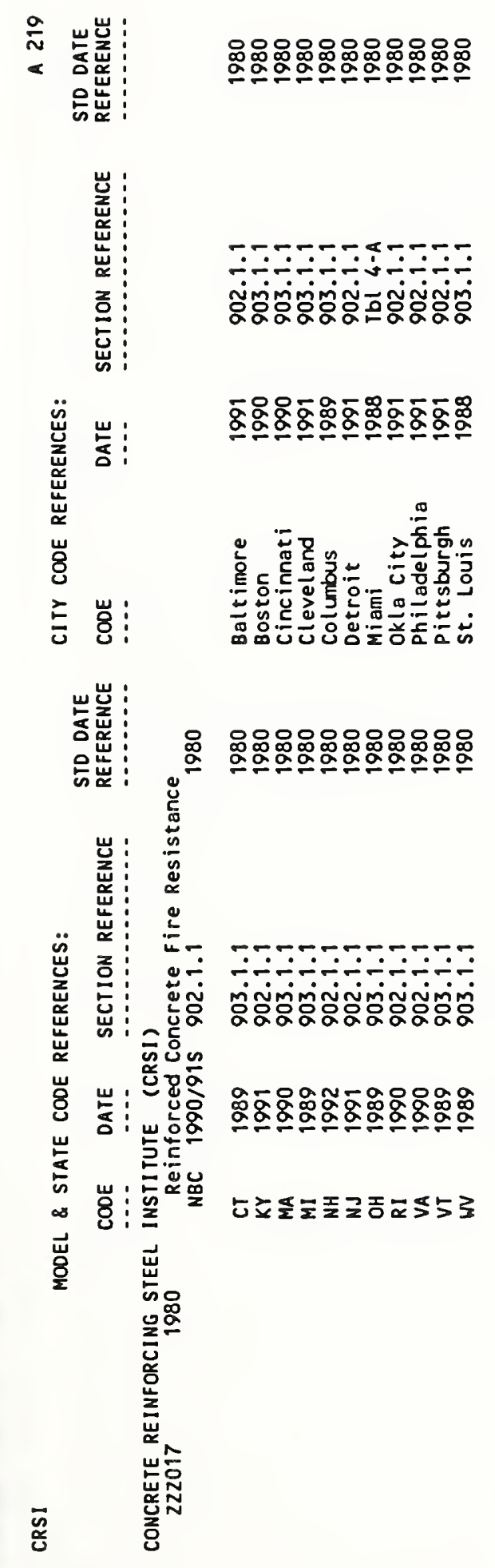


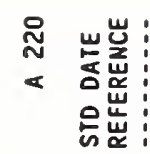

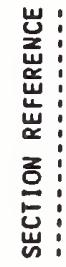

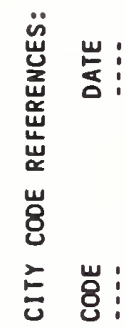

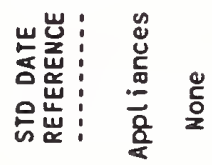

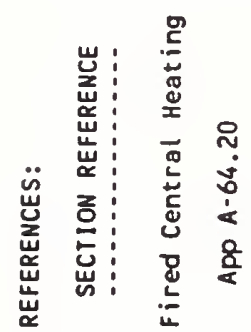

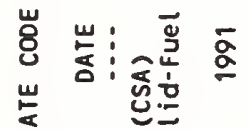

否

\&

㟧 㟧

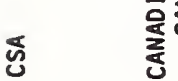




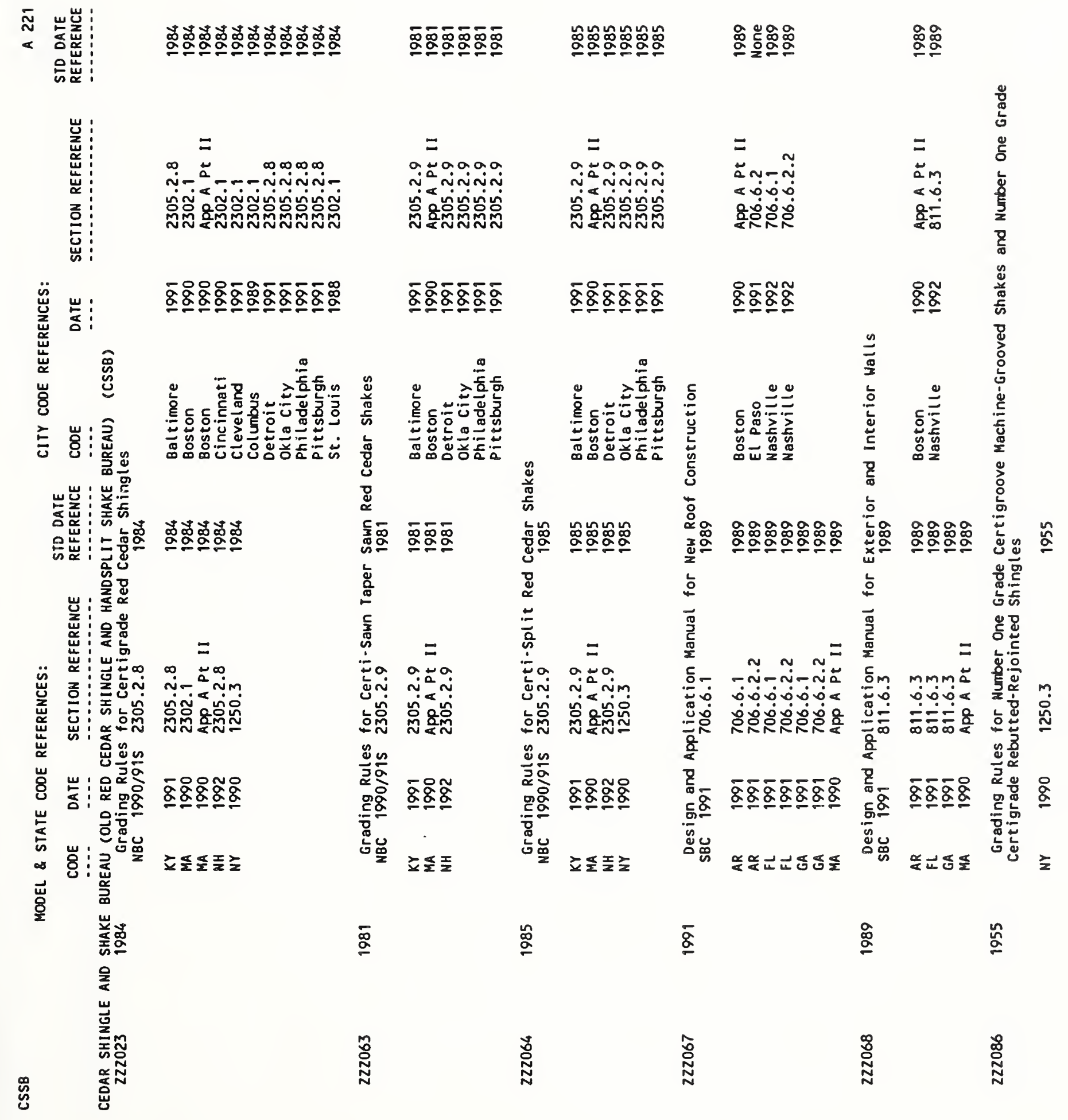




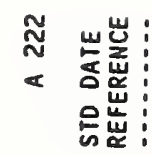

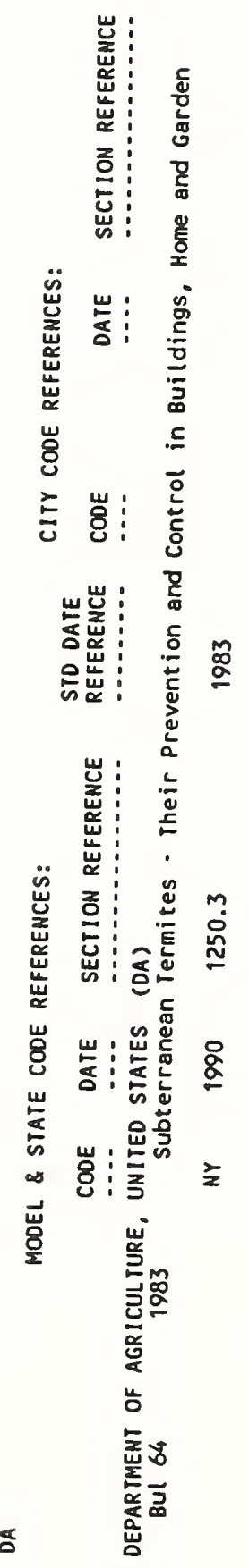




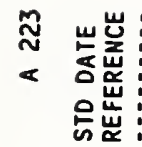

:

芯岕:

ठ্ّ

岂

岁岕: 崖

端:

至

ư

胥 i

出

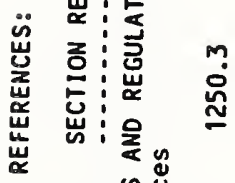

岁出: 出喜

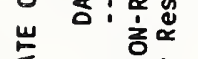

占

\&

㟧

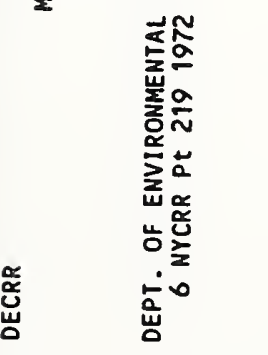




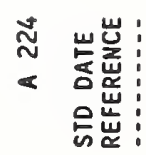

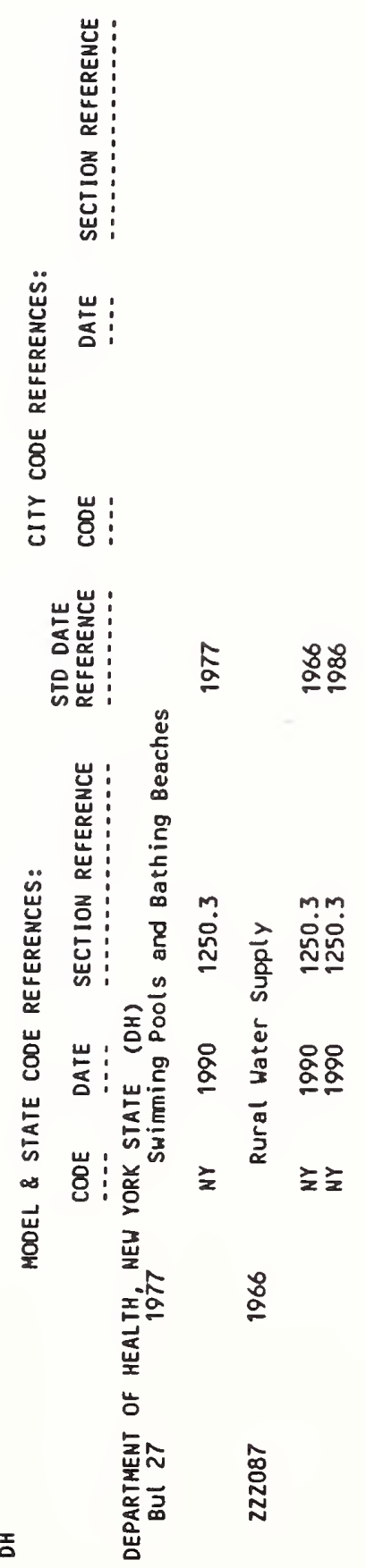




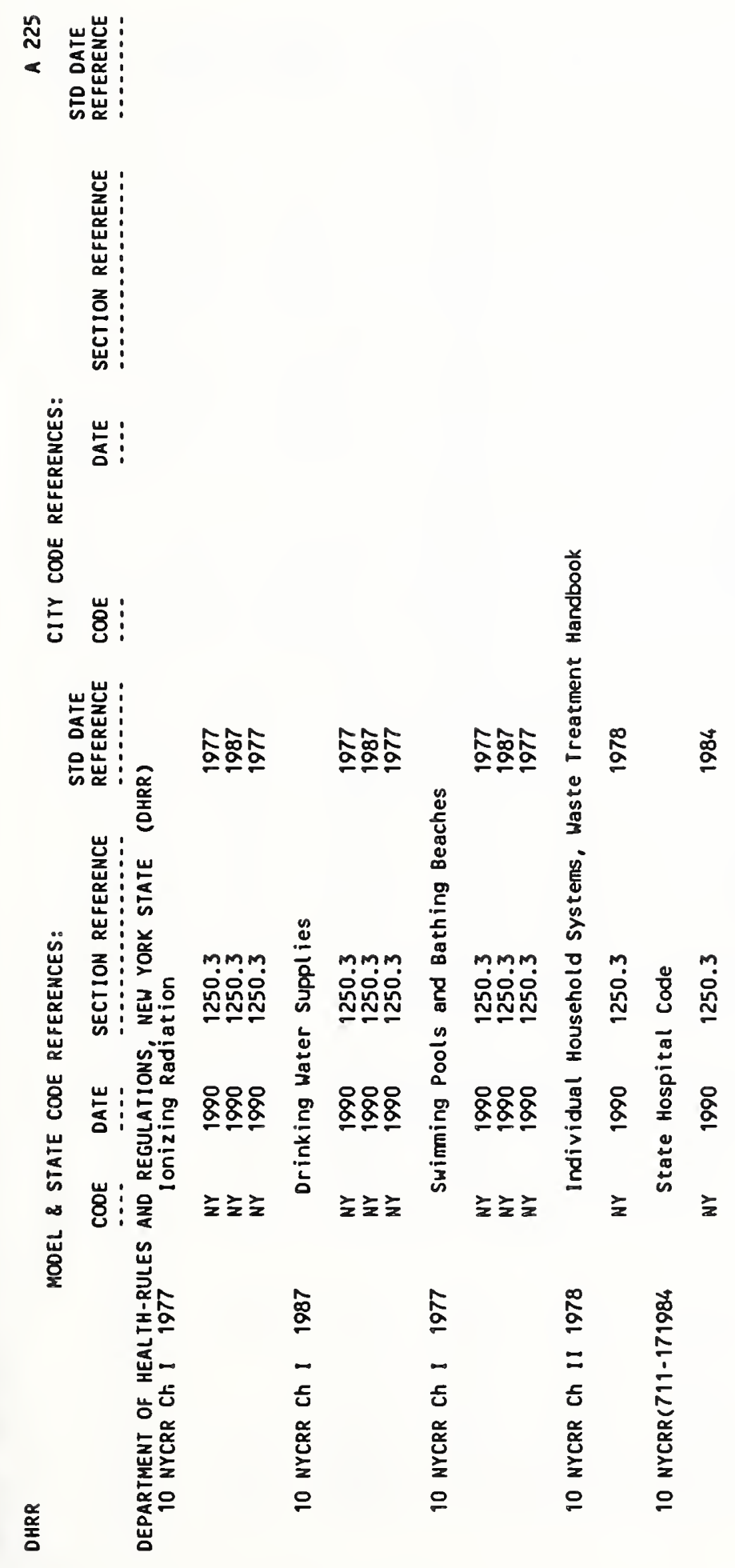




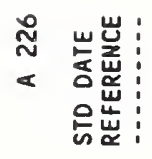

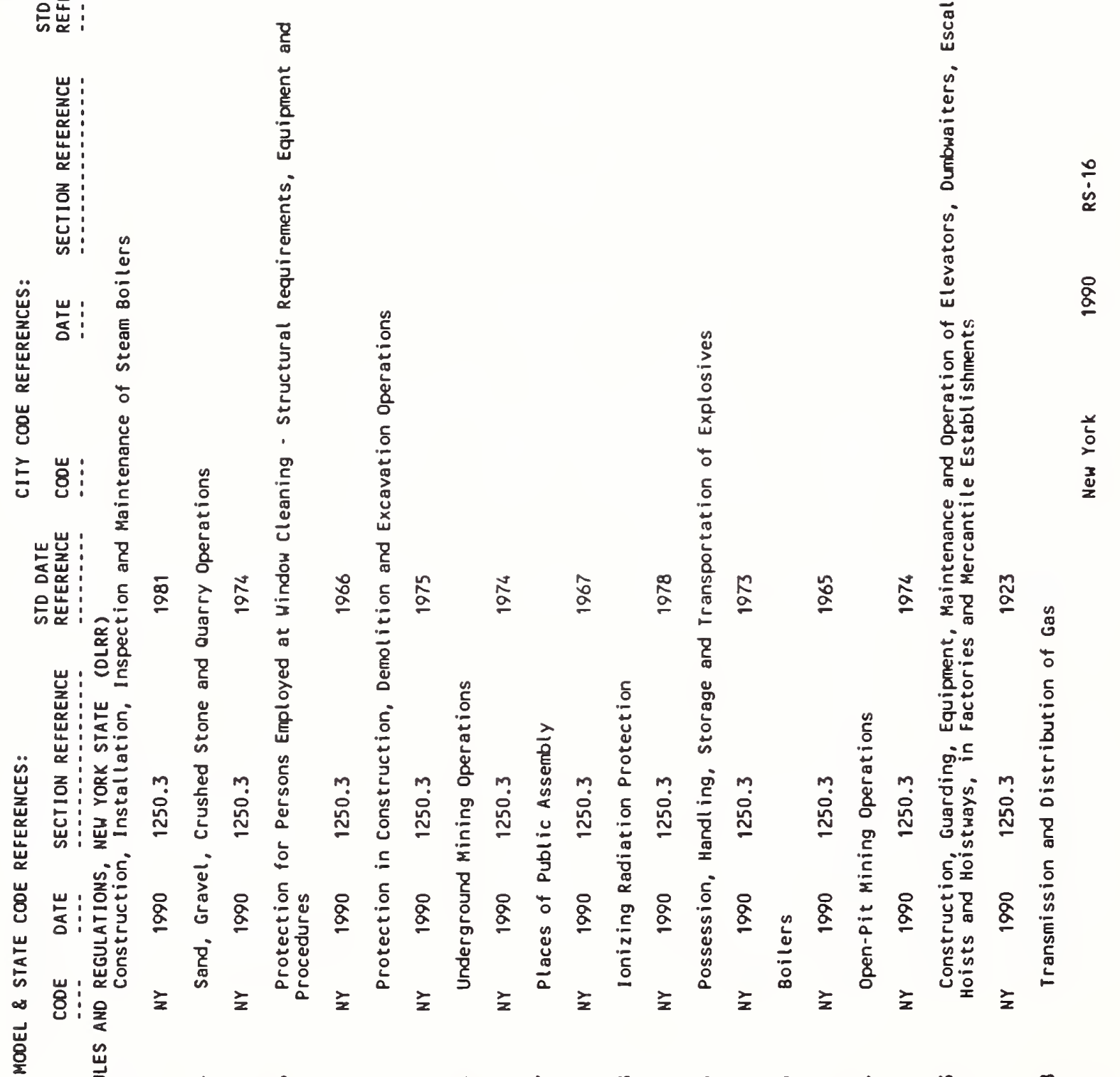

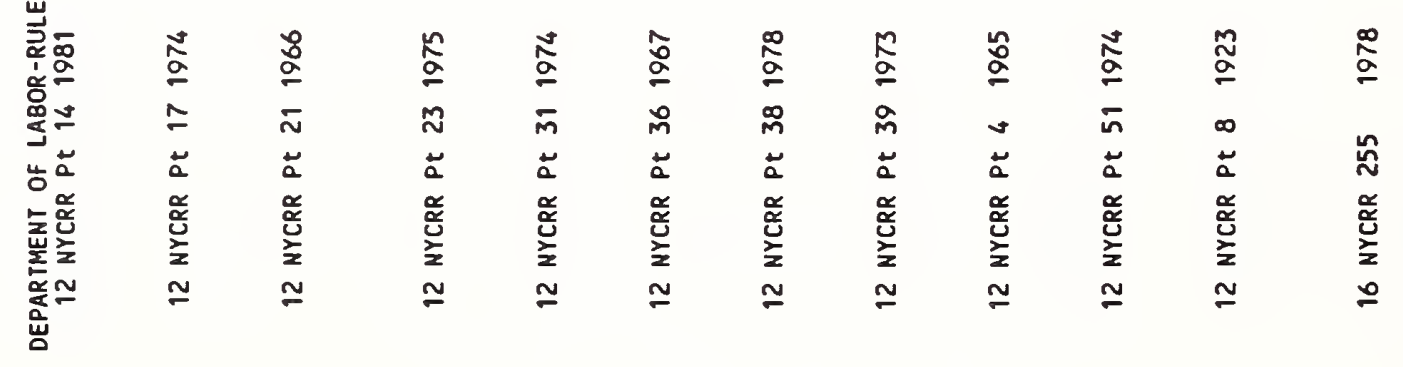




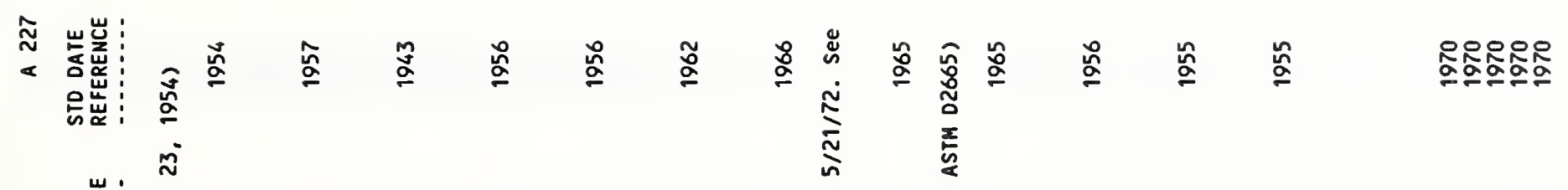

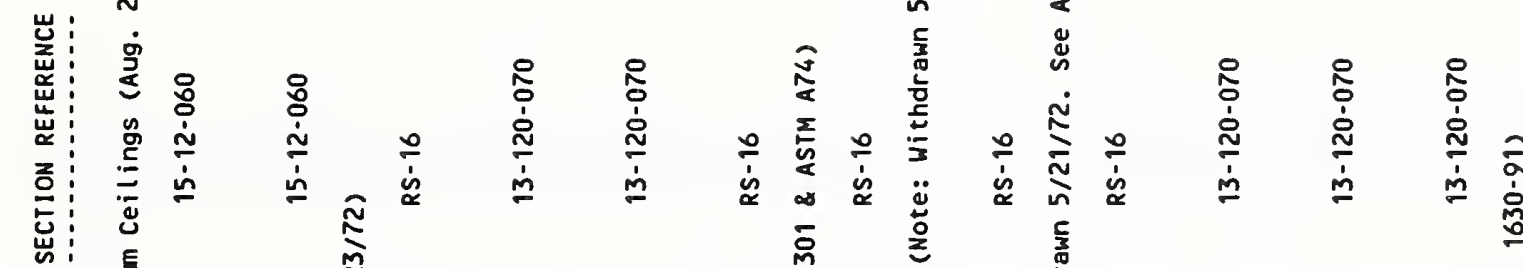

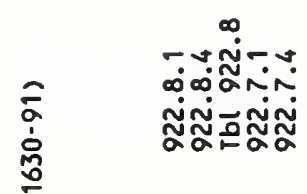

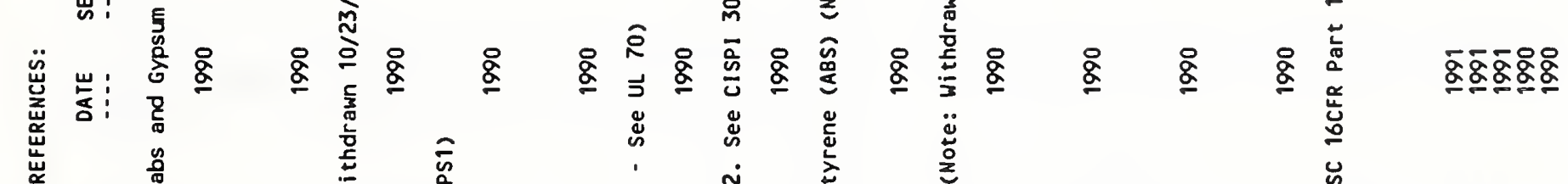

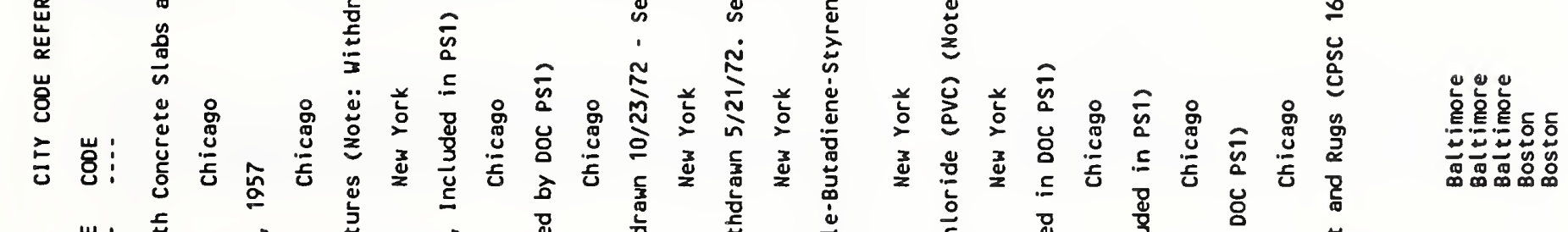

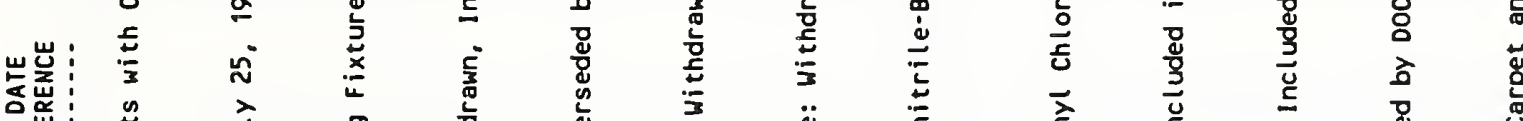

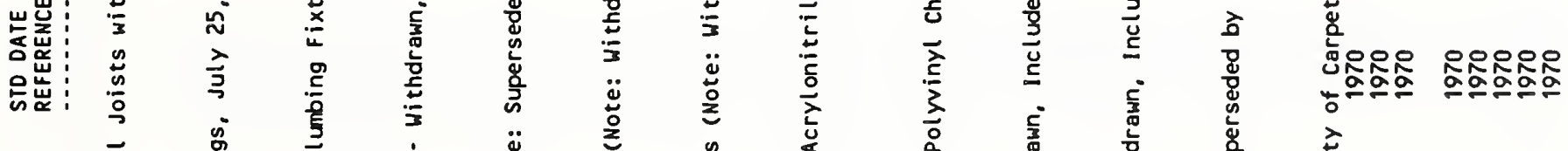

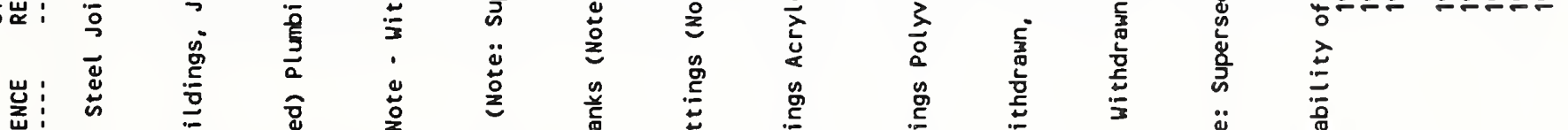

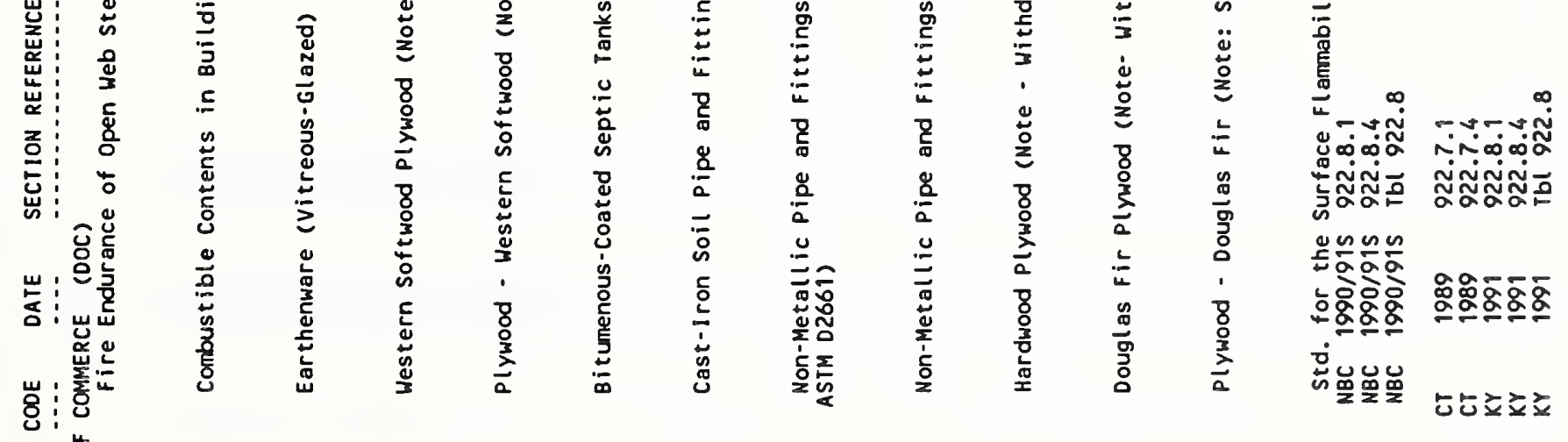

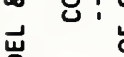

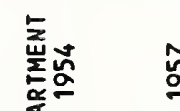

$\stackrel{2}{2}$

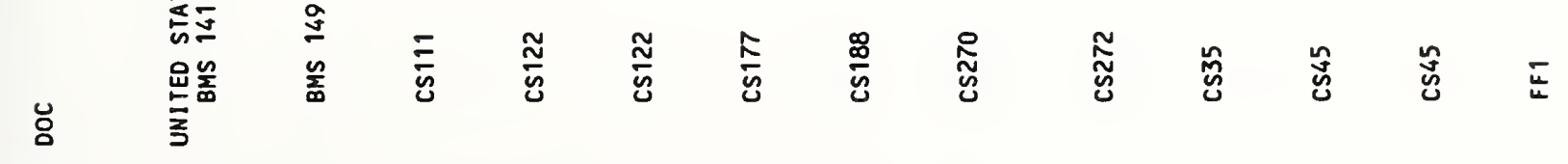


ฐึ

崖

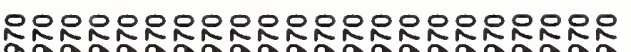
的空:

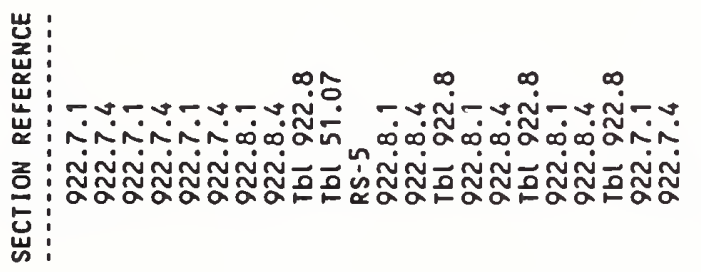

苍 s:

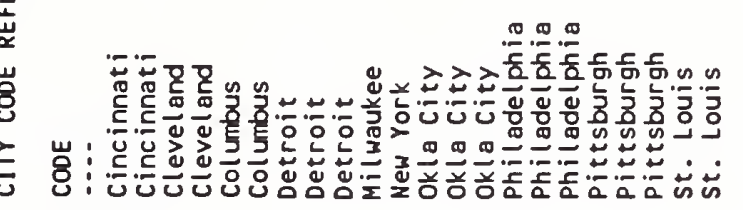

容 :

젼

i

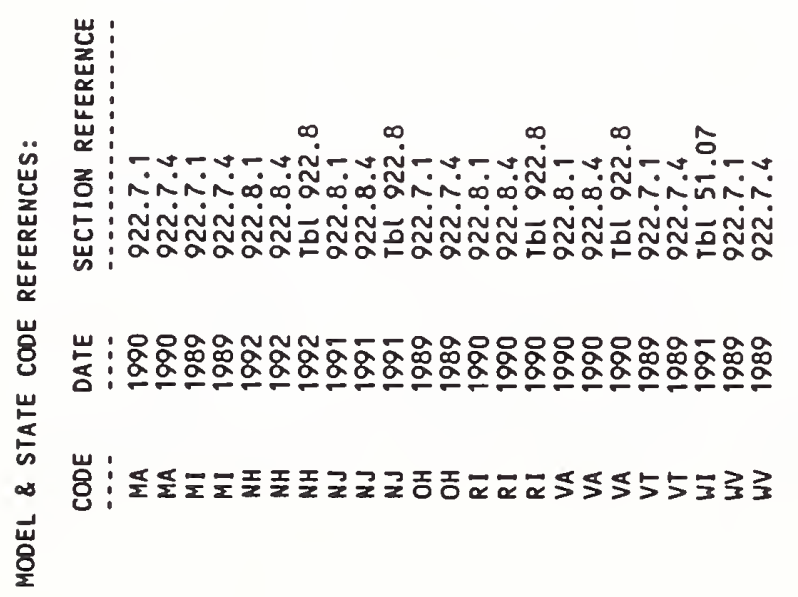

芒

음

$\stackrel{8}{\circ}$

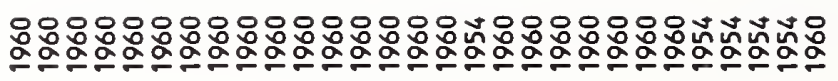

舷

-

u

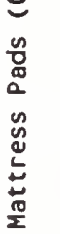

$\frac{\mathrm{d}}{\frac{\mathrm{a}}{2}}$

密

品

莣

。

:

离

号

ธ

它

$\stackrel{\widetilde{N}}{\underline{\alpha}}$

䒚 ojom

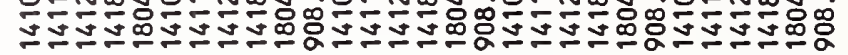

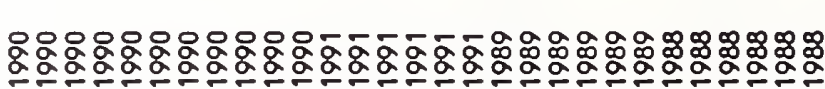

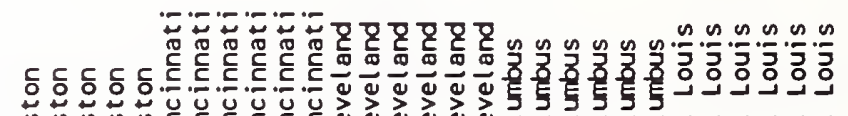

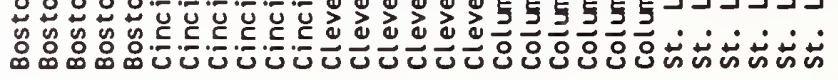

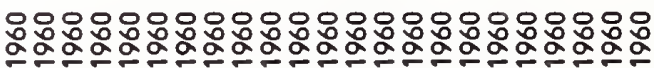

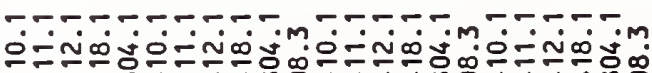

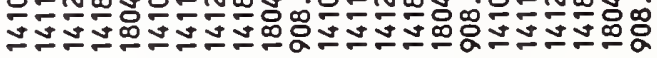

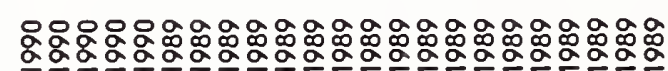

동

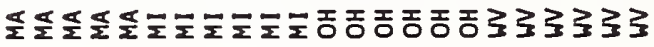




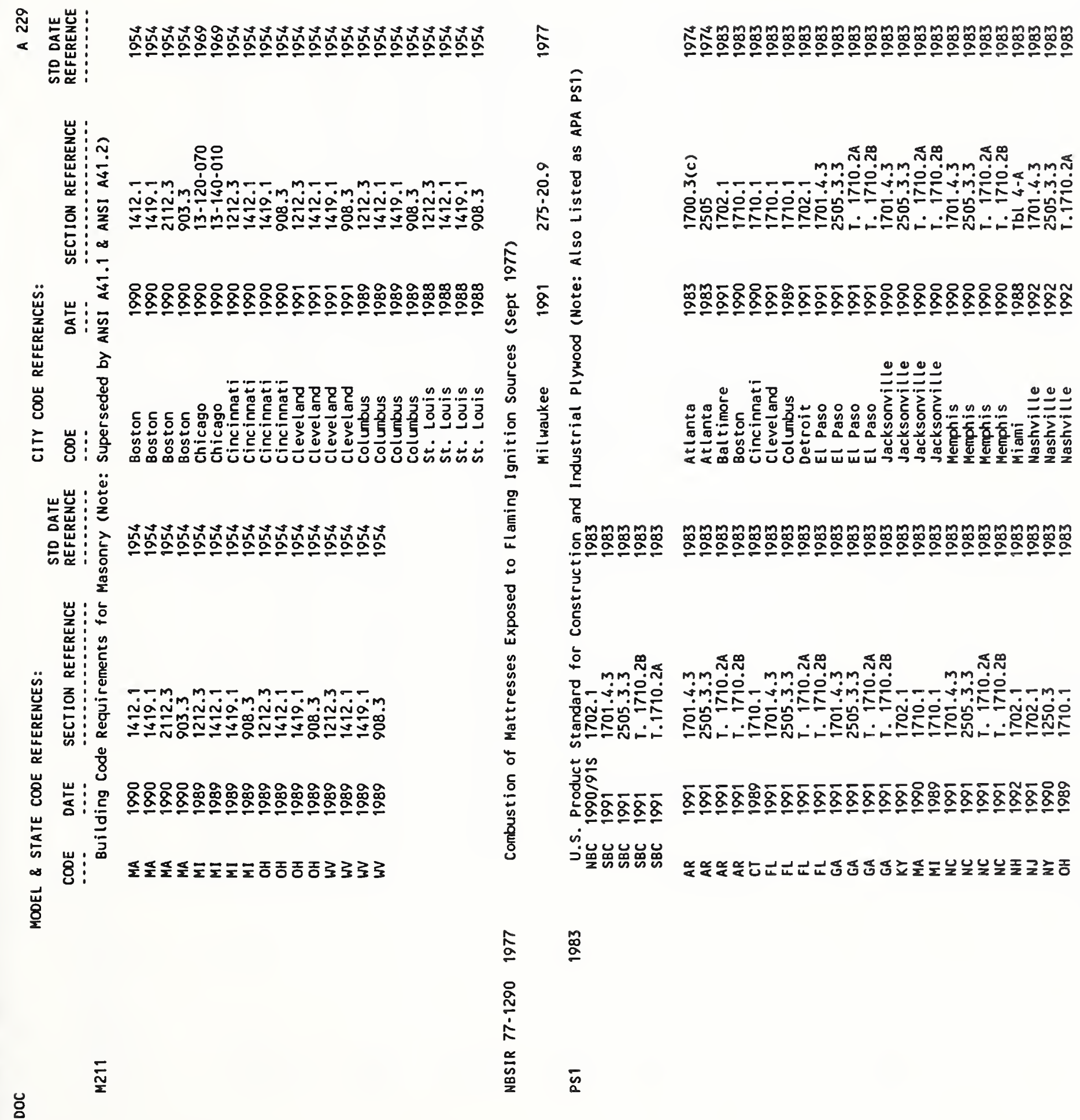


只 uㅗㅁ

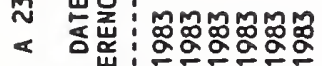
은ㄹ⿺

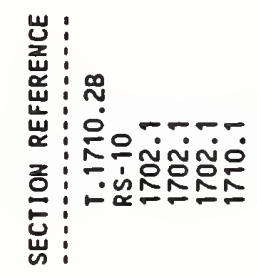

$\underset{u}{u}$

岁 峞

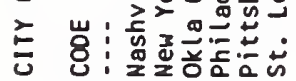

崖:

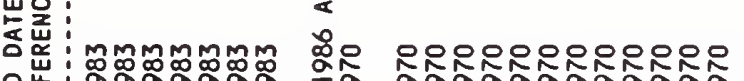

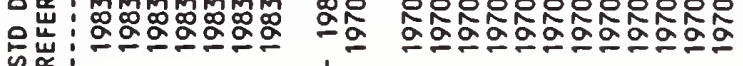

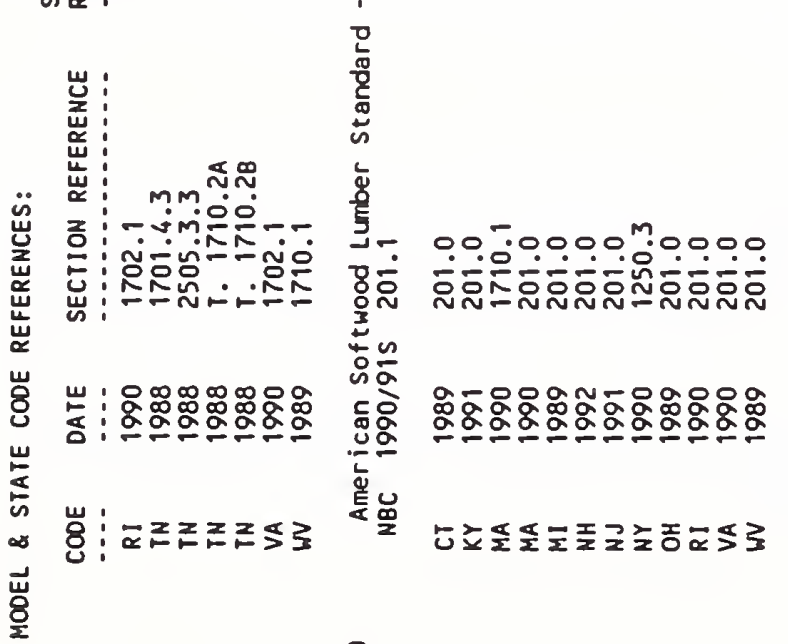

它

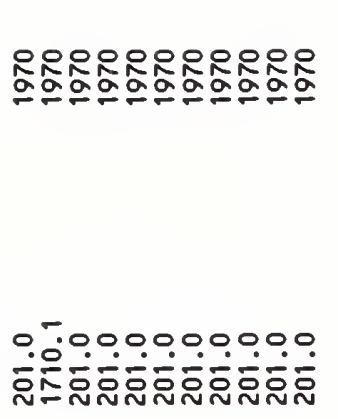

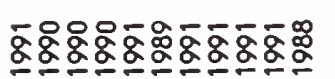

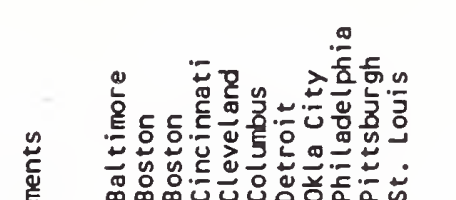

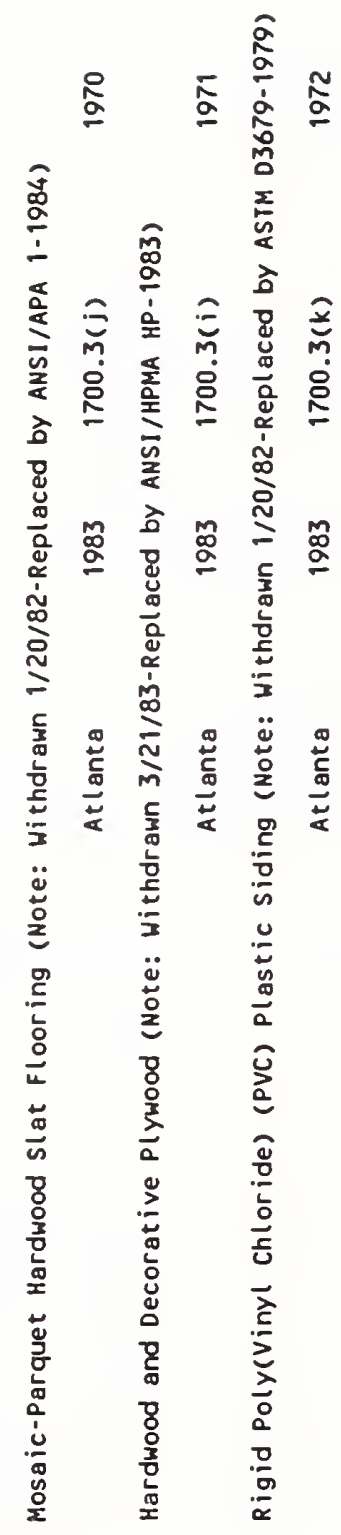

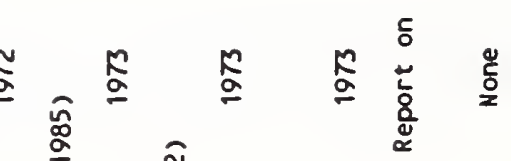

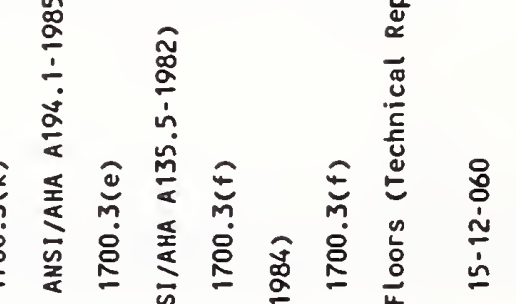
डิ 茎

古 宮

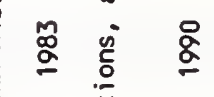
줓 은 高

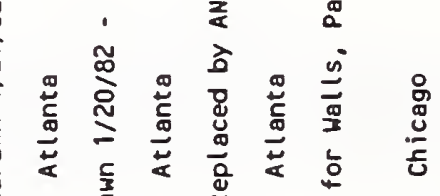
문 产 כ 兽

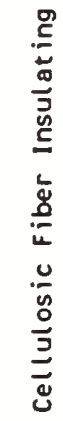
흘 䒓 范 点 בั

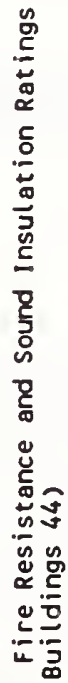
$\underline{2}$

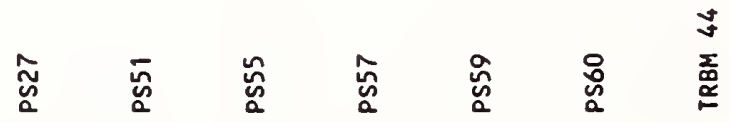

잉

๕̊ 


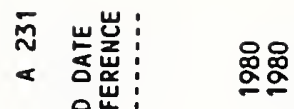
㩆 :

岩 :

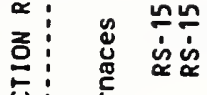

出:

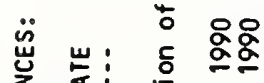

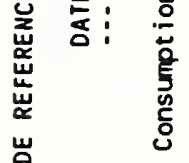

岁

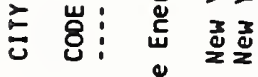

닌 :

它总

응년

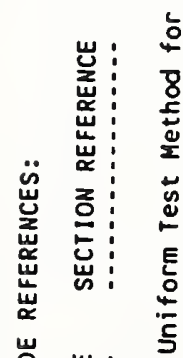

岁枈:

岕 w, 岁家

œ

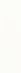

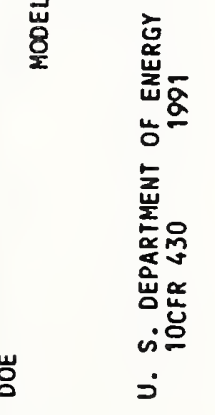




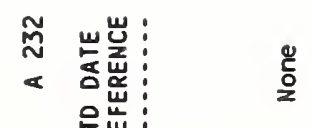

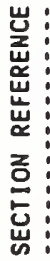

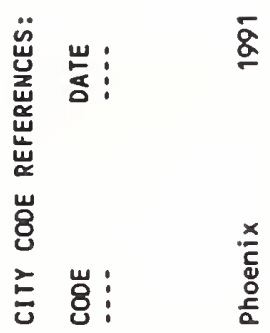

崖岸

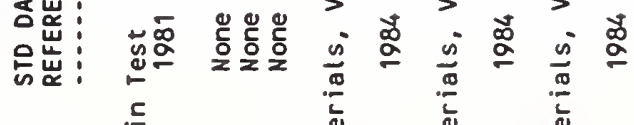

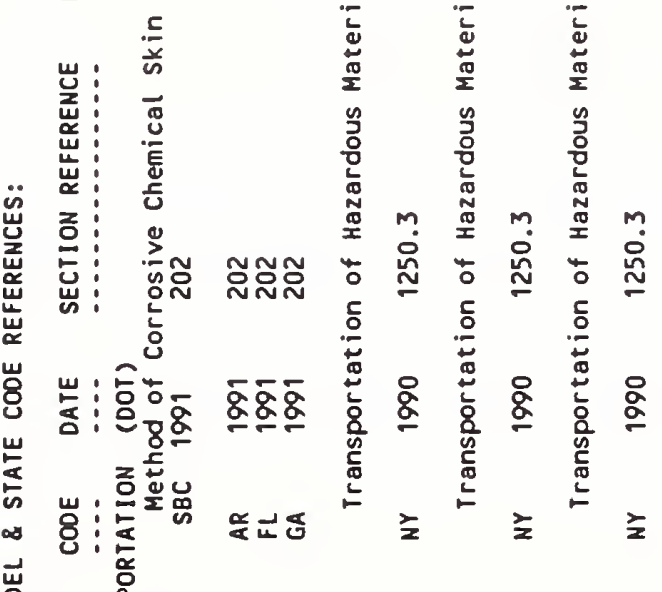

㟧

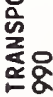

늠

$\bar{\alpha} \quad \bar{\alpha} \quad \bar{g}$

เ

起

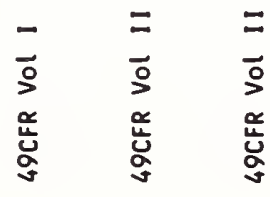




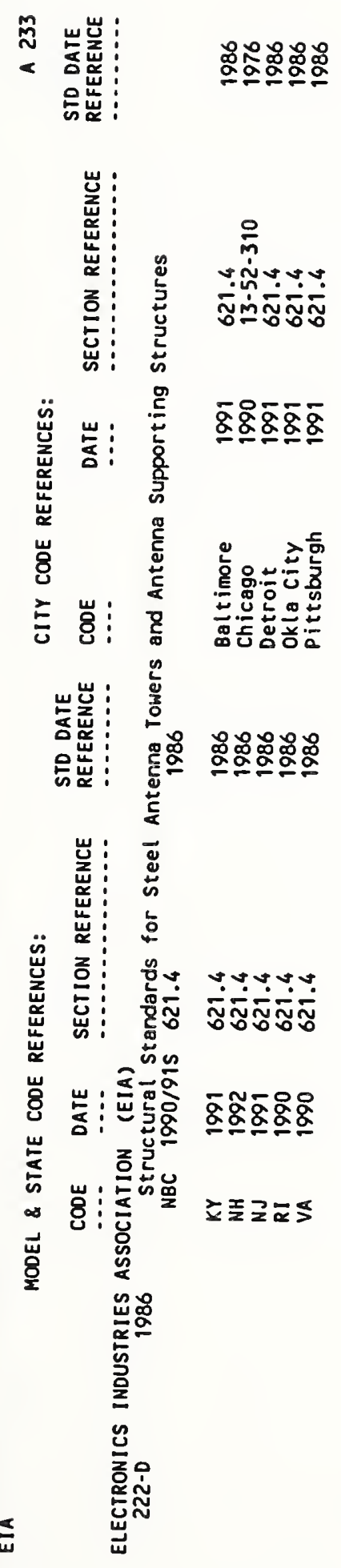




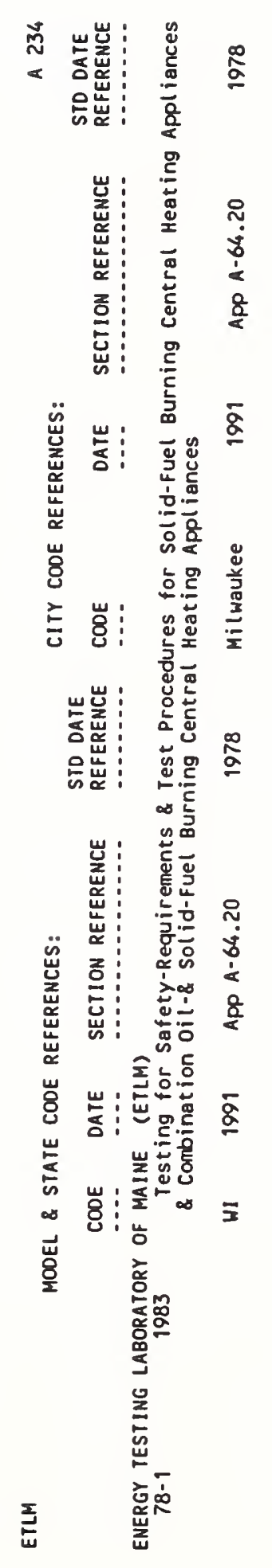


等岕:

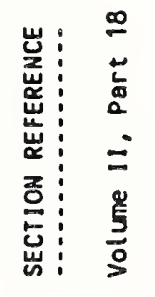

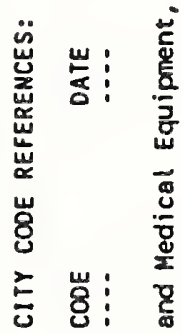

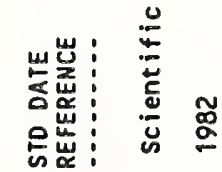
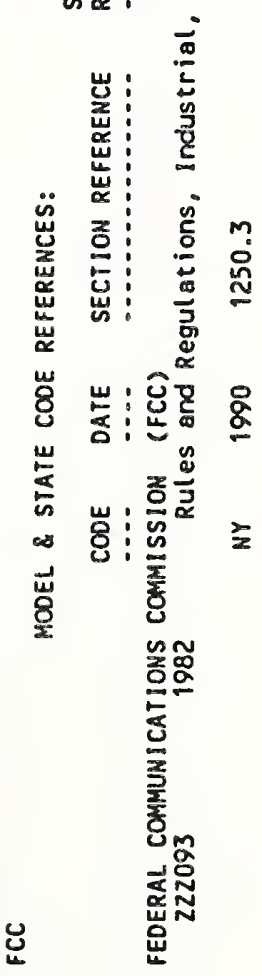


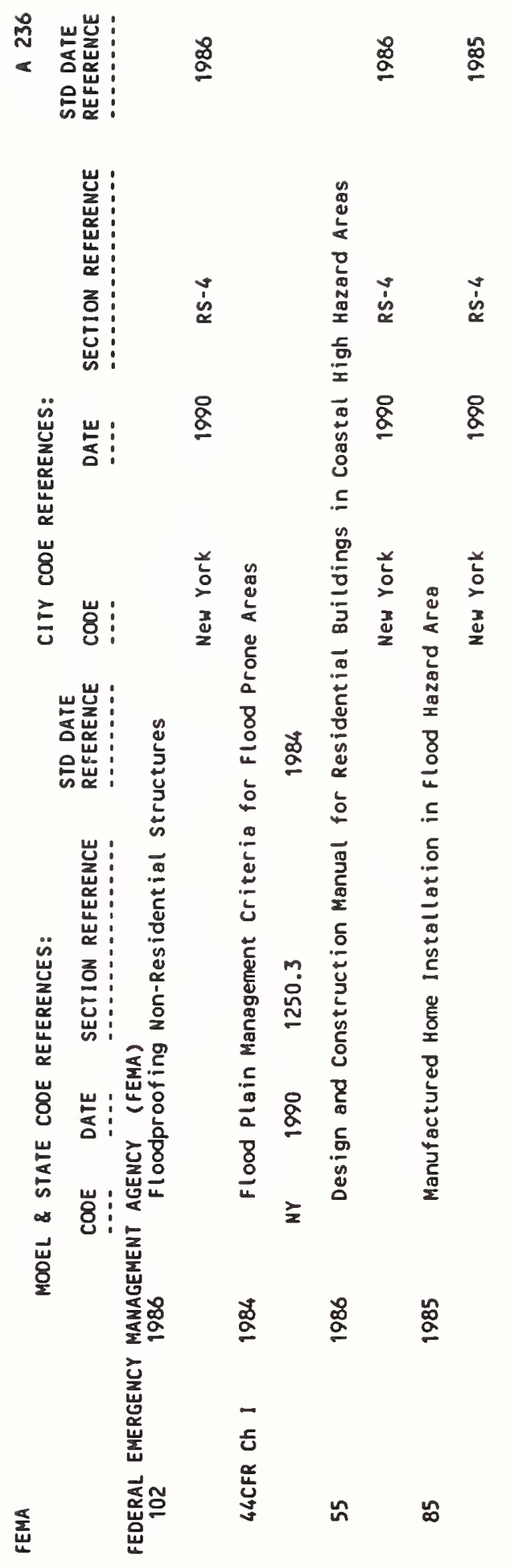




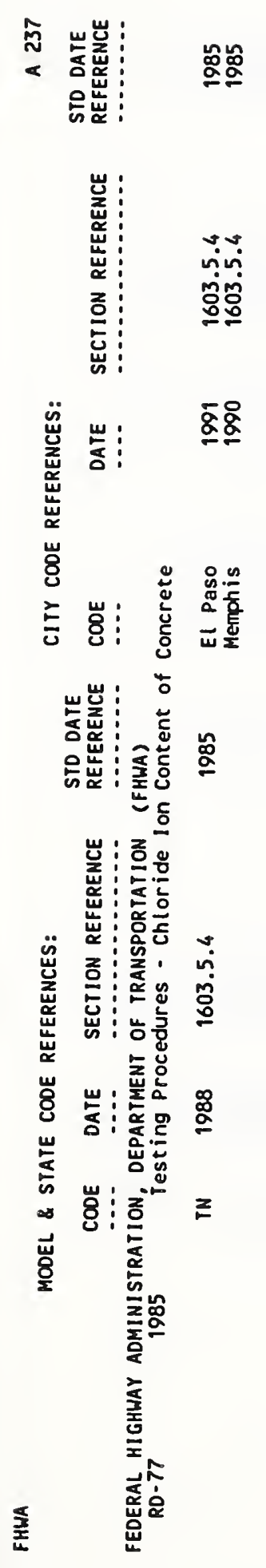




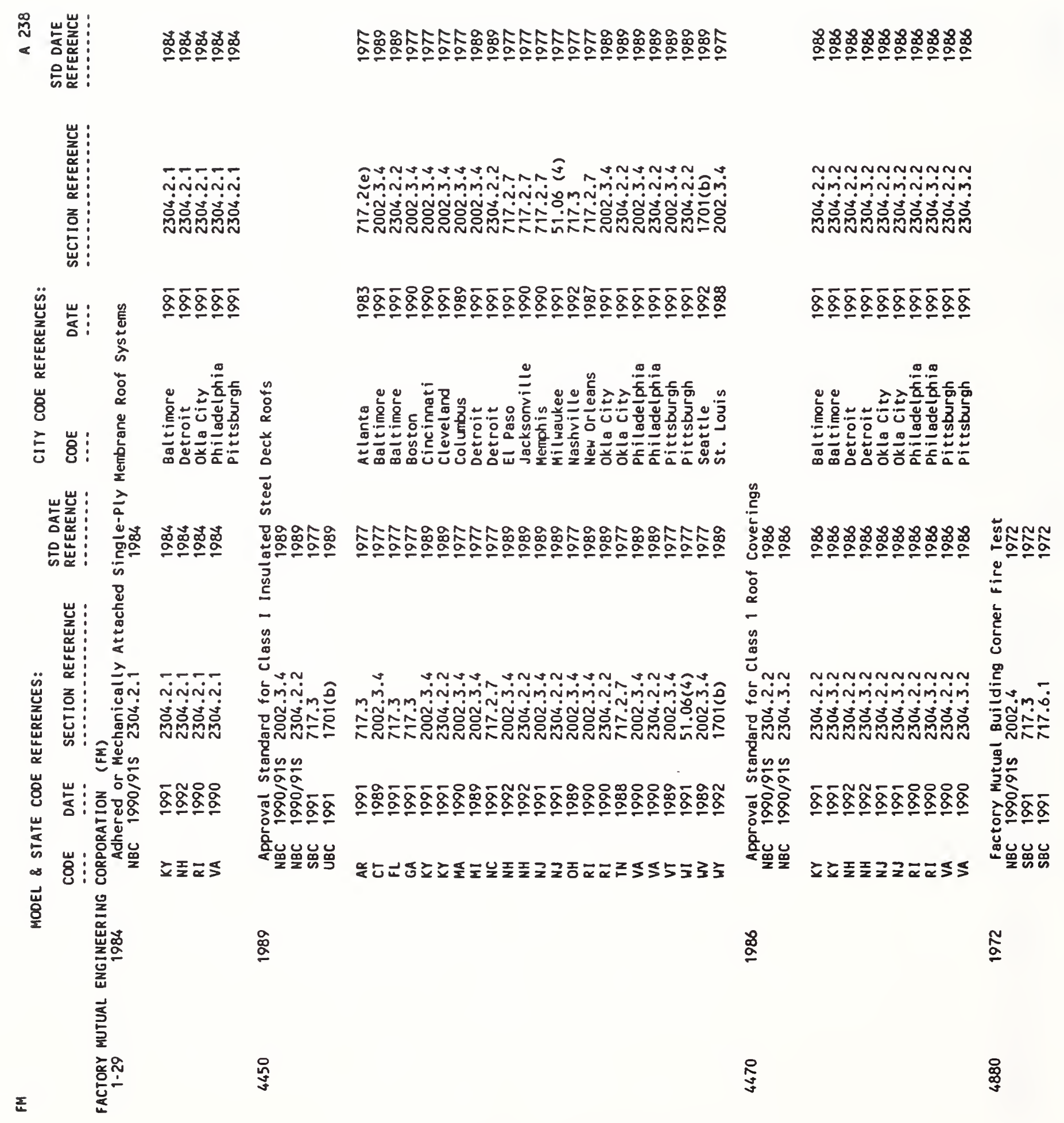




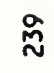
的崖

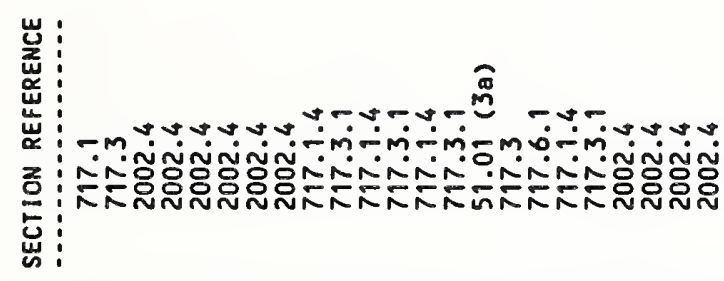

\footnotetext{
岀
}

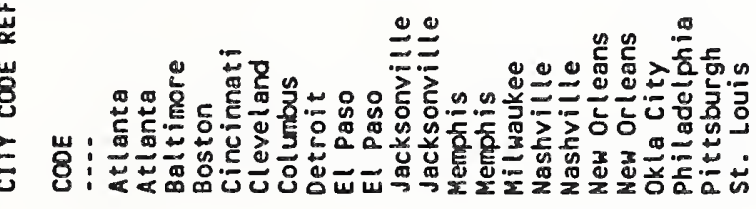

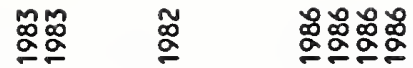

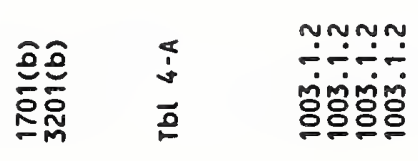

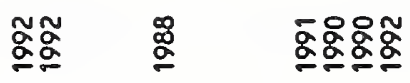

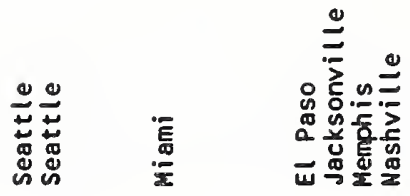

岕:

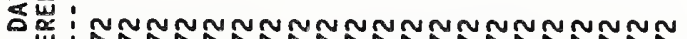

宽:
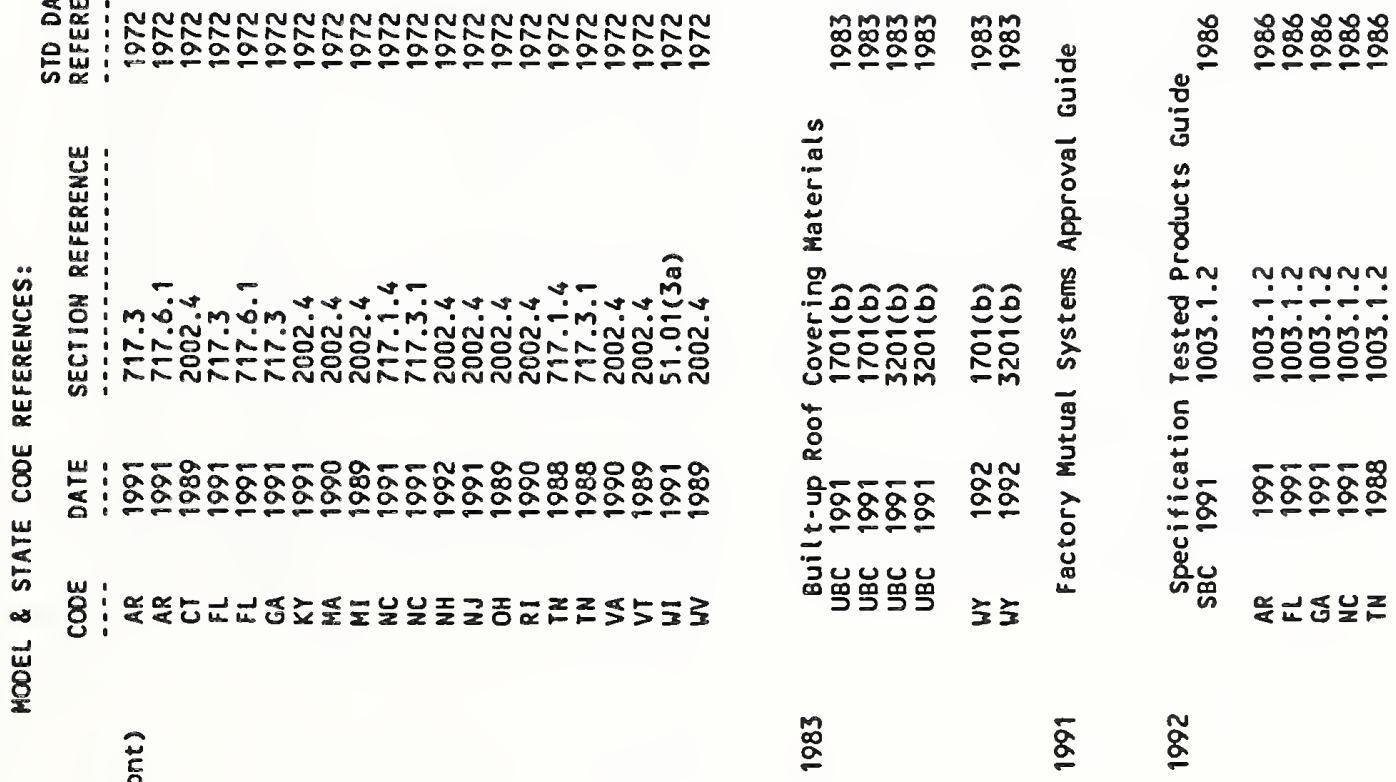

莒

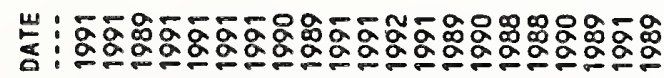

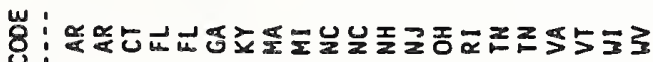

㟧

$$
8
$$

$$
\text { 更 }
$$

I

品

结

$\underset{\substack{0 \\ a}}{\stackrel{0}{0}}$ 
号 岕: 若

< 对 :

息岕:

岕両:

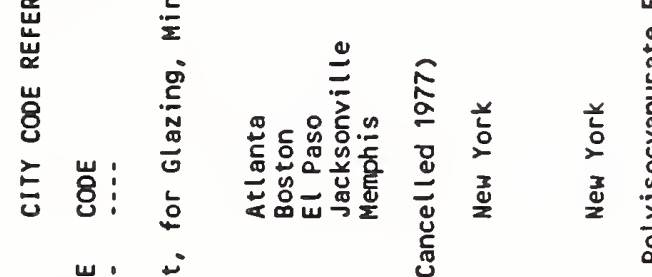

岁岁: 蓄

这岀: ‘

运: 정

i⿱

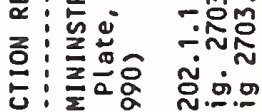

出: 定施

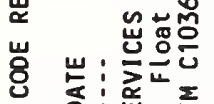$$
\text { . }
$$

山 : : 这心

鳏

: 荧

똔지

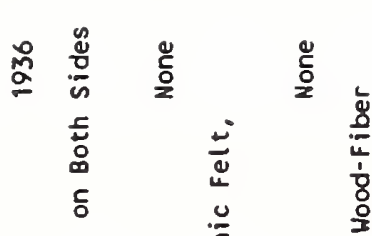

مَّ人

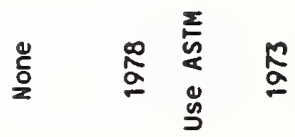

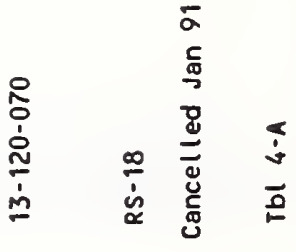

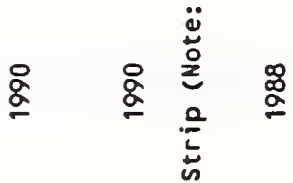

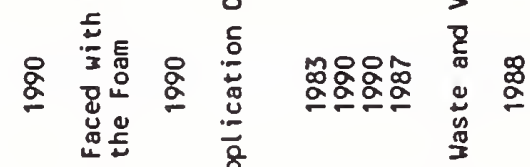

$\frac{5}{5}$

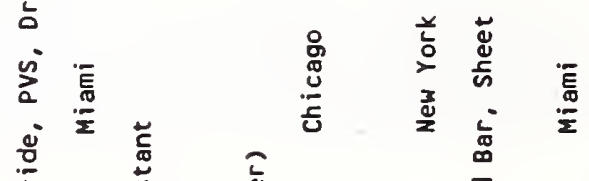

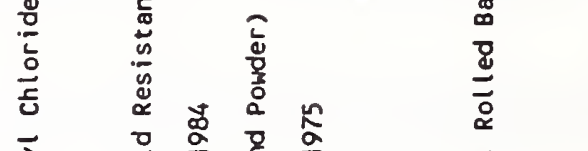

4

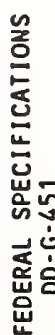

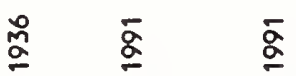

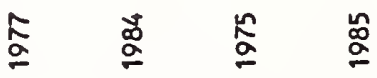

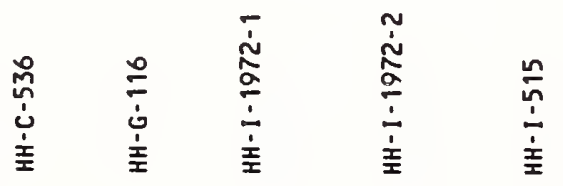

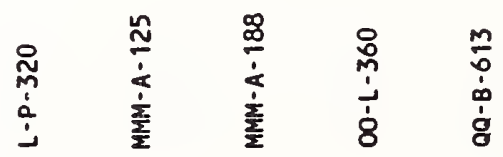




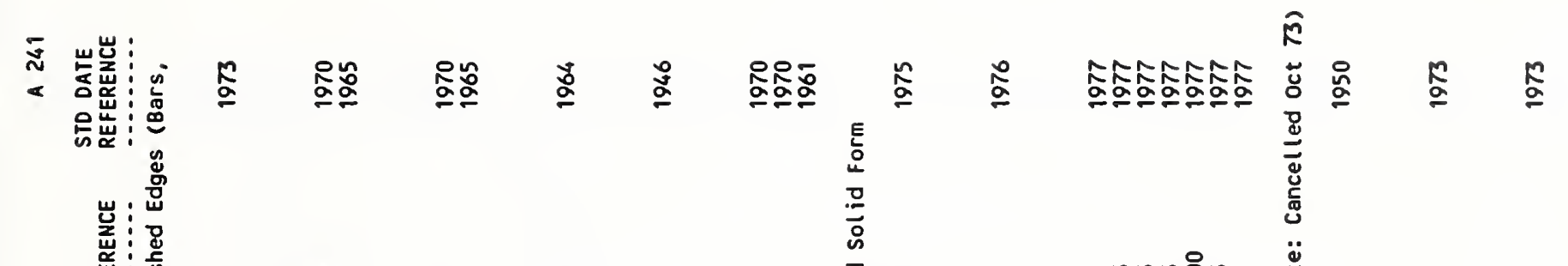

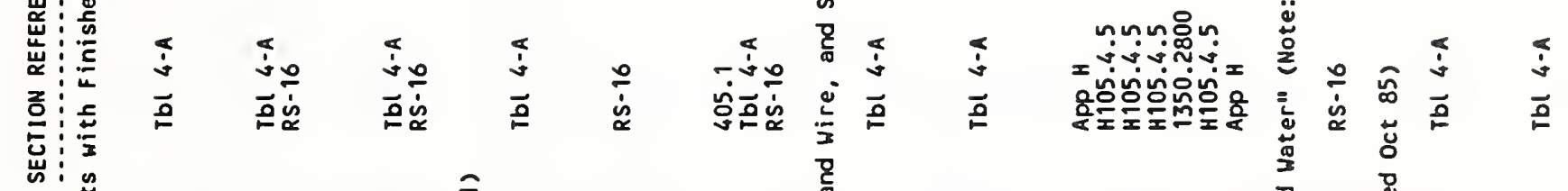

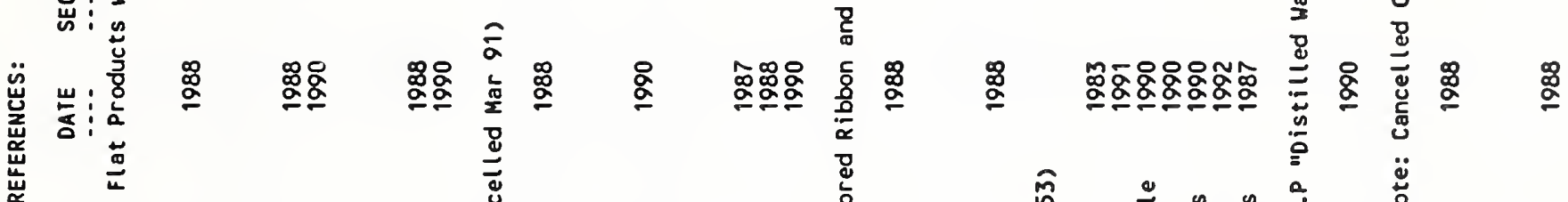

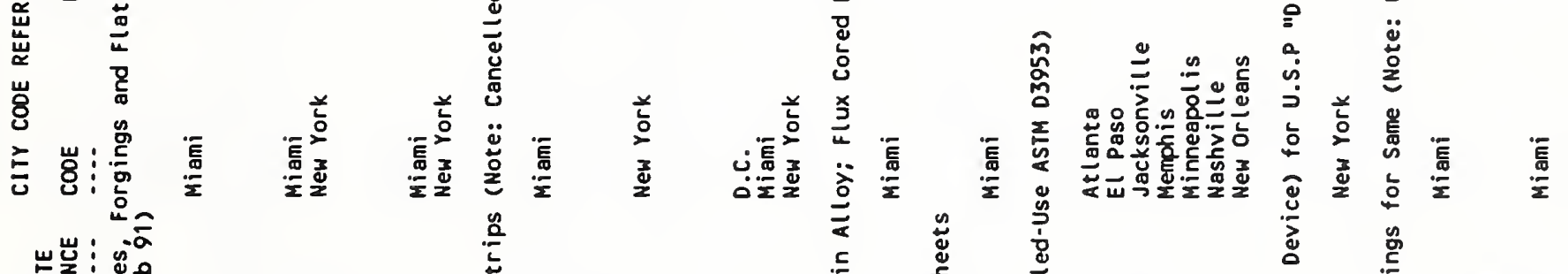

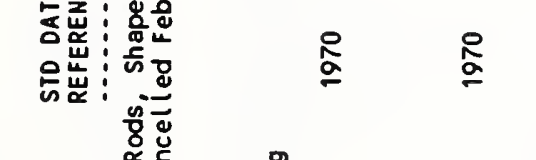

焉崖:

岁:

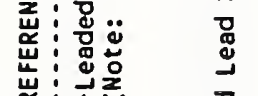

范

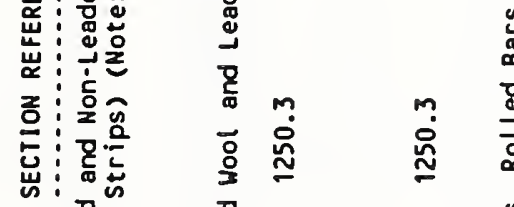

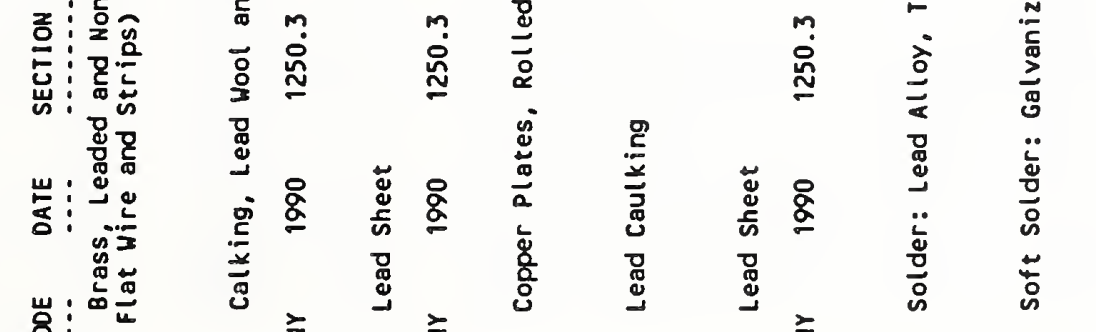

ठ이

续

(1)

$\stackrel{2}{\circ} \stackrel{2}{\circ}$

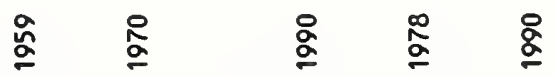

$\frac{n}{a}$

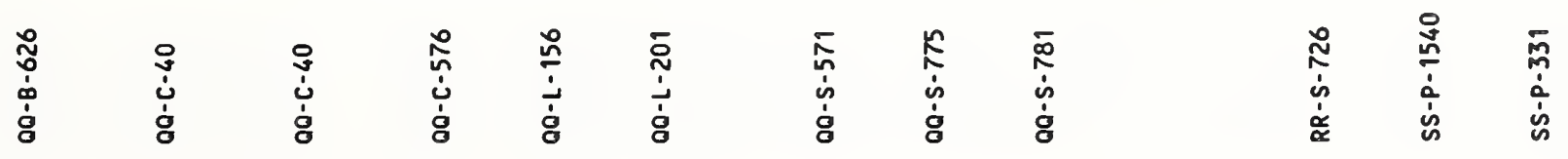




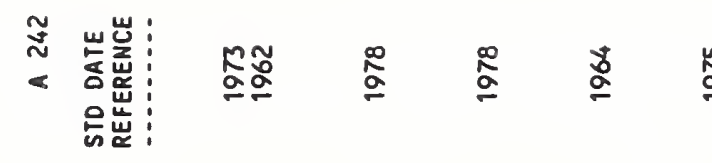

I

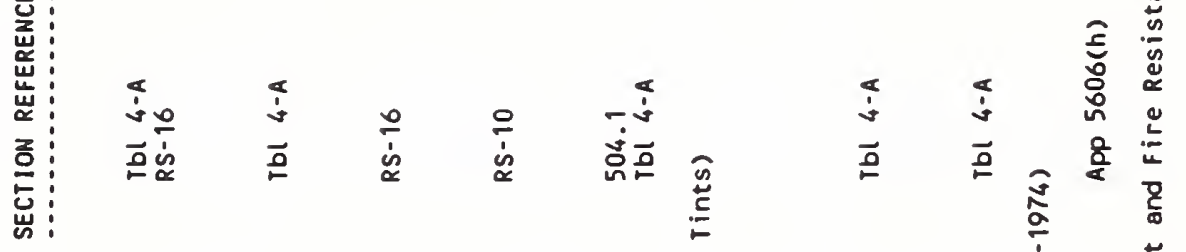

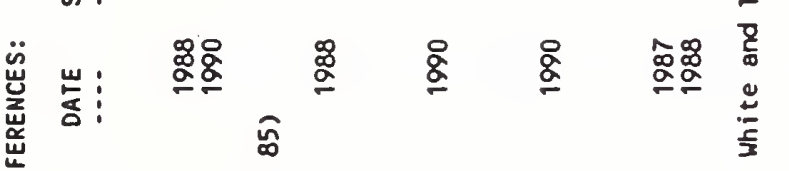

宸

岩

站

㟧

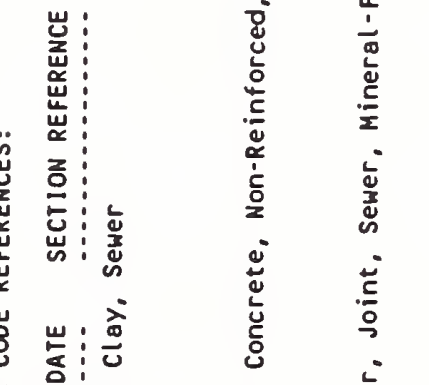

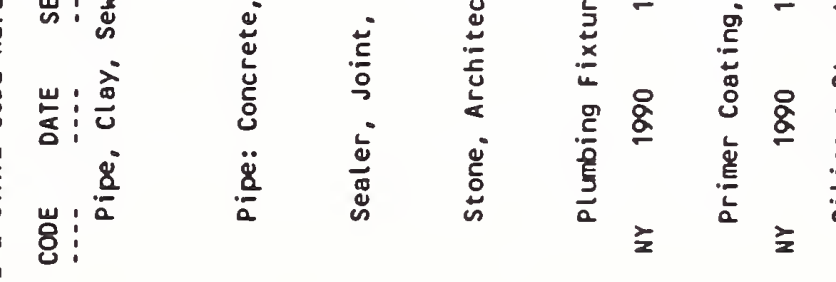

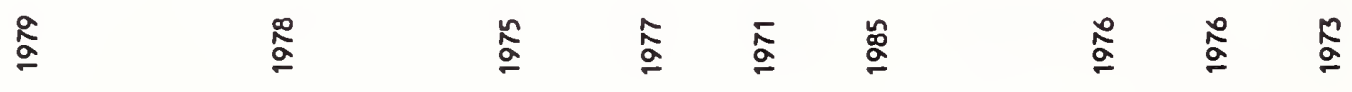

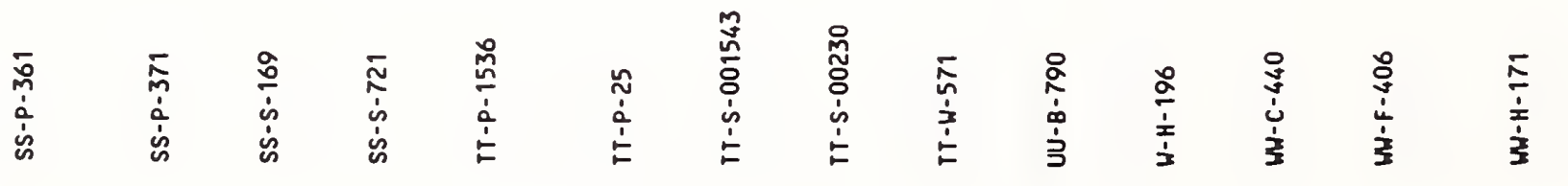

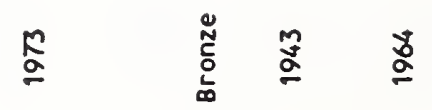

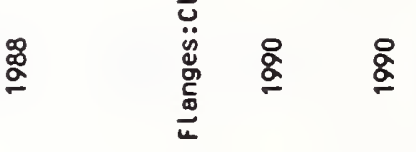

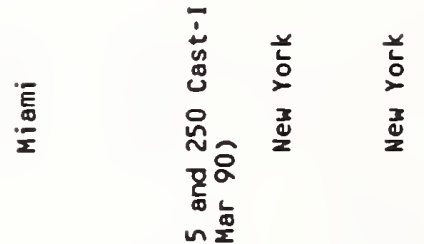

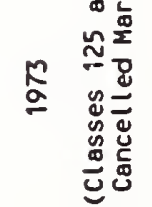

$\stackrel{\infty}{\circ}$

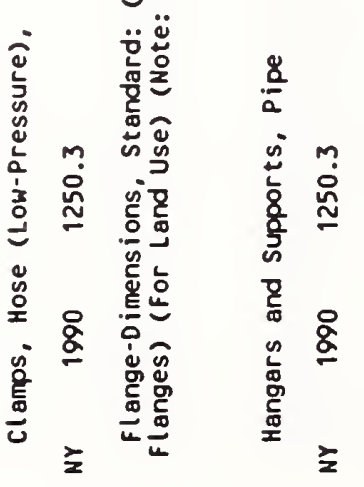

$\stackrel{\infty}{\alpha}$ 


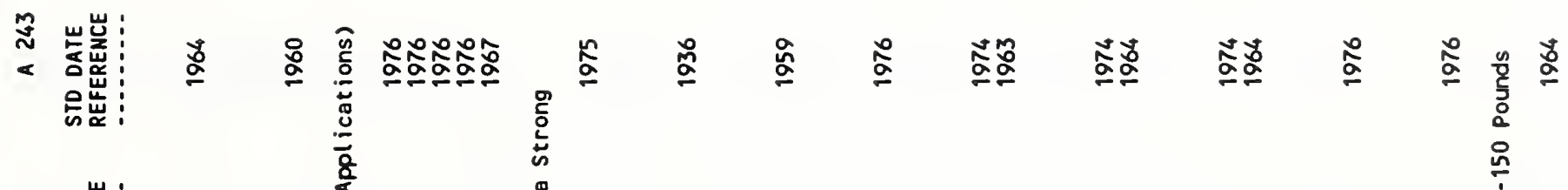

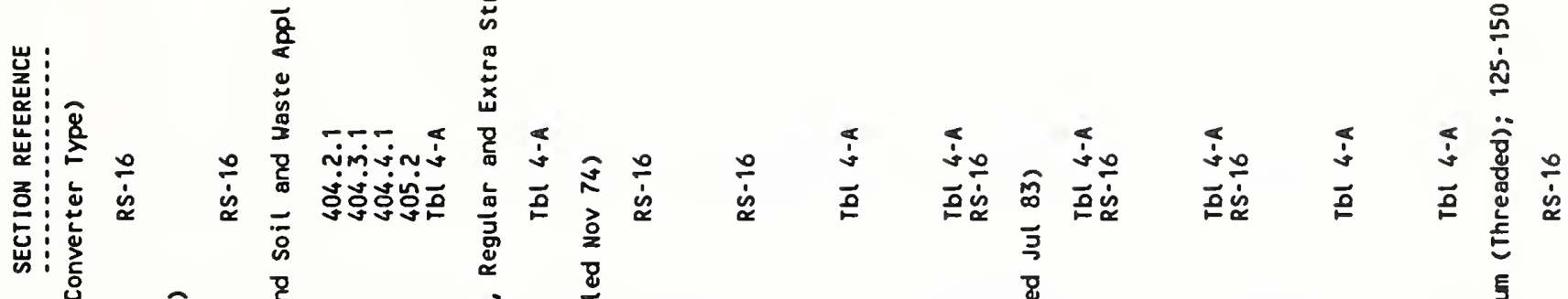

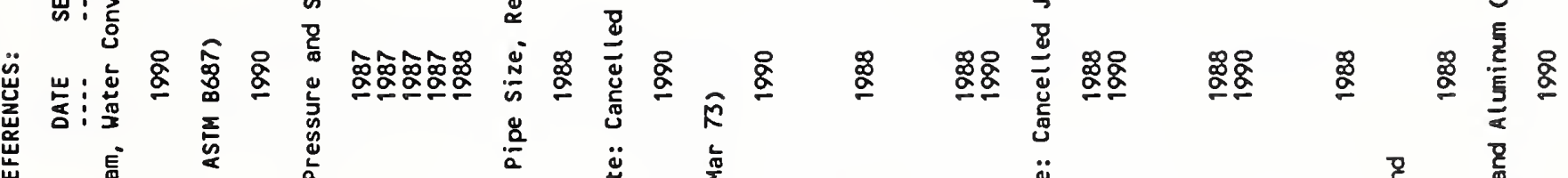

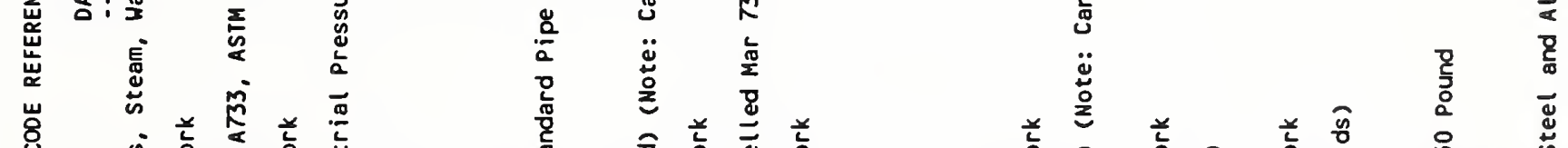

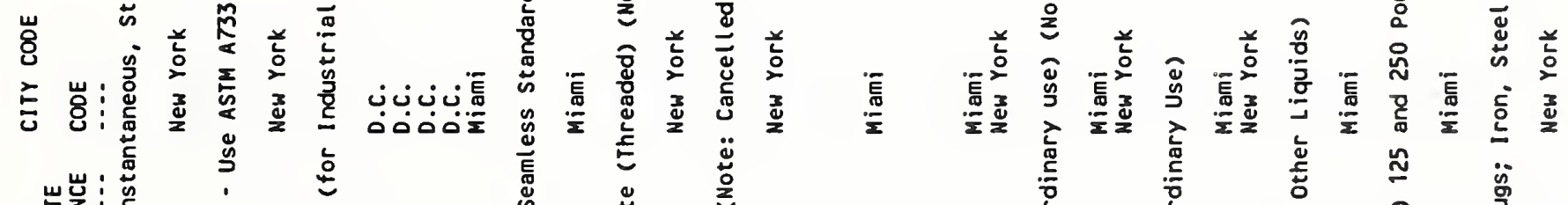

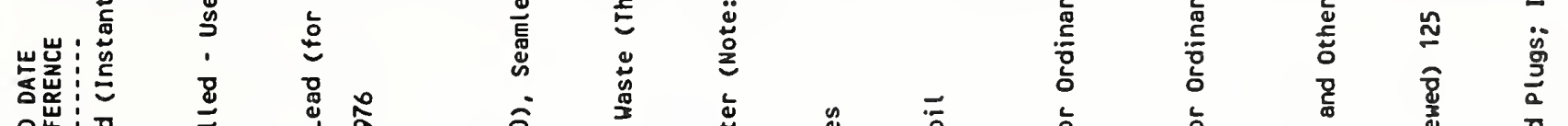

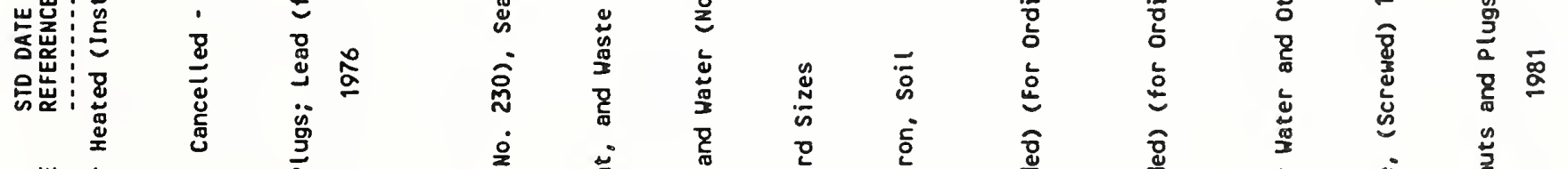

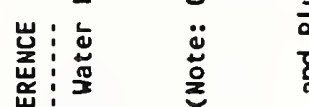

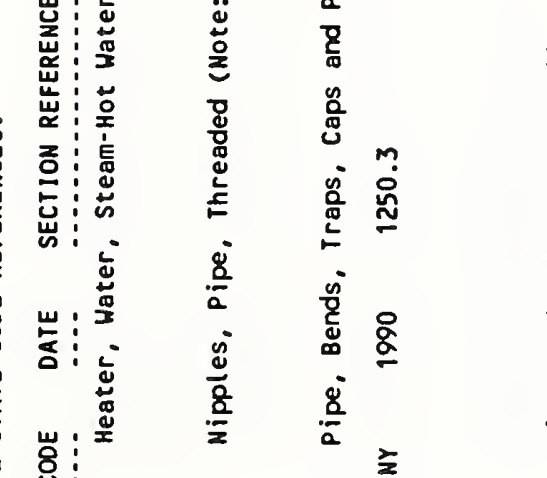

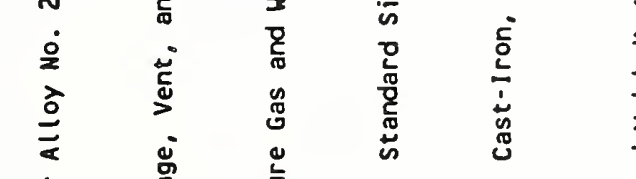

莒
g

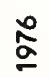
$\stackrel{\aleph}{2}$
高

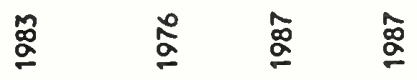

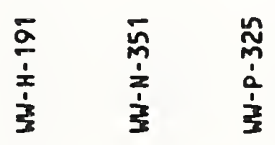

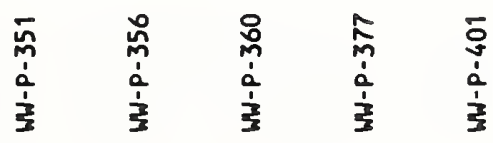

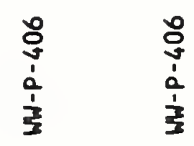

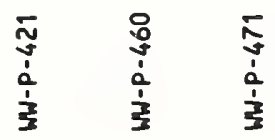




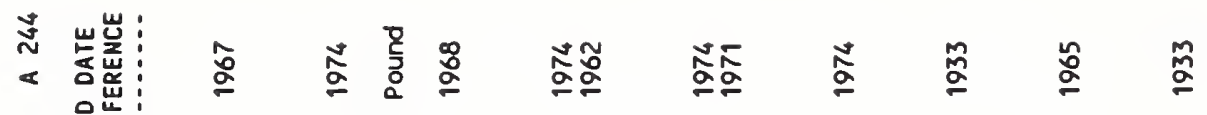

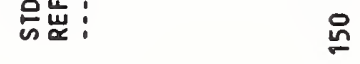

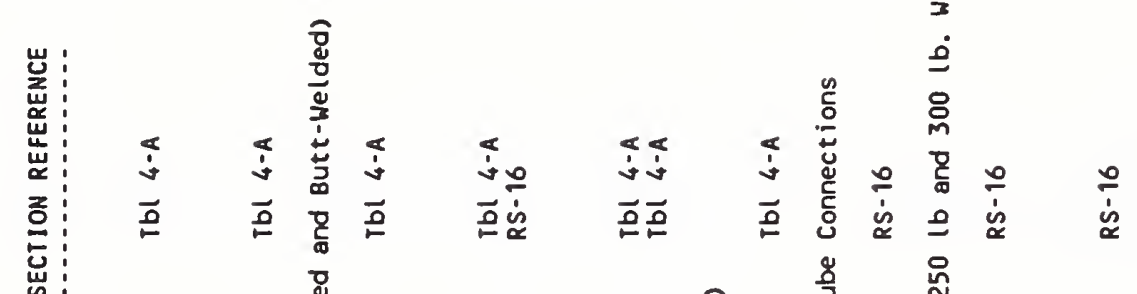

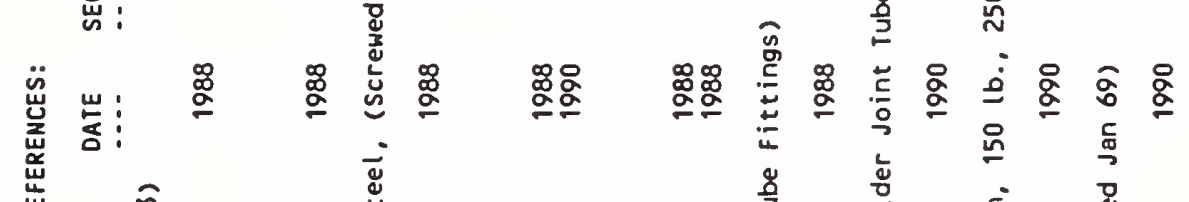

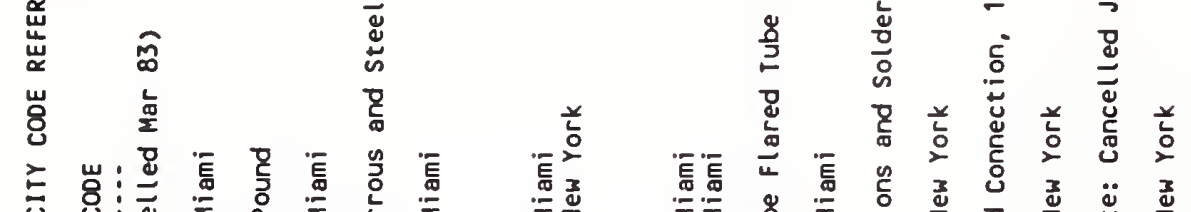

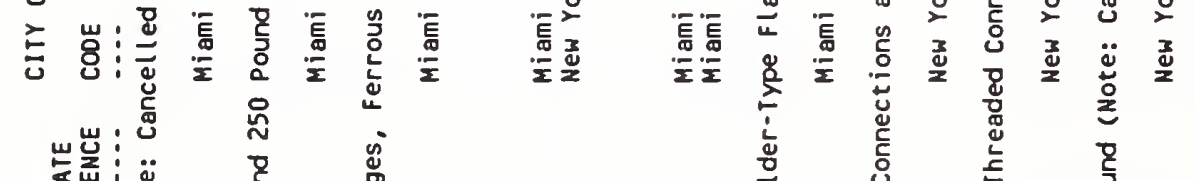

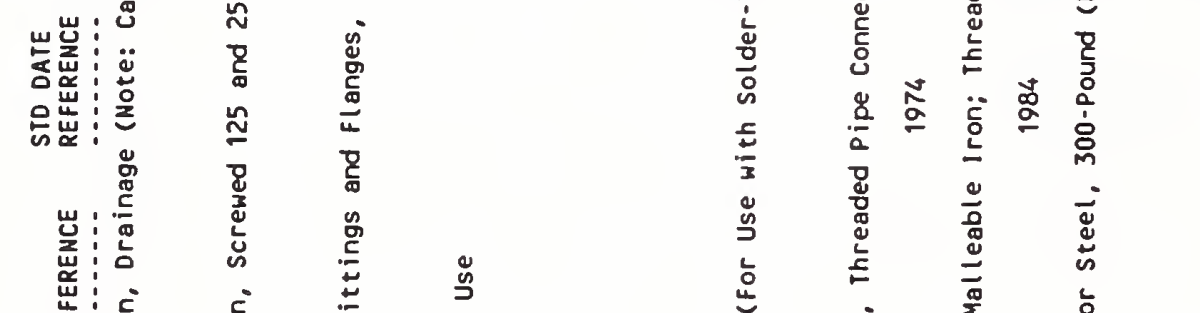

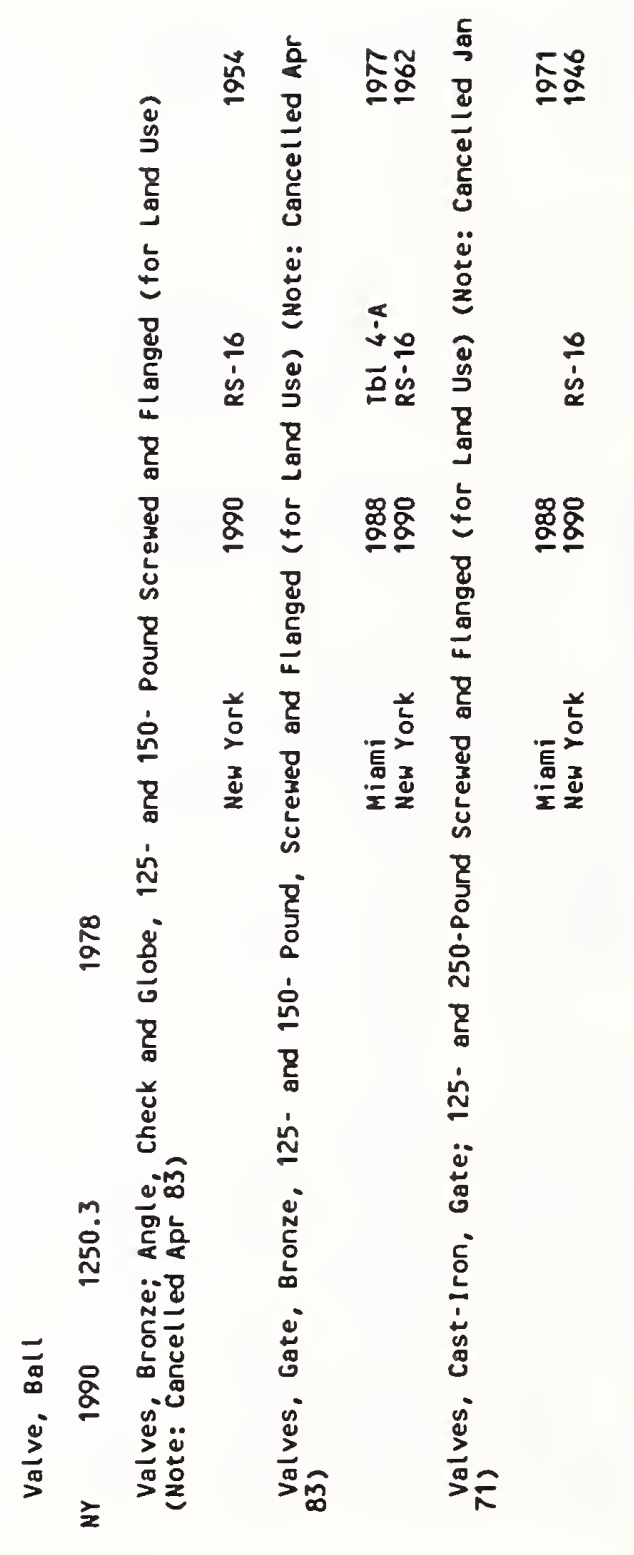

营

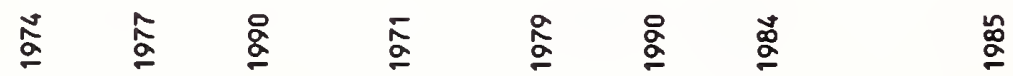

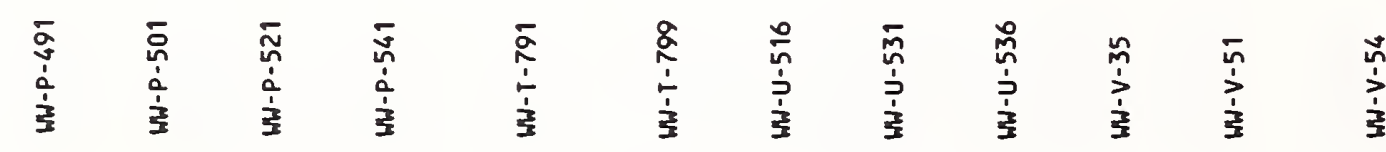




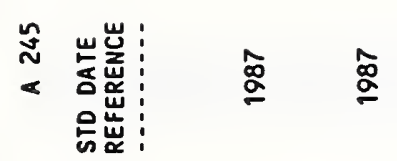

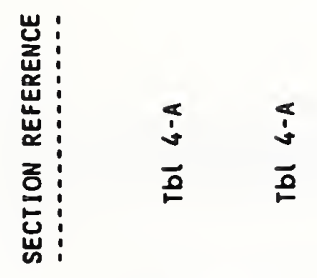

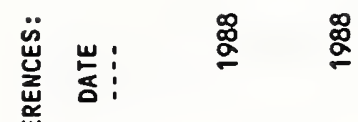

岕

뭉

志啰焉焉

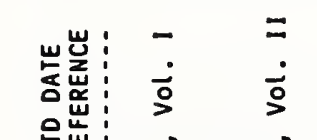

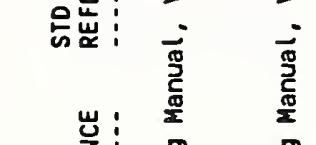

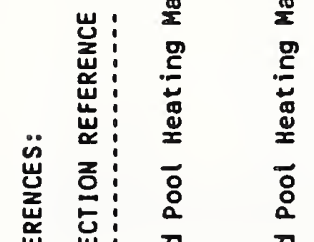

岕出: 蛋 总

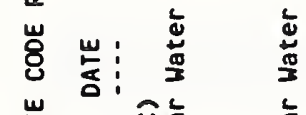

岕

㟧 惢

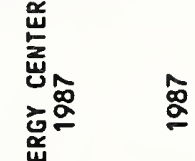

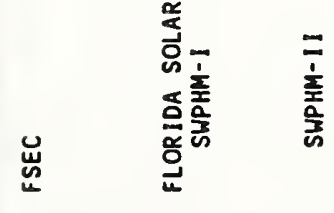




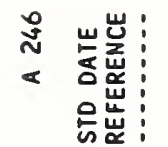

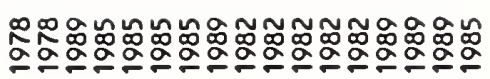

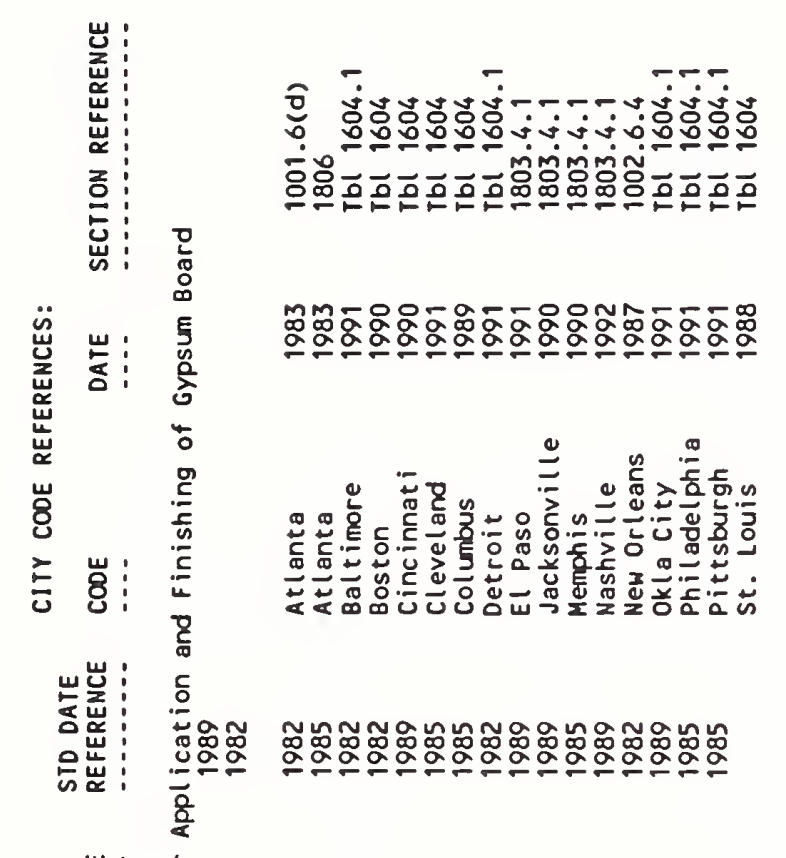

$\ddot{\ddot{u}}$

$\stackrel{5}{\circ}$

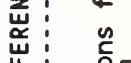

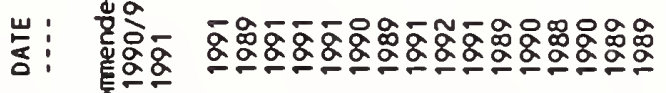

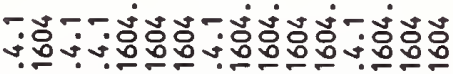

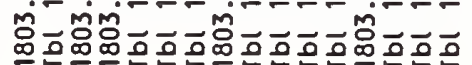

울

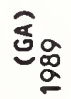

$$
\begin{aligned}
& \text { 줄 }
\end{aligned}
$$

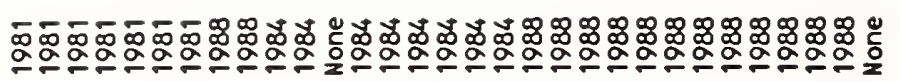

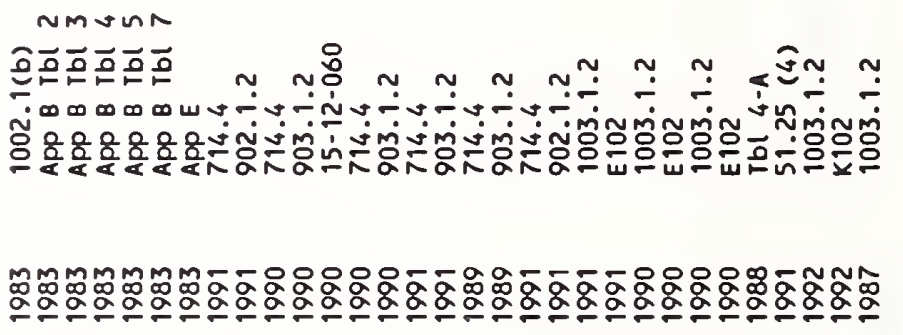

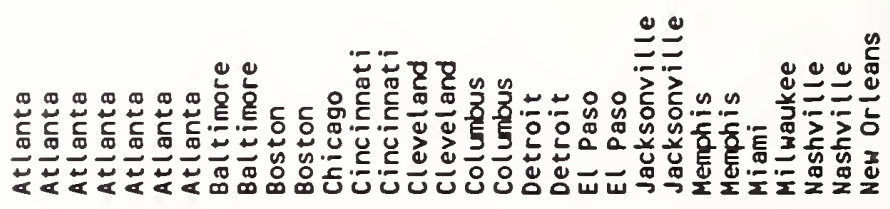

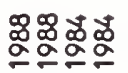

它

蛋

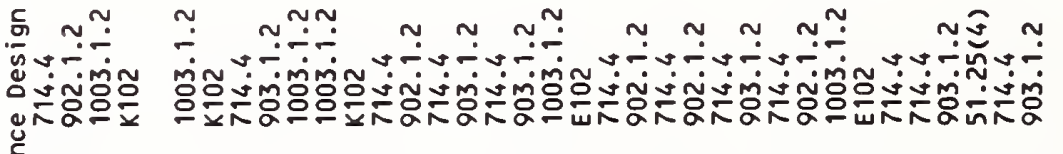
产的

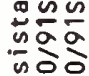

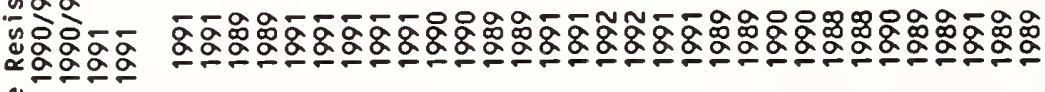

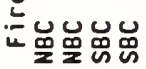

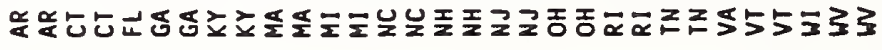

$\stackrel{\infty}{\mathscr{\Xi}}$

응 


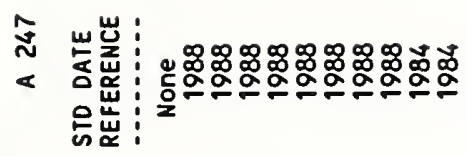

每:

흔

出!

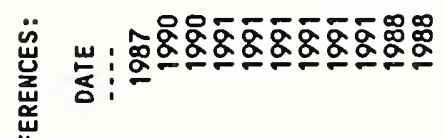

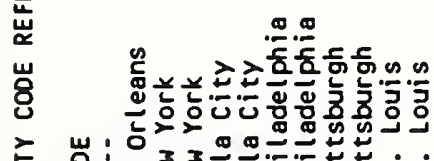

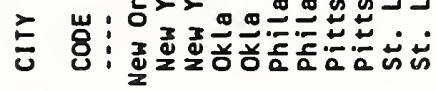

岁岕:

的岕!

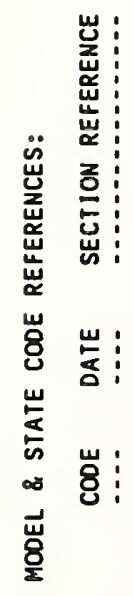

㝕

๘ 


\section{䒺

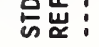

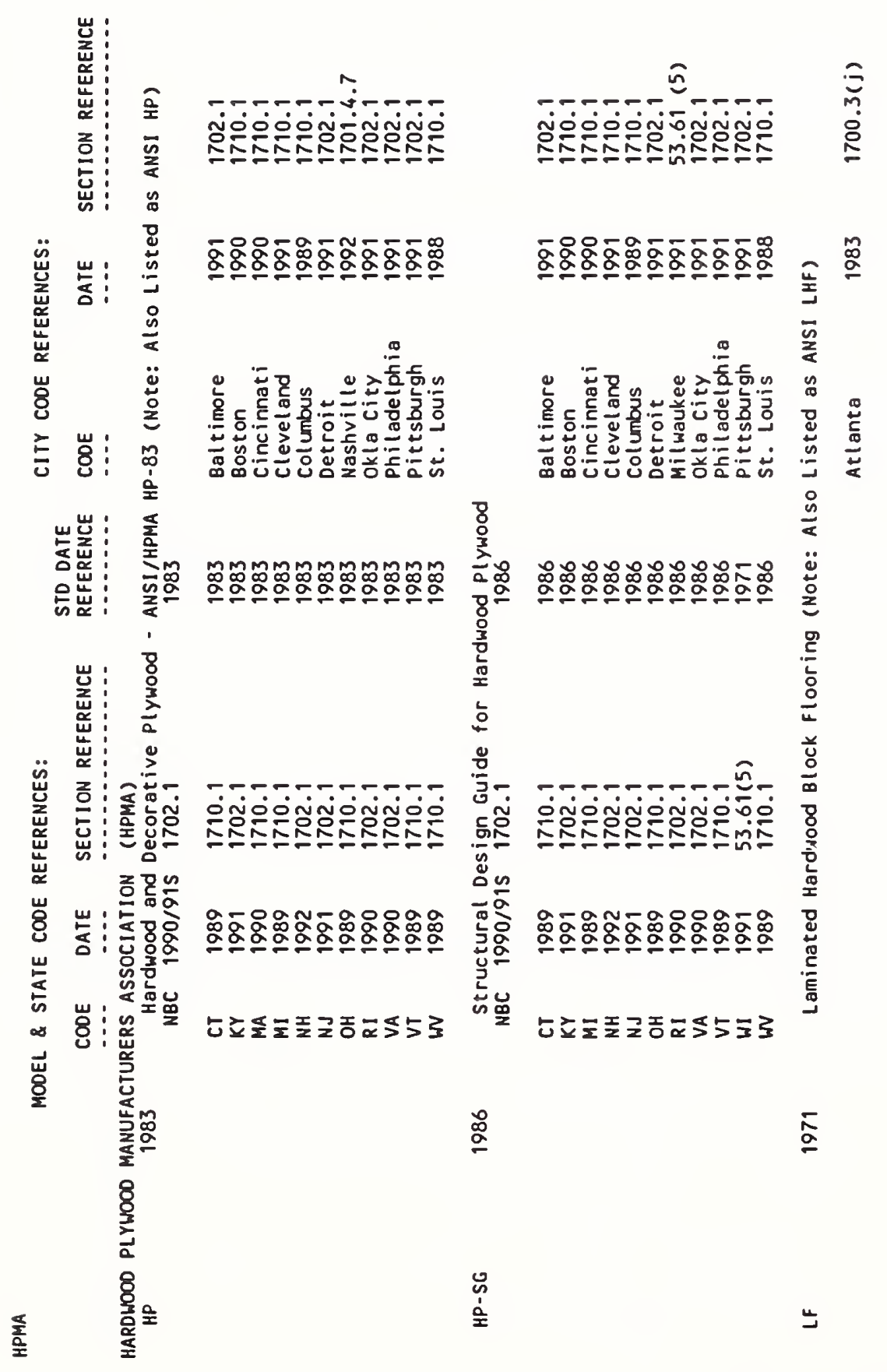




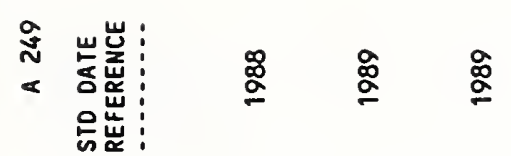

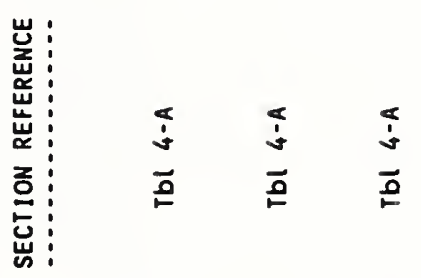

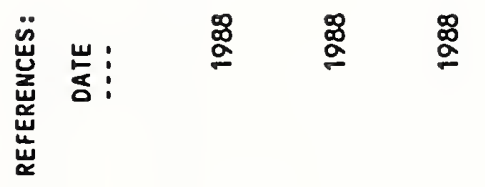

岁

岕岁

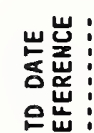

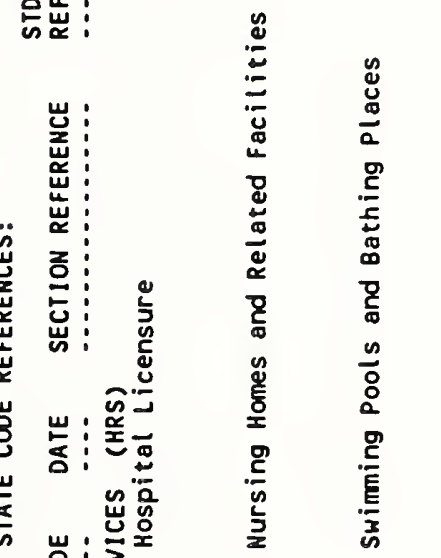

\&

일 흘

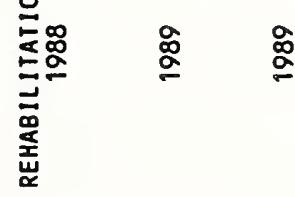

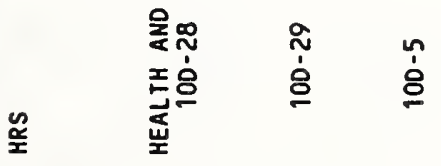




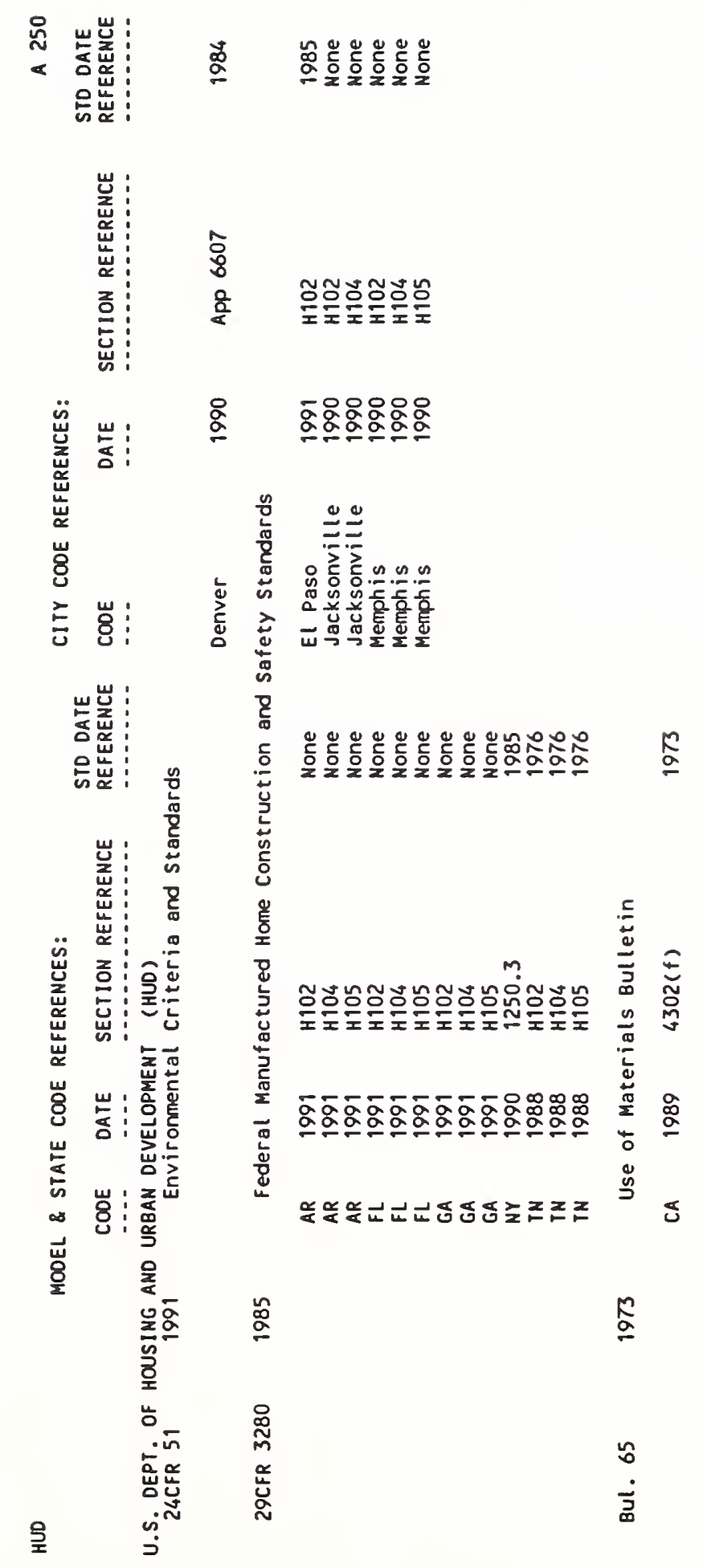


ॠ

< 就

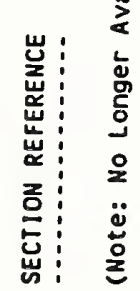

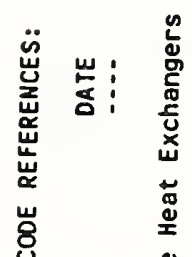

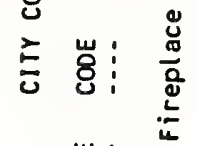

岁:

喘

号岕: :

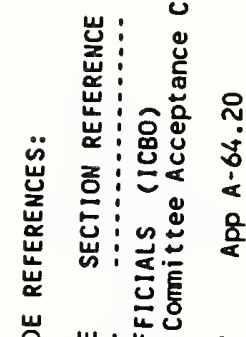

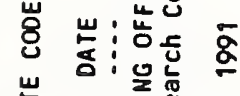

崖

$\infty$ ठ일

㟧

,

్

总

홍

高

용

홍 


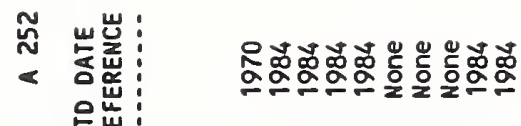 点岕 ?}

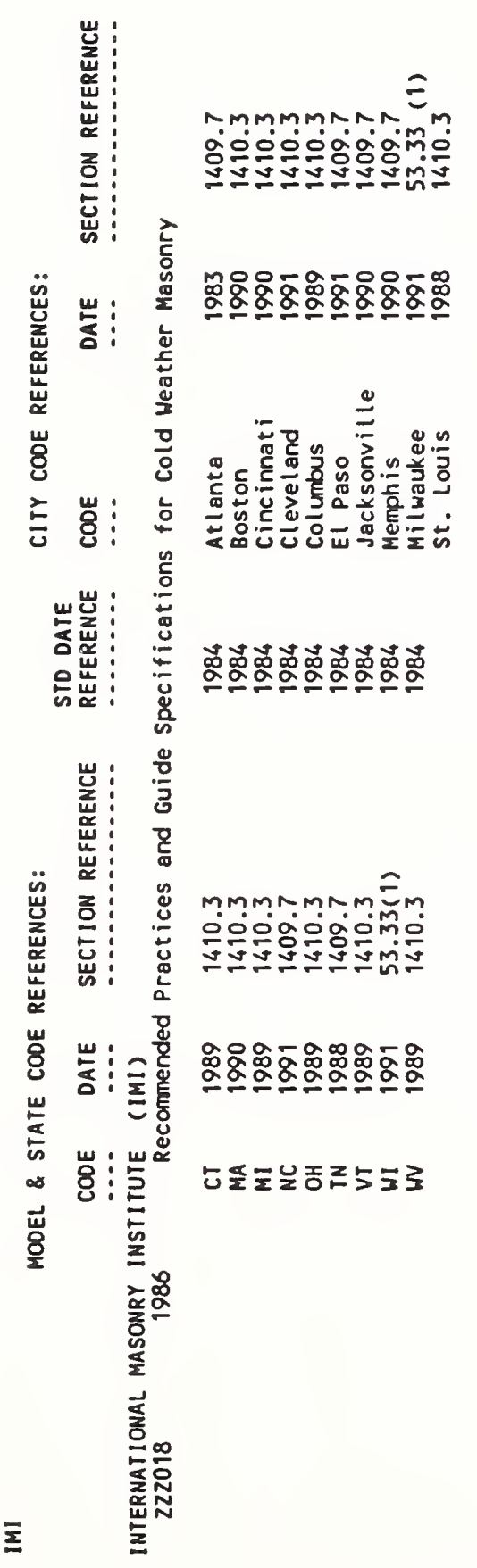




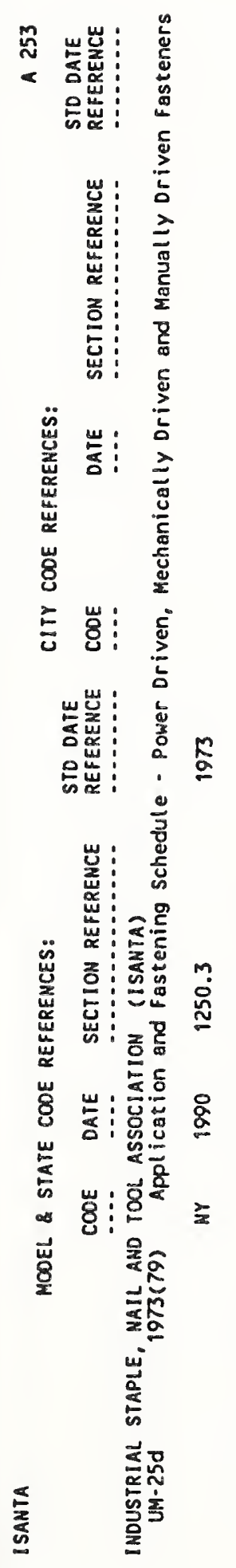




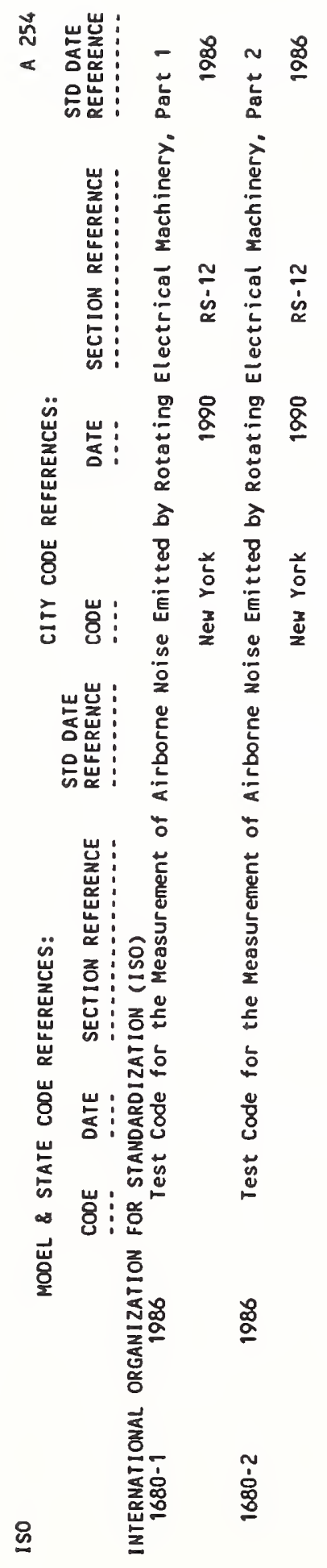




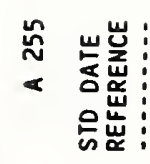

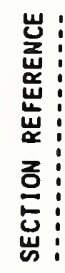

苞岕:

잉

ప

w岂: 岩

稆!

送岕:

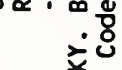

넝

总:

总:

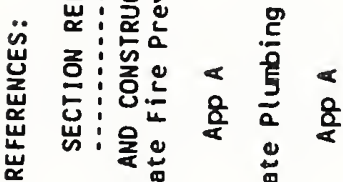

崩芯芯

峉

岕岕岕蒂

뺑 옹

ㅎㅇㅛ 용

춘웡 용

这苔

站

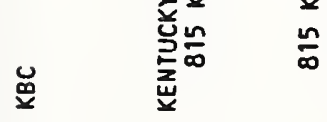




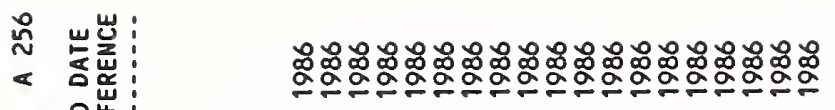

的㟔:

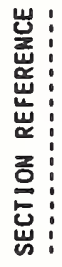

芯出

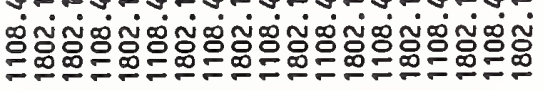

$\frac{\omega}{\alpha}$

岩

突岁

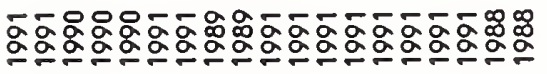

㟧:

喿蛋

온

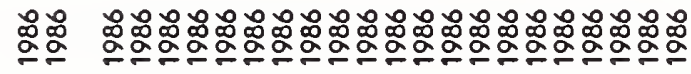

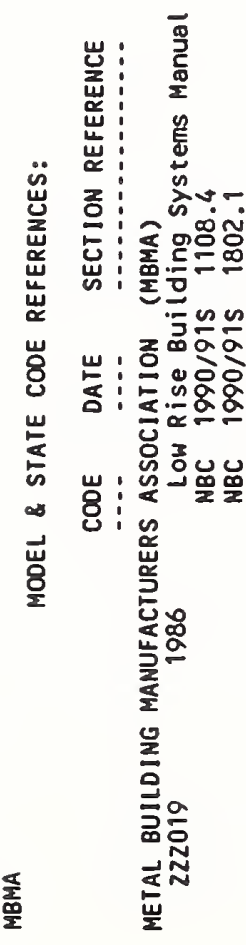

क.

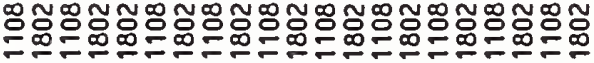

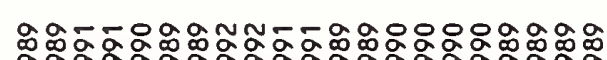

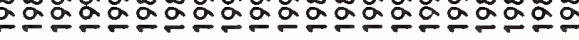

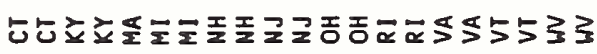



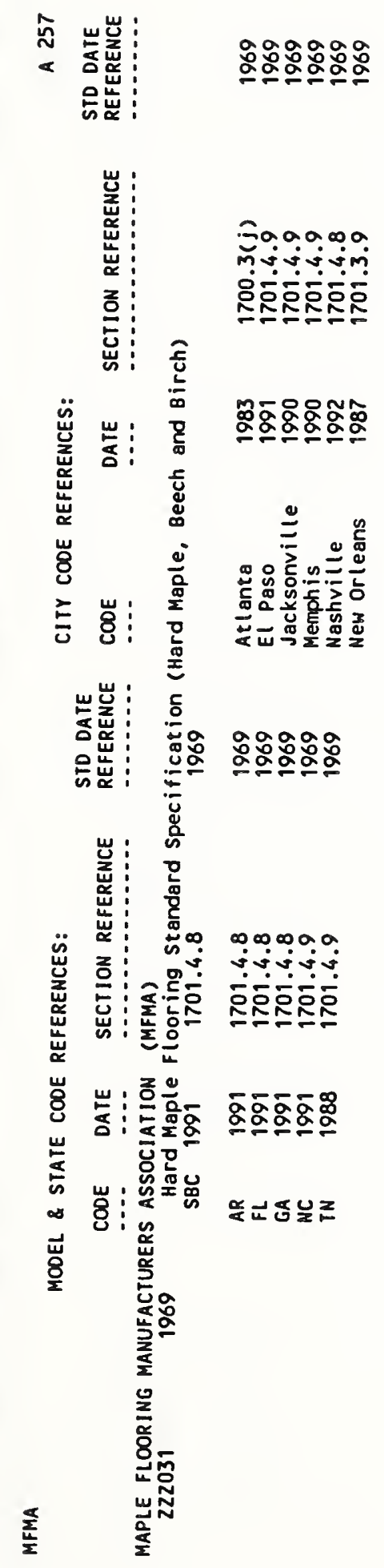


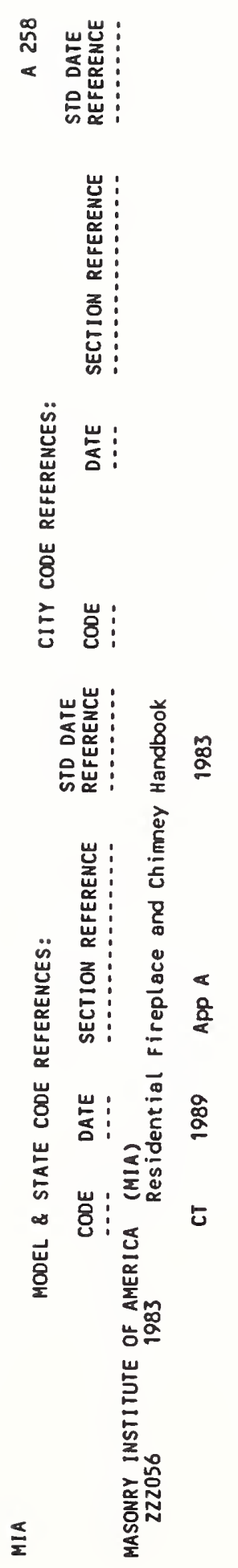


端

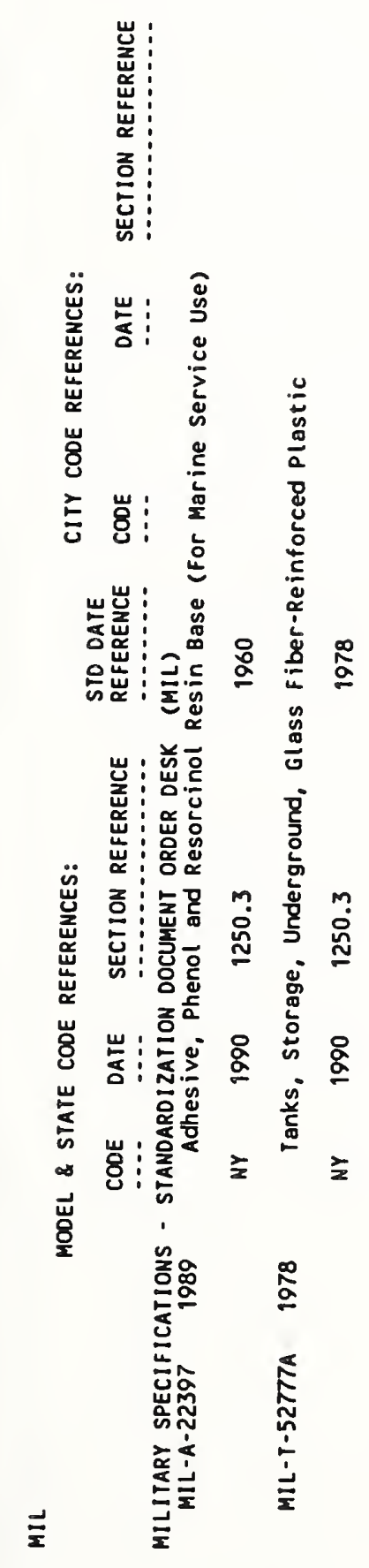




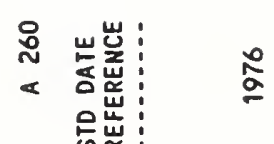

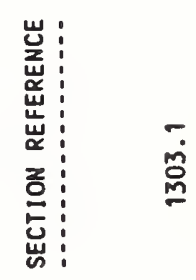

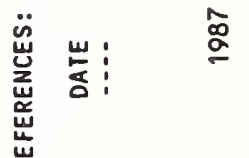

岁

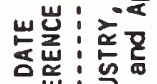

到

的岕: 美。

岁总

焉:

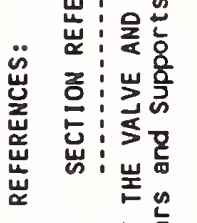

岁宸: 范高

崖告总

告

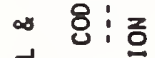

㟧

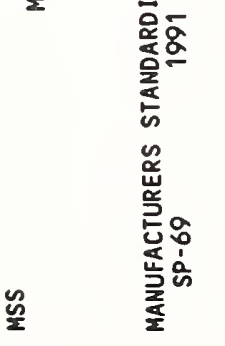




\section{赔}

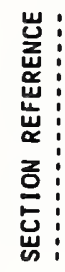

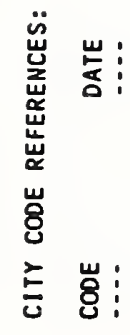

岂凯

品岀:

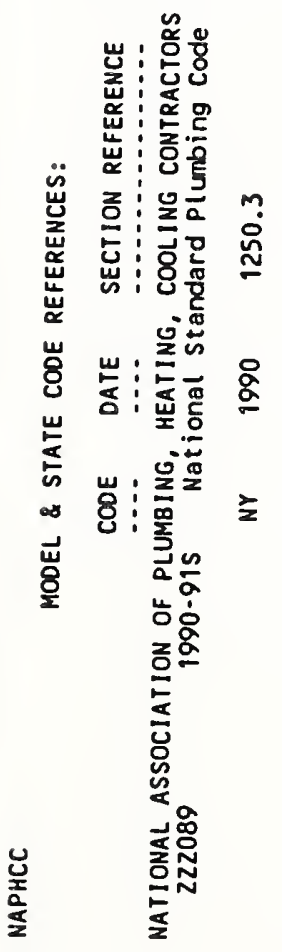



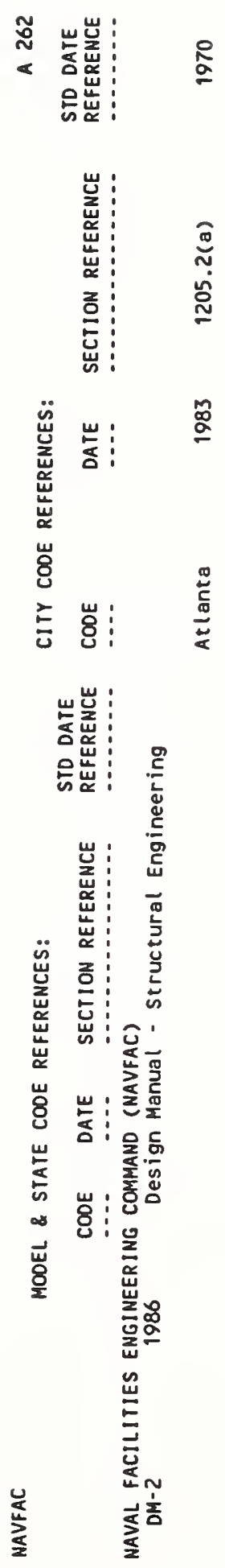


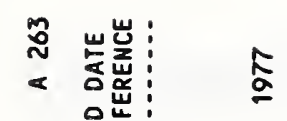
은 岃 !
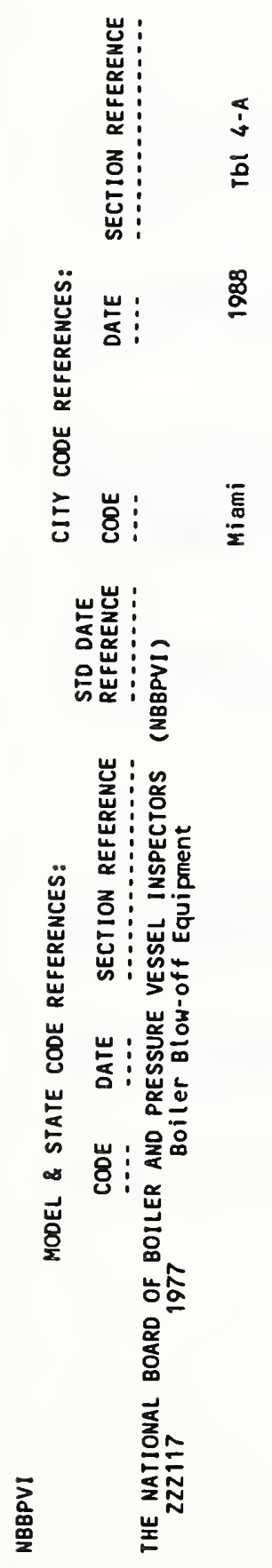


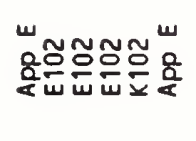

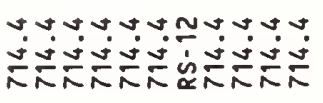

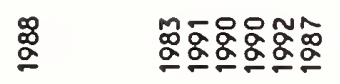

$$
\text { 容: }
$$

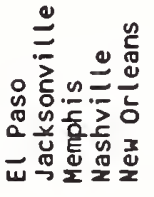

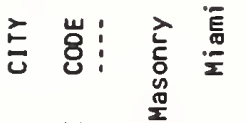

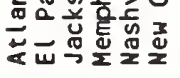

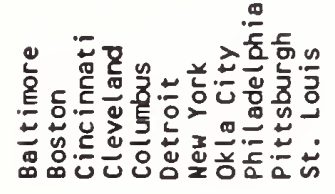

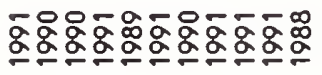

崖:

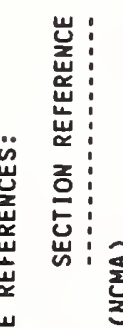

岁崖:

흘

ö:

종

齿

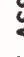

产.

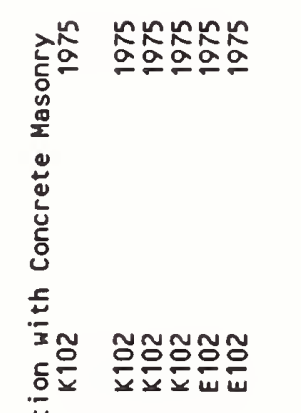

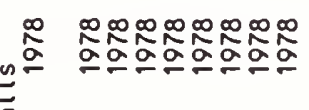

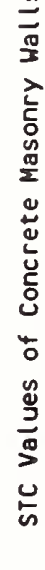

눈정눈

$\stackrel{\mathfrak{n}}{\underline{\alpha}}$

$\stackrel{\infty}{a}$

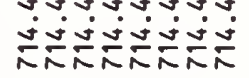

ton

要然

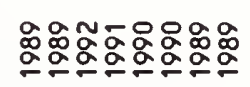

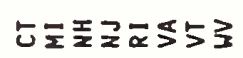

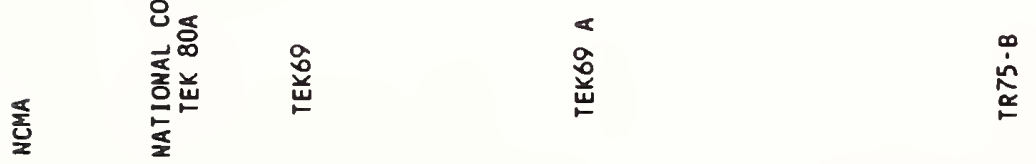

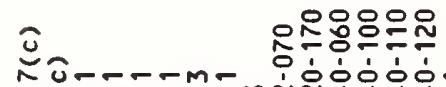

m.

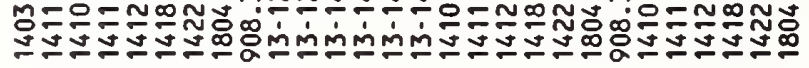

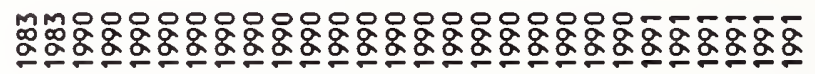

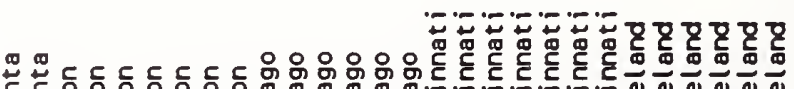

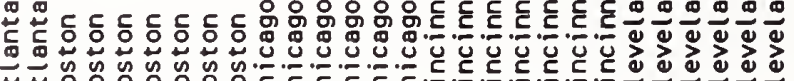

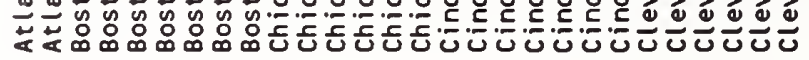

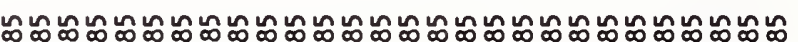

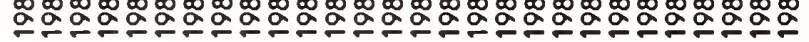

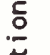

飠

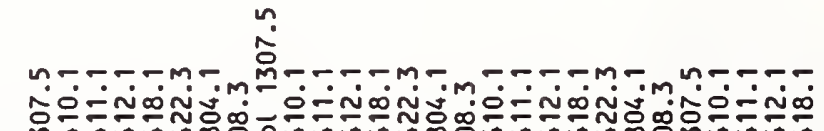

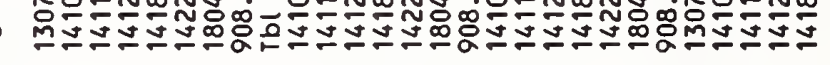
定

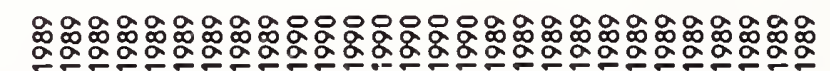
递

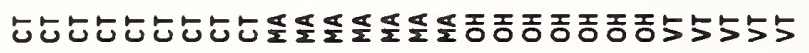


命 颌:
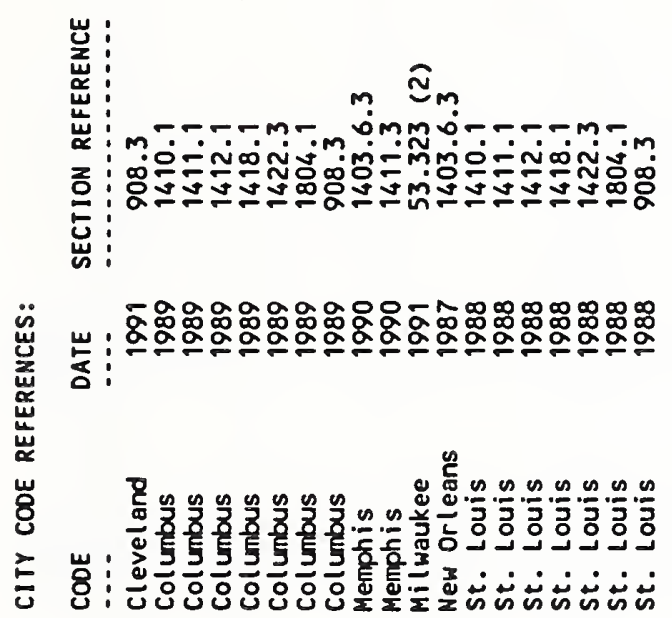

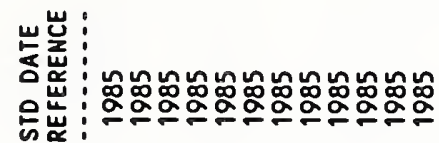

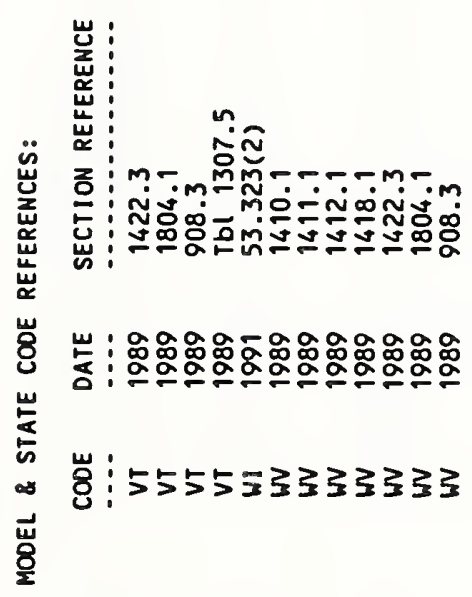

옹

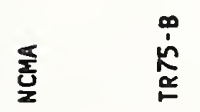




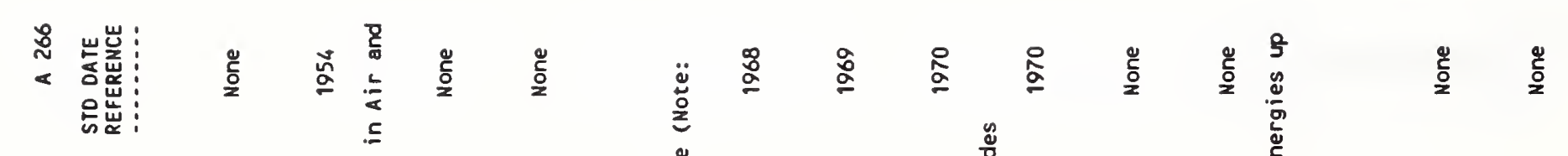

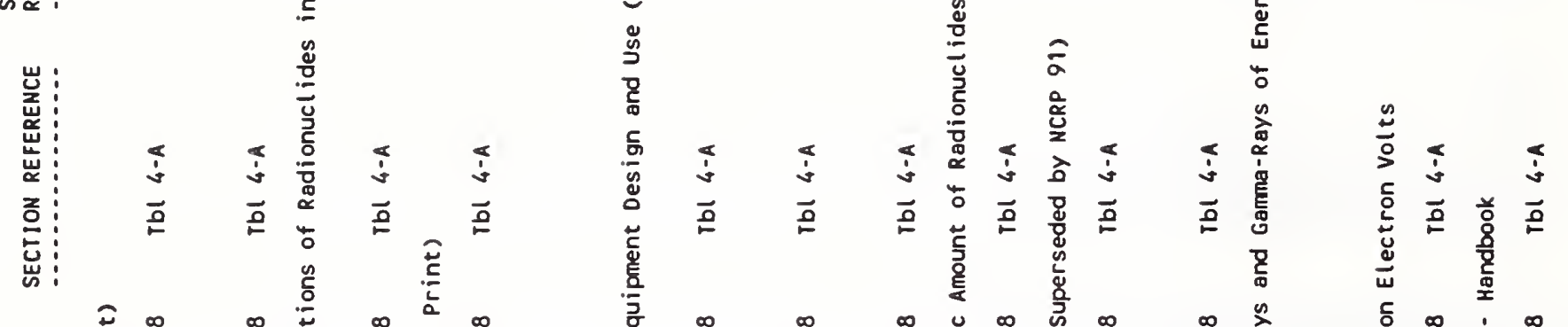

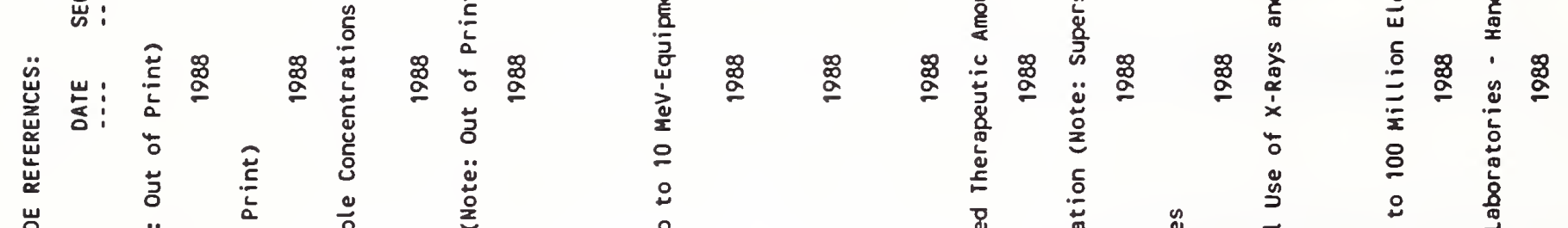

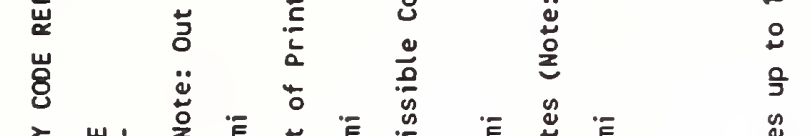

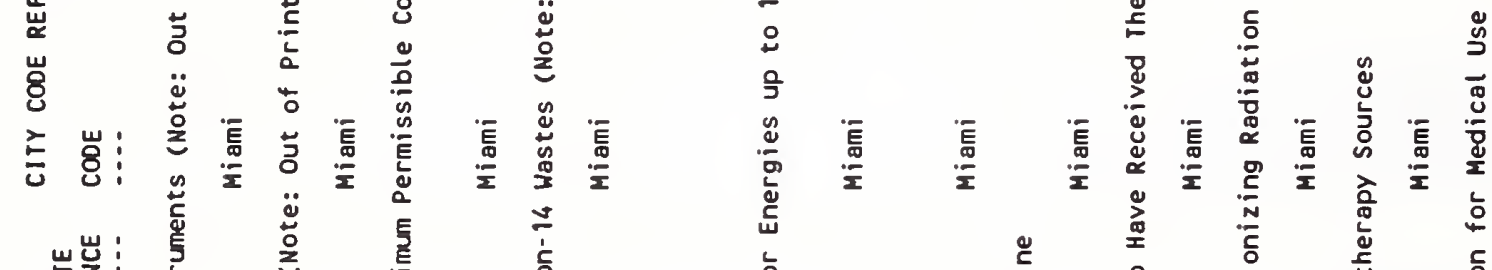

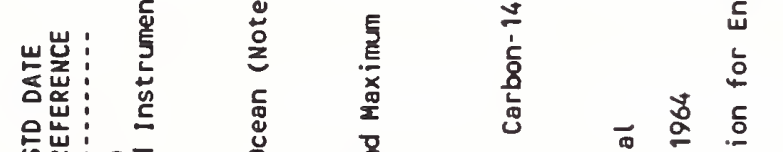

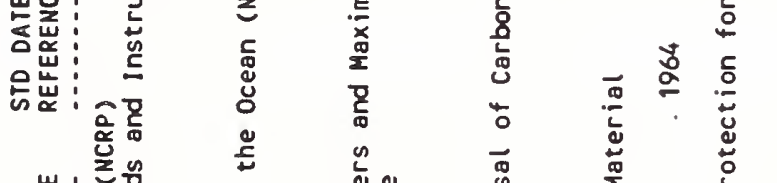

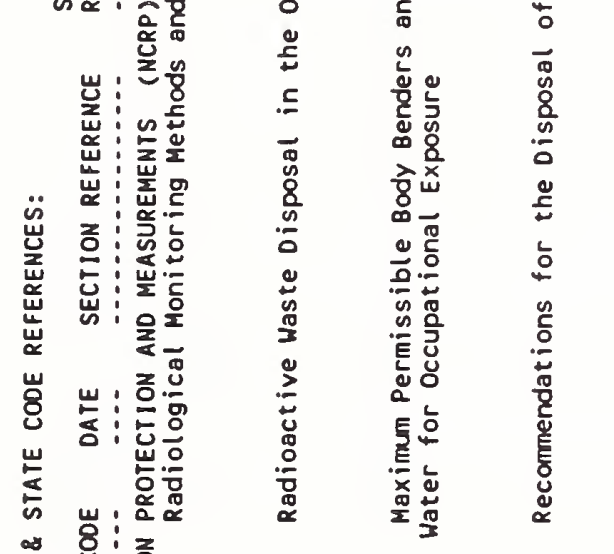

䆑

言

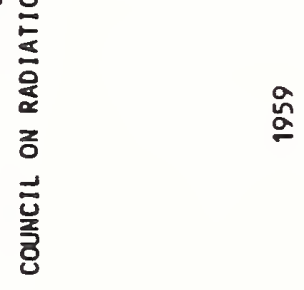

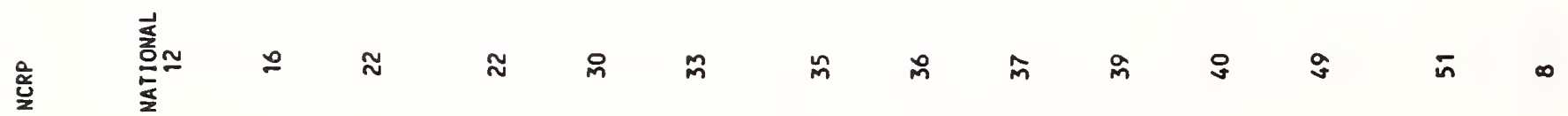




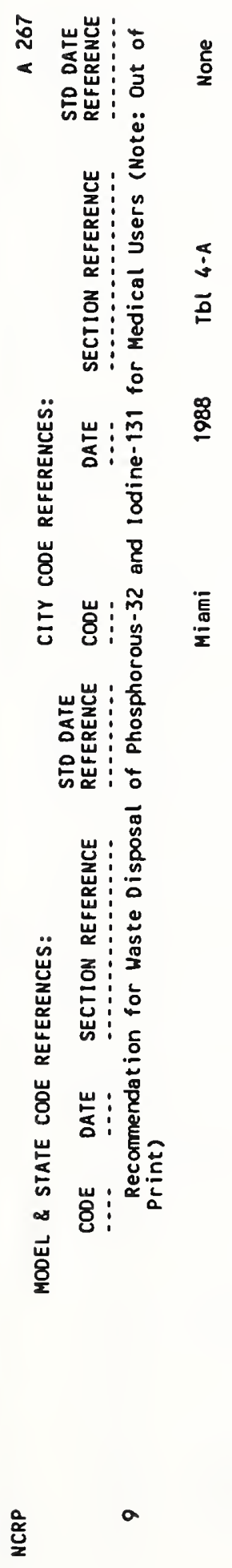




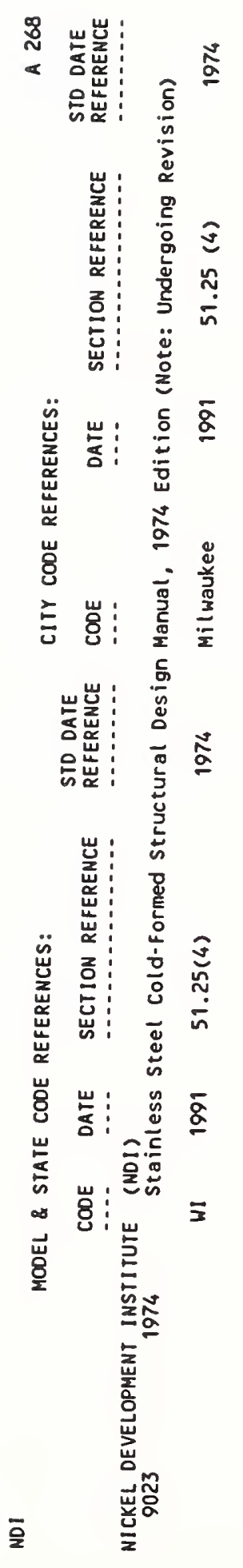




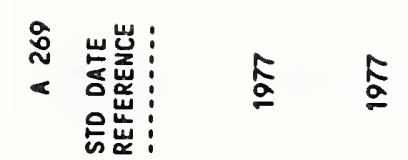

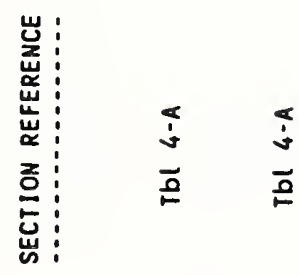

总

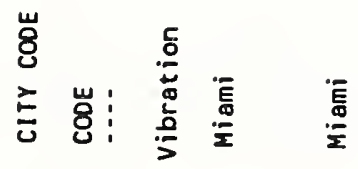

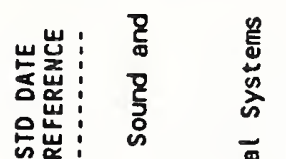

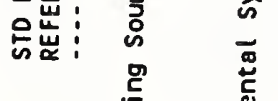

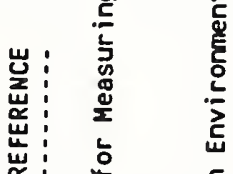

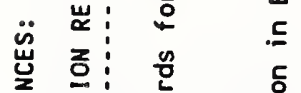

总

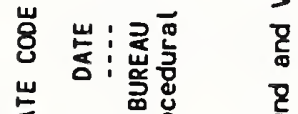

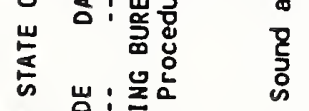

일

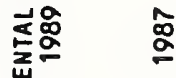

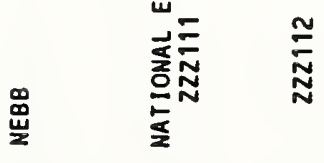




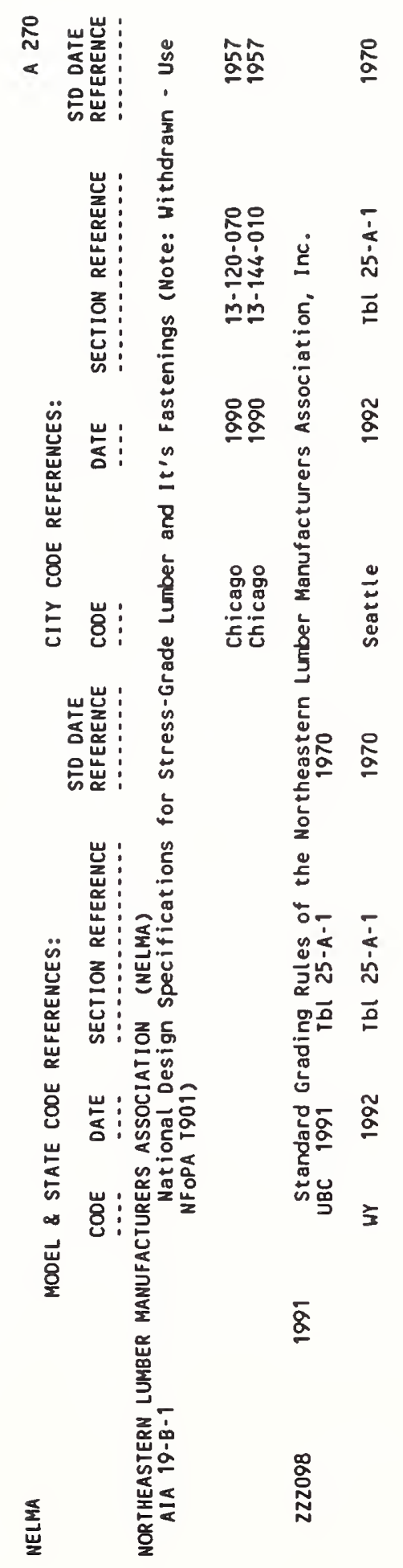




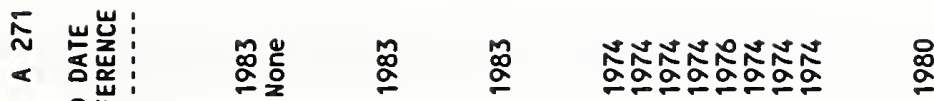

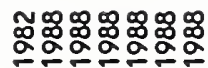

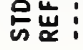

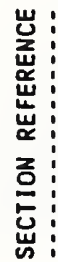

岂

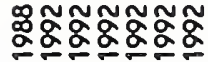

$\stackrel{\omega}{\sim}$

岁

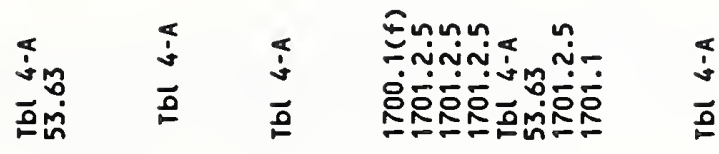

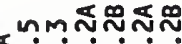

inimiogicis

오은든

ธุ

$\frac{6}{u}$

岕岕

:

西

究

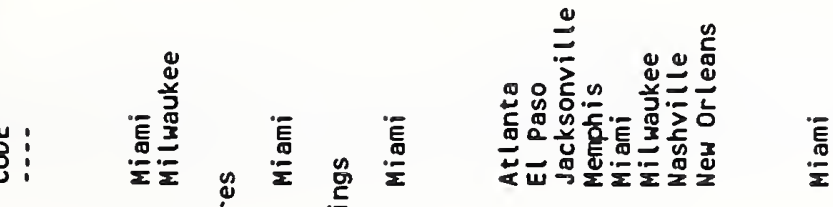

$\frac{\bar{E}}{\frac{\bar{\sigma}}{2}}$

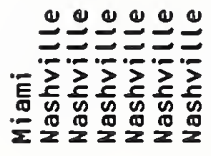

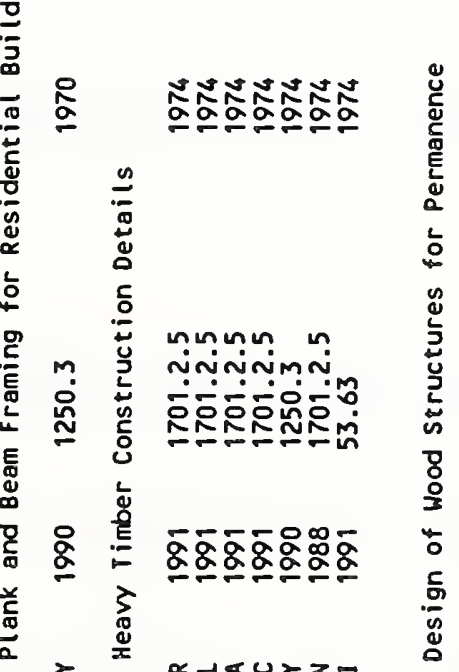

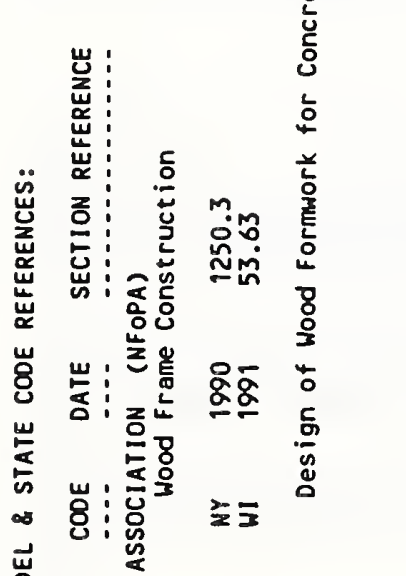

유웜

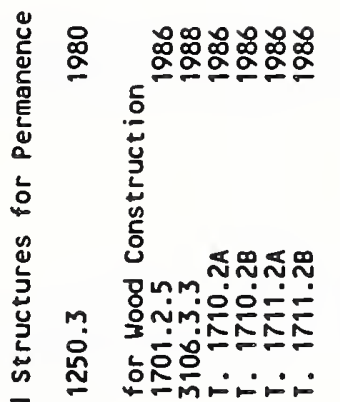

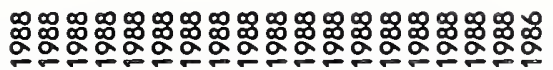

产

$\frac{\pi}{2}$

\begin{tabular}{|c|c|c|c|}
\hline$\stackrel{\mathrm{a}}{\stackrel{\mathrm{g}}{\mathrm{g}}}$ & 咲 & $\stackrel{\circ}{\stackrel{\alpha}{\alpha}}$ & 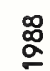 \\
\hline 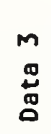 & 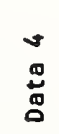 & $\begin{array}{l}n \\
\mathbb{D} \\
\stackrel{\Delta}{0}\end{array}$ & 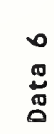 \\
\hline
\end{tabular}




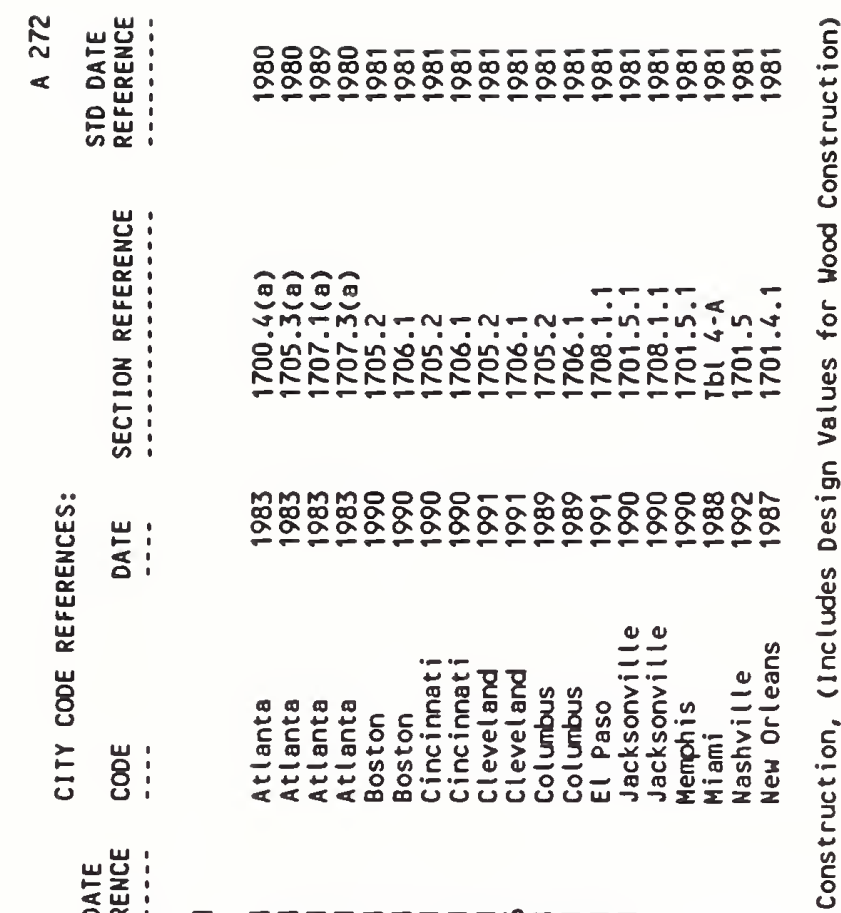

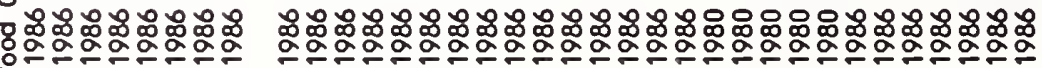

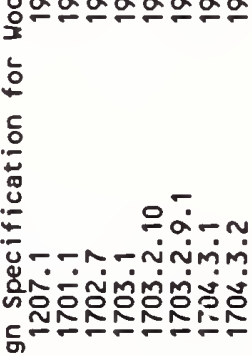
sencescosen

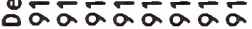

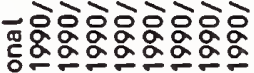

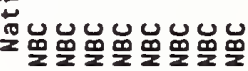

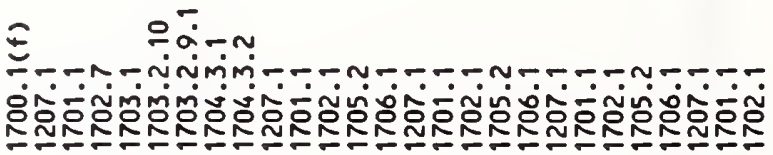

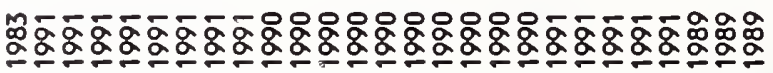

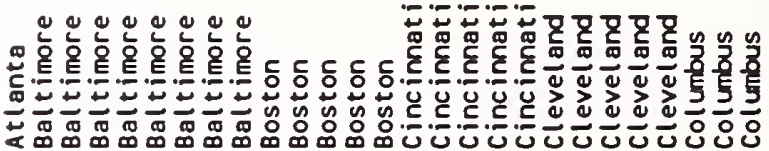

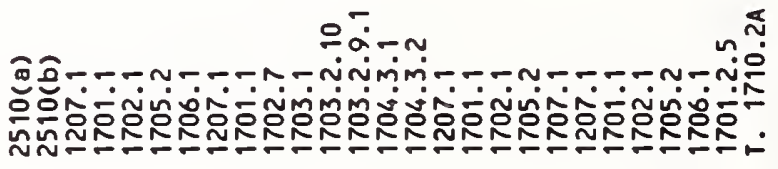

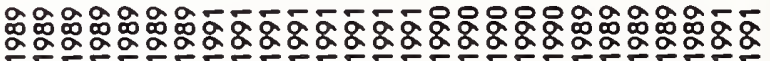

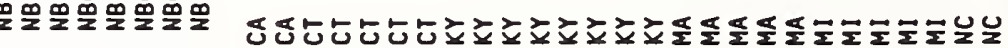

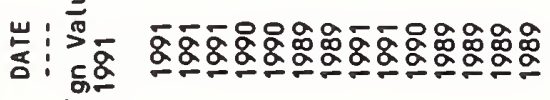

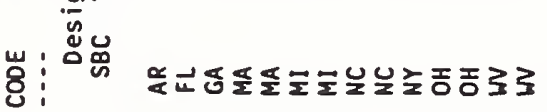


疋 은

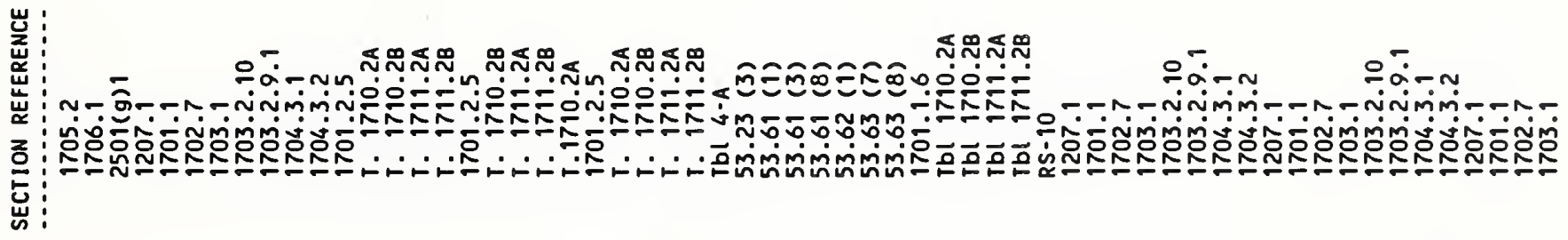

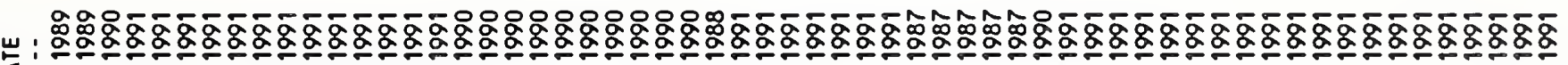
ธ。:

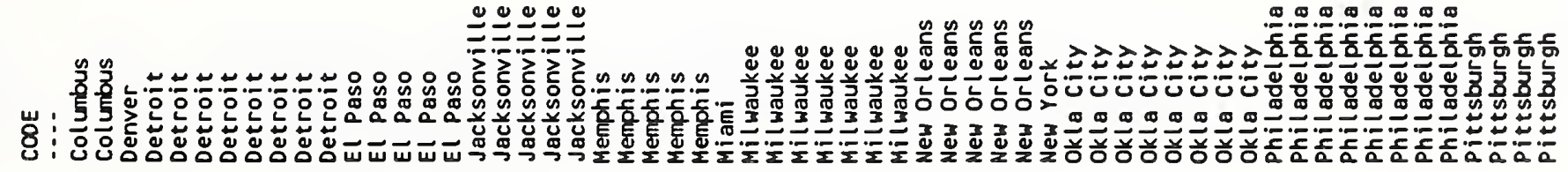

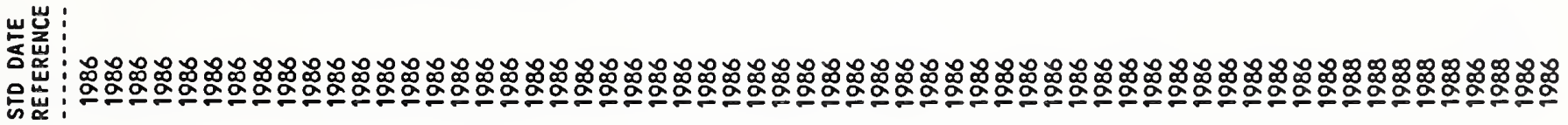

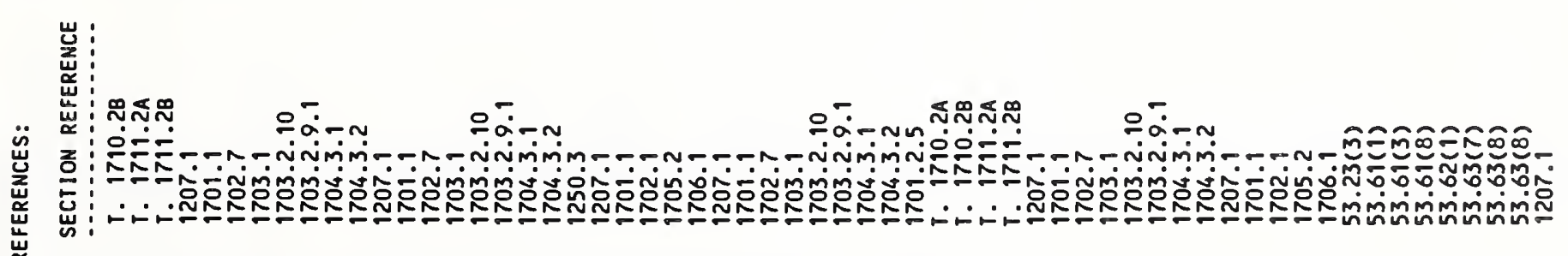

范

岁 :

蕛

홍 


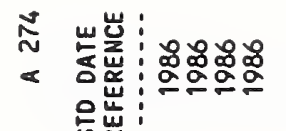

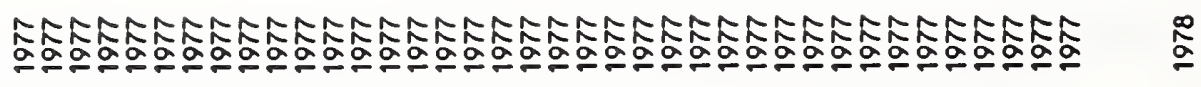

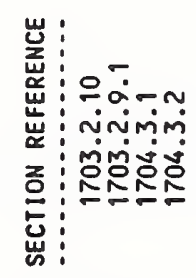

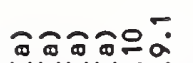

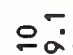

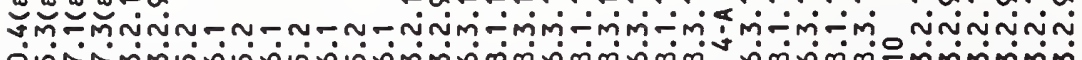

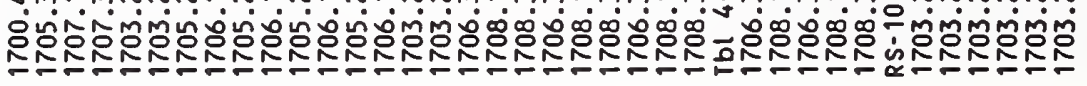

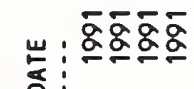

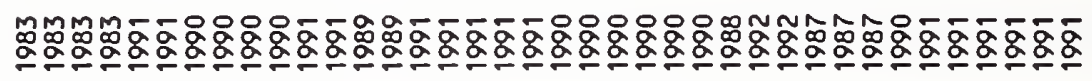

$\stackrel{\mathscr{B}}{\stackrel{\Xi}{\sigma}}$ o

宸

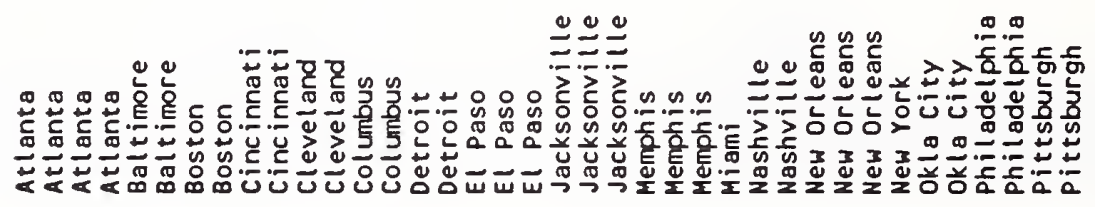

떤

o

"4

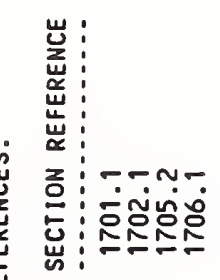

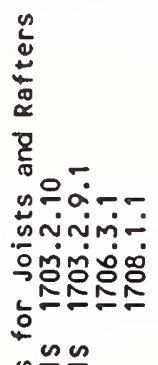

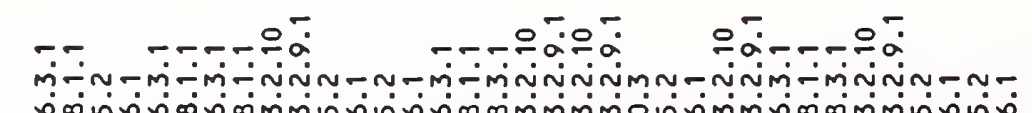

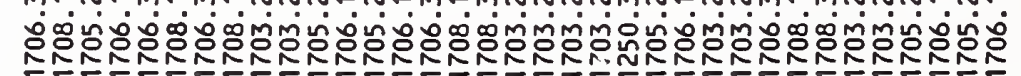

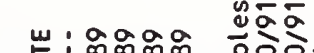

岁: 高器器

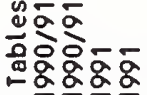

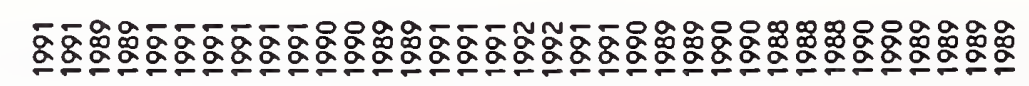

岁: 3333 总眊兽总惫

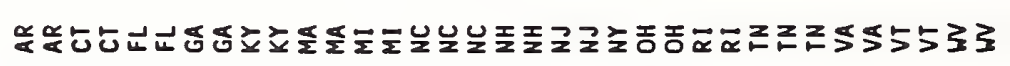

할

$\tilde{\underline{z}}$

$\underset{\check{\alpha}}{\check{\alpha}}$

$\stackrel{\check{\alpha}}{\circ}$

造 
ํㅜㄴ

:

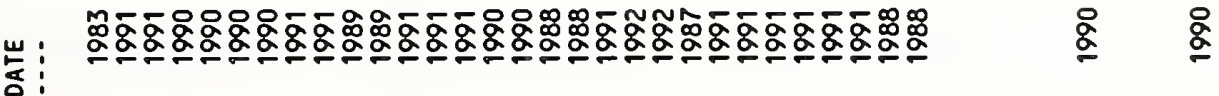

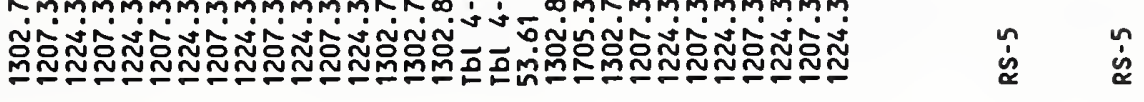

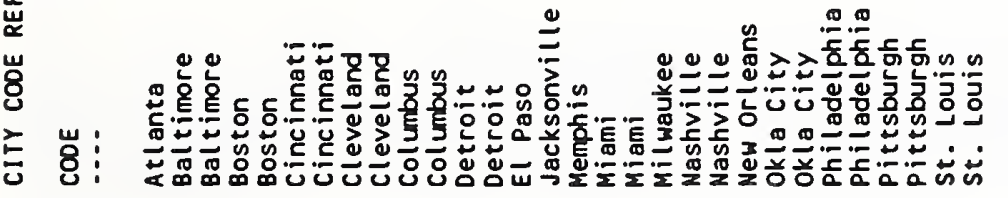

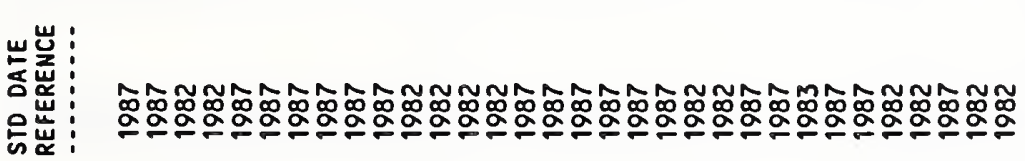

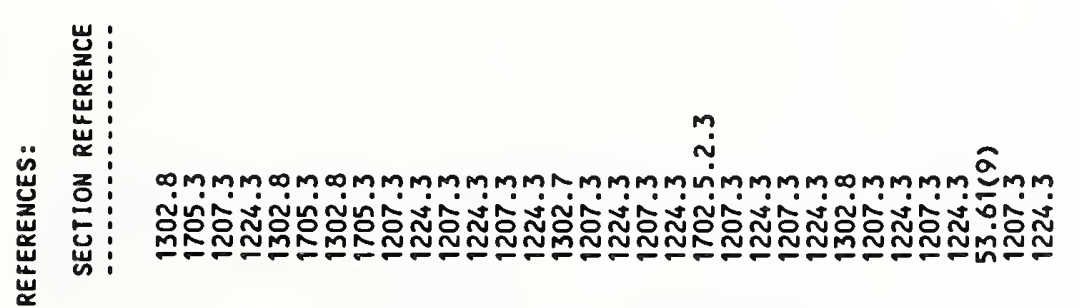

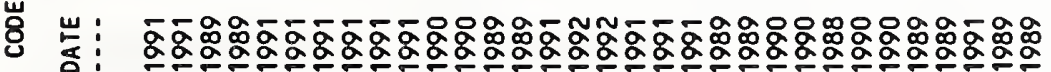

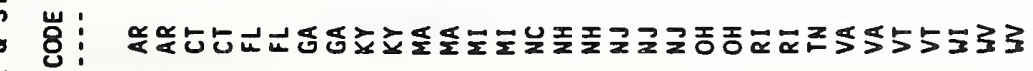

કั

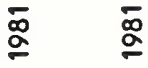

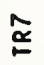

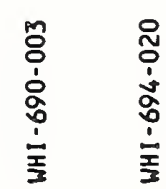




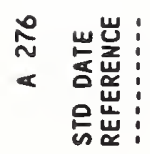

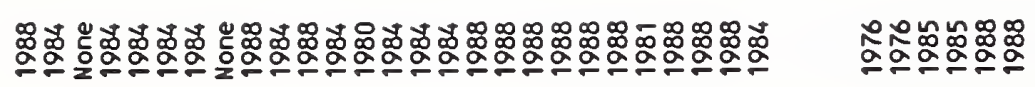

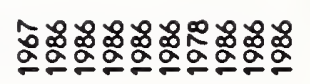

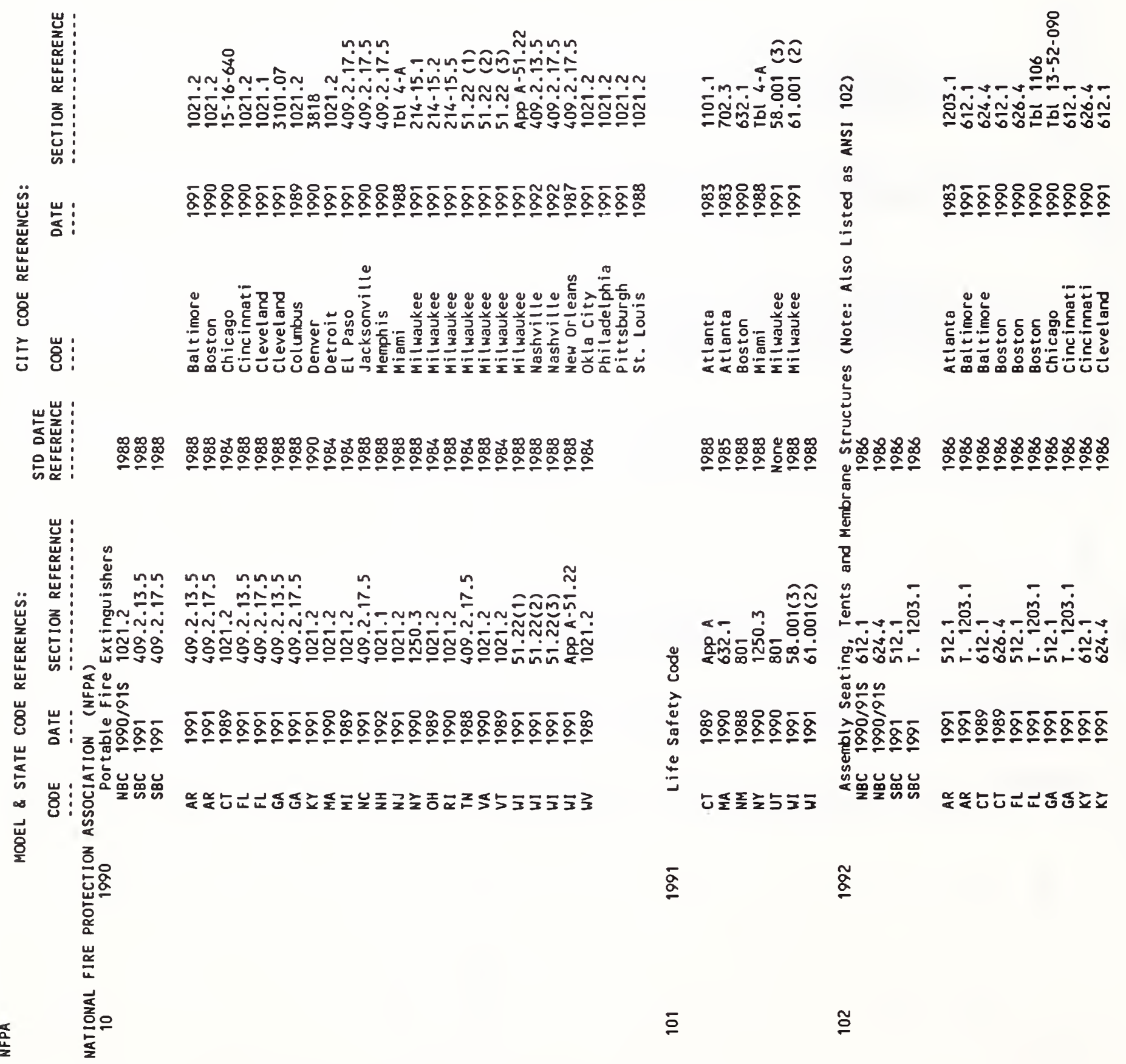


$\underset{\sim}{\kappa}$

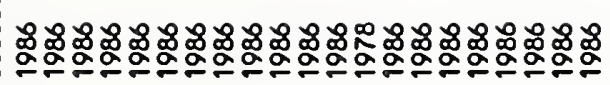

은

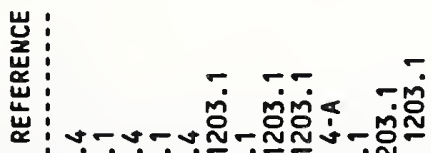

ว

出:

芦 व:

焉

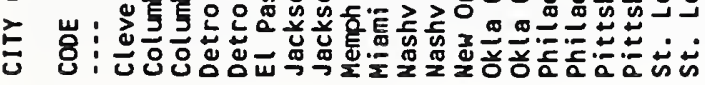

뜬 秽

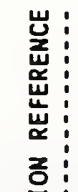

일.

点

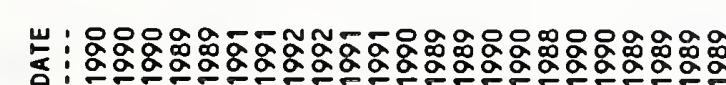

崖

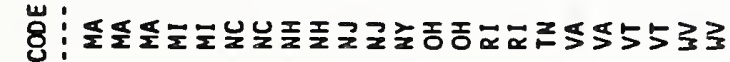

픙

它

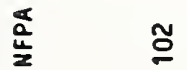

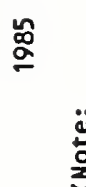

定

융

ํㅡㄴำ

ตุตั่

용요

$\stackrel{\Xi 0}{g}$

原

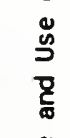

品昌

든돈

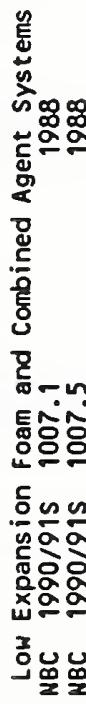

$\stackrel{\circ}{\sigma}$

$\stackrel{\mathscr{\Xi}}{2}$

$\cong \quad$ ๓

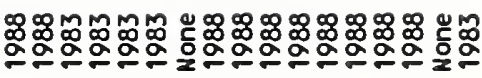

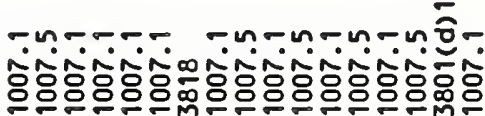

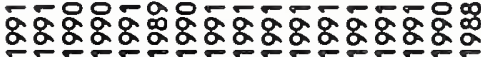

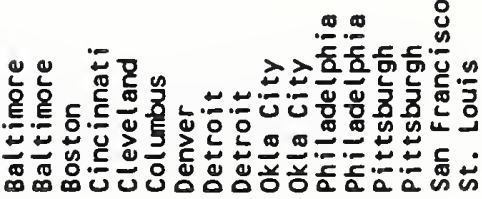

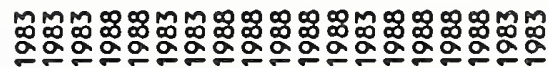

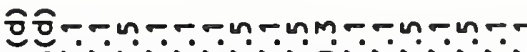

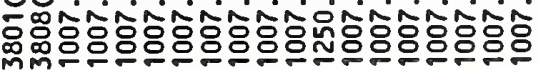

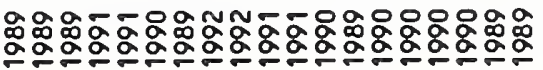

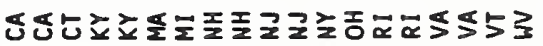




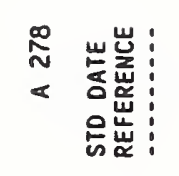

-n-7- -n nn-m

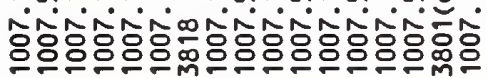

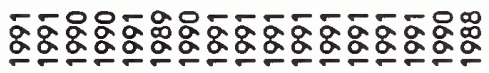

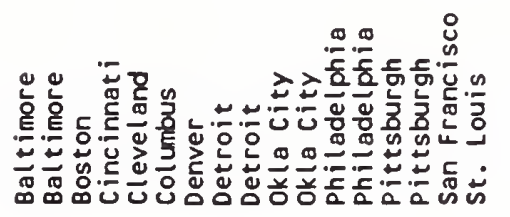

岕 :

突:

岀:

z:

点: क

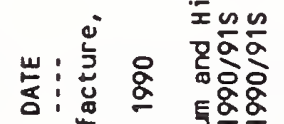

(2)

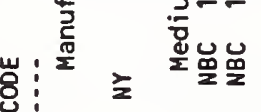

$\stackrel{8}{2}$

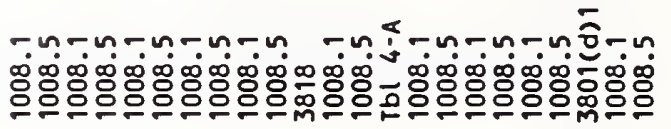

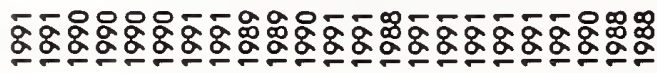

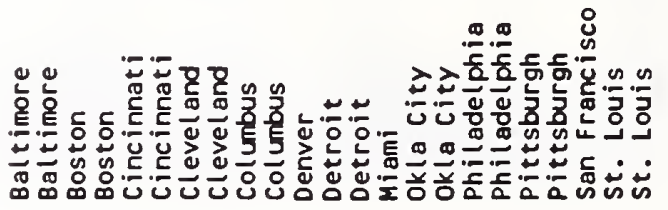

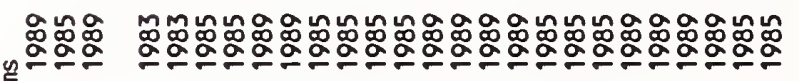

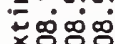

ชิำn-n-n-n-n-nm-n-n-n-n

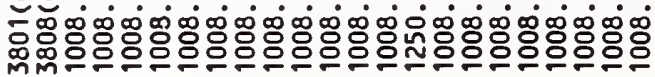
ํำ 중ㅎํ의

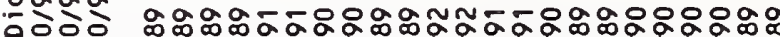

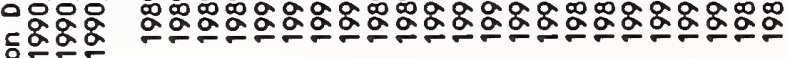
哥眨罢罢

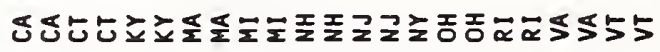

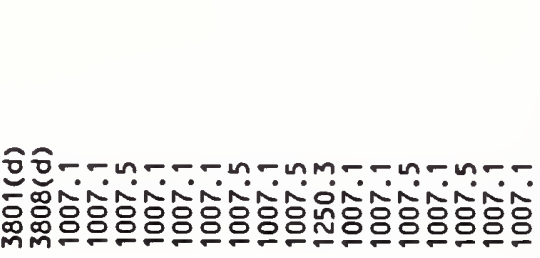

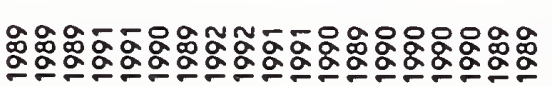

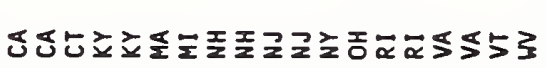

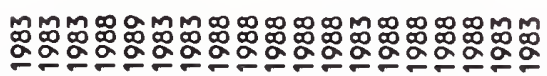

$\stackrel{\circ}{\circ} \stackrel{\circ}{\circ}$ is 몰

音

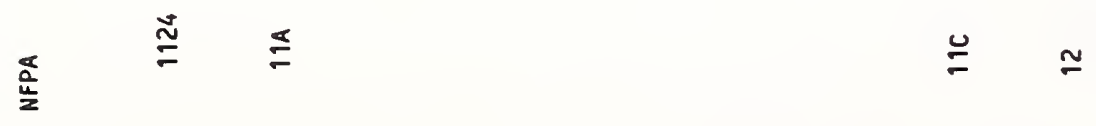


$\stackrel{8}{i}$

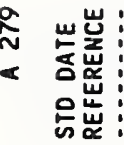

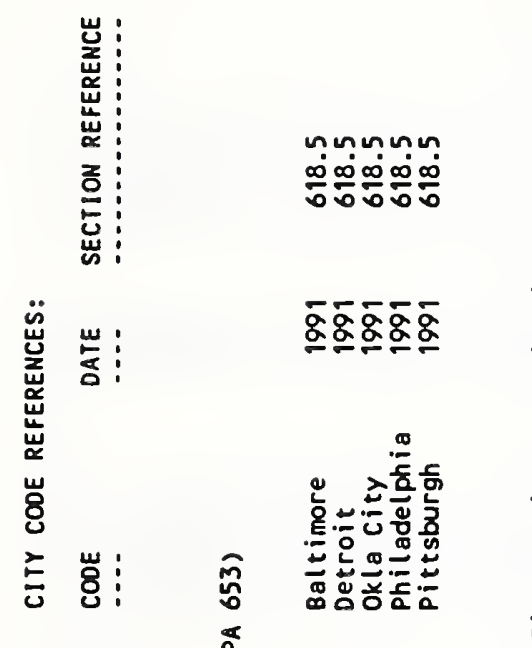

山岕:

은

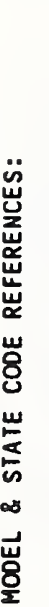

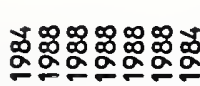

$\leqslant \frac{\infty}{\infty} \div \frac{\infty}{\infty} \div \frac{\infty}{\infty}=\frac{\pi}{\infty}$

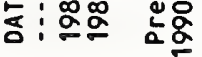

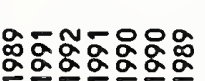

: $: 33$ 㟧

$8: 33$

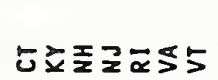

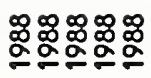

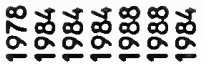

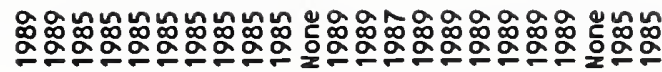

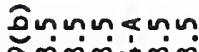

$\sigma \infty \infty \infty \dot{j} \infty \infty$

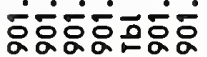

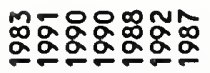

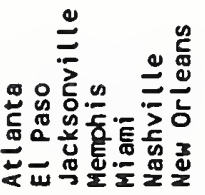

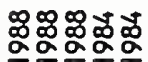<smiles>C1CCCCC1</smiles>

学

웅

蒫

nunumu

$\infty \infty \infty \infty \infty$

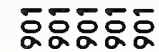

$\omega: 00$

容

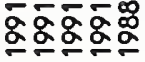

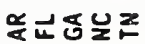

\section{$4 \frac{2}{2} \frac{5}{a}$}

$\bar{g}$

$\stackrel{\circ}{\circ}$

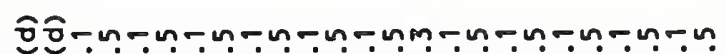

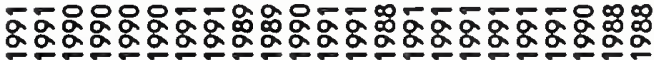

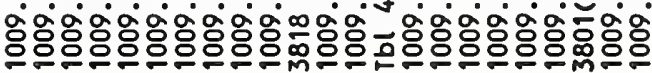

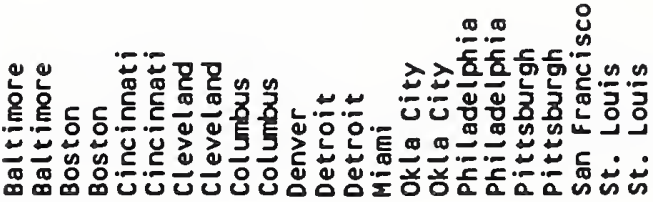

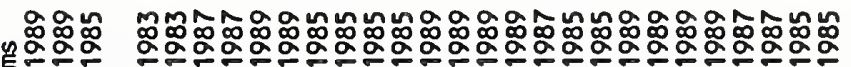

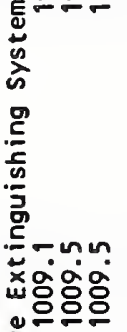

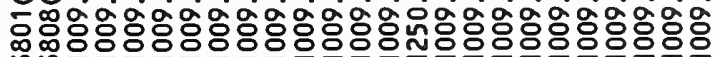

잏ㅎㅎㅎㅇ

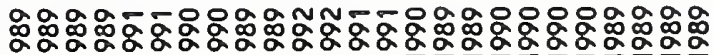

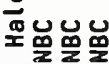

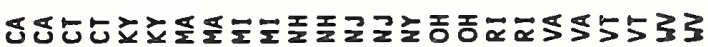

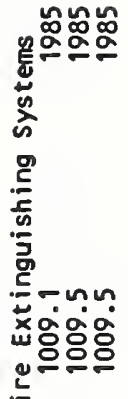

un un

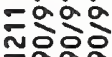

cو

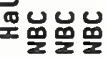

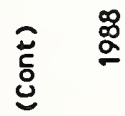

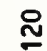

$\overline{\text { }}$

ฐ 


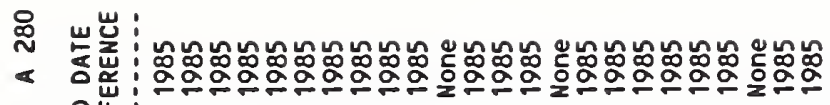

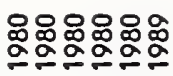
它岕:
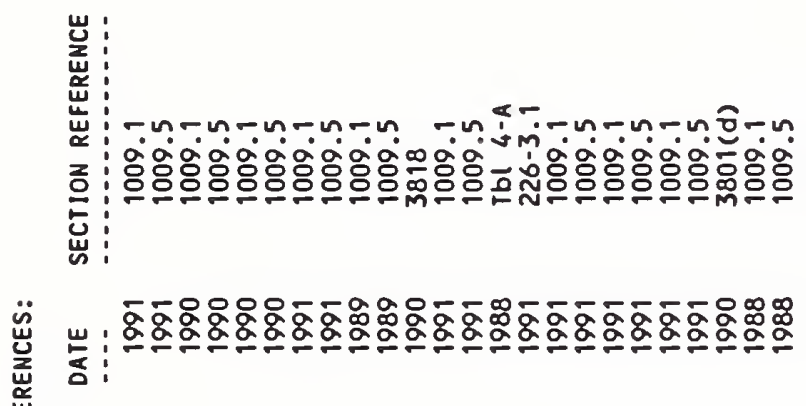

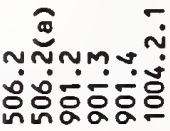

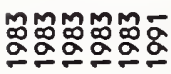

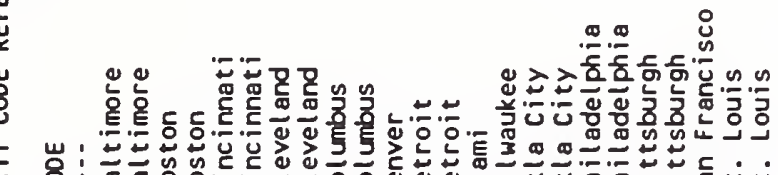

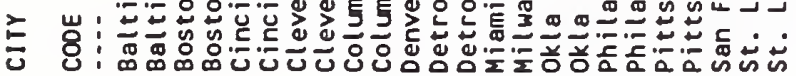

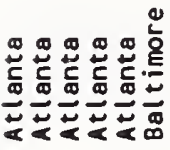

岂:

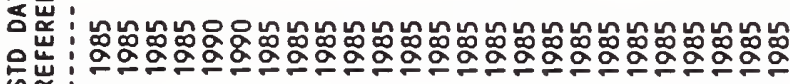

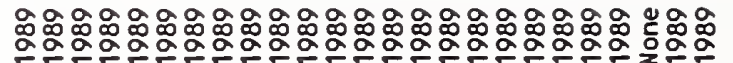
惫

$\ddot{\circ}$

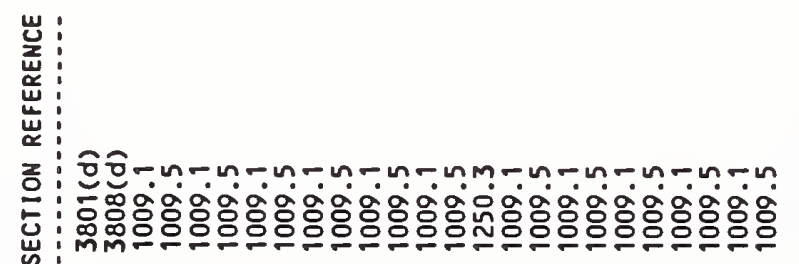

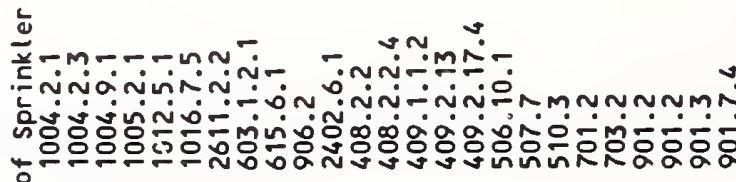

岁

岁

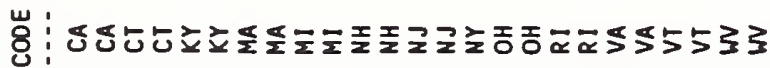

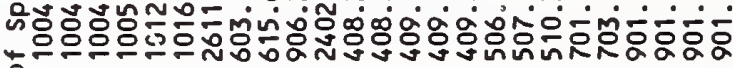
-

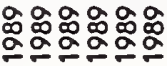

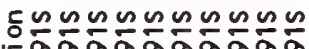

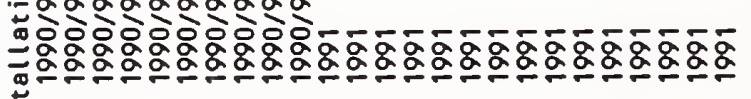

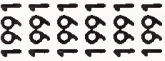

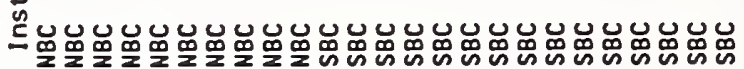

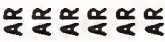

㟧

ํㅗㅇ

2

荘

$\stackrel{\circledast 刃}{\simeq}$

$m$ 


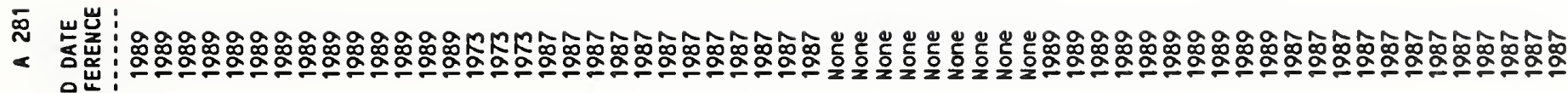
鹃: 岂:

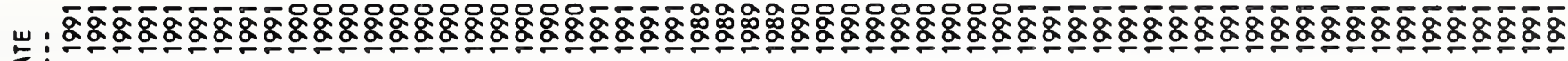
客:

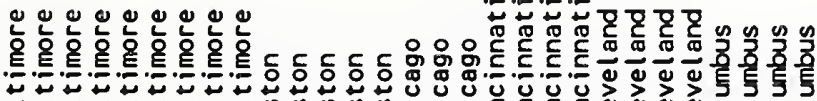
애ํํํำ vinjo-0.jm

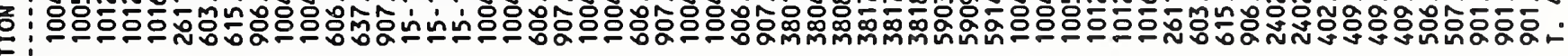

ठㄴ.

峞:

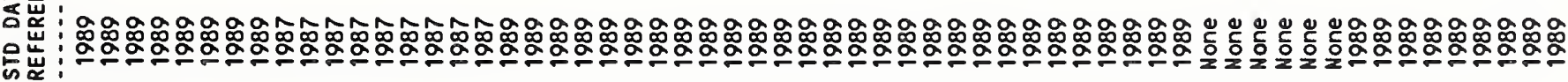

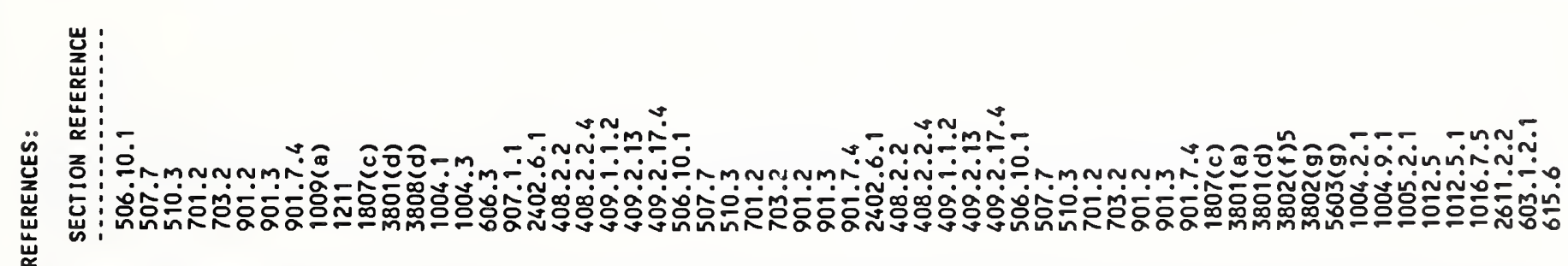

岩 :

ठั山 


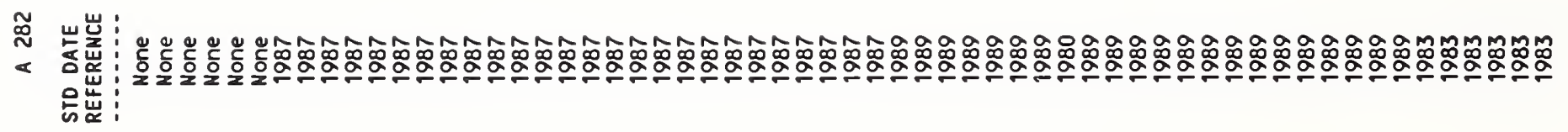

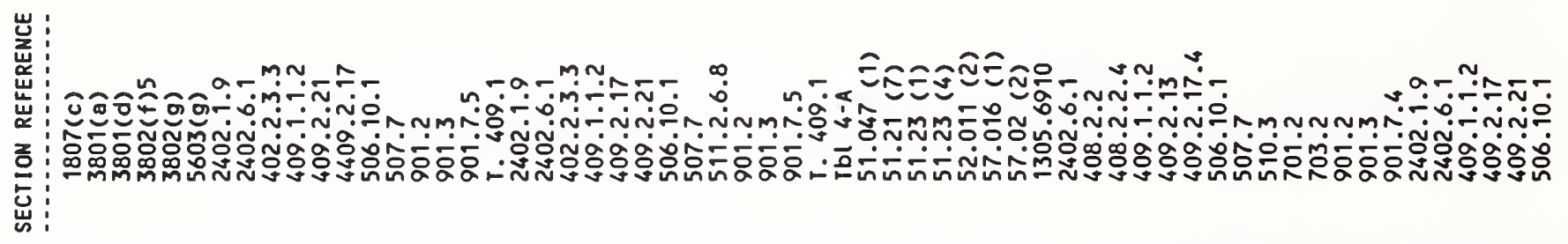

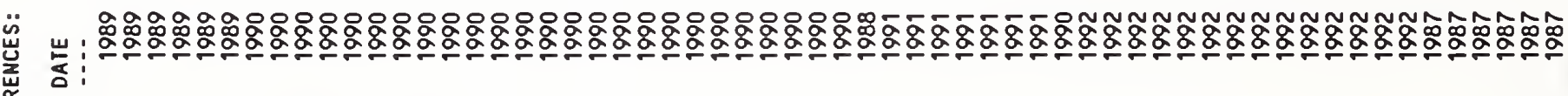

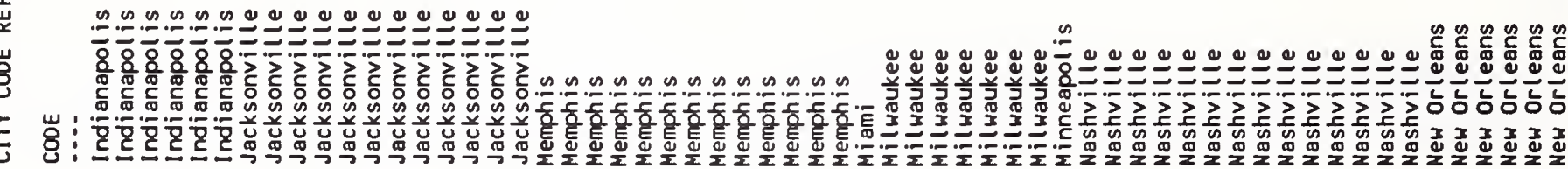

岁:

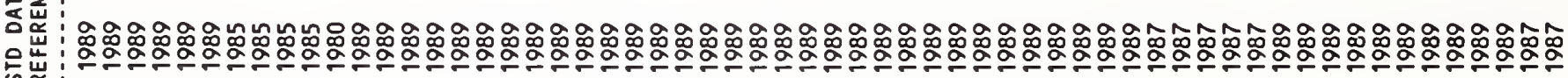

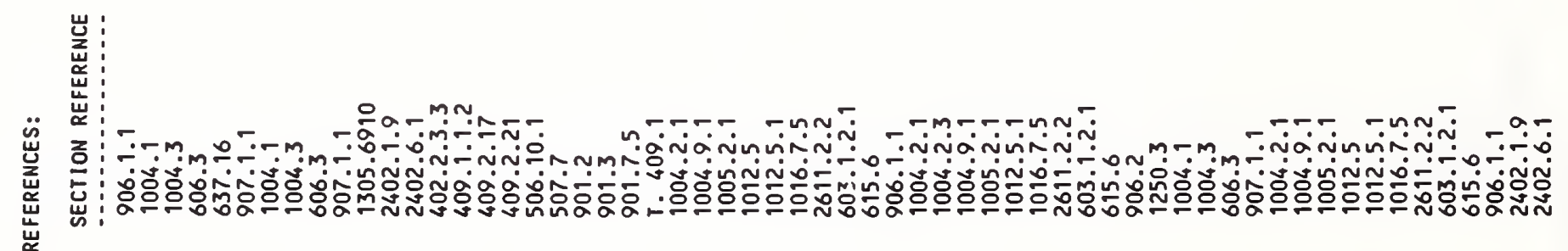

岁 崖

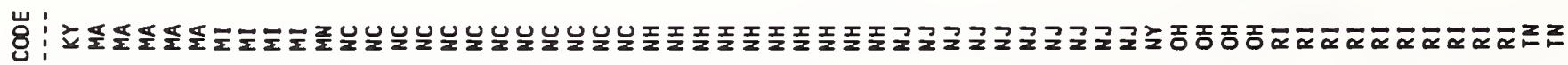

它

m 

这岕:

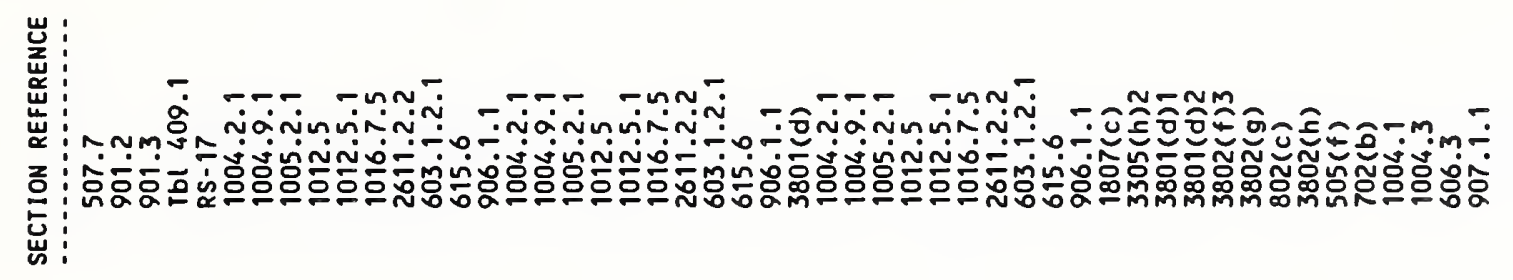

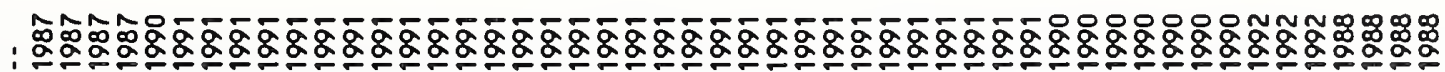

얋요 宁:

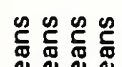

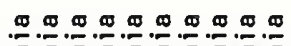 \\ 过通品品品

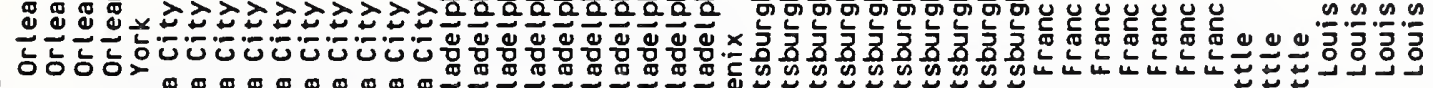

与

떤

ธิّ

눈 :

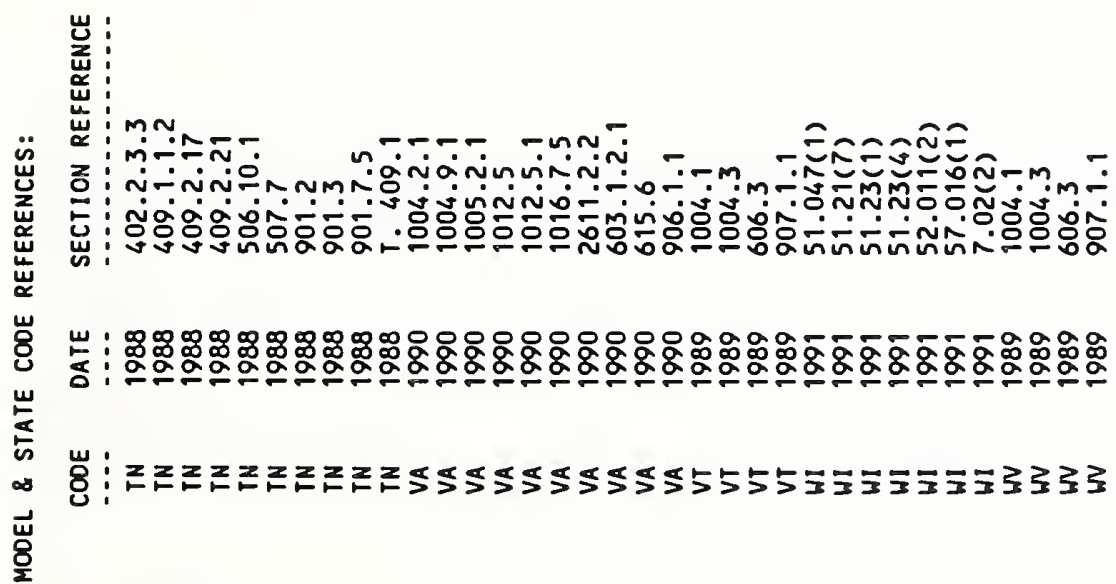


胥

崖

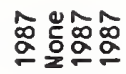

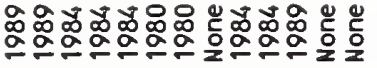

เิ อิธูป

๔ ⿻上丨

흘

出:

岂

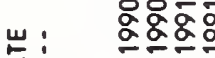

咅

엉

¿

垴

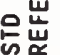

山.

:

=

㟧

z: ลิ ลิธิ

맏 बํำ

出:

या के

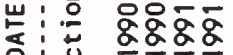

(⿸丆口

岁:

준도코

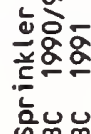

훌

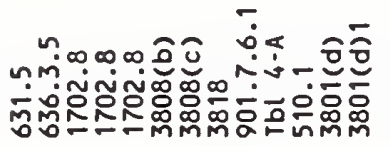

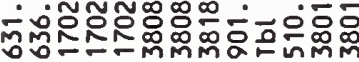

$00-a 0000 n-0$

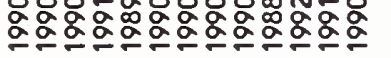

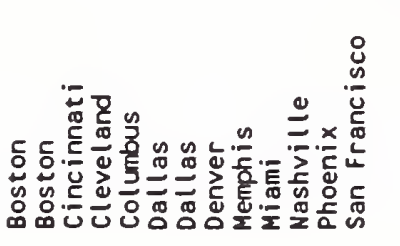

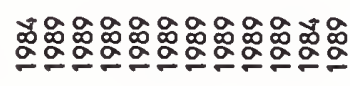

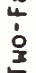

कृष

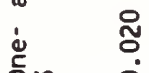

¿̊.

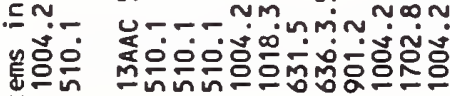
के

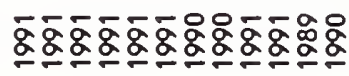

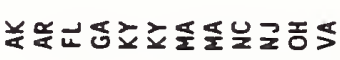

$\bar{g}$

$\stackrel{m}{m}$

$\frac{5}{\frac{0}{2}}$

ิㅗ

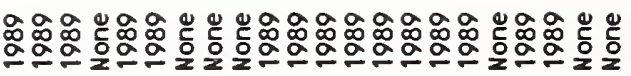

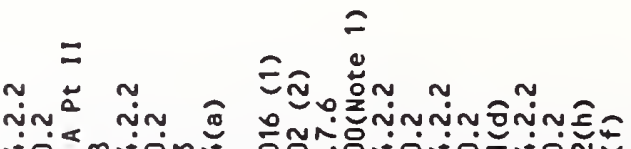

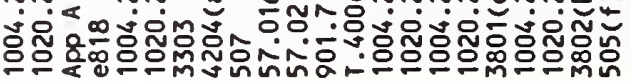

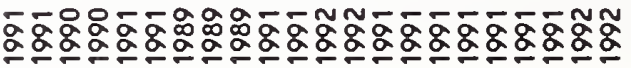

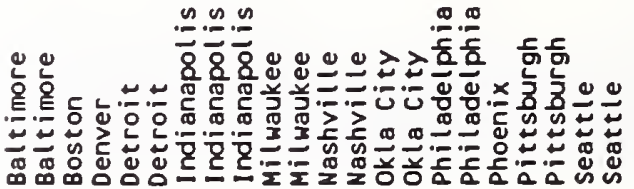
3

$\stackrel{\leftrightarrow}{\square}$

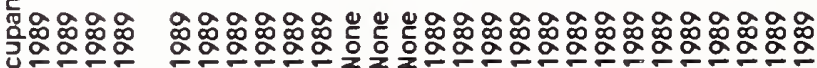

유용

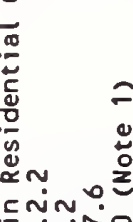

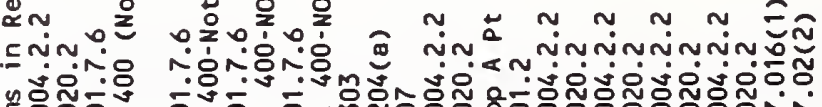

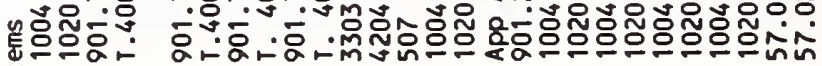
in $\frac{\pi}{2} \frac{\omega}{\alpha}$

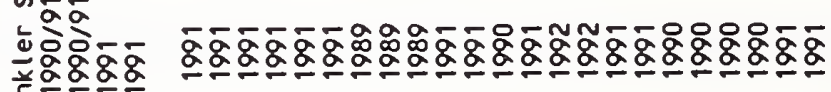

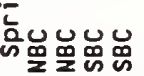

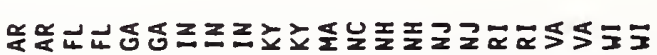

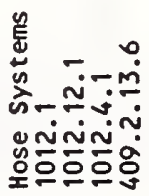

ㅌㅗㄸㄸㅡ

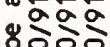

흥혛ㅎㅎㅎㅎㅎ

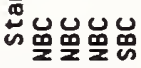

$\bar{g}$

g̊ 


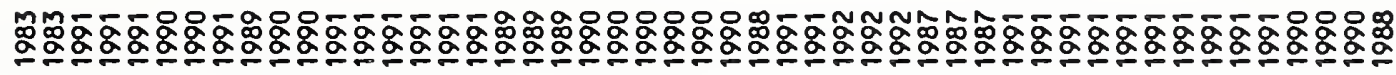

s̄효

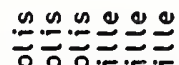

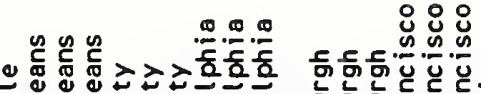

w岂 :

各总

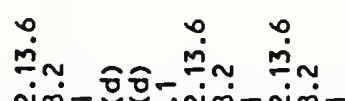

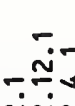

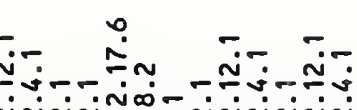<smiles>CC1(C)C2=CN=C1C2</smiles>

No-

동

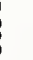

똔:-

sos

岁: 飠怘

\section{$\stackrel{\alpha}{x} \frac{\alpha}{\alpha}$}

岂 
总容容

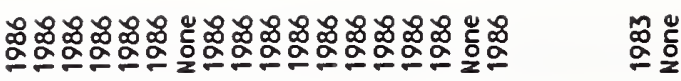

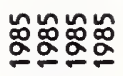
옹년:

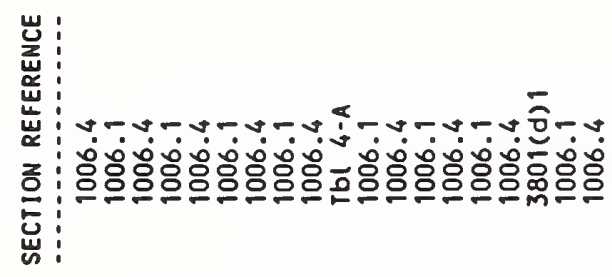

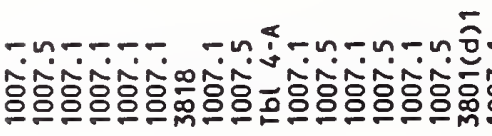

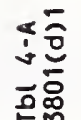

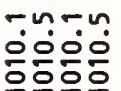

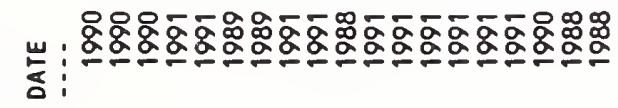

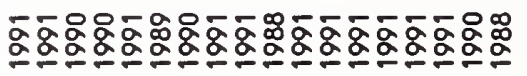

జ̊요

종모용요

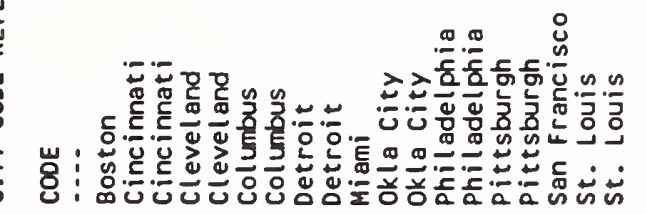

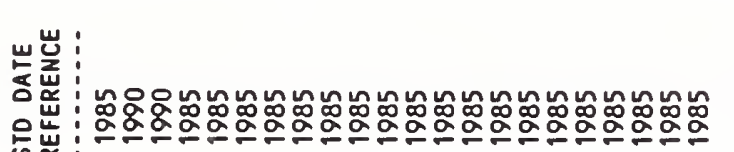

.

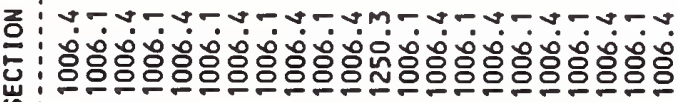

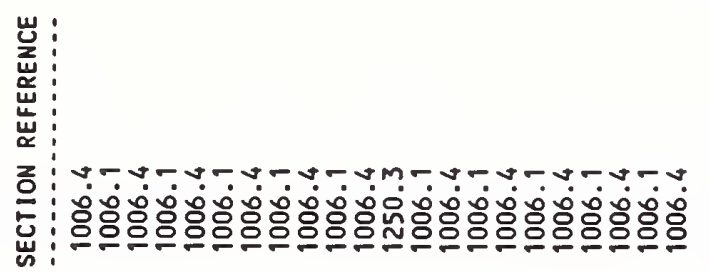

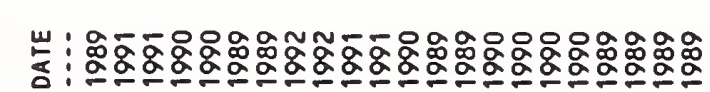

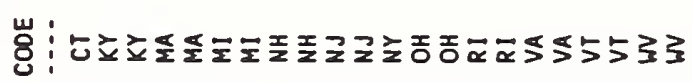

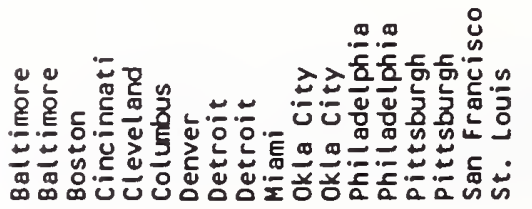

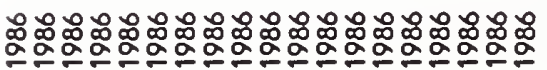

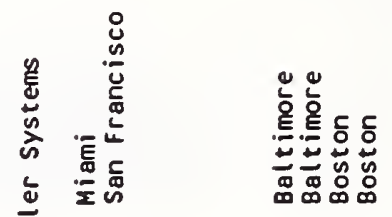

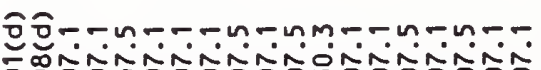

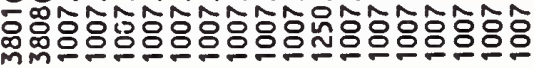

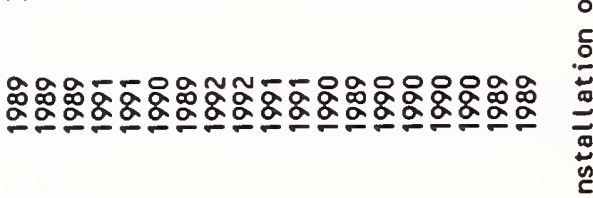

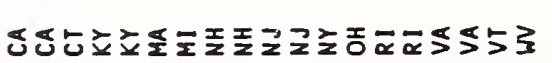

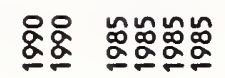

离

을

จิธ

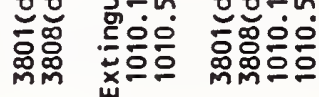

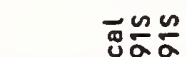

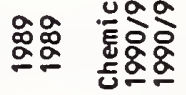

怘高高

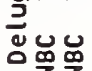

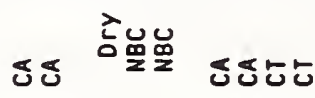

๕ัญ

$\bar{\Sigma}$

$\stackrel{8}{\circ} \stackrel{\circ}{\circ}$

㕍 
命

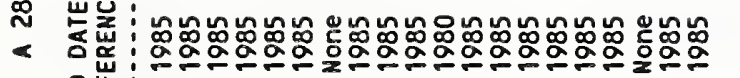
员营:

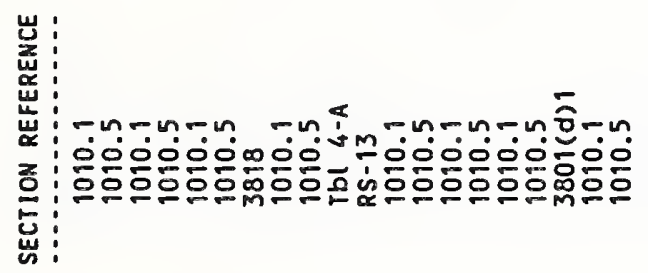

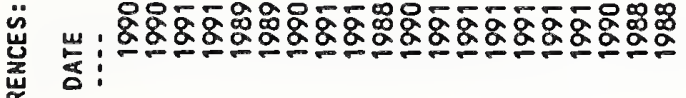

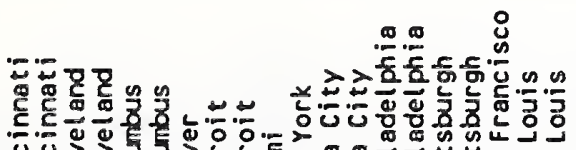

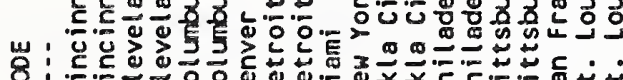

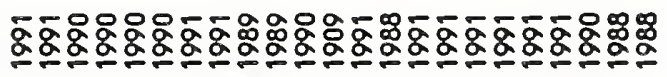

世岁 :

ธิ

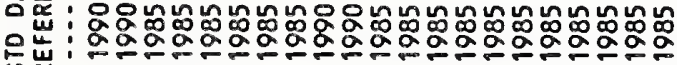
w
崖:
岃 :
z: :

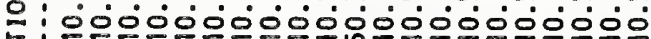

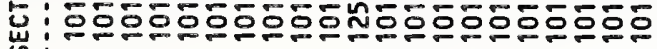
岕

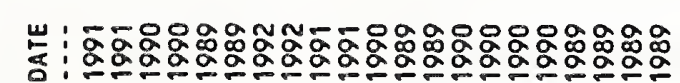

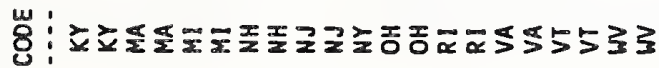

它

ఖ

옹

密

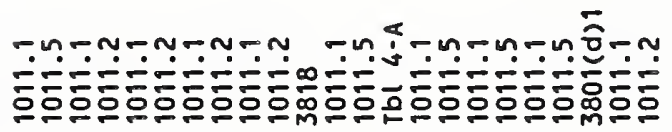

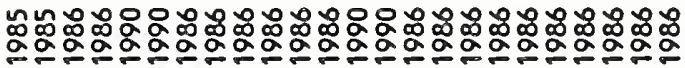

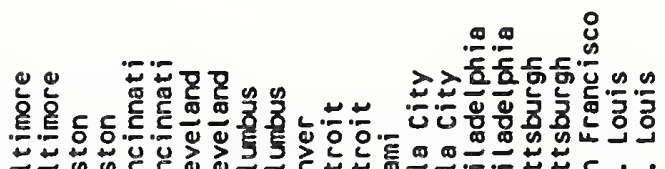

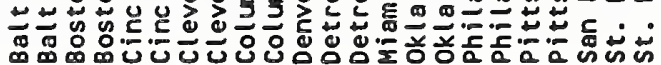

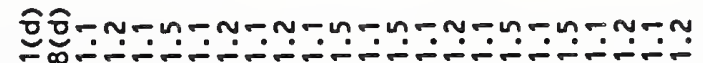

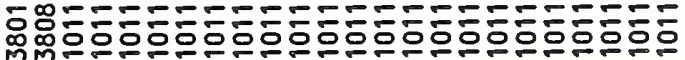

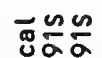

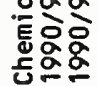

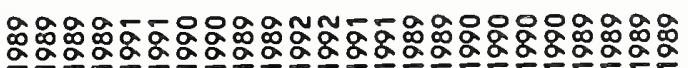
范嵔崖

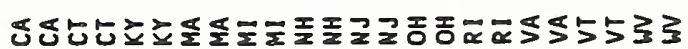




\section{备 㟯:}

岕:

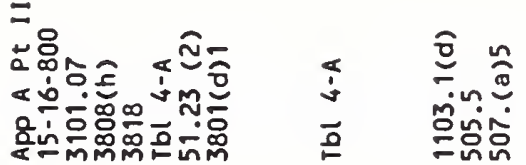

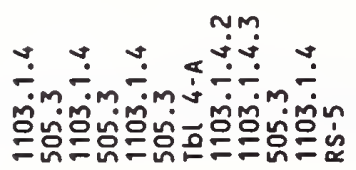

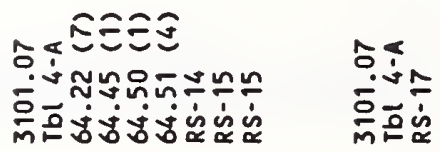

:

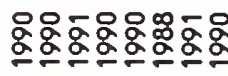

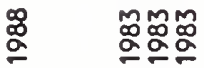

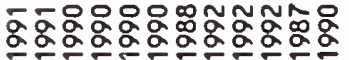

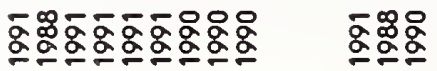

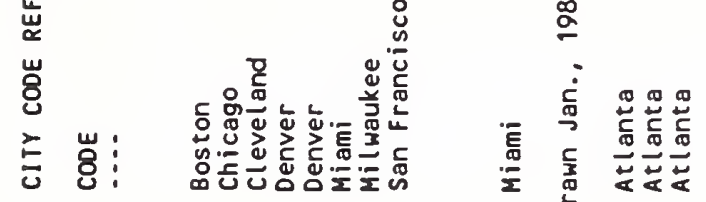

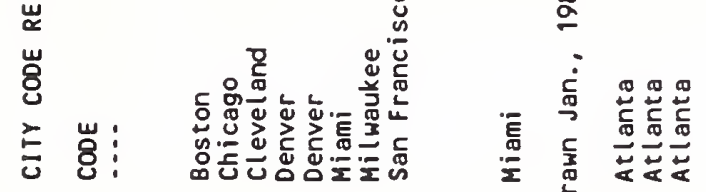

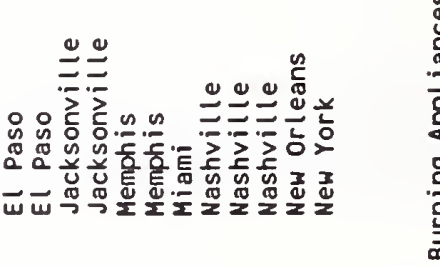

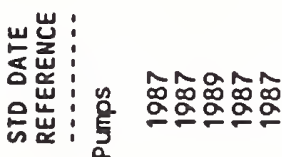

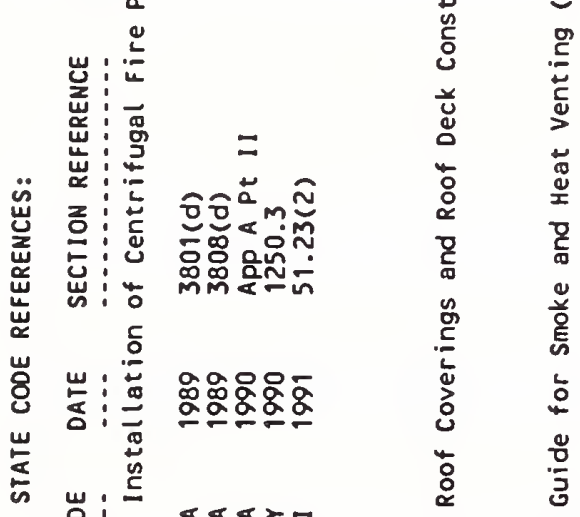

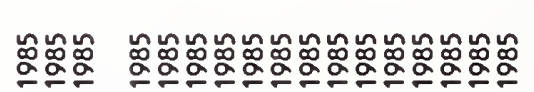

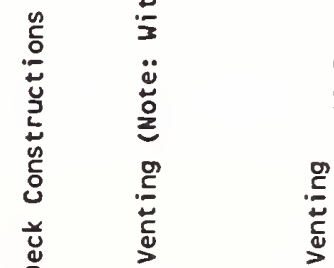

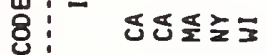

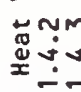

ษm

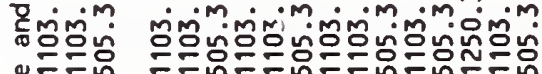

产

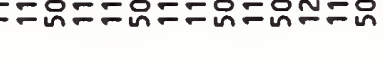

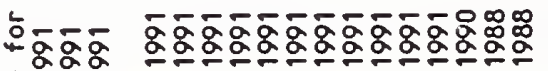

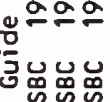

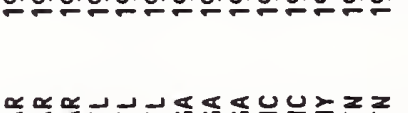

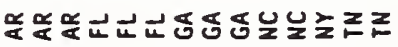

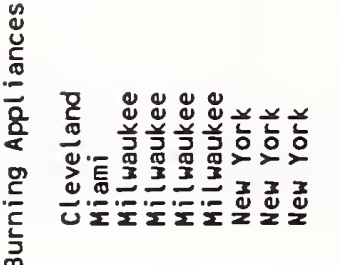

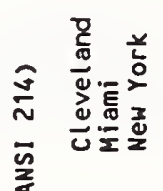

훌

$\stackrel{\circ}{g}$

$\approx$

$\bar{\sigma}$

$\underset{\Xi}{\stackrel{8}{\alpha}}$

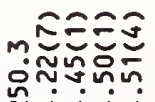

:

总 喿

o

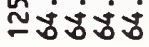

웋ㅎㅎㅎㅎㅎㅎㅁ

든

文ミミミ

岁

范

용

离

$\stackrel{\text { ळ̊ }}{\circ}$

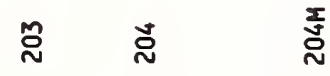

$\bar{N}$

$\frac{\pi}{2}$

ㅇ

蛋

$\sim$ 


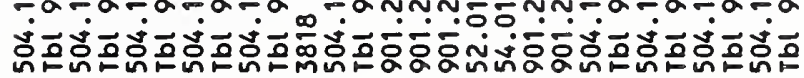

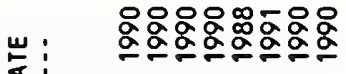

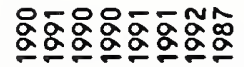

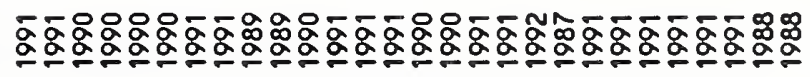

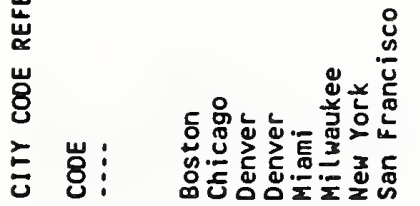

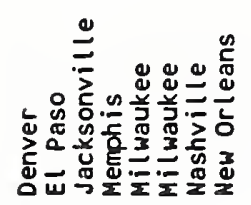

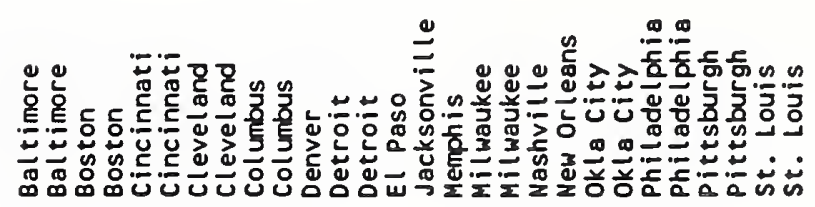

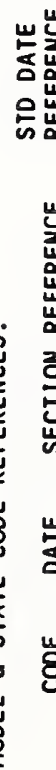

$\frac{\sigma}{\underline{a} \hat{a} \tilde{a} \tilde{a}}$

ติ

ฆัฒ̊:

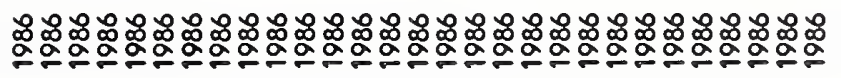

$=$

a

z:

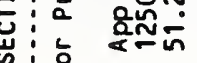

岁: 营

离

岁: $:^{\frac{0}{3}}$ 또로

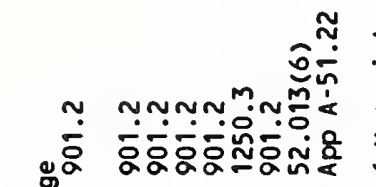

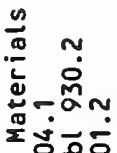

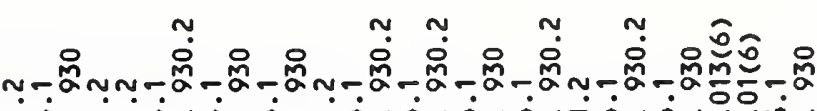

离

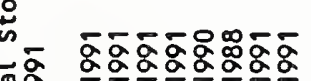

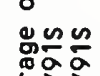

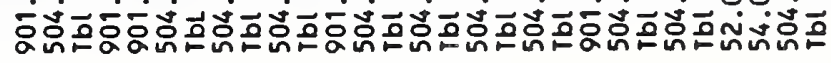

क्षे

宏山顿岩々ミミミ

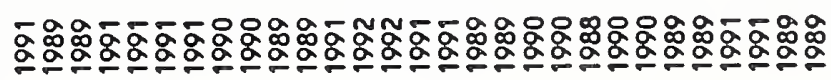

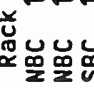

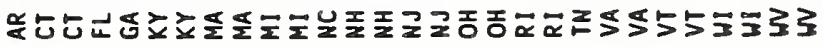

$\stackrel{\text { ळ }}{\underline{a}}$

$\stackrel{8}{2}$

$\bar{\alpha}$

$\frac{\sqrt{3}}{x}$

$\approx$

$\bar{n}$

$\frac{0}{n}$ 


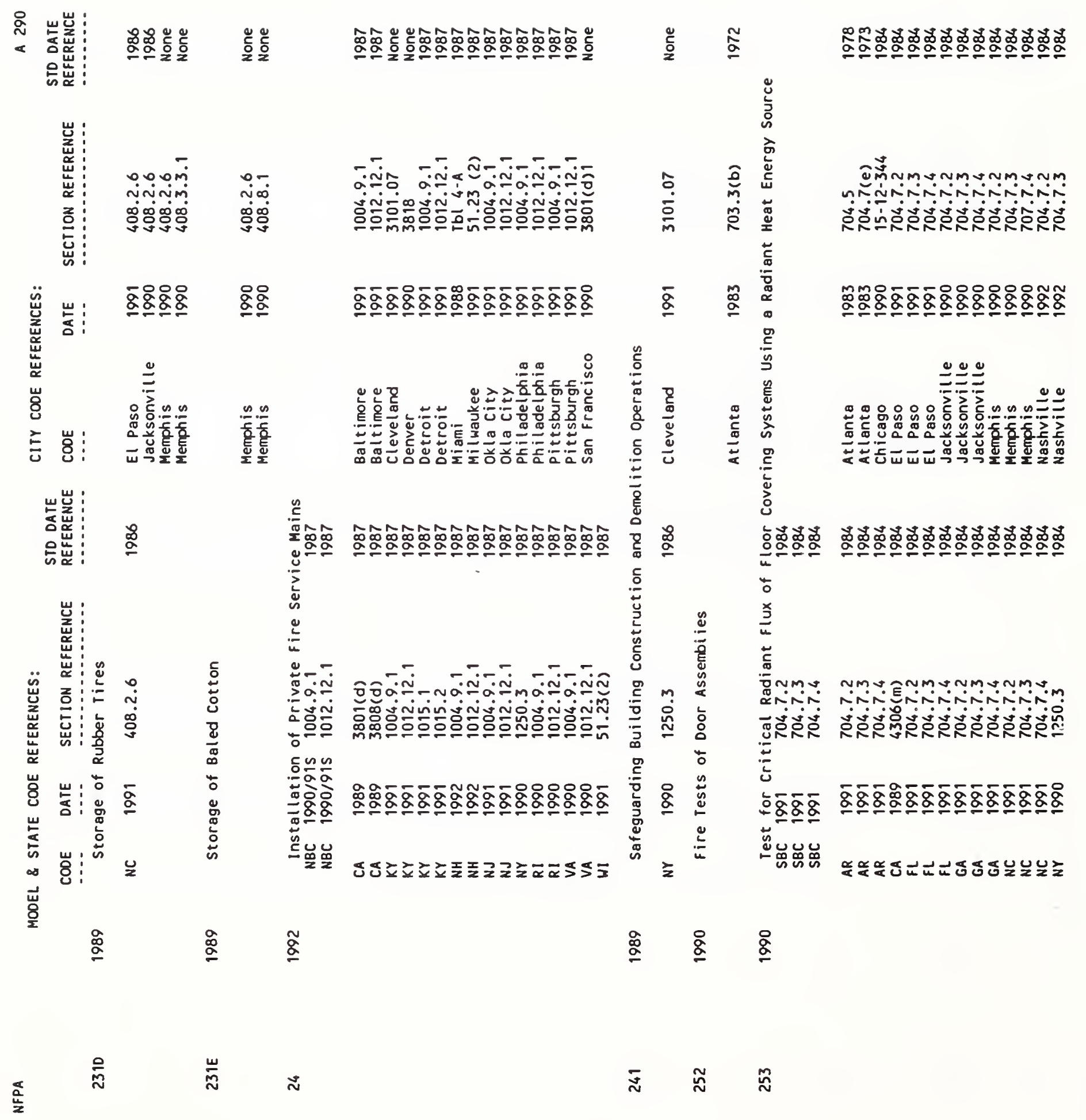




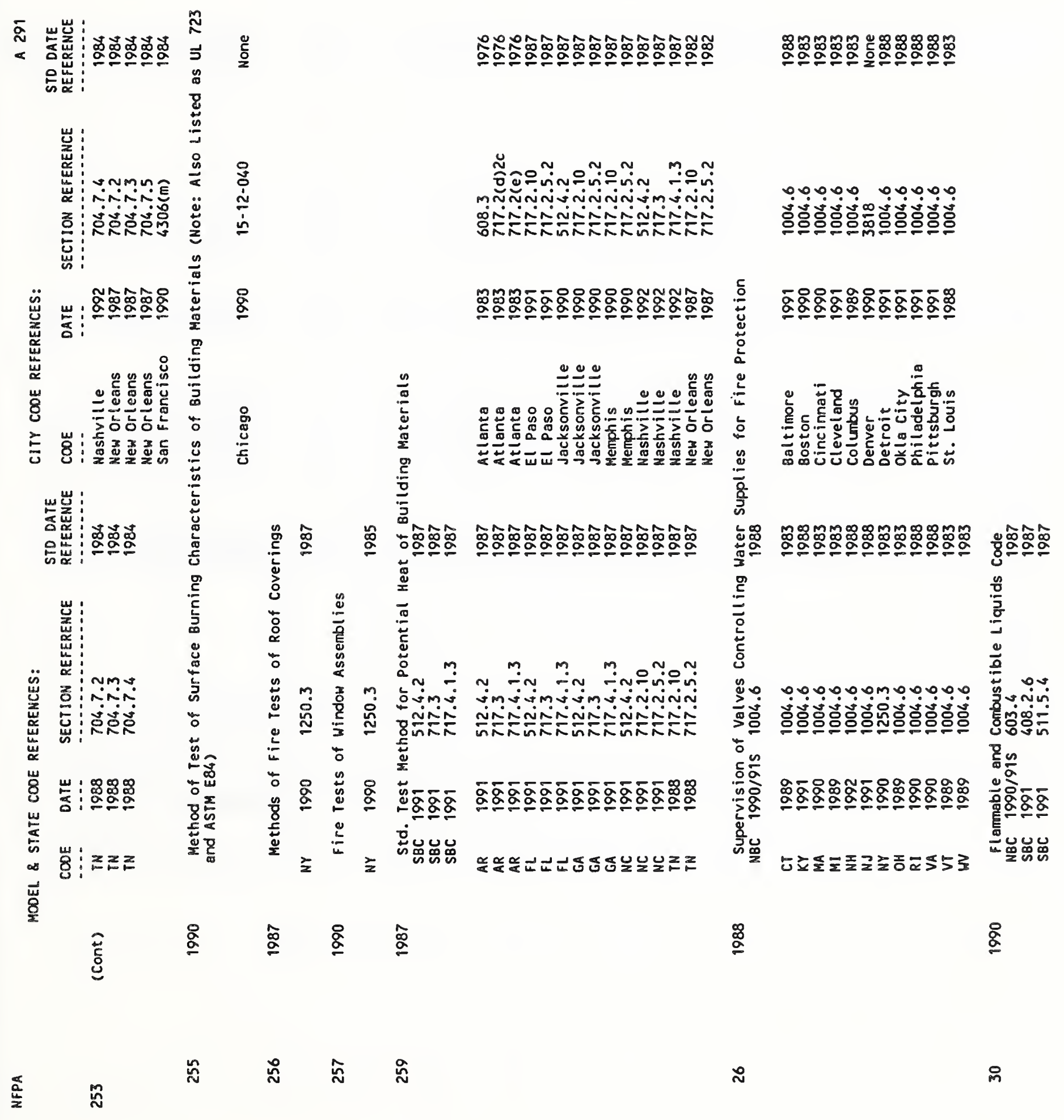




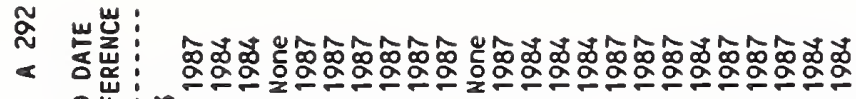

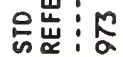

嵳:

z $=0.000$

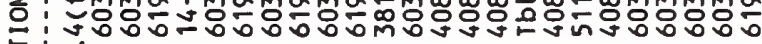

出行

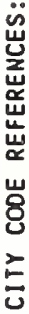

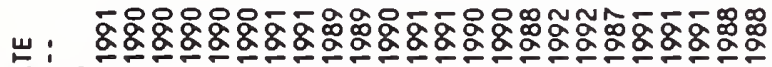

的

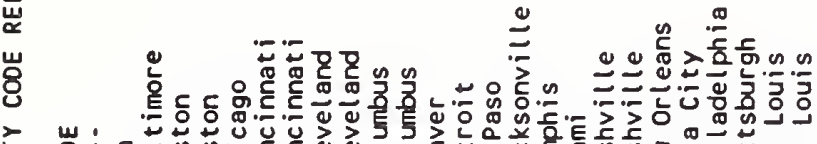

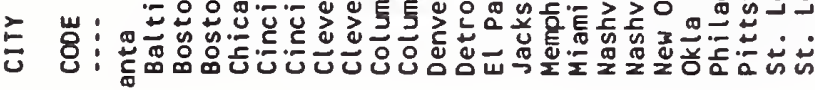

㟧

送焉

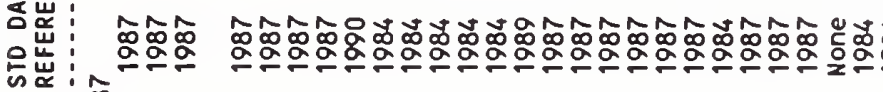
产

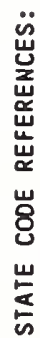

㟧 :

:

品

年 每

岩 $\underline{2}$

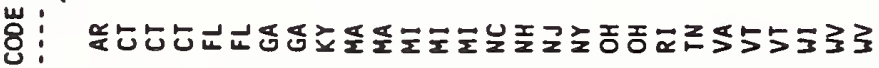
$\stackrel{\infty}{4}$

产

$\frac{d}{\frac{a}{2}}$

요

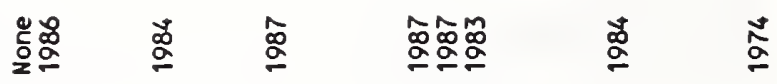

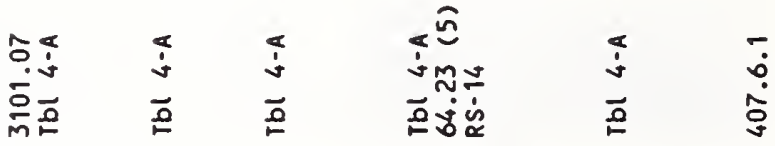

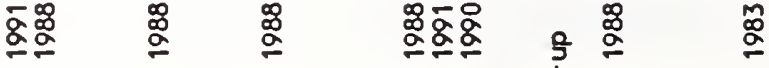

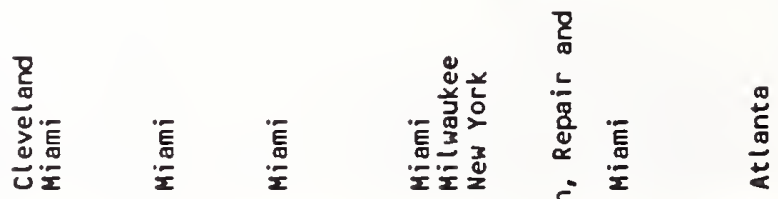

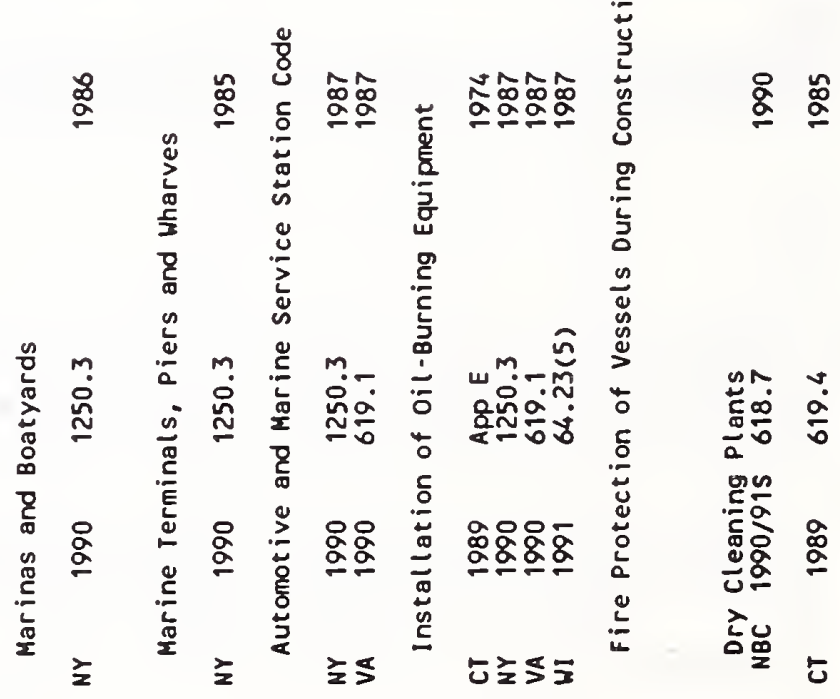

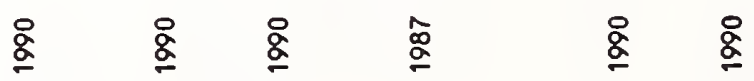

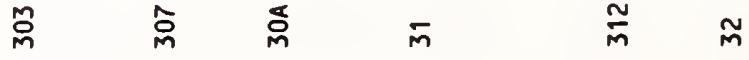


品

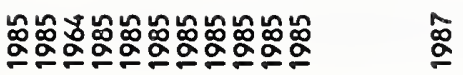

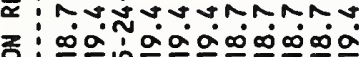

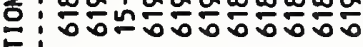

岕:

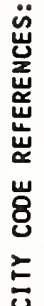

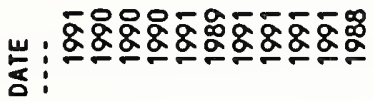

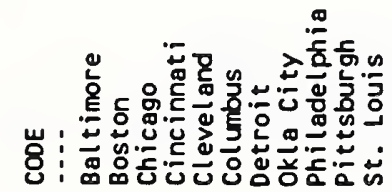

띤

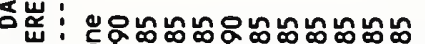

นิ

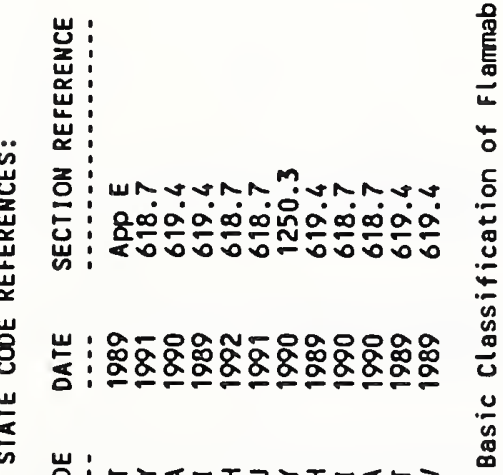

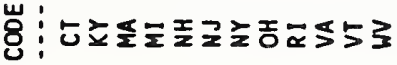

옹

$\stackrel{g}{g}$

$\bar{m} \quad m$

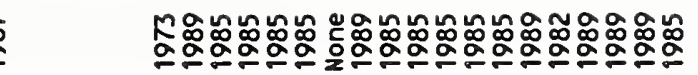

$\sum^{3}$

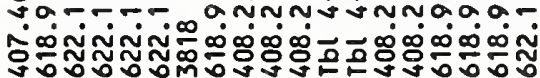

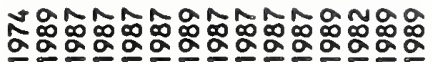

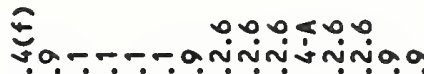

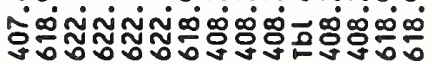

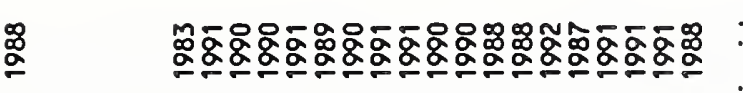

$\frac{2}{3}$

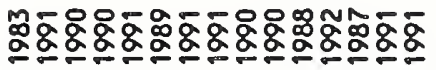

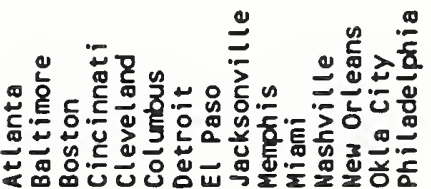

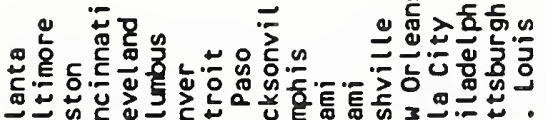

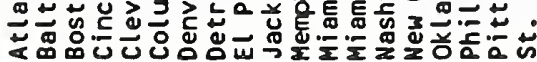

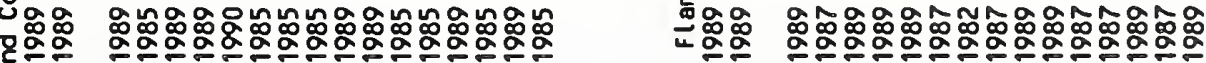
$\stackrel{\text { s. }}{2}$

岁

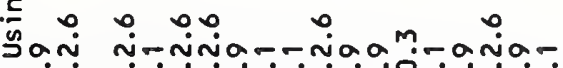

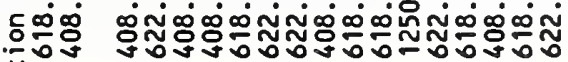

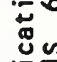

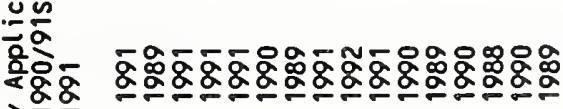
질 婄霅品

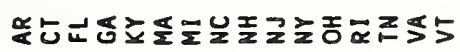



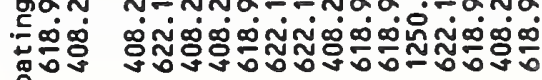

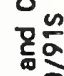

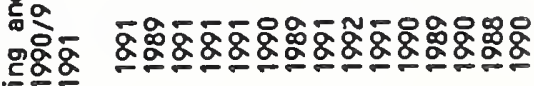
遂兽品

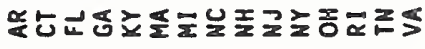


敢 은눈:

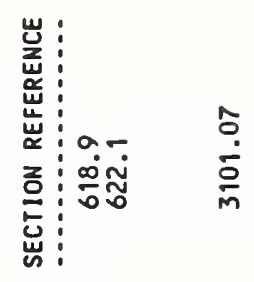

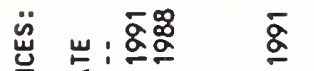 ¿ :

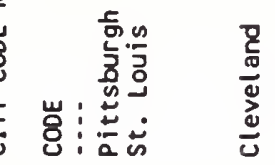

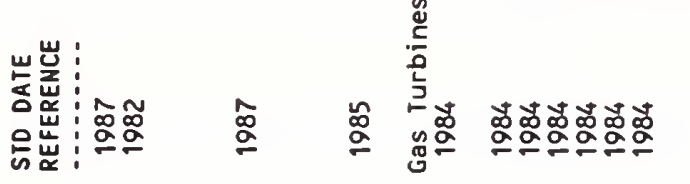

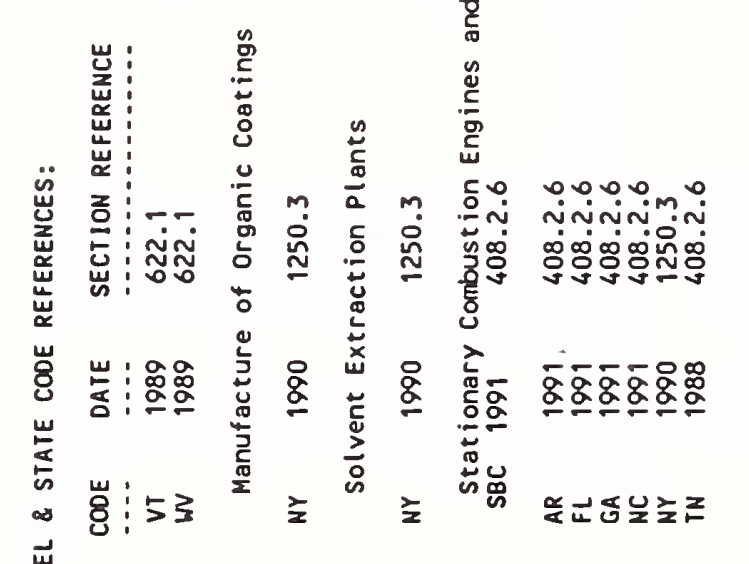

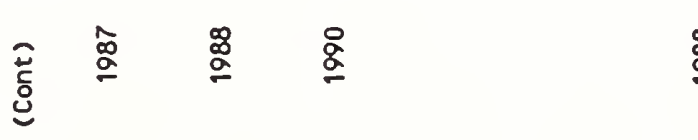

1)

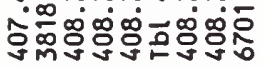

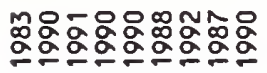

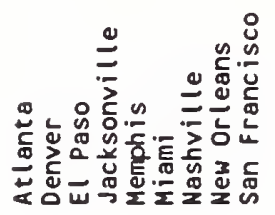

$\cong \stackrel{m}{\stackrel{m}{m}-1}$

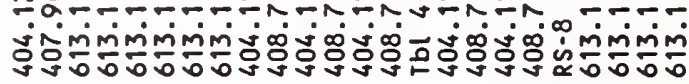

产

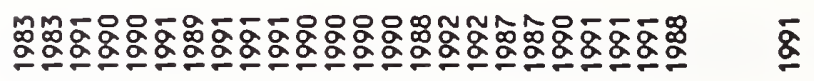

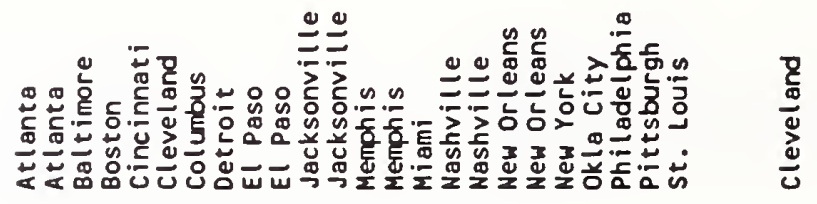

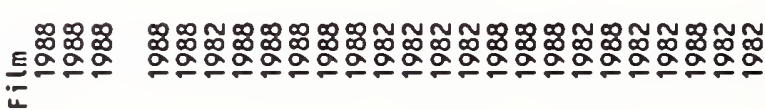

임 $\stackrel{0}{3}$ -

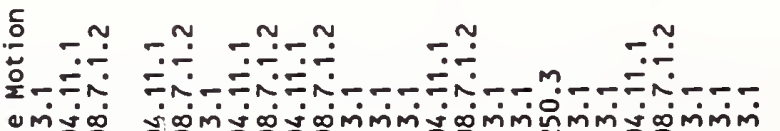

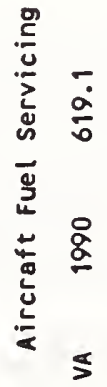
产总品

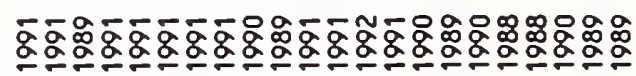

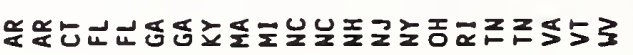
兽

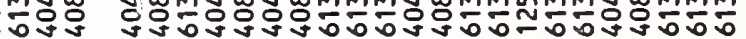
울 $\stackrel{0}{\circ}$ 


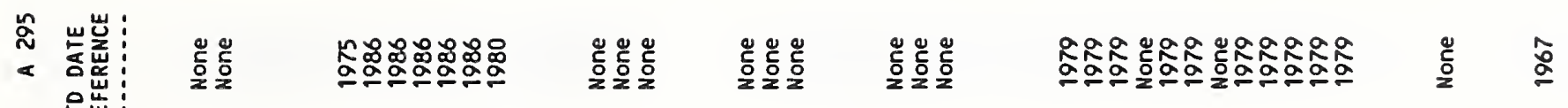

I

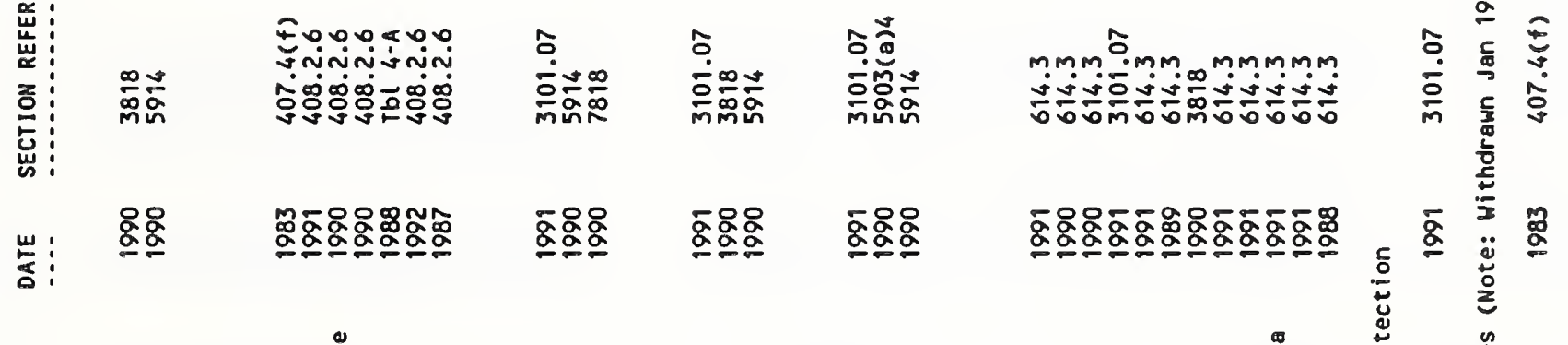

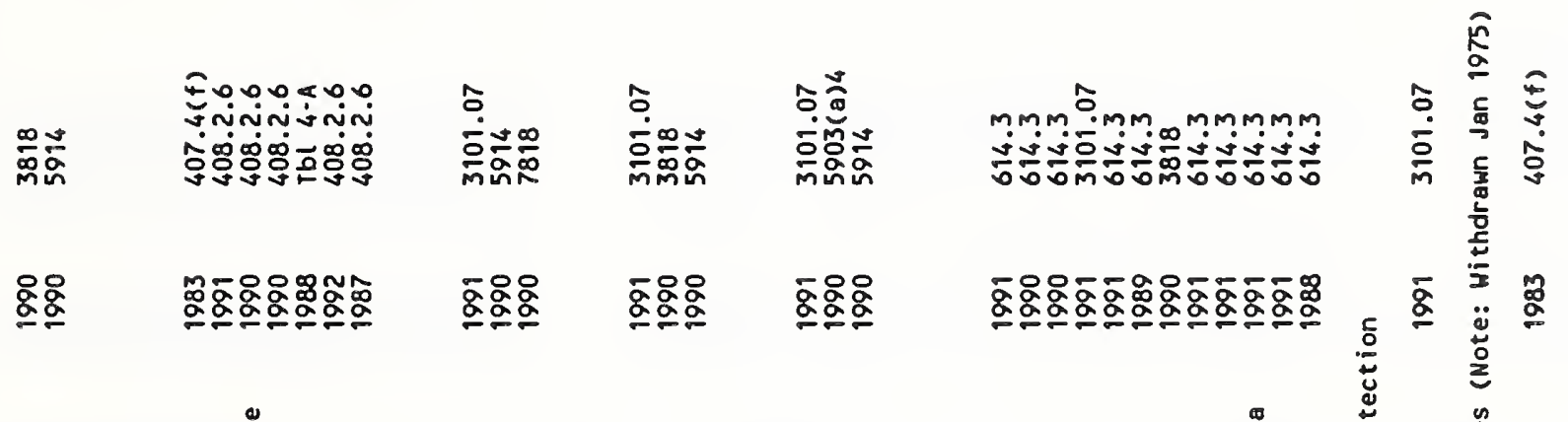

$u$
ü
岁

$\bar{z}$

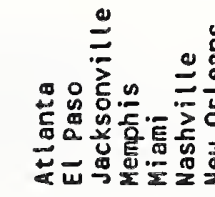

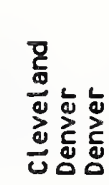

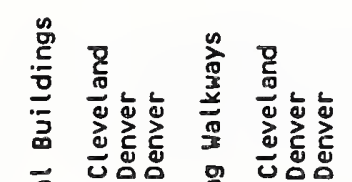

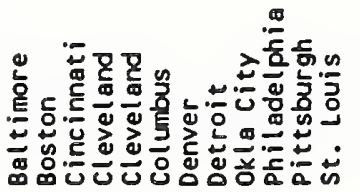

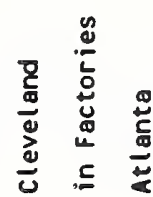

颜

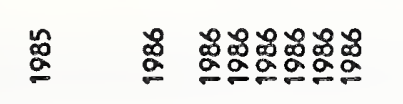

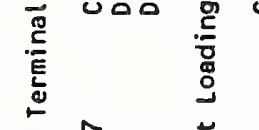

。ัّ

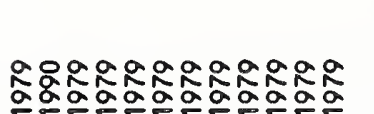

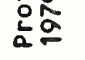

蛋

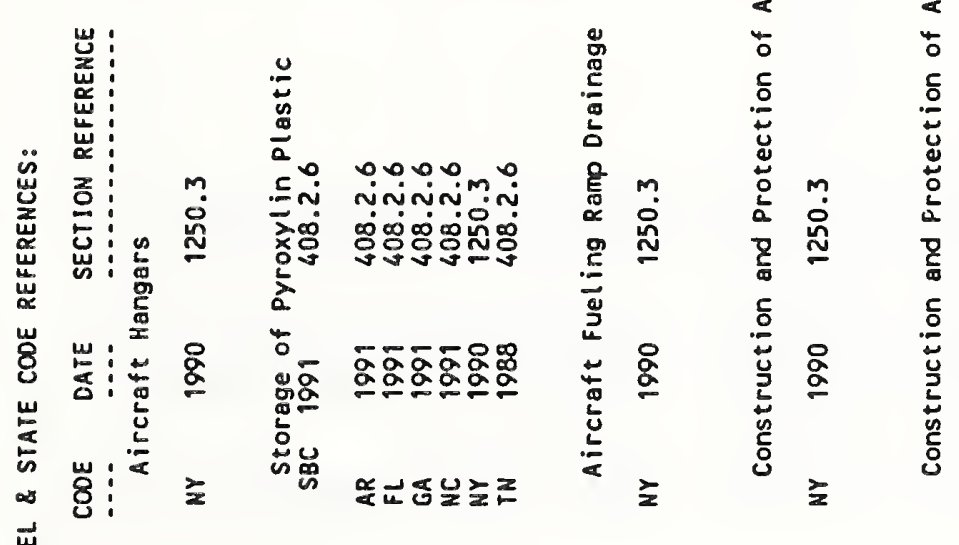

竞

范

$\stackrel{8}{2}$

善 $\quad \stackrel{\mathrm{a}}{\mathrm{g}}$

$\stackrel{8}{\circ}$

高

$\frac{\mathrm{x}}{\mathrm{a}}$

字 岁

$\frac{n}{3}$

$\stackrel{\circ}{3}$

$\bar{\xi}$

$\stackrel{\infty}{3}$

$\frac{9}{3} \approx$ 
E

unmunNunannmunmunm

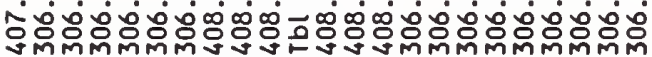

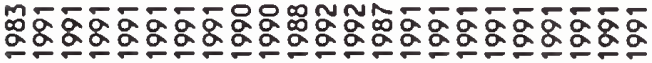

峞:

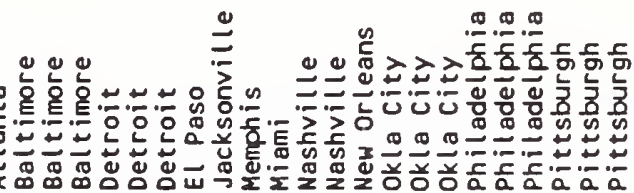

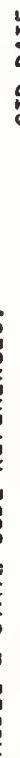

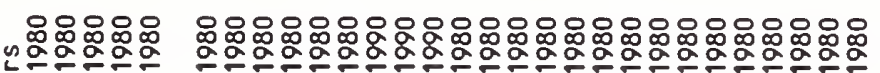

ب :

:

崖:

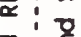

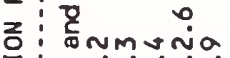

व்ं்

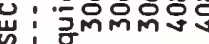
Jases

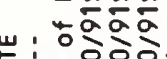

농 :영영형

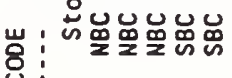

용

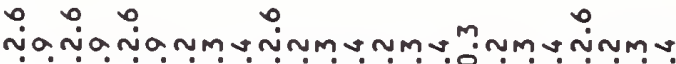

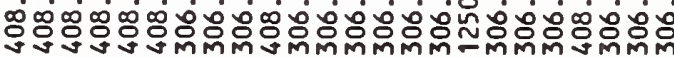

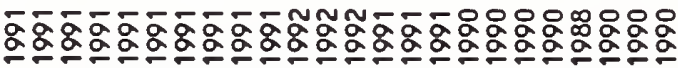

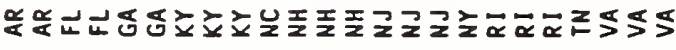

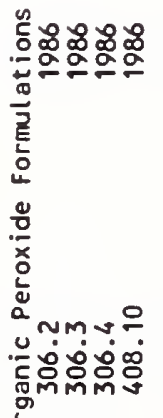

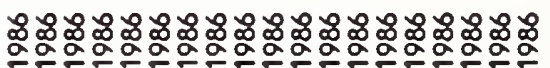

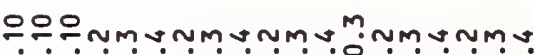

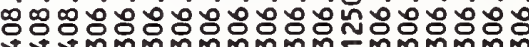
ठังun

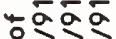 \\ 형영영

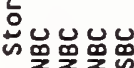

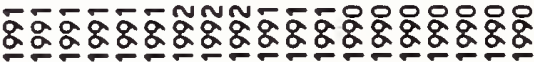

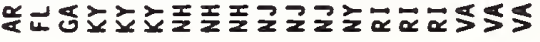




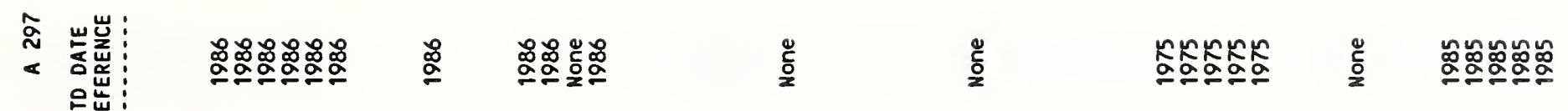

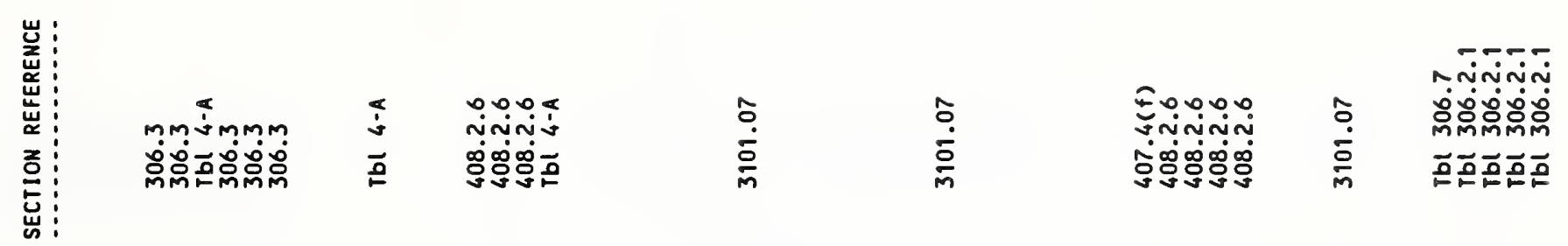

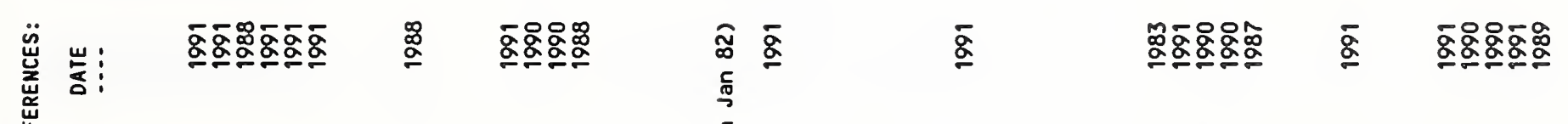

这

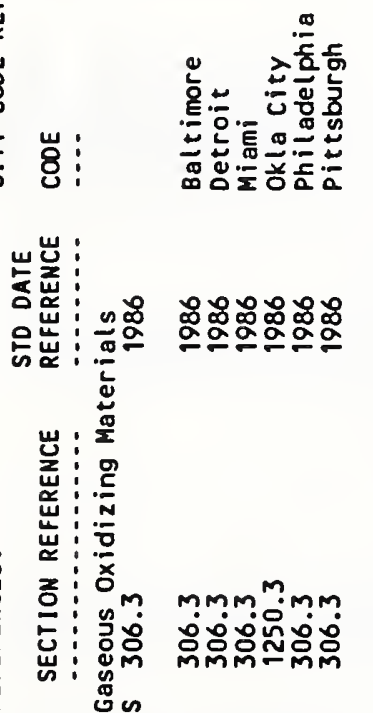

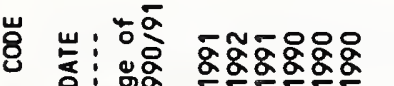

닝

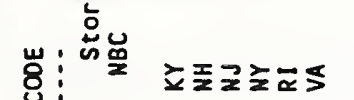

䒾

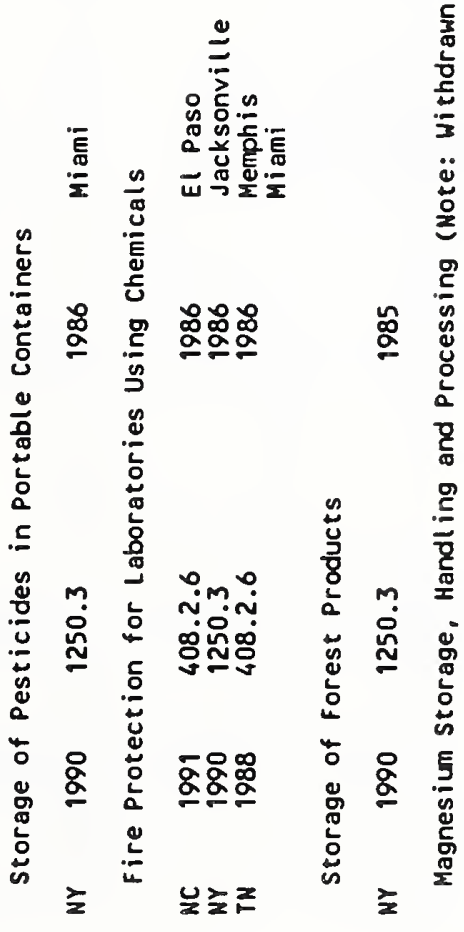

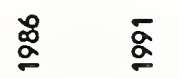

ㅇำ

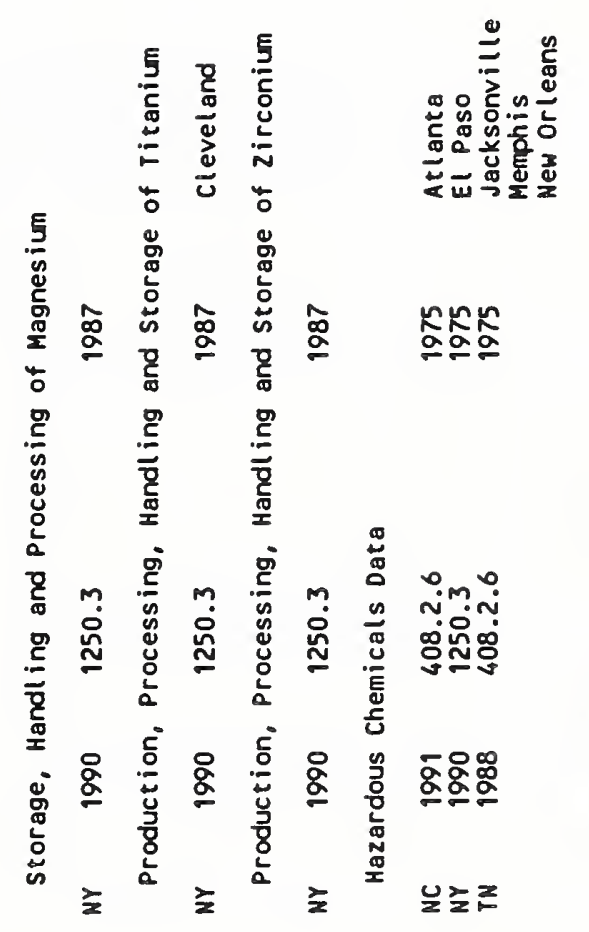

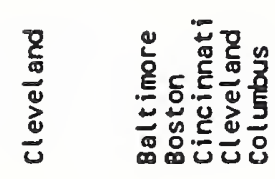

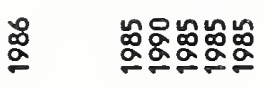

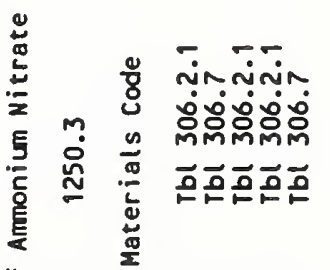

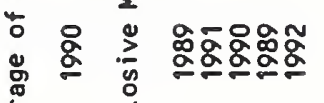

总 $\frac{0}{2}$

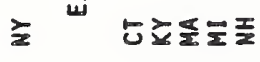

§

ఏ

是
ஓู่ ะู 


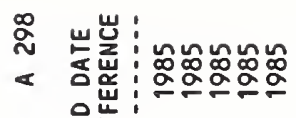
的峞!
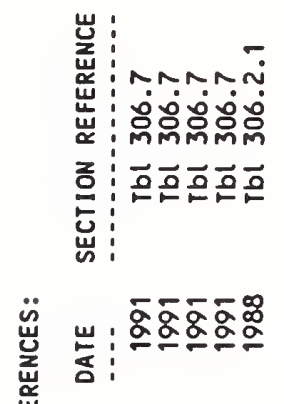

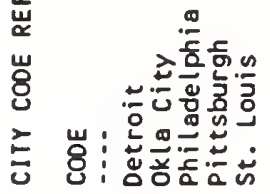

닌 :

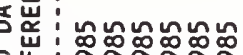
ํㅗㄴ

茩

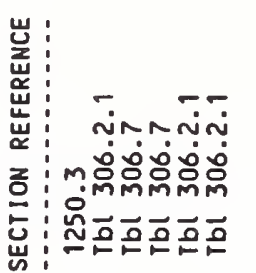

心!

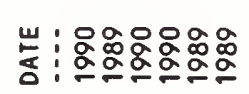

岁: ‘すテミらる

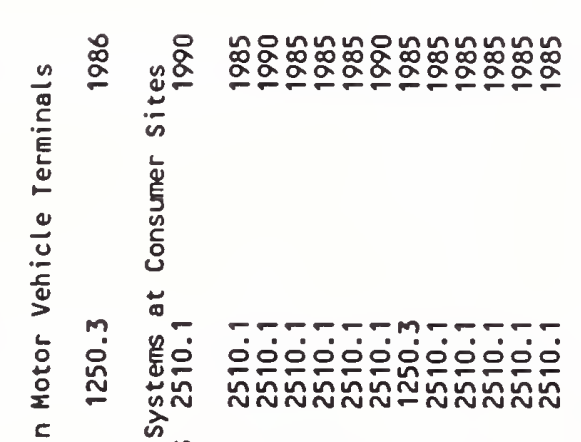

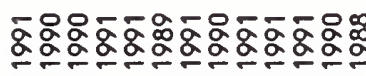

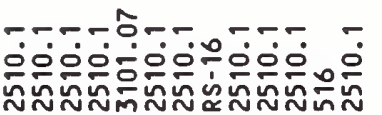

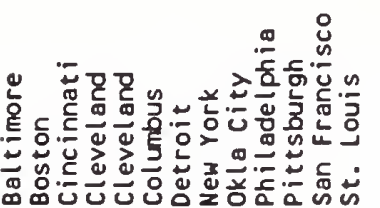

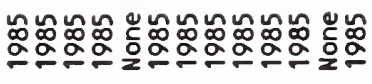

$\stackrel{\underline{0}}{\square}$

定

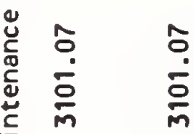

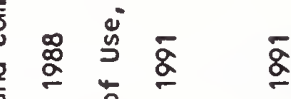

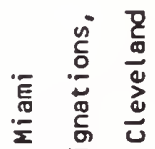

空

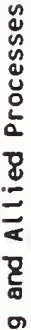

- \&

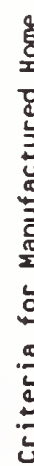

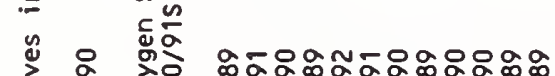

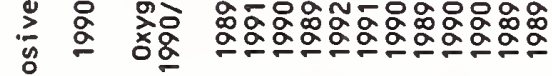

这, 茅品

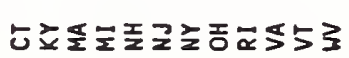

它 $\stackrel{\circ}{\varrho}$

$\stackrel{\infty}{\leftrightarrow}$ ถ

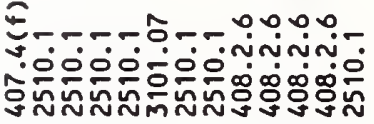

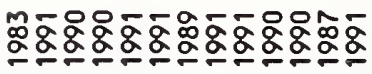

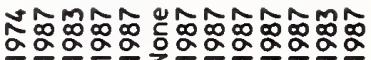

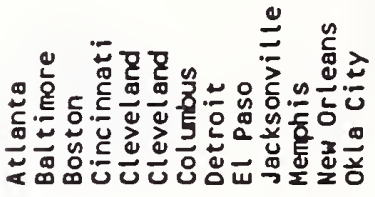

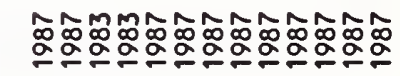




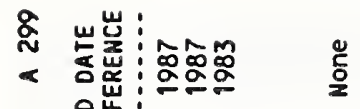

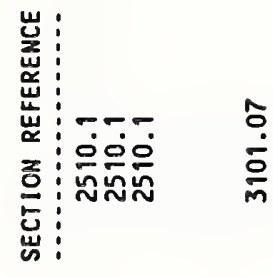

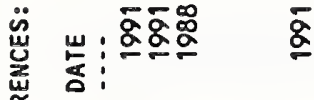

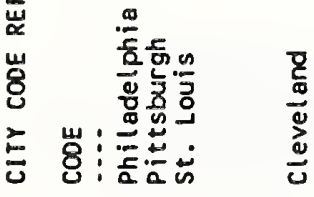

嵌

号岕:

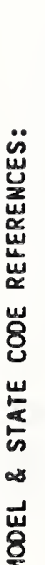

嵌 $\quad \frac{\stackrel{5}{5}}{a}$

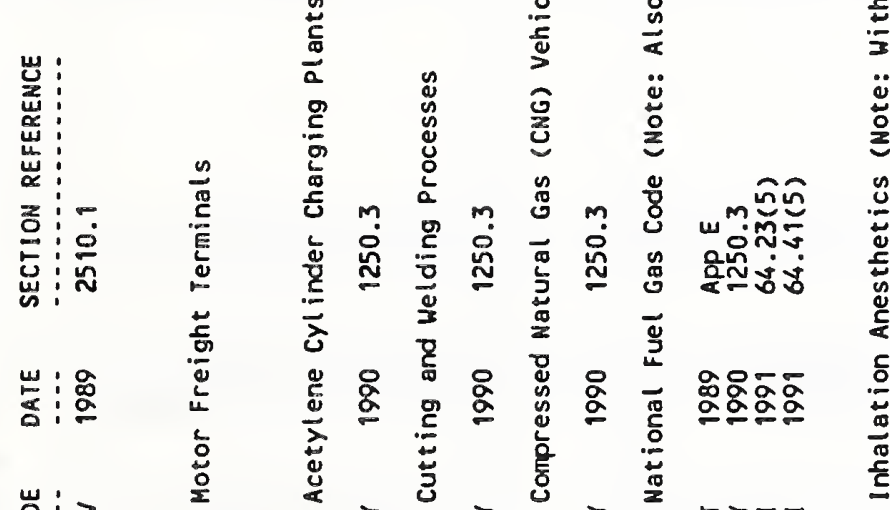

岁:

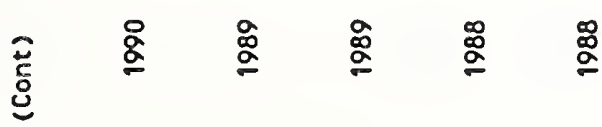

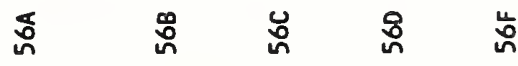

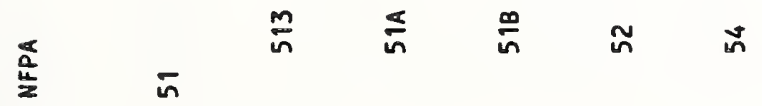

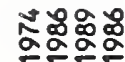

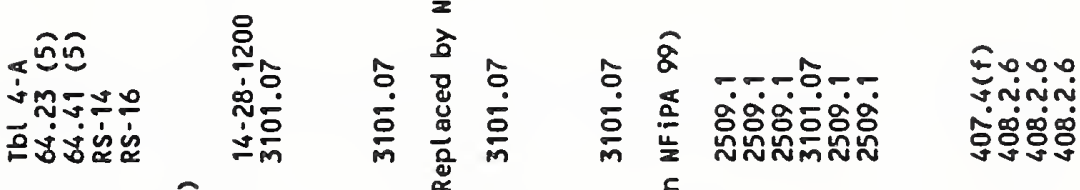

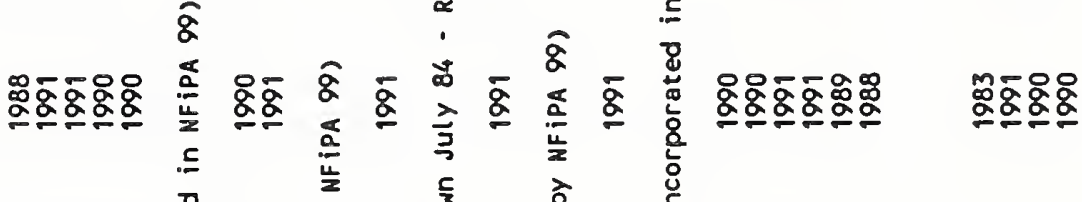

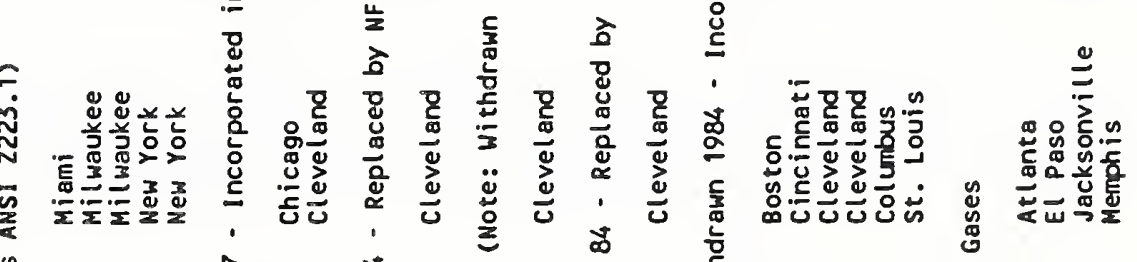

む)

这

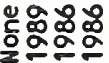

๗๕ฒฒ

要

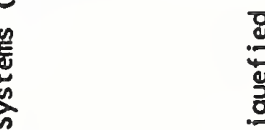

$\frac{\frac{0}{3}}{\frac{\sigma}{2}}$

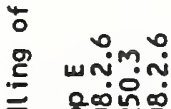

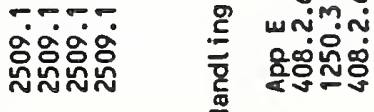

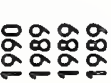

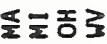

낭으를 


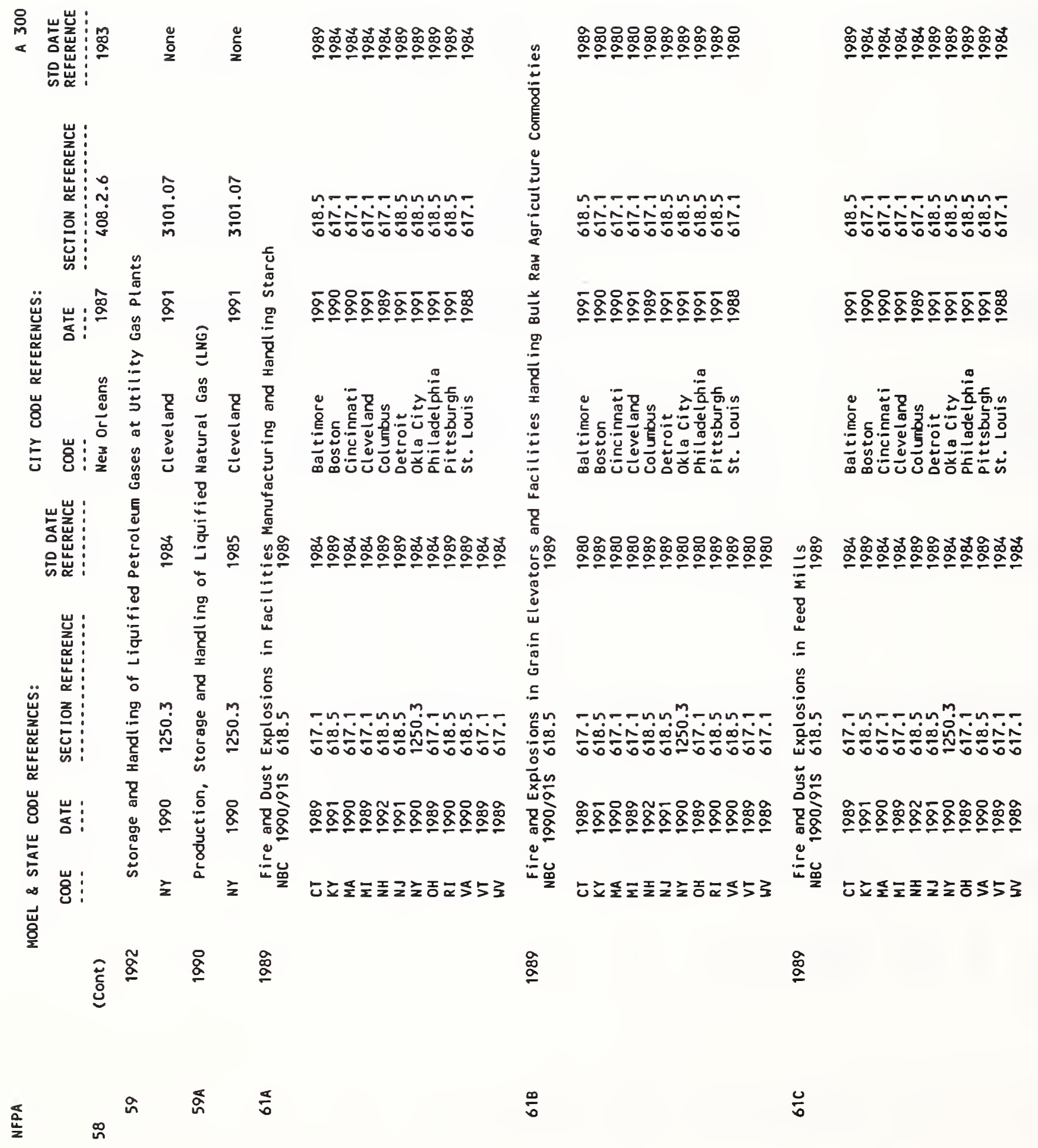




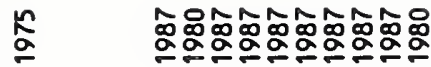

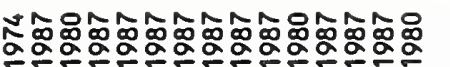

$\subseteq$
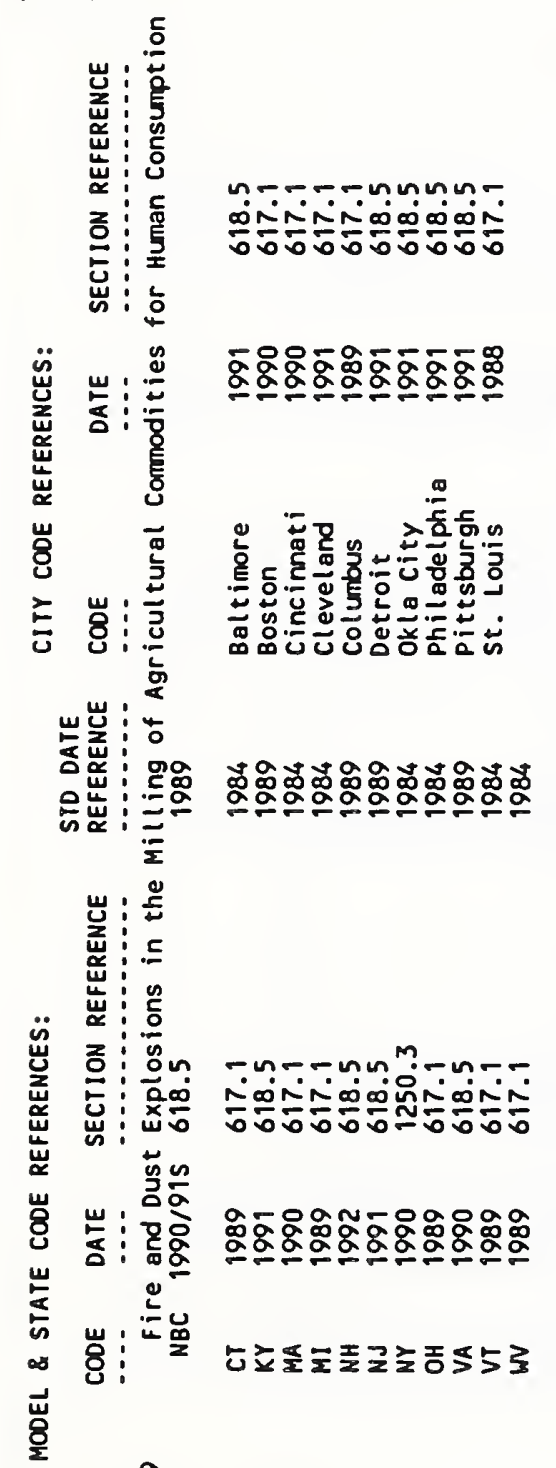

范

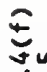

0.00

Gn-F--nninininun-

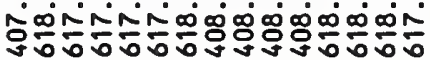

ลำำ

츠ํํำ

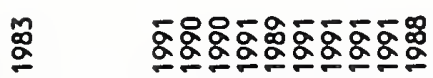

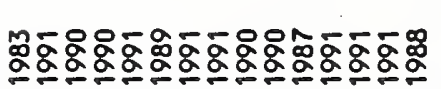

응ㅎㅀㅇㅡ

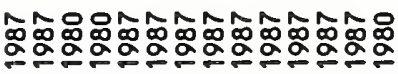

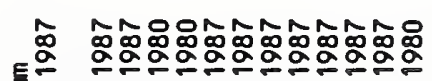

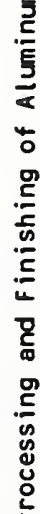

$\stackrel{\circ}{\alpha}$

ฒ̊

ฒิ

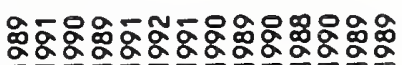

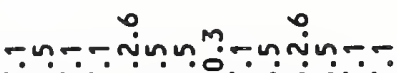

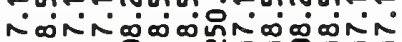

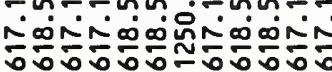

$5555 \% 55 \div 5 \div 8555$

$\operatorname{inin}$

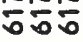

ติ

눔

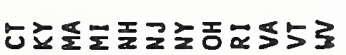

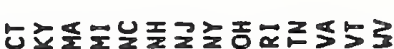

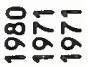

영영<smiles>CC(C)(C)C1CCCCC1</smiles> 
0000

n-tr-uninininimunt

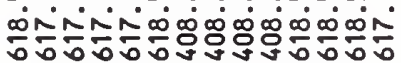

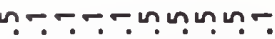
$\frac{\infty}{0} \frac{\infty}{0} \frac{0}{0} \frac{0}{0} \frac{\infty}{0} \frac{\infty}{0} \frac{\infty}{0} \frac{0}{0}$

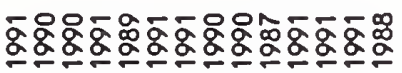

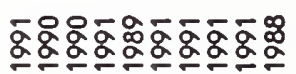

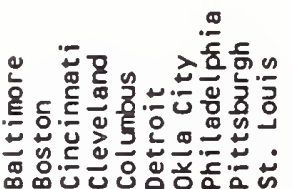

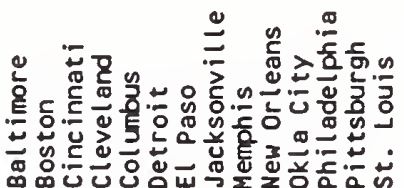

ㄴ:

:

岁

峟:

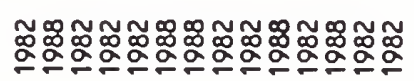

$\stackrel{\infty}{2}$

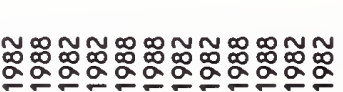

号

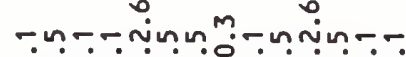

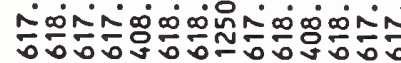

出:

드

แ. 등

岁 $\vdots \vdots$

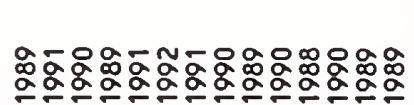

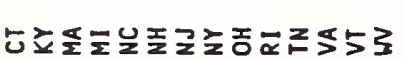

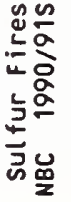

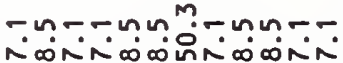

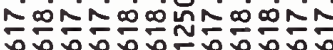

to

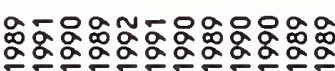

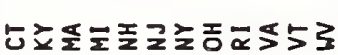

$\underset{\Xi}{\infty}$

ڤ્ळ

$\stackrel{\mathscr{B}}{\circ}$

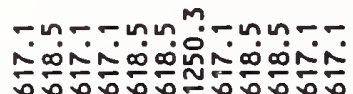

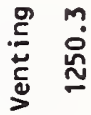

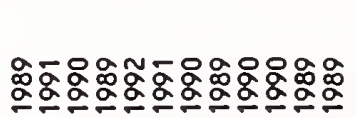

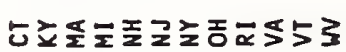

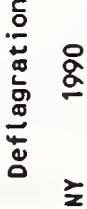

$\nwarrow$ 
畩

焉:

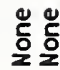

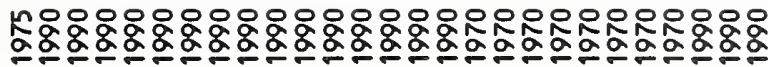

$\hat{a}$

$\dot{\circ} \frac{\infty}{m}$

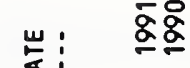

安:

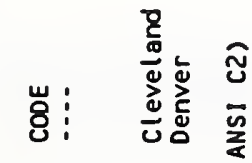

:

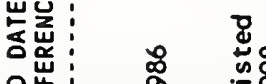

的岕:

岕:

总:

压

ơn?

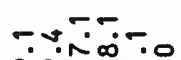

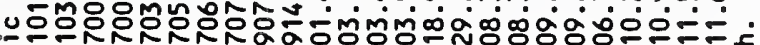

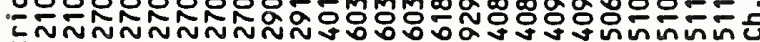

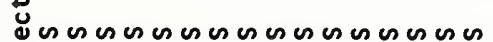

藏:

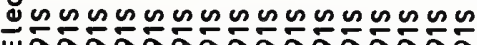

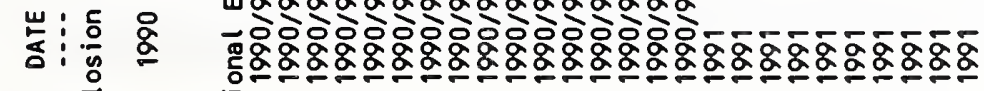

8

:

$i^{2} \Sigma$

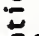

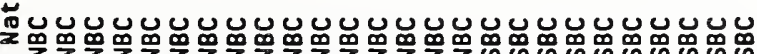

울

$\approx$

$\frac{a}{2}$

8

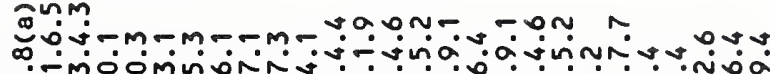

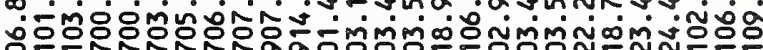
Иักลกลกลกลกล์

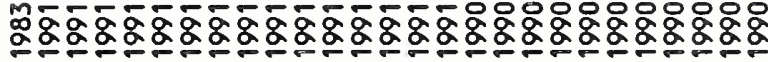

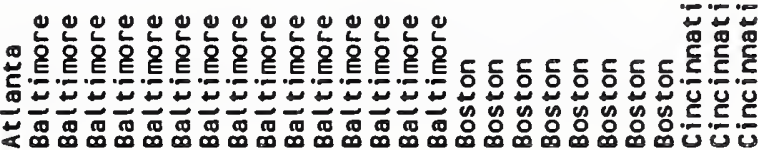

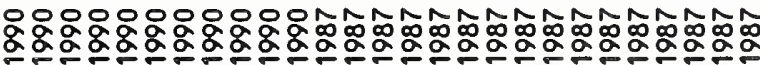

:

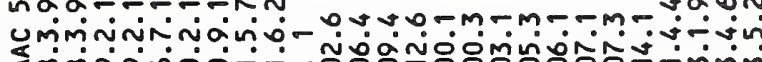

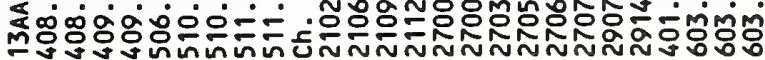

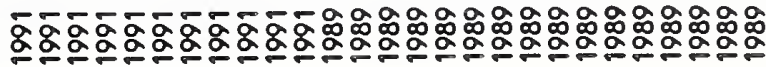

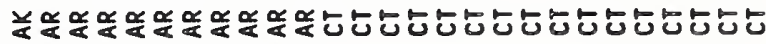

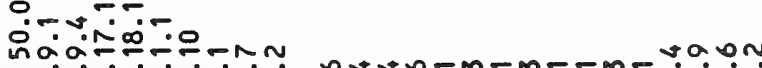


눙 定岩: 嵌:

ï

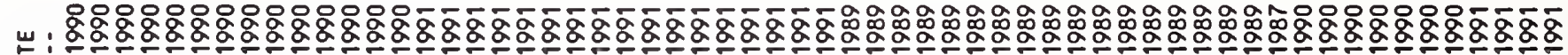
$\overleftarrow{8}$

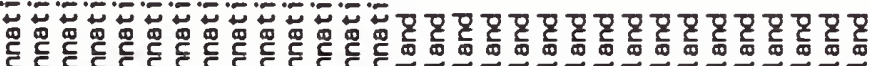

س.

$\because 0.0 \sim$

v.o.

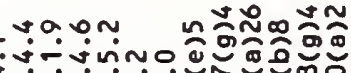

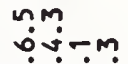

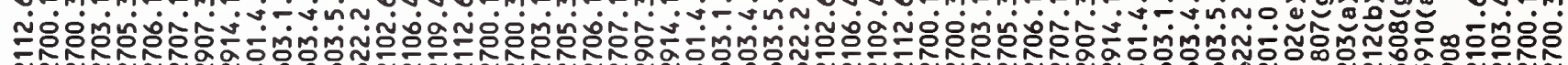

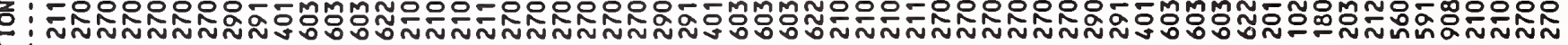

山्:

돈 은 $\frac{w}{4}:$

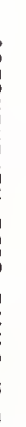

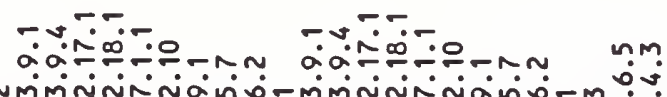

ล்ก-

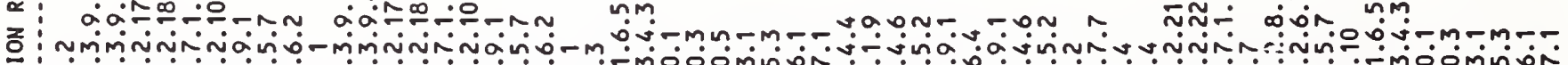

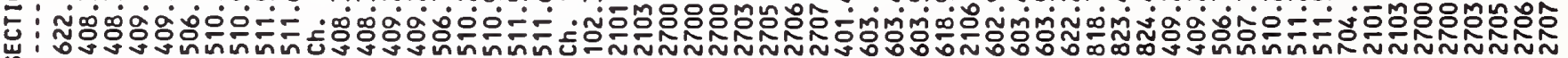
岕:

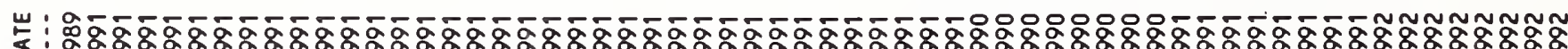

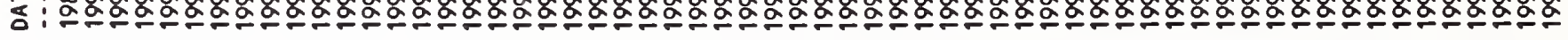

岁

옹

$\stackrel{\S}{\underline{4}}$

$\stackrel{P}{R}$ 
닌

范密 웅⿺ㅝㄴ

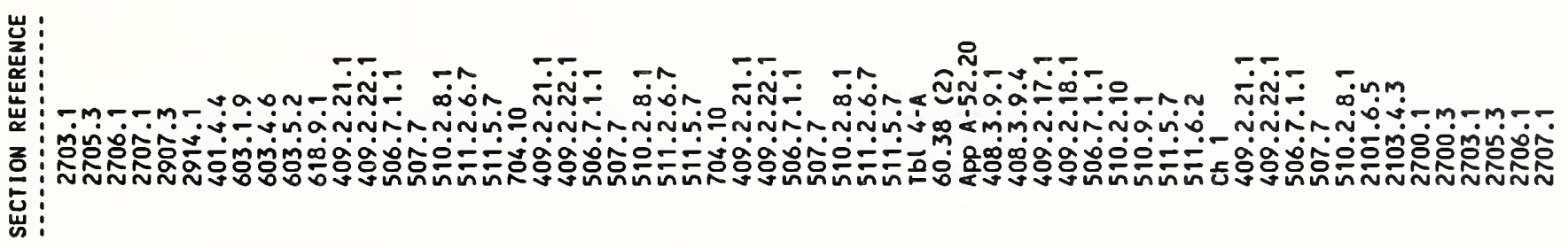

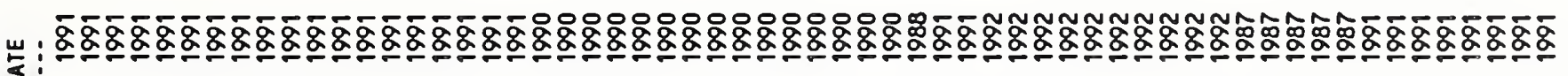
s:

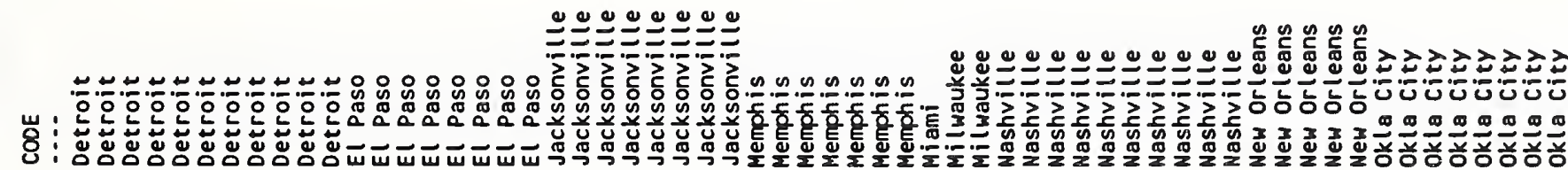

嵌岕:

它

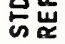

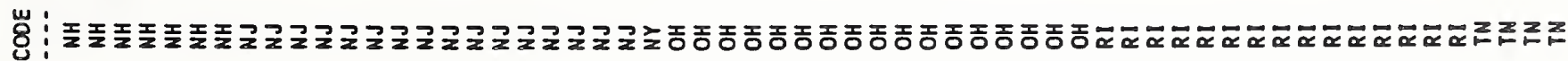


흘 蒫 î́m

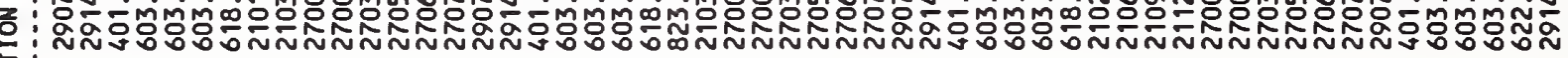
出:

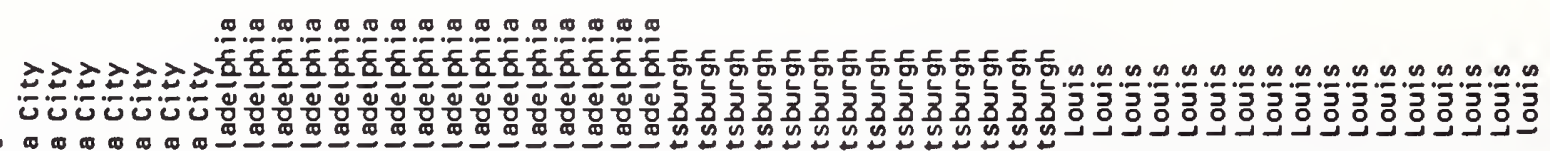

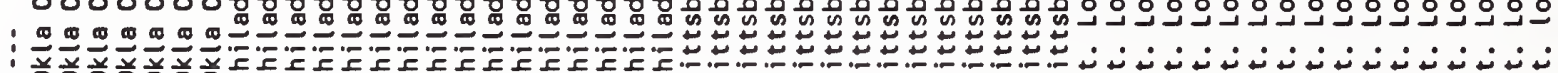

웡 不

는

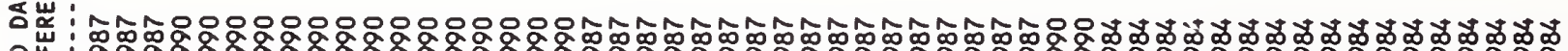

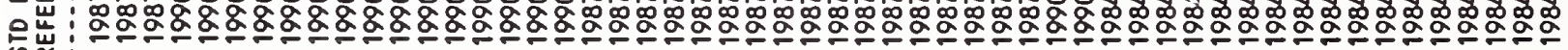

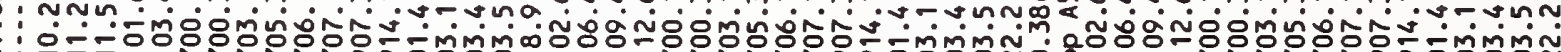

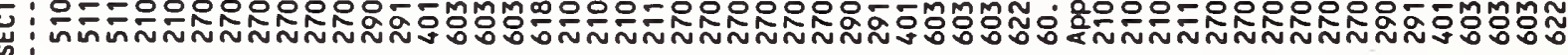

닌 : జ:

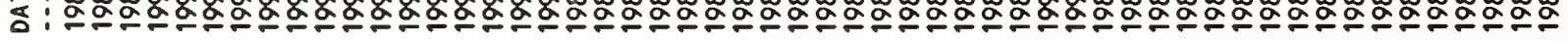

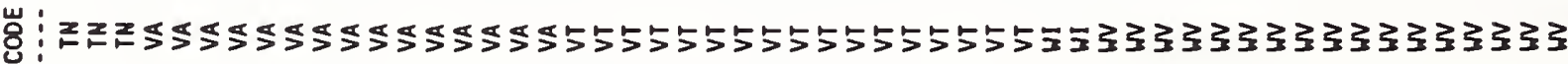




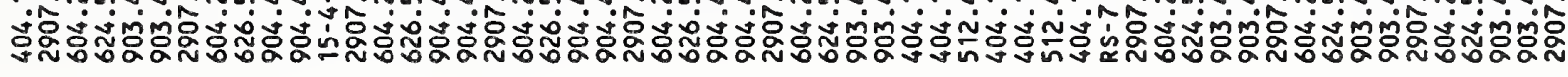

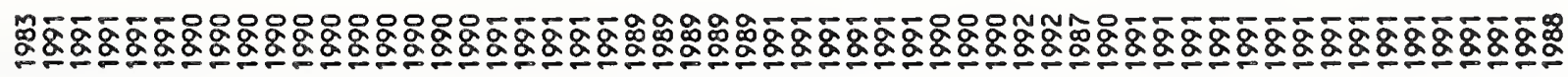

씰

:

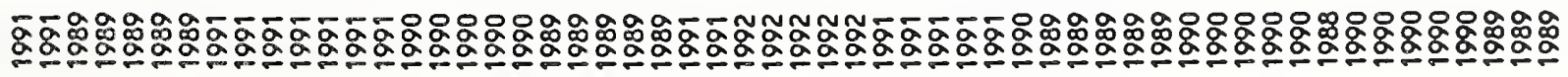

岁: 总总品

8

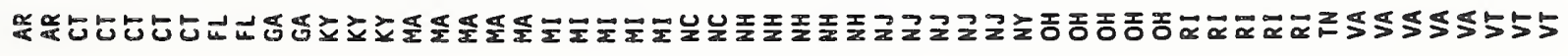

莟 


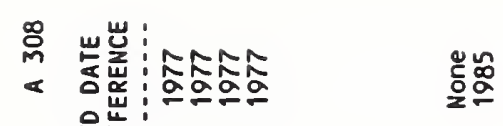
은

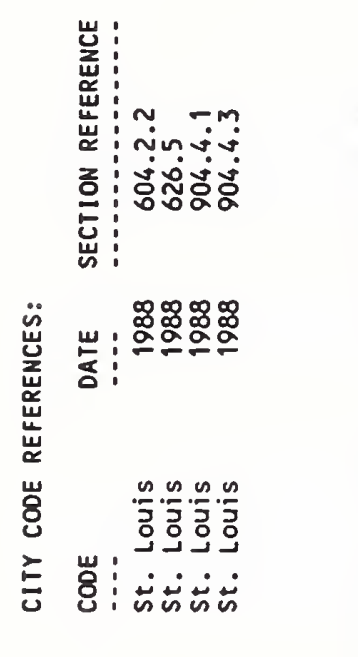

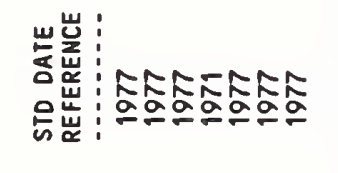

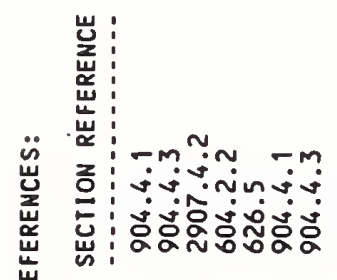

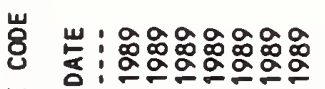

$8: 5533333$

巳ั

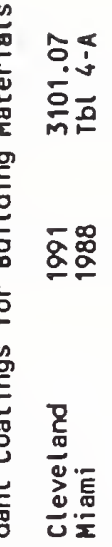

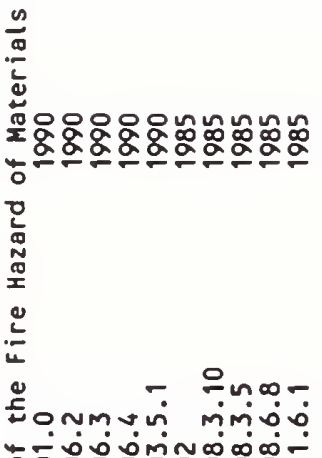

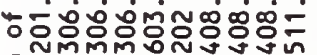
(5)

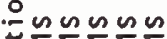

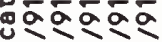

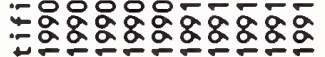

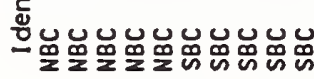

$\stackrel{0}{2}$

$\stackrel{m}{2}$

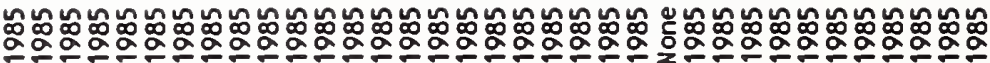

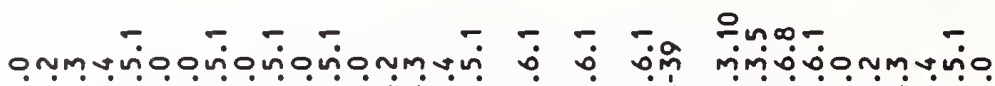

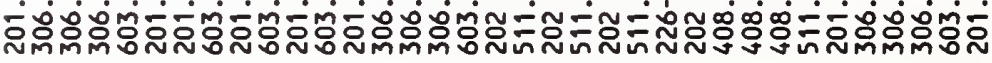

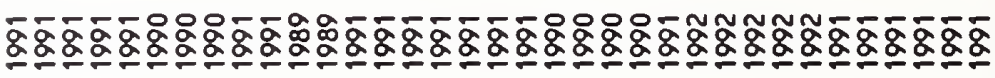

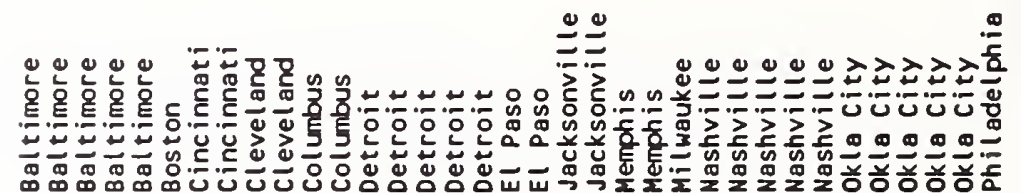

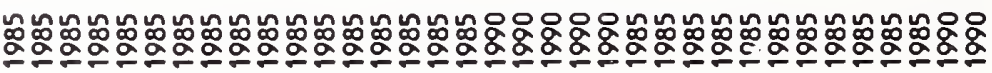

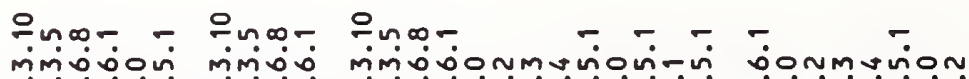

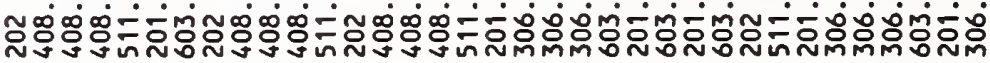

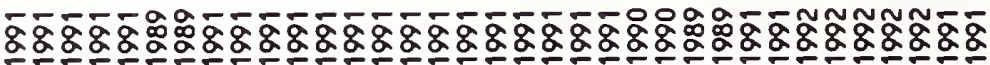

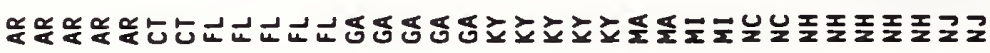


尾

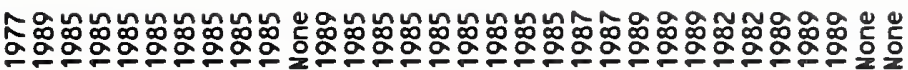

은현

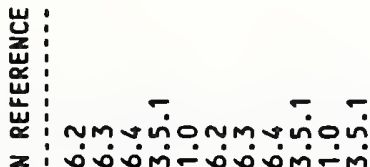

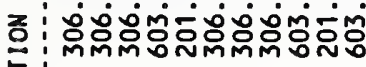

:

芯 岸

站

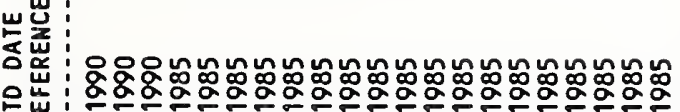

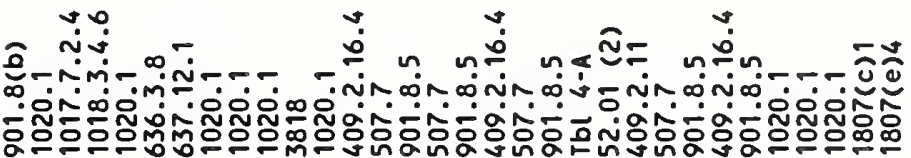

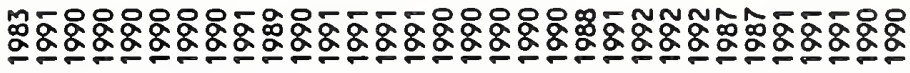

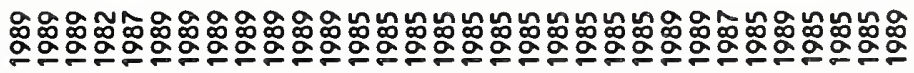

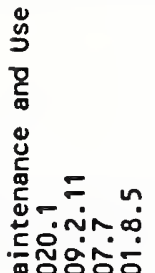

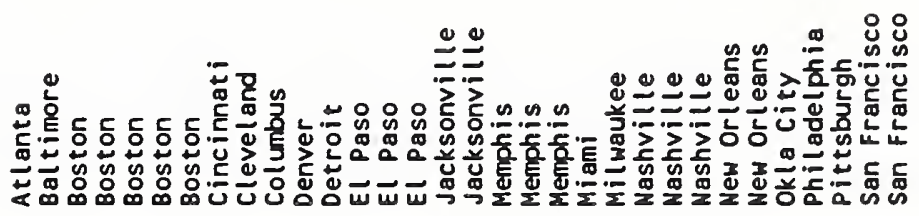

岂:

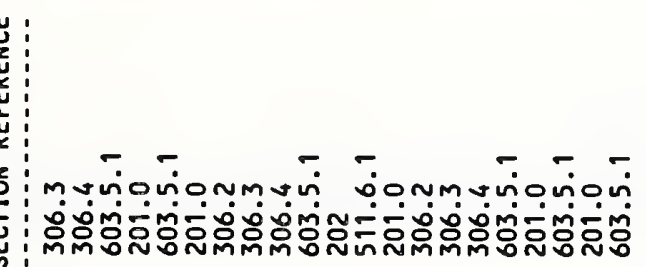

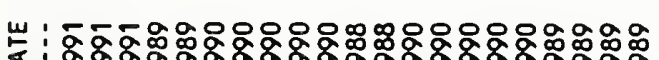

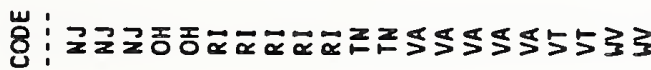
ธำ 


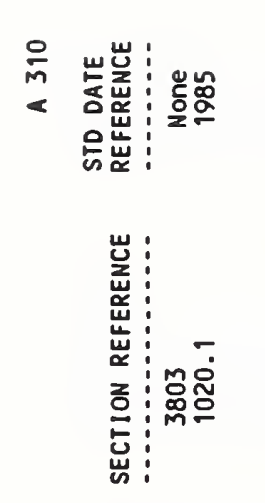

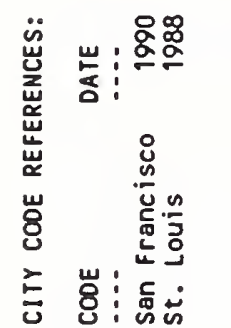

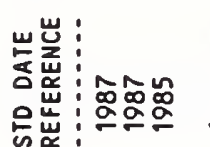

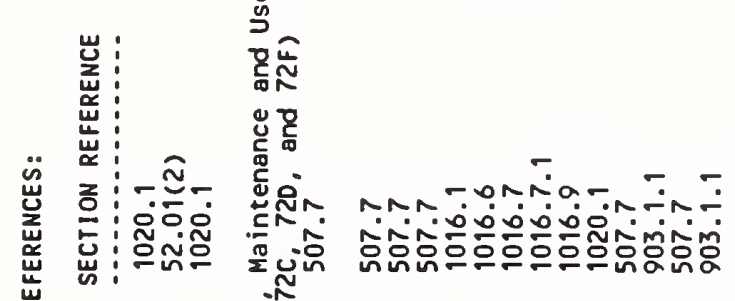

ठํ

峁

$\frac{5}{\infty}$

岁

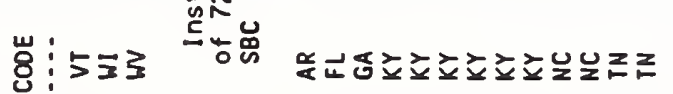

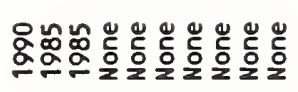

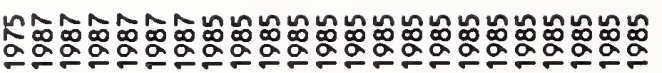

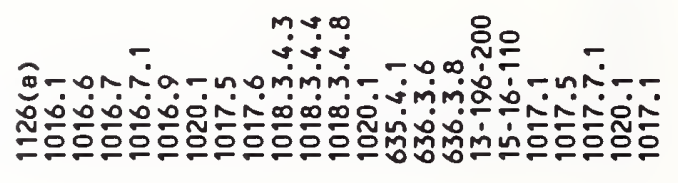

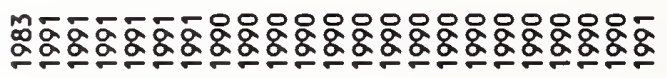

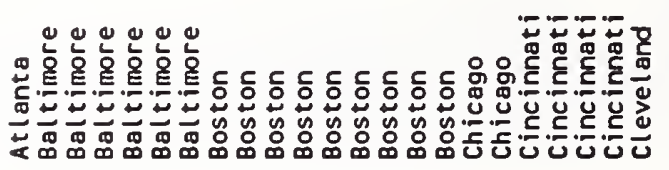

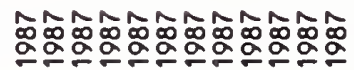

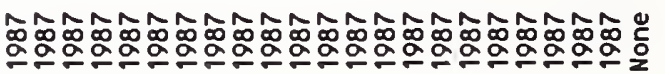

ơ

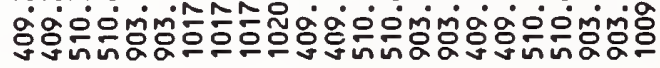

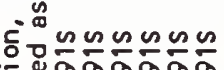

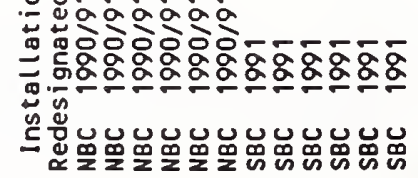

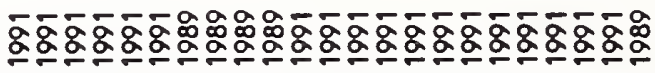

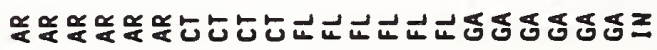

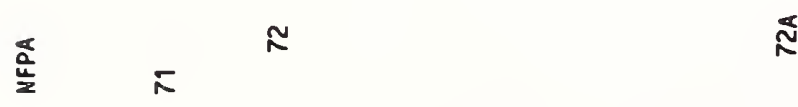




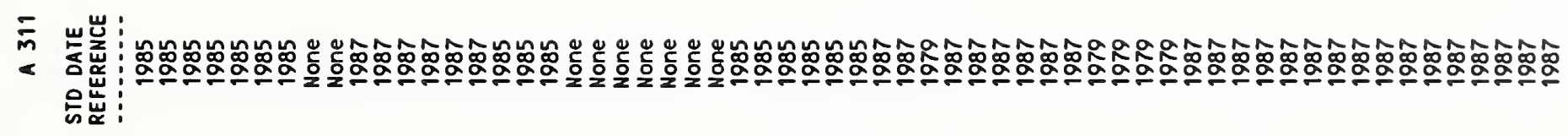

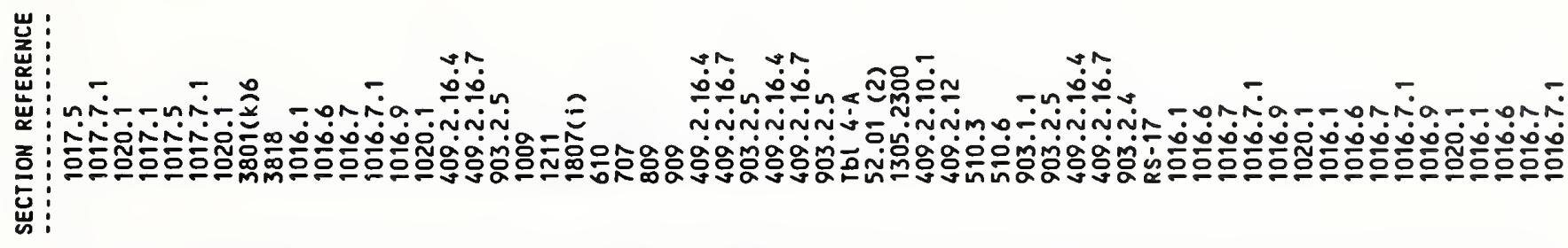

芦 :

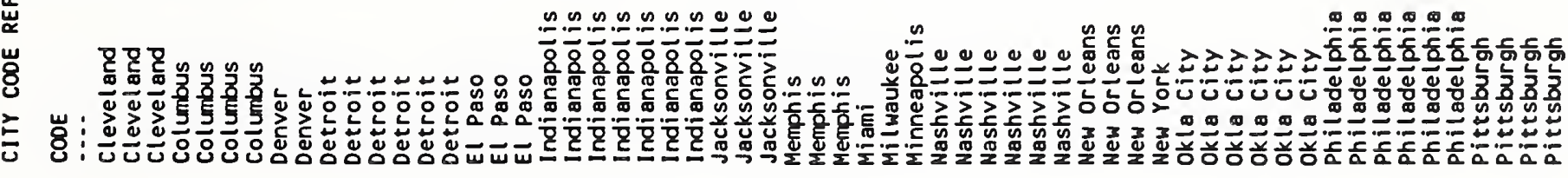

шبّ:

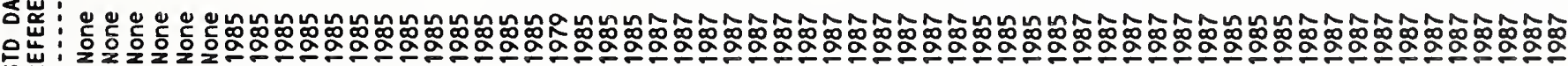
的:

岕:

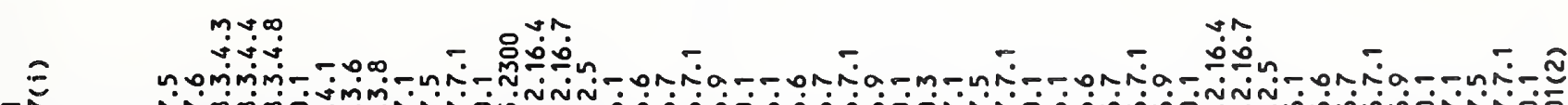

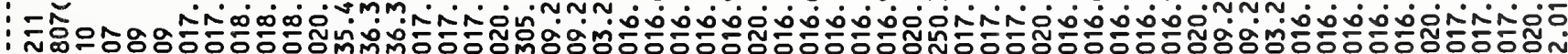

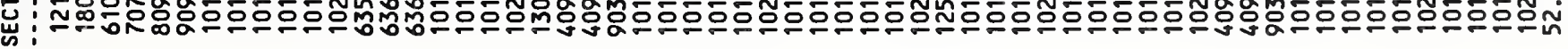

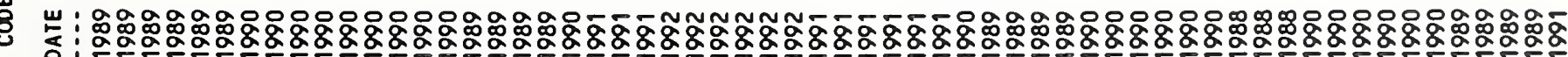

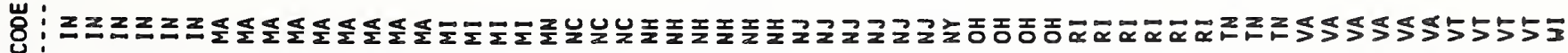

ڤ્ટ 


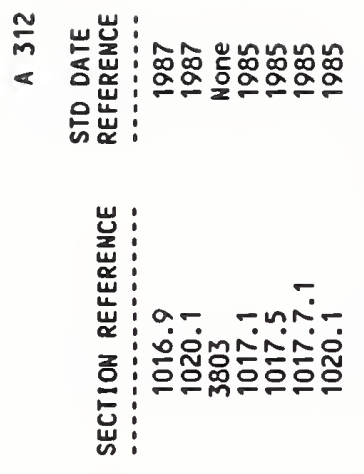

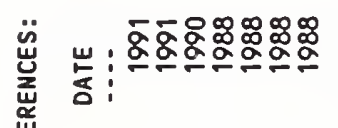

$\stackrel{\frac{\omega}{\alpha}}{\simeq}$

ํㅗㅇ

ㄴ.

ड 8 8:

崖:

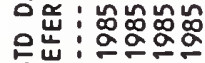

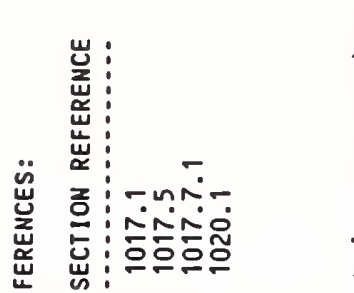

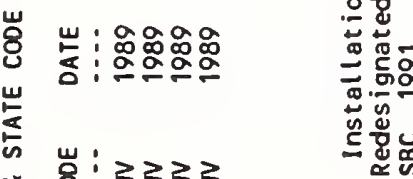

岁: 弦弦经

풍

ثิํํำ

起

$\stackrel{\mathbb{N}}{\sim}$

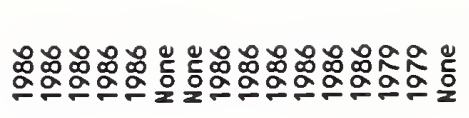

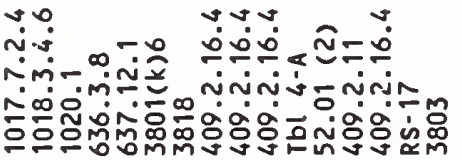

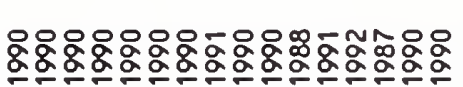

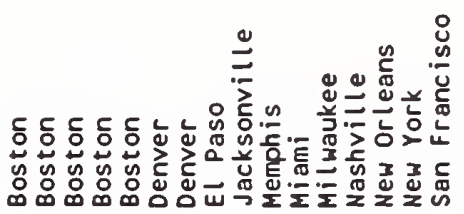

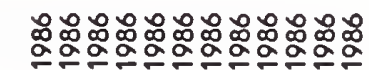

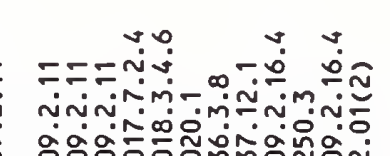

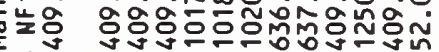

离

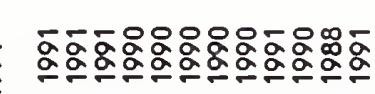

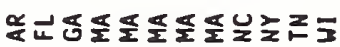

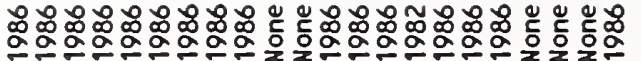

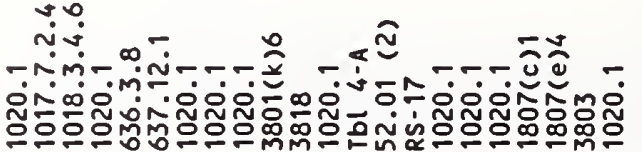

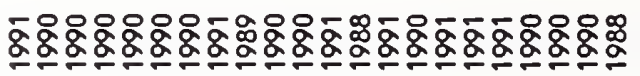

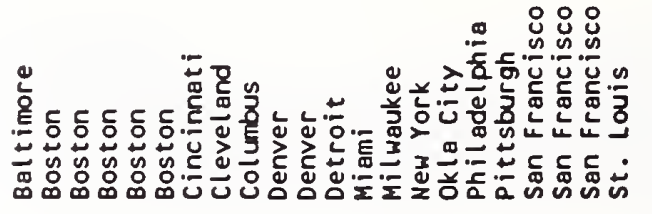

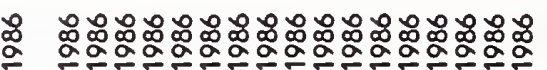

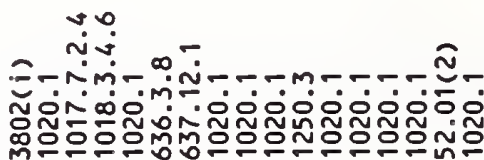

ci

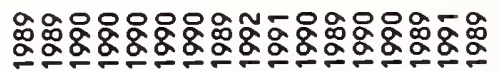

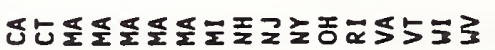

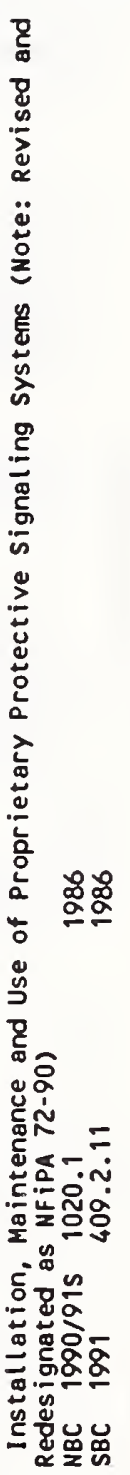




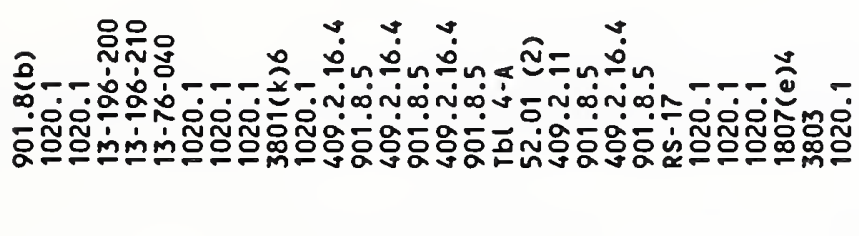

蒿 w岂 :

家:

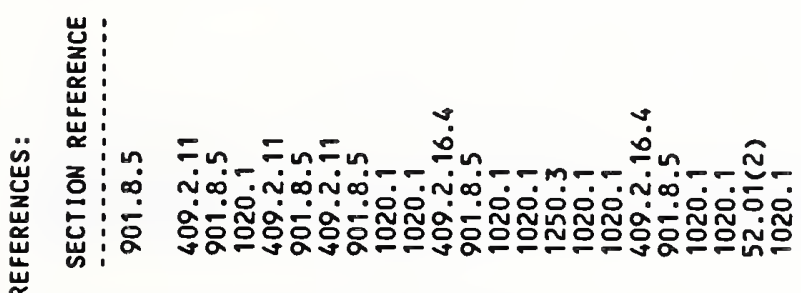

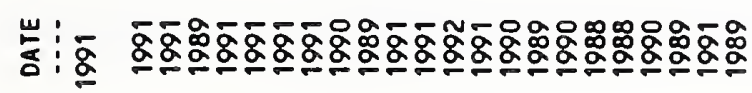

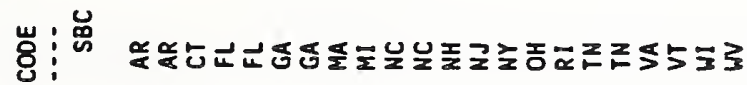

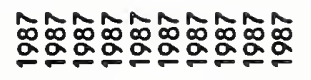

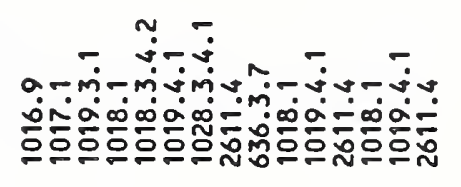

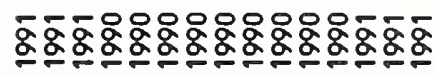

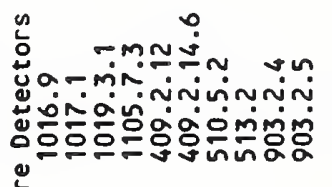
느는

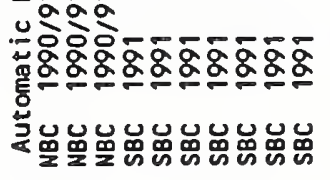

$\stackrel{20}{2}$

亗

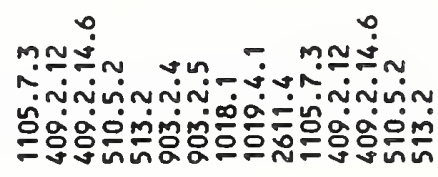

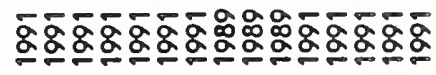

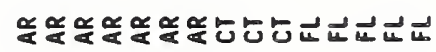




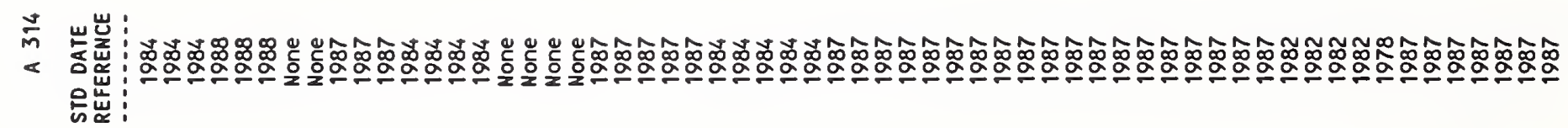

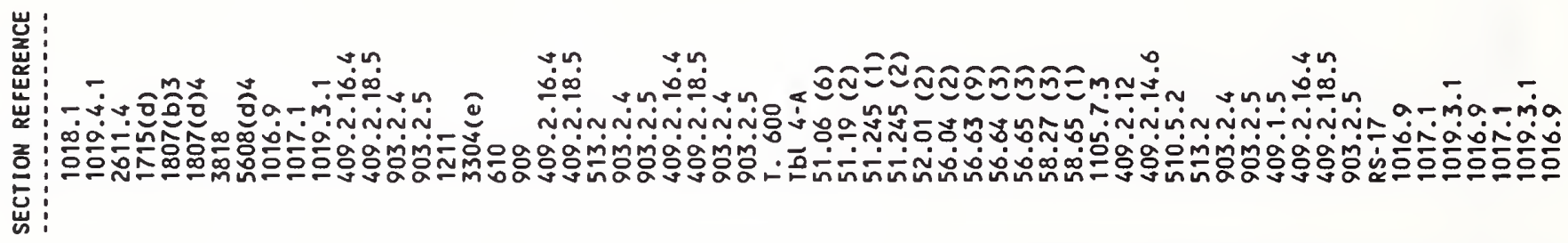
苮

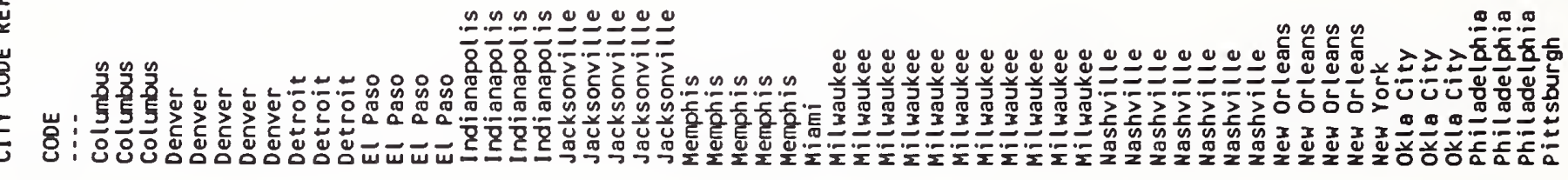

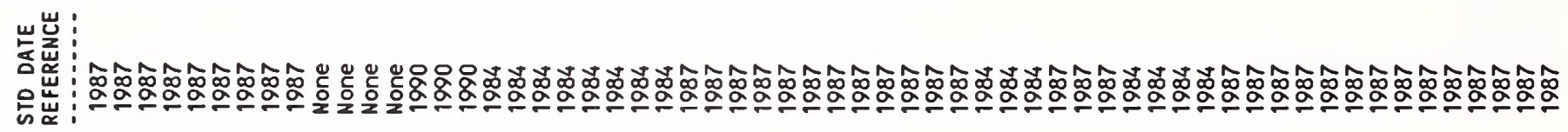

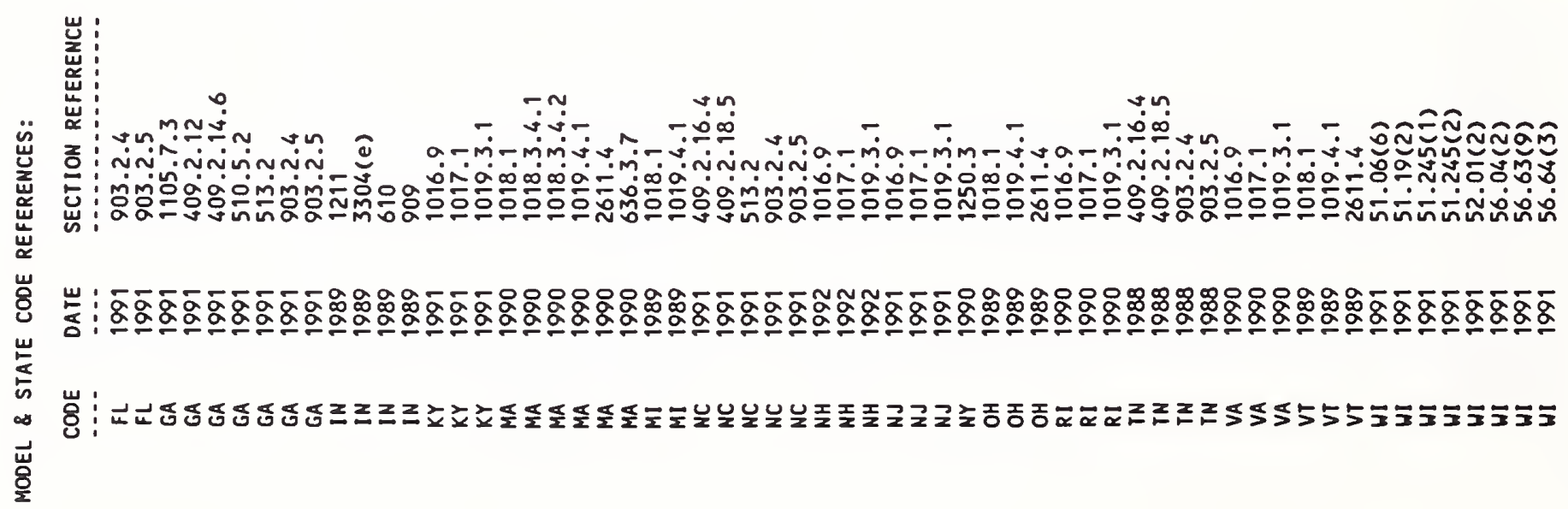
蒿 崖 


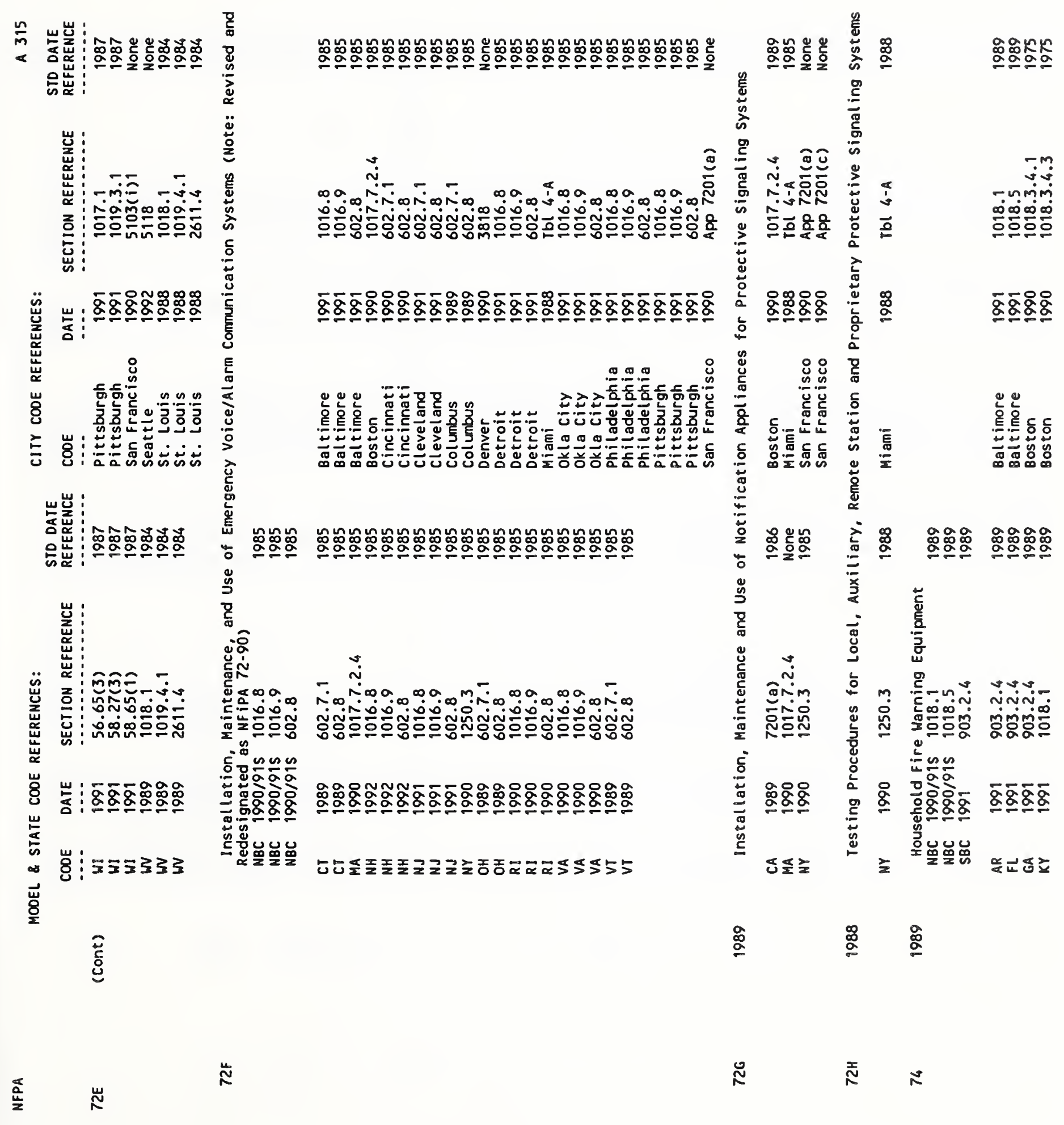


$\stackrel{\circ}{m}$

岕: 宫紊:

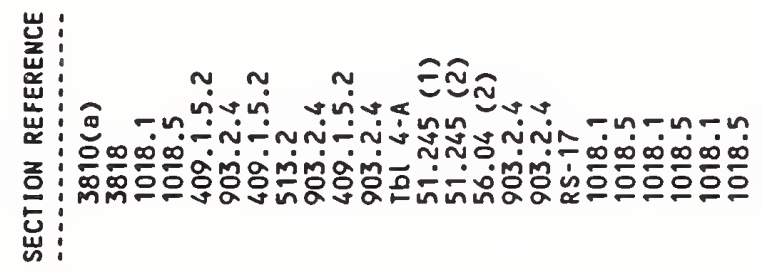

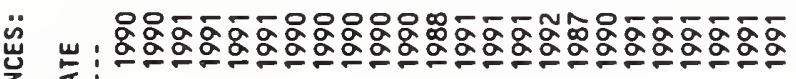
ธ。:

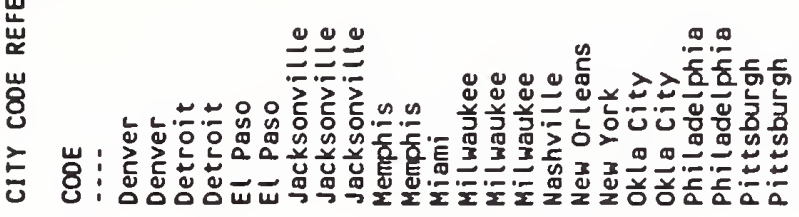

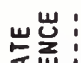

ธ。ّ

운

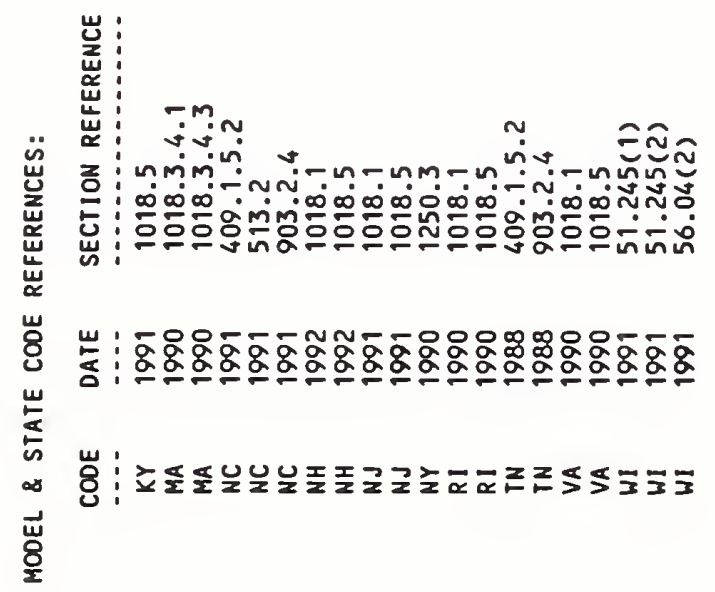

㝕

$\frac{a}{\frac{a}{2}}$

$\operatorname{s}$

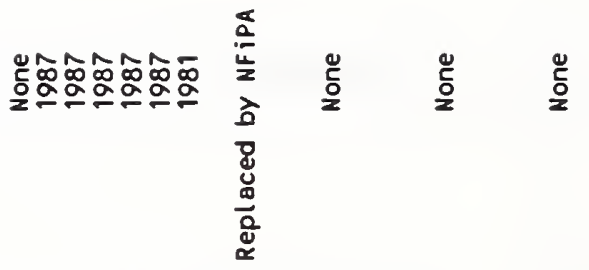

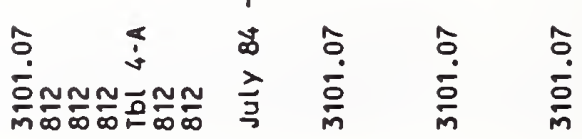

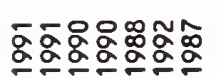

$\bar{\sigma} \quad \bar{\sigma} \quad \bar{\sigma}$

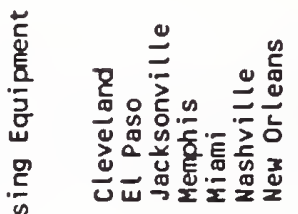

苟

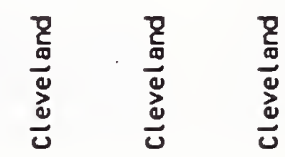

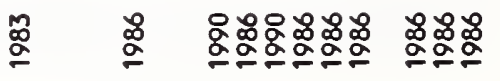

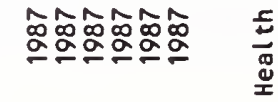

$\stackrel{5}{2}$

畩

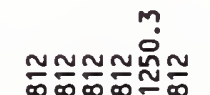

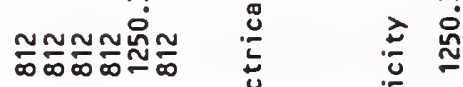

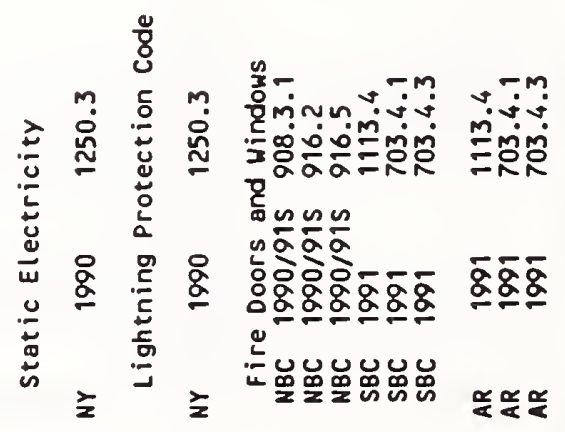

$\stackrel{a}{\stackrel{a}{\alpha}}$

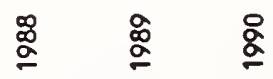

$\kappa$

న

^

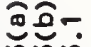

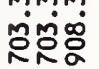

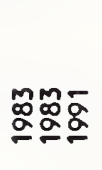

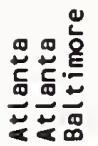

또용
ᄃ

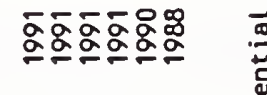

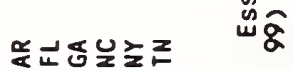

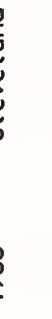


岕:

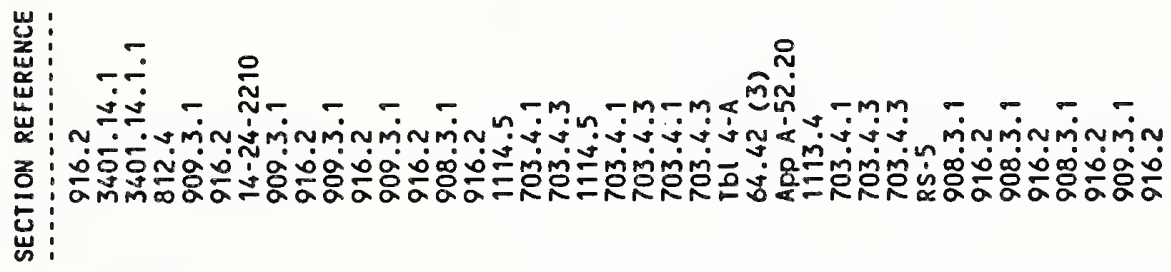

$\ddot{\text { ï }}$

空

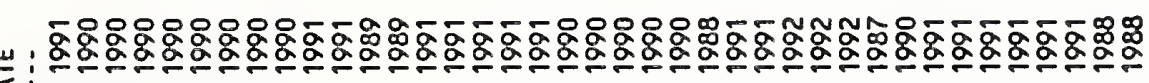
:

幽

ठ․
的岕

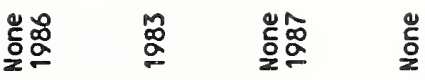

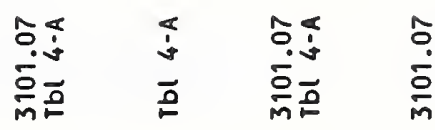

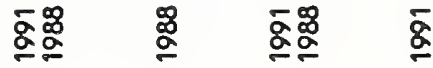

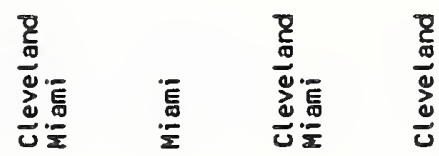

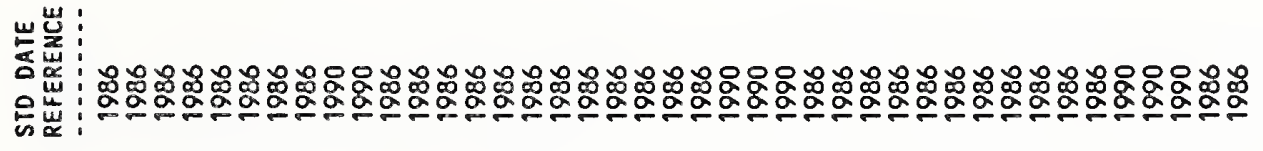

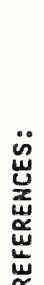

岁

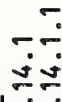

źn mo

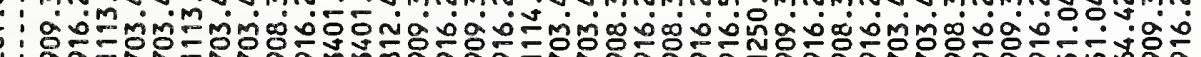

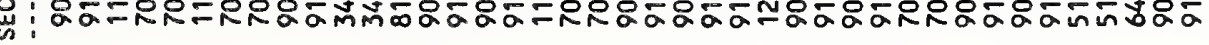

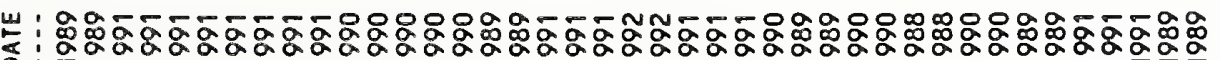

:

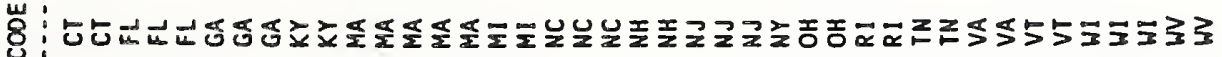

㟧

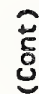

$\bar{g}$

$\bar{\infty}$

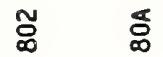

$\bar{\infty}$ 


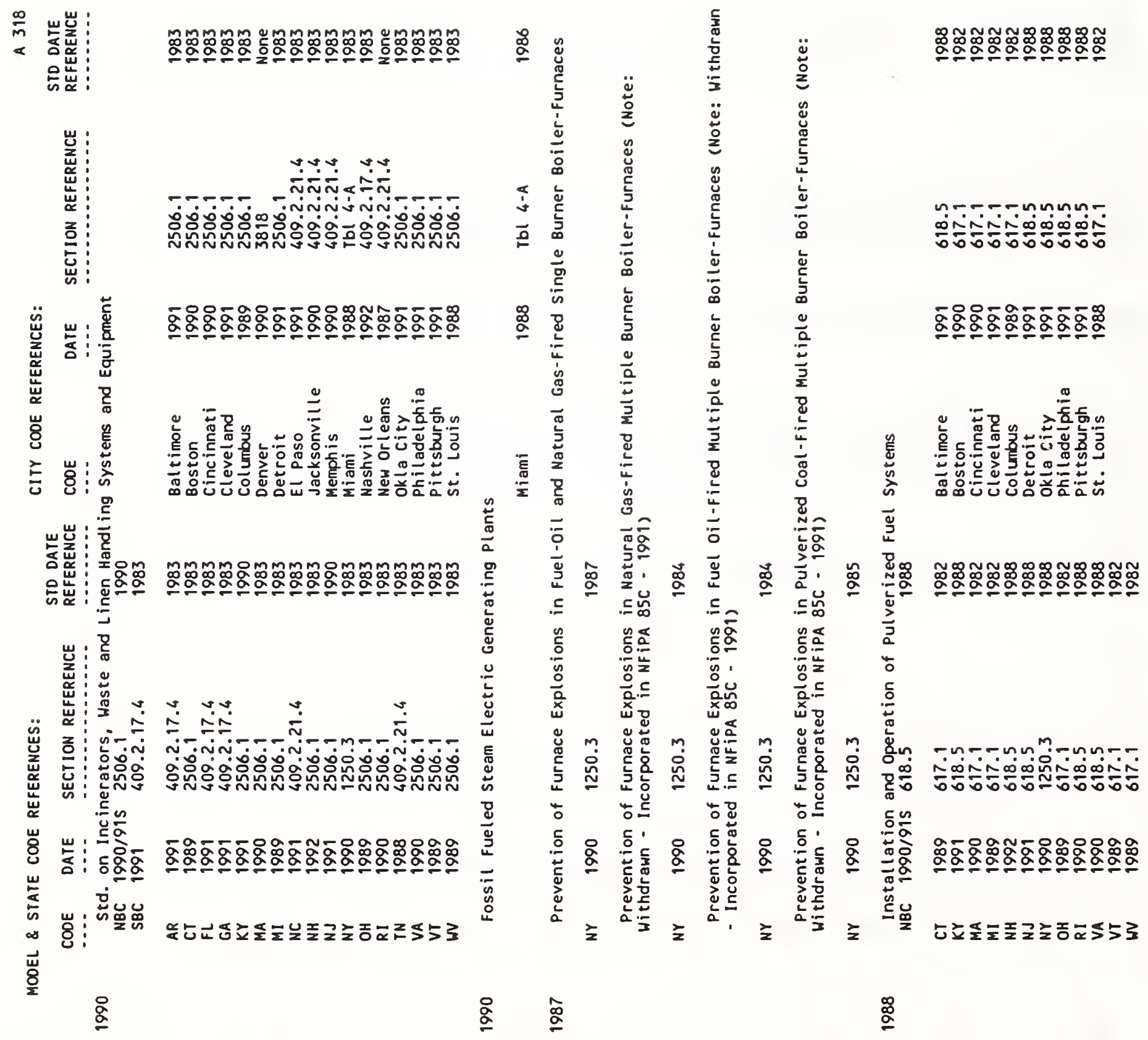




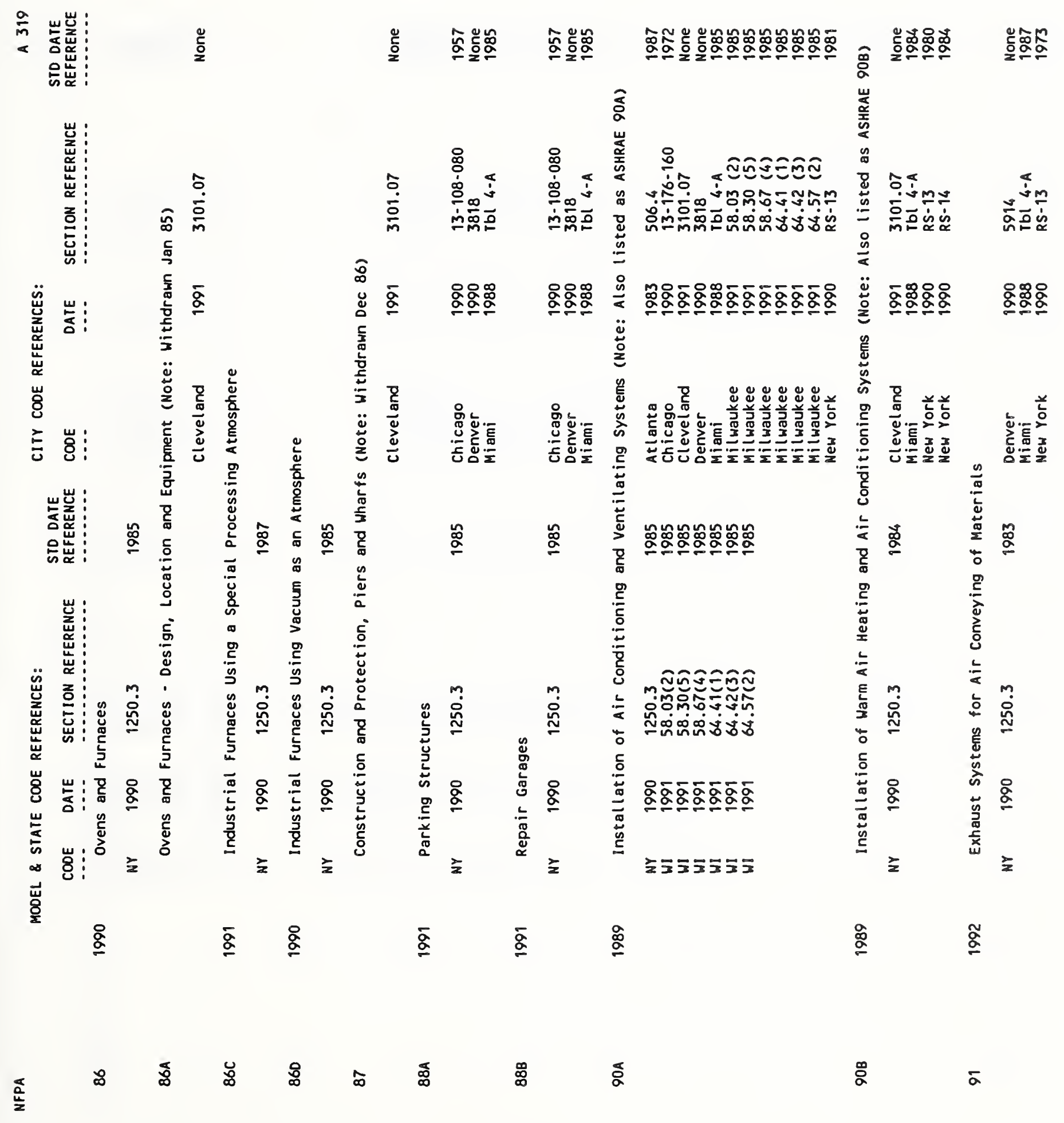




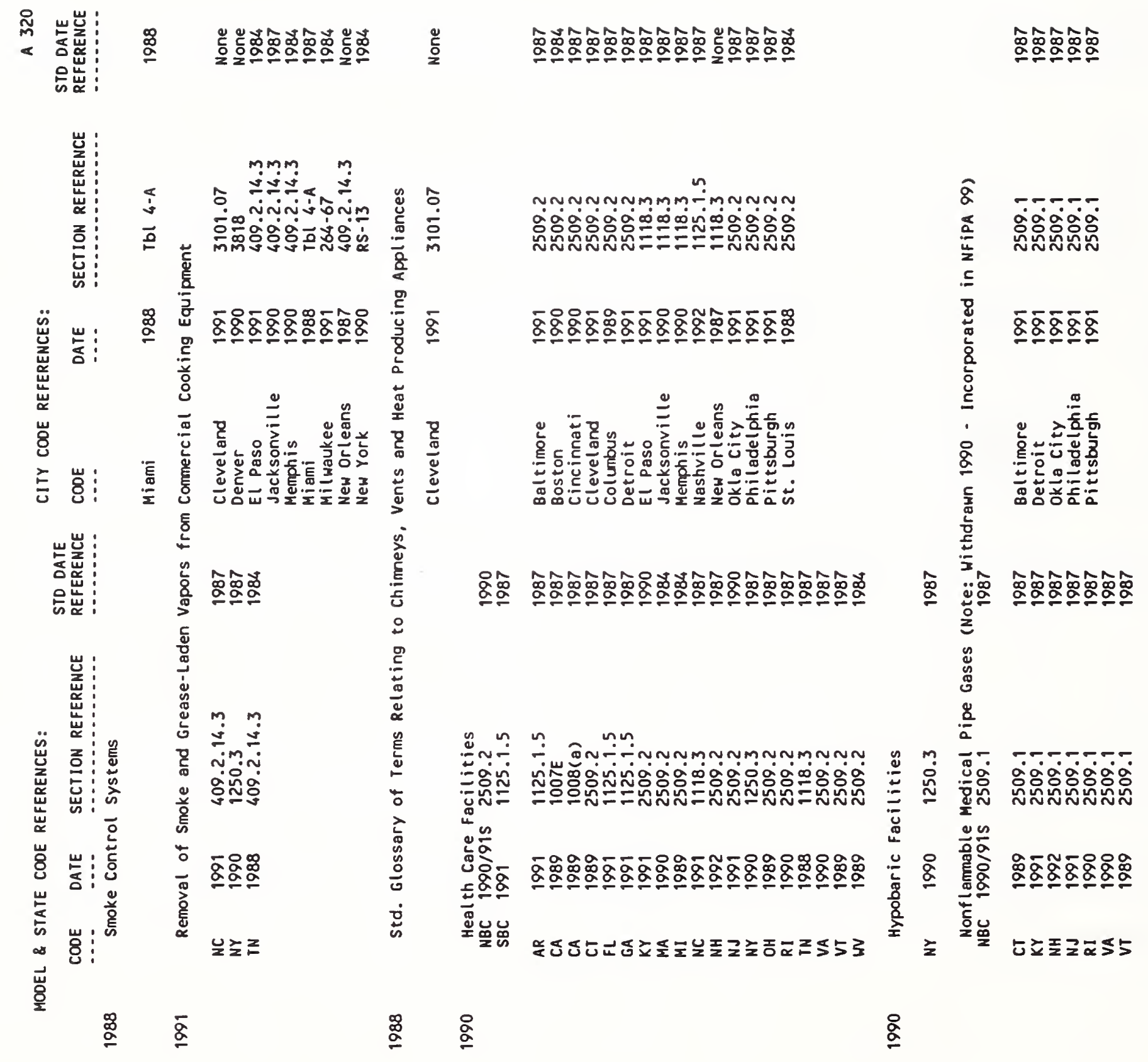

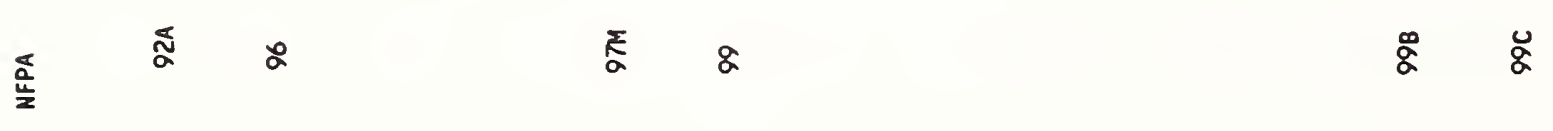




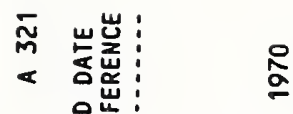

은

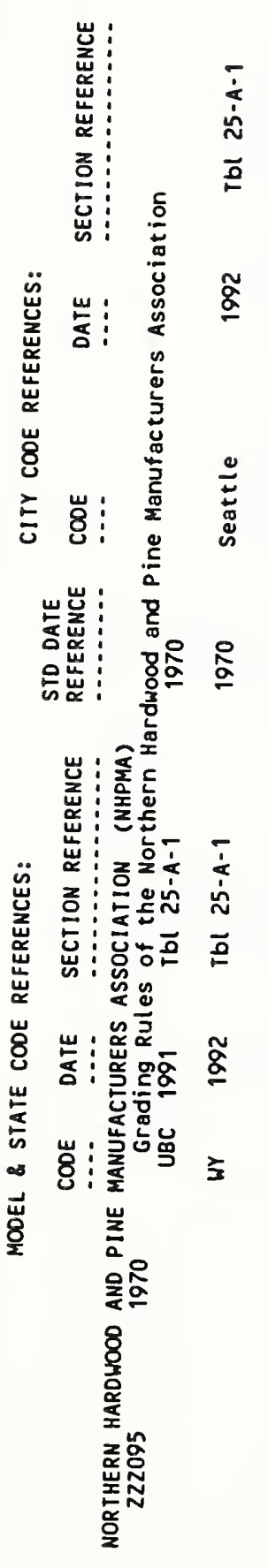




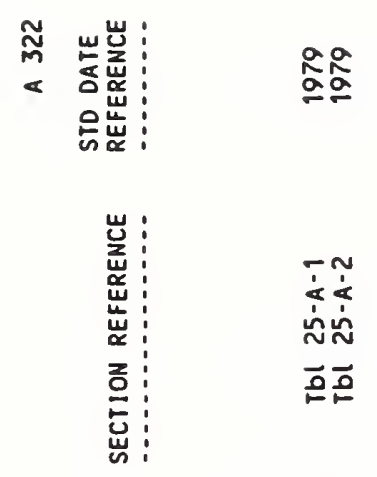

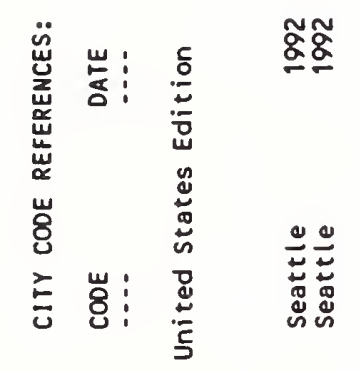

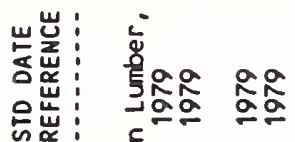

山 要

总

蛋: Lั0

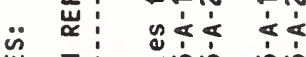

嵌

出: 옹문 음

ชั แ : ชิ์

u

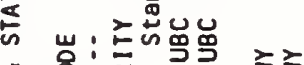

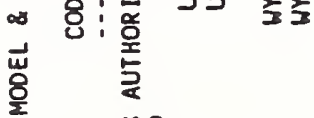

농ำ

通

嶧

芯部 
焦岕:

क㟔:

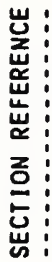

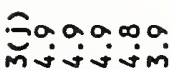

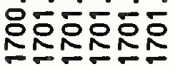

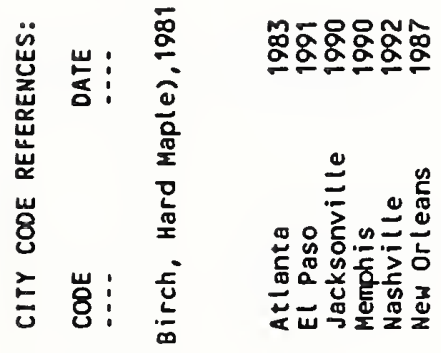

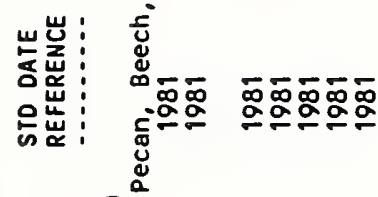

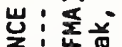

要:

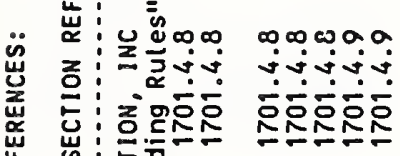

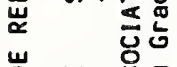

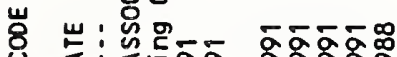

잉

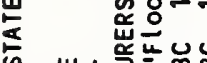

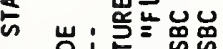

ه

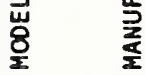

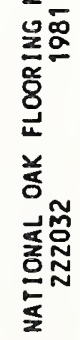

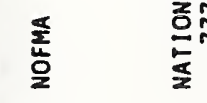




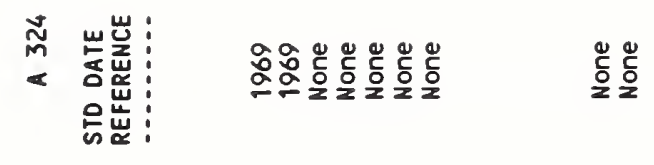

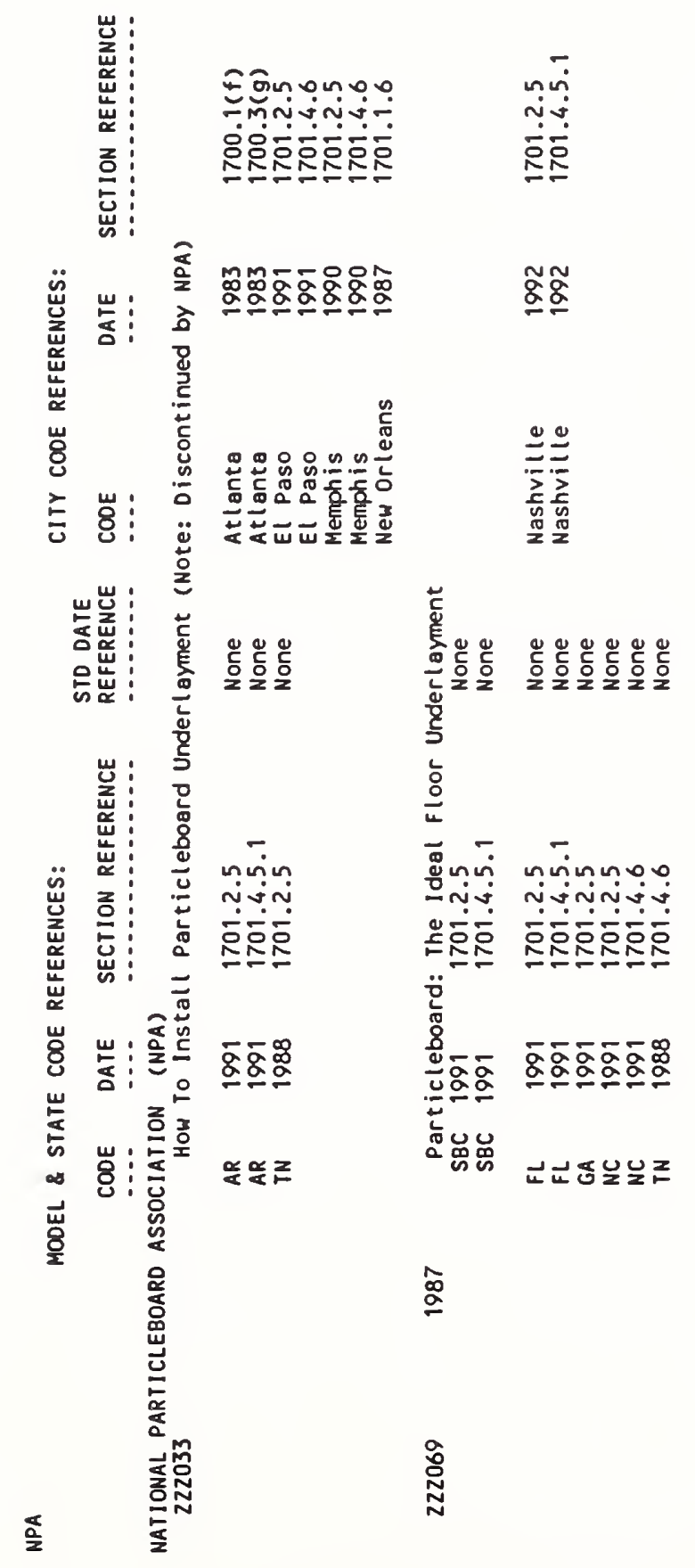




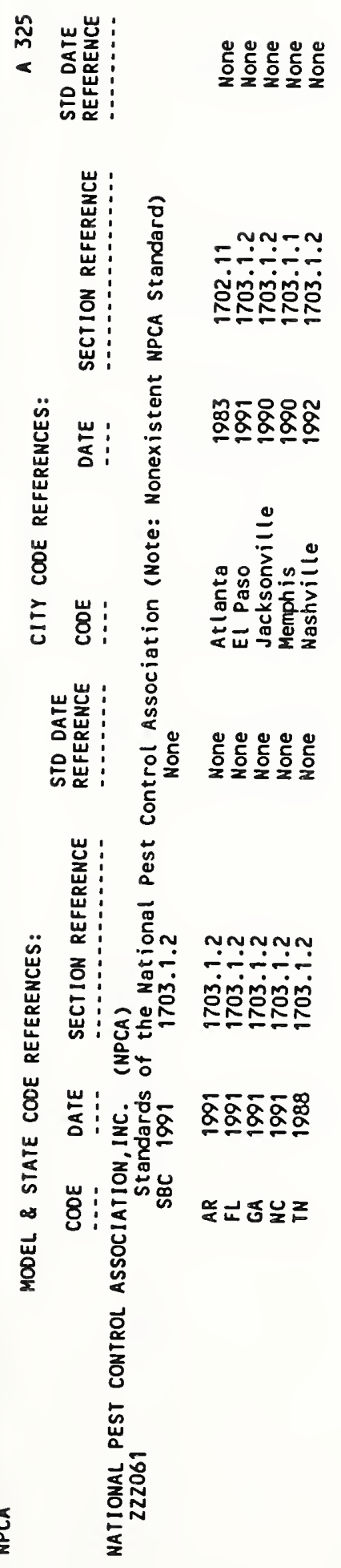




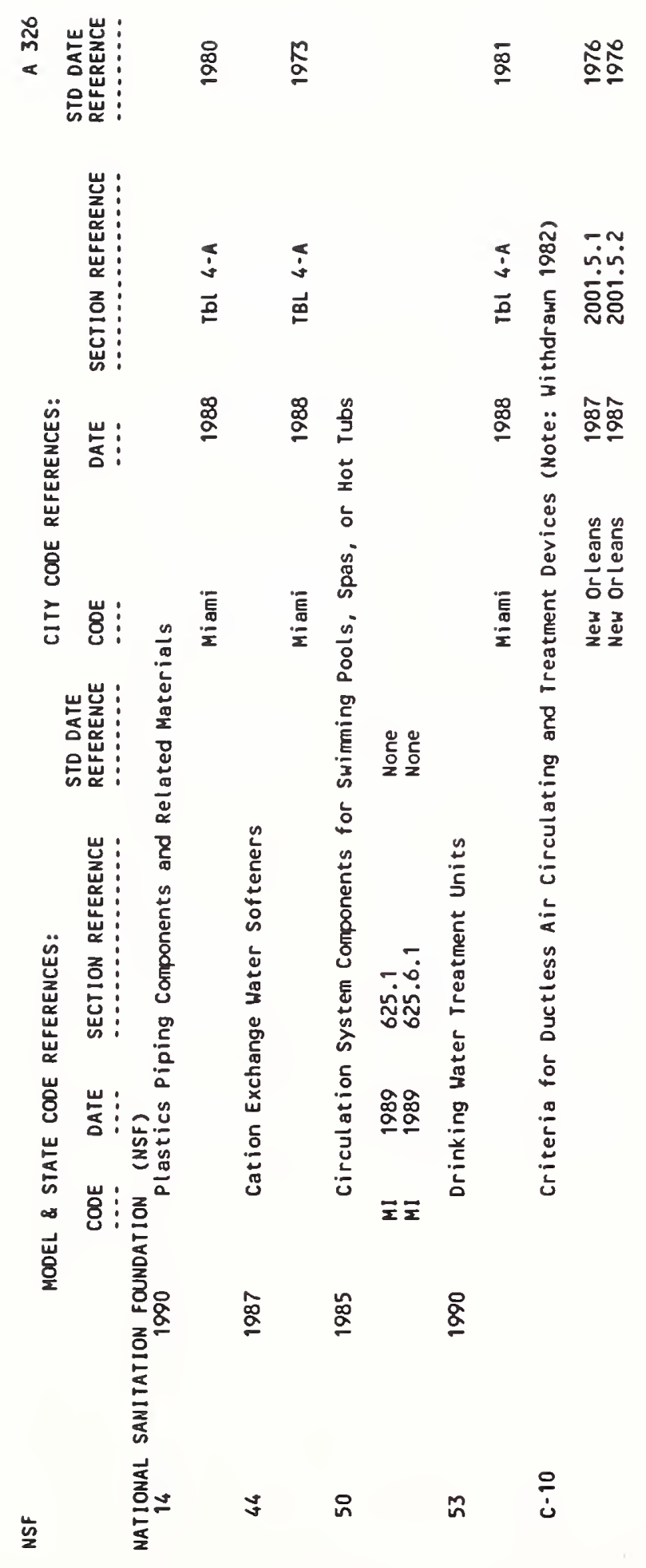




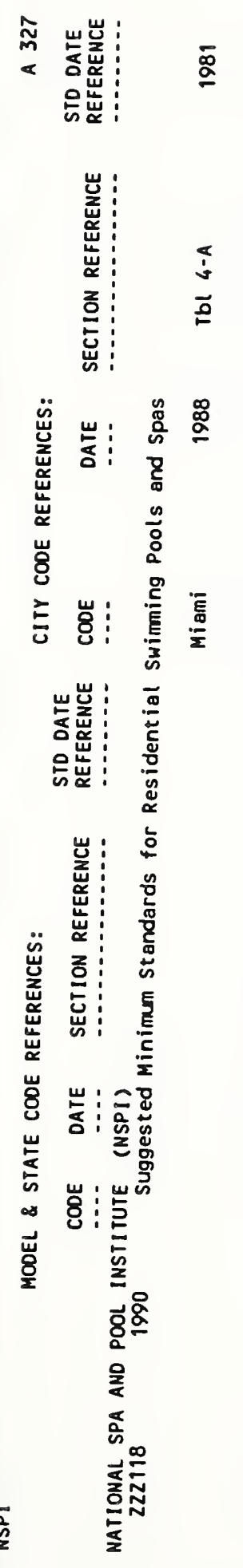




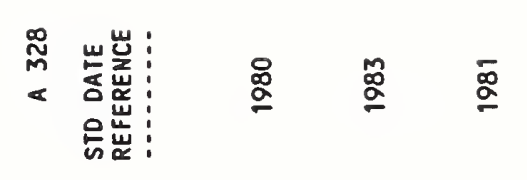

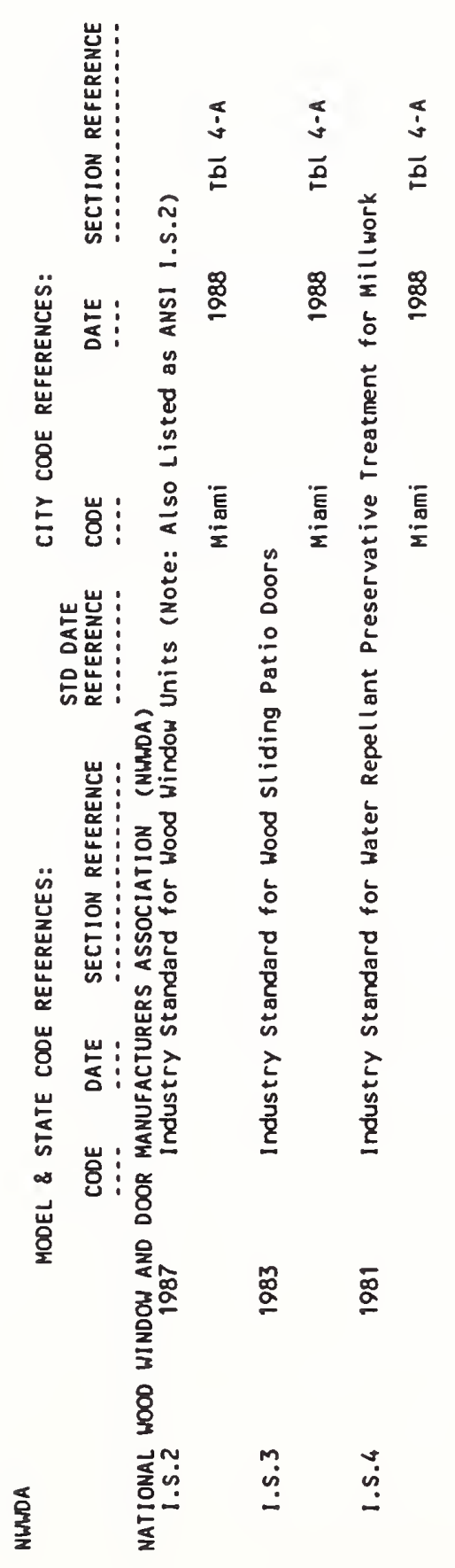




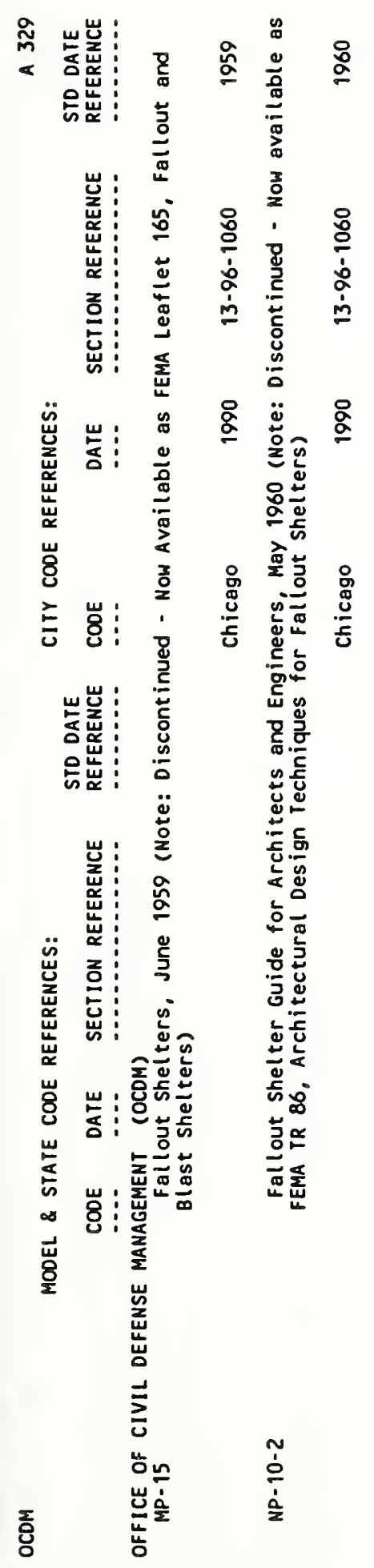




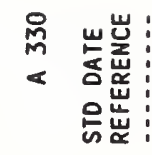

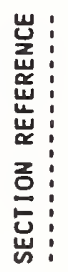

岕岕

岂

こ

닐

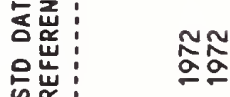

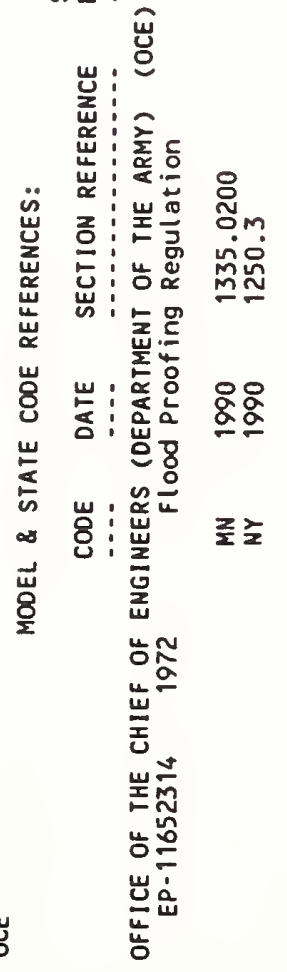




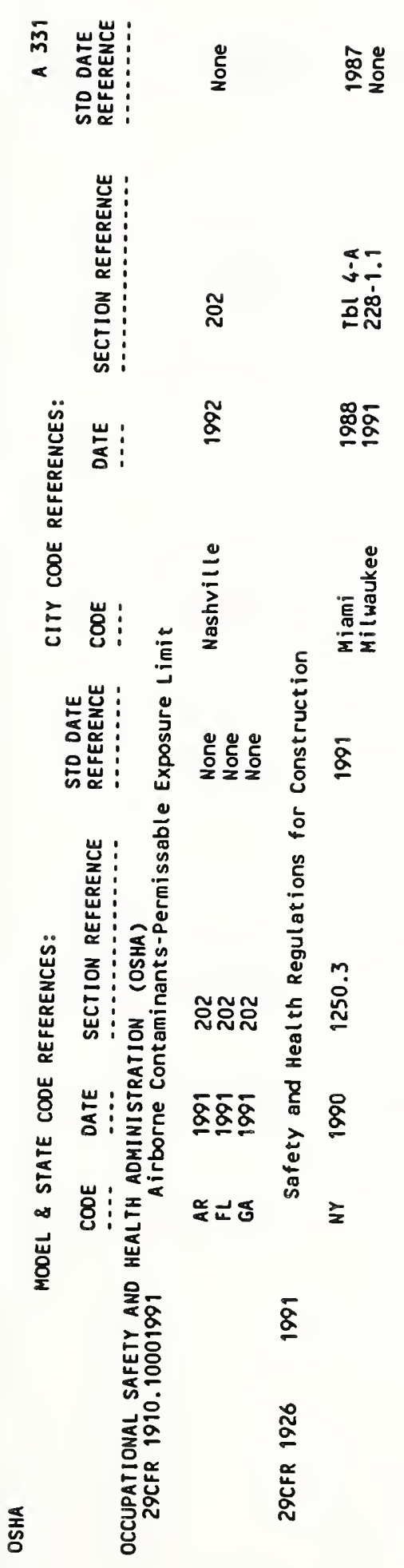




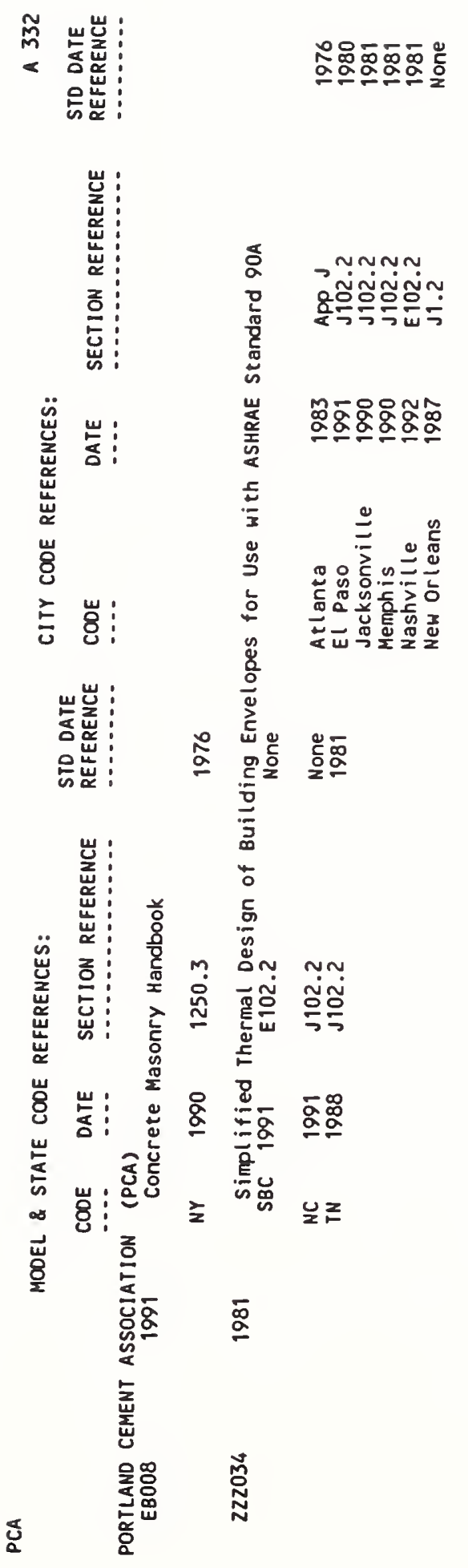


虽

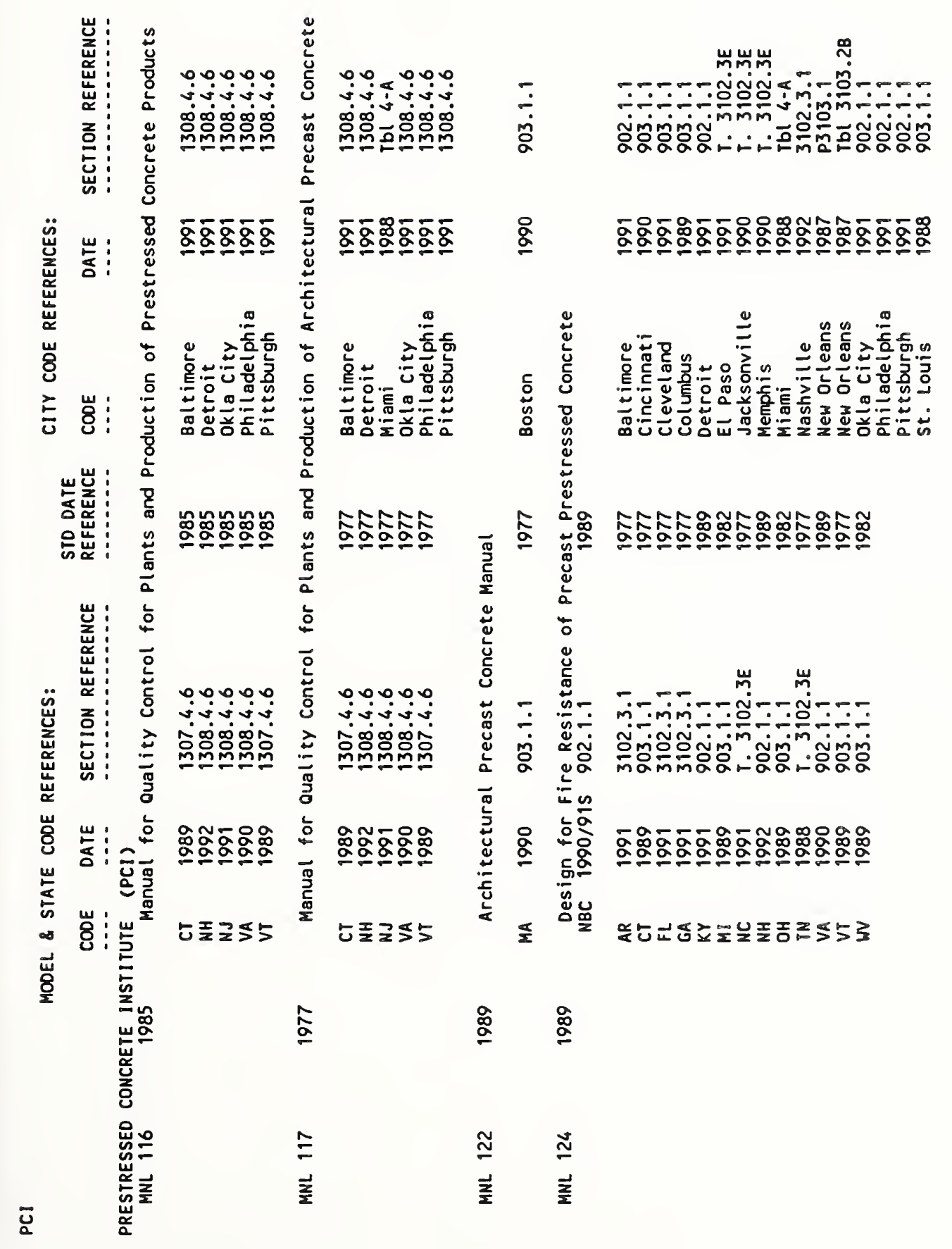




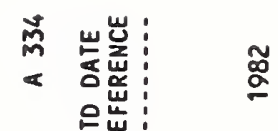

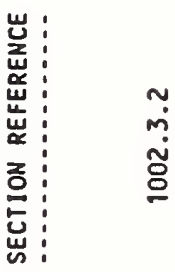

蒿宸:

응

흔 岁:

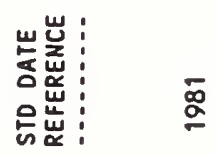

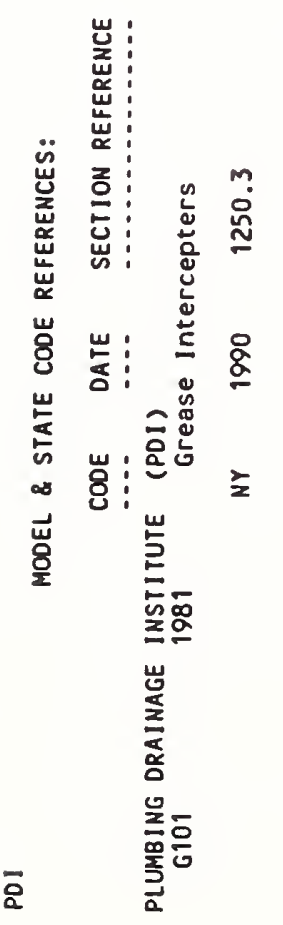




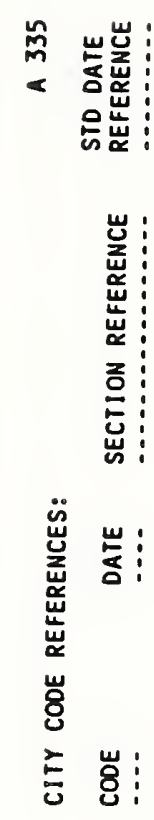

w

눙

乩岕:

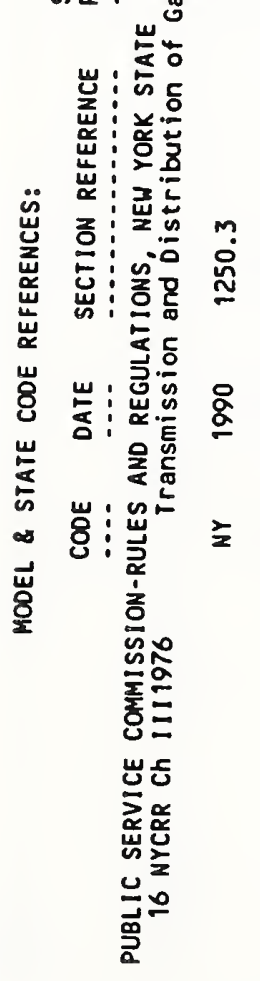


尽 w岂:

< 短 是压:

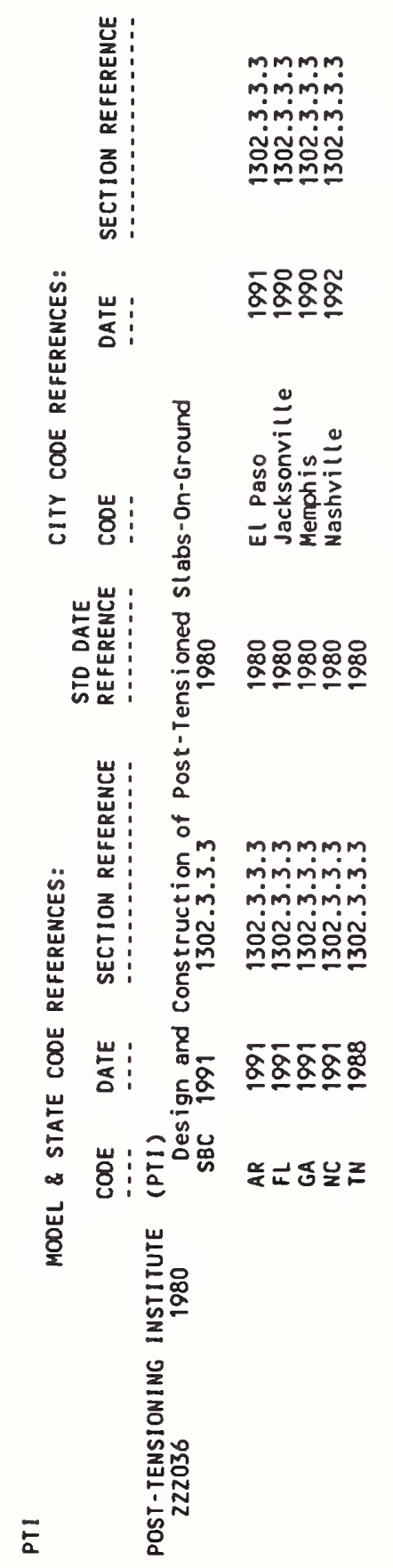




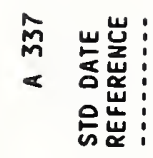

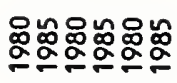

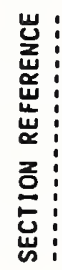

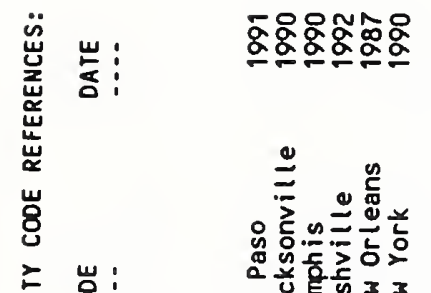

引㟯:

岂:

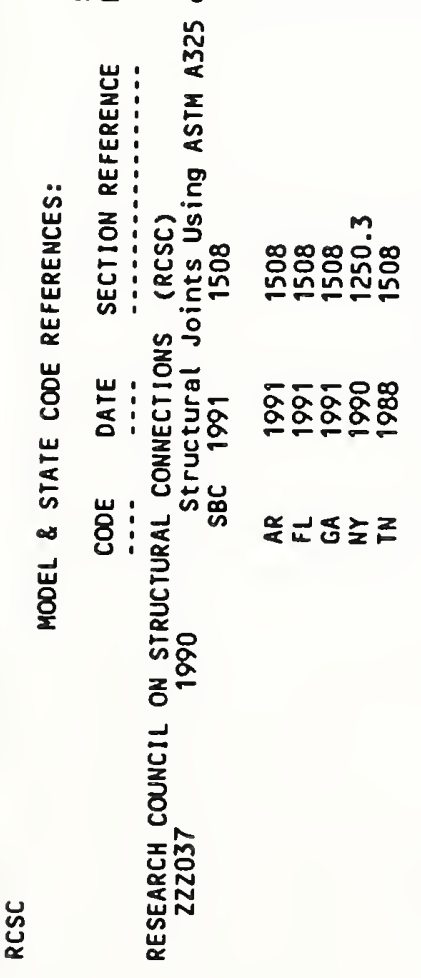




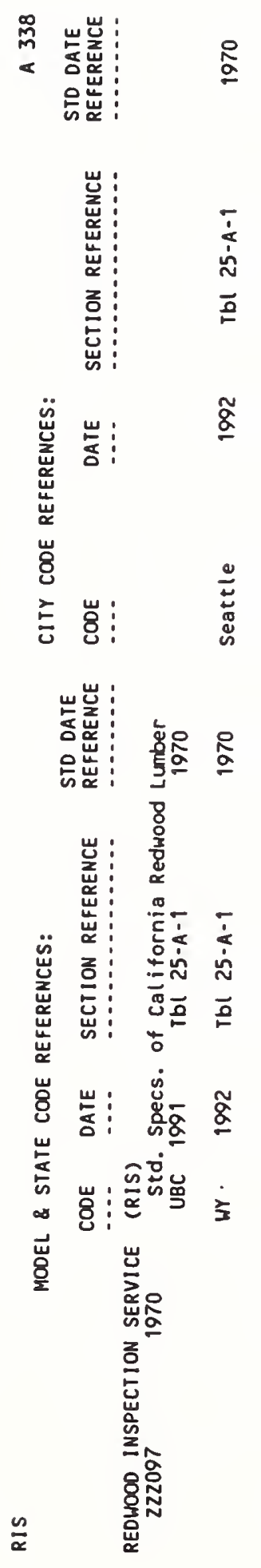




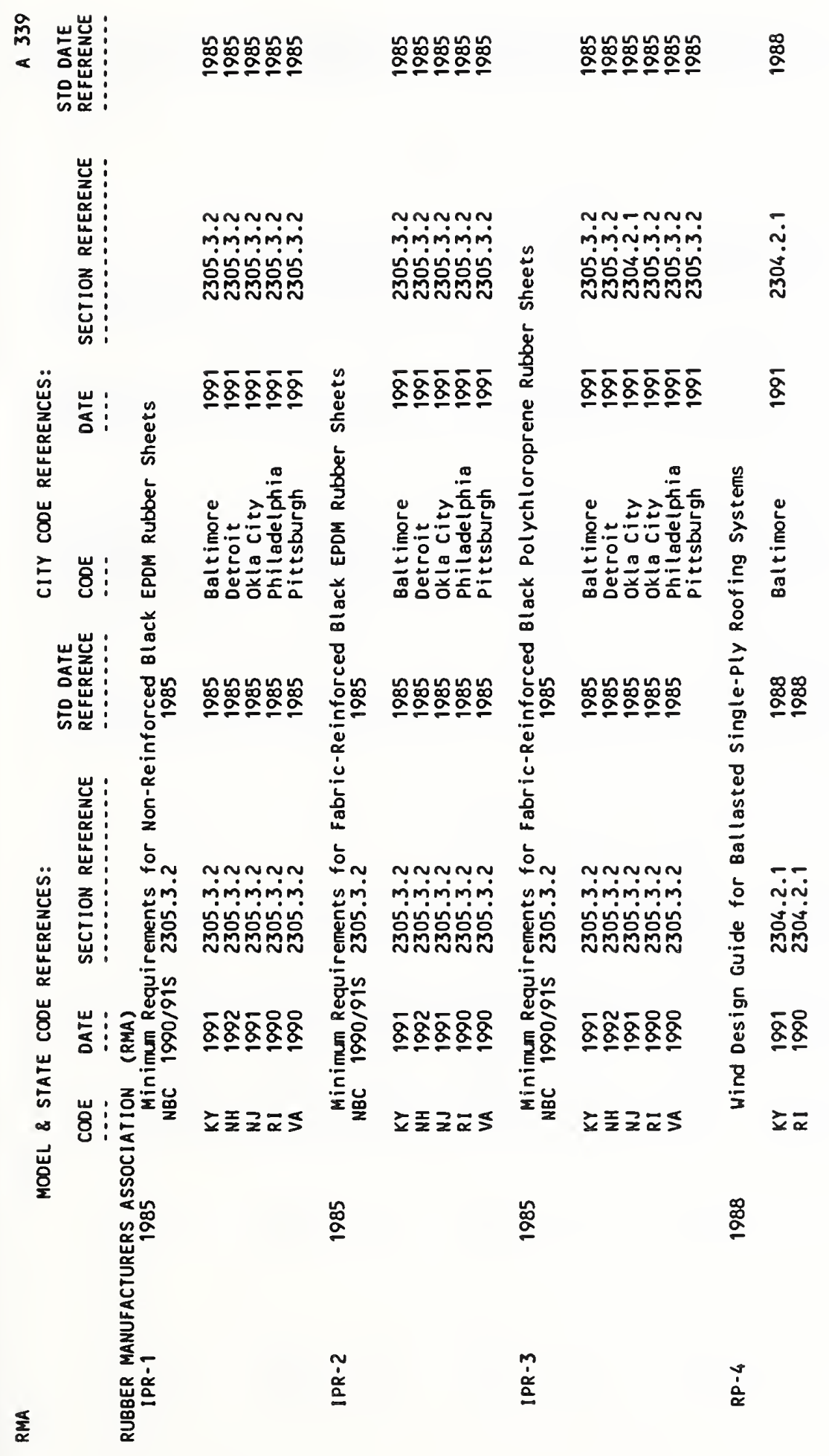




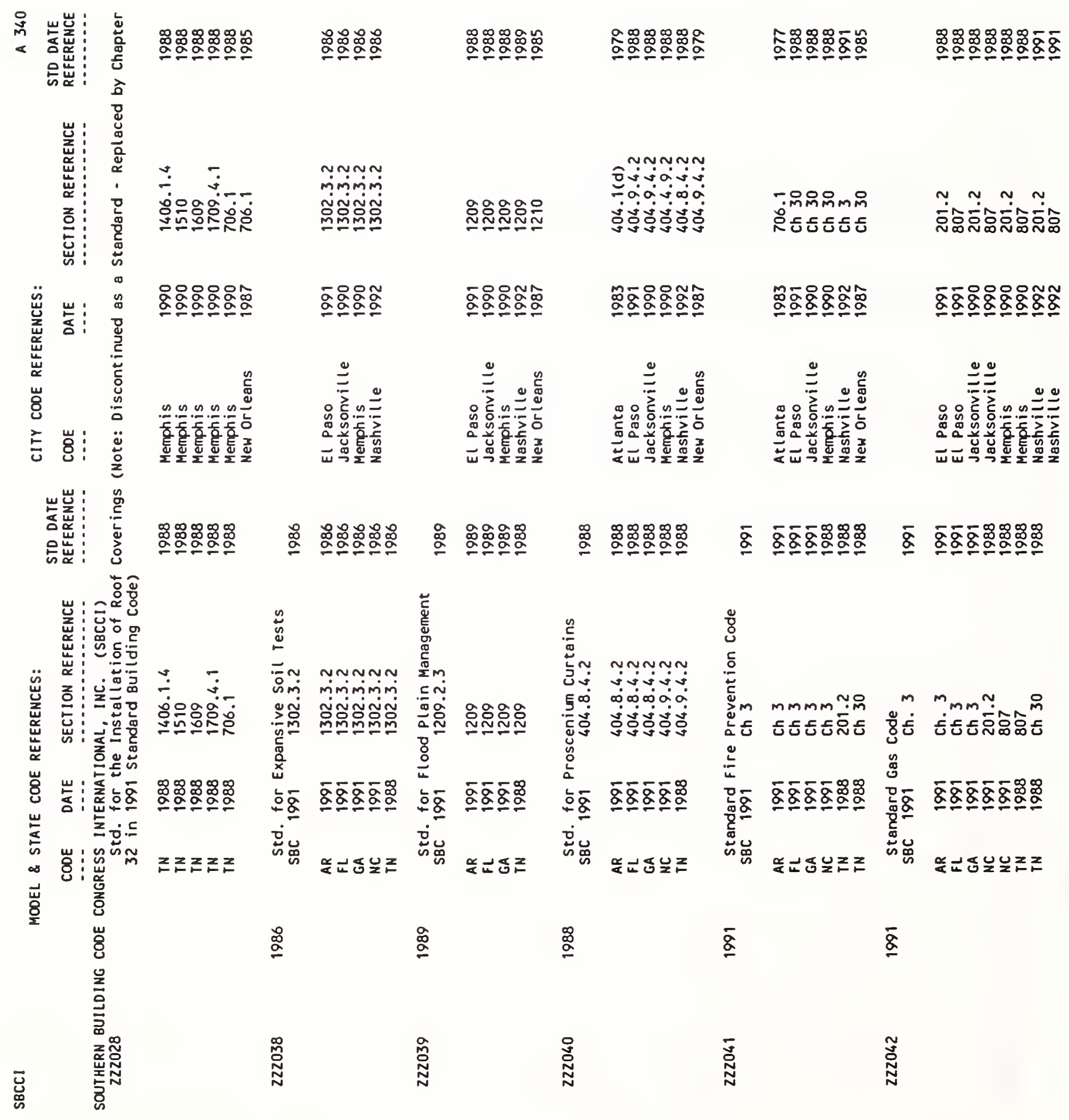




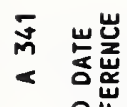

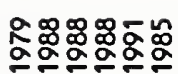

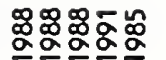

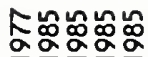

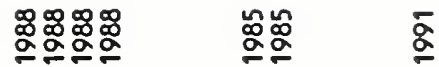

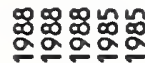

옹⿺ㅇ

岕

ํํํํำm

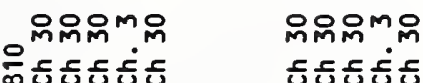

든던더

ตำำกำ

:

点:

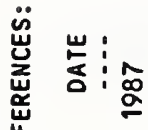

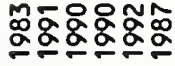

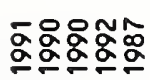

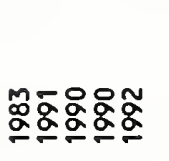

nann

¿-:

ลิ̃

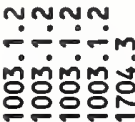

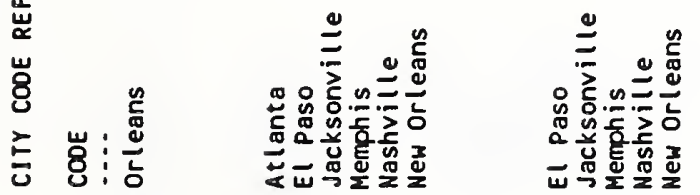

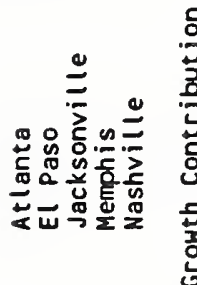

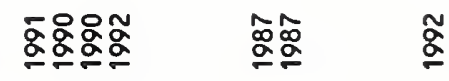

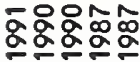

㟧 :

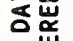

온

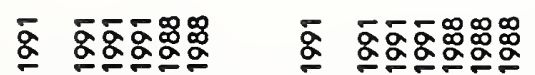

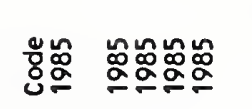

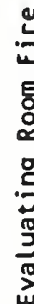

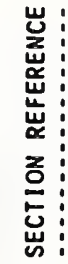

8ั

बim mmmmo

落 $\mathrm{mmmm}_{\mathrm{mm}}$

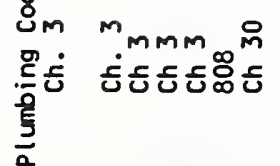

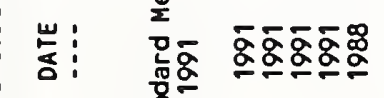

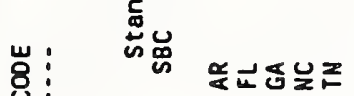

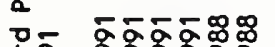

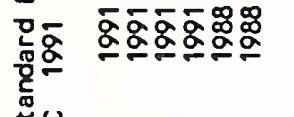

离䭂

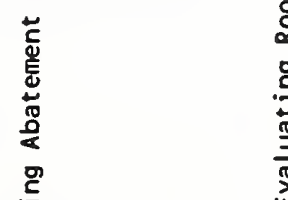

产 $\overline{\underline{\alpha}}$

$\bar{\alpha}$

$\stackrel{\leftrightarrow}{\stackrel{\circ}{\circ}}$

$\stackrel{\dddot{8}}{\mathrm{~g}}$

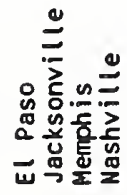

夢总

줄

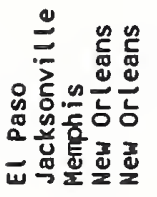

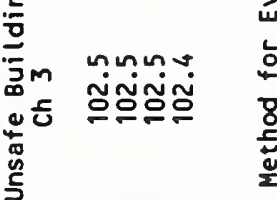

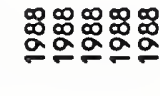

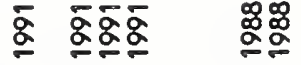

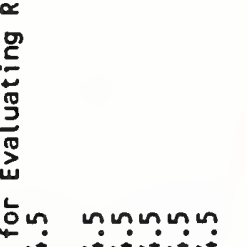

.

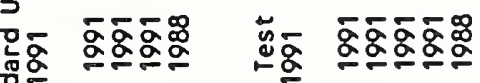

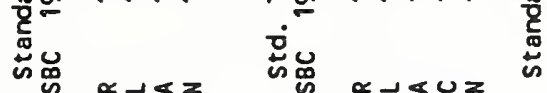

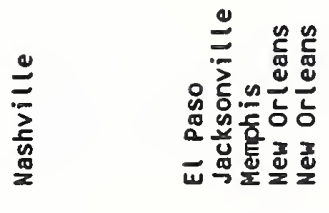




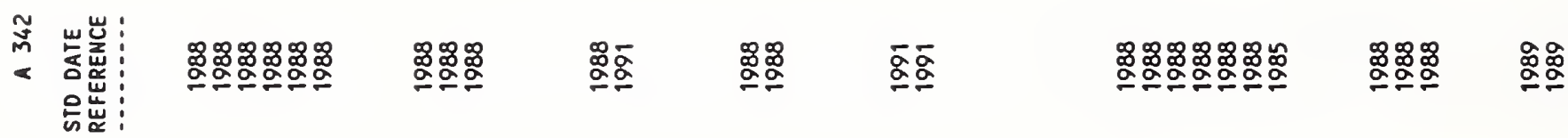

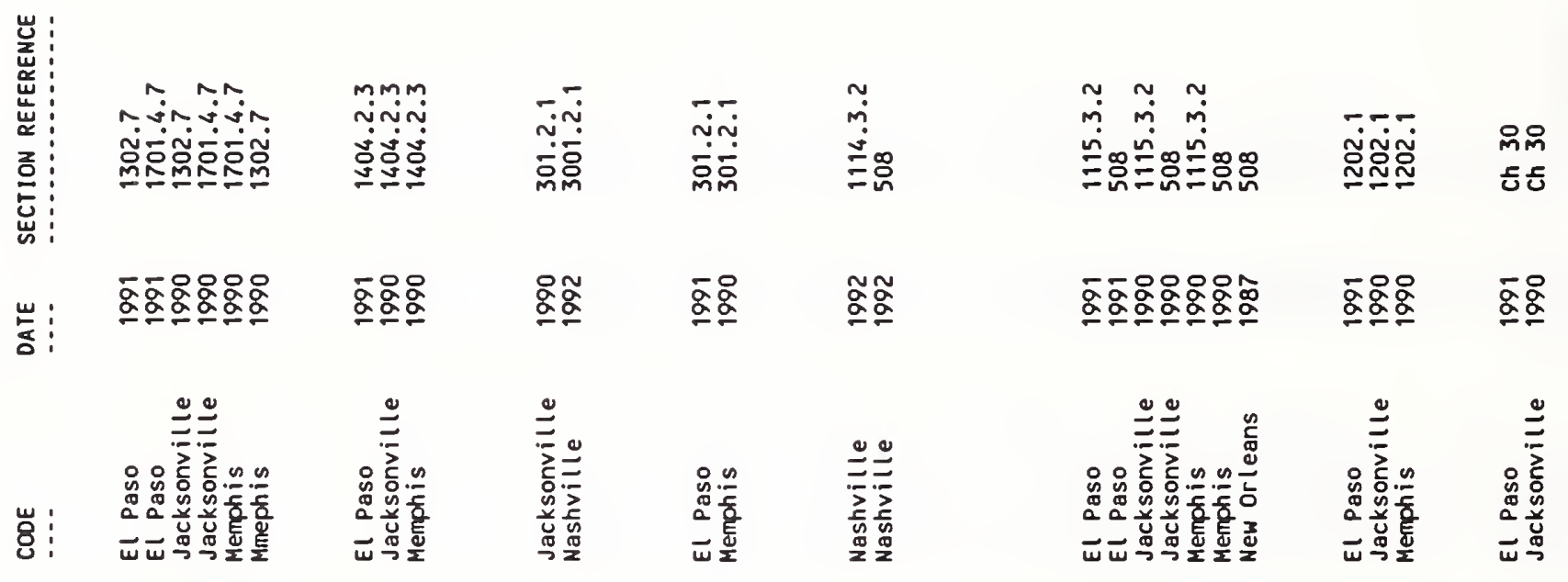

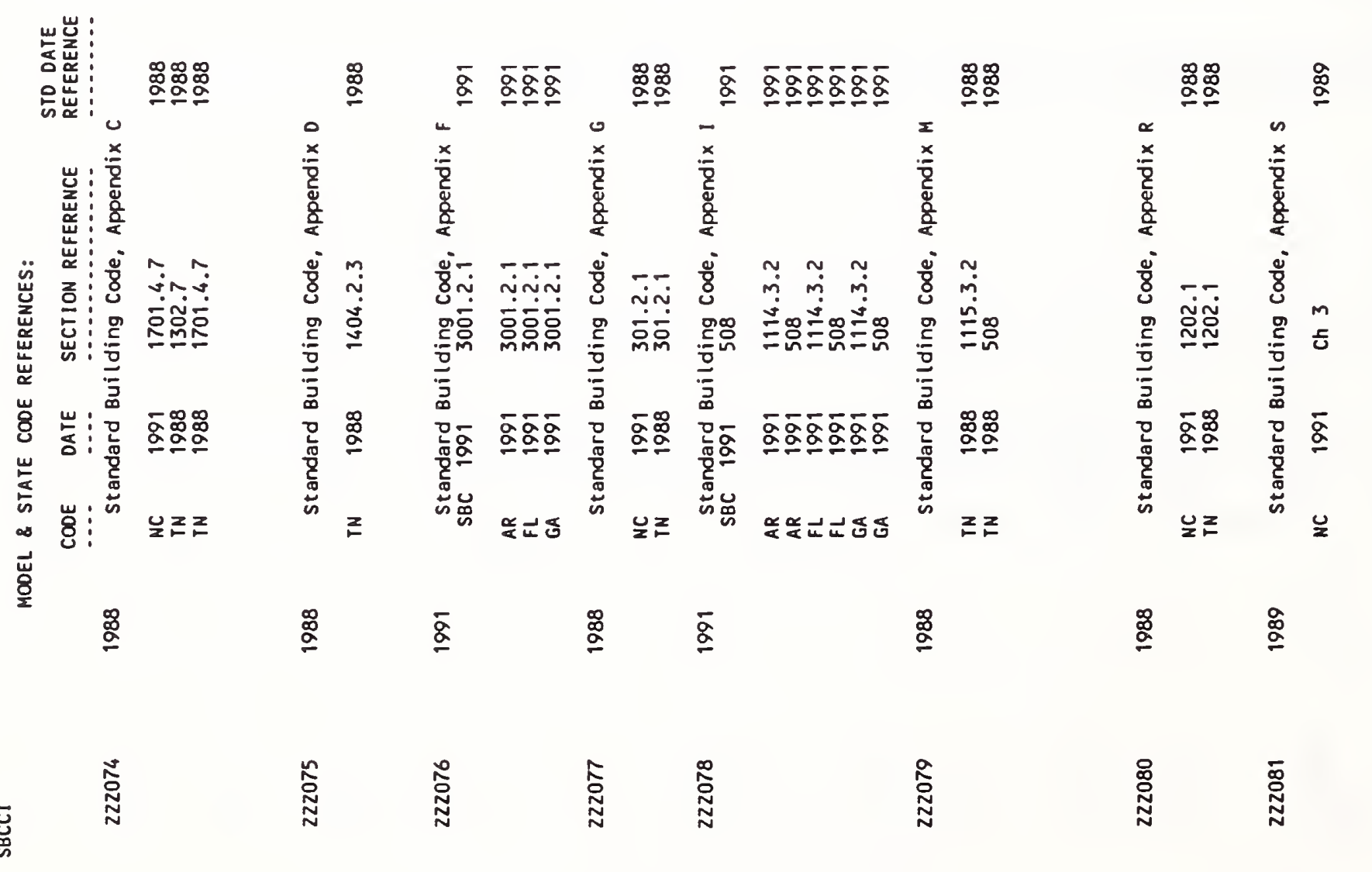




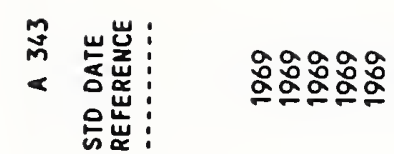

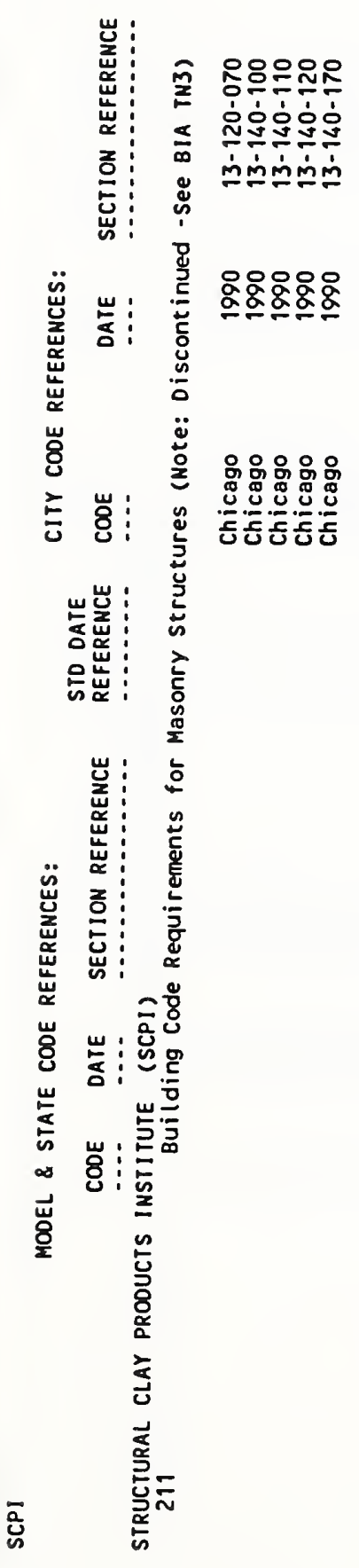




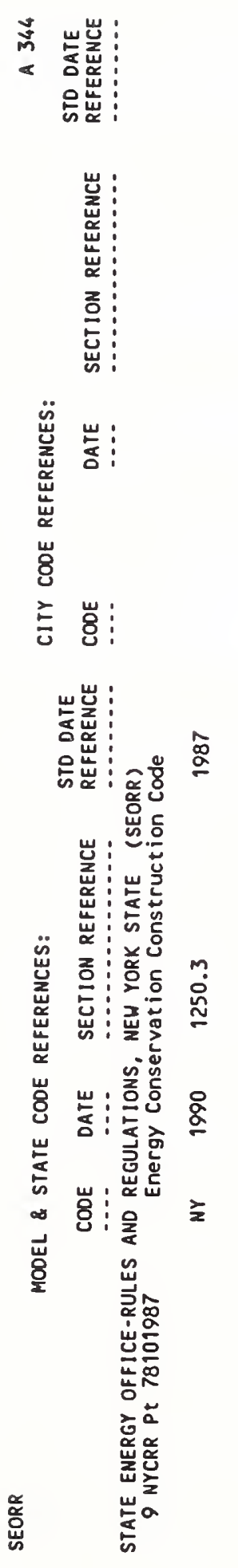




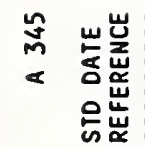

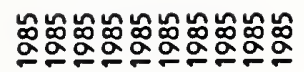

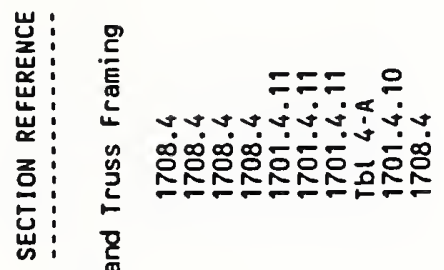

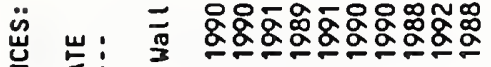

a:

$\stackrel{\circ}{\circ}$

㫕

乙

二 8:

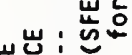

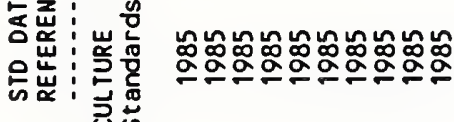

w , 중항

密:

(4)

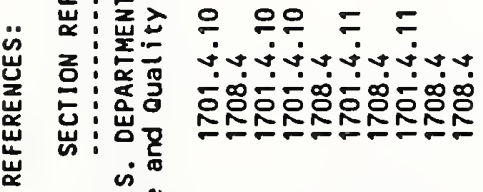

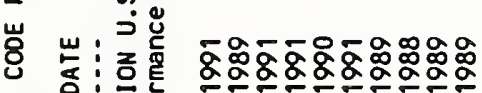

炭 造它

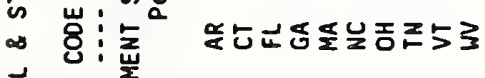

요

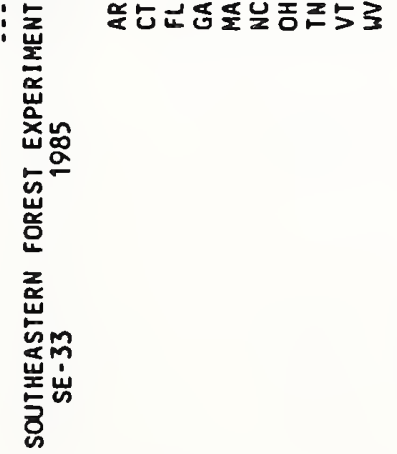




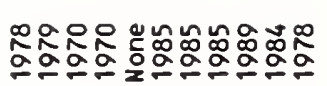

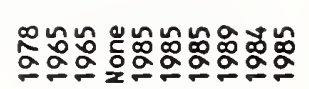

8

$\stackrel{\infty}{a}$

융응유ำ

웅유

ำ-

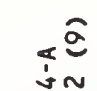

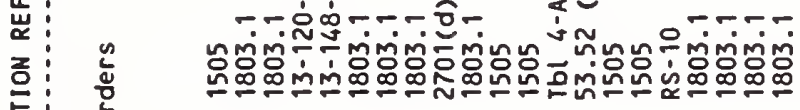

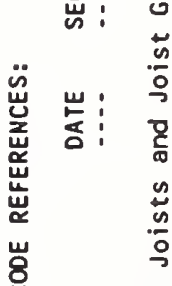

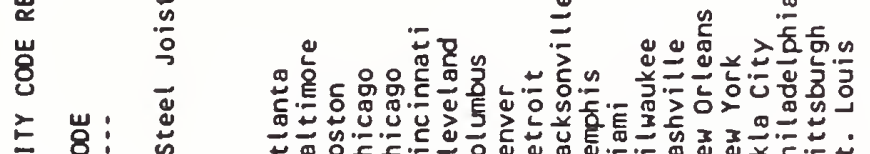

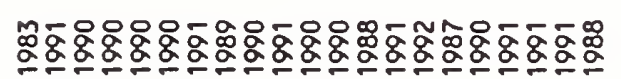

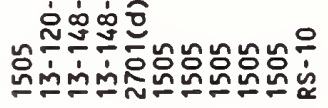

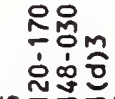

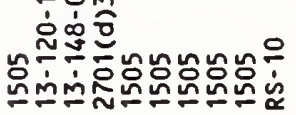

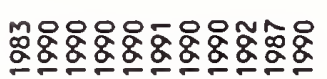

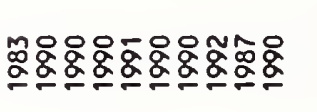

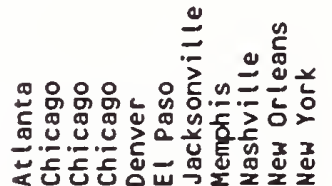

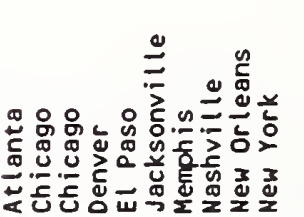

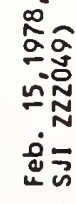

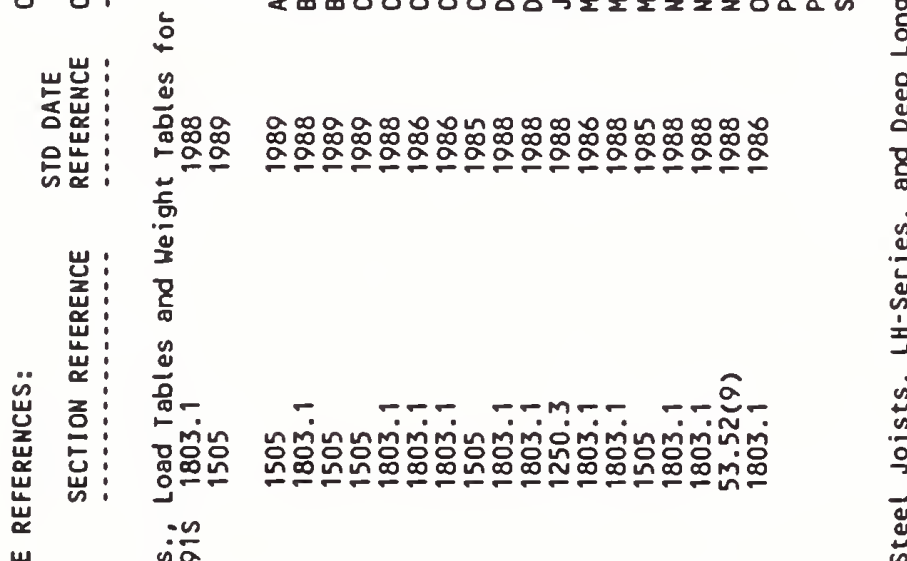

岁

崖

$\infty$

풍

㟧兽品 )

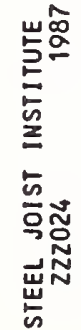

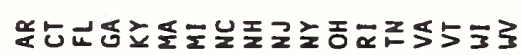

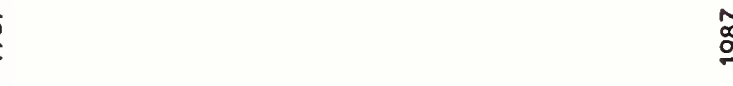

$\underset{\substack{0 \\ N}}{N}$

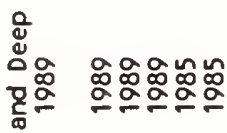

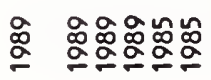

造

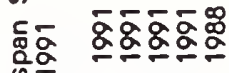

뚠뜬몬

$\stackrel{\circ}{\circ}$

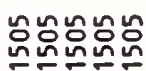

$\frac{2}{4}$

sis

후음

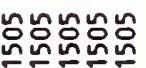

is

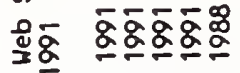

岁品

운떵문즈

$\underline{\underline{\alpha}}$

$\stackrel{\stackrel{0}{\circ}}{\circ}$
क्ष

$\stackrel{5}{\circ}$

?

$\frac{0}{\dot{4}}$

$\stackrel{ }{2}$

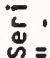

x.

s.

웅

뭉

8ू워

实

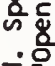

放

号

No
N
$N$ 


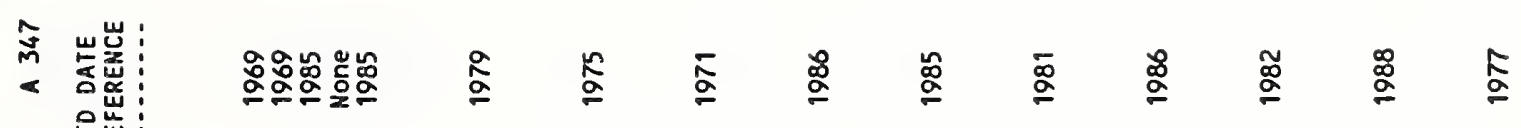
㜅:

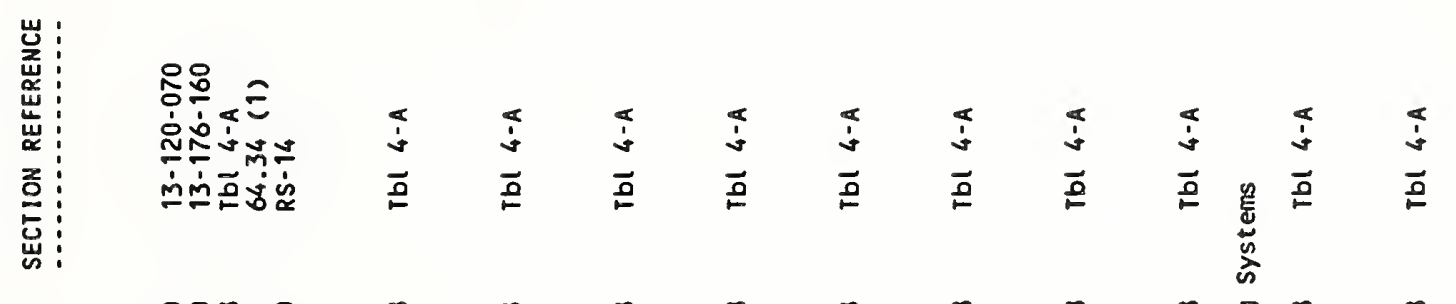

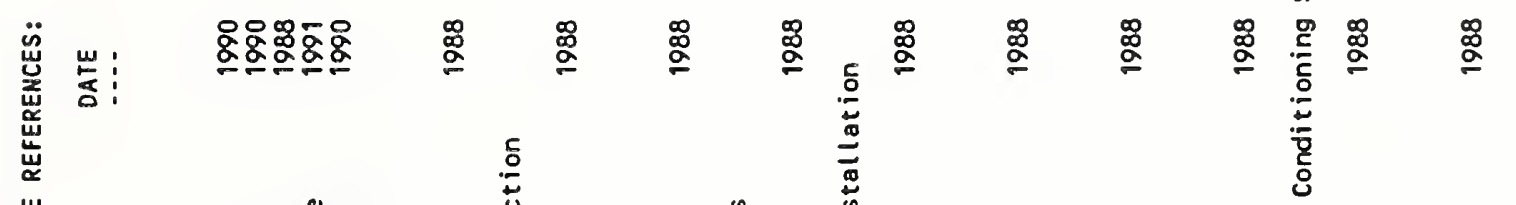

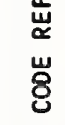

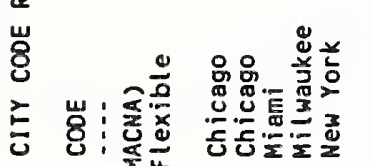

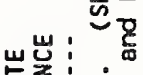

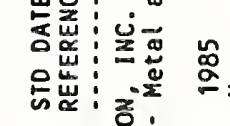

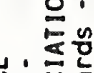

渠

*

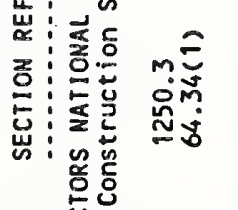

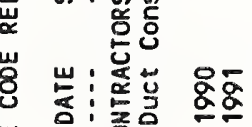

出

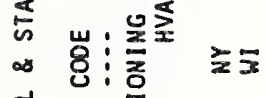

咅<smiles>[12BH]</smiles>

焉

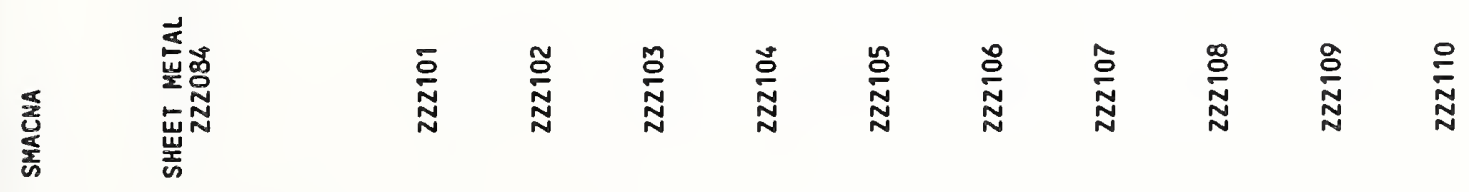




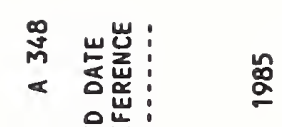

:

岕

岃

z흘

岕:

苞崖:

$\underset{w}{\stackrel{w}{\alpha}}$

岂 농

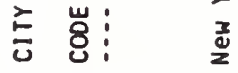

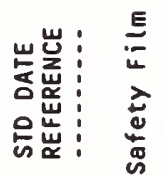

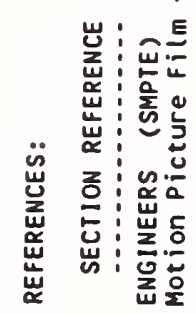

岁 容: 웅.

崖苛离

5 㟧:

㟧

ठั

는흐

흠

แ

旁管紊 


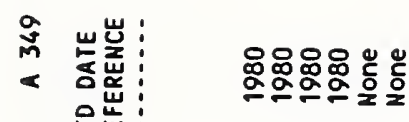

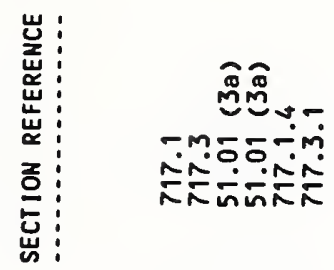

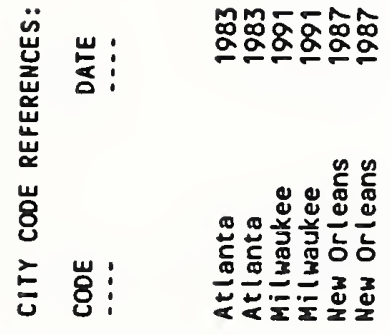

논

额:

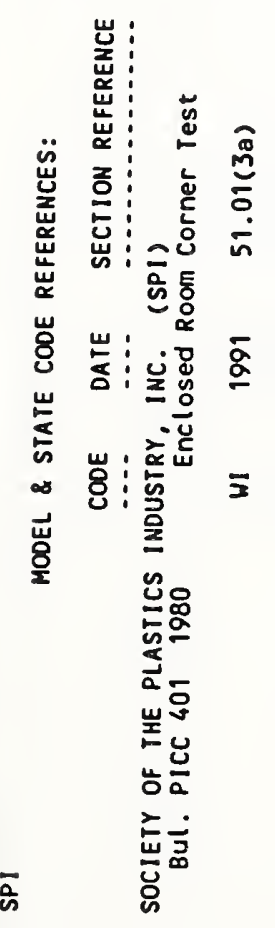



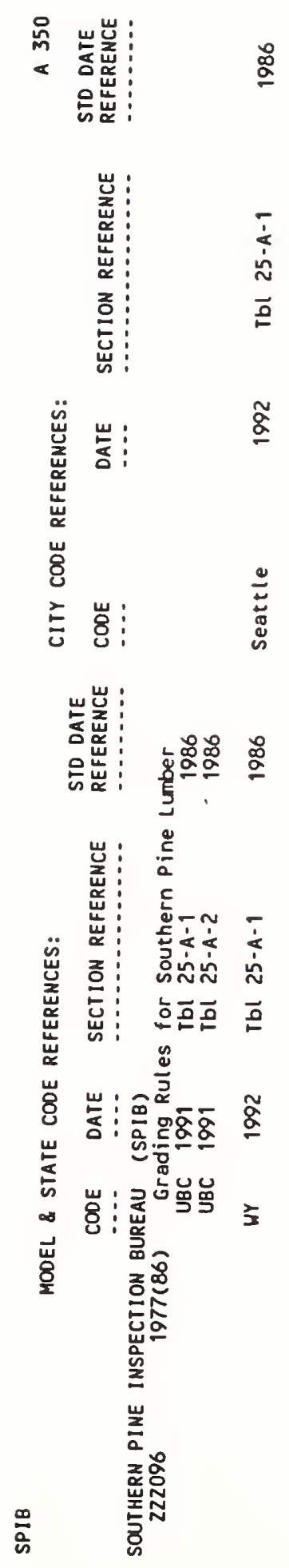


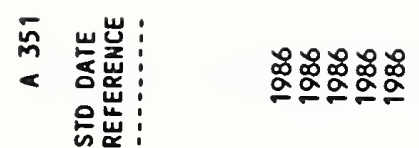

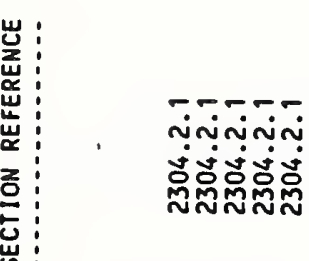

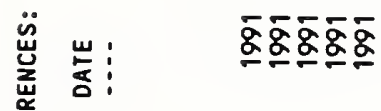

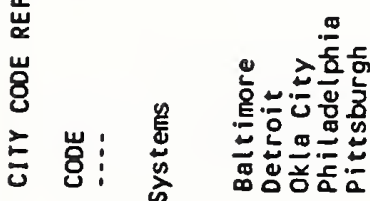

㟯岕:

운

:

总

zo

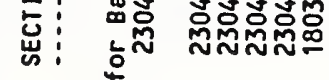

品

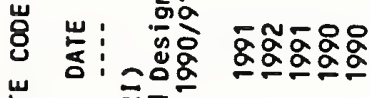

压

各

$\overrightarrow{\mathrm{w}}$

咅

ล้ำ

$\bar{\alpha}$

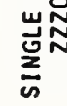




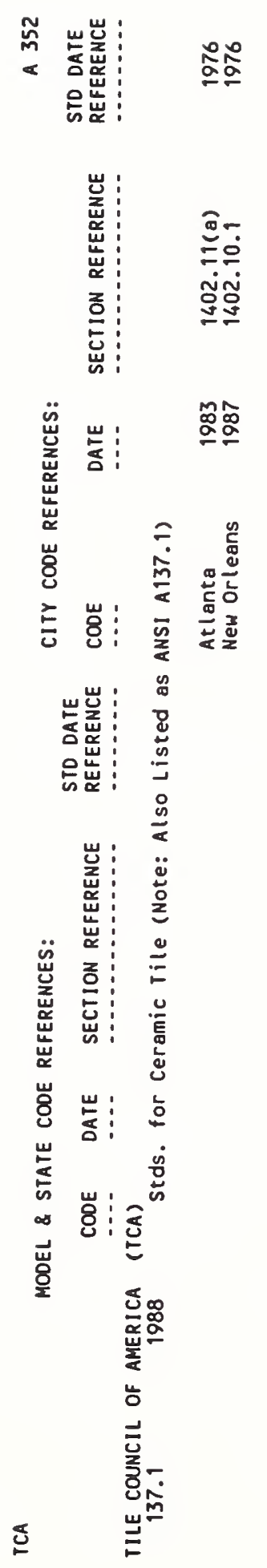




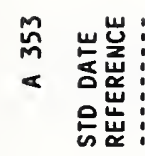

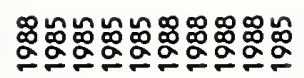

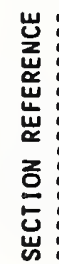

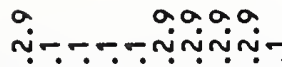

ทำiniviที่

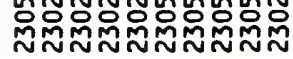

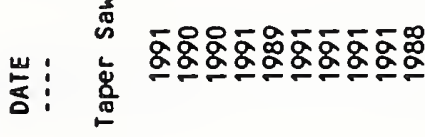

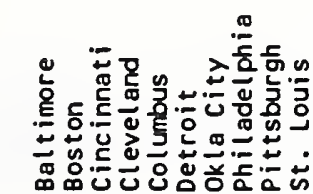

岩:

은

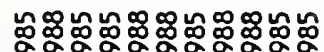

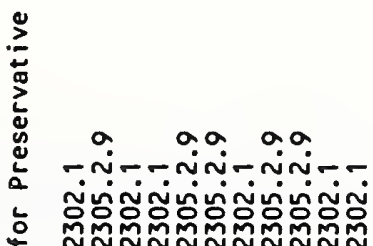

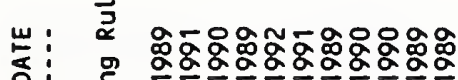

吾

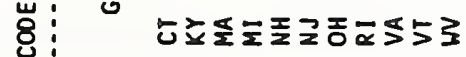

岁苍

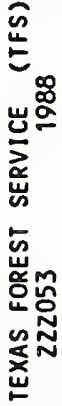




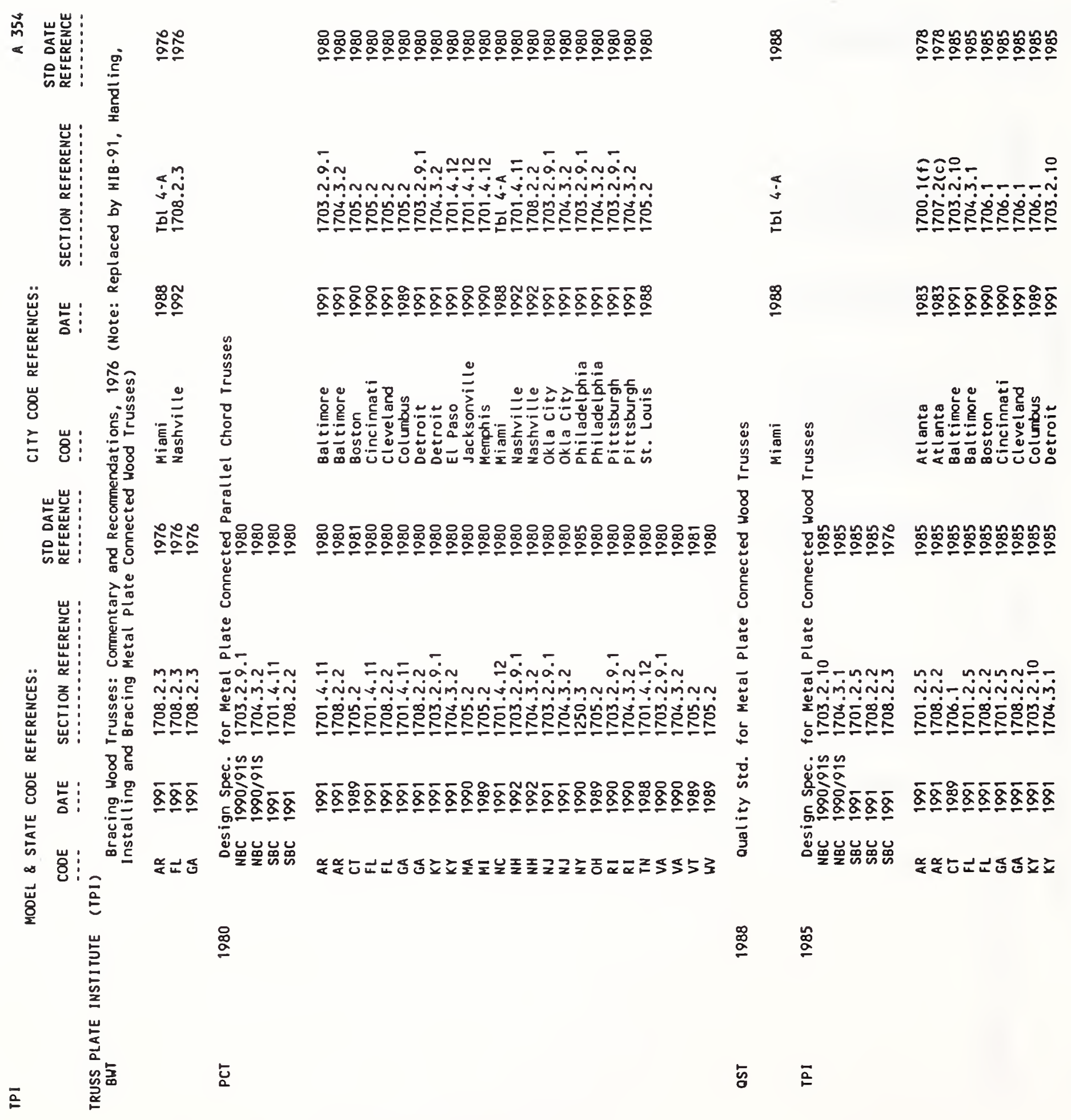



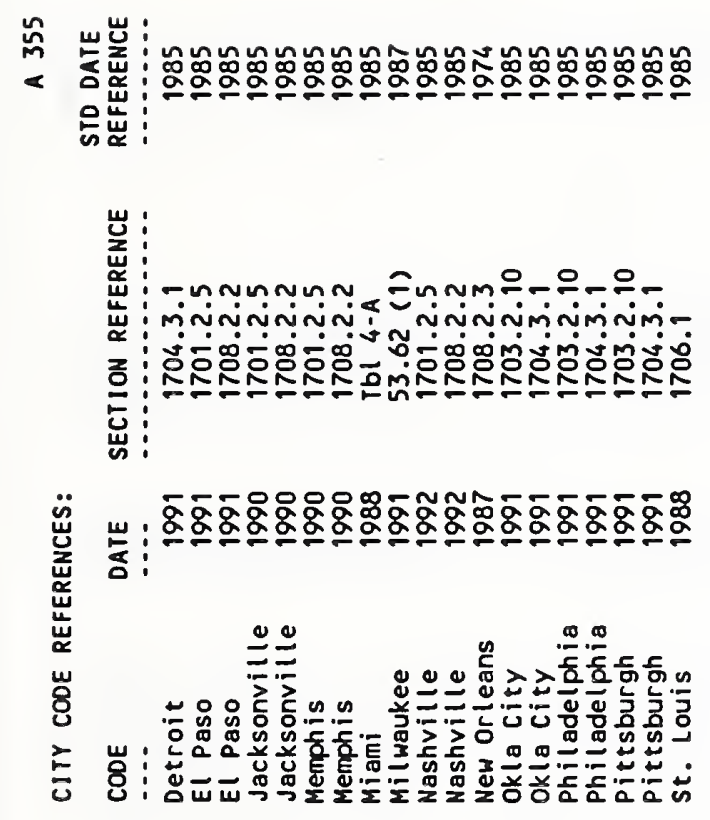

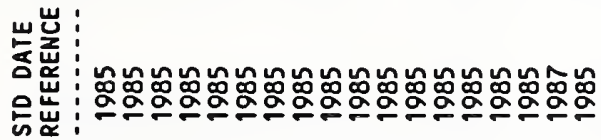

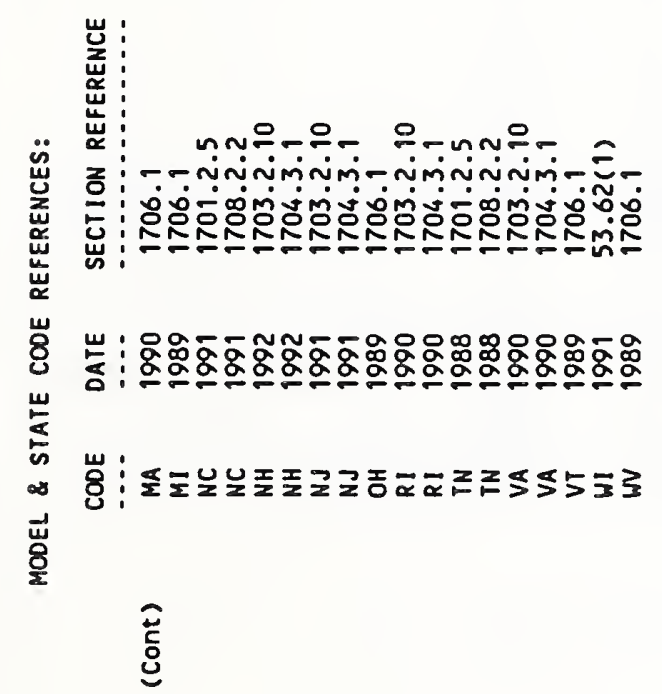

$\overline{0} \quad \bar{a}$ 


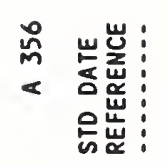

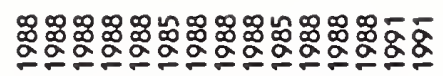

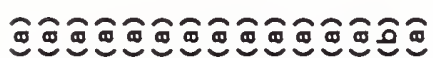

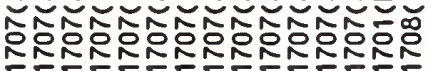

흠:

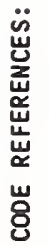

崖:

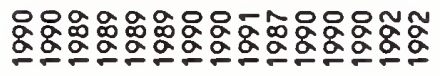

ร

떤

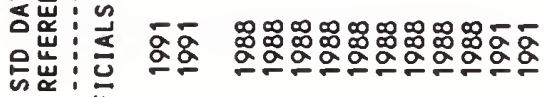

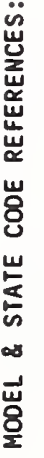

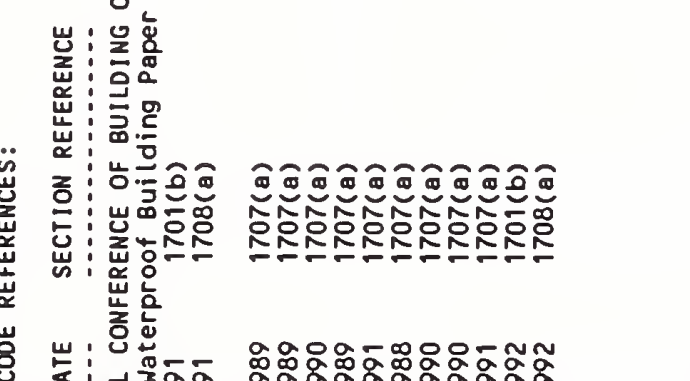

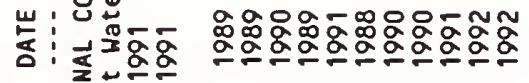
증ㅎㅎㄴ

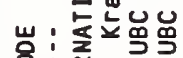

峝:

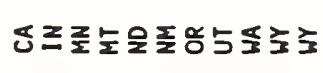

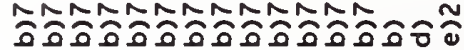

กำกำกำกำกำ

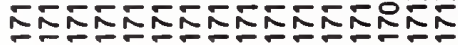

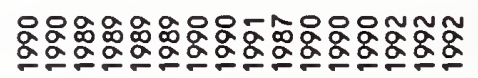

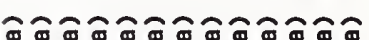

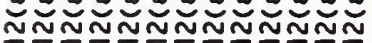
ミミミミミミミミミミミミミ

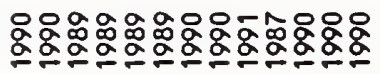

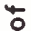

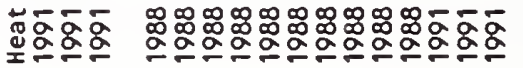

离

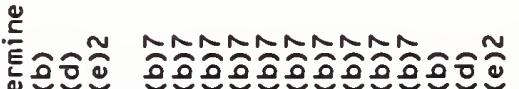

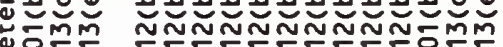

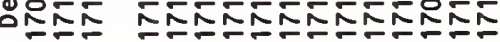
웅

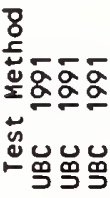

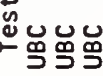

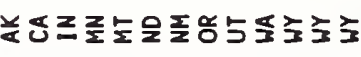

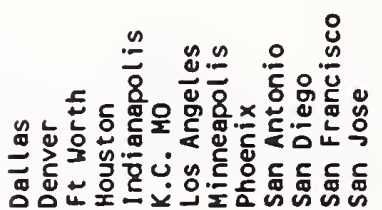

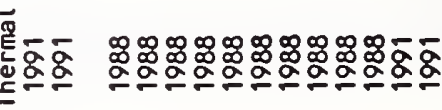

¿

.

$\frac{\mathrm{m}}{\mathrm{g}}$

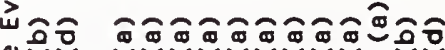

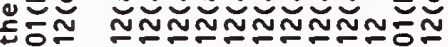

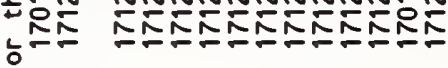

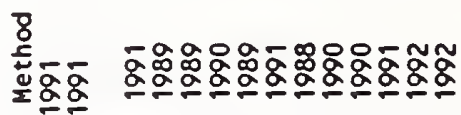

嘔品品

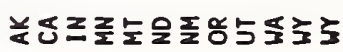

$\stackrel{\sim}{\check{I}}$

$\dot{m}$ 


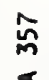

崖总:

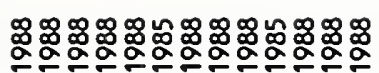

战岕 :

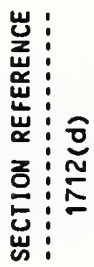

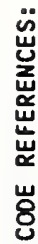

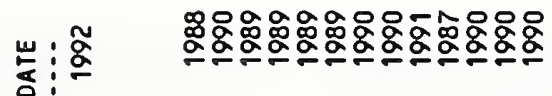

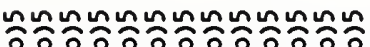

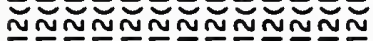

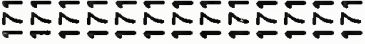

$\tau$ ᄂ

Ј

世 بّ :

究总

的峞

要

年

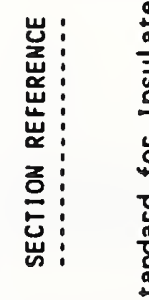

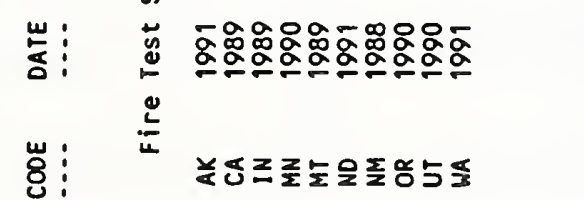

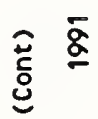

nunununununn

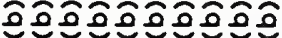

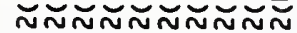

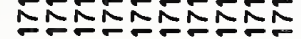

ஐ̊:

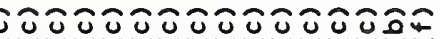

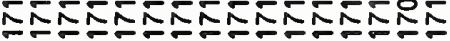

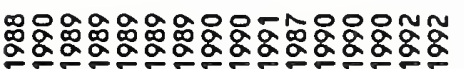

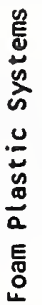

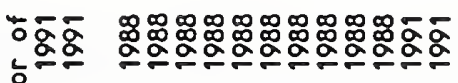

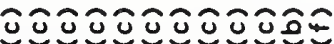

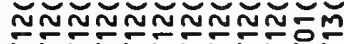

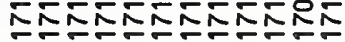
$\stackrel{4}{\cong}$

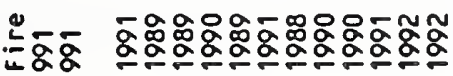
\&品品

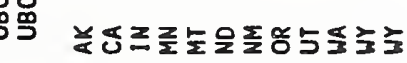

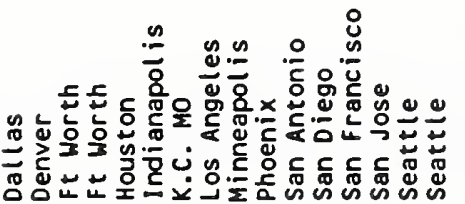

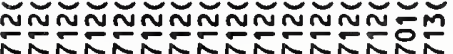

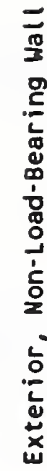

\section{¿}

$\stackrel{8}{5}$

要

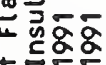
$\div$

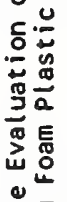

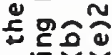

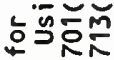
is 范 प卷衣各

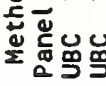

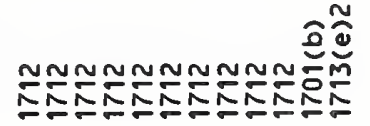

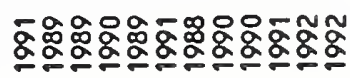

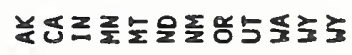

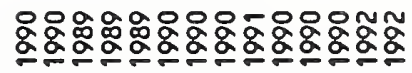

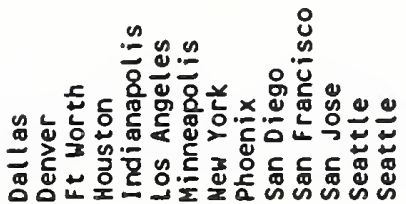

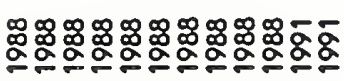

ำกำกำกนกำกำกำ

$\bar{\alpha}$

$\bar{\alpha}$

อิธิ์ 


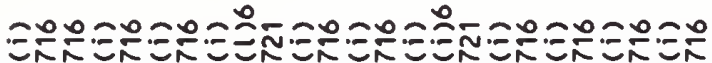

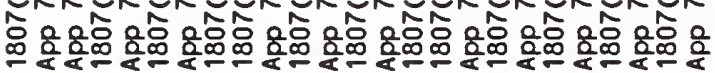

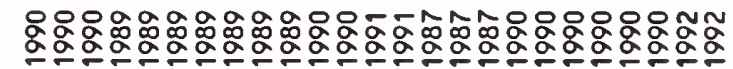

过品

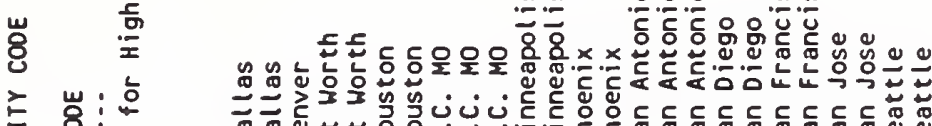
志菏:

뜬

安崖

은 崫: : :

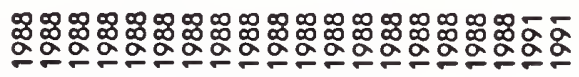

岁 : 㟧

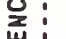

品: 蓄

崖:

충: 휠:=

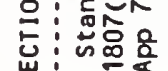

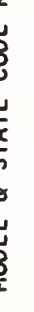

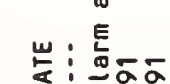

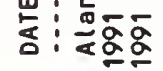

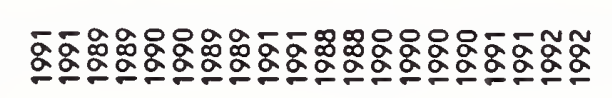

岩娣

岂:

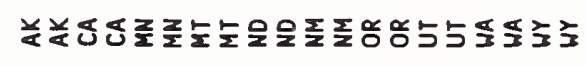

$\bar{\alpha}$

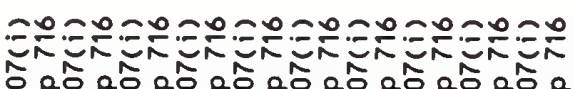

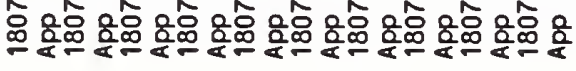

号

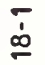

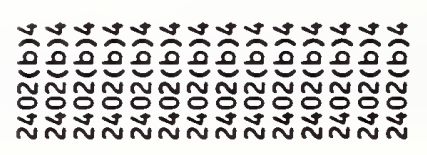

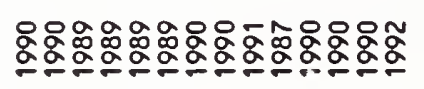

ㄴ.

$\stackrel{-}{\frac{0}{5}}$

予-

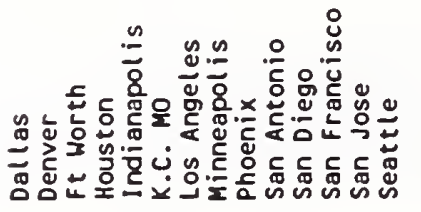

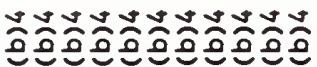

กิก

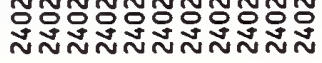

는

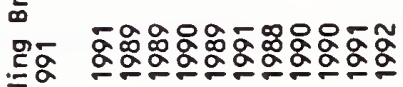

두웜

可鮙

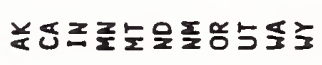

$\bar{\alpha}$

i

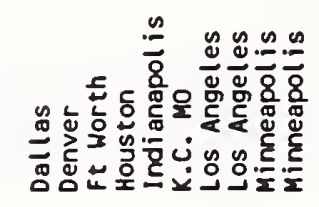

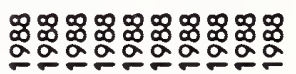

ํำำํํํำํํํำ

$\stackrel{\mathscr{\Xi}}{2}$

$\frac{m}{\dot{i}}$

웅ํํํํำำ

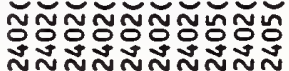

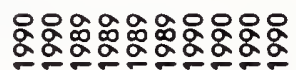

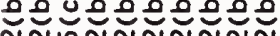

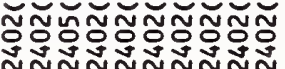

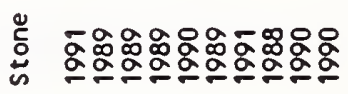
芯

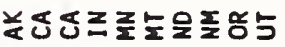

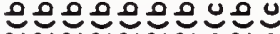



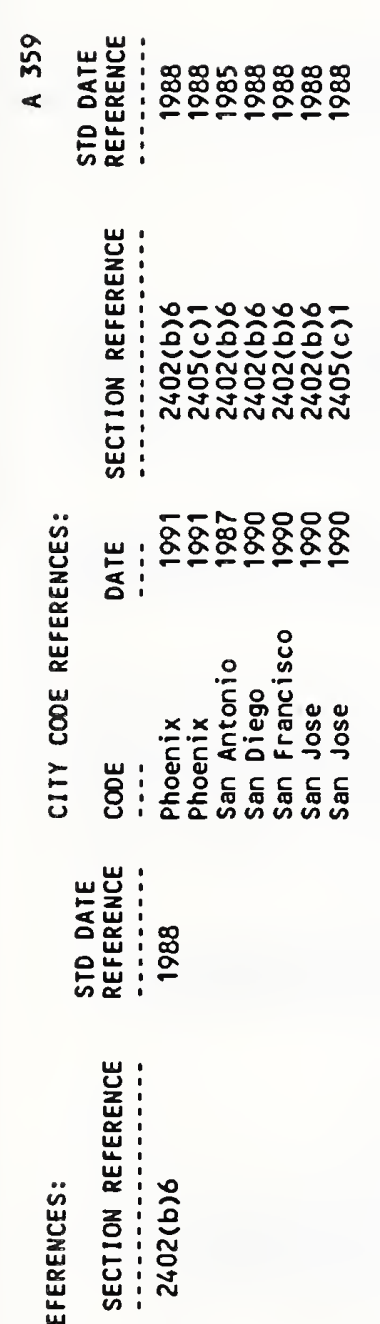

to

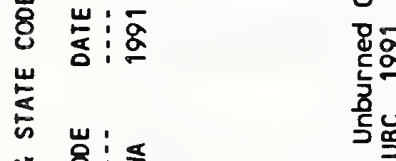

$\infty$

해을

言

$\stackrel{m}{\stackrel{m}{\sim}}$

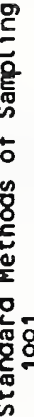

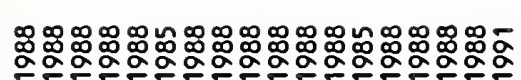

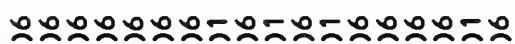

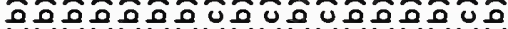

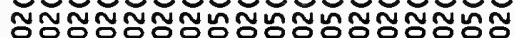

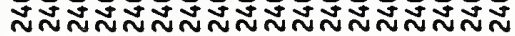

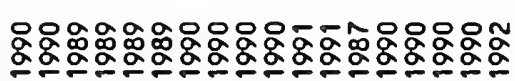

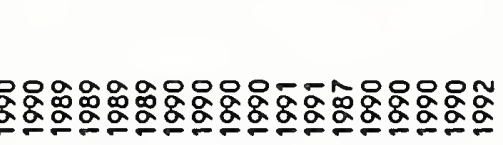

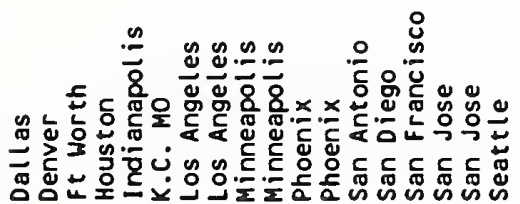

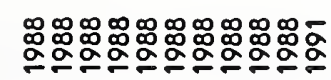

응ํㅇㅇํㅇํㅇㅇํ으

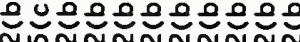

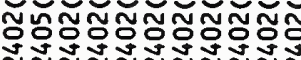

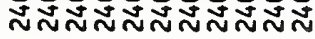

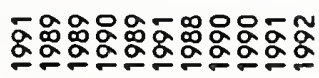

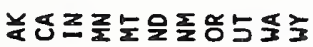

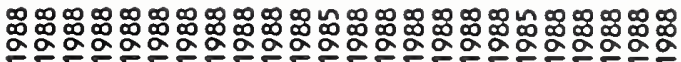

을.

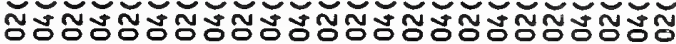

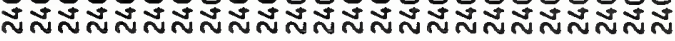

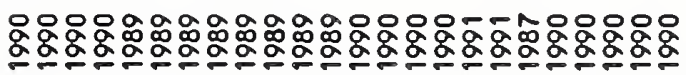

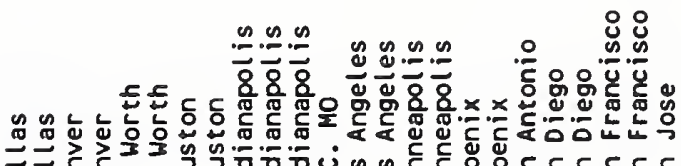

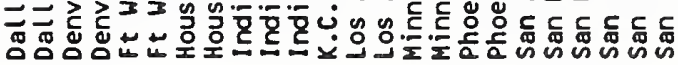

$=$

范

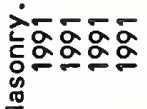

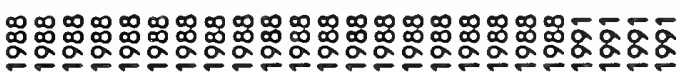
高

蒙

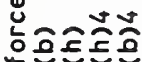

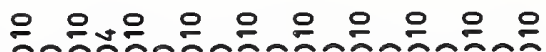

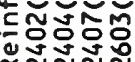

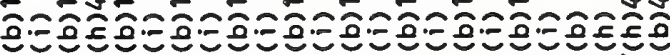

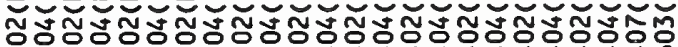

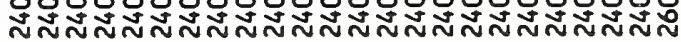
芫

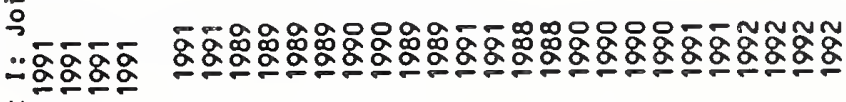

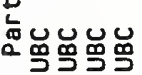

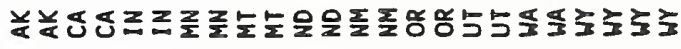

$\bar{\alpha}$

$\stackrel{n}{\sim}$ 
蛅:

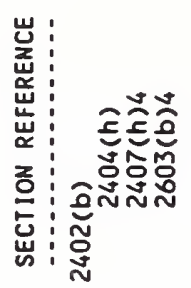

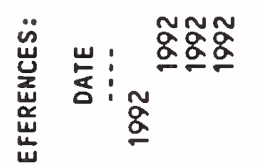

岩

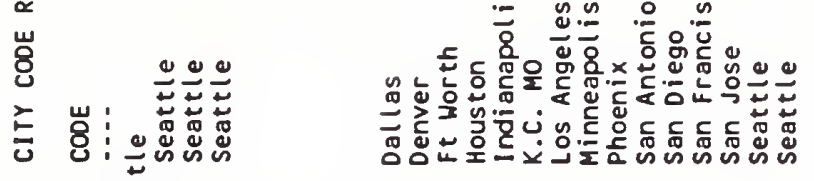

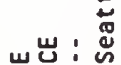

岕密:

舟岕:

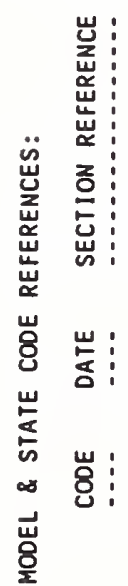

突

夢
nNNNNNNNNNNNNN<

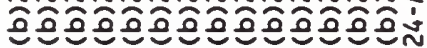

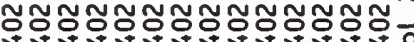

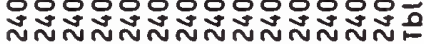

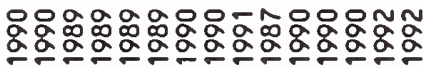

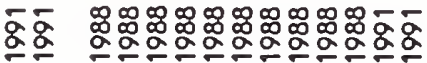

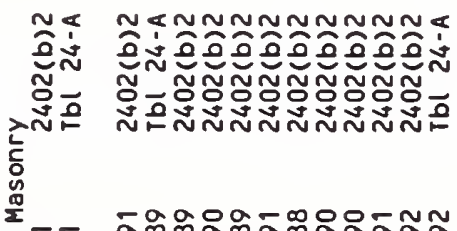

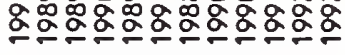

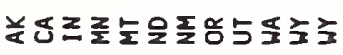

$\bar{\Phi}$

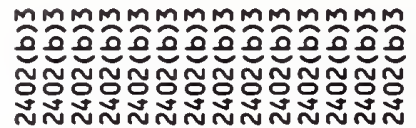

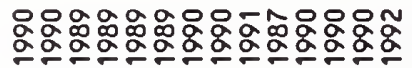

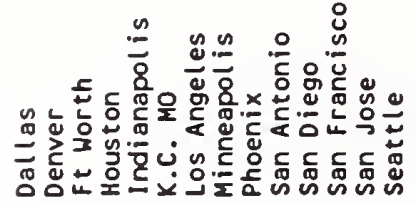

пмmmмmmmmmmmm

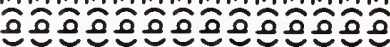

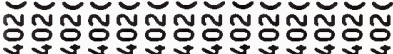

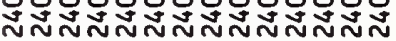

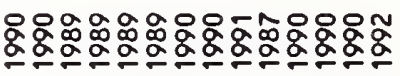

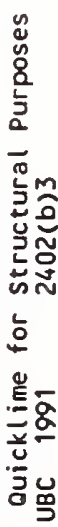

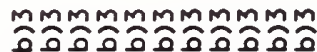
㑔页

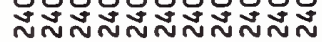
¿ 嬊

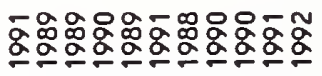

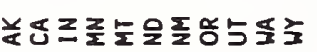

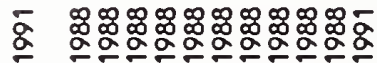

迹

¿ mmmmmmmmmmm

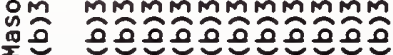

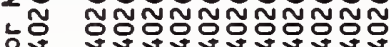

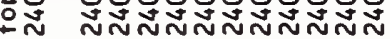
.E

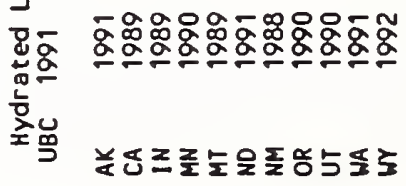




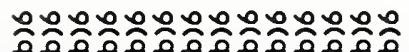

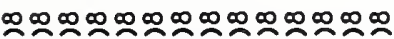

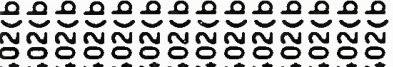

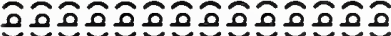

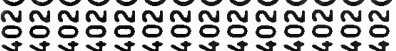

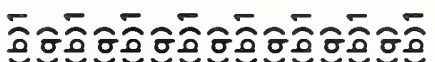

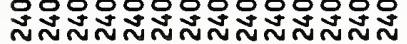

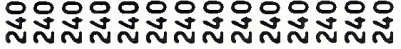
กั

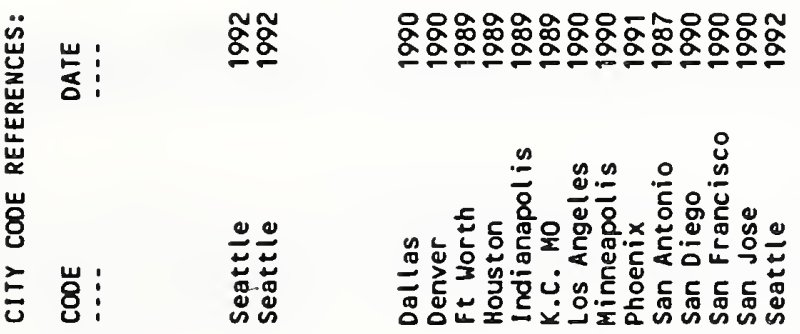

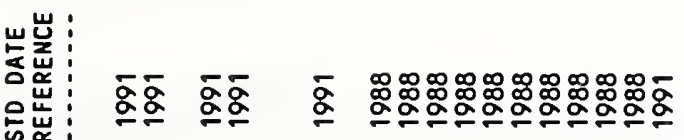

\footnotetext{
岁
}

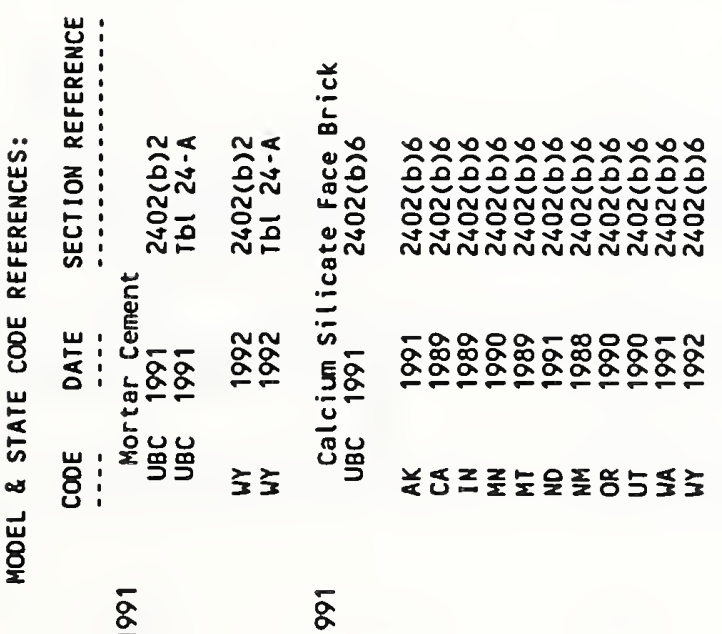

号

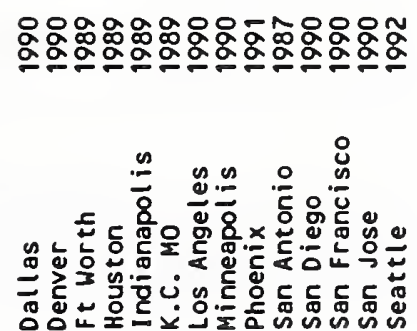
능

용

焉

농

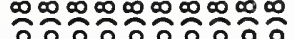

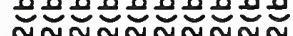

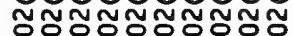

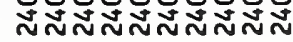

$\overline{5}$

宫

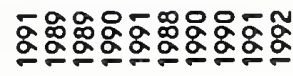

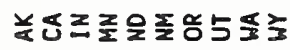

$\bar{g}$

$\stackrel{\sim}{\dddot{2}}$

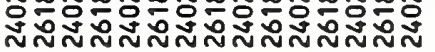

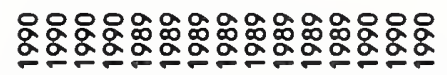

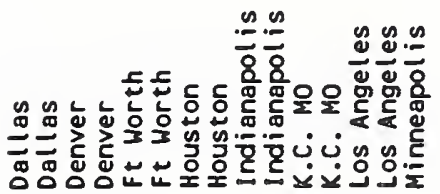

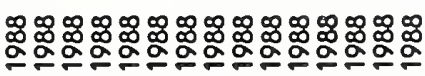

㐫

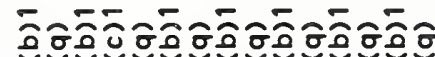

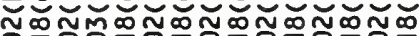

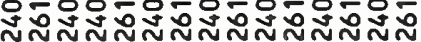

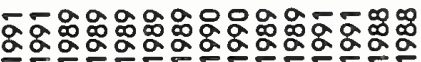

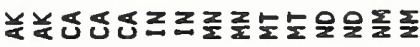

$\stackrel{\mathscr{B}}{2}$

$\bar{\sim}$ 


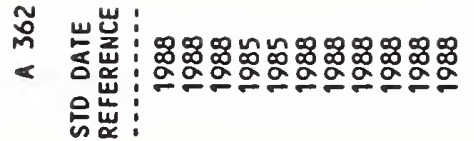

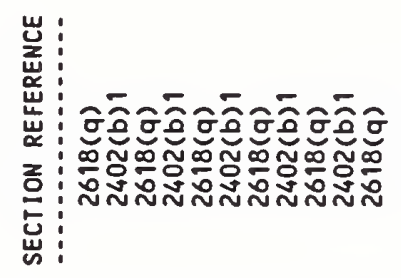

苨
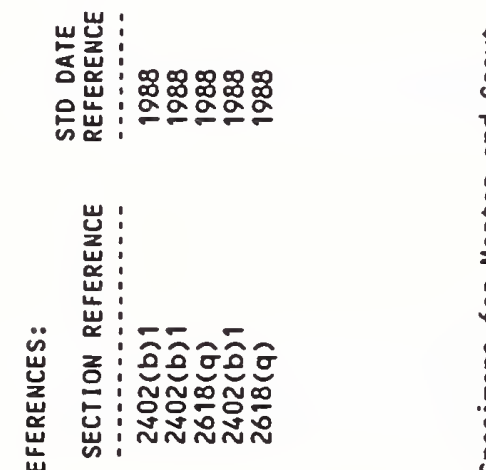

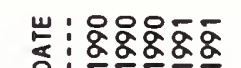

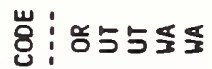

옹

мммммммммммимм s.

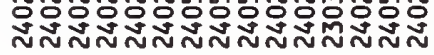

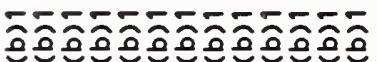

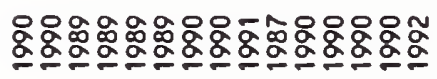

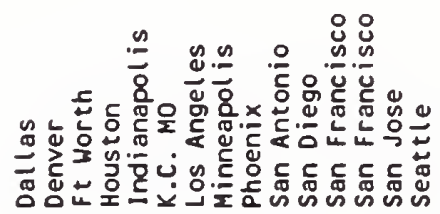
\$ån:

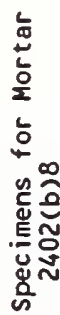

范 क्ष 品

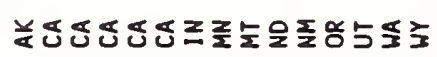

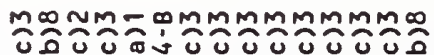

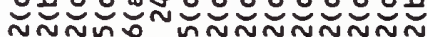

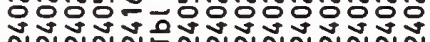

ลำ

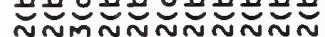

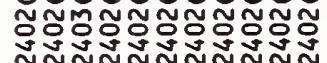
b 造

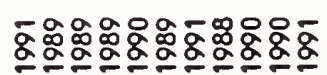

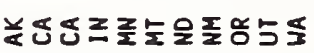

巨్

$\stackrel{\bar{m}}{\stackrel{\sim}{\sim}}$

$\stackrel{\mathscr{g}}{\mathbb{g}}$

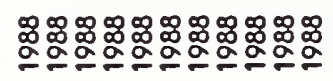
\%ับํำ

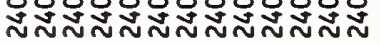

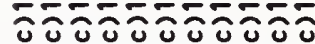

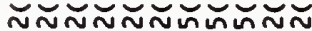

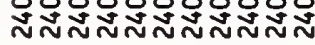

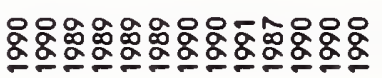

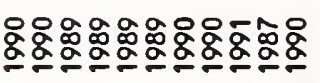

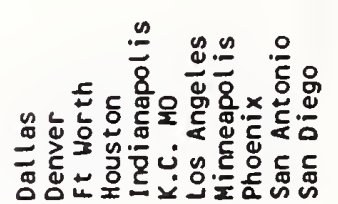

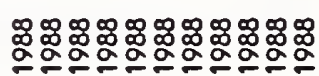

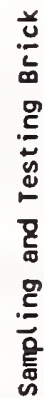

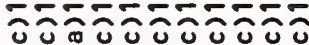

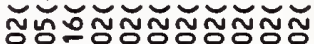

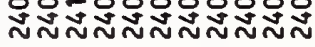

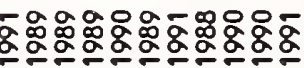

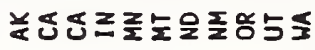

$\bar{\Phi}$

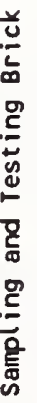

\section{(n)}

s. 
농:영

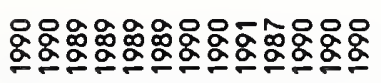

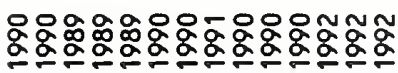

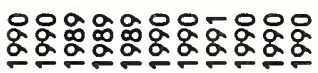

용요용

o.

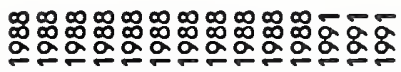

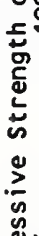

产

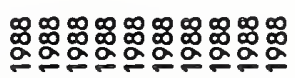

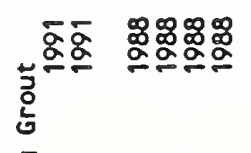

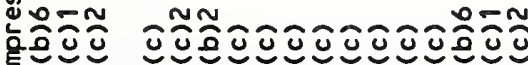

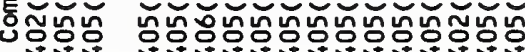

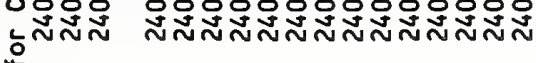

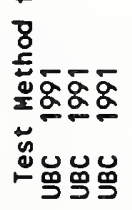

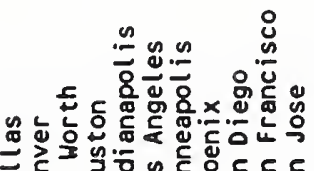

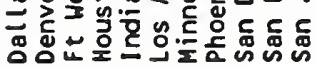

.

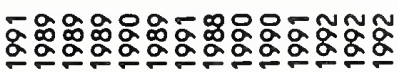

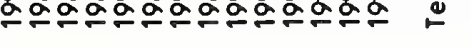

这

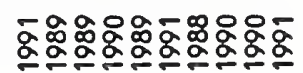

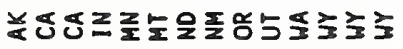

里

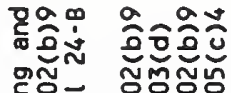

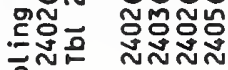
言

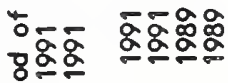

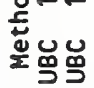
壴前芯 
m

峲 路 !

岁 :

แัน

世 อิ

岁:

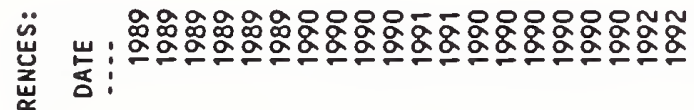

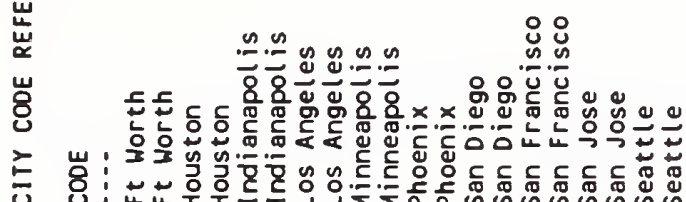

引

w岂 :

ర్జ

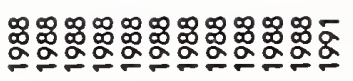

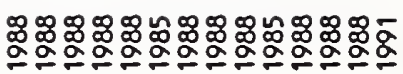

$\overline{\bar{\alpha}}$

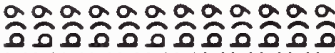

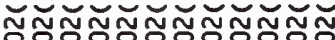

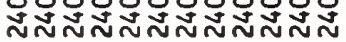

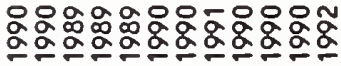

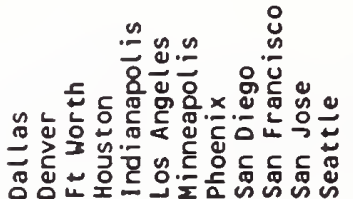

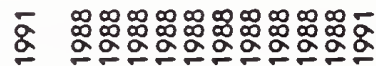

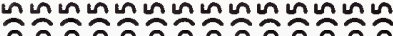

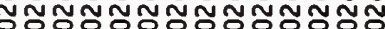

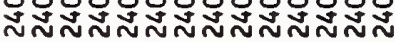

$\tilde{\sigma}$

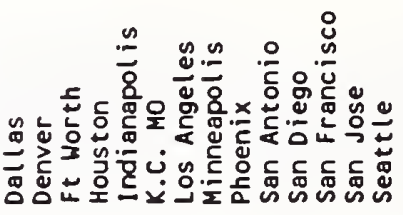

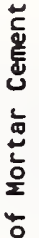

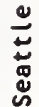

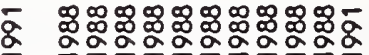

志

$\bar{g}$

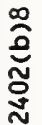

ì

z

은

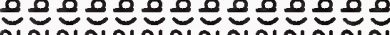

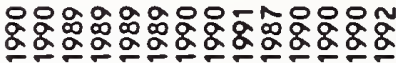
?

可

䓛

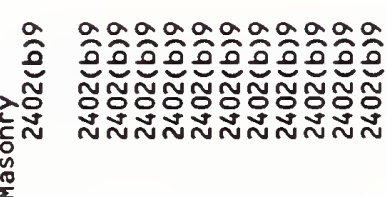

总

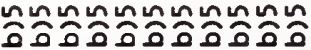
พีกNำ

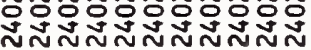

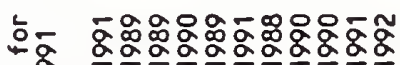

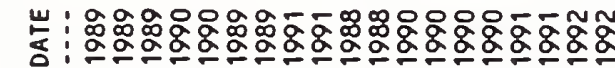

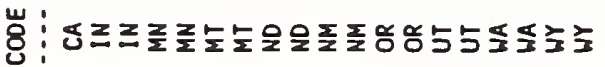

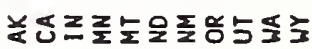

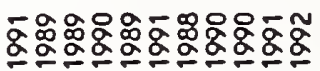
蒙品

dั.

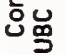

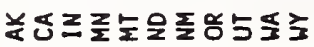

풀



$\bar{g}$

$\bar{g}$

$\bar{\sigma}$

$\stackrel{\stackrel{m}{*}}{\stackrel{m}{\sim}}$

$\stackrel{a}{\sim}$

$\stackrel{m}{\sim}$

i̊ 


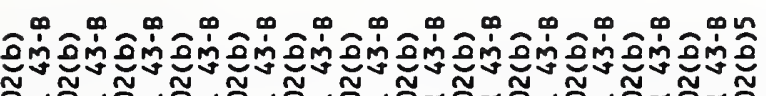

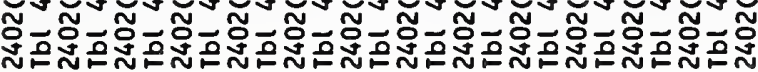

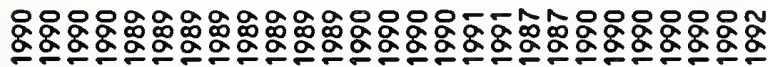

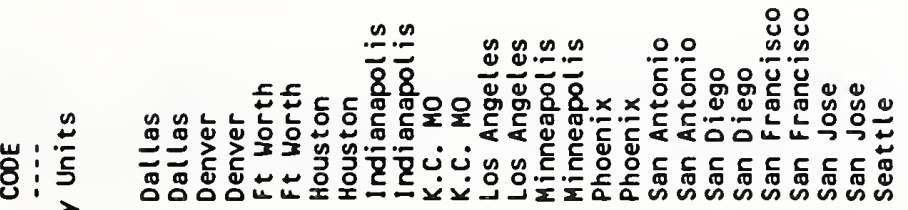

u :

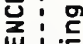

焉

虫的

zoิ

: :

䓛:

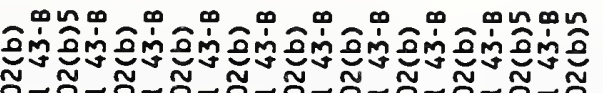

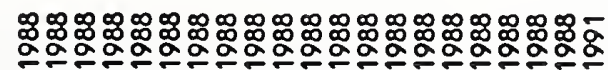

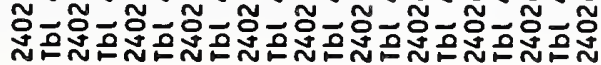

世 : 군

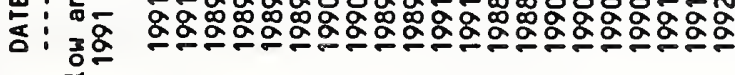

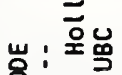

8

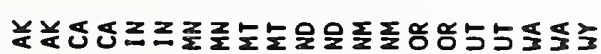

$\bar{g}$

$\bar{g}$

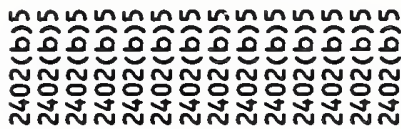

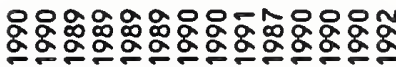

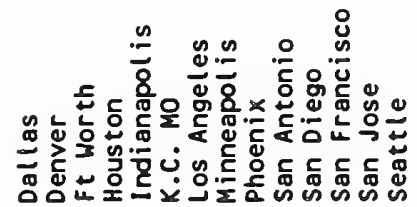

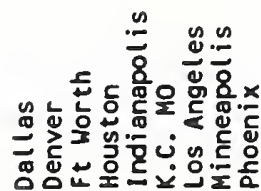

赵

논

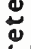

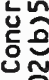

แnนnนnununun

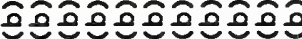

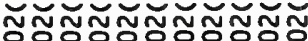

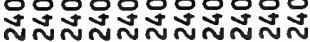

要

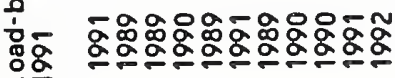

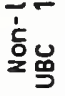

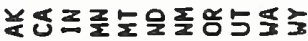

$\stackrel{\leftrightarrow}{5}$

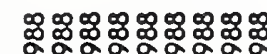

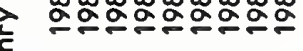

n-nnannan

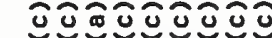

พกับกักสก

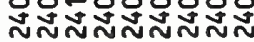

둠

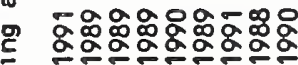

言

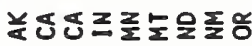

$\bar{g}$

i̊

ì 

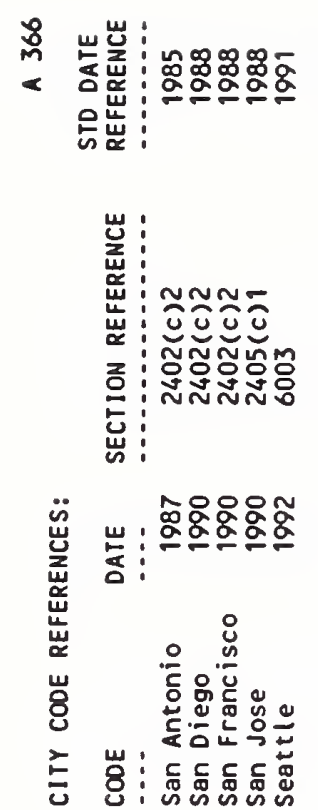

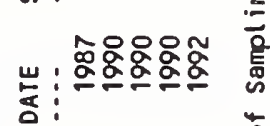

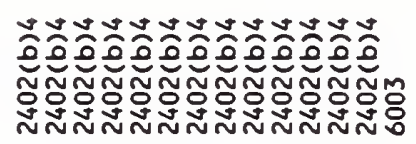

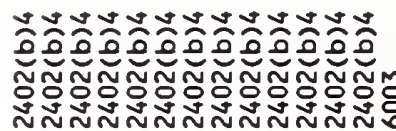

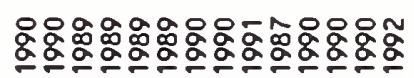

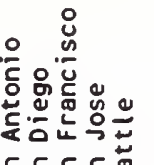

岩: 品品品品嵒

닐

球崫:

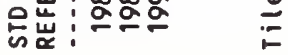

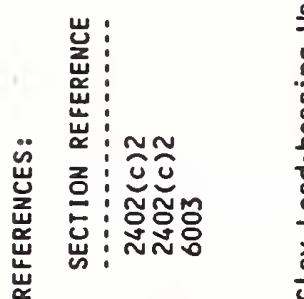

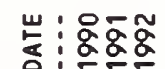

岁:

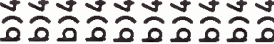

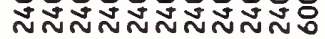

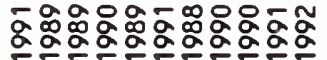

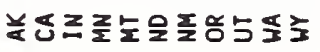

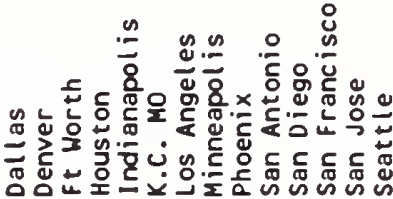

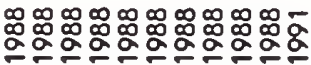

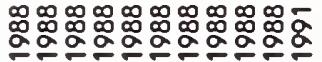

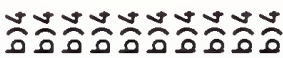

D

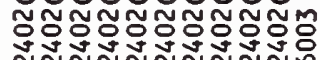

NNNNNNNNNNO

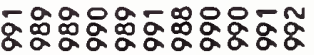

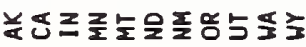

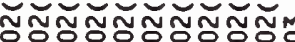

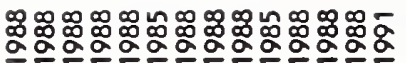

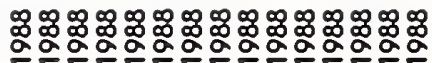

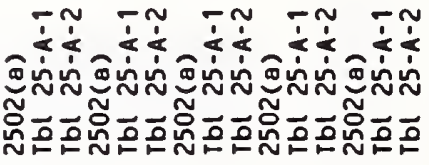

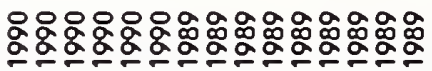

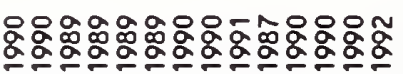

$\bar{g}$

$\bar{g}$ $\therefore \approx \frac{2}{8}$

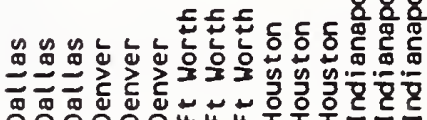

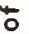

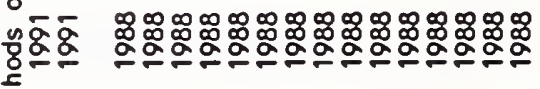
$\frac{4}{2}$

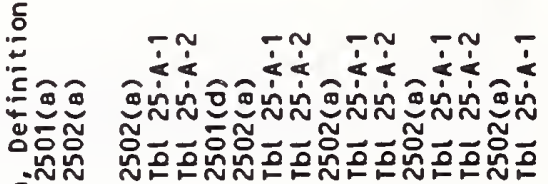
हn

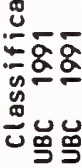

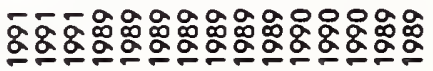

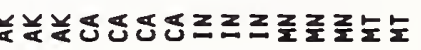

ฒั

思京

$\stackrel{\infty}{\sim}$

ì
宽 
i̊

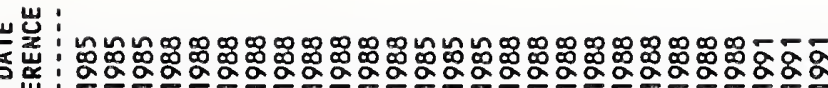
它崖:

㟧

崫

๕

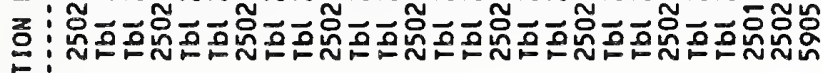

出:

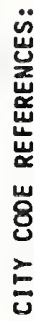

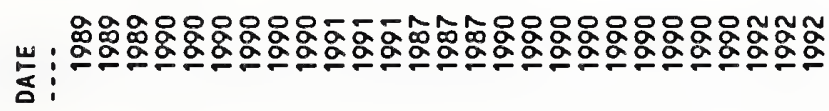

\&

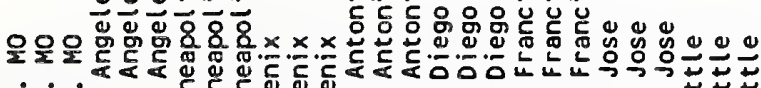
岁

岕 :

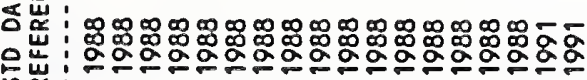

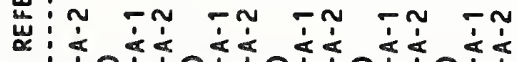

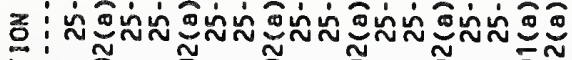

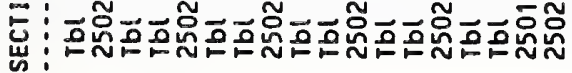

$\ddot{8}$

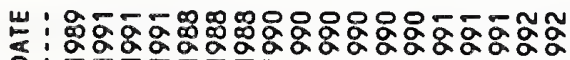

炭

苍

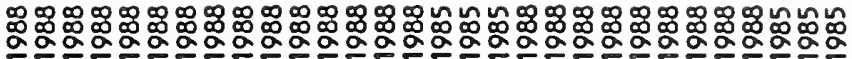

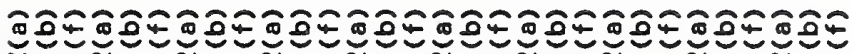

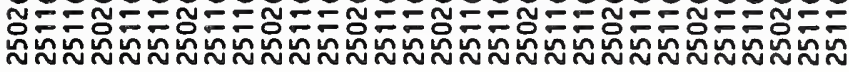

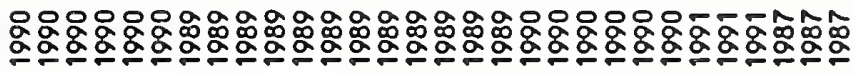

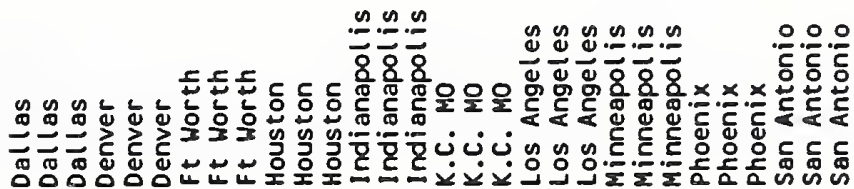

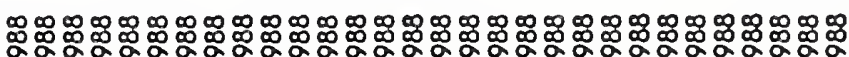

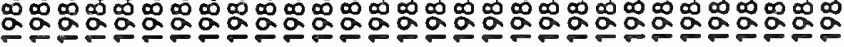

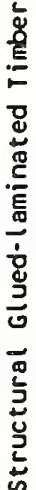

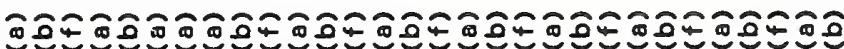
กับำㄴ.

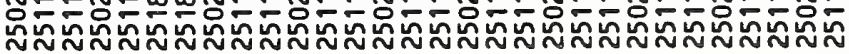

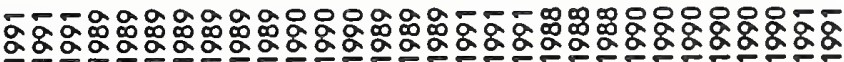

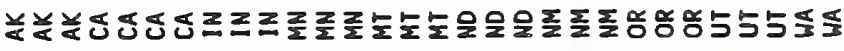

$\stackrel{\mathscr{\Xi}}{\circ}$

๗ํํ 
总

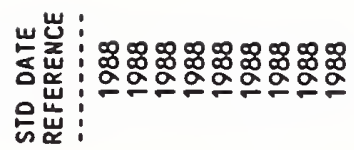

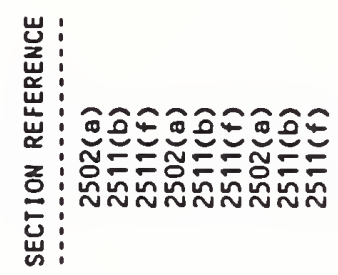

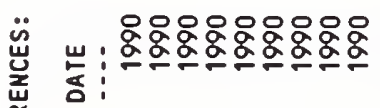

엉요

岁

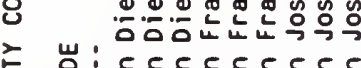

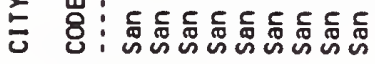

岕:

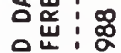

综岕:

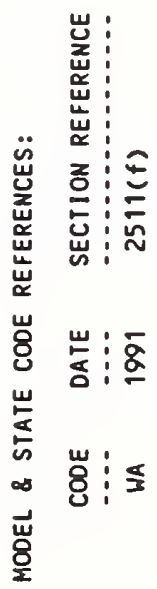

홍

$\stackrel{\mathscr{\Xi}}{\stackrel{0}{g}}$

$\overline{\check{n}}$

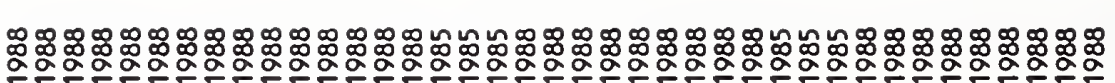

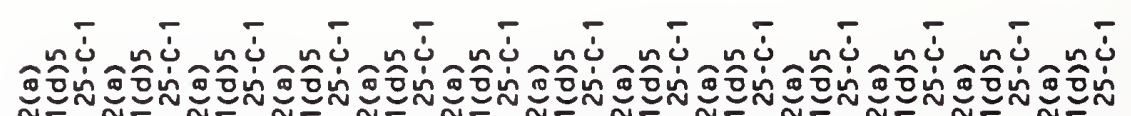

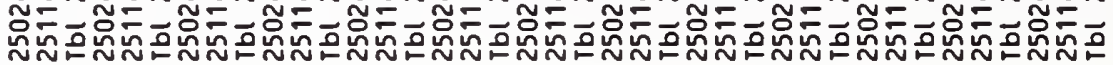

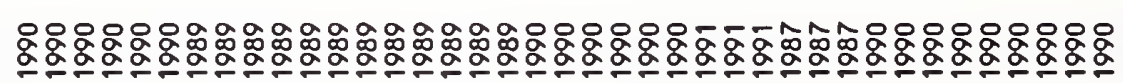$$
\text { 西 }
$$

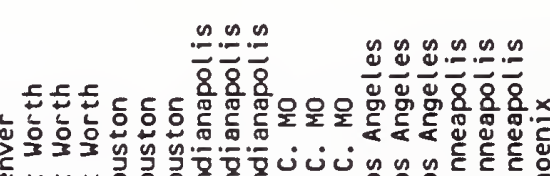

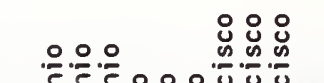

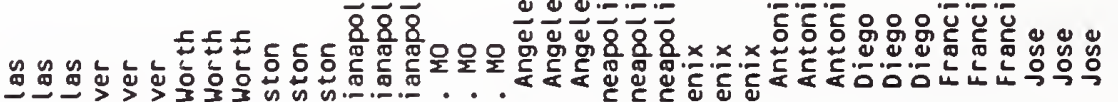

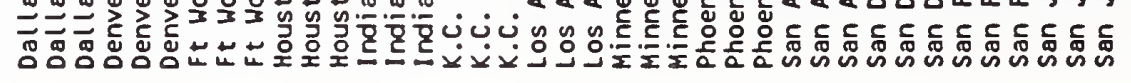

망

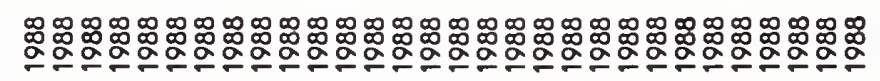

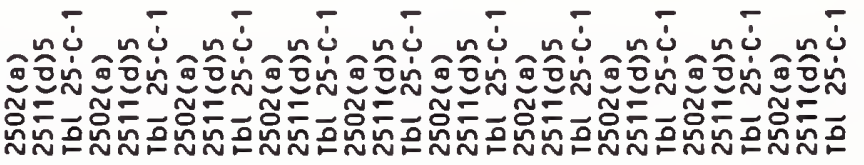

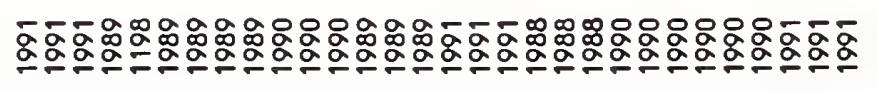

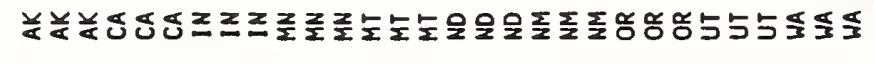

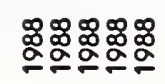

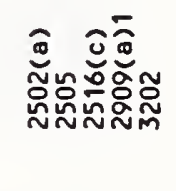

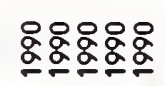

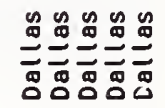

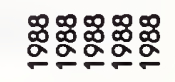

兽

$\stackrel{\mathscr{\Xi}}{\stackrel{8}{ }}$

$\stackrel{\simeq}{ }$ 


\section{它}

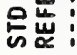

:

苕 紊:

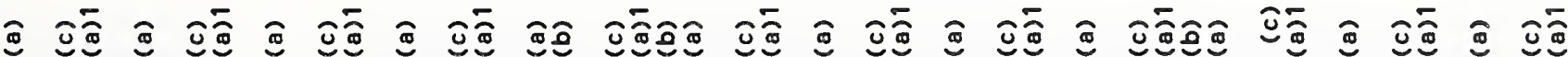

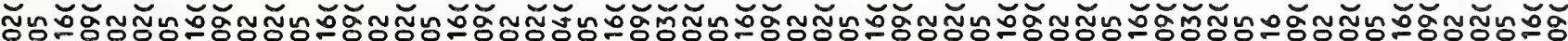

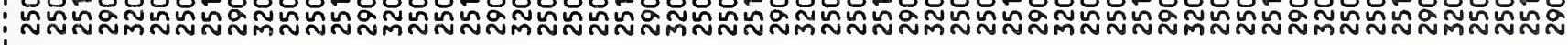

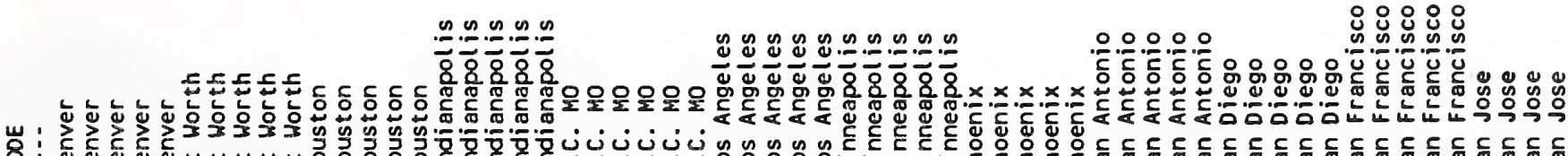

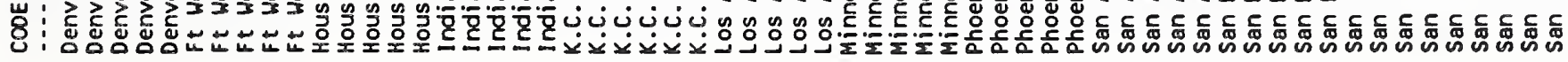

넚

ర్య

它岕 :

$\ddot{w}$

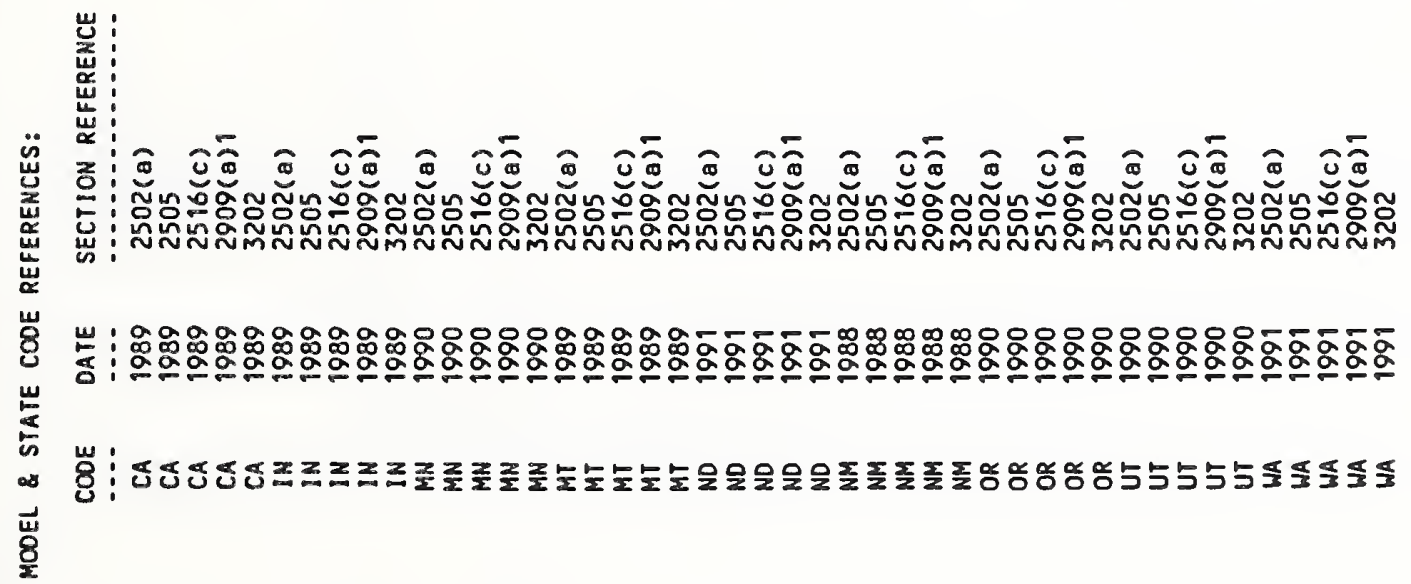

总

$\stackrel{\text { 岁 }}{\stackrel{\sim}{\sim}}$ 


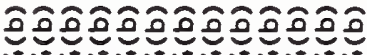

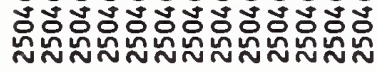

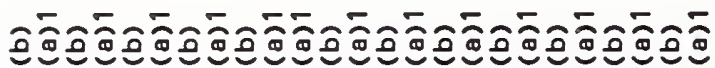

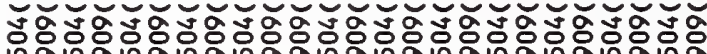

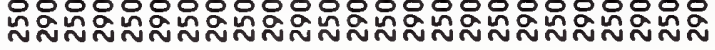

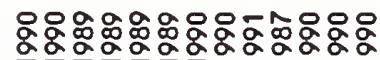

:

\begin{abstract}
$$
\text { (1) }
$$
\end{abstract}

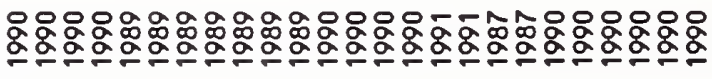

崖岕

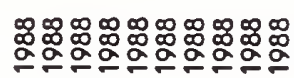

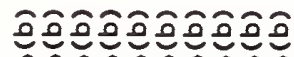

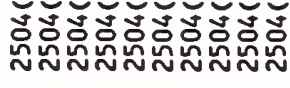

岕:

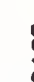

ب.:

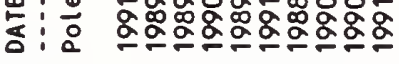

.

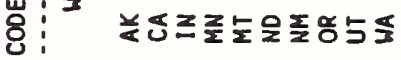

$\stackrel{\mathscr{a}}{\underline{\alpha}}$

$\stackrel{\not ̊ g}{\circ}$

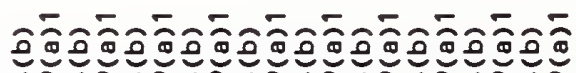

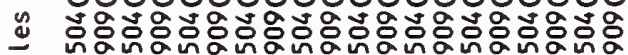

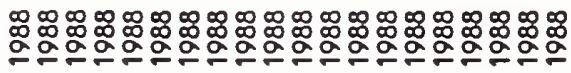

\section{$\bar{\alpha} \bar{\alpha} \bar{\alpha}$}

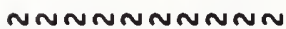

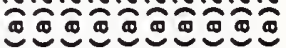

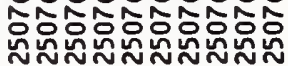

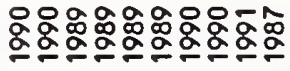

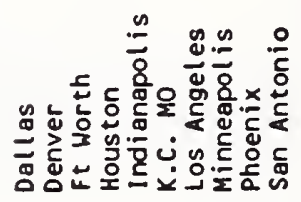

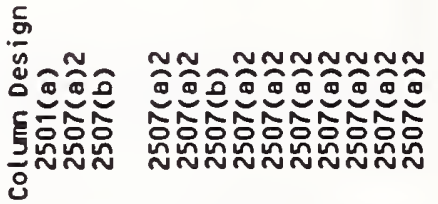
है

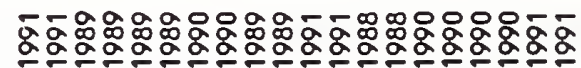

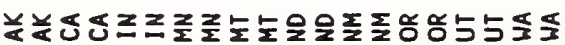

7

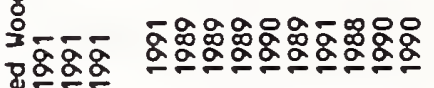
政 in

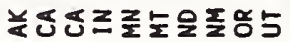




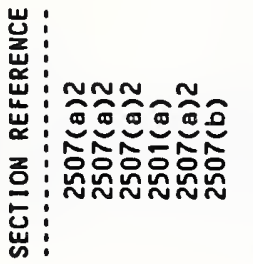

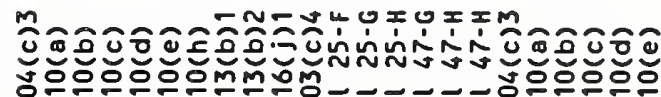

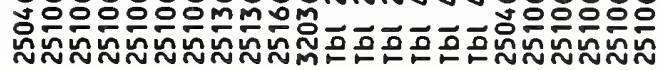

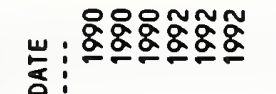

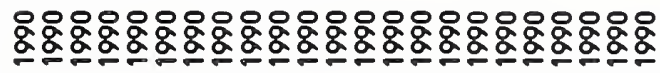

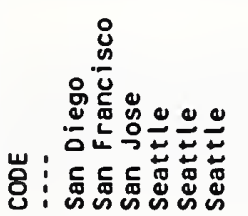
岂岂

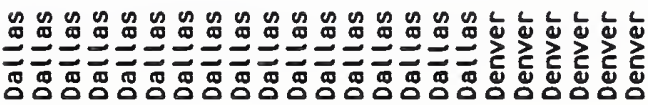

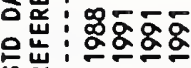

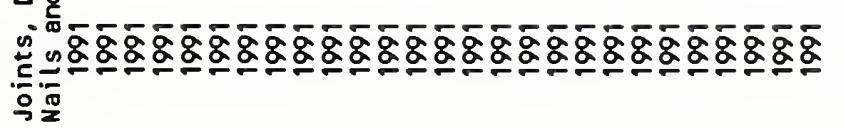

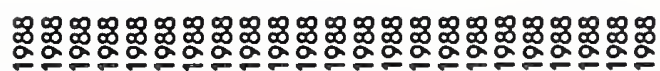

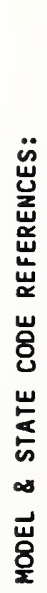

:̈

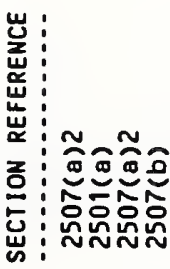

总畕

욤

邹

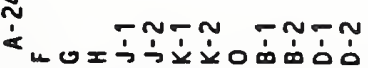

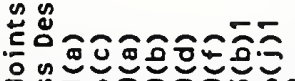

ì

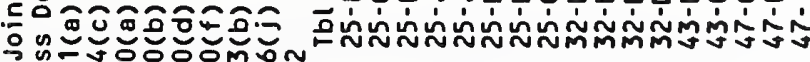

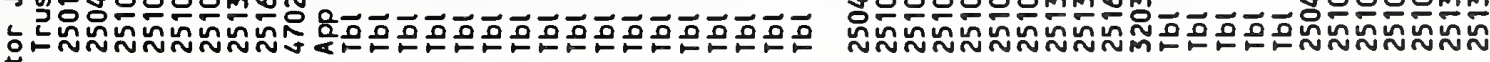

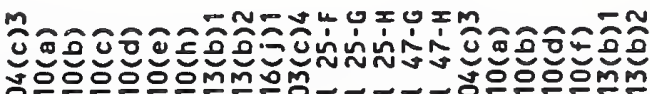

แะร 능응

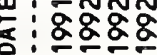

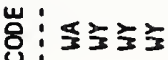

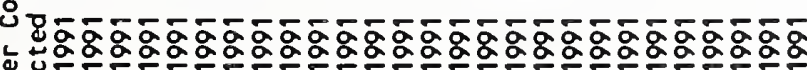

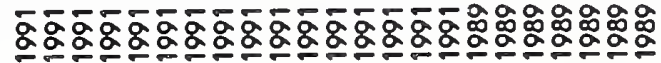
要要

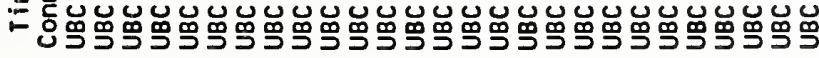

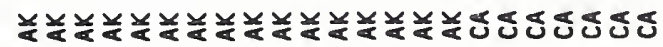<smiles>[CH]=C</smiles>

$\frac{\sim}{\grave{n}}$

$\stackrel{n}{\stackrel{n}{\sim}}$

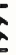




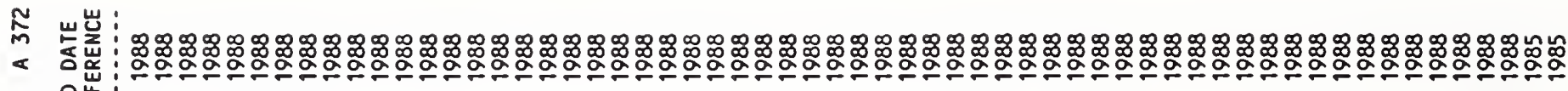
踤:

䕉:

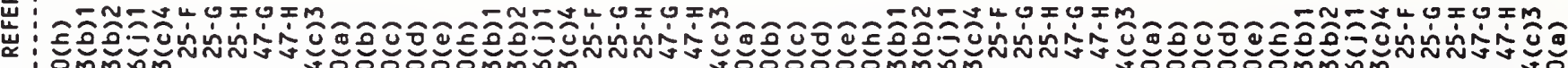

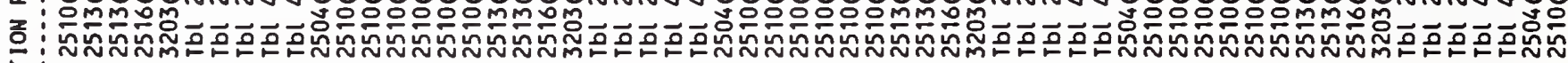
跑: 苞

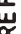

岁

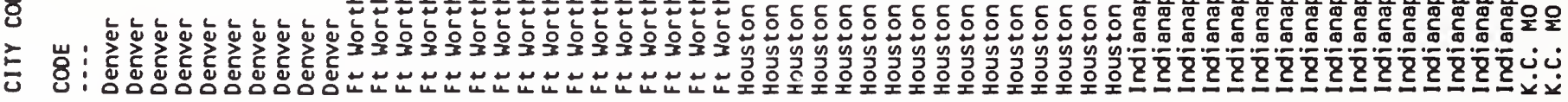

崖:

焉 눈

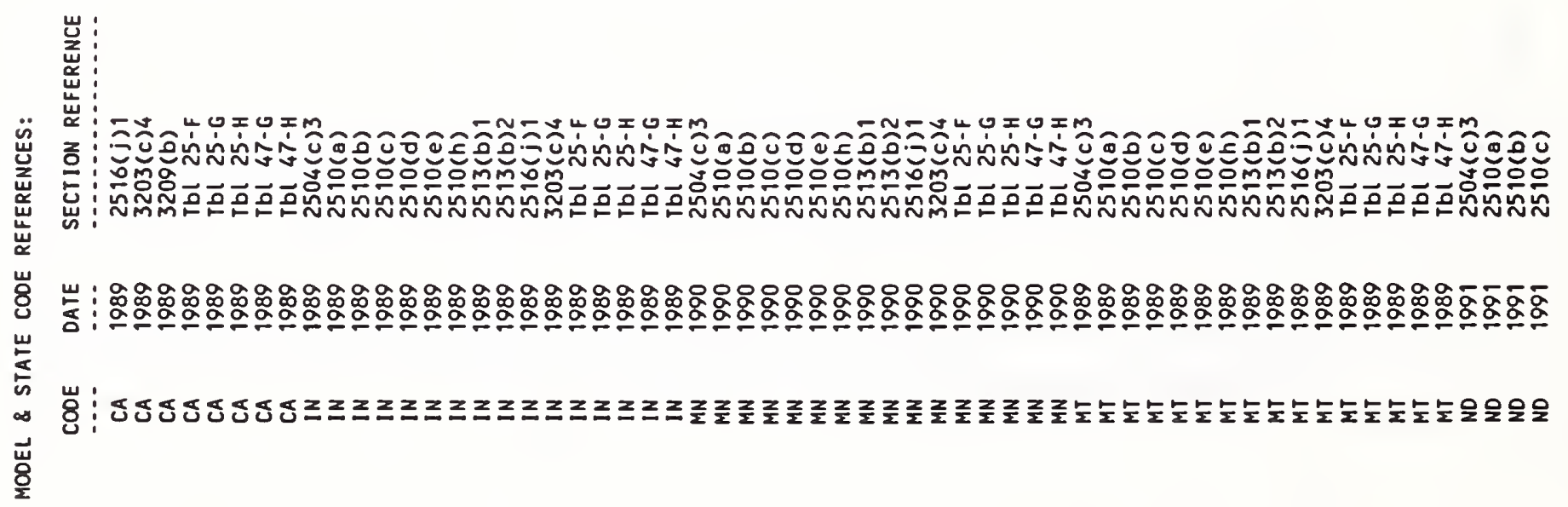

옹

茴 


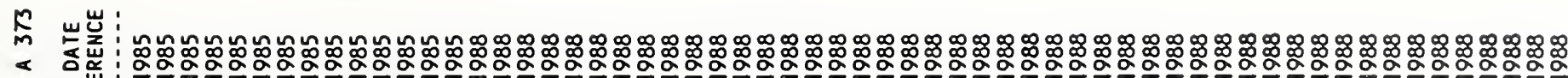
呈䋇 :

岂

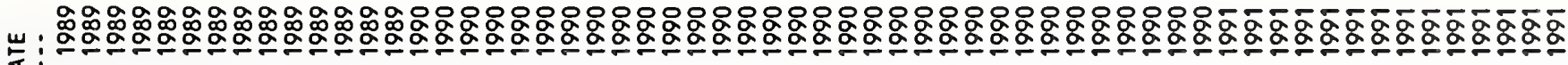
s:

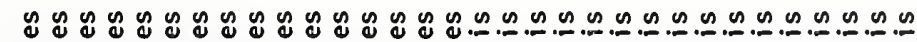

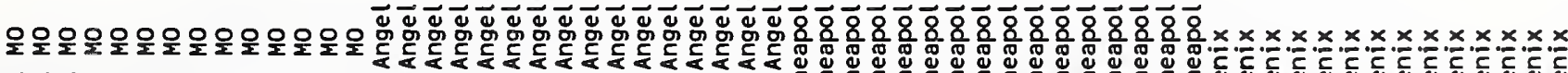

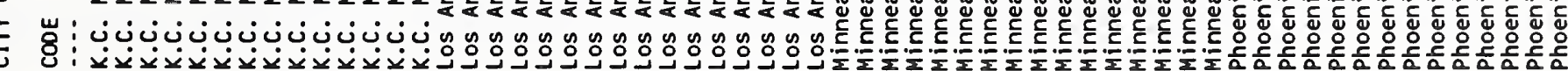

岁:

完离:

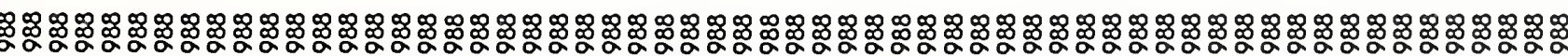

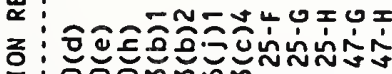

:

-

-

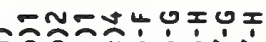

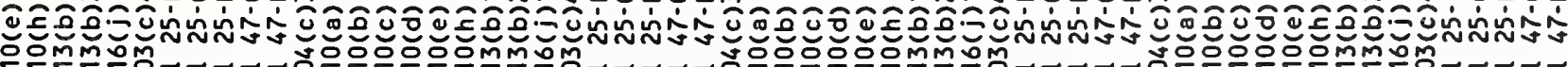

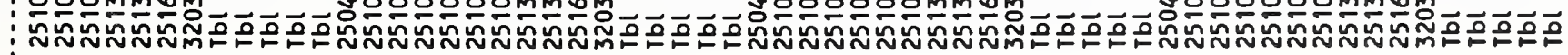

는

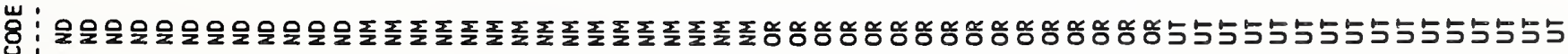

옹

茴

$\stackrel{\sim}{\dot{\sim}}$ 


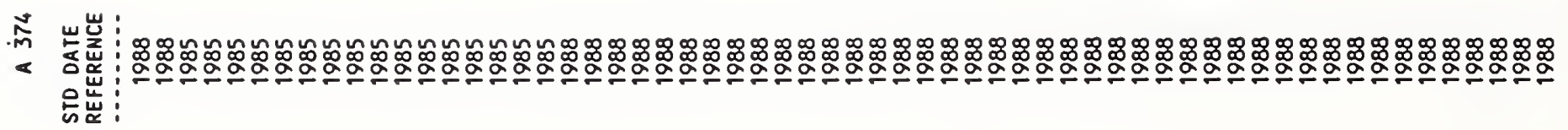

岂:

崖

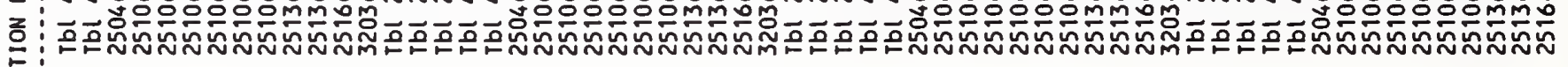
出:

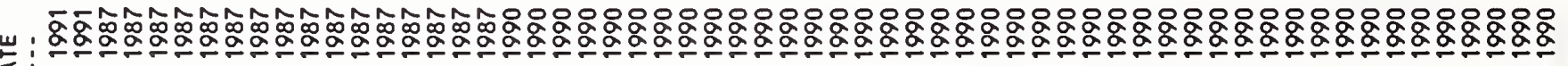
б:

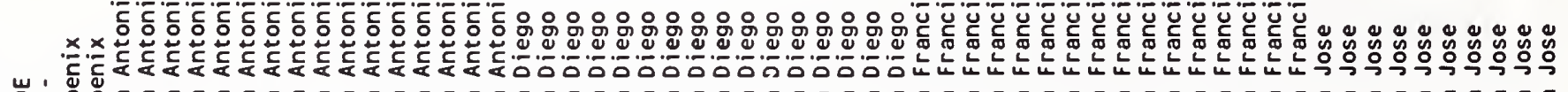

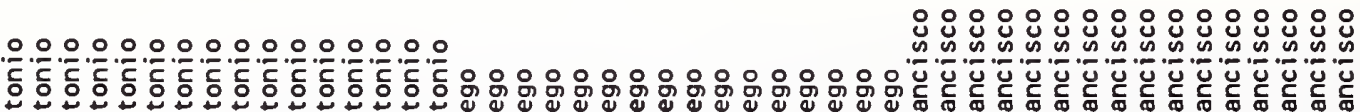
至

崖 :

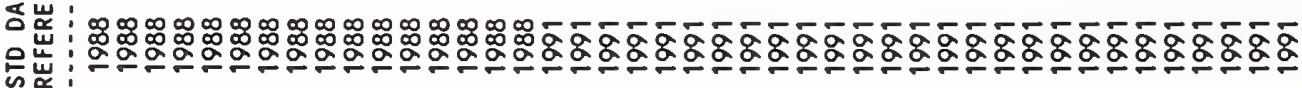

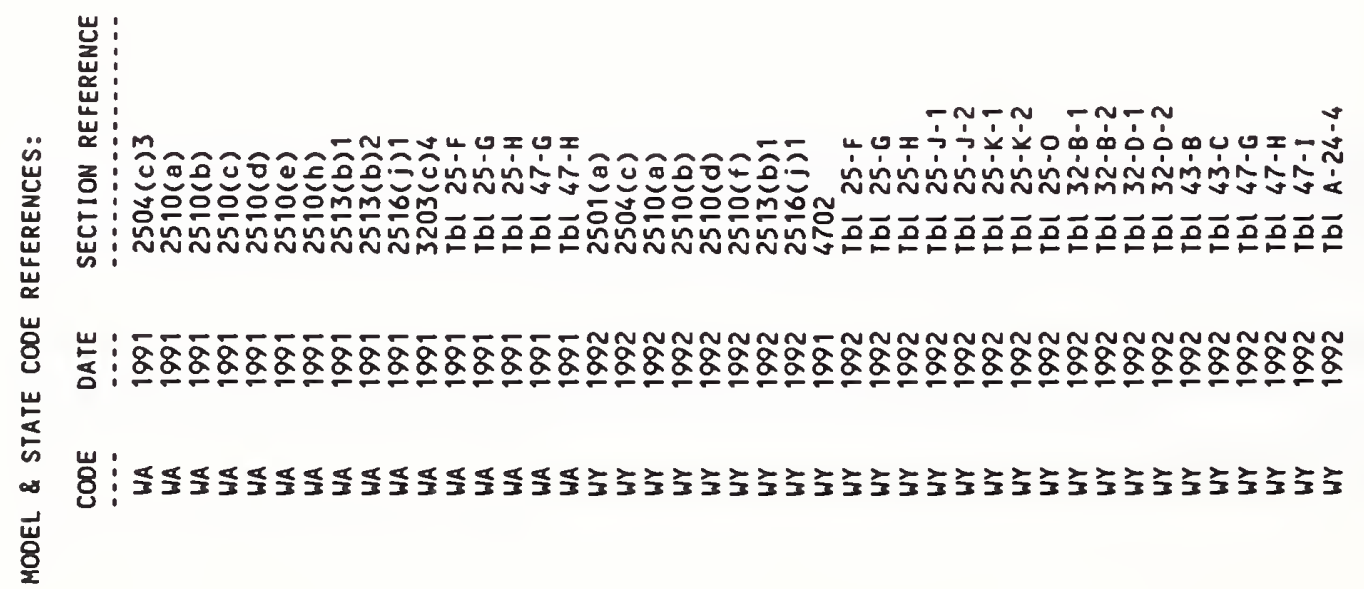

突

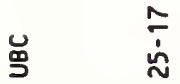




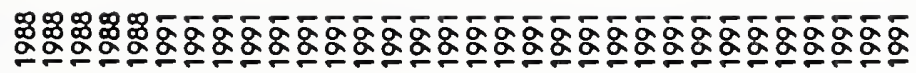
응누

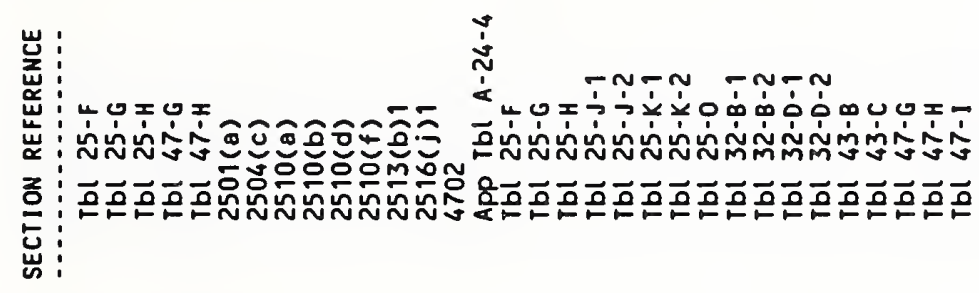

菏

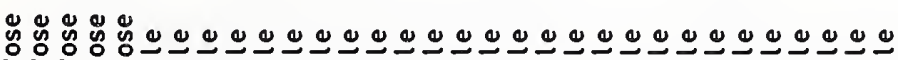
ర్ 。 岕岕

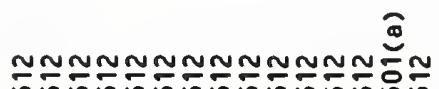

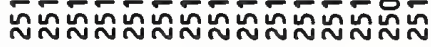

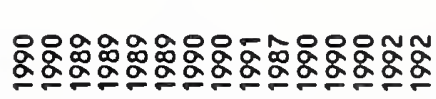

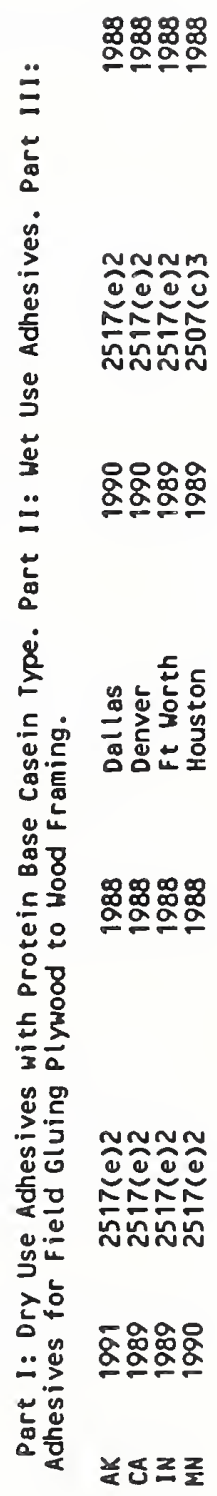

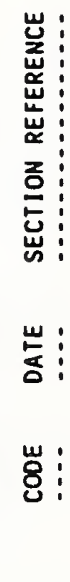

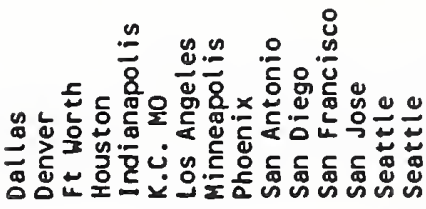
$\frac{1}{3}$

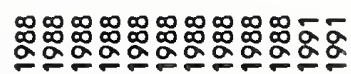

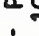

\$

لئ

ํㅗㅇ

$\bar{\alpha}$

$\underset{8}{\stackrel{8}{2}}$

ڤ்

$\stackrel{\infty}{\grave{n}}$ 


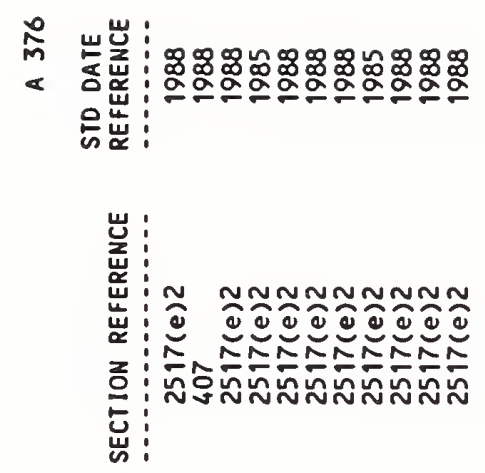

蒿

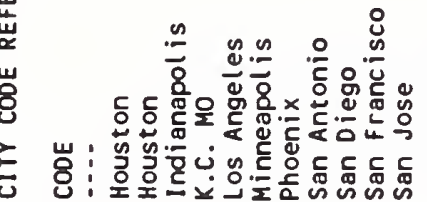

㟧:

s.

눈

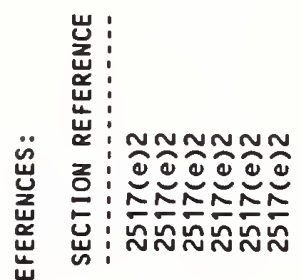

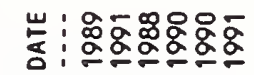

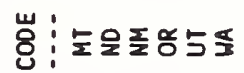

芒

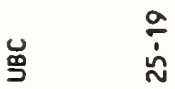

\section{: \$:

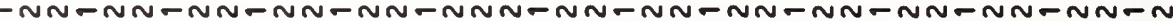

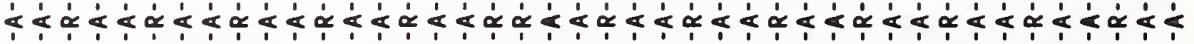

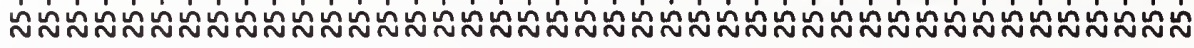

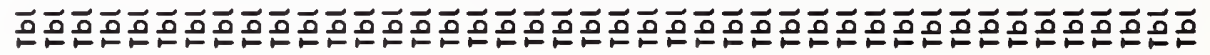

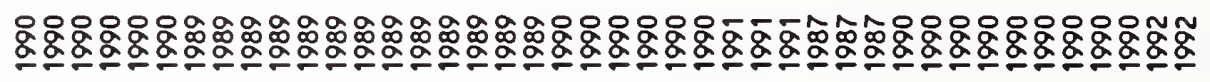

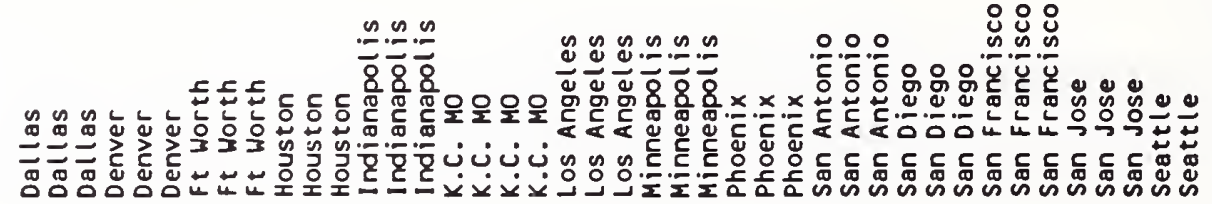

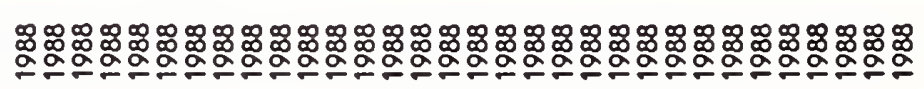

눙

宁的

ic

ผัญ

matn -nn-nn-nn-nn-nn-nn-nn-nn-nn-nn-n

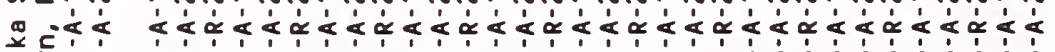

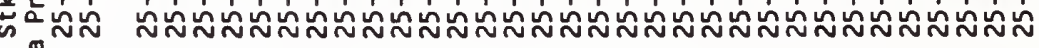

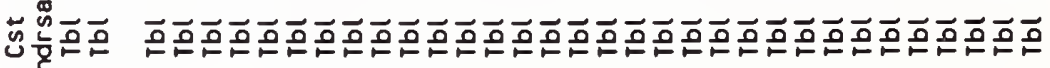

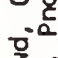

总

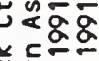

ب

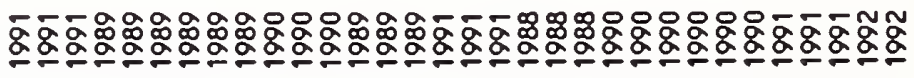

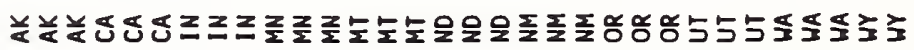




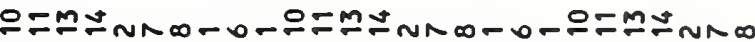
$\dot{j} \dot{\alpha} \dot{\alpha} \dot{\alpha} \dot{\alpha} \dot{\alpha} \dot{\alpha} \dot{\alpha} \dot{\alpha} \dot{\alpha} \dot{\alpha} \dot{\alpha} \dot{\alpha} \dot{\alpha} \dot{\alpha} \dot{\alpha} \dot{\alpha} \dot{\alpha} \dot{\alpha} \dot{\alpha} \dot{\alpha} \dot{\alpha} \alpha \dot{\alpha} \dot{\alpha} \alpha \dot{\alpha} \alpha \dot{\alpha} \alpha \dot{\alpha}$ فُ

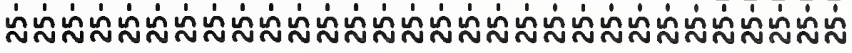

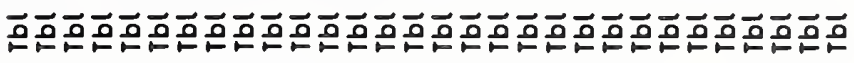

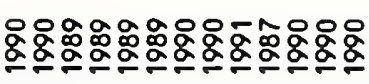

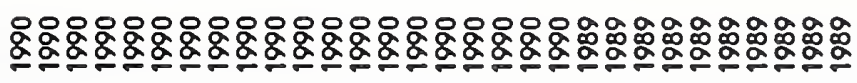

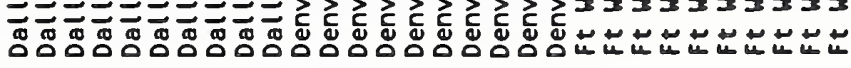

:

离

岂: :

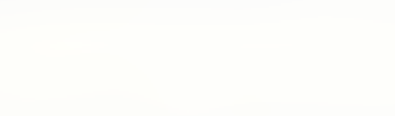

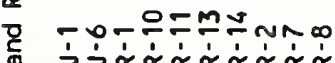

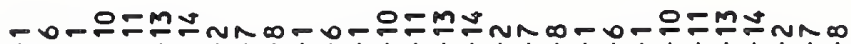

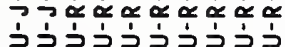

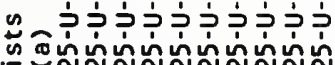

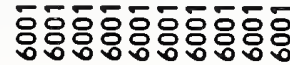
วับ

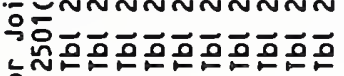

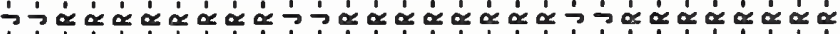

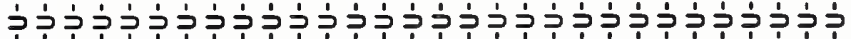

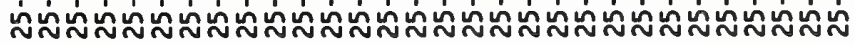
$\stackrel{2}{2}$

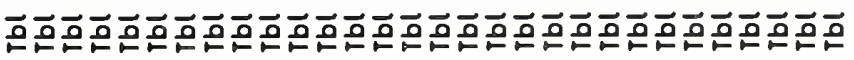
\&

岁

: : 品

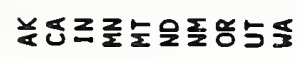

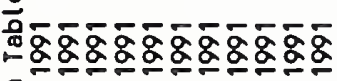

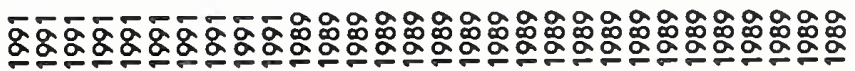

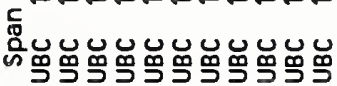

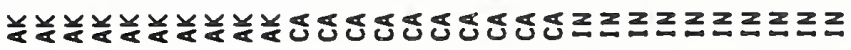


岕:

: 은

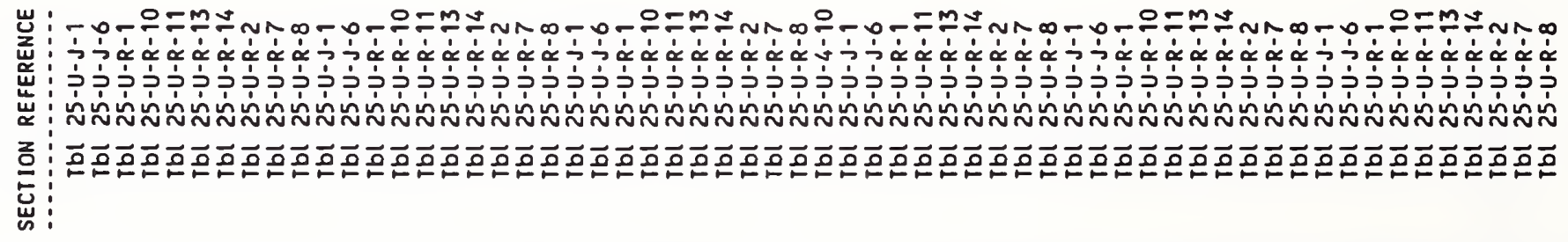

س : :

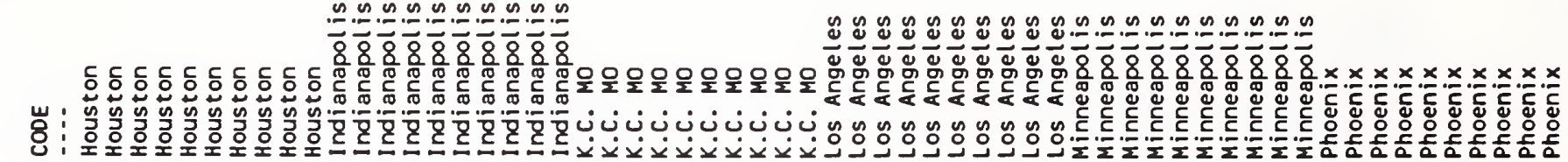

世닌

通

宸

بّ山⿸丆口

$\underset{\sim}{\sim}:$

$0-m v$

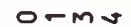

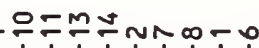

으ำำ

으ำ논

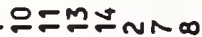

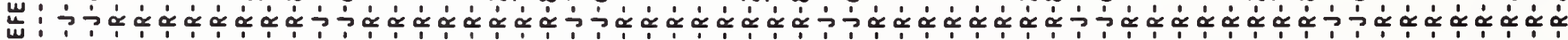
أخ

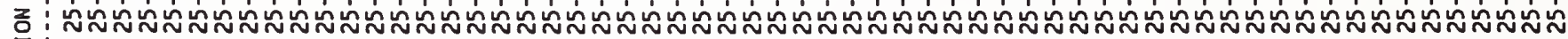

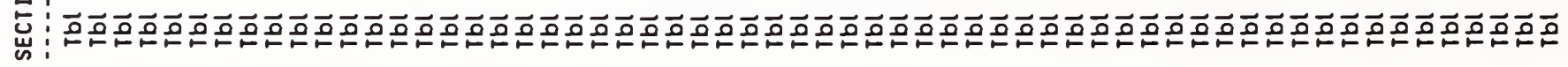

范

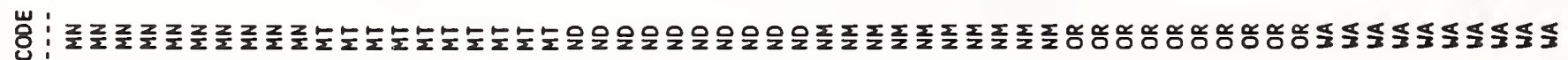

ટ્

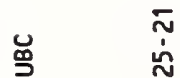


닐

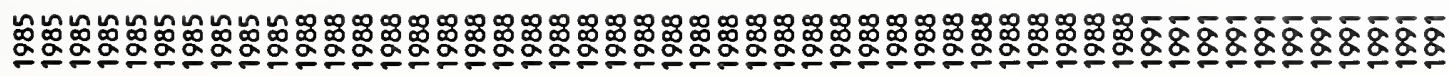

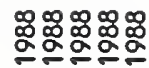
㩆崖:

岁 : -

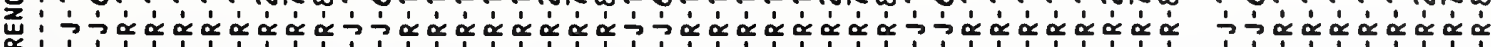

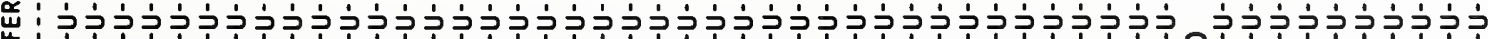

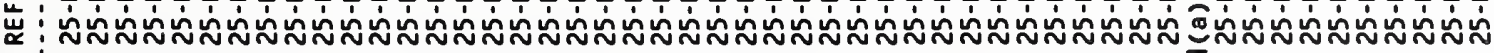

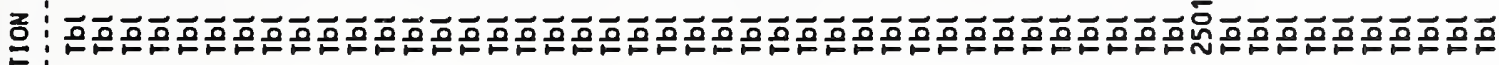
崫:

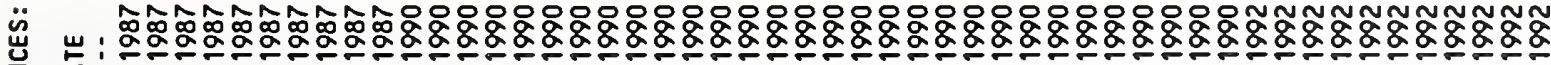
安:

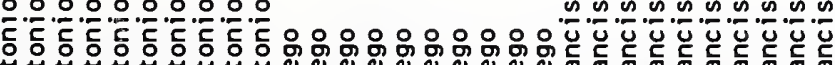

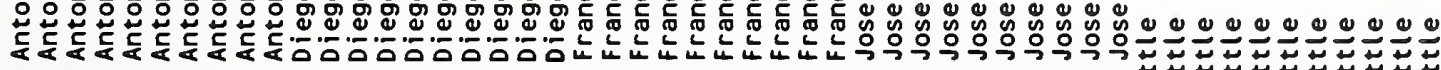

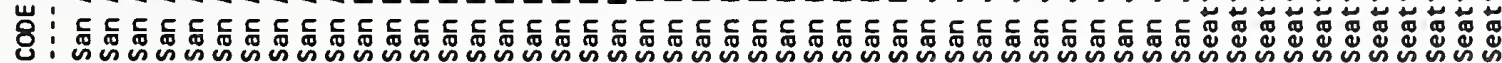

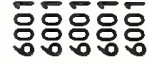

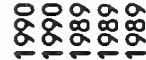

岕:

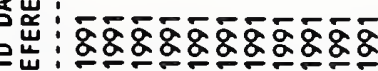

山् :

:

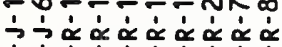

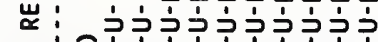

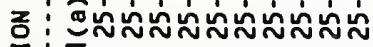

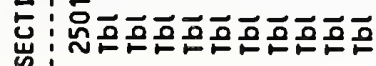

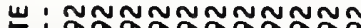

ธุ․

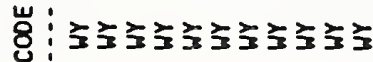

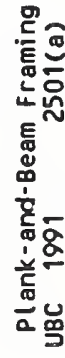

흏ㅎㅇㅎㅎㅇ

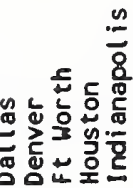

ت̈

울

巳ั

\&

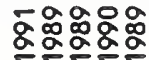

눙저조롤

జ

$\bar{\sim}$

$\approx$ 
啹 岕:

㖉 趾:

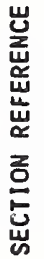

岂

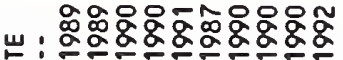
a :

岕:

济

केष:

嵌

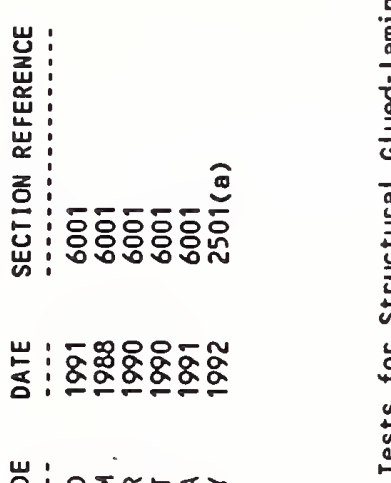

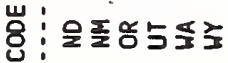

ક્ષ

$\stackrel{9}{\mathfrak{g}}$

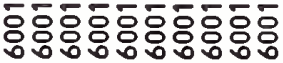

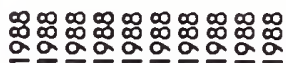

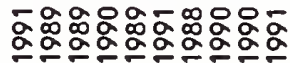

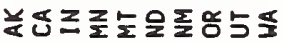

$\stackrel{m}{\mathfrak{n}}$

$\widetilde{\sim}$

กิ

:

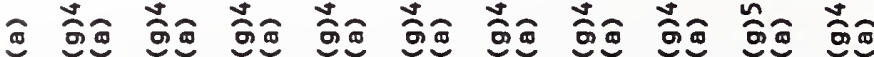
สับำง

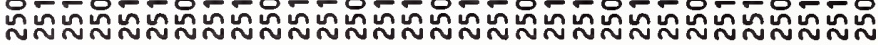

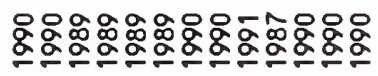

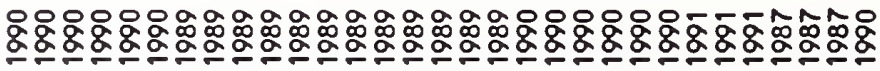

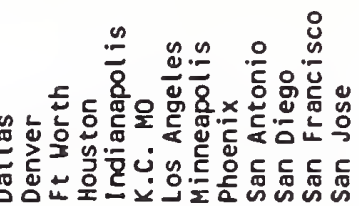

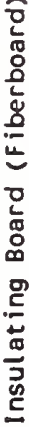

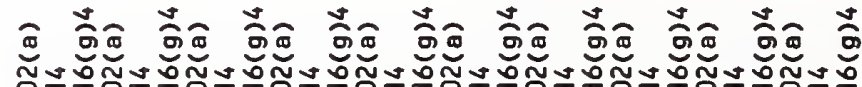

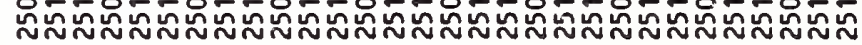

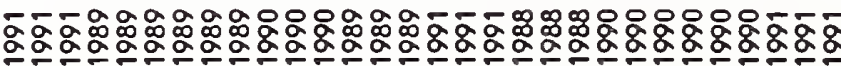

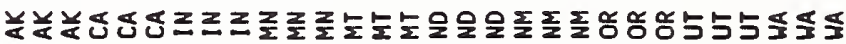


兽

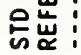
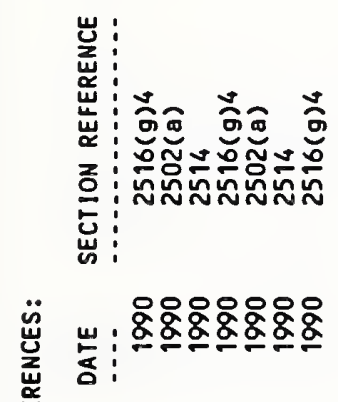

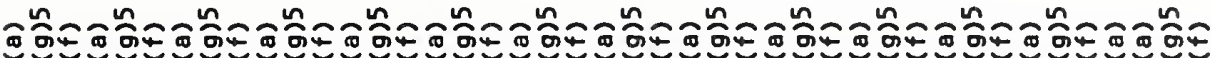

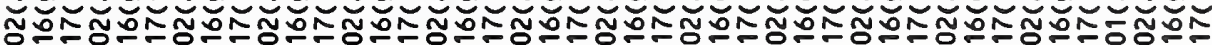

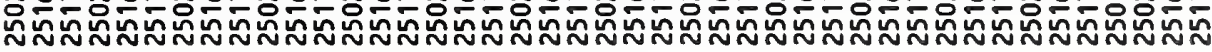

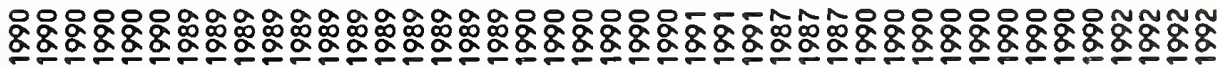

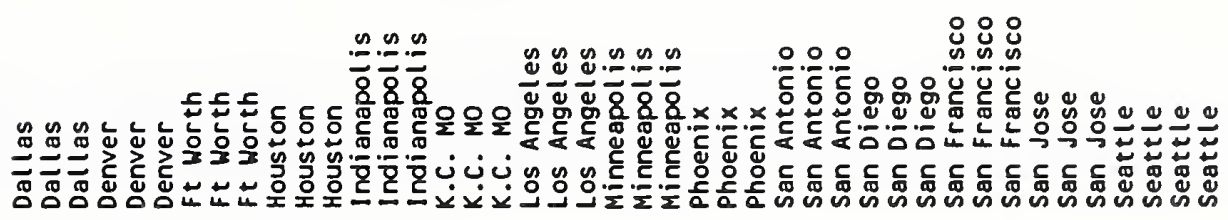

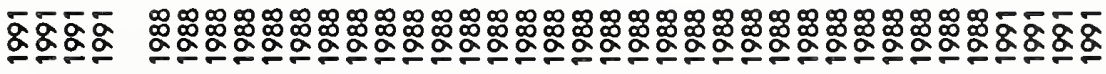

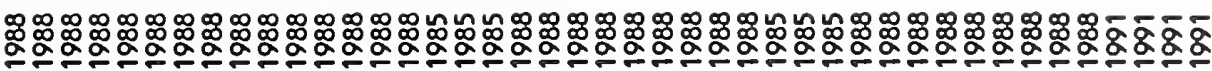

通品

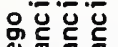

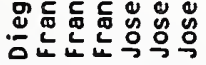

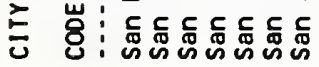

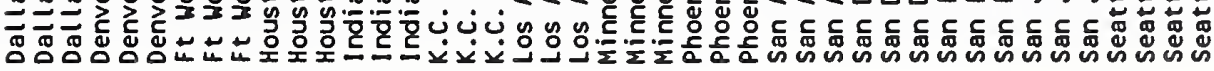

!

㟧:

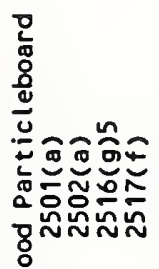

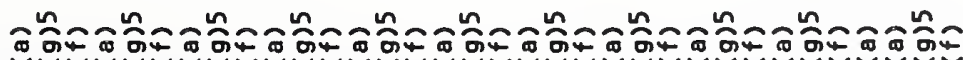

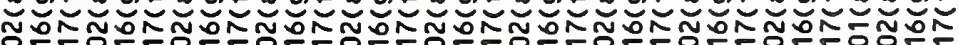

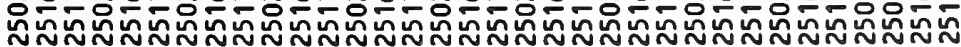
ס

岩

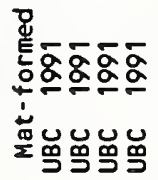

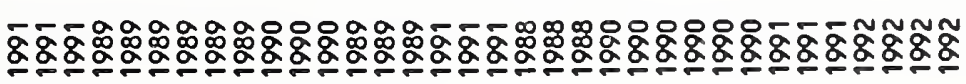

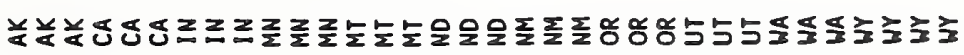




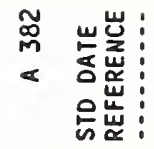

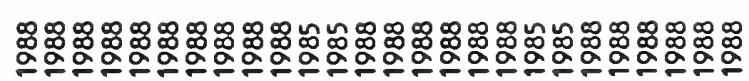
๔

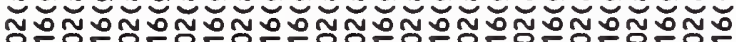

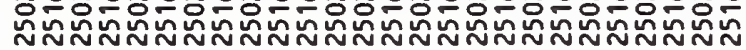

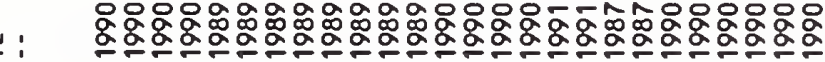
d! 崖

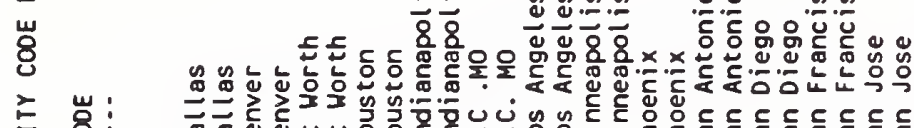

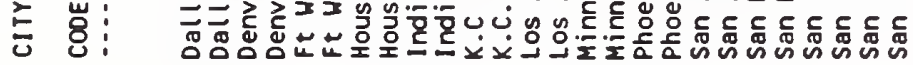

崖岕

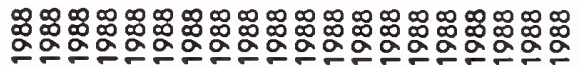

๓⿳亠े冋

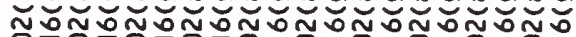
:

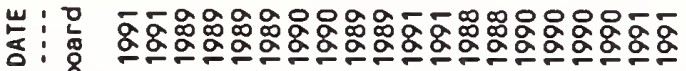
,

\&:

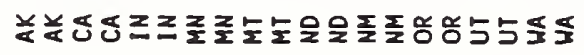

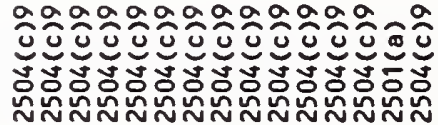

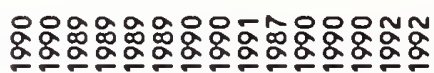

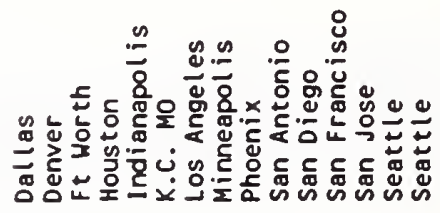

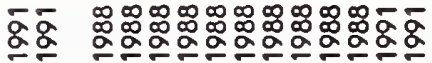

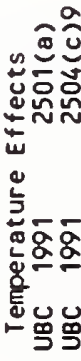

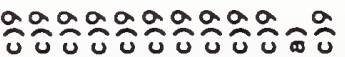

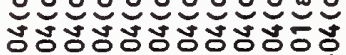

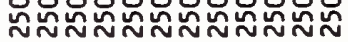

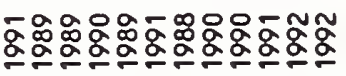

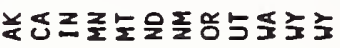

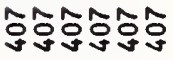

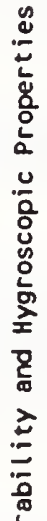

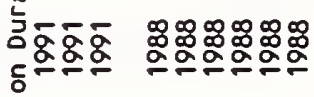

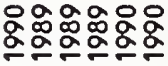

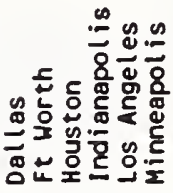

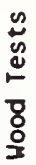

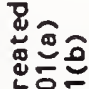

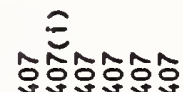

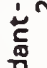

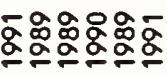

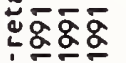

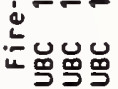

눙저ㄴㅡㅗ로도을

怘

$\bar{g}$

$\bar{\Xi}$ 
m

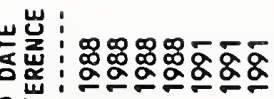

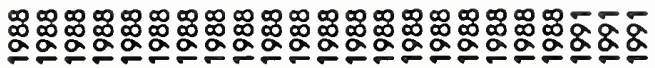

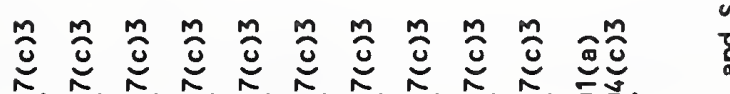

:

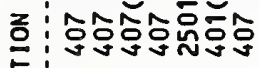

出:

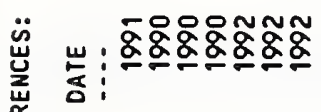

崫

岁

¿

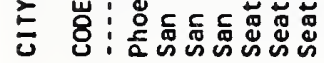

世岕

范崫

은

$\bar{\alpha} \bar{\alpha} \bar{\alpha}$

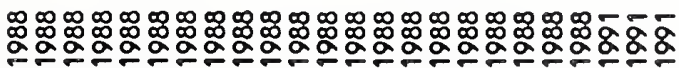

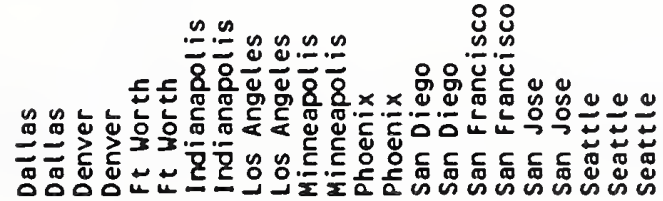

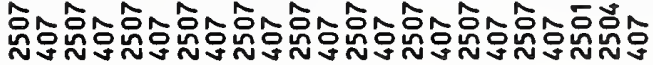

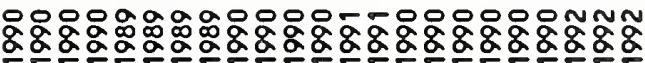

马

동
产

z ฮิ

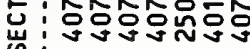

ठㅇㅇ

แ :

ธุ :

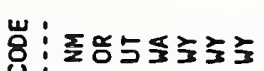

8

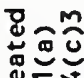

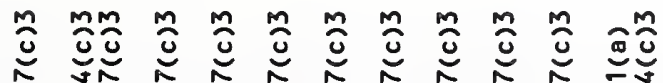

i

พื:

10010000000010000

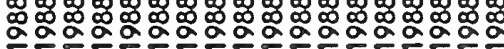

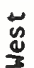

䛎-

๙

ณก๊

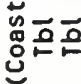

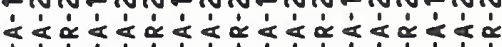

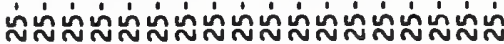

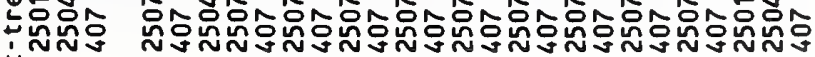
密

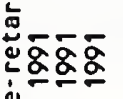

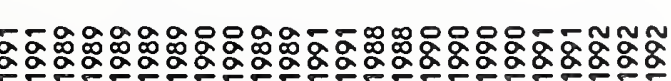

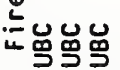

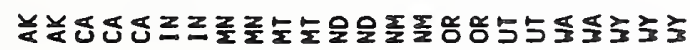

$\therefore$

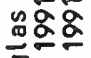

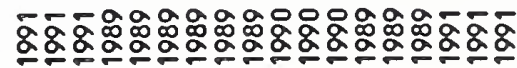

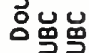

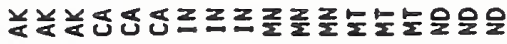

ธِّ

임

న

$\dot{ஸ}$

$\dot{m}$ 
品

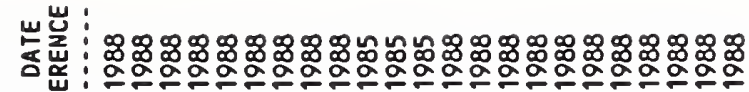
은

莀

Didn ए : $<<\alpha<<\alpha<<\alpha<<\alpha<<\alpha<<\alpha<<\alpha<<$

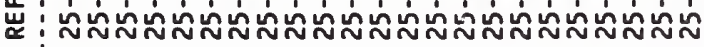

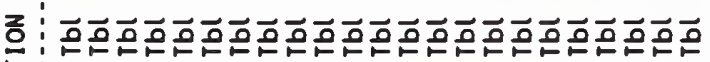
出:

芯

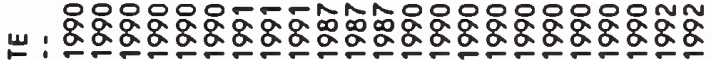
各:

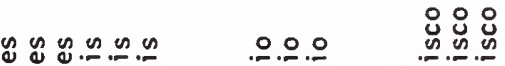

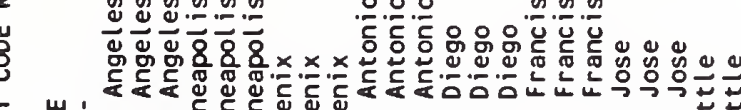

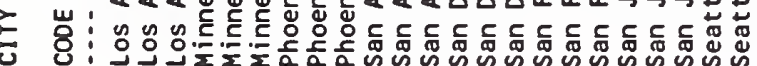

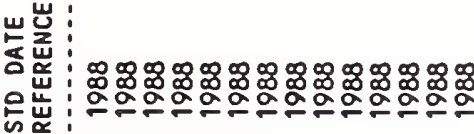

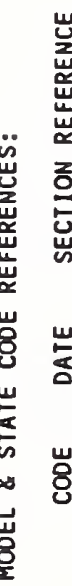

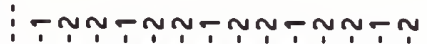

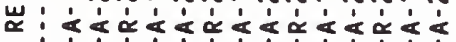

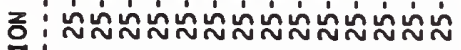

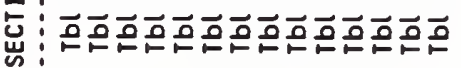

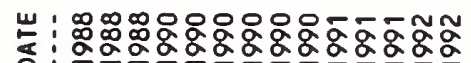

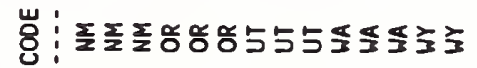

ํㅗㅇ

$\bar{\xi}$

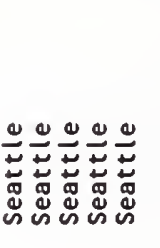

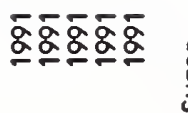

弯

ฮิํํำํํำ

$\frac{9}{8}$

웅ํㅁㅇํㅇㅇㅛ

กัฒ令

กักกั

gू잉

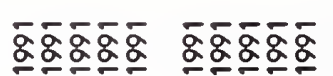

孪

督

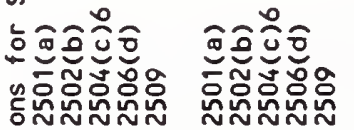

$\frac{5}{2}$

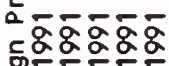

ก N N N

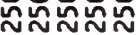

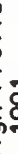

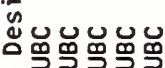

go $\alpha \underline{\alpha} \alpha \underline{\alpha}$

空空学方

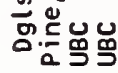

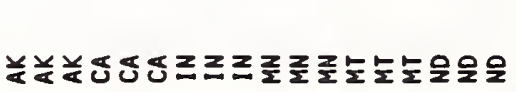

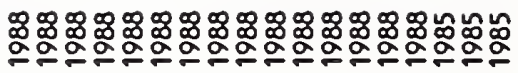

- hn$<<\alpha<<\alpha<<\alpha<<\alpha<<\alpha<<\alpha$

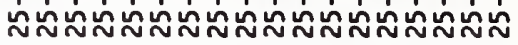

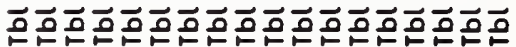

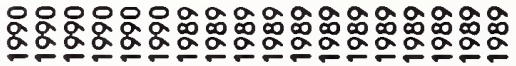

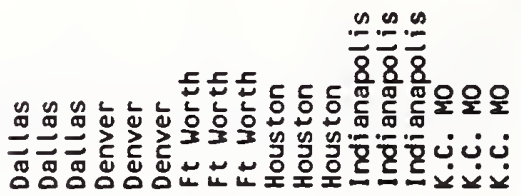

는

늗 토

은돈

: 는

$\stackrel{5}{\Sigma}$

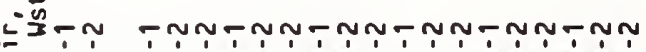

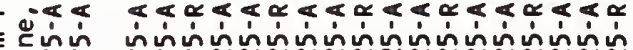

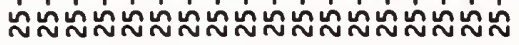

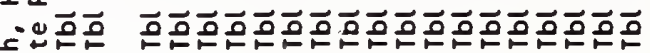
家至

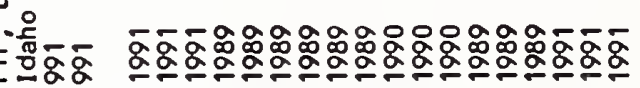
$-\underline{\underline{\alpha}}$

$\underset{\mathscr{\Xi}}{\stackrel{\infty}{\underline{0}}}$

ฒั

ฝ่ 


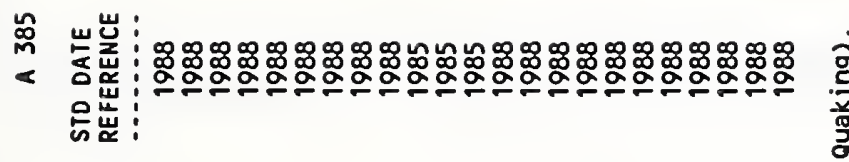

岁:

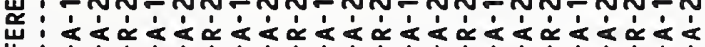

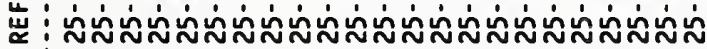

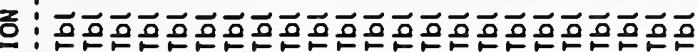
惫:

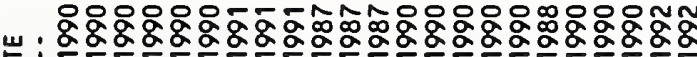
客:

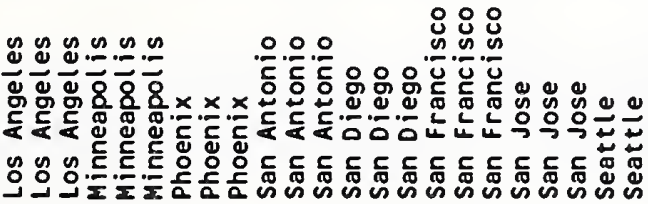
岀 :

S 佔 :

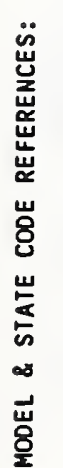

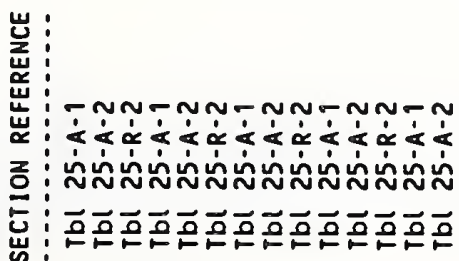

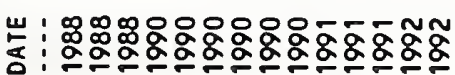

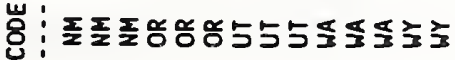

ํํㅇ

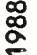

品

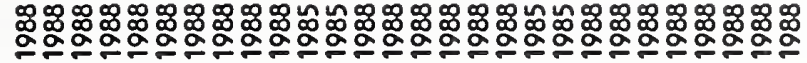

Tn-

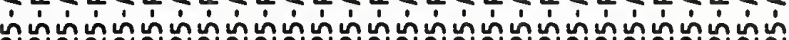

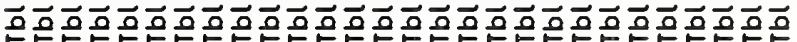

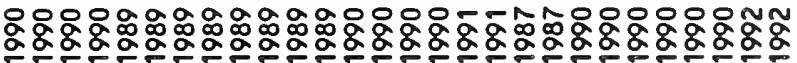

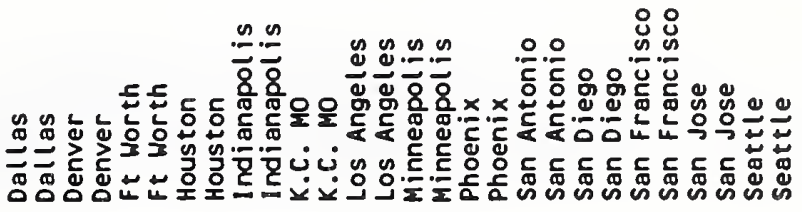

$\infty$

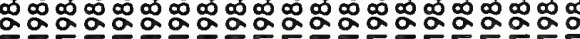

$\frac{\text { I }}{a}$

$\stackrel{2}{\frac{5}{3}}$

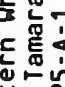

- ก-

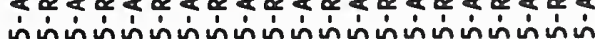

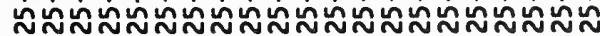

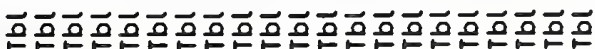

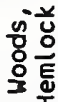

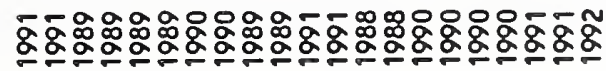

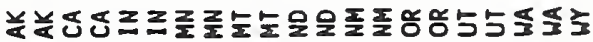




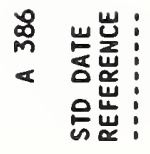

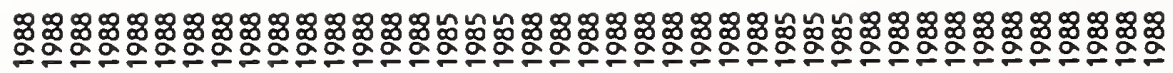

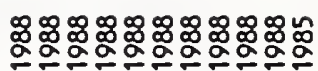

:

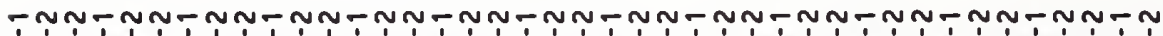

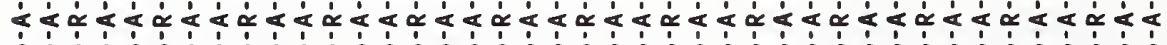

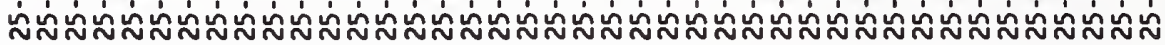

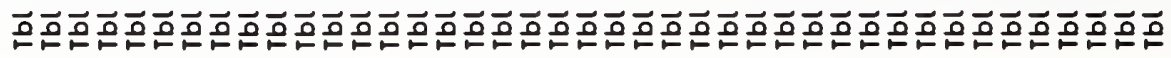

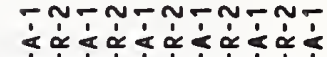

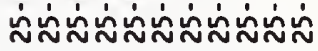

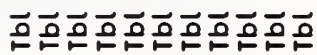

:

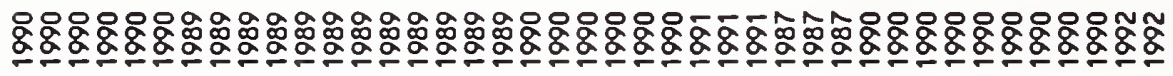

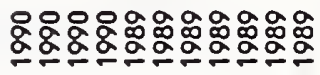

$\stackrel{4}{\simeq}$

岁

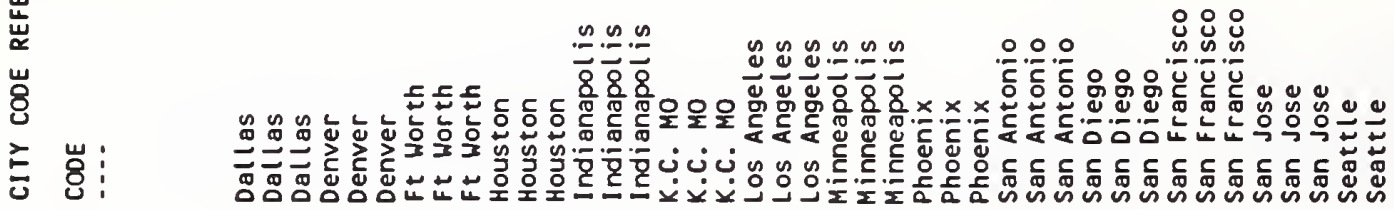

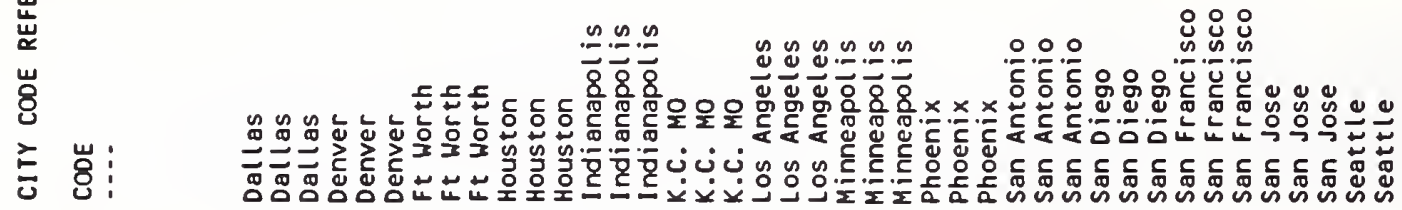

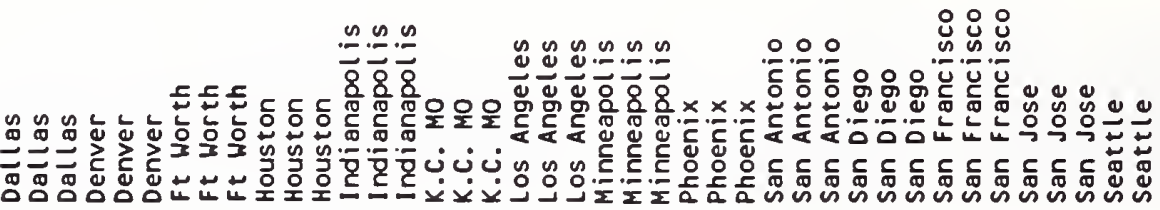

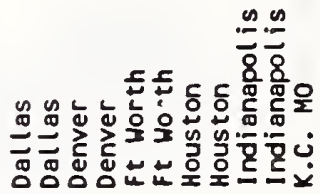

س 岂:

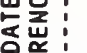

:

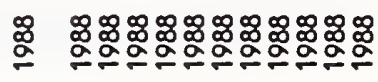

응

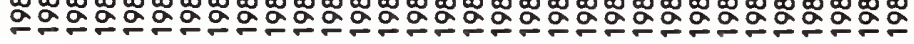

芩

㟥

-n -nn-nnenn-nn-nn-nn-nn-nn-nn-nn-n

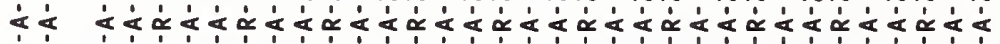

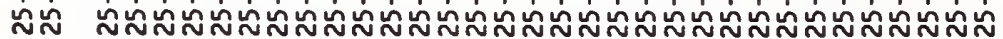

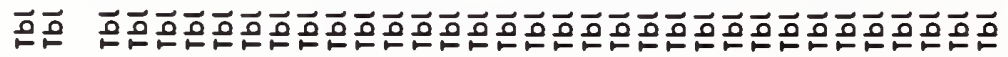

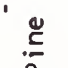

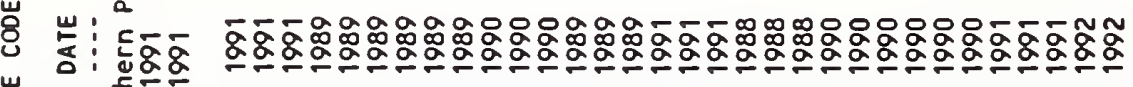

, 容邑怘

8

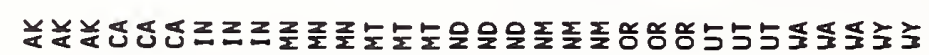

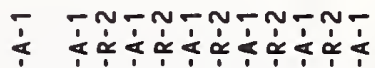

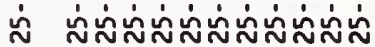

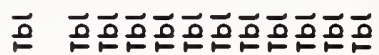

$\stackrel{\Xi}{\square}$

$\stackrel{\Xi}{\Xi}$

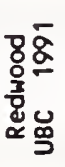

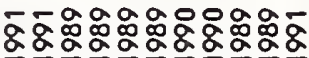

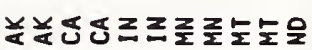




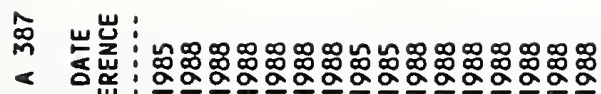

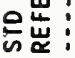

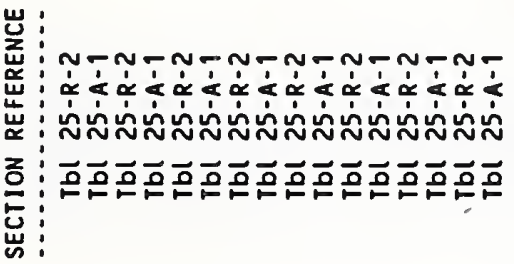

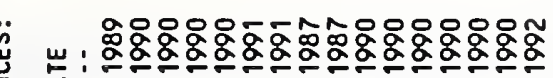
ธ。:

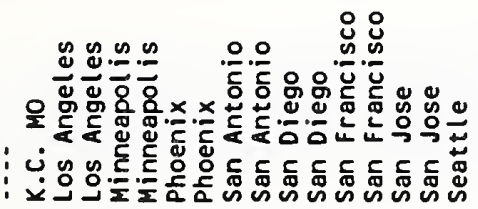

崖:

政

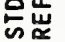

:

崖

ind

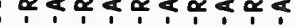

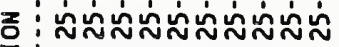

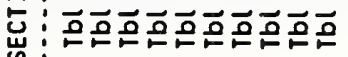

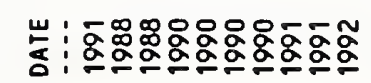

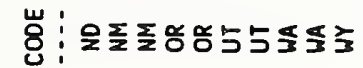

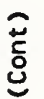

ळ̊

$\ddot{\infty}$

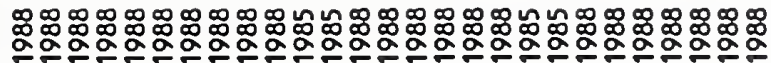

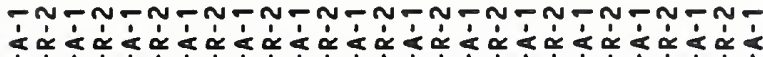

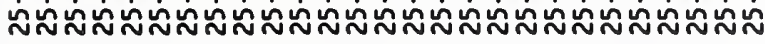

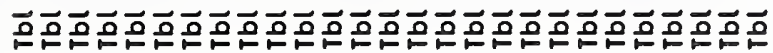

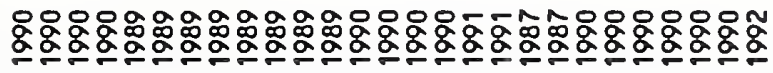

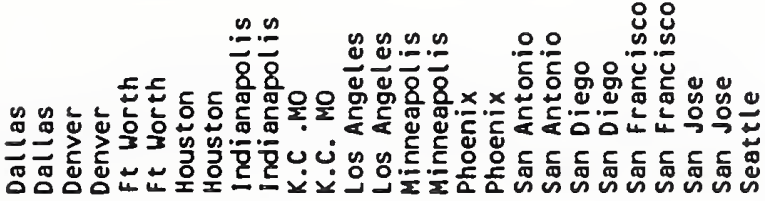

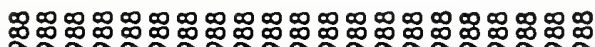

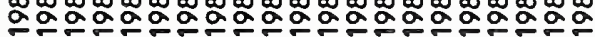

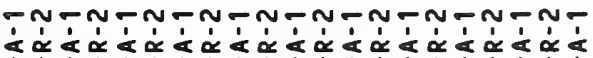

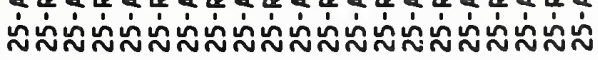

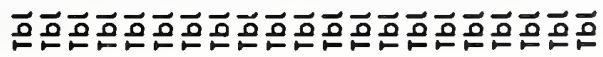

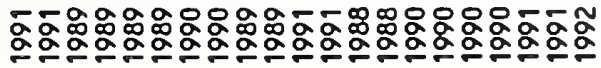

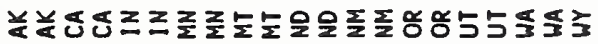

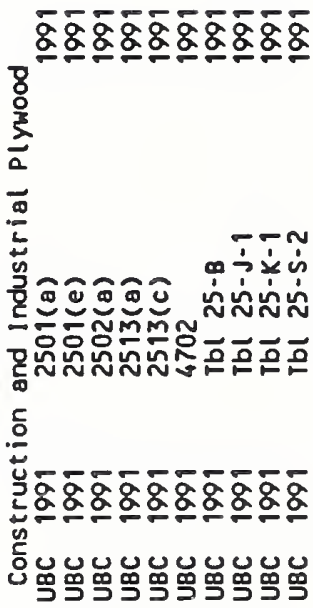

$\bar{g}$

ฌิ 


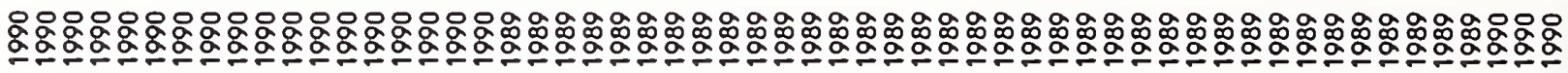

岁

崖 :

崖:

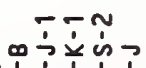
$\sin$

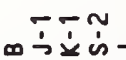
$\bar{i} \dot{i} \tilde{\dot{x}}$

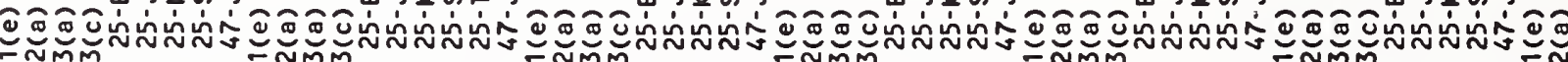

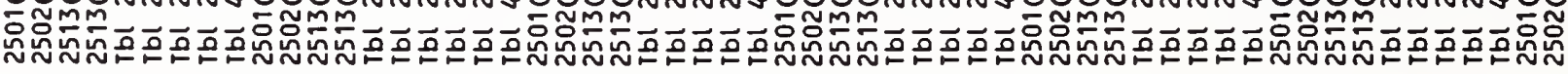

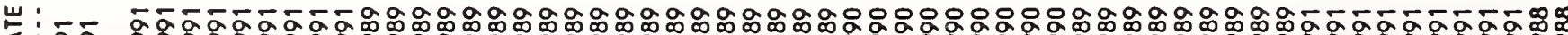
20으.

岁: 瞢岇

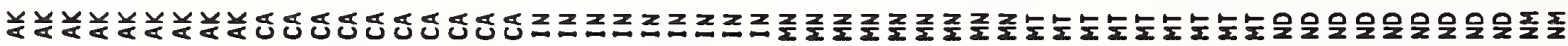

કัญ 


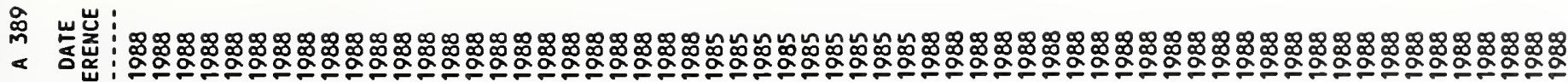
它峞:

疋

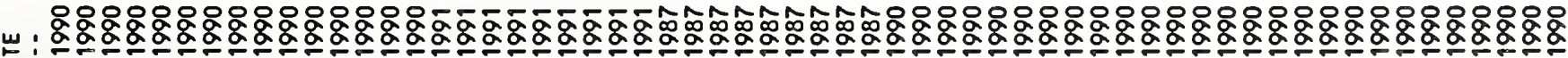
ธ์

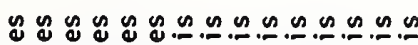
宁宁药

$\dot{\sim} \infty \dot{\dot{x}} \dot{x}$

$\div \div+\frac{1}{10}$

$T \div \widetilde{1}$

$\infty \dot{x} \dot{1}$

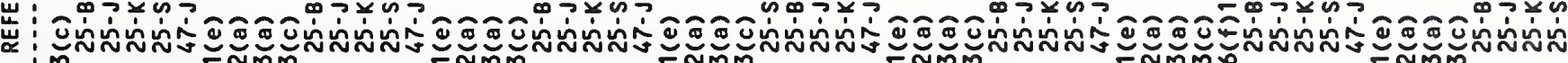
을 : 岕:

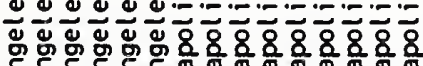

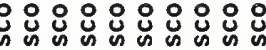

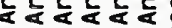

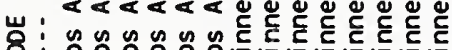

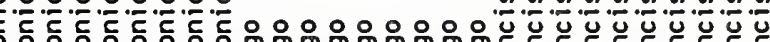

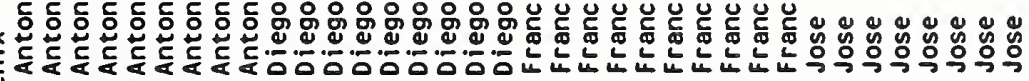

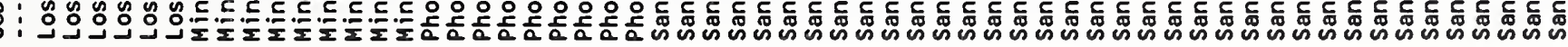

㟧

任

芯

岕 :

눈 : : :

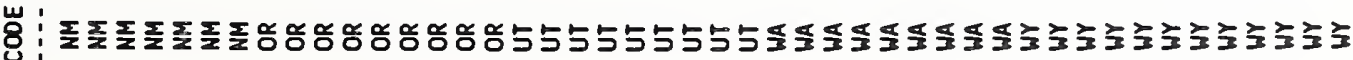

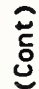




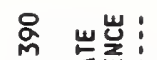

$<$ o 运岕:
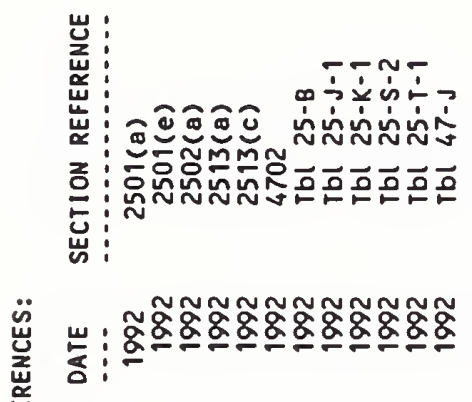

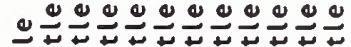
士

岕岕:

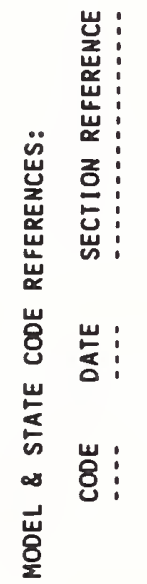

¿̊

产 $\quad \stackrel{a}{\dot{n}}$

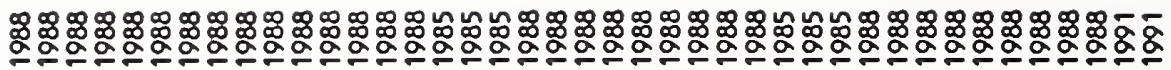

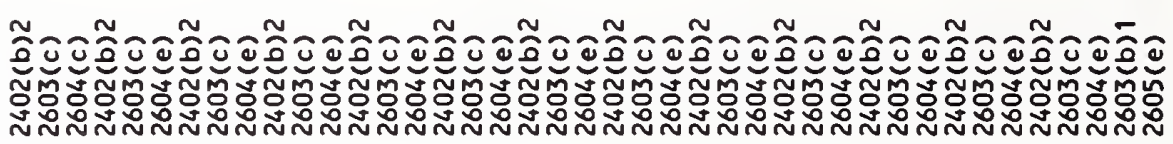

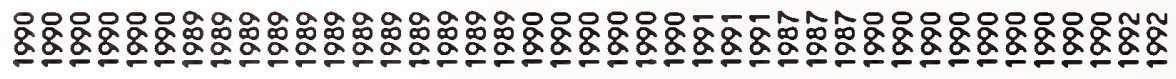

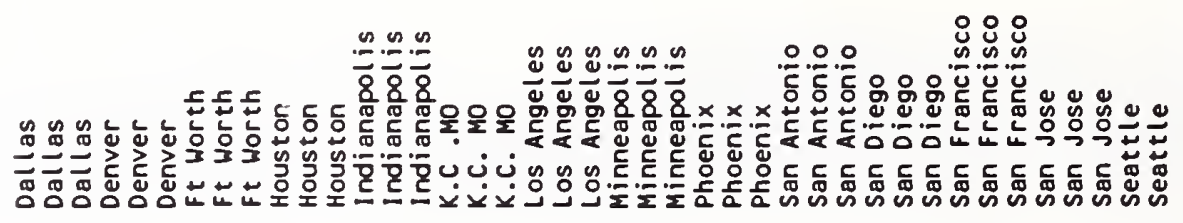

窎

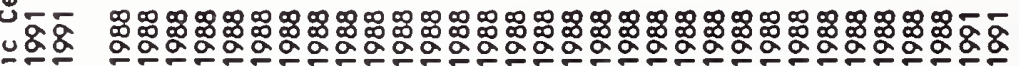

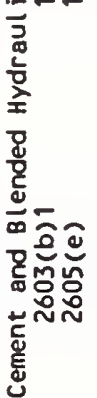

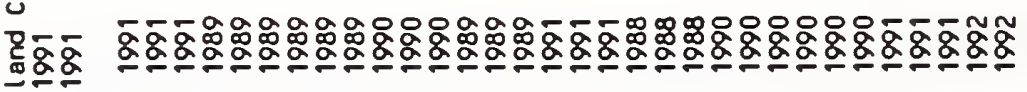

高鲜

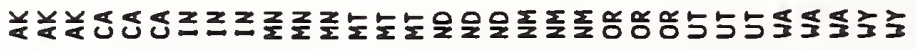

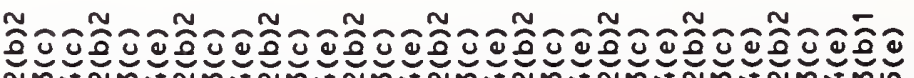

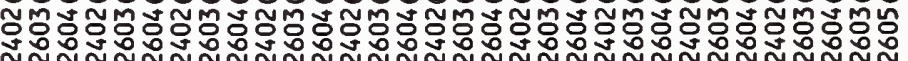

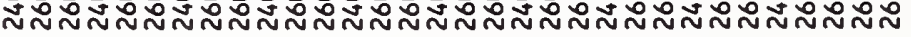

$\bar{\Xi}$

$\overline{\dot{s}}$ 
mMMMMำ

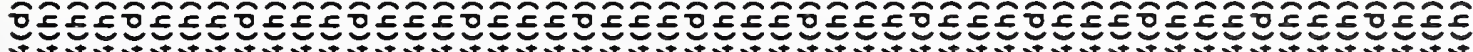

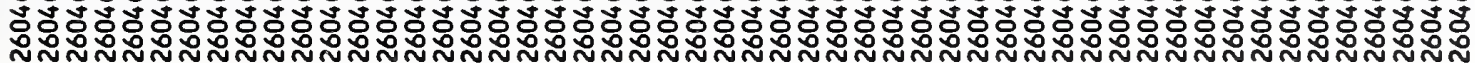

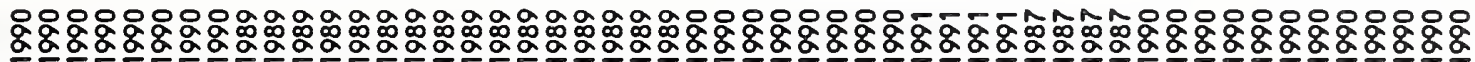

崖!

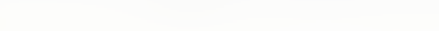

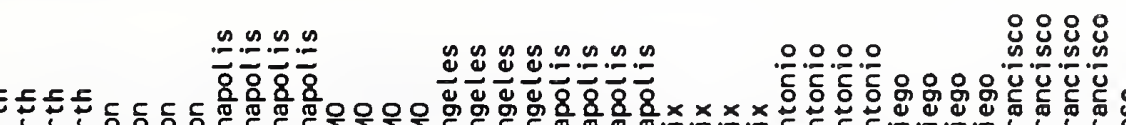




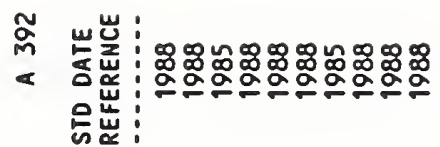

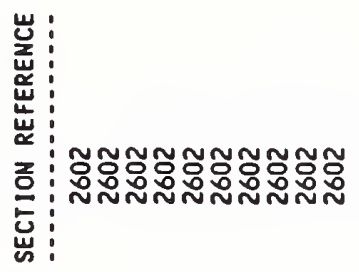

芦 w :

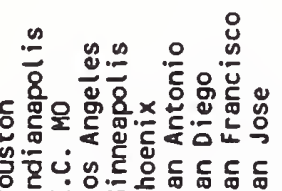

岂 :

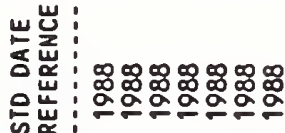

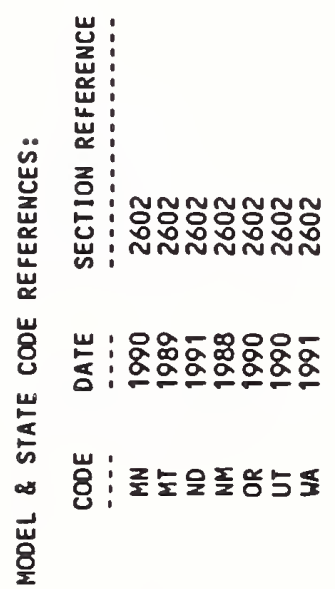

ํํํ

บ

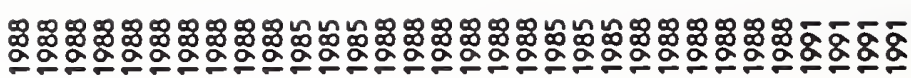

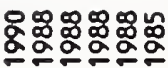

F

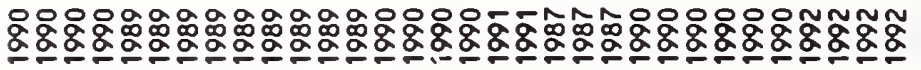

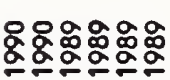

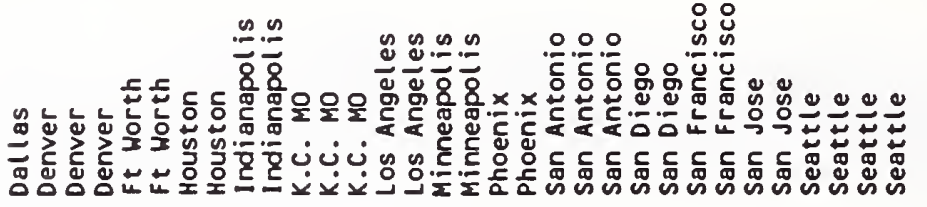

$\frac{\frac{2}{x}}{\frac{x}{x}}$

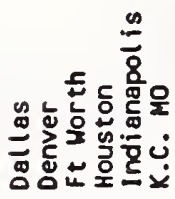

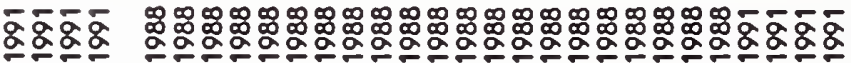
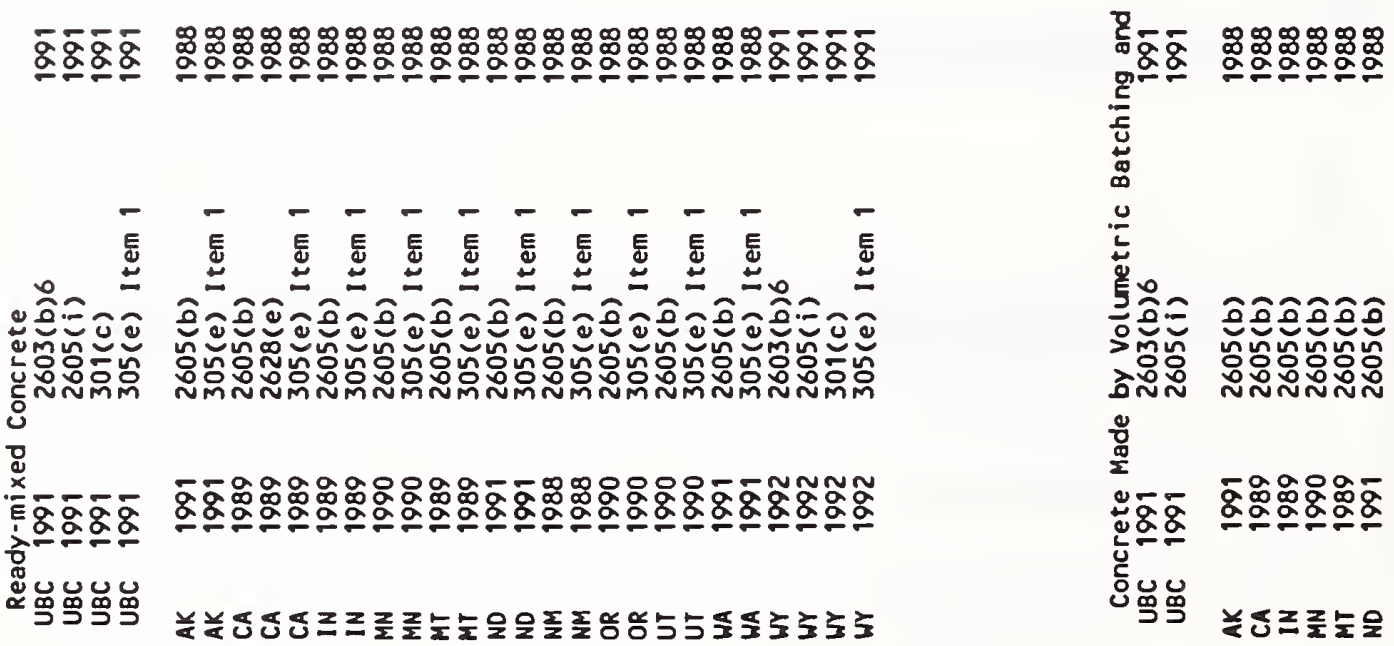

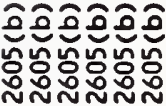

足

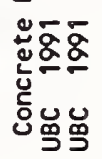

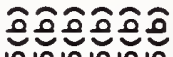

눙유윰유

วิกีำ

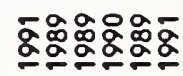

는즈줄노우

$\bar{g}$

$\bar{\xi}$

$\frac{m}{\dot{s}}$

จั 


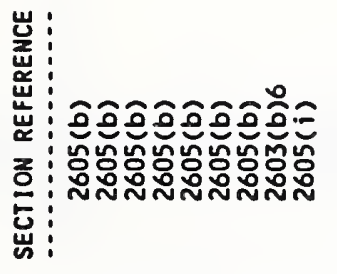

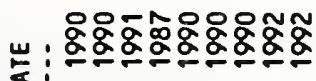
客:

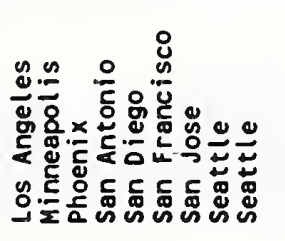

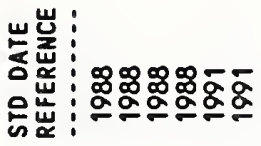

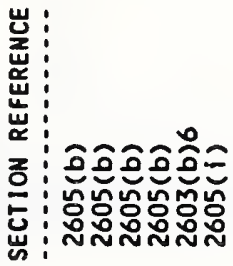

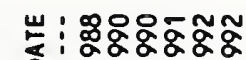

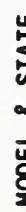

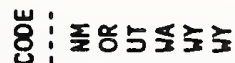

突

$\bar{g}$

$\frac{1}{\dot{n}}$

总 흠

宙

ษัँ 命

$\frac{n}{\dot{2}}$

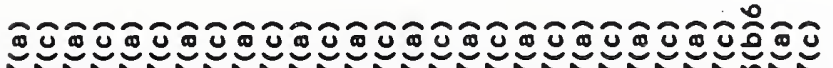

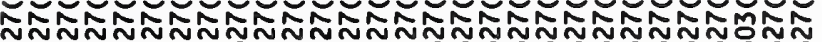

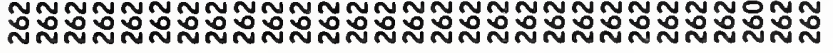

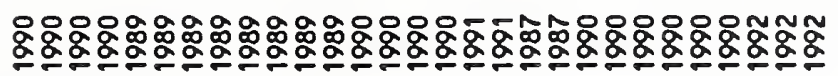

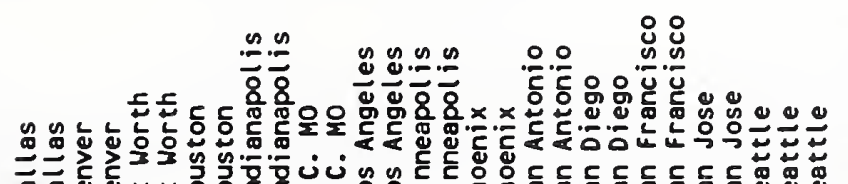

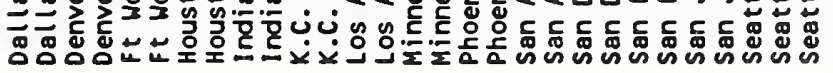

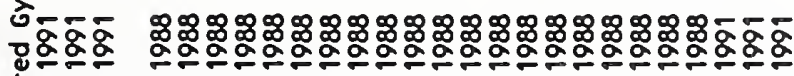

บัำำ

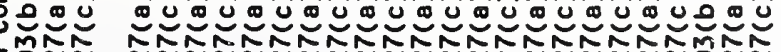

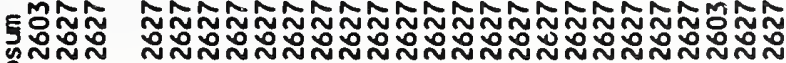

8

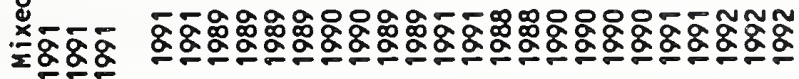

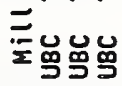

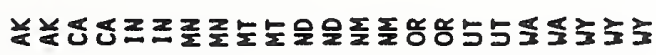

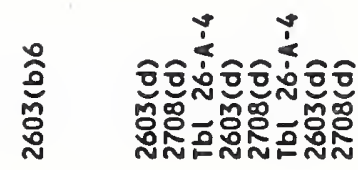

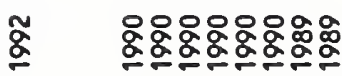

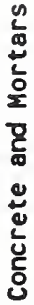

告

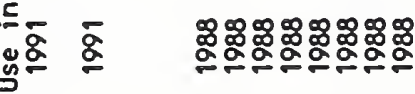

๖

品

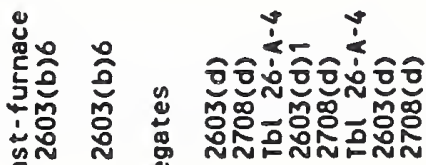

$\frac{\infty}{\infty}$

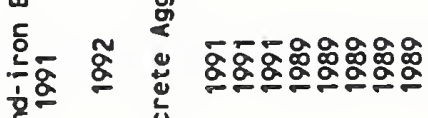

宅势

立

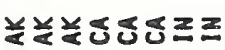

$\stackrel{\sim}{\dot{\delta}}$ 

战

u 崖:

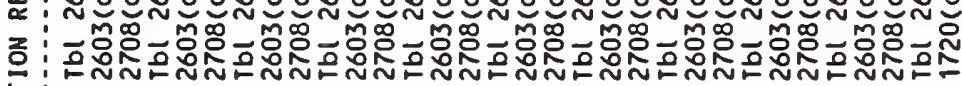
总:

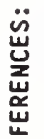

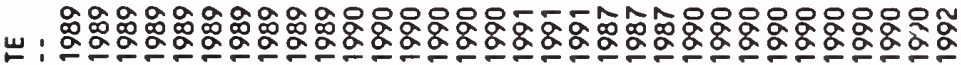
究

$\stackrel{u}{\longleftarrow}$

岁

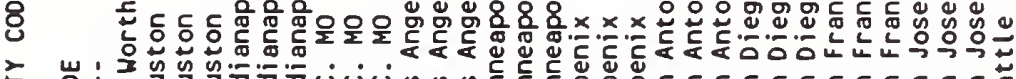

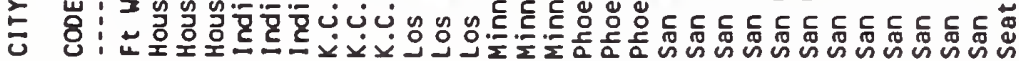

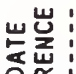

更出

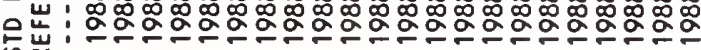

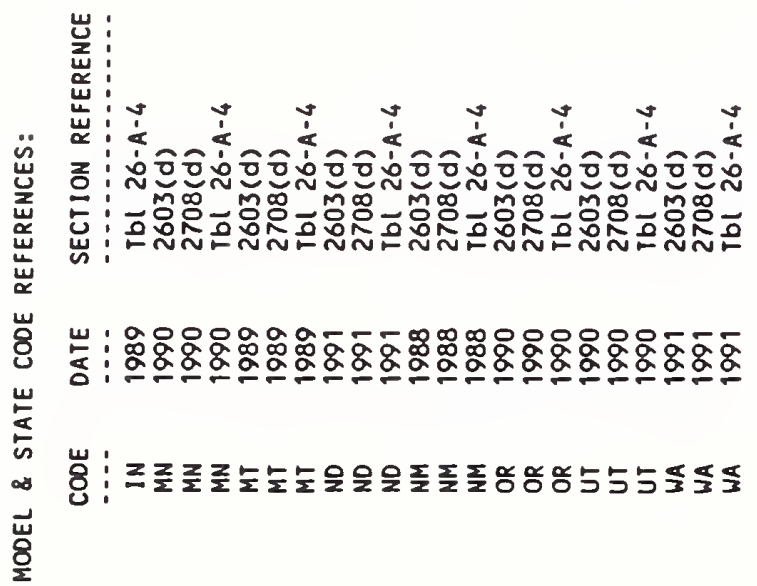

¿̊.

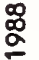

罗

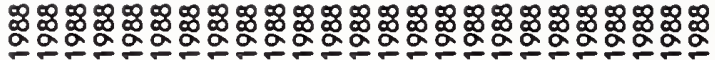

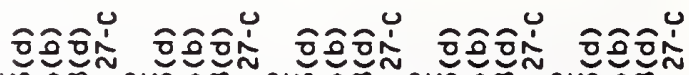

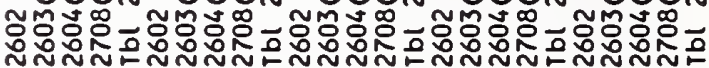

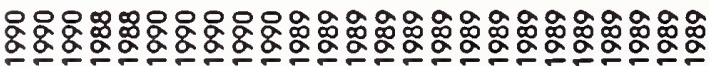

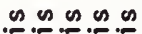

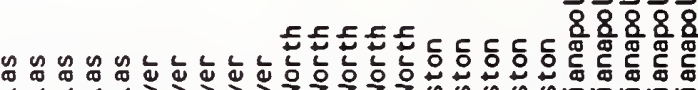

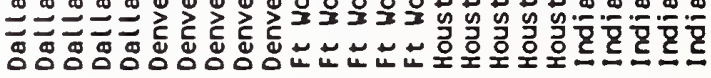

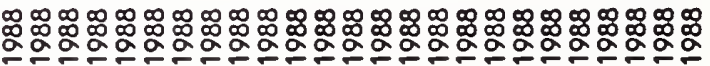

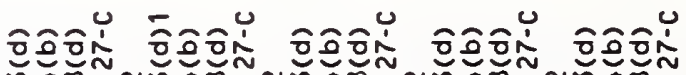

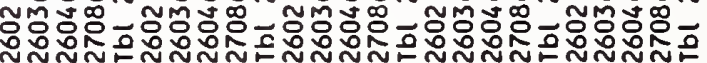

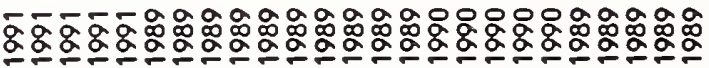

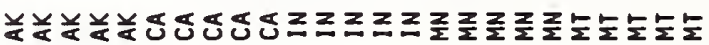


은

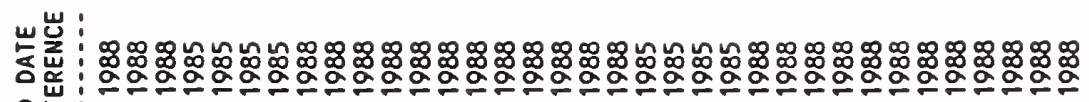
的岕:

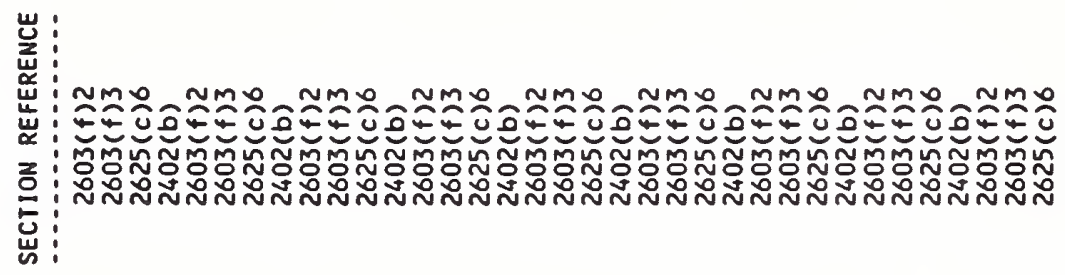

范 농

湈

\section{$\therefore \approx n$ \\ \&\&\&呦电}

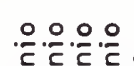

足施怘怘

岁

는

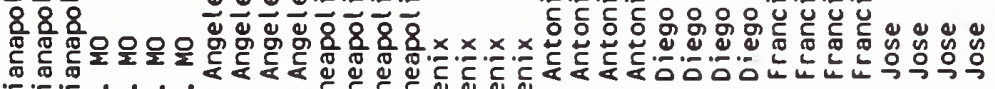
岩: :

峞岂

రీ

눈

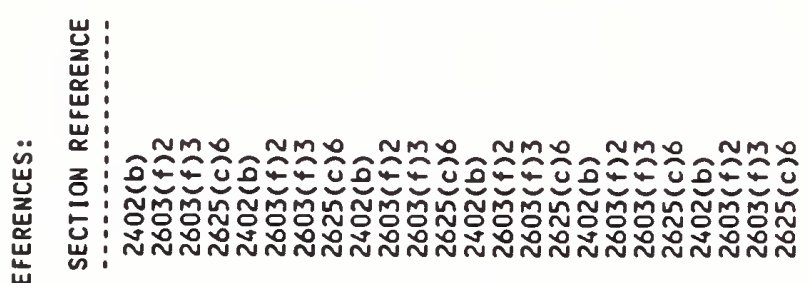

峞

峞

㟧

岩 $\vdots$ :

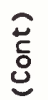

$\stackrel{8}{\stackrel{8}{g}}$

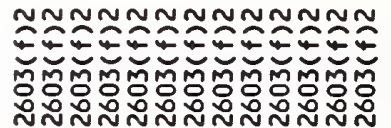

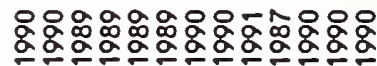

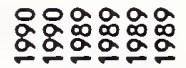

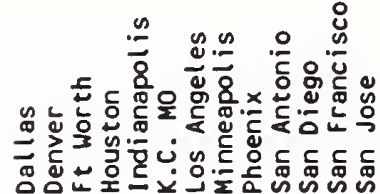

D

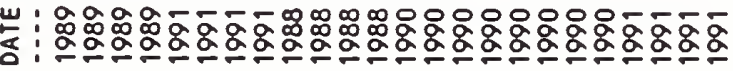

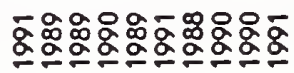

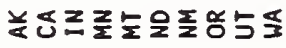

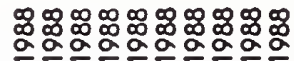

$\simeq \simeq \simeq \simeq \simeq \simeq \simeq \simeq \simeq \simeq$

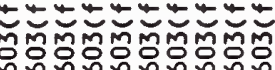

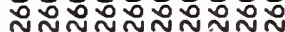

$\stackrel{\sim}{\sim}$

$\stackrel{\circ}{\text { ¿ }}$

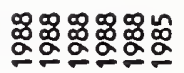

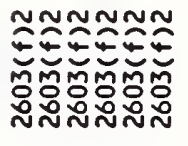

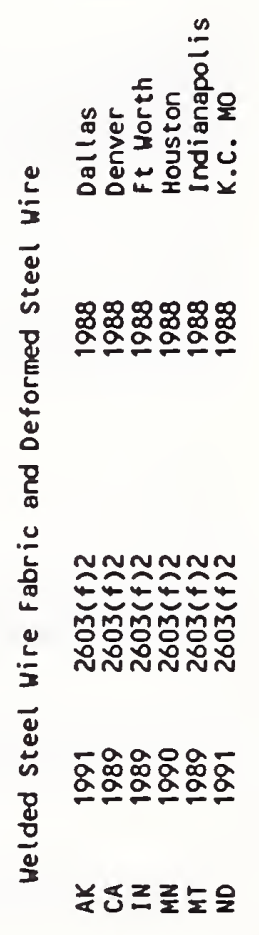

吕

芩

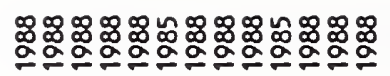




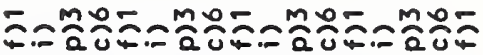
m

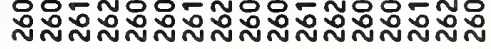

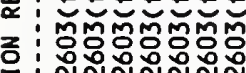

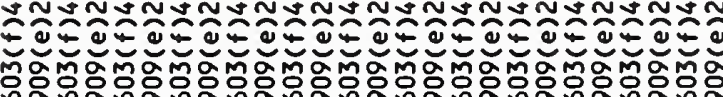

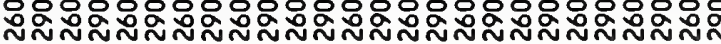

양영홍영영영

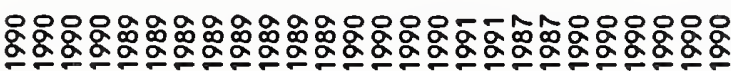
s:

\section{\& $\cong \quad$ @

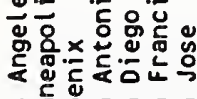

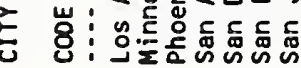

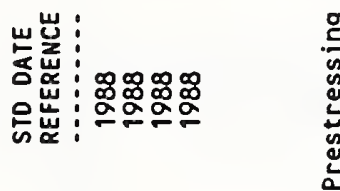

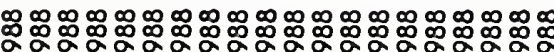

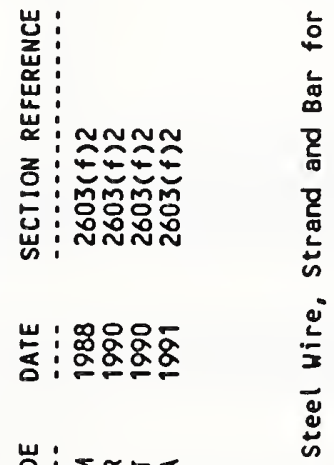

8

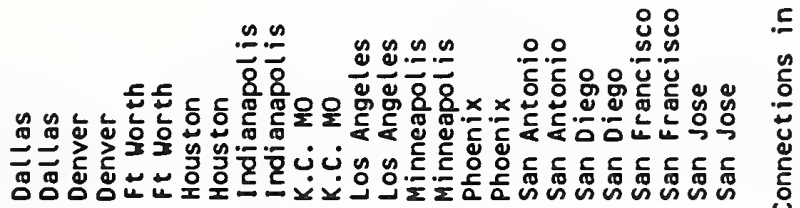

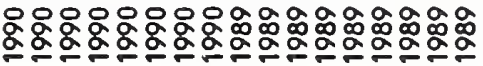

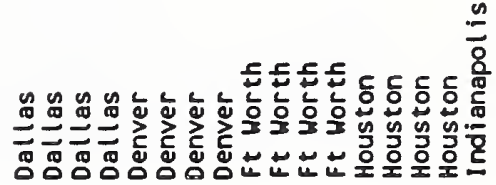

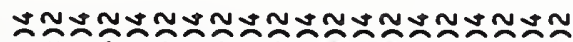

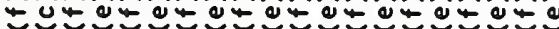

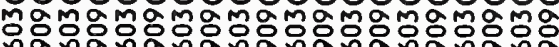

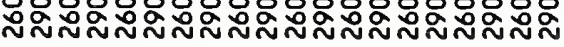

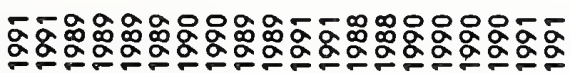

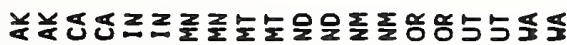

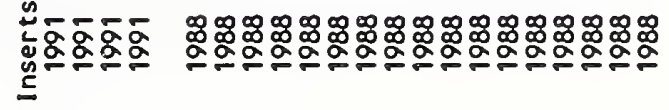

ฐँّ

¿ั

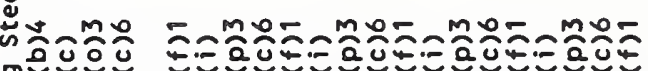

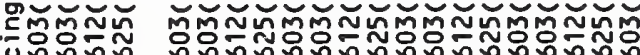

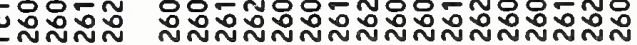

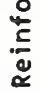

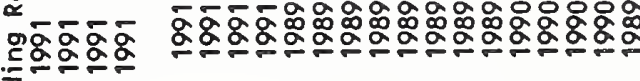
\% 品品品品

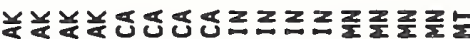

$\stackrel{\mathscr{\Xi}}{\square}$

$\bar{\alpha}$

号

$\hat{\grave{n}}$

$\stackrel{\infty}{\dot{0}}$

i̊n 
$\stackrel{\infty}{\circ}$

岕:

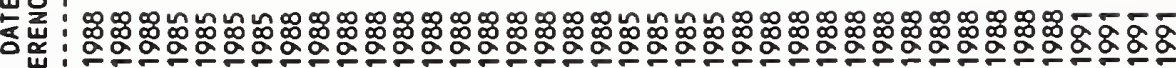
은

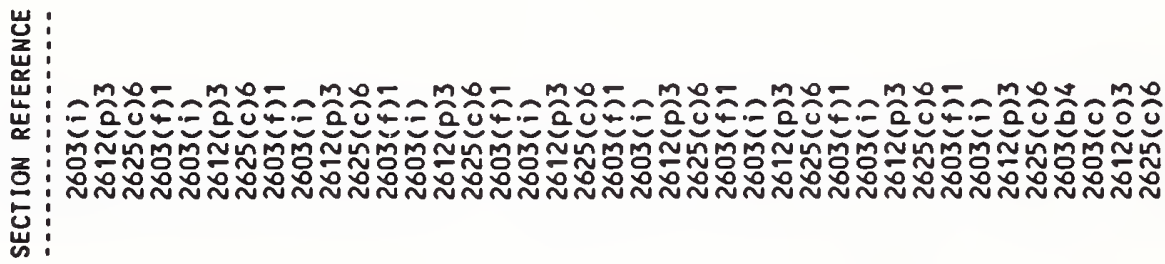

崖:

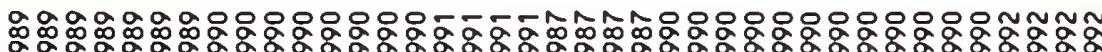
宅

\section{$\because 2$.}

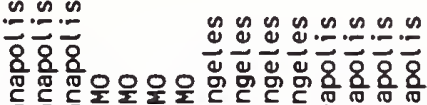

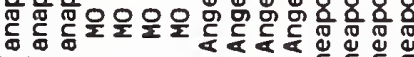

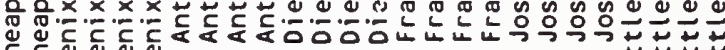

こ

世눈

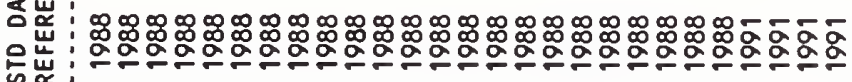

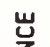

宸

崖

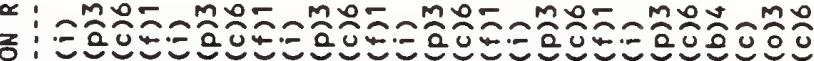

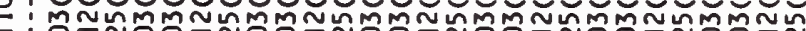

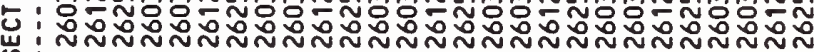

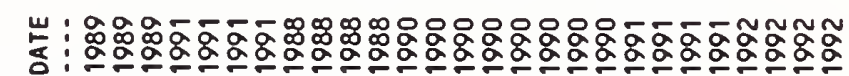

崖

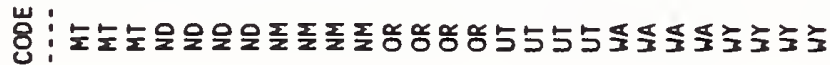

용

仓ั̊

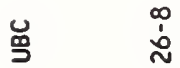

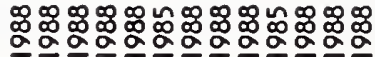

ติติติติติติติติ

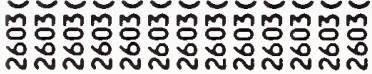

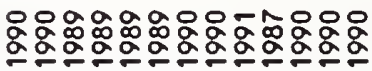

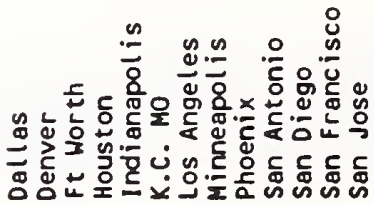

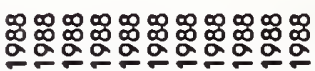

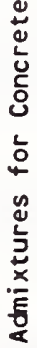

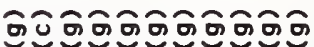

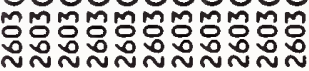

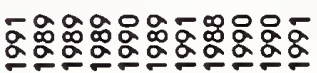

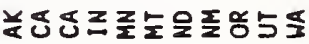

离

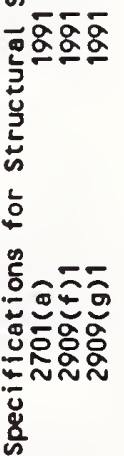

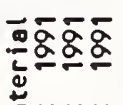

娄茴品品

$\stackrel{\infty}{\infty}$

\&

ó 


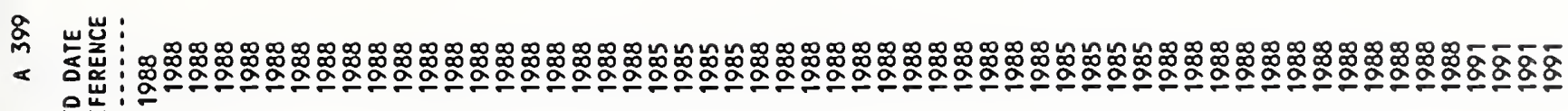

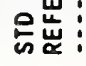

岕:

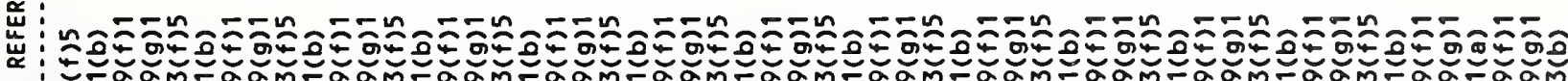
z: 岕:

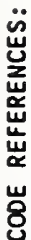

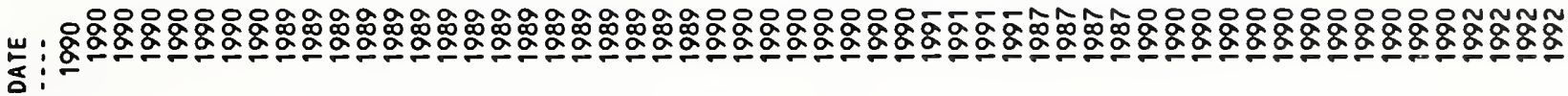

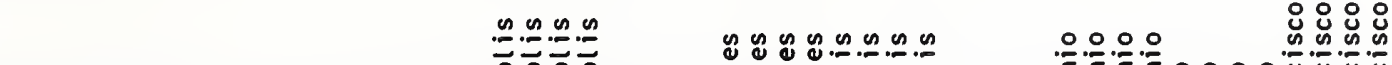

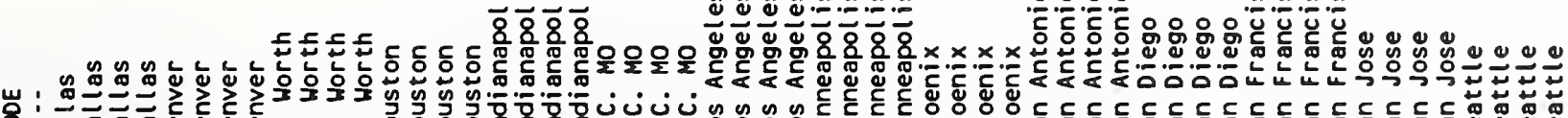

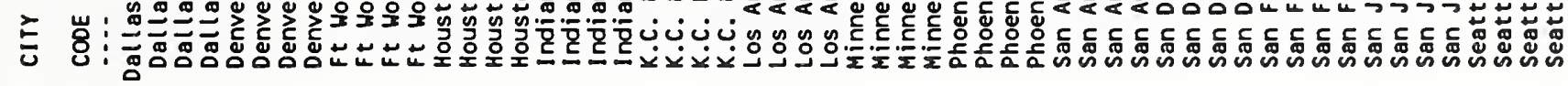

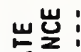

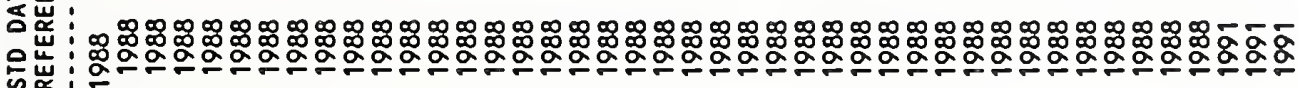

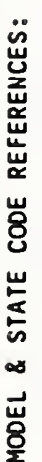

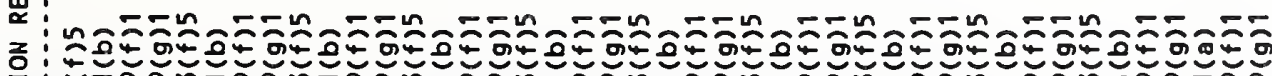

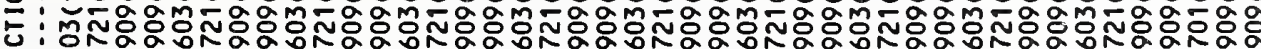

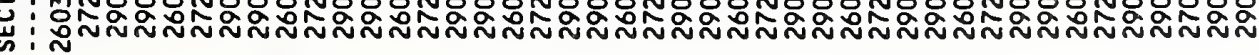

岩 :

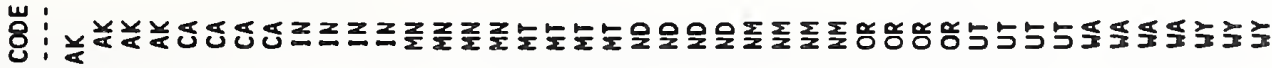

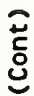

$\dot{\grave{\sim}}$ 


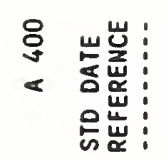

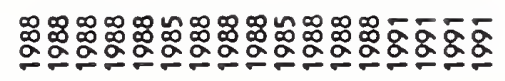

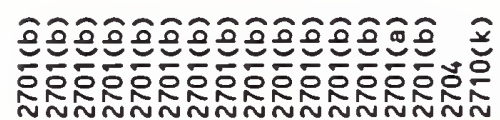

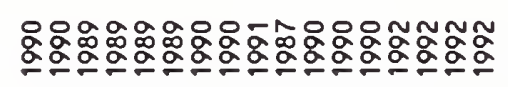

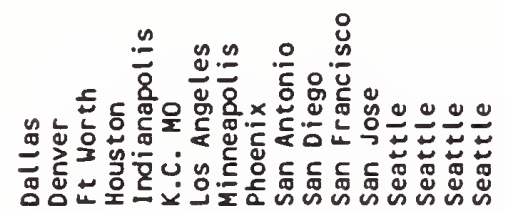

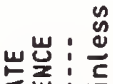

:

范

넌

㱏:

崖:

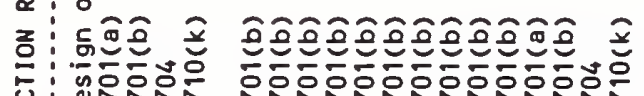

แ 寒

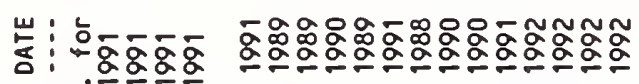

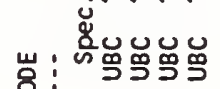

ठ이:

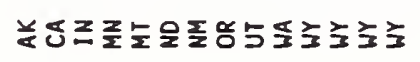

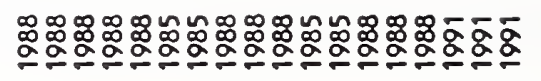

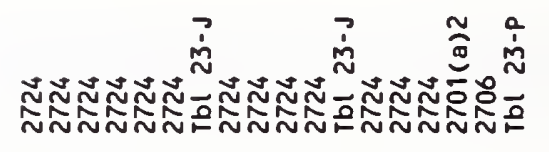

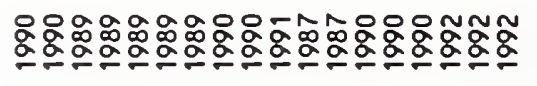

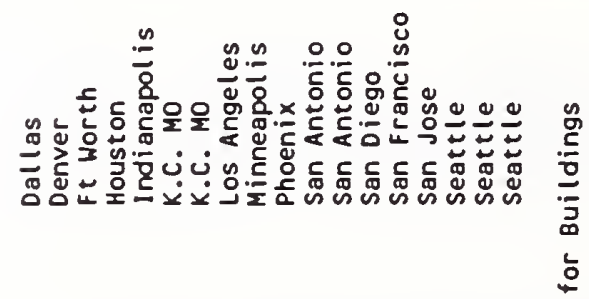

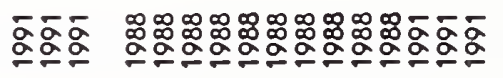

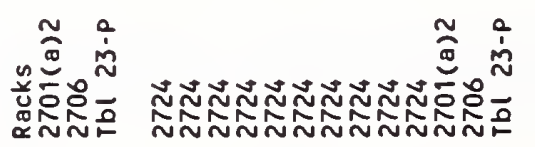

葛

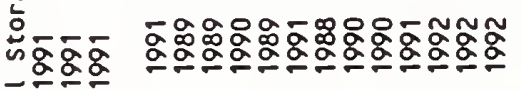
ळँ 芯员罗品

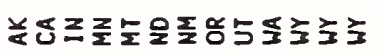

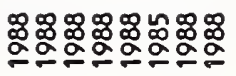

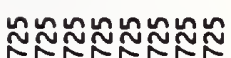
NลNลNลNล

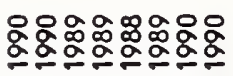

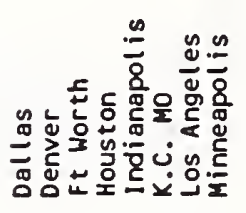

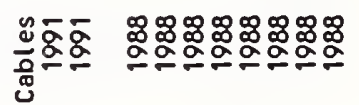

离

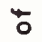

气ิำ

뜸옹

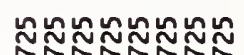
ลัNลNลกับ $\frac{2}{4}$

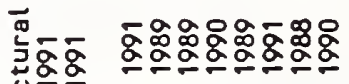
บ흥 究品品

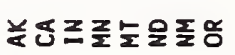

$\bar{g}$

$\bar{g}$

$\bar{a}$

$\frac{}{2}$

$\bar{\Sigma}$

$\frac{\sim}{\sim}$ 


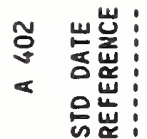

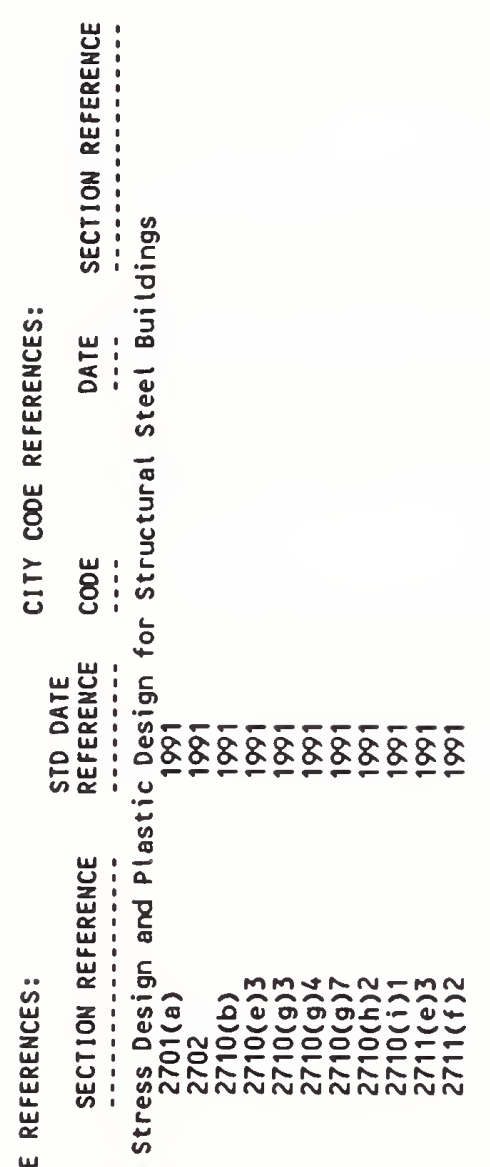

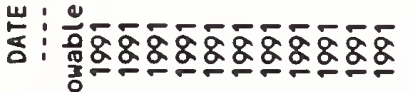

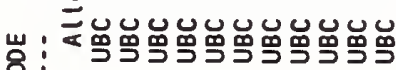
ठ․

$\bar{g}$

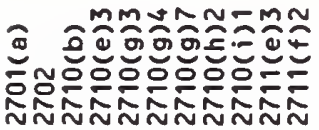

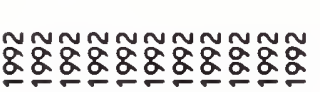

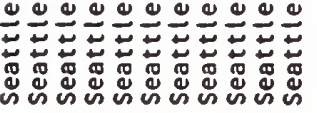

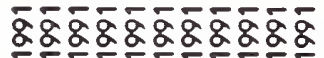

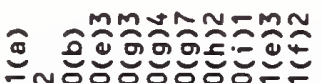

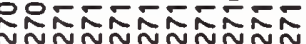

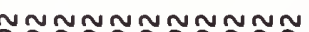

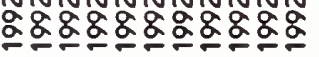

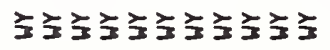

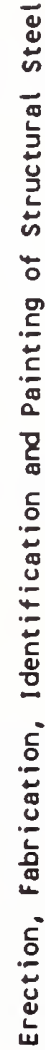

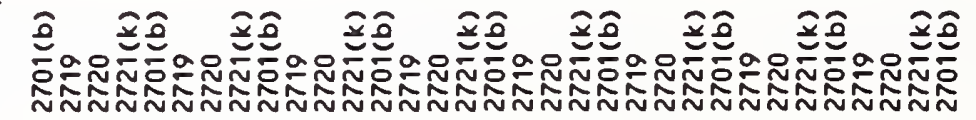

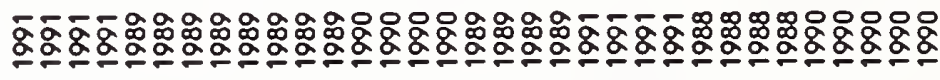

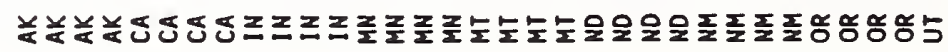

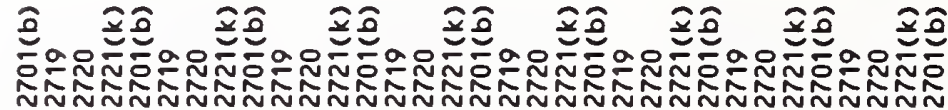

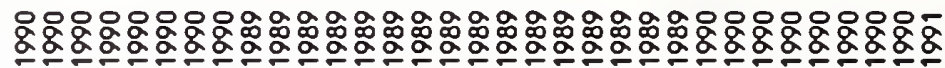

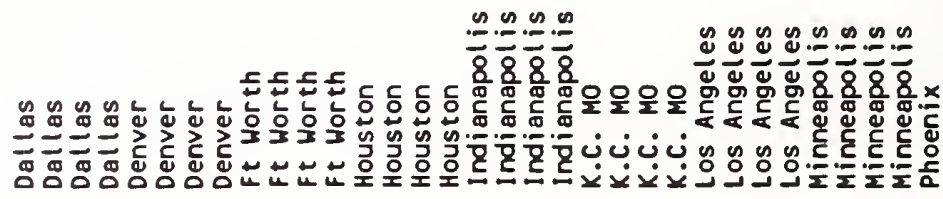

றீறை

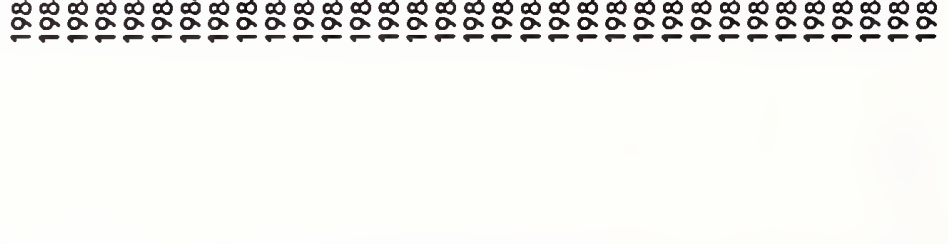




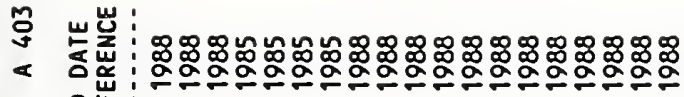

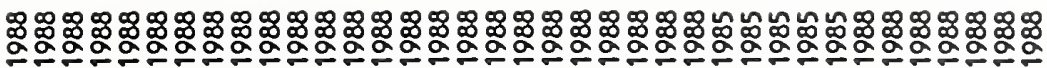
돈 :

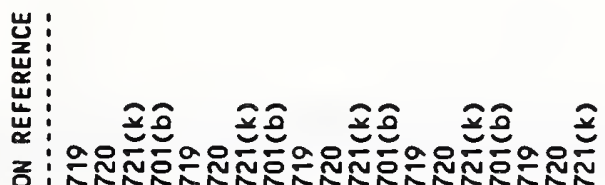

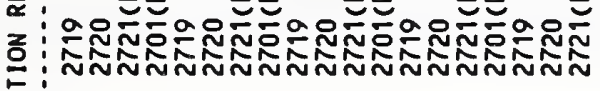
出

岂

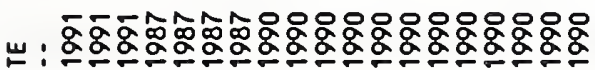
定:

峞

焗

济

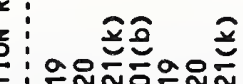

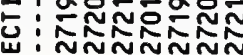

Minn

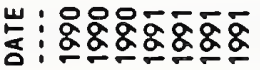

岁:

总

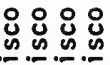

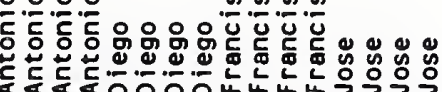
x.x.

至

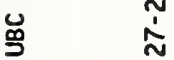

产

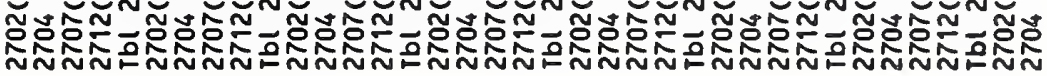

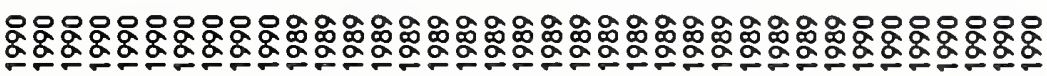

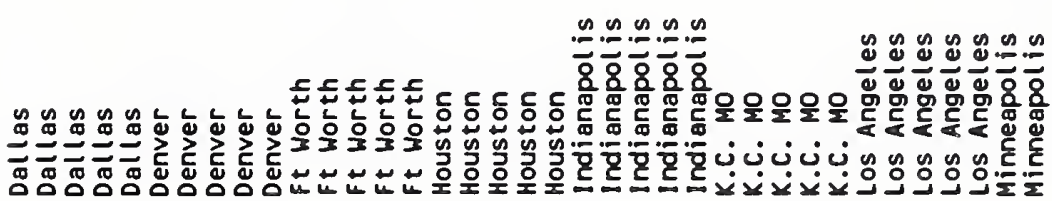

등

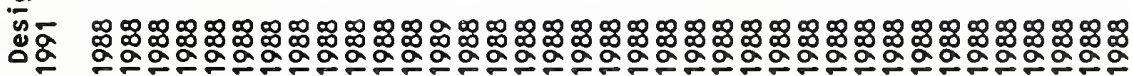
ब

妾

ธิ㇒ 等

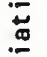

位

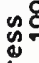

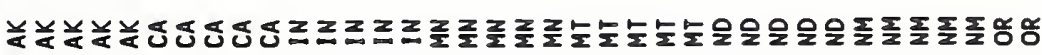

$\bar{\Phi}$

$\stackrel{m}{i}$ 


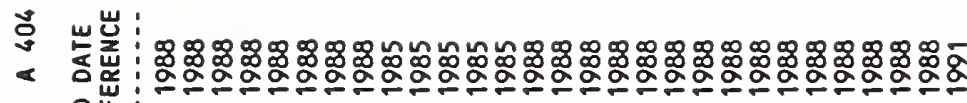

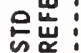

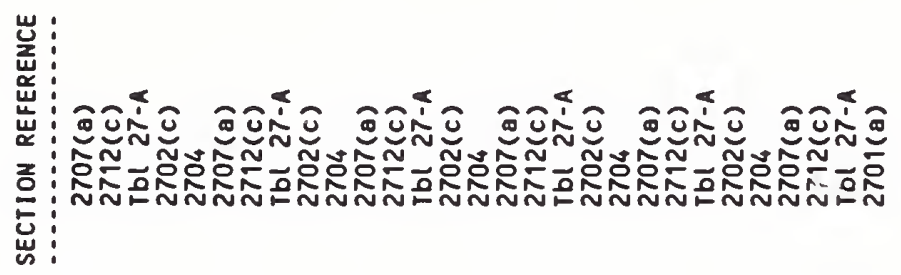

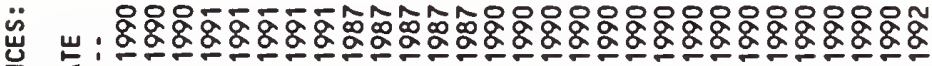
范

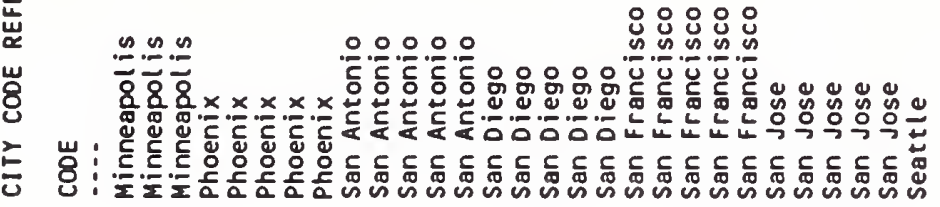

窟:

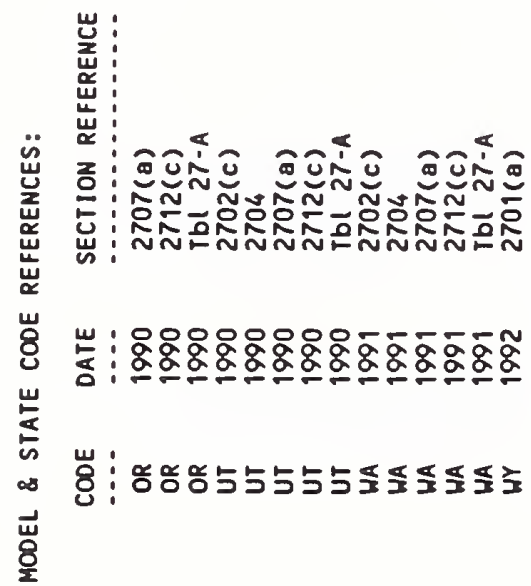

ํㅗㅇ

品 $\quad \stackrel{m}{ }$

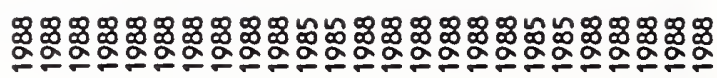

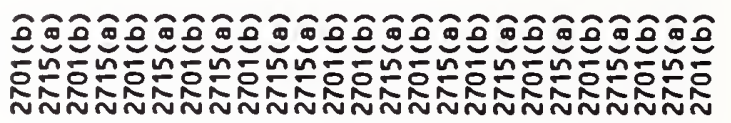

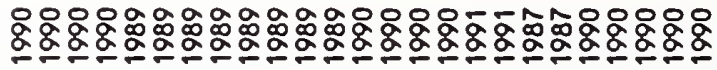

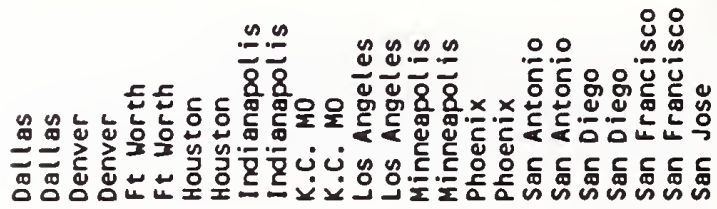

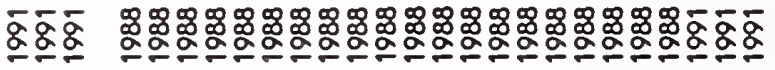

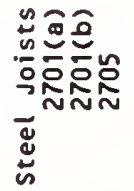

童京兗京 영놈욤

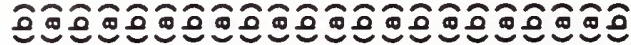

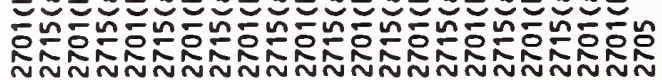

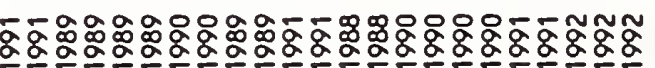

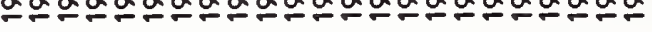

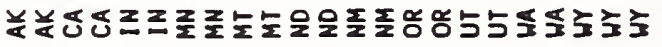

$\check{g}$

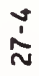


岁:

< 诧岕:

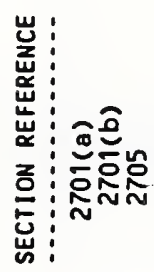

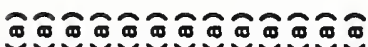
mong

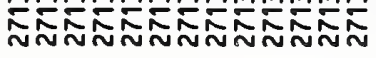

䓛

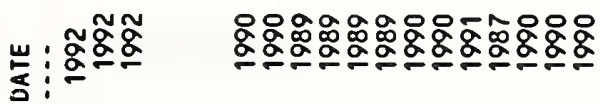

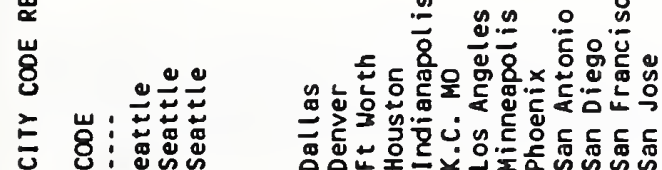

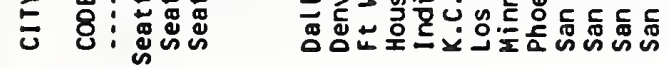

至:

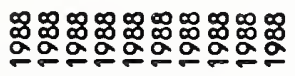

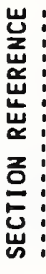

崖

:

풀

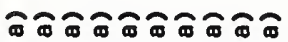
minmmmmmmm

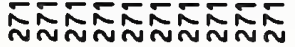

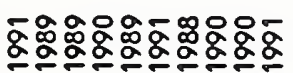

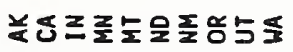

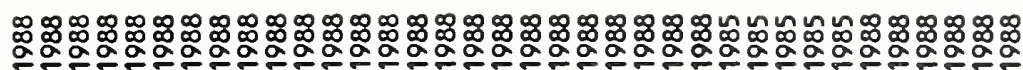

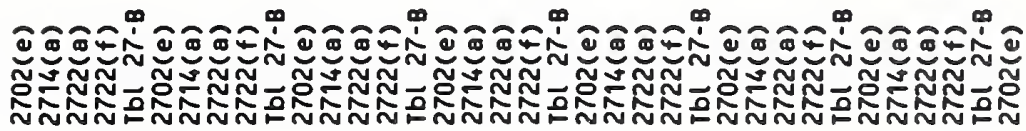

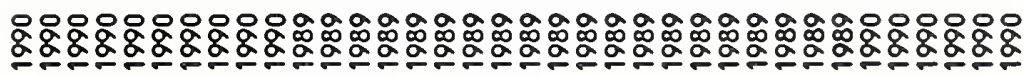

$n \cong n$ n

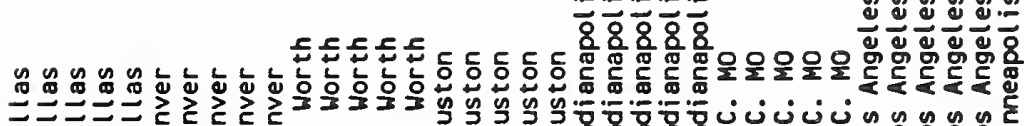

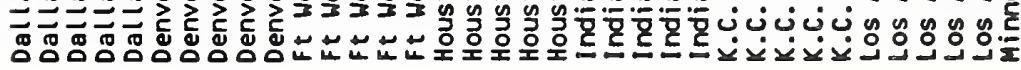

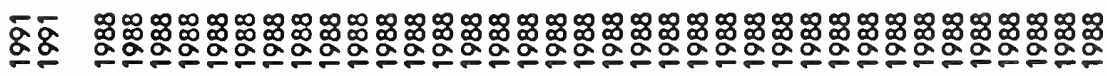

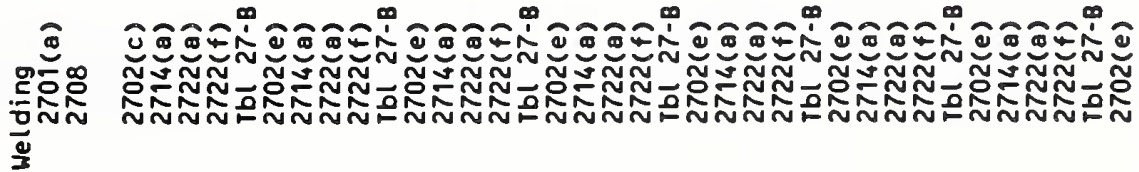

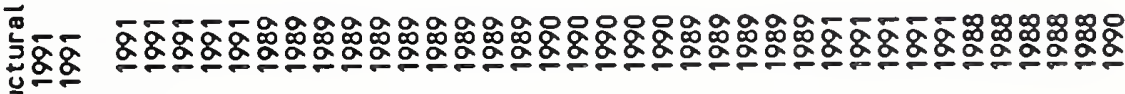

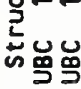

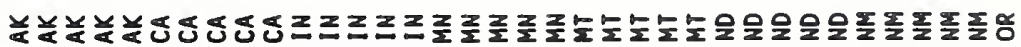


号 的炭:

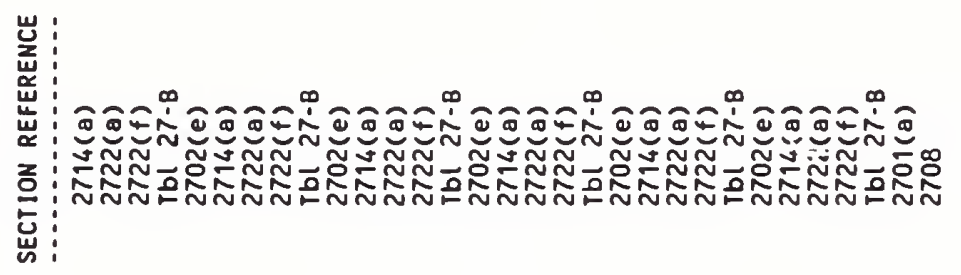
莖

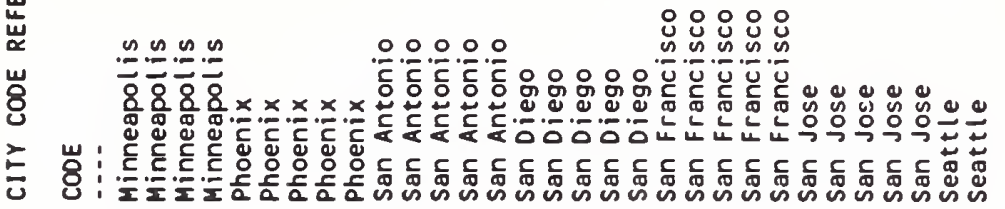

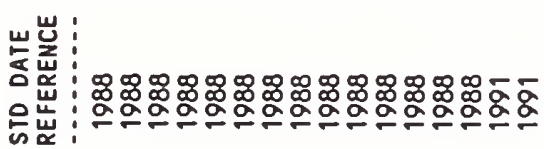

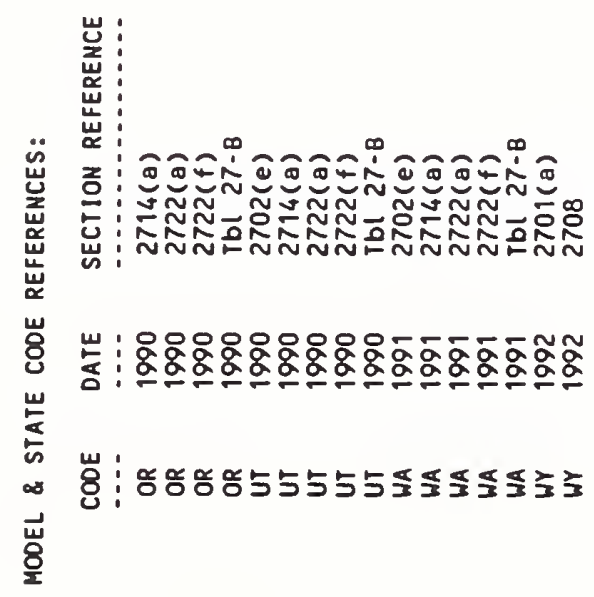

芒

$\begin{array}{ll}\stackrel{0}{y} & \stackrel{0}{N}\end{array}$

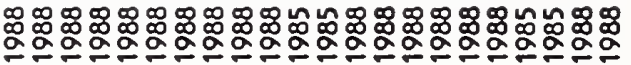

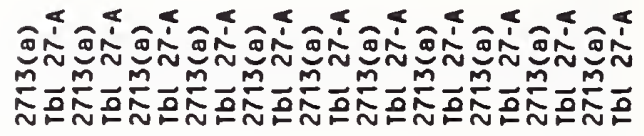

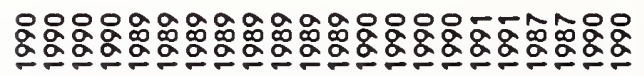

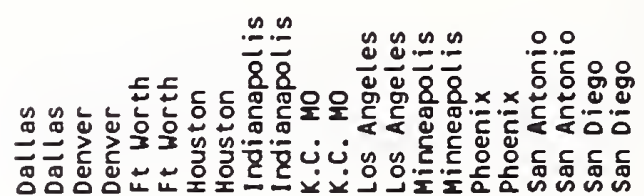

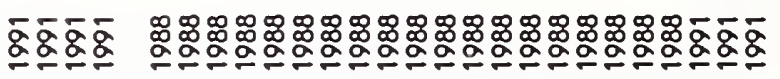

尊

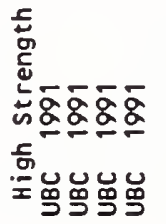

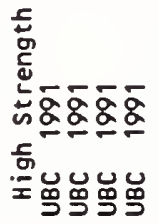

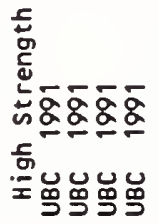

g

$\tilde{n}$

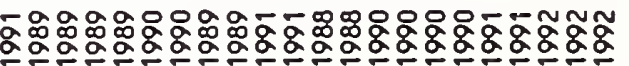

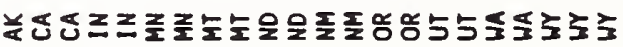

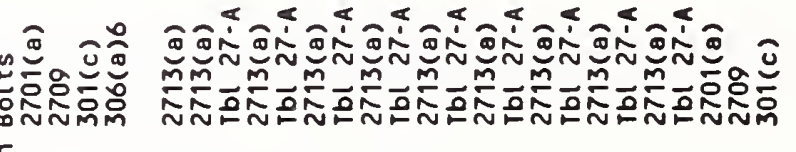



: 迹㟋:

\section{岂}

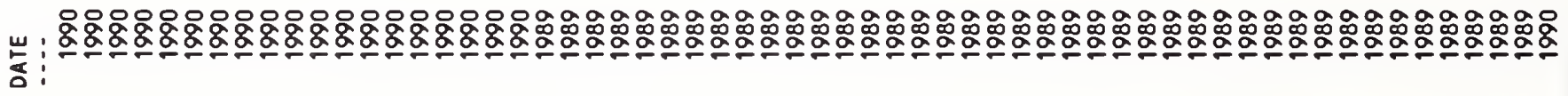

岁

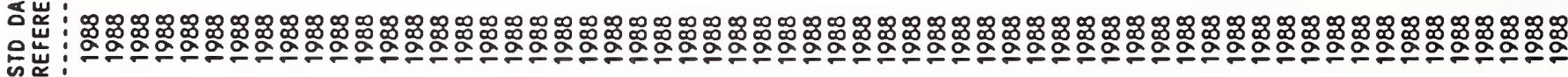

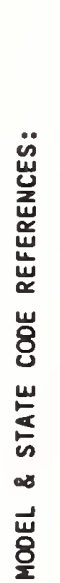

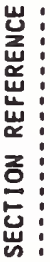

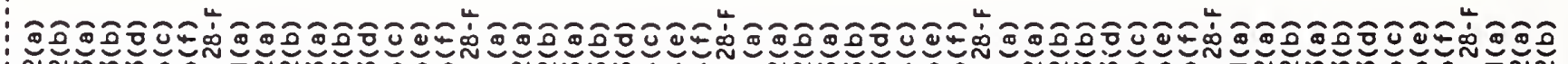

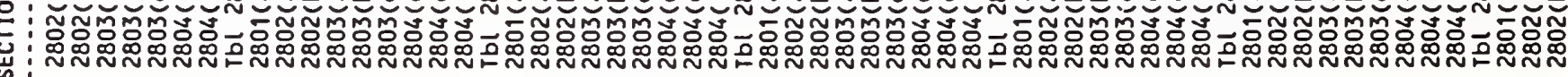
岩: 㟶:

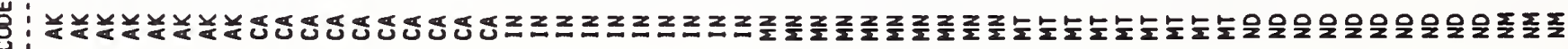

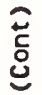

$\stackrel{\bar{m}}{\stackrel{\dot{m}}{\mathrm{~m}}}$ 


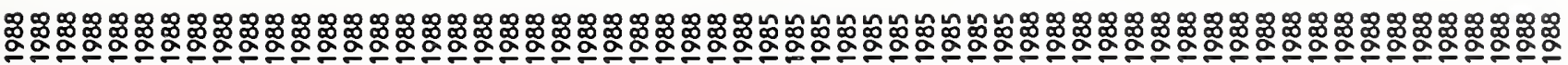
锷

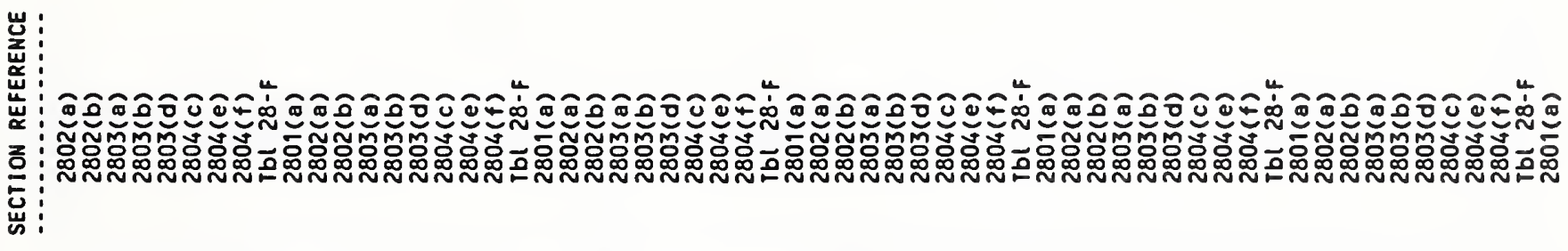

苨 安:

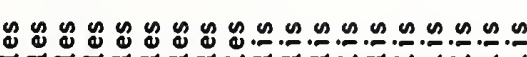

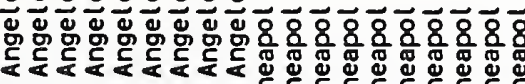

용ํㅇㅇㅇㅇㅇㅇㅇㅇㅇำ

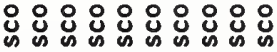

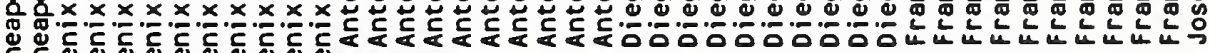

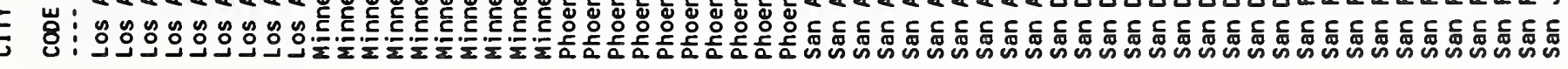

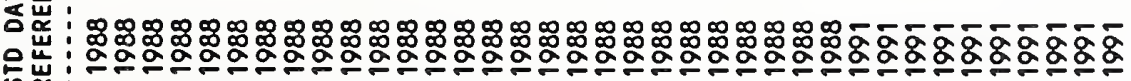

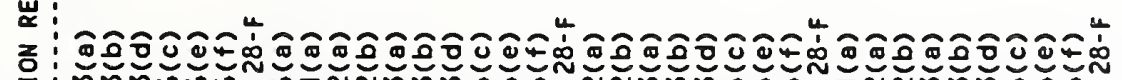

:

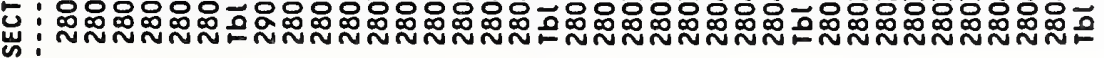

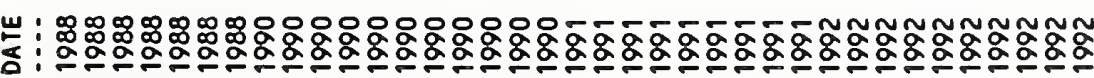

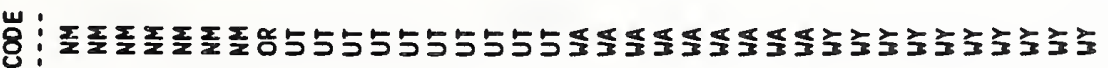

옹

市 
$\div$

崽: 忨峞: :

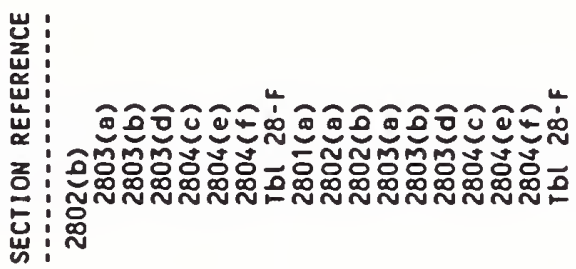

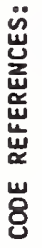

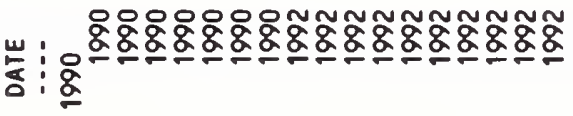

خ 山.

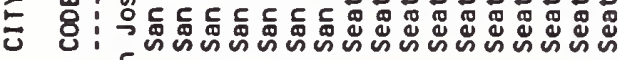
w 니 : Ф

它希

唒岕:

家

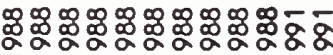

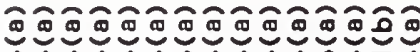

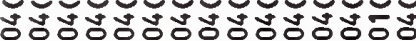

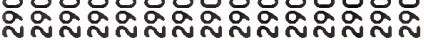

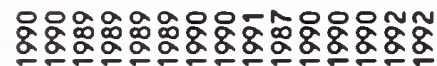

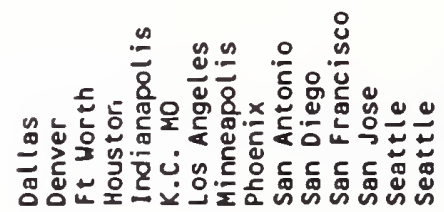

的

岕:

岁 :

突

$\bar{g}$

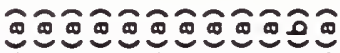
T身

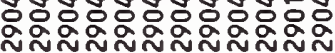

ํํำ

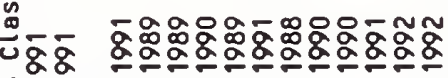

드음

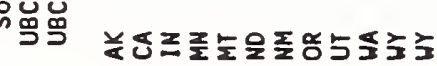

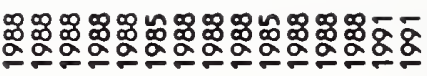

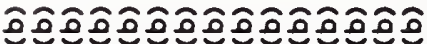

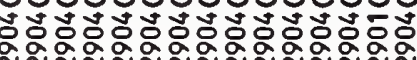

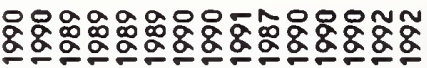

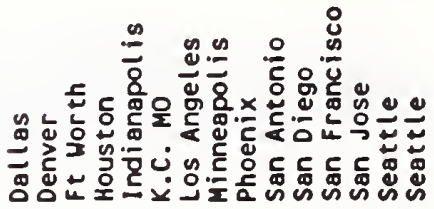

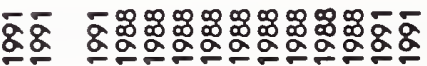

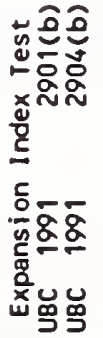

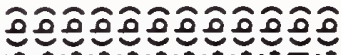

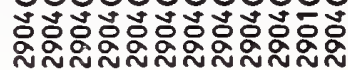

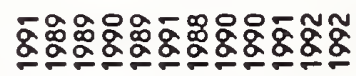

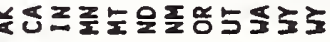

品 $\quad \overline{\dot{\infty}}$

$\overline{\dot{\alpha}}$

$\tilde{a}$ 


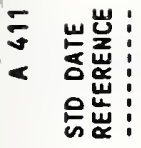

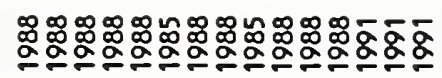

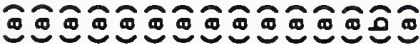

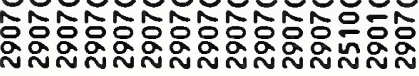

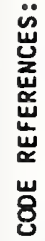

岕:

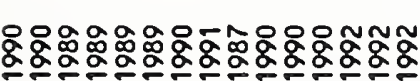

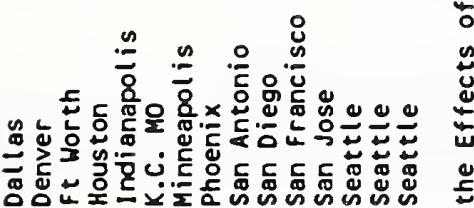

赔

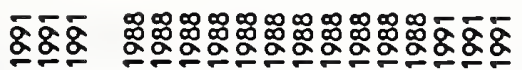

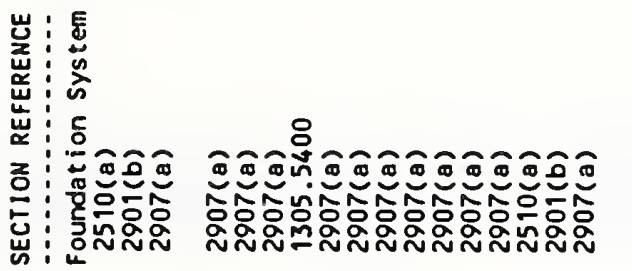

8

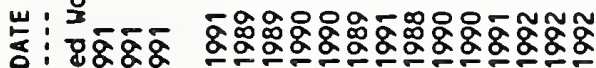

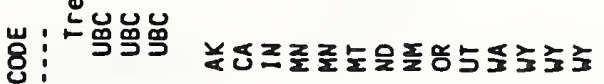

$\bar{g}$

$\bar{\Phi}$

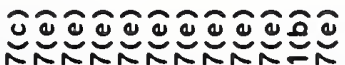

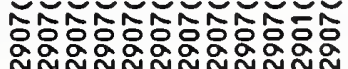

$\frac{2}{20}$ $\frac{\mathbf{m}}{\mathrm{s}}$

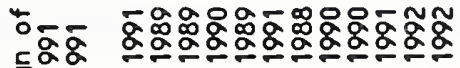

두요

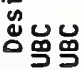

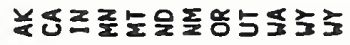

\section{0
0
0
0
0
0
0
0}

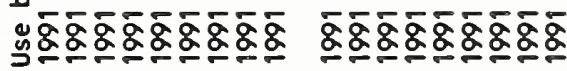
웅

巡

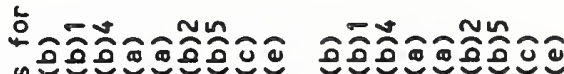

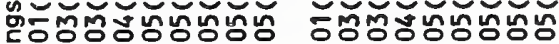

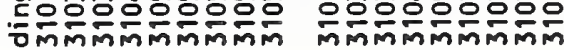
要

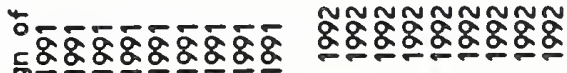

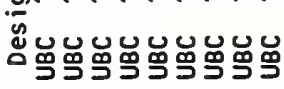

うううらうううらう 


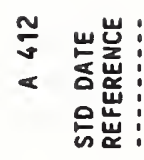

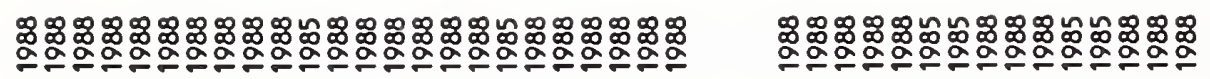

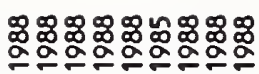

岂:

$\ddot{\ddot{u}}$

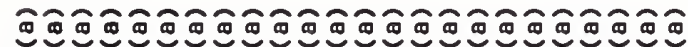

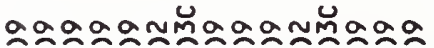

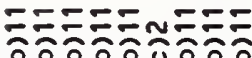

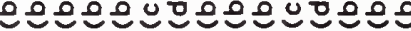

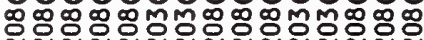

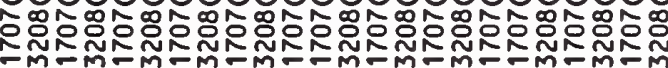

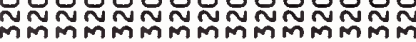
o.

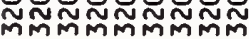

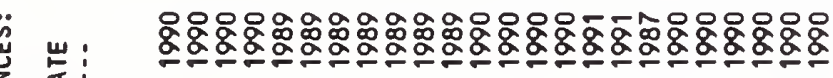

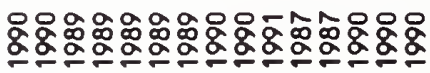

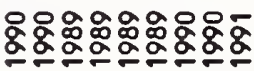

亭

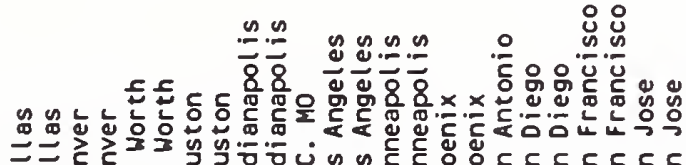

岩:

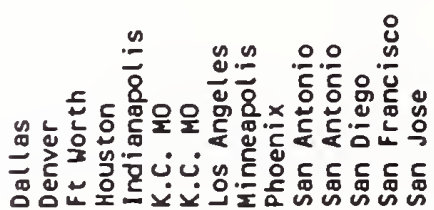

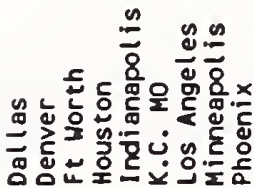

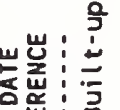

은

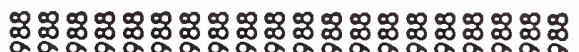

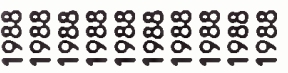

$\bar{\Phi} \bar{\alpha} \bar{\alpha} \bar{Q}$

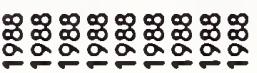
(2)

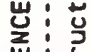

w

㟧

:

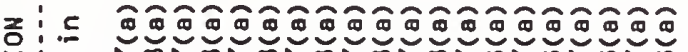

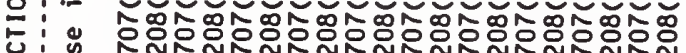

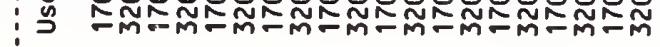

은

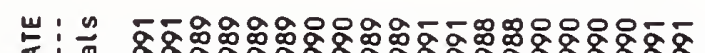

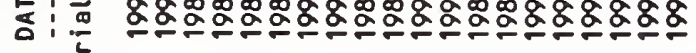

山.

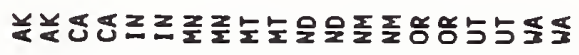

爱

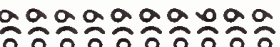

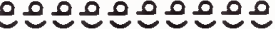

\%ㅇㅇㅇㅇㅇㅇㅇㅇㅇㅇㅇㅇㅇㅇㅇ

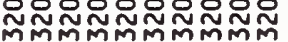

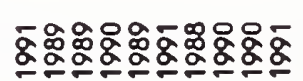

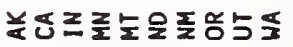

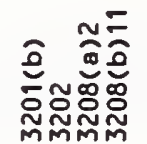

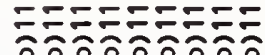

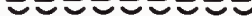

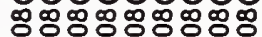

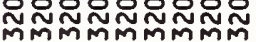

$\stackrel{8}{9}$

홓ㅎㅇ

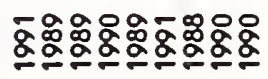

8

罗罪罗罗

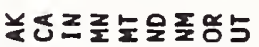

\&

\&

$\stackrel{\$}{2}$

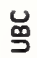

$\bar{\sim}$

ํㅜํ

$\bar{\sim}$ 


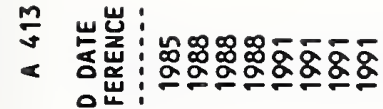
絬:

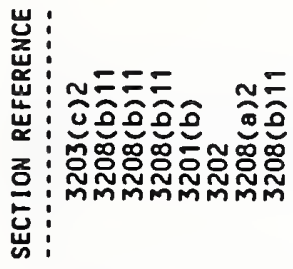

蒿

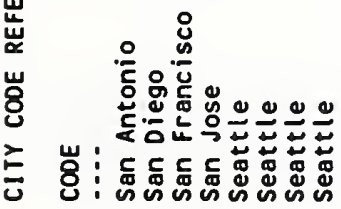

岂

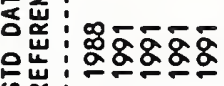

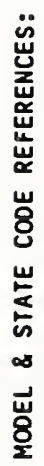

:

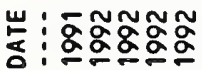

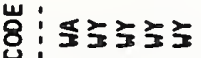

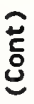

ळ

$\frac{\sim}{\dot{m}}$

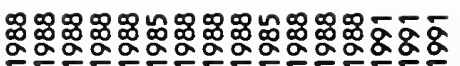

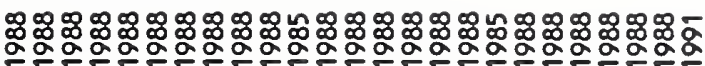

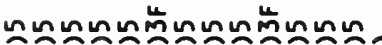

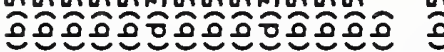
mo: ల్లైల్లుల్n

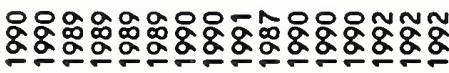

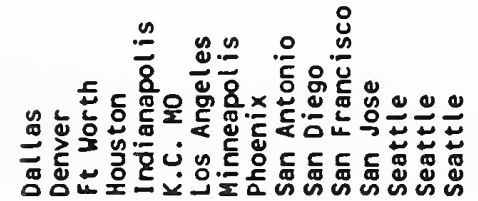

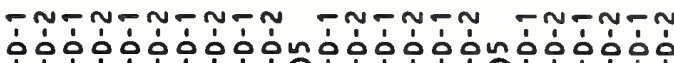

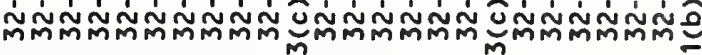

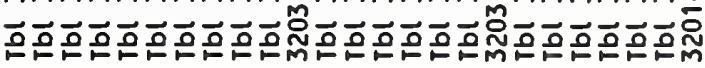

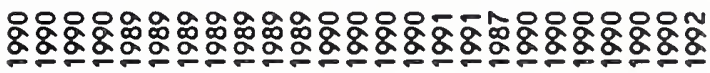

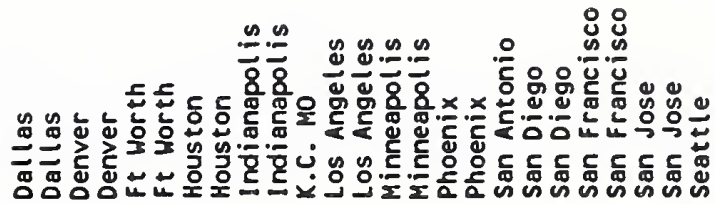

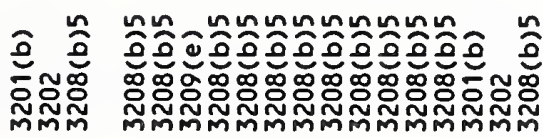

$\stackrel{ \pm}{=}$

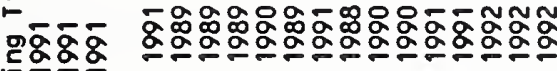

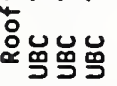

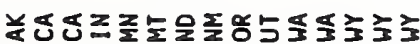

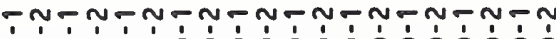

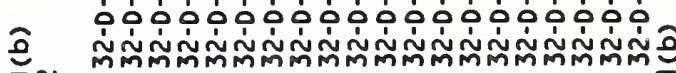

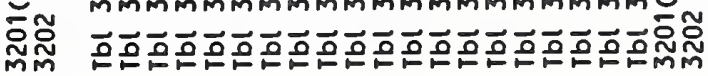

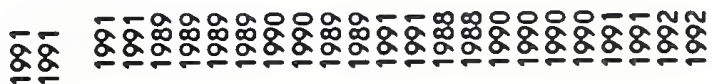
空品

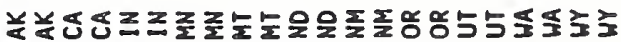

$\bar{\xi}$

$\frac{m}{\dot{m}}$ 


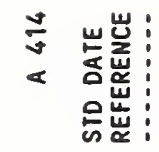

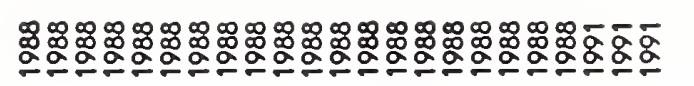

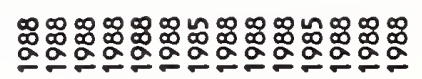

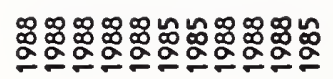

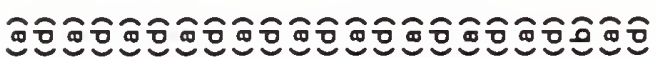

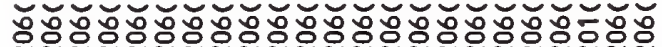
m̄ñ

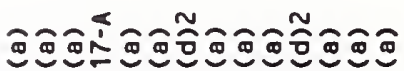

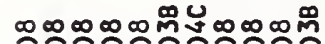

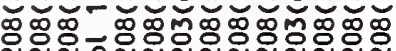

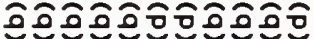

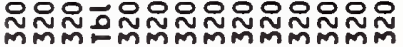
o.

긍 :

出:

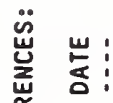

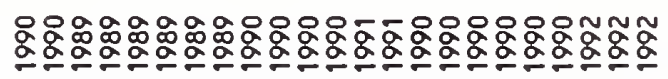

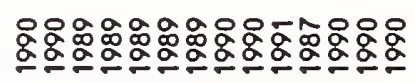

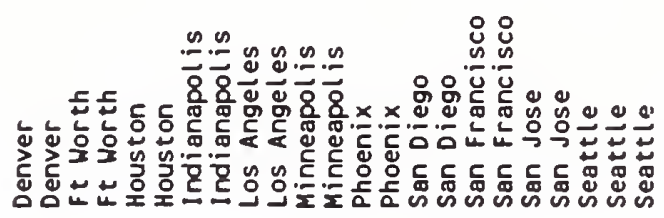

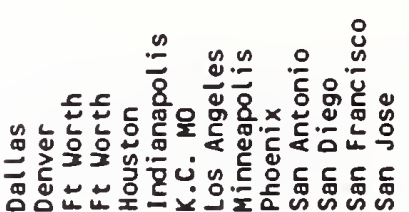

흥:

峞

作

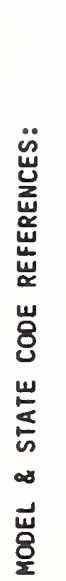

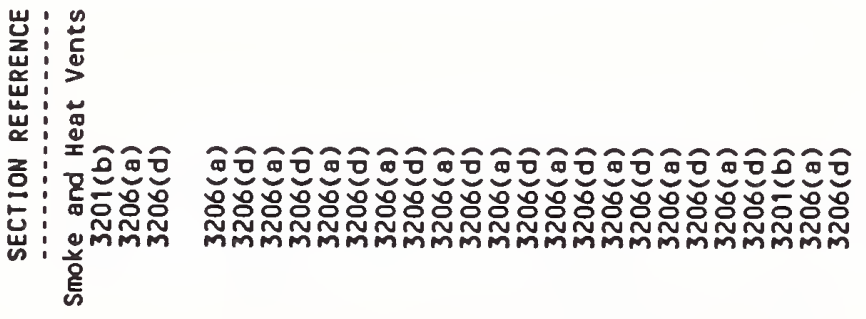

岁

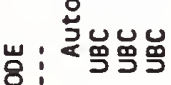

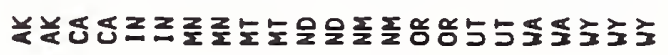

$\bar{g}$

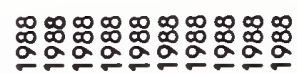

$\stackrel{\leftarrow}{\Phi}$

。ั

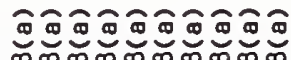

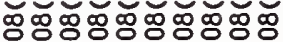

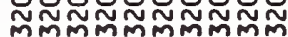

弯

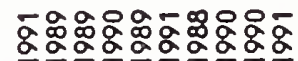

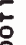

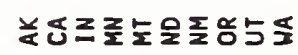

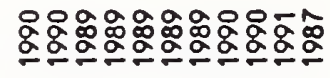

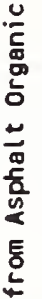

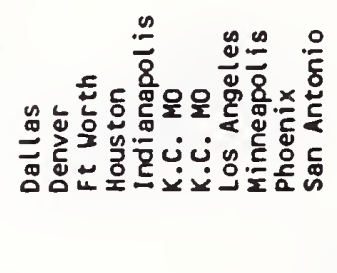

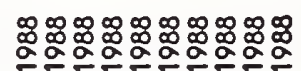

\&

$\frac{5}{5}$

$\infty \infty \infty \infty \infty \infty \infty$

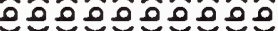

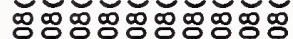

m̄nलำ

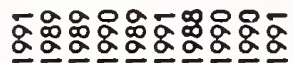

兽

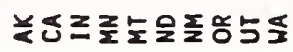

$\underset{\Xi}{0}$

$\stackrel{8}{2}$

$\tilde{\tilde{m}}$

$\dot{m}$ 


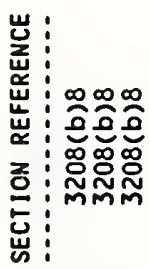

岂

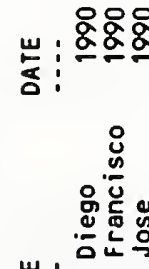

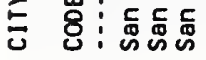

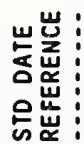

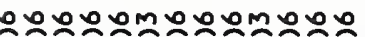

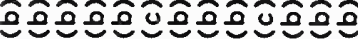

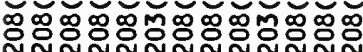

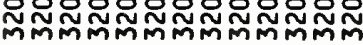

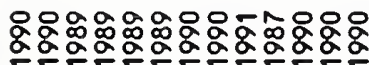

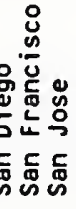

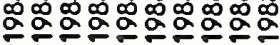

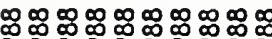

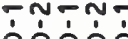

шищц шищ

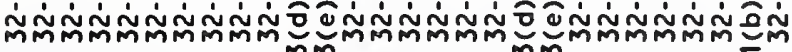

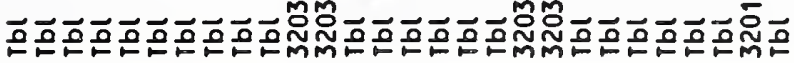

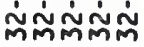
믄듀윰은

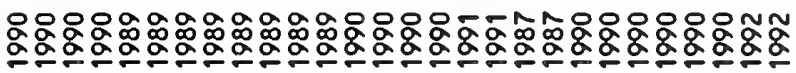

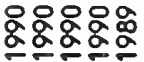

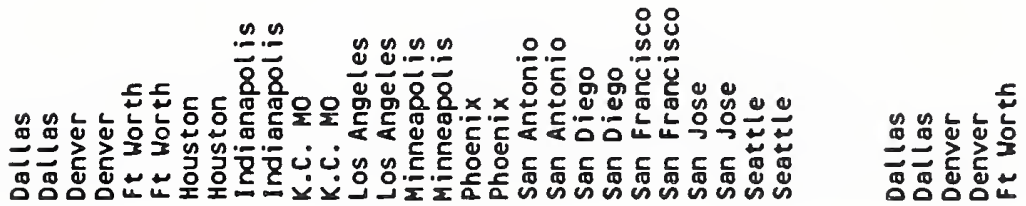

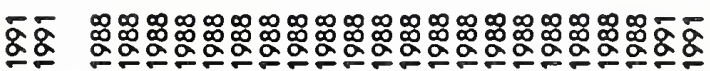

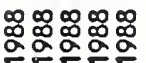

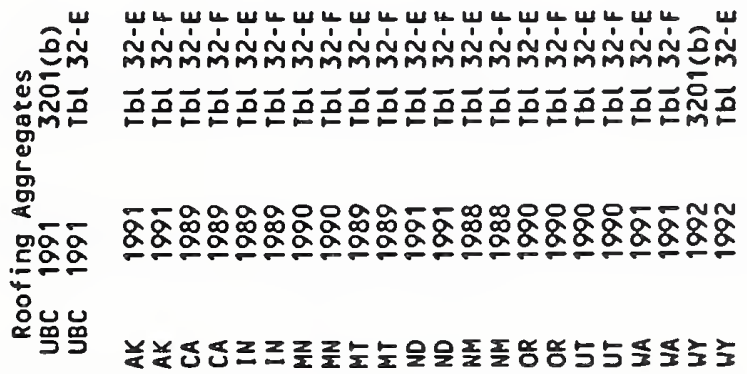

-NT N

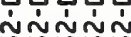

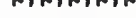

은은은은온

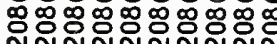

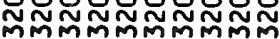

岕:

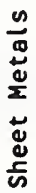

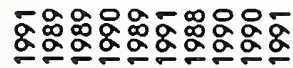

岁 :

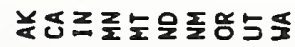

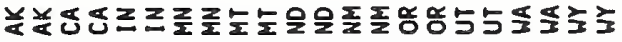

遂遂代 
$\frac{1}{4}$

岕: : 它岕:

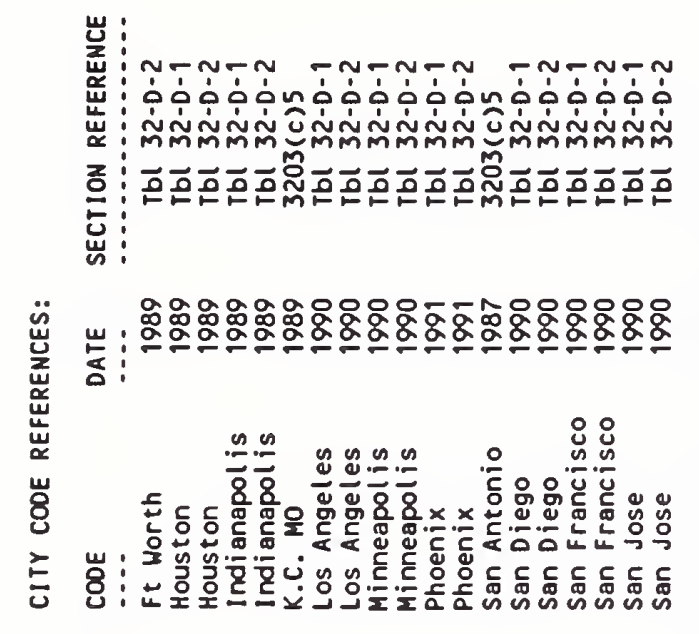

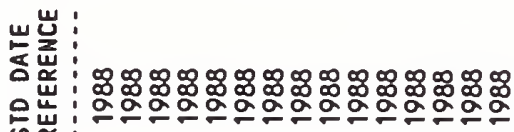

m

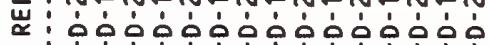

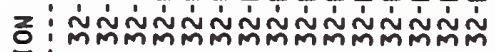

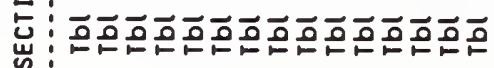

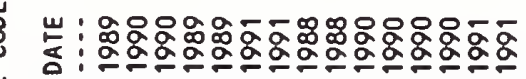

营

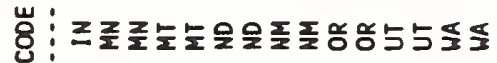

울

宅

芩 $\stackrel{0}{\dot{2}}$

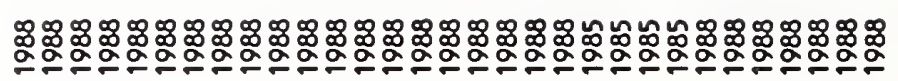

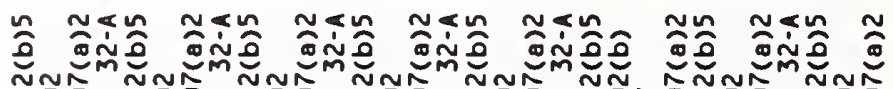

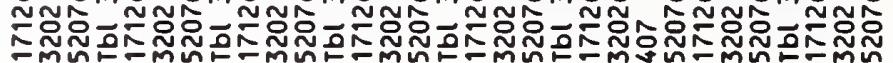

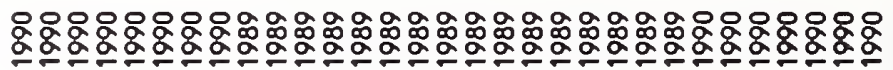

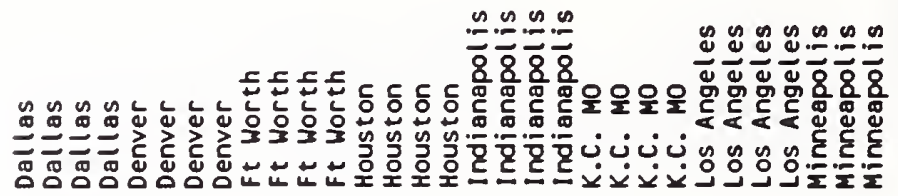

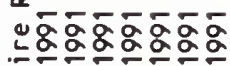

$\stackrel{4}{\frac{1}{5}}$

$\frac{\text { o }}{\frac{5}{E}}$

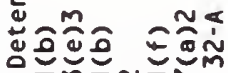

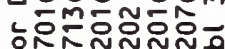

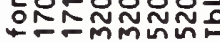

은

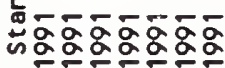

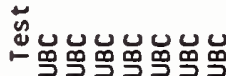

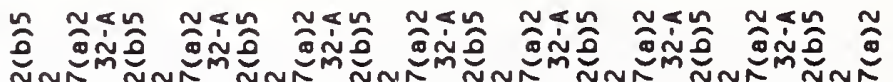

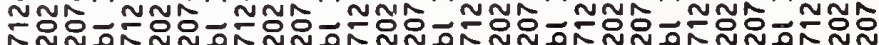

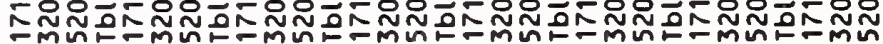

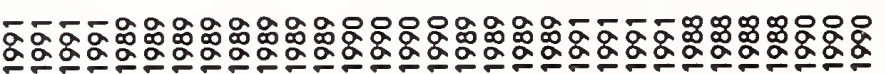

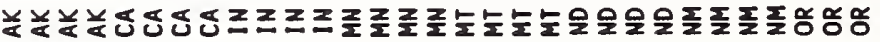

: 
我岕: 은

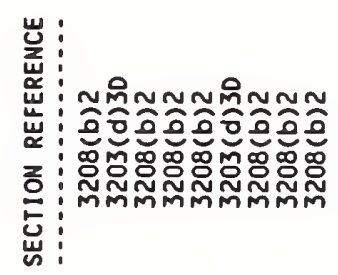

蒿 $\underset{u}{u}$

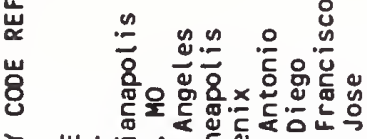

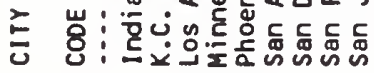

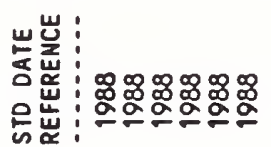

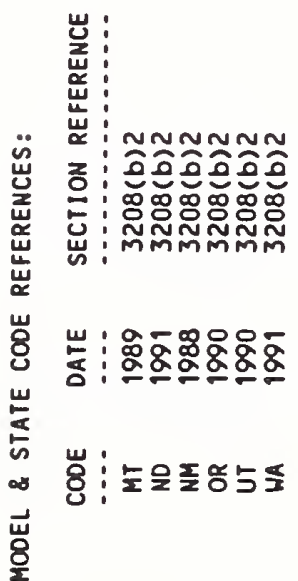

동

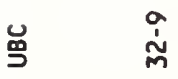

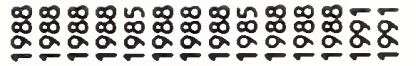

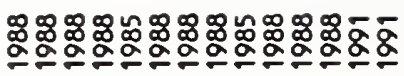

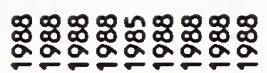

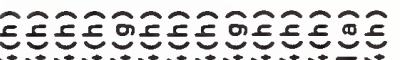

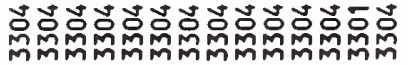

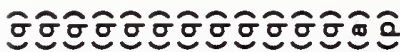

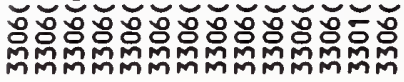

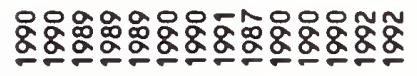

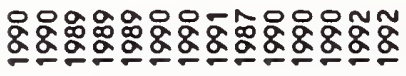

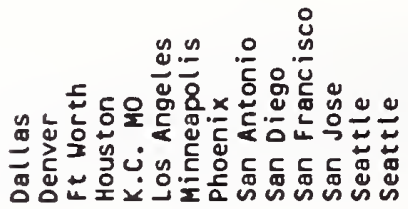

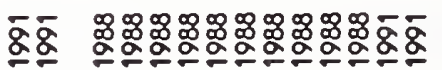

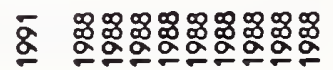

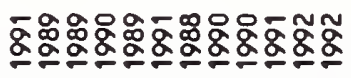

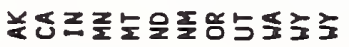

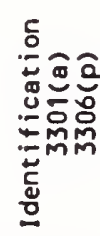

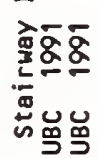

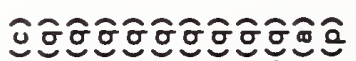

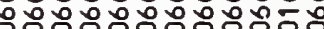

minmminmmminm

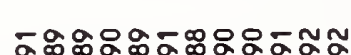

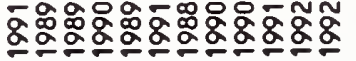

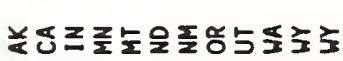

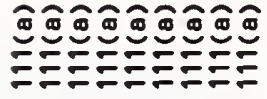
운운운운운운은연연

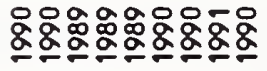

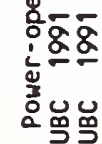

$\bar{\alpha}$

$\bar{m}$

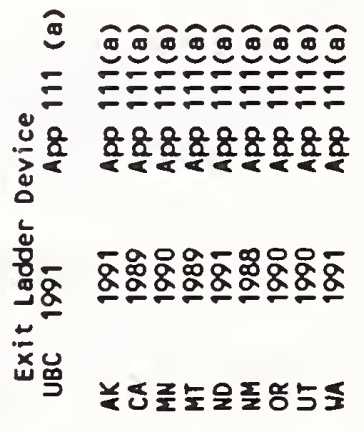

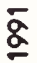

m

$\stackrel{m}{m}$ 


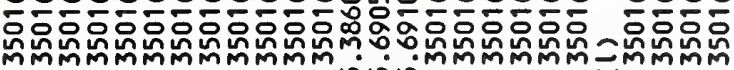

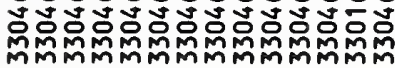

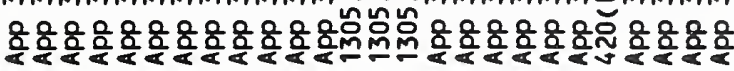

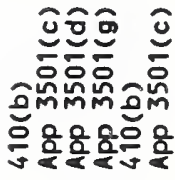

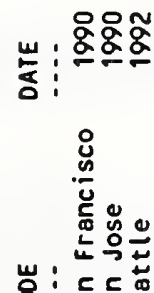

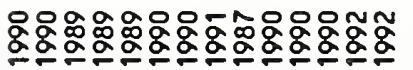

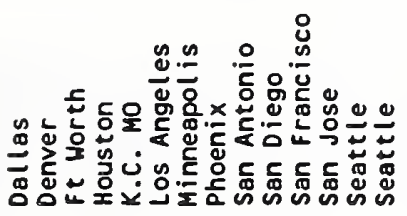

世

察:

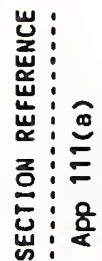

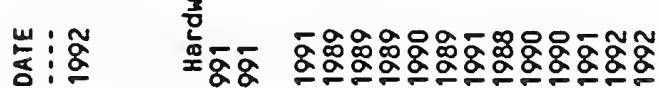

$\underline{\delta}: \underline{\alpha}$

岁

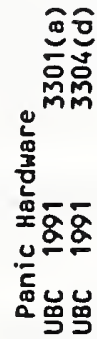

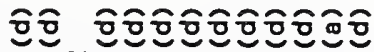

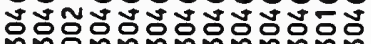
m̄ñ

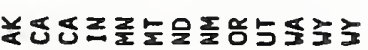

\section{高

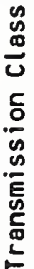

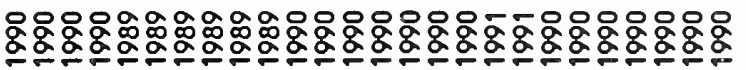

है 8

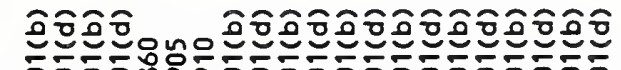

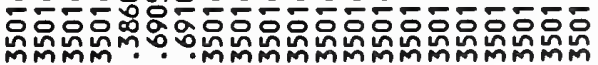

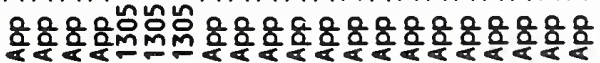

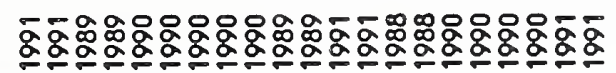

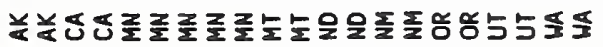

웡용

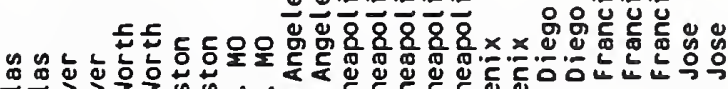

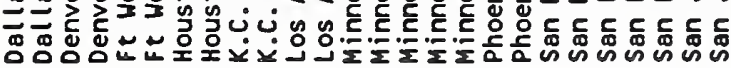

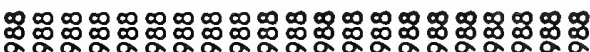

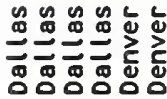

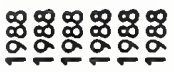

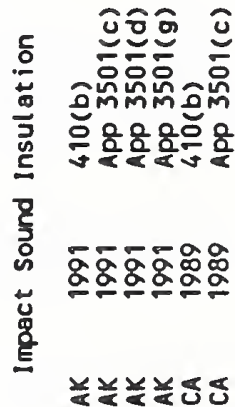

$\stackrel{8}{g}$

$\tilde{n}$

옹영영영영요 
莳 澏:

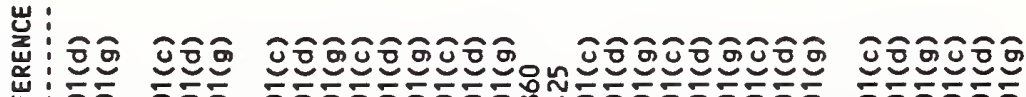

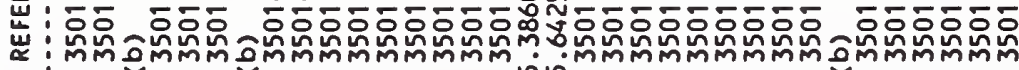

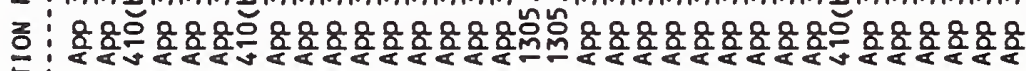
总:

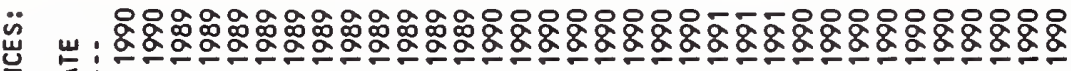
a :

웅

こ

崖岕

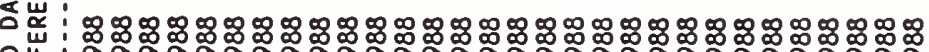

눈

:

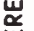

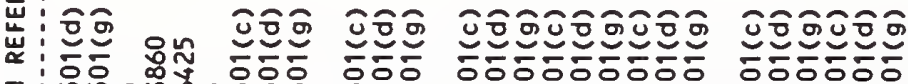
흘

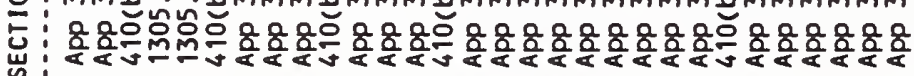

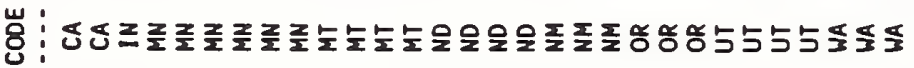

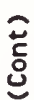

诰 $\quad \stackrel{n}{m}$

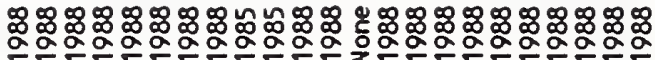

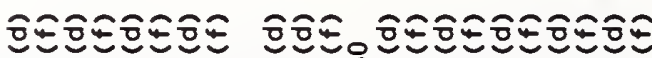

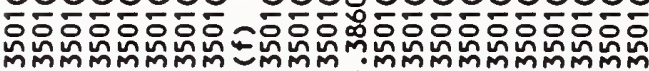

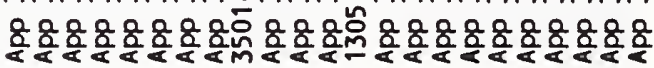

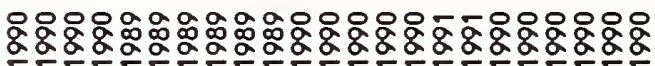

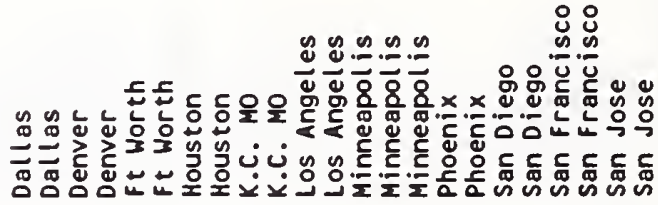

:

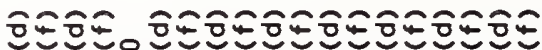

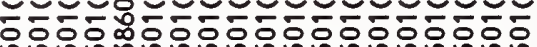

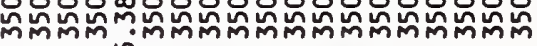

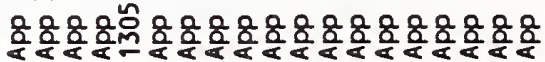

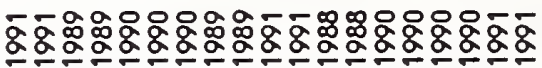

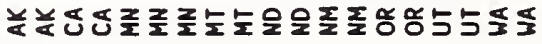




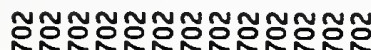

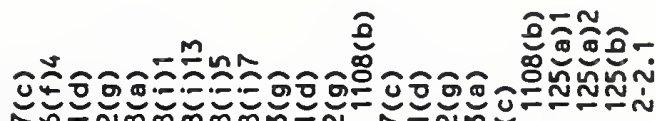

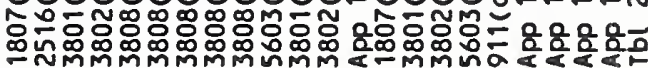
MMMMMMMMMMMMM

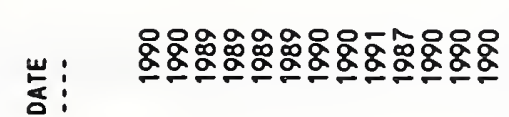

京

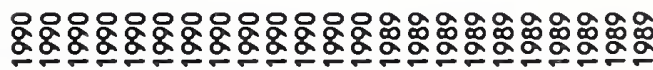

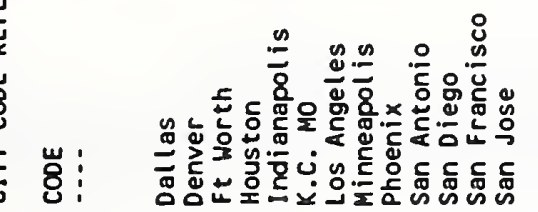

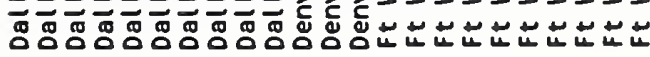
ప్

$\stackrel{\mathscr{8}}{\underline{\alpha}}$

$\bar{g}$

$\overline{\dot{m}}$

$\overline{\dot{m}}$

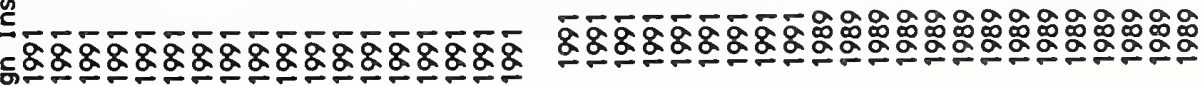

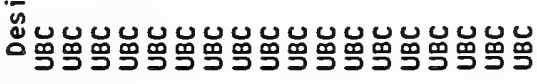

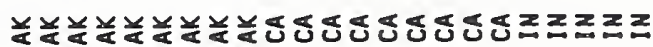



崖 岂:

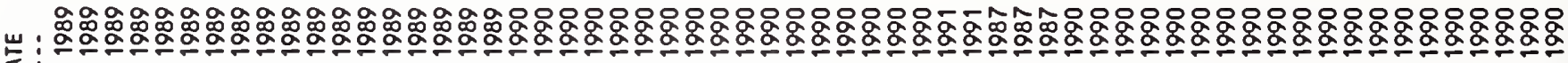
ธ:

르응으

ํㅜㅇㅇㅡ

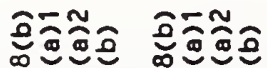

응

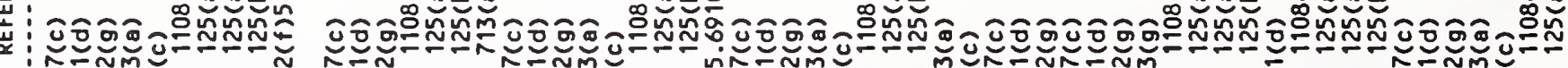

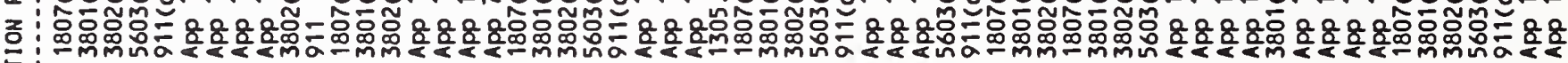

8

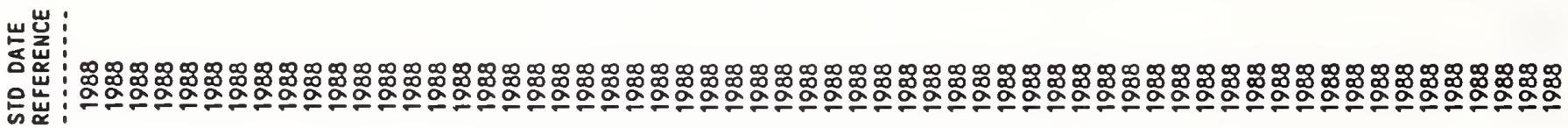

岂:

崖:

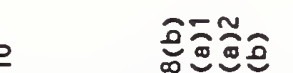

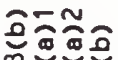

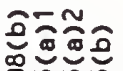

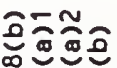

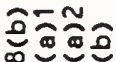

อิ

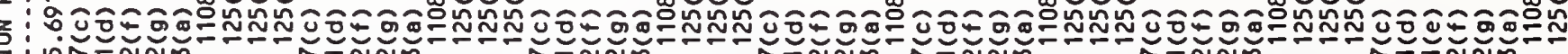

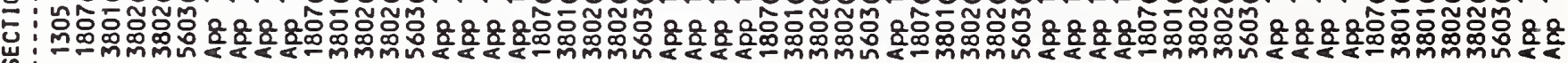

世

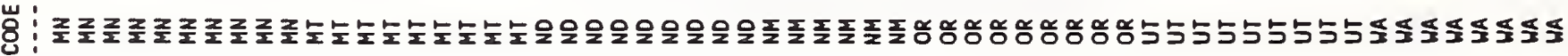

总

茴 $\quad \overline{\dot{m}}$ 

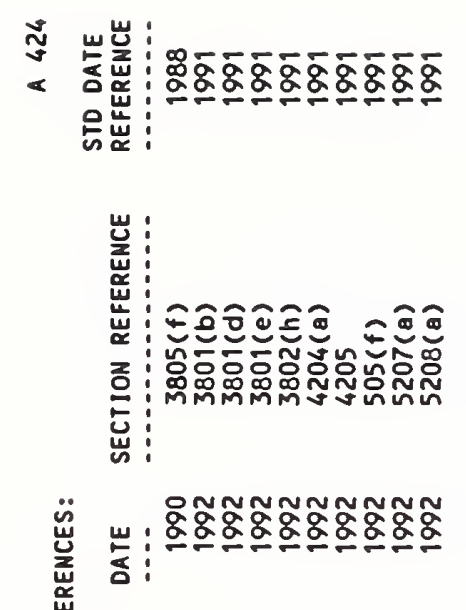

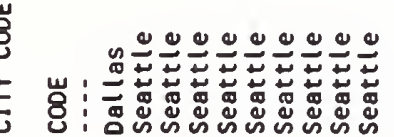

岕:

容:

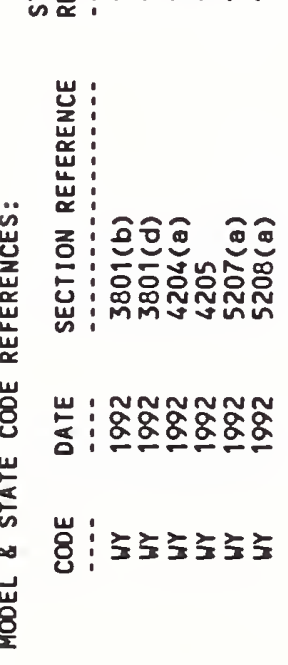

芒

号

ì

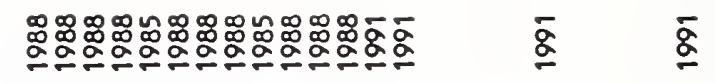

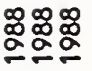

8

\%ั

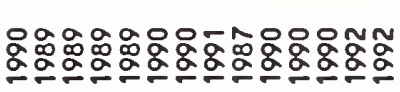

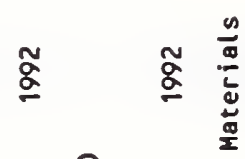

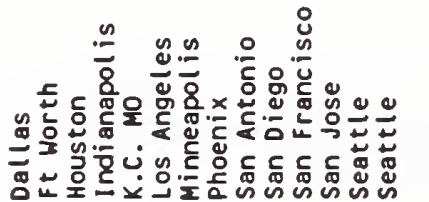

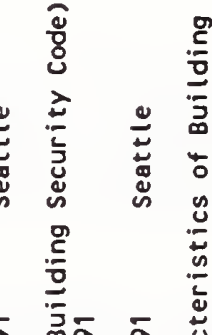

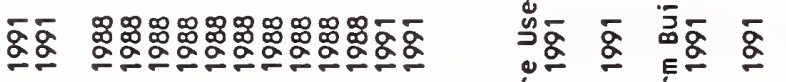

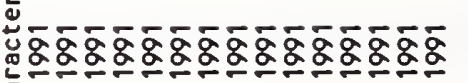

ํํำ

드르

양융요

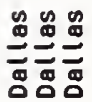

ఖ̊జ్ఞి

.?口.

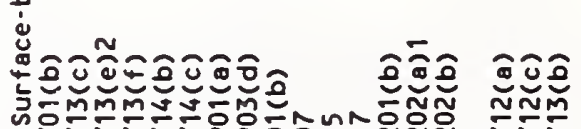

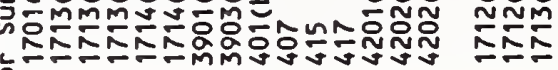
$+$

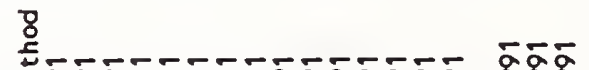

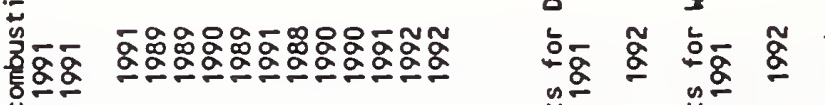

罗品

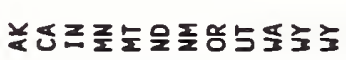

$\bar{\Phi}$

$\bar{g} \quad \bar{g} \quad \bar{g}$

$\bar{j}$

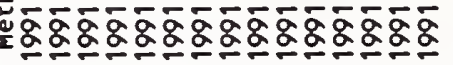

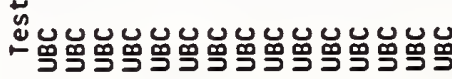

gूळ

$\dddot{2} \geq$

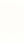

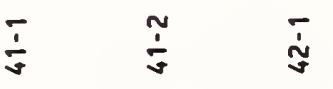




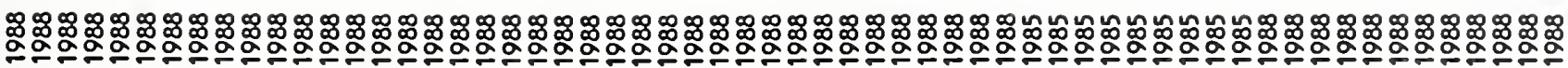
次芯 :

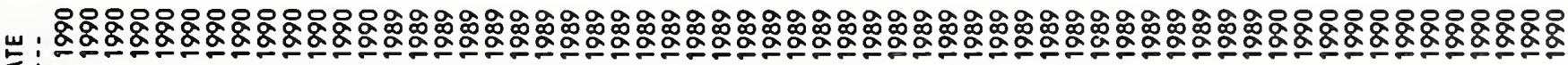

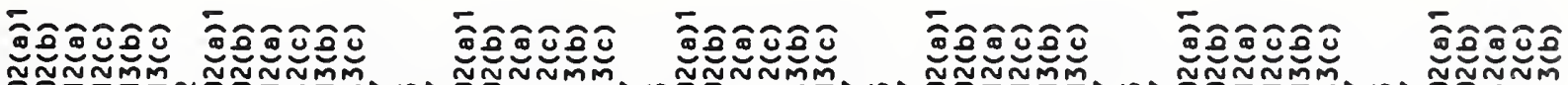
等 :

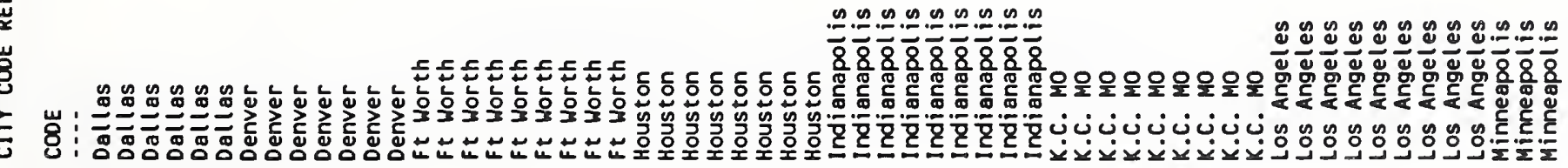

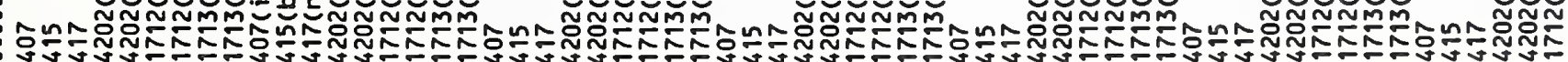

岁

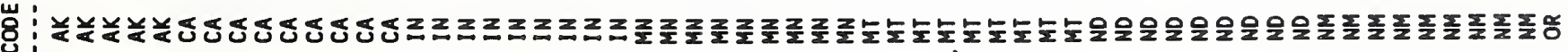

ญ્

$\underset{\sim}{\grave{s}}$ 


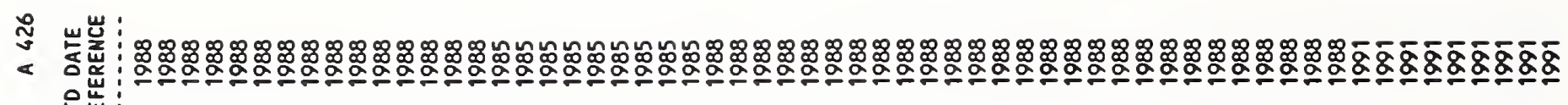
은

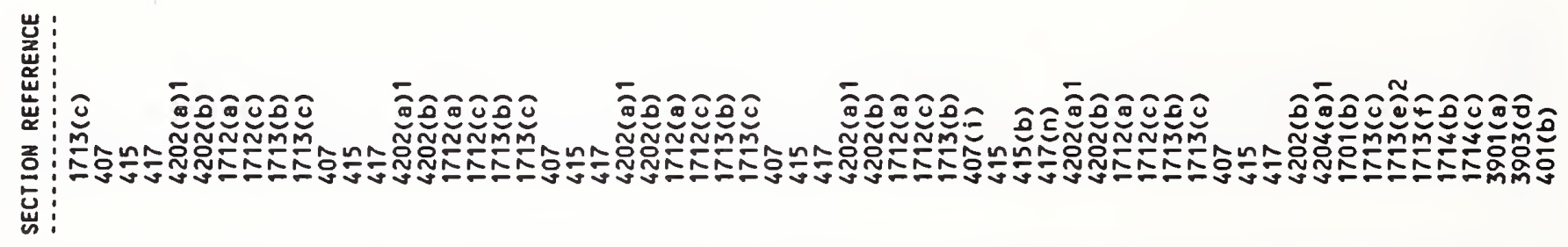
蒒

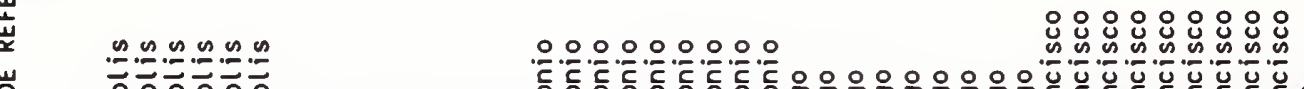

岁 至

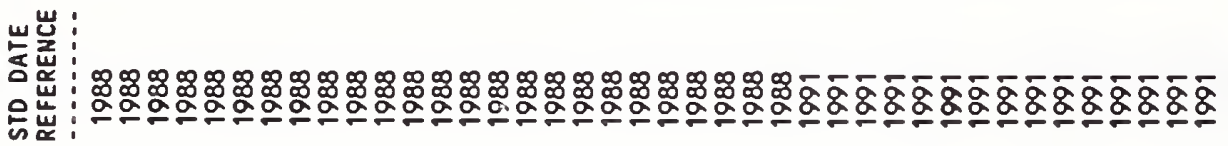

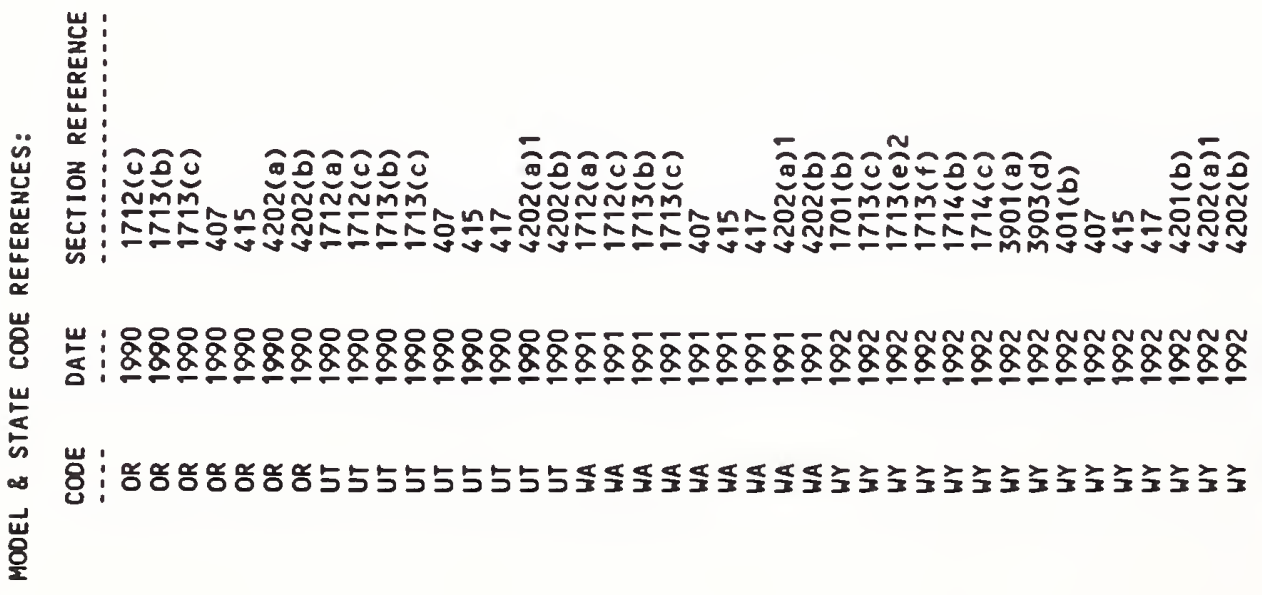

؛े

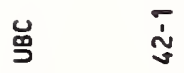




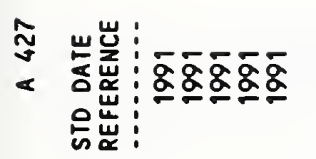

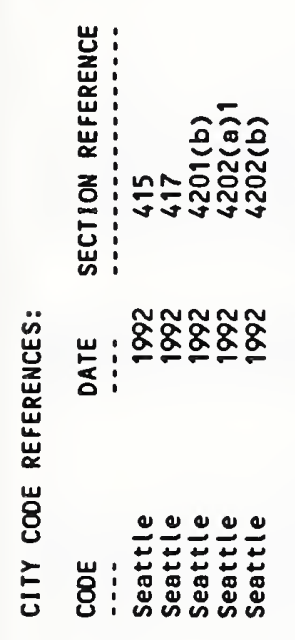

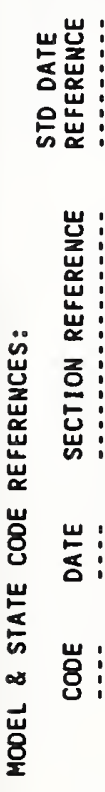

$\underset{\bar{c}}{\bar{c}} \bar{g}$

$\dot{\dot{\sim}} \quad \stackrel{\tilde{\sim}}{\dot{\sim}}$

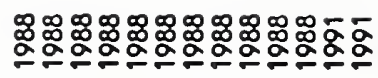

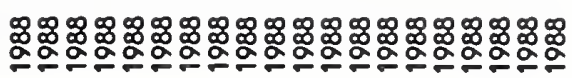

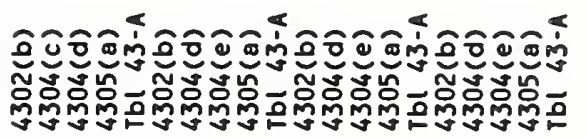

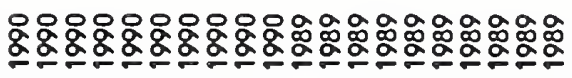

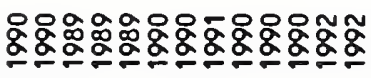

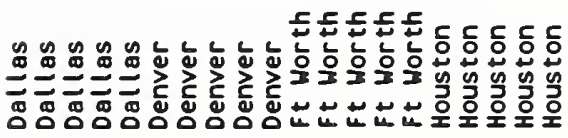

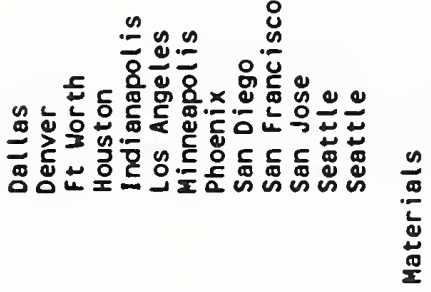

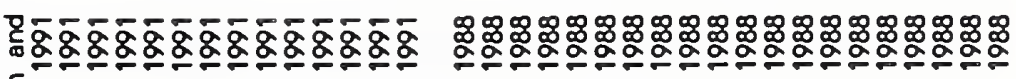
总

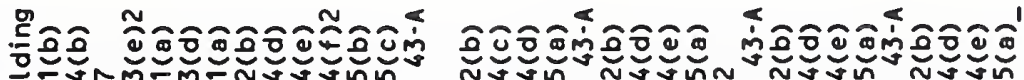

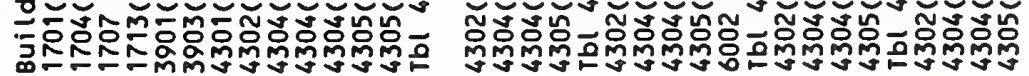

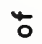

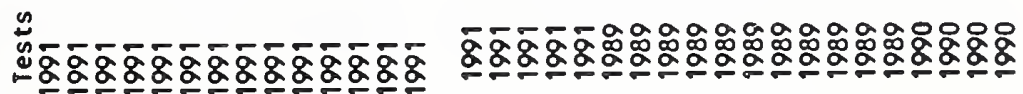
ㄴ.

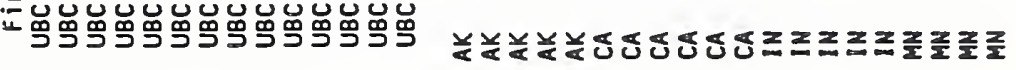

$\bar{g}$

$\overline{\dot{v}}$ 


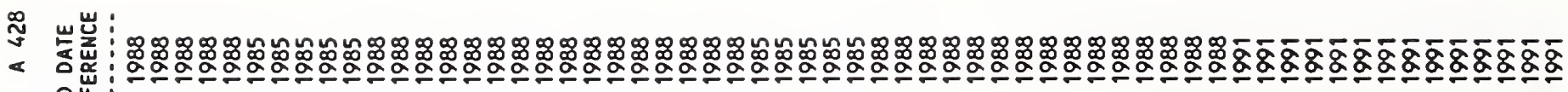
过崖:

禽

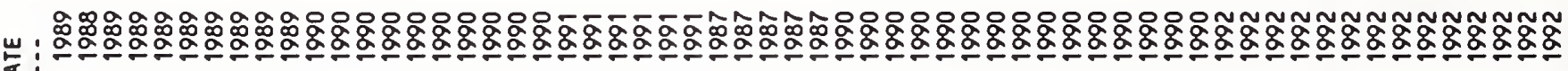
ธㄴ

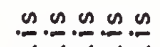 \\ $8 \overline{8} \frac{8}{8}$

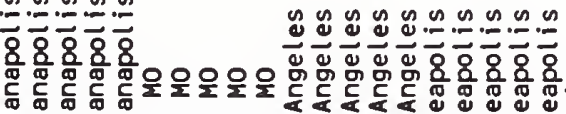

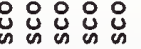

岁

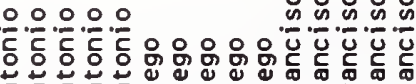

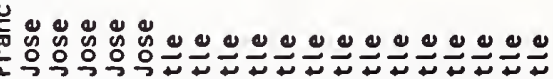

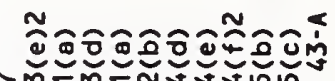

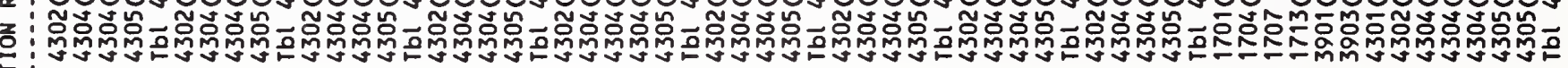
定:

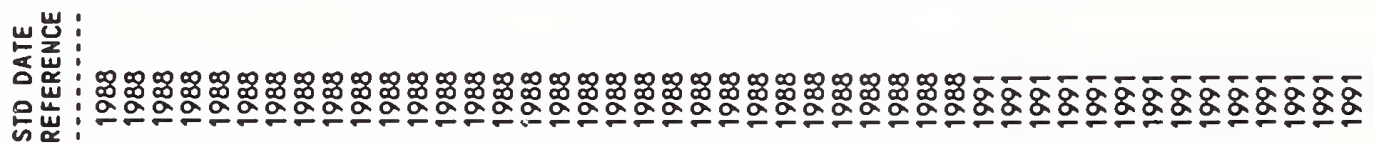

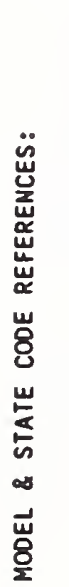

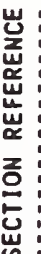

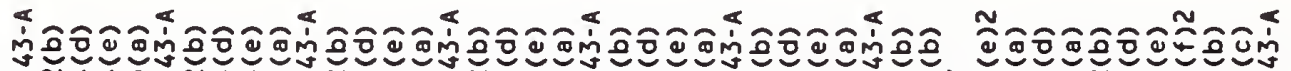

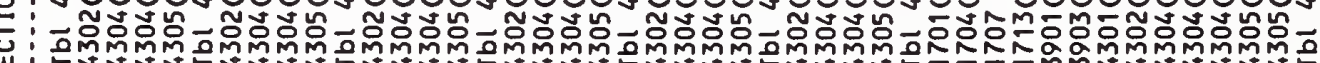

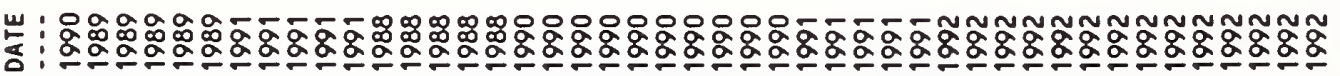

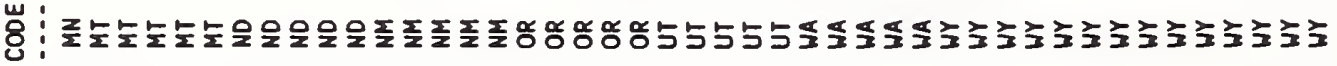

莣

$\stackrel{\mathscr{m}}{\dot{m}}$ 


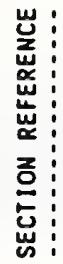

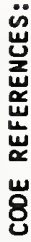

$\infty \infty \infty \infty \infty \infty \infty \infty \infty \infty$

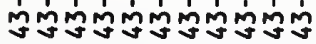

$\infty \infty \infty \infty \infty \infty \infty \infty \infty \infty$

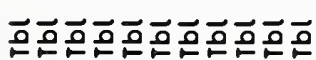

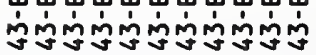

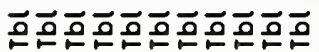

ํํำ

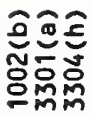

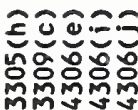

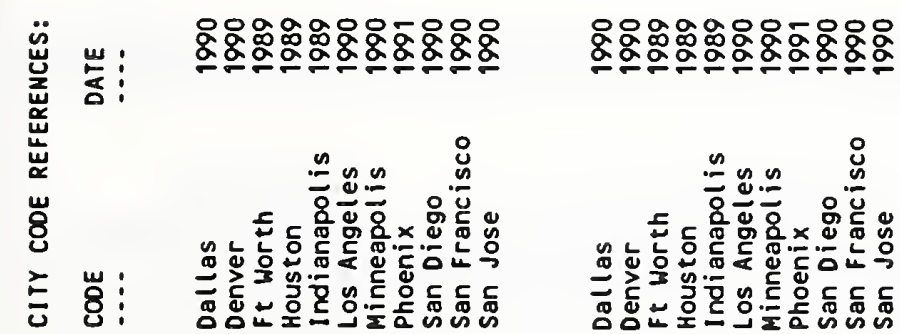

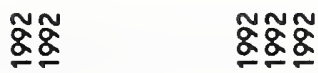

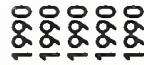

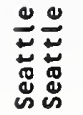

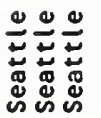

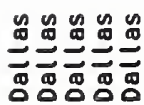

岕鵋?

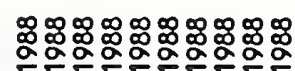

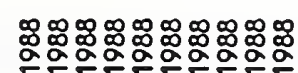

范

온

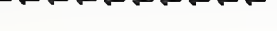

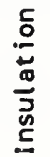

$\infty \infty \infty \infty \infty \infty \infty \infty \infty$

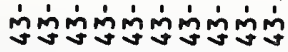

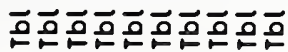

㞧

=

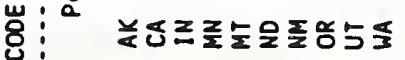

产:

ㄴ.

$\stackrel{\varpi}{\varpi}$

$\stackrel{\varpi}{\stackrel{\Xi}{\varrho}}$

曲

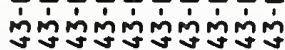

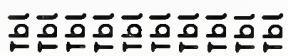

$\overline{\bar{\alpha}} \overline{\underline{\alpha}} \overline{\mathrm{g}} \overline{\mathrm{g}}$

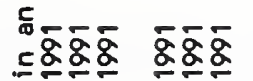

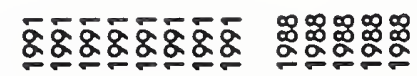

茄

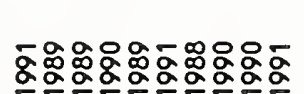

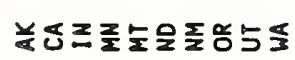<smiles>[AlH][TlH]</smiles>

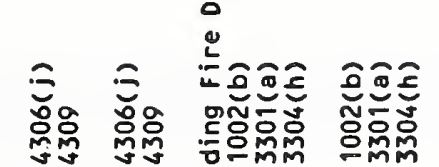

$\stackrel{8}{=}$

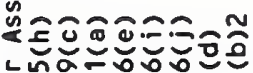

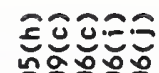

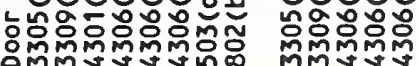
约 के ¿

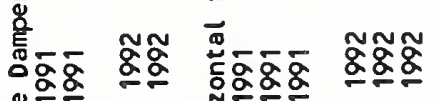

范.

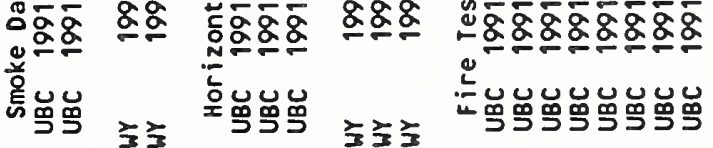

$\bar{\alpha} \bar{\alpha} \bar{\alpha} \bar{\alpha} \bar{\alpha} \bar{\alpha}$

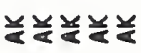

$\frac{}{\dot{m}}$

$\overline{\dot{m}}$

$\stackrel{\sim}{\dddot{m}}$

$\frac{m}{\dot{y}}$

ํํำ 


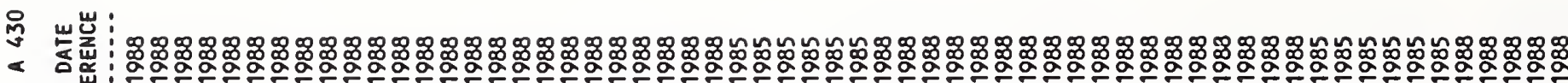
员岕:

岂:

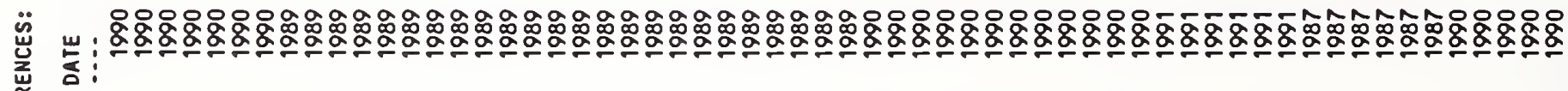

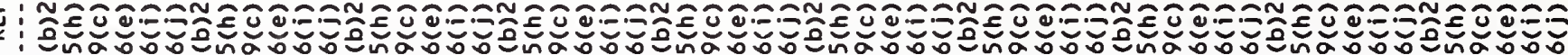

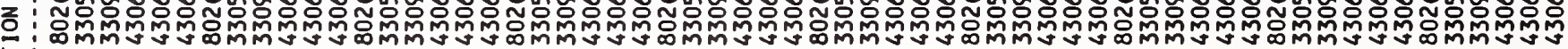

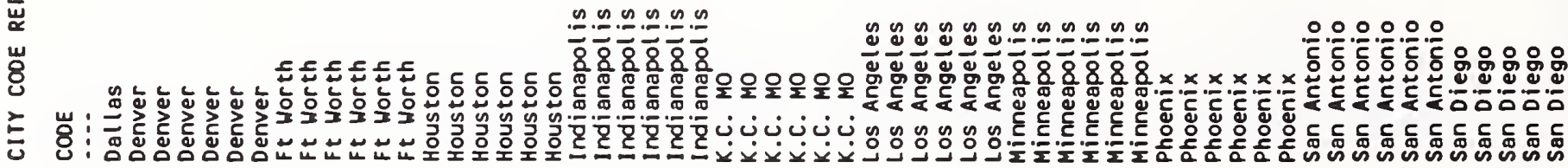

岂:

运崖?

:

넣

岂

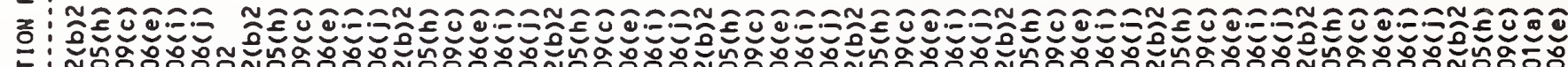

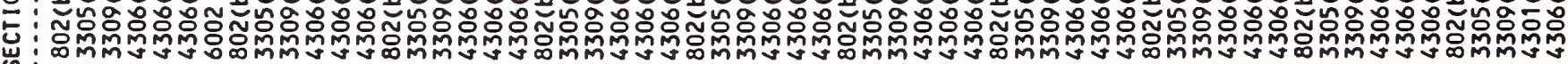

느 : -

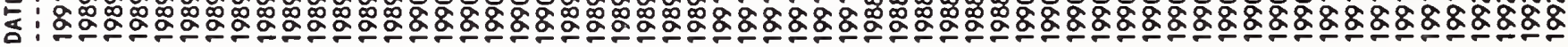

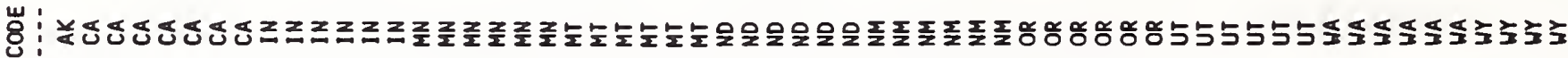

突

品 $\quad \tilde{m}$ 
: 䟲:

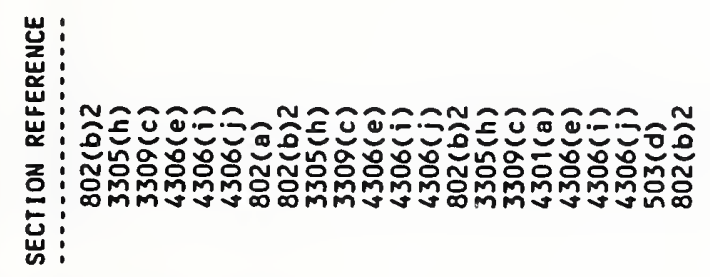

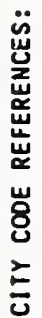

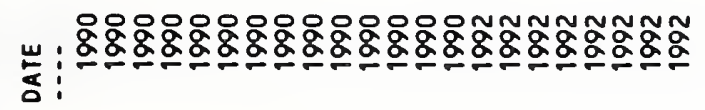

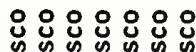

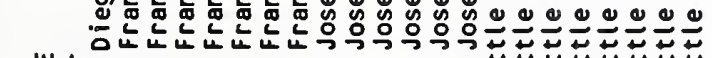

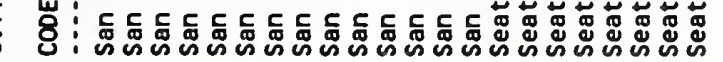

世ّ山ّ:

员:

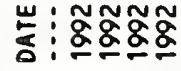

岁: ‘亏亏方

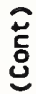

ஸ்

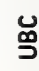

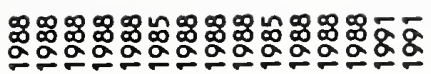

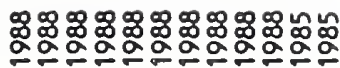

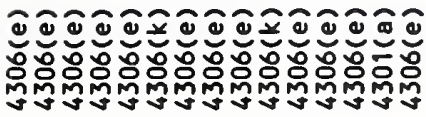

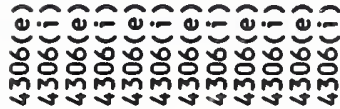

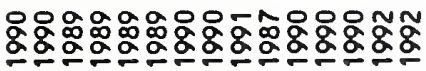

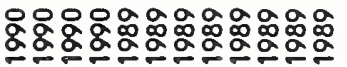

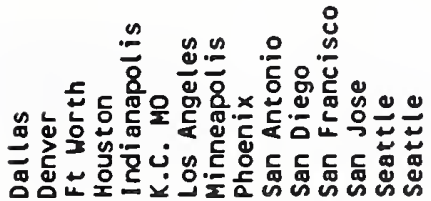

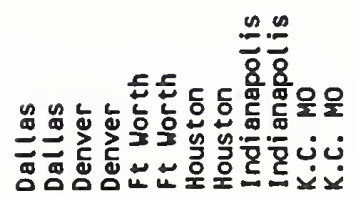

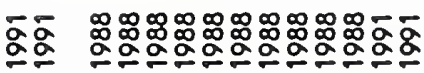

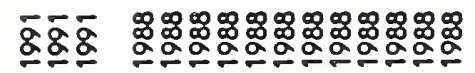

总

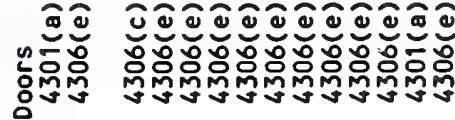

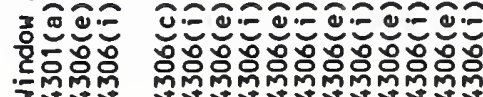

$\stackrel{5}{5}$

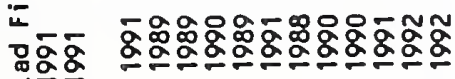

氙出品

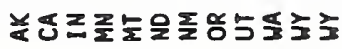

$\div$

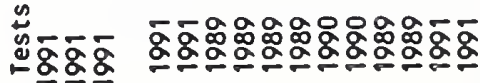

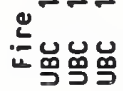

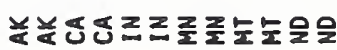

$\bar{\alpha}$

$\bar{\alpha}$

$\stackrel{m}{\tilde{g}}$

崩 


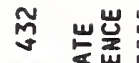

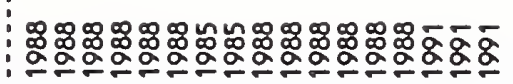
옹 :

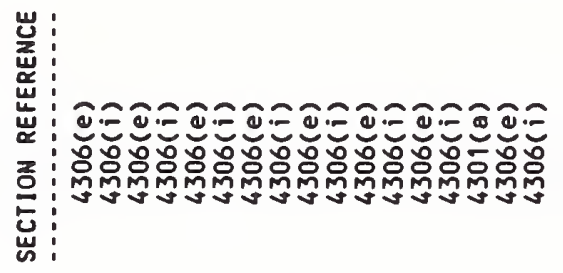

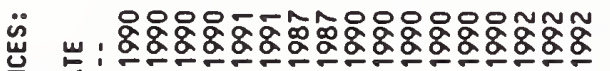
: :

岂 :

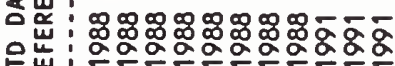

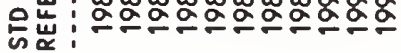

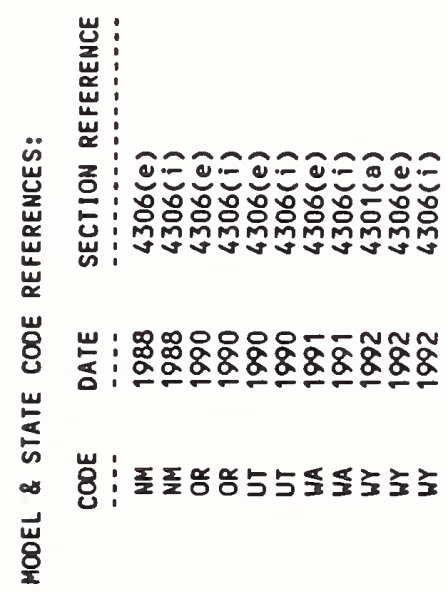

옹

萝 守

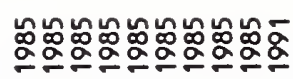

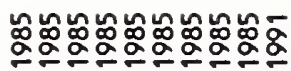

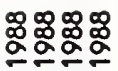
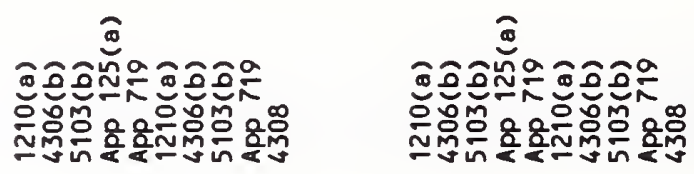

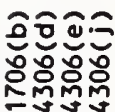

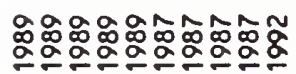

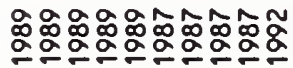

얗ㅇㅎㅇㅇㅛ

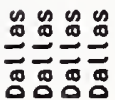

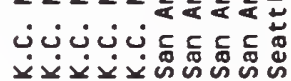

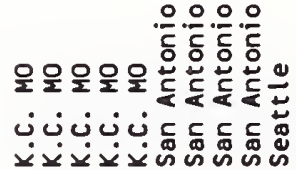

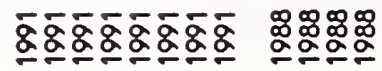

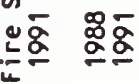

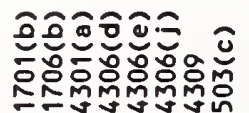

อิํํำ:

ธิ์

茄蛋

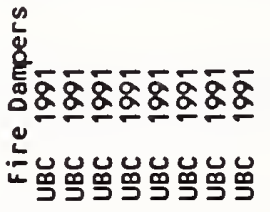

$\bar{\alpha} \bar{\alpha} \bar{\alpha} \bar{\alpha}$

\section{å}

离哥 跑

递

较

$\bar{\Phi}$

$\bar{\Phi}$ 


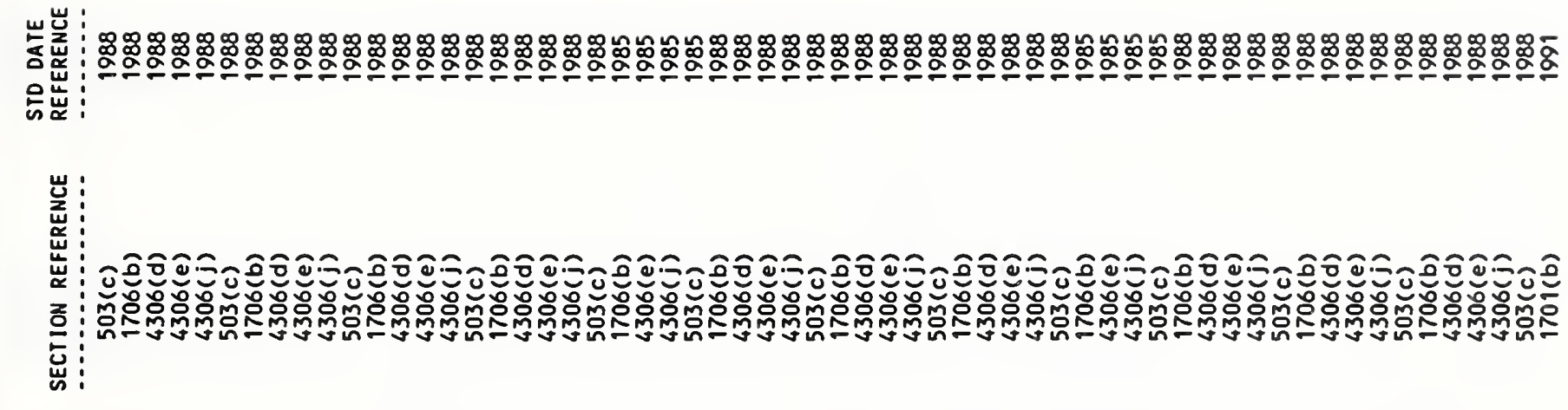
蔩

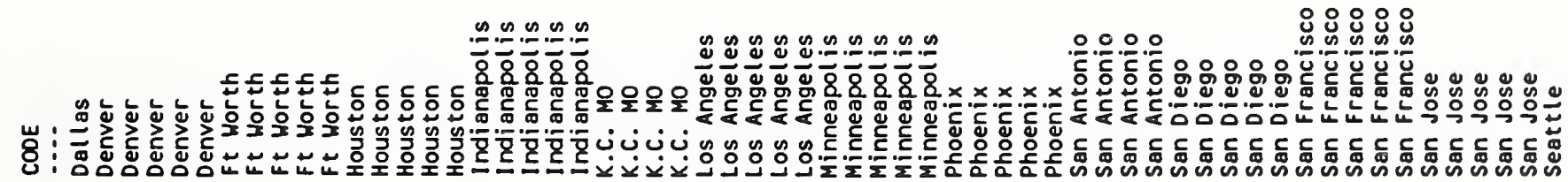
宸: $:$ :

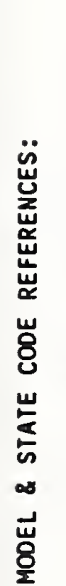

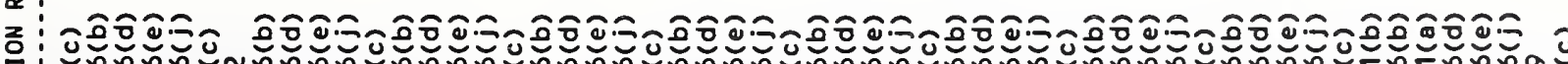
出 :

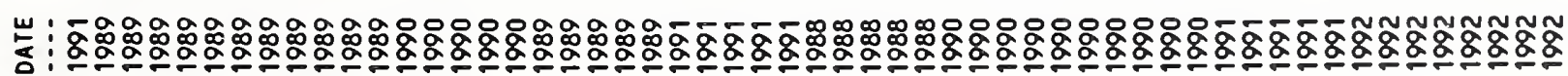

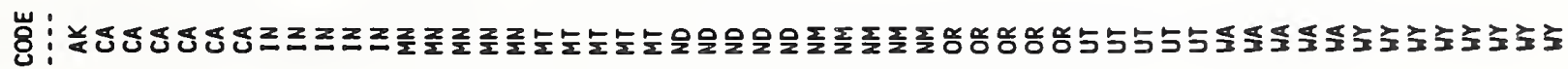


苟 岕:

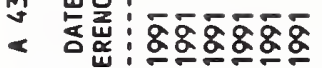

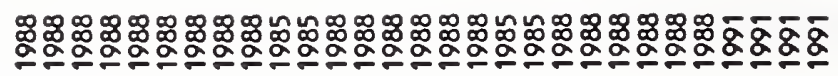

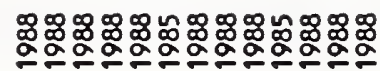

은

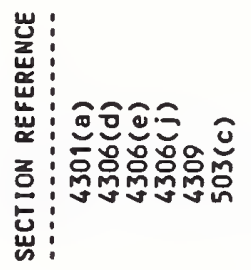

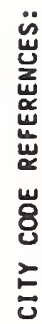

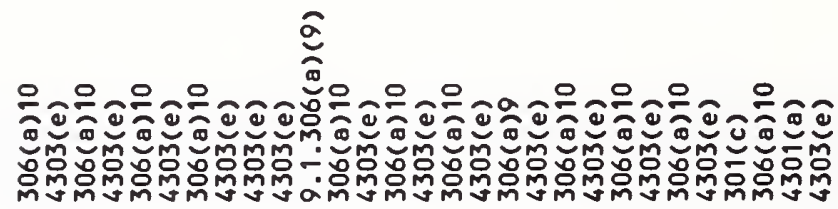

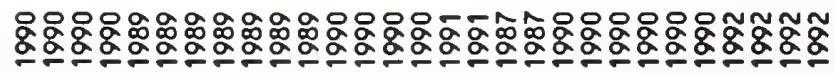

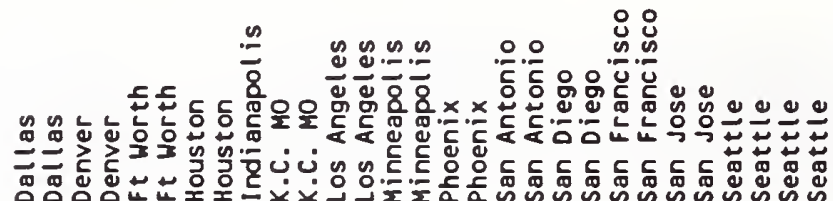

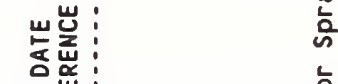

苟

$\infty$ 8ु :

岁:

a!
\&: :

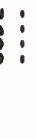

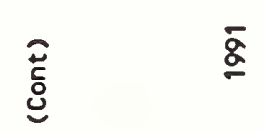

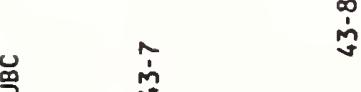

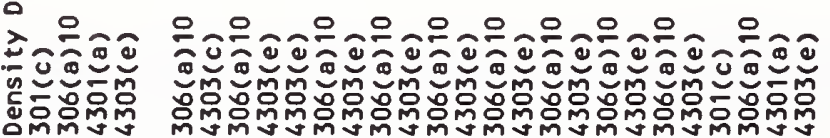

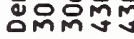

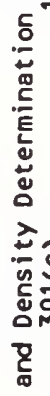

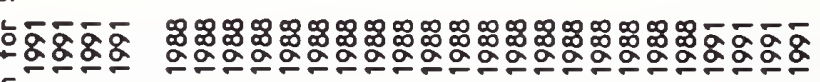

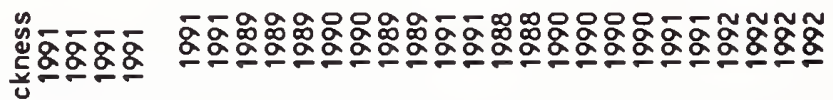

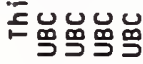

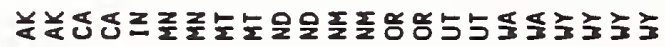

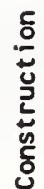

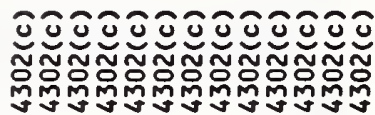

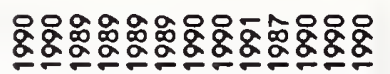

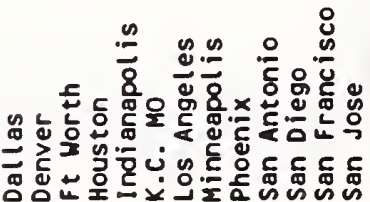

ذँّ ¿

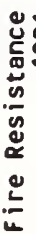

$\stackrel{9}{\circ}$

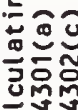
ธั

¿

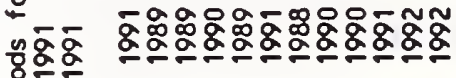
莣品品

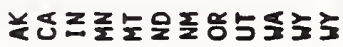

$\bar{\Xi}$

iे 


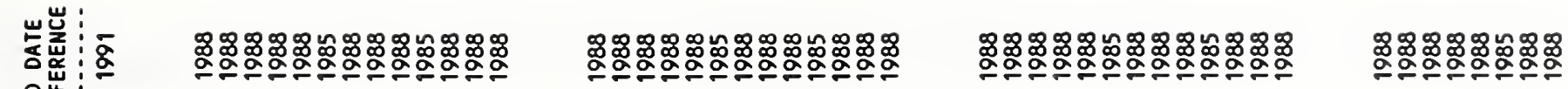
欲:

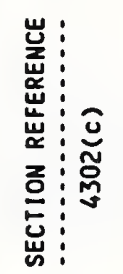

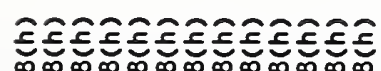

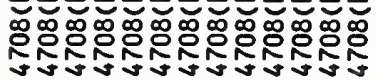

芦

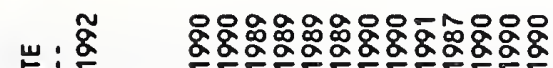
E:

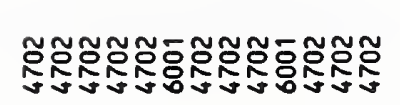

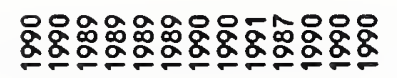

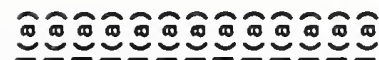

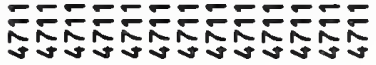

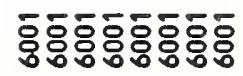

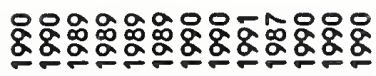

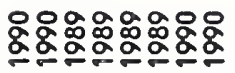

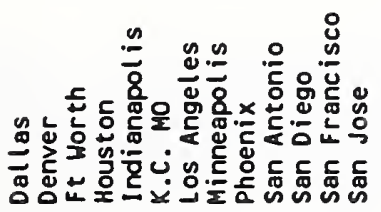

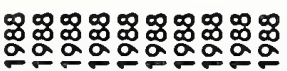

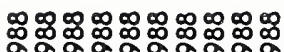

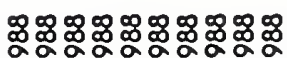

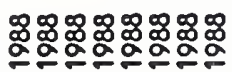

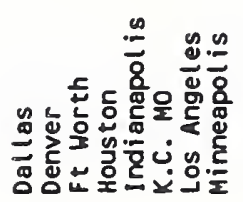

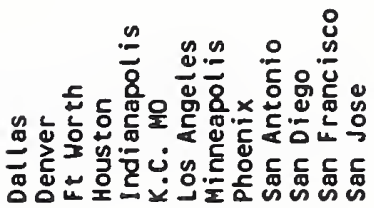

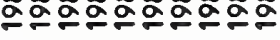

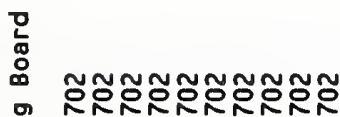

:

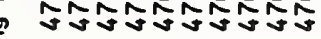

㟶

ㄴ:

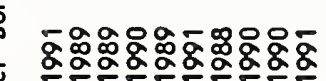

紊

:

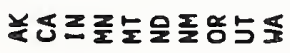

:

נִ

芯

ळ

$\stackrel{\mathbb{8}}{\stackrel{8}{\circ}}$

$\stackrel{\varpi}{2}$

$\dot{i}$

$\stackrel{ }{i}$

$\check{\xi}$

$\stackrel{\frac{1}{3}}{\frac{1}{3}}$

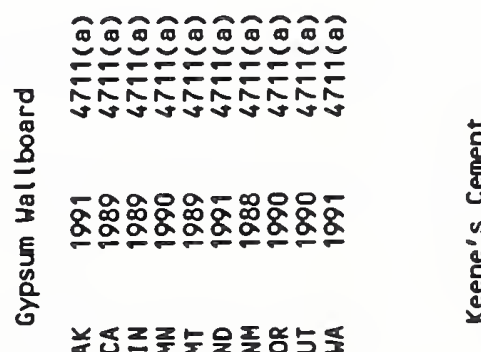

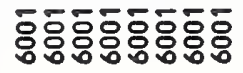

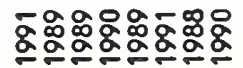

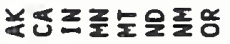


\%

< 它岩?

㟧

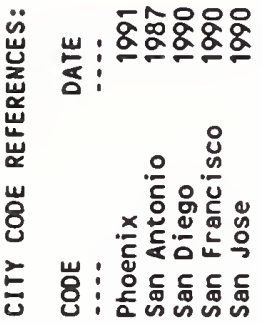

岕岕

空: :

论岕: 음

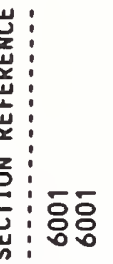

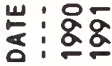

岁:

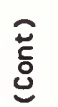

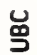

$\frac{\mathfrak{j}}{\dot{j}}$

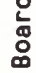

$\stackrel{\mathscr{0}}{=}$

$\stackrel{\sim}{\stackrel{5}{5}}$

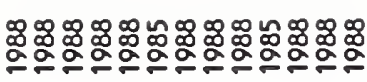

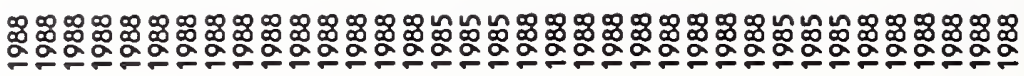

กำกำ

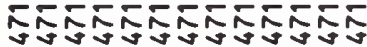

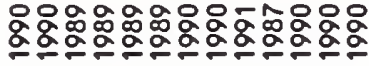

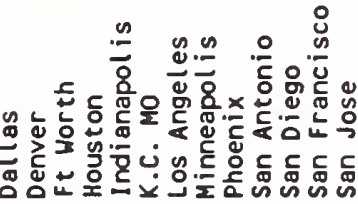

:

$\frac{\text { ? }}{\mathrm{g}}$

nNกNNNNNNN

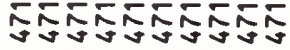

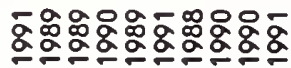

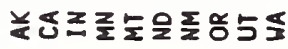

$<\infty u<\infty u<\infty u<\infty u<\infty u<\infty u<\infty u<\infty u<\infty u<\infty u<\infty u<\infty u$

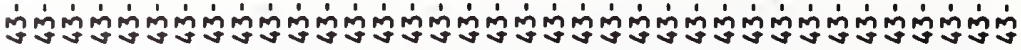

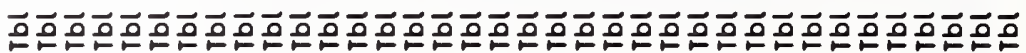

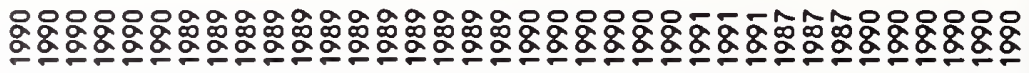

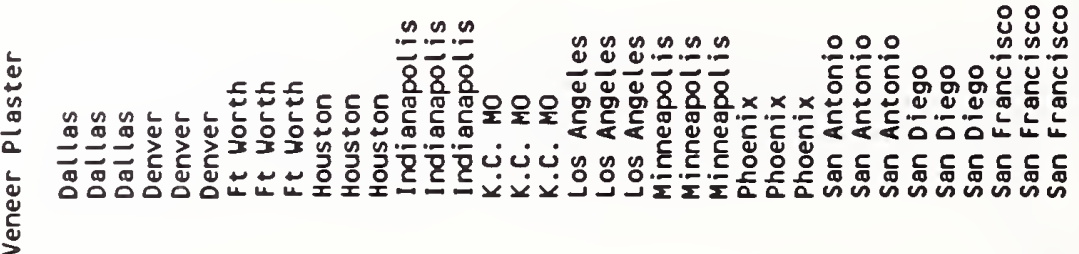

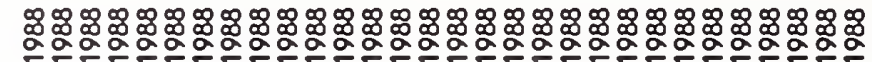

亩

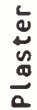

$<\infty u 0<\infty<\infty u<\infty u<\infty u<\infty u<\infty u<\infty u<\infty u<\infty 0$

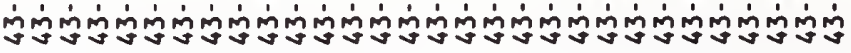

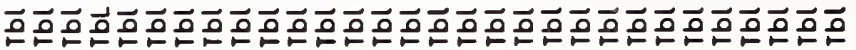

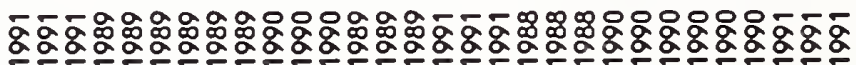

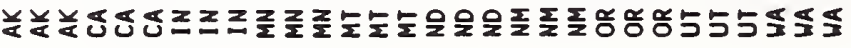



赔: 恼:

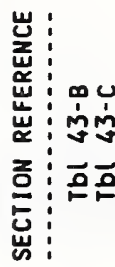

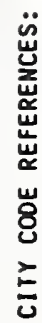
a

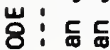

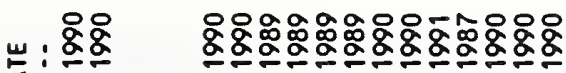

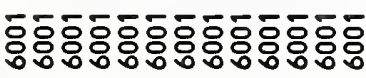

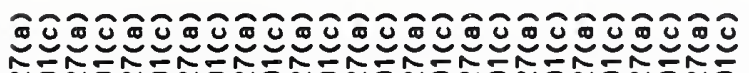

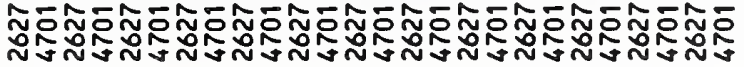
A

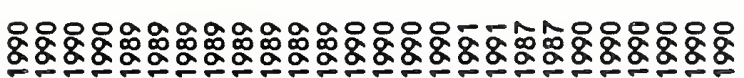

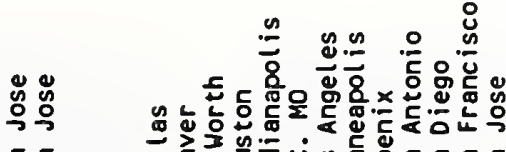

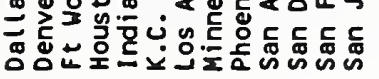

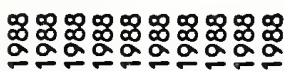
is

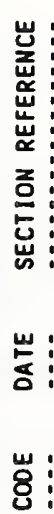

$\frac{w}{\Delta}$

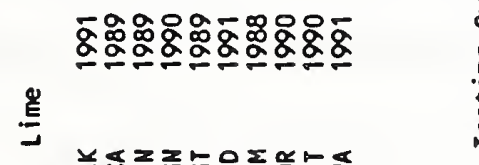

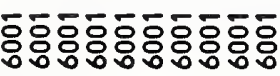

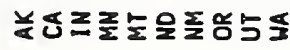

莺

ळ

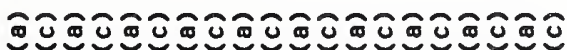

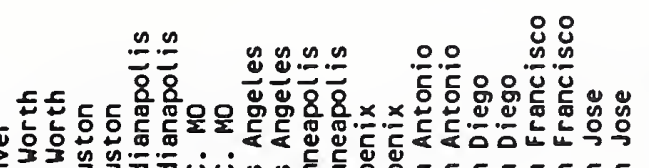

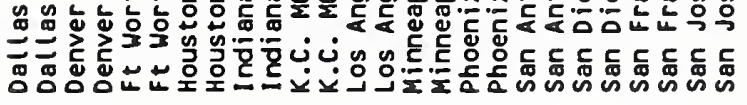

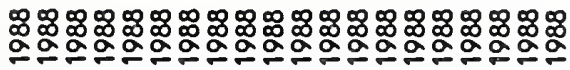

苑

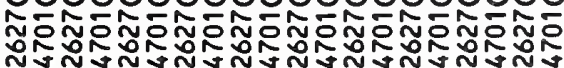

衰

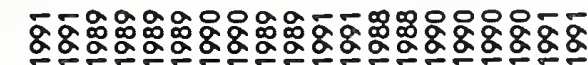

$\stackrel{\mathscr{s}}{2}$

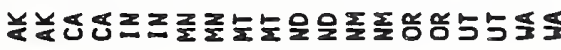

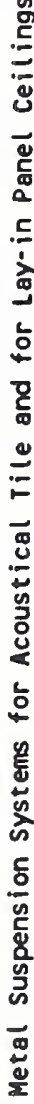

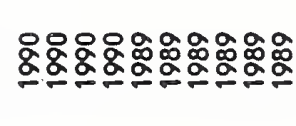

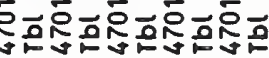

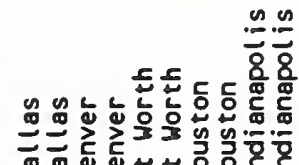

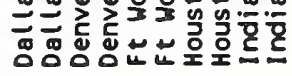

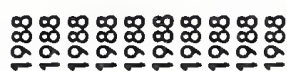

อ

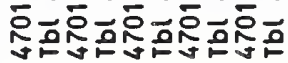

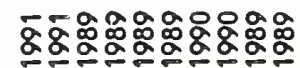

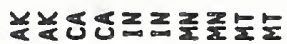

$\stackrel{\dddot{8}}{\stackrel{8}{2}}$

ก

$\frac{1}{5}$

$\frac{\infty}{5}$ 


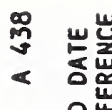

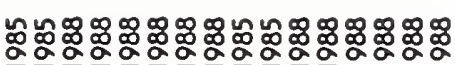

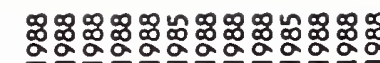

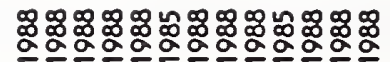
: å: 은
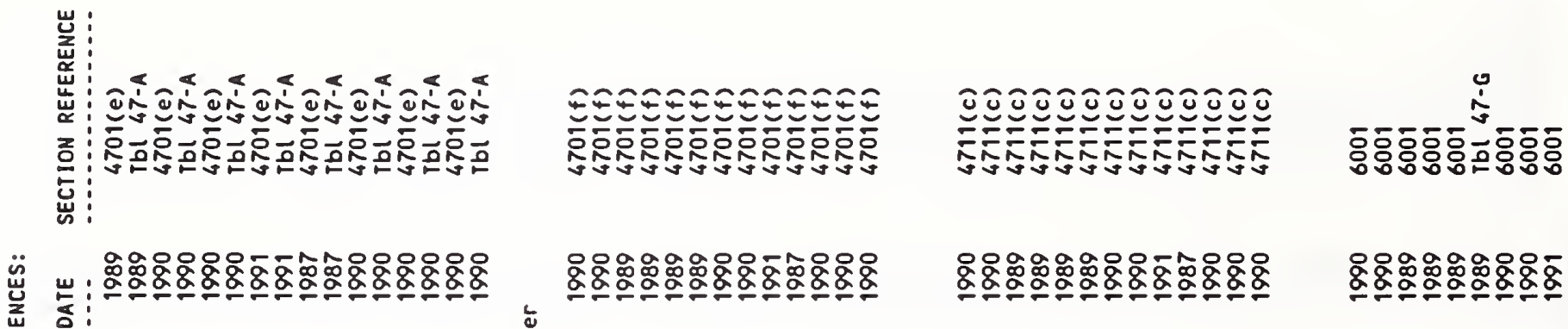

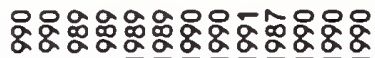

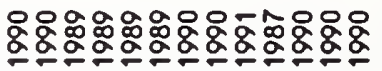

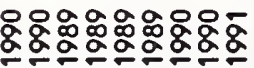

늘

芯

은

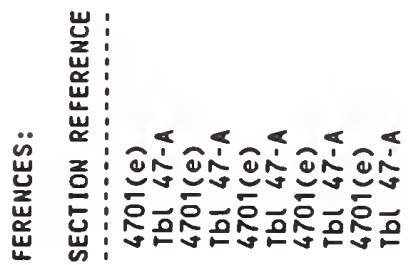

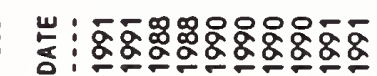

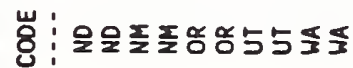

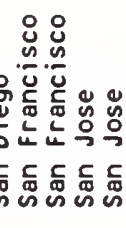
옹

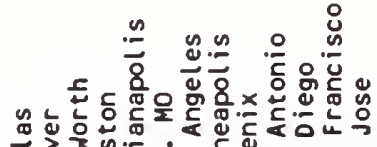

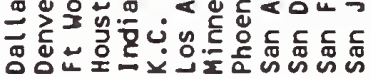

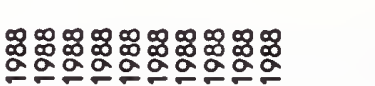

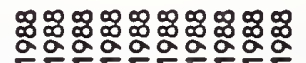

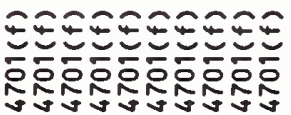
¿

음

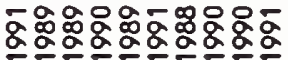

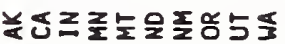

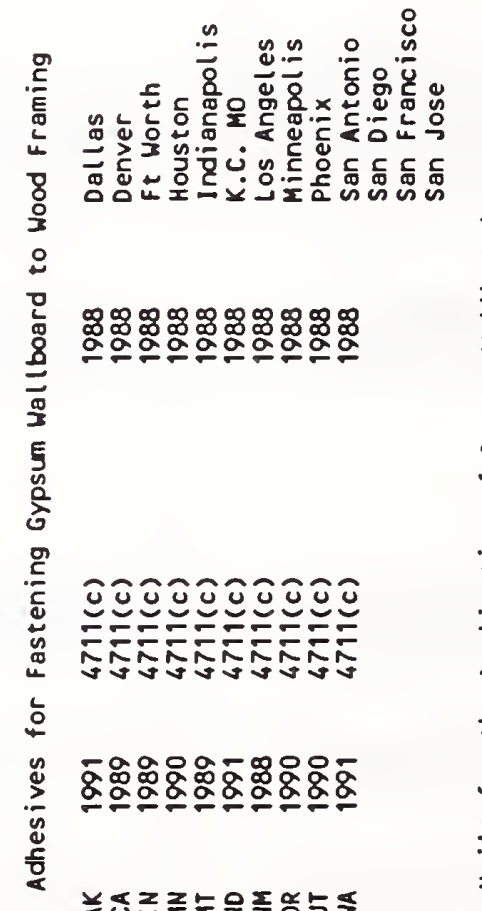

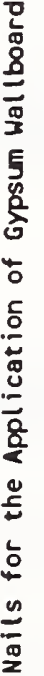

జ:ma:

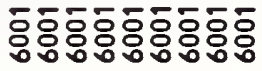

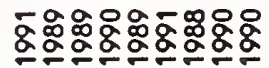

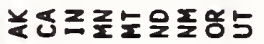

总

ळ̊

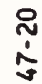

ָิ

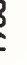

$\sim$

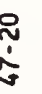

曾

$\frac{a}{5}$

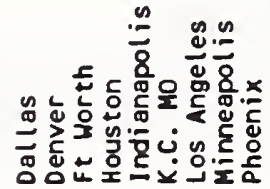<smiles>C1CCCC1</smiles>

竞

$\stackrel{\Xi}{\circ}$ 


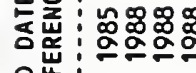
옸

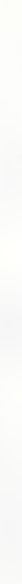

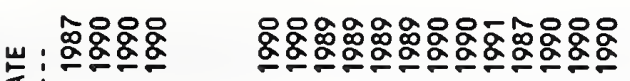
:
س

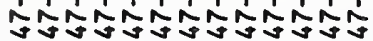

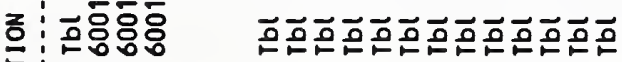

岕:

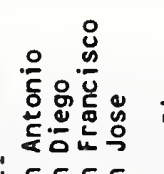

㟧

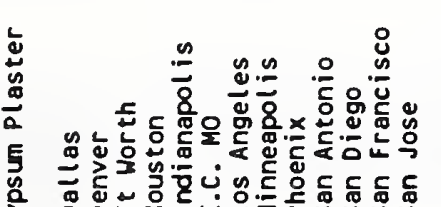

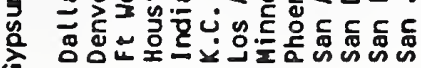

눙

:

س س س س س س س س س

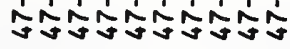

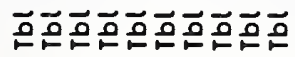

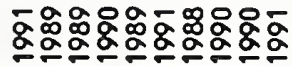

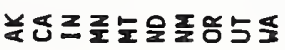

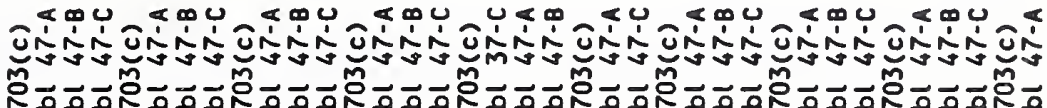

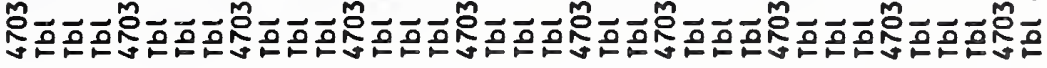

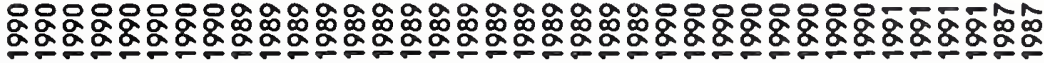

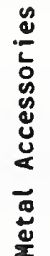

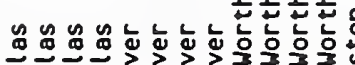

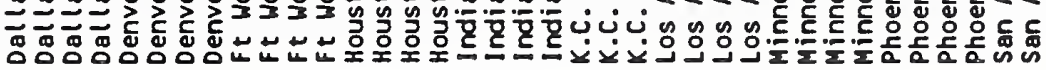

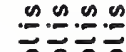

$8 \overline{8} 8 \frac{8}{8}$

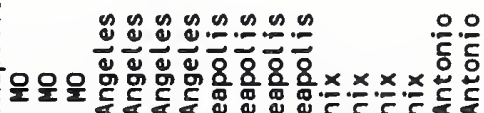

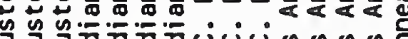

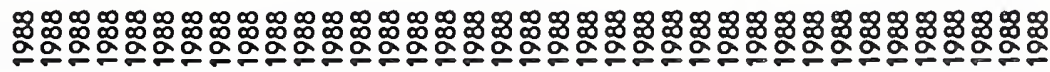

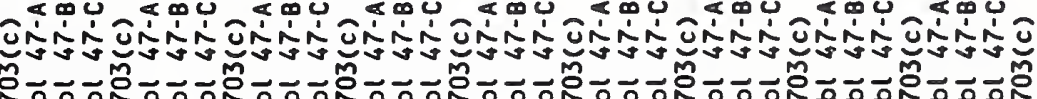

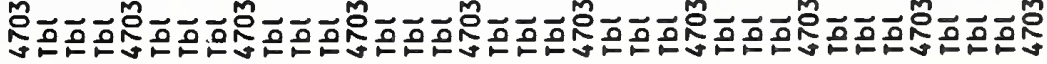

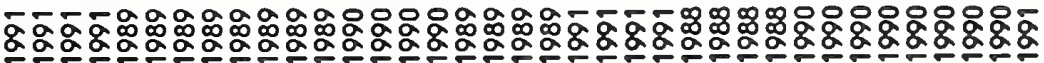

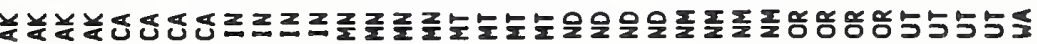


品

w岁:

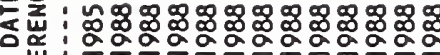
象岕:

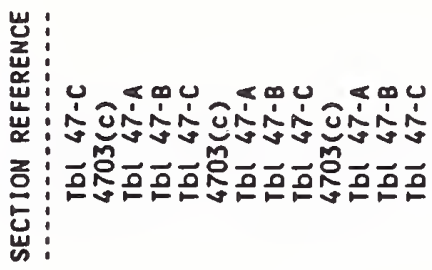

ن ن ون ب

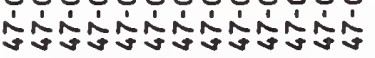

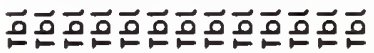

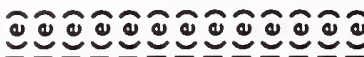
:

형영영영영영영영영영영

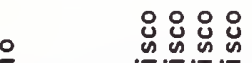

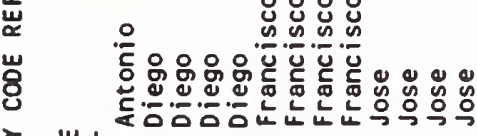

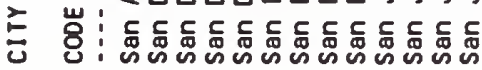

崖岂 :

ơ

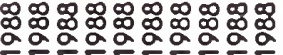

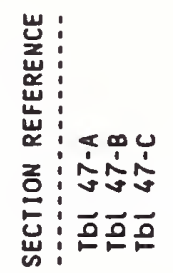

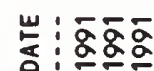

岁:

宅

$\stackrel{\mathscr{0}}{\underline{\sigma}}$

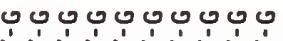

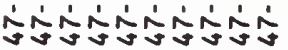

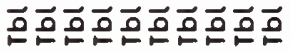

雚

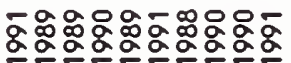

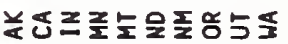

$\stackrel{n}{\stackrel{1}{5}}$

$\stackrel{8}{\stackrel{5}{*}}$

$\mathfrak{s}$

$\stackrel{\text { : }}{2}$

ळ

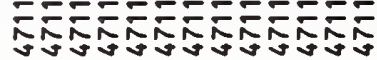

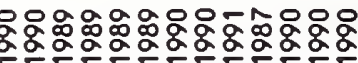

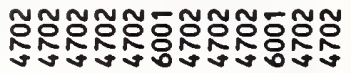

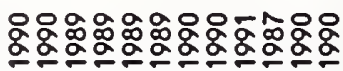

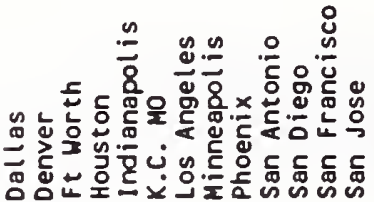

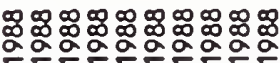

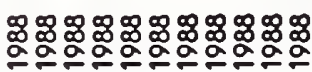

さすすすすすすすすす

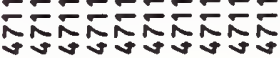

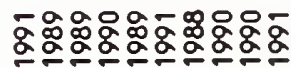

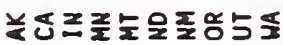

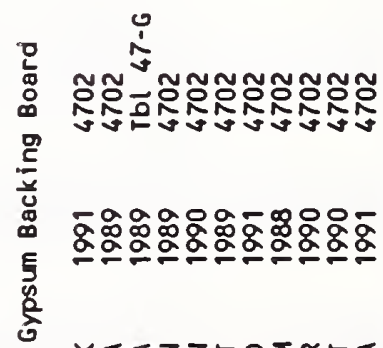

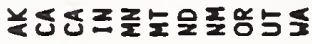

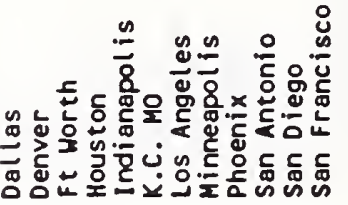

品 


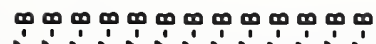
fos

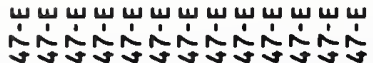

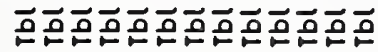

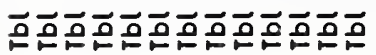

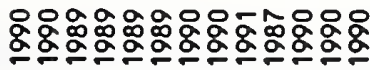

峉:<smiles></smiles>

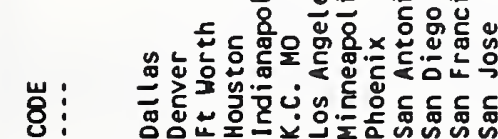

焉

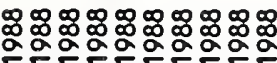

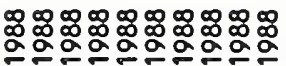

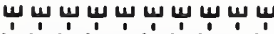

$\infty m \infty m \infty \infty \infty \infty m \infty$

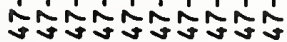

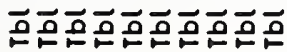

لـ

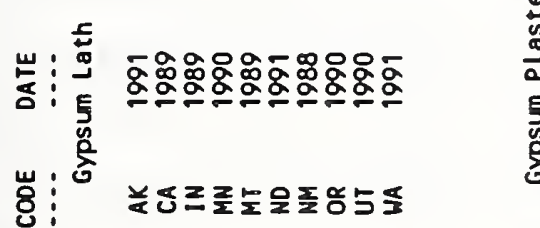

$\stackrel{\mathscr{\Xi}}{\circ}$

$\stackrel{\infty}{\stackrel{\Xi}{ٌ}}$

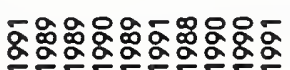
है

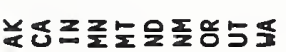

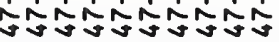

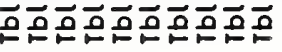

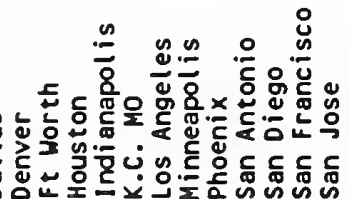

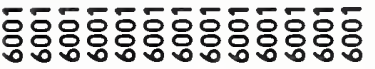

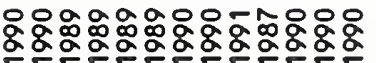

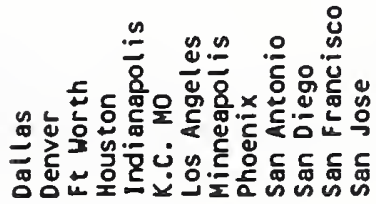

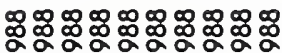
근.

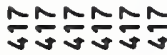

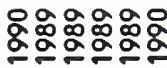

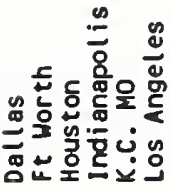

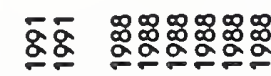
욜 章

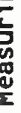

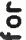

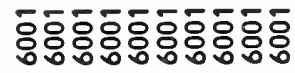

密

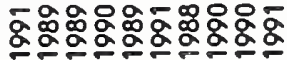

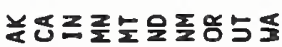

Eิ

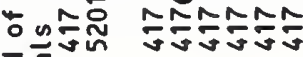

(8)

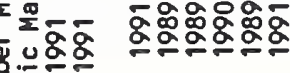

实.

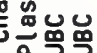

넌즈졸노을

$\stackrel{ฒ}{\stackrel{\Xi}{2}}$

$\bar{g}$

$\overline{\dot{n}}$

ஸ் 
Э : 柁岕 :

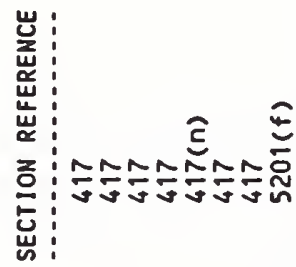

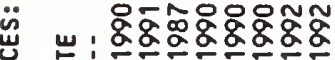
造:

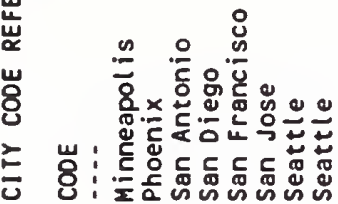

w는

ธิ

完岩:

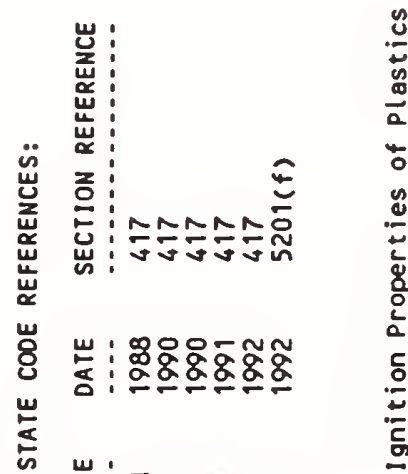

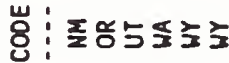

풍

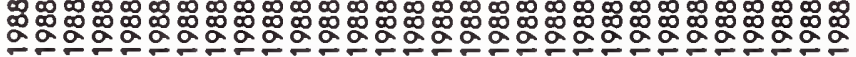

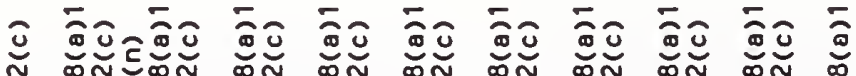

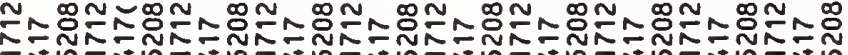

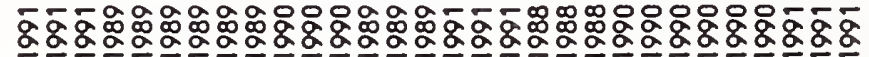

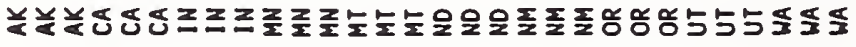

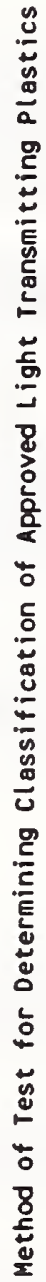

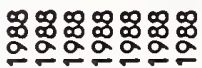

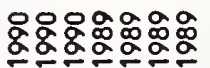

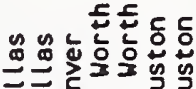

क्ष

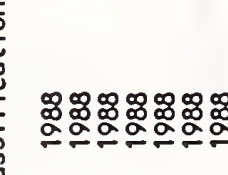

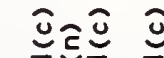

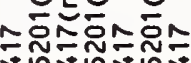

형영영영

ํㅏㅄㄸㅀミำ

$\stackrel{\mathscr{8}}{\square}$

$\stackrel{9}{\circ}$

$\dot{\sim}$

罗 $\tilde{\sim}$

กั

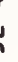




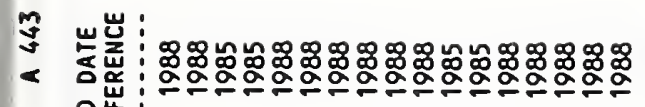

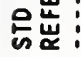

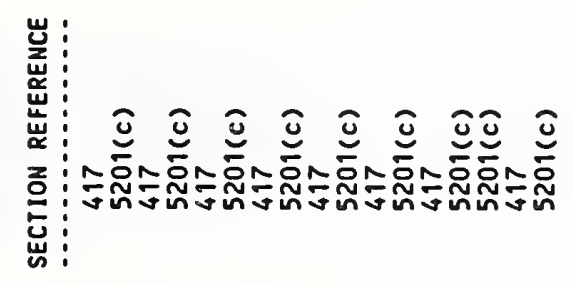
莣

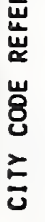

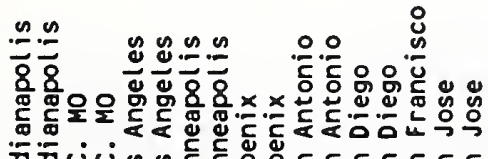

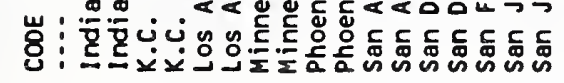

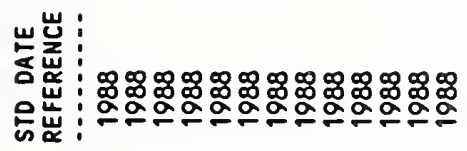

:

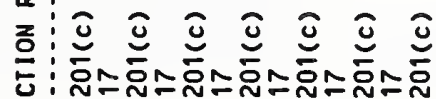

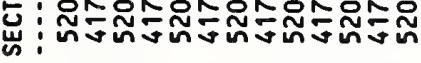

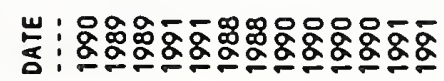

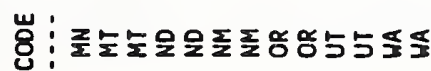

혈

ํㅗㅇ

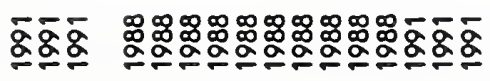

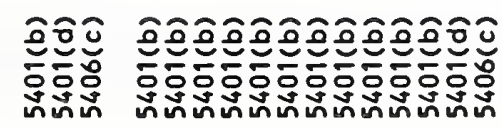

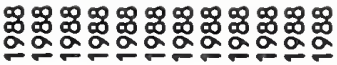

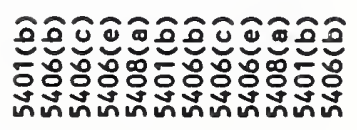

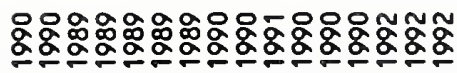

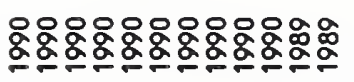

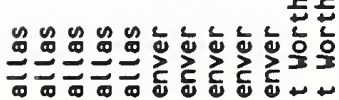

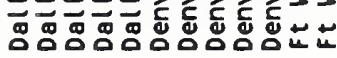

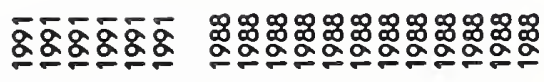

$\bar{g}$

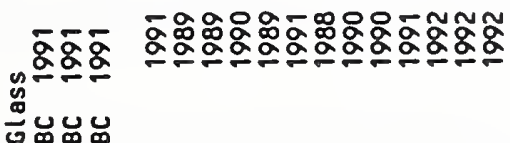

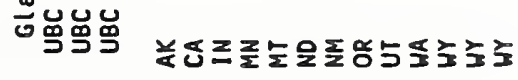

is

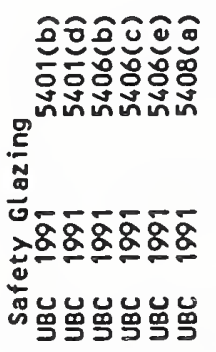

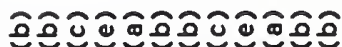
둥ㅎㅇㅇㅇㅇㅇㅇㅇㅇㅇㅇㅇㅇㅇ

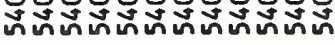

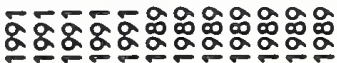

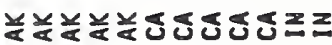

$\bar{\sigma}$

in 


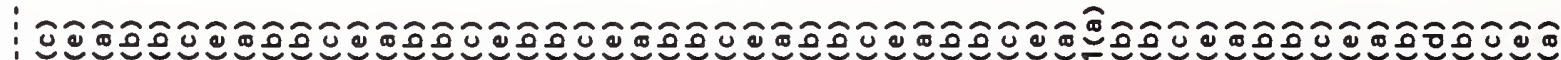

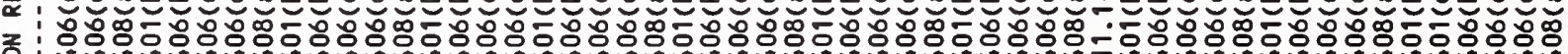
吾 惫:

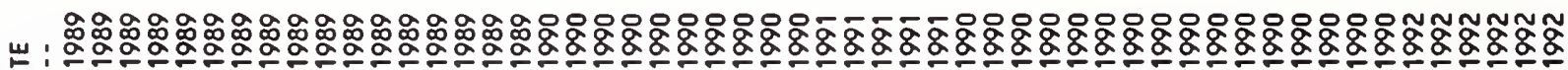
客:

w岕:

要崖:

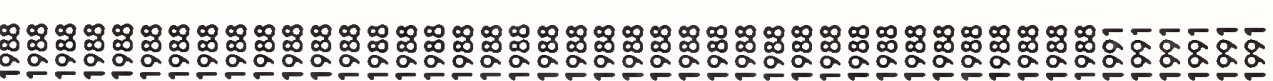

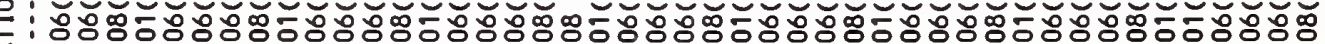

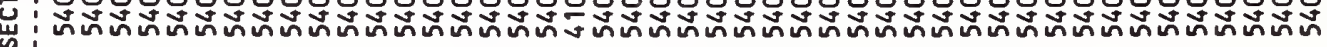

س : :a

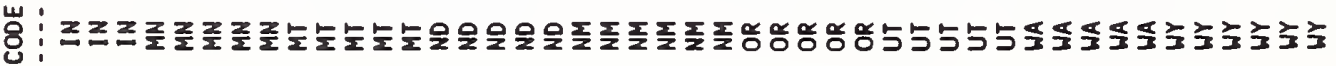




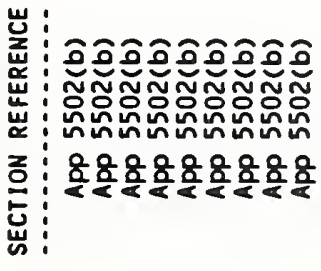

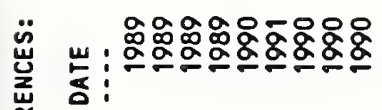

崫

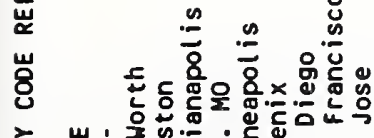

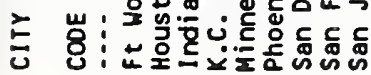

范: 은

要

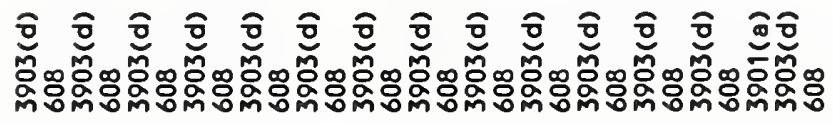

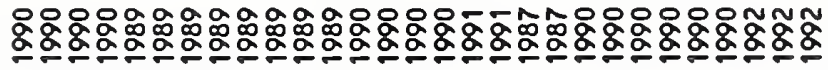

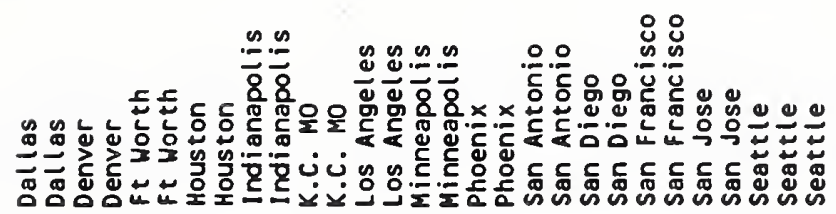

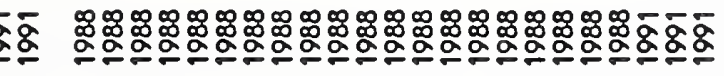

ล ํํํํํํํํำ

แ

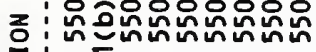

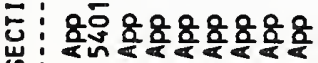

w)

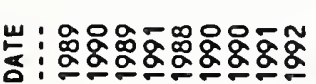

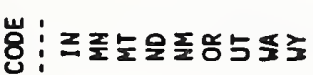

总

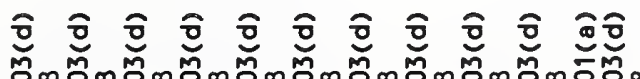

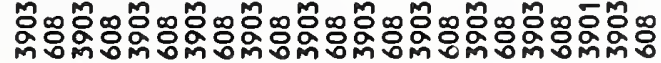

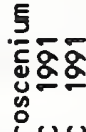

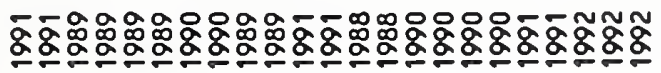

a昂罗

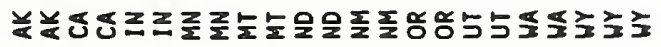

วองององององอิ

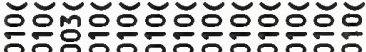

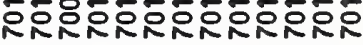

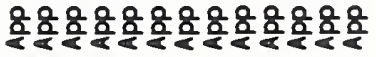

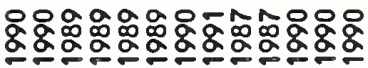

站

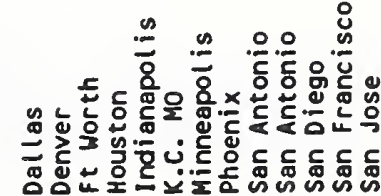

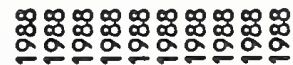

อิฮิฮิฮิฮฮิ

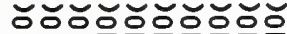

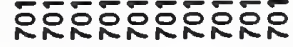

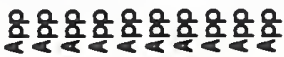

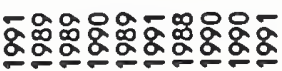

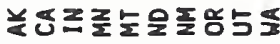

$\stackrel{\mathscr{\Xi}}{\stackrel{\Xi}{2}}$

$\bar{i}$ 


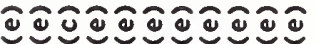

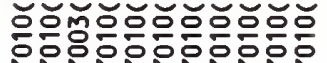

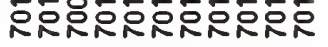

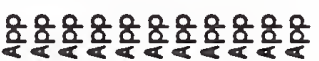

岕

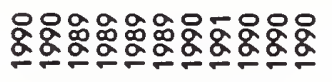

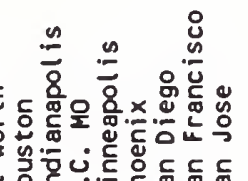
它岁:

惫

岁

$$
\text { 要 }
$$

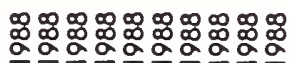

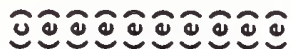
흥응으응흐응응ㅇ

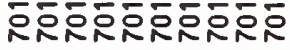

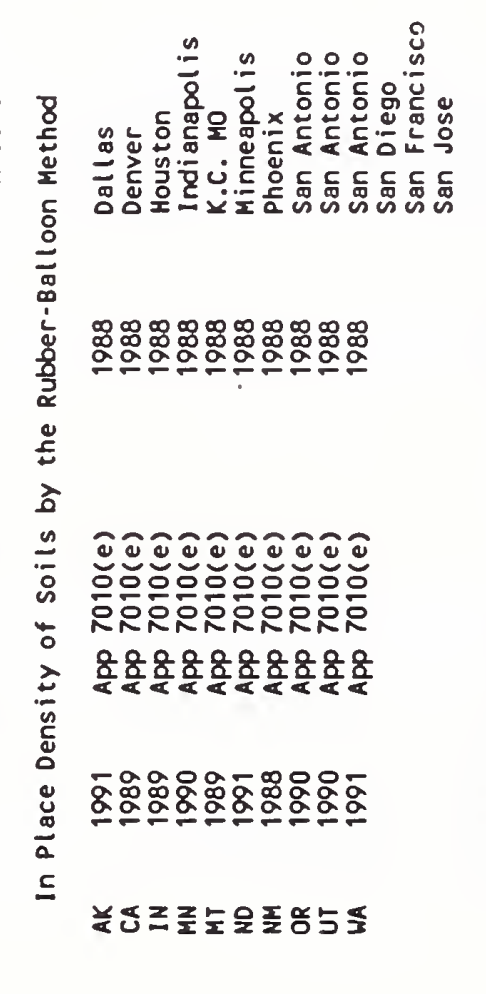

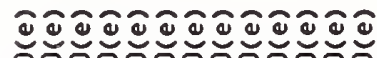

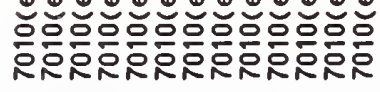

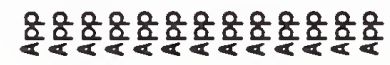

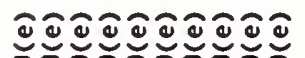

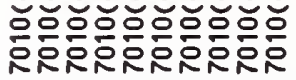

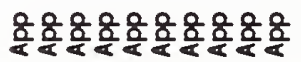

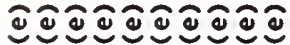

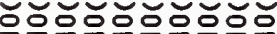

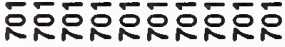

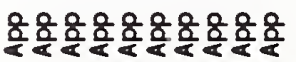

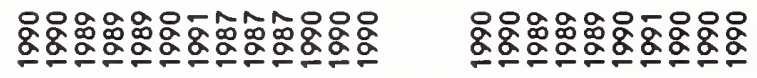

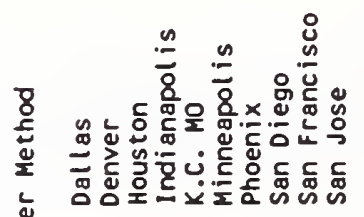

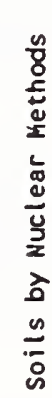

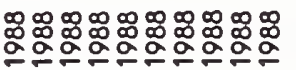

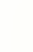

g

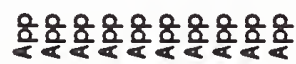

눙

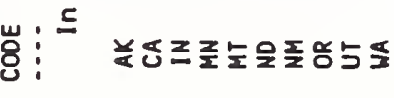

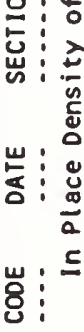

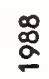

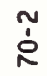

$\dot{R}$

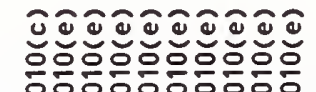

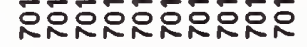

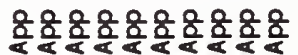

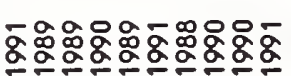

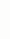
ᄃ

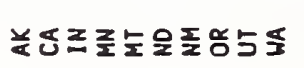

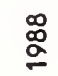
$\stackrel{8}{2}$

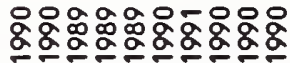

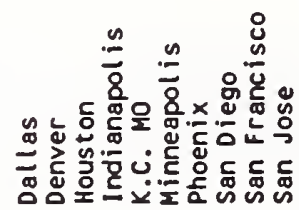

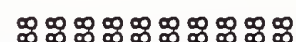
జ్0జ (1)

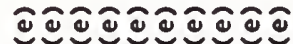

ธธธธธธธธธธ

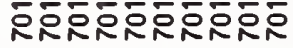

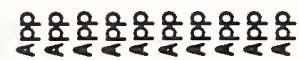

c

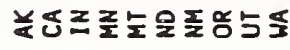




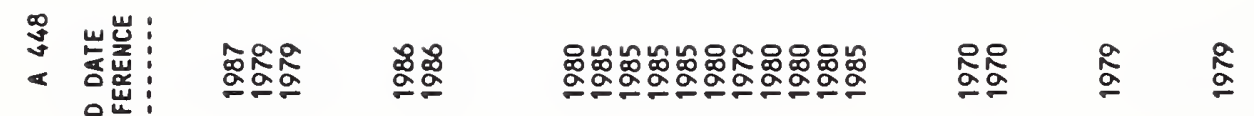

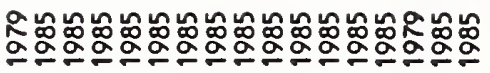

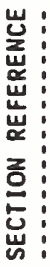

岂

똥

商 $\bar{g} \underline{2}$

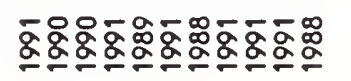

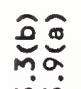

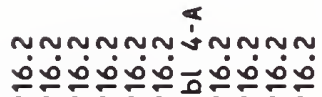

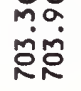

$\stackrel{v}{\dot{\omega}} \quad \frac{\square}{\dot{s}}$

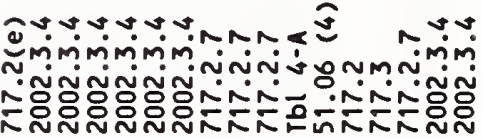

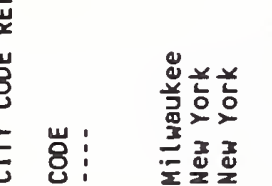

$\bar{u}$

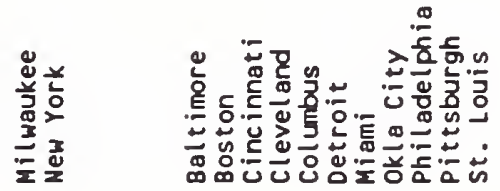

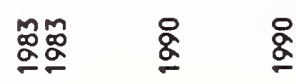

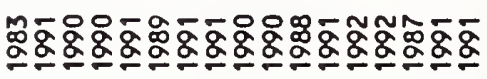

㟧:

柆

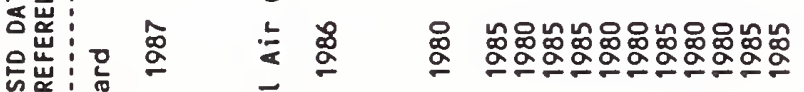

㟧:

کั.

ï

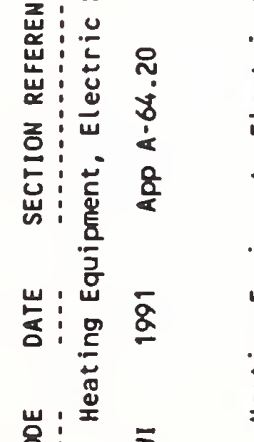

岁: ${ }^{x}$

ळ)

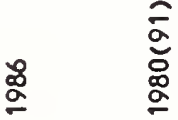

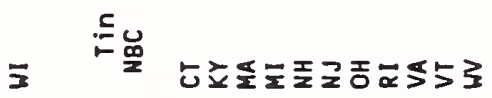

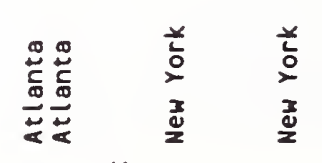

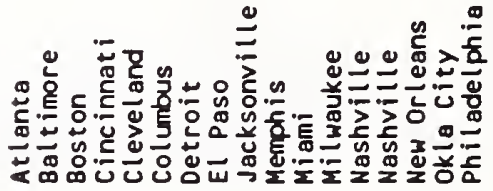

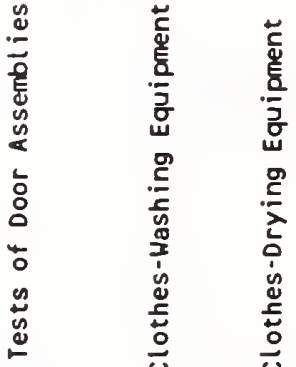

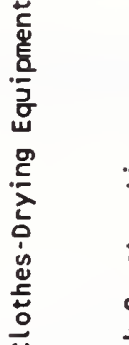

ळ్

等

ปั

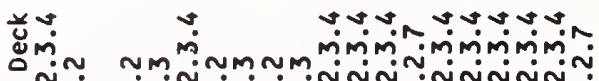

。铰

$\div \frac{4}{5}$

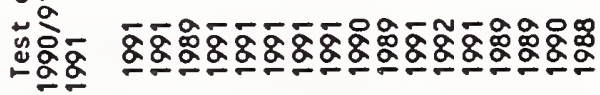

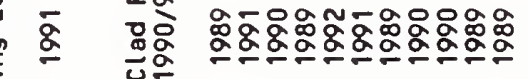

些品

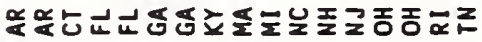

离

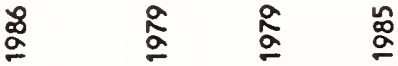

ฐับ ฐ ฐ

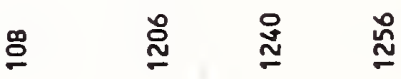

5 
行 닙

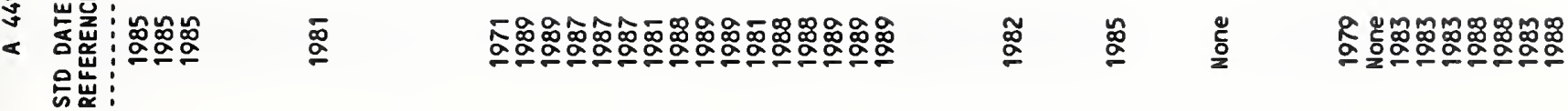

焉:

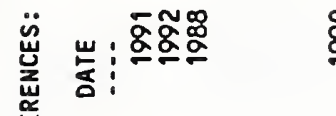

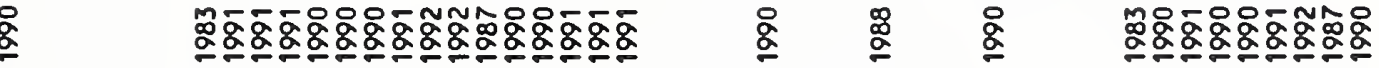

$\stackrel{\frac{\omega}{\sim}}{\sim}$

岁

岩

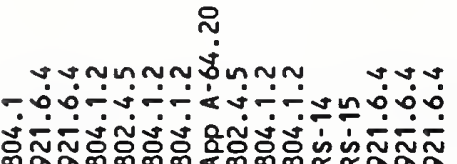

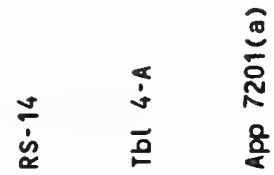

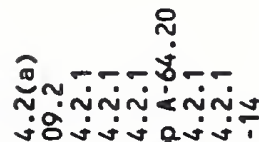

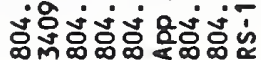

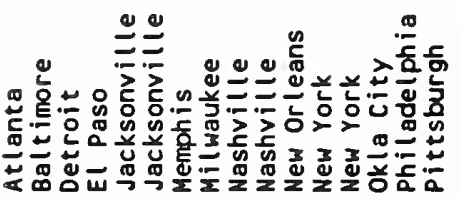

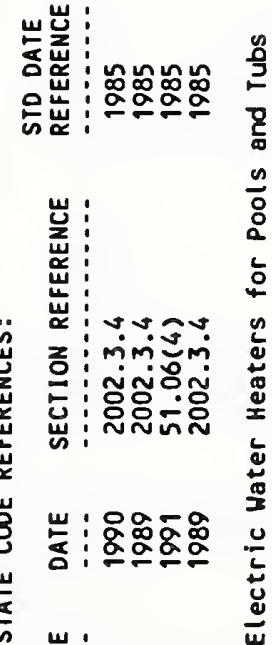

$8: \$ 553$

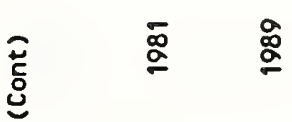

$\stackrel{\overline{2}}{\simeq}$

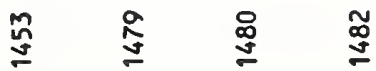

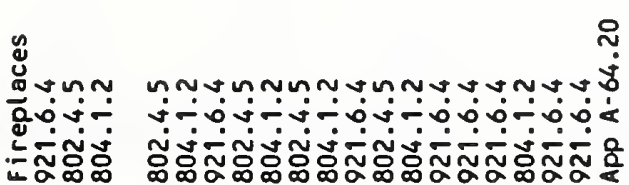
혼

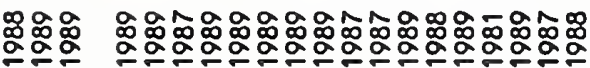

$\stackrel{\circ}{\circ}$

ᄒ

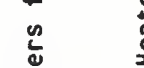

离

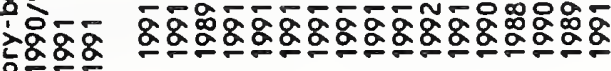

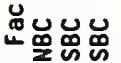

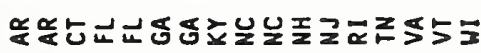

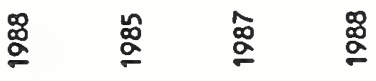

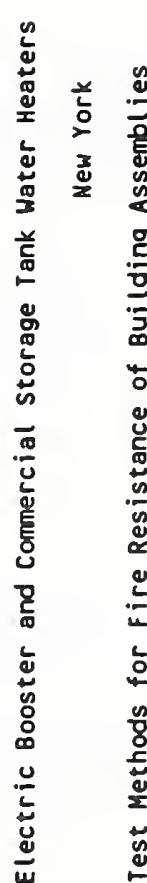

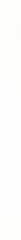

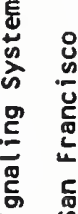

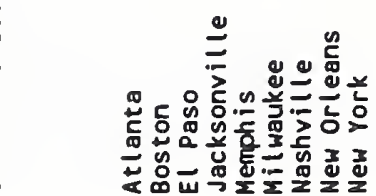

$\stackrel{8}{2}$

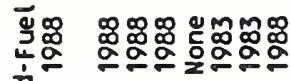

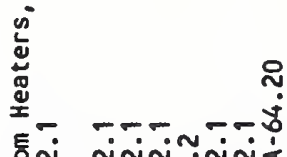

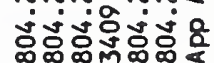

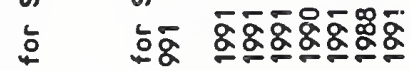

ฏ 


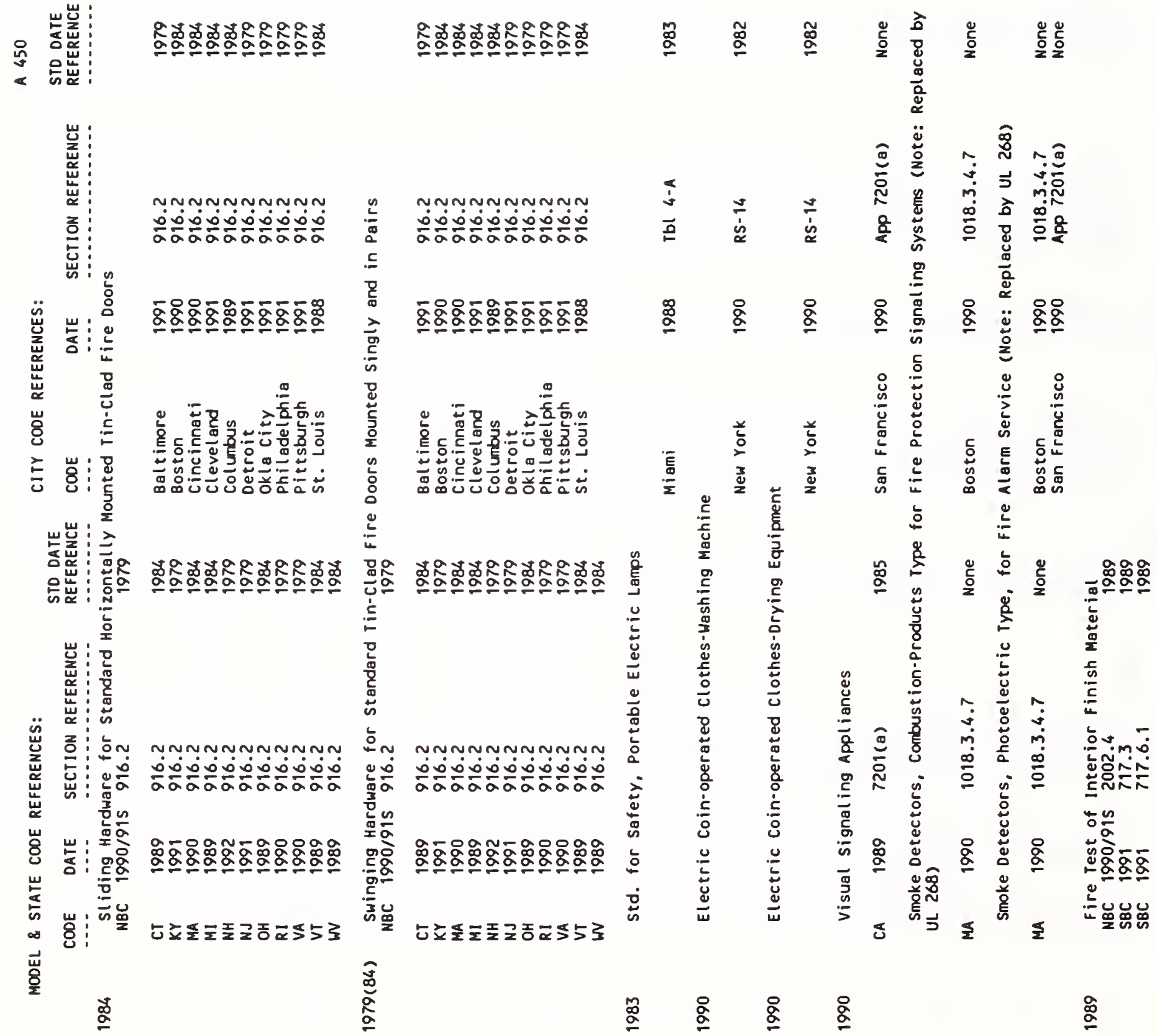




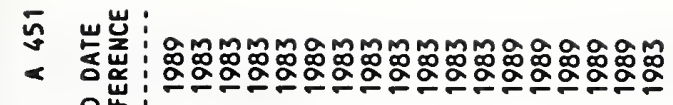
品崖:
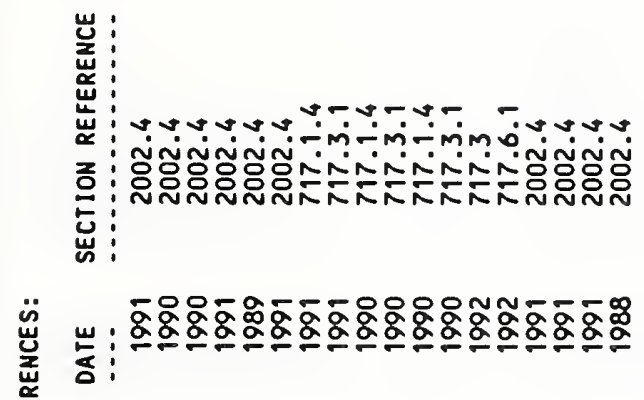

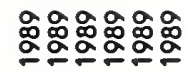
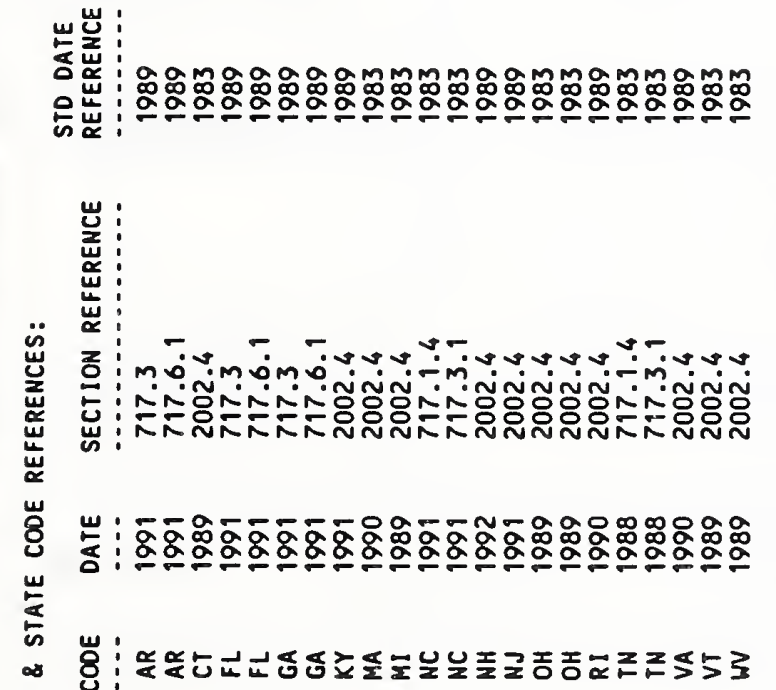

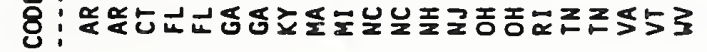

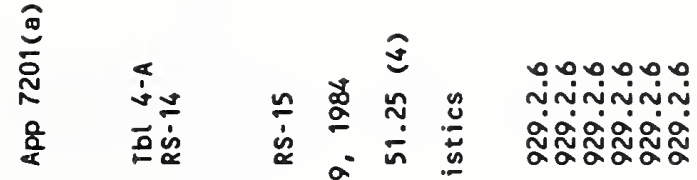

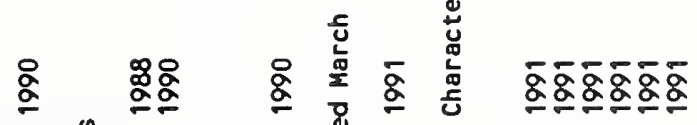

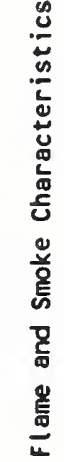

ฒ

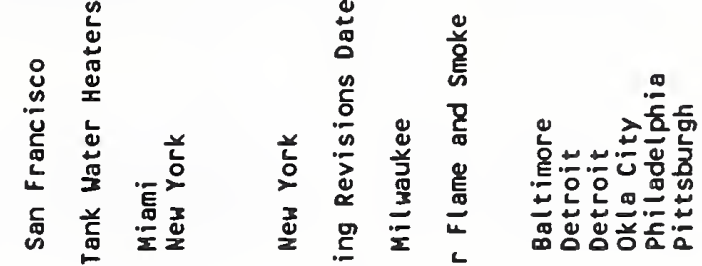

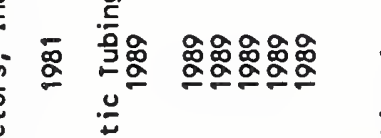

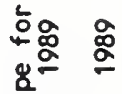

ब

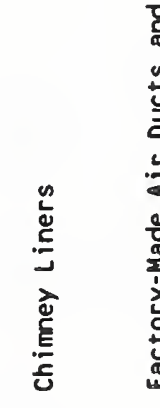

કั

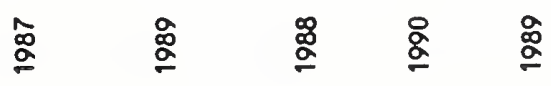

$\stackrel{\circ}{\stackrel{\alpha}{g}}$

$5 \quad \stackrel{n}{I}$

$\stackrel{E}{E} \quad \underline{\Xi} \stackrel{\Xi}{\Phi}$

⿷匚 


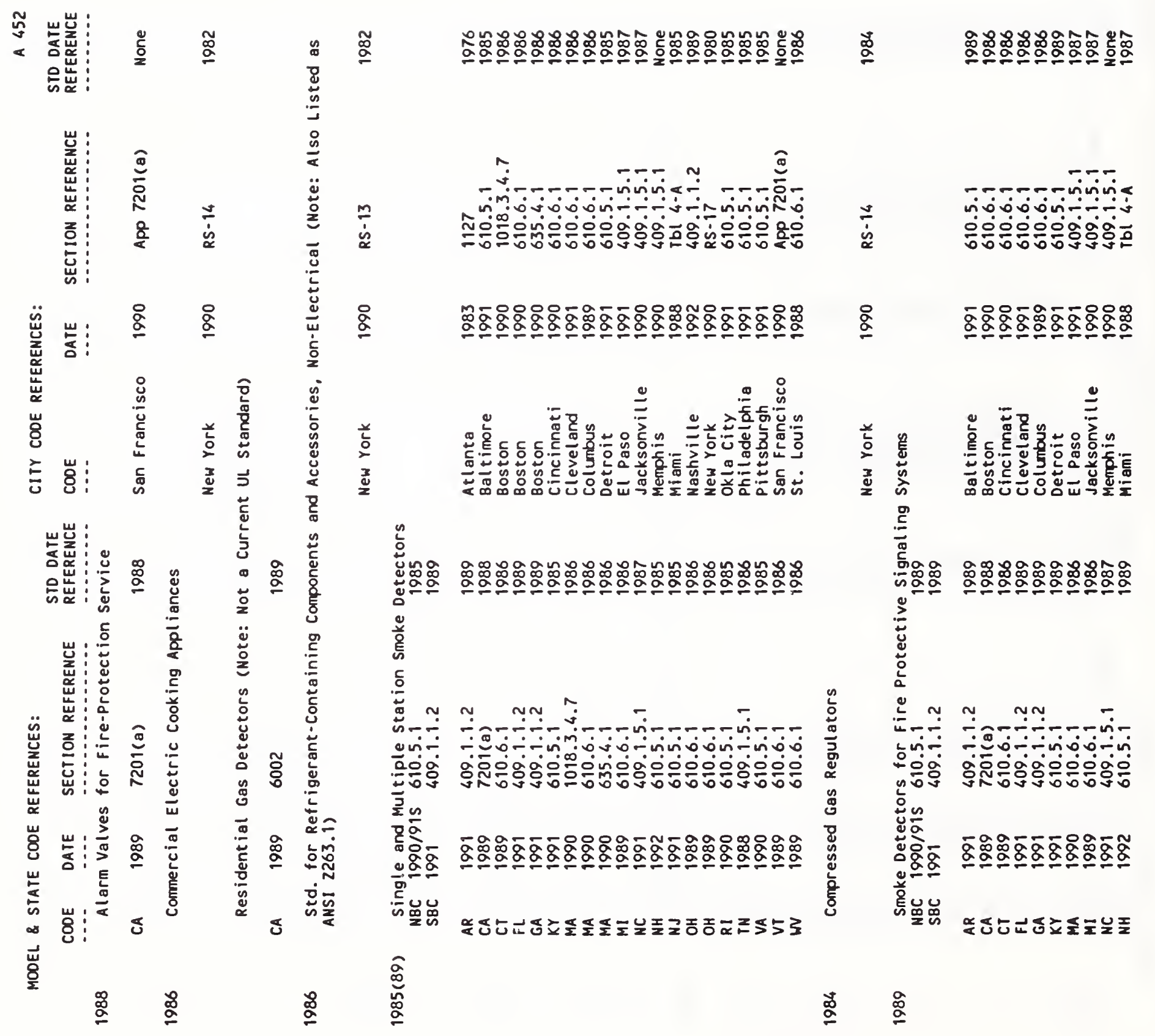

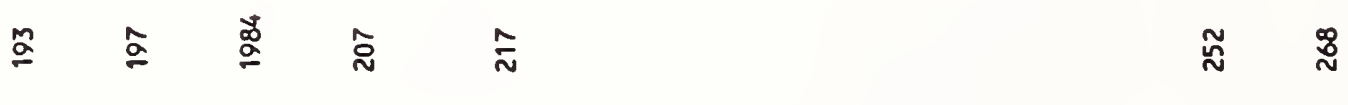




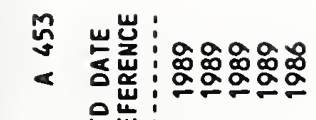
铈:

崫:

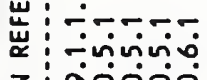

증

峞

岀 :

尊

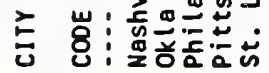

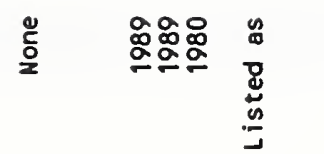

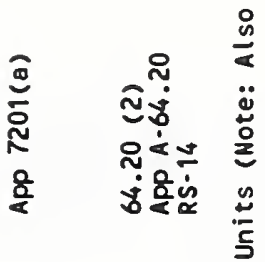

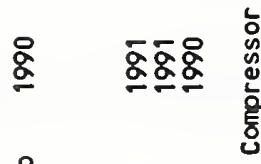

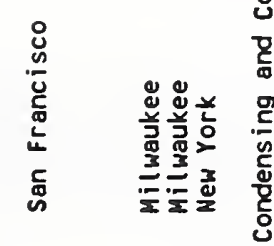

삔

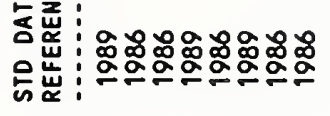

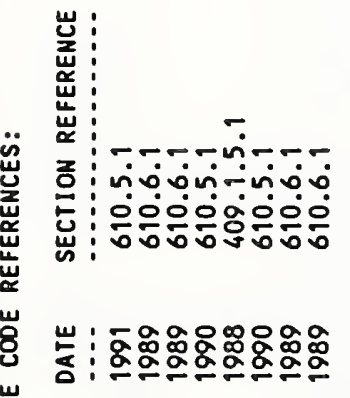

战

高

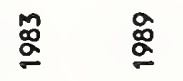

舜 ¿ $\stackrel{\square}{\square}$

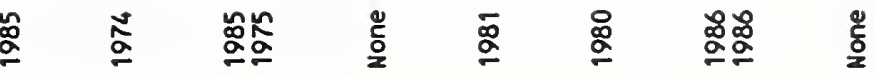

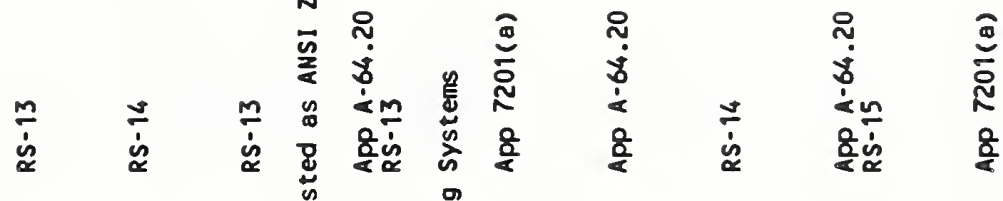

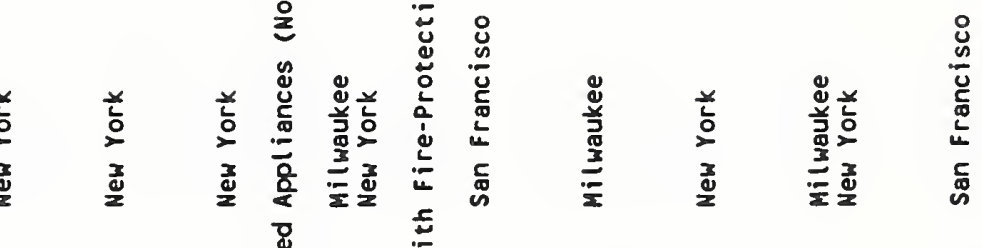

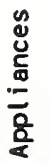

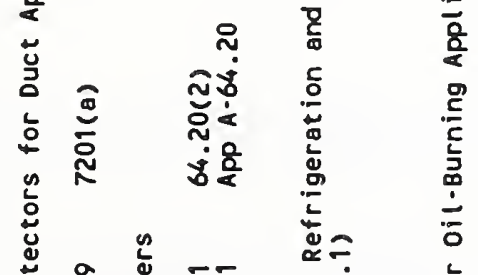

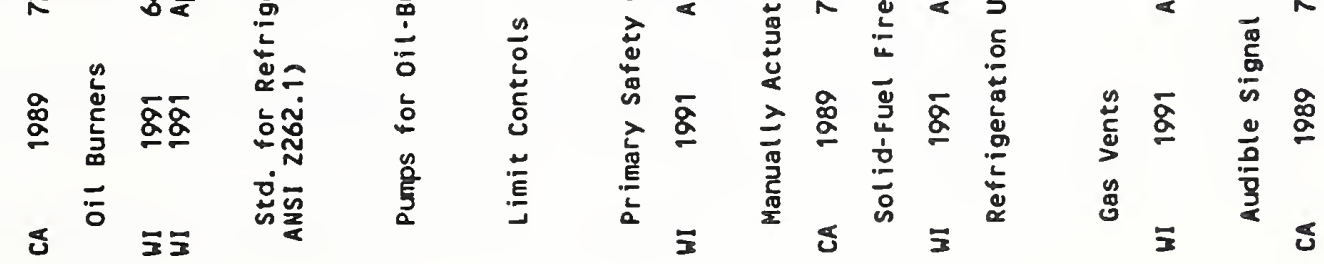

然

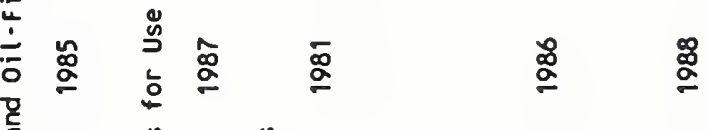

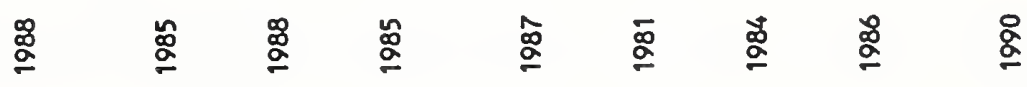

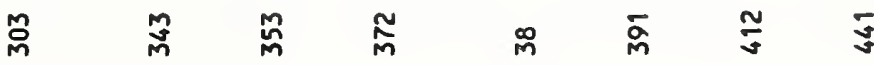

$\bar{\xi}$ 


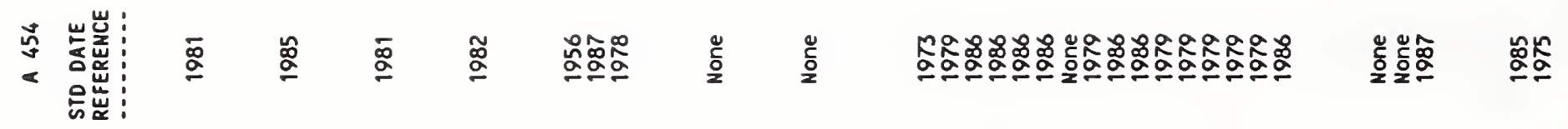

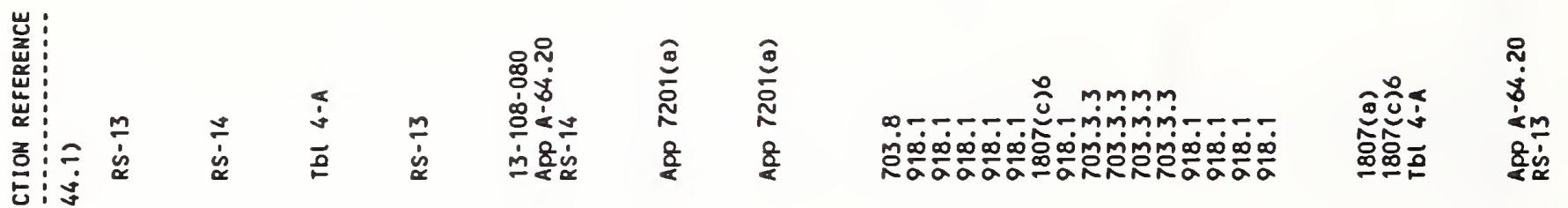

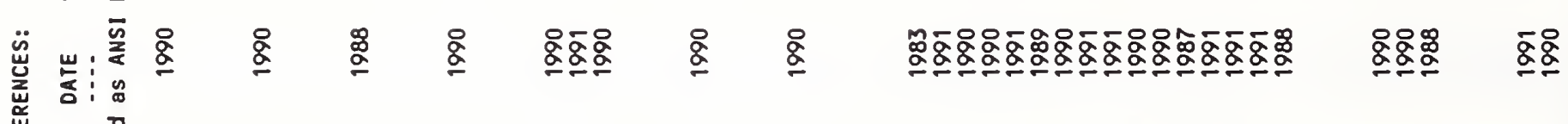

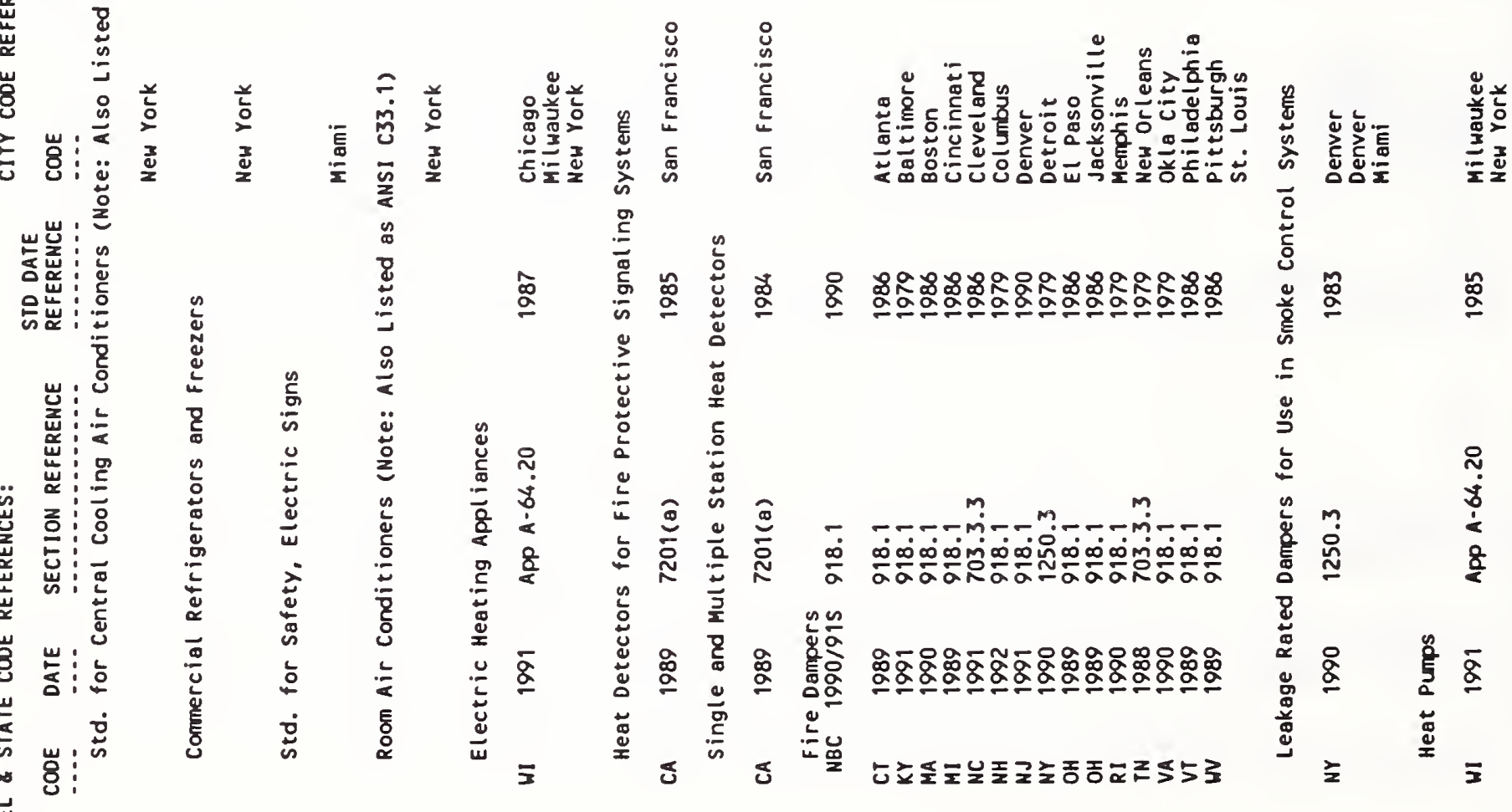
莒

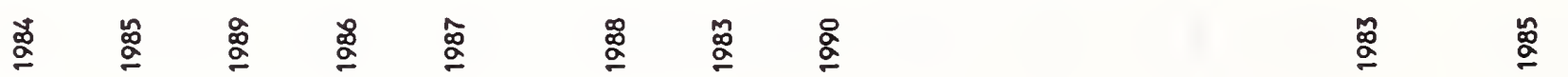

औ 


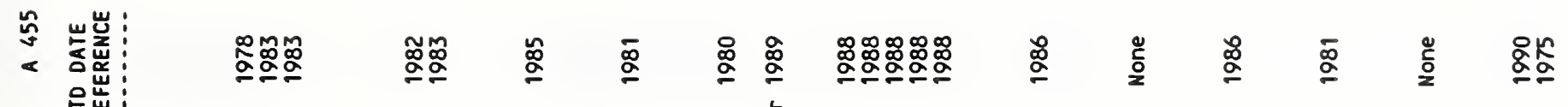
은

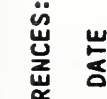

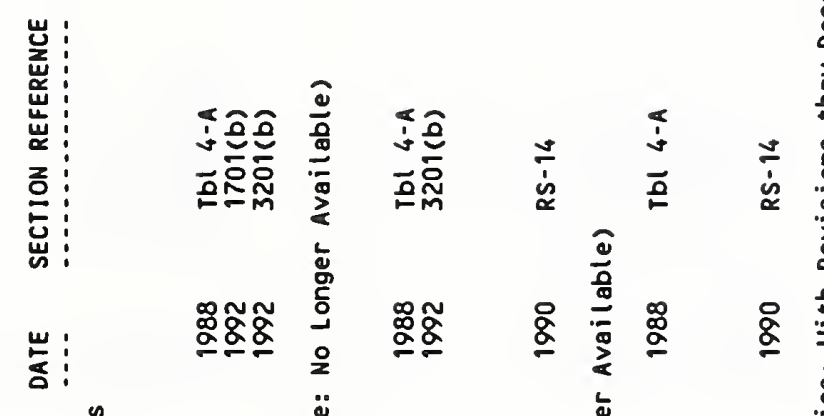

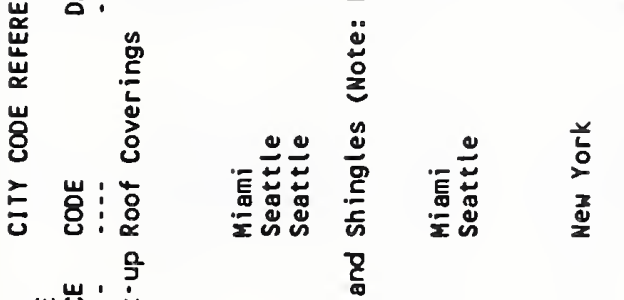

政:

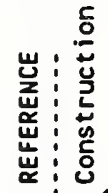

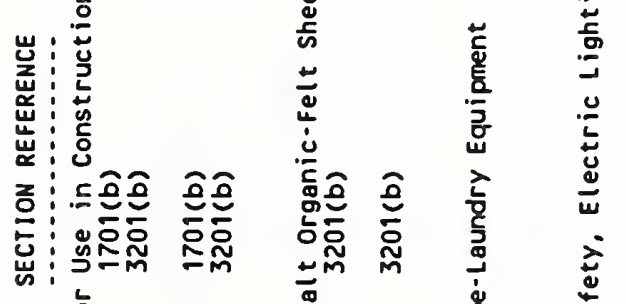

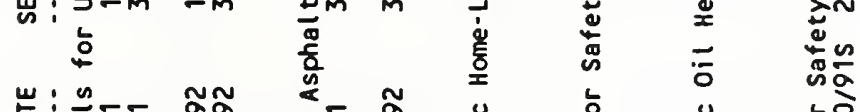

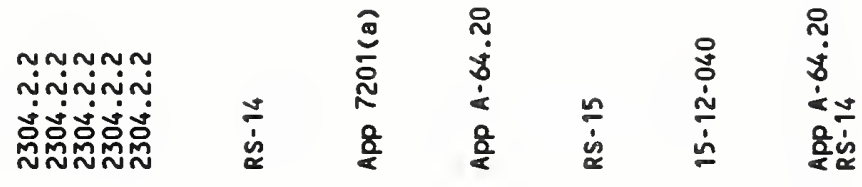

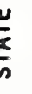

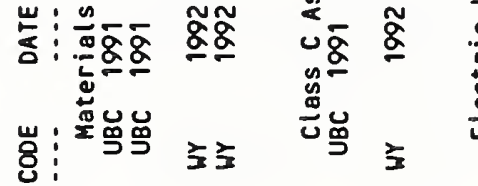

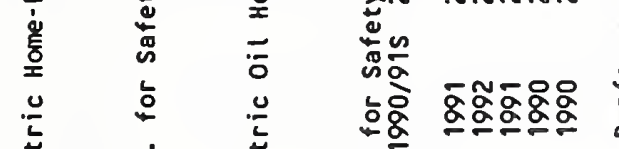

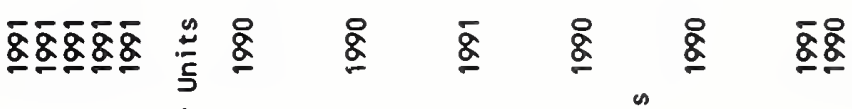

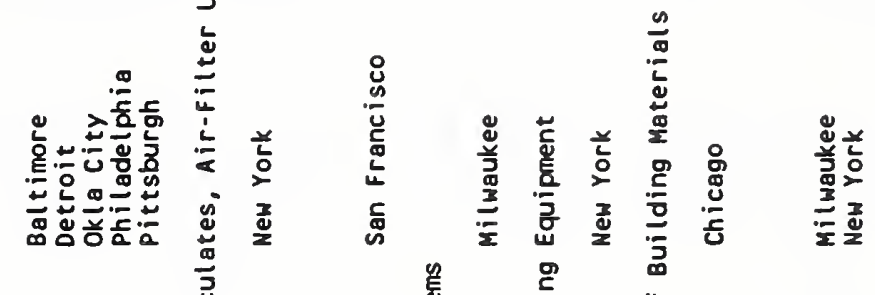

a)

言

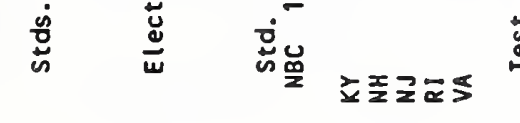

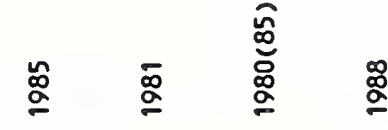

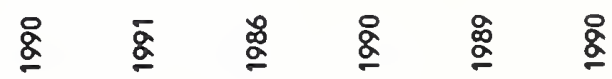

ฒ

8

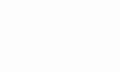

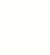

ฐ

荅总的芯总

ఖ 


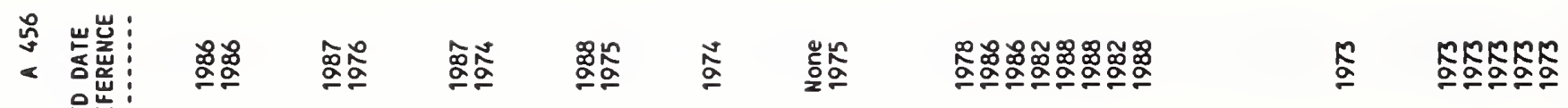
呟岕

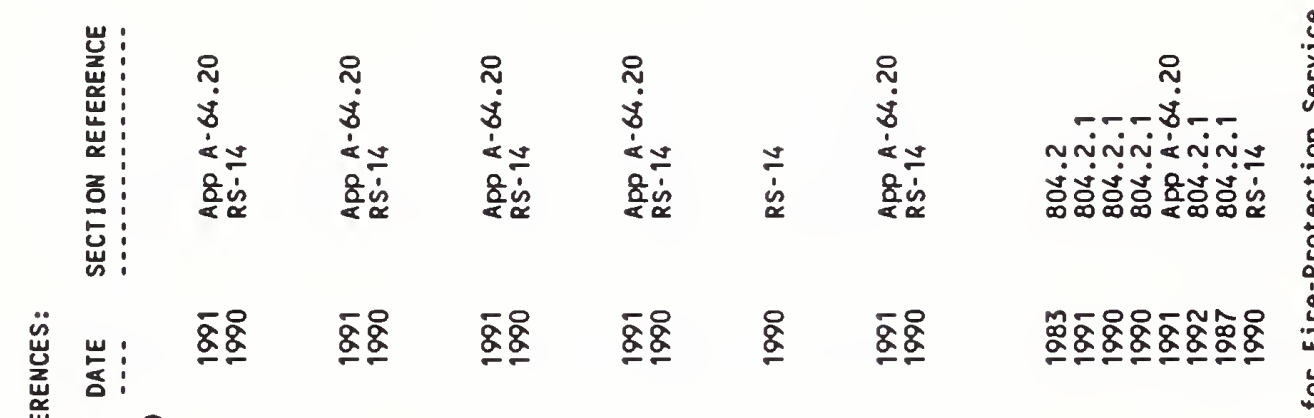

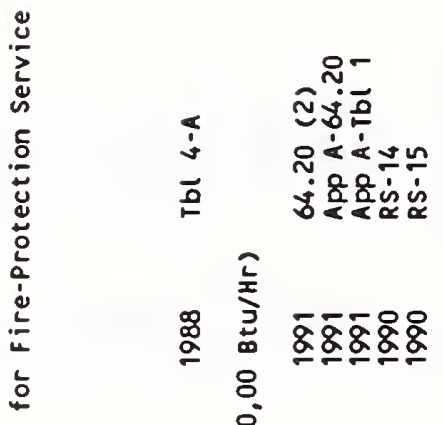

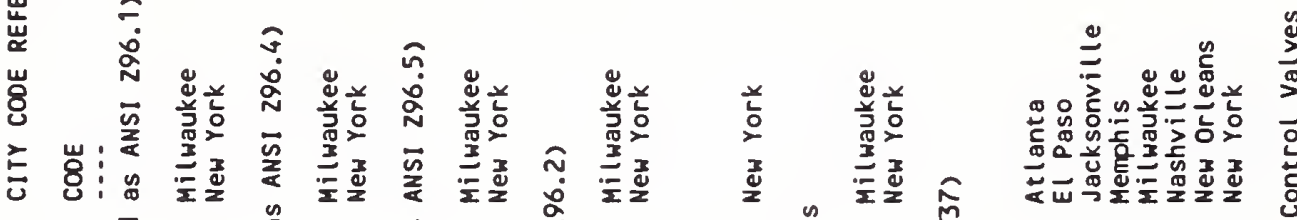

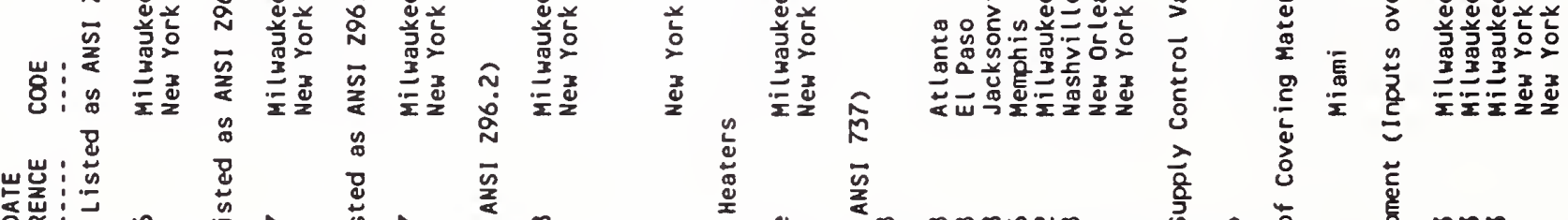

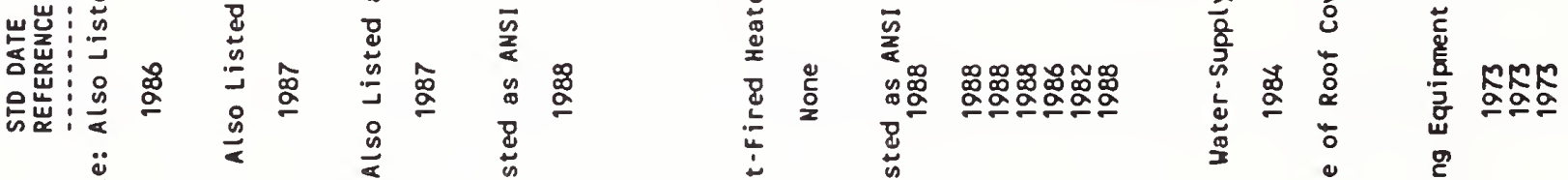

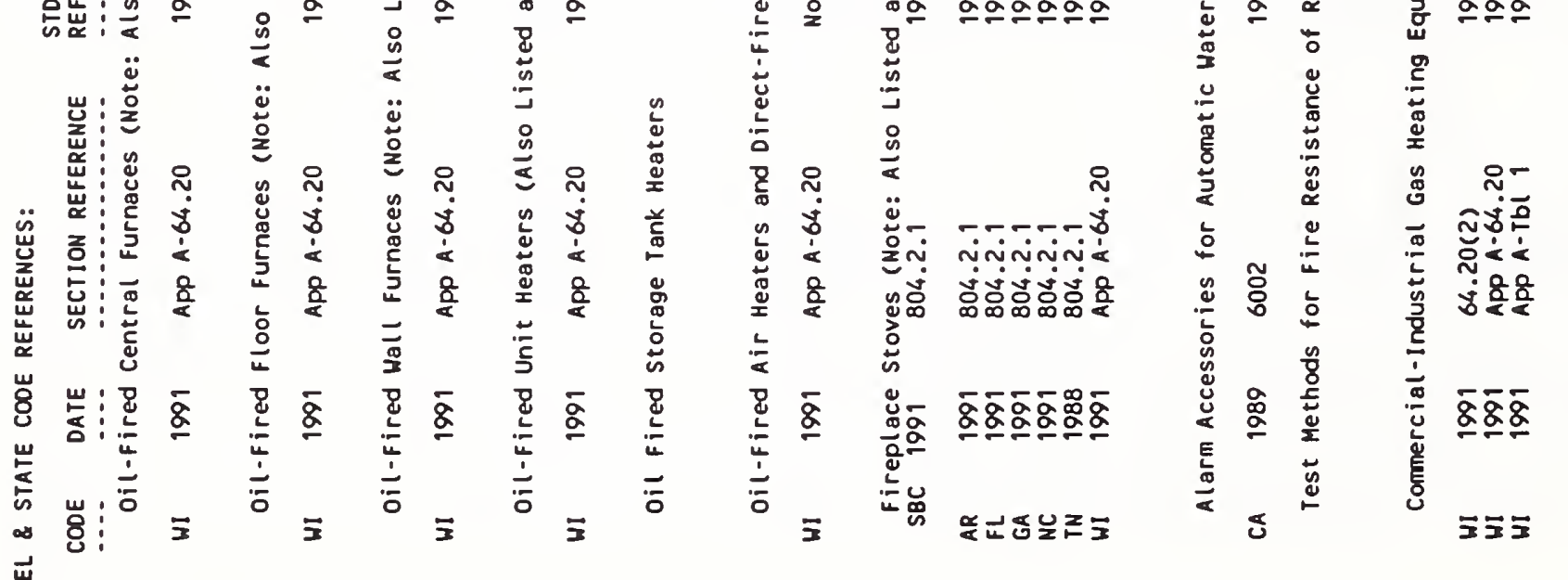

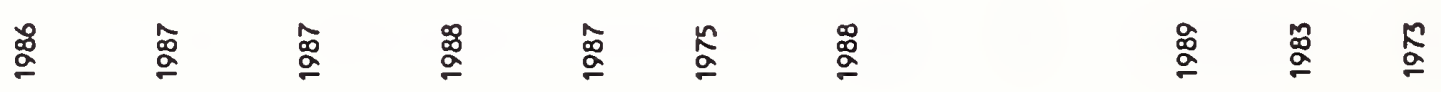

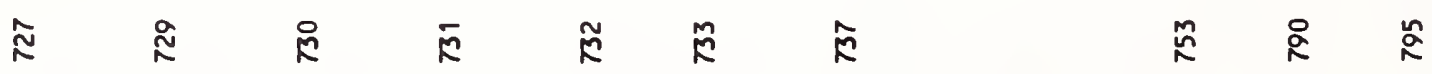




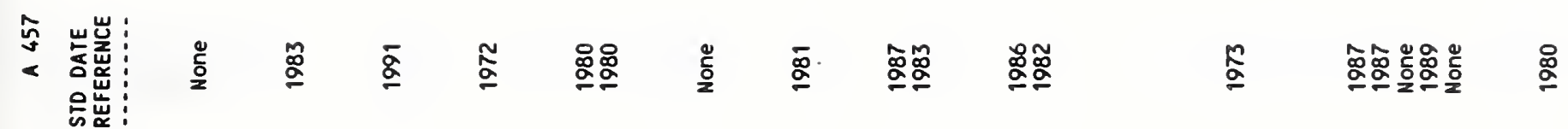

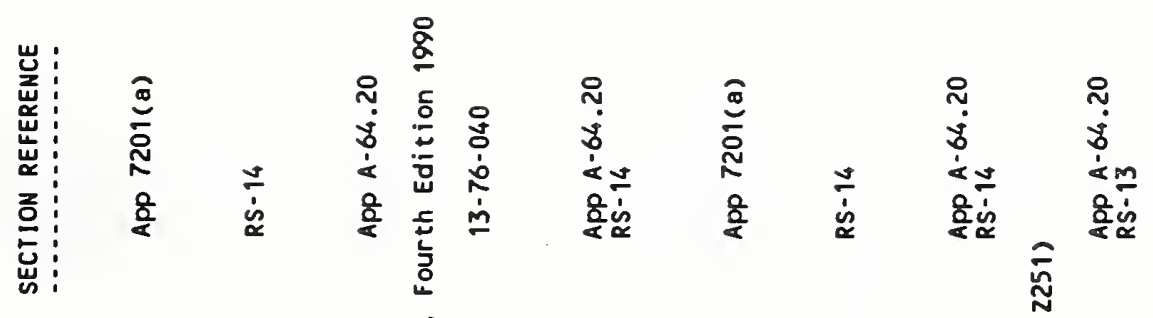

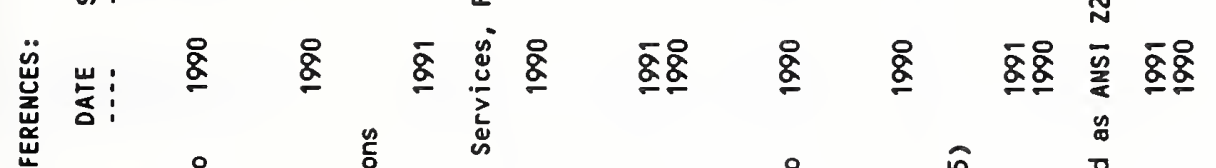

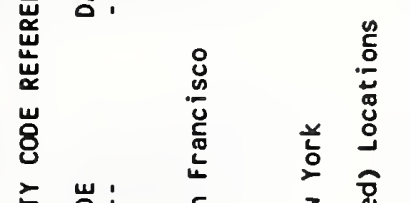

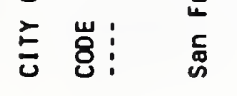

党:

章

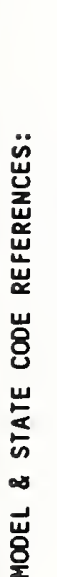

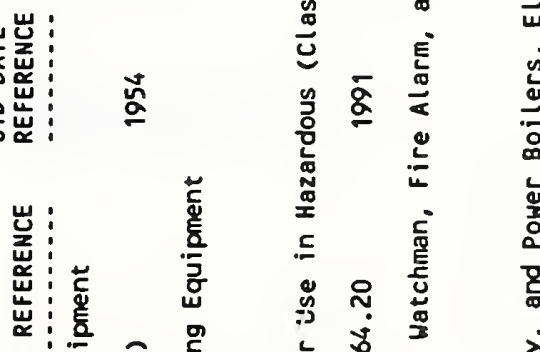

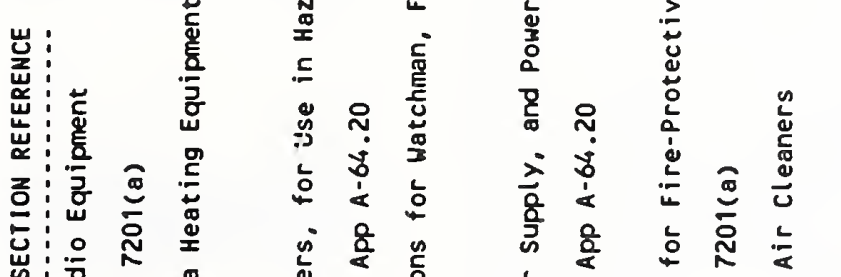

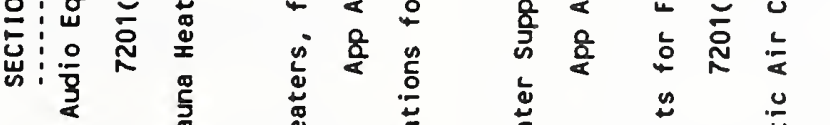

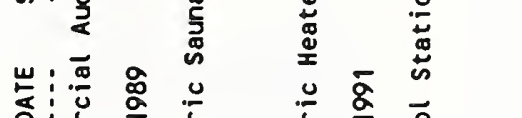

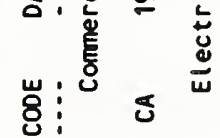

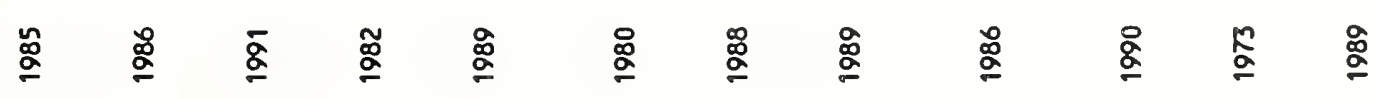

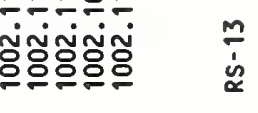

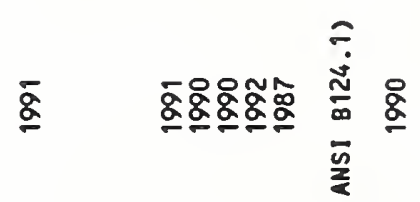

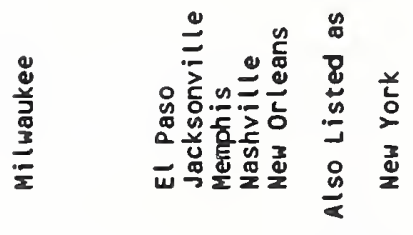

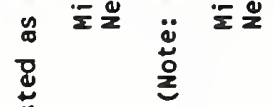

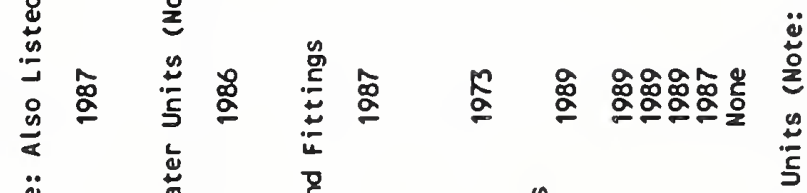

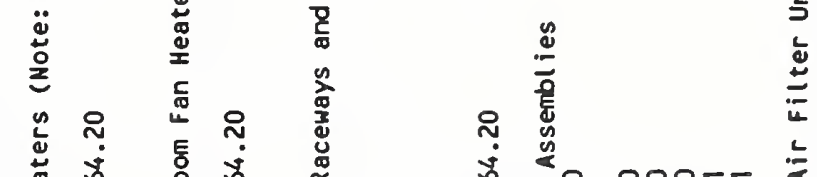

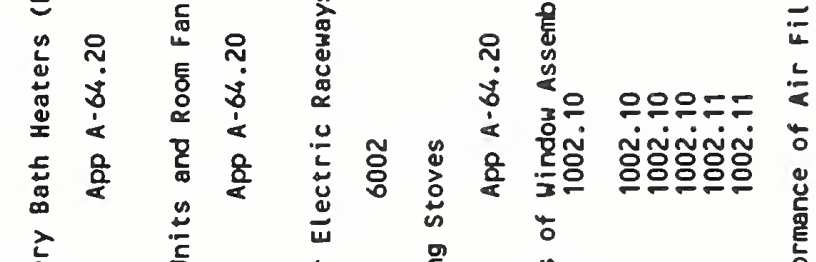

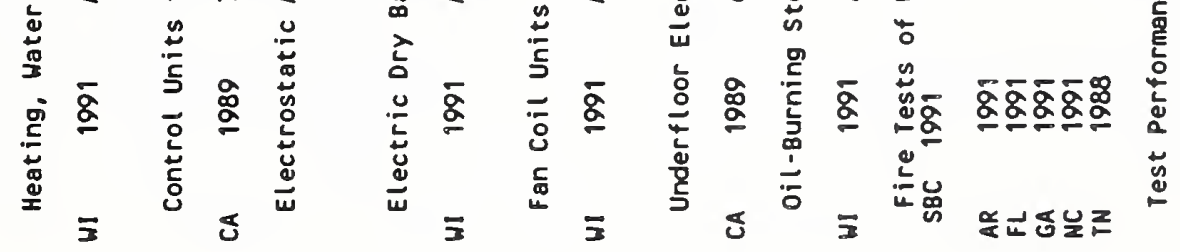




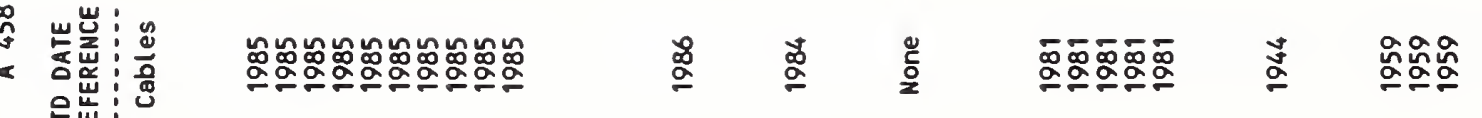

崖:

w:

z:

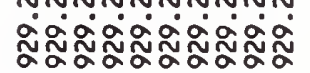

ㄷ:

总: :

芦总

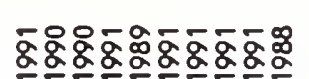

$\underset{\sim}{\sim}$

峁

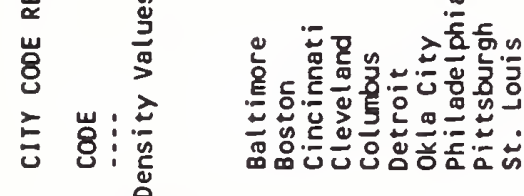

w ب. :

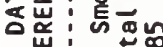

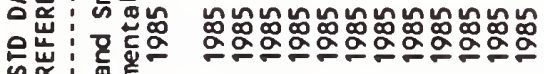

돈

놀: g둔

뚠: 은

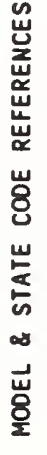

중:

: toñ ññññññññn

ㄴ.

出: 능

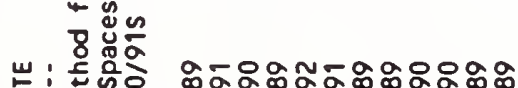

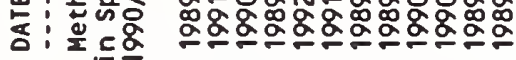

岩: 总总品

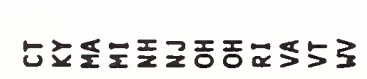

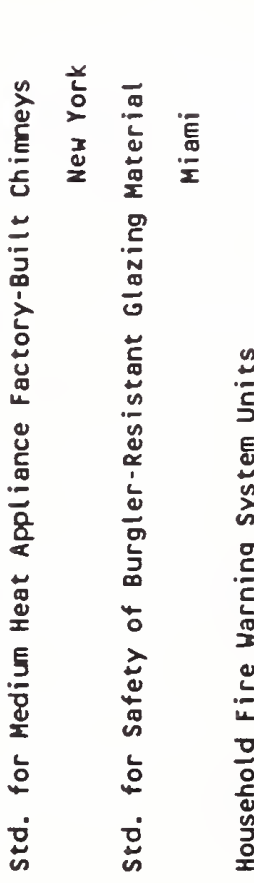

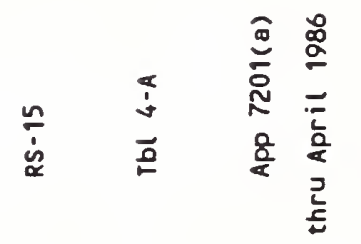

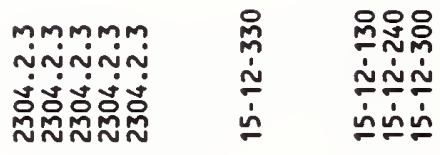

\& $\stackrel{8}{2} \stackrel{\circ}{\circ}$

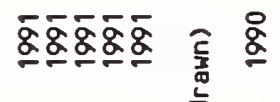

엉 $\frac{2}{5}$

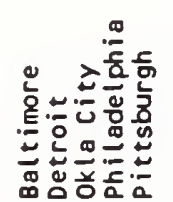

媳

岕

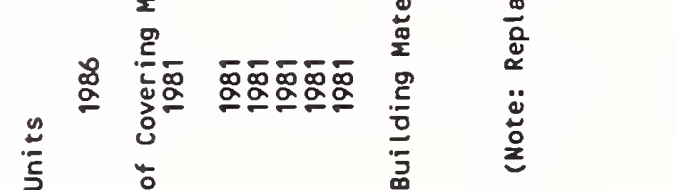

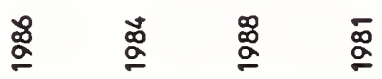

mmmmm?

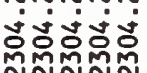

กั

索办

\%

๔

そ졸중

.

$\bar{g}$

웅

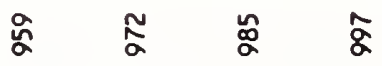

항ㅇㅇㅇ

$\frac{6}{2}$$$
\text { (n) }
$$

今

든돈

$\ddot{n}$

蒙

: å:2:

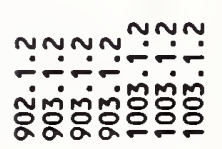

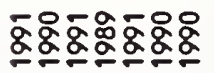

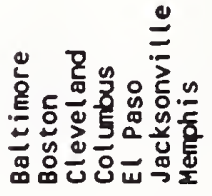

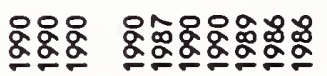

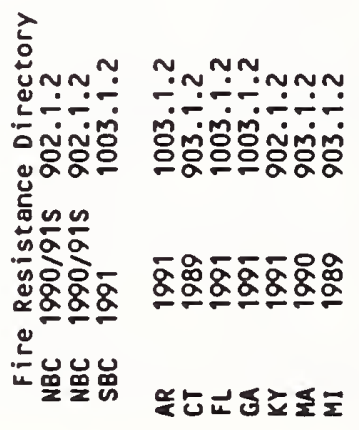

$\bar{g}$

$\begin{array}{lll}\bar{n} & \bar{N} \\ \dot{\Xi} & \tilde{N} & \text { N }\end{array}$ 


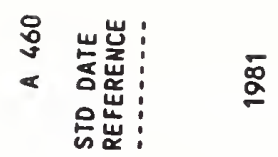

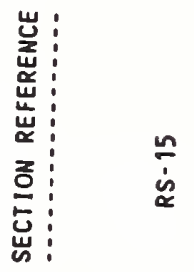

芯岕:

㟧

岂:

号岕: 它

क幽: 苛

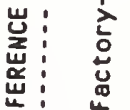

:

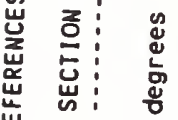

可品

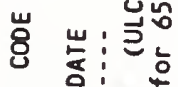

岩

\& 岁过的

㟧 :

울

峞

墒

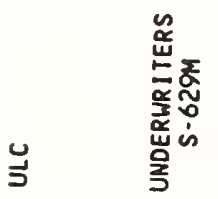




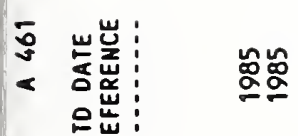

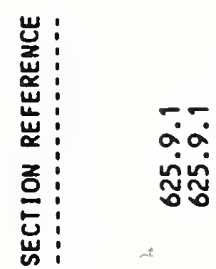

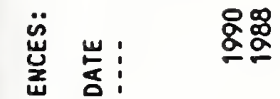

岕

岁

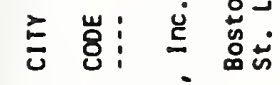

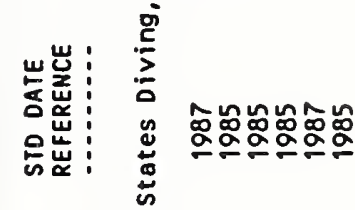

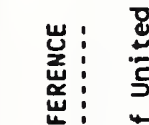

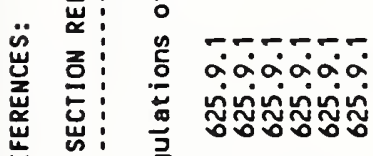

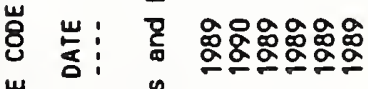

岸

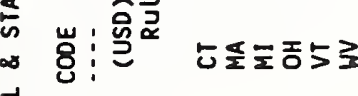

㟧 $\quad$ i⿱人

일

i

$\stackrel{\leftrightarrow}{2}$

농

$\stackrel{\text { on }}{=}$

율츄 


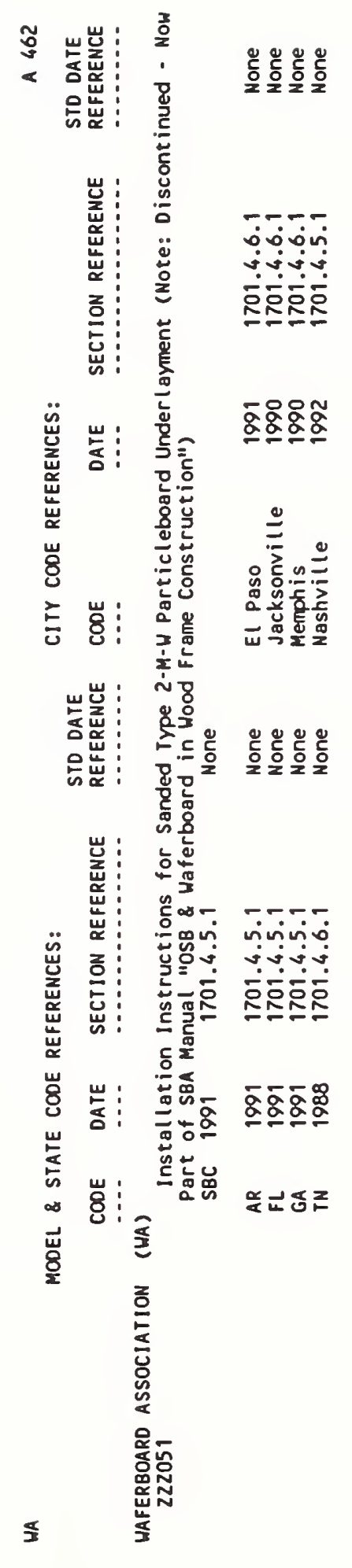


今ै

战

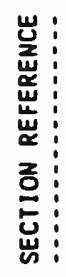

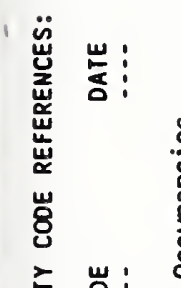

言岁:

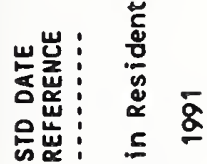

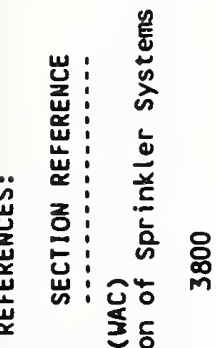

恧岩:

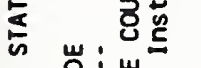

屯

일 을

害-

岕

증

編 
诂岕岂

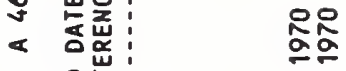

象岕:

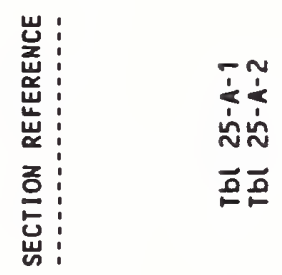

苞崖:

怘

岩

닌

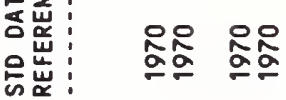

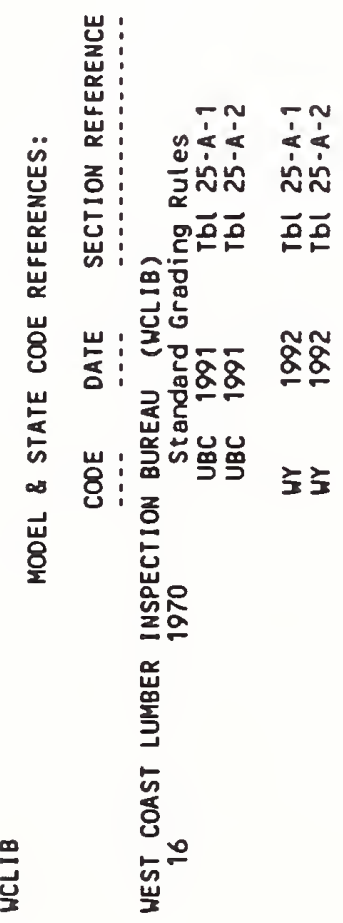




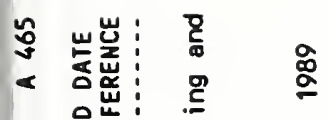

:

触:

岀: 西

ż̃ㅇ 合

总:

芯总

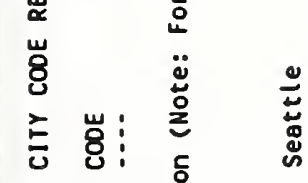

世岂: ‘

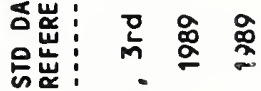

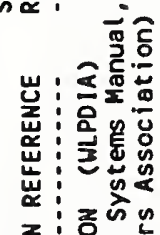

出

岕: 岩苔它

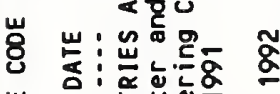

峞

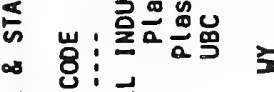

紊

옹ㅁㅀㅇ 

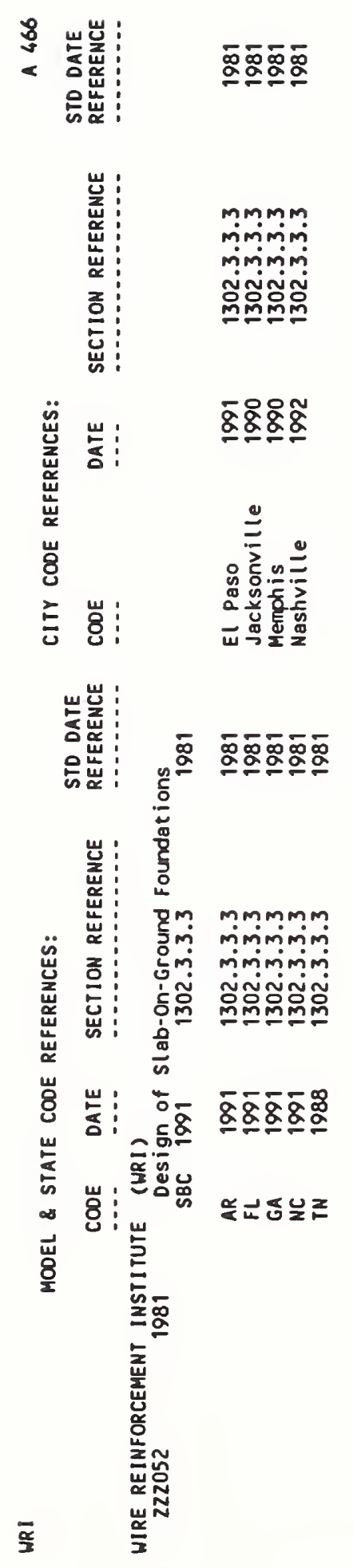


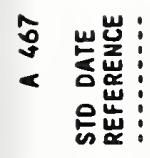

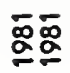
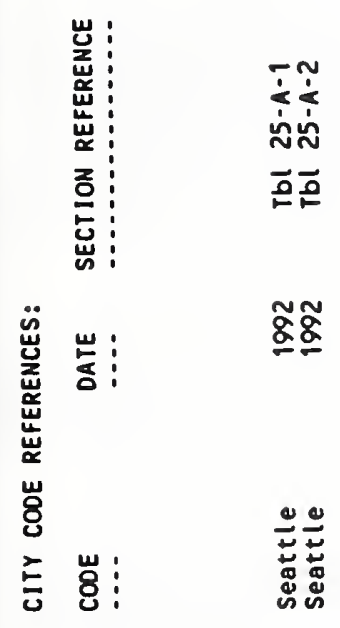

는

额:

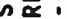
㟧
宸
ï 岕

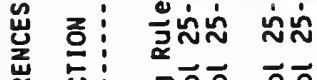
总

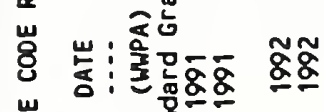
崖, 总密品

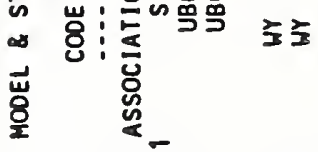
농

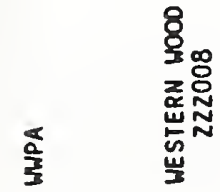





\section{NIST-114A}

(REV. 3-90)
U.S. DEPARTMENT OF COMMERCE NATIONAL INSTITUTE OF STANDARDS AND TECHNOLOGY

BIBLIOGRAPHIC DATA SHEET
1. PUBUCATION OR REPORT NUMBER NISTIR 4952

2 PERFORMING ORGANIZATION REPORT NUMBER SEPTEMBER 1992

3. PUBLCATION DATE

4. TITLE AND SUBTITLE

Standards Referenced in Model, State, and City Building Codes

5. AUTHOR(S)

John N. Brewer, Jr.

6. PERFORMINQ ORGANIZATION (IF JOINT OR OTHER THAN NIST, SEE INSTAUCTIONS)

U.S. DEPARTMENT OF COMMERCE

MATIONAL INSTITUTE OF STANDAROS AND TECHNOLOQY

CANTHERSBURO, MD 20890

7. CONTRACT/GRANT MUMBER

8. TYPE OF AEPORT AND PERIOO COVERED

9. SPONSORINQ ORGANIZATION MAME AND COMPLETE ADDRESS (STREET, CITY, STATE, ZIP)

11. ABSTRACT (A 200-WORD OA LESS FACTUAL SUMMAAY OF MOST SIGNIFICANT INFORMATION. IF DOCUMENT INCLUDES A SIQNIFICANT BIBUOQRAPHY OA UTERATURE SURVEY, MENTION IT HERE)

This publication provides a listing of the standards that are referenced in the building codes promulgated by: (1) the three model building code organizations; i.e., Building Officials and Code Administrators International, Inc. (BOCA), International Conference of Building Officials (ICBO), and the Southern Building Code Congress International, Inc. (SBCCI); (2) the 29 states that have mandatory state building codes; and (3) 35 selected U.S. cities. In addition to identifying each standard referenced in the above-named codes, this publication lists the current date of the standard, its title, the codes referencing it, the date of the code, the locations within the code where the standard is referenced, and the date of the standard referenced in the code. This publication is intended to provide assistance to the building community in updating, using and maintaining the standards referenced in building codes.

12. KEY WOADS (6 TO 12 ENTRIES; ALPHABETICAL ORDER; CAPITALZE ONLY PROPEA MAMES; AND SEPARATE KEY WORDS BY SEMICOLONS)

Building codes; building regulations; building regulatory systems; building technology; standards; standards organizations 

